
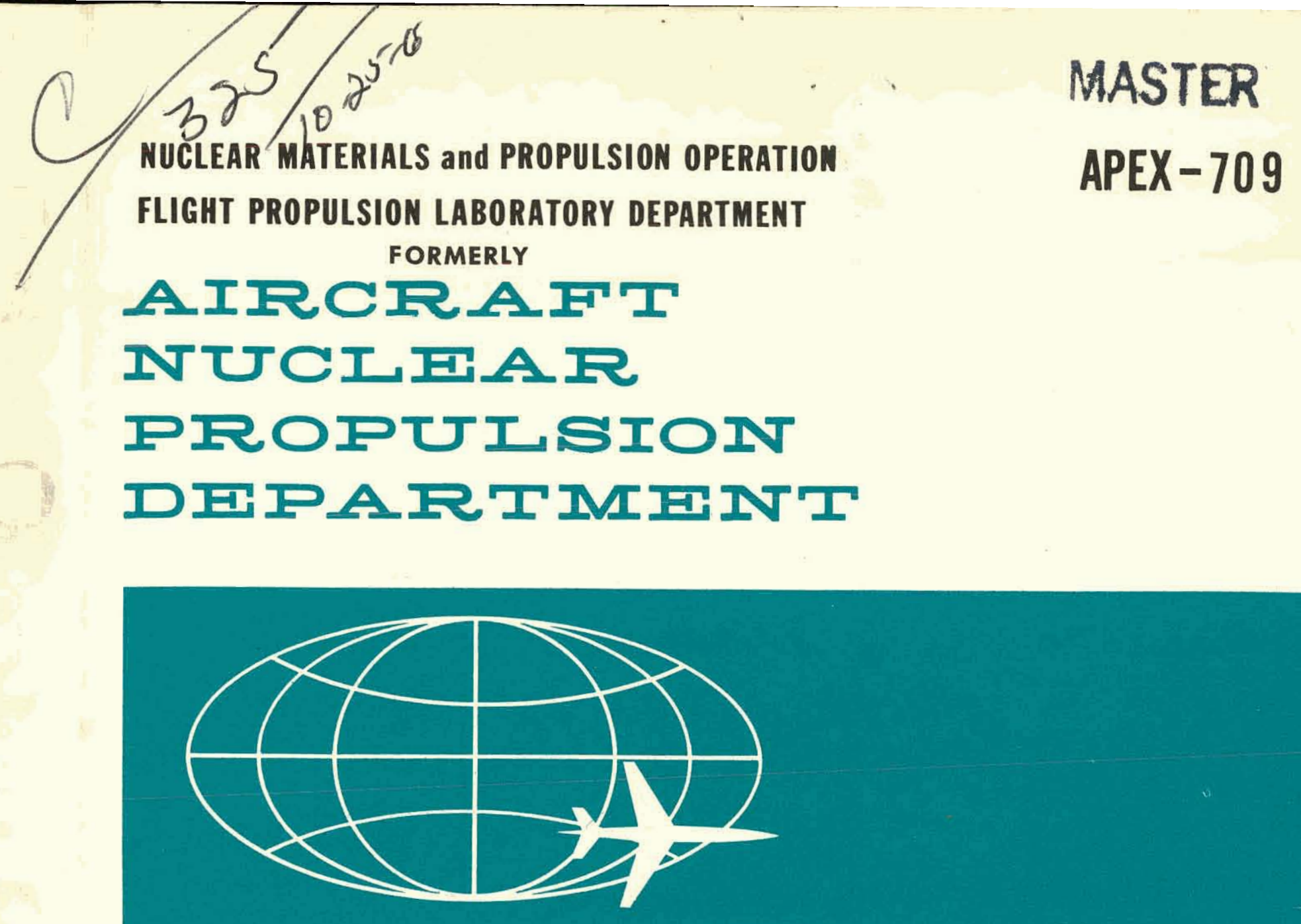

Twenty-Five Group Reactor Nuclear Data Tape

Neutron Cross Sections

J.W. Zwick

T. J. Kostigen

\title{
REPRODUCIBLE COPY
}

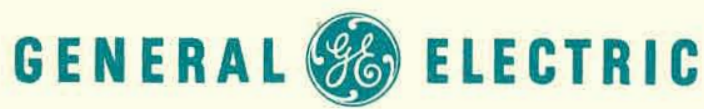




\section{DISCLAIMER}

This report was prepared as an account of work sponsored by an agency of the United States Government. Neither the United States Government nor any agency Thereof, nor any of their employees, makes any warranty, express or implied, or assumes any legal liability or responsibility for the accuracy, completeness, or usefulness of any information, apparatus, product, or process disclosed, or represents that its use would not infringe privately owned rights. Reference herein to any specific commercial product, process, or service by trade name, trademark, manufacturer, or otherwise does not necessarily constitute or imply its endorsement, recommendation, or favoring by the United States Government or any agency thereof. The views and opinions of authors expressed herein do not necessarily state or reflect those of the United States Government or any agency thereof. 


\section{DISCLAIMER}

Portions of this document may be illegible in electronic image products. Images are produced from the best available original document. 


\section{LEGAL NOTICE}

This report was prepared as an account of Government sponsored work. Neither the United States, nor the Commission, nor the Air Force, nor any person acting on behalf of the Commission or the Air Force:

A. Makes any warranty or representation, express or implied, with respect to the accuracy, completeness, or usefulness of the information contained in this report, or that the use of any information, apparatus, method, or prosess disclosed in this report may not infringe privately owned rights; or

B. Assumes any liabilities with respect to the use of, or for damages resulting from the use of any information, apparatus, method, or process disclosed in this report.

As used in the above "person acting on behalf of the Commission or Air Force" includes any employee or contractor of the Commission or $\mathrm{A}$ ir Force to the extent that such employee or contractor prepares, handles, or distributes, or provides access to, any information pursuant to his employment or contract with the Commission or Air Force.

Printed in USA. Price $\$ 4.50$. Available from the

Office of Technical Services

U.S. Department of Commerce Washington 25, D.C. 
APEX-709.

(Informal)

UC-34 (16th Ed.)

TID 4500

\section{TWENTY-FIVE GROUP REACTOR NUCLEAR DATA}

TAPE NEUTRON CROSS SECTIONS

J。W。Zwick

T. Jo Kostigen

June 13, 1961

UNITED STATES AIR FORCE

UNITED STATES ATOMIC ENERGY COMMISSION
CONTRACT NO. AF 33(600)-38062

CONTRACT NO. AT $(11-1)-17$

\section{GENERAL (G60) ELETRIC}

NUCLEAR MATERIALS AND PROPULSION OPERATION

(FORMERLY AIRCRAFT NUCLEAR PROPULSION DEPARTMENT)

CINCINNAT I 15, OHIO

PUBLISHED

July 1961 
9 Aberdeen Proving Ground

1 Aorojet-General Corporation

1 Aerojet-General Nucleonics

1 AFPR, Boeing, Beattle

2 AFPR, Lockheed, Marlette

2 Ar Force Speciel Weapons Center

2 ANP Project Office, Convalr, Fort Worth

1 Alco Prochuots, inc.

1 Alls-Chalmers Manufacturing Company

1 Alli-Chalmers Manufacturing Compeny, Washington

1 Allison Division- GMC

1 Argonne Cencer Research Hospital

10 Argonne National Laboratory

1 Armour Research Foundation

1 Army Balliatic Minsile Agency

4 Army Chemical Center

1 Army SIgnal Research and Development Laboratory

1 Atomic Bomb Casualty Commission

1 AEC Sclentific Representative, France

1 AEC sclentific Representative, Japan

3 Atomic Energy Commisolon, Washington

4 Atomlc Energy of Canada Limited

4 Atomlcs Internstional

2 Babcock and wilcox Company

2 Battelle Memorial Institute

4 Brookhsven National Laboratory

Brush Beryllium Company

1 Bureau of Shipe (Code 1600)

1 Bureau of Yarde and Docke

1 Carnegie Institute of Technology

1 Chlcago Operation Office

Chicago Patent Group

1 Columbia Univerelty (Havens)

1 Columbia Unversity (SOO-187)

1 Combustion Engineering, inc.

I Combustion Engineering, Inc. (NRD)

1 Convalr-General Dynamice Corporation, San Dlego

5 Defence Research Member

Defense Atomlc Support Agency, Washington

2 Department of the Army, G-2

3 duPont Company, Alken

1 duPont Company, Wilmington

1 Edgerton, Germeshausen and Grier, Inc., Las Vegas

I Frankford Arsans]

1 Franklin Institute of Pennoylvanla

General Atomic Divialon

2 General Electric Company (ANPD)

6 General Electrlo Company, Rdohlend

1 General Electric Company, St. Peteraburs

1 General Nuclear Engineering Corporation

1 Gibbe and Cox, Inc.

1 Glastone, Samuel

2 Coodyear Atomic Corporation

1 Grand Jinotion Operations Otfles

1 Hughes Alroraft Company

2 lowe state Univeralty

2 Jet Propulaion Laboratory

3 Knolls Atomio Powor Laboratory

2 Los Alemon solentufio Laboratory

Los Alamos Sclentific Laboratory (Beeonnke)

1 Lovelace Foundation

1 Maritime Adminitutration

1 Marquardt Corporation

1 Martin Company

1 Massichusetts Institute of Technology (Profio)

1 Massachusett Institute of Teohnology (Thompeon)

2 Midwestern Univereltied Research Aasoolation

1 Mound Laboratory

NASA Lewis Resenrch Center

2 National Bureau of Bandard.
National Bureau of Standande (Lbrary)

National Lead Company of Otso

Naval Modical Research Institute

Naval Research Laboratory

New Brunswick Aree Office

New York Operations Onfice

Now York Univeralty (Fioher)

New York Univernity (Rlchtmyer)

Northern Research and Engtneering Corporation

2 Nuclear Devalopment Corporntion of Americe

Nuclear-Materialo and Equipment Corporstion

Nuclear Metale, tho.

Oak Radge Institute of Nuclear studie

15 Offlice of Naval Researcb

Office of Naval Research (Code 422)

Office of Ormance Regearch

1 Office of the Chlef of Naval Operation:

1 Ondnence Materials Rescurch Office

1 Ordance Tank-Automotivo Command

Patent Branch, Washington

1 Pennsylvanie State University (Blenchand)

1 Phlllp Petroloum Company (NRT:

Power Reactor Development Compen

Pratt and Whitney Aircraft Divislon

1 Princeton Univerelty (White)

2 Public Health Service

1 Publlc Health Sorvice, Las Vegas

1 Public Health Service, Montgomery

Quartermaster Research and Development Command

Renseselner Polytechnic Institute

Bundia Corporation. Albuquerque

States Merine Lines, Inc.

1 Scevens Inntiturte of Technology

1 Etrategic Air Command

Sylvania Electric Products, Ino.

Teohnical Researoh Group

1 Tennossee Valley Authority

Texne Nuclear Corporation

The Surgeon Generol

Union Carbide Nuclear Company (ORCDP)

6 Union Carbide Nuclear Company (ORNL)

1 Union Carblde Nucleur Compuny (Pudroah Plant)

USAF Project RAND

U. 8. Ceological Survey, Denver

1 U. 8. Geologicel Burvey, Menlo Park

1 U. 8. Geological Burvey, Neval Gun Feotory

U. 8. Geologioal survey, Weshington

1 U. 8. Naval Ortnence Laboratory

1 U. 8. Naval Posteraduate 8chool

2 U. 8. Naval Rediological Defense Inboratory

1 U. 8. Petent Orfioe

1 University of Callfornla at Los Angeles

2 Undversity of Cellfornia, Berkeley

4 University of Callfornia, Livermore

1 Univereity of Cellfornia, San Franclaco

1 Unlversity of Puerto RIco

1 University of Roohenter

2 Univeralty of Rocheater (Marshaks

1 University of Waahington (Geballe)

1 Unlveruity of Washington (Robdo)

1 Walter Roed Army Medical Center

1 Watortown Arsemal

1 Westinghouse Bettla Atomlc Power Laboratory

2 Westinghouse Electric Corporation

6 Wright Air Development Divislon

1 Yale Univerolty (Brelt)

1 Yale University (Schults)

1 Yankee Atomic Electrle Company

325 Office of Technical Information Extention

75 Office of Technical Services, Washington 
J. R. Beeler

V. P. Calkins

C. L. Chase

E. B. Delson

M. B. Goldstein

G. G. Leeth

L. Lomen

W. H. Long

J. E. MacDonald

H. F. Matthiesen

F. W. Mezger

J. W. Morfitt - ITS

R. E. Motsinger
W. E. Niemuth

R. E. $\operatorname{Re} 1 d$

C. S. Robertson

A. J. Rothstein

J. D. Selby

R. M. Schuh

J. D. Simpson

G. Thornton

J. W. Zwick (2)

Library - ITS

Reports \& Publications

Reports Library (12)

(54326)

Atomlc Energy Commission

U.S. Air Force, Wright Air Development Division (WCLPU)

Aerojet - General Nucleonics, S.T. Friedman

APED, J. We1 1, R. M, Cohen

Atomics International, Canoga Park, California - E.R. Specht

Babcock \& Wilcox Co. - W. L. Orr

General Atomics, San Diego, Callfornia - J. M. Krase

HAPO - B, H, Duane J. E. Faulkner, E. G. Dawson

Internuclear - E. E. Mason

KAPL - D. S. Selengut

LRL, L1vermore - R. N. Stuart

Martin Co., Nuclear Division - J. E. Long, F. D. Wenstrup

ORNL - A. D. Callihan

TEMPO - P.G.FIscher

Westinghouse, APD - E. I, Langford $P$. $G$. Lacey

\section{ABSTRACT}

A compilation is presented, in the twenty-five group Reactor Nuclear Data Tape (RNDT) format, of neutron cross sections for elements of major interest for GE-ANPD reactor analysis.

The tabulated data is a reproduction of neutron cross section information contained on the Reactor Nuclear Data Tape, which has been recently prepared. A brief outline of methods used in processing of the cross sections is also included.

\section{ACKNOWLEDGMENTS}

We wish to thank Dr. P. G. Fischer and W. B. Henderson for many discussions and suggestions. Thanks are due to D. E. Wetzel for computing the cross sections of hydrided zirconium and to $F$. D. Wenstrup for assistance in placing the cross sections on the Reactor Nuclear Data Tape. 
Page

1.0 Introduction.

2.0 Cross Section Format

3.0 Sources of Cross Section Data $\quad$ - $\quad$ • $\quad$ - . . 7

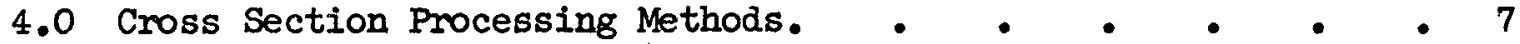

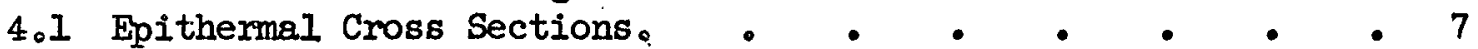

4.2 Thermally Averaged Cross Sections - . . . . 08

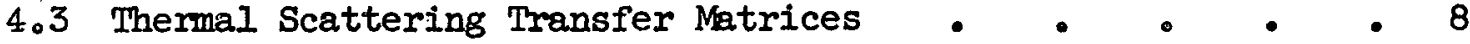

4.4 High Energy Scattering Transfer Matrices. - . . . 8

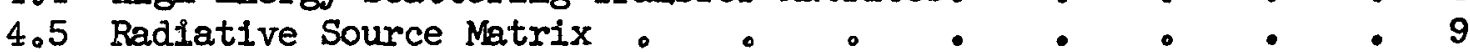

4.6 Resonance Data . . . . . . . . . 10

5.0 References ......... .11

6.0 Reactor Nuclear Data Tape Neutron Cross Section Data. . . 13

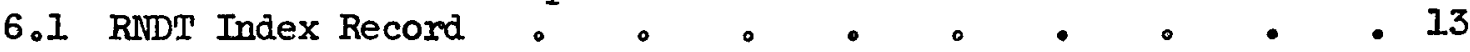

6.2 RNDT Group Structure Boundary Energies, Corresponding

Iethargies and I/v Factors $0.0^{\circ} \cdot{ }^{\circ} 14$

6.3 RNDT Energy Levels and Corresponding Lethargles . . . 15

6.4 Uranium-235 Fission Spectral Data • • • • . . 16

6.5 Microscopic Cross Section Data. . . . . . . 24

Uranium-235

Uranium-238 39

Plutonium-239 : 53

Nickel 63

Iron 0.0 .0 .074

Chromium 0.0 .0 .0 .086

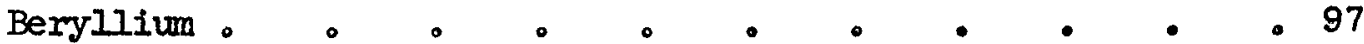

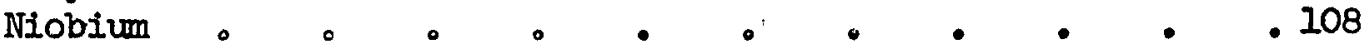

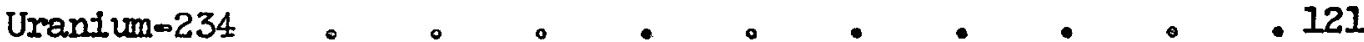

Uranium-236 $\quad \circ \quad \cdot \quad \cdot \quad \cdot \quad \cdot \quad \cdot \quad \cdot \quad \cdot \quad \cdot \quad \cdot 131$

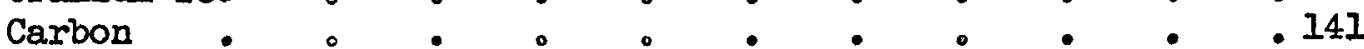

Hydrogen (gas) :

Boron-10 $: 0.0166$

Dxygen 0.177

Aluminum : 0185

Yttrium 0.196

Zirconium . 0.0208

Manganese . 0.0 .0 .0223

Cobalt

Ifthium-6. 246

Iithium 0.0257

Boron. 0.0268

Thorium-232

Cedolinium

Europium $0.0^{\circ} \cdot 0^{\circ} \cdot{ }^{\circ}$

Samarium-149 311

Rhodium

Platinum

Xenon-135

Tantalum

6.6 Macroscopic Cross Section Data.

Hydrided Zirconium 


\subsection{INTRODUCTION}

Neutron cross sections form the basic physical data on which all nuclear design work is based. It is therefore necessary to constantly revise and improve basic neutron cross section data on all materials of interest in reactor technology. Accurate neutron cross sections coupled with improvements in computational techniques and the availability of larger, faster and more efficient computers offer the possibility of more exact theoretical predictions of reactor parameters and their behavior.

As a part of the effort to improve the reliability of theoretical predictions of reactor parameters, a program was initiated to improve the physical model to be used in reactor analysis calculations. The revised GE $-A N P D$ multigroup energy lattice, the corresponding Reactor Nuclear Data Tape (RNDT) 4,18 system and the diffusion theory Program ODD $^{27}$ which uses these data were designed to provide the basis for an improved nuclear analysis procedure.

In conjunction with this effort basic neutron cross section data on elements of major interest in reactor nuclear calculations have been processed into the Reactor Nuclear Data Tape format. Cross sections, in the RNDT structure, of the following materials have been placed on magnetic tapes for use in multigroup or multilevel diffusion theory nuclear reactor calculations:

\section{Microscopic Cross Sections}

Uranium-235

Uranium-238

Plutonium-239

Nickel

Iron

Chromium

Beryllium

Niobium

Uranium $=234$

Uranium-236
Carbon

Hydrogen (gas)

Boron-10

Oxygen

Aluminum

Yttrium

Zirconium

Manganese

Cobalt

Iithium -6
Iithium

Boron

Thorium-232

Gadolinium

Europium

Samarium-149

Rhodium

Platinum

Xenon-135

Tantalum

\section{Macroscoplc Cross Sections}

Hydrided zirconium

Other Data

U-235 fission spectral data

Group boundary energies and corresponding level energies, $1 / v$ factors

Nuclear data contained on the RNDT tape are reproduced in this report. Microscopic cross sections are given in barms; macroscopic cross sections in inverse centimeters. 


\subsection{CROSS SECTION FORMAT}

The RNDT format retains all of the data format of the earlier NDT, $i_{0} e_{0}$ the format used in $A P E X-515^{8}$, so that the tape could be used by other ANPD nuclear analysis programs; this results in actually the same data being presented in several ways. Thus, for high energies where inelastic scattering occurs, a scatter transfer matrix is given, and the same data are represented in terms of an average logarithmic energy decrement, $\xi$, since this latter is the format used by Programs $C_{2}{ }^{16}$ and $\mathrm{G}_{2}{ }^{1}$ for example. Likewise, at low energies, conventional ANPD machine programs allow representation only of energy loss reactions (slowing down) so that the thermalization region is represented by an overlaping of a Maxwellian and a slowing down flux with a temperature dependent cut-off. Such a representation requires complete Maxwellian averaged cross sections $(0-10 \mathrm{ev})$ for various temperatures. Program $\mathrm{ODD}^{17}$, however, provides for both energy gain and loss reactions in the thermalization region with temperature independent group boundaries. The lowest group $(0-0.0253 \mathrm{ev})$ only is now Maxwell averaged and a complete five by five scatter transfer matrix must be given for each temperature. Thus, Maxwellian averaged cross sections are separately given for the energy ranges $0-10 \mathrm{ev}$ and $0.0 .0253 \mathrm{ev}$ for each of ten temperatures.

Cross section detall is based upon twenty-four epithermal groups (levels) from $12.84 \mathrm{Mev}$ to $0.0253 \mathrm{ev}$, and one thermal group corresponding to one of the ten temperatures: $68,500,1000,1500,2000,2500$, $3000,3500,4000$ and 4500 degrees Fahrenheit. This group structure provides a more detailed energy lattice coverage of the resonance region ( 1 to $100 \mathrm{ev}$ ) and near source region ( 1 to $100 \mathrm{kev}$ ) than the nineteenlevel Nuclear Data Tape $(\mathrm{NDT})^{8,14}$ energy lattice, and it covers more adequately the basic needs in epithermal, resonance and fast reactor systems. Furthermore, the RNDT energy lattice structure is designed to produce group-averaged cross sections consistent for use with both multigroup and multilevel calculations. This is achieved by flat-flux averaging (in lethargy) the cross sections over a band extending from the midpoint of the lethargy interval below to the midpoint of the Interval above each level, and ascribing the averaged value to the level in question, ioe.

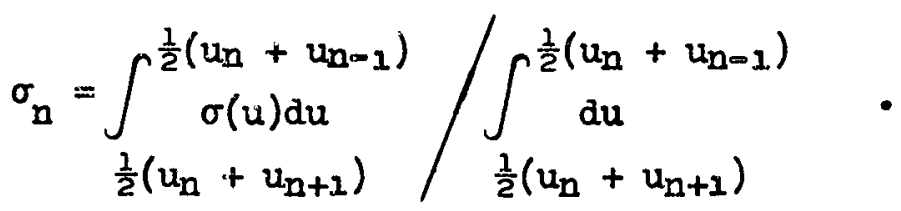

Iethargy levels are defined by

$$
u_{n} \equiv \ln \left(10^{7} / E_{n}\right), 0<n \leq 24,
$$

where $E_{\mathrm{n}}$ is the neutron energy, in electron volts, corresponding to the $n$-th level。

The RNDT crass section compilation consists of: 
(1) Partial cross sections at each of the twenty-four epithermal groups (levels) and thermal cross sections averaged over a Maxwellian distribution for ten temperatures from $680 \mathrm{~F}$ to $45000 \mathrm{~F}$.

$\begin{array}{ll}\sigma_{s} & \text { scattering cross section } \\ \xi \sigma_{s} & \text { slowing-down cross section } \\ \sigma_{t r} & \text { transport cross section } \\ \sigma_{a} & \text { absorption cross section } \\ \left(\xi \sigma_{s}\right)_{1} & \text { firstmoment slowing mown cross section } \\ v \sigma_{f} & \text { fissionmproduction cross section }\end{array}$

(2) Near-thermal zeroth-moment scattering transfer matrices for each of the ten temperatures from $68^{\circ} \mathrm{F}$ to $4500^{\circ} \mathrm{F}$, coupling the bottom five energy groups $(0-1 \mathrm{ev})$.

(3) High energy scattering matrix providing for down-scattering from each energy level to all. lower levels。

(4) Radiative source matrix, $\sigma\left(E_{n} / E_{\gamma}\right)$, which provides data on gamma ray production by neutron capture, neutron inelastic scattering, and by neutron induced fissioning.

(5) Resonance data, if applicable, for the energy region $1 \mathrm{ev}<\mathrm{E}<$ $600 \mathrm{ev}$.

(6) The U-235 fission spectral data:

U-235 prompt fission spectrum,

U-235 total fission spectrum,

U-235 fission spectra for each of the six delay groups.

\subsection{SOURCES OF CROSS SECTION DATA}

The basic cross section data were taken partially from CaFINE manylevel nuclear cross section data tape 2,15 and partially from more recent published information. Available data on high energy phenomena of anisotropic scattering and inelastic scattering were incorporated in the compilation.

The data used in preparing the CoFINE nuclear data tape and those on elements for which cross section information was obtained directly from other published sources are described in detail elsewhere $6,15,20,21,22$ and will not be discussed here.

\subsection{CROSS SECTION PROCESSING METHODS}

\subsection{Epithermal Cross Sections}

Energy-band averaged partial cross sections, $\sigma_{s}, \xi \sigma_{s}, \sigma_{t r}, \sigma_{a}$ and $v \sigma_{f}$, at each of the twenty -four epithermal groups (levels) were computed, for a number of elements with Program C-FINE (ANP Program No. 180) 12 from C-FINE manyolevel nuclear cross section data tape. For elements, on which basic cross section information was obtained directly from 
sources other than the C-FINE data tape, group-averaged cross sections were calculated, in the resonance region, with ANP Program No. $181^{9}$. ANP Program No. $42 I^{3}$ was employed to calculate, at energies outside the resonance region, groupmaveraged values of the absorption cross section, and AIV Program No. $335^{5,10}$ was used to compute energy-band values of the scattering cross section, slowing-down cross section and transport cross section.

\subsection{Thermally Averaged Cross Sections}

Thermal cross sections averaged over a Maxwellian distribution in the energy range $10^{-4} \mathrm{ev}$ to $10 \mathrm{ev}$ for temperatures of $65,500,1000$, 1500, 2000, 2500 and 3000 degrees Fahrenheit were taken, for a number of elements, from APEX $5.15^{8}$. For these elements, the thermal cross sections for temperatures of 3500,4000 and 4500 degrees Fahrenheit were set equal to the value at $3000 \mathrm{~F}^{\circ}$. When this is the case, an appropriate footnote will so indicate.

For other elements, ANP Program No. $421^{3}$ was employed to compute thermal cross sections averaged over a Maxwellian distribution for all ten temperatures from $68^{\circ} \mathrm{F}$ to $4500^{\circ} \mathrm{F}$ 。 If an element had resonances close to the thermal region, ANP Program No. $181^{9}$ was used instead.

The thermal cross sections preceding each of the thermal scattering transfer matrices resulted from averaging over a Maxwellian distribution in the energy range $10^{-4} \mathrm{ev}$ to $0.0253 \mathrm{ev}$.

\subsection{Thermal Scattering Transfex Matrices}

Nearothermal zeroth-moment scattexing transfers in the energy groups from $10^{-4} \mathrm{ev}$ to about $\mathrm{l} \mathrm{ev}$, for each of the ten temperatures from $68^{\circ} \mathrm{F}$ to $4500^{\circ} \mathrm{F}$, were generated from a monoatomic gas model (ANP Program No. 396 $)^{19}$ except for hydrided zirconium where the bound oscillator model of McReynolds, et al.e, was used ${ }^{13}$. These thermal transfer matrices provide for both up-and down-scattering, and can be used in calculations of neutron thermalization.

The group to group transfers were obtained by averaging over a flatin-lethargy flux for the four highest energy groups and over a Maxwellian characterized by the material temperature in the lowest energy group.

\subsection{High Energy Scattering Transfer Matrices}

The high energy scattering transfer matrices provide for downscattering from each energy level to lower levels. They were computed from experimental information on inelastic scattering and elastic scattering, using the statistical model of nuclear level transitions (ANP

Program No. 335 $)^{5}, 10$, except in the case of oxygen, for which sufficient experimental data are available on the cross sections for inducing individual transitions.

The values used for the parameter " $a$ " which enters into the expression for nuclear temperature required in the statistical model calculations, are given in references 15 and 20 . 
Exit energy distributions for neutrons scattered by oxygen through three known states of the 0-16 nucleus with excitation energies of 6.14 , 6.9 and $7.1 \mathrm{Mev}$ have been computed in the following mannert:

(1) The angular distribution of inelastically scattered neutrons is assumed to be isotropic in the center-of-mass system. No measured data is available, but this assumption is usually valid for incident energies up to several times the excitation energy. Designating the excitation energy by $\epsilon_{1}$ for state 1 , then

$$
\sigma_{\text {in }}^{i}\left(E \rightarrow E^{\prime}\right)=\frac{(A+I)^{2}}{4 A}\left[1-\frac{A+1}{A} \frac{\epsilon_{1}}{E}\right]^{-\frac{1}{2}} \frac{d E^{8}}{E} \sigma_{\text {in }}^{i}(E),
$$

where $A$ is the scatterer to neutron mass ratio.

(2) Partial cross sections, $\sigma_{\text {In }}^{i}(E)$, for excitation of state 1 , were obtained from BNL-325, Supplement 1, Second Edition ${ }^{11}$, as follows:

(a) The cross section for excitation of the $6.14 \mathrm{Mev}$ state is given from threshold $(6,52 \mathrm{Mev})$ to $10 \mathrm{Mev}$, and the cross section was assumed constant from $10 \mathrm{Mev}$ to $12.84 \mathrm{Mev}$, the upper limit of group 1 .

(b) The combined $6.9,7.1 \mathrm{Mev}$ state curve given from 7.7 to 9.4 Mev was divided using the relative excitation probabilities of 14 and 4 respectively measured by Hall and Bonner ${ }^{7}$ at 8.5 Mev. The cross sections were extrapolated to zero at the corresponding thresholds and assumed constant from 9.4 to $12.84 \mathrm{Mev}$.

(3) First Legendre moment scatter transfer cross sections were computed using the relation

$$
\mu=\frac{A+I}{2} \sqrt{\frac{E^{\prime}}{E}}-\frac{A^{2}}{2(A+I)}\left(I-\frac{A+1}{A} \frac{\epsilon_{1}}{E}-\frac{1}{A^{2}}\right) \sqrt{\frac{E}{E^{\prime}}},
$$

where $\mu$ is the cosine of the laboratory scattering angle.

(4) Multigroup transfer cross sections were computed by integrating over the exit energy group and averaging over the incident group with a l/E flux.

\subsection{Radiative Source Matrices}

Rediative source matrices, $\sigma\left(E_{n} / E_{7}\right)$, provide cross section data on the production of gamma rays by radiative capture of a neutron, by inelastic scattering of a neutron, and by neutron induced fissioning. These cross sections for the production of gamma rays were processed by Mary S. Ferry ${ }^{6}$, and are tabulated, for individual elements, as a function of photon energy bands and neutron energy groups.

\footnotetext{
This information was supplied by Dr。 $P_{\circ} G_{0}$ Fischer in a private comunication.
} 
A discussion of the methods used in generating the radiative source matrices and a summary of data are given in reference 6.

\subsection{Resonance Data}

In the energy range $1 \mathrm{ev}<\mathrm{E}<600 \mathrm{ev}$, the Reactor Nuclear Data Tape contains resonance data required in calculating energy self-shlelding effects and resulting reduction in resonance absorption:

$\mathrm{E}_{\mathrm{o}}$ resonance energy (ev)

$I_{0}$ resonance integral (barns)

$\sigma_{0}$ peak cross section (barns)

$R$ Doppler temperature coefficient $\left({ }^{\circ} \mathrm{K}^{-1}\right)$

$\eta \quad$ number of neutrons produced per neutron absorbed.

Of these $E_{0}$ was supplied as imput to Program RNDP ${ }^{18}$, the program which was used to compile the tape from imput supplied nuclear data. Resonance data $E_{0}, \Gamma_{n}, \Gamma_{Y}, \Gamma_{F}$ and $g$ supplied as input were used by this program in computing the four quantities $I_{0}, \sigma_{0}, R$ and $\eta$ for each resonance:

$$
\begin{aligned}
& I_{0}=\frac{2 \pi^{2} Q K_{O}^{2} g}{E_{O} \Gamma}\left(\Gamma_{\gamma}+\Gamma_{F}\right) \Gamma_{n}, \\
& \sigma_{0}=\frac{2 E_{O} I_{0}}{\pi \Gamma}, \\
& R=\frac{4 A N^{Q} E_{O} k}{A \Gamma^{2}}, \\
& \eta=\frac{v \Gamma_{F}}{\Gamma_{\gamma}+\Gamma_{F}},
\end{aligned}
$$

where

$\Gamma_{n}$ is the neutron width $(\mathrm{ev})$ at the resonance energy $\mathrm{E}_{\mathrm{O}}$

$\Gamma_{\gamma}$ is the radiation width (ev)

$\Gamma_{F}$ is the fission width (ev)

$\Gamma=\Gamma_{\mathrm{N}}+\Gamma_{\gamma}+\Gamma_{\mathrm{F}}$ is the total width (ev)

$g$ is the statistical weight factor
$Q=\frac{A+A_{N}}{A}$ is the factor relating energy in the laboratory coordinate system to energy in the center-of-mass system

$A$ is the atomic weight (In $A_{0} M_{0} U_{0}$ ) of the scattering nucleus

$A_{N}$ is the neutron mass (in A.M.U. )

$\lambda_{0}=\frac{4.5517359 \times 10^{-10}}{\sqrt{E_{0}}}$ is neutron wave length $/ 2 \pi$ 
$v$ is the number of neutrons emitted per fission.

$k$ is Boltzmann's constant.

The resonance data stored on RNDT is used by Program ODD 27 in calculating self-shlelded resonance absorption and Doppler broadening effects.

\subsection{REFERENCES}

1. Campbell, Do Jo, "Program G-2", XDC 58-4-63, April 4, 1958.

2. Cooper, Jo R. and Henderson, Wo Bo, "Nuclear Data Tape Program with Fine Energy Detail", XDC 60-8-69, May 13, 1960。

3. Cooper, Jo Ro and Henderson, W० Bo, "A Program for the Calculation of Average Thermal Cross Sections on the IBM-704 (ANP Program 421)", XDC 60.5-51, March 15, 1960。

4. "Englineering Progress Report No. 36", APEX-36, June 1960, p. 150.

5. "Englneering Progress Report No. 33", APEX-33, September 1959, p.99。

6. Ferry, M。 So, "Production Cross Sections for Gama Rays (Program GAMMA-P)", XDC 60-11-138, November 30, 1960。

7. Hall, H。 E。 and Bonner, ToWo, Muclear Physics 14, 295 (1959)。

8. Henderson, W。 B。 and Stanley, M。 Jo, "Cross Sections for Reactor Analysis", APEX-515, May 1957.

9. Henderson, W. B. and Sullivan, R。 E., "Breit-Wigner Program (ANP Program No. 181)", APEX-640。

10. Henderson, Wo Bo, "Distribution of Scattered Neutrons (AIIP Program No. 335)", APEX-64I。

11. Hughes, D。 Jo, Magurno, $B_{0} A_{0}$ and Brussel, Mo Ko, "Neutron Cross Sections", Supplement No. I to BNL-325, Second Edition, January 1, 1960 。

12. Klingenberg, E。 W。 and Henderson, W。 Bo, "Program C-FINE", XDC 59-12-230, December 22, 1959。

13. McReynolds, A。 Wo, Nelkin, Mo So, Rosenbluth, Mo N. and Whittemore, Wo Lo, "Neutron Thermalization by Chemically Bound Hydrogen and Carbon", Proceedings of the Second Geneva Conference 16, 297 (1958)。

14. Paine, Ko, "Nuclear Data Tape Program (704 Program NDTP)", XDC 58-2-204, December 20, 1957 .

15. Sullivan, R. Io, Cooper, Jo Ro and Henderson, Wo Bo, "Reactor Neutron Cross Sections in Fine Energy Deta11", APEX-704. 
16. Vollenweider, D. B., "Bare Reactor Program (704 Program C-2)", XDC 57-10-10, July 30, 1957.

17. Wenstrup, F。D. et alo, "Program ODD - A One-Dimensional Multigroup Diffusion Theory Code for the IBM-7090", APEX-637.

18. Wenstrup, F。 D. et alo, "IBM-7090 Programs to Compile and Modify a Nuclear Data Tape", APEX-708.

19. Wigner, E. Po and Wilkins, J. E., "Effects of Temperature of the Moderator on the Velocity Distribution of Neutrons with Numerical Calculations of $\mathrm{H}$ as Moderator", AECD-2275 (1944).

20. Zwick, J. Wo, "Neutron Cross Sections for Rhodium and Tantalum", XDC 61-4-35, April 4, 196l。

21. Zwick, J. W., "Neutron Cross Sections for Samarium-149 and Natural Samarium", XDC 61-1-113, November 15, 1960.

22. Zwick, J. Wo, "Revised Cross Sections for Chromium, Iron and Nickel", XDC 60-1-139, January 1960。 
6.0 REACTOR NUCLEAR DATA TAPE NEUTRON CROSS SECTION TABUIATION

6.1 Reactor Nuclear Data Tape Index Record

DATE 02110161

I NDEE

DAJA

BLOCK NO.

\section{LEVELS}

\begin{tabular}{|c|c|c|c|}
\hline BLOCK NO. & CODE NO. & DESIGNATION & RECORD NO. \\
\hline $\begin{array}{r}1 \\
2\end{array}$ & $\begin{array}{r}500.0000 \\
92.2350\end{array}$ & $\begin{array}{l}35 \text { SPECTRUM } \\
\text { URANIUM } 235\end{array}$ & $\begin{array}{r}3 \\
4 \\
\end{array}$ \\
\hline 3 & 92.2380 & URANIUM 238 & 8 \\
\hline 4 & 94.2390 & PLUION 239 & 12 \\
\hline 5 & 28.5900 & NICKEL & 15 \\
\hline 6 & 26.5600 & IRON & 18 \\
\hline 7 & 24.5200 & CHROMIUM & 21 \\
\hline & $-4 \frac{4}{41.93000}$ & $\begin{array}{l}\text { BERYLLIUM } \\
\text { NIOBIUM }\end{array}$ & $-\frac{24}{27}$ \\
\hline 10 & 500.2350 & 35_PRQMPI & 31 \\
\hline 11 & 501.2350 & 35 CELAY 1 & 32 \\
\hline 12 & 502.2350 & 35 DELLAY 2 & 33 \\
\hline 13 & 503.2350 & 35 DELAY 3 & 34 \\
\hline 14 & 504.2350 & 35 DELLAY_4 & $3 \underline{2}$ \\
\hline 15 & 505.2350 & 35 DELAY 5 & 36 \\
\hline 16 & 506.2350 & 35 DELAY 6 & 37 \\
\hline 17 & 92.2340 & URANIUM 234 & 38 \\
\hline 18 & 92.2360 & URANIUM 236 & 41 \\
\hline 19 & $6.120 \mathrm{c}$ & CARBON & 44 \\
\hline 20 & 1.1000 & H_EREE & 47 \\
\hline 27 & 5.1000 & BORON 10 & 51 \\
\hline 22 & $8=1600$ & OXYGEN & 54 \\
\hline 23 & 13.2700 & ALUMINUM & $5 \overline{7}$ \\
\hline 24 & 39.8900 & YIIRIUM & 60 \\
\hline 25 & 40.9100 & ZIRCONIUM & 63 \\
\hline 26 & 140.1910 & $4-H-1-2 R$ & 67 \\
\hline 27 & 25.5500 & MANGANESE & 72 \\
\hline$-\frac{28}{2}-$ & $-27=\frac{5900}{3.6000}$ & COBALL & $-8 \frac{10}{80}-$ \\
\hline 30 & 3.7000 & LI THIUM & 83 \\
\hline $3 i$ & 5.1100 & BORON & $\overline{8} \overline{6}$ \\
\hline 32 & 90.2320 & THORIUM 232 & 88 \\
\hline $3 \frac{3}{3}$ & 64.1570 & GADOLINIUM & 91 \\
\hline 34 & $63=1520$ & EUROPIUM & 95 \\
\hline$-\overline{3} 5$ & 62.1490 & SA $\bar{M} \bar{A} \bar{I} \cup \bar{M} 14 \overline{9}$ & ${ }^{-} \overline{9} \overline{9}^{-}$ \\
\hline 36 & 45.1030 & RHODIUM & 103 \\
\hline & 78.1950 & PLATINUM & $10 \overline{0}$ \\
\hline 38 & 54.1350 & XENON-135 & 110 \\
\hline $\begin{array}{l}39 \\
40 \\
\end{array}$ & $\begin{array}{l}73.1810 \\
0 .\end{array}$ & $\begin{array}{l}\text { TANTALUM } \\
000000000000\end{array}$ & $\begin{array}{l}111 \\
114\end{array}$ \\
\hline
\end{tabular}


6.2 Reactor Nuclear Data Tape Group Structure Boundary Energies, Corresponding Lethargies and 1/v Factors LOWER UPPER IV FACTOR GROUP _. _ENERGY__ (EY_) LEIHARGY (2200

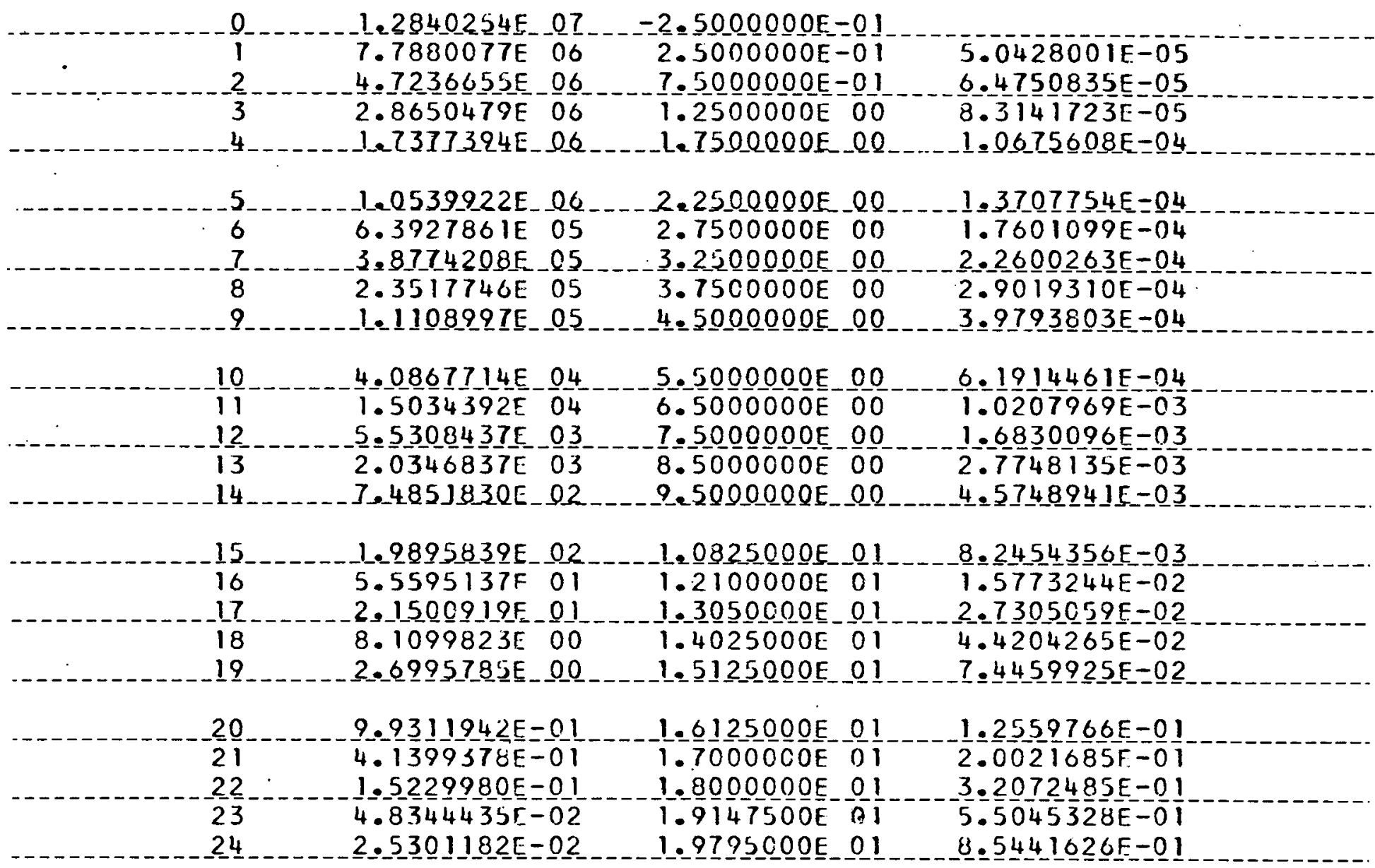


$\because 3$ Reactor Nuclear Data Tape Energy Levels and Corresponding Lethargies

1.EKEL

ENERGY $(E Y)$

LE IHARE $\underline{Y}$

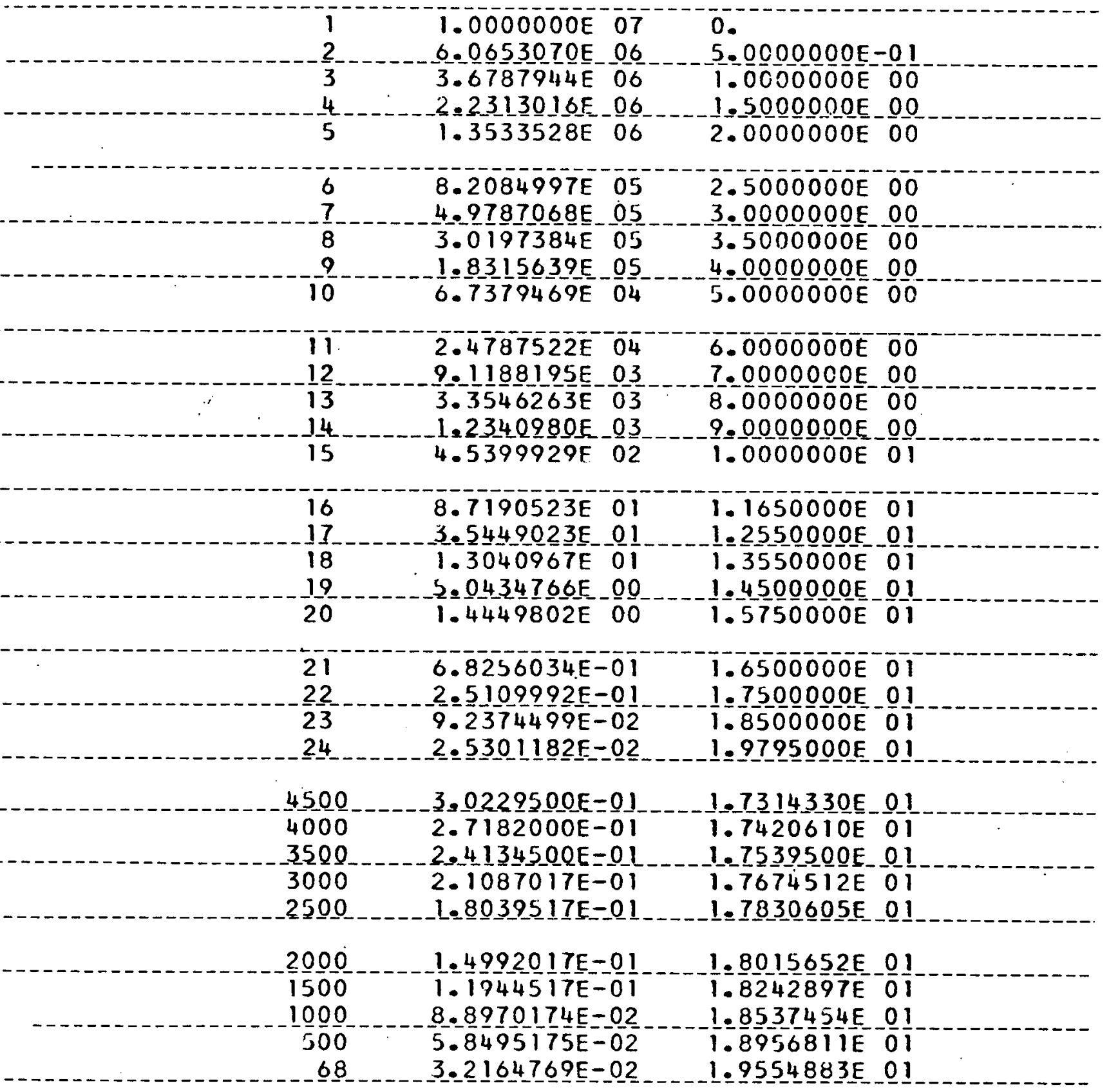


C.4 U-235 Fission Spectral Data

DATE 12107160

DESIGNATION

35 PROMPT
DELAY

FRACTION

$9.9343 \mathrm{E}-0 \mathrm{I}$
PRECURS OR MEAN LIFE

LEVEL

ENERGY

SPECIRUM

$\frac{1}{2}$
$-\frac{3}{4}$
5

$1.000 E-07$

$1.2128500 E-02$

6.065E 06

$1.1298960 \mathrm{E}-01$

3.679 E 06

$3.2997910 \mathrm{E}-01$

$2.231 \mathrm{TE} 06$

$4.6580859 \mathrm{E}-0 \mathrm{I}$

1.353 EE 06

4.2 $193690 \mathrm{E}-01$

$\begin{array}{rr}\frac{6}{7} & 8.208 E \\ 4.979 E & 05 \\ -\frac{3}{9} & \frac{3}{9}=020 \mathrm{E}-05 \\ 10 & 0.832 \mathrm{E}-05 \\ 10\end{array}$

$2.9330140 \mathrm{E}-01$

$1.7435500 \mathrm{E}-0 \mathrm{I}$

$9=4492098 \mathrm{E}-02$

$4.1751800 \mathrm{E}-02$

1.2 2 77 1 100E -02

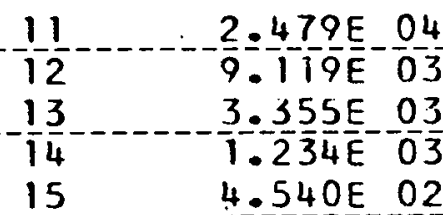

$2.8765000 E-03$

$6.4959999 \mathrm{E}-04$

$1.4560000 E-04$

$3.2499999 \mathrm{E}=05$

$6.0999999 \mathrm{E}=06$

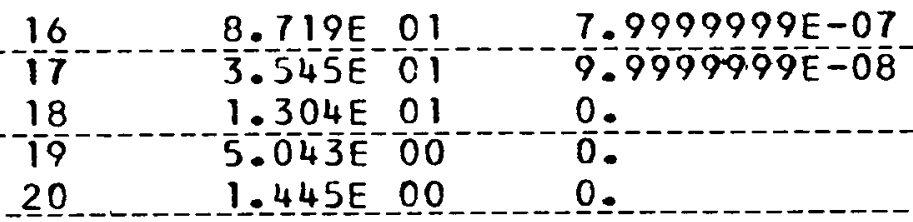

$-\frac{2}{2} 1$
22
$-\frac{23}{24}-\frac{6}{2}=\frac{826}{5} 11 E=0.01$
$2.530 E-02$

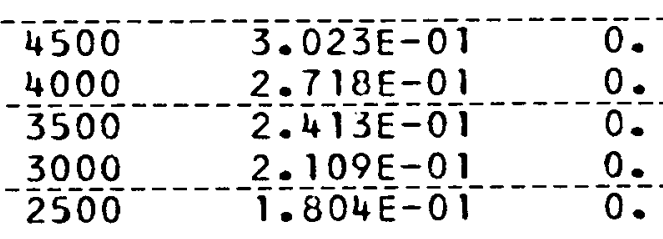

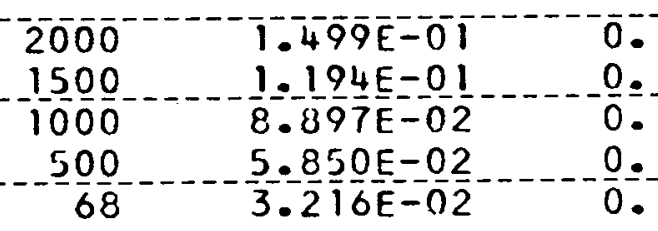


DAIE $12 / 07160$

DELAY
PRECURSOR

\begin{tabular}{|c|c|c|}
\hline LEVEL & ENERGY & SPECTRUM \\
\hline $\begin{array}{r}1 \\
-2 \\
3 \\
-4 \\
-4\end{array}$ & $\begin{array}{l}1.000 \mathrm{E} \\
0.065 \\
3.079 \mathrm{E} \quad 06 \\
2.231 \mathrm{E}-06 \\
1.353 \mathrm{E}-06\end{array}$ & $\begin{array}{r}1.2048800 E-02 \\
1.1224720 E=01 \\
3.2781140 E-01 \\
-\frac{4}{4}=6312220 E=01 \\
4.2181799 E-01\end{array}$ \\
\hline $\begin{array}{c}6 \\
-7 \\
8 \\
-9 \\
10\end{array}$ & 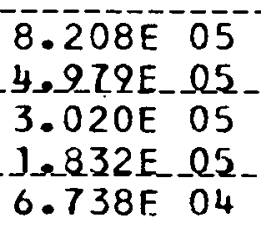 & $\begin{array}{r}2.9531380 \mathrm{E}=01 \\
1.7627380 \mathrm{E}=01 \\
9.5595700 \mathrm{E}-02 \\
-4.2160000 \mathrm{E}=02 \\
1.2548800 \mathrm{~F}-02\end{array}$ \\
\hline $\begin{array}{r}11 \\
-12 \\
13 \\
-14 \\
-15\end{array}$ & $\begin{array}{l}2.479 \mathrm{E} \quad 04 \\
9.1119 \mathrm{E}-03 \\
3.355 \mathrm{E}-3 \\
1.234 \mathrm{E}-03 \\
4.540 \mathrm{E}-02\end{array}$ & $\begin{array}{l}2.8805000 \mathrm{E}-03 \\
-6.4850000 \mathrm{E}=04 \\
1.4510000 \mathrm{E}-04 \\
-3.2399999 \mathrm{E}=05 \\
5.9999999 \mathrm{E}-06\end{array}$ \\
\hline $\begin{array}{l}16 \\
17 \\
18 \\
-19 \\
20\end{array}$ & $\begin{array}{l}8.719 \mathrm{E} 01 \\
3.545 \mathrm{E} 01 \\
1.304 \mathrm{E}-01 \\
5.043 \mathrm{E} \quad 00 \\
1.445 \mathrm{E} .00\end{array}$ & $\begin{array}{l}7.9999999 \mathrm{E}-07 \\
9.9999999 \mathrm{E}-08 \\
0.0 \\
0 .\end{array}$ \\
\hline $\begin{array}{l}21 \\
22 \\
-23 \\
-24 \\
-2\end{array}$ & $\begin{array}{l}6.826 E-01 \\
2.51 L E=01 \\
9.237 E-02 \\
2.530 E=02\end{array}$ & $\begin{array}{l}0 . \\
0.2 \\
0 . \\
0 .\end{array}$ \\
\hline $\begin{array}{r}-4500 \\
4000 \\
-3500 \\
3000 \\
2500 \\
-250\end{array}$ & $\begin{array}{l}-3=023 \mathrm{E}=01 \\
2.718 \mathrm{E}-01 \\
-\frac{2}{2}=413 \mathrm{E}=01 \\
2.109 \mathrm{E}-01 \\
1.8004 \mathrm{E}-01\end{array}$ & $\begin{array}{l}0 . \\
0 . \\
0 . \\
0 . \\
0 .\end{array}$ \\
\hline $\begin{array}{r}2000 \\
1500 \\
1000 \\
500 \\
608\end{array}$ & $\begin{array}{l}1.499 \mathrm{E}=01 \\
1.194 \mathrm{E}-01 \\
8.897 \mathrm{E}=02 \\
5.850 \mathrm{E}-02 \\
3.216 \mathrm{E}-02\end{array}$ & $\begin{array}{l}0 \\
-0 \\
0 \\
0 \\
0 \\
0= \\
0 \\
0 .\end{array}$ \\
\hline
\end{tabular}




\begin{tabular}{|c|c|}
\hline DESIIGNAIION & $\begin{array}{l}\text { DELAY } \\
\text { FRACIION }\end{array}$ \\
\hline _QELAY 1 & $-2 \cdot 3000 E-04$ \\
\hline LEVEL & SPECTRUM \\
\hline$-\frac{1}{3}$ & $\begin{array}{l}1.000 \mathrm{E} 07 \\
-6.006 \mathrm{E}-06 \\
3.679 \mathrm{E}-06 \\
-\frac{2.231 \mathrm{E}-06}{1.353 \mathrm{E}-06}\end{array}$ \\
\hline $\begin{array}{c}6 \\
-\frac{7}{8} \\
-9 \\
10 \\
-9\end{array}$ & 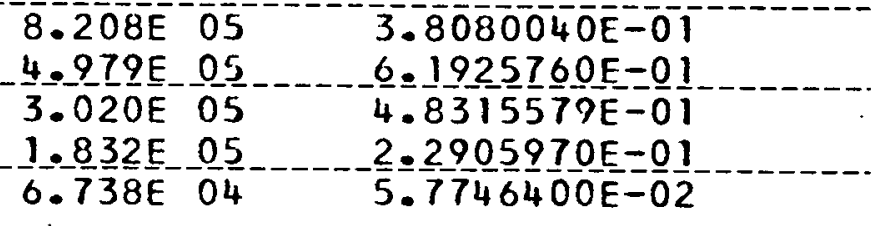 \\
\hline $\begin{array}{l}11 \\
12 \\
13 \\
13 \\
-15\end{array}$ & 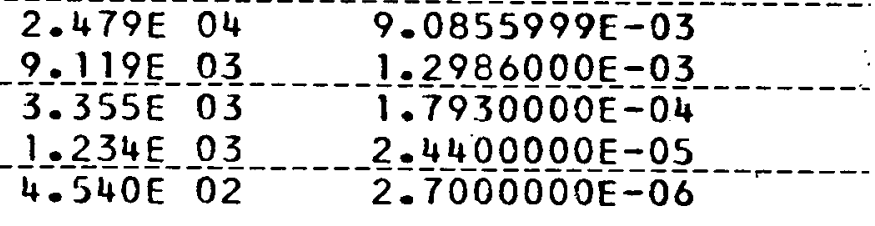 \\
\hline $\begin{array}{l}16 \\
17 \\
-18 \\
-19 \\
20\end{array}$ & 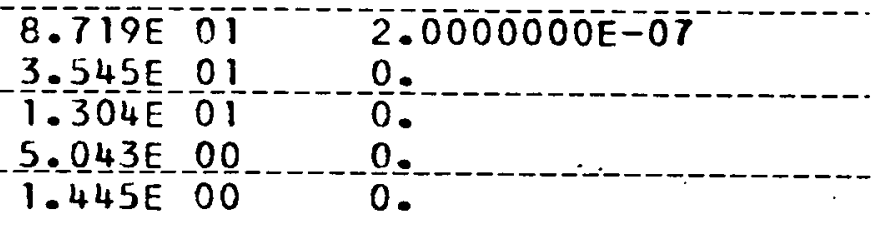 \\
\hline $\begin{array}{r}21 \\
2 \frac{2}{23} \\
23 \\
24\end{array}$ & $\begin{array}{l}6.826 \mathrm{E}=01 \\
2.311 \mathrm{E}=01 \\
9.237 \mathrm{E}-02 \\
2.5330 \mathrm{E}=02\end{array}$ \\
\hline $\begin{array}{l}-4500 \\
-4000- \\
-3500 \\
3000- \\
2500 \\
-\end{array}$ & $\begin{array}{l}3.023 E=01 \\
2 \cdot 718 E=01 \\
2=413 E=01 \\
-2.109 E=01 \\
1.804 E-01\end{array}$ \\
\hline $\begin{array}{l}2000 \\
1500 \\
1000 \\
500 \\
608 \\
\end{array}$ & $\begin{array}{l}1.499 \mathrm{E}=01 \\
1.194 \mathrm{E}-01 \\
8.897 \mathrm{E}-02 \\
5.850 \mathrm{E}-02 \\
3.216 \mathrm{E}-02\end{array}$ \\
\hline
\end{tabular}


DATE $12 / 07 / 60$

DELAY

FRACTION

$1 . \overline{2} 00 \overline{\mathrm{E}}-0 \overline{3}$
PRECUURSOR

MEAN LIFE

35 DELAY 2 1.4200E-03

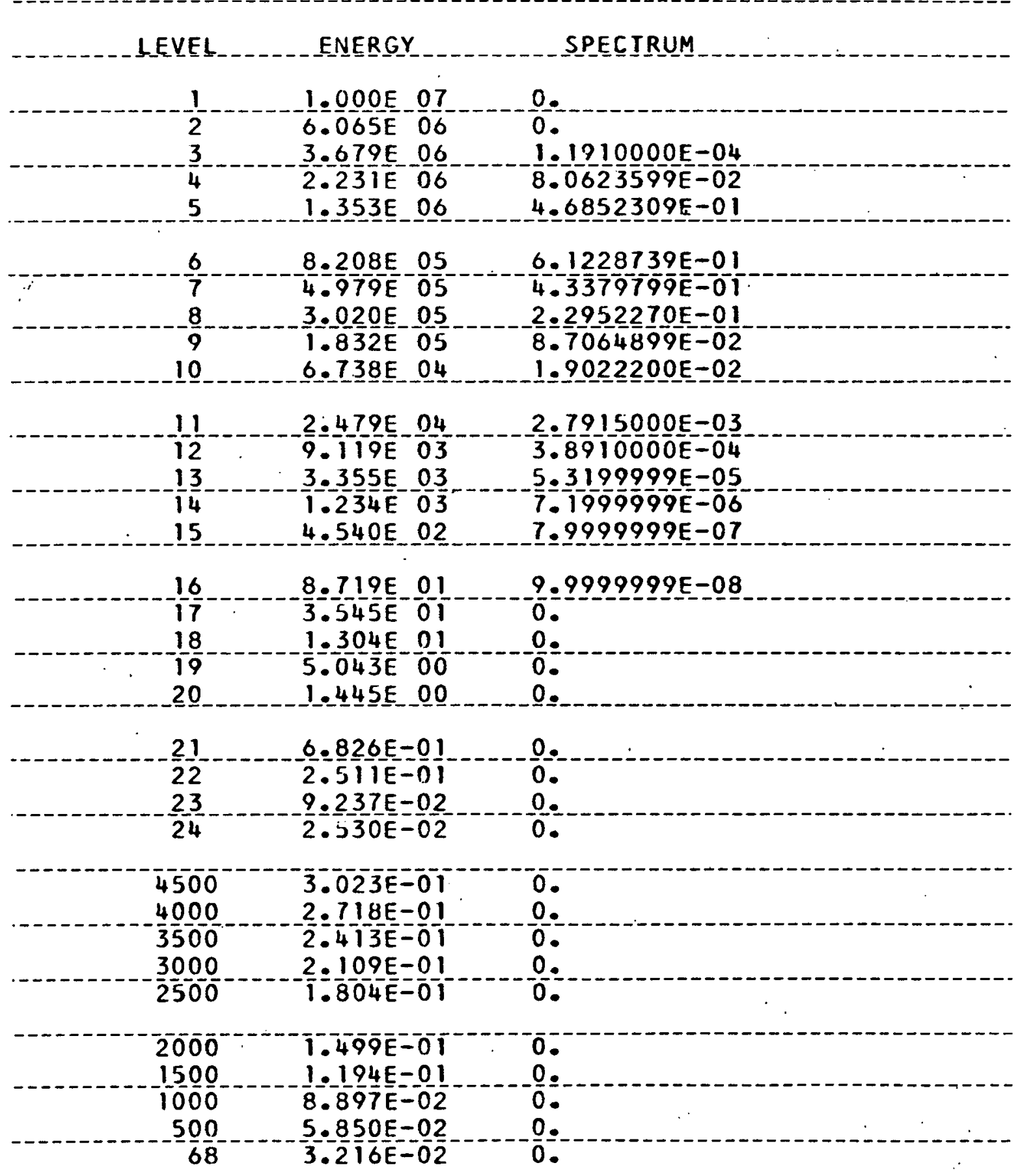




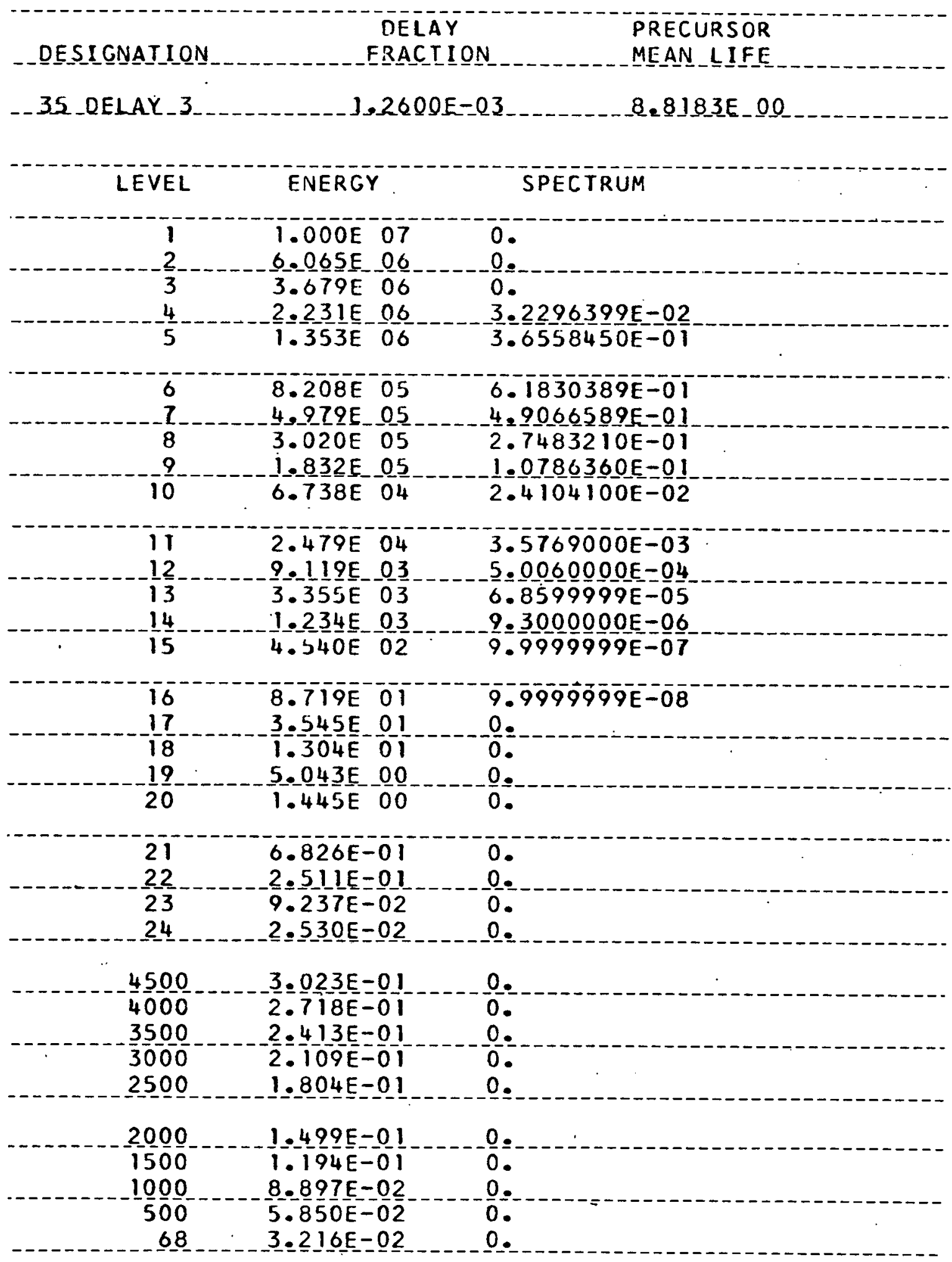


DESIGNATION

35 DELAY 4
DELAY

FRACTION

$2.6400 E-03$
PRECURSOR MEAN LIFE

$3.2680 \bar{E}$ OO.

LEVEL

ENEBGY

SPECIRUM

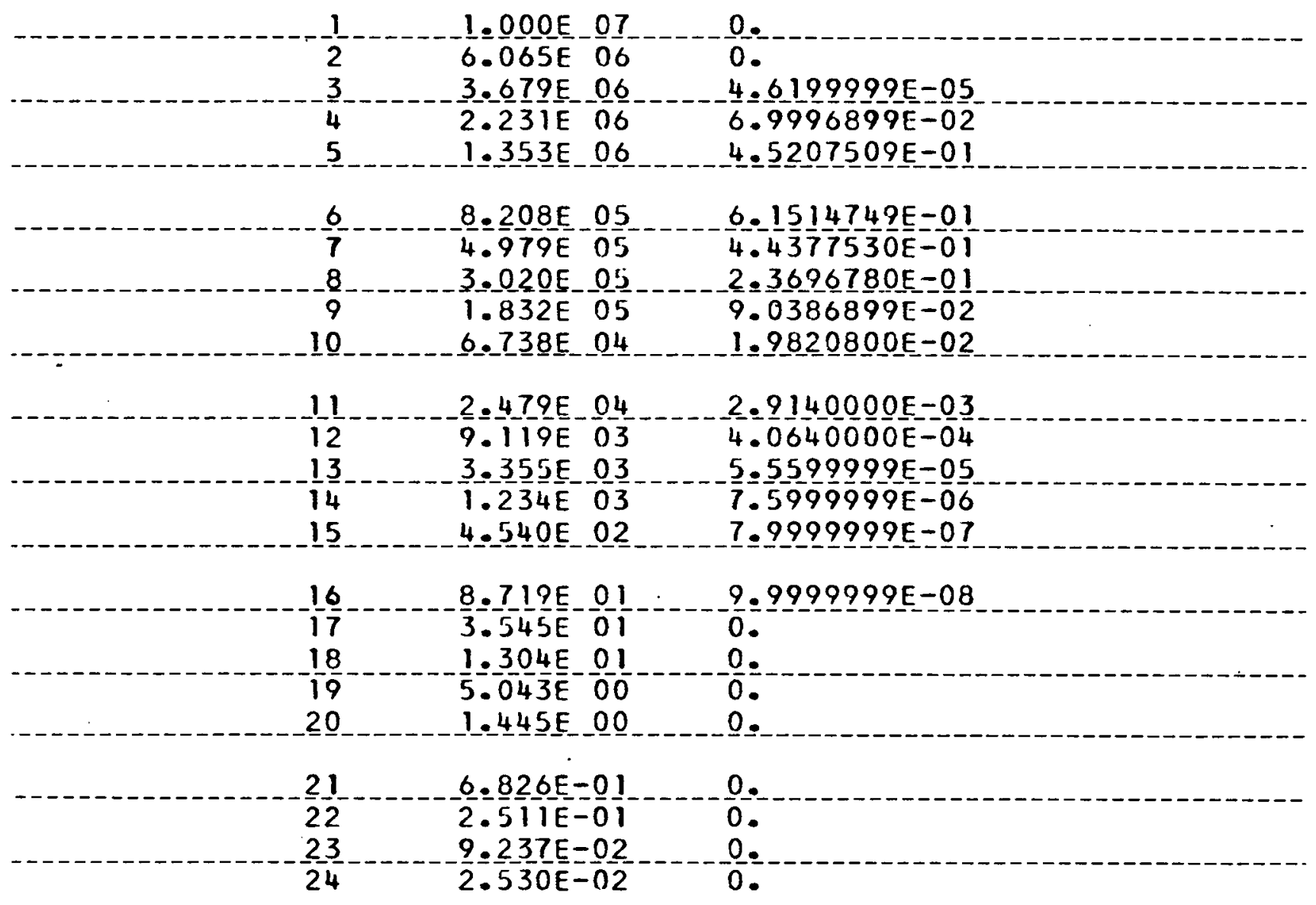

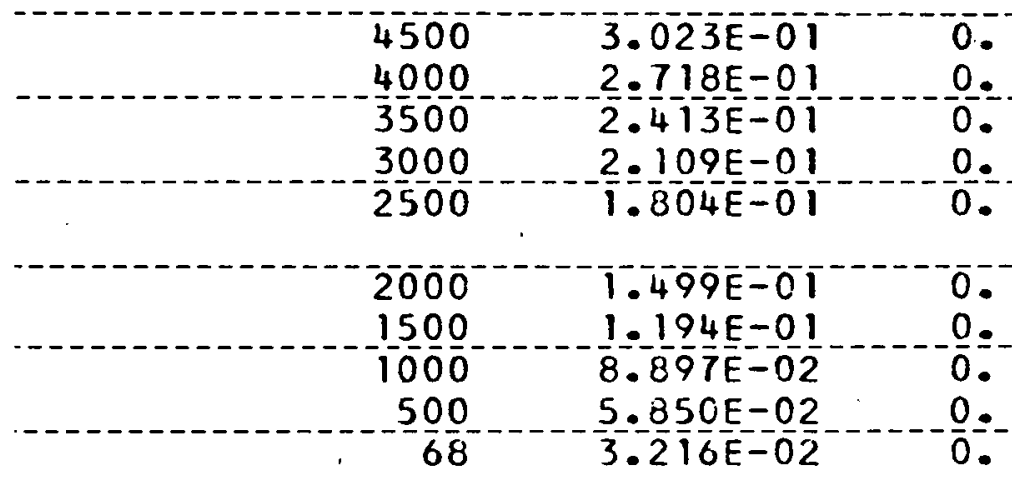


DAIE 12/07/60

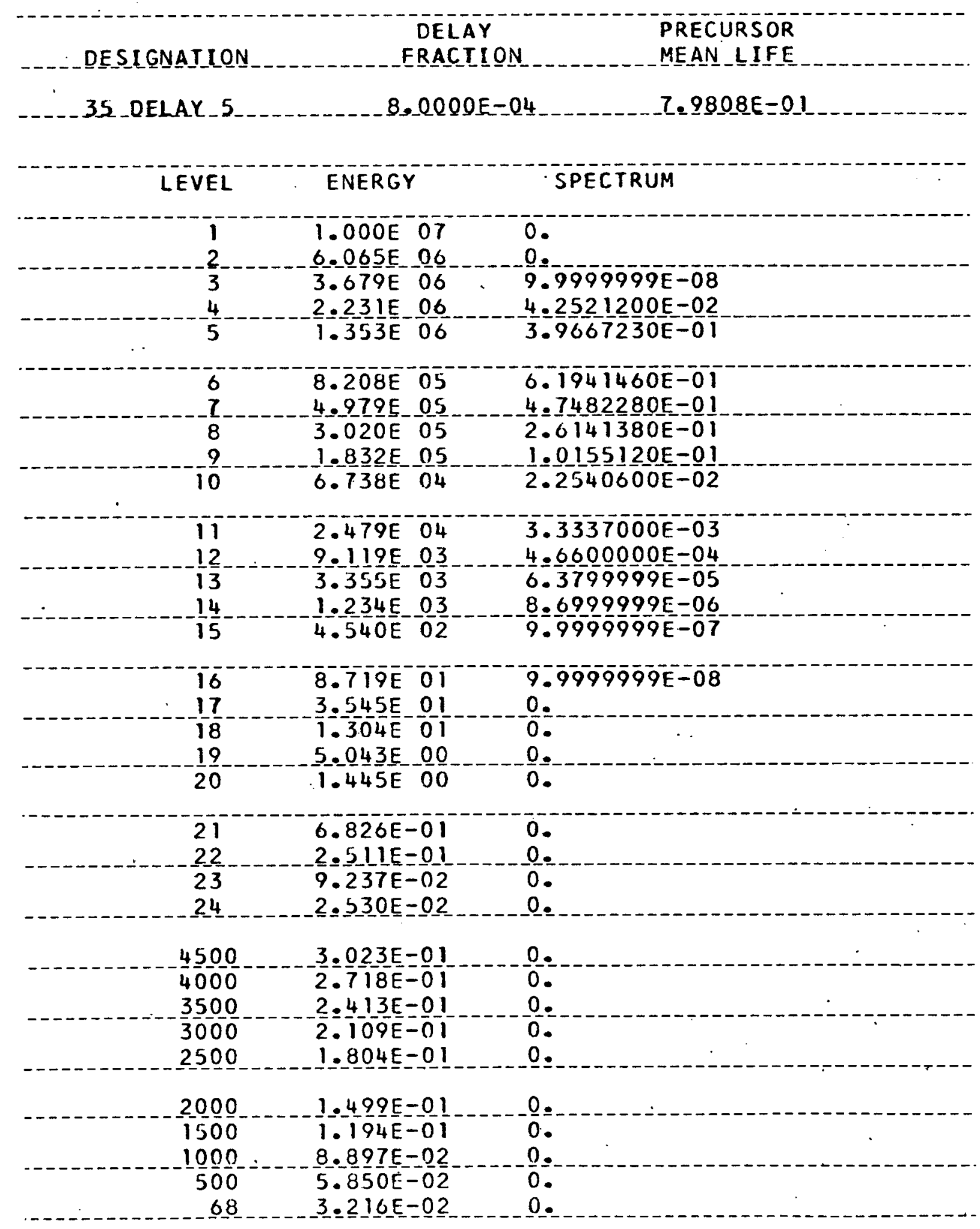




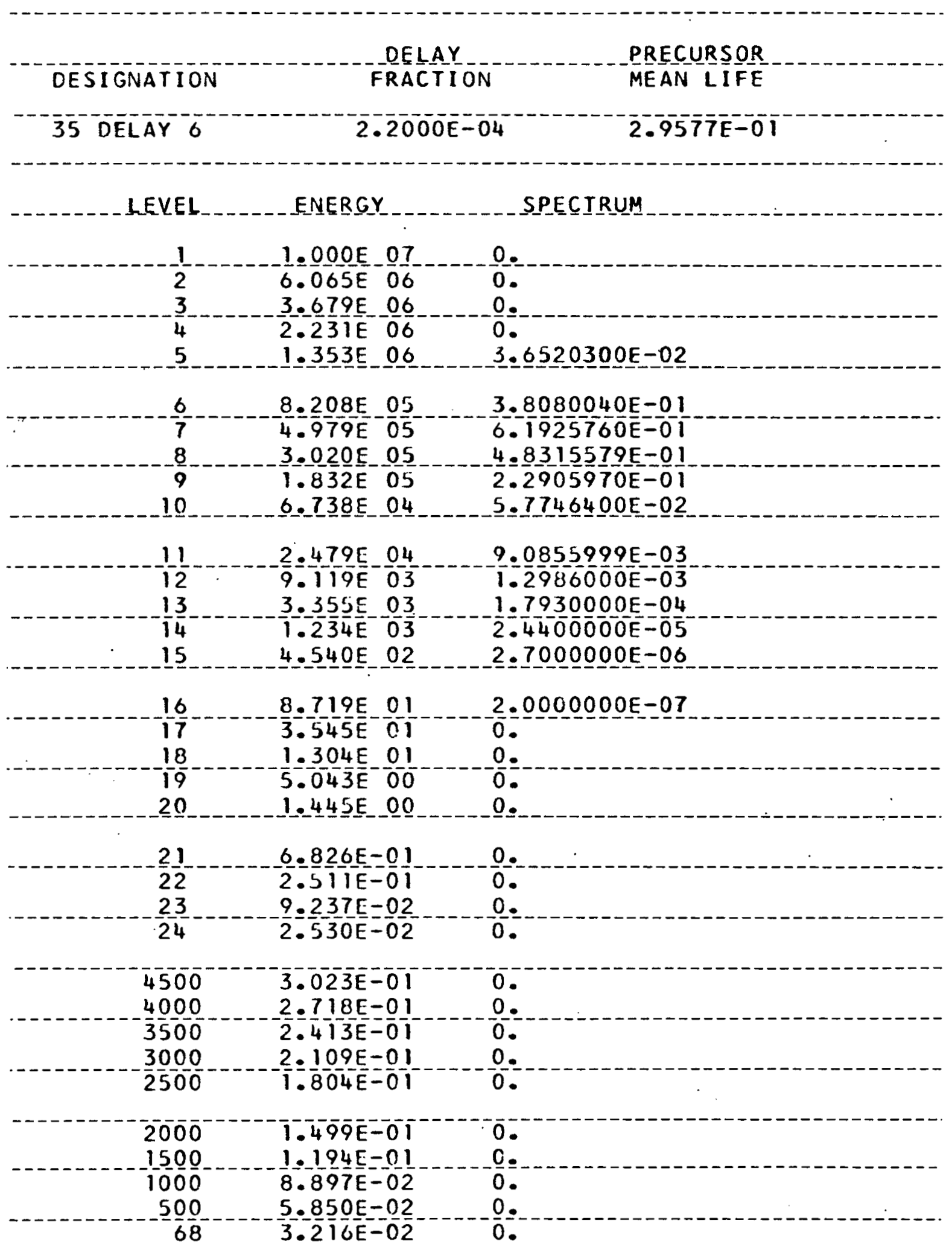


6.5 - Micresconic Cxoss Sections

DATE. DEC O1 1960

DES_LGNATIQN URANIUM 235
CODE NO.

92.2350
DENSIIY FACIOR

$3.90255 E_{-} 02$
SIGMA

LEVEL _ ENERGY _ _ SCATIER
SIGMA
SIGMA TRANSPQRI A ABSORPIION
XII SIGMA

SCATIER 1

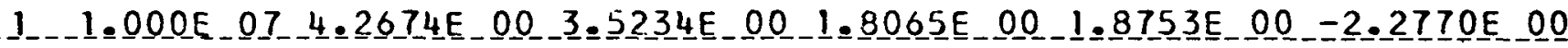

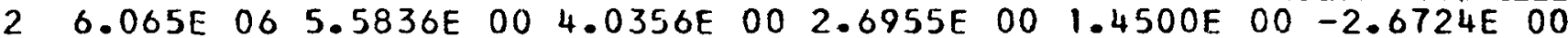

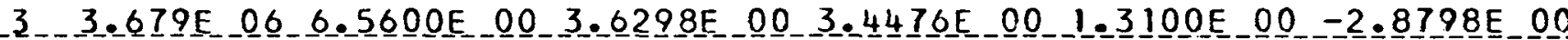

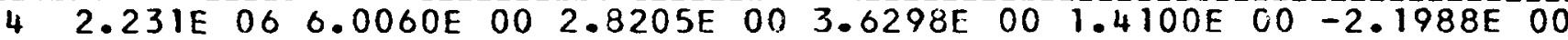

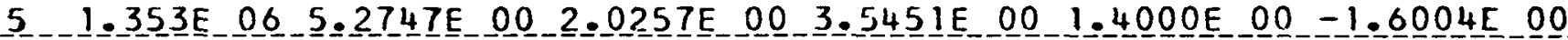

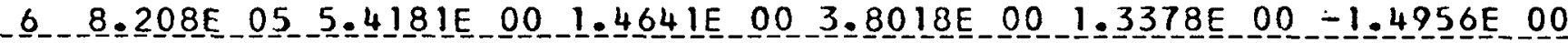

7 4.979E $056.2158 \mathrm{E} 001.2757 \mathrm{E} 00 \quad 4.5950 \mathrm{E}$ 00 $1.4239 \mathrm{E}$ OD $-1.4997 \mathrm{E} 00$

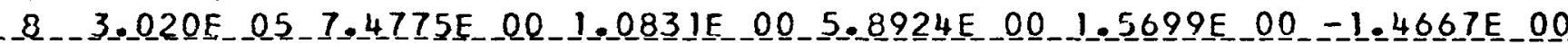

9 1.832E $059.0132 \mathrm{E}$ O0 7.9060E-01 7.7903E $001.8439 \mathrm{E}$ OD $-1.1316 \mathrm{E} 00$

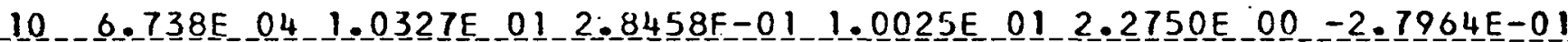

$11-2.479 E-041.0462 E_{0} 01.8 .9530 E-021.0431 E 01-3.6903 E-00-2.8119 E-02$

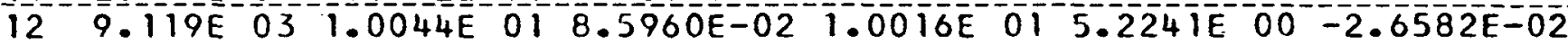

$13 \quad 3.355 E \quad 03 \quad 1.0034 E \quad 018.587$ IE-02 $1.0005 E \quad 01$ 7.3008E $00-2.6554 E-02$

$140.234 \mathrm{E} 031.1925 \mathrm{E} 0 \mathrm{1} 1.0206 \mathrm{E}-011.189 \mathrm{E}-011.0816 \mathrm{E}-1-3.1559 \mathrm{E}-02$

15 4.540E 02 1.3353E 01 1.91428E-01_1.3315E 01 2.3062E $01-3.5337 \mathrm{E}-02$

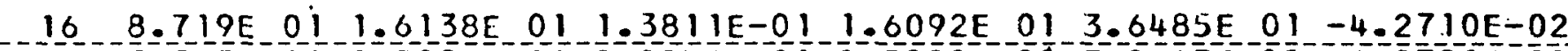

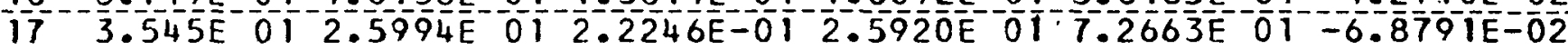
$\begin{array}{lllllllllllll}18 & 1.304 \mathrm{E} & 01 & 1.1277 \mathrm{E} & 01 & 9.6508 \mathrm{E}-02 & 1.1245 \mathrm{E} & 01 & 1.0987 \mathrm{E} & \mathrm{C} 2 & -2.9843 \mathrm{E}-02\end{array}$

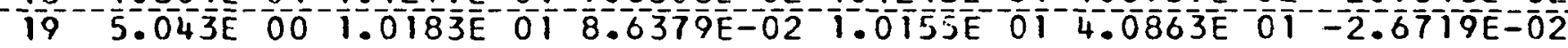
20 1.445E 00 1.1605E 01 9.8435E-02 1.1572E $01.4 .2582 \mathrm{E} 01-3.0447 \mathrm{E}-02$

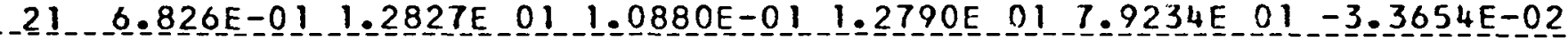
22 2.5 IIE-0 $1.3767 \mathrm{E}-1.1678 \mathrm{E}-0 \mathrm{~T} 1.3728 \mathrm{E} 012.0812 \mathrm{E}-02-3.6122 \mathrm{E}-02$ $239.237 \mathrm{E}-02 \quad 1.4351 \mathrm{E} 01,1.2173 \mathrm{E}-011.4311 \mathrm{E} 013.3004 \mathrm{E}-02-3.7653 \mathrm{E}-02$ $242.530 \mathrm{E}-02 \cdot 1.4600 \mathrm{E} 01-1.2385 \mathrm{E}-011.4559 \mathrm{E} 01-5.6816 \mathrm{E} 02-3.8307 \mathrm{E}-02$

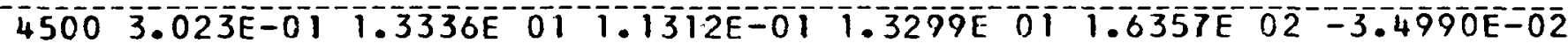

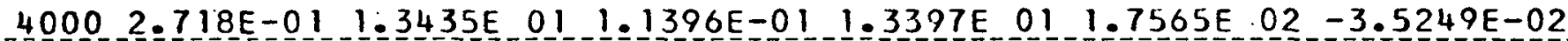

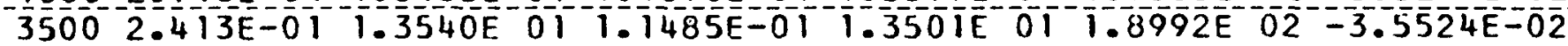
$30002.109 E-01 \quad 1.3651 E-01 \quad 1.1579 E-01 \quad 1.3612 E \quad 01-2.0703 E-02-3.5816 E-02$

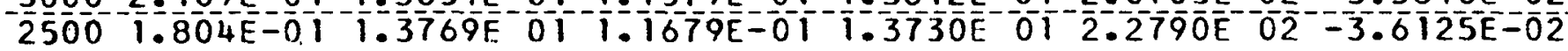

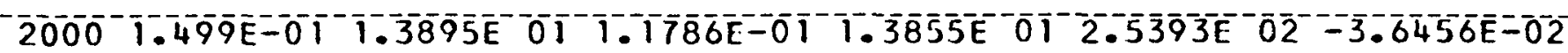
$15001.194 E-011.4030 E 01$ 1. $1901 E-01$ 1.3990E $012.8778 E-02-3.6808 E-02$ $1000-8.897 \mathrm{E}-021.4180 \mathrm{E}$ O 1 1.2028E-01 $1.4139 \mathrm{E}$ 0 1 3.3612E $02-3.7202 \mathrm{E}-02$ $500 \quad 5.850 E-02 \quad 1.4355 E \quad 01 \quad 1.2176 E-01 \quad 1.4314 \mathrm{E} \quad 014.2093 \mathrm{E}-02-3.7662 \mathrm{E}-02$

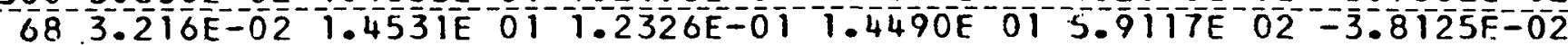


-DESIGNATLON CODE NO. DENSI IY FACTOR _. URANIUM_ 235 92.2350 $3=90255 E_{-} 02$

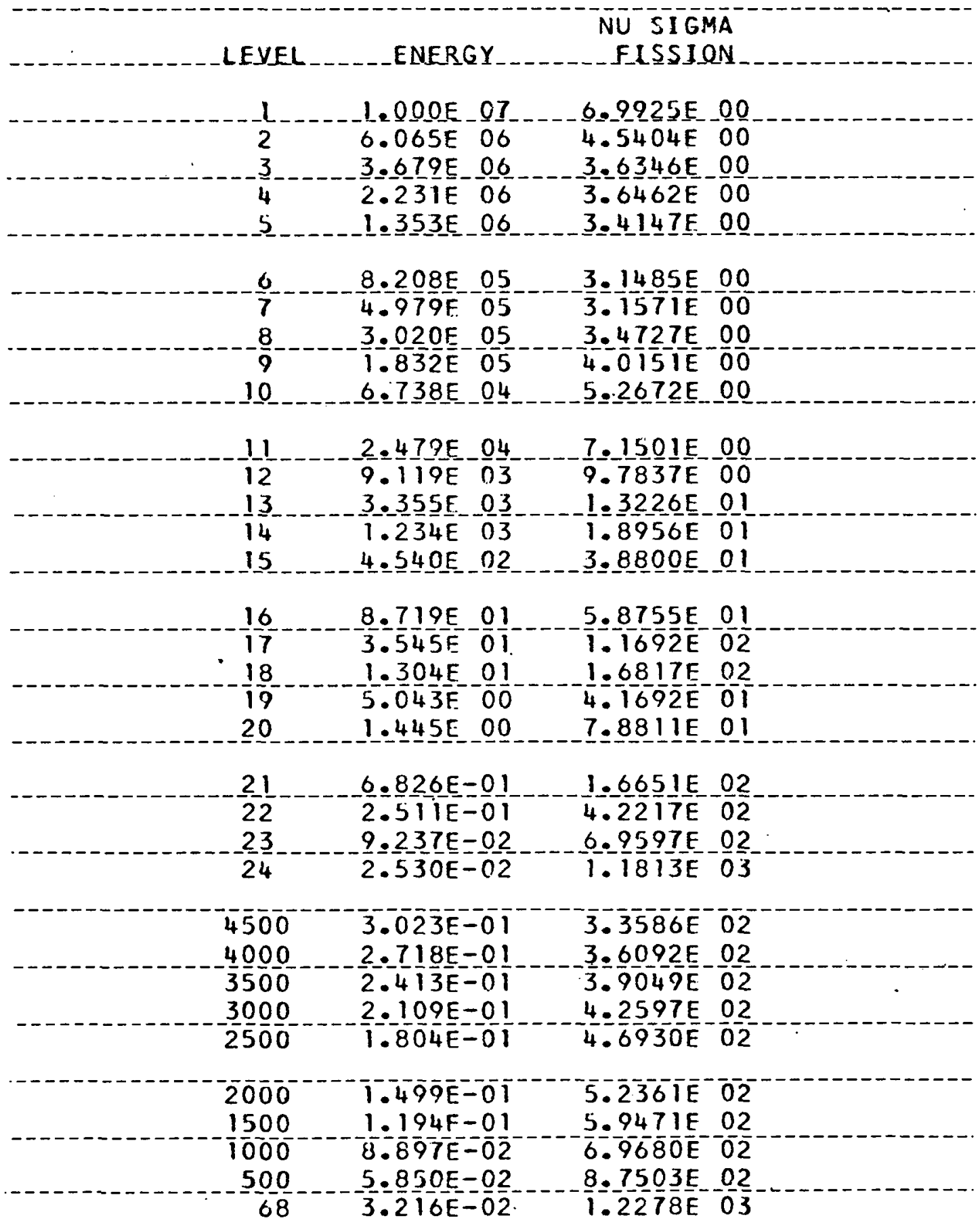


URANIUM 235

IHERMAL_IRANSFER MAIRICES.

IEMPERAIURE _. - SIGMA

SIGMA

S IGMA

NU SIGMA

DEG. F.

SCATTER

TRANSPORT

ABSORPIION

FISSION

$4.5000 \mathrm{E} 03$ 1.5266E 01

$1.5223 E 01$

$9.4 \overline{3} 8 \overline{\mathrm{E}} 0 \overline{2}$

$1.9530 \mathrm{E} 03$

\section{SIGMA $\frac{N 1}{S}-\frac{N 2}{N}-\frac{1}{N 2}$}

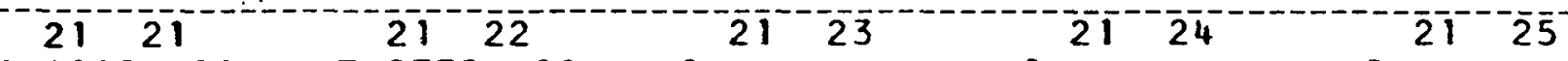

1.2029E 01

2221

$22 \quad 22$

2223

$22 \quad 24$

$22-25$

$7.433729=$

$1.2511 E-01$

$5=\frac{39}{23}=1 \mathrm{E}=\mathrm{-O}$

$3.2044 E=06$

$23^{-25}$

-.-.

$1.6640 \mathrm{E}-00$

$1=2 \frac{28}{24}-\frac{2}{2}=01$

$4.6070 E-01$

$1.5072 \frac{5}{24}-02$

$0 .-\overline{2} 5^{-1}$

$5.8352 E-0.05$

$4.0938 E 00$

$9.0732 E-00$

$1.6364 E 00$

0.

0.

$3.1019 E-01$

4.0124E 00

$1.0943 E$

IEMPERAIURE

DEG. F.

4.00OOE 03
SI GMA

SCATTER
SIGMA

TRANSPORT
SIGMA

ABSORPTION
NU SIGMA

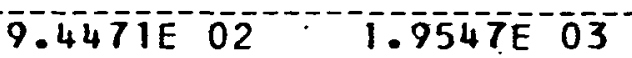

SIGMA S (N1/N2)

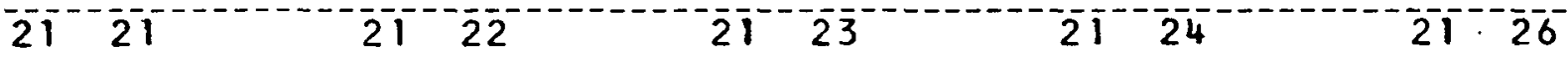
$1.1971 E-01$ 2221

0.

$\overline{2} 2-1 \overline{3}$

$0 .-24$

으.

$6.6 \frac{4}{2} 55 E-01$

1. $2 \frac{25}{2} 77 \mathrm{E}-0$

$5.5054 \mathrm{E}-01$

$1.3563 E-06$

$1.5468 \mathrm{E}-00$

$1.24066 \mathrm{E}$

$24-2 i$

2422

2423

$4.5436 E=01$

2424

0.

$26-21$

2. 054 트는 05

$3.8851 \mathrm{E} 00$

$-9=\frac{3}{26}-\frac{1}{2}-0 \mathrm{E}$

$-2 \frac{3}{24}-\frac{36}{26}-0$

0.

0 .
1.58405
$2.3944 E-01 \quad 3.8469 E \quad 00$ 
URANIUM 235

THERMAL TRANSFER MATRICES

SIGMPERATURE
SIGMA $\begin{gathered}\text { SIGMA } \\ \text { SIGMA }\end{gathered}$

\begin{tabular}{|c|c|c|c|c|}
\hline$-21-21$ & $-21-22$ & $-2 L$ & --21 & $-21-27$ \\
\hline $1.1892 \mathrm{E} \quad 01$ & $9.3479 E-01$ & 0. & 0 & 0. \\
\hline $\begin{array}{c}5.8011 E-01 \\
23-21\end{array}$ & $\begin{array}{l}1.2641 \mathrm{IE} 01 \\
23\end{array}$ & $\begin{array}{l}5.6801 E-01 \\
2323\end{array}$ & $\begin{array}{c}4.7133 \mathrm{E}-07 \\
23\end{array}$ & 0.23 \\
\hline 0.2 & $\begin{array}{r}1.4213 E \\
24\end{array}$ & $\begin{array}{l}1.2533 E^{2} 01 \\
24.23\end{array}$ & $\begin{array}{c}4.4896 \mathrm{E}-01 \\
2424\end{array}$ & $\begin{array}{c}9.7505 \mathrm{E}-03 \\
2427\end{array}$ \\
\hline & $\begin{array}{r}5.7018 E-06 \\
27\end{array}$ & $\begin{array}{r}3.6589 \mathrm{E} 00 \\
27 \quad 23\end{array}$ & $\begin{array}{r}9.5787 \mathrm{E} 00 \\
27 \\
-24\end{array}$ & $\begin{array}{l}1.5252 \mathrm{E} \\
2 \mathrm{Z}\end{array}$ \\
\hline 0. & 0. & $1.7582 E-01$ & $3.6597 \mathrm{E} 00$ & $1.1316 \mathrm{E} 01$ \\
\hline
\end{tabular}

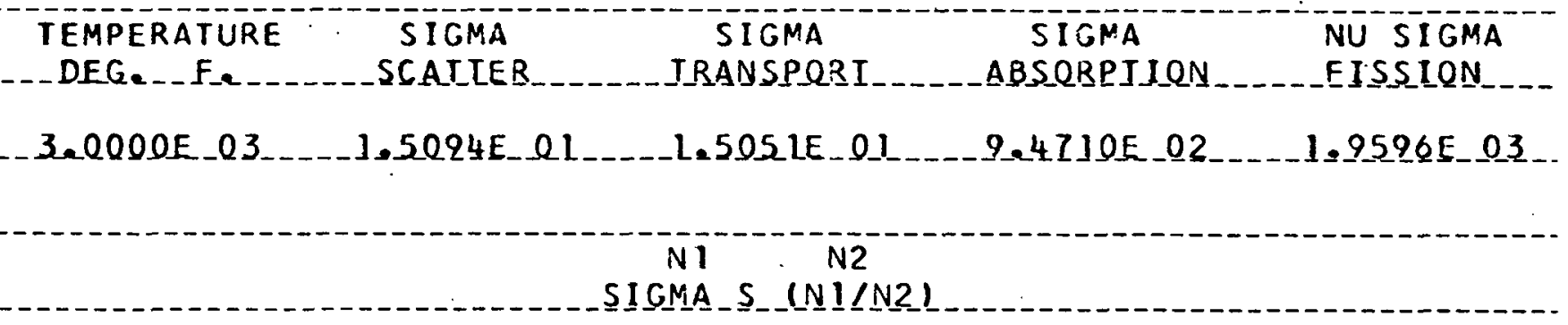

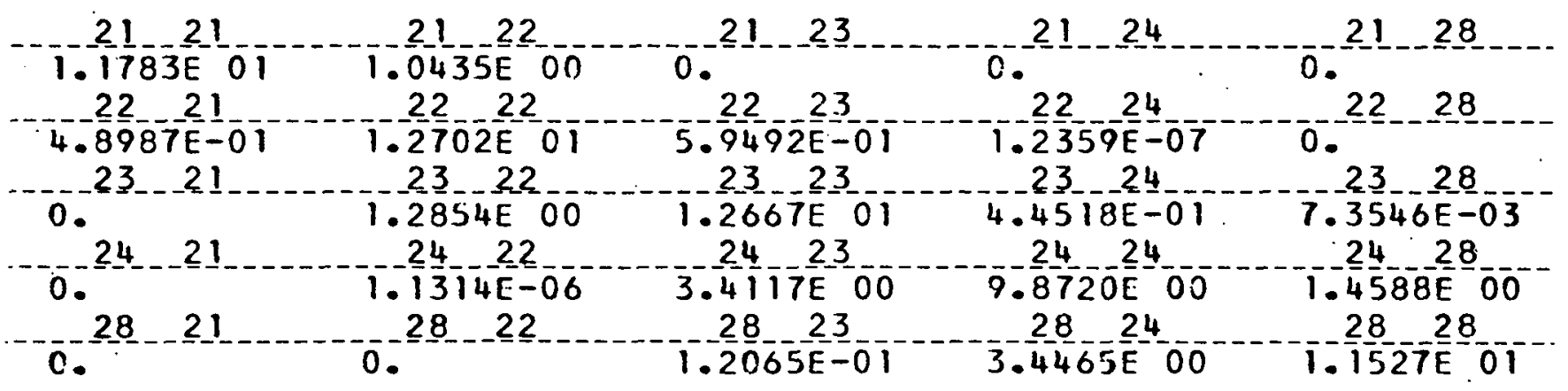


URANIUM 235

_LHERMAL_IRANSFER_MAIRICES _

-IEMPERAIURE
DEG. F. FIGMA
SCATTER

$2.5000 \mathrm{E} 0 \overline{3}$
$1.50 \overline{3} \overline{\mathrm{E}} \mathrm{0} \overline{\mathrm{I}}$
SLGMA

TRANSPORT
SIGMA ABSORPTION
NU SIGMA

FISSION

SIGMA S $(N) / N 2)$

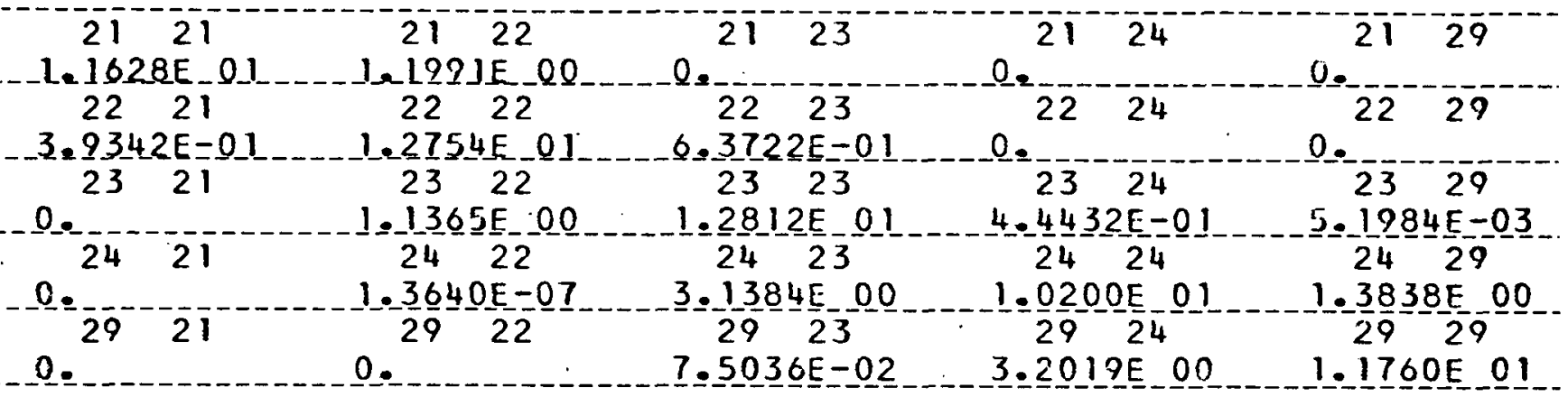

IEMPERAIURE DEG. $F$.

$1.4994 \mathrm{E} 01$

$9.4891 \mathrm{E} 02$

$1.9634 \mathrm{E} 03$

\section{SIGMA S (N IIIN2)}

SIGMA TRANSPORT

$1.49 \overline{7} \overline{0} 01$
SIGMA ABSORPTION

$9.514 \overline{B E} 02$
NU SIGMA

FISSION

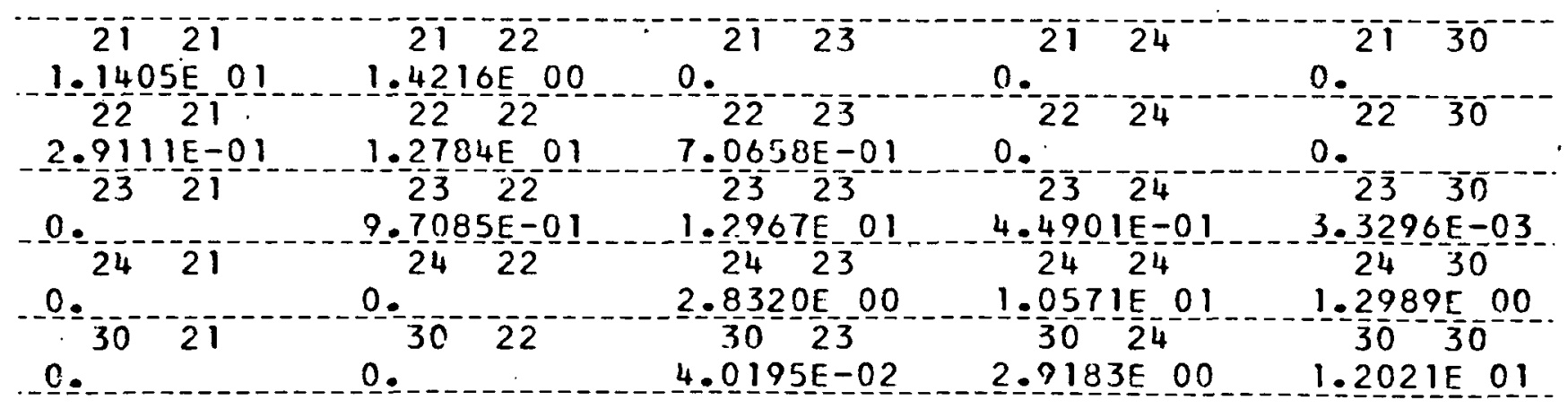


URANIUM_235

THERMAL TRANSFER MATRICES

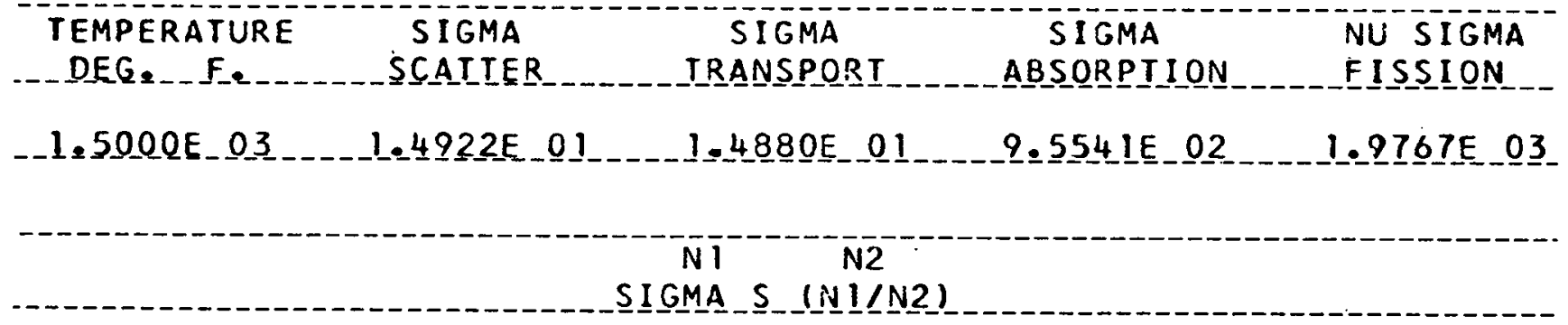

\begin{tabular}{|c|c|c|c|c|}
\hline $\begin{array}{l}21 \\
-1231 \mathrm{I}\end{array}$ & $-21-22$ & $21-23$ & -21 & 21 \\
\hline $\begin{array}{l}-1231 \mathrm{E} 01 \\
-22 \quad 21\end{array}$ & $\begin{array}{l}1.5963 E 00 \\
22 \\
22\end{array}$ & 0.22 & 0.2 & ${ }_{22}^{0 .} 3$ \\
\hline $\begin{array}{l}8563 \mathrm{E}-01 \\
23 \quad 21\end{array}$ & $\begin{array}{c}1.2765 E 01 \\
23\end{array}$ & $\begin{array}{c}8.2879 \mathrm{E}-01 \\
2323\end{array}$ & 0 & 0. \\
\hline & $7.8257 \mathrm{E}-01$ & $1.3134 \mathrm{E} 01$ & $4.6514 E-01$ & $1.8146 \mathrm{E}-03$ \\
\hline 0 & $-24-22$ & $-\frac{24}{4} \div=\frac{23}{F}$ & $-\frac{2}{0}-50-24$ & $-24-31$ \\
\hline 7 & 31 & 3123 & 31 & 31 \\
\hline & 0 . & $1.6770 E-02$ & $2.5849 \mathrm{E} 00$ & $1.232 \mathrm{JE}$ \\
\hline
\end{tabular}

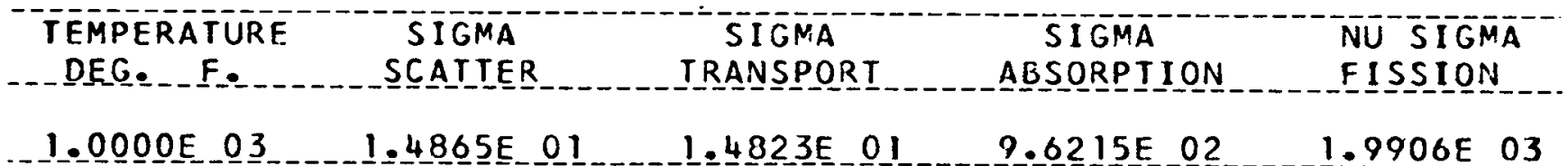

$N 1$
SIGMA S (N1/N2)

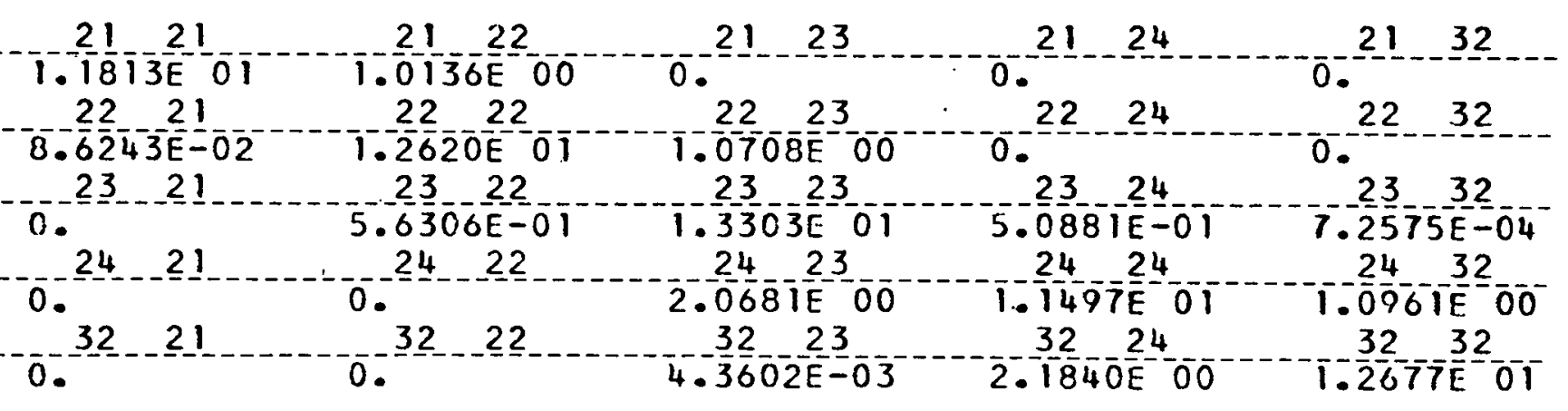




\section{URANIUM 235}

IHERMAL IRANSFER MAIRICES -

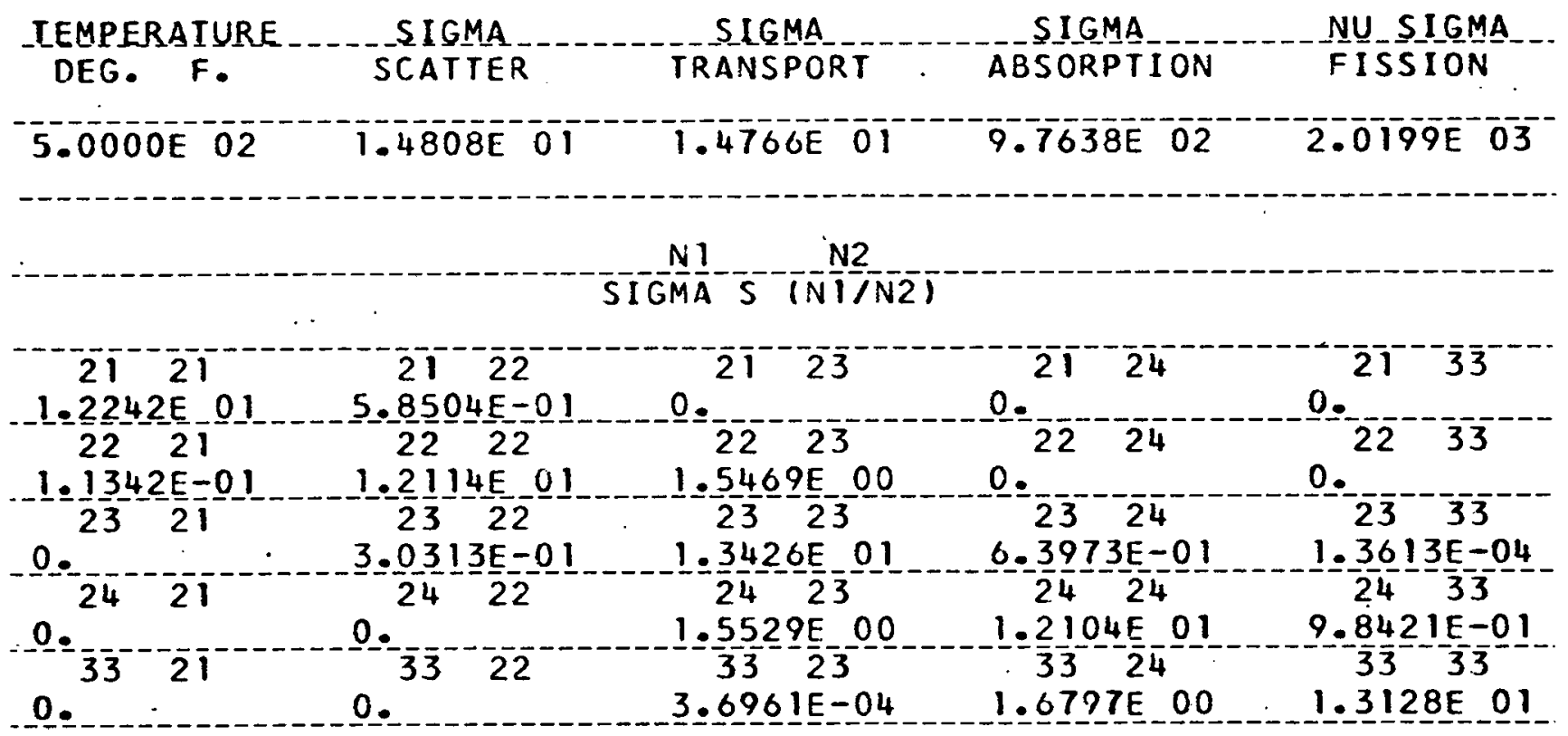

IEMPERAIURE

DEG. $F$.

$6.8000 \mathrm{E} O \mathrm{I}$
SIGMA

SCATTER
SIGMA

TRANSPORT
SIGMA ABSORPTION
NU SIGMA

FISSION

\section{SIGMA S (N $1 \frac{N}{N}$}

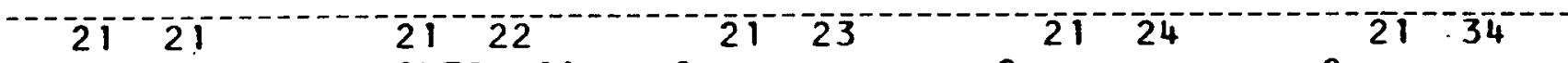

$1.2222 \mathrm{E}-01$

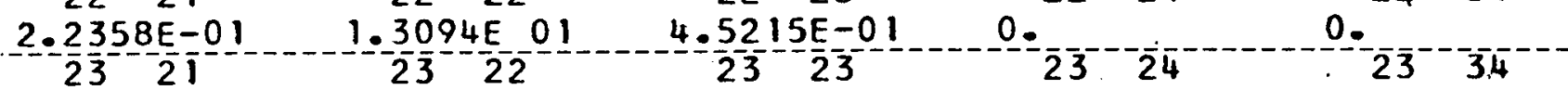

$0 .=2.0153 E-02 \quad 1.3196 E-01 \quad 1.0961 E-00 \quad 3.3100 E-06$

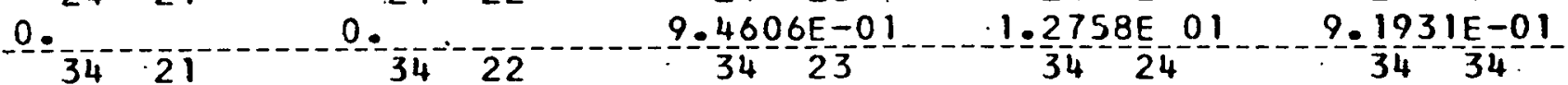

-

ㅇ..

$2.0326 E-06$

$1.0814 \mathrm{E} 00$

$1=3680 E$ 요 


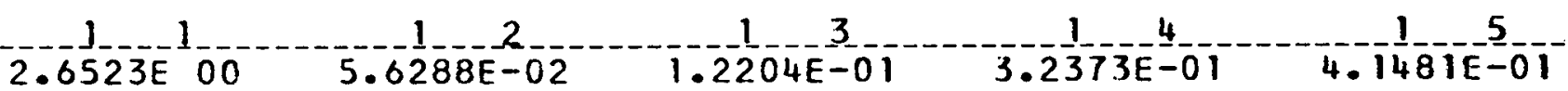
$2.6523 E 000.628$ $3.3208 \mathrm{E}-01 \mathrm{1}-9723 \mathrm{E}-019.7316 \mathrm{E}-02$ $-1$ $2.2541 \mathrm{E}-03$ $-\frac{1}{5787}-\frac{16}{6}-08$ $3.1267 \mathrm{E}-04$ $4.33 \frac{1}{3} 86 \mathrm{E}-05$ 0. - 5 $5.0790 \mathrm{E}-01$ 10 $3.212 \mathrm{IE}-02$ $1.8408 \mathrm{E}-06$ 3 $1.9993 E-01$ $5.049 \frac{2}{10}-0$ $3.4958 \mathrm{E} 00$ $3.4359 \bar{E}-01$ $5.3622 \mathrm{E}-02 \quad 1.5357 \mathrm{E}-02$ 4. $481 E-01$ $5.7750 \mathrm{E}-06-\frac{1}{8.5069 \mathrm{E}-07}$

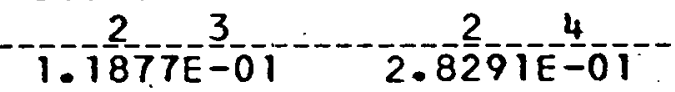
3

$1.6276 \mathrm{E}-0115.1049 \mathrm{E}-\mathrm{O}$ -..-3 14 $2.0670 \mathrm{E}-05$ $3-15$ $4.8168 \mathrm{E}-03$ $6.7356 \mathrm{E}-04$ $1.4238 \mathrm{E}-07$ 0 . 3 3 $5.4878 \mathrm{E}-01$ $1.8 \frac{2}{4} \overline{3 E}-\frac{8}{0}$ $1.0762 \mathrm{E}-0 \mathrm{O}$ 0 . $3.0476 \mathrm{E}-06$ $7.8551 \mathrm{E}-03$ $9.3737 \mathrm{E}-05$ 0 . $\frac{3}{2.35}=\frac{16}{6}-07$ $4.1358 E^{-4} 00^{-16}-\frac{4}{3} 160 \mathrm{E}-01$ -.-

3. $1494 \mathrm{E}-01$ $2.3056 \frac{4}{2}-04$ 40 0. 5

0.5

$4.249 \overline{1 E}-01$ $2.2870 \mathrm{E}-0 \mathrm{3}$ 0. $4.189-6$ - 60 $2.2518 \mathrm{E}-0 \overline{2}$ 0. $2.1556 \mathrm{E}-\mathrm{O} 1$ $-7.2 \frac{4}{170}-\frac{10}{0}-02$ $3.082 \overline{3}=05$ $4.5 \frac{4}{4}-7 \frac{15}{E}-06$ $7.3 \frac{6}{19}-\frac{16}{\mathrm{E}}-\overline{0} \overline{7}$ $-\frac{5}{3}-1$ 0 . 5 $-5-29$ $-\frac{9}{-0}$ 513 $3.2279 \mathrm{E}-04$ $-5$ 0. 2 0 . $4.0 \frac{6}{6} 65 \mathrm{E}-0 \mathrm{7}-3.5 \frac{6}{545 \mathrm{E}-0 \mathrm{O}}$ $-\frac{3}{4}-37-\frac{7}{1 E}-0$ - 3 - 12

1. $109 \overline{1 E}-0 \overline{3}$ 0 4 $1.2 \frac{2}{49}-\frac{1}{\mathrm{E}}-05$ $4.4606 \mathrm{E} \cdot 00$ 
32

_URANLUM 235.

SIGMA SO (NI/N2)

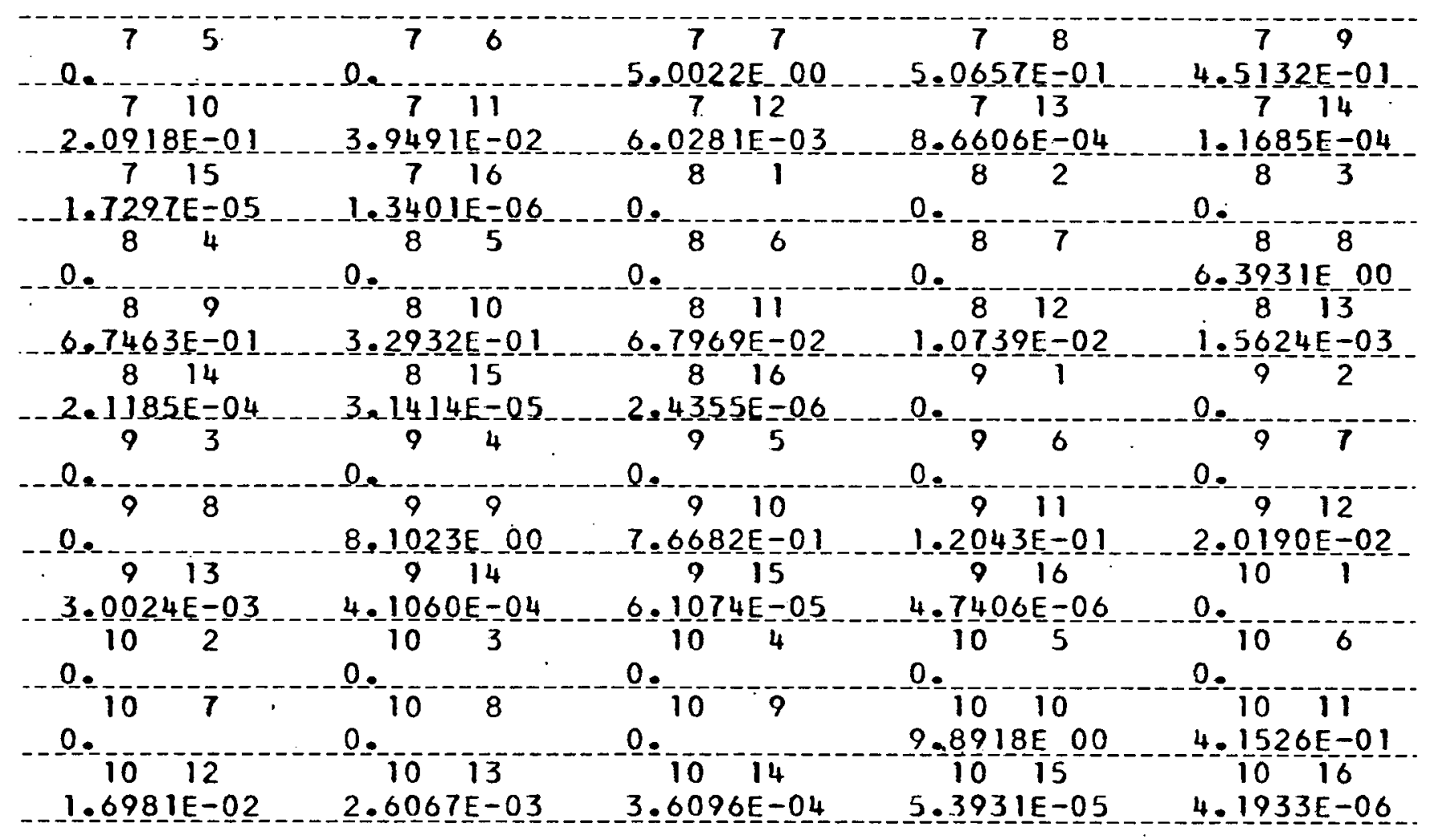


URANIUM 235

_. RESONANCE _... RESONANCE ENERGY INTEGRAL

CROSS SECTION

R

$7.3 \overline{7} \overline{4} \overline{\mathrm{E}}-0 \overline{5}$

$1.5513 \mathrm{E}=03$

4. $1529 E-04$

$1.6980 \mathrm{E}=04$

$5.7634 \mathrm{E}-04$

$5.9296 E-00$

$3.1400 E-00$

$3.6100 E$ OO

$4.8400 \mathrm{OE} 0 \mathrm{O}^{4} 4.6 \overline{3} 3 \mathrm{E}$

$5.4500 E$ EO

$6.1200 E$ OO

$6.4000 E$ OOO

7.1000 E 00

$\overline{8} . \overline{8} \bar{O} \bar{O} \overline{\mathrm{E}} \mathrm{O} \overline{\mathrm{O}}$

9.2 270OE 00

$9.7400 E$ OO

$1.0150 E$ OI

$1.0640 E$ OI

T.TTOE OT

$1.1660 \mathrm{E}$

$1.2400 \mathrm{E} 01$

$1.3400 E$ O 1

$1.3800 \mathrm{E}-\mathrm{T}$

$\overline{1.4} \overline{1} \overline{1 O E} \overline{0} \bar{T}$

$1.4600 E \quad 01$

T. $5500 E$ OT

$1.6100 \mathrm{E}-01$

$1.6800 \mathrm{E} 01$

$1.810 \overline{O E}$ O

1.8700E 01

$1.9300 \mathrm{E}$ O 1

2.0600E 01

2. 110OE O

2. $2 \overline{1} \bar{O} \overline{O E} \bar{O}$

2.2900E 01

$2.350 \mathrm{O}$ O

2. $4400 E \quad 01$

$2 \cdot \overline{5} \bar{O} \overline{O E} \bar{O} \overline{1}$

$2 . \overline{8} \overline{00} \bar{O} \mathrm{O} \overline{1}$

$2.6500 E$ O 1

$2.7300 \mathrm{E} 01$

2. 7900 E 01

3.0700E 01
$1.4585 \mathrm{E}-00$

$1.4980 \mathrm{E} 00$

$1.5206 \mathrm{E} \quad 01$

$4.4609 \mathrm{E} 00$

3. $124 \mathrm{E} 01$

$3.0647 \mathrm{E} \quad 00$

$7.4604 \mathrm{E}-01$

$1.2110 E$ OO

$5.9402 \mathrm{E}-01$

$\overline{7} . \overline{7} \overline{3} \bar{O} \overline{\mathrm{E}}-\overline{\mathrm{O}} \overline{\mathrm{I}}$

$8.7303 E$ O 0

$1.7602 \mathrm{E}$ 0 1

$8.4022 E-01$

$1.6066 \bar{E} 0 \overline{0}$

$2.0 \overline{7} \overline{8} \overline{\mathrm{E}} 0 \overline{0}$

$1.6236 \mathrm{E} 00$

$2.3250 E^{\circ}$

2. $2278 \mathrm{E}$ OO

2.0899E 00

2. $9 \overline{309 E}$ OO

1.272 IE 00

$1.7294 \mathrm{E} 01$

$1.7576 \mathrm{E} \quad 00$

$4.2128 \mathrm{E} 00$

3. $96 \overline{\mathrm{TE}}-\overline{0} \mathrm{I}$

$2.6153 \mathrm{E} \quad 00$

$6.5675 \mathrm{E}-00$

$1.4986 \mathrm{E} \quad 00$

$1.226 \overline{5} \mathrm{O} \overline{\mathrm{O}}$

$9.4 \overline{10} \overline{8 E}-\overline{0} \overline{1}$

1. $4584 \mathrm{E} 00$

$3.0326 \mathrm{E}-01$

$2.0809 E \quad 00$

$9.5320 E-01$
$1.1541 E 02$

$1.1445 E$. 02

$7.1825 \mathrm{E} 01$

$1.7259 E \quad 02$

$3.0407 E$ 02

$5.6215 E-01$

$6.4828 E$ O 1

1.0626 트 03

$3.5936 \bar{E}-02$

1. $\overline{\overline{2}} \overline{\mathrm{T}} \overline{\mathrm{TE}} \overline{0} \overline{3}$

$2.0067 E \quad 02$

$5.1380 \mathrm{E} 01$

$8.6885 \mathrm{E} \quad 01$

$4.469 \mathrm{EE} 0 \mathrm{~T}$

$6.12 \overline{1} \overline{\mathrm{O}} \mathrm{O}$

$9.5892 \mathrm{E} \quad 02$

$2.0040 \mathrm{E} 0 \mathrm{3}$

$7.9576 \mathrm{E} \quad 01$

$1.5657 \bar{E}-02$

$\overline{2} . \overline{0} \overline{9} \overline{3} \overline{\mathrm{O}} \mathrm{0} 2$

$1.6737 \mathrm{E} 02$

$2.5414 \mathrm{E}-02$

$2.5292 \mathrm{E} \quad 02$

$2.4756 \mathrm{E} 02$

$3 . \overline{3} \overline{30 E} 0 \overline{2}$

$1.6787 \mathrm{E}-02$

$1.7247 \mathrm{E} 03$

$2.5508 E$ E.2

$6.224 \mathrm{IE} 02$

$6 . \overline{19} \overline{35 E} \overline{0} \overline{1}$

4.205IE 02

$1.0704 \mathrm{E}-\overline{0} 3$

2.5740E 02

$2.1857 \mathrm{E}-02$

1.7 İ⿴囗十 02

$2.7186 E_{1} 02$

$5.849 \mathrm{TE} 01$

4. $0709 E-02$

$2.0599 E-02$
$3.2196 \mathrm{E}-03$

$9.9046 E=04$

1. Tि $\mathrm{TE}=03$

$2.7728 E-03$

$3 . \overline{3} 214 \mathrm{E}-0 \mathrm{3}$
1. $6807 \mathrm{E}-03$

1. $8430 \mathrm{E}-03$

$1.9332 \mathrm{E}=03$

2.0 $\overline{7} \overline{9} \overline{\mathrm{C}}=0 \overline{3}$

$3.7600 E-03$

$3.7985 E=03$

$2.4324 E-03$

$2.5009 \bar{E}=03$

$2 . \overline{5} \overline{4} \overline{2 E}=\overline{0} \overline{3}$

2.6447E-03

$2.8 \overline{0} \overline{3} \bar{E}=\overline{0} 3$

$2.9091 E-03$

$3.0352 E-03$

$\overline{3} .25 \bar{T} \overline{\mathrm{E}}-0 \overline{\mathrm{O}}$

3. $3838 E-03$

$1.8725 \mathrm{E}-03$

3. $7155 \mathrm{E}-03$

$3.7593 E-03$

4.000 $9 \overline{9 E}-\overline{0} \overline{3}$

4. $1024 E-03$

4. $107 \overline{4}=0 \overline{3}$

4. $3939 E-03$

$4.5612 \bar{E}=03$

$4.6 \overline{94} \overline{\mathrm{E}}=\overline{0} \overline{3}$

$4.7652 E-03$

4. $9847 E-03$

\section{ETA}

$8.0000 \bar{E}-\bar{O}$

$3.1818 \mathrm{E}=01$

7.0000E-0I

$8.1818 E=01$

$6.8750 E-01$

$3.6 \overline{170 \bar{E}}=0 \overline{1}$

$6.6667 E-01$

$6.6667 \mathrm{E}-01$

4.8276E-01

$4.6429 \bar{E}-01$

$7.0000 \bar{E}=0 \overline{1}$

$6.6667 \mathrm{E}-01$

$1.7696 \mathrm{E}-03 \mathrm{3}-6.666 \overline{7 E}-01$

$6.6667 E-01$

$6.66 \overline{6} \overline{\mathrm{E}}=\overline{0} \overline{1}$

$6 . \overline{6} \overline{6} \overline{7} \overline{\mathrm{E}}-\overline{0} \overline{\mathrm{T}}$

$2.6866 \mathrm{E}-0.1$

$4.4 \overline{1 B E}=01$

$6.6667 E-01$

$6.6 \overline{6} \overline{7} \overline{\mathrm{C}}=\overline{0} \overline{1}$

$\overline{6} . \overline{6} \overline{6} \overline{\overline{7}} \overline{\mathrm{E}} \overline{\mathrm{B}}=\overline{0} \overline{1}$

$6.6667 E-01$

$6 . \overline{6} \overline{6} \overline{7} \overline{\mathrm{E}}=\overline{0} \overline{1}$

$6.6667 E-0$

$6.6667 \mathrm{E}=0 \mathrm{~T}$

$6.666 \overline{7 E}=01$

$6.6667 \mathrm{E}-01$

$7.5000 \bar{E}-01$

$6.6667 \mathrm{E}-01$

$6.6667 \bar{E}=01$

$6 . \overline{6} 6 \overline{6} \overline{\mathrm{E}} \overline{\mathrm{E}}=\overline{0} \overline{\mathrm{T}}$

$6.6667 E-01$

$6.6 \overline{6} \overline{7} \overline{\mathrm{E}}=\overline{\mathrm{E}}=\overline{\mathrm{T}}$

$6.6667 \mathrm{E}-01$

$6.6 \overline{6} 6 \overline{7 E}=\overline{0} \overline{1}^{-}$

$6.6667 \mathrm{E}=0 \overline{1}$

$\frac{6.6667 E}{6.6} \frac{6}{6} \frac{01}{6 \mathrm{~F}}=01$

$\frac{6}{6.66667 E-01}$ 


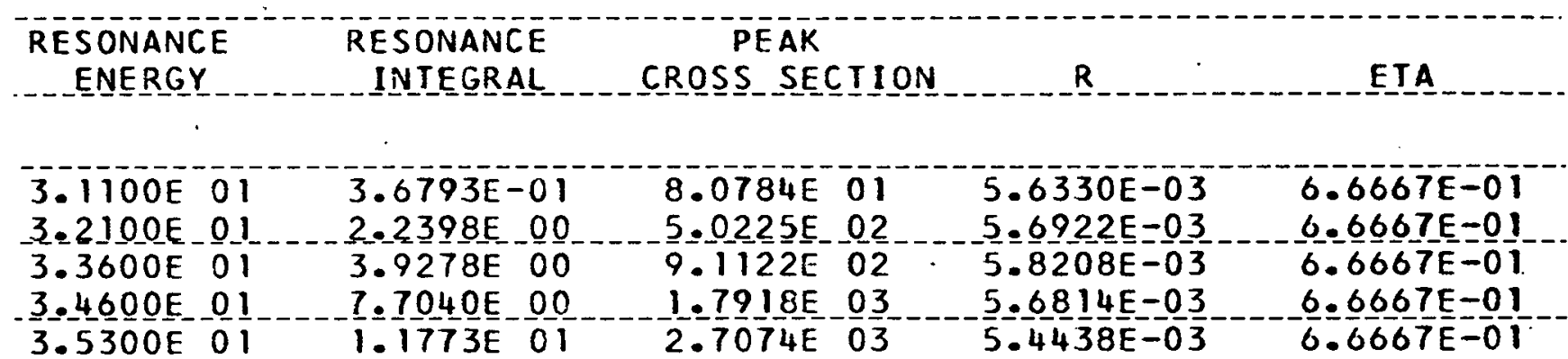


URANIUM 235

TRANSFERS FROM GROUPS 1 THROUGH 34 TO GROUPS 1 THROUGH 11

$$
\text { Ni } 2
$$

SI GMA N,G (NIIN2)

$0.12 .6856 \mathrm{E}-02-6.6 \frac{1}{246 E-02} 2.7 \frac{1}{2} 14 \mathrm{E}-01$

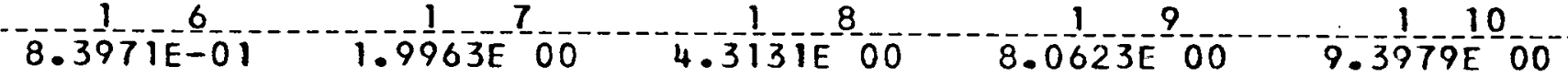

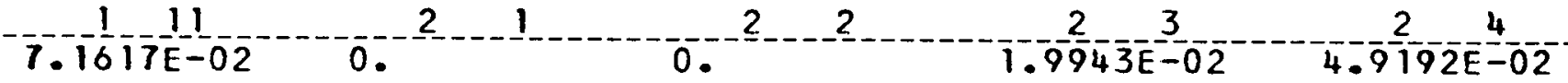

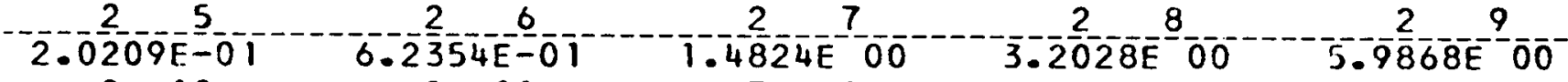

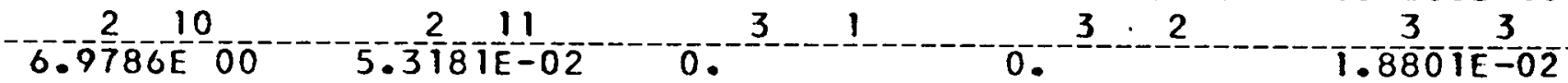

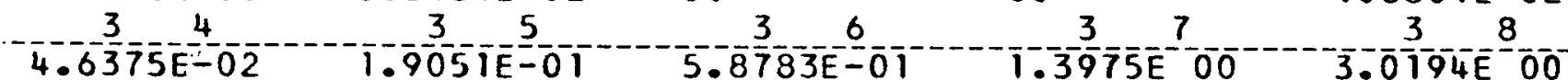

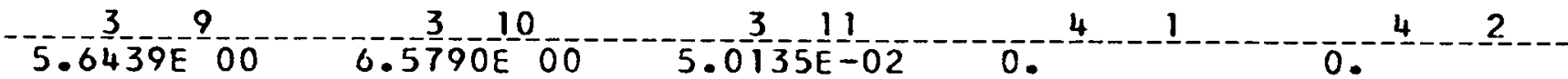

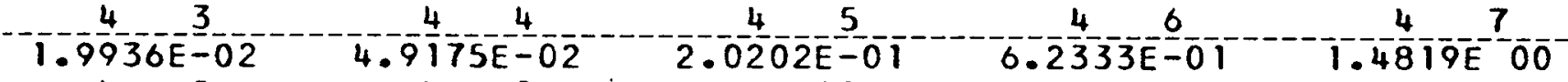

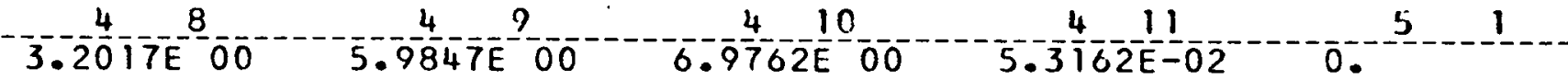

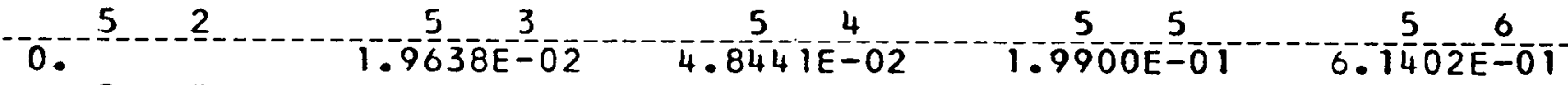

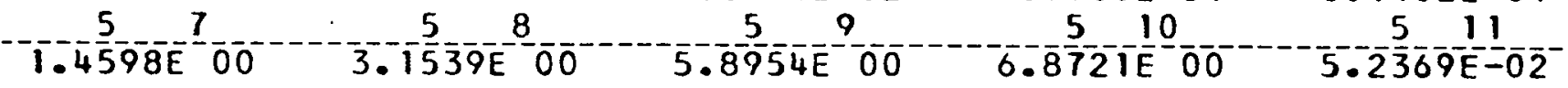
-0.

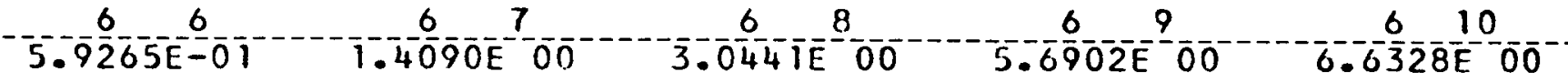

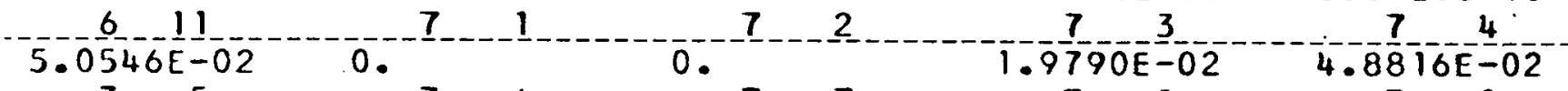

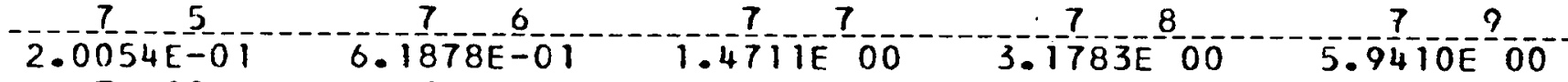

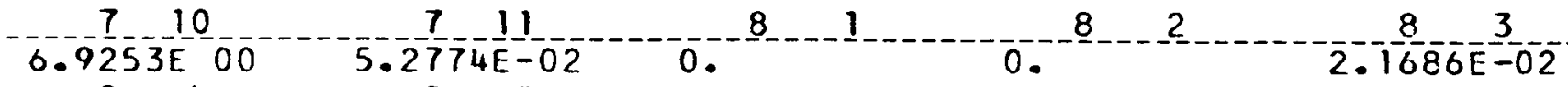

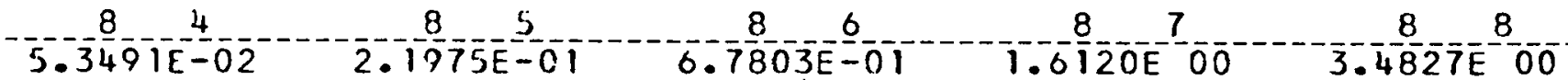

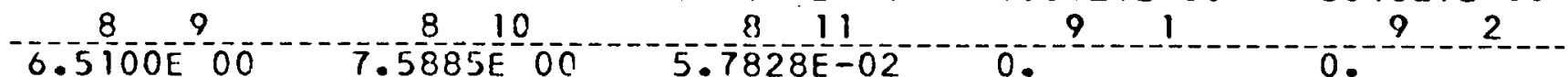

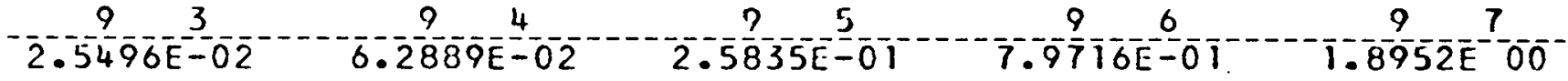

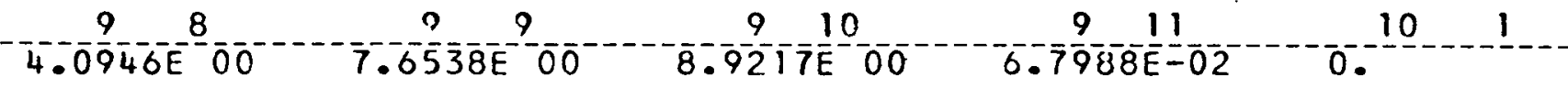


URANLUM 235.

N1

SIGMA N,G (NIIN2)

10210301010

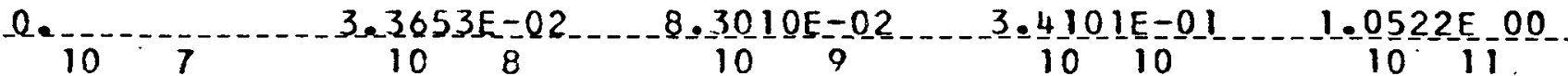

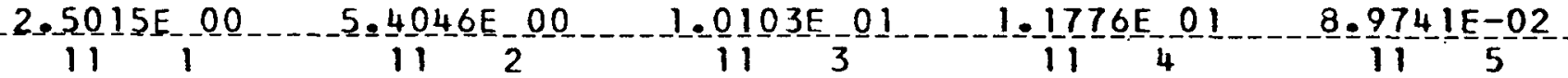

Q.

$1.4655 \mathrm{E}-00$

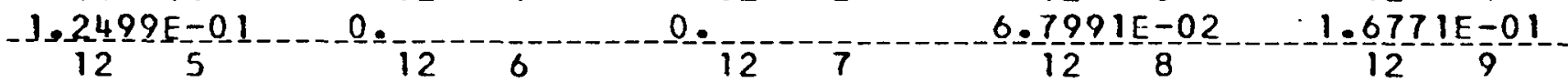

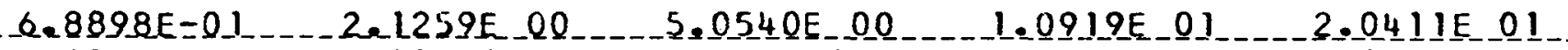
1210
1211
131
132
133

2.3792E_01__- 1.813]E-01___ 0 .

$13 \quad 4 \quad 13 \quad 5$

$13-6$

0.

$13-\overline{7}$

$9.3337 E-02$

2. $3023 \mathrm{E}=01$

13

1310

1311

$6.9380 E$. 00

138 $2.8020 E_{3} 01-3.2662 E_{4} 01$

14

$1.4990 E-01$

0.

0.

$1.3825 E-0$

$3.4 \frac{4}{14} 01 E-0$

$1.4009 E_{-10} 00$

$4 \cdot 3225$

$14-7$

148

4. $1502 E$

$4=8377 \mathrm{E}$

$-142-11$

$1.0276 E$ 요.

.2. 2202 E

15 - 3

$\left.3=\frac{6}{15} 606 \mathrm{E}-0\right)$

0.

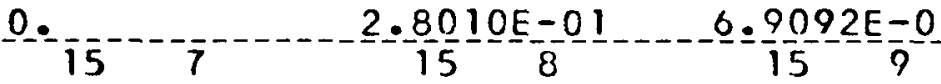

2.082IE 01

$8.4087 E \quad 01$

$2.8384 \mathrm{E} \quad 00$

$15-10$

$8.7579 \mathrm{E} 00$

$$
16
$$

0 .

16 .

$1.3976 \mathrm{E} \quad 01$

0.

163

$9.8018 E_{-} 0$ 1511

1611

1. 1920 E OO

3.32260

4. $4699 \mathrm{BE}-01$

164

7.4694 E-01. 175

$8.9607 \mathrm{E} \quad 00$

1710

$Z=\frac{1}{17} 860501$

1.1026 E 0

$4.5294 \mathrm{E} 00$

1711 184

o.

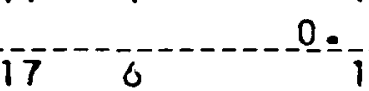

17

$.3418 \mathrm{E} \quad 02$

$16-10$

$2288 \mathrm{E} 00$

189

18

$6.5731 E$ OI

$.8428 \mathrm{E}-0$

$1.5641 E_{4} 02$

$1.3264 \mathrm{E}-0$

18 10

18 i

$1.420 I E C 2$

$2 \cdot \frac{18}{17} 12 E_{9} 00$

3.92955

4. $5805 E$ E 02

0.

i

2.6546 튼

1.3090E 00

$4.0927 \mathrm{E} \quad 01$

9. $7300 \mathrm{E}$ O 1

2. $1022 E \quad 02$

3 -4906E 00

ㅇ. 
URANIUM 235

NI N2

SIGMA N,G (NIIIN2)

\begin{tabular}{|c|c|c|c|c|}
\hline $4.8909 \mathrm{E}-01$ & $1.2064 E^{-4} 00$ & $4.956 \mathrm{IE} 00$ & $1.5292 E^{6} 01$ & $3.6356 \mathrm{E} 01$ \\
\hline 8 & $19 \ldots 2$ & 10 & 19 & 20 \\
\hline $\begin{array}{l}7.8548 \mathrm{E} \\
20\end{array}$ & $\begin{array}{c}1.4682 \mathrm{E} \quad 02 \\
20\end{array}$ & $\begin{array}{l}1.7115 E^{2} 02 \\
20\end{array}$ & $\begin{array}{l}1.3042 \mathrm{E} \\
20\end{array}$ & 0.20 \\
\hline & $5.7386 E-01$ & $1.4155 E_{0} 00$ & $5.8151 E 0$ & $1.7943 \mathrm{E} 01$ \\
\hline $.2657 \mathrm{E} 01$ & $9.2162 \mathrm{E} 0 \mathrm{I}$ & $1.7227 \mathrm{E} \quad 02$ & $2.0081 E 0$ & $1.5303 E$ \\
\hline & & $1.1411 \mathrm{E} 00$ & $2.8148 \mathrm{E} 00$ & $1.1563 \mathrm{E} 01$ \\
\hline$-\frac{21}{-5679[0}-61$ & $-3 . \frac{21}{482} \bar{E}^{-7}-1$ & $1.8 \frac{21}{32} 6 E^{8} 02$ & $3 . \frac{2}{3} 56 E^{9} 0$ & $-\frac{2}{3}-\frac{1}{9}-1 \frac{10}{10}$ \\
\hline $21_{\ldots} \ldots 11$ & 22 & 23 ? & 22 & 22 \\
\hline $\begin{array}{l}3.0430 \mathrm{E} 00 \\
22\end{array}$ & 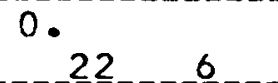 & 0.22 & $\begin{array}{l}3.0167 \mathrm{~B}_{2} 00 \\
22\end{array}$ & $\begin{array}{l}7.4411 \mathrm{C}^{0} \\
22\end{array}$ \\
\hline $\begin{array}{c}3.0569 \mathrm{E} 01 \\
2210\end{array}$ & $\begin{array}{c}9.4321 E ~ \\
22\end{array}$ & $\begin{array}{c}2.2424 \mathrm{E}, 02 \\
23\end{array}$ & $\begin{array}{l}4.8448 \mathrm{E} 02 \\
23\end{array}$ & $\begin{array}{l}9.0560 \mathrm{E}_{3} \\
23\end{array}$ \\
\hline $\begin{array}{l}.0556 E^{-} 03 \\
23\end{array}$ & $\begin{array}{c}8.0444 \mathrm{E} 00 \\
235\end{array}$ & 236 & 0 & $4.7394 \bar{E}$ \\
\hline $\begin{array}{r}1.1690 \mathrm{E} \\
23\end{array}$ & $\begin{array}{c}4.8026 \mathrm{E} O 1 \\
23 \quad 10\end{array}$ & $\begin{array}{c}1.4818 \mathrm{E} \\
23 \quad 11\end{array}$ & $\begin{array}{c}3.5229 E 02 \\
24\end{array}$ & $\begin{array}{c}7.6114 E \\
24\end{array}$ \\
\hline $.4228 \mathrm{E}$ & $1.6585 \mathrm{E} 03$ & $1.2638 \mathrm{E} 01$ & 5 & 0 \\
\hline $7.7934 \mathrm{E}^{-} 0$ & $1.92224 E^{-2}$ & $7-\frac{24}{8973}=5$ & $-\frac{2}{4} 367 E^{6}$ & 24 \\
\hline 24 & 24 & 10 & $24-11$ & 25 \\
\hline $\begin{array}{l}1.2516 \mathrm{E} \\
25\end{array}$ & $\begin{array}{c}2.3396 E 0 \\
25\end{array}$ & $\begin{array}{r}2.7272 E 0 \\
25\end{array}$ & $\begin{array}{l}.0782 E \\
25\end{array}$ & \\
\hline & $\begin{array}{l}1.2917 E_{2} 01 \\
25\end{array}$ & $\begin{array}{l}3.1861 \mathrm{E} \\
25\end{array}$ & $1.3089 \mathrm{E} 02$ & $4.0386 E$ \\
\hline $\begin{array}{l}9.6014 \mathrm{E} 02 \\
26\end{array}$ & $\begin{array}{c}2.0744 \mathrm{E} 03 \\
26\end{array}$ & $\begin{array}{c}3.8776 E 03 \\
26\end{array}$ & $4.5200 \mathrm{E} \quad 03$ & $\begin{array}{c}3.4445 \mathrm{E} \\
26 \mathrm{~S}\end{array}$ \\
\hline & & $1.2926 \mathrm{E} 01$ & $3.1885 E 0$ & $1.3099 \bar{E} 02$ \\
\hline $4.0416 \mathrm{E} 02$ & $9-\frac{26}{9086 \bar{E}}=\frac{7}{02}$ & $-2.0 \frac{26}{760 E^{-}}-\frac{8}{0}$ & $3 . \frac{26}{80}-95$ & $\frac{26}{6}-\frac{10}{3}-\frac{10}{\mathrm{~F}}$ \\
\hline $26-11$ & 27 & 27 & $27 \quad 3$ & 27 \\
\hline $\begin{array}{c}3.4470 \mathrm{E} O \\
275\end{array}$ & 27 & & $\begin{array}{r}-294 \mathrm{E} \\
27\end{array}$ & $\begin{array}{c}1922 \mathrm{E} \\
27\end{array}$ \\
\hline.$\overline{3} 1 \overline{14 E}$ & $\begin{array}{l}4.0463 E^{2} \\
27\end{array}$ & $9.6197 \mathrm{E} 02$ & $2.0784 \mathrm{E} 0 \overline{3}$ & $3.8850 \bar{E}^{-} 0 \overline{3}$ \\
\hline$-\frac{2}{5}$ & $x^{-1}$ & 10 & & $1 . \overline{2} \overline{59} \overline{0 E}$ \\
\hline
\end{tabular}


-URAVLUM_-232.

N 1

N2

SIGMA N,G (NIINZ)

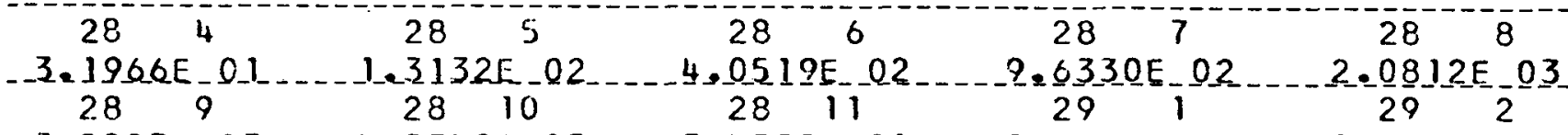

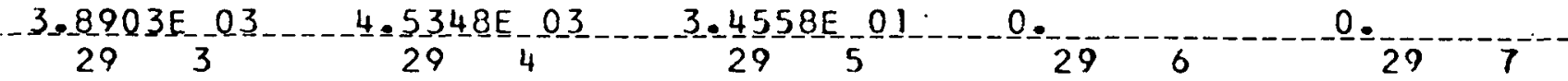

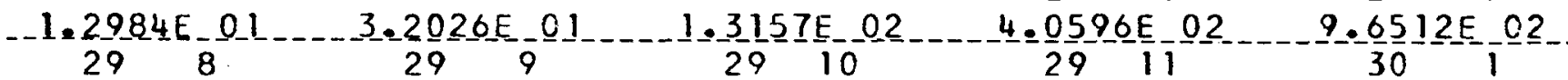

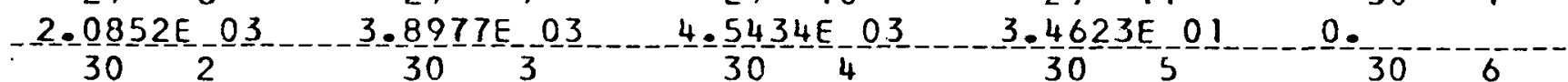

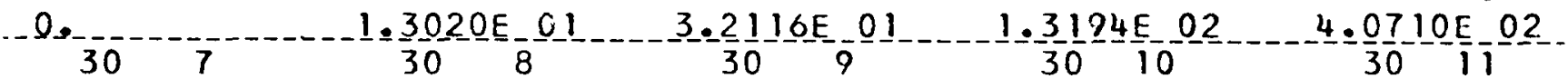

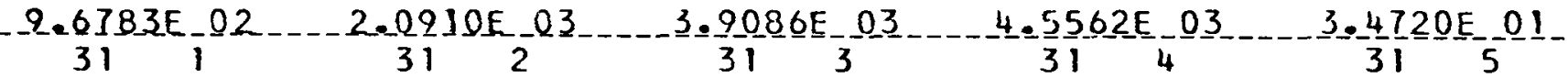

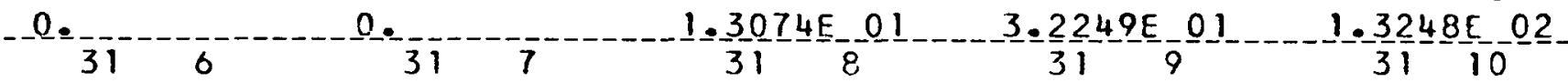

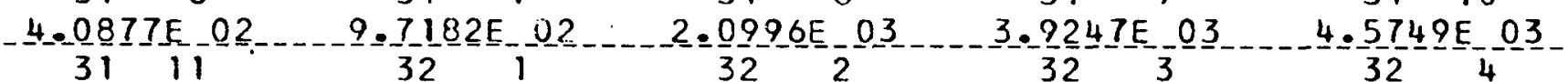

$3.4863 E_{5} 01$

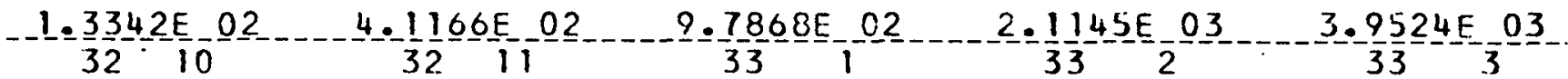

$-4.6072\left[\begin{array}{l}3 \\ 3\end{array}\right.$

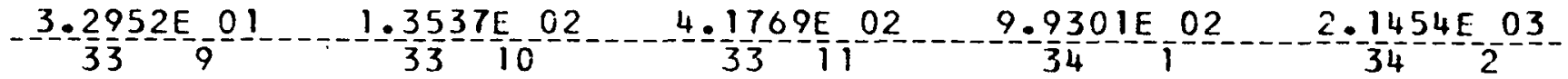

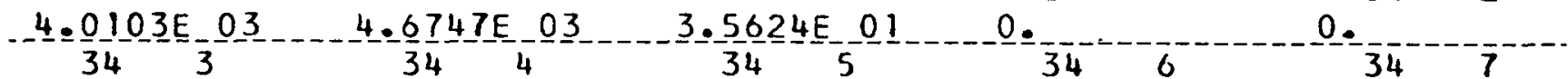

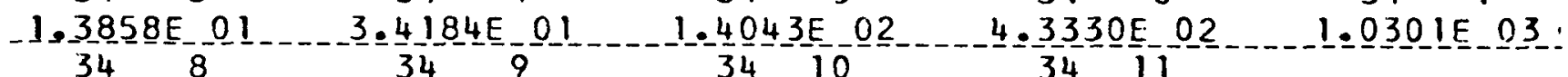

-2.2257E 03 
DES_LGNATION CODE NO.

DENSIIY EACIOR

URANIUM 238

92.2380

$3=95248 E-02$

SIGMA
LEVEL ENERGY SIGMA

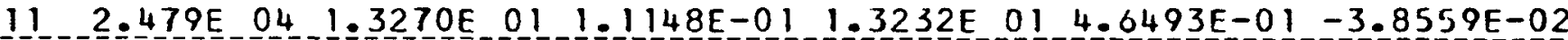

$129.119 \mathrm{E} 031.4059 \mathrm{E} 011.1810 \mathrm{E}-011.4020 \mathrm{E} 010.3497 \mathrm{E}-01-4.0422 \mathrm{E}-02$

$13 \quad 3.355 E \quad 03 \quad 1.5074 E$ O 1 1.2662E-01 $1.5032 E$ O1 $9.9544 E-01-4.3340 E-02$

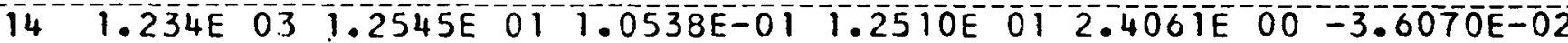

15 4.540E 02 1.8221E $01 \quad 1.5305 E-01 \quad 1.8170 E$ O $01 \quad 5.9857 E$ E0 $-5.2388 E-02$

$16 \quad 8.719 E \quad 01 \quad 5.2305 E$ OI $4.3936 E-01 \quad 5.2158 E$ OI $2.1926 E \quad 01-1.5039 E-01$

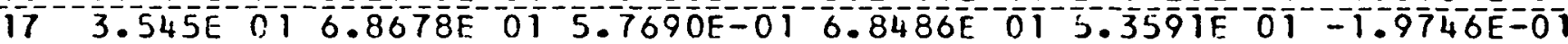
$18-1.304 \mathrm{E}-012.9210 \mathrm{E} \quad 01-2.4536 \mathrm{E}-012.9128 \mathrm{E} \quad 010.6547 \mathrm{E} \quad 01-8.3981 \mathrm{E}-02$

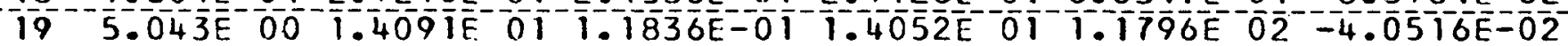

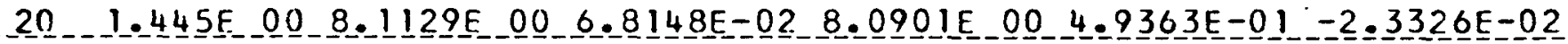

$21-6.826 E-018.2894 E-006-9631 E=02-8.2662 E-00-6.0122 E-01-2.3833 E-02$ 22 2.51TE-01 8.3449E 00 7.0097E-02 8.3215E 00-8.8630F-01-2.3993 -02 $23-9.237 \mathrm{E}-02 \quad 8.3671 \mathrm{E} \quad 007.0283 \mathrm{E}-02$ - $8.3436 \mathrm{E} \quad 00 \quad 1.4741 \mathrm{E} \quad 00-2.4057 \mathrm{E}-02$ $242.530 \mathrm{E}-028.34 \mathrm{TPE} 007.0072 \mathrm{E}-028.3185 \mathrm{E} 002.269 \mathrm{TE} 00-2.3984 \mathrm{~F}-02$

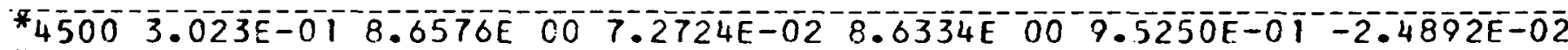
* $40002.718 \mathrm{E}-018.6576 \mathrm{E}$ 00 $7.2724 \mathrm{E}-02 \quad 8.6334 \mathrm{~F} \quad 00 \quad 9.3250 \mathrm{E}-01-2.4892 \mathrm{E}-02$

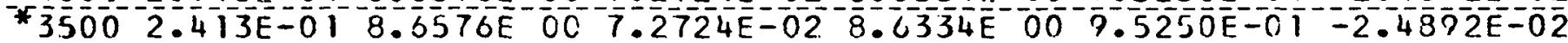
* $30002.109 \mathrm{E}-018.6576 \mathrm{E} \quad 007.2724 \mathrm{E}-02 \quad 8.6334 \mathrm{E} \quad 00 \quad 9.5250 \mathrm{E}-01-2.4892 \mathrm{E}-02$

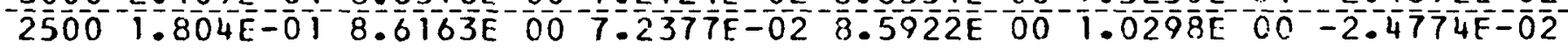

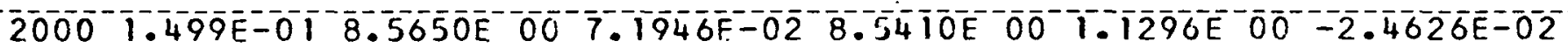

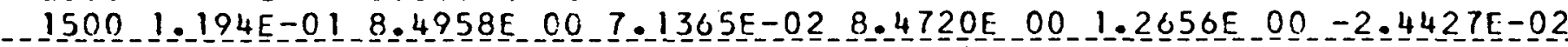
$1000-8.897 \mathrm{E}-02-8.3968 \mathrm{E} \quad 00-7.0533 \mathrm{E}-02-8.3733 \mathrm{E} 001.4664 \mathrm{E} 00-2.4143 \mathrm{E}-02$ 500 5.850E-02 8.2340E 00 6.9166E-02 8.2109E 00 1.8085E $00-2.3674 \mathrm{E}-02$ $68-3.216 \mathrm{E}-027.9182 \mathrm{E}-006.6513 \mathrm{E}-02-7.8960 \mathrm{E} 00-2.4389 \mathrm{E} 00-2.2766 \mathrm{E}-02$

Thermal cross sections taken from APEX-515.

NOTE: For a summary of the sources of data see APEX-704. 


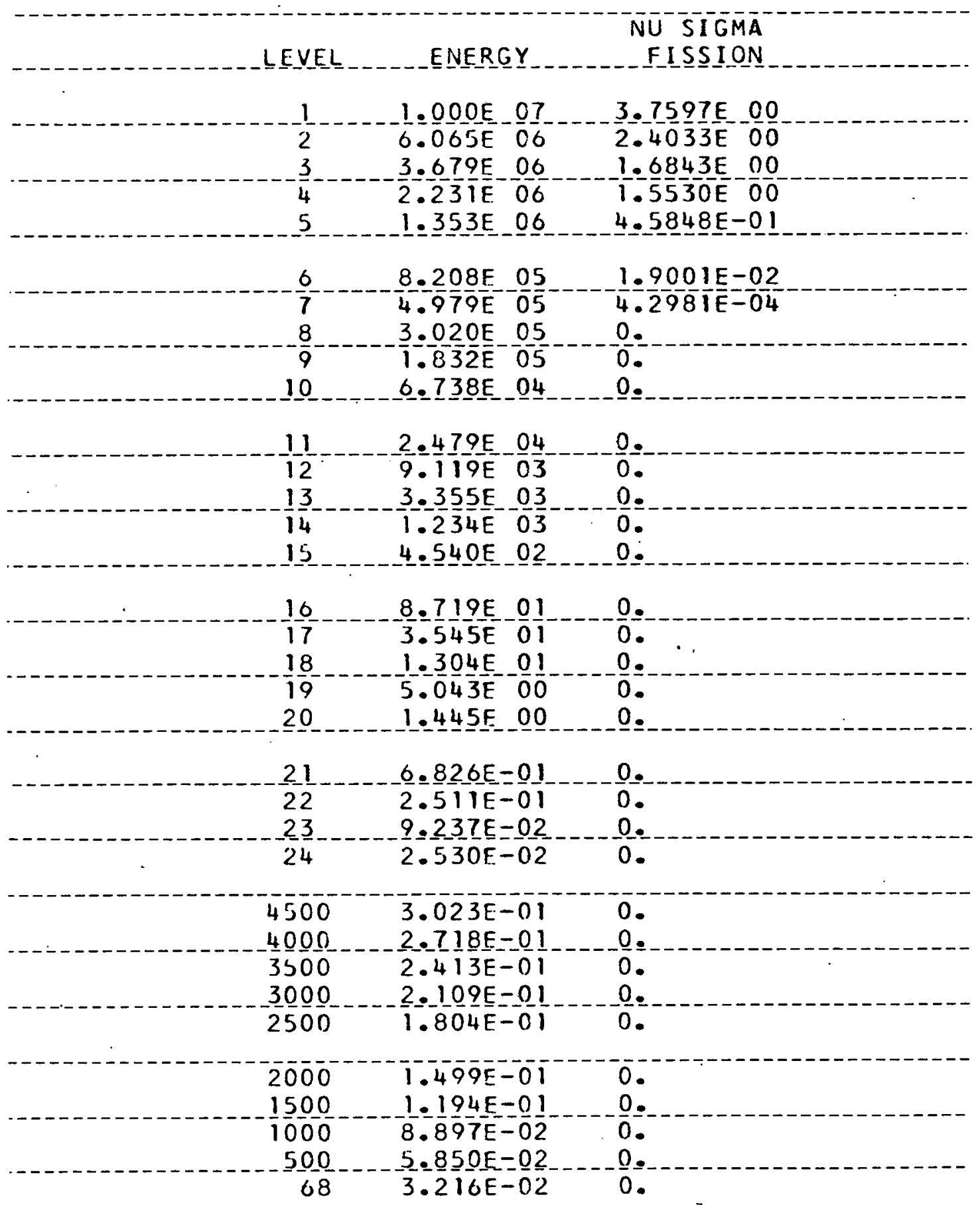


IEMPERA IURE DEG. $F$. $4.5000 \overline{0} 0 \overline{3}$
SIGMA

SCATIER
SIGMA

TRANSPORT
SIGMA ABSORPTION
NU SIGMA

FISSION
$9.8453 \mathrm{E} 00$

\section{0.}

SIGMA S (N $\frac{N}{1}(N 2)$

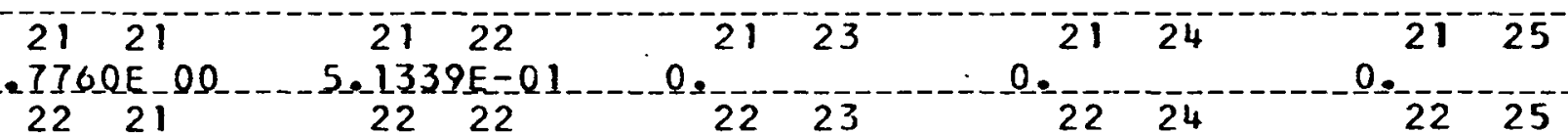

$4.4836 E=01$

2321

$24-21$

$25-21$

- 0.

2322

$9.6448 E=01$

2422

$2.9808 \mathrm{E}-0.5$

2522

0 .

\section{3}

$7.1703 \mathrm{E}-00$

2423

2.3264 E 00

2523

$1.9518 E=01$
$1.7500 E-06$

$$
2324
$$

2.6733E-01

2424

$5=1991 \mathrm{E}-00$

2524

$2.5850 E-00$
$23-25$

8. 5627 IE- 03

2425

9. $3 \frac{3090 \mathrm{E}-01}{25}$

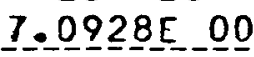

IEMPERAIURE

DEG. $F$.

$4.0000 \bar{E}$ O3
S I GMA

SCATTER

$9.8 \overline{36} \mathrm{BE} 0 \mathrm{C}$
SIGMA TRAN SPORT

$9.8088 \overline{0} 0$
SIGMA

ABSORPTION

$3.6423 \bar{E}$ OO
NU SIGMA

FISSION

\section{SIGMA $-\frac{N}{S}-\left(N \frac{N}{1}-\frac{1}{N}\right)$}

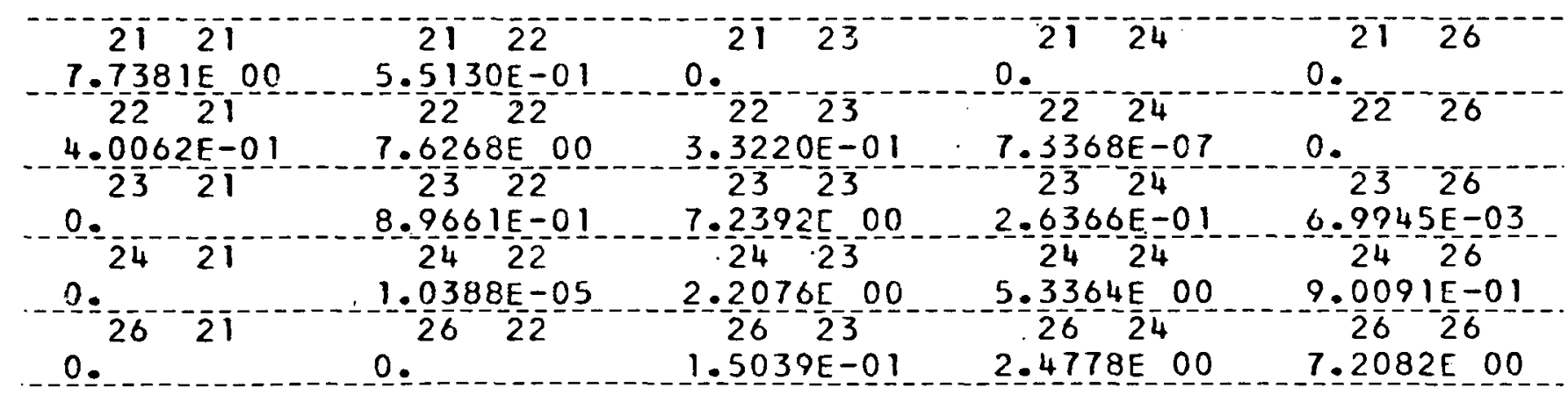


URANIUM 238

THERMAL TRANSFER MATRICES

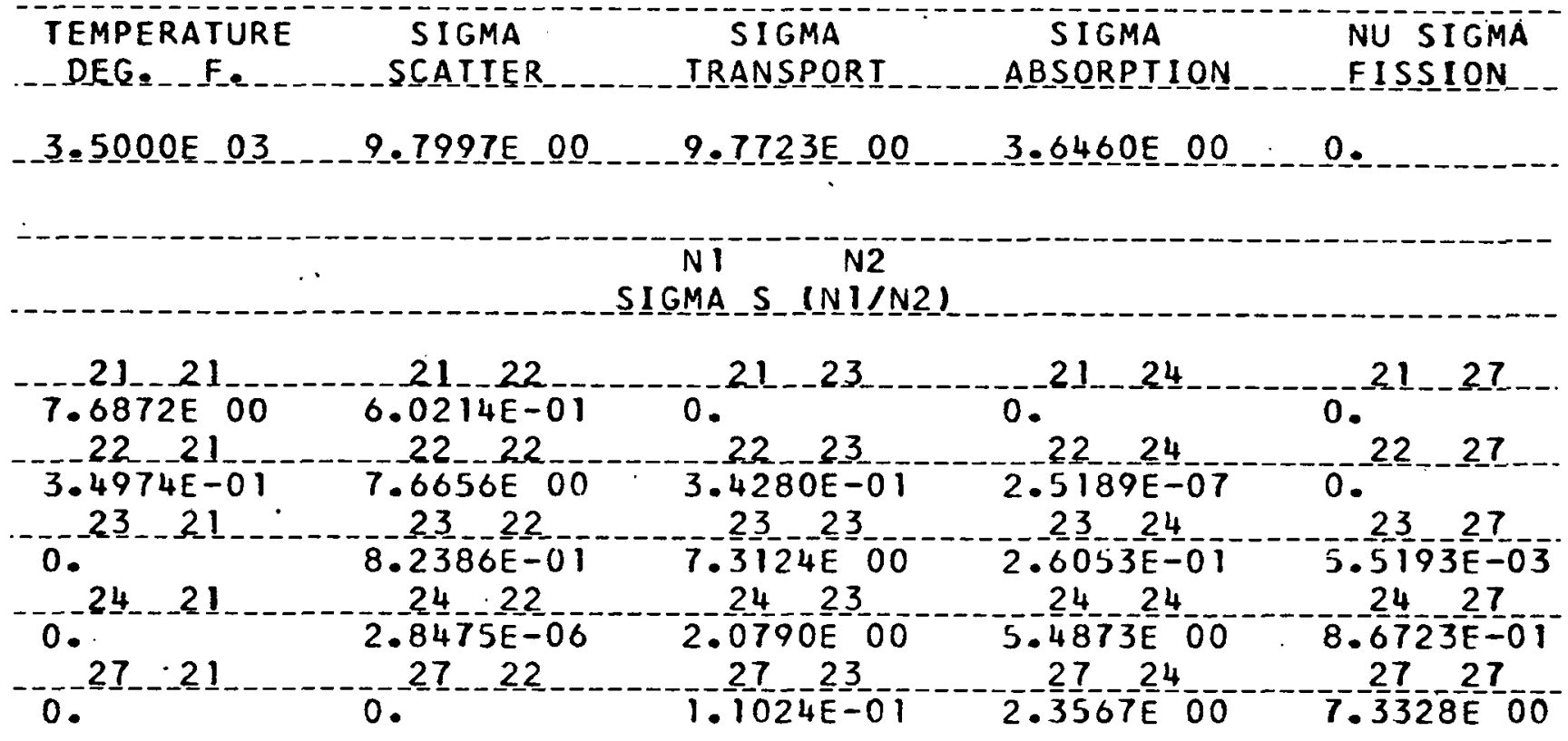

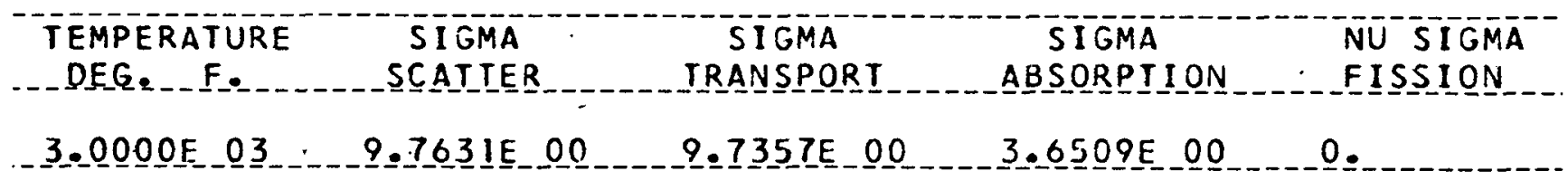

ni

SI GMA S (N1/N2)

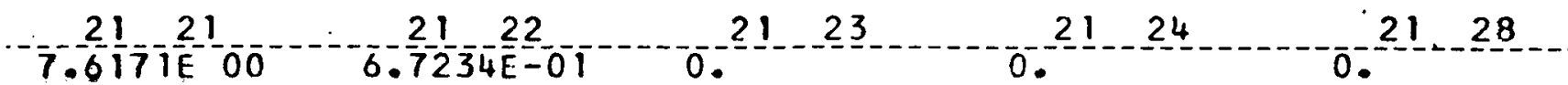

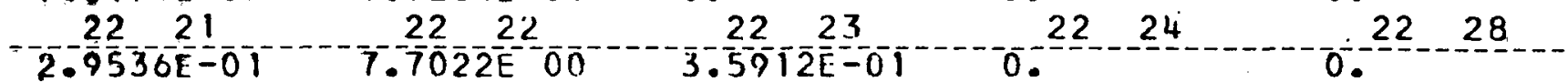

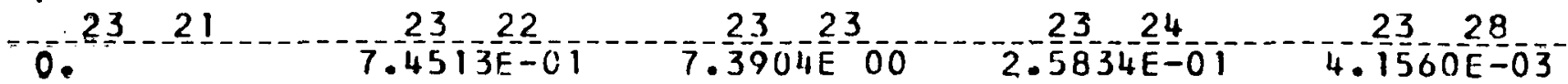

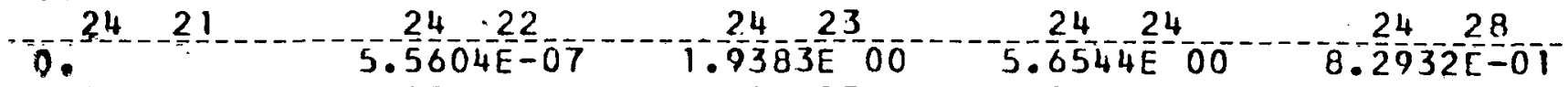

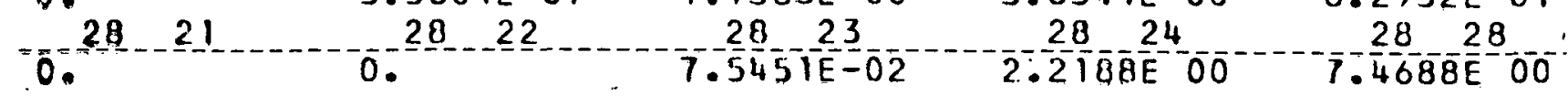


URANIUM 238

_IHERMAL_IRANSEER_MAIRICES

_ IEMPERA IURE

SI GMA

SIGMA

SIGMA

DEG. $F$.

SCATIER

TRANSPORT

ABSORPTION

NU_ SIIGMA

$2.5000 \mathrm{O} O \overline{3}$

$9.7263 \mathrm{E} 00$

$9.6991 \mathrm{E} 00$

$3.65 \overline{7} \bar{E} 00$

FISSION

SIGMA S $\frac{N 1}{N I / N 2)}$

$21^{2} i^{2} 1^{2} 21^{2} 3^{-24}$

7.5166E 00

22,21

$22 \quad 22$

$22-2 \overline{3}$

ㅇ. $22-24$

$22-29$

$-2.3724 \mathrm{E}=0 \mathrm{~L}$

$=\frac{8}{23} 7 \frac{75}{23}=0$

$23-24$

0

$0.0 .5888 E-01$

$7=\frac{4}{2} \frac{74}{4}-\frac{E}{2} 300$

$2.5 \frac{7}{24}-\frac{60}{24}=0$

$2.9288 E-03$

$-0 .-\frac{1}{2} \overline{1}$

292

1.78299600

$5.8411 E 00$

$7.8650 E-01$

-

0.

$4.6791 \mathrm{E}-02$

7.

IEMPERAIURE

DEG. F. SCATTER

$2.0000 \mathrm{E} 03$
SI IGMA

$9.6895 \mathrm{E}-0 \mathrm{O}$
SIGMA

TRANSPORT
S IGMA

ABSORPTION
NU SIGMA

FISSION

SIGMA S (NINA

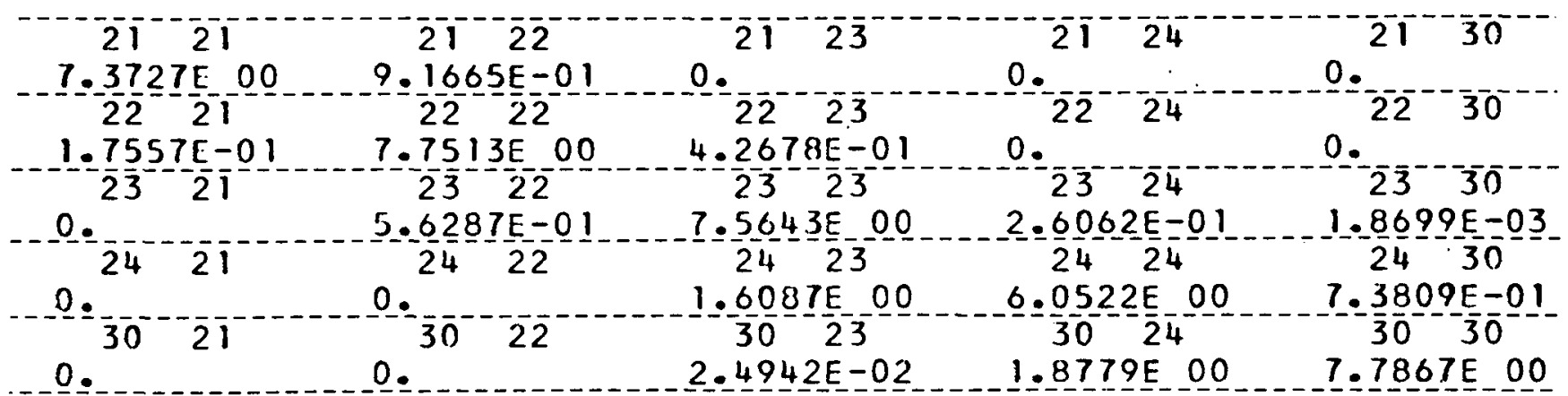


URANIUM 23

THERMAL TRANSFER MATRICES

TEMPERATURE
DEG SIGMA
1.5000E_03

$\overline{\mathrm{N}} \overline{\mathrm{T}}-\overline{\mathrm{N}} \mathrm{2}$

SIGMA S (iv1/N2)

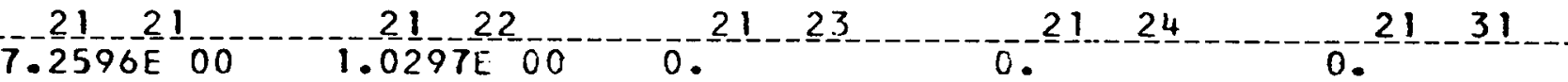

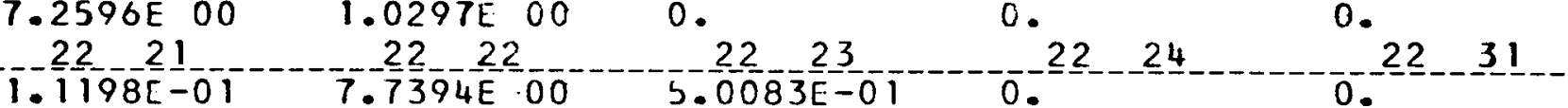

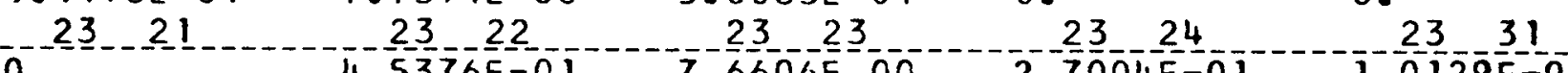

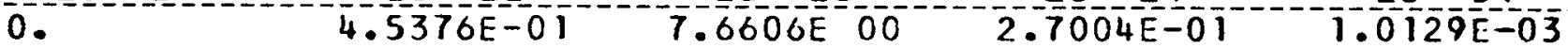

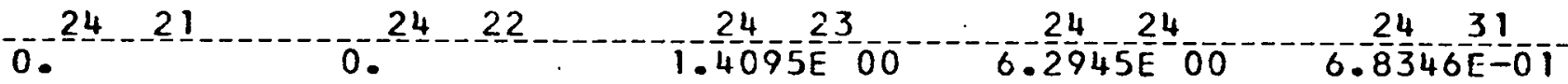

0.

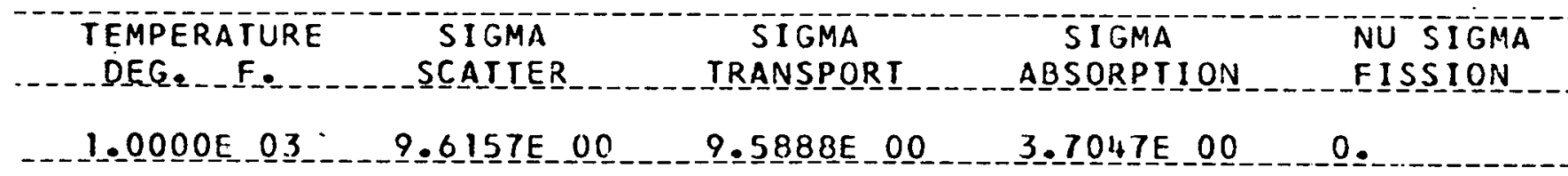

SIGMA S $(N 2$ I 1 N2)

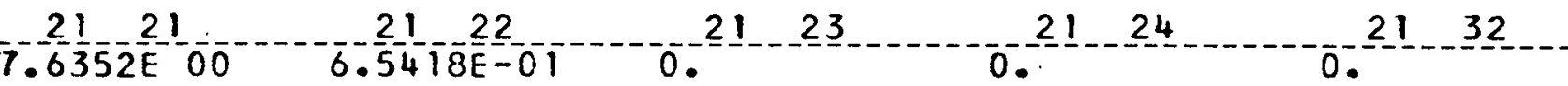

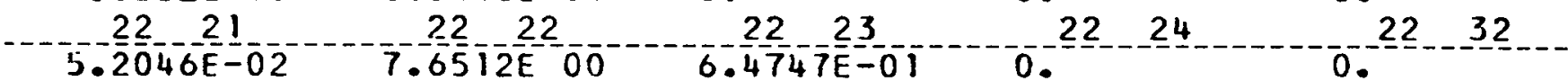

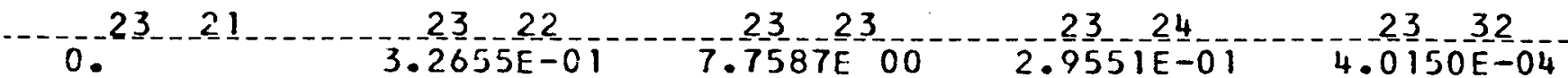

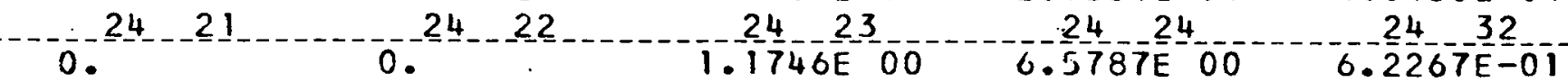

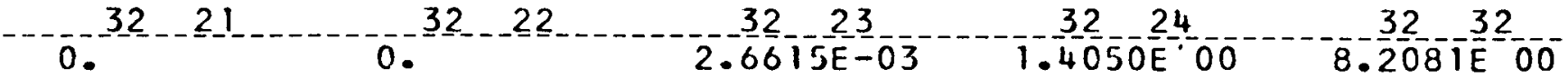


URAN IUM 238

IHERMAL_ IRANSEER_MAIRICES.

- LEMPERAIURE SIGMA

SIGMA

SIGMA NQ

DEG. F. SCATTER

TRANSPORT

ABSORPTION

FISSION

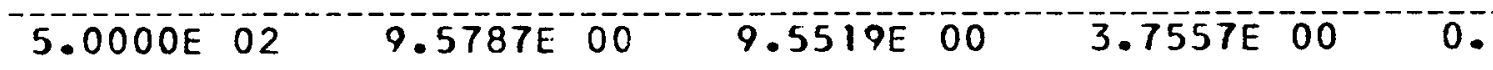

\section{$\left.S I G M \frac{N}{S}-(N) \frac{N}{S}-1 / 2\right)$}

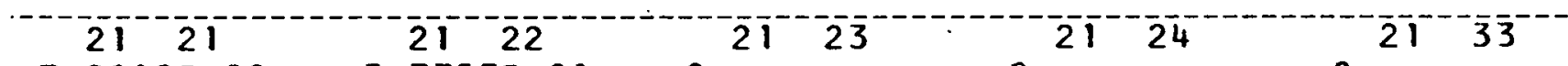
T.9118E_OO 2221 $22 \quad 22$ 을

I. $3442 E$ 2322 $9=\frac{36}{2} \frac{15}{2}=-0$ O. ㅇ.. $23-\frac{1}{1}$ .0. 24 $1.7586 E-01$ $7.8292 \mathrm{E}-00$ O. $\frac{}{2}--\frac{-}{24}$ 2233 - $0 .-\frac{1}{3} \overline{3}-$ $0 .-\frac{1}{3}-\frac{22}{22}$ $8.8204 \mathrm{E}-01$

3. $-7184 E-01$ $2 \overline{3}-\overline{3} \overline{3}$ 으. …

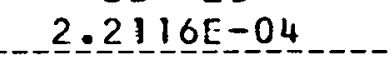
$6=\frac{92}{3} \frac{3}{3}-\frac{E}{2}-00$ $7=\frac{38}{24}-\frac{4}{33}-05$ (1) (1) 1.0805E 00 5. $-\frac{5}{3} \frac{9}{3} 13 E-01$ 8.4980으으.

- TEMPERAIURE

DEG. F.

$6.8000 \overline{0} 0 \overline{1}$
S I GMA

SCATTER
SIGMA TRANSPORT
SIGMA ABSORPIION
NU SIGMA.

FISSION

\section{SIGMA $\frac{N 1}{S}\left(N \frac{N 2}{N}-N 2\right)$}

$21^{2} 1^{-1} 1^{2}$

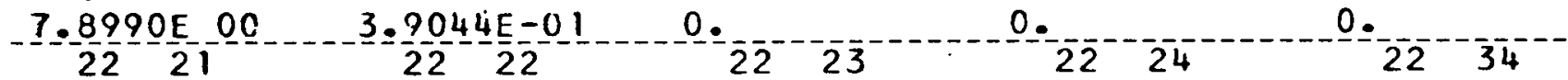

$1 \cdot \frac{3}{2} \frac{5}{3}-\frac{8}{2} \overline{1}-01$

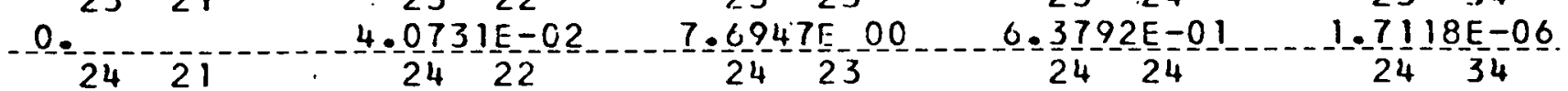

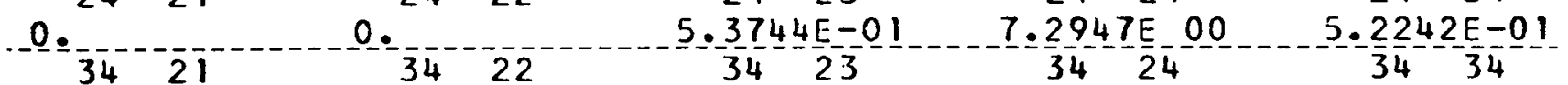
O. 
URANIUM 238

TRANSFERS FROM GROUPS I THROUGH 1 I TO GROUPS I THROUGH 16

$\mathrm{N} 1$ N2

SIGMA_SO (NIIN2)

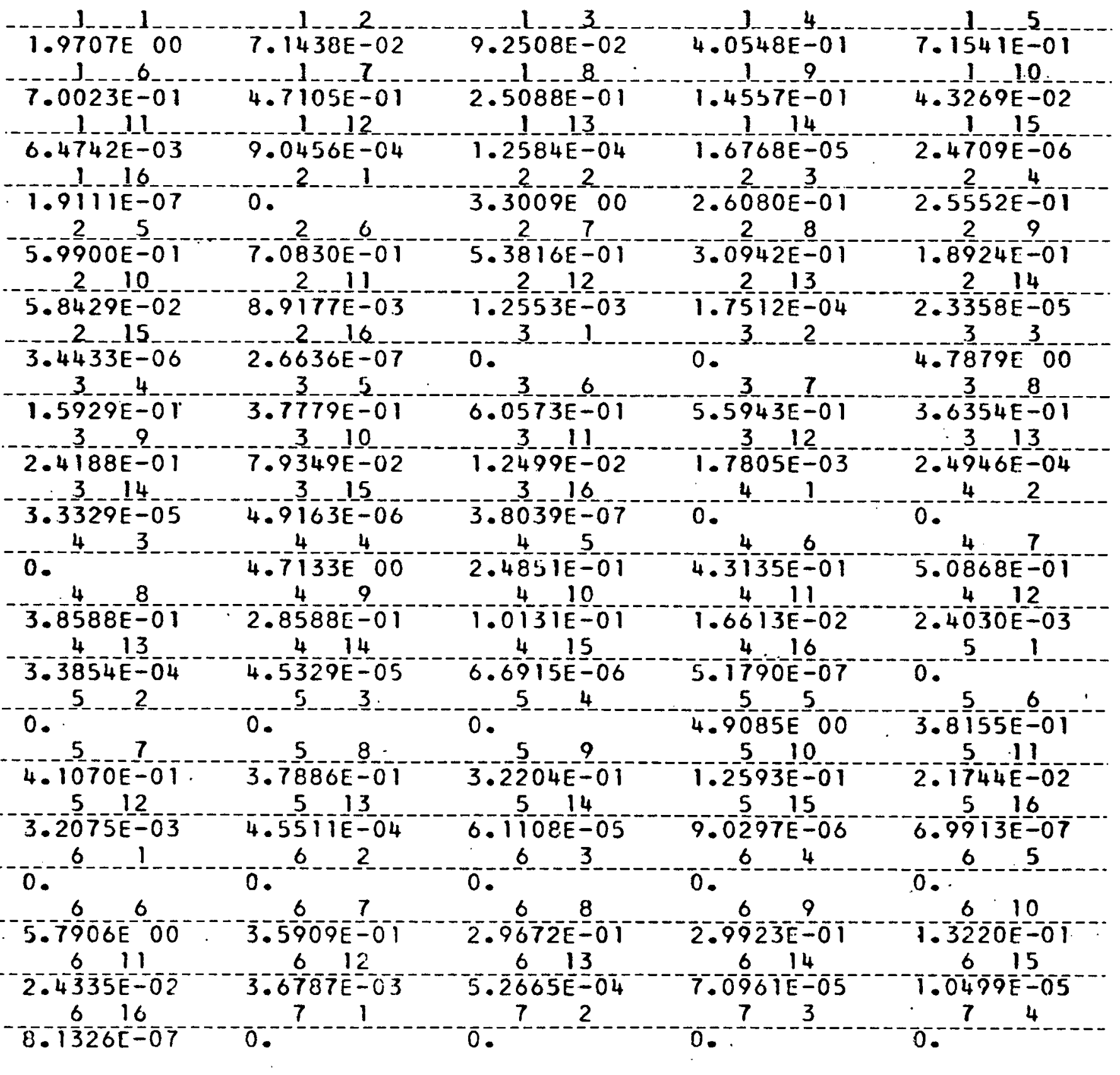


-URANIUM 23요...

SIGMA

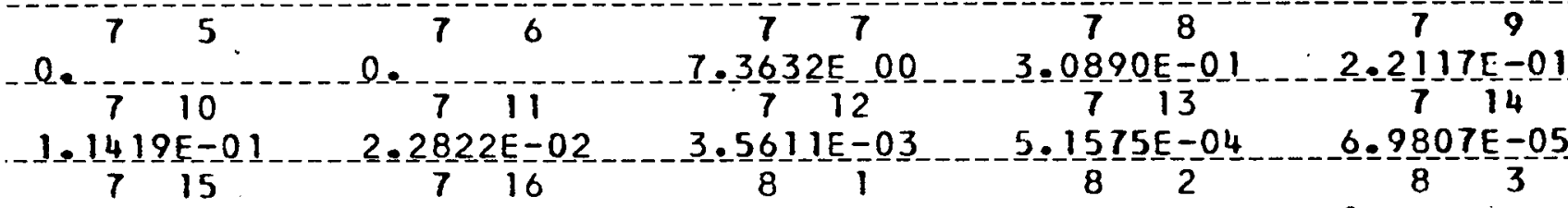

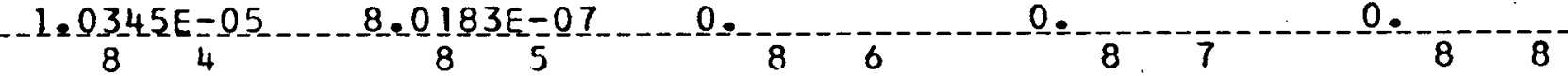

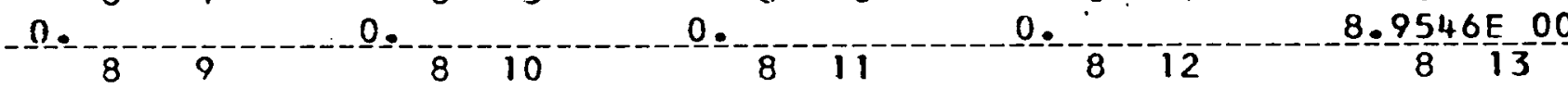

$2.480 .6 \mathrm{E}-01$

-6.4989E=05 $9.6508 \mathrm{E}=06$

$\begin{array}{cccccccc}9 & 3 & 9 & 4 & 9 & 9 & 9 & 9\end{array}$

$-0.01 .0537 E-01 \quad 4=0970 E=01 \quad 1.5751 E-02-2.7348 E-03$

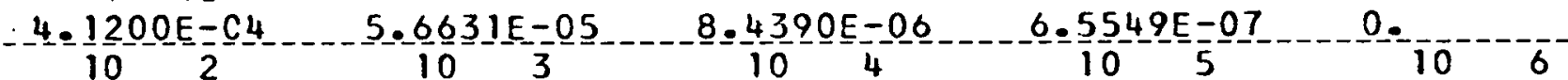

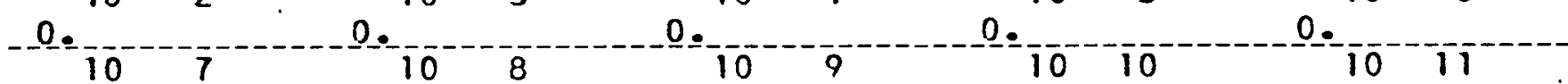

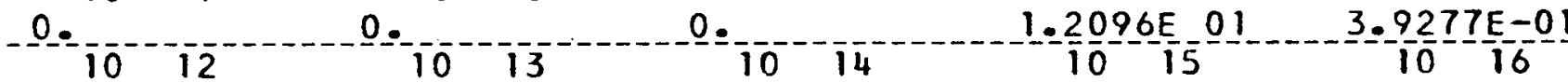

$1.8350 E-03 \quad 2.8988 E-04 \quad 4.0597 E-05 \quad 6.0905 E-06-4.7429 E-07$

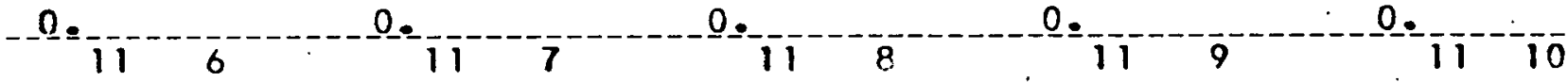

$00.111^{0}=11^{-11}$

$1.2931 E-01$ 
URANIUM 238

BESONANCE
ENERGY
INIESONANCE
INIER


URANIUM 238

TRANSFERS FROM GROUPS 1 THROUGH 34 TO GROUPS 1 THROUGH 11

SIGMA $N, G(N I T N 2)$

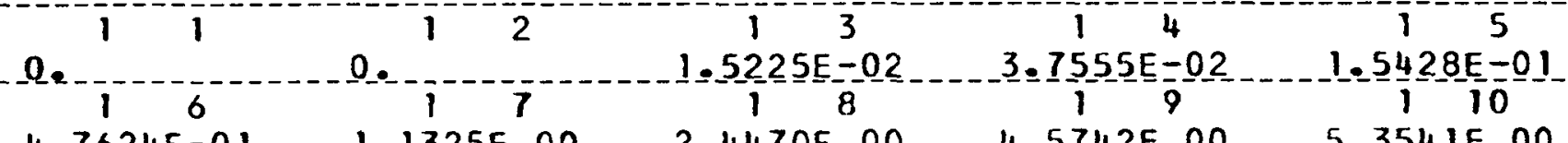

$4.7624 E-01 \quad 1=1325 E_{1} 00 \quad 2.4470 E-00 \quad 4.5742 E-00 \quad 5.3541 E 00$

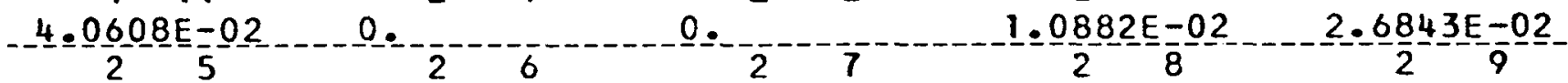

$1.1028 \mathrm{E}-01$

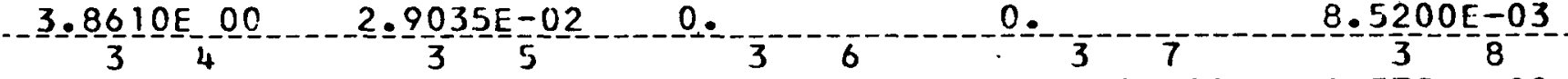

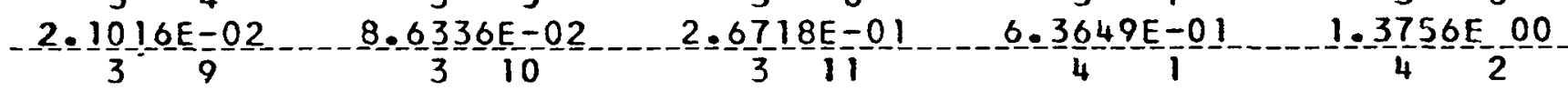

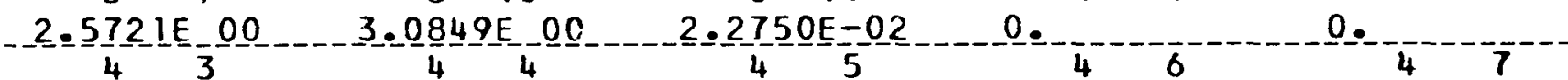

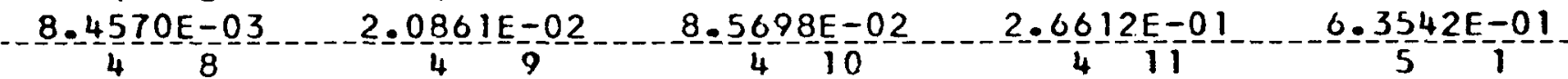

$1=37399 E_{2} 00$

‥

2. $0895 E-01$

D.

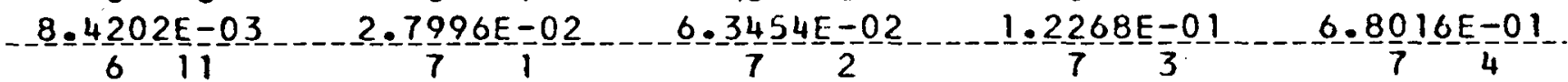

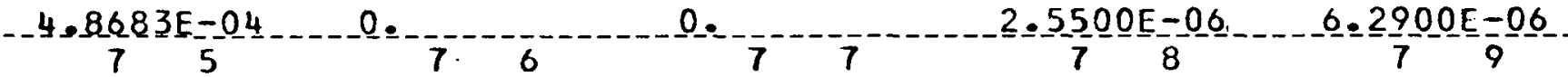

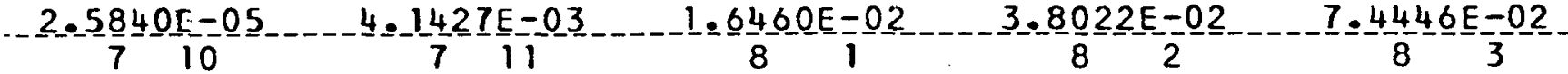

$5.3193 E-01$

-0.

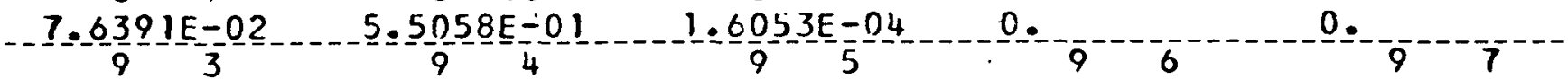

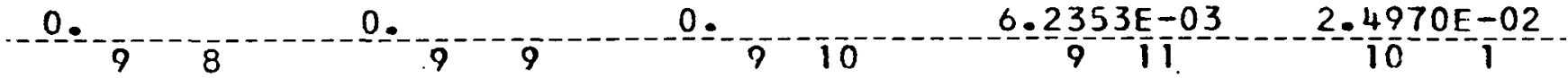

$5.7723 E-02 \ldots 1.1308 E-01 \ldots$ 
URANIUM 238

$\mathrm{N} 1 \mathrm{~N} 2$

SIGMA N,G (NIIN2)

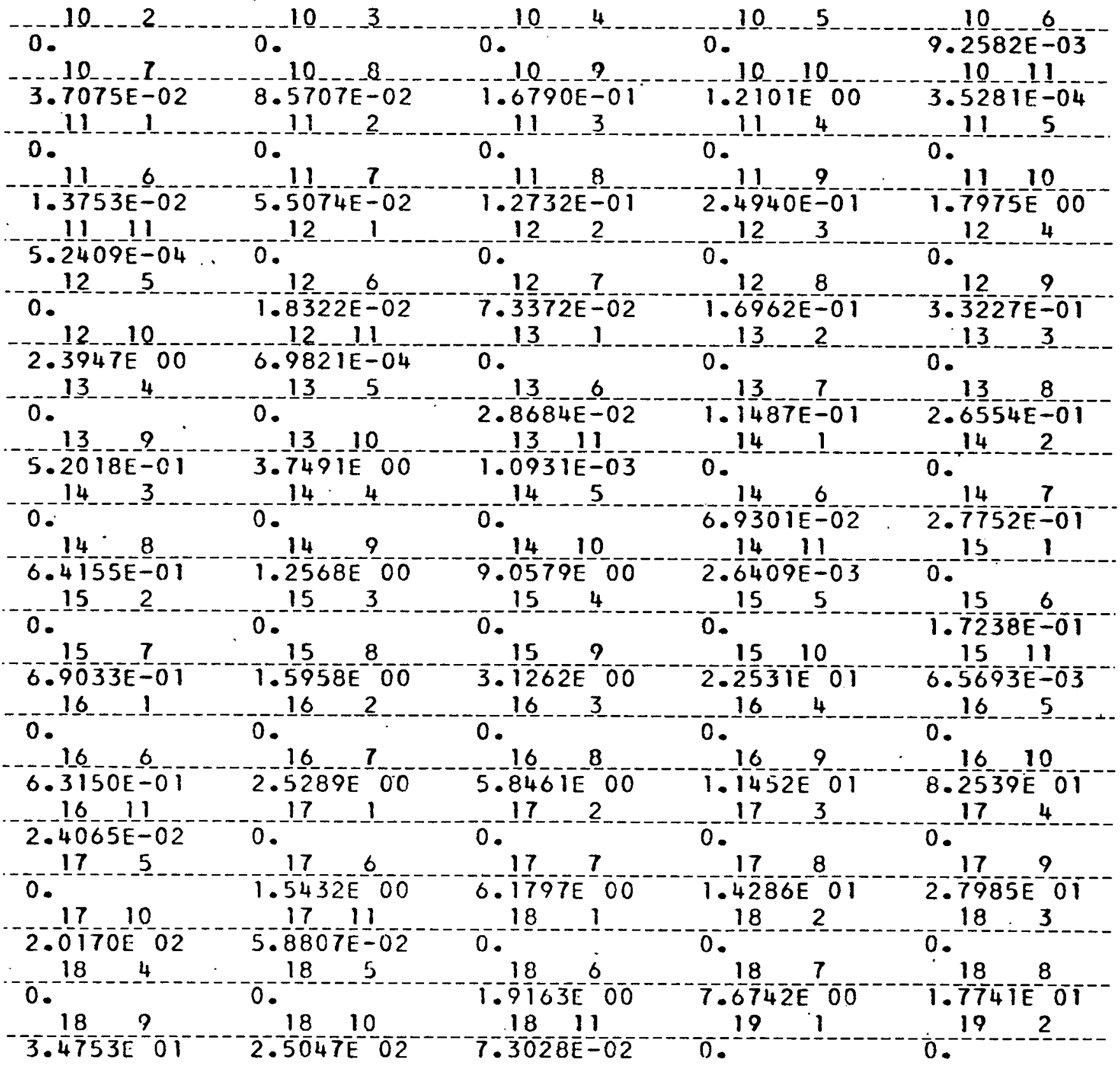


URANIUM 238

N1 1

\section{SIGMA N,G (NI/N2)}

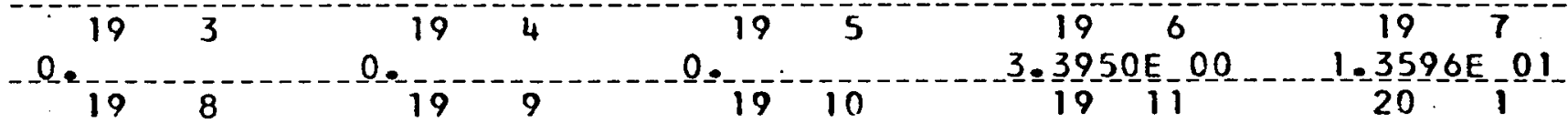

3. $1429 E$ E 01

6.15686

$4.4374 E-02$

ㅇ.

202

$\frac{1}{20}-68$

204

20

20

$-0=-20$

$0 .-20$

20

ㅇ..

$1.4213 E-02$

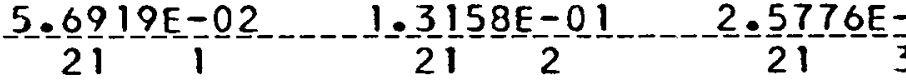

0.010

으.

ㅇ. 2011

$-1.7312 \mathrm{E}=02$

6. $2327 E-02$

1. $\frac{8}{2} 577 \mathrm{~T}-\frac{0}{4} 0$

$5=4165 E-04$

2111

22

$.6026 \mathrm{E}=0.1$

2

215

-6. 5972E=0

0.

0 .

22

(1)

210 (2)

$2.5521 E-02$

Q. 22

$\frac{1}{22} 395 E-01$

2. 2627 IE_OO

2210

22

23 -

2

0 .

224

$22-9$

3. $3357 E$ EO

9.7257E-04

0

232

4. 6283 E -01

$$
23
$$

235

$4.2444 E-0$

0

23

- 0.

$23^{10}$

$1=\frac{69}{2} 97 \mathrm{E} E=01$

23

239

5. 5477 E 00

$1.6175 E-03$

0.-

$24-16$

$3.9293 \mathrm{E}-01$

$-7.69726 E-01$ . 0.

$24-8$

$24^{-9}$

24

6. $6 \frac{6}{2} 661 E-02$

0 .

$24-7$

으. $\frac{1}{25} 172$ E -0.1

0. $2 \frac{2089 E}{3}$

$8.7129 \mathrm{E}$

$2.5403 E=03$

$2.6695 \mathrm{E}-01$ 257

0.

258

0.

0.

_4. 1967E-01

$9=7017 E=01$

$1.9005 E-00$

$25-10$

25

$1.0480 E-01$ 2511 26 $26 \quad 2$

$0 .-16$

$1.36 \frac{36}{26}-\frac{0}{4}$

$3.9937 \mathrm{E}-03$

- D. 26

26

$\frac{7}{7}$

8

26

0

$1.0488 E-01$

26 i1

$4 . \frac{200}{27} \mathrm{IE}=01$

$9.7094 E-01$

$1.9020 E-00$

$26-10$

3. 9968 E -03

$27^{-6}$

ㅇ.-

$-\overline{2}$

$1.3708 \mathrm{E}-01$

ㅇ..

$27^{-10}$

$1.0499 E-01$

$4 \cdot \frac{204}{28} \mathrm{E}-01$

$9 \cdot \frac{7}{2} \frac{193 E-01}{2}$

0.

$27^{-19}$

_ 1.3722 E_01

4.0009E-03

ㅇ.

ㅇ.

$1.9039 \mathrm{E} 00$

0 . 
URANIUM 238

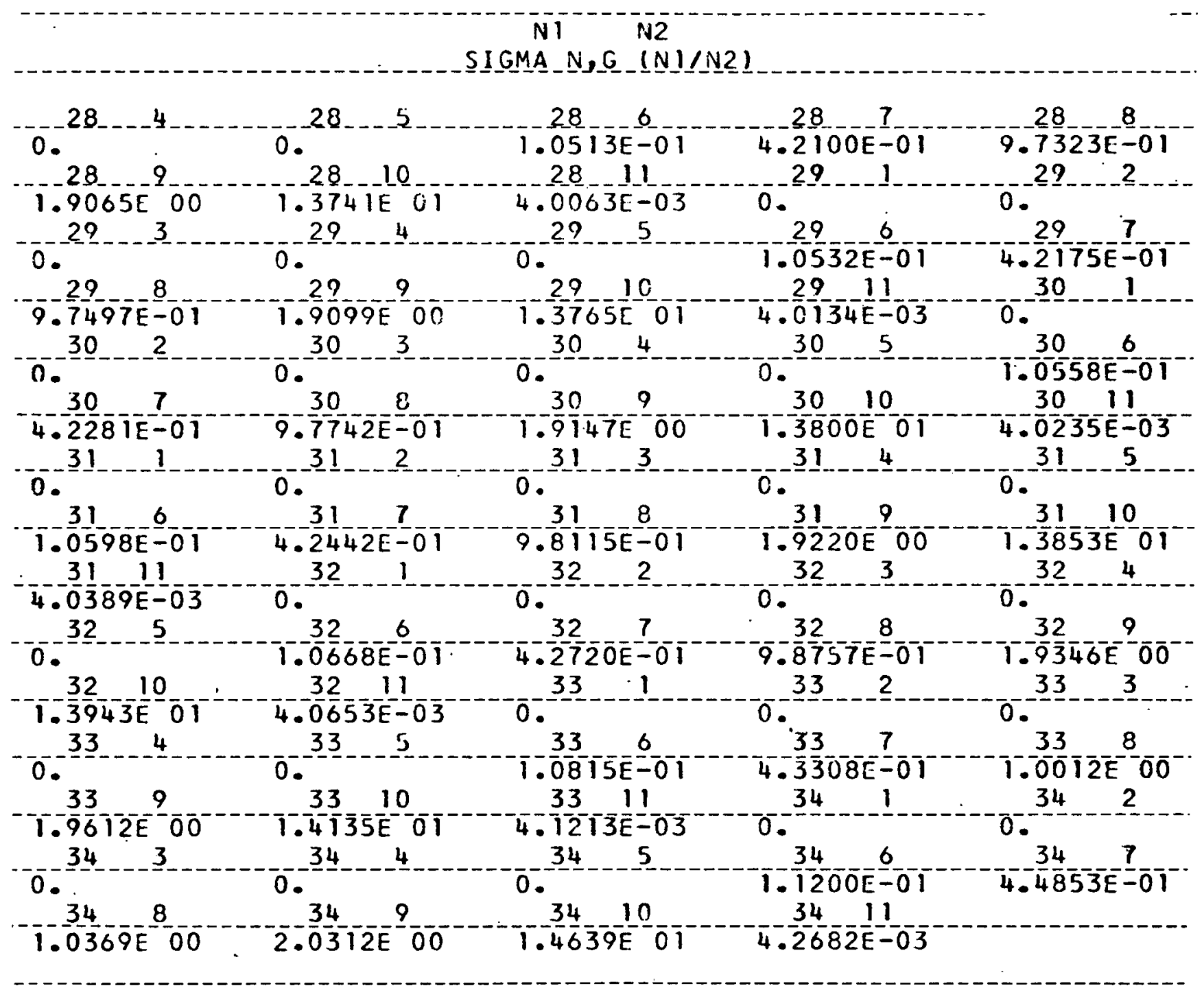


DAIE_DEC 01 1960.

DESIGNATION

PLUTON 239
CODE NO.

94.2390
DENSITY FACTOR

$3.9691 \overline{1 E} 02$
SIGMA

LEVEL ENERGY SCATTER
XI_SIGMA SCATTER
S I GMA TRANSPORT
SIGMA ABSORPIION
XII SIGMA SCATTER I

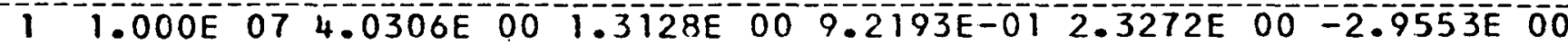
2 6.065E 06 5.3809E $00 \quad 2.3094 \mathrm{E} \quad 00 \quad 1.7899 \mathrm{E} 00 \quad 1.9331 \mathrm{E} 00-3.4139 \mathrm{E}-00$

$3.679 \mathrm{E} 06$ 6.0440E $002.8105 \mathrm{E} 002.6017 \mathrm{E} 00$ 1.8717E $00-3.2725 \mathrm{E} 00$

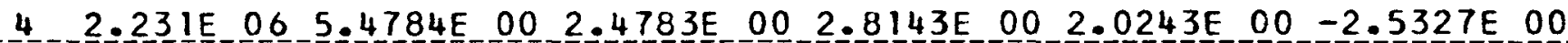

$51.353 \mathrm{E} 065.0682 \mathrm{E} 00$ 1.7420E $003.1722 \mathrm{E} 00$ 1.9965 $00-1.8026 \mathrm{E} 00$

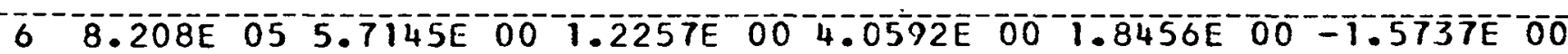
7 - 4.979E 05 - $6.7357 E 00 \quad 7.4301 E-015.5480 E 00 \quad 1.8581 E 00-1.1291 E 00$

8 3.020E 05 7.7366E 00 3.7484E-01 7.2383E 00 1.9316E $00-4.7374 \mathrm{E}-01$

$9 \quad 1.832 \mathrm{E} 05$ - $8.8781 \mathrm{E} 00 \quad 1.1917 \mathrm{E}-01$ 1 $8.7330 \mathrm{E}-00 \quad 2.0556 \mathrm{E} 00-1.3792 \mathrm{E}-01$

$106.738 \mathrm{E} 041.0112 \mathrm{E} 018.5951 \mathrm{E}-02 \mathrm{2} 1.0084 \mathrm{E}-12.4064 \mathrm{E} 00-2.6917 \mathrm{E}=02$

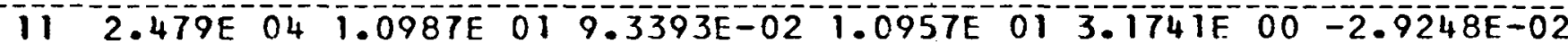

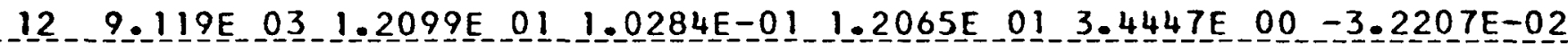

$13-3.355 \mathrm{E} 031.3313 \mathrm{E} 0 \mathrm{1} 1.1316 \mathrm{E}-011.3275 \mathrm{E} 0 \mathrm{1} 4.7924 \mathrm{E} 00-3.5438 \mathrm{E}=02$ $14-1.234 \mathrm{E} 03 \quad 1.0701 \mathrm{E} 01.9 .0958 \mathrm{E}-02 \quad 1.0671 \mathrm{E} 01 \quad 7.5652 \mathrm{E}-00-2.8484 \mathrm{E}-02$ $15-5.540 \mathrm{E} 021.0785 \mathrm{E} 019.1675 \mathrm{E}-02-1.0755 \mathrm{E} 01.9796 \mathrm{E}-0 \mathrm{1}-2.8709 \mathrm{E}=02$

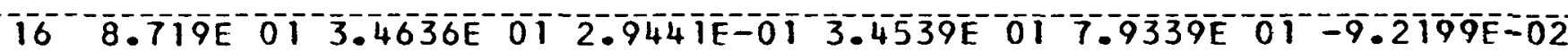
$17 \quad 3.545 \mathrm{E}$ ol $2.1478 \mathrm{E}$ ol $1.8256 \mathrm{E}-01$ 2.1418E $01 \quad 0.5651 \mathrm{E} 01-5.7170 \mathrm{E}-02$

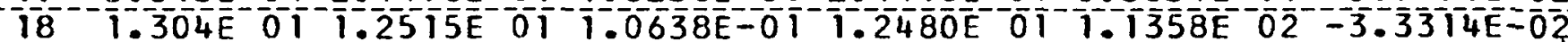

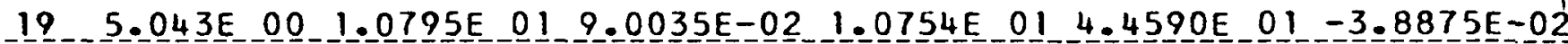
$201.445 \mathrm{E} 001.2092 \mathrm{E} 011.0086 \mathrm{E}-011.2047 \mathrm{E} 012.2500 \mathrm{E}-01-4.3548 \mathrm{E}-02$

$210.826 \mathrm{E}-011.4196 \mathrm{E}$ O $1.1840 \mathrm{E}-011.4142 \mathrm{E} 011.6703 \mathrm{E} 02-5.1123 \mathrm{E}-02$

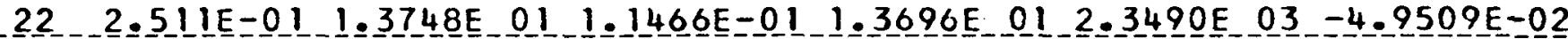
$239.237 \mathrm{E}-02 \quad 1.0455 \mathrm{E}$ 01 $8.7200 \mathrm{E}-02$ 1.0415E $016.7335 \mathrm{E} 02-3.765$ IE-02

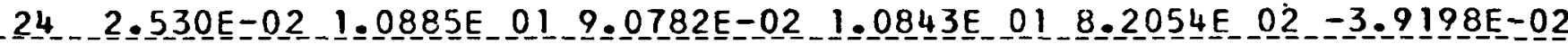

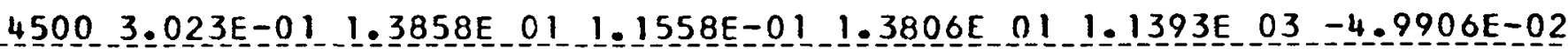
$40002.718 \mathrm{E}-0 \mathrm{1} 1.379 \mathrm{TE} 011.1502 \mathrm{E}-011.3739 \mathrm{E} 0 \mathrm{I} 1.2323 \mathrm{E}-03-4.9663 \mathrm{E}-02$ $3500 \quad 2.413 E-01 \quad 1.3658 E$ ol $1.1391 E-01 \quad 1.3606 E$ O 1 1.3262E $03-4.9186 E-02$ $30002.109 \mathrm{E}-0 \mathrm{I} 1.3432 \mathrm{E} 011.1203 \mathrm{E}-01 \mathrm{1} .3381 \mathrm{E} 01-1.4129 \mathrm{E} 03-4.8372 \mathrm{E}=02$ $25001.804 \mathrm{E}-01$ 1.3077E 01 1.0907E-01 $1.3028 \mathrm{E} \quad 011.4773 \mathrm{E} \quad 03-4.7093 \mathrm{E}-02$

$20001.499 E-01$ 1.2556E 01 1.0473E-01 1.2509E 01 1.4923E 03 - $4=5219 E-02$ $15001.194 \mathrm{E}-0 \mathrm{I} 1.1859 \mathrm{E}$ 0I $9.8913 \mathrm{E}-02$ 1.1814E $011.4163 \mathrm{E}-03-4.2708 \mathrm{E}-02$ $10008.897 E-02 \quad 1.1089 E \quad 01 \quad 9.2491 \mathrm{E}-02 \quad 1.1047 \mathrm{E} \quad 01 \quad 1.2084 \mathrm{E} \quad 03-3.9935 \mathrm{E}-02$ $5005.850 E-021.0620 E$ O1 $8.8572 E-021.0579 E$ OI $9.2654 \mathrm{E} 02-3.8244 \mathrm{E}-02$ 68 3.216E 02 1.0759E $018.9732 \mathrm{E}-02$ 1.0718E 01 8.9489E $02-3.8743 \mathrm{E}-02$ 


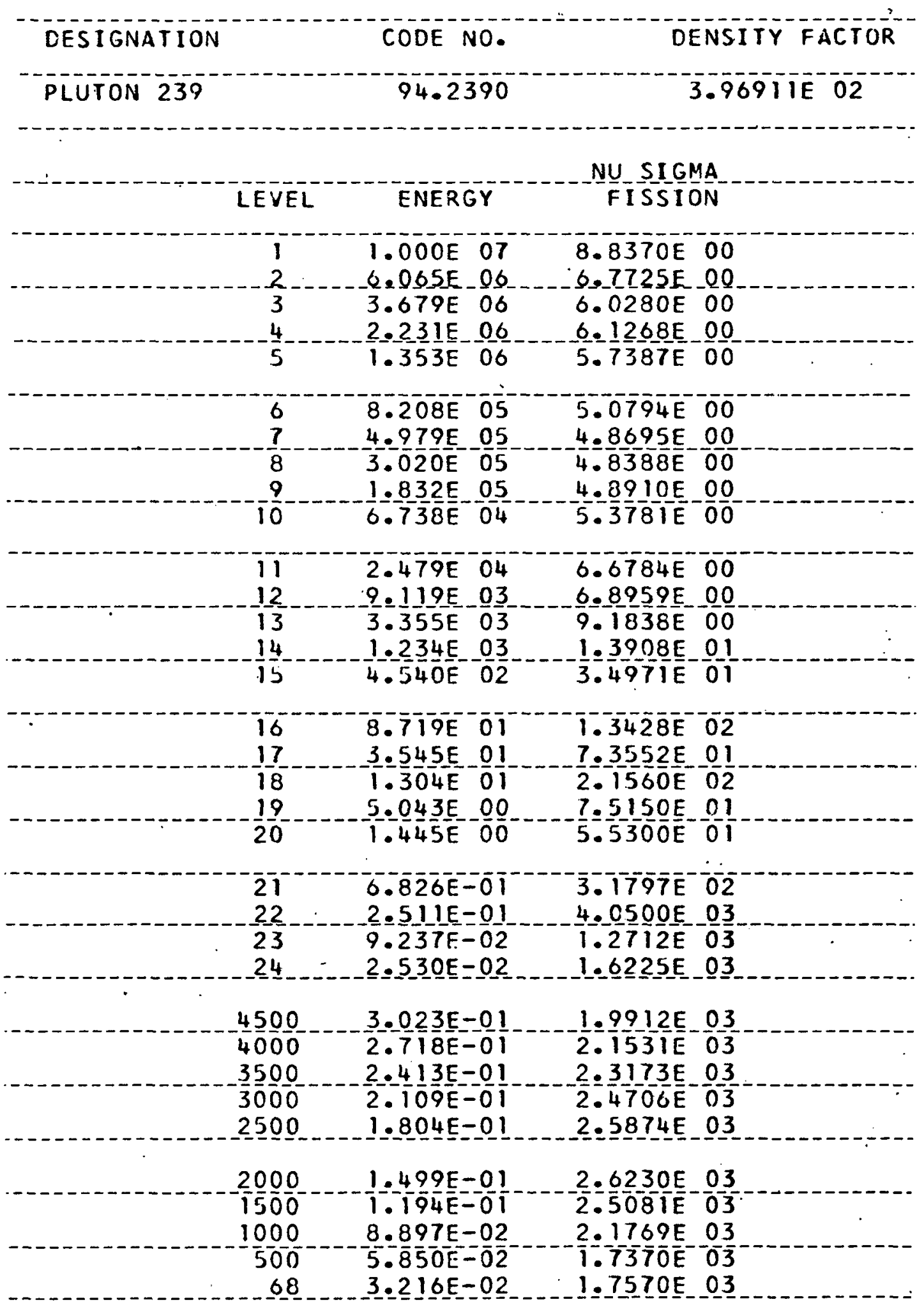


PLUION 239

THERMAL TRANSFER MATRICES

TEMPERATURE DEG. .. F. $--4.5000 E_{-} 03$
SIGMA SCAIIER Le1409E_0]
SIGMA IRANSPQQRI 1.1377E_Q1
SI GMA ABSORPIION
NU SIGMA FISSION
N 1

N2

SIGMA_S_(N1/N2) $1.2487 E$ E 03

\begin{tabular}{|c|c|c|c|c|}
\hline 2$]_{-}-21$ & $21-22$ & 21 & $21 \ldots 24$ & 21 \\
\hline $\begin{array}{l}.3318 E \\
-22\end{array}$ & $\begin{array}{r}8.7796 E-01 \\
22\end{array}$ & $0.22 \quad 23$ & 0.22 & 0. \\
\hline $\begin{array}{l}7.3710 E-01 \\
-23 \\
-21\end{array}$ & $\begin{array}{r}1.2502 \mathrm{E} 01 \\
23\end{array}$ & $\begin{array}{r}5.3498 \mathrm{E}-01 \\
23\end{array}$ & $\begin{array}{r}2.7680 E-06 \\
23 \quad 24\end{array}$ & \\
\hline & $1.2024 \mathrm{E} 00$ & $8.9 .628 E 00$ & $3.3344 \mathrm{E}-01$ & $1.0591 \mathrm{E}-02$ \\
\hline & $\begin{array}{l}3.7231 E-05 \\
25\end{array}$ & $\begin{array}{l}3.0292 E 00 \\
-25\end{array}$ & $6.7915 E 00$ & $\begin{array}{l}1.2126 E 00 \\
25\end{array}$ \\
\hline
\end{tabular}

TEMPERATURE
SIGMA

\section{Ni N2}

SI GMA_S_(N] IN2)

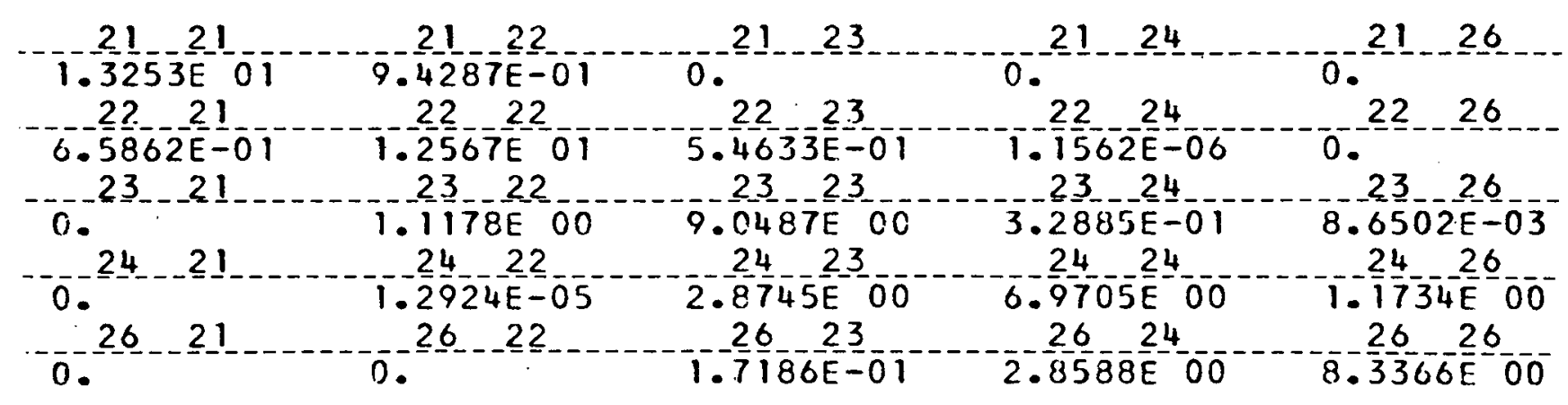


PLUTON 239

._LHERMAL_JRANSEER_MAIRICES_

- IEMPERATURE DEG. F.
SIGMA.

SCATTER
SLGMA TRANSPORT
SIGMA $\triangle B S O R P T I O N$
NU SLLMA FISSION

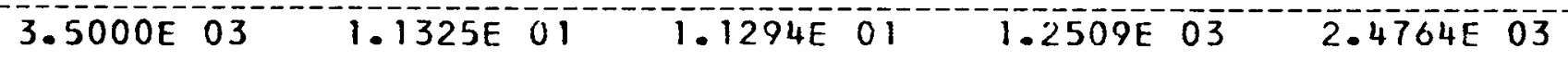

\section{SI GMA S $(N) / N 2)$}

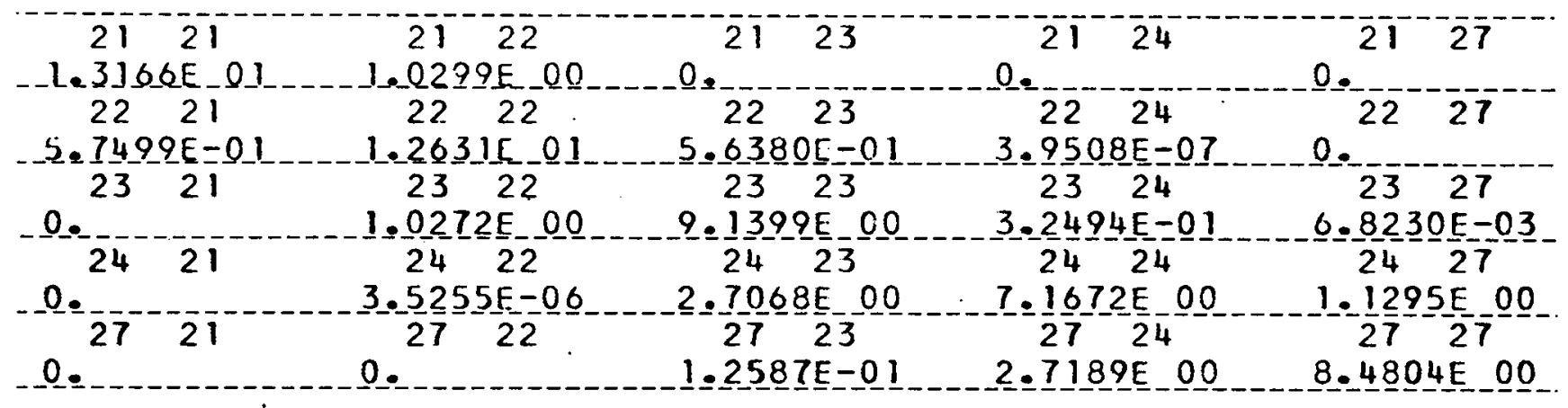

TEMPERAIURE

DEG. $F$.

\section{$3.0000 \mathrm{E} 03$}

SI GMA SCATTER
SIGMA TRANSPORT
SIGMA ABSORPTION
NU_ SIGMA

FISSION

\section{I.T283E OT \\ 1. $1252 \mathrm{E} 01$ \\ $1.2525 \mathrm{E} 0 \overline{3}$ \\ $2.4796 \mathrm{E} 03$}

\section{SIGMA S (NIIN2)}

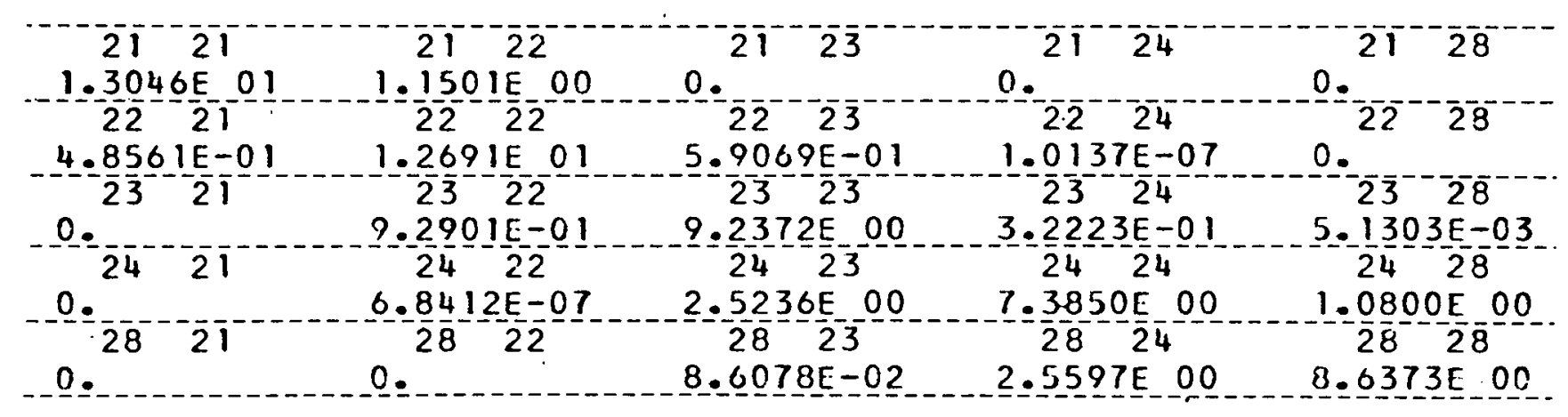


PLUION_239

THERMAL TRANSFER MATRICES

TEMPERATURE
STAMA
SIGEG

\begin{tabular}{|c|c|c|c|c|}
\hline$-21-21$ & $1.3222 \mathrm{E} \quad 00$ & 0 & 0 & 0 \\
\hline $\begin{array}{l}28 \\
-22 \\
-2\end{array}$ & $\begin{array}{r}32225 \\
22 \\
-\end{array}$ & 0.22 & 22 & 22 \\
\hline $3.9006 \mathrm{E}-01$ & $1.2742 \mathrm{E} 01$ & $6.3292 \mathrm{E}-01$ & 0. & 0. \\
\hline-23 & $8.2149 E-01$ & $-9-\frac{23}{34} 15 \mathrm{E}-\frac{23}{00}$ & $3 .-2 \frac{2}{164 E-01}$ & $-\frac{23}{3.6120-\frac{29}{E}-03}$ \\
\hline 24 & 24 & 24 & 24 & $24 \quad 29$ \\
\hline$-\leq 1$ & 0.29 & $\begin{array}{l}2.3212 E \\
29\end{array}$ & $\begin{array}{c}7.6283 E 00 \\
29\end{array}$ & $\begin{array}{c}1.0241 \mathrm{E} 00 \\
2929\end{array}$ \\
\hline & & $5.3306 \mathrm{E}-0$ & & $8.8104 \mathrm{E} 0 \mathrm{C}$ \\
\hline
\end{tabular}

TEMPERATURE
SIGMA

ni

SIGMA S (N1/N2)

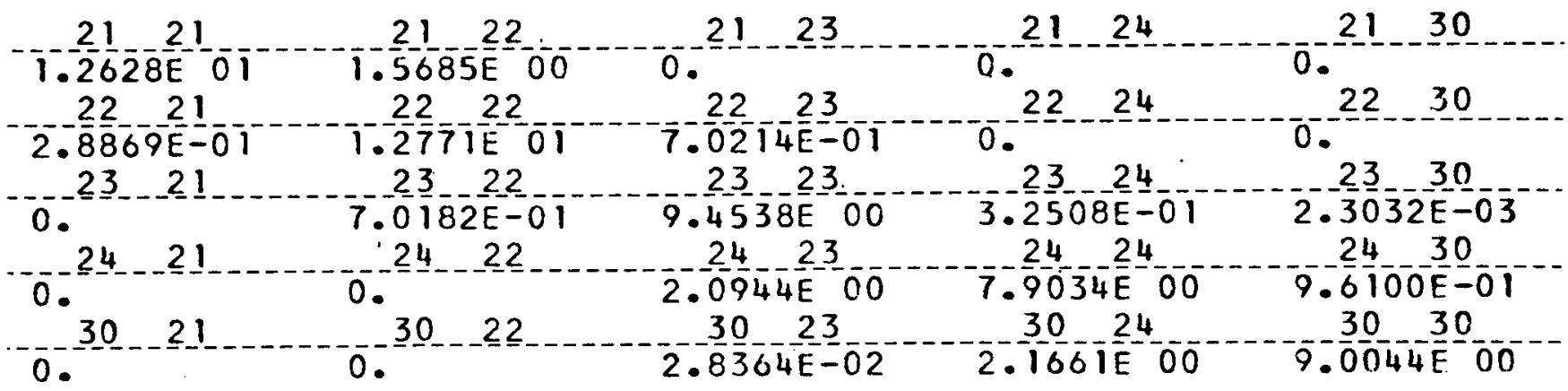


58.

PLUTON 239

.IHERMAL IRANSEER MAIRICES.

_. LEMPERATURE

DEG. F. SCATTER

$1.5000 \mathrm{E} 0 \overline{3}$
1. $1 \overline{15} \overline{7} \overline{0} \mathrm{~T}$
SIGMA TRANSPORT
SIGMA ABSORPTION
NQU SIGMA

FISSION

\section{SIGMA S (N IIN}

$1.2624 \mathrm{E} 03$ 2.4995E 03

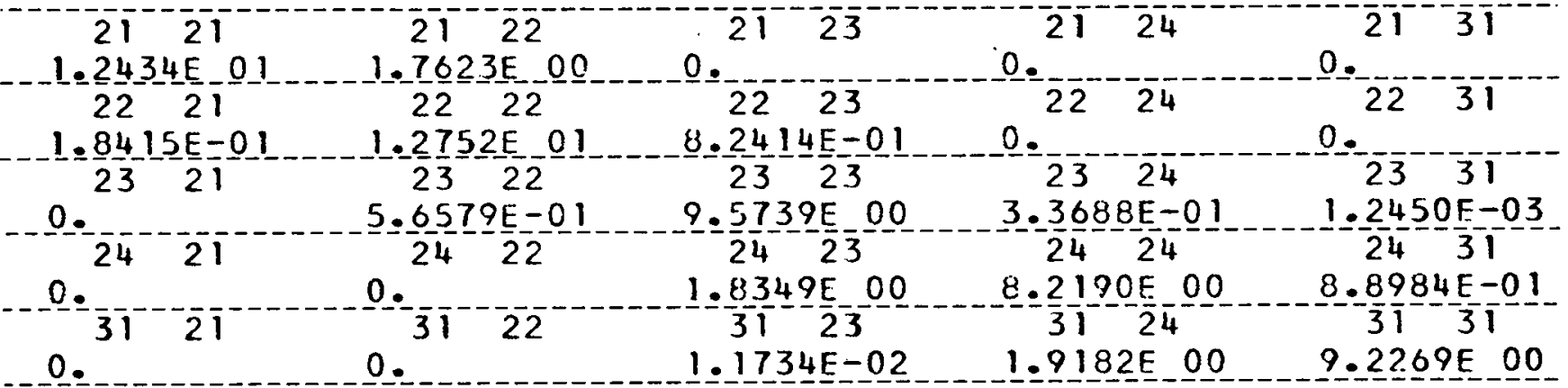

IEMPERAIURE

DEG. $F$.

$1.0000 \mathrm{E} 0 \overline{3}$
SIGMA

SCATTER

I. IISE $\mathrm{G}$
SIGMA TRANSPORT
SISMA ABSORPTION
NU SIGMA

FISSION

\section{$1.0000 \mathrm{C}$ (}

SIGMA S $\left(\frac{N}{N}-\frac{N}{N}-N 2\right)$

$1.1084 \mathrm{E}^{-} \mathrm{1}$


PLUTON 239

THERMAL TRANSFER MATRICES

TEMPERATURE
DEG SIGMA

\begin{tabular}{|c|c|c|c|c|}
\hline 21 & 21 & 23 & 21 & $21 \quad 33$ \\
\hline $\begin{array}{lll}35 & 20 & 01\end{array}$ & $6.4627 E-01$ & 0.0 & 0 & 0. \\
\hline $1.1291 \mathrm{E}-01$ & $1.2100 E .01$ & $1.5414 E 00$ & 0 & 0 \\
\hline & $2.1933 E-01$ & $9.7839 \mathrm{E} 00$ & $4.6408 \mathrm{E}-01$ & $-8.9729 \frac{23}{E-05}$ \\
\hline $24 \quad 21$ & 24 & 24 & 24 & $24 \quad 33$ \\
\hline & 0.33 & $\begin{array}{r}1483 \mathrm{E} 00 \\
-33 \\
2.4802 \mathrm{E}-04\end{array}$ & $\begin{array}{l}9.0377 \mathrm{E} 00 \\
1 . \frac{33}{2}-265 \mathrm{E}-00\end{array}$ & $\begin{array}{r}7.2796 \mathrm{E}-01 \\
9.8268 \mathrm{3} \\
9.00\end{array}$ \\
\hline
\end{tabular}

SEMPERATURE
SIGMA
DEG

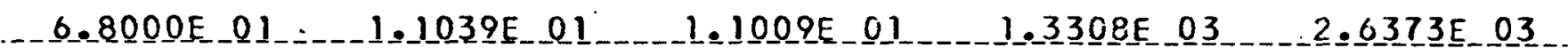

Ni

S1GMA S (N IIN2)

\begin{tabular}{|c|c|c|c|c|}
\hline 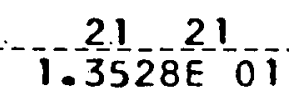 & $21-22$ & $21-23$ & 21 & $21-34$ \\
\hline $\begin{array}{l}3528 \mathrm{E} \\
22 \\
22\end{array}$ & $\begin{array}{l}E-1 \\
22\end{array}$ & 22 & 22 & 22 \\
\hline 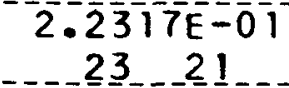 & 1.307 O 11 & $4.5100 \mathrm{E}-0 \mathrm{~T}$ & & 0 \\
\hline & $5.0813 \mathrm{E}-02$ & $9.6154 \mathrm{E} 00$ & $7.9656 \mathrm{E}-01$ & $2.0410 E-06$ \\
\hline & 0 & $-0.2479-23$ & $9.5211 E 00$ & $6.8024 \mathrm{E}-01$ \\
\hline 0 & 0. & $1.2801 E-06$ & $8.0295 \bar{E}-01$ & $1.0236-\frac{34}{2}-0$ \\
\hline
\end{tabular}




\section{SIGMA $S 0(N 1 / N 2)$}

11013

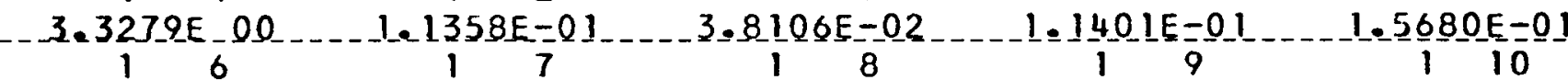
. $1.3090 \mathrm{E}-01 \ldots$

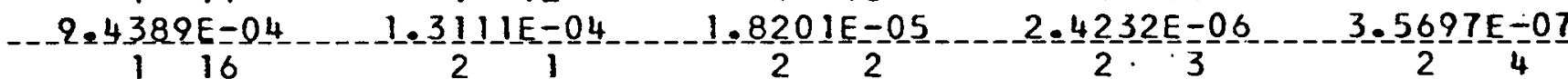
-2. $7 \frac{6}{2} 07 \mathrm{E}-08$

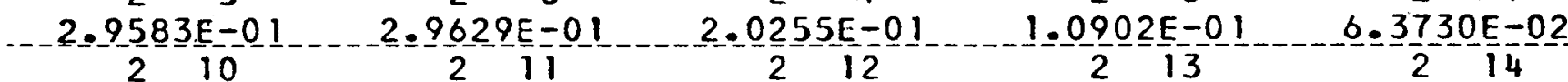

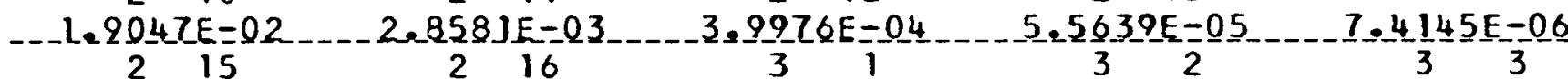

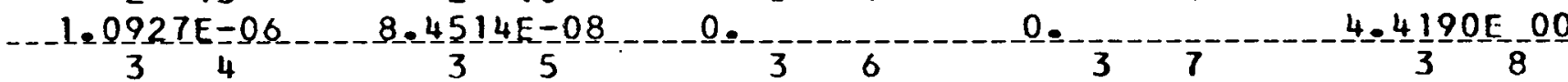

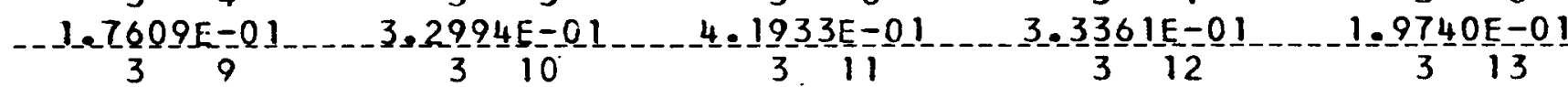

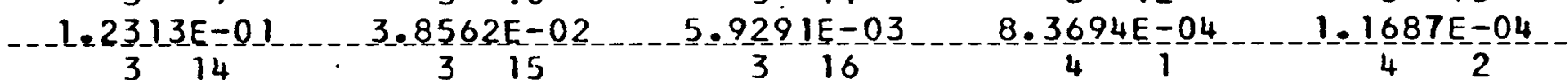

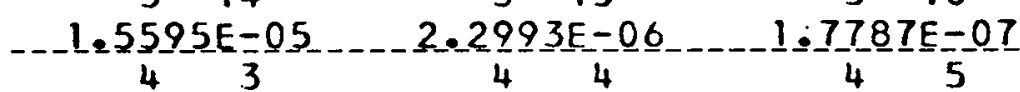

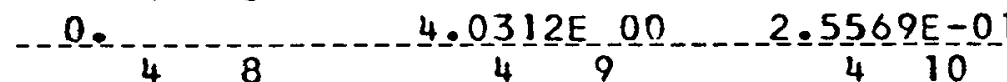
$2.4095 \mathrm{E}-01$ $1.7560 \mathrm{E}=04$

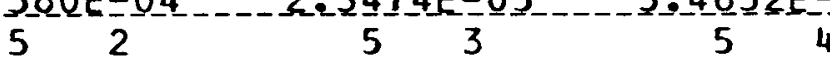

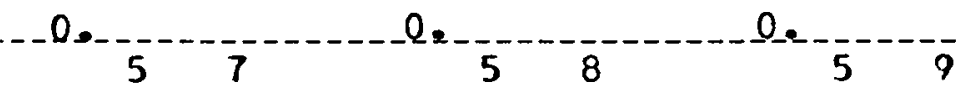

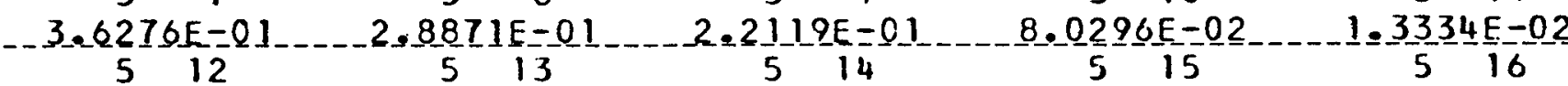

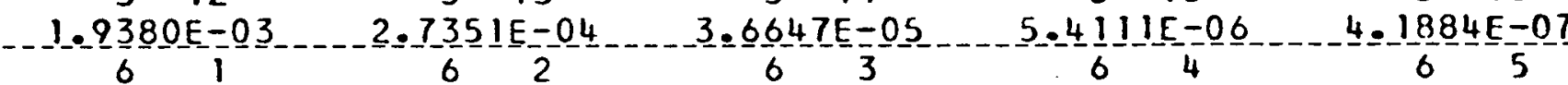
0 . $0.0-100$ $0.000-10$ -4.6486E $0003.8844 \mathrm{E}-01 \ldots 2.9258 \mathrm{E}-01 \quad 2.5909 \mathrm{E}-01$ $1.829 \mathrm{IE}-02$ 2.7139E-03 3.8590E-04 $5.1857 \mathrm{E}-05$ 6
6 0 $-14-6$ 0 $3.6382 \mathrm{E}=01$ $8.7536 E-03$ $2.6798 \mathrm{E}=0 \mathrm{Z}$ 55 $3.7192 \mathrm{E} 000$ 
N1 N2

SIGMA_SO_ (NIIN2)

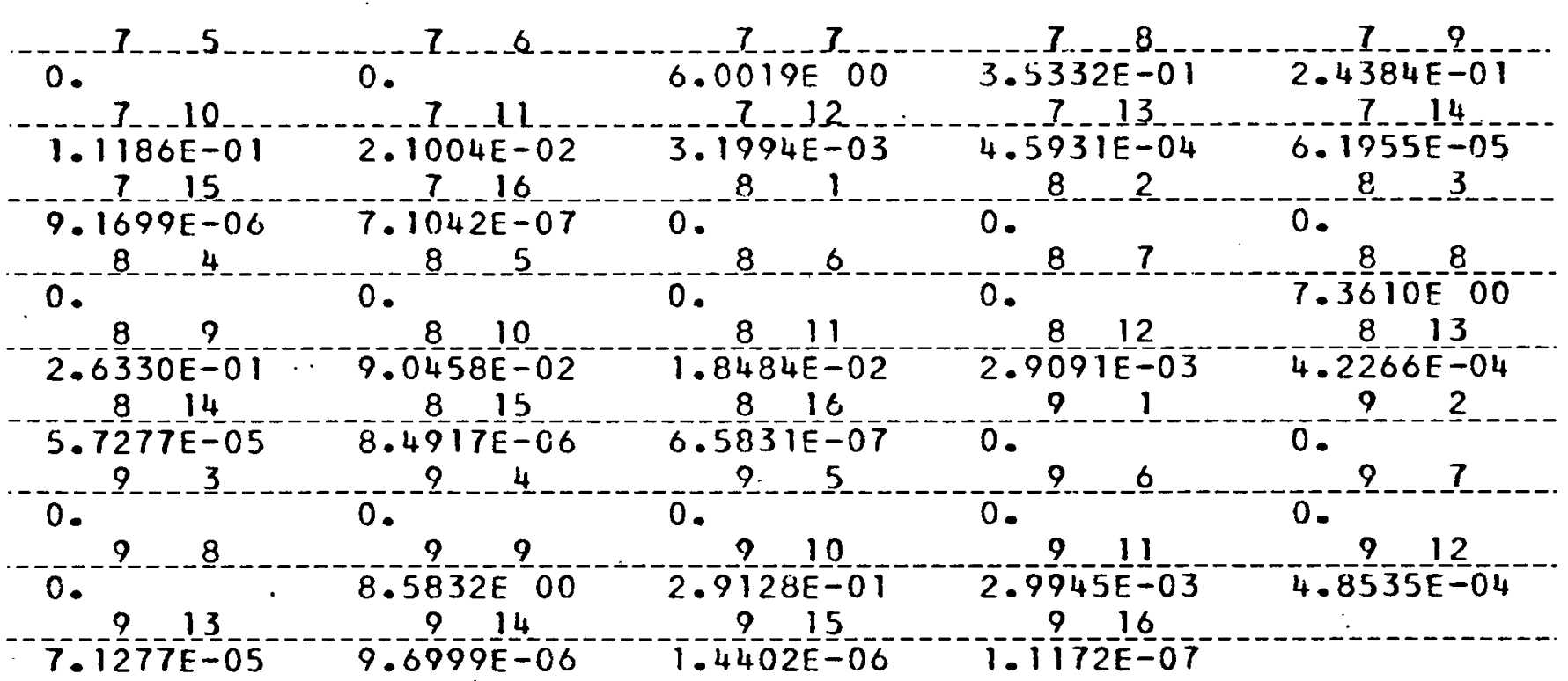


PLULON 239

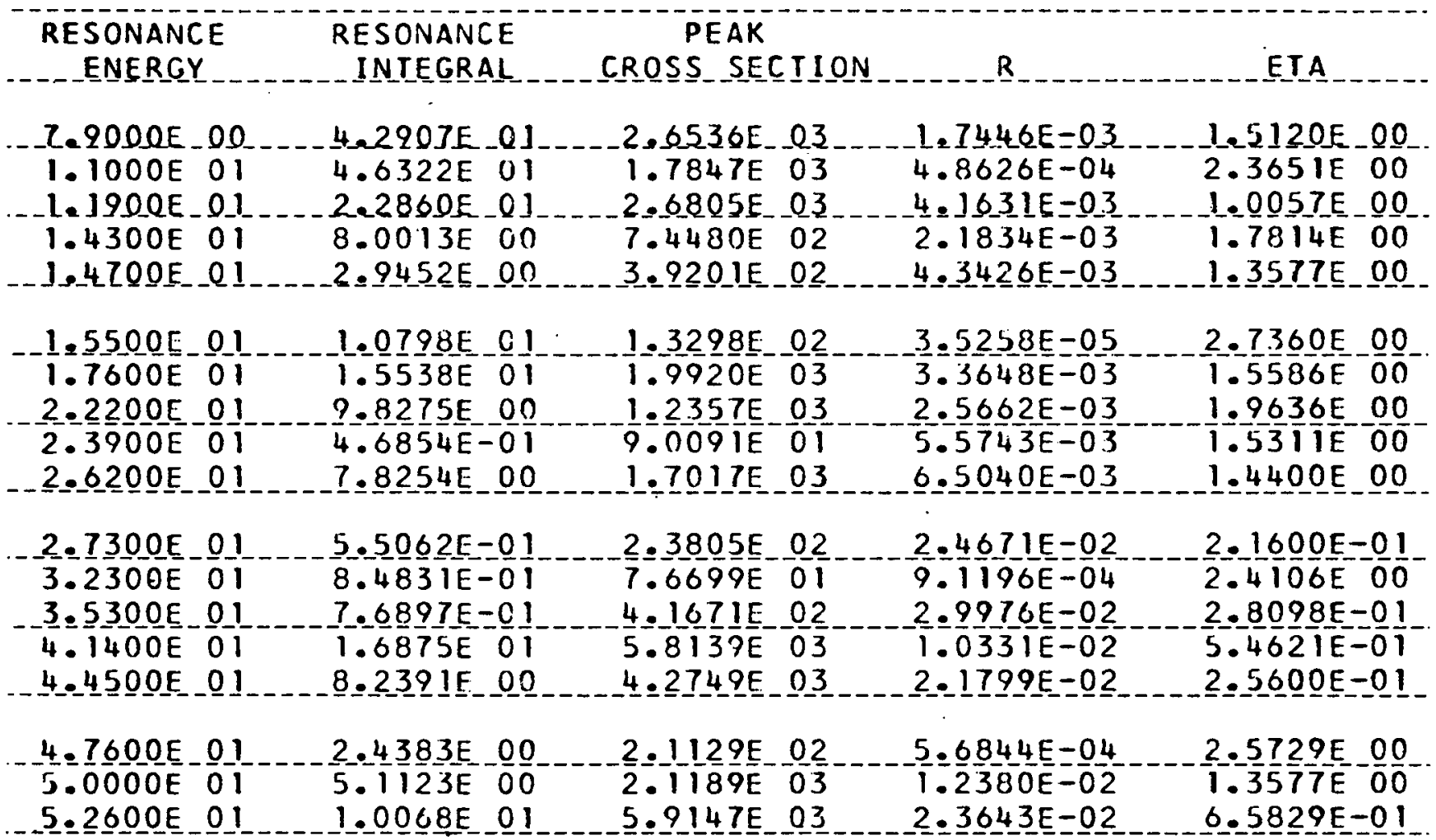


DESIGNAIIION

NI $\underline{C}$ KEL
CODE_NO.

28.5900
DENSIIYY FACIOR

$9 \cdot 7 \underline{4} 65 \underline{4} \underline{E}$

\section{SIGMA XI SIGMA SIGMA SIGMA XIT SIGMA}

_EVEL__ENERGY _ _ SCA I IER _ _. SCA I IER _ TRANSPORI _ ABSORPIION _SCAITER I

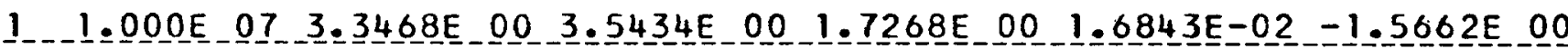

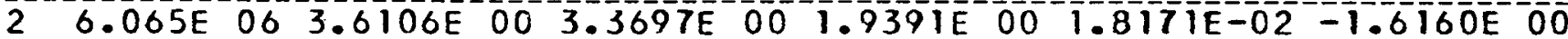

3 3.679E 06 3.4 104E 00 2.6464E $002.3021 \mathrm{E} 00 \quad 1.7150 \mathrm{E}-02-1.0715 \mathrm{E} 00$

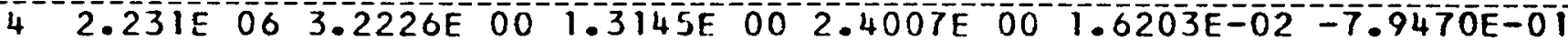

5 1.353E 06 3.2580E 00 3.1851E-01 2.5198E $001.6393 \mathrm{E}-02-7.1375 \mathrm{E}-01$

6 - $80208 \mathrm{E}-053.3982 \mathrm{E}-00 \quad 1.0135 \mathrm{E}-01 \quad 2.7794 \mathrm{E}-00 \quad 1.7076 \mathrm{E}-02-5.9825 \mathrm{E}-01$

$7.979 \mathrm{E} 053.9376 \mathrm{E} 001.2479 \mathrm{E}-0 \mathrm{1} 3.4082 \mathrm{E} 002.0004 \mathrm{E}-02-5.1182 \mathrm{E}-01$

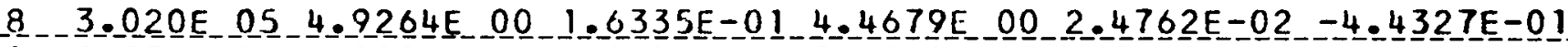

$9 \quad 1.832 \mathrm{E} 05 \quad 6.0904 \mathrm{E} 00.2 .1176 \mathrm{E}-015.8152 \mathrm{E} 00 \quad 3.0609 \mathrm{E}-02-2.6603 \mathrm{E}-01$

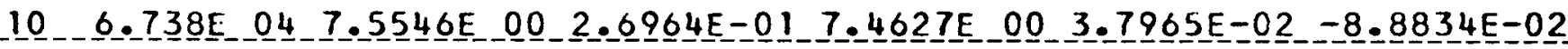

11 2 $479 \mathrm{E}-04-2=4875 \mathrm{E}-01$

$129.119 \mathrm{E} 032.1487 \mathrm{E} 017.6707 \mathrm{E}-012.1229 \mathrm{E} 011.0765 \mathrm{E}-01-2.4928 \mathrm{E}-01$

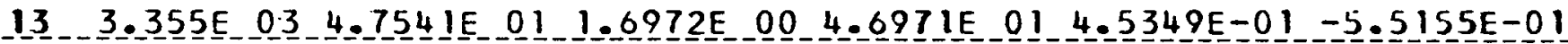

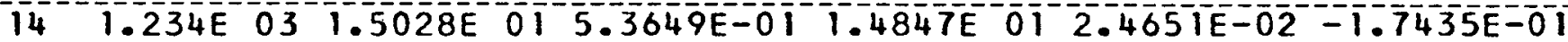

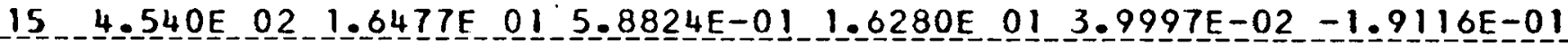

$16-8.719 E-011.7029 E-01-6.0792 E-01-1.6824 E-0.17 .7134 E-02-1.9756 E-01$

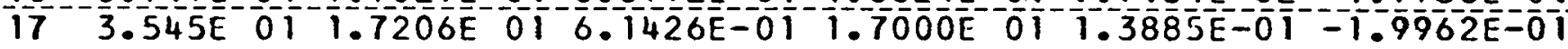

18 1.304E $011.7250 \mathrm{E} 01.6 .1581 \mathrm{E}-011.7043 \mathrm{E} 01-2.1954 \mathrm{E}-01-2.0012 \mathrm{E}-01$

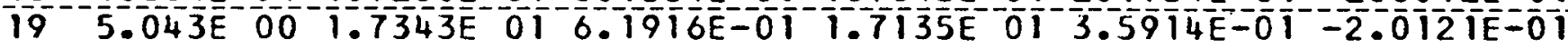

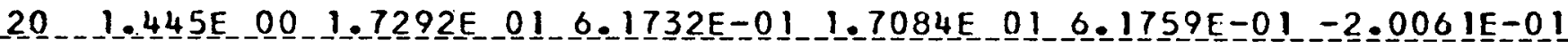

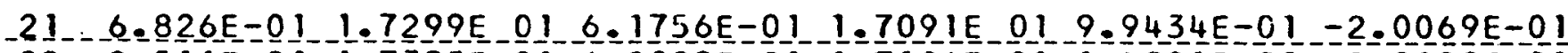
$222.511 \mathrm{E}-011.7395 \mathrm{E} 016.2099 \mathrm{E}-011.7186 \mathrm{E} 011.3801 \mathrm{E} 00-2.0180 \mathrm{E}-01$ $23-9.237 E=02-1=7218 E-01-6.1467 E=01-1.7011 E-01-2.6670 E-00-1.9975 E-01$ $242.530 \mathrm{E}-021.6716 \mathrm{E} 015.9478 \mathrm{E}-011.6516 \mathrm{E} 014.1045 \mathrm{E} 00-1.9337 \mathrm{E}-01$

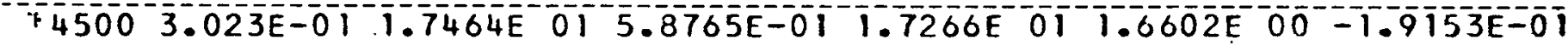

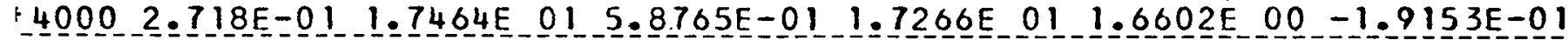

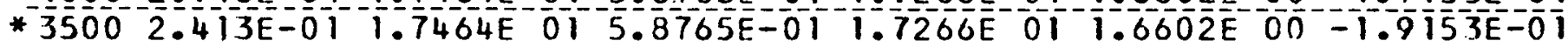

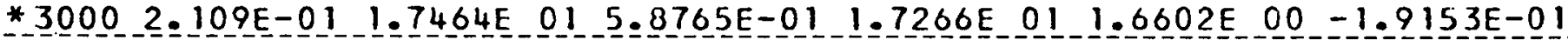

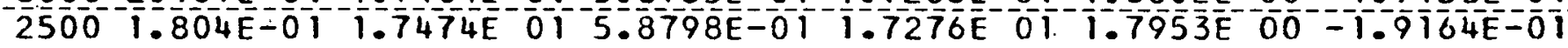

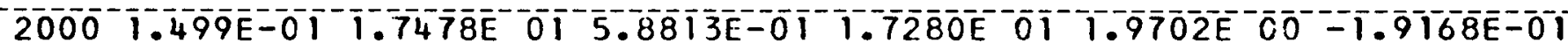
$15001.194 \mathrm{E}-01$ 1.7467E $015.8774 \mathrm{E}-01$ 1. $7269 \mathrm{E} 01$ 2.2084E $00-1.9155 \mathrm{E}=01$

$10008.897 \mathrm{E}-021.7433 \mathrm{E} 015.8659 \mathrm{E}-011.7235 \mathrm{E} 012.5605 \mathrm{E} 00-1.9118 \mathrm{E}-01$ 500 5.850E-02 1.7372E 01 5.8454E-01 1.7175E 01 3.1620E 00 -1.9052E-01 $683.216 \mathrm{E}-02-1.7274 \mathrm{E} 015.8126 \mathrm{E}-011.7078 \mathrm{E} 014.2713 \mathrm{E} 00-1.8944 \mathrm{E}=0 \mathrm{I}$

* Thermal cross sections taken from XDC 60-1-139.

NOTE: For a sumary of the sources of data see APEX-704 and XDC 60-1-139. 
NICKEL

_LEERMAL_IRANSEER_MAIRICES

.LEMPERAIURE

SI GMA

SIGMA

DEG. $F$.

SCATTER

TRANSPORT

SIGMA

NU SIGMA -

$4.5000 \mathrm{E} 03$

$1.857 \overline{T E}$ OI

$1.8 \overline{36} 7 \mathrm{E}$ O

ABSORPTION

FISSION

4.5000E O3

NI

SIGMA S INI/N2)

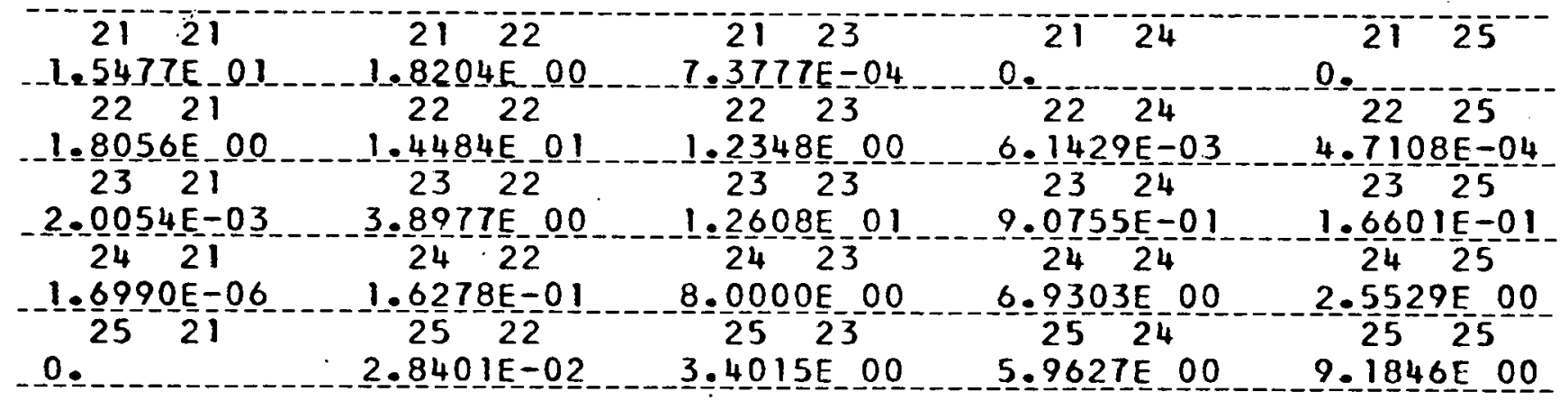

- IEMPEERAIUURE

DEG. F.

$4.0000 \mathrm{E} 03$
S IGMA

SCATTER

$\overline{1} . \overline{3} \overline{\mathrm{IE}} \overline{\mathrm{O}} \overline{\mathrm{T}}$
SI IGMA TRANSPORT

1.8ิउ3E
$6.1775 \mathrm{E} 00$
0 . 
NICKEL

\section{THERMAL TRANSFER MATRICES}

TEMPERATURE
SIGMA

3.5000E 03 1.8103E_01 _ 1.7898E 01

$\mathrm{N} \overline{\mathrm{N}} \mathrm{N}$

SI GMA S

\begin{tabular}{|c|c|c|c|c|}
\hline $\begin{array}{l}-21 \\
1.5239 \mathrm{E} \\
22\end{array}$ & $2.0598 \mathrm{E} 00$ & $-\frac{21}{2.7665 E-04}$ & 0 & 0 \\
\hline $\begin{array}{l}3962 \mathrm{E} 00 \\
23\end{array}$ & $1.4831 \mathrm{I} 01$ & $1.2754 \mathrm{E} 00$ & $3.4695 \mathrm{E}-03$ & $1.7584 \mathrm{E}-04$ \\
\hline $\begin{array}{l}0151 E-04 \\
24 \\
2\end{array}$ & $\begin{array}{c}3.3249 \mathrm{E} 00 \\
2422\end{array}$ & $\begin{array}{c}1.3153 E \\
2421\end{array}$ & $\begin{array}{c}8.9769 E-01 \\
2424\end{array}$ & $\begin{array}{l}1.354 \\
24\end{array}$ \\
\hline$\leq-$ & $\begin{array}{c}6.8786 \mathrm{E}-02 \\
7.8719 \mathrm{E}-0 \overline{3}\end{array}$ & $\begin{array}{l}7.3395 \mathrm{E} 00 \\
2.5119 \mathrm{E} 00\end{array}$ & $\begin{array}{l}7.5113 \mathrm{E} 00 \\
5.8066 \mathrm{E}\end{array}$ & 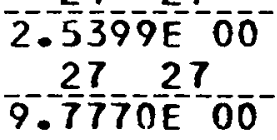 \\
\hline
\end{tabular}

TEMPERATURE
SIGMA

3.0000E 03 1.7864E OI 1.7661E_01 $0.1970 E 00$

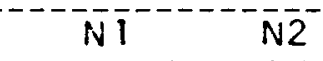

SIGMA S (NIIN2)

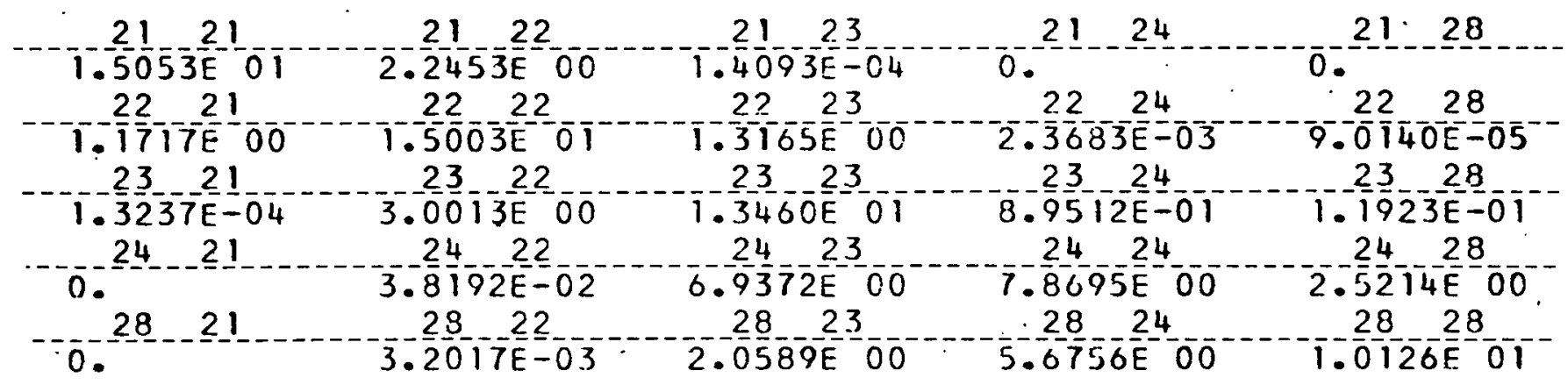


NICKEL

_LHERMAL_IRANSFER_MAIRICES

_- IEMPERAIURE

SIGMA

SLGMA

SISMA

NU_ SIGMA

DEG. F.

SCATTER

TRANSPORT

ABSORPTION

FISSION

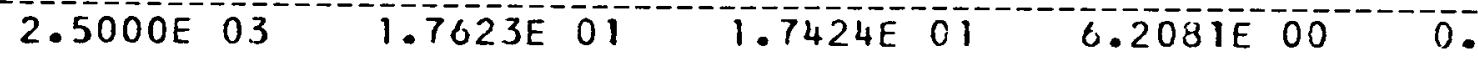

$\operatorname{SIGMA} S\left(\frac{N}{N}-\frac{N 2}{N}\right.$
$2 i$
$21-2 \overline{2}$
$2 \overline{1}^{-1}-\overline{3}$
$2 i^{-14}$
$\overline{2} \overline{1}^{--} \overline{2} \bar{q}$

$.4794 E_{-1} 0.1$

$2.5048 E-00$

$22 \quad 22$

-5.8476 든 -05

22,23

0.

$22 \quad 21$

$1.5163 E-01$

$1.38 \frac{3}{2} 29 \frac{\mathrm{E}}{23}-00$

$1=\frac{4}{2} 385 E-03$

2229

$-9 \cdot 3344 \mathrm{E}=0$

2322

1. 37966트이

$8 \cdot \frac{96}{2}-5 E-01$

$3.82 \frac{1}{2} 15 E-0$

3. $1280 E-0$

2421

$2.64560 \mathrm{E} O 0$

$24-23$

$1 .-7 \frac{89}{2}-\frac{4 E}{22}-0$ ?

$6.4730 \mathrm{E}-00$

$3 \cdot \frac{29}{2} 913 \mathrm{E}-00$

$1.0243 \mathrm{E}-0$

$-0 \cdot 29-\frac{1}{21}$

29
$9.9850 \mathrm{E}-04$

1.6080500

$5.4912 \mathrm{E} \quad 00$

$2.4908 E \quad 00$

$-\frac{4}{2} 908 \frac{9}{29} \div$

- 0.

9.98506-04

IEMPERAIURE

SI SMA

SIGMA

SISMA

NU SIGMA

DEG. $\mathrm{F}$.

SCATTER

TRANSPORT

ABSORPTION

FISSION

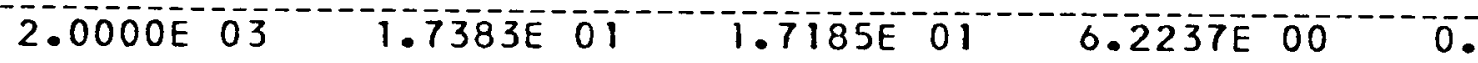

SIGMA S INITN2I

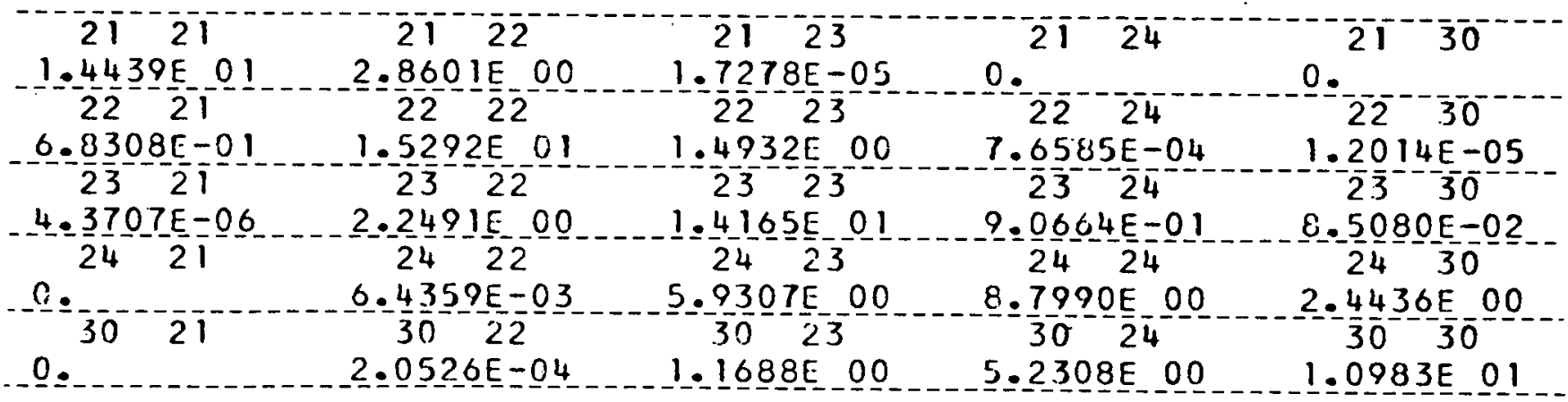


NI CKEEL

THERMAL TRANSFER MATRICES

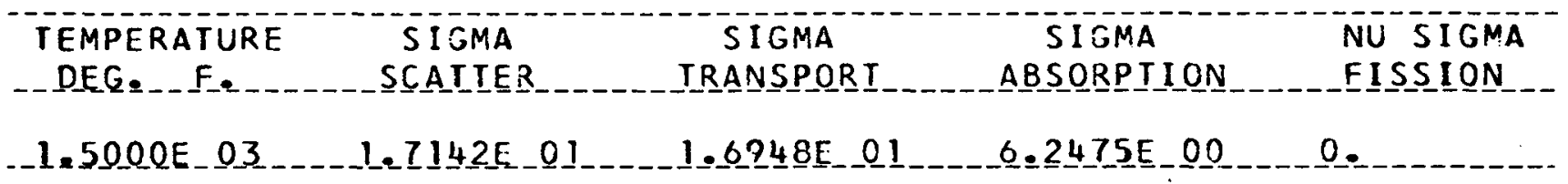

$\mathrm{N} \mathrm{N}^{-12}$

SIGMA S (NIIN2)

\begin{tabular}{|c|c|c|c|c|}
\hline-21. & $21-.22$ & $21-23$ & 24. & 21 \\
\hline $\begin{array}{r}1.4240 E 01 \\
22\end{array}$ & $\begin{array}{c}3.0591 E 00 \\
22 \quad 22\end{array}$ & $\begin{array}{c}2.5362 E-06 \\
22\end{array}$ & & \\
\hline $4.2862 E-01$ & $1.534 \mathrm{JE} 01$ & $1.6866 \mathrm{E} 00$ & $3.0607 \mathrm{E}-04$ & $2.2468 \mathrm{E}-06$ \\
\hline 2 & 22 & $23-23$ & 24 & 31 \\
\hline $2.4627 \mathrm{E}-07$ & $1.7992 \mathrm{E} 00$ & $1.4569 \mathrm{E} 01$ & $9.3472 E-01$ & $0.7212 \mathrm{E}-02$ \\
\hline 0. & $1.4761 \mathrm{E}-03$ & $-5.2835 \mathrm{E} 00$ & $9.4286 \mathrm{E} 00$ & $2.3729 E 0$ \\
\hline ניב & 31 & 23 & 31 & 31 \\
\hline 0 & $2.0603 E-05$ & $7.5607 E-01$ & $4.8576 E \quad 00$ & $1.1528 \mathrm{E}$ \\
\hline
\end{tabular}

TEMPERATURE

DEG _ F.

$1.0000 E \quad 03$
SIGMA

SCATIEER

$1.6905 E$ 이
SI GMA

IRANSPORI

$1.6713 E$ O 1

$\mathrm{N} 1 \mathrm{~N} 2$

SI GMA S (N] I IN2) $\therefore 21-21$

$1.547 \mathrm{E} 0 \mathrm{O}$

$22-21$

$1.9410 \mathrm{E}-01$

-..23 3 - 21

0.

..-24_-2 1

0.

0.32
21

$1.2782 \mathrm{E} 00$

$24-22$

$1.4118 \mathrm{E}-04$

$5.0397 \mathrm{E}-07$
21

2.05930

$23-23$

$1.5002 \mathrm{E} 01$

$24-23$

$4.4828 \mathrm{E} 00$

$3.946 \frac{32}{4 E-0}$
S IGMA

ABSORP IION

$0.2885 E$
NU. SIGMA

FI SSION

으. 
NICKEL

IHERMAL_ TRANSFER MAIRICES

IEMPERAIURE
DEG. FIGMA
F. SCATTER

$5.0000 \mathrm{E} 02 \mathrm{1}-6679 \mathrm{E} 01 \mathrm{1}$

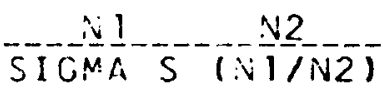

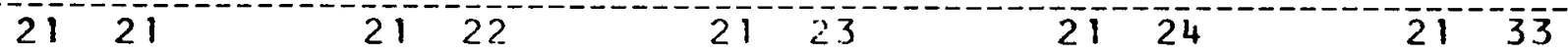

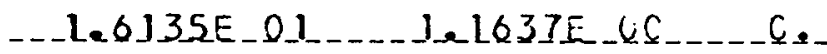

$.89845-01$

$2321 \quad 2322$

2323

$1.53892 \underline{0} 01$

2423

C.

$22-24$

$3.8093 \mathrm{E}=06$

2324

0.

$23^{-13}$

2422

1.- $2 \frac{1}{24} 06 E-240$

2.99770E 02

2433

$3.4240 \mathrm{E}-00$

3323

$-0=33-21$

1. $5929 E=06$

3322

$1.2625 E=01$

$1.1353 E$ 이

3324

- 0

0.

$3.4521 E 00$

$2.1226 E 00$

$33-33$

1. $3101 E$ 으.

TEMPERAIURE

DEG. $F$.

SIGMA

SCATIE?
SIGMA

TRA USPORT
SISMA

AOSORPTION
NU SIGMA

FISSION

\section{SIGMAS TNINZT}
$212 i$
$212 z$
2123
2124
$-1.6185 \mathrm{~F} 01-1.1139 \mathrm{E}-0$
$22-2 \overline{3}$
0.25
2134
$2.9993 E-01$
1.6333501
$7.2644 \mathrm{E}-01$
2323
ก.
23.24
0.
$22^{--} 34^{-\cdots-}$

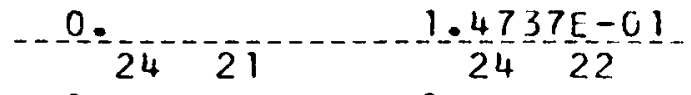
$1.3 \frac{3}{24}-\frac{4}{23}=01$
$1.06777 \frac{C}{24}$
$23-34$
$2 \cdot 34-\frac{1}{2}$
3422
$2.0970 E$ 0O
1.2723E 0
$1.3550 E-02$
3424
$-2 \frac{27}{24} 6-5=02$
……
0 .
$2.2472 \mathrm{E} 00$
1. $\frac{99915}{34} 00$
$1.4269 E$ OI 


\section{NICKEL}

TRANSFIRS FROM GROUPS I THROUGH 5 TO GROUPS 1 THROUGH 16

$\mathrm{NI} N 2$

SIGMA_SO_(NIIN2)

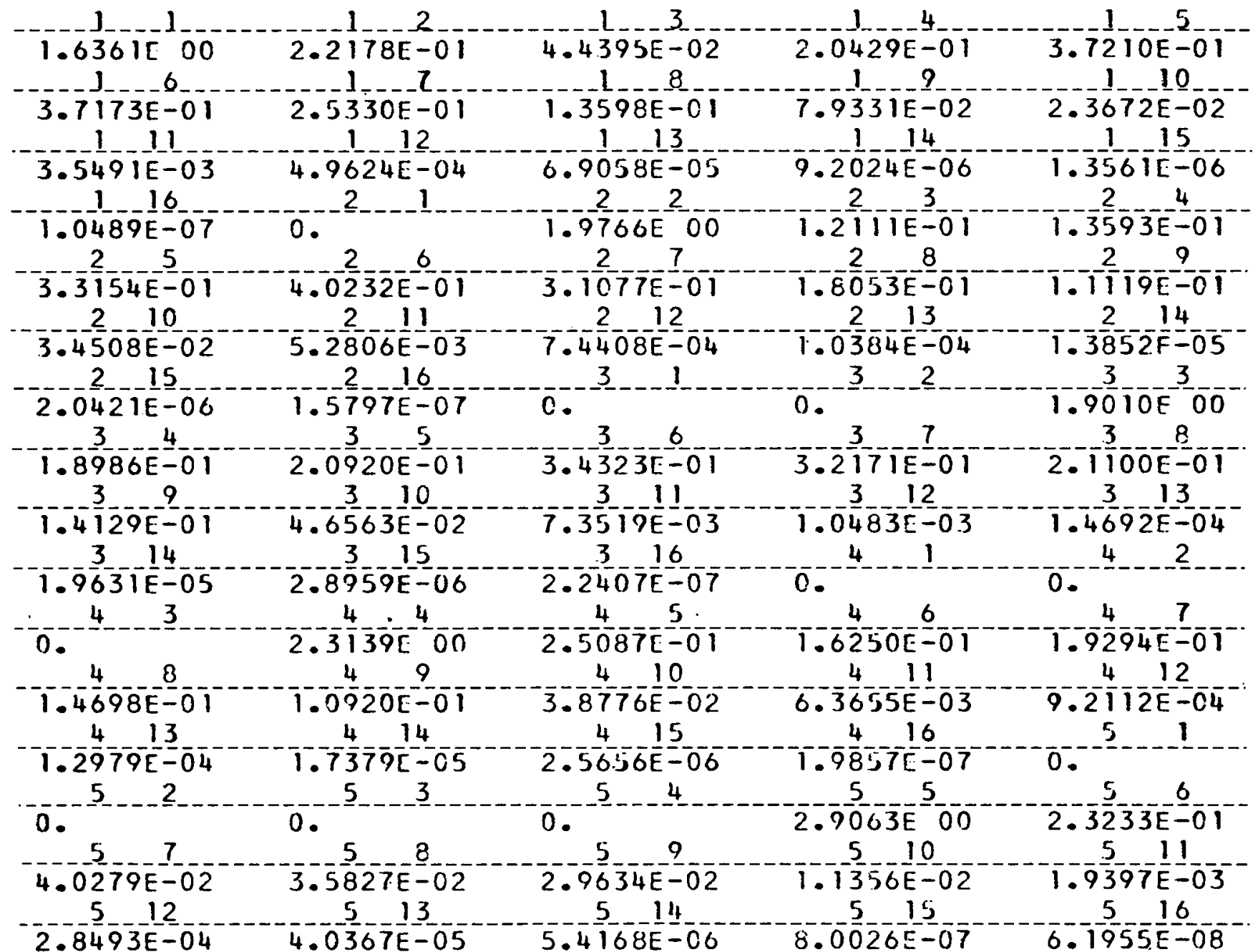


NICKEL

TRANSFERS FROM GROUPS I THROUGH 34 TO GROUPS I THROUGH 10

N1

SIGMA N,G (NIINZ)

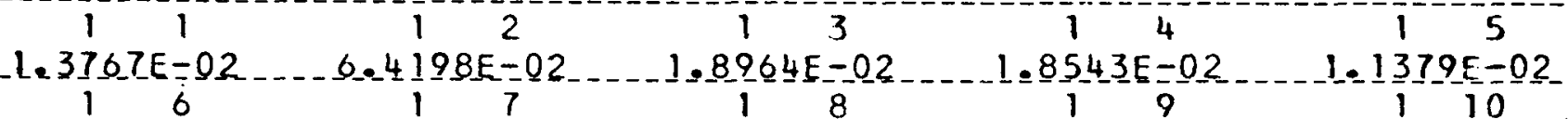
$7 \cdot 1643 E-03 \quad-2.8095 E-03 \quad 5.6 \frac{1}{2} 90 E-04 \quad 1.8827 E-00$

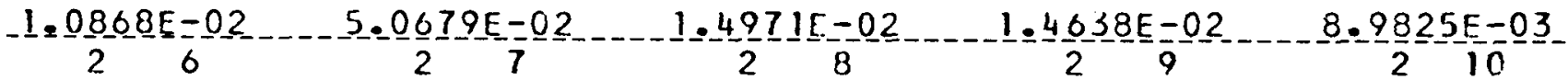
$5.6556 E-03$

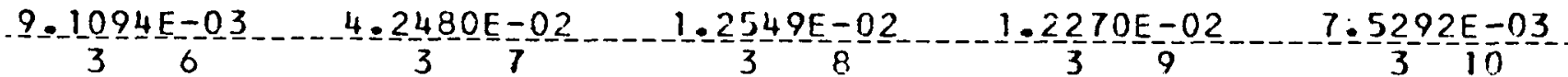

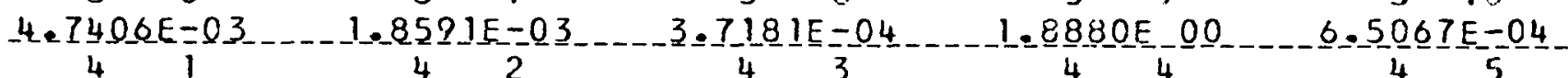

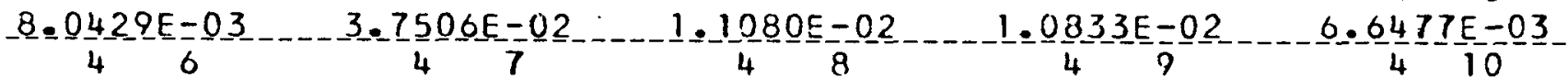

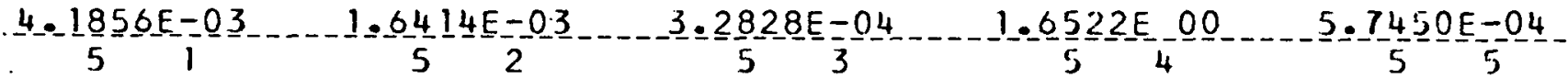
I. $396-1 E=03-3.4490 E-02-1.0188 E-02-9.9621 E-03 \quad 0.1131 E=03$

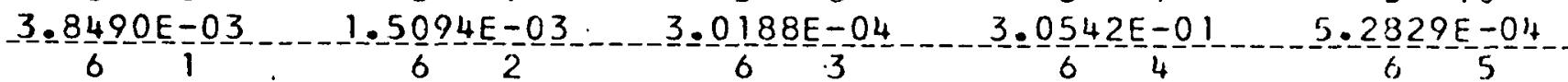
$7.1681 E-03 \quad 3.3427 E-02 \quad 9.8744 E-03 \quad 9.6550 E-03 \quad 0.9247 E-03$ $3.7303 E-03 \quad 1.4629 E-03 \quad 2.9258 E-04 \quad 4.3886 E-04-5.1201 E-04$

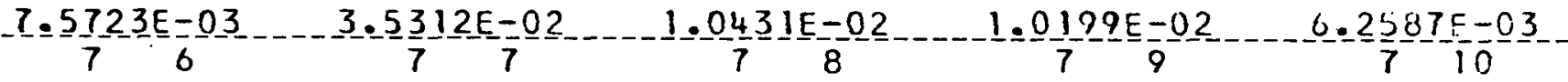
$3.9407 E=03 \quad 1.5454 E=03 \quad 3.0907 E-04 \quad-2.0301 E-04 \ldots$ $8.2327 E-03 \quad \ldots 3.8391 E-02 \ldots 1.1341 E=02 \ldots 1.1089 E-02 \ldots$ $4.2844 E-03 \quad 1.6801 E-03 \quad 3.3603 E-04 \quad 5.0404 E-04 \quad 0.8805 E-04$ $6.9386 E-03 \ldots .2357 E-02 \ldots .5583 E-03 \quad 9.3439 E-03$ $3.6109 E-03 \quad 1=4160 E-03 \quad 2=8321 E-04 \quad-4.2481 E-04 \quad-4.9562 E-04$ $3.2655 E-02 \quad 1.5228 E-01 \quad 4.4984 E-02 \quad 4.3985 E-02 \quad 2.6990 E-02$ I. $6994 \mathrm{E}=02$ 
NI CKEL

N 2

SIGMA N,G (N)/N2)

\begin{tabular}{|c|c|c|c|c|}
\hline 11 & -11 & 11 & 11 & \\
\hline $0705 E-0$ & $4.9920 E-01$ & $1.4747 \mathrm{E}-01$ & $1.4419 E-01$ & $8.8480 \mathrm{E}-02$ \\
\hline 11 & 11 & 11 & $11 \quad 9$ & $11 \ldots$ \\
\hline $\begin{array}{l}5709 E-0 \\
12\end{array}$ & $2.1847 E-02$ & $4.3694 \mathrm{E}-03$ & $6.5540 \mathrm{E}-03$ & $7.6464 \mathrm{E}=0$ \\
\hline $4 E-1$ & 12 & $-\frac{12}{2}$ & 12 & 12 \\
\hline $\begin{array}{l}+E-C \\
-6\end{array}$ & $4.2857 E-01$ & $2660 E-01$ & $2379 E-01$ & $5961 E-0$ \\
\hline $\bar{F}$ & $1.8756 E-02$ & $3.7512 \bar{E}-03$ & $5.6267 E-03$ & $6.5645 E-0$ \\
\hline 1 & 13 & 3 & 13 & 13 \\
\hline & $1.8051 E 00$ & $5.3322 E-01$ & $5.2137 E-0$ & $3.1993 \mathrm{E}-0$ \\
\hline-0 & $\frac{1}{80}$ & $-\frac{1}{57}$ & $\frac{13}{369}$ & $\frac{13}{76}-\frac{10}{9}=-1$ \\
\hline
\end{tabular}

$2.1034 E-02-9.8089 E-02-2.8976 E-02 \quad 2.8332 E-02-1.7386 E-02-$

$1.0946 E-02-4.2927 E-03 \quad-\frac{1}{8} .5855 E-04 \quad 1.2878 E-03-1.5025 \frac{1}{E}-03^{-}$

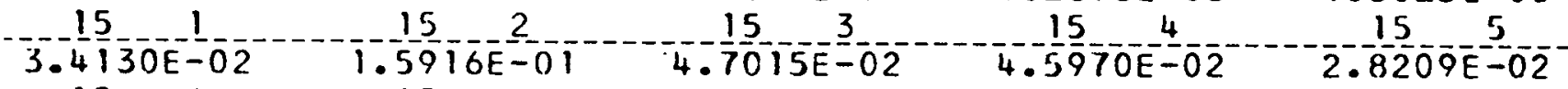

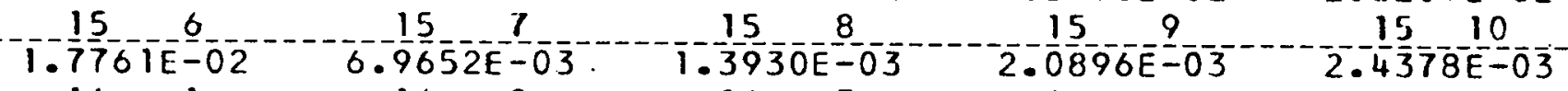

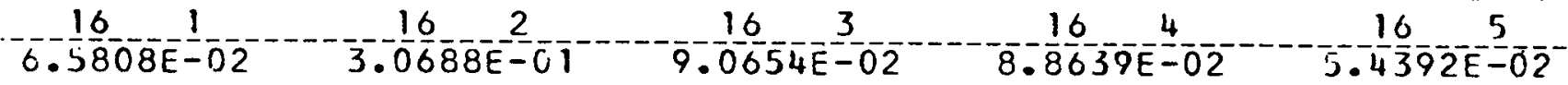

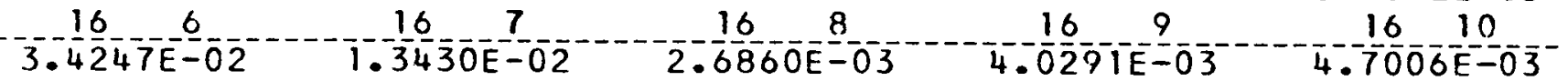

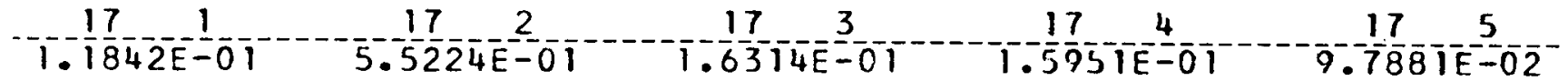

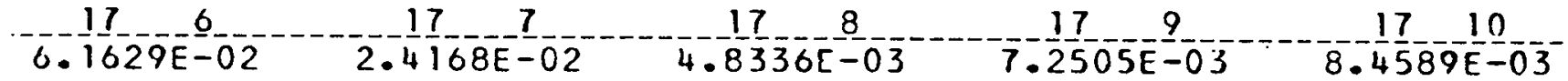

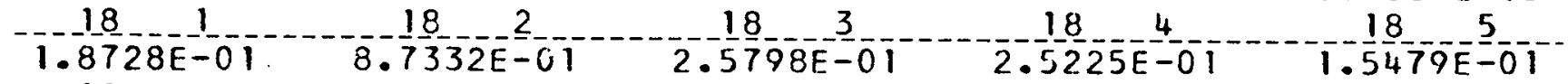

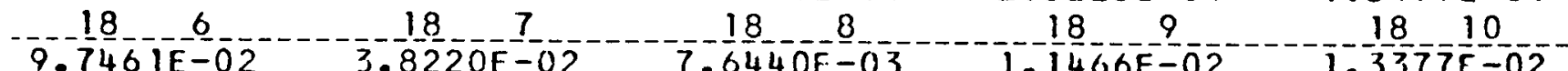

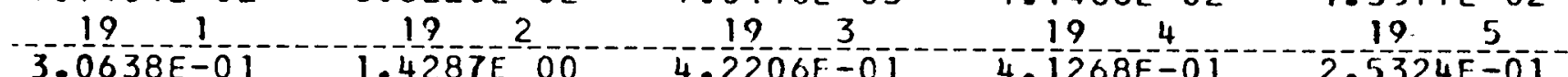

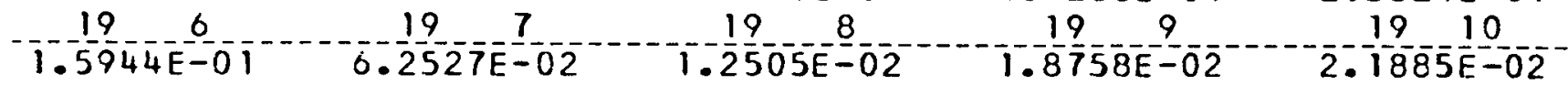

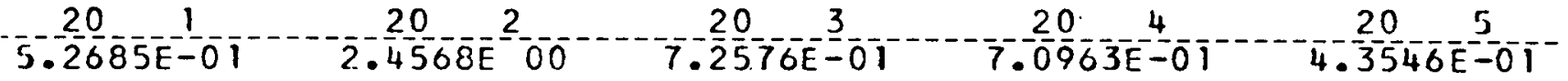

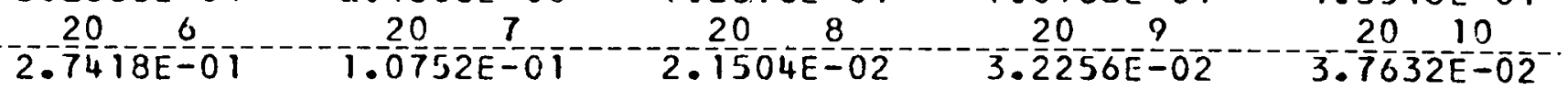


-NICKEL.

N1

N2

SIGMA N,G (NIIN2)

211212135

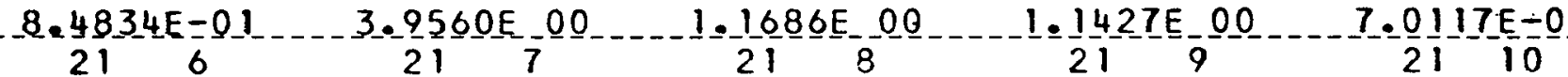
4.4 $148 E-01 \ldots 1.7313 E-01 \ldots 3.4626 E-02$ 22

$1.3481 E$ 226 22 22 3

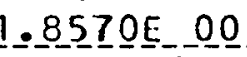
228 $22-7$ $5=\frac{502}{2}-\frac{3}{3}=\frac{-02}{3}$ $-\frac{75}{23} \leq 1 E-$ 23 1 $1.0611 E_{7} 01$ $3 .-\frac{1}{2} 346 E-00$ 4. 643 BE -01 242 $9.2877 E=02$ 4.82005 O 0 248 $1.4281 \mathrm{E}-01$ 247 $7.1407 E-01$ 252 253 $7.5761 E$ OQO 258 257 $1.1224 E$ EO $2=2448 E=01$ 263 262 7.58200 E 00 267 1. $1233 \underline{2}$ E 00 $2.2465 E-01$ 27 7. $5902 E$ OOO 278 $\left.2.5694 E_{-1}\right]$ 277 $1.1245 E-00$ 2. $2490 E=01$ 283 $2.5726 E_{-1} 01$ $1.1259 E-00$ 292 $2.2517 E=01$ 293 $7.6138 \mathrm{E}-00$ $29-\frac{2}{7}$ $1=1280 \mathrm{E}$ On $2=2559 E=01$ $.5838 E 01$ $-5 \cdot \frac{5}{3} \underline{0} 0 \mathrm{ZE}-\frac{0}{6}$ $2-\frac{5}{3} \frac{8}{0} 3 \underline{E}-\frac{0}{7}$ $30-18$ $2.2615 E=01$ $22 \frac{1}{4}$ $6.0595 \mathrm{E}-02$ 22 1. $11142 E-00$ $22 \frac{1}{10}$ $22-\frac{8}{9}$ $9.6 \frac{6}{23}-\frac{0}{5}-0$ $8.2 \frac{2}{2} 334 E-02$ $3.0648 E-00$ $1=\frac{8}{2} \frac{808 E}{10}=0$ $1.3932 E=01$ 244 $4.7129 E-00$ 249 2. $1422 \mathrm{E}-01$ 25 $7.4078 E_{0} 00$ 25 $3.3672 \mathrm{E}=01$ $26-\frac{1}{4}$ $7.4 \frac{1}{26} 35 E-00$ $3.3 \frac{3}{2} \frac{2 B E-01}{4}$

$7.4216 E$ 27

9 $3.3734 E-01$ 284 7. $430 \underline{0}$ E 00 28 3. $3776 E=01$ 294 $7.4446 E-00$ $3.3839 E-01$ $7 \cdot \frac{4}{3} 629 \mathrm{E}$. 00 $3.3922 E=01$ $1.6253 \mathrm{E}-01$ 245 $24 \quad 10$ $2.4993 E-01$ $25-5$ 4. 54 57E 00 2510 26 10 $3.9314 E-01$ $27 \quad 10$ $3=9357 E=01$ 285 4.5598 E 00 2810 $3=-9406 \frac{6}{29}-0$ $2.8920 \mathrm{E} 00$ 3.-92 $284 E-01$ 4. 5492 E 00 $27-5$ 4. 55 4_IE_oㅇ.

5. $5270 E$ 296

$2.8763 \mathrm{E}$ OOO 1_-1307E_00. 
NICKEL

N1 N2

SIGMA_N,G_(N)IN2)

\begin{tabular}{|c|c|c|c|c|}
\hline 31 & 31 & 31 & 31 & 31 \\
\hline $.5620 E 00$ & $2.5937 \mathrm{E} 01$ & $7.6619 \mathrm{E} \quad 00$ & $7.4917 \mathrm{E} 00$ & $4.5972 E \quad 00$ \\
\hline 3] & 31 & $3 L_{\ldots} .8$ & $31 \ldots$ & 31 \\
\hline $.8945 E 00$ & $1.1351 E 00$ & $2.2702 E-01$ & $3.4053 E-01$ & $3.9729 E-01$ \\
\hline .5 & $-32-z^{2}$ & -32 & 32 & 32 \\
\hline 32 & $\begin{array}{l}2.6108 E \text { O } \\
32\end{array}$ & $\begin{array}{c}7.7125 E 00 \\
32\end{array}$ & $\begin{array}{c}7.5411 \mathrm{E} 00 \\
32\end{array}$ & $\begin{array}{c}4.6275 E 00 \\
3210\end{array}$ \\
\hline $2.9136 \mathrm{E} \quad 00$ & $1.1426 \mathrm{E} 00$ & $2.2852 E-01$ & $3.4278 E-01$ & $3.9991 E-01$ \\
\hline & 33 & 33 & $3 \underline{3}$ & 33 \\
\hline $5.6755 \mathrm{E} 00$ & $2.6466 \mathrm{E} 01$ & $7.8183 \mathrm{E} 00$ & $7.6445 \mathrm{E} 00$ & $4.6910 \mathrm{E} 00$ \\
\hline 33 & -33 & $\ldots 3$ & 33 & 33 \\
\hline $2.9536 E 00$ & $1.1583 \mathrm{E} 00$ & $2.3165 E-01$ & $3.4748 \mathrm{E}-01$ & $4.0539 E=01$ \\
\hline 84 & $--34-2$ & -34 & --34 & -34 \\
\hline 34 re 6 & $\begin{array}{c}2.84091 \\
34\end{array}$ & $\begin{array}{c}8.0968 E \quad 00 \\
348^{2} 8\end{array}$ & $\begin{array}{c}1.9169 E^{34}, 90 \\
9\end{array}$ & $\begin{array}{c}4.8581 E 00 \\
3410\end{array}$ \\
\hline $3.0588 \mathrm{E} \quad 00$ & $1.1995 \mathrm{JE} 00$ & $2.399|\mathrm{E}-0|$ & $3.5986 E-01$ & $4.1983 \mathrm{E}-0 \overline{1}$ \\
\hline
\end{tabular}


DAIE_ DEE _ O1_ 1960

DESIGNATION

IRON
CODE NO.

$26 \cdot 5600$
DENSITY FACTOR

$\overline{9} \cdot \overline{2} \bar{Y} \overline{8} \overline{3} \overline{0} \bar{O}$

\section{LEVEL ENERGY SCATTER SCATTER TRANSPORT ABSORPTION SCATTER}

1 1.000E $073.2668 \mathrm{E}$ OO $3.3578 \mathrm{E} 00 \mathrm{1} .6488 \mathrm{E} 00 \mathrm{1} .6439 \mathrm{E}-02-1.5609 \mathrm{E} 00$

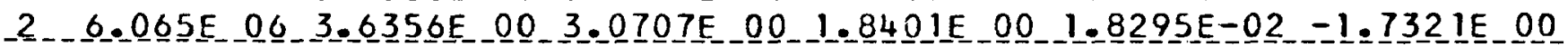

$3 \quad 3.679 \mathrm{E} 06 \quad 3.4717 \mathrm{E}$ OO $2.4741 \mathrm{E} 002.2951 \mathrm{E} 001.7488 \mathrm{E}-02-1.1351 \mathrm{E} 00$

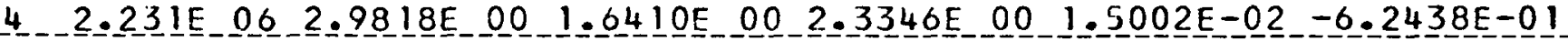

5 1.353E $062.7415 \mathrm{E} 00-0588 \mathrm{E}-012.3297 \mathrm{E} 001.3801 \mathrm{E}-02-3.9722 \mathrm{E}=01$

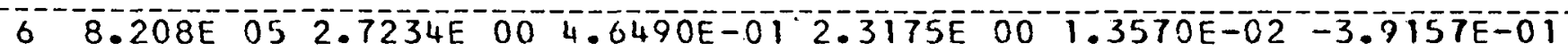

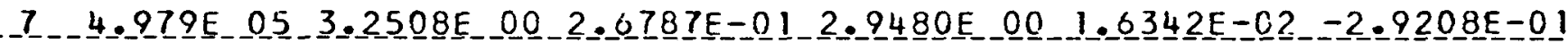

$83.020 E$ OS 3.0188E $001.0577 \mathrm{E}-012.5602 \mathrm{E}$ 00 $1.5164 \mathrm{E}-02-4.4240 \mathrm{E}-01$

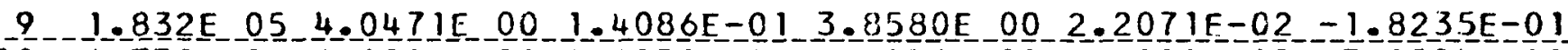

$106.738 \mathrm{E} 046.1814 \mathrm{E}$ 00 $2.2070 \mathrm{E}-016.1072 \mathrm{E}$ 00 $2.4200 \mathrm{E}-02-7.1594 \mathrm{E}-02$

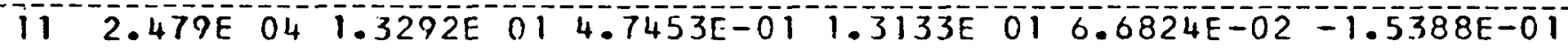

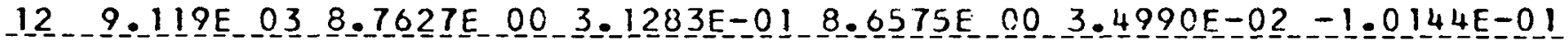

13 3.355E $035.9979 \mathrm{E} 002.1412 \mathrm{E}-015.9259 \mathrm{E} 00 \mathrm{1}-1600 \mathrm{E}-02-6.9436 \mathrm{E}-02$

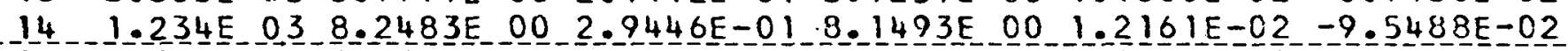

$154.540 \mathrm{E} \quad 021.0714 \mathrm{E} 013.8248 \mathrm{E}-011.0585 \mathrm{E}-1 \mathrm{1} 2.0920 \mathrm{E}-02-1.2403 \mathrm{E}-0 \mathrm{1}$

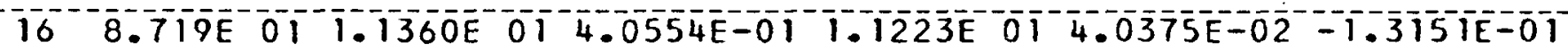

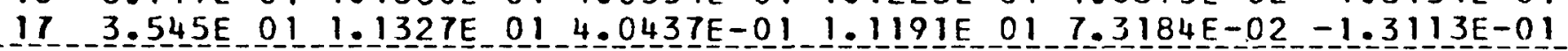

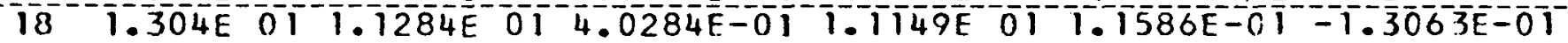

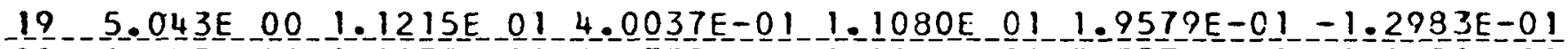

$201.445 \mathrm{E}$ OD $1.1135 \mathrm{E} 013.9753 \mathrm{E}-011.1002 \mathrm{E} 013.3376 \mathrm{E}-01-1.2891 \mathrm{E}-01$

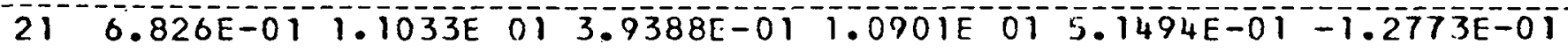

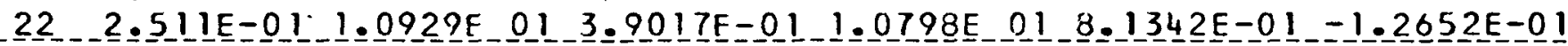

$239.237 \mathrm{E}-021.0721 \mathrm{E} 013.8275 \mathrm{E}-011.0593 \mathrm{E} 01 \mathrm{1} .3976 \mathrm{E} 00-1.2412 \mathrm{E}-01$

24 2.530E-02 1.0657E 01 3.8045E-01 1.0529E 01 2.1667E $00-1.2337 E-01$

*4500 3.023E-01 1.0820E 01 3.8248E-01 1.0691E 01 8.7077E-01-1.2445E-01 * $40002.718 \mathrm{E}-011.0820 \mathrm{E} 013.8248 \mathrm{E}-011.069 \mathrm{IE} 018.7077 \mathrm{E}-0 \mathrm{1}-1.2445 \mathrm{E}=1$ *3500 2.413E-01 1.0820E 01 3.8248E-01 1.0691E $01.8 .7077 \mathrm{E}-01-1.2445 \mathrm{E}-01$

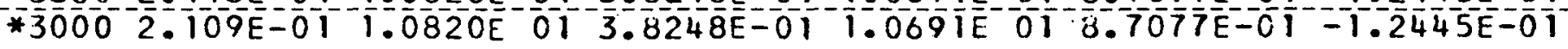
2500 1.804E 01 1.0810E $013.8215 E-011.0681 E 019.4168 E-01-1.2435 E-01$

$20001.499 E=01-0.0800 E 013.8179 E-01-1.0671 E 01-1.0334 E 00-1.2424 E=01$ $15001.194 \mathrm{E}-011.0785 \mathrm{E}$ 01 $3.8126 \mathrm{E}-011.0657 \mathrm{E} 011.1585 \mathrm{E} 00-1.2406 \mathrm{E}-01$

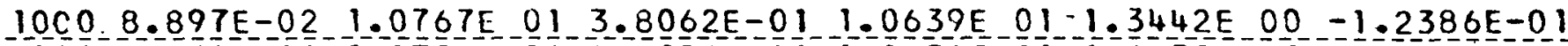

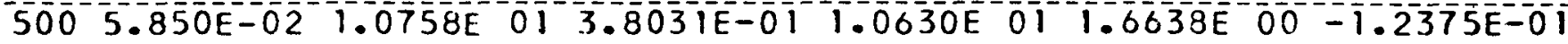

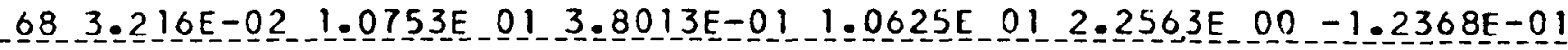
*Thermal cross sections taken from XDC 60-1-139.

NOTE: For a summary of the sources of data see APEX-704 and XDC 60-1-139. 
I R.ON

THERMAL TRANSFER MATTRICES

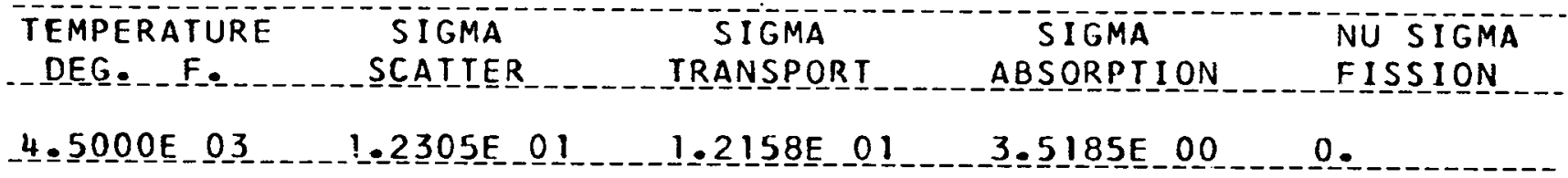

\section{$\bar{N} \overline{1}-\bar{N}$ \\ SIGMA S (NIIN2)}

\begin{tabular}{|c|c|c|c|c|}
\hline $2 J_{-\ldots} 21$ & 21 & 23 & 21 & 21 \\
\hline $8462 \mathrm{E} 00$ & $1.1864 \mathrm{E} 00$ & $6.1536 E-04$ & $1.0089 \mathrm{E}-07$ & 0. \\
\hline $1620 E 00$ & $9.0592 E$ OD & $7.9340 \mathrm{E}-01$ & $4.4996 E-03$ & $3.7573 E$ \\
\hline 23 & 22 & 23 & 23 & 2 \\
\hline $.6562 \mathrm{E}-03$ & $2.4851 \mathrm{E} 00$ & $7.7896 \mathrm{E} 00$ & $5.7349 \mathrm{E}-0 \mathrm{i}$ & 1.0950 \\
\hline 24 & -24 & 23 & 24 & 24 \\
\hline $2.0504 E-06$ & $1.2170 E-01$ & $5.1786 \mathrm{E} \quad 00$ & $4.3459 \mathrm{E} 00$ & 1.63388 \\
\hline & $3 E$ & $.3731 \mathrm{E} 00$ & $.25381 \mathrm{E}-20$ & \\
\hline
\end{tabular}

TEMPERATURE SIGMA SIGMA SIGMA NU SIGMA _. DEG _ _ F_. _. _. SCAIIER _. _. IRANSPORI $\triangle B$ SORPIION FI SSION

4..0000E 03 1.2.2141E 01 1.1997E_01 3. 5214 E 00 0.

\section{SI GMA S (N I IN2)}

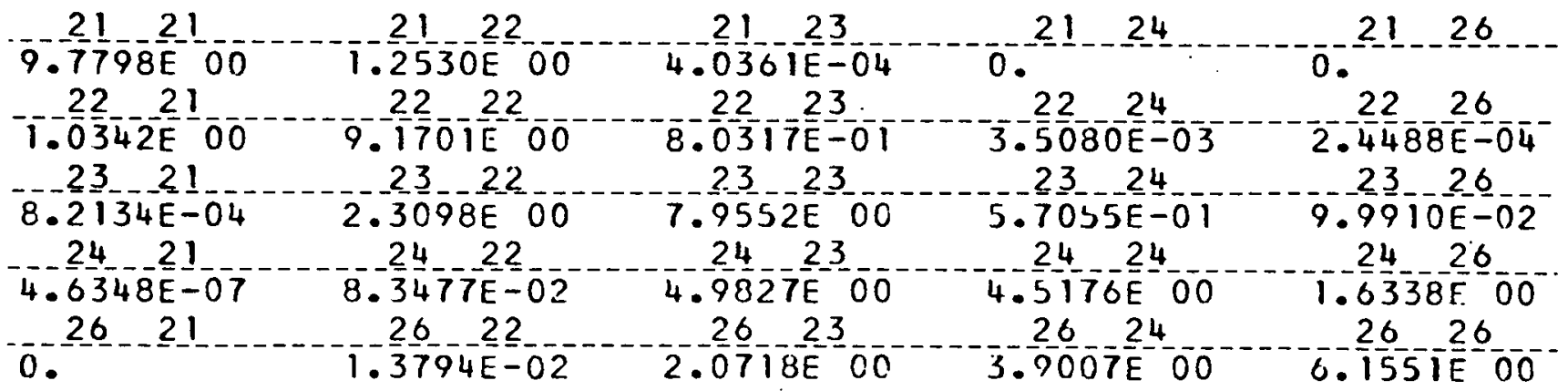


IRON

_THERMAL IRANSFER _MAIRICES

- LEMPERAIURE

SIGMA

SIGMA

DEG. F. SCATTER

IRANSPORT

SIGMA

NU_ SIGMA

$3.5000 \bar{O} 0 \overline{3}$

1. $1977 \mathrm{E}$ OI

$1.1834 \mathrm{E} 01$

$3.5250 E 00$

FISSION

-

NI

N2

SIGMA S (N)/N2)

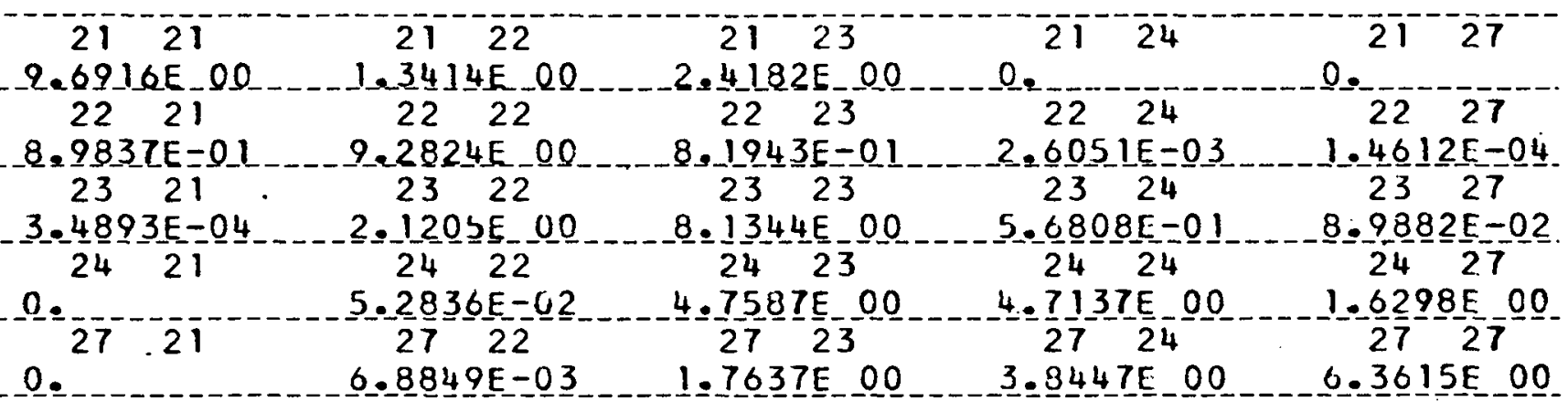

- TEMPERAIURE

DEG. F.
SIGMA

TRANSPORT
SIGMA

ABSORPTION
NU SIGMA

FISSION

\subsection{E 03 T.181TE OT $1.1670 \mathrm{O}^{-1}$}

$21^{2} 1^{-12} 1^{2}$

$9.5 \frac{7}{2} 17 \mathrm{E}-00$

$3.52 \overline{9} \overline{\mathrm{E}} \overline{\mathrm{O}} \mathrm{O} \overline{\mathrm{O}}$

o.

$7 \cdot \frac{5}{2} 387 E-01$

- I. $1924 E-04$

$1.9143 E$ EO

을

2422

$2.9892 E-02$

$-\underline{0}$
$28-22$

2. 8883 E
$8.4568 \frac{8}{2}-01$

$8.3295 \mathrm{E}-0 \mathrm{O}$ 2423

4.5O I JE 00

2823

$1.4514 E$ OO
$0 .-24$

$1.8087 E-03$

$5.6682 E=01$

2424

4.9408E 00

2824

3. 7636 트 OO $22-\frac{1}{28}$

$7 \cdot-\frac{70}{2} \frac{3}{3}-\frac{05}{28}$ 7.-9421E-0? 2428

$1.6 \frac{6}{2} \frac{204}{2}=0$ 6. $5931 E$ OO 
IRON

THERMAL TRANSFER MATRICES

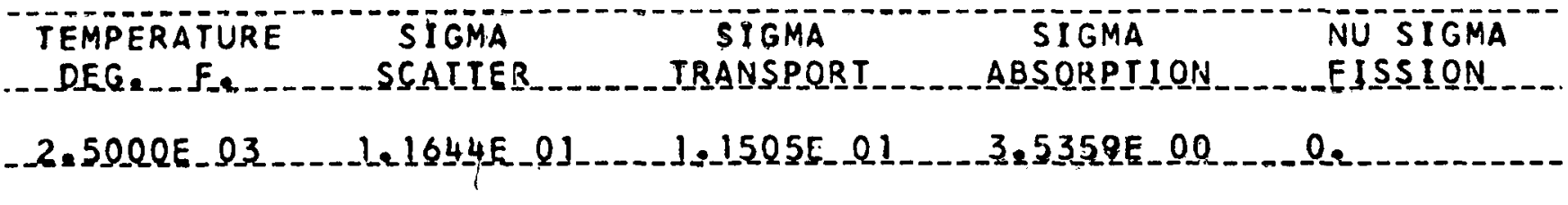

$\bar{n} 1-\cdots$

SI SMA S. (NIINN2)

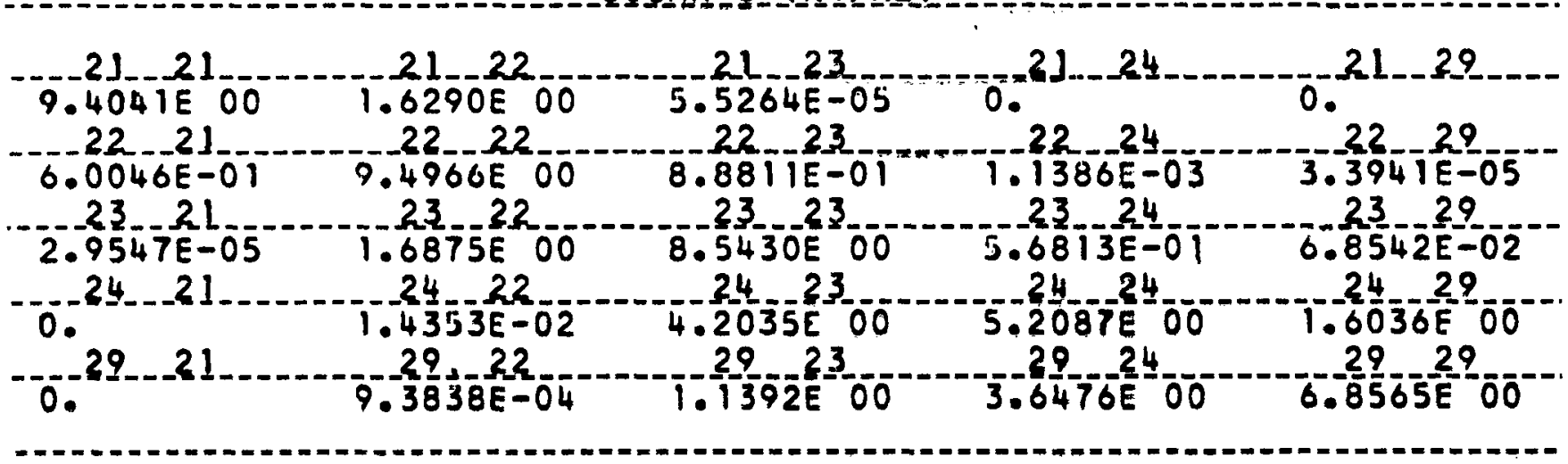

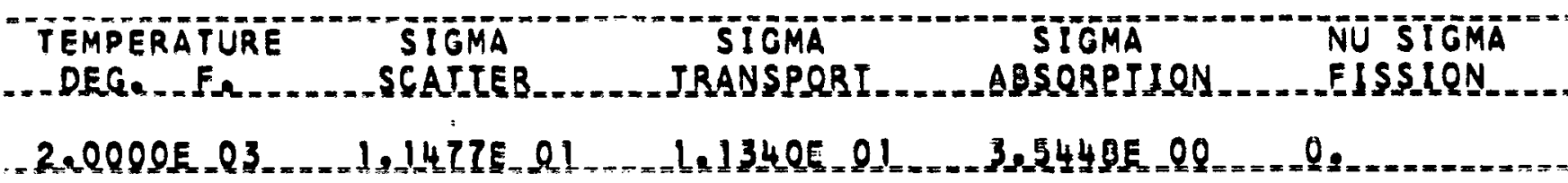

Ni $1==== \pm \bar{N}$

SIGMA $S=(N) \perp N 2)$

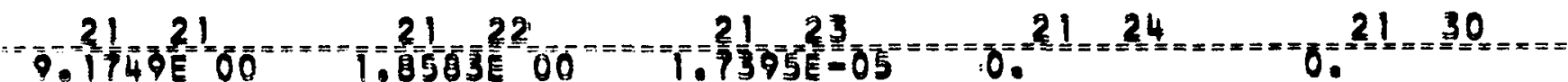

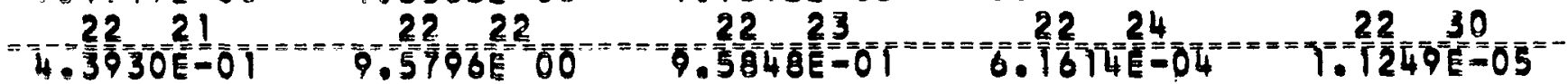

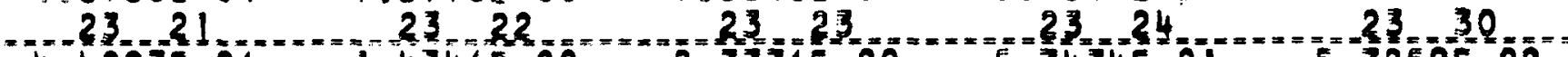

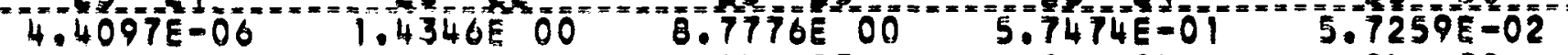

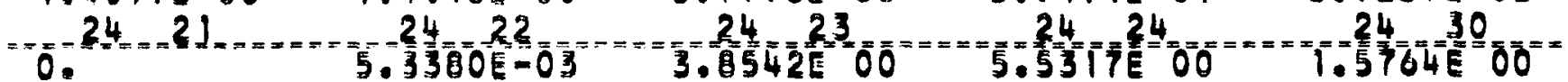

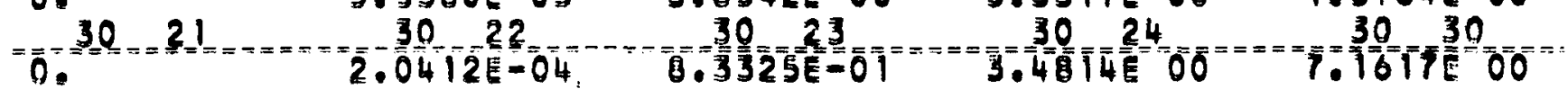


I RON

_IHERMAL_ IRANSFER MAIRICES

TEMPERAIURE

DEG. $F$.
SI GMA

SCATTER
SIGMA TRANSPORT
SIGMA ABSORPTION
NU SIGMA_FISSION

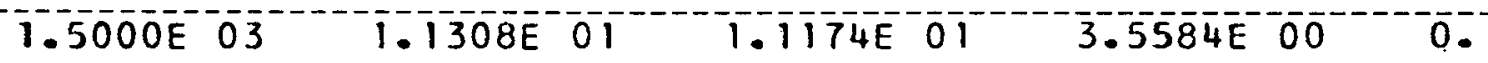

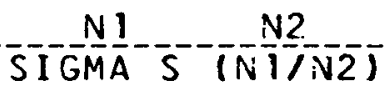

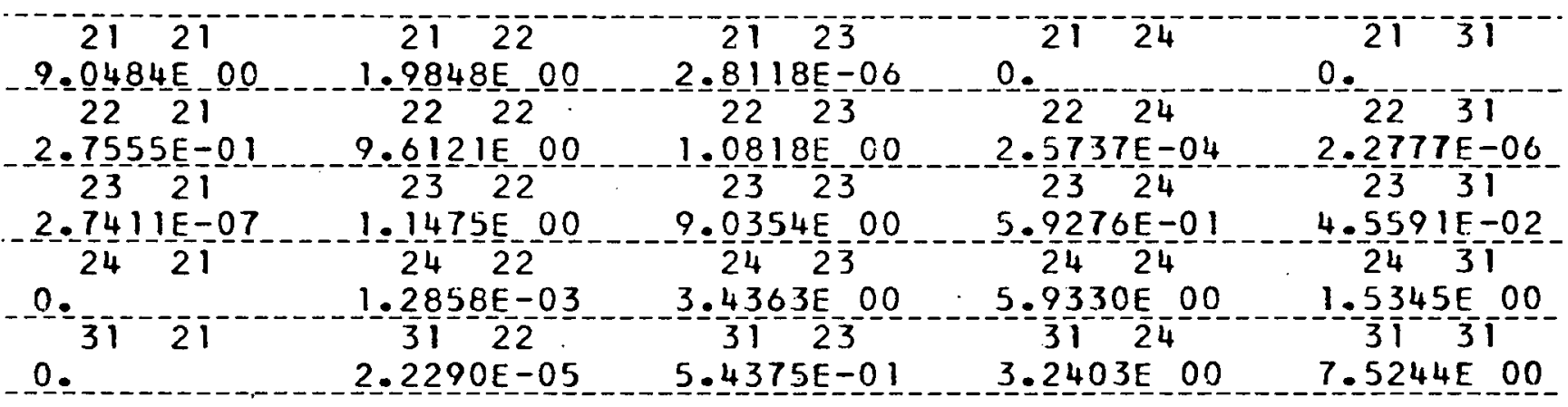

TEMPERAIURE DEG. $F$. $1.0000 \mathrm{E} 0 \overline{3}$
SIGMA

SCATTER
SIGMA TRANSPORT
SIGMA

ABSORPTION
NU SIGMA

FISSION

1.114TE OT 1.1008E OT 3.5817E 00

\section{SIGMA S (NIIN2)}

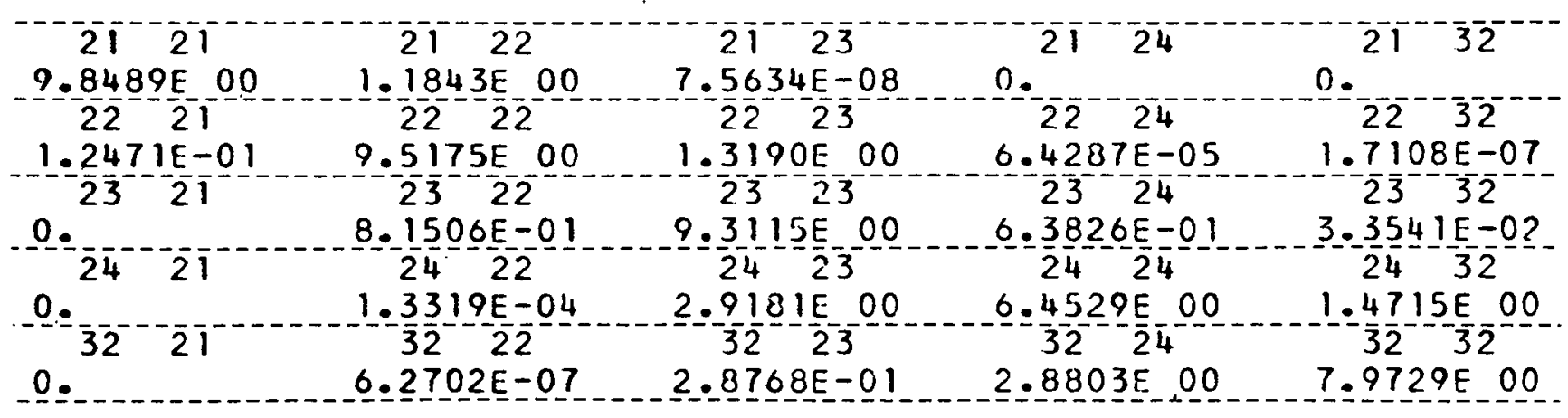


IRON

THERMAL TRANSFER MATRICES

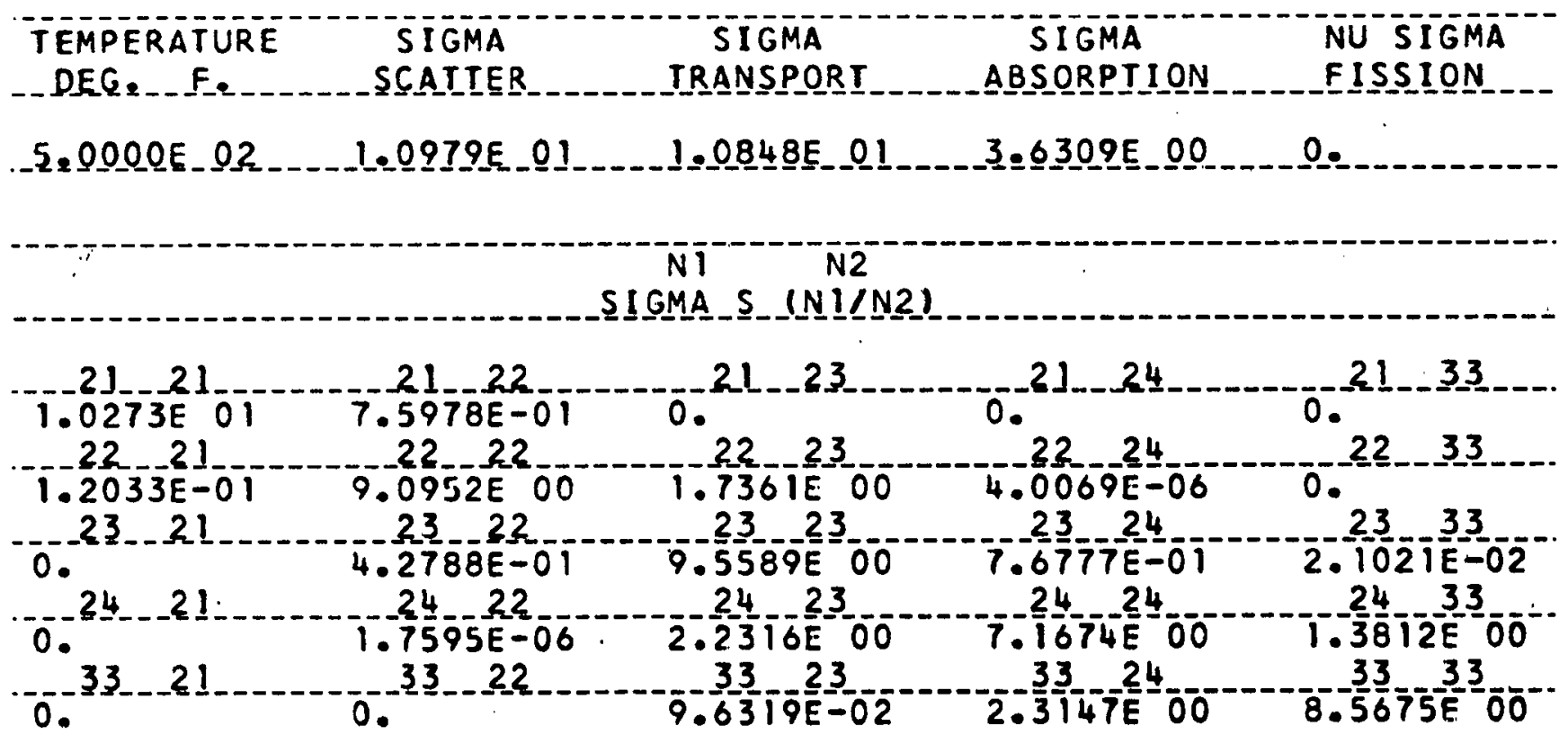

TEMPERATURE
DEGE_FA STGMA

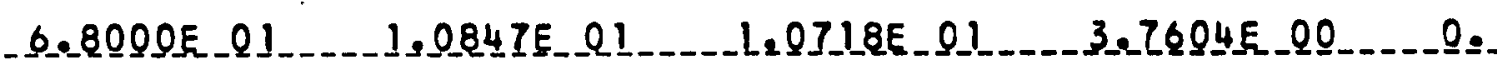

Ni' $\overline{1}$

SIGMA_S_(N1/N2)

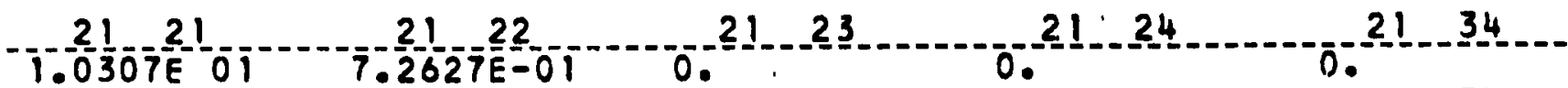

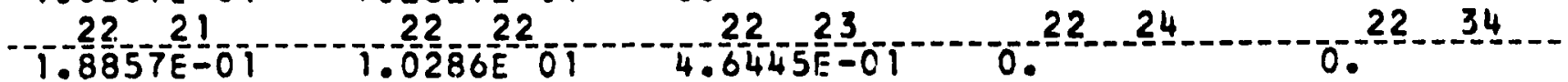
23
0.

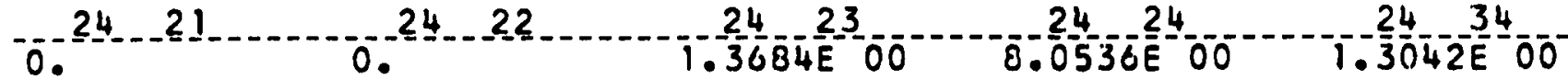

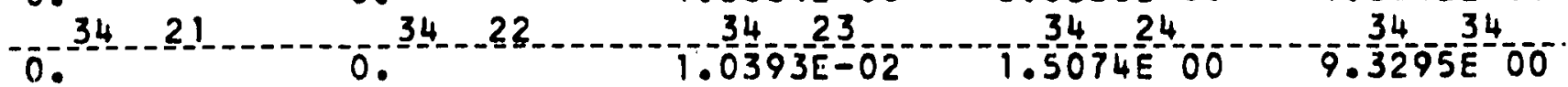


SIGMA 50 (NIIN2)

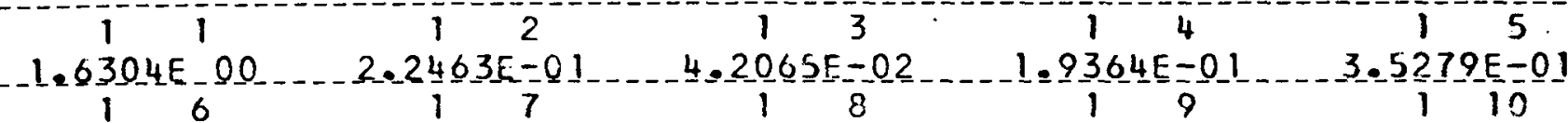
3.5249E-01 2.4021E-01 1.2897E-01 $1.5240 E-02$ $\therefore 3.3662 E=03-4.7068 E-04-6.5501 E=05 \quad 28.7264 E-06 \quad 1.2863 E-06$

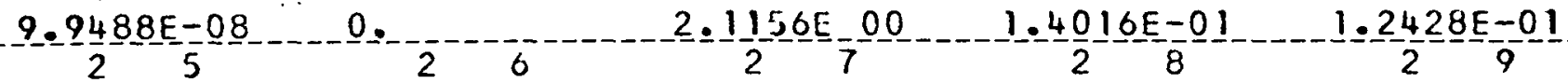
-3.0265E-01 $203.6690 \mathrm{E}=01 \quad 2.8323 \mathrm{E}-01 \quad 1.6447 \mathrm{E}-01$

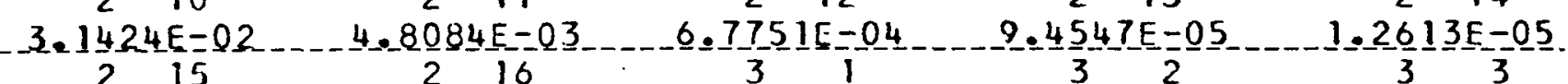

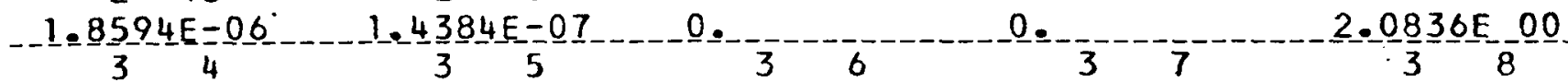
$1.8850 E-01 \ldots 1.9435 E=01 \ldots 3.2060 E=01 \quad 3.0157 E-01$ $1.3295 E=01 \ldots 4.3866 E-02$ _ $6.9302 E-03$ $314 \quad 3 \quad 15 \quad 316$ $-1.8512 E-05-2.7307 E-06-2$ O.

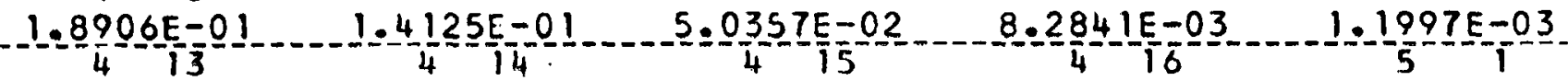

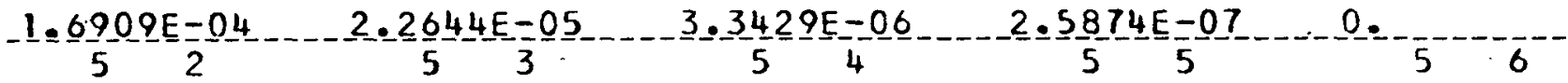
0 . $1.4 \frac{9}{5} 5 \mathrm{E}-01$

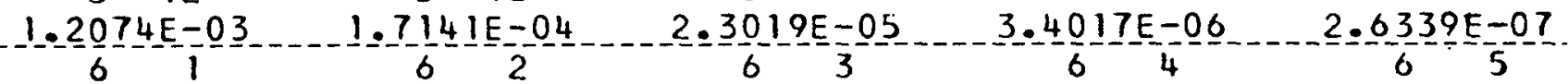

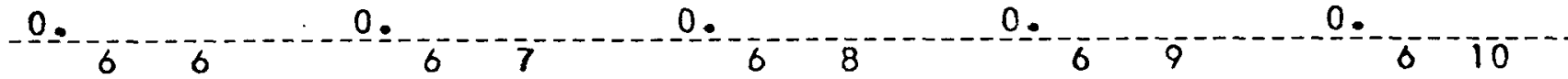
-2.2931E 00 - $2.11425-01 \quad 8.4901 E-02 \quad 8.6820 E-02 \quad 3.8738 E-02$ $7.1684 E-03 \quad 1.0858 E-03 \quad 1.5556 E-04 \quad 2.0 .966 E-05 \quad 3.1023 E-06$ $2=4032 E-07$ 0. 0. 


\section{1.}

\section{IRON}

N1

SIGMA SO_(N1/N2)

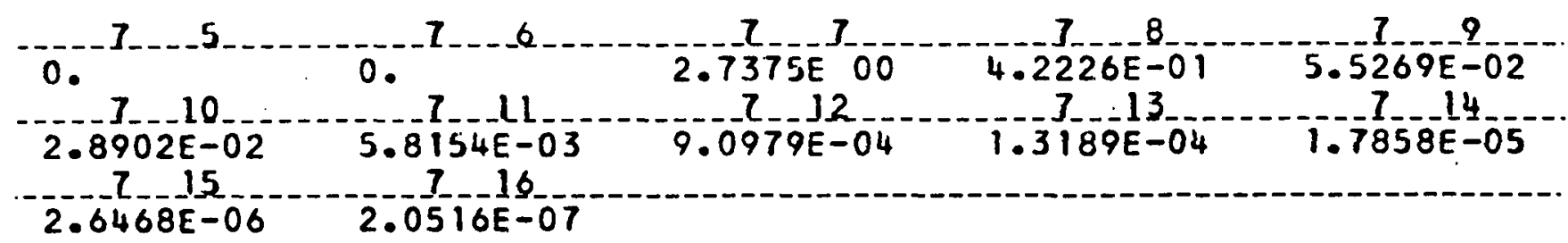


SIGMA N,G (NIIN2)

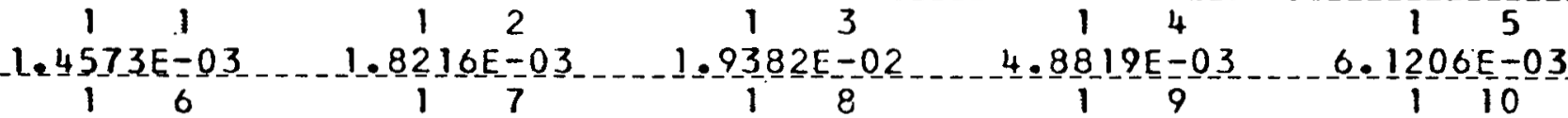

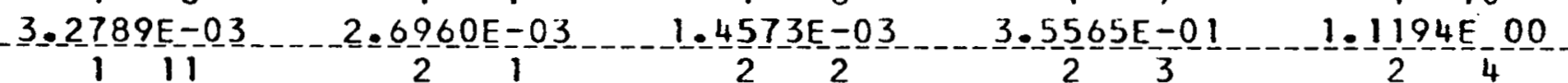
$1.2987 E-02-1.1357 E-03 \quad 1.4196 E-03 \quad 1.5105 E-02 \quad 3.8046 E-03$ $4.7700 E-03 \quad 2.5553 E-03 \quad 2.1011 E-03 \quad 1.1357 E-03$ 1. $15 \frac{15}{3} 16 \mathrm{E}-0 \mathrm{O} \quad 1.0121 \mathrm{E}-02$ $3.1512 E=03-3.9508 E-03-2.1165 E=03-10-7402 E-03-29-4066 E-04$

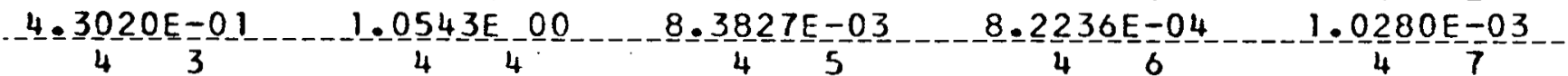
$1.0937 E-02 \quad 2.7549 E-03 \quad 3.4539 E-03 \quad 1.8503 E-03$ $8.2236 E-04 \quad 8.8065 E-02 \quad 7.7663 E-01 \quad 7.3285 E-03 \quad 7.5061 E-04$ $9.3826 \mathrm{E}-04$ $1.3886 E-03 \quad 7.5061 E-04 \quad 1.1259 E-03 \quad-4.8360 E-01 \quad 6.6890 E-03$ $1.0253 E-03 \quad 1.2816 E-03 \quad 1.3636 E-02 \quad 3.4347 E-03-4.3062 E-03$

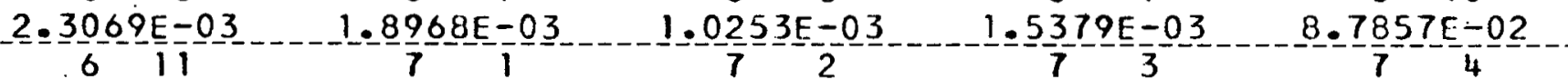
$9.1367 E-03-1.1542 E-03 \quad 1.4427 E-03 \quad 1.5351 E-02 \quad 3.8606 E-03$ $4.8476 \mathrm{E}-03$ $2.8855 E-04 \quad 1.0286 E-02 \quad 1.2841 E-03 \quad 1.0051 E-03 \quad 1.7078 E-02$ $4=3017 E-03 \quad-5=3931 E-03 \quad 2.8892 E-03 \quad 2.3755 E-03 \quad 1.2841 E-03$ $1.9261 E-03 \quad 3.2102 E-04 \quad 1.1443 E-02 \quad 1.3198 E-03 \quad 1.6498 E-03$ $1.7554 E-02 \quad 4.4214 E-03 \quad 5.5433 E-03 \quad 2.9696 E-03 \quad 2.4417 E-03$ 1. 3198E-03 1.9797E-03 - 3.2996E -04 _ 1.1762E-02 _ 4.8830E-03 
I RON

N1 N2

SIGMA N,G (NIIN2)

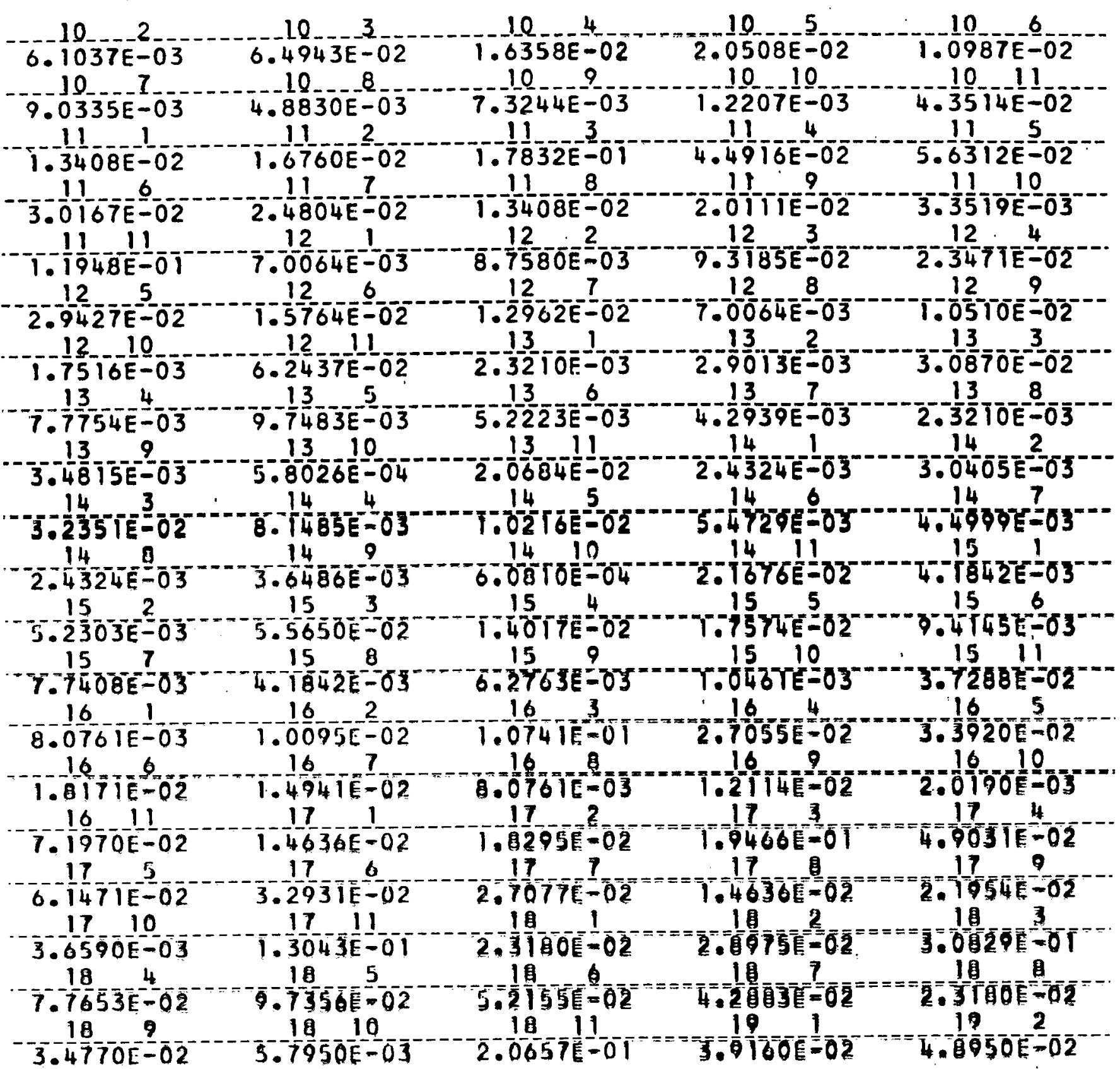


$\underline{\text { R }} \underline{\mathrm{N}}$

N 1

SIGMA N,G (NITNZ)

$19-19019-1906079$

5.2083E-01 _ 1.3119E-01 $1.6447 \mathrm{E}-01$

$19-801909$

1910

$\varepsilon=8110 E=02$

$7.2446[-02$

$3.9160 E-02 \quad 02.8740 E-0 ?$

$3.48 ? 7 E-01$

$6=\frac{6}{20} 60 \mathrm{E}-02$

$8=\frac{3}{2} 250 E-02 \quad-8 \cdot \frac{8}{2} 791 E-01$

$2.80396-01$

$1.5021 E-01$

$1.2351 E-01$

$\frac{0014 \mathrm{E}-0}{3}$

$1.6690 \mathrm{E}-02$

$5.9493 E-0$

$1.0298 E-01$

$1.3696 \mathrm{E} 00$

$21-4$ 215

2. $317 I E-01$

2111

22

22

$-4498 E-0$

4. $3252[-01$

9. $1770 E-01$

$1.6268[-01$

$2.0335 E-01$

$=5447 E-01$ 210 225 226 22 22

$2.5745 \mathrm{E}-02$

6. 8326 EE-O

$3.6603 \mathrm{E}=01$

$3.00966=01$

$2=1636 \mathrm{E}-00$

$5.4498 E-01$

$22 \quad 10$

4. $=0670$ 으-02

1. $4497 E$ 은

234

$9.36660 \mathrm{E}-01$

1. $1743 \mathrm{E}$ ON

$2.7960 E-01$

1. $-\frac{6}{2} \frac{2}{3} 68 E-01$

$2.4402 E-01$

239

. $4.1 \frac{1}{2} \frac{4}{4} 0[-01$

$6.9900 E-02$

$6.2910 \mathrm{E}=\mathrm{O}$

2430
$7509 E \quad 00$

$24-8$

4. $3240 E=01$

$.4483 E 00$

$2.4917 E-00$

$3.4930 E-01$

$3.7 \frac{1}{2} \frac{1}{3}$ E 00

$$
252
$$

$8.4950 E=0$ 249

$1.8 \frac{1}{2} 4-1 \mathrm{E}-\mathrm{CO}$

$6.4860 E-01$

$1.0810 E-01$

254

23

5. 172626 E 01

$2.7960 \mathrm{E}-01$ 257

9.0387500

$1.2573 E$ O 00

26

258

$2.2 \frac{7}{25} \underline{0} 7 E-00$

4. $3240 E-01$

$2 \frac{1}{4}-6$

$5.4050 E-0$

$6.8000 E-01$

$6.79600 \mathrm{E}=0$

1. $0194 E_{3}$ On

9.7290E-01

24 i

$7.9994 \mathrm{E}-01$ 266

$1.5300 E$ OO

8. $5000 \mathrm{E}-\mathrm{C}$

9.0440500

$3=\frac{8}{25} 33 \underline{E}-00$

$6.7960 \mathrm{E}-01$

$2.8543 E$ EO

1. $5291 E$

2510

$1.6990[-0$

$0.0562 E 00$

2611

$0.0598 E$ ED

$1.2580 \mathrm{E}$ on

$26-\frac{1}{3}$

$2 .-\frac{2}{2} \frac{7}{6}$ OE $-\frac{O}{9}$ 265 275

$2.8594 \mathrm{E} 00$

27
$0.80805-01$

$27^{10}$ 27

$0.8000 E=0$

1. 02000

2.8560 E 00

$1.7020 E-01$

$1.5318 \mathrm{r}$ on

2711

$8 \cdot \frac{5}{2} \frac{1}{7}$ NEE $=01$

$9.0546 E-00$ $26 \quad 10$ $0.0669 \mathrm{E} \quad 00$

1.2525500

$6.8080 E-01$

$=\frac{700 E-0}{27}$

01 
IRON

N1 N2

SIGMA N,G (NI/N2)

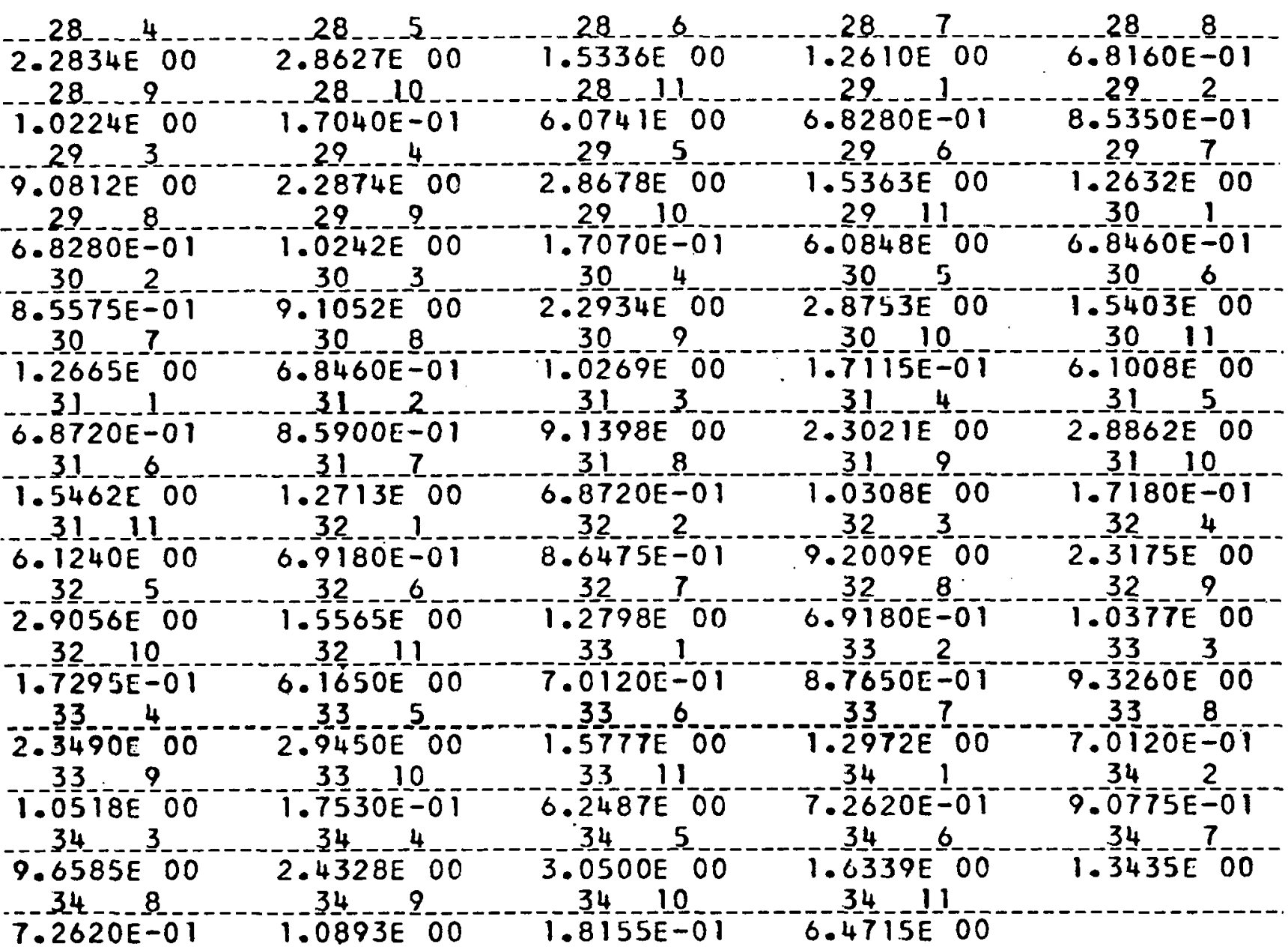


FAIE_LEC OI 1960

DESIGNATION

CHROMIUM
CODE NO.

24.5200
DENSITY FACTOR

$8.63279 E^{\circ}$ OI
SIGMA LEVEL ENERGY SCATTER
XI_SIGMA SCATTER
SIGMA TRANSPORT
SIGMA ABSORPTION
XII SIGMA SCATTER 1

1 1.000E $073.2179 \mathrm{E} 00 \quad 3.0634 \mathrm{E} \quad 00 \quad 1.5262 \mathrm{E} \quad 00 \quad 1.6193 \mathrm{E}-02-1.6293 \mathrm{E} \quad 00$

2 - 6.065E_06_3.5840E_00_2.7681E_Q0_1.7260E_00_1.8039E-02_-1.7895E_00

3 3.679E $06 \quad 3.7598 E$ OO $2.3515 E$ OO $2.3779 E$ O0 $1.8913 E-02-1.3310 E$ OO

$4-2.231 \mathrm{E}-06-3.5300 \mathrm{E}-00-1.6052 \mathrm{E}-0002.7005 \mathrm{E}-00-1.7759 \mathrm{E}-02-7.9892 \mathrm{E}=01$

$51.353 \mathrm{E} 062.7906 \mathrm{E} 003.1714 \mathrm{E}-012.1646 \mathrm{E} 001.4037 \mathrm{E}-02-6.0291 \mathrm{E}-01$

$6 \quad 8.2085052 .7980 E 008.3374 \mathrm{E}-02-2.2868 \mathrm{E} 00-1.4082 \mathrm{E}-\mathrm{C} 2-4.9229 \mathrm{E}-0 \mathrm{~T}$

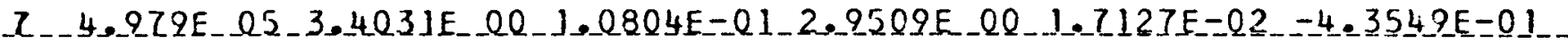

$83.020 E$ O5 2.7910E OO $9.2480 E-02$ 2.5286E $001.4230 E-02-2.5269 E-01$

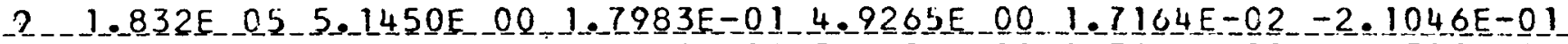

$10 \quad 6.738 E$ O4 5.5301E $00 \quad 1.9749 E-015.4628[00 \quad 2.7806 E-02-6.4793 E-02$

$112.479 \mathrm{E} 043.1300 \mathrm{E}$ 00 $1.1174 \mathrm{E}-013.0925 \mathrm{E} 00 \quad 1.5758 \mathrm{E}-02-3.6176 \mathrm{E}-02$

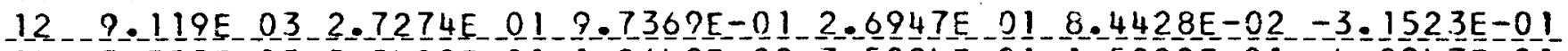

$133.355 \mathrm{E} 033.5429 \mathrm{E} 011.2648 \mathrm{E} 003.5004 \mathrm{E} 01 \mathrm{1} 1.5220 \mathrm{E}-01-4.0947 \mathrm{E}-01$

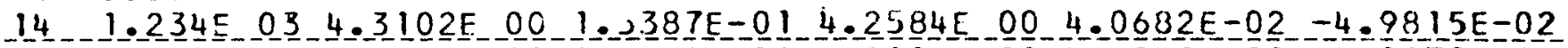

$154.540 \mathrm{E} 024.2638 \mathrm{E}$ OO $1.5222 \mathrm{E}-014.2126 \mathrm{E}$ 0Q $4.6248 \mathrm{E}-02-4.9279 \mathrm{E}=02$

16 8.7 विE OT

17 3.545E $014.2601 \mathrm{E} O \mathrm{C}$ 1.5208E-01 4.2089E $009.0077 \mathrm{E}-02-4.9236 \mathrm{E}-02$

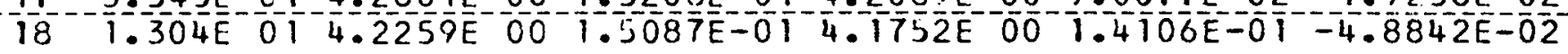

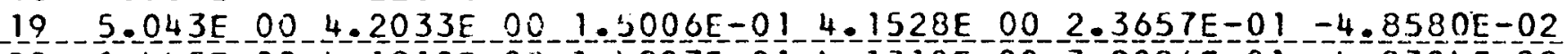

$20-1.445 \mathrm{E} 004.1812 \mathrm{E}$ 00 $1.4927 \mathrm{E}-014.1310 \mathrm{E} 00 \mathrm{3} .9086 \mathrm{E}-0 \mathrm{1}-4.832 .4 \mathrm{E}-02$

$210.826 \mathrm{E}-014.0883 \mathrm{E} 00 \mathrm{1}-4595 \mathrm{E}-014.0392 \mathrm{E}$ 00 $6.2304 \mathrm{E}-0 \mathrm{1}-4.7251 \mathrm{E}-02$

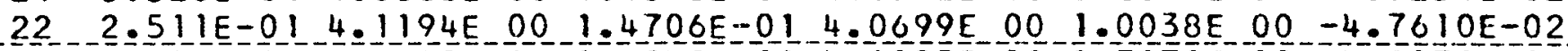

$239.237 \mathrm{E}-024.1592 \mathrm{E} 00-1.4848 \mathrm{E}-014.1093 \mathrm{E} 001.7171 \mathrm{E} 00-4.8071 \mathrm{E}-02$

$24-2.530 E-02$ 4.1273E 00 1.481 IE-01 4.0775E 002.6488E 00 -4.7958E-02

*4500 3.023E-01 4.0496E $00 \quad 1.5378 E-01 \quad 3.9977 E$ E $001.0483 E 00-5.0006 E-02$

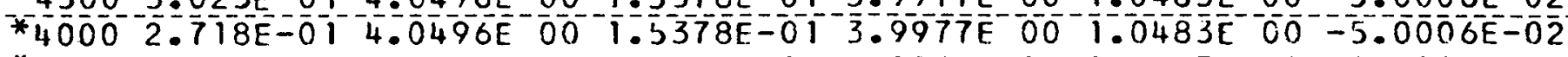

*3500 2.413E-01 4.0496E $001.5378 \mathrm{E}-01 \quad 3.9977 \mathrm{E} \quad 001.0483 \mathrm{E} 00-5.0006 \mathrm{E}-02$

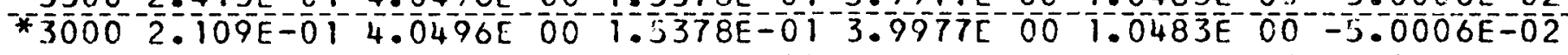

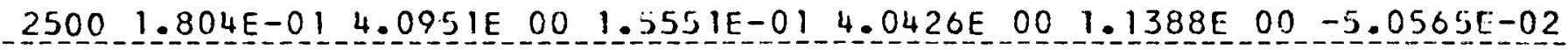

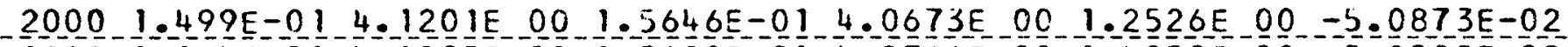

$15001.194 \mathrm{E}-014.1295 \mathrm{E}$ OQ $1.5682 \mathrm{E}-014.0766 \mathrm{E}-001.4058 \mathrm{E} 00-5.0988 \mathrm{E}-02$

$10008.897 E-024.1283 E-00 \quad 1.5677 E-014.0753 E 00 \quad 1.6328 E 00-5.0979 E-02$

$500-5.850 \mathrm{E}-02-4.1134 \mathrm{E}$ 00 $1.562 \mathrm{TE}=0 \mathrm{~T}-4.0606 \mathrm{E}$ 00 $2.0241 \mathrm{E}$ 0D $-5.0756 \mathrm{E}-02$

$683.216 E-024.0633 E$ O $0.1 .5430 E-014.0112 E$ O0 2.758.1E $00-5.0170 E-02$

*Thermal cross sections taken from XDC 60-1-139.

NOTE: For a summary of the sources of data see APEX-704 and XDC 60-1-139. 
CHROMIUM

THERMAL TRANSFER MATRTICES

SEMPERATURE
SIGMA $\begin{gathered}\text { SIGMA } \\ \text { SIGEG }\end{gathered}$

SIGMA S- $(N 1$ IIN2)

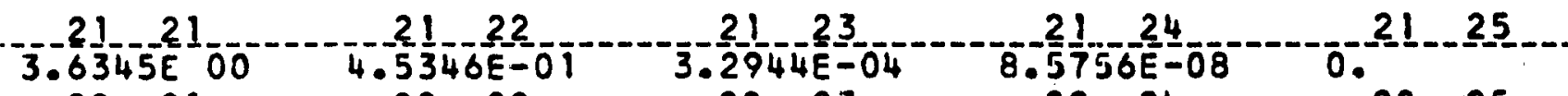

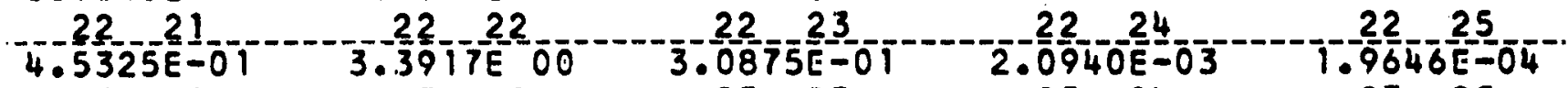

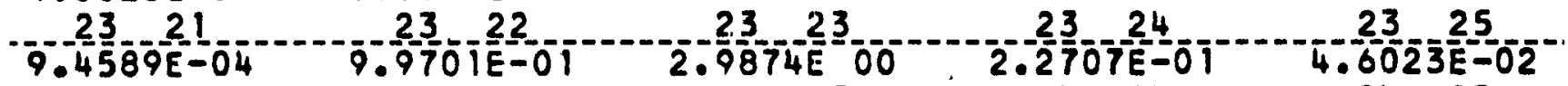

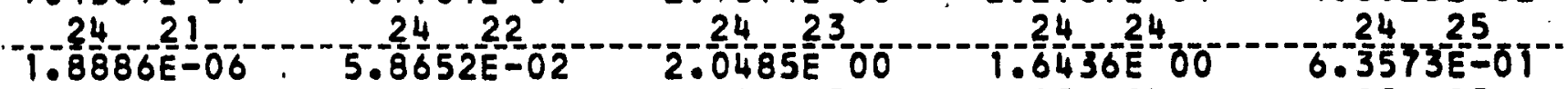

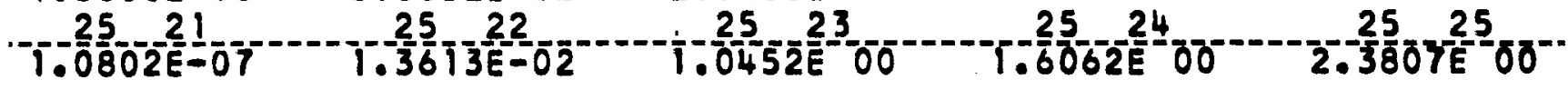

'TEMPERĀTURE

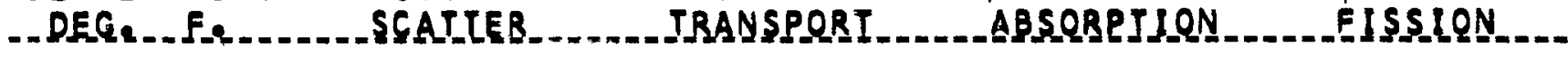

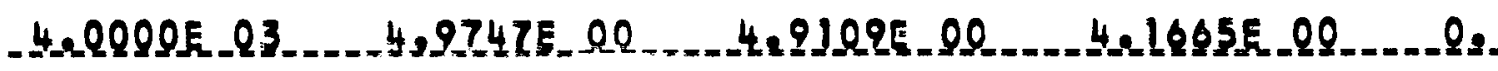
SIGMA.S-WIINRL

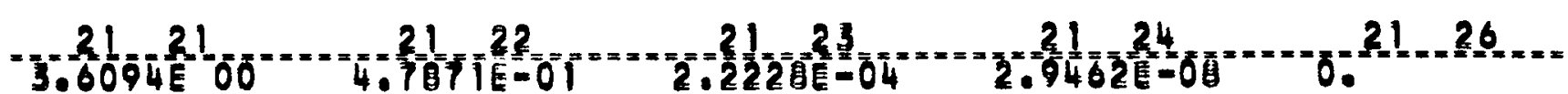

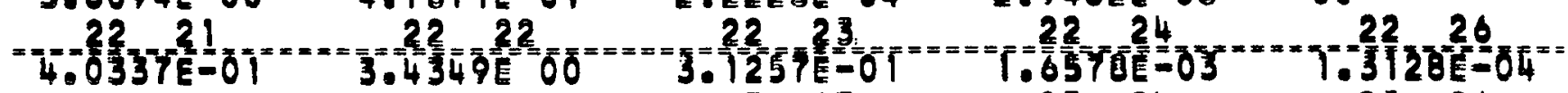

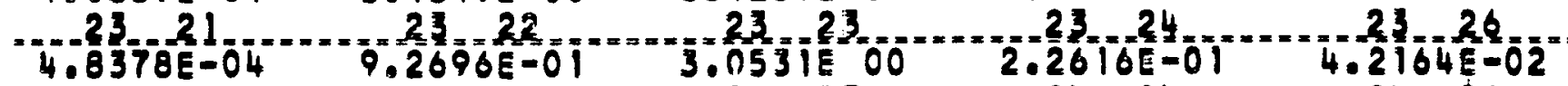

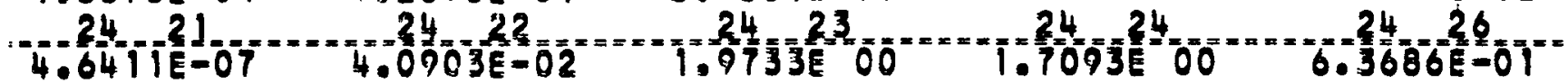

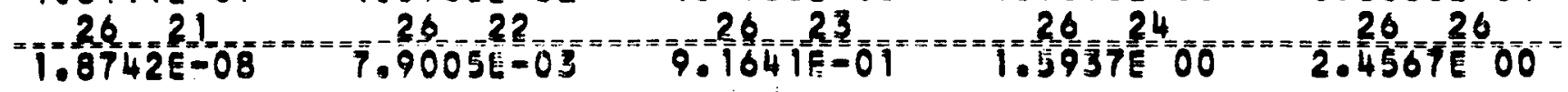


CHRQMIUM

\section{THERMAL TRANSFER MATRICES}

TEMPERATURE
SIGMA
DEG _F FIGMA

\begin{tabular}{|c|c|c|c|c|}
\hline 21 & $21--22$ & $21-23$ & --21 & 0 \\
\hline $.4674 \mathrm{E} 0$ & $0.2091 E-01$ & $3.5016 \mathrm{E}-05$ & & 0. \\
\hline $3409 E-C$ & $3.5624 \mathrm{E} 00$ & $3.4543 \mathrm{E}-01$ & $5.7804 E-04$ & 2.053 \\
\hline $23-21$ & 22 & 23 & 23 & 23 \\
\hline $\begin{array}{c}2.0239 E-0 \\
24 \quad 21\end{array}$ & 6.7759E-01 & $\begin{array}{c}3.2872 E \\
2423\end{array}$ & $2.2588 E-01$ & 2.939 \\
\hline & $7.6119 E-03$ & $1.6707 E 00$ & $1.9748 E 00$ & 6.2932 \\
\hline & $.1227 E-04$ & $5.1269 \mathrm{E}-01$ & $1 E-100$ & 2.7 \\
\hline
\end{tabular}

SEMPERATURE
SIGMA

$2.0000 E_{03} 03.6844 E_{0} 00$

SIGMA S

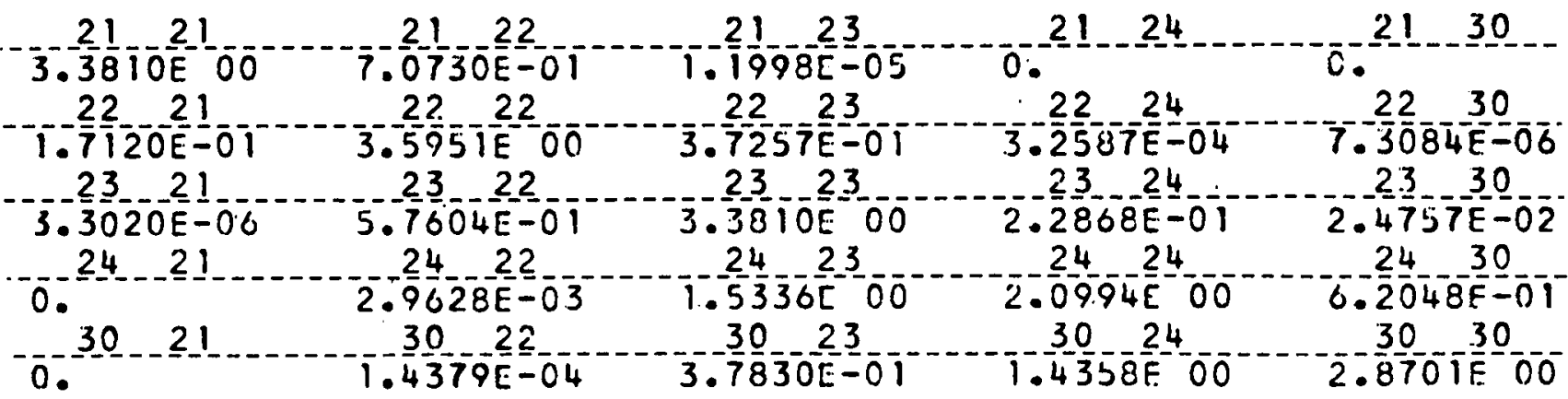


CHROMIUM

_LHERMAL_IRANSFER_MAIRICES

-_LEMPERATURE

DEG. $F$.

SIGMA

SIGMA

SICEMA

NU_SLLEM .

SCATTER

TRANSPORT

AESORPTION

FISSION

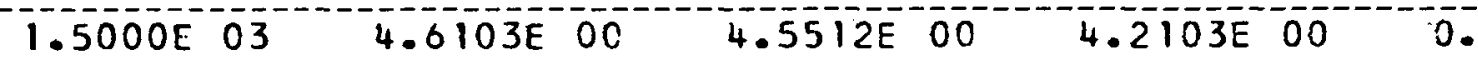

SIGMA

$N 1$

2121

$3.3343 \mathrm{E} 00$

2221

$1.0733 \mathrm{E}=01$

2321

2. 34 39E $=07$ 2421

$0 .=\frac{24}{31}$ ㅇ.
2122

]. $5395 \mathrm{E}-01$

$22 \quad 22$

3.6083E 00

2322

$4=6073 E=01$

2422

7. $6278 E-04$

3122

1. $7596 E-05$
$2 \overline{1} 2 \overline{3}$

$2.2026 E=06$

$22 \quad 23$

4. $2004 E=01$ 2323

$3=4843500$ 2423

$1.3689 E_{0} 00$

31.23

2.4986 트이
$2 \overline{24}$

$0=$

$.4454 E-04$

2324

$2.3600 E-01$

2424

$2.2547 E 00$

3124

$1.3408 E 00$
$2 i^{-} \overline{1}$

o.

2231

1.6471E-06

2331

$1.9928 E-02$ 2431 6. $06096-01$

3131 3.0196E 00

IEMPERATURE

DEG. $F$.

$1.0000 \overline{0}$ O
S I GM:A

SCATTER

$4.5 \overline{357 \mathrm{E}} 0 \mathrm{O}$
SIGMA TRANSPORT

$4.4775 \mathrm{E} 0 \mathrm{O}$
SIGMA

ABSORPTION

$4.2379 \bar{E}^{-10} 0$.
NU SIGMA

FISSION

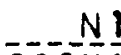

N2

SIGMA S (NTINZ)

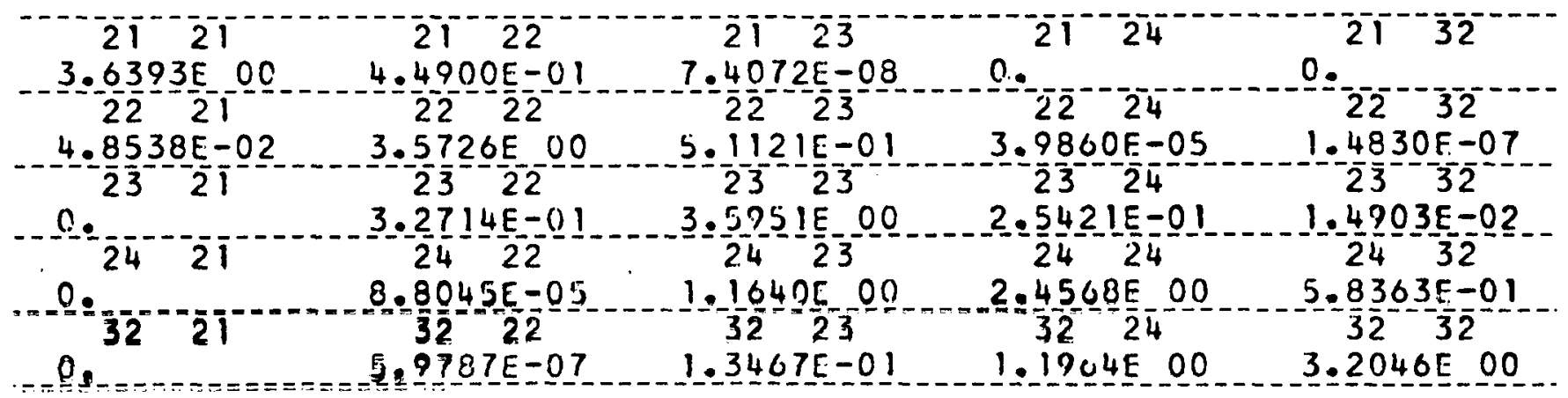


CHROMLUM

THERMAL TRANSFER MATRICES

TEMPERATURE
SIGMA

$\mathrm{N} 1-1 \overline{\mathrm{N}}$

SIGMA_S_. $(N 1<N 2)$

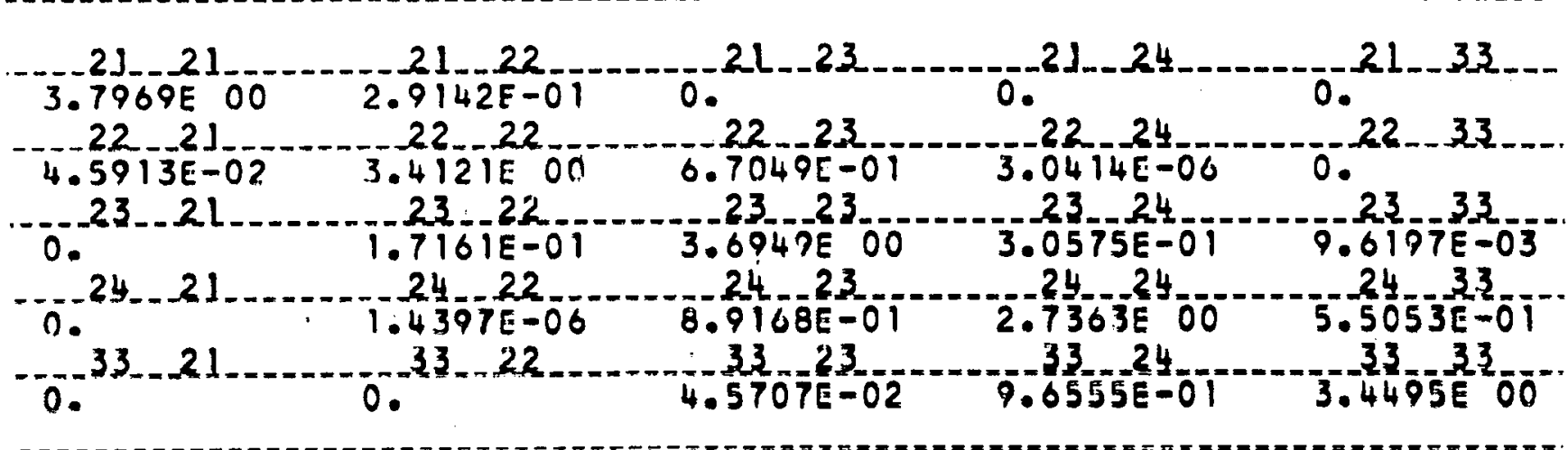

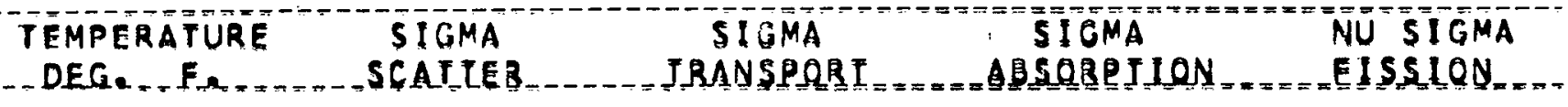

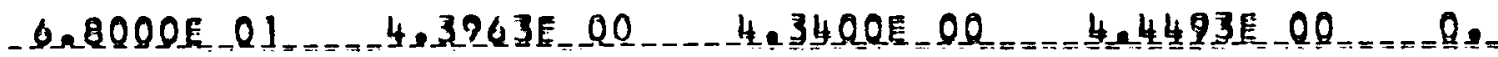

\section{SIGMA S NLILNLL}

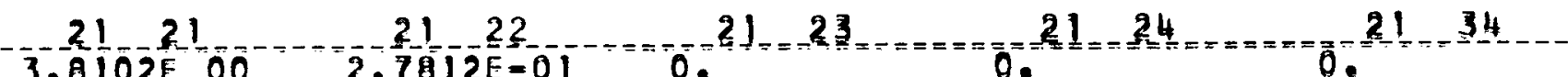

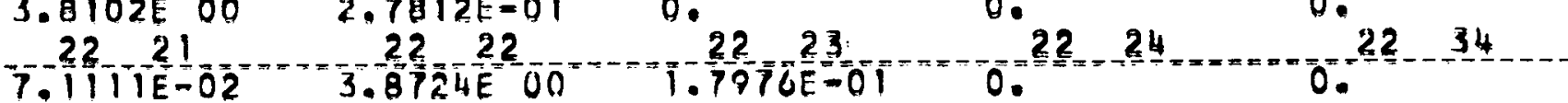

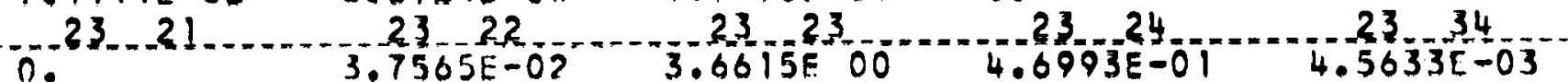

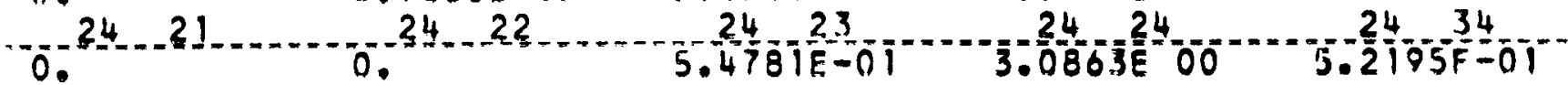

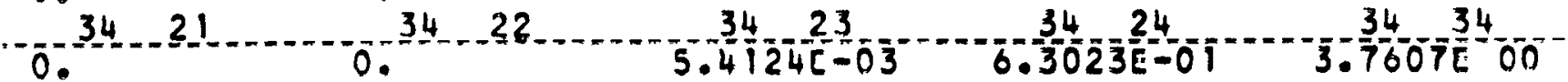


CHROMIUM

TRANSFFRS FROM GROUPS I THROUGH 5 TO GROUPS I THROUGH 16

SIGMA SO (NITN2)

110

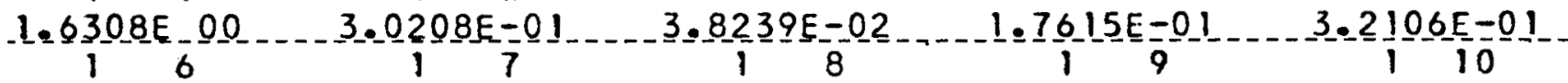
$3.2088 E-01 \quad 2.1871 E-01 \quad 1.1744 E-01 \quad 13.8519 E-02-2.0447 E-02$ $3.0657 E-03 \quad 4.2867 E-04 \quad 25=9655 E-05 \quad 2.9494 E-06 \quad 1.1715 E-06$

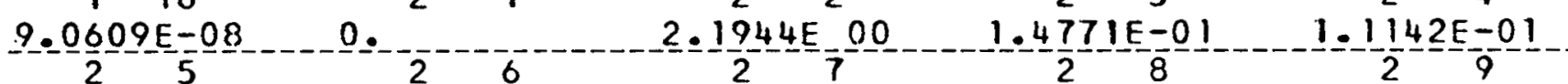
$2.7198 \mathrm{E}-01 \quad 3.3025 \mathrm{E}-01 \quad 2.5520 \mathrm{E}-01 \quad 1.4828 \mathrm{E}-01$ $2.8353 E-02-4.3321 E-03-6.1142 E-04-8.5326 E-05-1=1383 E-05$

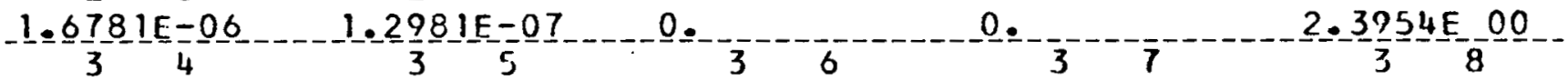
$2.2493 E-01-1.8357 E-01 \quad 3.0404 E-01 \quad 2.8674 E-01$

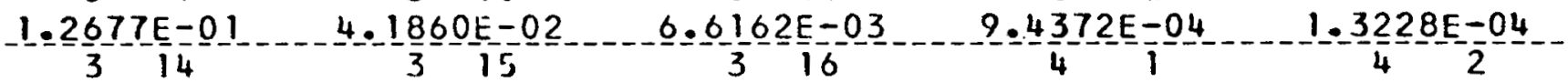
$1.7677 \mathrm{E}-05$ $0.42 .4752 E-00 \quad 2.4125 E-01 \quad 1.9884 E-01$ $1.8251 E-01 \quad I .3613 E-01 \quad 4.8479 E-02 \quad 7.9702 E-03 \quad 1.1540 E-03$

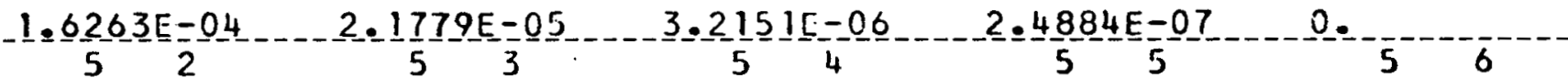

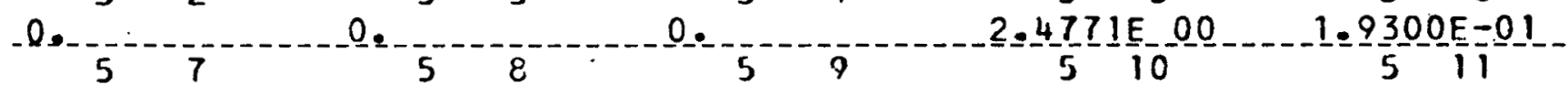
$4.1235 E-02 \quad 3.6147 E=02 \quad 2.9582 E=02 \quad 1.1248 E-02 \quad 1.2133 E-03$ $2.8061 E-04 \ldots 3.9732 E=05 \quad 5.3304 E=06 \quad 7.8743 E-07$ 
CHROMIUM

TRANSFERS FROM GROUPS 1 THROUGH 34 TO GROUPS 1 THROUGH 11

$\mathrm{N} 1 \mathrm{~N} 2$

SIGMA N,$G[(N] / N 2$ )

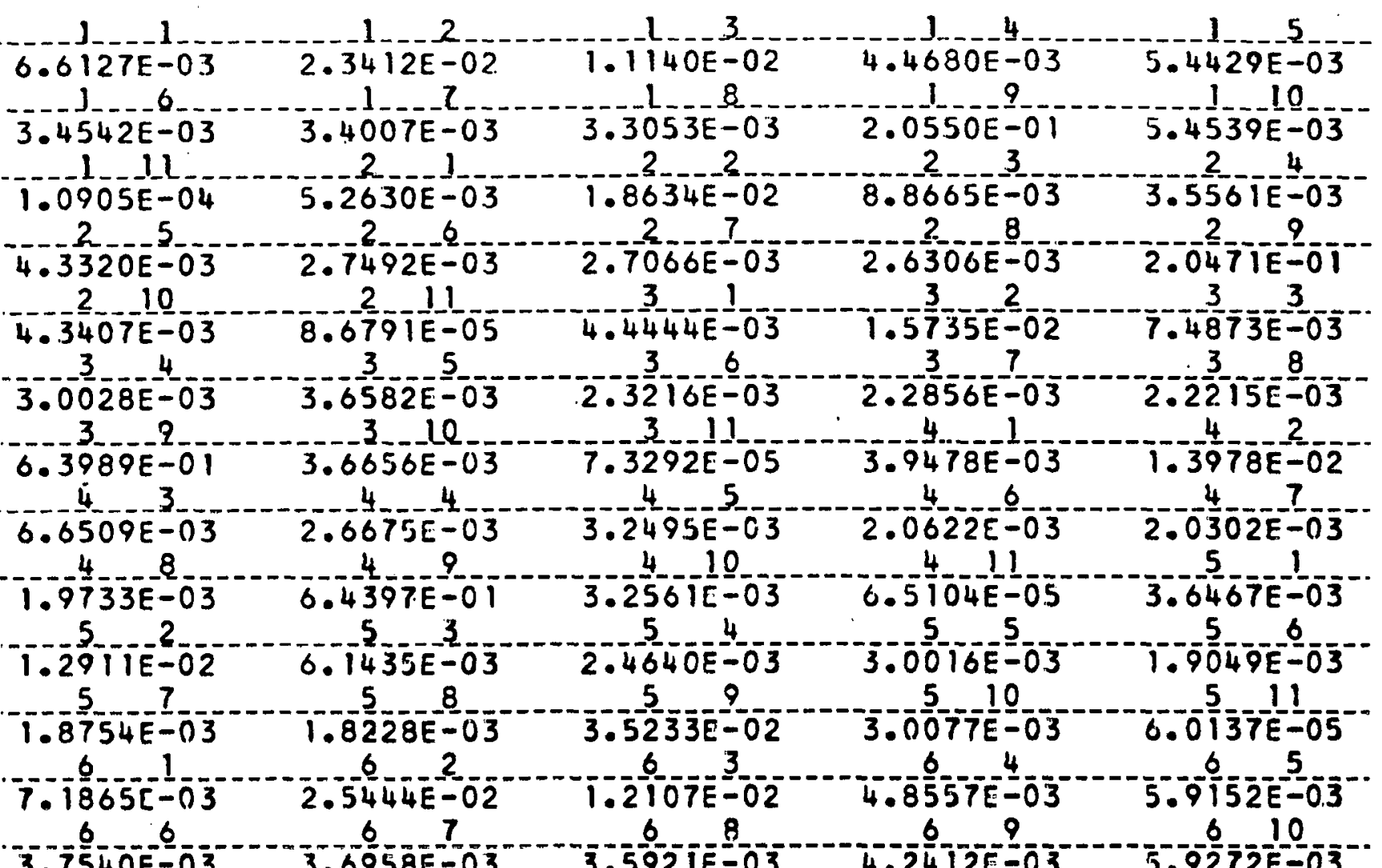

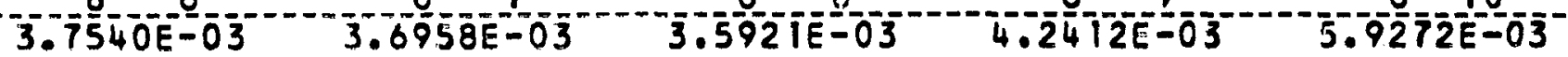

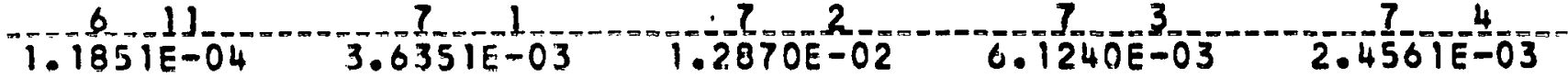

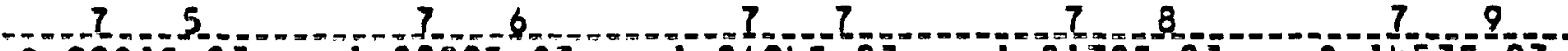
$2.9921 E-03 \quad 1.8989 E-03 \quad 1.8694 E-03 \quad 1.8170 E-03-2.1453 E=03$

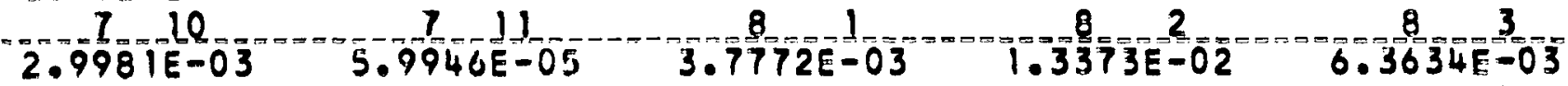

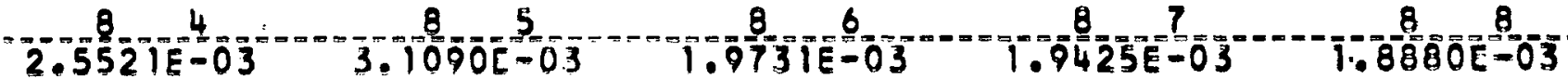

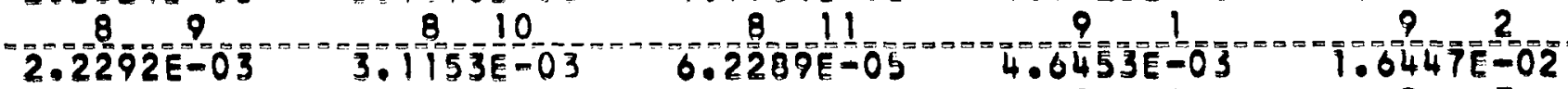

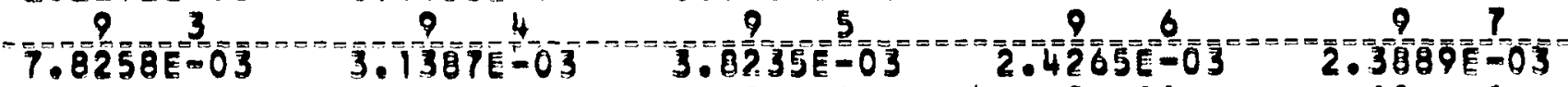

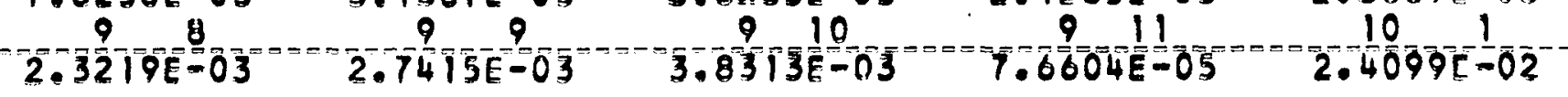


CHROMI UM

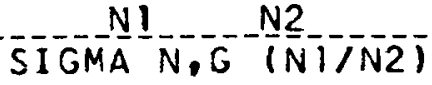

$10-10-3$

8. $\begin{gathered}5325 \mathrm{E}-02 \\ 10\end{gathered}$

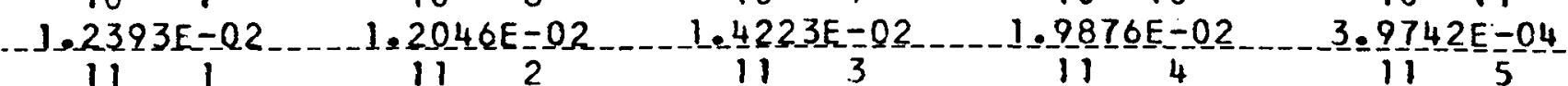

- $1.3593 E=02 \ldots-4.8126 E=02 \ldots-2900 E=02 \ldots-0.1844 E=03 \ldots$
116
117
118
119
1110

I- 1005E $=03$

$6.9904 E-03$

$6.7943 E=03$

8. 022 2IE -03

1. $1211 E-02$

1111

121

122

123

124

$-2.2416 E=0$

125

$I .2694 \mathrm{E}=02$

2. $5738 E=01$

$12 \frac{7}{7}$

1.22 $247 E=01$

128

$4=9118 E=02$

$3.3384 \mathrm{E}=\Omega 2$

1210

3. $2973 E=02$

13

. $30965-0$

L. $1988 \mathrm{E}=03$

$13 \mathrm{~S}$.

134

1. $02729 E=01$

B. $8487 \mathrm{~J}=02$

139

1310

136

1311

$2.1597 E-03$

$$
144
$$

145

$2.8804 E-02$

$14 \quad 10$

149

2. $0653 \mathrm{E}-02$

153

$2=\frac{88}{15} 63 \mathrm{E}=02$

6.702 IE -02

$2=\frac{68}{15} 0 \mathrm{E}=02$

L. $9885 \mathrm{SE}=02$

162

$2.3478 \mathrm{E}=02$

163

1. $0142 E=01$

$16 \quad 8$

$1 \frac{13}{16}-4 E=\frac{0}{7}$

$3.0091 E=02$

17 1

172

$2=\frac{74}{17} 32 E=\frac{0}{7}$

176

$3.9846 E=02$

$17-\frac{04}{11}$

$1.2777 E=03$

18 5

$1 \cdot \frac{2}{18} \frac{1}{8} 6 E=\frac{0}{6}$

$6.3397 \mathrm{E}=02$

$18-\frac{3397}{11}=0$

$18=10$

$2.00145=03$
132

4.636ZZE $=01$

137

6. $7348 E=02$

141

129

4.2202E-02

133

2. $2063 E-01$

138

$6.5459 E-02$

$3=4995 \mathrm{E}-02$

140

$1.8280 \mathrm{E}=02$

1411

$5=7710 E=04$

155

$3.2745 \mathrm{E}=02$

15 10

3.2811 IE $=02$

4. 0676 E $=02$

169

142

1.23 20 EE $=01$

147

1. $79997 E-02$

151

$3=9783 E-02$

$2.0781 E-02$

$6.5605 E-04$

16

4. $9552 E-02$

3. $-5529 E-02 \quad 4.9652 E-02$

1. $3053 E-01 \ldots .5 .2352 E-02$

3. $8728 \mathrm{E}-02 \quad 4.5726 \mathrm{E}-02$ $18=-2.0 .5$ 4.2969E-01 $18-7$ $2=0446 E-01$ $6.2413 \mathrm{E}=02$ $0.0662 E-02$ 7_-20 $22 E-01$ 2. 03 3 1 IE $=01$
$7.1625 \mathrm{E}-02$

$1.0010 E-01$

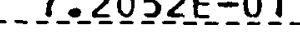


CHROMIUM

N1 N2

SIGMA_N,G (N]IN2)

-.-19_-_3

$3.4284 E-01$

$-19$

$1.0172 \mathrm{E}-01$

20

$1.1904 \mathrm{E} 00$

$-20$

20

$1.7291 E-01$

- -21

$5.3586 E-0$

$-2-\frac{2}{2} \frac{1}{9} \overline{9}=\mathrm{6}^{\prime}$

- -2 J

$8.8368 E-03$

7. $2208 \bar{E}-\overline{-}$

$22-10$

$7.1224 E-0$

$9.9786 \mathrm{E}=0$

23

$8.7158 \mathrm{E}-01$

$3 . \frac{24}{3} \frac{3}{3} \mathrm{E}^{-3} \mathrm{D}$

T.

$-\ldots 25$

$1.2678 \mathrm{E} 0$

$-\bar{T} .84 \overline{14} \overline{\mathrm{E}}$

26
$-5833 \mathrm{E}$

-26

$1.8718 \mathrm{E}$

$-5.9092 \mathrm{E}-02$

5.2755
$=.9530 \mathrm{E}$

2710

2.9580 हO
12

$1.3750 E-01$

19

$1.2010 E-01$

20

$5.6643 E-01$

20

$1.6806 \mathrm{E}-01$

$1 . \frac{2}{8} 97 \overline{2}=\frac{2}{0}$

$2.7557=-7$

-.22

$8.6357 \mathrm{~F}-0 \mathrm{~T}$

$4.511 O E-01$

$22-11$

$1.4241 \mathrm{E}-02$

23

$5 E^{5}$

2310

$1.2180 \mathrm{O}$ Ō

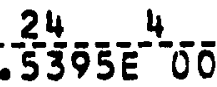

249

$-\frac{24}{3447 E}=00$

$0.0324 E$ OO

.750 OE

26 03

1. $.842 \overline{8}$ -

12

$1.675 \mathrm{JE}-01$
$1.12 \quad \mathrm{~J} 0$

$1.6785 E-01$

$2.2718 \mathrm{E}-\mathrm{O} 1$

$1.9843 \mathrm{E}-01$

$-\frac{2}{0} \frac{1}{2} 75 \bar{E}-0$

21

$2.6784 \bar{E}=01$

22

$3.0575 \mathrm{E} 00$

$-\frac{22}{4.44}=\overline{0}=-7$

$1 . \frac{23}{4768}-1-1$

7.

$23 \quad 11$

$2 . \frac{2}{4} 5 \overline{4} \bar{E}=02$

$-24-5$

I. $7554 E^{2}-00$

T.84921

$2.4104 \mathrm{E}$ O

2. 25 1 $32 \mathrm{E}^{\circ}$

19

$1.0630 \mathrm{E}-01$

19

$3.3560 E-03$ 20

$3.3622 E-01$

$2.7675 \mathrm{E}-01$

$1.7563 \mathrm{E}-\mathrm{O} 1$

$-200$

$2.773 \mid E-01$

20

$5.5446 E-03$

$3 .-2 \frac{1}{2} 07=-\frac{4}{-0}$

3. $\frac{2}{16} \frac{1}{5}=-9$

$4 . \frac{2}{4} 107 \bar{E}-01-$

223

$4 .-\frac{2}{4} 196 \frac{10}{2}=01-$

$1.4548 \mathrm{E} 0$

22

$4.3164 \mathrm{E}-0$

$5 .-\frac{2}{2} \frac{3}{88} \bar{E}^{2} \overline{0}$

$2 \frac{22}{834}=-\frac{4}{9}-0$

$5.0965 E^{-2}-01$

$2 . \frac{23}{48} 80 E^{3}-{ }^{3}$

$7 .-\frac{2}{5} \frac{3}{49}=\frac{7}{1}=0$

238

$24-1$

$2.2785 E$ OO

$8.0 \frac{24}{0} 70 \mathrm{E}^{2}$

1. $\frac{24}{902}=0$

1. 24 TITE ${ }^{7}-00^{-}$

$3.7574 \mathrm{E}=02$

3. $5857 \mathrm{C}^{1}$

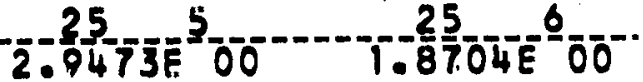
$2.9533 \frac{10}{10}$ $5.90495-02$

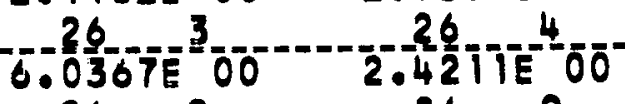
$2-\frac{26}{94} 94 \mathrm{E}^{5}-00$ i- 260

$2.1147 \mathrm{C}^{2} 0$

$-2610$ $3.5876=00$

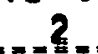

273

2.9554 270

06

$5.9763 E=02$

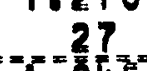

T. $\frac{27}{85}$

28
.

r: 28
8

$50^{-60}$

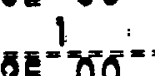

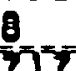


CHROMIUM.

STI

N2

SIGMA N,G (NIINZ)

$288^{4} 285$

2270E 00 _. $2.9565 E_{0} 00$

289

2. $1198 \mathrm{E}$-00

293

$0.0628 E_{-} 00$

298

$1.7988 E$

$30 \quad 2 \quad 303$

$1.2772 E-01$

30

- $1.8551 E$ IE 00

31

$3.6211 E 00$

316

$1.8916 \mathrm{E} \quad 00$ 31 i

$5 \cdot \frac{97}{32} 16 E-02$

$3.0004 E-00$

$.0065 \mathrm{E} 00$

$\overline{3} \overline{3}$

2810

$2.9625 \mathrm{E}-00$

294

$2.4316 E$

299

$6=0773 E-00$

1. $-8031 E-00$

1.2821E 0

317

$.8622 E 00$

32

$3.6452 \mathrm{E} 00$

$32-6$

$1.9041 E$

$6.0113 \mathrm{E}-02$

$3.0415 E$ OO

$33-10$

$33^{-1} 9^{-1}$

$-2=\frac{1807 E}{34}-0$

3. $0476 E$ EO

344

$2=58566-00$

$2.2584 E \quad 00$
$1.8763 E_{0} 00$ 2811

$5.9234 E-02$

295

$2.9622 \mathrm{E} 00$

2910

$2.96815-00$

$2.4374 \mathrm{E}-00$

2. 1289 E -00

6.10055

318

$1=\frac{8}{32} 100 E-00$

$1.2906 E$ E 01

32 7

33

$3.6951 \mathrm{E} 00$

3.3

$1.9302 \mathrm{E} 00$

$33-11$

$6.093605-02$

345

$3.1498 E \quad 00$

3410

3.1561 트이
287

$1.8472 E_{-} 00$

$29 \quad 1$

$3.5988 \mathrm{E} \quad 00$

296

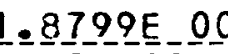

2911

$5 \cdot \frac{9}{30} \frac{3}{7} \mathrm{TE}-\mathrm{O}-2$

2.9692500

2. $9752 E$ E 00

314

$2.4467 E$

31

2. $1371 \mathrm{E}-00$

$\frac{1}{32} 10 E-00$

$-\frac{82}{3} 3$-OE ${ }^{2}$

$1.3083 E_{7} 01$

$9003 \mathrm{E} 00$

34 T 00

$3 . \frac{82}{3} \frac{67}{6}-0$

$1.9989 \mathrm{E} \quad 00$

34 i1

$6.3106 E-02$
288

$1.7954 E$ Q 00

292 $1.2742 \mathrm{E} O 1$ 297 .8507트은 (3) $3.6074 E-00$ $1.8844 \mathrm{E} 00$ 5.9489E-02 315 $2.9806 \mathrm{E} \quad 00$ 3110 2.98666E 00 2.4630 E 00 $2.1513 E 00$ 6.225 IE 00 1.8470500 $34-2$ 1. $3549 E$ 요. 1.9679600 
DATE DEC OI 1960

DES_LGNAILLON

BERYLLLI U UM
CODE NO.

DEENSIIYY_EACI_OR

4. 9000
$1 \cdot 4963 \underline{6} \underline{E}-01$

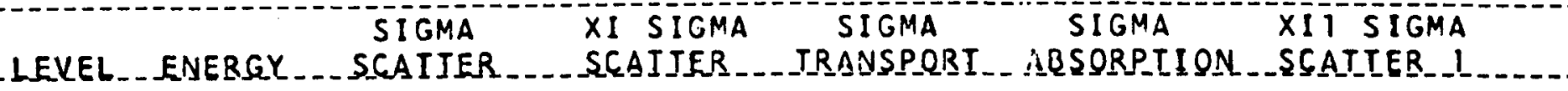

$1.000 E-07-1.6412 E-00-5.6050 E=01-8.2000 E-012.3578 E=02-6.4490 E-01$

$20.065 \mathrm{E} 06 \mathrm{1} .7819 \mathrm{E} 008.2710 \mathrm{E}-019.4580 \mathrm{E}-01-4.7408 \mathrm{E}-02-6.5660 \mathrm{E}-01$

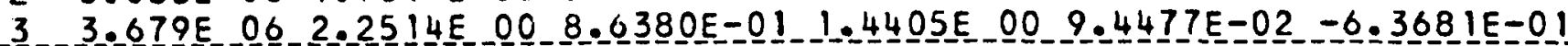

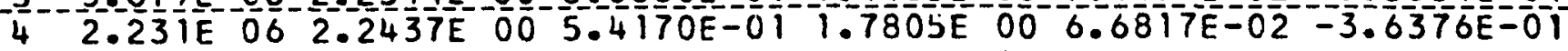

$5 \quad 1.353 \mathrm{E}$ 06 2.5043E 00 4.2460E-01 1.8456E 00 1.2182E-02 -5.1729E-01

$6 \quad 8.208 E 05 \quad 3.5611 E-007.4320 E-01 \quad 3.0829 E-009.0399 E-05-3.7554 E-01$

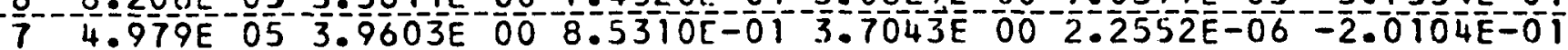

8 3.020E $05-4.1120 E-00-8.3870 E=01-3.8220 E 00-2.8959 E-06-2.2774 E-01$

$90.832 \mathrm{E} 054.9644 \mathrm{E}$ O0 $1.3196 \mathrm{E}$ 00 4.5972E $003.9111 \mathrm{E}-06-2.8837 \mathrm{E}-01$

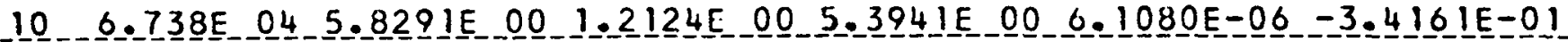

$112.479 E \quad 045.9200 E 001.2314 E$ E $005.4783 E 00 \quad 8.9769 E-06-3.4687 E-01$

$129.119 \mathrm{E} 035.9525 \mathrm{E} 001.2382 \mathrm{E} 00-5.5083 \mathrm{E} 00 \mathrm{1} .3173 \mathrm{E}-05-3.4884 \mathrm{E}=\overline{\mathrm{O}}$

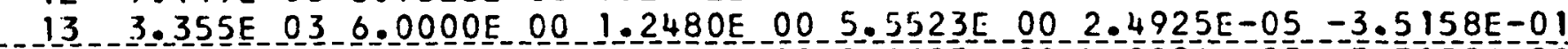

$1401.234 \mathrm{E} 036.000 \mathrm{OE} 00 \mathrm{1}-2480 \mathrm{E} 00 \mathrm{5} .5523 \mathrm{E}-00-4.0296 \mathrm{E}=05-3.5158 \mathrm{E}=01$

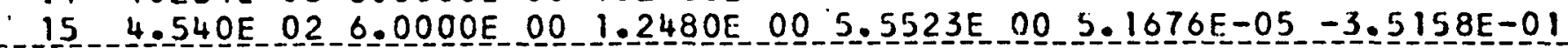

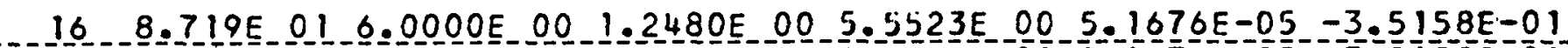

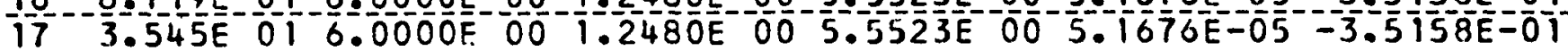

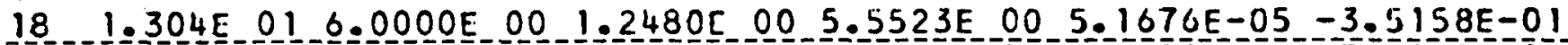

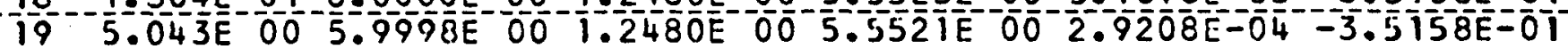

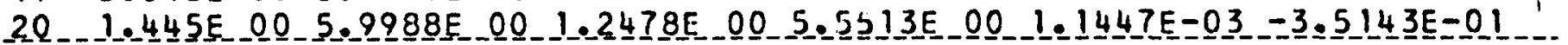

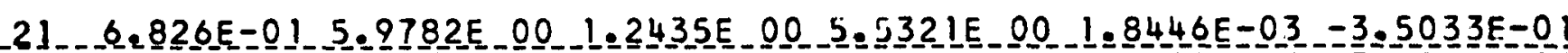

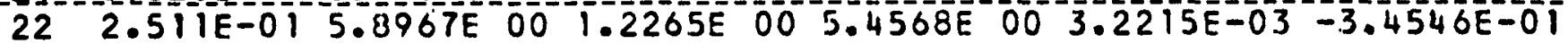

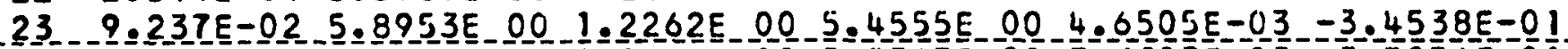

$242.530 \mathrm{E}=02-5.9827 \mathrm{E}-00-1.2444 \mathrm{E} 00-5.5363 \mathrm{E} 00-7.3323 \mathrm{E}-03-3.5056 \mathrm{E}-01$

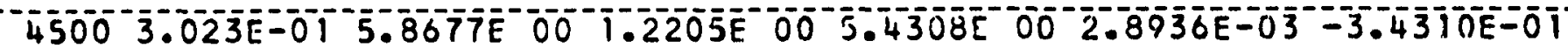

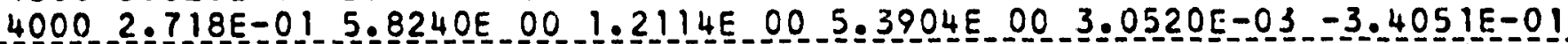

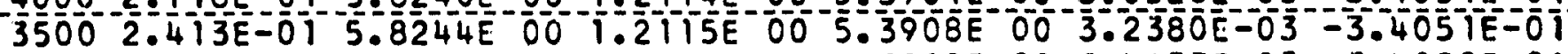

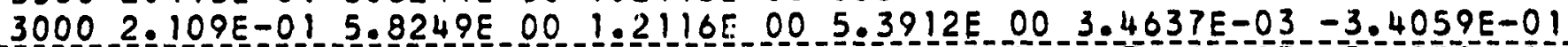

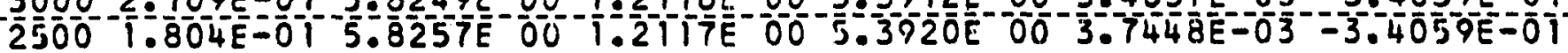

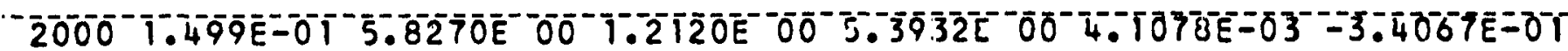
1500 1. $194 \mathrm{E}=01$ 1 $5.8292 \mathrm{E}$ 00 $1.212 \mathrm{SE}$ 00 5.3952E 00 4.6022E-03-3.4083E-01

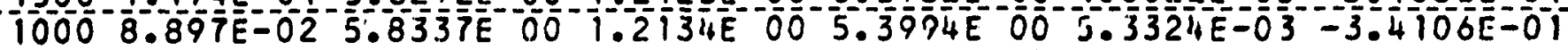
500 5.85OE-02 5.8449E 00 1.2 I57E 00 5.4097E $00.0 .5764 \mathrm{E}-03-3.4177 \mathrm{E}-01$

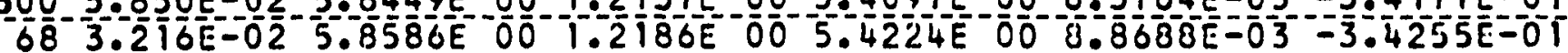

NOTE: For a summary of the sources of date see APEX-704. 
BERYLLIUM

_IHERMAL_IRANSFER_MAIRICES_

IEMPERAIURE

SIGMA

SIGMA

SIGMA

NU_ S_IGMA

DEG. $F$.

SCATTER

TRANSPORT

ABSORPTION

FISSION

$4.5000 E \quad 03$

$1.0569 \mathrm{E} \mathrm{OI}$

$9.7819 E 00$

$1.3429 \mathrm{E}-02$

0

N1

SIGMA S (NI/N2)

2121

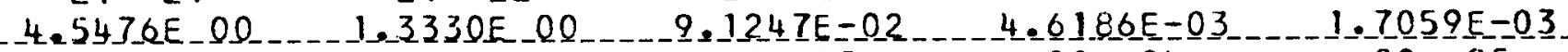
$22 \quad 21$
$22 \quad 22$
$22 \quad 23$
$22 \quad 24$
2225

$1.3654 E_{0} 00$

2321

$23 \quad 22$

$8.4108 E-01$

2323

$6.4124 E=02$

$2.8938 \mathrm{E}=02$

3. 0850 OE_ 00

$24 \quad 21$

$24 \quad 22$

$2.2541 E-00$

2324

2325

$1.00009 E=0$.

1. $4251 \mathrm{E} 00$

2423

4.0949 $0-01$

$2.0628 E-01$

$25 \quad 21$

2522

$2.83645-00$

$1=\frac{0}{2} \frac{104}{2}=00$

$6.0458 E-01$

$1.2784 \mathrm{E}=01$

2. $0616 \mathrm{E}$ OQD

4.5518 [ 00

1.90이트음

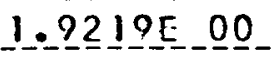

IEMPERAIURE

DEG. $F$.

$4.000 \bar{O}=\bar{O}$
SIGMA

SCATTER
SIGMA

TRANSPORT
SIGMA

ABSORPIION
NU SIGMA

FISSION
$9.4323 \mathrm{E} 00$
$1.34 \bar{O} \bar{E}-0 \overline{2}$
0 .

SIGMA $\left.-\frac{N}{S}-\left(\frac{N}{2}\right)-N 2\right)$

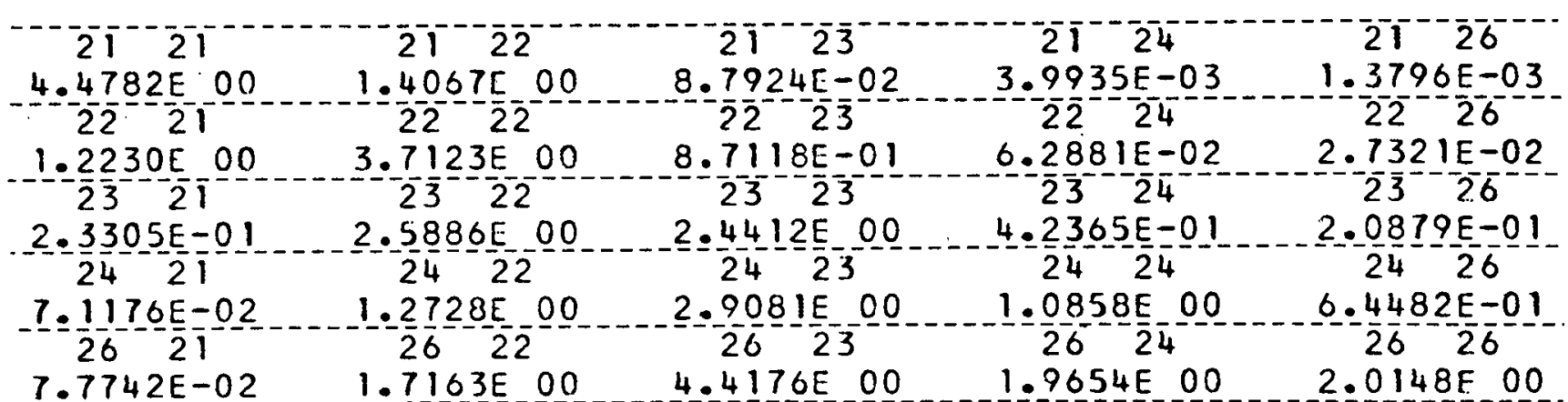


BERYLLI UM

THERMAL TRANSFER MATRICES

IEMPERATURE
DEEG. SIGMA

SIGMA S $(N 1$ N 1 N2)

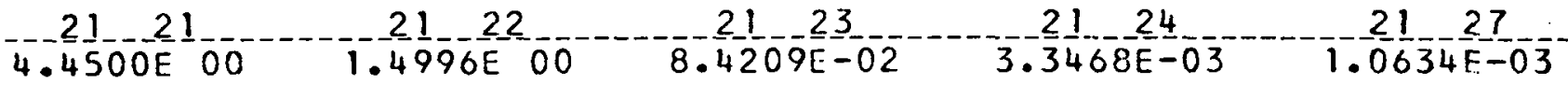

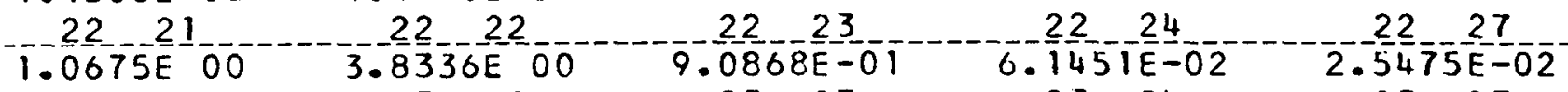

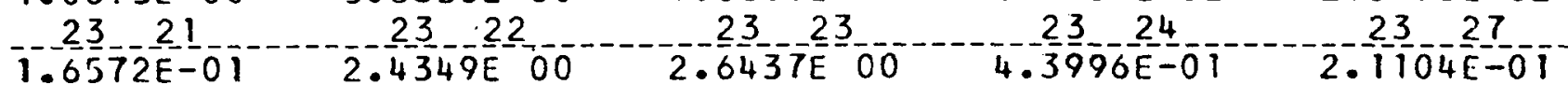

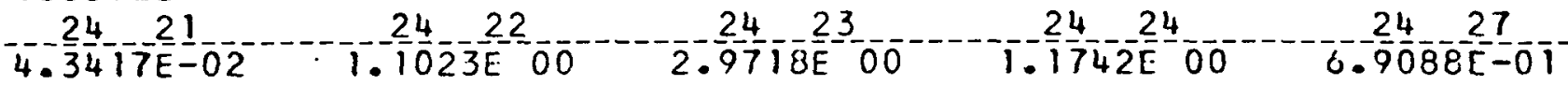

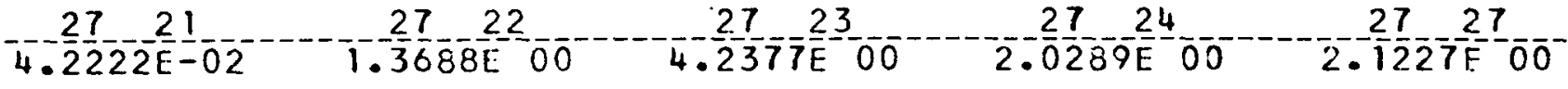

SEMPERATURE
SIGMA

$\mathrm{N} 1 \mathrm{~N}^{-1}$ SI GMA S (NIIN2)

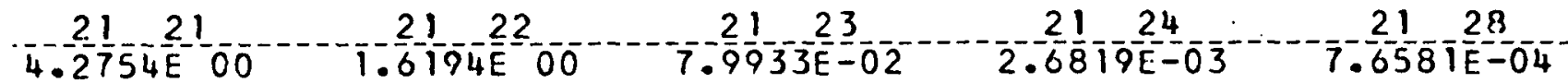

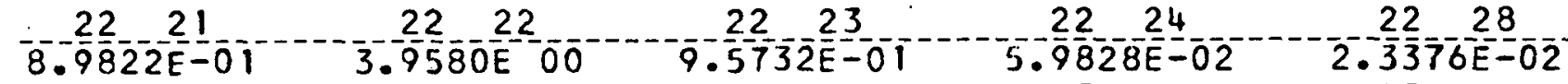

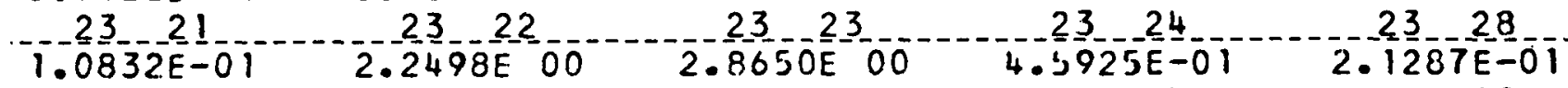

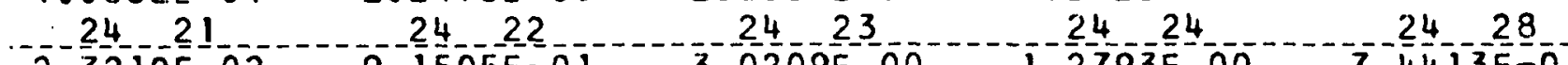

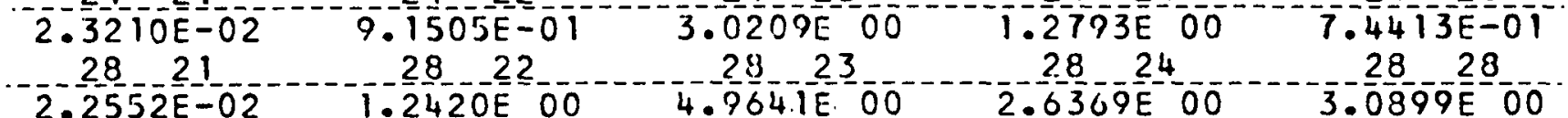


BERYLLIUM

IHERMAL_IRANSEER_MAIRICES_-

.LEMPERA IURE _... _SIGMA

DEG. F. SCATTER

SIGMA

TRANSPORT

SIGMA

NU_ SIGMA

2.5000 E $0 \overline{3}$

$8.9678 \mathrm{E}$ OO

$8.2997 \mathrm{E}$ OO

$1.3496 \mathrm{E}-02$

FISSION

.

$N 1$

N2

SIGMA S (NTINZ)

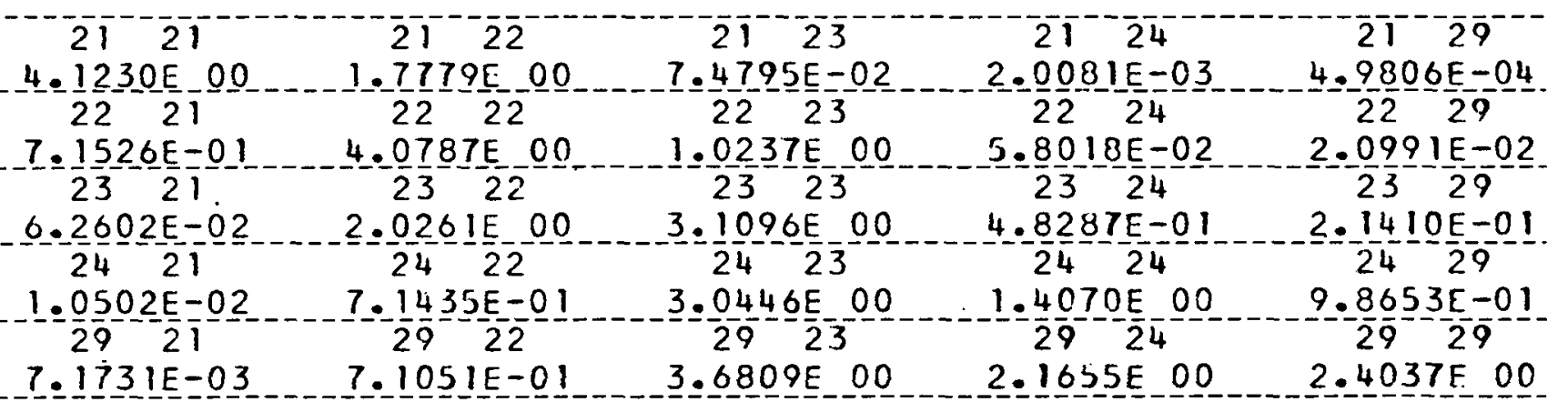

- IEMPERAIURE

DEG. $F$.

$2.0000 E^{-13}$
SIGMA

SCATTER
SIGMA TRANSPORT
SICMA

ABSORPTION
NU SISMA.

FISSION

$8.5223 \mathrm{E} \quad 00 \quad 7.8874 \mathrm{E} O 0 \quad 1.3530 \mathrm{E}-02 \quad 0$

\section{$\left.\operatorname{SigMA}-\frac{N}{S}-\left(\frac{N}{N}\right)-N 2\right)$}

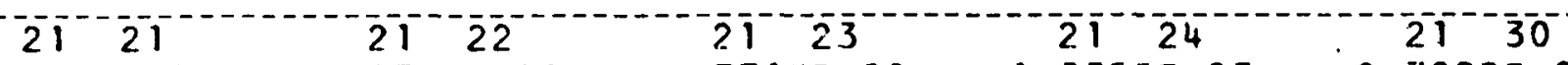

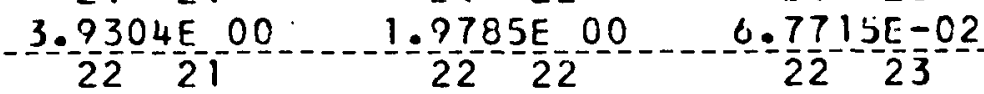

$1.3 \frac{3}{2} 55 \mathrm{E}-03$

$2 \cdot \frac{72}{2} \frac{29}{2}-\frac{8}{30}-04$

S. $-\frac{2}{2} \frac{0}{3} 88 \mathrm{E}-01$

$4 .-\frac{1}{2} \frac{80}{3}-\frac{8 E}{2} 2$

$2 \cdot \frac{9}{2} \frac{85}{4} 5-02$

$1.7539 \mathrm{E} O 0$

2422

$\frac{1207}{2.3}-\frac{5}{2}=$

$3.3838 E 00$

2423

$3.0250 E-00$

3. 2625500
$5.6039 \mathrm{E}-02$

$2 \frac{1}{2} \overline{4}$

$5: 1337 E=01$

2424

$.5663 E 00$

30
1. $8278 E-02$

2. $1444 E-01$ $24-35$

8. $8053 E-01$.
$1.8191[-03$

4. $3065 E-01$

2.2332 E OO

2.594IE 00 
BERYLLI I UM

\section{THERMAL TRANSFER MATRICES}

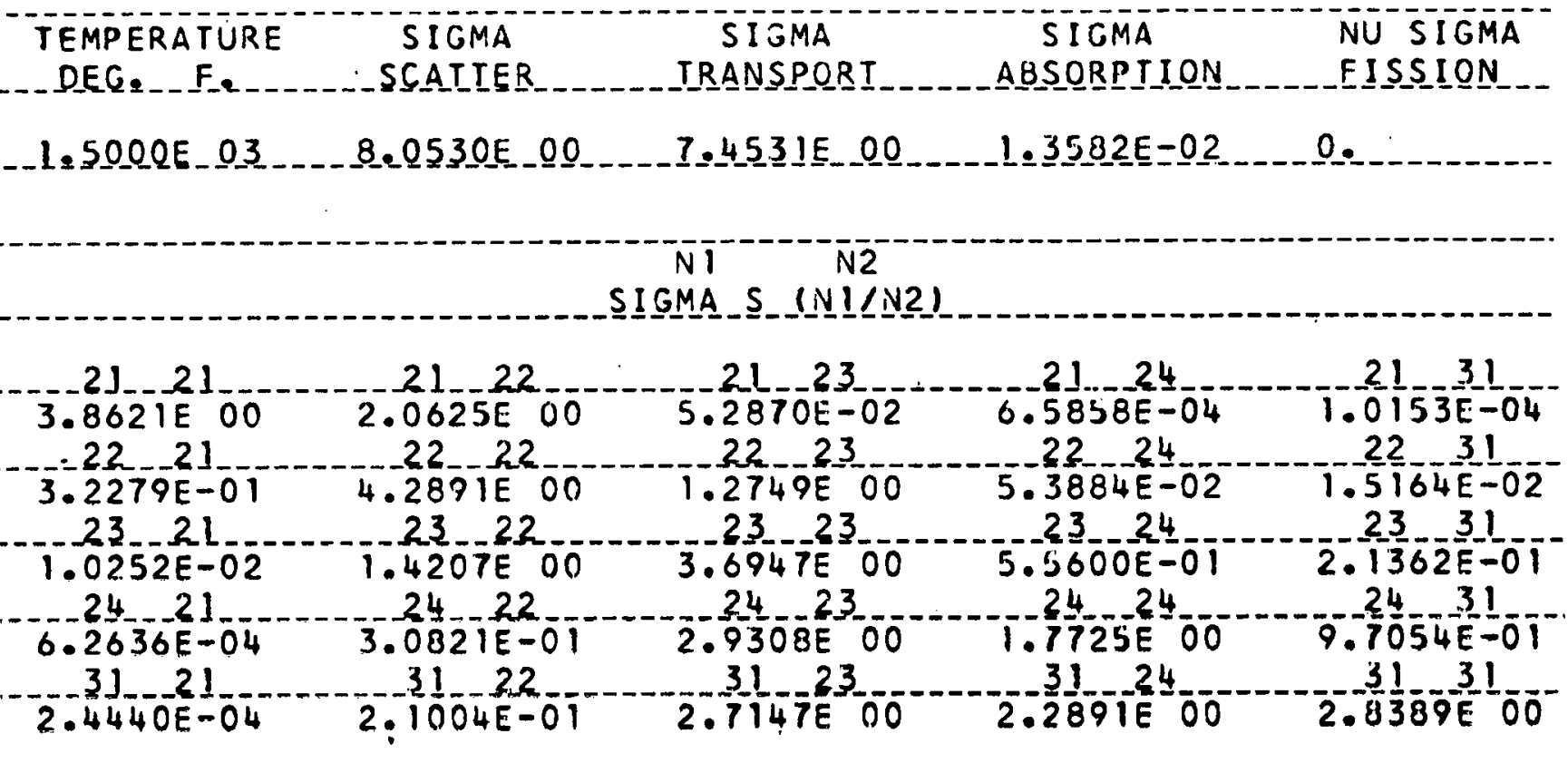

TEMPERATURE
SIGMA

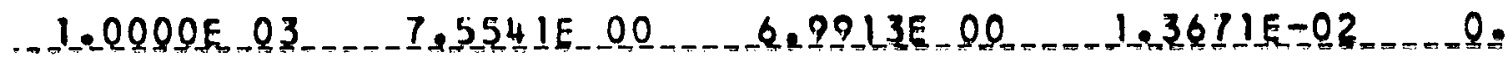

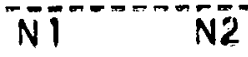 \\ S! GMA S $(N ! I N 2)$}

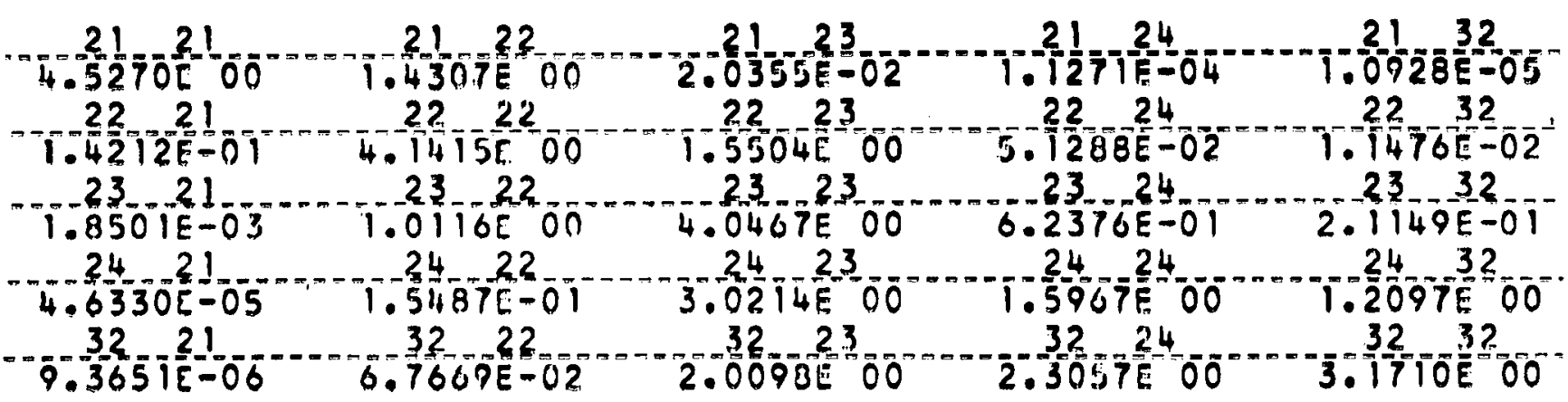


BERYLLIUM

_IHERMAL_IRANSFER_MAIRICES.

_ LEMPERAIURE

SIGMA

SIGMA

SIGMA

NU SICMA

DEG. F.

SCATTER

TRANSPORT

ABSORPTION

FISSION

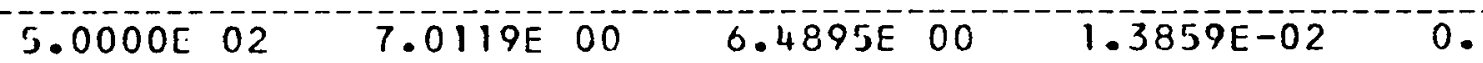

NI

$\mathrm{N} 2$

SIGMA S (NI/N2)

2121

$4.6498 E_{-} 00$

2221

$6.7557 E=02$

2321

-6.7974E-05

2421

$\frac{0.2}{3} \overline{3}-\frac{1}{2}$

……
$2 i^{2} 2$

$1,3212 E-00$

$22 \quad 22$

3. $2 \underline{2} 2 \underline{0} \underline{0 E}-\underline{0} \underline{0}$

2322

$5.1996 \mathrm{E}-01$

$24 \quad 22$

3. $2923 \mathrm{EE}-02$

3322

8. $0672 E-03$
$212 \overline{3}$

$7.1451 \mathrm{E}=03$

$22 \quad 23$

$2.0565 E$

2323

$4.4075 E$ OO

2423

$2.4244 E$ OOO

3323

$1.14668 \mathrm{E}$
$2 i^{24}$

7. $5845 \mathrm{E}=06$

$22 \quad 24$

$4=4823 E=02$

2324

7. $5866 \mathrm{E}-01$

2424

$2=1892$ E 00

3324 2.197IE 00
$2 \overline{1}^{-1} \overline{3}$

0.

$22 \quad 33$ $6.5972 E-03$ $-\frac{5}{2} \frac{9}{33}$ 2.09 I I E-0 I 2433 I. $33 \underline{3} 1 \mathrm{E} \quad \underline{0}$ $33-3 \overline{3}$

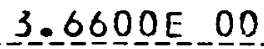

TEMPERAIURE

DEG. F.

$6.8000 E$ OI
S I GMA

SCATTER
SIGMA

TRANSPORT
SIGMA

ABSORPTION
NU SIGMA

FISSION

STGMA S (N)

$5.9384 \mathrm{E}^{-} 00^{-1.435} \overline{\mathrm{E}}-02 \mathrm{C}$

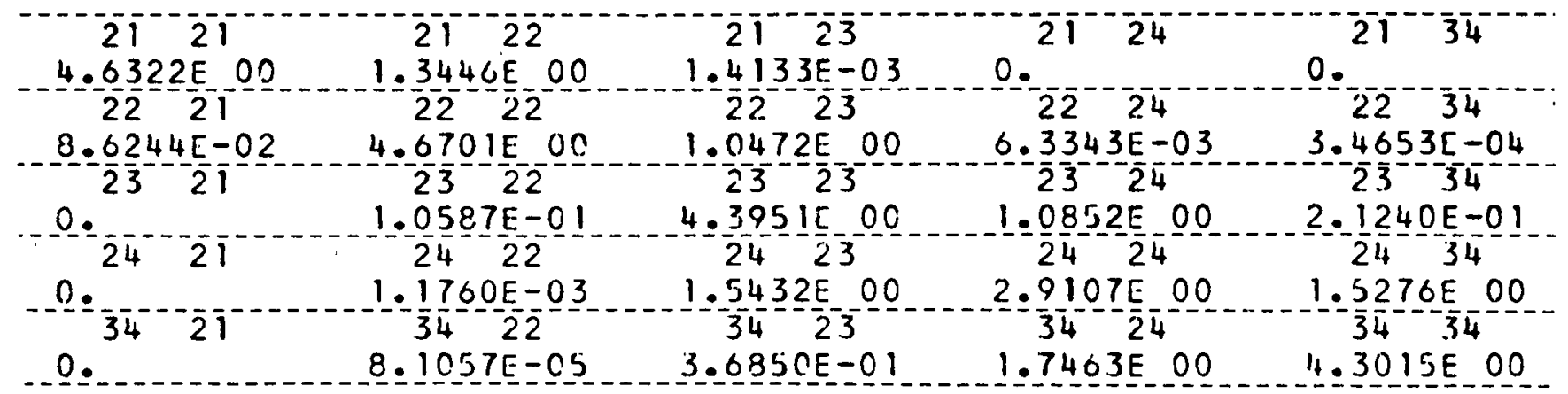


BERYLLIUM

TRANSFERS FROM GROUPS I THROUGH 4 TO GROUPS 1 THROUGH 21

$\mathrm{N1} N 2$

SIGMA_SO_- (N] IN2)

$1.0564 E^{-1} 00^{3} 3739 E-0101.3393 E-01-2.1680 E-01-2.5359 E-01$ $1.0452 E-0101.3696 E-02 \quad 8.3073 E-03 \quad 6.7567 E-033.8237 E-03$ $101-12 \ldots 13$ $1.4067 E-030.1748 E-04 \quad 1.9037 E-04 \quad 7.0033 E-05 \quad 2.9924 E-05$

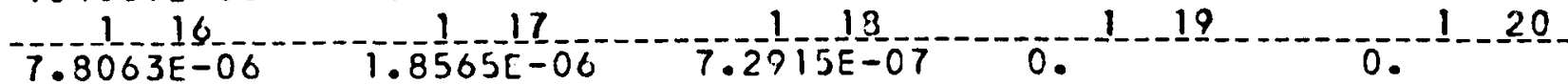

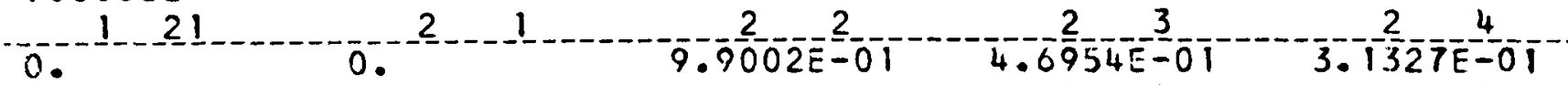

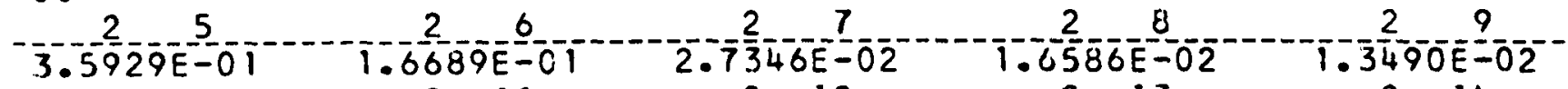
$7.6342 E-03-2.8085-03-1.0332 E-03 \quad 3.8008 E-04-1.3982 \frac{1}{2}-04$ $-\frac{2}{5.9745 E-05} 1.5 \frac{2}{586 E-05}-\frac{2}{3.7065 E-06} 1.4558 E-06-5.8819 E-07$

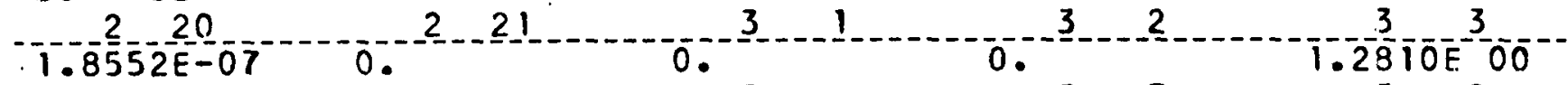

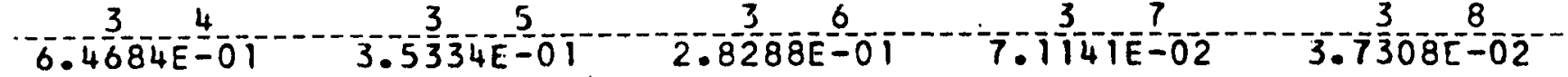

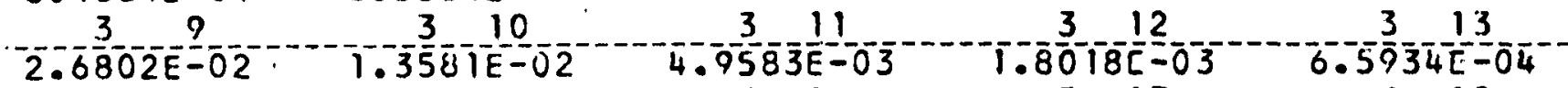

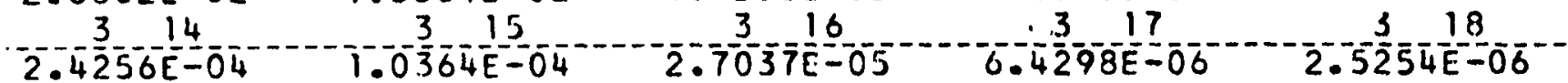

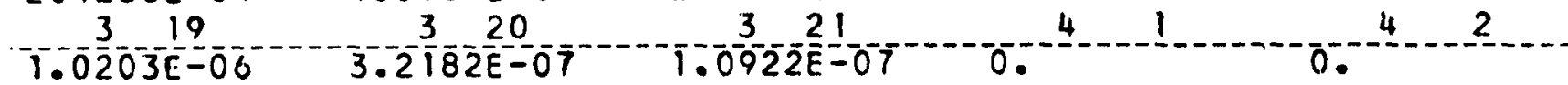

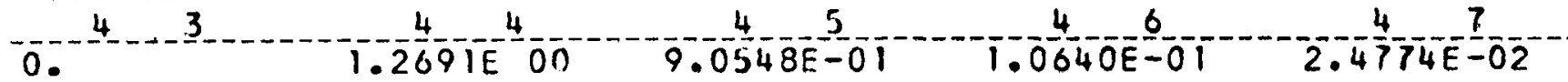

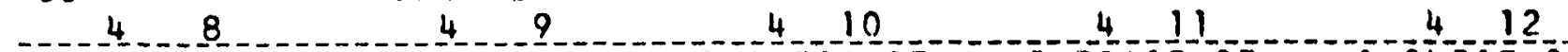
$1.7667 E-02 \quad 1.5899 E-02 \quad 8.1430 E-03-3.3861 E-03 \quad 1.0437 E-03$

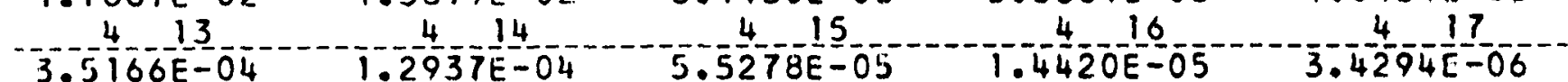

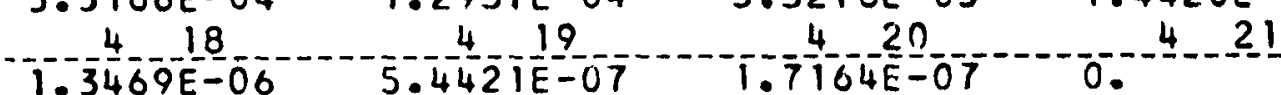


DERYLLIUM

TRANSFERS FROM GROUPS I THROUGH 34 TO GROUPS I THROUGH 11

SIGMA N,G (NIIN2)

Q. 1 1

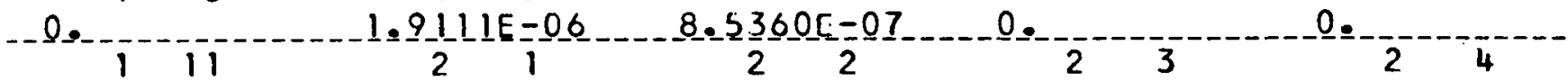
$1.8773 E-07$ -

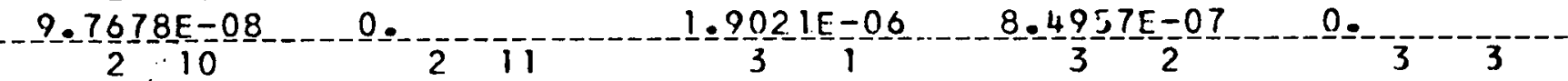

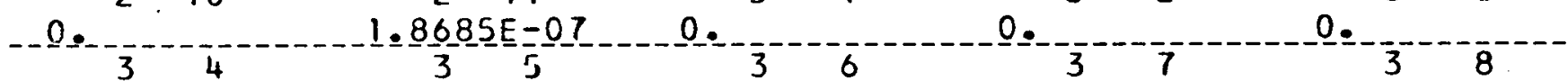

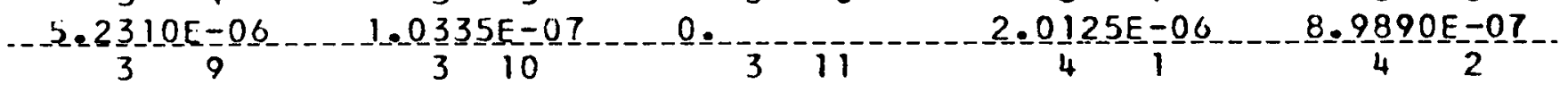
C. -0.

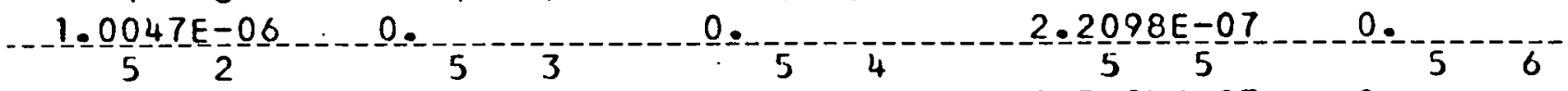

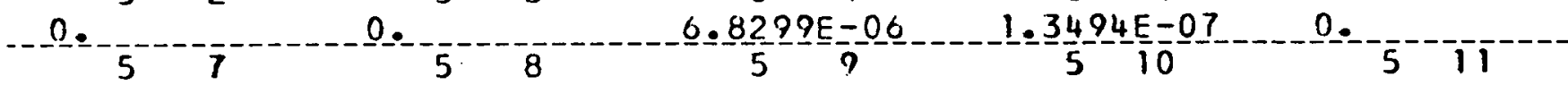

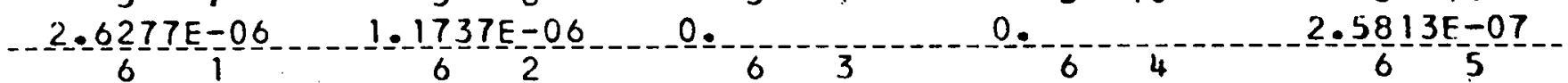
C.

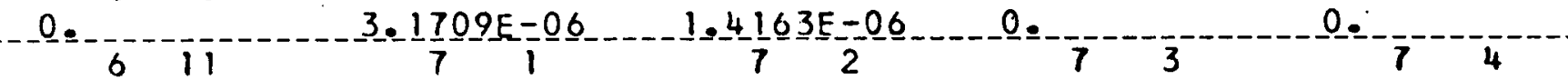

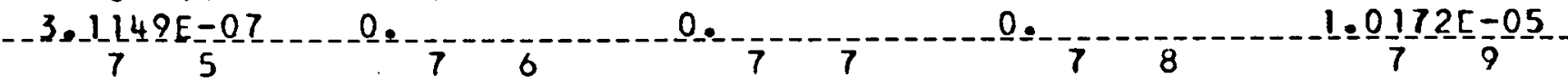

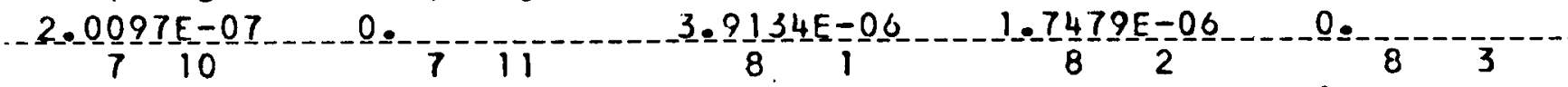

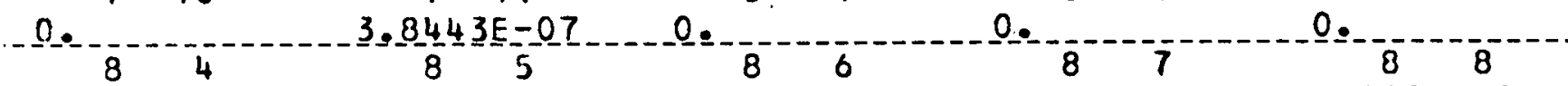

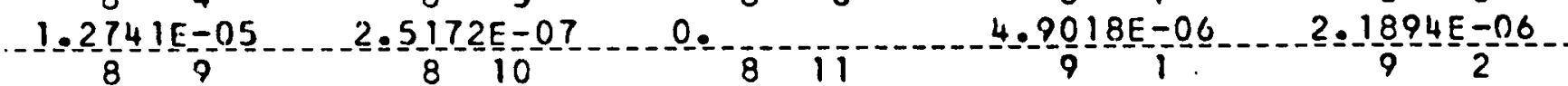

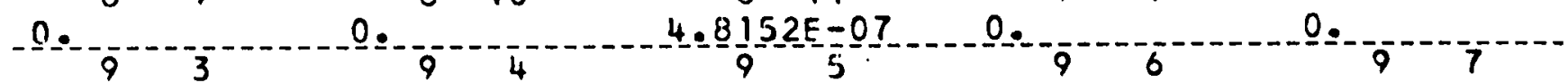

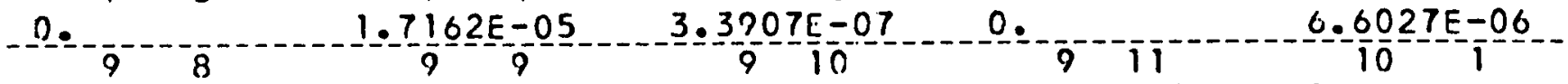
2.9491E 06 . 
BERYLLIUM

N1 N2

SIGMA_N, G_ (NIIN2)

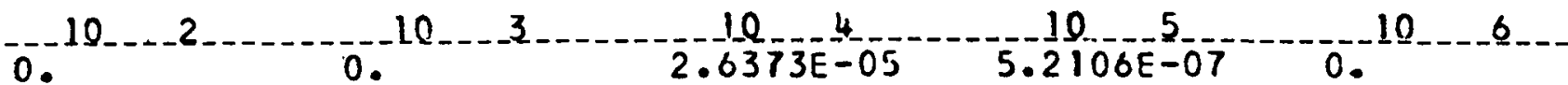

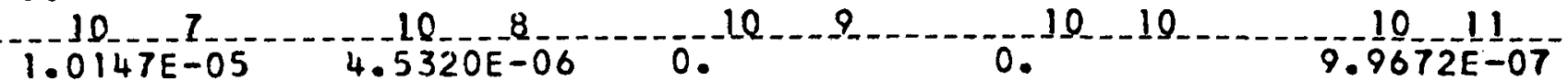

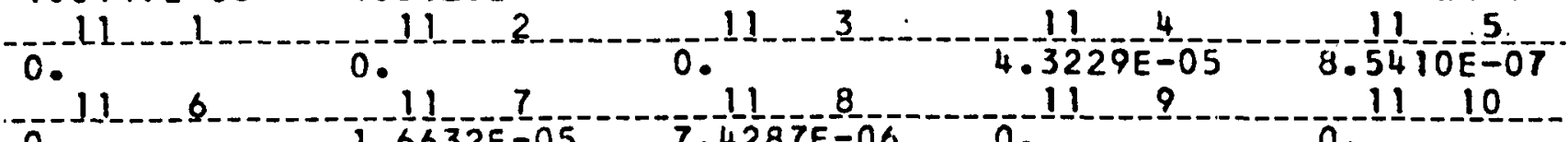

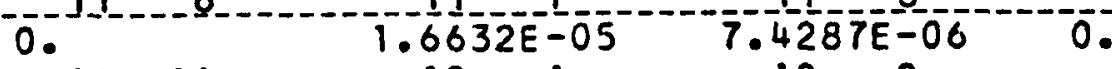

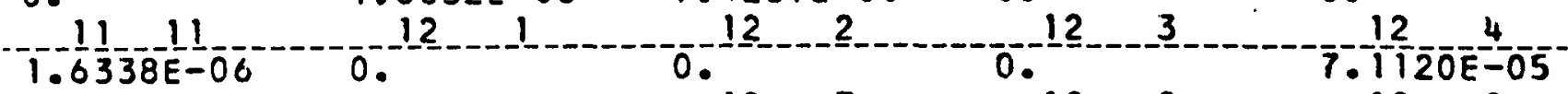

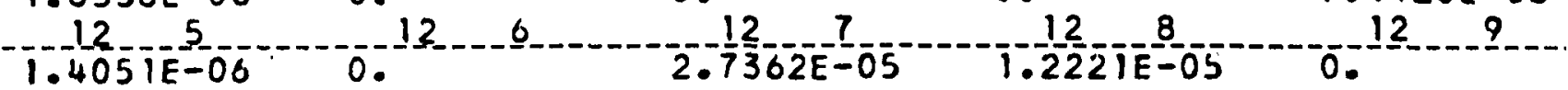

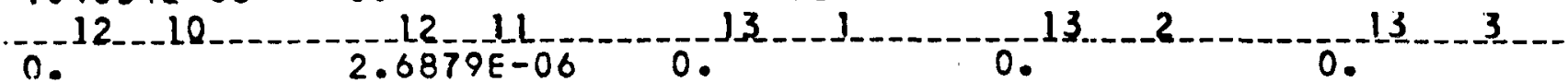
1.1716E-04 2.3148E-06 0 . 13 0. 14 1.

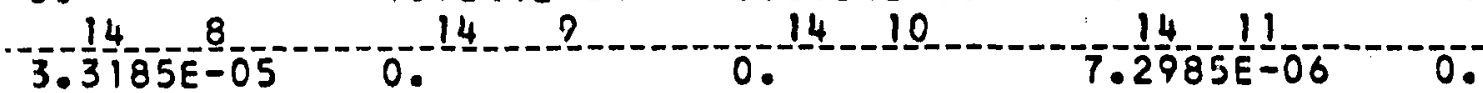

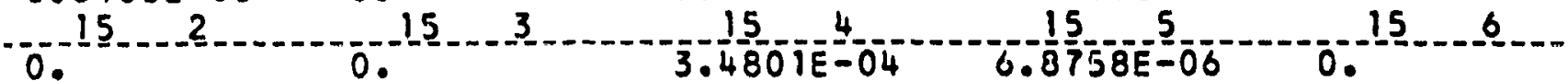

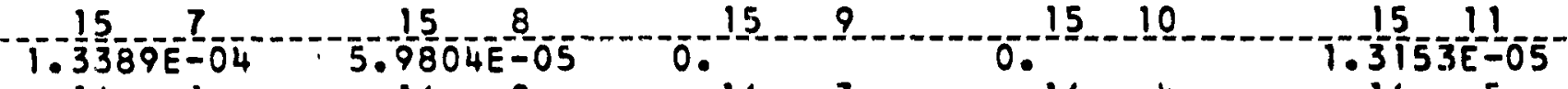

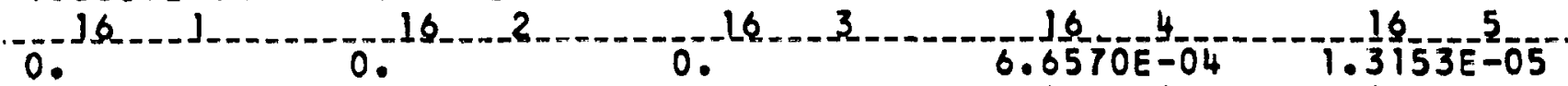
0.

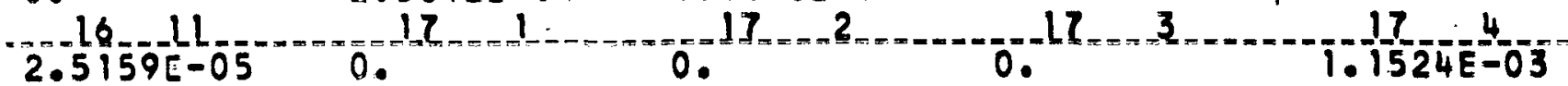
$=-\frac{17}{2.2768 E-05}$ 0.

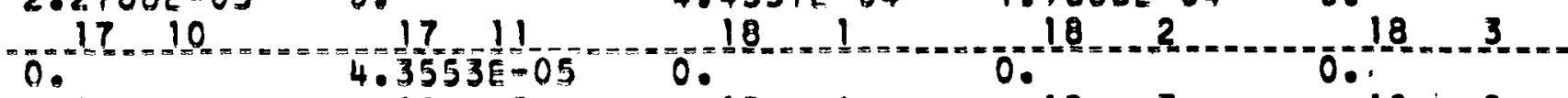

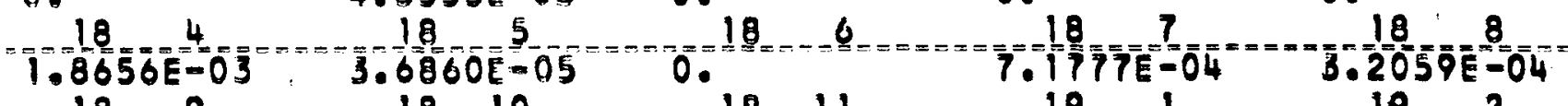
O. o. $18+10$ $\begin{gathered}10 \\ 7.0508 \frac{1}{2}-05\end{gathered}=-=0=$ o. 
-BERYLLLIUM _

\section{SIGMA N,G (NIIN2)}

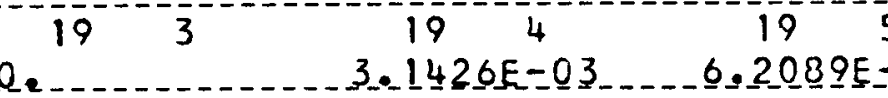

$198019 \quad 1910$

$5.4003 E-04 \ldots 0.020$
Q. $20-\overline{8}$ $5.30095-0$

$9-1092 E-04$

0.

$21^{-\cdots}$

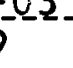

21

$.4521 E=0.3$

222

0.

$.2077 E=03$

$3.2511 \mathrm{E}=03$

\section{1}

22

$3.1936 E=0$

$22-6$

$2.6743 E=04$

0.

2211

$5 \cdot-\frac{1}{2} \frac{1}{3} 575=0$

Q. 23

$2.3232 E=02$

4..5899E-0 04

${ }^{0} 239$

23,10

$0 .-24$

$3.6060 E-02$

23

0.

$23^{6}$

$-0$.

2311

$8.7800 E=04$

$21^{-7}$

1911

$-1877 E=0$

$20915-03$

$.0473 E-04$ 2010

8. $-4502 E-03$

$2.0034 E=04$

\section{8}

6. $1967 E=03$

249

$7=\frac{12}{24} 46 \frac{6}{10}=04$

Q. 25

0.257

2. $1806 E=02$

Q.

0

4

5. 667 JE $=02$

Q.

22

o.

22

3

$2.3260 E=03$

50

0 .

$20-1$ $20^{-1-1}$ 
BERYLLIUM

N1 N2

SIGMA_N,G_(N)/N2)

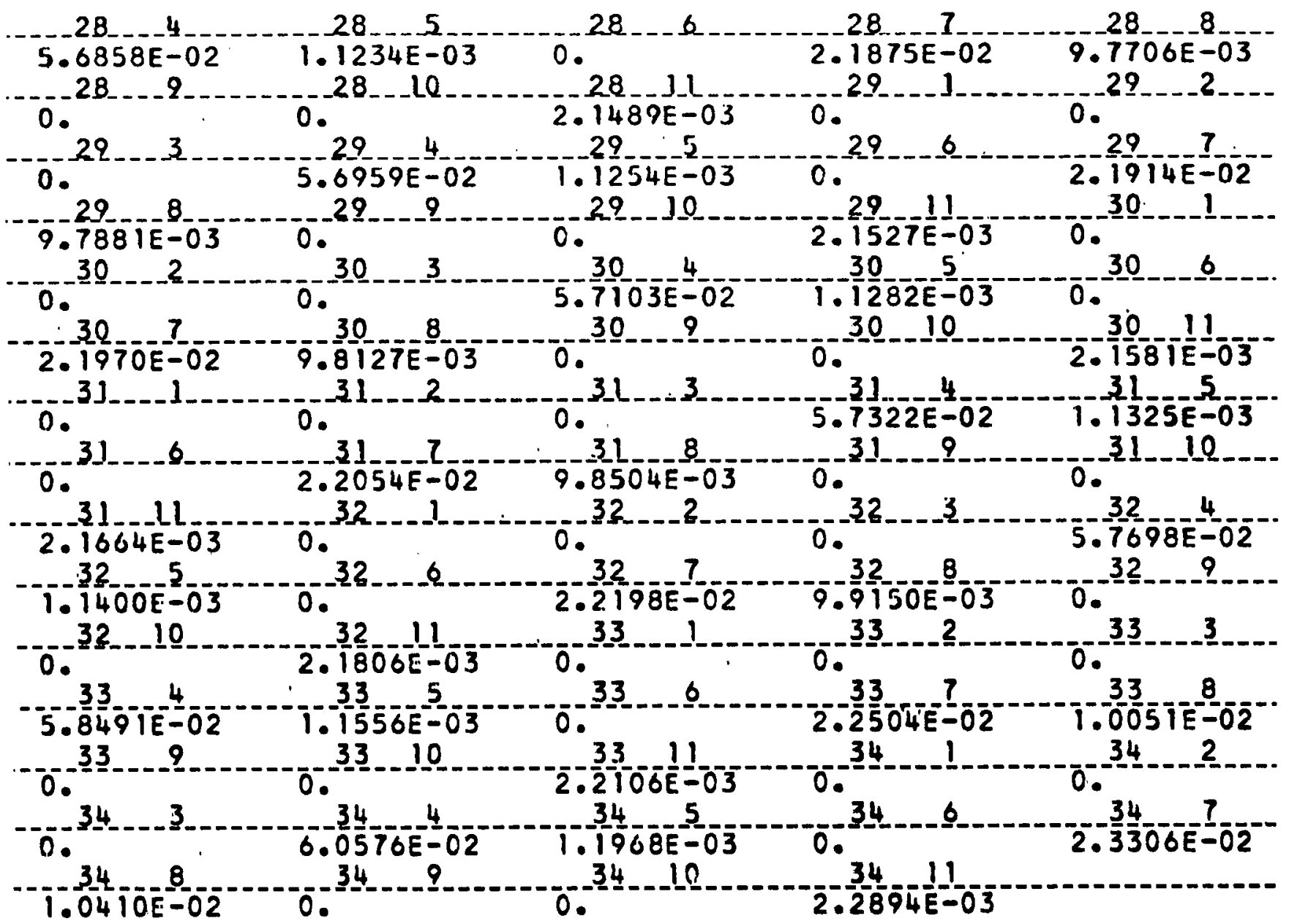


DAIE_DEC_01 1960

DESIGNATION NIOBIUM
CODE NO.

$4 \overline{1.9300}$
DENSITY FACTOR

$1.5 \overline{4} \overline{5} \overline{\mathrm{T}} \mathrm{E}-\overline{0}$

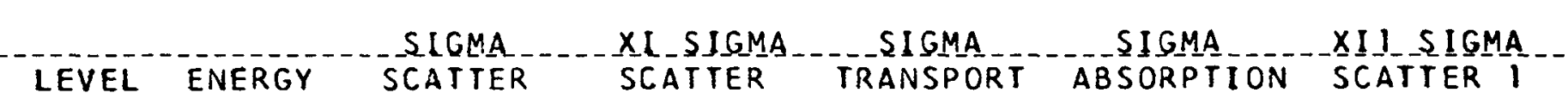

1 1.000E $073.9282 \mathrm{E} 003.3687 \mathrm{E} 001.9084 \mathrm{E} 009.3198 \mathrm{E}-02-1.9821 \mathrm{E} 00$

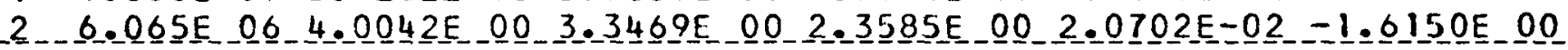

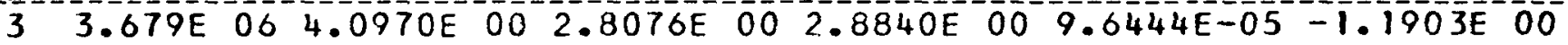

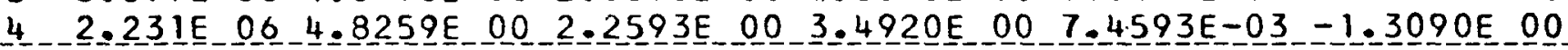

$51.353 \mathrm{E} 06 \quad 6.1264 \mathrm{E} 001.7832 \mathrm{E} 004.1984 \mathrm{E} 00 \mathrm{1}-2523 \mathrm{E}-02-1.8920 \mathrm{E} 00$

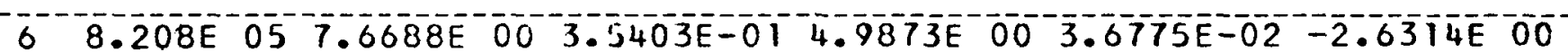

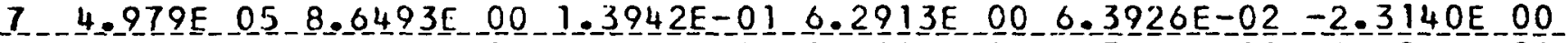

8 3.020E $058.9213 \mathrm{E} 00-1.6245 \mathrm{E}-017.4107 \mathrm{E} 006.7725 \mathrm{E}-02-1.4824 \mathrm{E} 0 \mathrm{O}$

9 1.832E $05990449 E-00-1.7547 E-01-8.1459 E-006.8582 E-02-8.8221 E-01$

$106.738 \mathrm{E} 048.4192 \mathrm{E}$ 00 $1.7124 \mathrm{E}-017.9411 \mathrm{E}-009.9494 \mathrm{E}-02-4.6917 \mathrm{E}-0 \mathrm{1}$

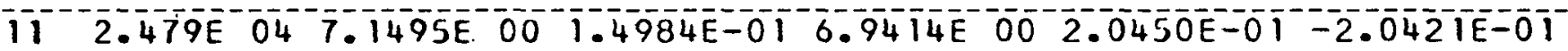

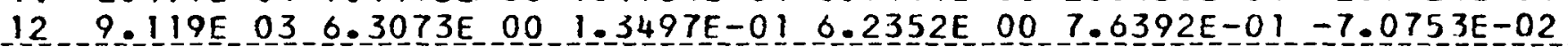

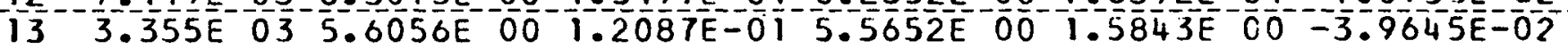

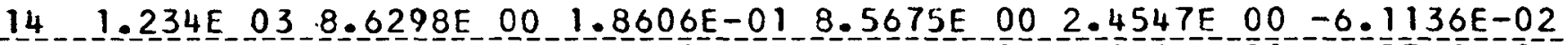

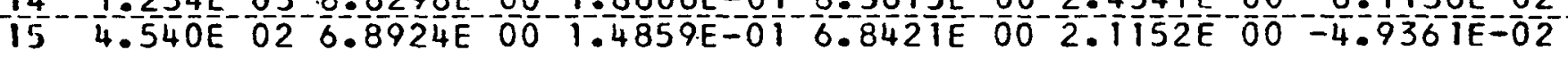

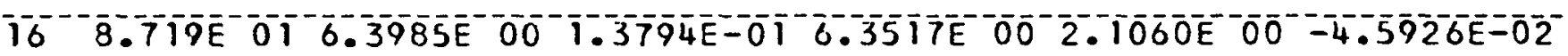

$17 \quad 3.545 E$ O $110.2504 \mathrm{E}$ 00 $1.3475 \mathrm{E}-01 \quad 6.2048 \mathrm{E} \quad 00 \quad 3.3034 \mathrm{E}-01-4.4748 \mathrm{E}-02$

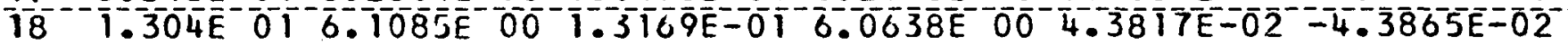

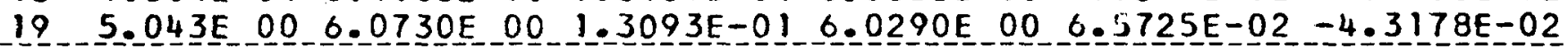

$201.445 \mathrm{E} 006.0134 \mathrm{E} 001.2964 \mathrm{E}-0 \mathrm{1}-5.9695 \mathrm{E}-001.2759 \mathrm{E}=0 \mathrm{1}-4.3080 \mathrm{E}-02$

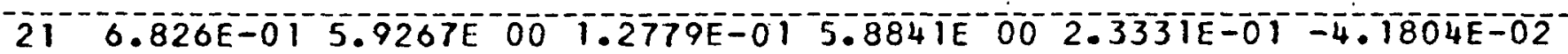

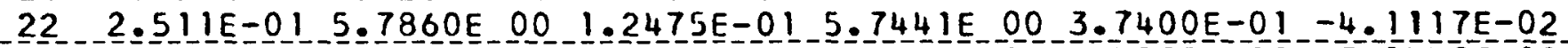

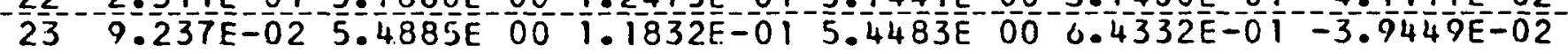

24 2.530E-02 5.1104E 00 1.1017E-01 5.0730E 00 9.9960E-01 -3.6701E-02

$4500 \quad 3.023 E-015.8157 E-001.2539 E-015.7736 E 003.3566 E-01-4.1314 E-02$

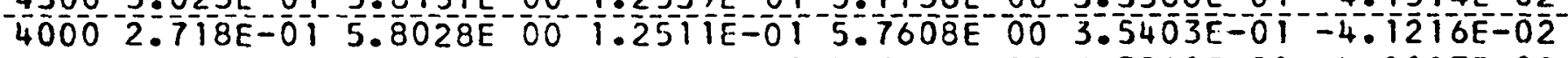
$35002.413 E-015.7868 E$ O $001.2476 E-015.7449 E$ O0 3.756 IE-01 $-4.1117 E-02$

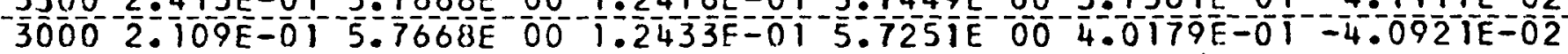
2500 1.804E-01 5.74 I IE 00 1.2378E-01 5.6998E 00 4.3440E-01 -4.0823E-02

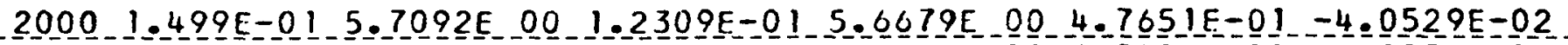
$15001.194 \mathrm{E}-015.6696 \mathrm{E}$ 00 $1.2224 \mathrm{E}-0150.0286 \mathrm{E} 00-5.3386 \mathrm{E}-01-4.0234 \mathrm{E}-02$ $10008.897 E-025.6219 E$ O $1.2121 E-015.5812 E$ O $00.1856 E=01-3.9940 E-02$ $5005.850 \mathrm{E}-025.576 \mathrm{TE} 00 \mathrm{1} .2022 \mathrm{E}-0 \mathrm{~T} 5.5357 \mathrm{E} 0 \mathrm{7} .6286 \mathrm{E}=0 \mathrm{~T}-3.9645 \mathrm{E}-02$

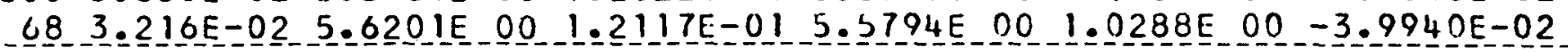

NOTE: For a summary of the sources of data see APEX-704. 
NI OBI

\section{THERMAL TRANSFER MATRICES}

TEMPERATURE
SIGAMA $\begin{gathered}\text { SIGMA } \\ \text { SIIGMA }\end{gathered}$

SIGMA S $N 2$

\begin{tabular}{|c|c|c|c|c|}
\hline$-21-21$ & & $-21-23$ & $21 \ldots 24$ & $21-25$ \\
\hline $\begin{array}{c}5.4277 E 00 \\
22\end{array}$ & $\begin{array}{r}4.9894 E-01 \\
22 \quad 22\end{array}$ & $\begin{array}{l}1.0661 E-05 \\
22\end{array}$ & 0.22 & \\
\hline $.7477 \mathrm{E}-01$ & $4.9866 \mathrm{E} 00$ & $3.2387 \mathrm{E}-01$ & $3.4771 E-04$ & $9.8879 \mathrm{E}$ \\
\hline $2.6265 \mathrm{E}-05$ & $9.7825 \mathrm{E}-01$ & $4.2384 \mathrm{E} 00$ & $2.4345 \mathrm{E}-01$ & $2.8343 \mathrm{E}-02$ \\
\hline & $8.2690 E-03$ & $-2.0192 \mathrm{E}-00$ & $2.3730 \mathrm{E}$ OO & $7.0990-\frac{25}{0}-0$ \\
\hline & $\frac{25}{6.5742 E-04}$ & $6.7335 E-01$ & $2.0446 \mathrm{E} 00$ & $3.72 \frac{25}{36 E} 0$ \\
\hline
\end{tabular}

SEMPERATURE
STAMA

$\mathrm{Ni}-\mathrm{N}^{2}$

SIGMA S $(N] / N 2)$

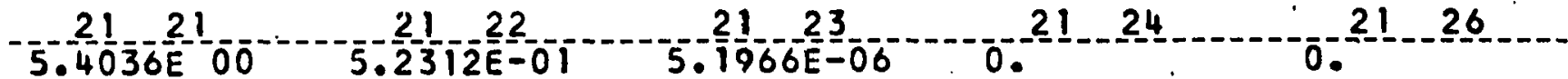

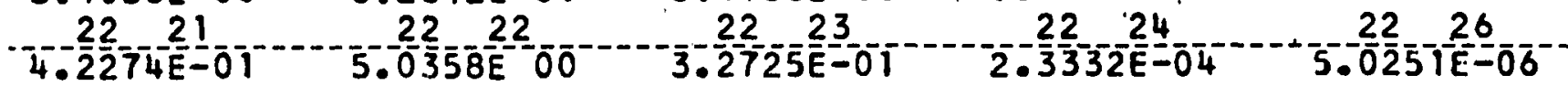

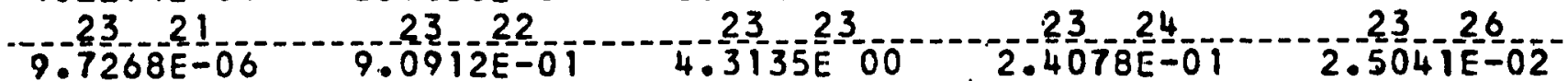

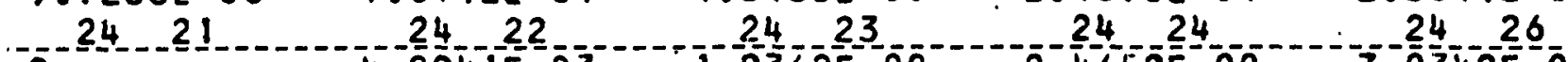

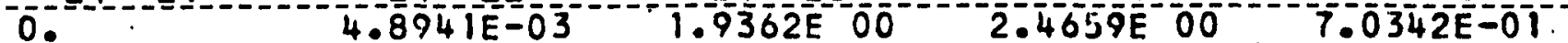

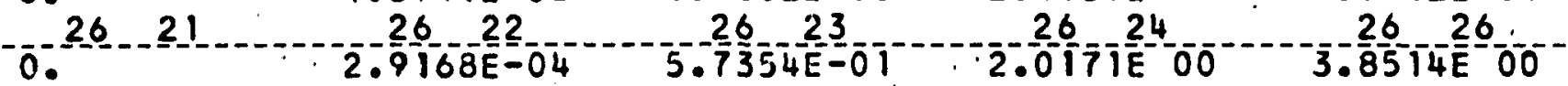


NIOBIUM

IHERMAL_ IRANSEER MAIRICES

- IEMPERAIURE SIGMA

SIGMA

SIGMA

NUY SICMA

DEG. F. SCATTER

TRANSPORT

ABSORPIION

FISSION

$3.5000 \mathrm{E} 030.4424 \mathrm{E} 00$ 6.3958E 00 $1.5607 \mathrm{E} 00 \mathrm{0}$

N

N2

SIGMA S (NTINZ)

2121

$-5.3709 E-00$ $22 \quad 21$

$3=6741 \mathrm{E}=0.1$ 2321

2.874 IE-00 2421

$-0 . \frac{0}{27}-\overline{2} \overline{1}$

- 0 .
5.558]E-01 $22 \quad 22$

5. $08 \underline{S} 2 \mathrm{E} Q 0$ 2322

$8.3463 E=01$ 2422 $2.5822 E-03$ $27 \quad 22$ $1.0767 E-04$
2123

$2.1567 E=06$ $22 \quad 23$ $3.3320 \mathrm{E}-01$ 2323 $4.3938[00$ 2423 $1.8430 \mathrm{E} \quad 00$ 2723 4. 737 ? $\mathrm{E}-01$
2124

O. $22 \quad 24$ $1.4406 \mathrm{E}=04$ 2324 2. $3834 \mathrm{E}-0$ ! 2424 $2 \cdot 5704 \mathrm{E}-00$ 2724 1.9777E_00
0. 2227 $2.2 \frac{20}{23}-\frac{8[-06}{27}$ 2. 16 으르는. 24 $-\frac{9}{2} 735 E-01$ 3.9908E 00
FEMPERAIURE DEG. $F$.

उ.0OOOE $0 \overline{3}$
SI GMA SCATTER
SIGMA TRANSPORT
SISMA ABSORPTION
NU SIGMA

FISSION

SIGMA $\frac{N !}{S}-\frac{N 2}{N I / N 2 T}$

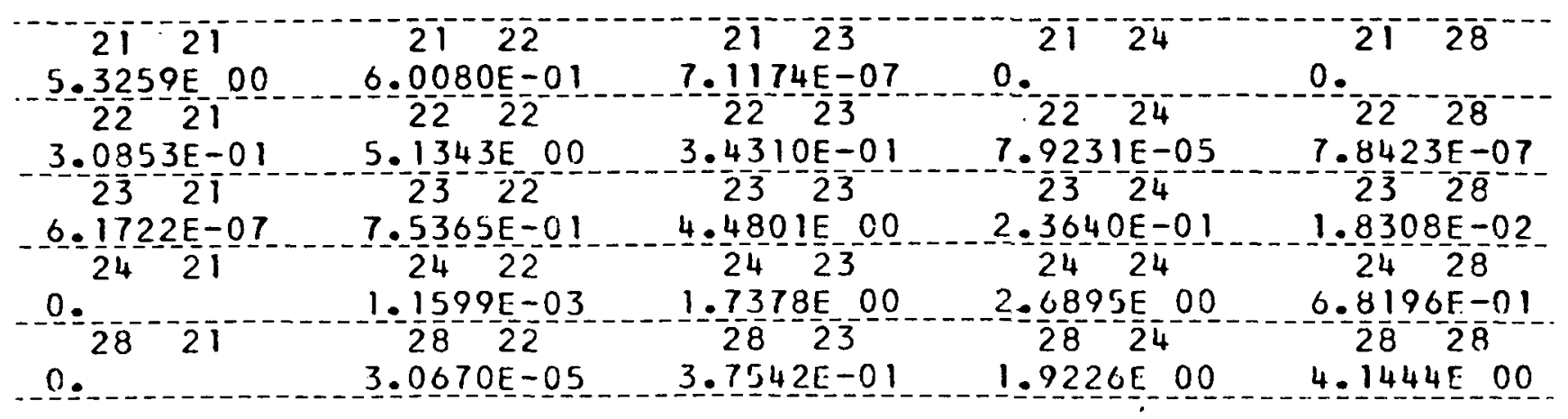


NI OBI UM

THERMAL TRANSFER MATTICES

TEMPERATURE
SIGMA $\begin{gathered}\text { SIGMA } \\ \text { SIGMATER }\end{gathered}$

\begin{tabular}{|c|c|c|c|c|}
\hline & $\frac{21}{6.6432 \mathrm{E}-01}$ & $\begin{array}{l}2 L-23 \\
1.6641 E-07\end{array}$ & $0.21 \ldots$ & 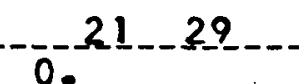 \\
\hline & $22-22$ & 22 & $-22 \quad 24$ & -.22 \\
\hline $\begin{array}{l}4596 \mathrm{E}-01 \\
23-21\end{array}$ & $\begin{array}{r}5.1806 \mathrm{E} \text { ON } \\
23 \quad 22\end{array}$ & $\begin{array}{l}3.5936 E-01 \\
23 \quad 23\end{array}$ & $\begin{array}{l}3.6790 \mathrm{E}-05 \\
23\end{array}$ & $\begin{array}{l}2.0519 \mathrm{E}-07 \\
23 \\
29\end{array}$ \\
\hline & $0.6465 \mathrm{E}-01$ & $\begin{array}{c}4.5734 E^{20} \\
2423\end{array}$ & $\begin{array}{l}2.3548 E-01 \\
24 \quad 24\end{array}$ & 1.4913 \\
\hline$=-$ & $\begin{array}{c}4.1146 E-04 \\
29 \\
5.9515 E-06\end{array}$ & $\begin{array}{l}1.6175 \mathrm{E} 00 \\
-\frac{29}{2.8041 \frac{23}{0}}\end{array}$ & $\begin{array}{r}2.8272 E 00 \\
1.89467 E 00\end{array}$ & $\begin{array}{l}6.6532 \mathrm{E}-01 \\
4 .-39154 \mathrm{E}-1 \\
0.00\end{array}$ \\
\hline
\end{tabular}

SEMPERATURE
SIGMA

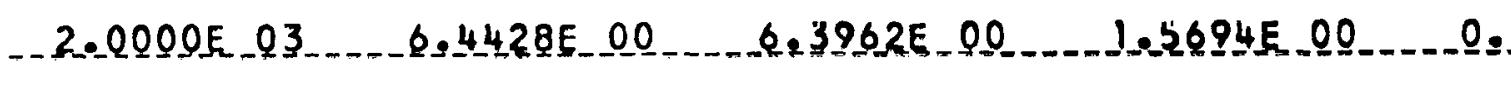

SIGMA $S$ (NIIN2)

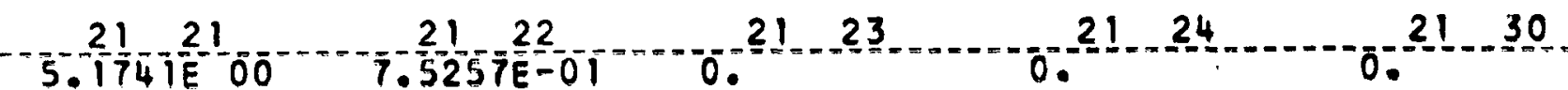

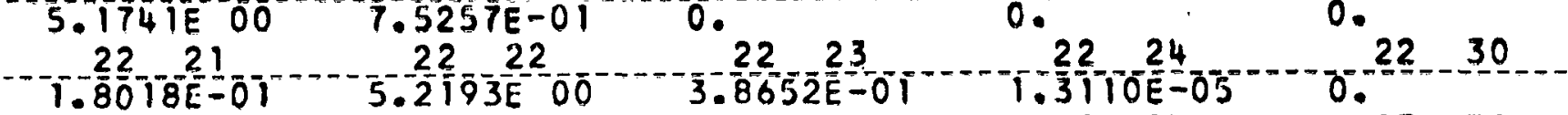

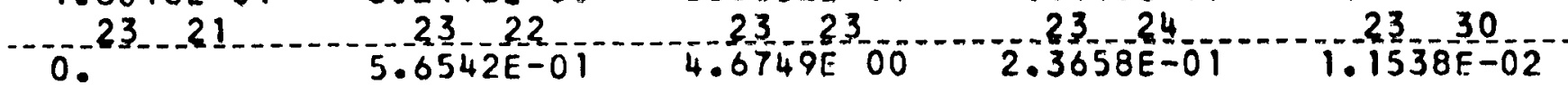

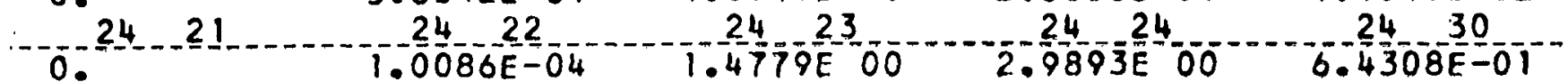

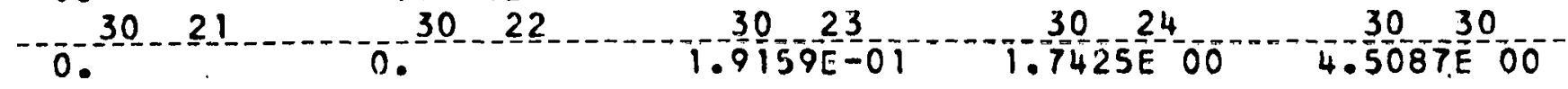


NIOBIUM

IHERMAL IRANSEER MAIRICES

TEMPERAIURE
DEG FI F.
1.5000 SCATIER 03

\section{SIGMA $\frac{N}{S}\left(\frac{N 2}{N} \frac{N}{N}-1\right)$}

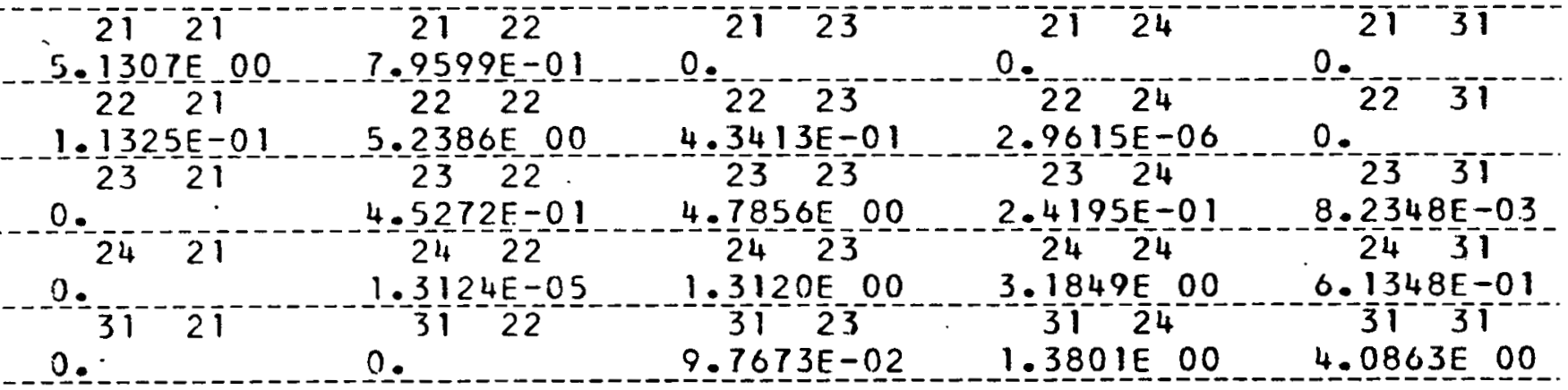

IEMPERAIURE

DEG. $F$.

$1.0000 \overline{0}$
SIGMA

SCATTER

$6.4437 \mathrm{E} 00$
SIGMA TRANSPORT
SIGMA ABSORPTION
NU SI GMA

FISSION.

\section{SIGMA $-\frac{N}{S}\left(\frac{N 2}{N}-\frac{1}{N}\right)$}

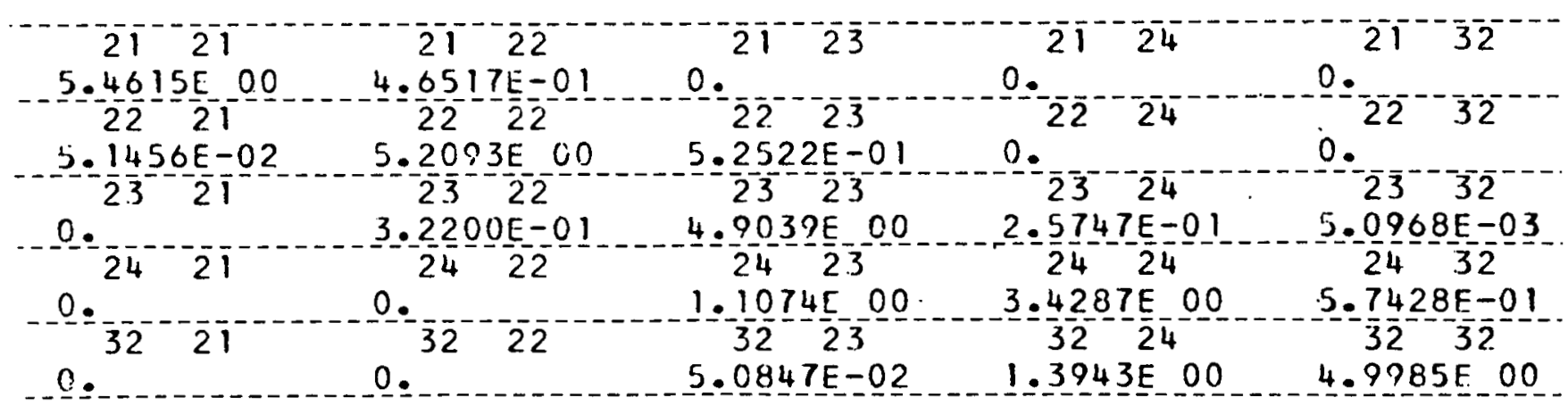


NI OBIUM

THERMAL TRANSFER MATRICES

TEMPERATURE
SCEG IGMA

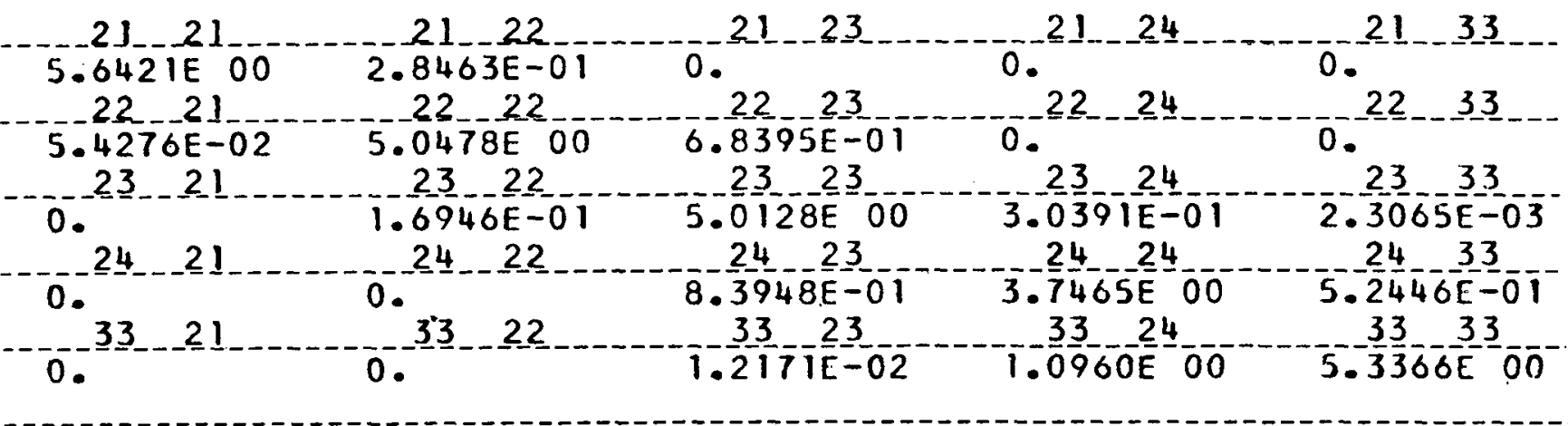

SEMPERATURE
SIGMA $\begin{gathered}\text { SIGMA } \\ \text { SIGMA }\end{gathered}$

$\mathrm{Ni}-\mathrm{N}^{2}$

SI GMA S (NIIN2)

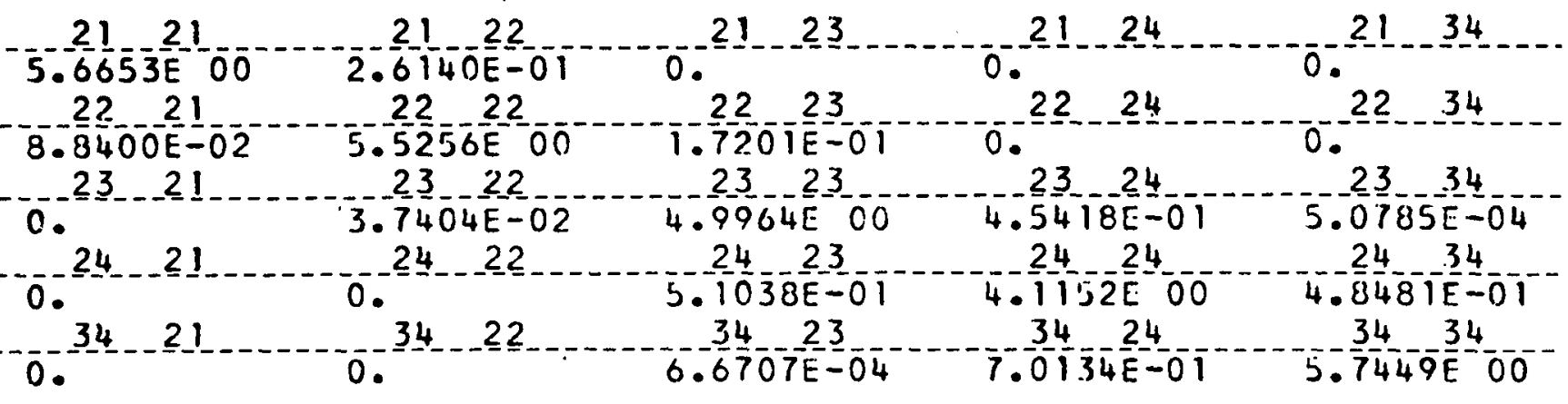


NIOB_LYM

TRANSFERS FROM GROUPS I THROUGH 6 TO GROUPS I THROUGH 21

N1 1

SIGMA SO (NI/N2)

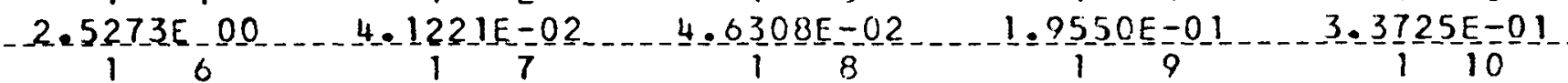
-3. 35 $78 \mathrm{E}-01$ - $3.3403 E=03$

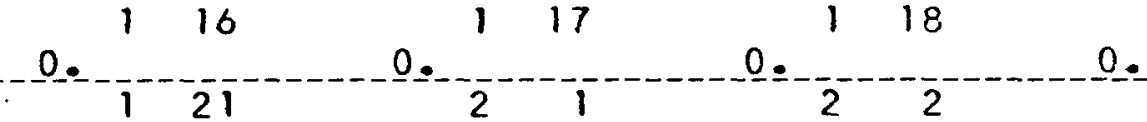

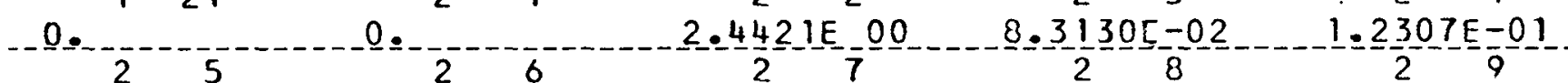
$3.0838 \mathrm{E}=0.1$ 210 $3.9345 E-01$

$3.7836 E=02$ 211 $3.1096 \mathrm{E}=01$ 215 $5.8302 E=03$ 212 $1.837 ? 96=01$ $7.9373 E-04$ 216 .2. $3696=06$ 220 2.21 ㅇ. $0 .-3$ $0 .-5$ 213 $1.1474 E-01$

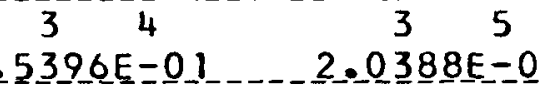
3 310 1.5662E $=01$ $5.4863 \mathrm{E}-02$ 314 3 15

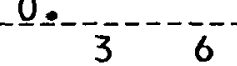
$1=0577-\frac{0}{18}=\frac{04}{8}$ 2.14 $2.2312 \mathrm{E}-05$ 319 $-0 .-1 \frac{1}{3}$ $-0$.

$2.660 \mathrm{JE}=0$ $3.2804 \mathrm{E}-06$

$$
30
$$

Q. 4

$3.2612 E_{-} 00$

$3.5308 E=01$

$0 .-\frac{1}{3}$ $1.4544[-05$ 413 $2.0400 E=01$ $8.7638 E=03$ $0 .-$ 3 3 414 316 $-3=\frac{41}{3}-\frac{192}{12}=0$ 2. $5930 \mathrm{O}=00$ $2.4546 E=04 \ldots 3,3885 E=05 \ldots-\ldots .9860 E=06$ $4 \quad 18$ 0. $4 \quad 19$ $2.5 \frac{3}{3}-\frac{5}{2}=0$ 0 . 4 $3.2885 E=01$ 410 $1.20960=03$ $2.2951 E-0$

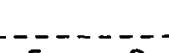
$0 .-5$ 0.000 .5 2. $9008[-0.1$ $2.5123 \mathrm{E}=03$ $2.7485 E-0$ 2. $736 \mathrm{LE}=02$ 0. 4 Q. 4 $2.6747 \mathrm{E}=01$ $1.6 \frac{1}{3} 99 \mathrm{E}-04$ $3.4115 E=04$ 420 411 ㅇ..4 
NIOBIUM

Ni

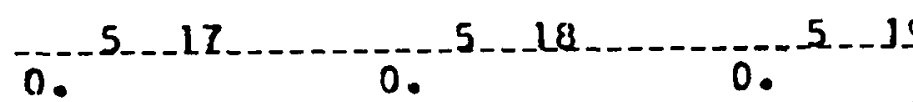

9 0.

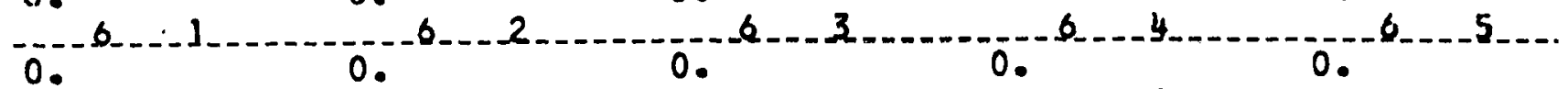
$7.1880 \mathrm{E} 00^{6}-5.548 \mathrm{E}-01$ 0. 6 $4.9457 \mathrm{E}-02$ $2.2379-\frac{6}{E}-02$ $4.1600 \mathrm{E}-03$ $6 \quad 12$ $4.9601 E-02$ -6 14 $-10 \frac{6}{3}-16-0$ $6.0879 E-04$ $8.3269 \mathrm{E}-0.5$ $1.1552 E-05$ $-15$ $1.3172 E-07$ 0 . 0 . 6 0. 6 $0 .-6-21$ 
NIOBIUM

RESONANCE RESONANCE
ENERGY PEAK

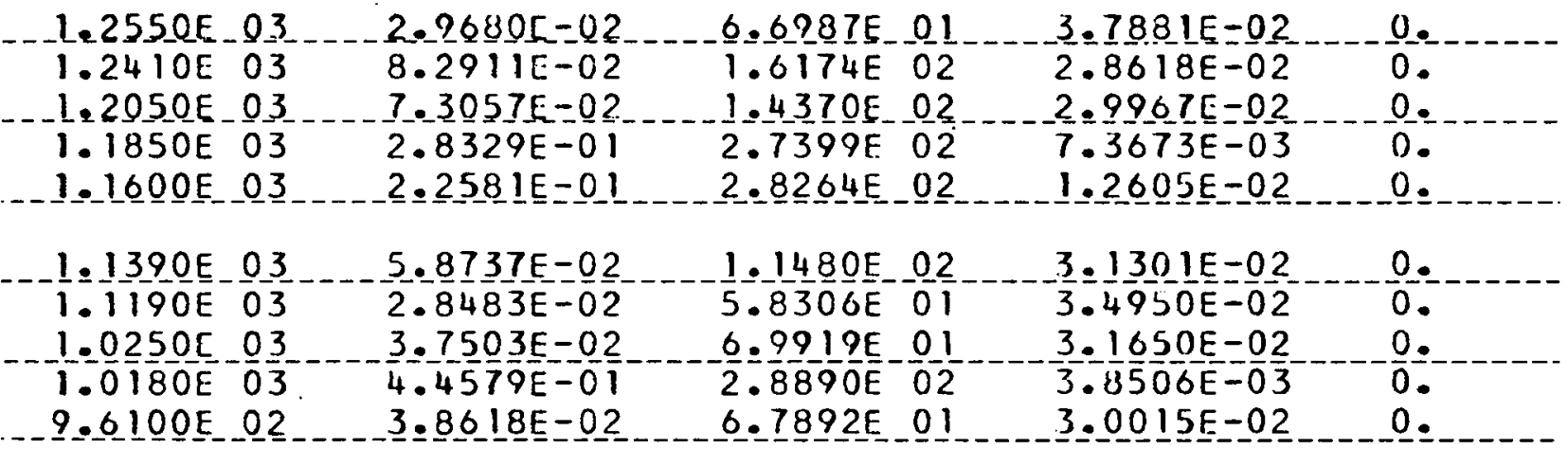

\begin{tabular}{|c|c|c|c|}
\hline $\begin{array}{r}9.4200 E-02 \\
9.2000 E-02 \\
7.6300 E-02 \\
7.4600 E-02 \\
7.2700 E-02\end{array}$ & $\begin{array}{r}4.6810 E=01 \\
2.0486 E-02 \\
1.9435 E=02 \\
-5.3454 E-01 \\
8.8792 E=02\end{array}$ & 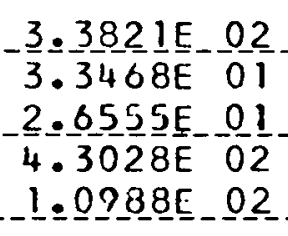 & $\begin{array}{l}5=1722 E=03 \\
2.7076 \mathrm{E}-02 \\
2 .=2836 \mathrm{E}=02 \\
8.1062 \mathrm{E}-03 \\
1.9659 \mathrm{E}-02\end{array}$ \\
\hline$\frac{6}{6}$ & $\begin{array}{l}4.4322 E-02 \\
3.2051 \mathrm{E}-02 \\
1.0775 \mathrm{E}-02 \\
2.5663 \mathrm{E}-02\end{array}$ & $\begin{array}{r}3.6916 \mathrm{E} \\
-3.2101 \mathrm{E} \\
2.8013 \mathrm{E}-0 \\
2.80\end{array}$ & \\
\hline
\end{tabular}

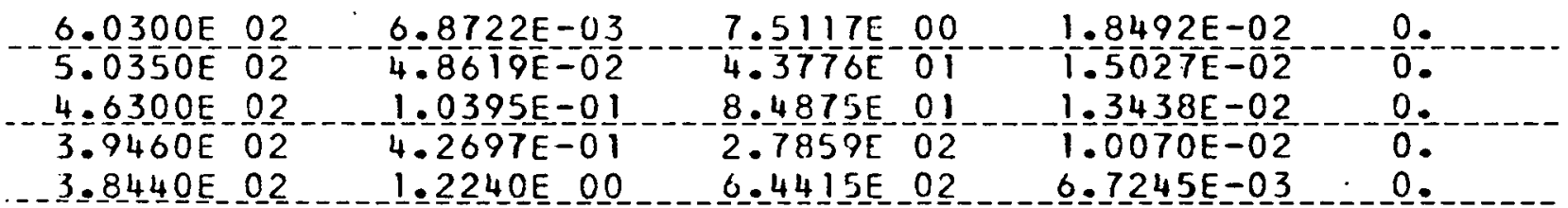

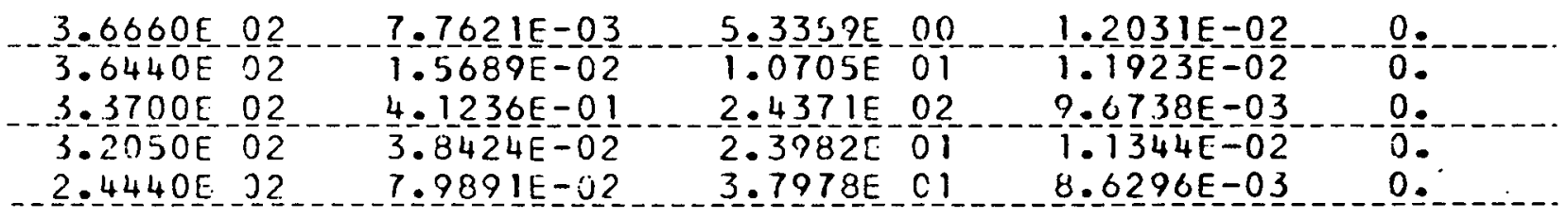

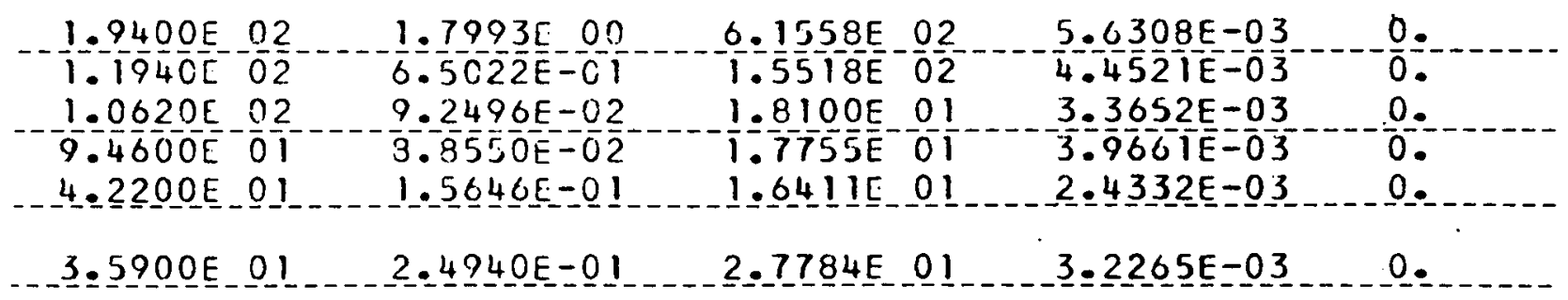




\section{NIOB I UM}

TRANSFERS FROM GROUPS 1 THROUGH 34 TO GROUPS I THROUGH 1

N1 N2

SIGMA_N,G_INIIN2 I

0.

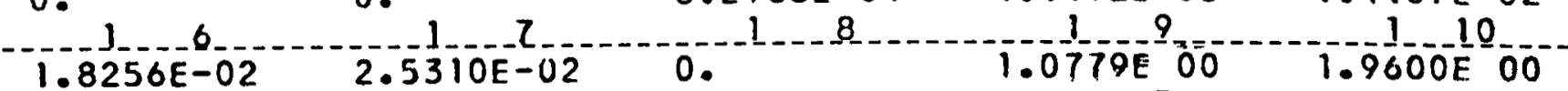

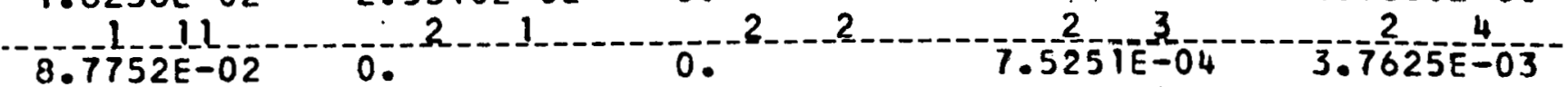

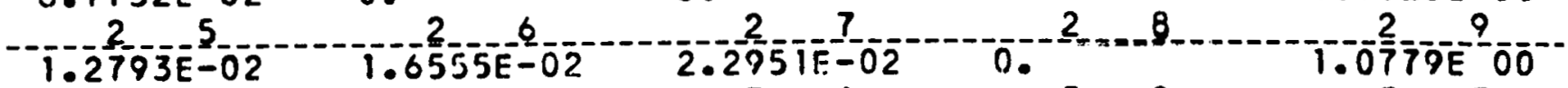

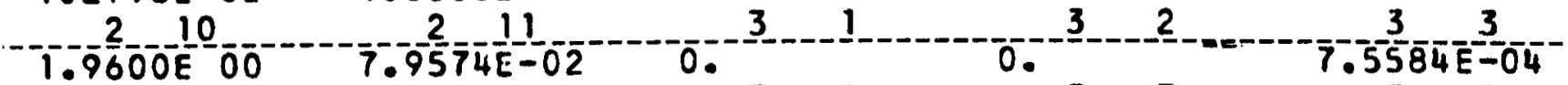

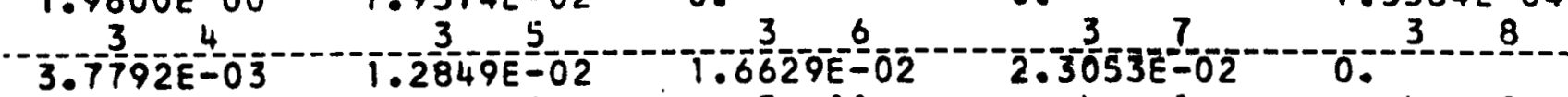
$1.0779 E^{2} 00^{-1.9600 E} 00^{3}$

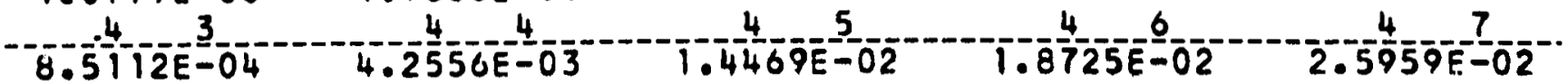

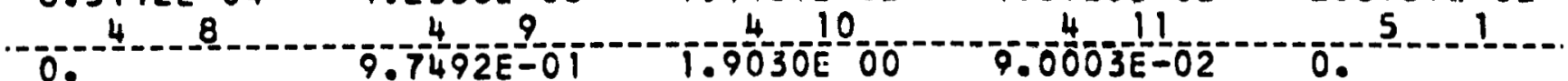
-

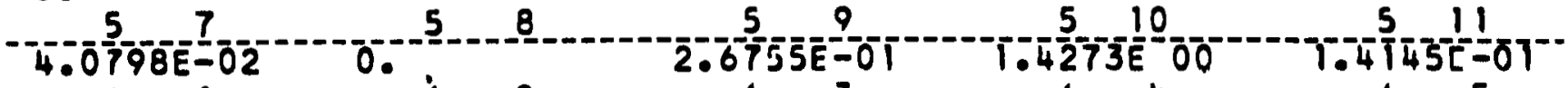
.

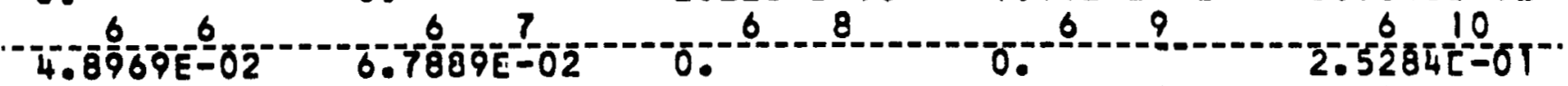

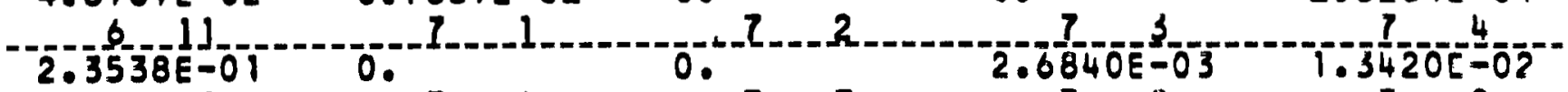

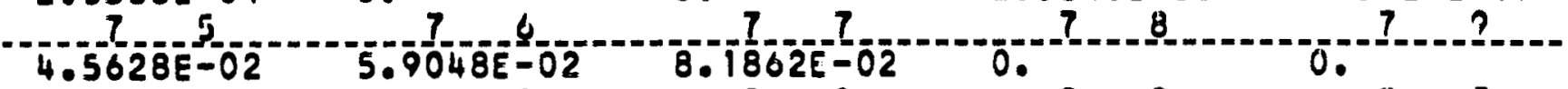
-

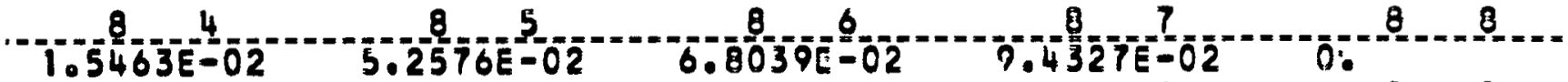

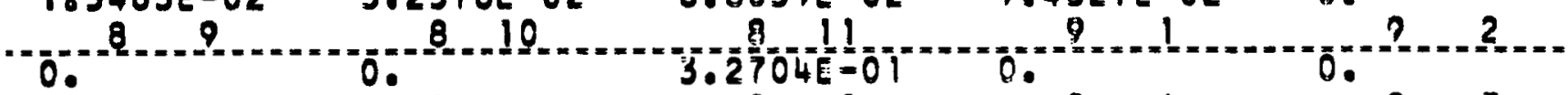

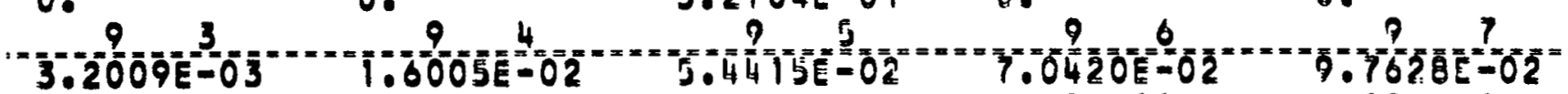
- 
N1 1

\title{
SIGMA N,G (NTINZT
}

$10-2$

ㅇ..

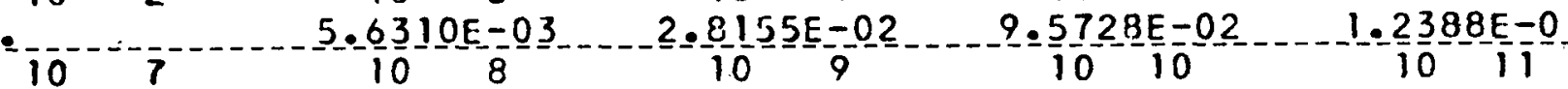
$10-7$. 10 .

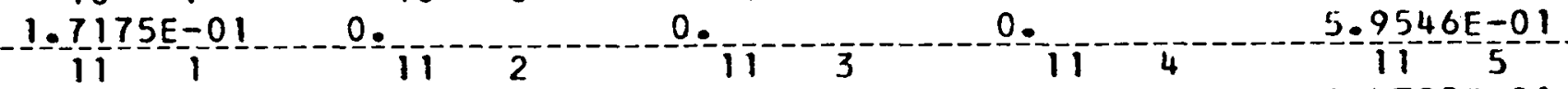

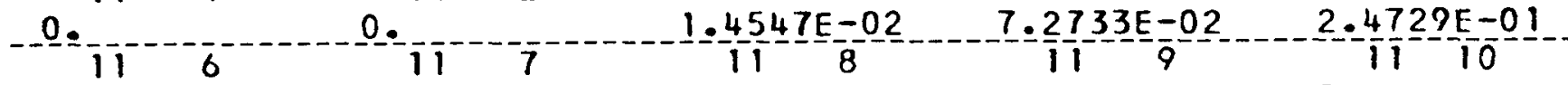
3.2.003E $01 \quad 4.4367 E_{1}-01 \quad 0$.

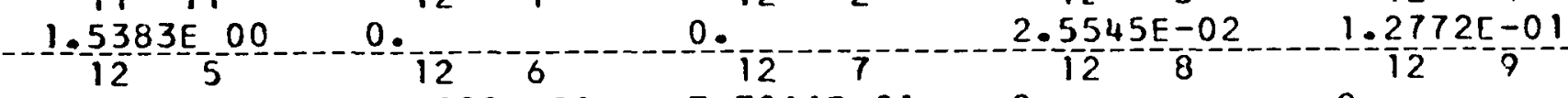
$4.3426 \mathrm{E}=01$ 1210 $0.13-2.7012 E-00 \quad 00$. $9.8021 E-02 \quad-3.3327 E-C 1$ 0.0 -3. $\frac{1}{14} \frac{5}{14}=02-1.5978 E-01 \quad 0.4327 E-01 \quad 7.0305 E-01 \quad 9.7468 E-01$ 0.

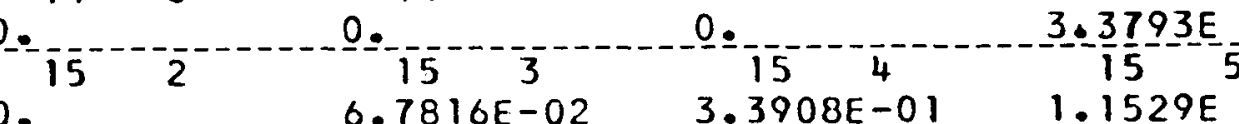

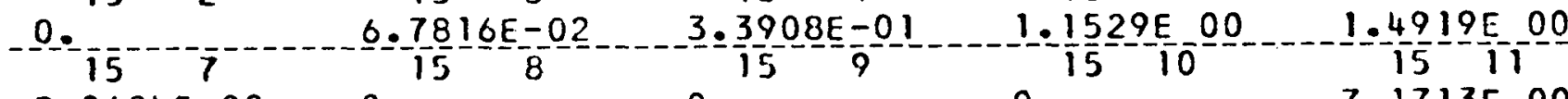

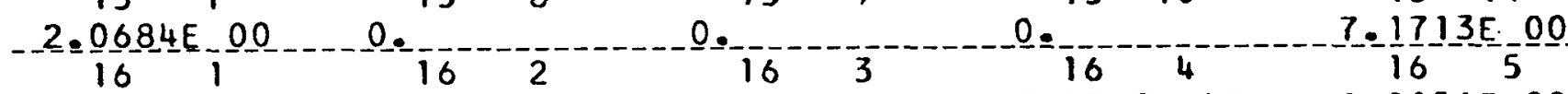

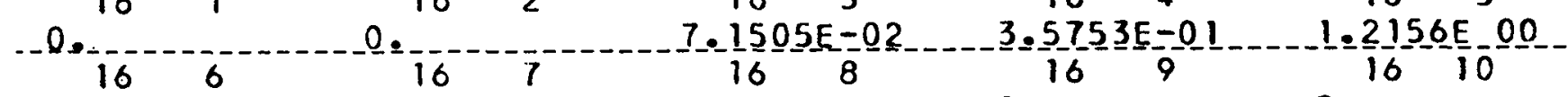

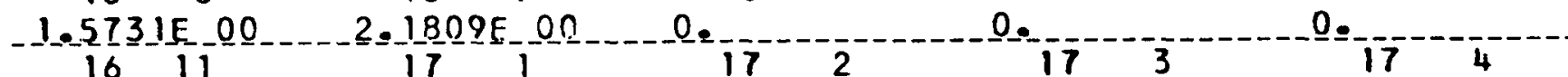

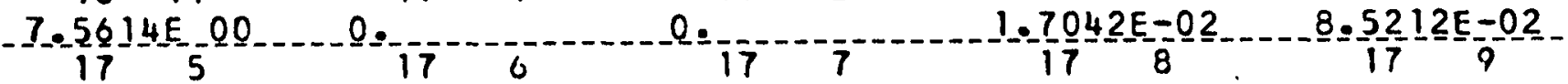
$2.8 ? 725-0$

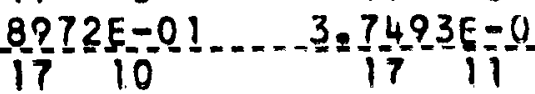
$\begin{array}{ll}17 & 10\end{array}$ 18 - 18 $1.0255 E-02 \quad 3.4868 E-02$ 5. 1980E $=01$ 0 $1.9604=02$ $1793 E-01$ 133

\author{
NIOBIUM
}


NIOB I UM

$\mathrm{NT}^{\mathrm{N} 2}$

SIGMA N,G (NIIN2)

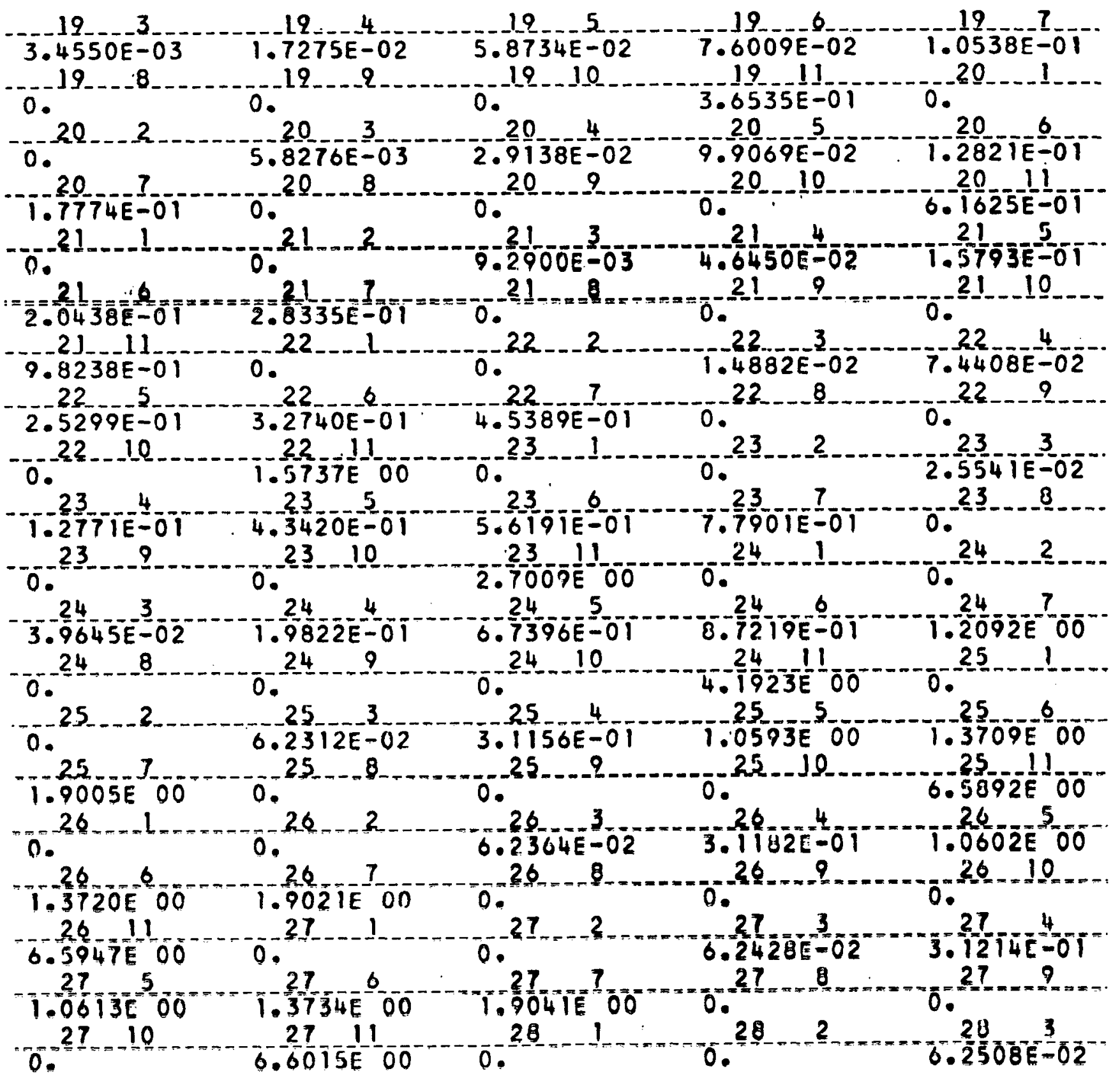


NIOBLUM -

SIGMA N.G (NITNZ)

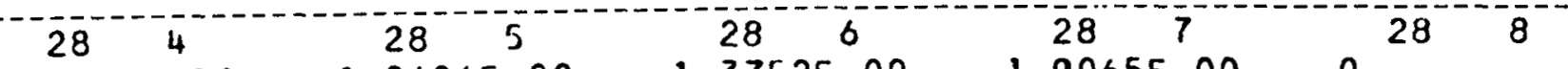

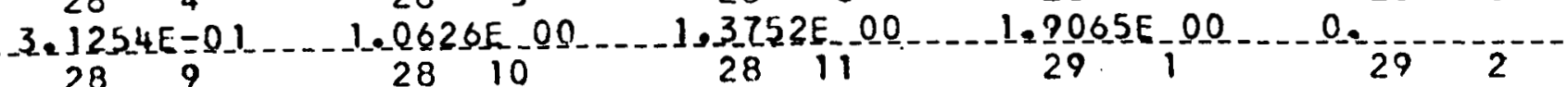

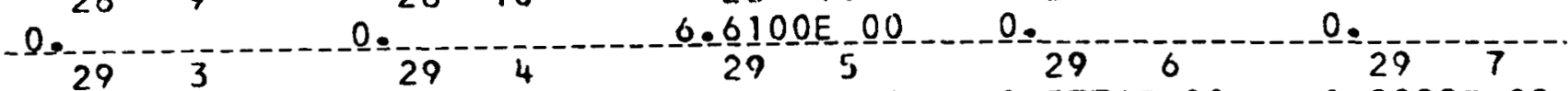

$6.2620 E-02$
29

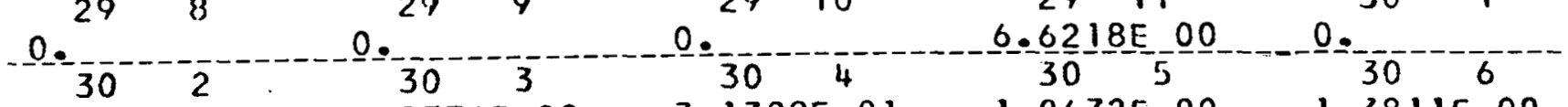

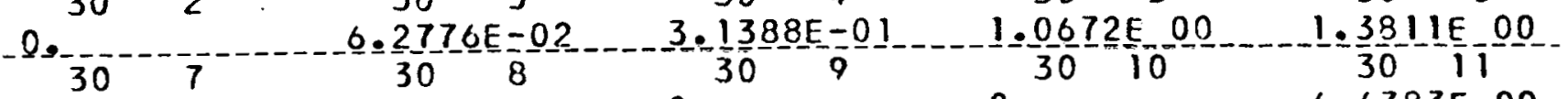

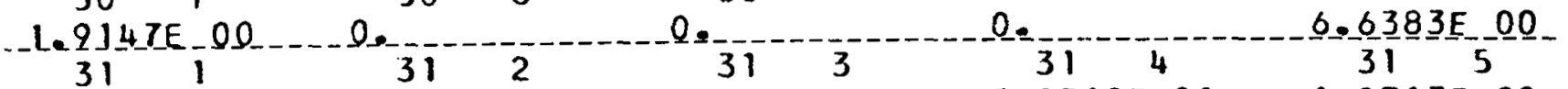

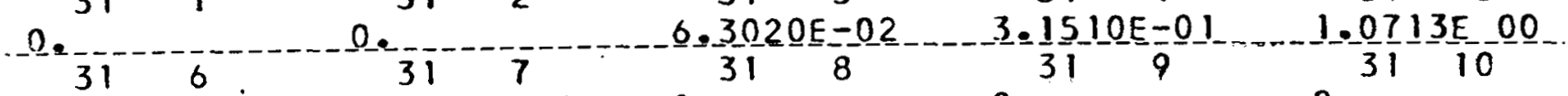

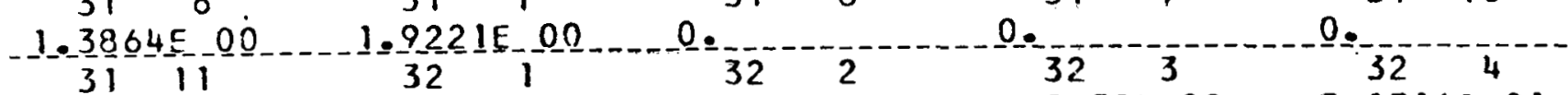

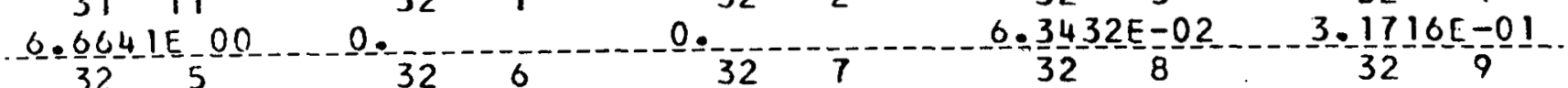

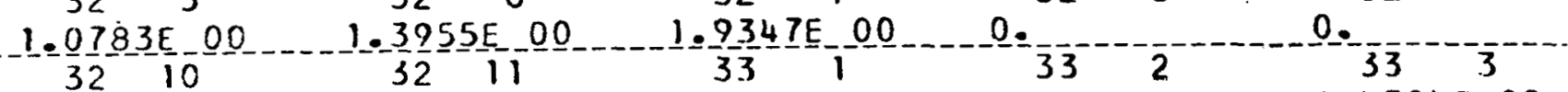

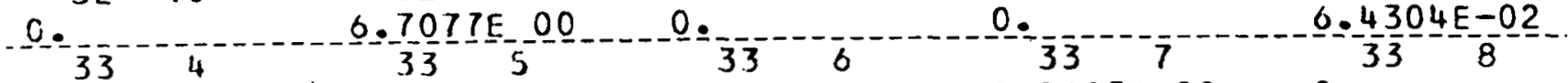

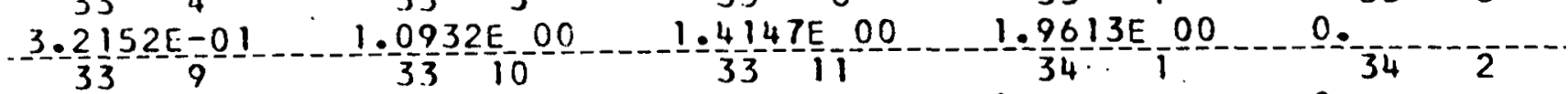

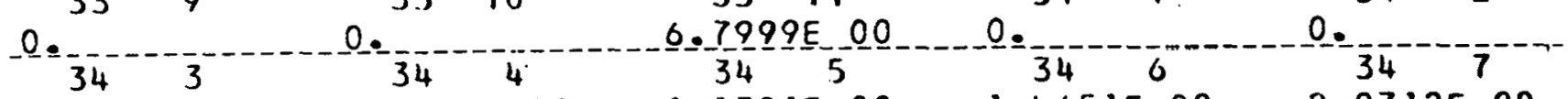

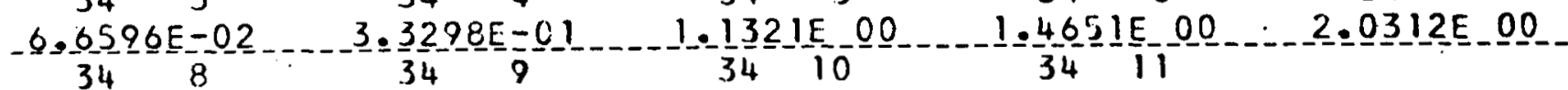

0 .

o.

7.0423E 00 
DAIE 12/08/60

DESIGNATION

URANIUM 234
CODE NO.

92.2340
DENSITY FACTOR

$\overline{3} . \overline{8} 8 \overline{5} \overline{\mathrm{O}} \overline{\mathrm{E}} \mathrm{0} \overline{2}$

\section{SIGMA}

LEVEL ENERGY

SCAT TER
XI_SIGMA SCATTER
SIGMA TRANSPORT
SIGMA ABSORPTION

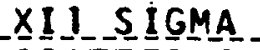
SCATTER 1

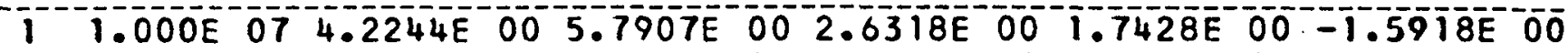

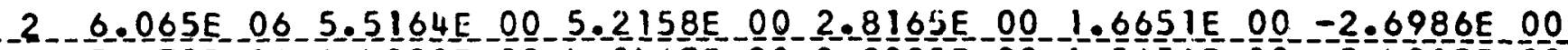

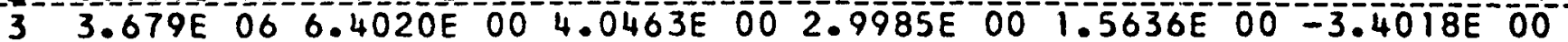

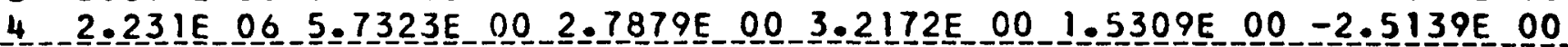

5 1.353E 06 5.2326E 00 1.8184E $003.5812 \mathrm{E} 00^{1.414} 1 \mathrm{E} 00-1.6505 \mathrm{E}$

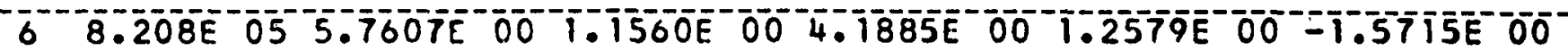

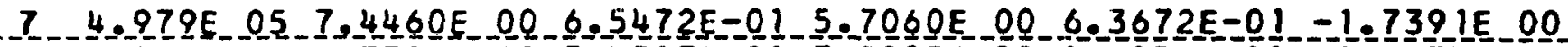

8 3.020E $058.7785 E$ O0 $3.4527 \mathrm{E}-017.0903 \mathrm{E}$ 00 $2.6054 \mathrm{E}-0 \mathrm{1}-1.6874 \mathrm{E}-00^{-}$

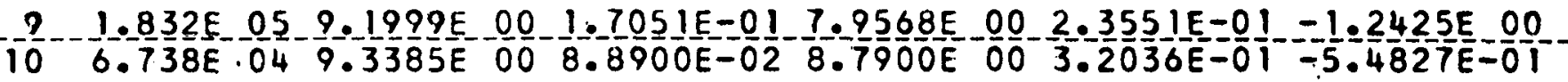

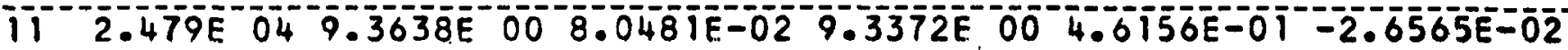

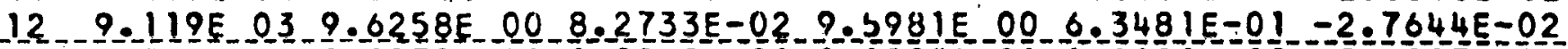

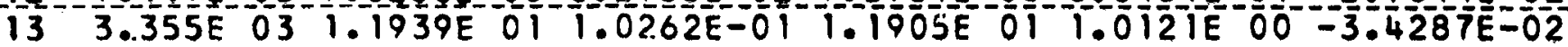

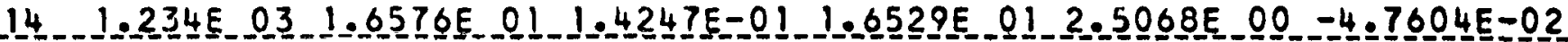

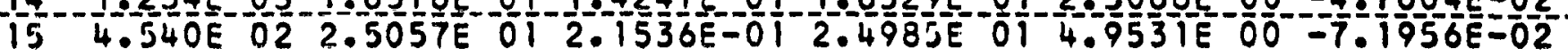

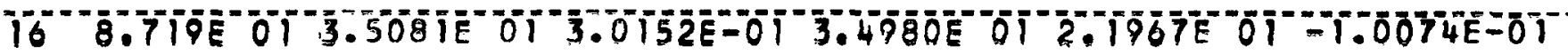

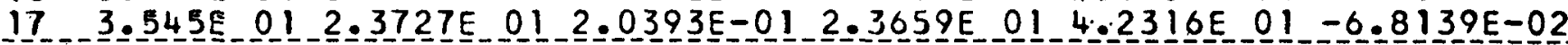

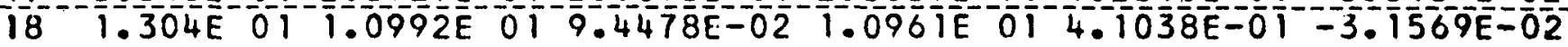

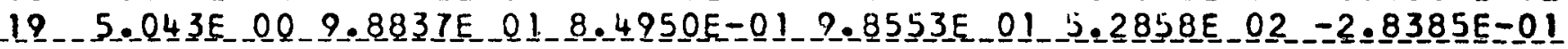

$20 \quad 1.445 \mathrm{E} 001.0972 \mathrm{E}$ 01 $9.4300 \mathrm{E}-02$ 1.0940E $010.1758 \mathrm{E} 00-3.1509 \mathrm{E}-02$

$2 \overline{1} 6.826 \mathrm{E}-0 \overline{1}$ 1.3772E OT

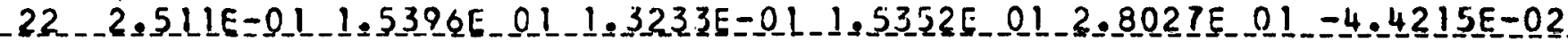

$239.237 \mathrm{E}-021.6277 \mathrm{E} 011.3990 \mathrm{E}-011.0231 \mathrm{E} 015.4915 \mathrm{E}$ 01 $-4.6745 \mathrm{E}-02$

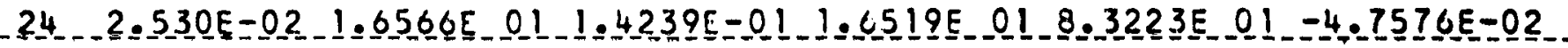

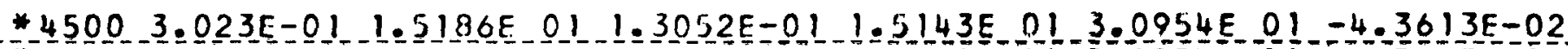

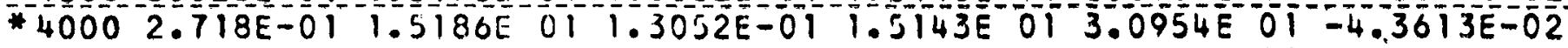

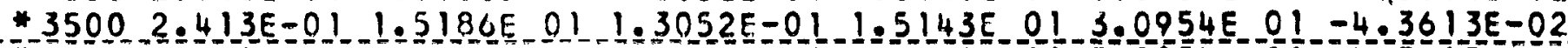

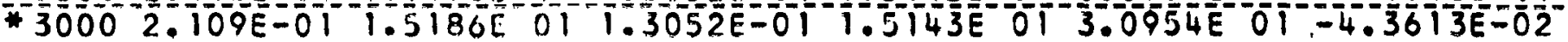
2500 1.804E-01 1.5397E 01 1.3234E-01 1.5353E 01 3.4261E $01-4.4218 E=02$.

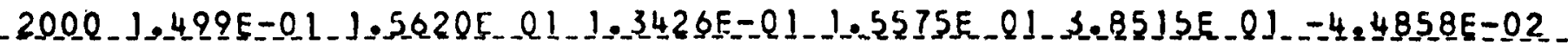
$15001.194 \mathrm{E}-011.5852 \mathrm{E} 01 \mathrm{1} .3625 \mathrm{E}-011.5806 \mathrm{E}$ 01 $4.4268 \mathrm{E} \quad 01-4.5524 \mathrm{E}-02$

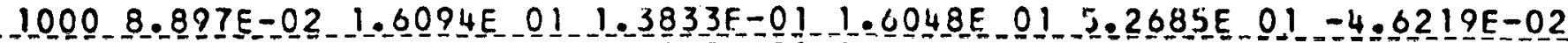

$5005.850 \mathrm{E}-021.6353 \mathrm{E} 011.4055 \mathrm{E}-011.6306 \mathrm{E}-016.6813 \mathrm{E}-1-16.6962 \mathrm{E}-02$

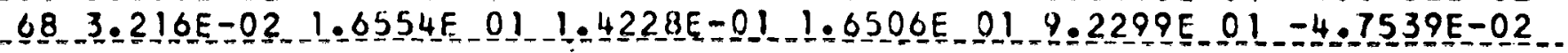

Thermal cross sections taken from XDC 59-11-72.

NOTE: For a summary of the sources of data see APEX-704. 


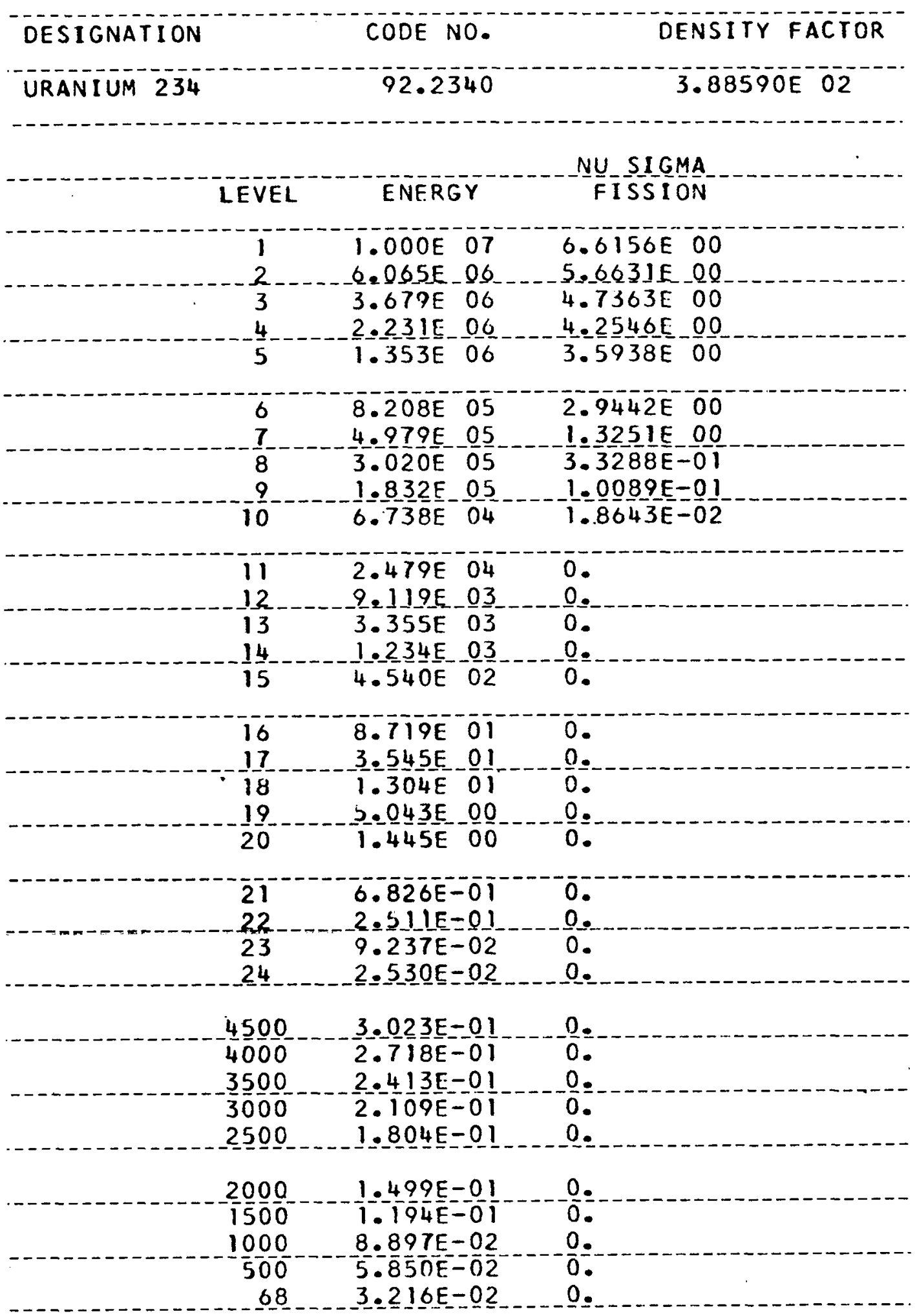


URANLUM_234

THERMAL TRANSFER MATRICES

TEMPERATURE
SIGMA

Ni

SIGMA_S_- (NILN2)

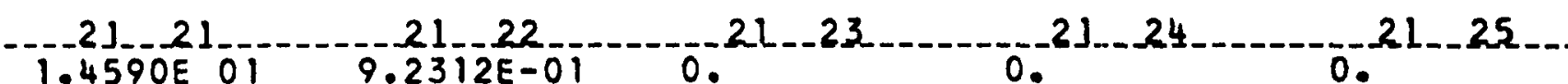

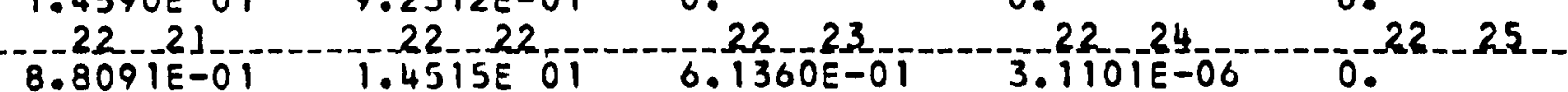

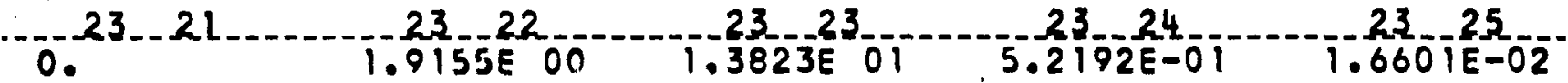

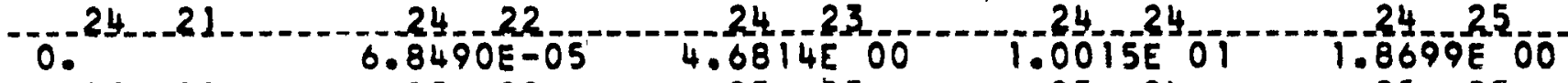

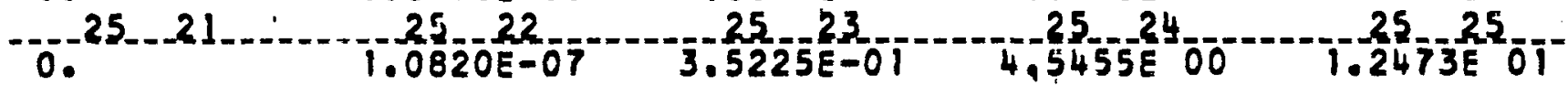

TEMPERATURE SIGMA SIGMA STGMA SIGMÁ

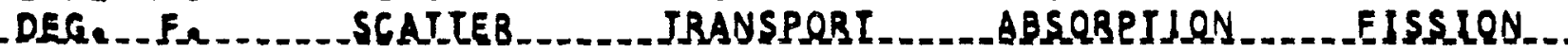
. 4.

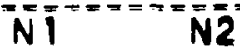
SIGMA $=$ S. $(N ! L N 2)$

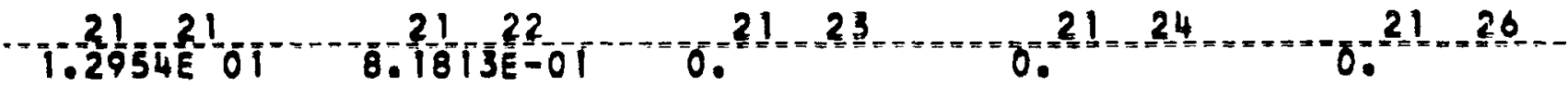

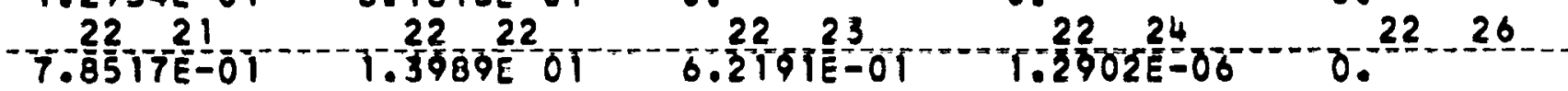

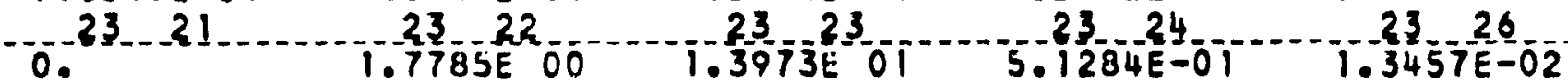

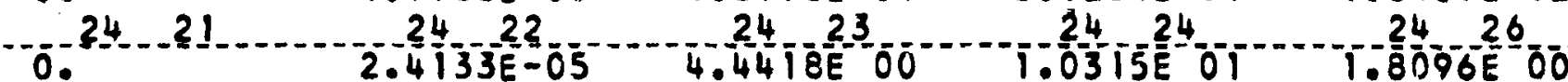

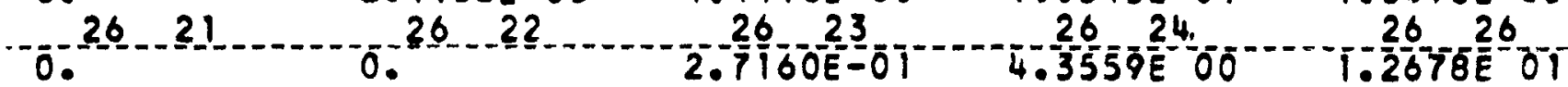


URANIUM 234

IHERMAL IRANSFER MAIRICES

-.LEMPERAIURE

DEG. $F$.

SIGMA

SIGMA

SIGMA

NU_SIGMA

SCATTER

TRANSPORT

ABSORPTION

FISSION

$3.5000 \mathrm{E} 03$ $1.7239 \mathrm{E} 01$

$1.7189 \mathrm{O}^{-}$

$1.4127 \overline{02}$

0.

\section{SIGMA $-\frac{N}{S}\left(\frac{N 2}{N-N 2)}\right.$}

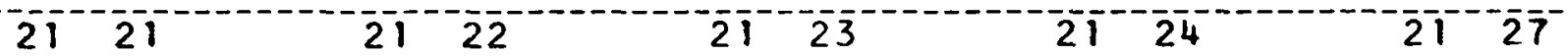

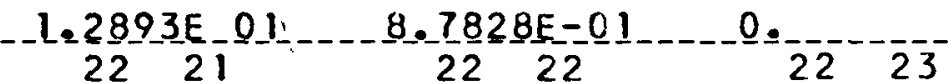

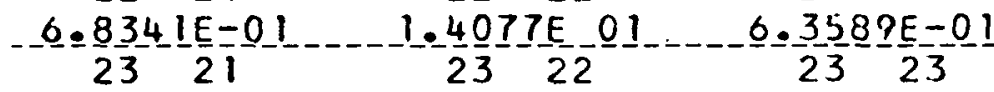

$1.4131 E-01$

24.23

0.

0

$4.3900 E-07$

$23-24-2327$

$24-21$

$-0$.

$24-6-16-00$

$6.7058 E-06$

$27 \frac{12}{22}$

o.

$4 \cdot 1821 \mathrm{E}-00$

2723

$1.9924 E-01$

$5.0437 \mathrm{E}-01$

24,24

$1.0513 \frac{3}{24}-02$

$=\frac{0}{2} \frac{642 E}{2}-\frac{0}{24}$

1. $-7417 E-00^{\circ}$

-

4. $1413 \mathrm{E} 00$

$1.2899 \mathrm{E} 01$
IEMPERAIURE

DEG. F.

$3.0000 \bar{E}-0 \overline{3}$
SIGMA

SCATTER
SIGMA TRANSPORT
SIGMA

ABSORPTION
NU SIGMA

FISSTION

$1.7123 \mathrm{E}^{\circ} \mathrm{O}$

SIGMA

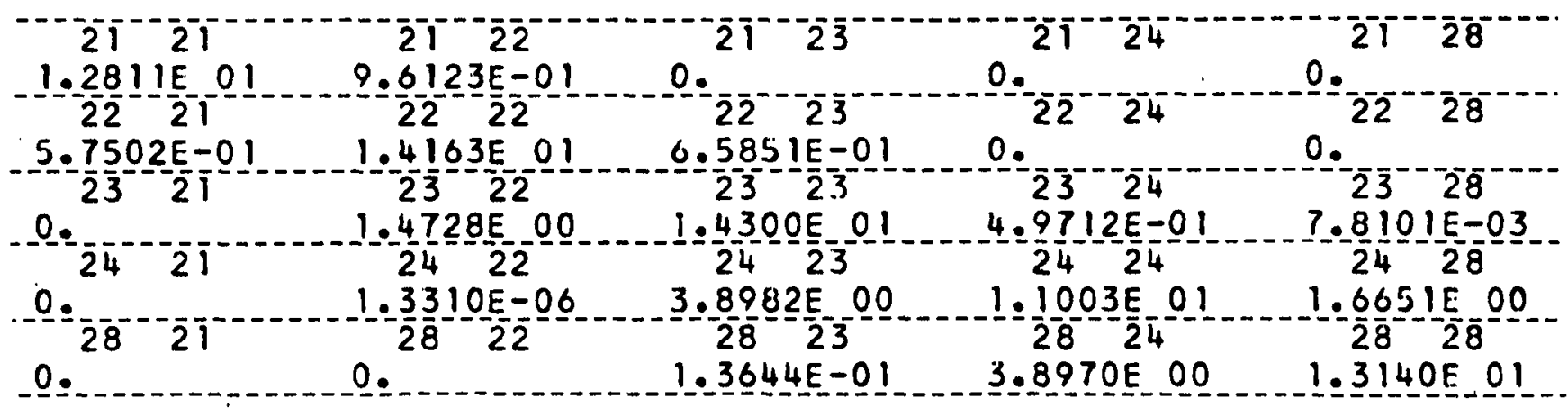


URANIUM 234

THERMAL TRANSAER MATTRICES

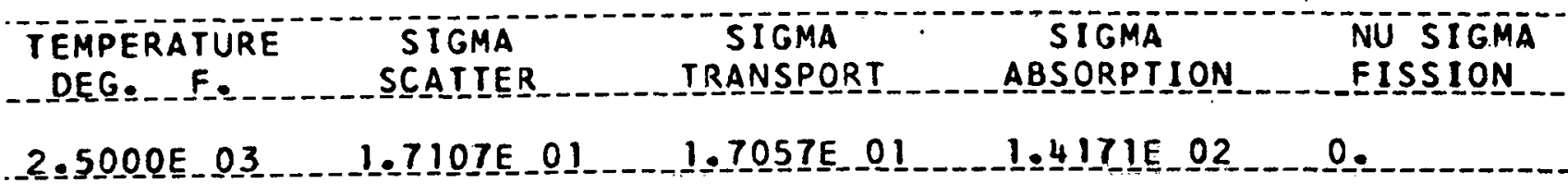

SIGMA_S (NIIN2)

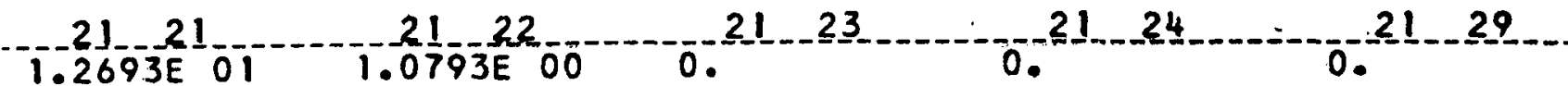

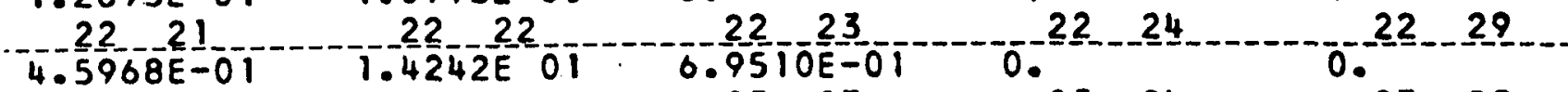

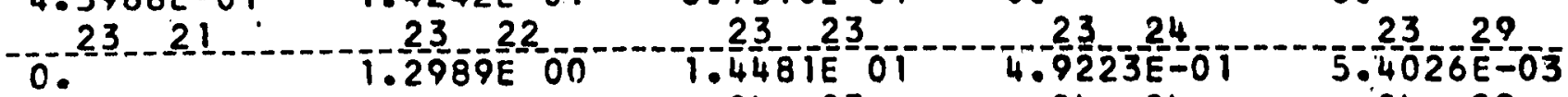

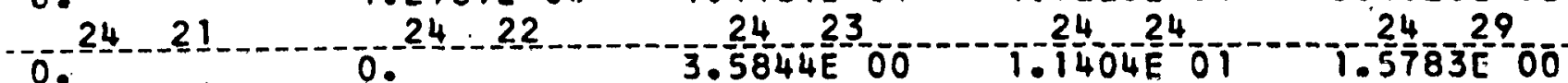

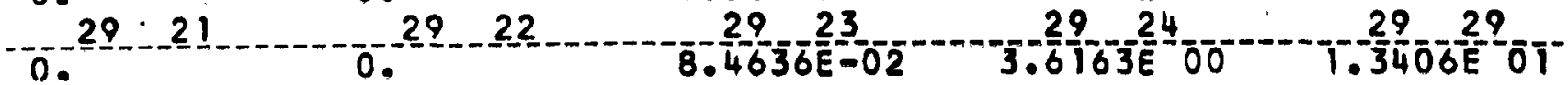

TEMPERATURE
STAMA

$$
\begin{gathered}
N 1 \\
\text { SI GMA_S_EI }
\end{gathered}
$$

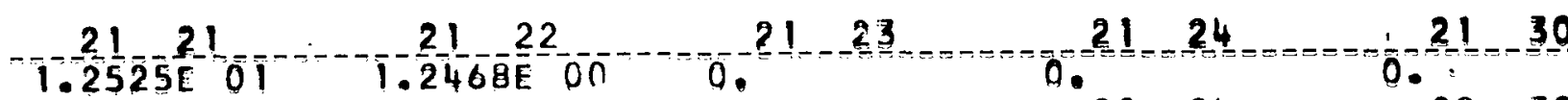

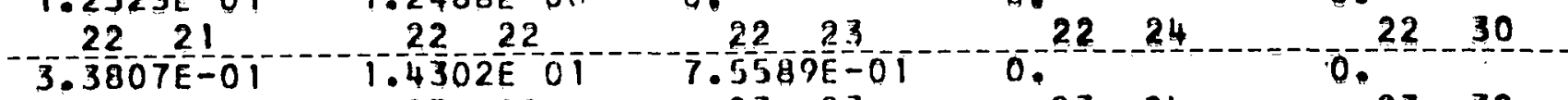

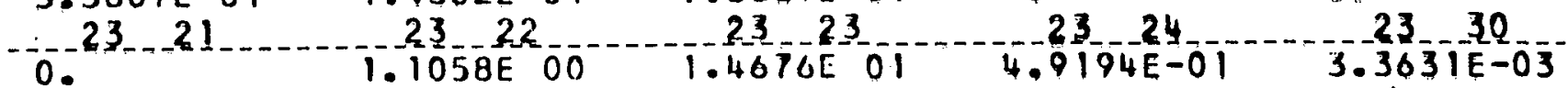

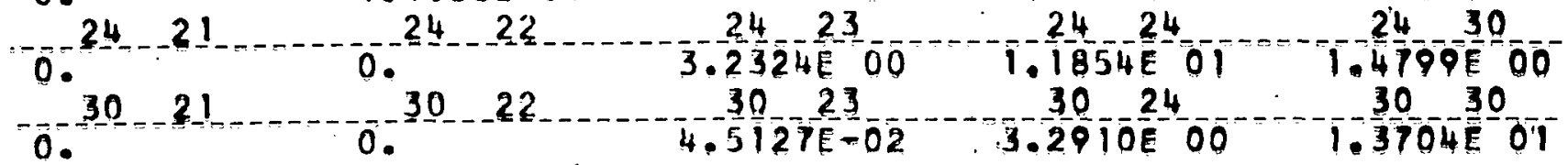


URANI UM 234

IHERMAL_IRANSFER MAIRICES

IEMPERAIURE

DEG. $F$.
SIGMA

SCATTER
SIGMA TRANSPORT
SIGMA ABSORPTION
NU_ SIGMA

FISSION

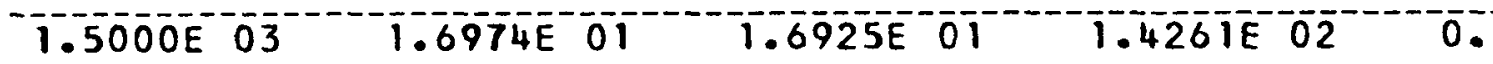

$\operatorname{SIGMA}$

$N \frac{1}{4}-$

N2

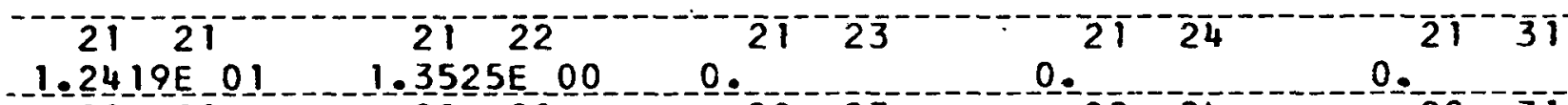

22

2222

2223

$22-24$

$22-3 i$

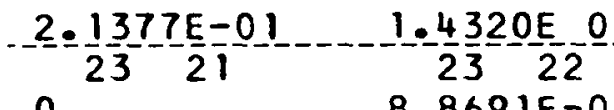

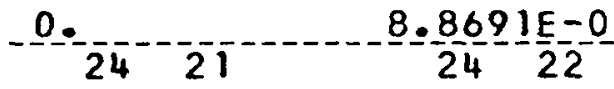

ㅇ.- $3 \overline{1}-\overline{2}$

$0 .-\frac{1}{3} \overline{1}$

8. $=\frac{62}{23} \frac{9}{2}-\frac{E}{2}-01$

ㅇ.

$23-\overline{24}$

․․․

o.

$1.4 \frac{8}{2} \frac{87 \mathrm{E}}{2} \mathrm{O}-1$

$5.0141 E-01$

$1 \cdot 7587 \mathrm{E}-03$

2.8296 E 00 3723

$1.2368 \mathrm{E}$ 이

2431

$1.8689 E=02$

$2.9083 \mathrm{E} 00$

1. $-\frac{36}{3} \frac{83 E}{3}-\frac{00}{1}$

$1.4047 \mathrm{E} \quad 01$

IEMPERAIURE

DEG. F.

SI GMA

SIGMA

SCATTER

TRANSPORT

SIGMA

NU SICMA

$1.0000 \bar{E} 0 \overline{3}$

$1.6908 \mathrm{E} 01$

$1.6859 \mathrm{E}$ OO

$1.4354 \mathrm{E} 02$

0

\section{SIGMA $\left.S(N) \frac{N}{N}\right)$}

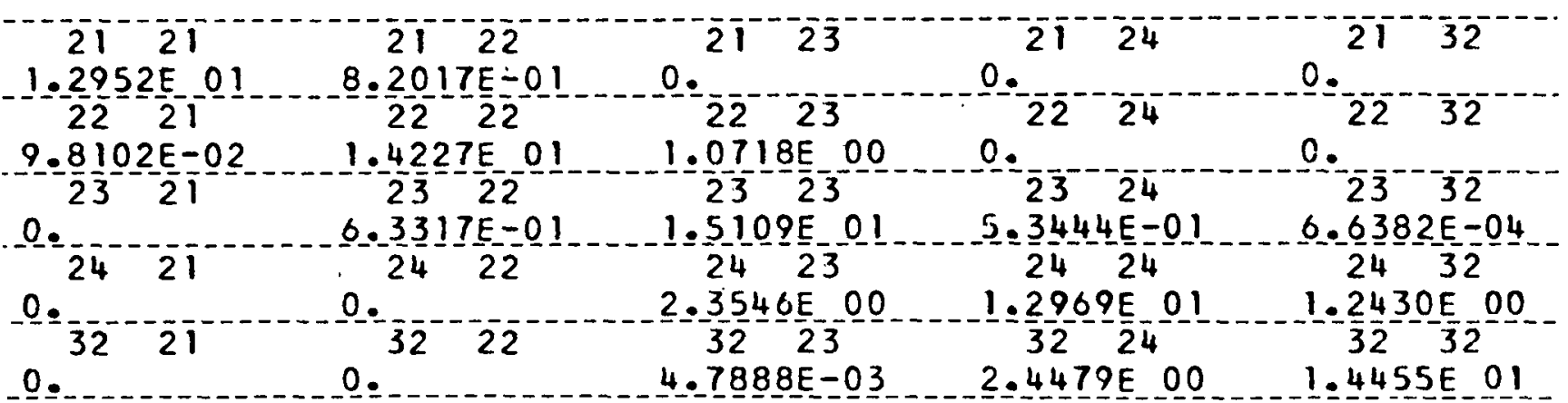


URANLUM 234

THERMAL TRANSFER MATRICES

TEMPERATURE
DEG SIGMA

$---21--21$.

$1.3305 E 01$

$1.2151 E-01$

- $23.2 \mathrm{~L}$

$0.24-21$

o.
$21 \ldots 22$

$4.6728 \mathrm{E}-01$ 22

1.3811 OI $23-22$

$3.3604 E-01$

$0.24 \ldots 22$

0.

$33 \ldots 22$ 0.
$22-23$
O. $21-23$

$1.4638 \mathrm{E} 00$

2]...24

0.

22

0 .

$23-23 \ldots-23-24$

$1.5300 \mathrm{O} O \mathrm{~T}$

24.23

$1.7628 \mathrm{E} 00^{\circ}$

$3.9191 \mathrm{E}-04$
$24-24$

$1.3694 \mathrm{E} T$

$1,86888 \mathrm{E}$ 00
$21-33$

0 . $22 \ldots 33 \ldots$ $23-33$ 23
$.1568 \mathrm{E}-04$ 1. 109 IE $1.4972 \mathrm{E}$ 01

\section{TEMPERATURE} . DEG. F. F. G.8000E OL... J $6284 E$ OL

\section{SIGMA} SCAT_LEB.
SIGMA JRANSPART
SIGMA
ABSOBRJION

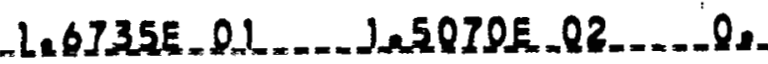

NU SIGMA

EISSLLN

\section{Ni \\ SIGMA.S._NILNRL}

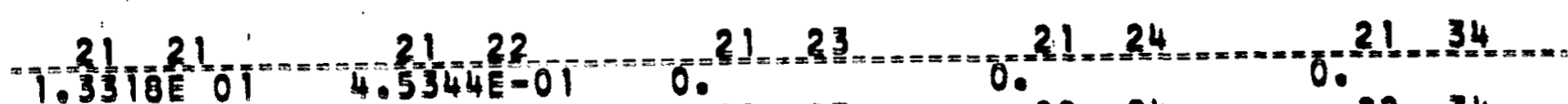

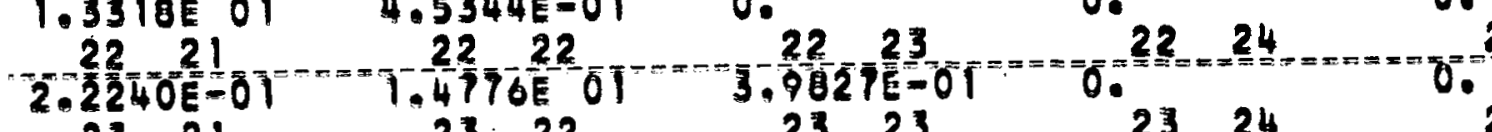

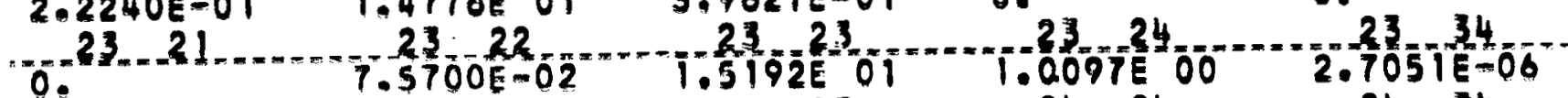

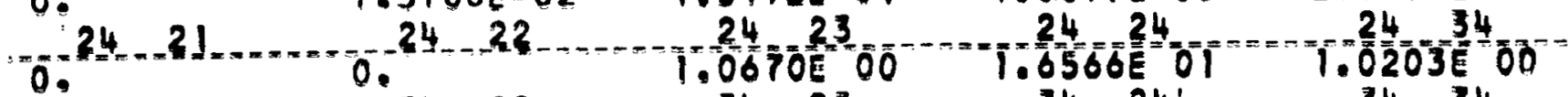

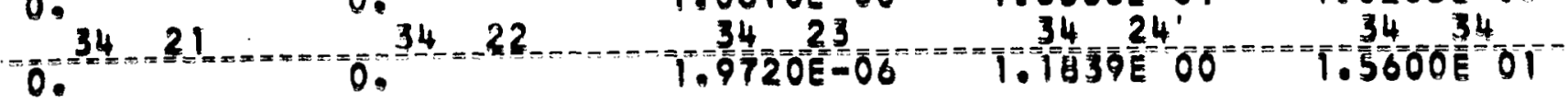


URANLUM_234

TRANSFERS FROM GROUPS I THROUGH 10 TO GROUPS I THROUGH 16

$\mathrm{N} 1$

SIGMA SO (NIIN2)

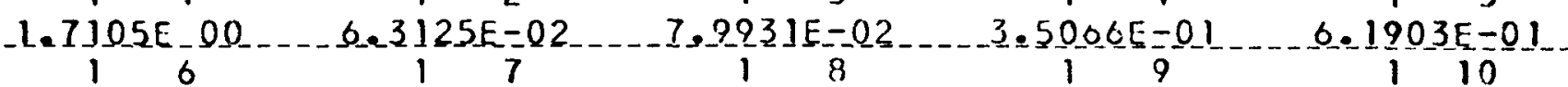

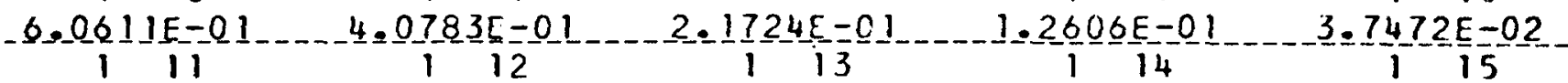
$-5.6070 E=03 \quad-7.8341 E=04-1.0899 E=04-1.4522 E-05-2=1229 E-06$ $1.4 \frac{8}{2} 68 \mathrm{E}-07 \quad 0$.

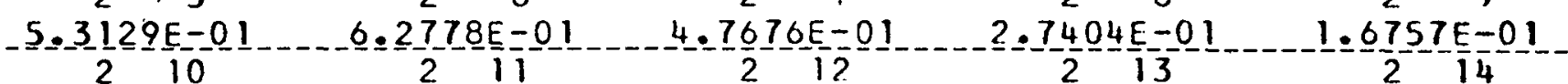

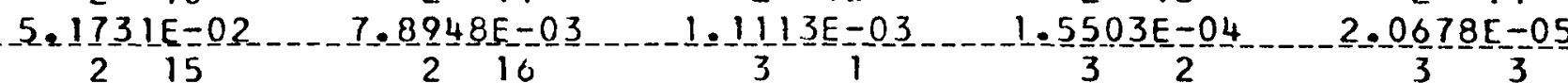
$3.0238 E=06 \quad 2.1181 E-07 \quad 0$ _

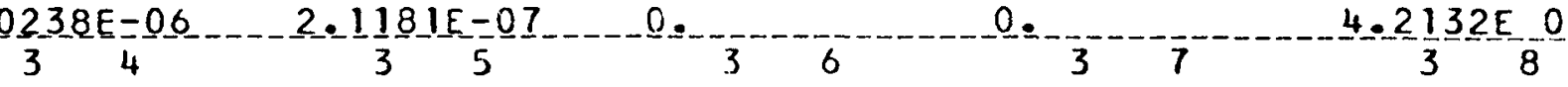
$1.4 \frac{1}{3} 25 \mathrm{E}-01$ $2=1546 E-01 \quad 7.0691 E-02 \ldots 1.1135 E-02 \quad 1.5864 E-03-2.226 E-04$ $2.9696 E-05$ -0. -3. $3010 E-01 \quad 2.4423 E-01 \quad-8.6466 E-02 \quad 1.4173 E-02 \quad 2.0496 E-03$ $-2=\frac{8}{5} 74 \mathrm{E}=04-3.8660 \mathrm{E}-05$

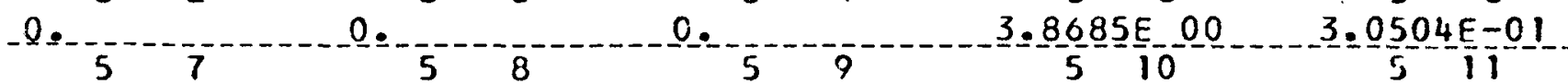
$3.27 \frac{8}{5} 12 \mathrm{E}-01$ $2.5617 E-03 \quad-3.6348 E-04 \quad-4.8805 E-05 \quad 7.1541 E-06-5.0160 E-07$

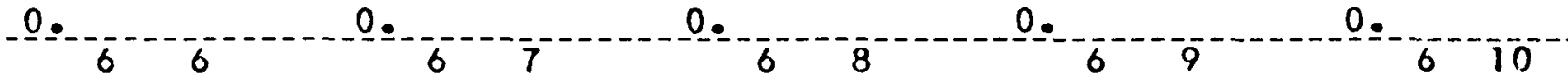
$4.7148 E-00 \quad 3.0161 E-01 \quad 2.4572 E-01 \quad 2.4820 E-01 \quad 1.0978[-01$ $2.0220 E-02 \quad 3.0574 E-03 \quad 4.3775 E-04 \quad 5.8983 E-05 \quad 8.6570 E-06$ 6. $0728 E-07$ $\underline{0 .}$ 0. 0. 
URANIUM $234^{-}$

N1 N2

SIGMA SO (N1_N2)

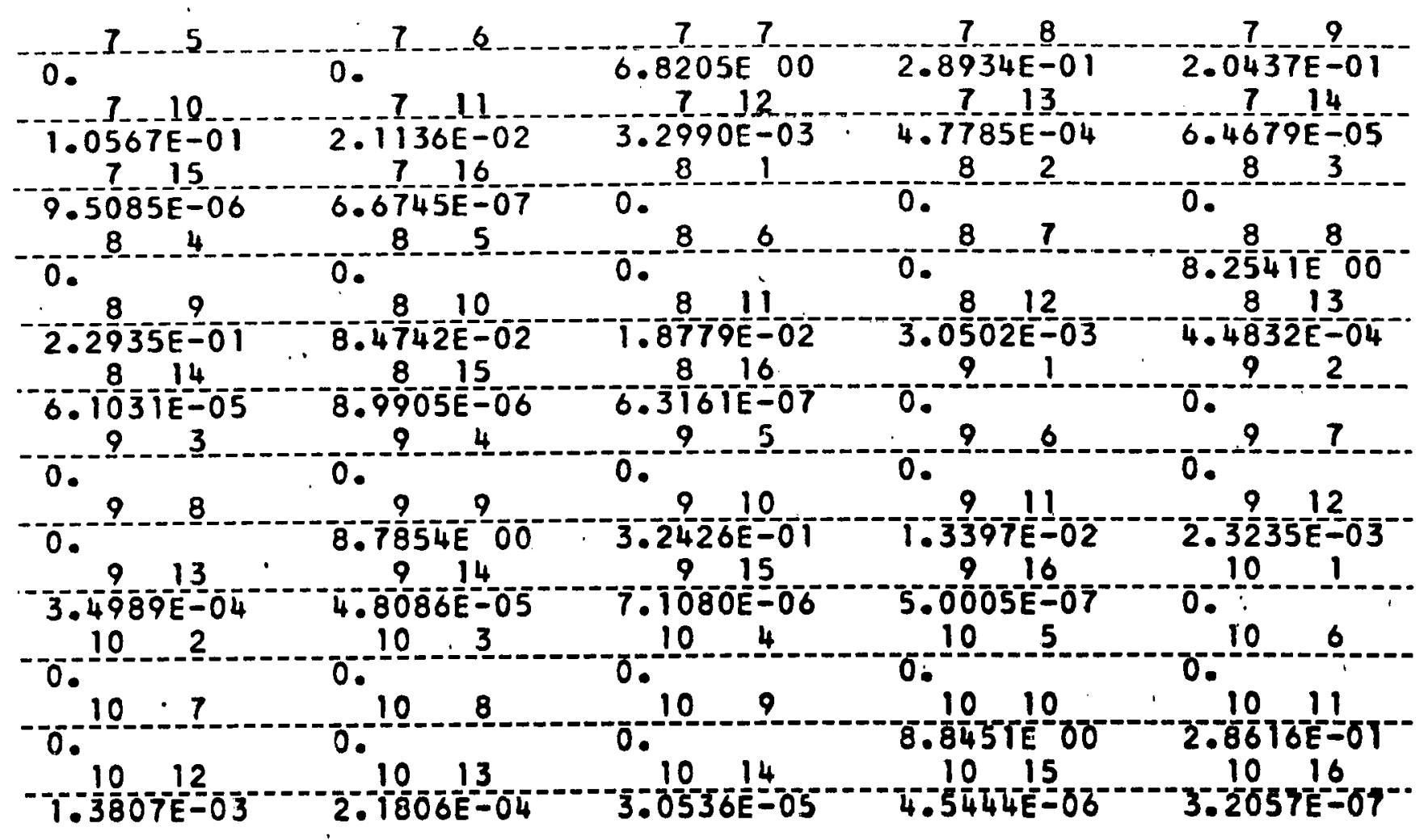


URAN I UM 234

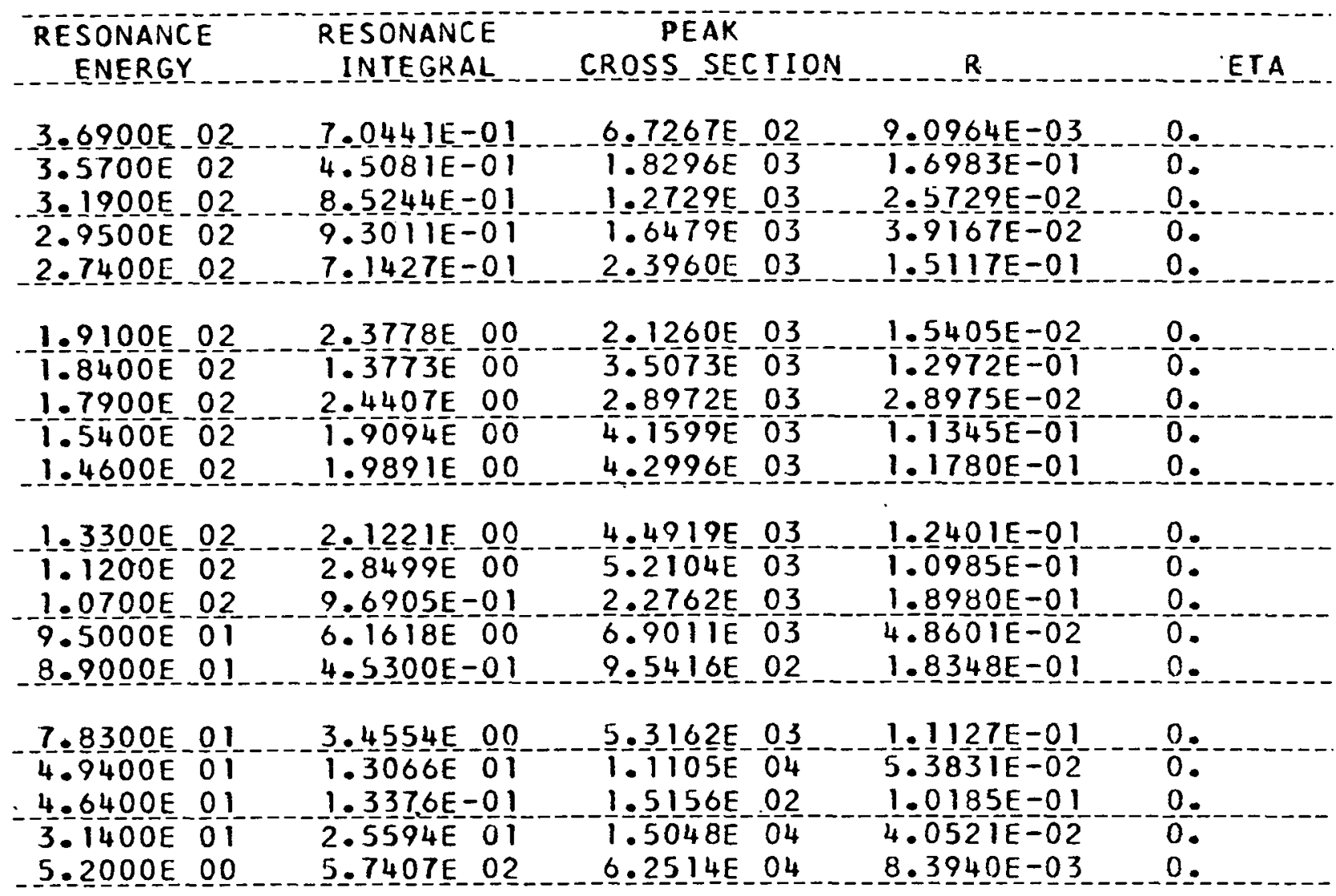


DESIGNAIION URANIUM 236
CODE NO. 92.2360
DENSITY FACIOR $3 . ? 1920$ E 02

\section{SIGMA}

SCAIIER
XI SIGMA

SI GMA

SIGMA SCAITER

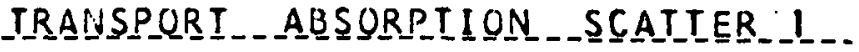

$1-1.000 E-0]-3.7484 E-00-5=0724 E-00-2.3311 E-002-2349 E-00-1-3191 E 00$ $26.065 \mathrm{E} \quad 06 \quad 6.0313 \mathrm{E} 00 \quad 5.6818 \mathrm{E} \quad 00.3 .0703 \mathrm{E}$ ON $1.1493 \mathrm{E}$ 00 $-2.7562 \mathrm{E}$ OO

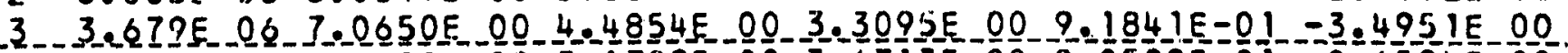

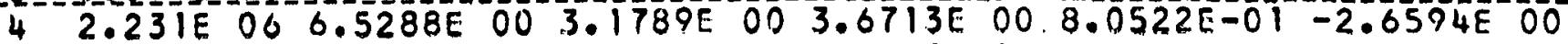

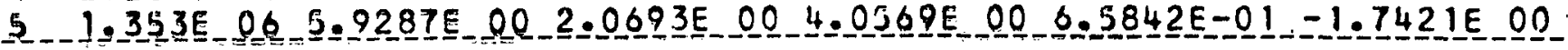

6 6.208E 05 -6.7007E $001.3511 E$ E $004=9149 E-0022.9473 E=01-1.7178 E 00$

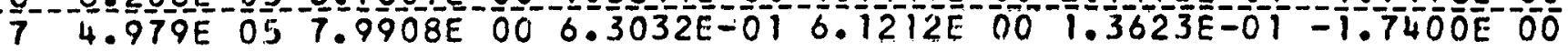

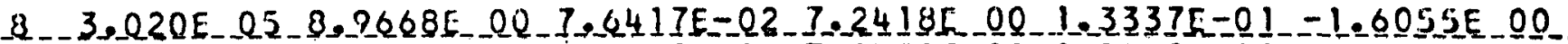
9 1.832E 05 9.1865E $007.8289 E-02$ 7.9452E 00 1.9862E-0 1 $-1.1552 E$ OD

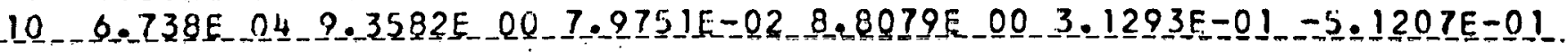

- $11.2 .479 E-04-9.3638 E-007.9799 E-02-9.3373 E-00-4=6156 E-01-2.4652 E=02$ 12 9. TIOE $039.6258 \mathrm{E}$ 00 $8.2032 \mathrm{E}-02$ 9.5984E $006.3481 \mathrm{E}-0 \mathrm{1}-2.5521 \mathrm{E}-02$

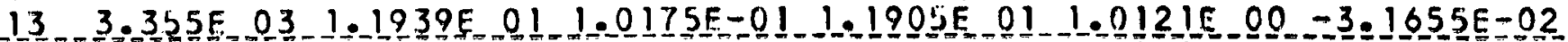

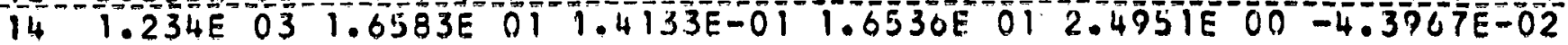
$15.4 .540 E$ 02 3.4623E 01 2.9506E-01 3.4524E 01 6.4670E 00 =9.1794E-02.

$16 \quad 8.719 E-015.2610 E 014.4835 E-015.2400 E 012.8172 E 01=1.3948 E-01$

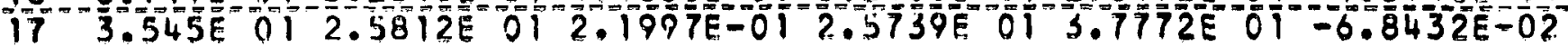
18 1.304E 01 9.2477E $007.8810 E-02.9 .2214 E 00.2 .1809 E=01-2.4518 E-02$

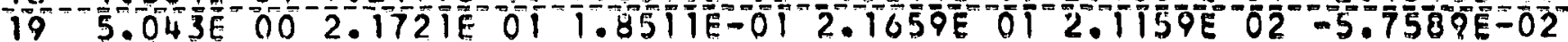

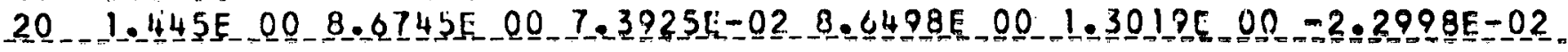

$21.6286 E=0109.10105007 .7560 E-02.9 .0750 E-00-1.7002 E-00=2.412 ? E=02$

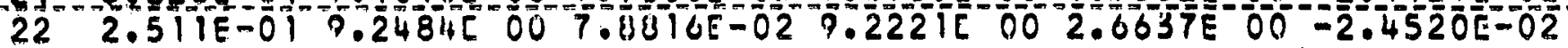
$23-9.237 E-02-9.3106 E 007.9346 E=02=2.2041 E-004.3600 E-00-2.4685 E-02$

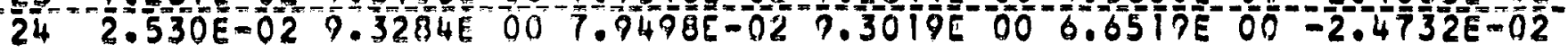

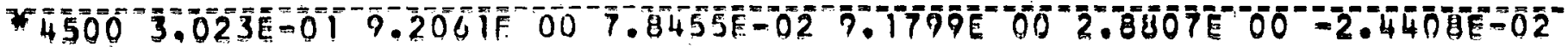
- $40002.718 E-019.20615-007.8455 E-02$ - $1799 E 002.8107 E-00-2.4408 E=02$

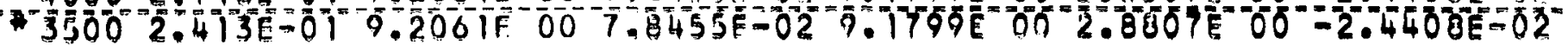
-3000 2.109E-01 ?.2001E 007.8455E-02 9.1799E 002.8807E 00-2.4408E-0?

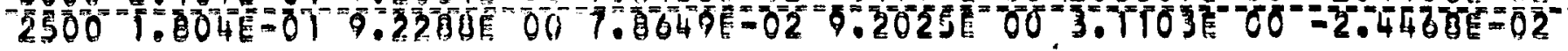

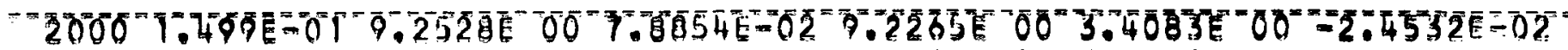

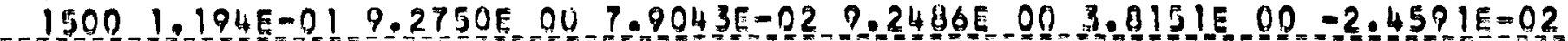

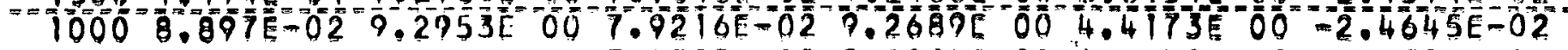
$=500.5 .850 E-029.3164 \mathrm{C} 007.9395 \mathrm{E}-02-9.2898 \mathrm{E}=00 \quad 5.4441 \mathrm{E}=00=2.4700 \mathrm{E}-02$

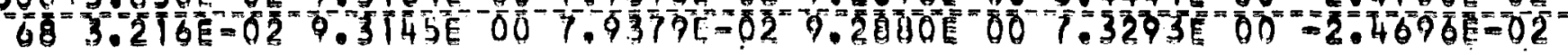

Thormal oross nections takon from XDC 50-11-72.

NOTE: For oumary of the oources of date see APEX-704. 


DESIGNAIION

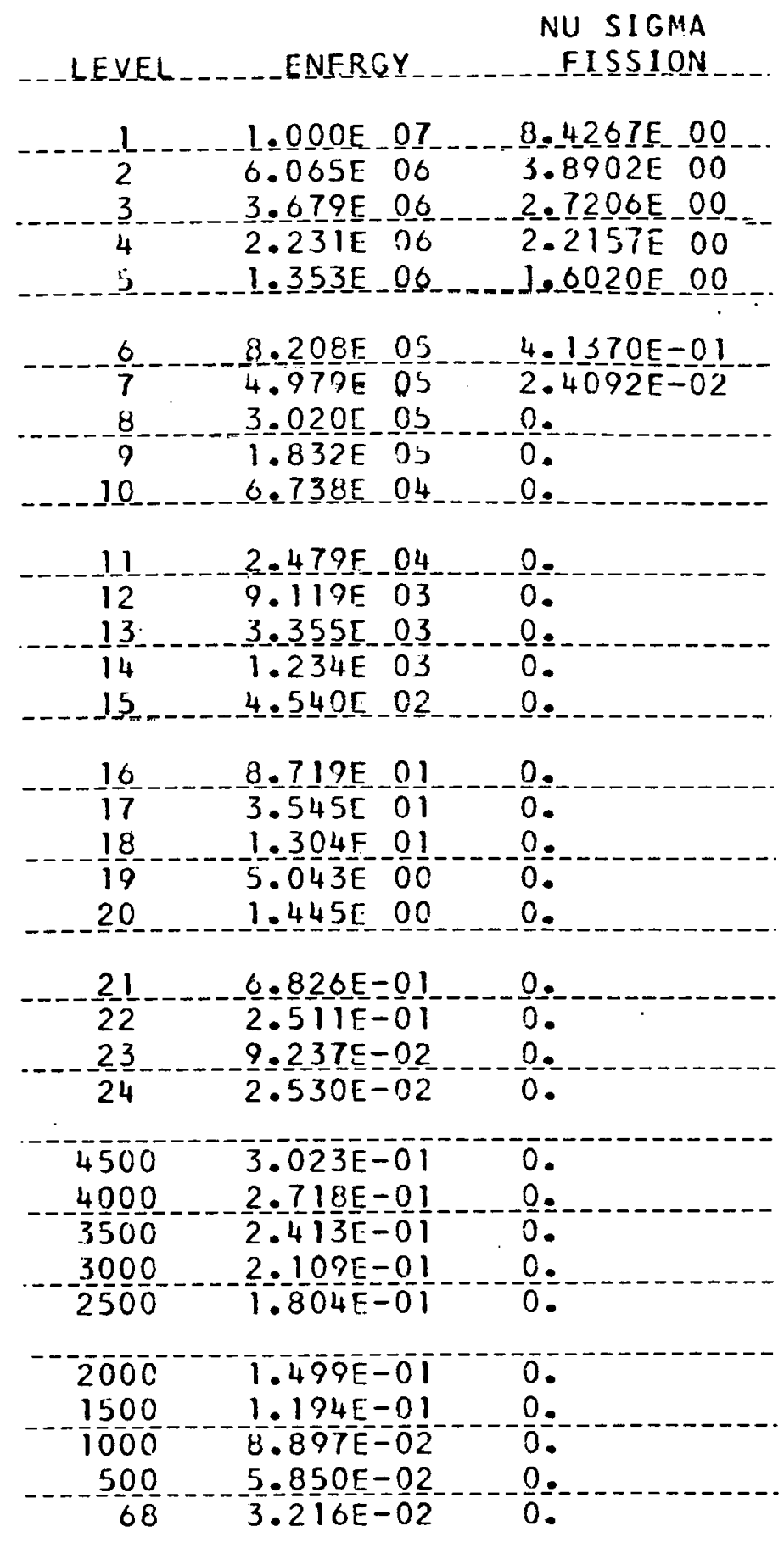


URANIUM 236

IHERMAL_IRANSFER_MAIRICES

- IEUPERAIURE DEG. $F$.

SIGMA

SIGMA TRANSPORT

SIGMA

NU SIGMA.

SCATTER

ABSORPTION

FISSION

$4.5000 \bar{O}$

$9.6707 \bar{E} 00$

$9 . \overline{4} \overline{36 E} \overline{0}$

$\overline{9} . \overline{40} \overline{0} \overline{6} \overline{\mathrm{O}} \overline{\mathrm{O}} \mathrm{O}$

$\overline{0}$.

\section{$S\left(G M \frac{N}{1}-\bar{S}-\left(N \frac{N}{1}-N 2\right)\right.$}

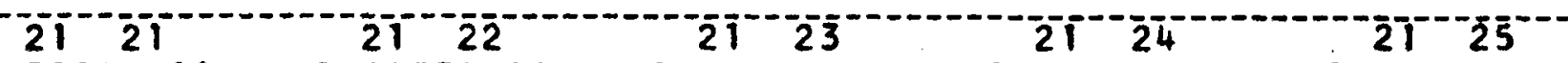

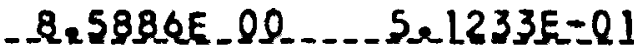

$22 \quad 2122$

$22-23$

$Q_{2}-2 \frac{2}{24}$

$22-15$

$-4.8857 E=9$.

$8.4 \frac{1}{3}-45-00$

$-\frac{4}{23}-\frac{2}{2}=\frac{5}{3}=0$

$1.6080 \mathrm{E}=06$

$2 \overline{3}^{--}-\overline{25}-\cdots$

$-9 .-25$

$1.06235=00$

$7.9 \frac{94975}{24}=0$

$2.8 \frac{89}{24}-\frac{15}{24}=01$

9. $-\frac{0719 E-03}{24}=03$.

$-0.25-3 i \frac{5273 E-05}{25}$

2.5972E 00

5.693 IE 00

I. $\frac{03825}{25}$ - 00

으.

0.

$1.9237 E-01$

$2.5236 E$ OO

6.9547E. 00

TEMPERAIURE - SIGMA
DEG.

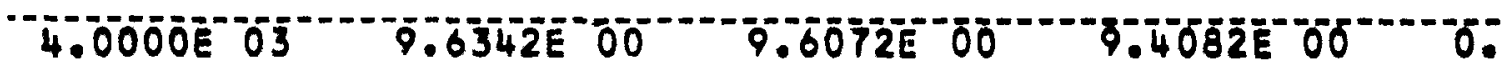

\section{SIGMA}

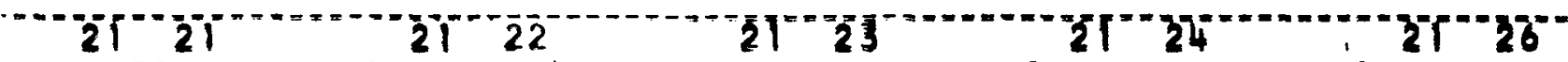

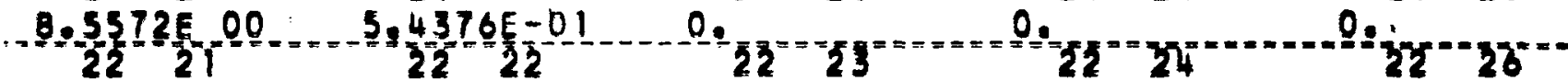

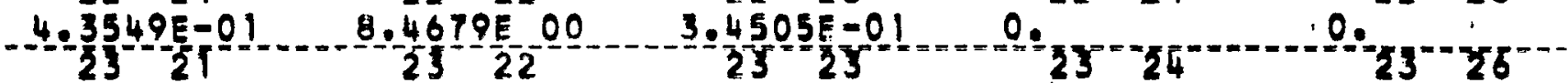

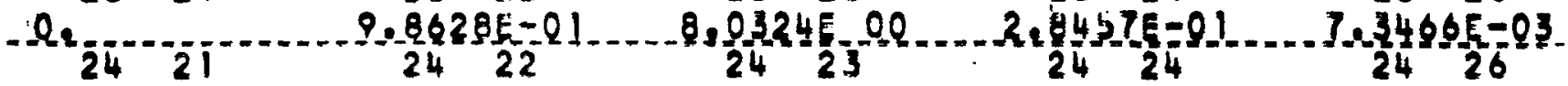

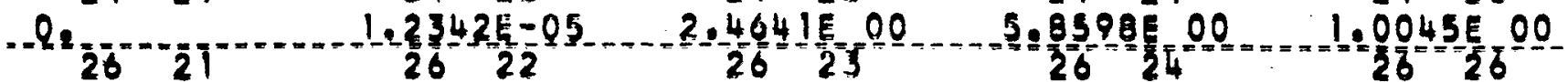

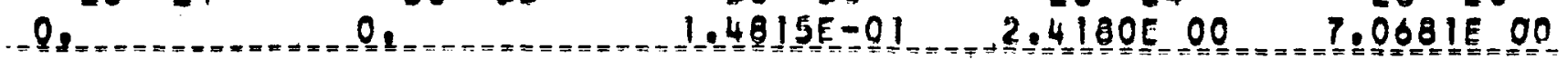


URANIUM 236

\section{THERMAL TRANSFER MATRICES}

SEMPERATURE
SIGMA

Ni

SI GMA S IN1/N2I)

\begin{tabular}{|c|c|c|c|c|}
\hline $5153 E 0$ & $5.8568 E-01$ & 0 & 0 & 0 \\
\hline 22 & $\begin{array}{l}0.85082-22 \\
22\end{array}$ & 22 & $22 \quad 24$ & $22 \quad 27$ \\
\hline $\begin{array}{l}3.9055 \mathrm{E}-01 \\
23 \quad 21\end{array}$ & $\begin{array}{c}8.5165 E 00 \\
2322\end{array}$ & $\begin{array}{c}3.5282 \mathrm{E}-01 \\
2323\end{array}$ & & 0. \\
\hline & $9.0484 E-01$ & $8.1202 \mathrm{E} 00$ & $2.7987 \mathrm{E}-01$ & $5.7293 E-03$ \\
\hline $2 ?$ & $\begin{array}{l}3.3990 E-06 \\
02 \\
0\end{array}$ & $\begin{array}{l}2.3199 \mathrm{E} 00 \\
27 \\
-0851 \mathrm{~F}-01\end{array}$ & $\begin{array}{c}6.0418 E \\
2 \frac{27}{2} 20\end{array}$ & $\begin{array}{c}9.6666 \mathrm{E}-01 \\
27 \quad 27\end{array}$ \\
\hline
\end{tabular}

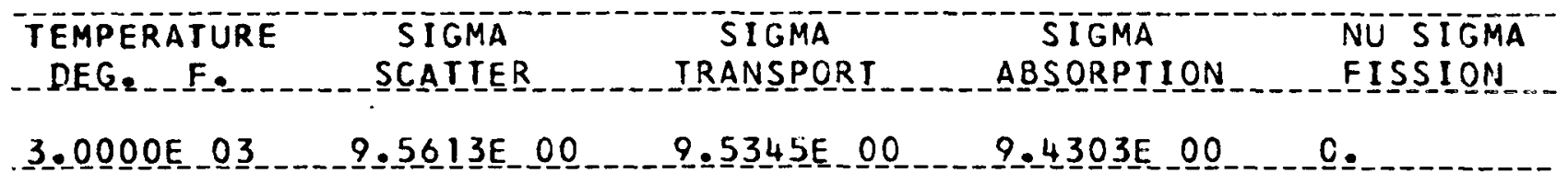

SIGMA S (NIIN2)

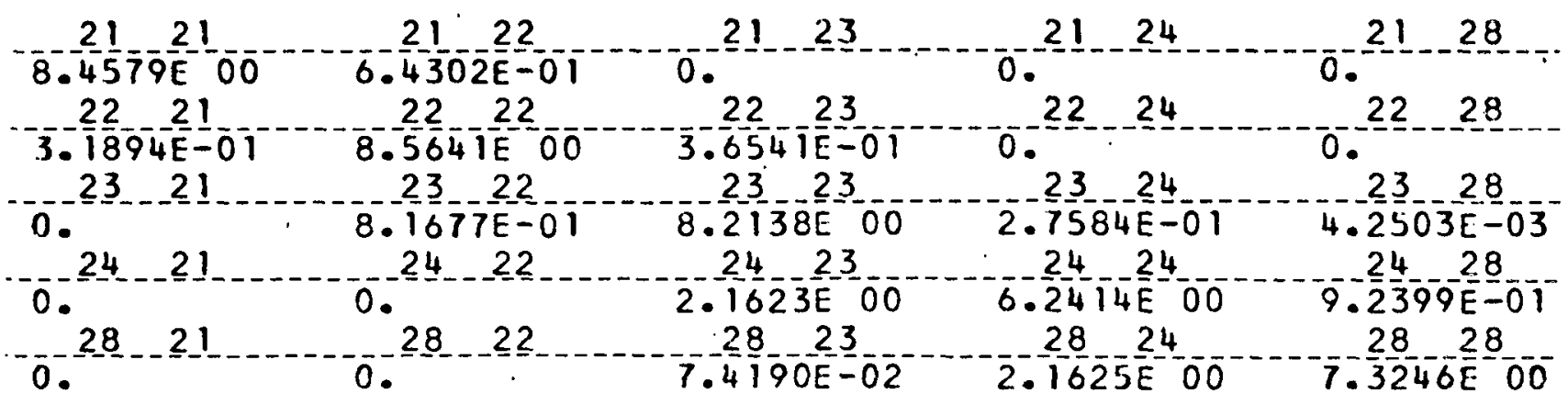


URANIUM 236

_HERMAL_IRANSFER_MAIRICES -

TEMPERATURE
DEG. F. SIGMA
SCATTER

$2.5000 \mathrm{E} 03$ 9.5247E 00 $9.4980 \mathrm{E}$ 00 $9.4470 \mathrm{E} 00^{-0}$

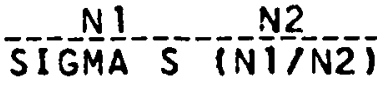

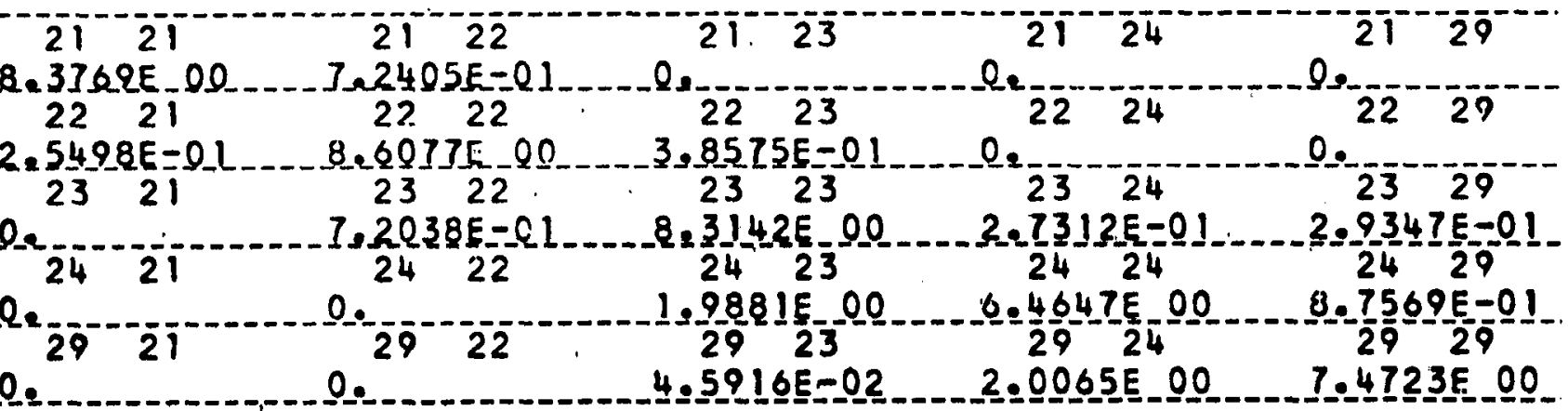

IEMPERAIURE DEG. F. $2.0000 \overline{0}-\overline{3}$
SIGMA SCA TTER
SLGMA TRANSPORT
SICMA ABSORPTION
NU_SIGMA. FISSION
$9.4881 \mathrm{E} 00$
$9.46750^{\circ}$
$9.470 \mathrm{BE}^{-} \mathrm{O} O \bar{O}$
0 .

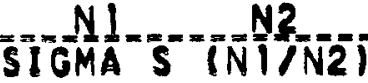

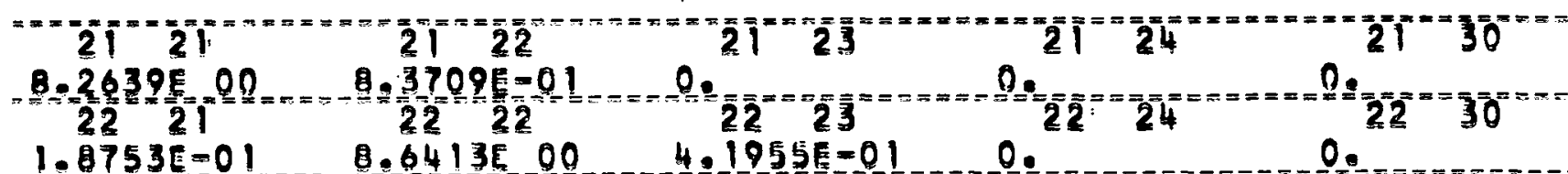

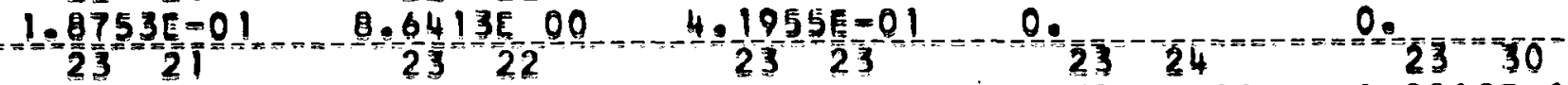

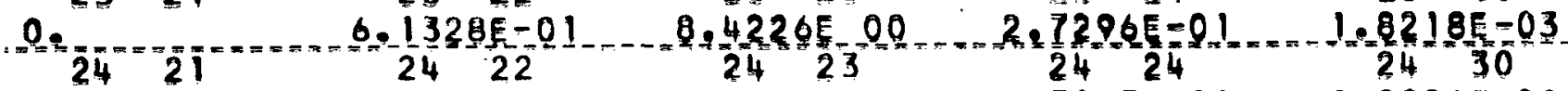

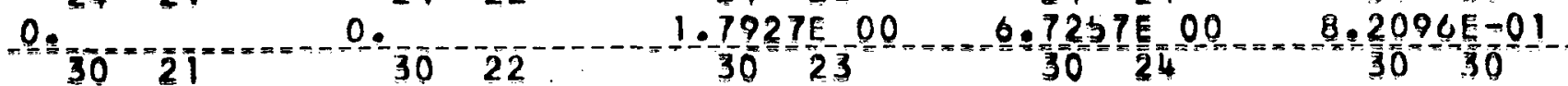
으. 
URANIUM_236

THERMAL TRANSFER MATRICES

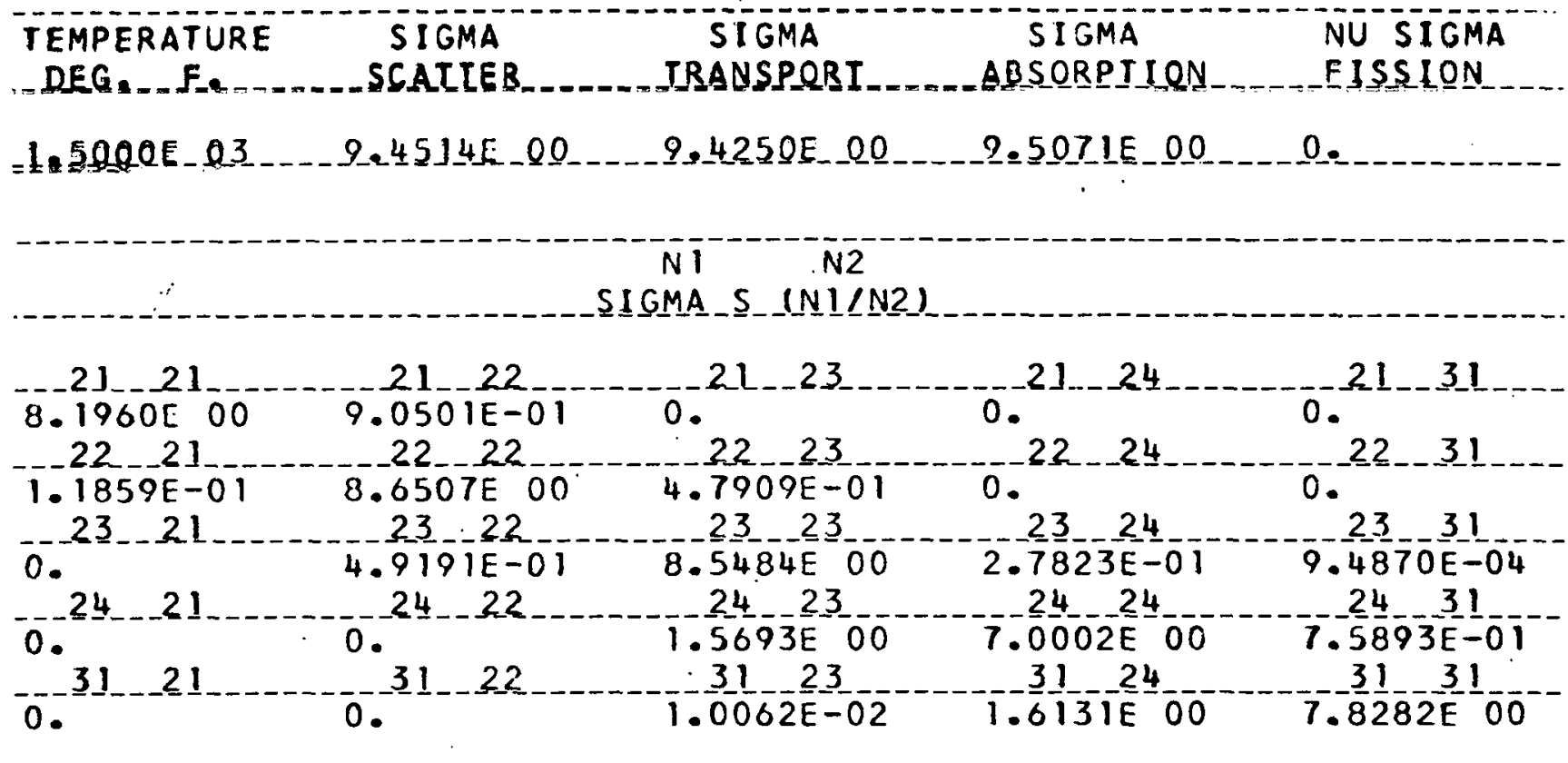

TEMPERATURE

DEG _ F.

SIGMA

1.000OE_03 _. 9.4147 E_OO
SIGMA IRANSPORI 9. $3883 E \quad 00$
SIGMA ABSORPIION 9.5694트으므.
NU SIGMA

EI SSION

\section{$\begin{array}{cc}N 1 & N 2 \\ \text { SIGMA S }(N ! I N 2)\end{array}$}

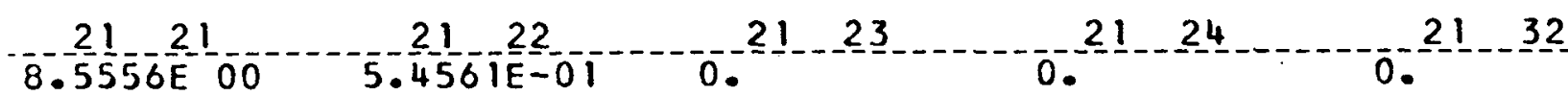
$22 \quad 21$
22
$-5 . \frac{22}{95}-\frac{2}{1}-\frac{3}{\mathrm{E}}-0$
- $2 \underline{2}-2$
$0^{-2}$
0.
3. $\frac{23}{12}-22$
$8.6625 \mathrm{E}-2$
$2.9660 \mathrm{E}-01$
$-\frac{23}{5} \frac{32}{80}-\frac{32}{0}-04$
$--24-21$
0. 24
1. $\frac{24}{30} \overline{7} \div \mathrm{2}$
$7-\frac{24}{3} 422-\frac{24}{3}-$
2432
- 32
0 . 32
$2 . \frac{3}{5} \frac{2}{9}-\frac{23}{2 E-0} \overline{3}$
$1 . \frac{32}{35} \overline{7} \div \frac{24}{0}-0$
$6.8937 \mathrm{E}=0 \mathrm{~T}$
$8.0545 \mathrm{E}-10$ 
URANIUM 236

IHERMAL IRANSFER MAIRICES.

-.LEMPERATURE SIGMA

SLGMA

SIGMA

NU_SIGMA.

DEG. F. SCATTER

TRANSPORT

ABSORPTION

FISSION

5.0000E 02 9.3780 00 9.3517E 00 9.7010E 00 0.

$\mathrm{N} 1$

,

SIGMA S (NITNZ)

2121

8.7895E 00

2221

$6.750 Z E=02$

2321

le.

2421

$332 i$

-.‥

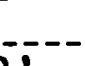

1
2122

3. $1148 \mathrm{E}=01$

2222

8.3676E 00

2322

$1.8642 E=01$

2422

$33^{22}$
$2 i^{2} 3$

$0 .-22-\frac{1}{2}$

$8.1336 \mathrm{E}=\mathrm{O} 1$

2323

$8.7682 \mathrm{E} 00$

2423

9.7757E $=01$

3323

2. $06048 \mathrm{E}=01$ 0. -

27

Q.

$23^{24}$

3. $5589 \mathrm{E}-01$

2424

7. 73 STZEE 00

3324 $1.0365 \mathrm{E}$
0.

$21-\overline{3} 3$ 2233 0. $23-33$ 6. $1188 E=05$ 2433 6. 15IIE-0! $33-33$ 8,34 I3E 00

TEMPERAIURE

DEG. $F$.

$6 . \overline{80} \bar{O} \overline{\mathrm{E}} \overline{\mathrm{O}} \mathrm{T}$
SIGMA

SCATTER
SIGMA TRANSPORT
SIGMA ABSORPTION
NU SIGMA

FISSION
$9.3463 \mathrm{E} 00$
$9.32010^{\circ}$
$1.00 \overline{7} \overline{\mathrm{O}} 0 \mathrm{~T}$
0.

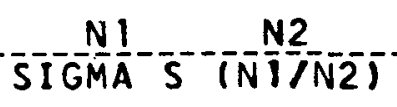

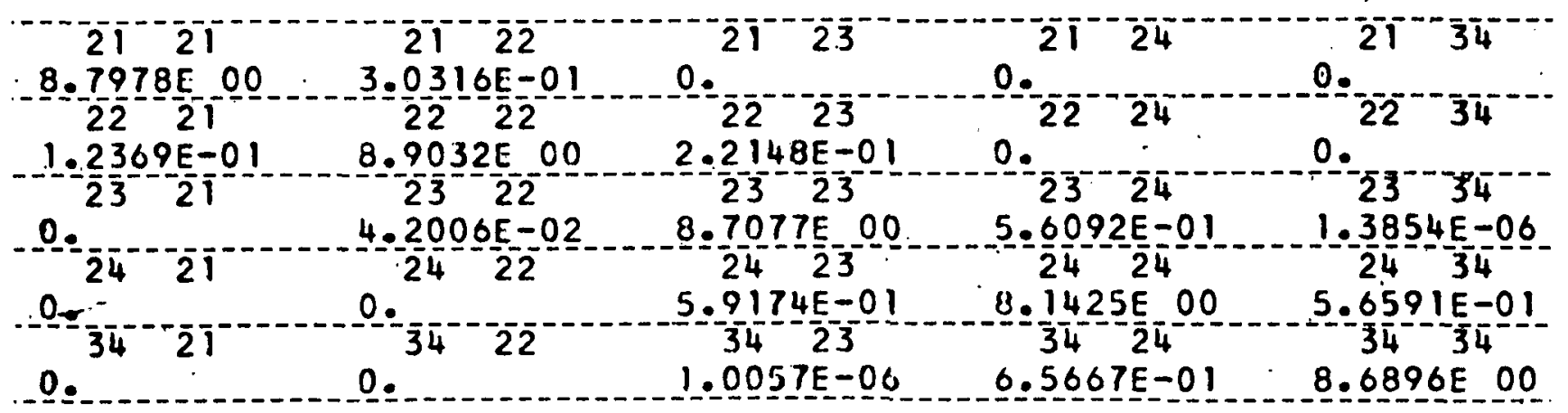


URANIUM 236

TRANSFERS FROM GROUPS I' TRROUGH 10 TO GROUPS I THROUGH 16

$\mathrm{N} 1 \quad \mathrm{N2}$

SIGMA_SO (N1/N2I

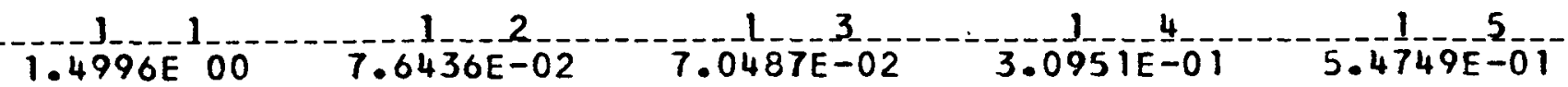

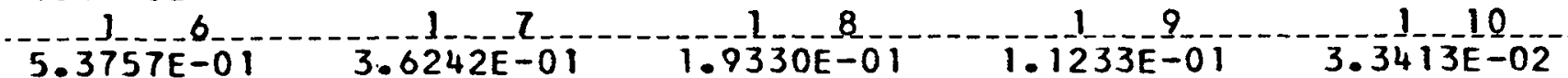
$-5.0455 E-03 \quad 7.1805 E-04 \quad 9.5597 E-05 \quad 1.3372 \frac{1}{1}-05-1.9132 E-06$

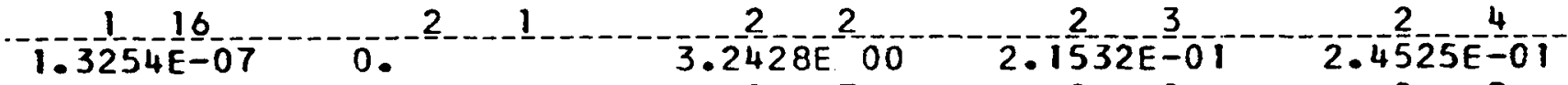

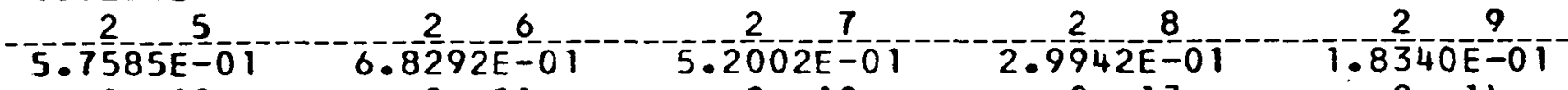

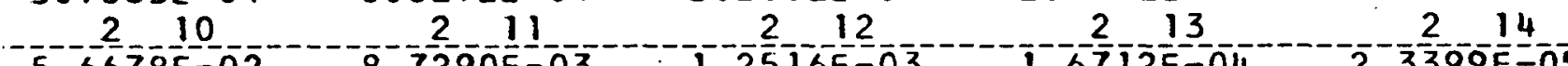
$5.6678 \mathrm{E}-02-8.7290 \mathrm{E}-03 \mathrm{3}-1.2516 \mathrm{E}-03^{-1.6712 \mathrm{E}-04} 2.3399 \mathrm{E}-05$

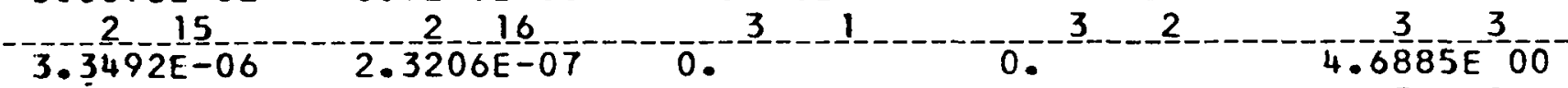
$1.7231 E-01-3.7075 E-01-5.9475 E-01-5.4995 E-01-3.5771 E-01$

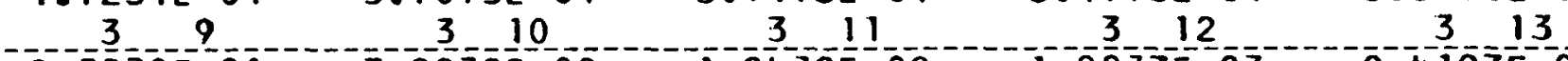

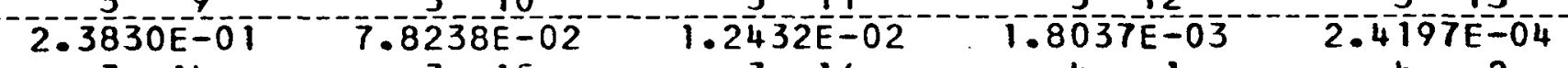

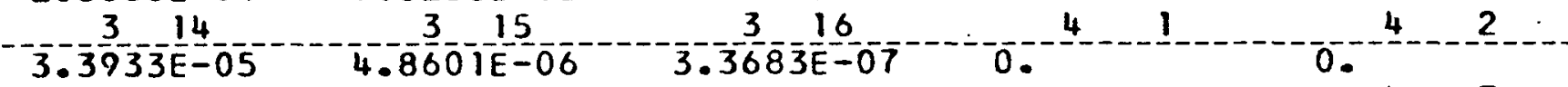

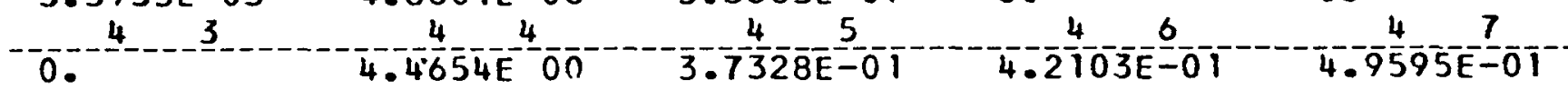

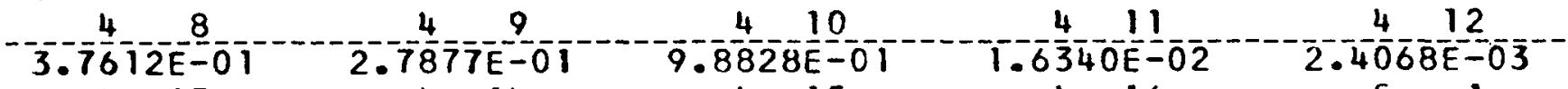

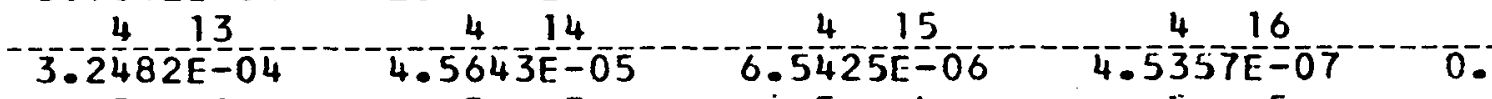
O.

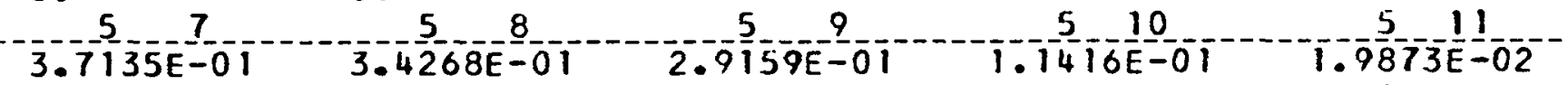

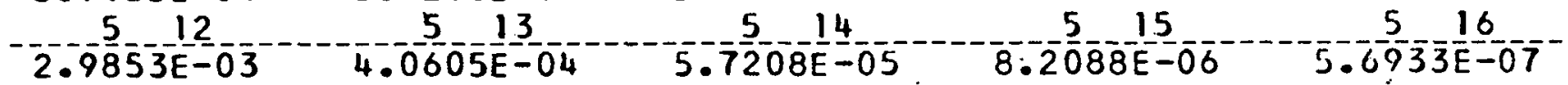

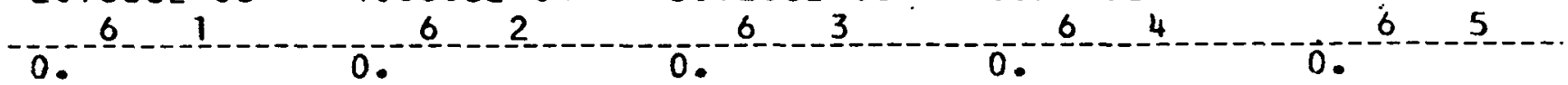

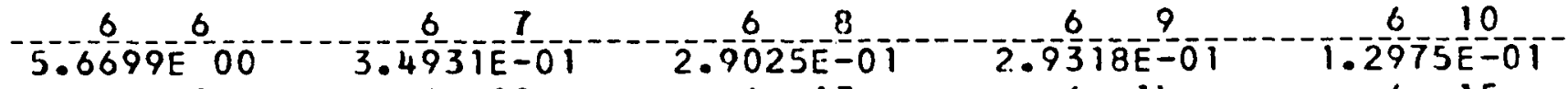

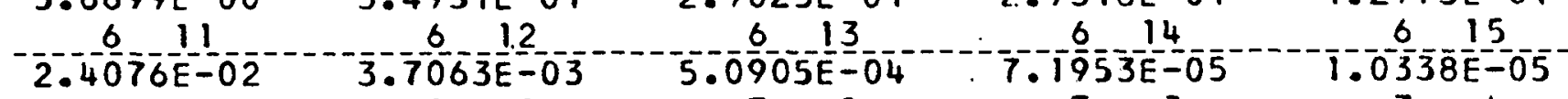

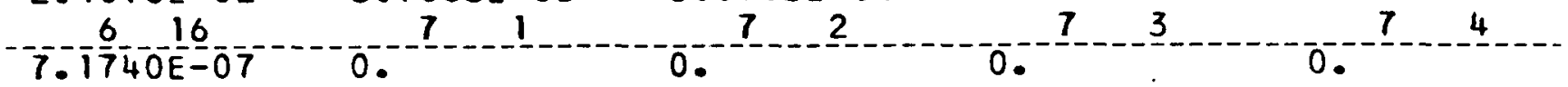


_URANLUM 236.

SIGMA SO (NIIN2)

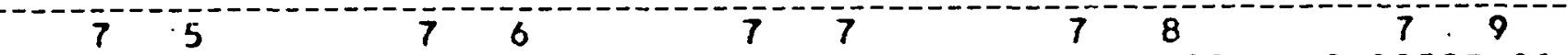
Q.

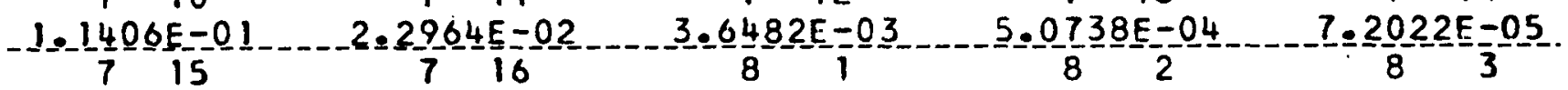

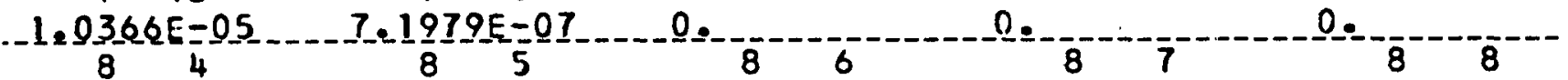

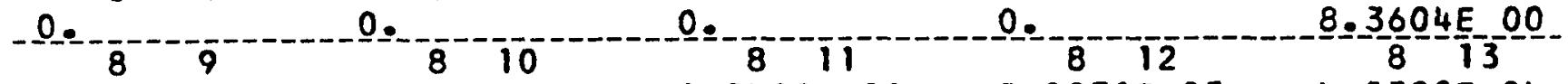

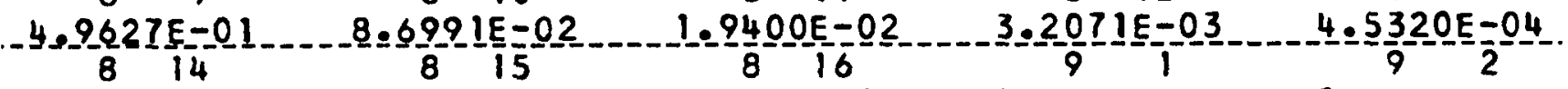

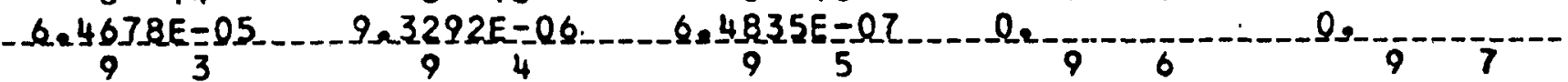

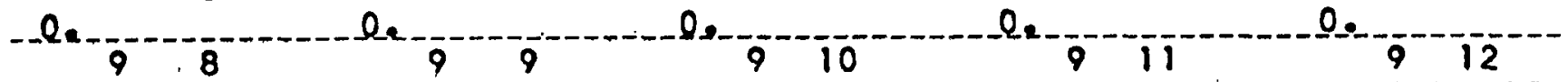

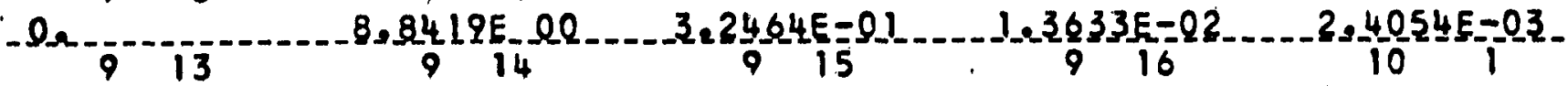

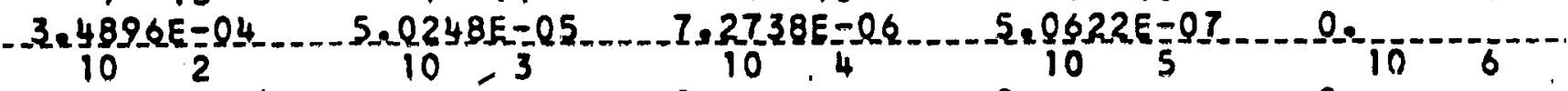
le 10 Q

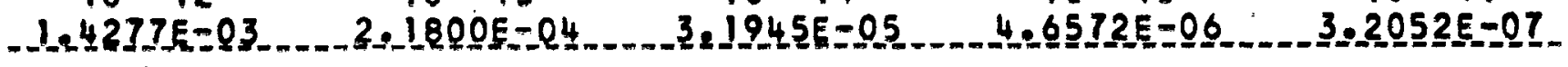


-BESONANCE RESONANCE PEAK ENERGY

INTEGRAL CROSS SECTION

$\mathrm{R}$

ETA

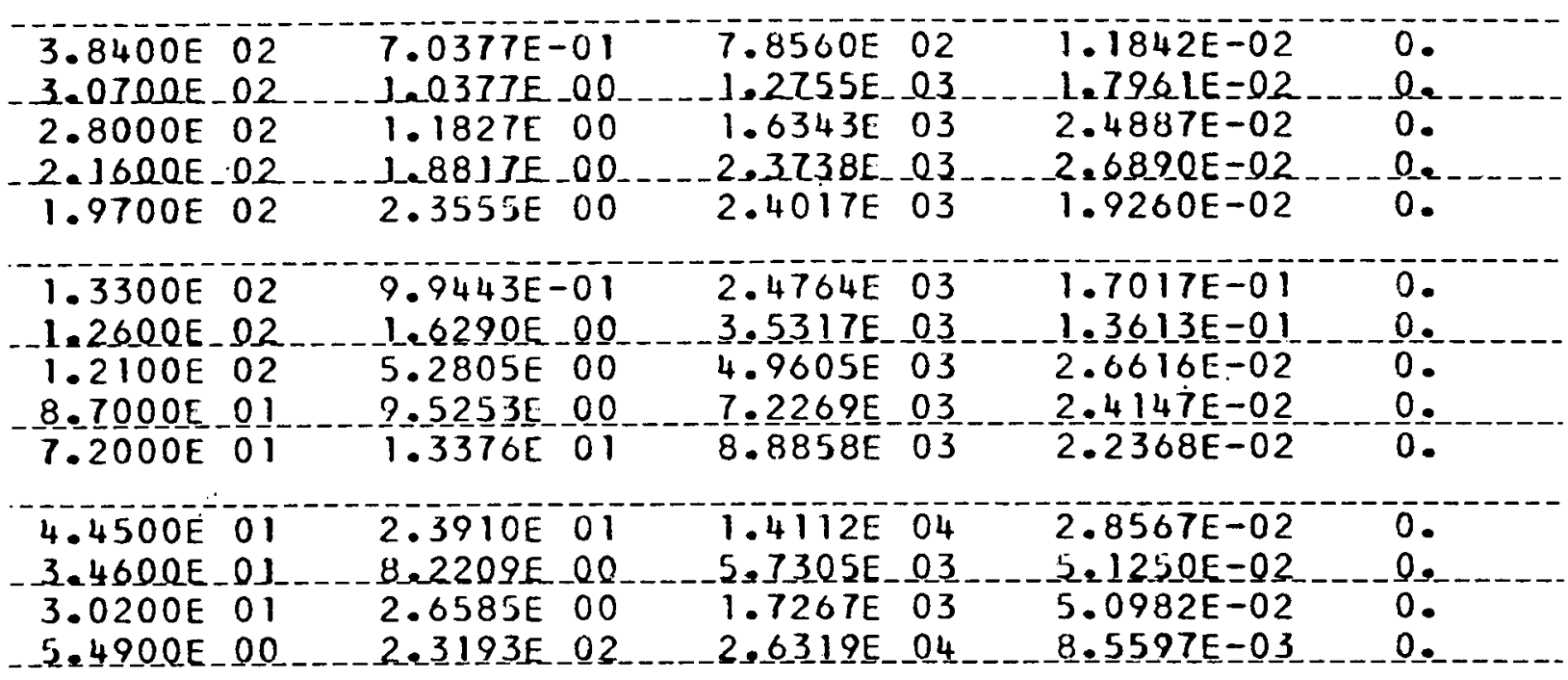


DALE $12 / 08 / 60$

DESIGNATION

CARBON
CODE NO.

6.1200
DENSITY FACTOR

$1.99230 \bar{O}$ O

SLGMA
LEVEL ENERGY SIGMA
SCATTER SIGMA
SCATTER TRANSPORT ABSORPTION SCATTER 1

$1.000 E$ OT $1.3220 E$ O0 $3.0628 E-019.2932 E-01-1.8216 \bar{E}-07-3.2874 E-01$

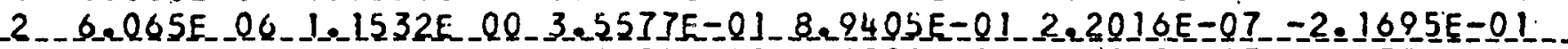

$3 \quad 3.679 \mathrm{E}$ O6 $1.9555 \mathrm{E}$ 00 $3.2082 \mathrm{E}-011.8901 \mathrm{E} 00 \quad 2.8269 \mathrm{E}-07-5.4756 \mathrm{E}-02$

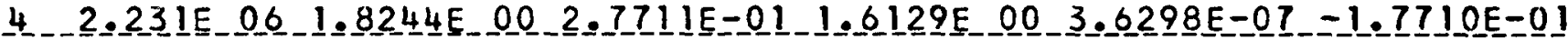

5 1.35.3E $062.2155 \mathrm{E} 003.4738 \mathrm{E}-012.0647 \mathrm{E} 004.6611 \mathrm{E}-07-1.2624 \mathrm{E}-01$

$6 \quad 8.208 \mathrm{E}$ 05 $2.8343 \mathrm{E} 00 \quad 4.5042 \mathrm{E}-01$ 2.6756E $005.9863 \mathrm{E}-07 \overline{-1.3288 \mathrm{E}-01}$

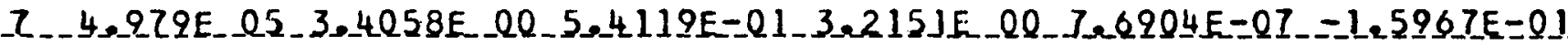

$8 \quad 3.020 E$ OS $3.9179 E$ O 00 6.2255E-01 3.6985E $00 \quad 9.8949 E-07-1.8367 E-01$

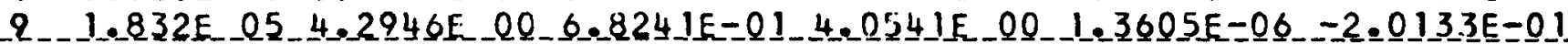

$106.738 E$ O4 4.5240E $007.1887 \mathrm{E}-014.2707 \mathrm{E} \quad 002.1132 \mathrm{E}-06-2.1209 \mathrm{E}-01$

$1^{2} 2.479 \mathrm{E} 044.6634 \mathrm{E} 007.410 \mathrm{IE}-014.4023 \mathrm{E} 007.0539 \mathrm{E}-06-2.1862 \mathrm{E}-0 \mathrm{~T}$

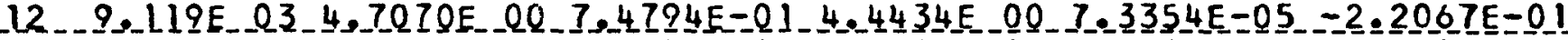

$133.355 E$ E $034.7159 E$ O0 7.4936E-01 4.4518E $00 \quad 1.6214 E-04-2.2109 E-01$

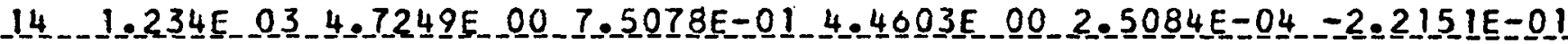

$15 \quad 4.540 E$ O2 $4.7352 E$ OO $7.5242 E-014.4700 E$ OO $3.5353 E-04-2.2199 E-01$

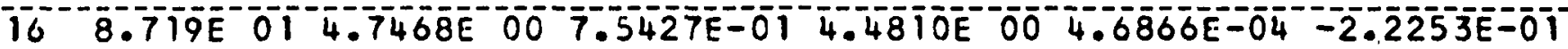

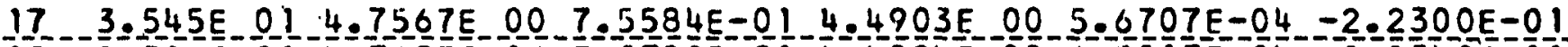

$18.304 \mathrm{E} 014.7653 \mathrm{E} 00-\frac{1}{7} .5720 \mathrm{E}-014.4984 \mathrm{E} 00-6.521 .7 \mathrm{E}-04-2.2340 \mathrm{E}=0 \mathrm{~T}$ 12 _5.043E_Q0_

$201.445 E \quad 004.7839 E \quad 007.6015 E-014.5160 E \quad 008.3674 E-04-2.2427 E-01$

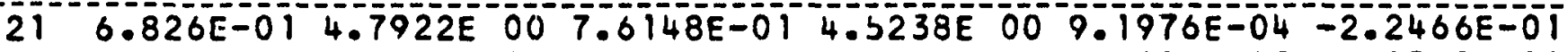

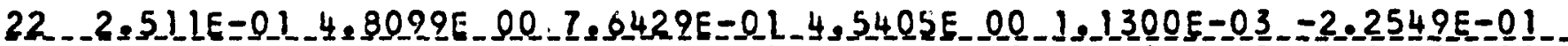
$23-9.237 \mathrm{E}-024.8293 \mathrm{E} 007.6737 \mathrm{E}-014.5588 \mathrm{E} 00 \mathrm{0} 1.8814 \mathrm{E}-03-2.2640 \mathrm{E}-01$

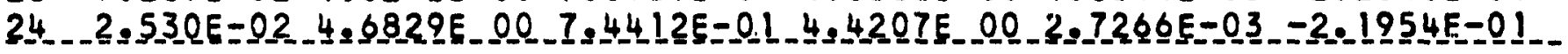

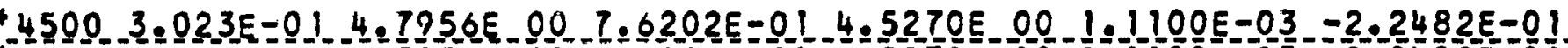
$40002.718 E-014.7956 E 007.0202 E-014.5270 E-00-1.7100 E-03-2.2482 E=1$

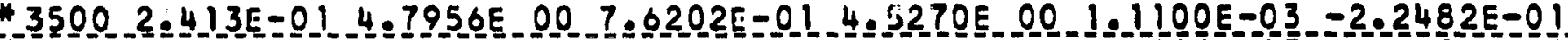

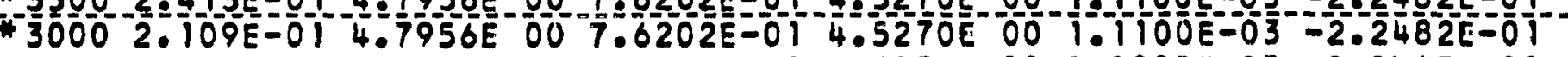

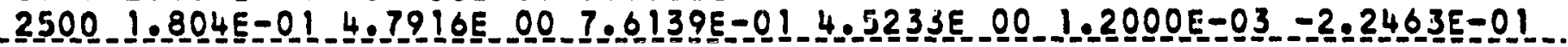

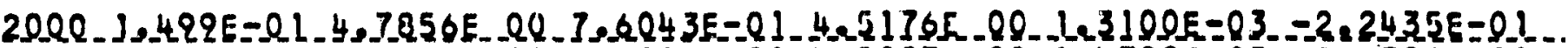
$15001.194 \mathrm{E}-01$ 4.7762E $007.5894 \mathrm{E}-01$ 4.5087E 00 1.4700E-0.3 - 2.2391. -01

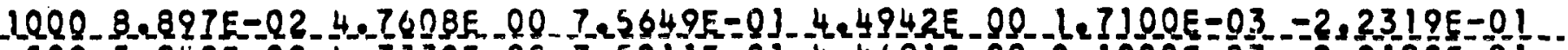
$5005.850 E-024.7332 E$ O0 7.5211E-014.4681E 002 . $1000 E-03-2.2190 \mathrm{E}-01$

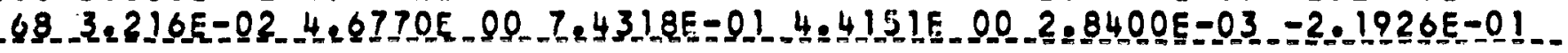

"Thermal cross noctions taken from APSX-515."

NOTE: For a sumary of the sources of data see APEX-704. 
CARBQN

THERMAL TRANSFER MATRICES

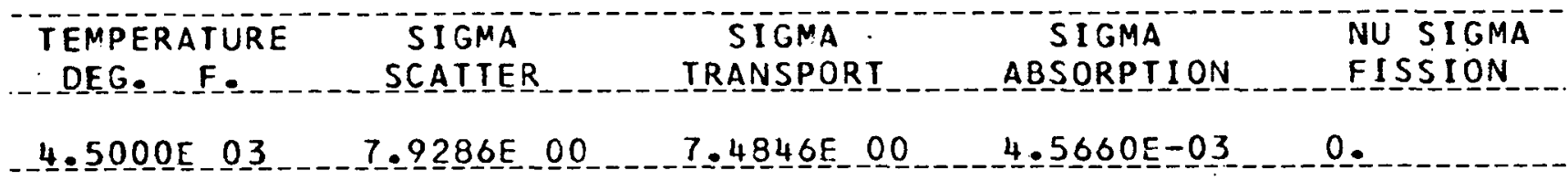

Ni $\bar{N}^{-12}$

SIGMA S (N1/N2)

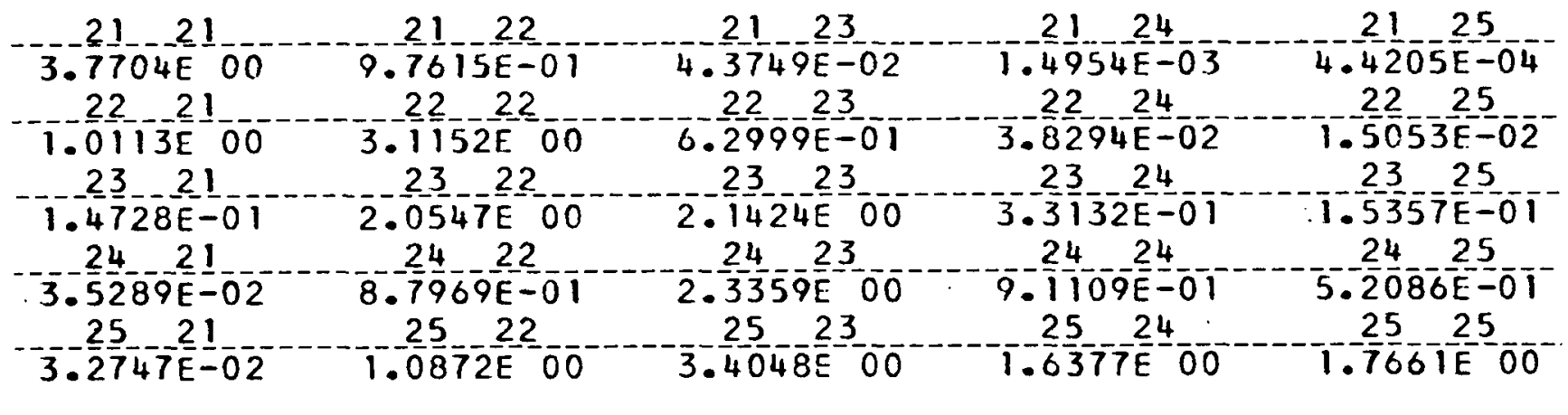

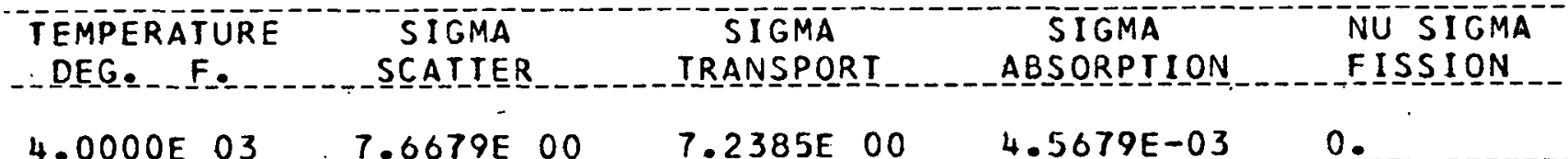

\section{SIGMA S_(NIIN2)}

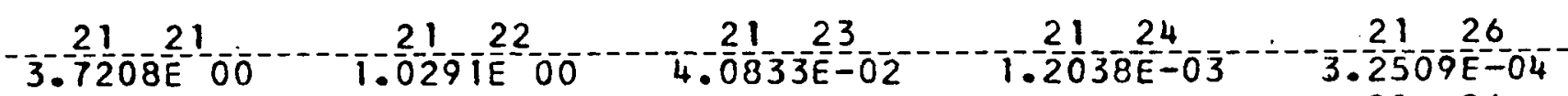

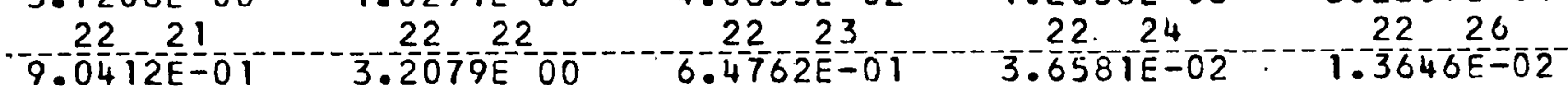

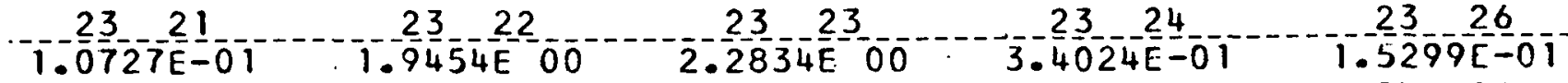

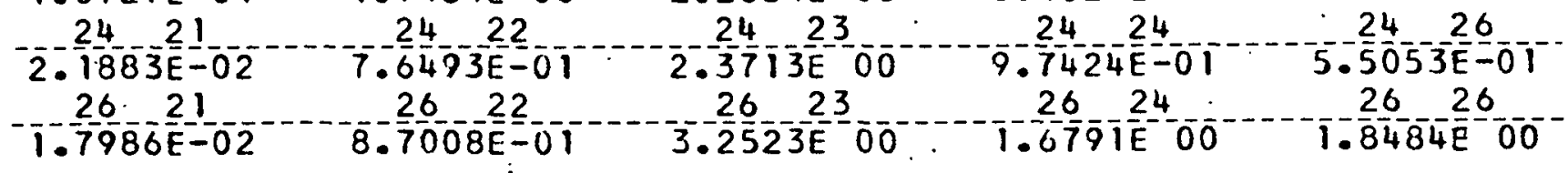


CARBON

THERMAL IRANSEER MAIRICES.

IEMPERAIURE

SIGMA

SIGMA

SIGMA

NU SIGMA

DEG. F. SCATTER

TRANSPORT

ABSORPTION

FISSION

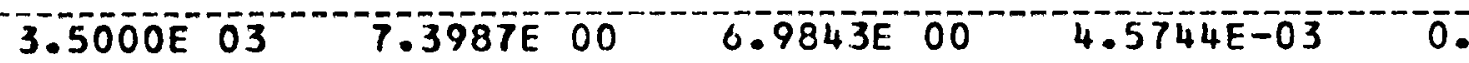

N1 $-N 2$

SIGMA S (NIINZ)

$2 i 2 i$

3.656926 .00

I. $8799 \mathrm{E}-01$

$23-21$

7.2994 E-02

2421

$1.2145 E-02$

$272 \mathrm{i}$

$8.6240 \mathrm{E}-03$
2122

$1.0965 E \quad 00$

$22 \quad 22$

3.3052500

2322

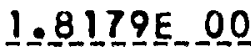

2422

6. $4257 E-01$

2722

$6.6258 E-01$
$21^{-2} 3$

$3.7650 E-02$ 2223

$6.69920 E-01$

2.4362500

2423

$2.3968 \mathrm{E} \quad 00$

2723

$3.0625 \mathrm{E} \quad 00$
2124

$9.2 \frac{4}{22} 20 \mathrm{E}-04$

$3=4670 \mathrm{E}=02$

2324

$3.5038 E=01$

2424

1.0477E 00

$27-24$

$1.7214 \mathrm{E} 00$
$217 \overline{7}$

$2.2319 E-04$ 2227 $-\frac{2}{2} \frac{1}{3}-7 E-02$ $1.5180 E-01$ 5. $8374 E-01$ $1.9436 E 00$

IEMPERAIURE

DEG. $F$.

उ.0000E $0 \overline{3}$
SIGMA

SCATTER
SIGMA

TRANSPORT
SI GMA

ABSORPTION
NU SIGMA

FISSION
$7.120 \mathrm{TE} 00$
6.7214500
$4.5804 E-0 \overline{3}$
0.

N1 N2

SIGMA S INITNZI

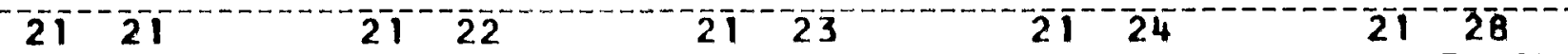

$3.5727 E \frac{00}{2}-1.1846 E-100$

$3.4 \frac{1}{2} \frac{11}{2}-\frac{02}{3}$

$0.0292 \mathrm{E}-0.04$

1.3897E-04

$0.0247 E-01$

$3.4051 E \quad 00$

$6.9225 E-01$

$3.2531 E-02$

2324

$22^{-28}$

$23-2 T$

2322

$23 \frac{23}{3}$

$1.6686 \mathrm{E}$ OO

$2.6034 E 00$

$3.6225 \mathrm{E}-01$

2421 2422 2423

2424

$23-28$

$5=7634 E=03$

5. 1469E-01

$2.4068 \mathrm{BE} \quad 0$

1. $\frac{13}{2} \frac{34}{8} \frac{\mathrm{E}}{2} \frac{00}{4}$

$.4984 F-01$ 28 i

$28-22$

2823

$1.7635 \mathrm{E} 00$

2428

$3.4162 E-03$

4. $7125 E-01$

$2.1207 E \quad 00$

6. $2115 E-01$

$28-2 \bar{B}$

$2.0553 E_{0} 00$ 
CARBON

THERMAL TRANSFER MATTRICES

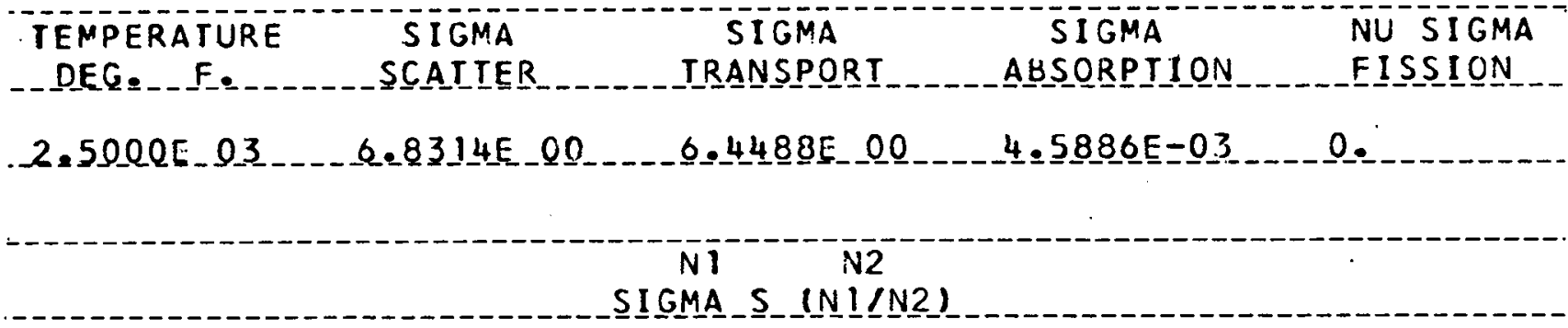

\begin{tabular}{|c|c|c|c|c|}
\hline 21 & $-21-22$ & $-21-23$ & -21 & -21 \\
\hline $\begin{array}{c}3.4592 \mathrm{E} \\
22\end{array} 00$ & $\begin{array}{l}1.3025 \mathrm{E} 00 \\
22\end{array}$ & $\begin{array}{c}3.0081 E-02 \\
2223\end{array}$ & $\begin{array}{c}4.2916 E-04 \\
2224\end{array}$ & $\begin{array}{c}7.4788 E-05 \\
2229\end{array}$ \\
\hline $5.2762 E-01$ & $3.5027 \mathrm{E} 00$ & $7.4001 E-01$ & $3.0125 \mathrm{E}-02$ & $8.7614 \mathrm{E}-03$ \\
\hline $2.4330 \mathrm{E}-02$ & $1.4928 \mathrm{E}$ on & $2.7885 \mathrm{E} 00$ & $3.7673 E-01$ & $1.4689 \mathrm{E}-01$ \\
\hline 24 & & 23 & 2 24 & 244 \\
\hline $\begin{array}{c}2.1691 E-03 \\
29 \quad 21\end{array}$ & 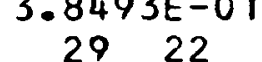 & $\begin{array}{c}2.3930 t \\
2923\end{array}$ & $\begin{array}{c}1.2392 \mathrm{E} \\
2924\end{array}$ & $\begin{array}{c}0.6362 E-01 \\
2929\end{array}$ \\
\hline $.0208 E-03$ & $3.0378 \mathrm{E}-$ & $2.5348 \mathrm{E} 00$ & $1.8028 \mathrm{E} 00$ & $2.1890 \mathrm{E} 00$ \\
\hline
\end{tabular}

TEMPERATURE
SIGMA

\section{Ni $N 2$ \\ SIGMA S (NIIN2)}

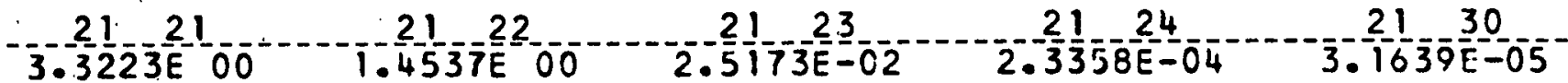

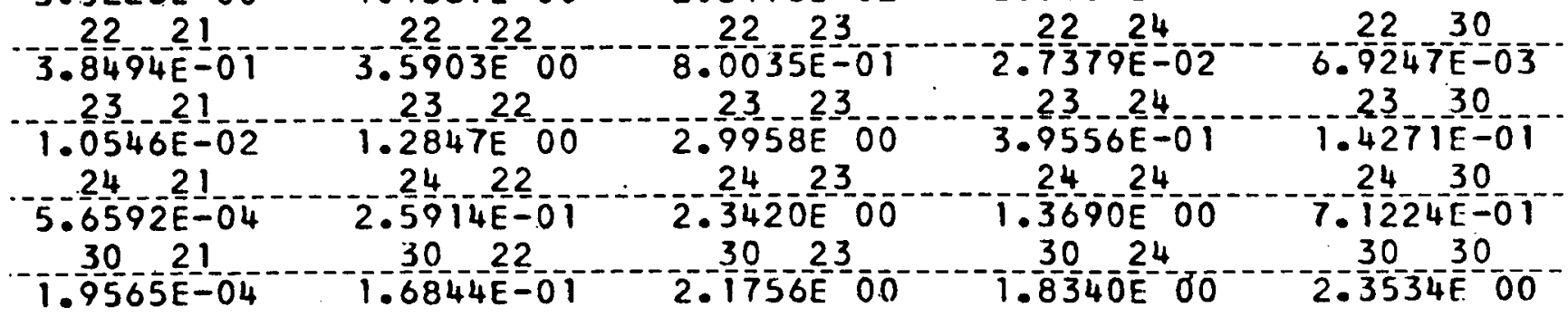


CARBON

_LHERMAL _ IRANSFER_MAIRICES_

- IEMPERATURE

SI GMA

SLGMA

SIGMA

NU SIGMA -

DEG. F. SCATTER

TRANSPORT

ABSORPTION

FISSION

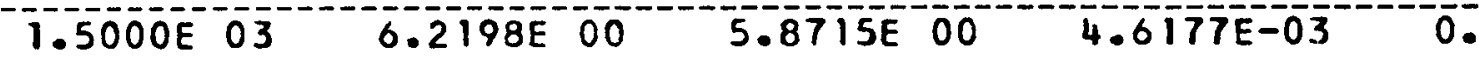

NI

$-\mathrm{N} 2$

SIGMA S (NTIN2)

21

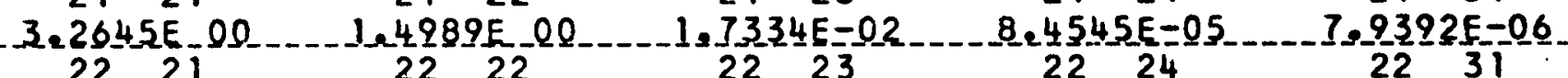

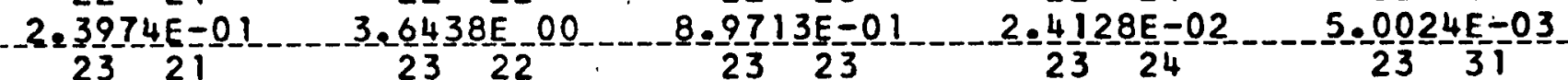

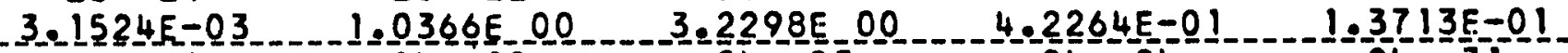

$2421 \quad 2422.2424$ $24-3 i$

$7.8355 E-05$ $1.7432 \mathrm{E}-05 \ldots$

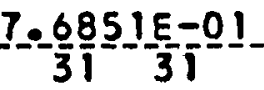

IEMPERAIURE DEG. F.

\$IGMA SCATTER
SLGMA TRANSPORT
SIGMA

ABSORPTION
NU SIGMA.

FISSTON

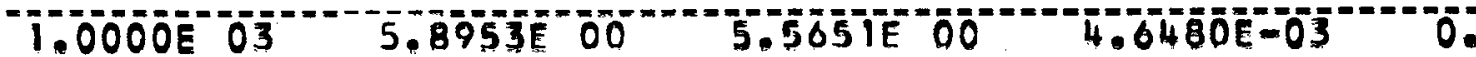

N1

SIGMA S $(N 1 / N 2)$

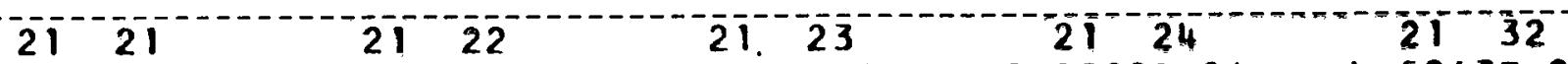

3.

$1.0690 \mathrm{E}-01$ 3.6109E 00 1.0690E $00 \quad 1.9951 \mathrm{E}-02 \quad 3.0330 \mathrm{E}-03$
$2 \overline{3} 2 \overline{1}$
23
$23-\frac{1}{2}$
$23-\frac{1}{4}$
23 32

$4.54760=04$

7. $3860 \mathrm{E}-01$

$3.4874 \frac{E}{24}-0$

$4.6846 E-01$

2424

1. $3030 \mathrm{O}-01$

$$
2421
$$

2422

$2.0256 \frac{0 E}{32}-0$

$1.76 \frac{36}{32} \frac{\mathrm{E}}{2} \mathrm{O}$

$24 \quad 32$

$-3.0937 E-06$

$5.8 \frac{8}{32} 12 E-0$

8. $3481 E-01$

$32-32$

$3.4146 E-07$

1.9307E $=02$

1.2216E 00

$1.8119 \mathrm{E} \quad 00$

$2.8425 \mathrm{E} \quad 00$ 
CARBQN

THERMAL TRANSFER MATRICES

TEMPERATURE
SIGMA
DEG SIGMA

N $\overline{1}-\cdots$

SI GMA S INIIN2)

\begin{tabular}{|c|c|c|c|c|}
\hline$-2 \frac{1}{0}-21$ & $-21-22$ & $21-23$ & $-21-24$ & 21 \\
\hline $\begin{array}{l}3.9346 \mathrm{E} 00 \\
22 \\
2\end{array}$ & $\begin{array}{c}8.5635 E-01 \\
22 \quad 22\end{array}$ & $\begin{array}{c}1.2205 E-03 \\
22 \quad 23\end{array}$ & $\begin{array}{c}2.0077 E-07 \\
22 \quad 24\end{array}$ & \\
\hline $5.8131 \mathrm{E}-02$ & $3.4050 \mathrm{E} 00$ & $1.3327 \mathrm{E} 00$ & $1.2911 \mathrm{E}-02$ & 1.110IE-03 \\
\hline $1.23-21$ & $-\frac{23}{3.8498 E-01}$ & $-3.7498 \mathrm{E}=00$ & $5.7069 \mathrm{E}-01$ & $-\frac{23}{1.2377}=33$ \\
\hline 24 & 24 & 24 & 24 & $24 \quad 33$ \\
\hline 0. & $\begin{array}{c}1.0538 \mathrm{E}-02 \\
1.6 \frac{3}{12}-\frac{22}{25-03}\end{array}$ & $\begin{array}{l}1.6533 \mathrm{E} 00 \\
6.43 \\
6.407 \mathrm{E}-01\end{array}$ & $\begin{array}{l}2.1022 \mathrm{E} 00 \\
1.6594 \mathrm{E}\end{array}$ & $\begin{array}{l}9.1691 E-0 \\
33 \\
3.2499-33\end{array}$ \\
\hline
\end{tabular}

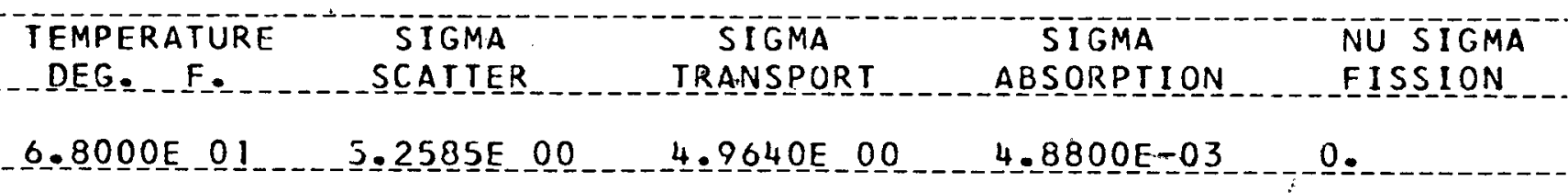

Ni $\mathrm{N} 2$

SIGMA S (N IIN2)

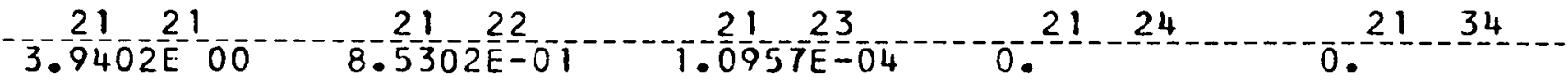

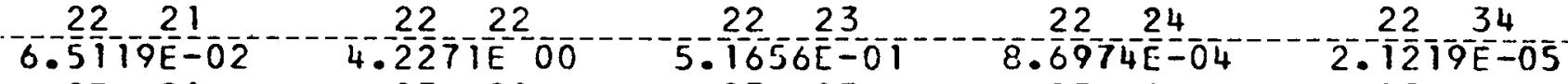

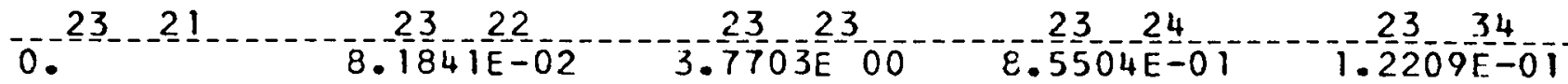

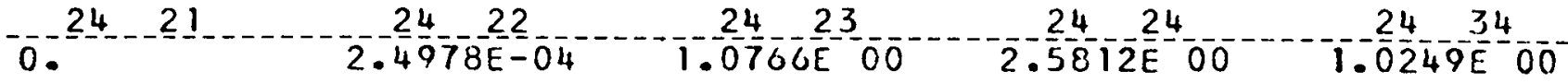

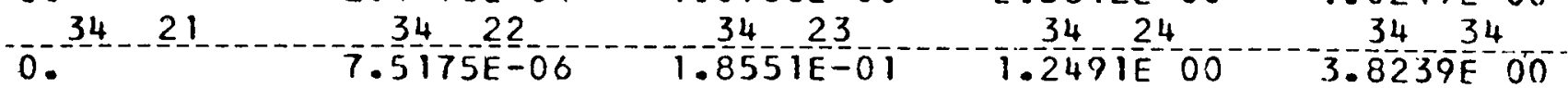


CARBON

TRANSFERS THOM GROUPS I THROUGH 2 TO GROUPS I THROUGH 21

SIGMA SO (NI/N2)

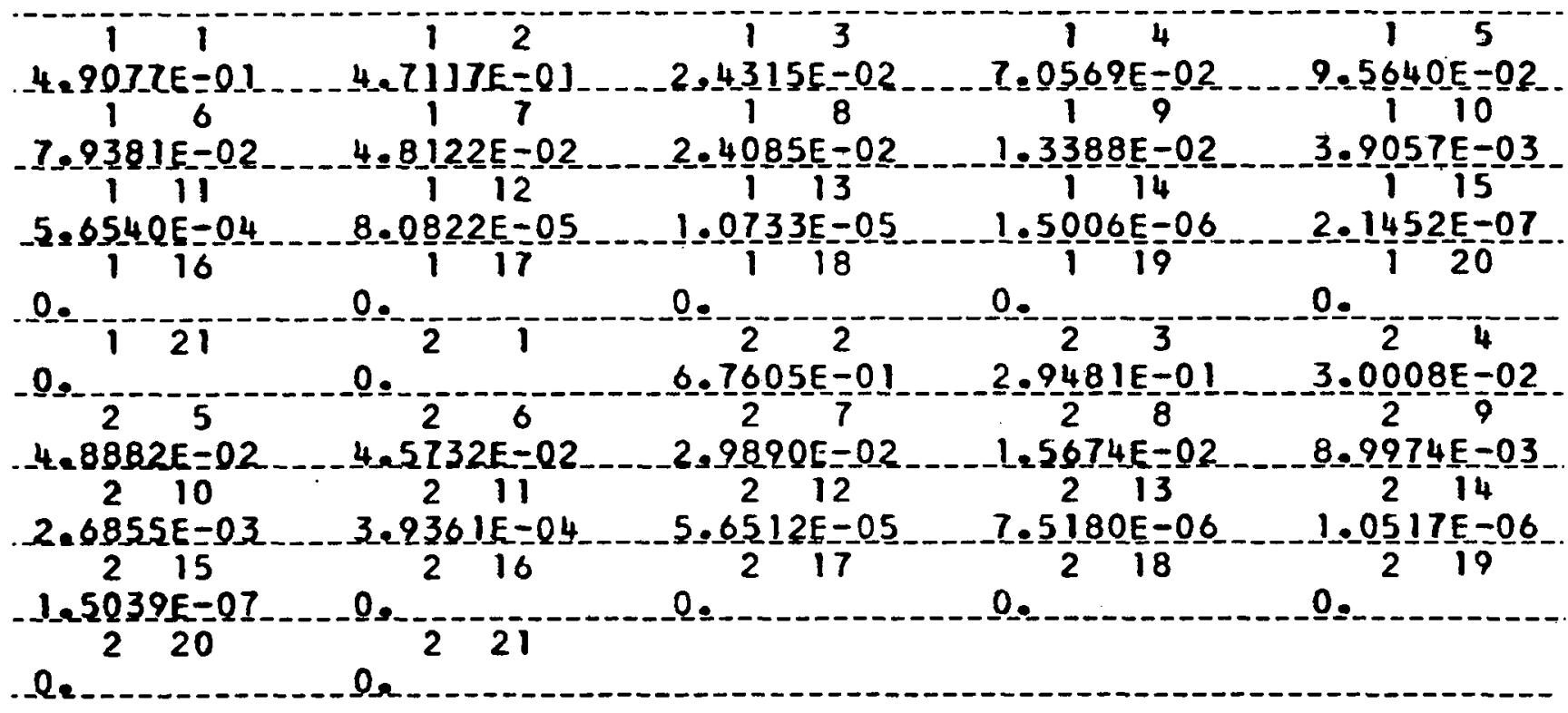


CARBON

TRANISFERS FROM GROUPS ' 1 THROUGH 34 TO GROUPS 1 THROUGH 11

N1 N2

SIGMA_N, G $(N) I N 2)$

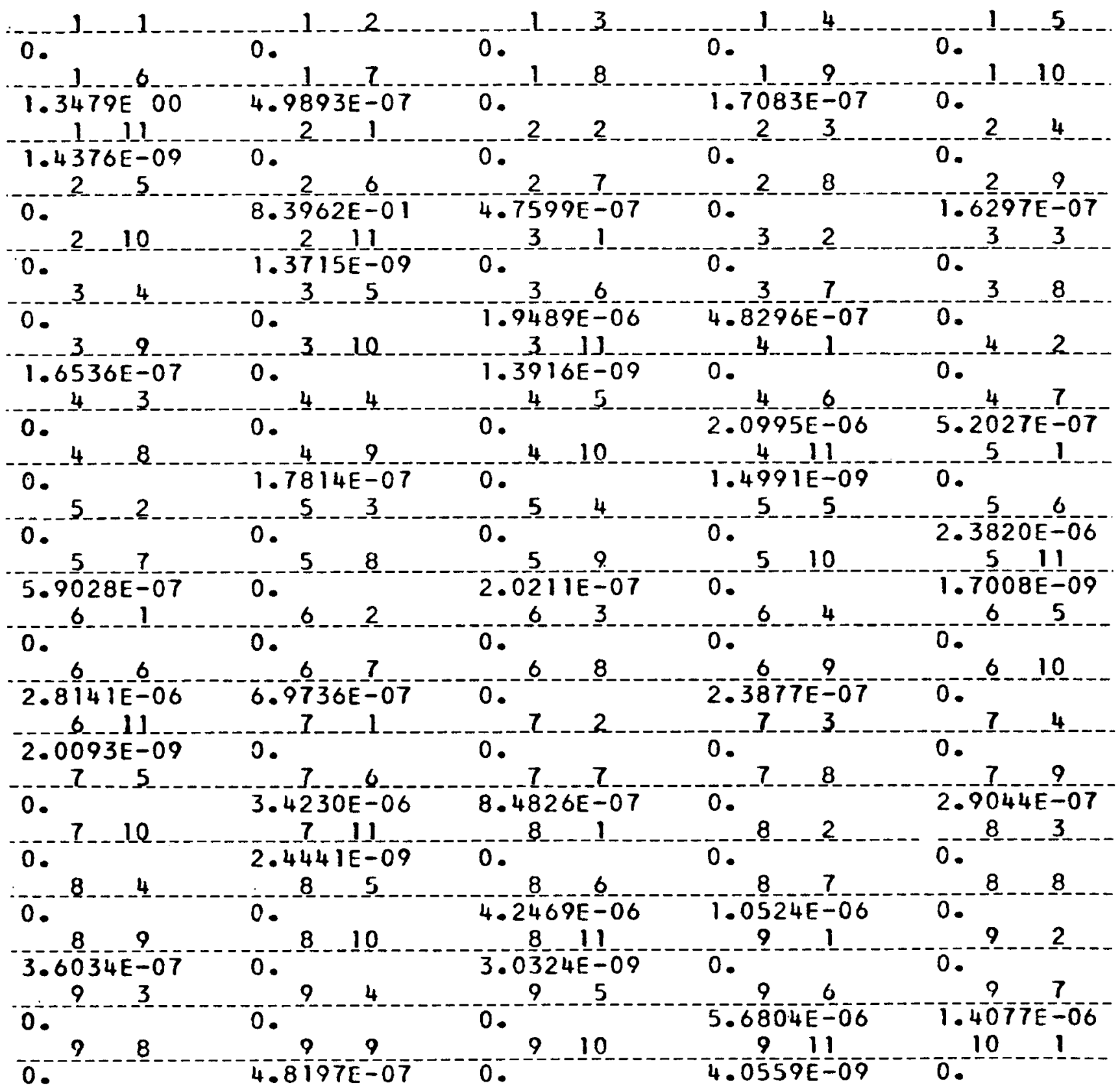


-CARBQN.

\section{SIGMA NIG $(N$ IIN2)}

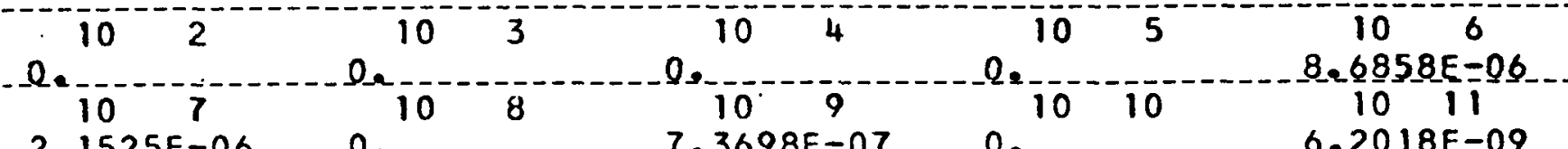

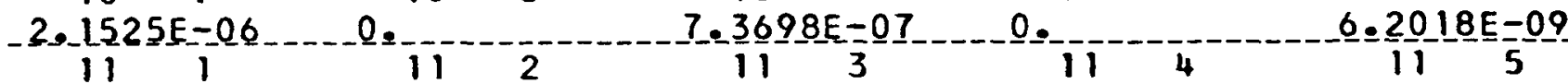

Q. 1100.110

$1.4204 E=05 \quad 3.5199 E-06 \quad-12=12052 E-06-12=0$.

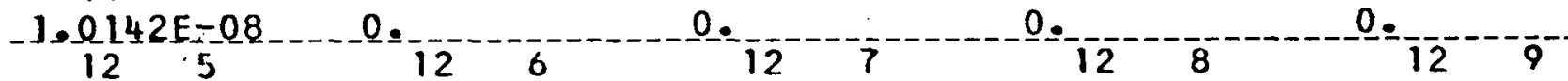

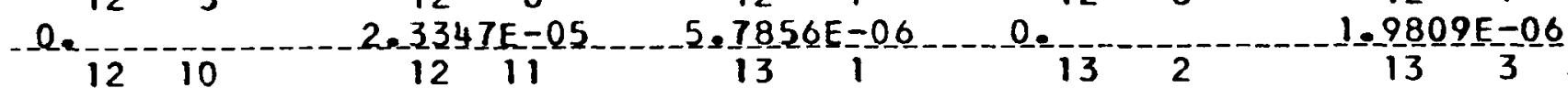

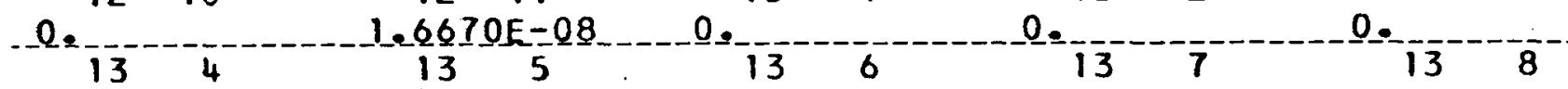

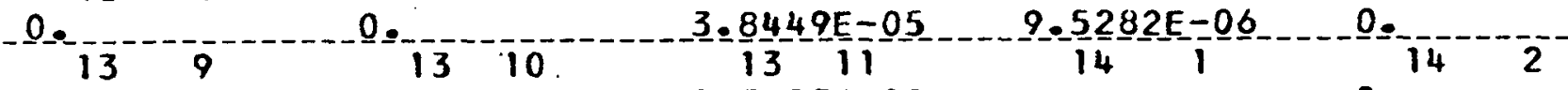

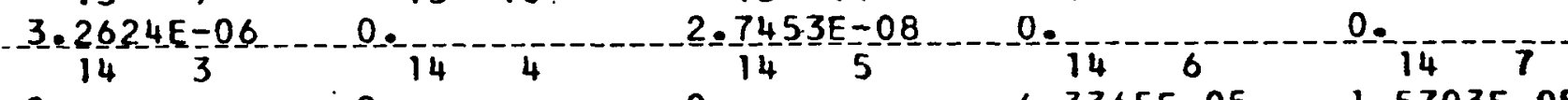

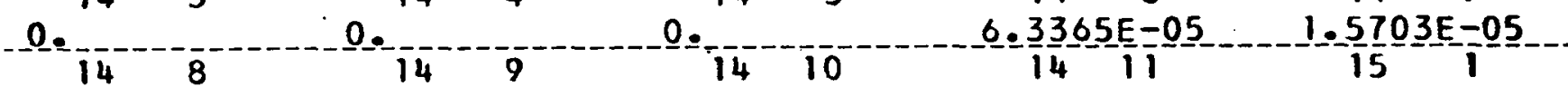

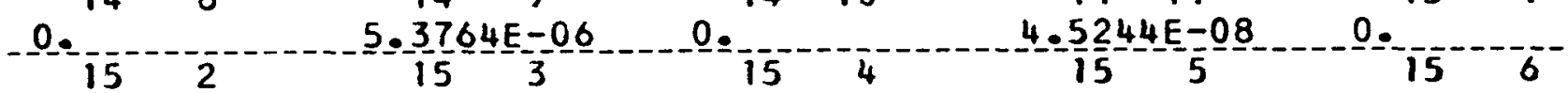

0. 15

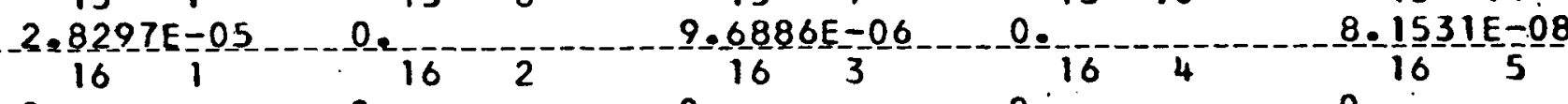

$16-0.0016-16$

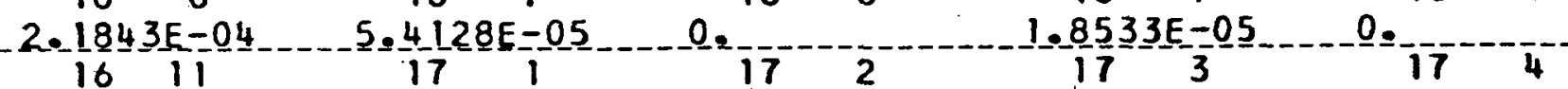

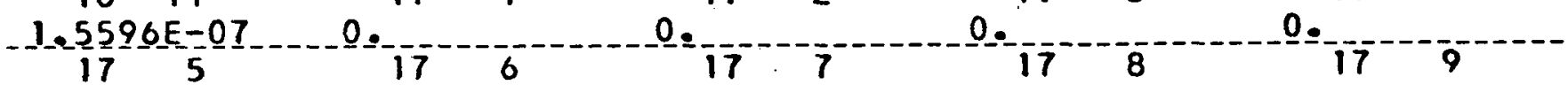

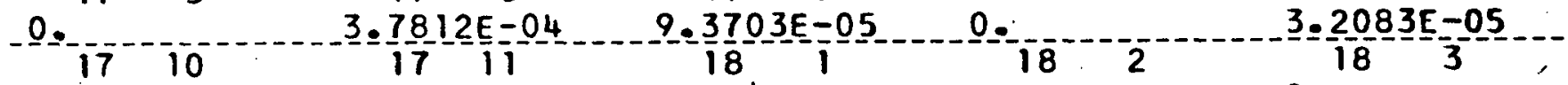

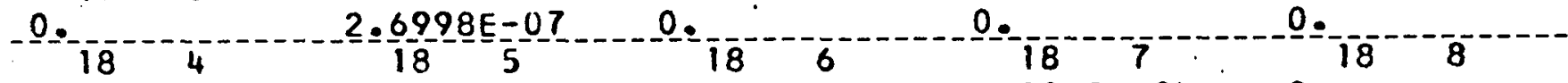

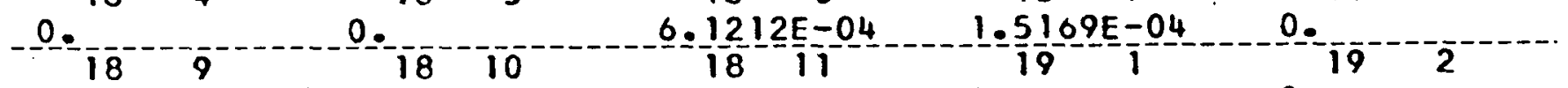

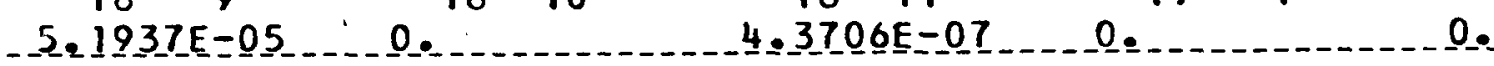




\section{CARBON}

\section{$\mathrm{N} 1 \mathrm{~N} 2$ \\ SI GMA N,G (NI]N2)}

0.

19

19

0 .

20

0 .

20

4.3100E-04

0 .

$-\frac{21}{7725}=\frac{6}{\mathrm{E}-0}$

0 .

19

19

- 21 - 11

22

0 .

$22-10$

0.

0.

23

$6.4676 \mathrm{E}-040$

0.

24

(-

0

25

0.

$25-7$

$4.6085 \mathrm{E}-03$

0 .

26

$1.86 \frac{26}{12}-\frac{6}{2}-02$

$26 \quad 11$

1. $32 \overline{8} \overline{\mathrm{E}}-0 \overline{5}$

0.275

$027-10$
0.

$8.7488 \mathrm{E}-05$

20

0 .

20

8

21

$6.8707 E-04$

0 .

$4.4413 \mathrm{E}-0 \overline{3}$

3. $17 \div 1 \mathrm{2}-11$

0 .

$19+10$

0 .

20

$120=9$

21

0.

22

0 .

1. $1006 \mathrm{E}-03$

0 .

-

$-\frac{23}{6}-\frac{2}{5} \overline{\mathrm{E}}-\frac{6}{-0} \overline{3}$

$-\frac{23}{44} \overline{2}-\frac{11}{6}-06$

0.24

$1.0039 E-03$

0 .

25

26

$4 \cdot 6 \frac{26}{12} \frac{7}{1 E-03}$

0.

$1 . \frac{27}{86} \overline{1} \overline{\mathrm{T}}-0 \mathrm{-0}$

$1 . \frac{27}{3} \frac{11}{30} \overline{3}-0 \overline{5}$
$24 \quad 10$

1. $5779 \overline{2}-0 \overline{3}$

0 .

0

0.

$4 .-6 \frac{7}{16} \overline{9} \bar{E}-\overline{0} \overline{3}$

0 .
25
19

$1.0311 E-03$

$19-11$

$7.3623 E-07$

20

$20-10$

0.

0. 21

21

$2.3525 \bar{E}-04$

$0.22-3$

22

0.

2

23

$1 . \frac{23}{8} 889 \bar{E}-03$

0.24

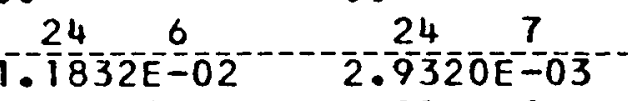
$24 \quad 11$

$8.4 \overline{4} \overline{80}=\bar{E}=\overline{0}$

0 .

25

$025-10$

26

$0 .-25$

$2.5552 \mathrm{E}-04$

-20 20.1

0.

$1 . \frac{20}{7392} \overline{\mathrm{E}}-0 \mathrm{0}$ $20-11$

$1.2418 \mathrm{E}-06$

0 .

0.

22

$3.7683 E-04$

23

0. 24

5

$1 .-\frac{25}{8} 57 \overline{7}-\frac{6}{02}$

$-\frac{25}{3} \frac{1}{2} 78 \frac{1}{\mathrm{E}}-05$

$026 \quad 5$

$1.5 \frac{26}{9} \overline{2} \frac{9}{0} \overline{3}^{-1}$

0. $27 \quad 3$

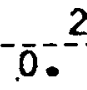

$1 . \frac{27}{580} \overline{8} \frac{9}{-1}-0 \overline{3}$

0.

278

283 
CARBON

SIGMA N.G (NITNZ)

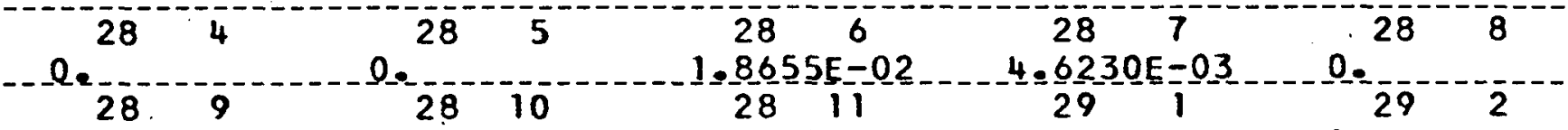
$\frac{1.5828 E-03}{3}$

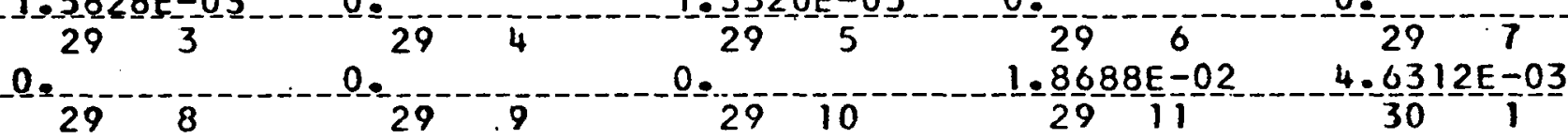

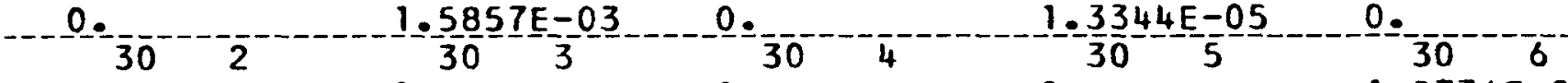

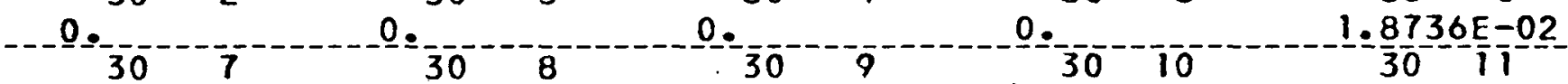

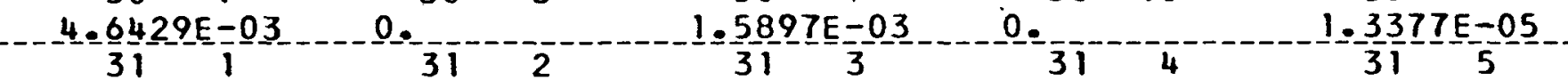

0 .

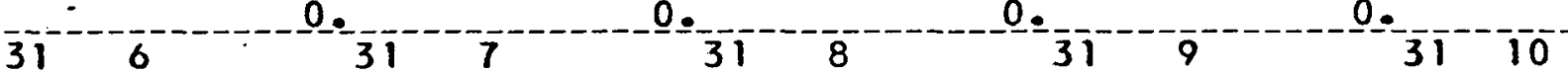

$1.8807 E-02-1,-60606 E-03$

$\overline{32}-\overline{2}$

$1-\frac{5}{3} \frac{9}{2}-\frac{8 E}{3}-\frac{03}{3}$

0.

$1.3429 \mathrm{E}=05$

325

0.

32

0.

0

ㅇ.

.8930 OE -02

$4.6912 E-03$

0.

$1.3517 E-0.5$

$\underline{0}-$

0.

$1.6062 \mathrm{E}-03$

- 0.

-

0.

$1=\frac{9191 \mathrm{E}}{33}=02$

4. $7557 E-03$

$1 . \frac{62}{34}-\frac{3}{5}=03$

0.

34

1. $3702 E-05$

$-\frac{7}{3}$

345

을

$34-\frac{1}{6}$

$34 \quad 10$

$1.9875 \mathrm{E}-02$

$34 \quad 11$

$0=-134$

$1.68664 E=03$

ㅇ.

1.4- 191E-0 5

33 3 $-\frac{1}{3}-\cdots$ 0. $34-{ }^{-\cdots}$ ㅇ.4. $9252 E=03$ - 0 . --- 
DATE $12108 / 60$

DESIGNAIION

H FREE
CODE NO.

1.1000
DENSIIY FACIQRR

$\underline{1}=\underline{6} 7 \underline{3} \underline{1} \underline{1} \underline{E}-\underline{0} \underline{0}$

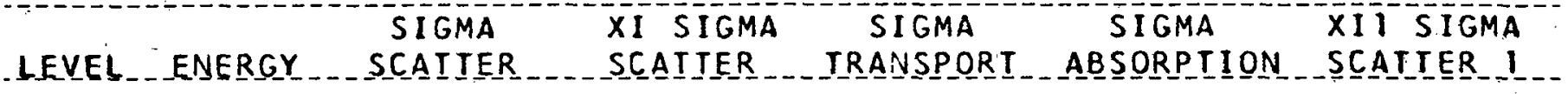

$1.1 .000 E-071.0441 E-001.0441 E-003.4758 E-01-1.7787 E-05-4.6435 E-01$

$26.065 \mathrm{E} 061.4139 \mathrm{E} 00-1.4139 \mathrm{E} 004.7069 \mathrm{E}-012.1498 \mathrm{E}-05-6.288 \mathrm{IE}-0 \mathrm{1}$

3 3.679E 06 1.9996E 00 1.9996E 00 -6.6566E-01 2.7604E-0 $-8.8928 E-01$

$42.231 \mathrm{E} 062.7149 \mathrm{E} 002.7149 \mathrm{E} 00-9.0379 \mathrm{E}-013.5444 \mathrm{E}-05-1.2074 \mathrm{E} 00$

5 1.353E 06 3.5490E 00 3.5490E 00 1.1815E 00 4.5515E-05 $-1.5784 E 00$

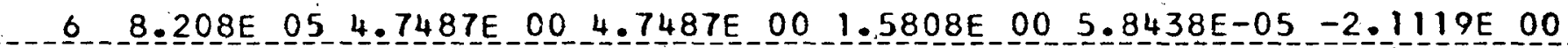

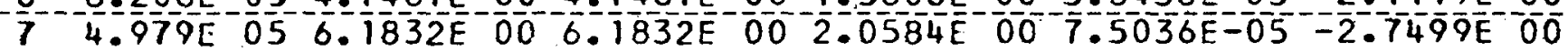

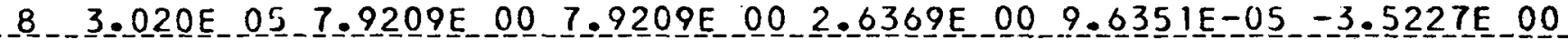

9 1.832E 05 1.0499E 01 1.0499E 01 3.495 IE 00 1.3248E-04 $-4.6692 \mathrm{E} 00$

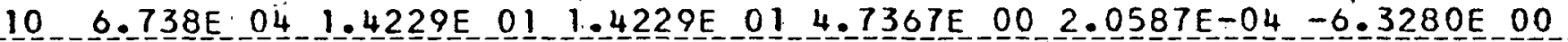

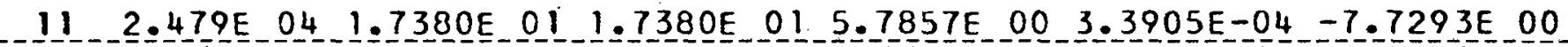

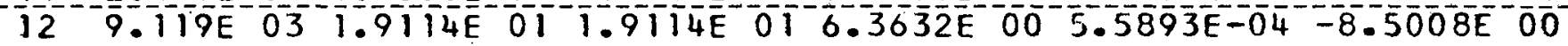

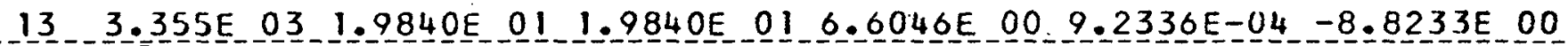

$140.234 \mathrm{E} 031.9998 \mathrm{E} 011.9998 \mathrm{E} 016.6575 \mathrm{E} 00$ 1.5221E $0 \overline{3}-8.8940 \mathrm{E} 00$

15 4.540E 02 1.9997E 01 1.9997E 01 6.6571E 00 2.7400E-03_-8.8934E 00

16 - $819 \mathrm{E} 01$ 1.9995E 01 1.9995E 01 6.6563E $00.5 .2417 \mathrm{E}-03-8.8923 \mathrm{E}-00$

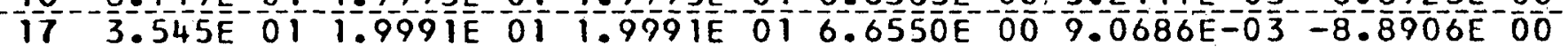

$\begin{array}{lllllllllllll}18 & 1.304 E & 01 & 2.0001 E & 01 & 2.0001 E & 01 & 6.6582 \mathrm{E} & 00 & 1.4671 \mathrm{E}-02 & -8.8949 \mathrm{E} & 00\end{array}$

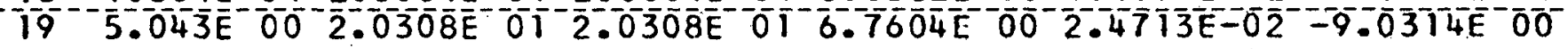

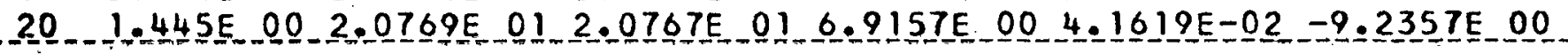

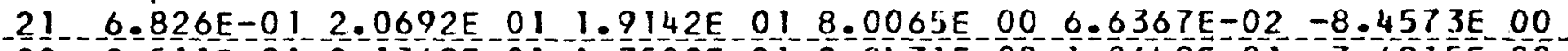

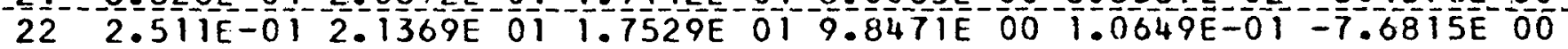

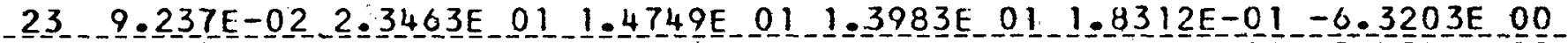
$24-\frac{1}{2} .530 \mathrm{E}-02-2.7344 \mathrm{E} 01-1.3134 \mathrm{E} 01.1 .9156 \mathrm{E} 01-2.8414 \mathrm{E}-0 \mathrm{1}-5.4584 \mathrm{E}-0 \mathrm{O}$

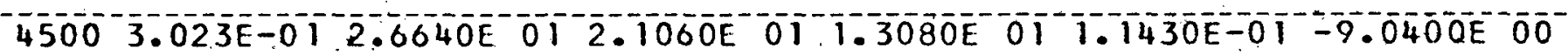

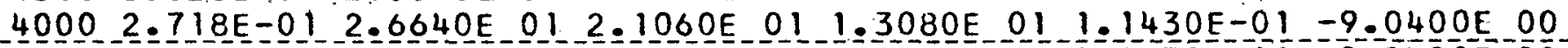

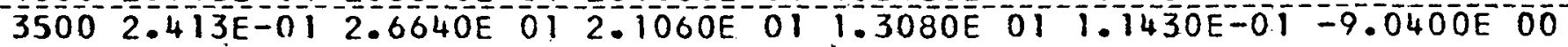
$30002.109 \mathrm{E}-012.6640 \mathrm{E}-012.1060 \mathrm{E}-01,1.3080 \mathrm{E} 01-1.1430 \mathrm{E}-01-9.0400 \mathrm{E}-00$

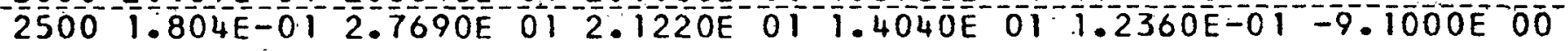

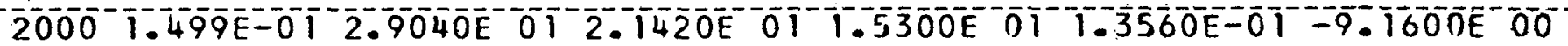

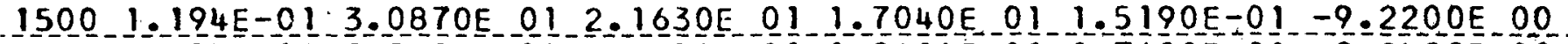
$1000-8.897 \mathrm{E}-023.3480 \mathrm{E}$ OI $2.1830 \mathrm{E}$ 01 $1.9620 \mathrm{E} 01 \mathrm{1.7600E-01-9.2400 \textrm {E } 0 0}$ 500 5.850E-02 3.7760E 01 2.1950E 01 2.3990E 01 2.1700E-01-9.1800E OO

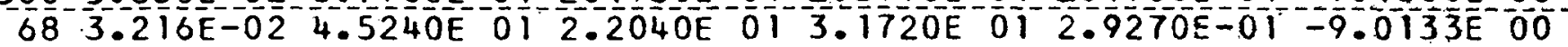

NOTE: For a sumary of the sources of data see APEX-704. 
$15 \underline{4}$.

H_FREE

THERMAL TRANSFER MATRICES

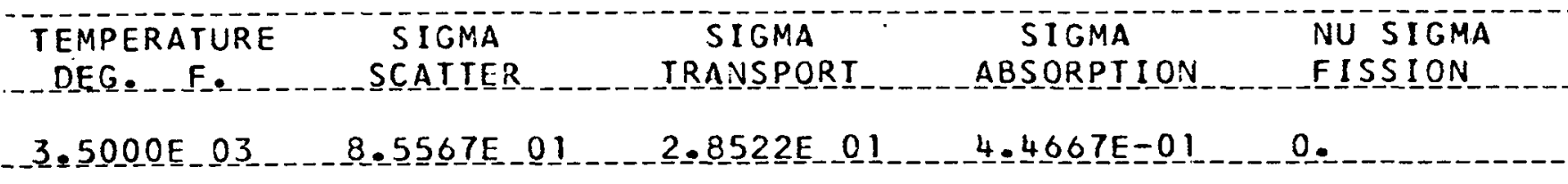

\section{$\mathrm{N} 1 \mathrm{~N} 2$}

SI GMA S_(N1_N2)

\begin{tabular}{|c|c|c|c|c|}
\hline-2$]_{-}-21$ & $21-22$ & 21 & 21 & 21 \\
\hline $1.1452 \mathrm{E} 01$ & $7.7619 \mathrm{E} 00$ & $2.3345 E 00$ & $3.5109 E-01$ & $2.2208 \mathrm{E}-01$ \\
\hline $5.8956 \mathrm{E} 00$ & $1.4280 \mathrm{E} 01$ & $6.0287 \mathrm{E} 00$ & $9.0606 \mathrm{E}-01$ & $5.7350 E-01$ \\
\hline $.0951 T^{2}-\frac{21}{.00}$ & 23 & $23-23$ & $23-24$ & $23-27$ \\
\hline $24-21$ & 24 & 24 & 24 & $\begin{array}{r}1 \\
24\end{array}$ \\
\hline $.45170,00$ & $2.0158 E$ OI & $1.9672 E^{0} 01$ & $5.6943 E 00$ & $3.9013 E 00$ \\
\hline $9 \mathrm{E} 00$ & $2.9650 \mathrm{E} 01$ & $2.8935 \mathrm{E} 0$ & $8.8602 \mathrm{E} \mathrm{O}$ & 8.6 \\
\hline
\end{tabular}

SEMPERATURE
SIGMA
SEEG SIGMA

Ni

SIGMA S (N1/N2)

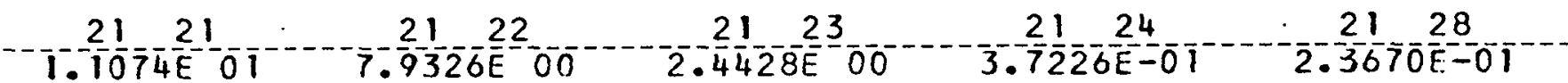

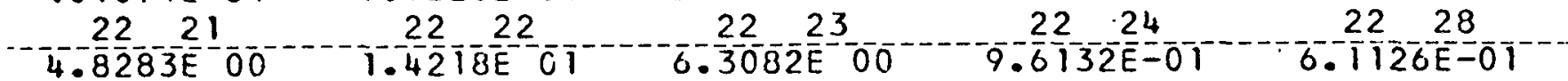
23
$3.7099 E$

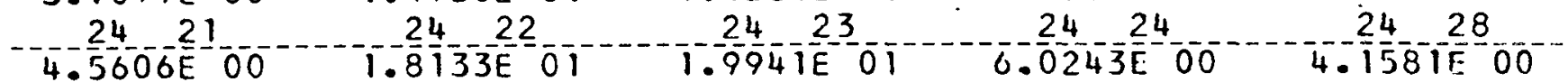

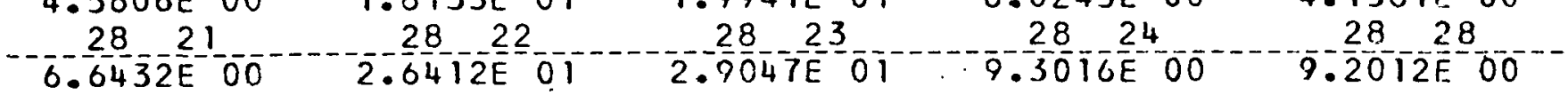


H FREE

- IHERMAL_IRANSFER_MAIRICES

IEMPERAIURE

SIGMA

SLGMA

DEG. F. SCATTER

TRANSPORT

SIGMA

ABSORPTION

NU SIGMA

$2.5000 \mathrm{E} 03$

$7.5159 \mathrm{E} 01$

$2.5053 E^{-} 01$

$4.4806 \overline{0}-01$

0 .

\section{$\operatorname{SIGMA} S\left(\frac{N}{2}-\frac{N 2}{1 / N 2}\right.$}

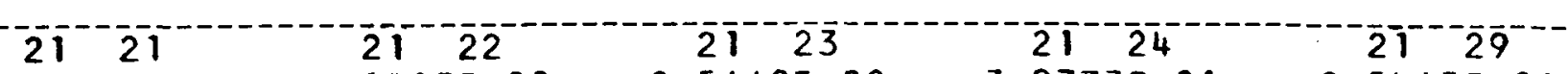

$1.0 \frac{0629 E}{22}-01$

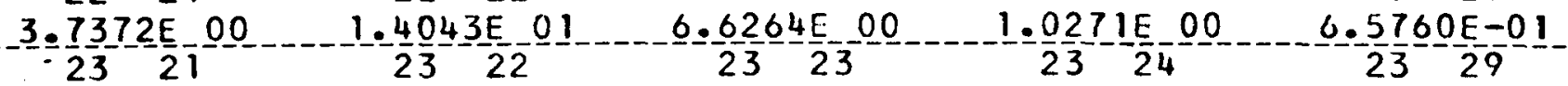

$-2.4359 \mathrm{E}-00 \quad 1.3232 \mathrm{E} O \mathrm{O} \quad 1.4863 \mathrm{E}-01 \quad 3.0526 \mathrm{E}-00 \quad 1.9544 \mathrm{E}-00$

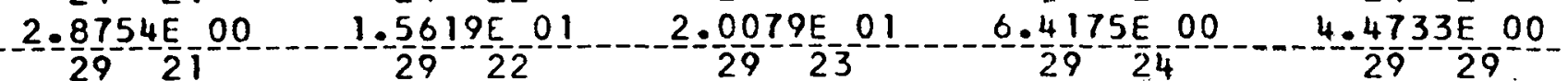

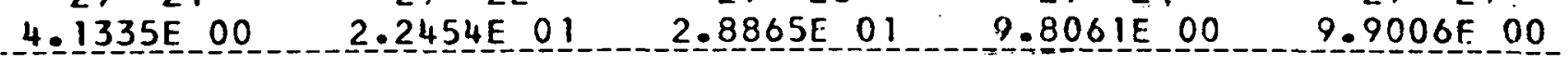

IEMPERATURE

DEG. $F$.
SI GMA

SCATTER
SIGMA TRANSPORT
SIGMA ABSORPTION
NU SICMA

FISSTON

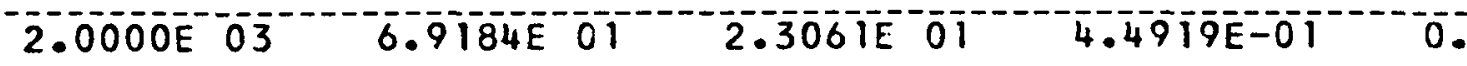

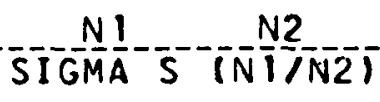

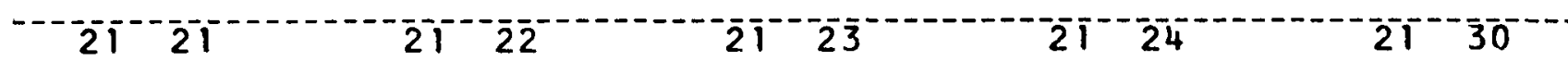
$-1.0111 \mathrm{E}-01 \quad 2.2614 \mathrm{E}-00 \quad 2.7071 \mathrm{E}-00 \quad 4.2916 \mathrm{E}-01 \quad 2.7740 \mathrm{E}-01$

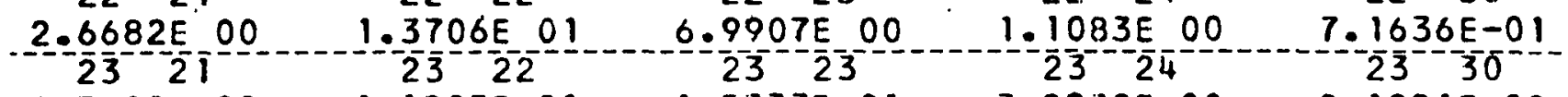

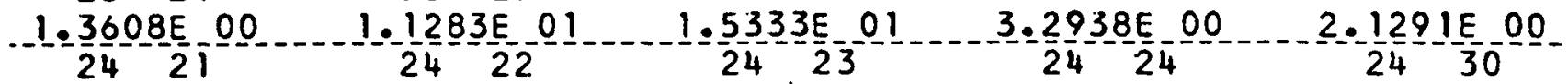

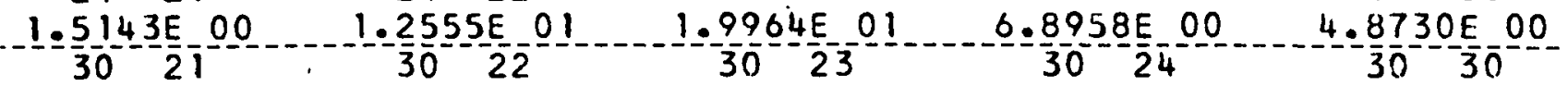

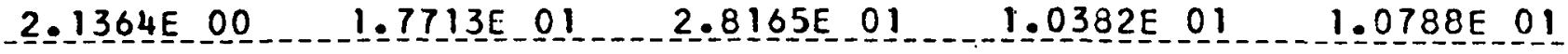


H_FREE

THERMAL TRANSFER MATRICES

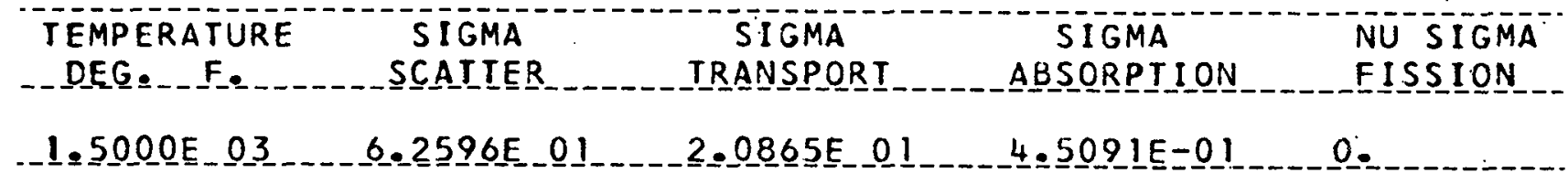

$\mathrm{N} 1$

SIGMA S $(N \underline{N} \mathbb{1}$ N2)

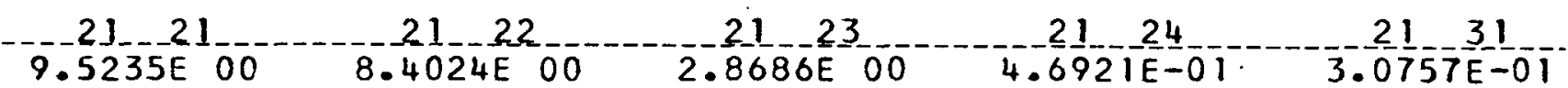

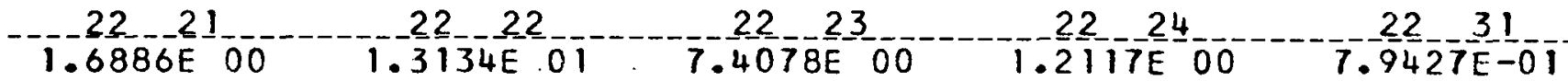
2321

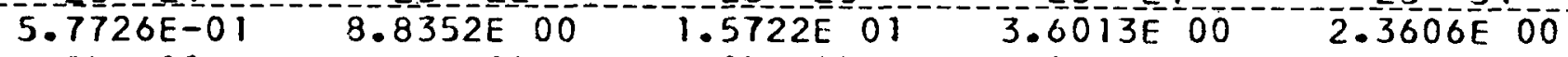

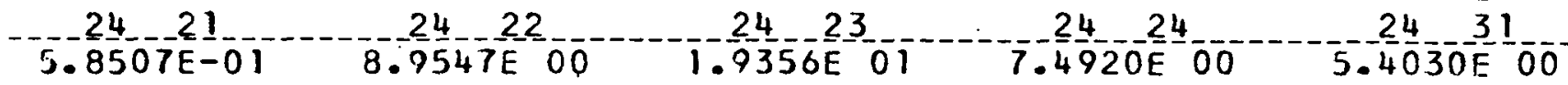

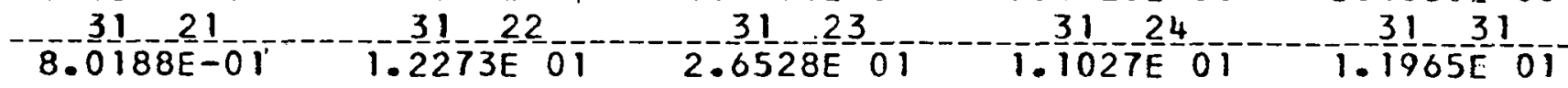

TEMPERATURE
SIGMA

_. 0000 E 03 - 5.5222E 01

Ni 2

SIGMA S_LILN2L

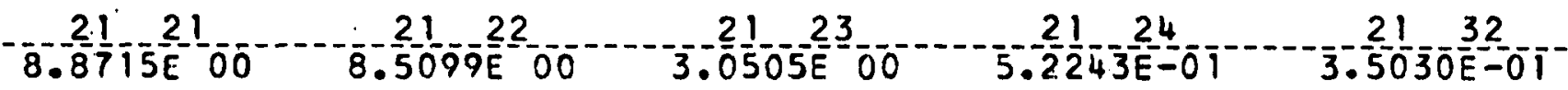

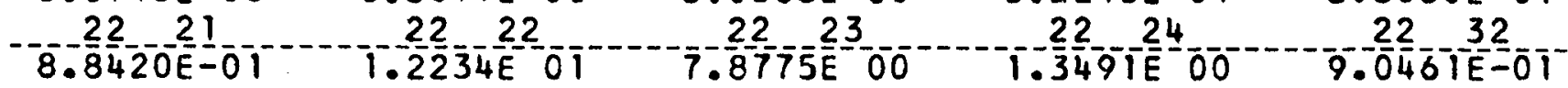

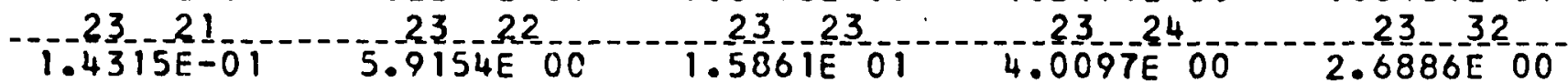

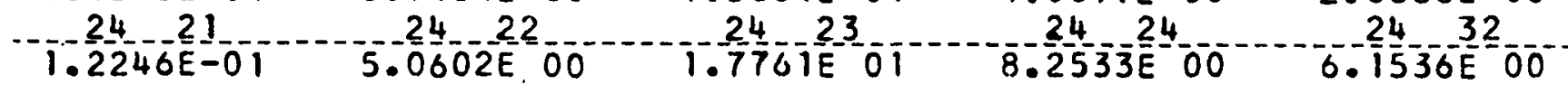

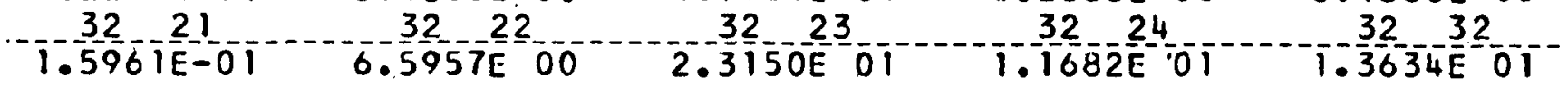


H FREE

_IHERMAL_JRANSFER_MAIRICES_

.. LEMPERAIURE SIGMA SIGMA SIGMA DEG . F. SCATTER TRANSPORT ABSORPTION NU_ SILMA... FISSION

\begin{tabular}{|c|}
\hline $5.0000 E \quad 02 \quad 4.6729 E \quad 01 \quad 1.5576 E$ O1 $4.6010 E-01 \quad 0$. \\
\hline
\end{tabular}

SIGMA S $\left(N \mid \frac{N}{N}\right)$

$21^{21} 21^{2} 21^{2} 3 \overline{1}^{2}$

$8.1681 E_{-} 00$

2221 8. $\$ 692 E_{-2} Q 0$

$3.2409 E$ EO $5=96760 E-01$ 4. $1756 E-01$

$3.3797 E=0$ 2321 $22 \quad 22$ $22 \quad 23$ $22 \quad 24$ $22 \quad 33$

9. $5650 E-03$ $24 \quad 21$ 1. $0918 E$ 이 8. 36923 E $1.5411 E 00$ 2324 $4.5802 E-00$ $1=\frac{53}{2} 10 E-\frac{01}{2}$ 2.8385 E 00 2422 1. 63 3 $58 \mathrm{E}-00$ 3322 $1.4132 \mathrm{E}-0$ 3323 1. $9133 \mathrm{E} \quad 00$ 1.6529트이
$9=2248 E 00$ 3324 $1.2016 E \quad 01$
1. $0783 E$ E 00 . 2333 $3.2048 E-0 O$ 7. $33 \underline{5} 2 \mathrm{E}$ OOO $3 \frac{3}{3}$

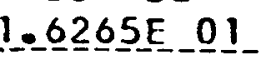
3...-. $6.4473 E-03$

$5=\frac{5}{3} \frac{12}{3}-\frac{3}{2}=0$

- $-4473 E=0$

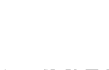

-.-

IEMPERAIURE

DEG. F.
SIGMA

SCATTER
SIGMA

TRANSPORT
SIGMA

ABSORPTION
NU SIGMA

FISSION

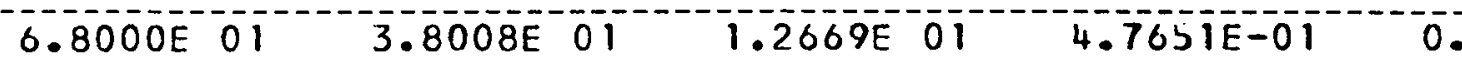

$N 1 \ldots \ldots$

SIGMA S INI/N2)

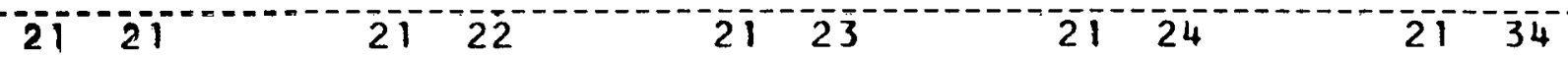

T. $-52 \underline{2} \underline{0 E}-\underline{0} \underline{0}$

22,1

8.5824 E 00

$3 . \frac{3}{2} \frac{73}{2}-\frac{5 E}{2}=0$

$6.8 \frac{8}{2} 5 \underline{2} \frac{1 E-0 !}{24}$

I. $22-200 E-01$

$-9 \cdot \frac{2}{2} \frac{7}{3}-\frac{5 E}{2}=02$

9.44660600

$-\frac{8}{2} \cdot \frac{7117 \mathrm{E}}{2} \mathrm{O}-0$

$1 . \frac{7}{2} \frac{7}{3} 02 \frac{E}{2} \frac{00}{4}$

$1.3432 E \quad 01$ 2423

7. $9731 \mathrm{E} 00$ 3423

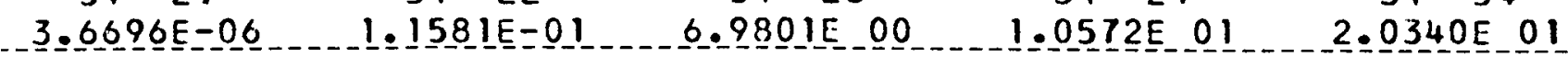

$5.2613 E$ - 00 2424

$1=00069 \frac{E}{3}-01$

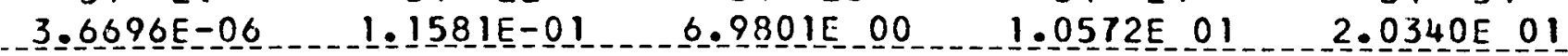

1. $-\frac{3}{2} \frac{4}{3}-\frac{\mathrm{OE}}{34}-\underline{0}$

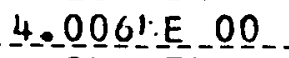
2434 9. $1698 \mathrm{E} \quad 00$ $\frac{1}{34}-\frac{2}{34}$ 
H FREE

TRANSFERS FROM GROUPS 1 THROUGH 20 TO GROUPS 1 THROUGH 25

$\mathrm{NI} N 2$

SIGMA_SO_ (N] /N2)

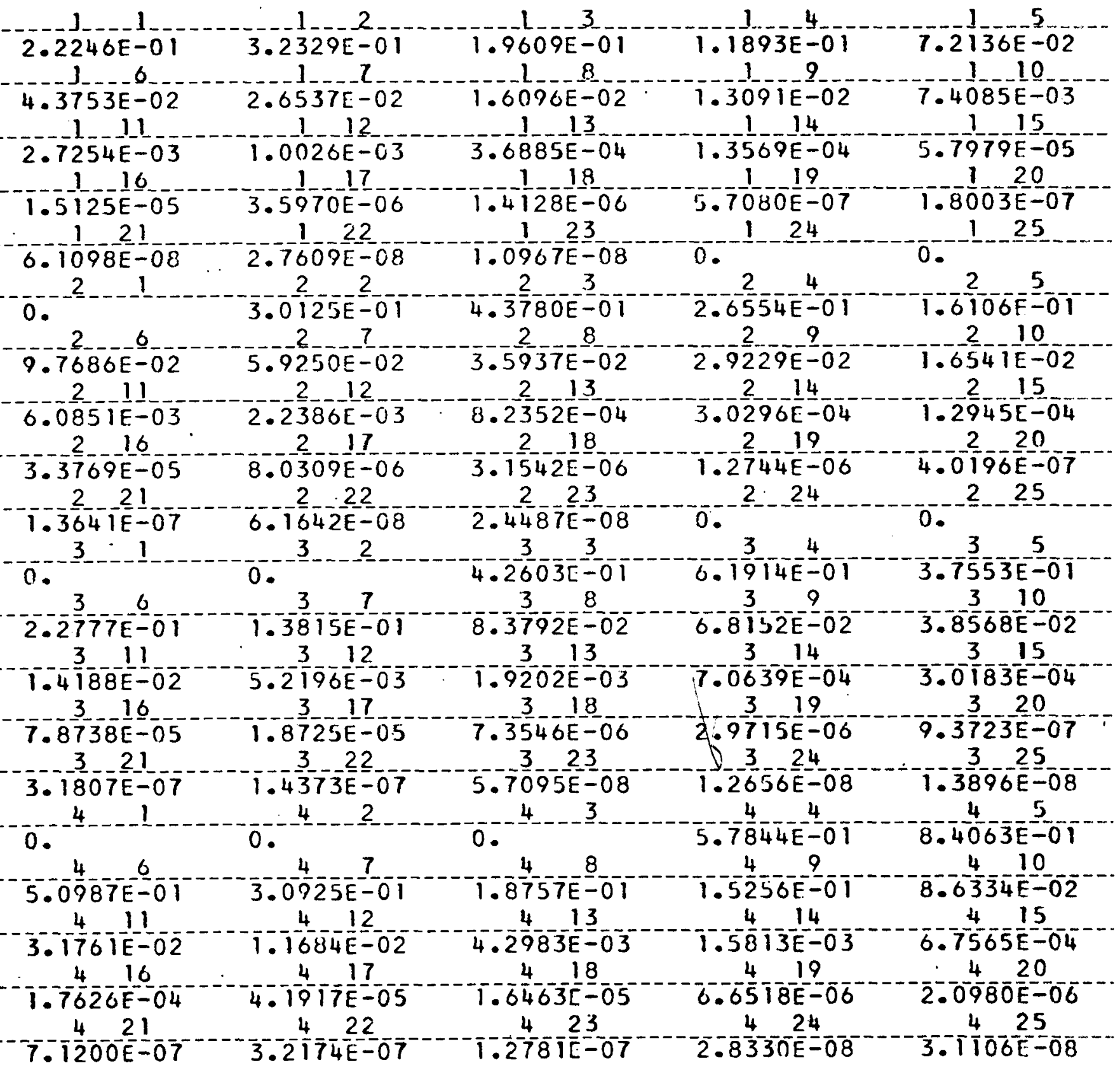


- H_FREE

$\mathrm{N} 1$

SIGMA SO (NI/N2)

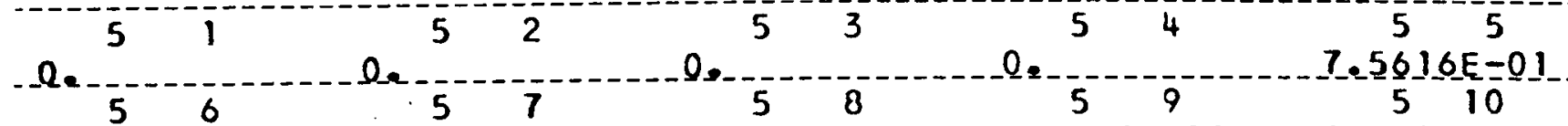

-1.0989E 00 - $6.6652 \mathrm{E}=01 \quad-4.0426 \mathrm{E}-01$

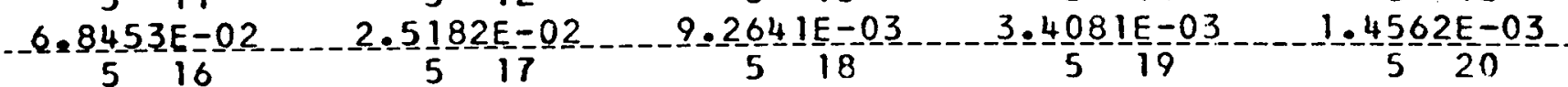

$3.7988 E-04-9.0342 E-05-3.5483 E-05-1.4336 E-05-4.5217 E-06$

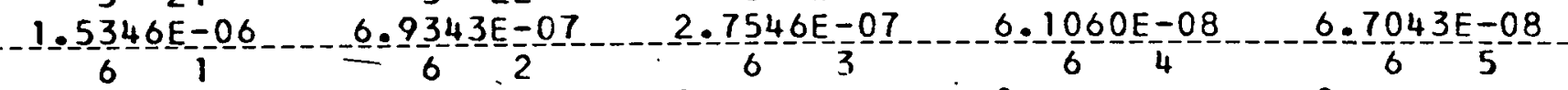

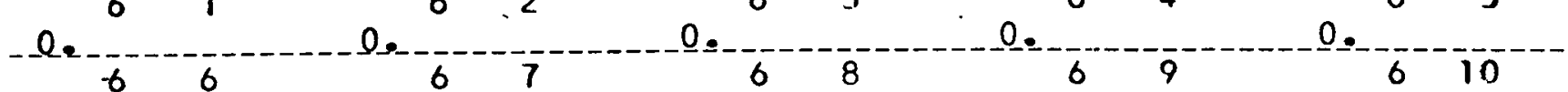

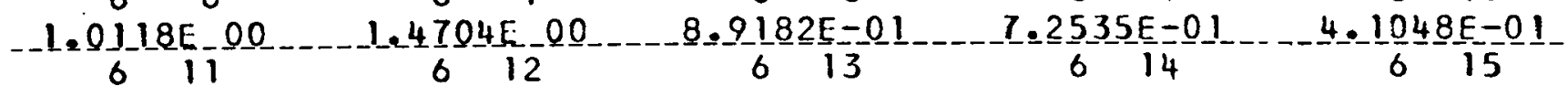

$5.5553 \mathrm{E}-02$

1.5 $101 E-0$

$5553 \mathrm{E}=-0$

2. 0437 JE -02

7. $5183 E-03$

$3=2124 E-03$

$8.3803 E=04$

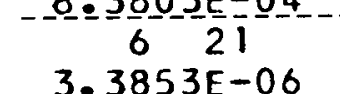

622

$7.8277 E-05$

619

620

$1.5297 \mathrm{E}-06$

623

$3.1626 \mathrm{E}=05$

$9 \cdot 9751 E-06$

0. 7 .

o.

$0 .-1.3174 E$ E 00

$6.0767 E-\frac{07}{7}$

$\left.1.3 \frac{3}{7} \mathrm{TOE}-\frac{0}{4}\right]$

- 3. 24 $2196 E=01$

1.1926E -01

ㅇ..

$7-\frac{1}{8}$

$0 .-7$

$1.4790[-0]$

716

$1.7991 \mathrm{E}=03$

721

7.2675E -06

$-0 .--\frac{1}{6}$

-0.

-6.8470E-0

$3.7998 \mathrm{E}-03$

$8-21$

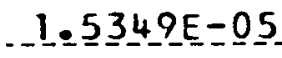

$4.2785 \mathrm{E}=04$

$=9.91466 \mathrm{E}=00$

1. $5572 \mathrm{E}-00$

0.

4.3874E-02

$1.6140 E=02$

8. $812 \frac{1}{7}=\frac{3 E-01}{15}$

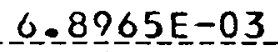

718

6.78960 E 05

723

724

20

722

$1=3046 E=06$

2. $89 \frac{9}{8} 17 \mathrm{E}-0 \mathrm{0}$

2. $1415 E-05$

$3.2840 E-06$

$0 .-$

0.

$0=-8$

1. 6876E 00

$8^{-19}$

9

3.28892000

$9.2664 E-02$

$3.4 \frac{089 E-02}{19}$

$19-02$
$19-04$

$3.5492 \frac{E}{8}-04$

$1=4340 E-04$

$3.1751 E-07$

$0=-10$ $1.8612 E=00$ 1.4566 E-02 $9.0365 E-04$ $2.7553 E=06$ $6.1075 E=07$ 4. 5229E-05 $6.9360 E=06$ $6.7059 E-0 ?$ 
H FREE

N1 N2

SIGMA SO_ (NIIN2)

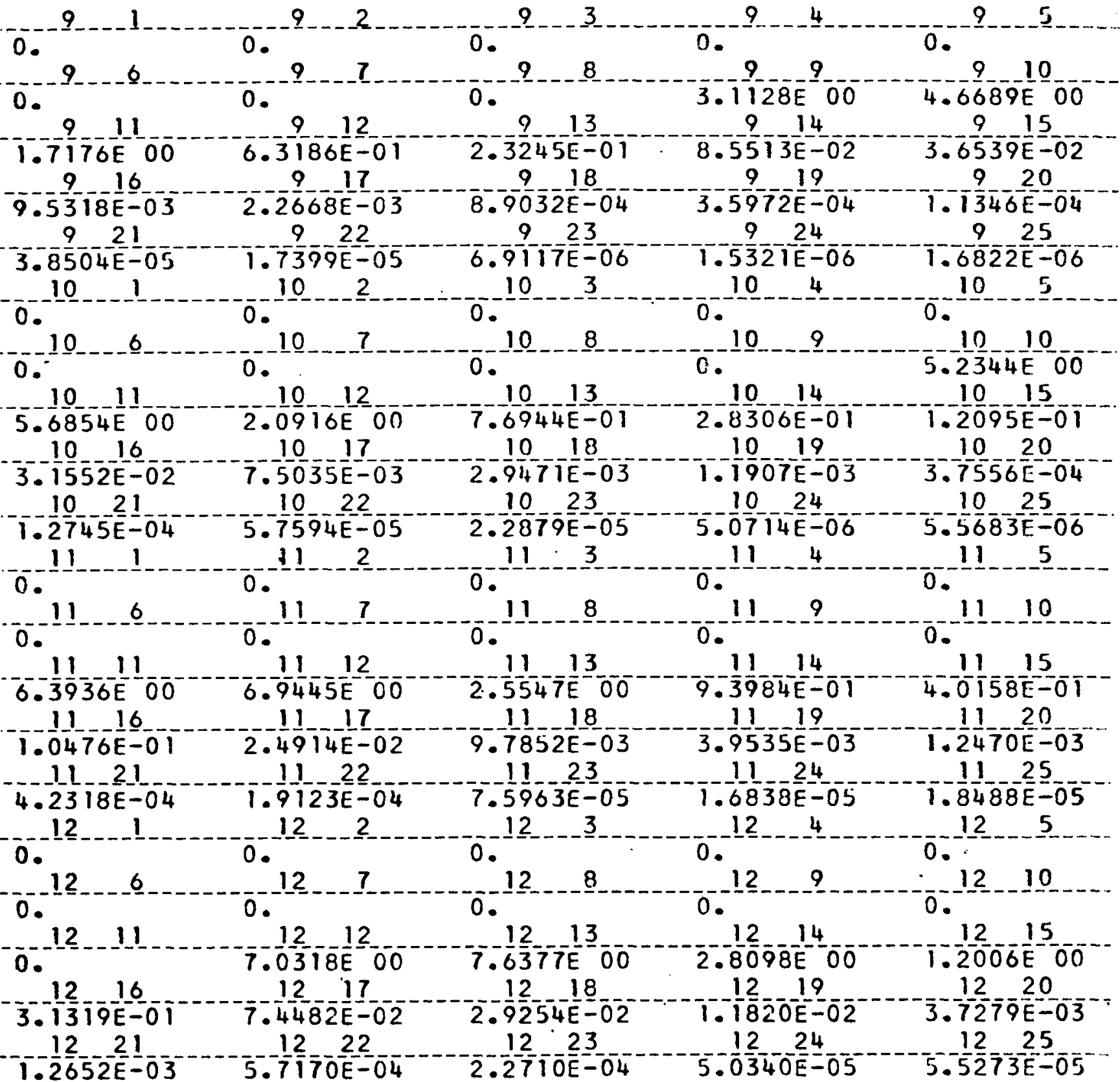


-H_FREE.

SIGMA SO (NIJN2)

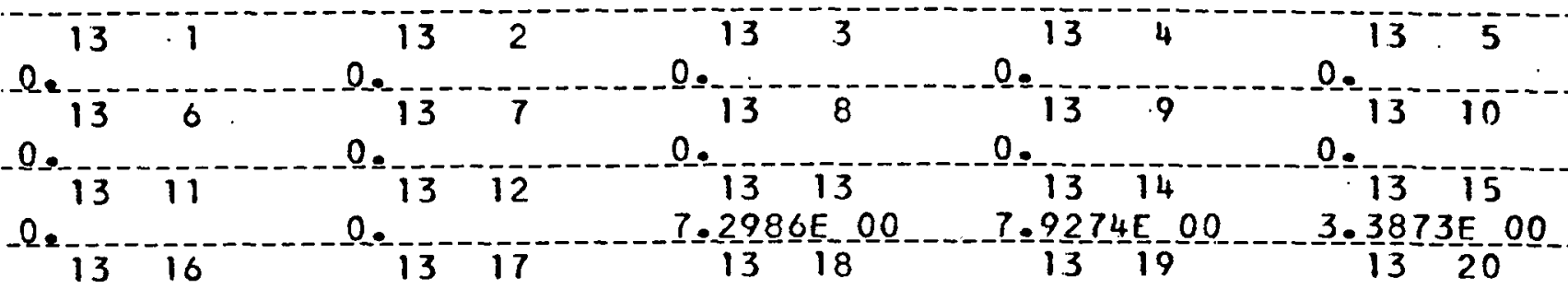
1321
$8.2536 \frac{6}{2}-02$
$13-24=0$
$13-\frac{1}{2}=-0$

$2=-1014 \mathrm{E}-\mathrm{E}=\mathrm{O}$

-3. $-5695 E-03$

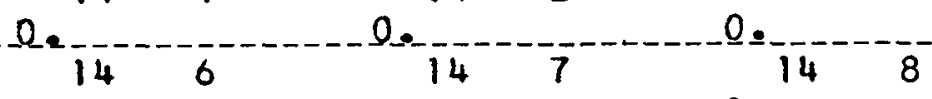

0.14 .11

1413

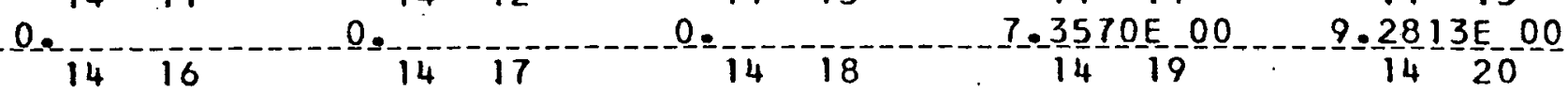

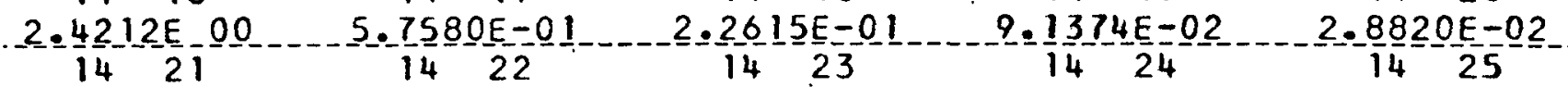

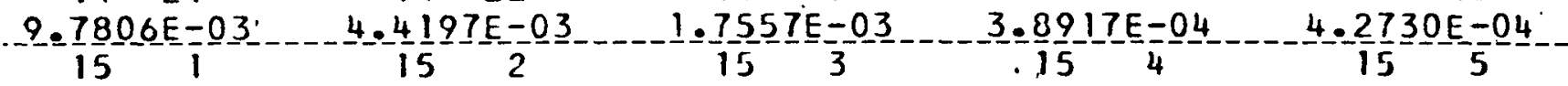

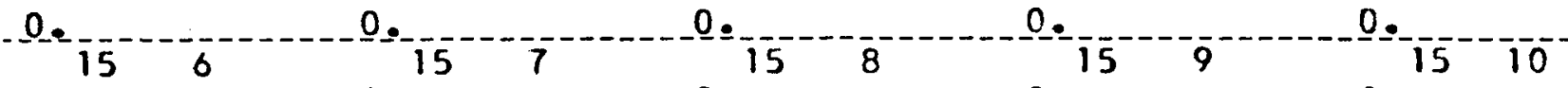

0. 15

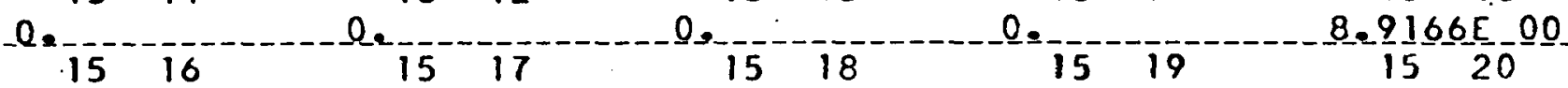

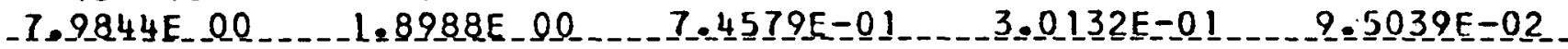

$$
1521 \quad 15 \quad 22 \quad 1523 \quad 15 \quad 24 \quad 15 \quad 25
$$

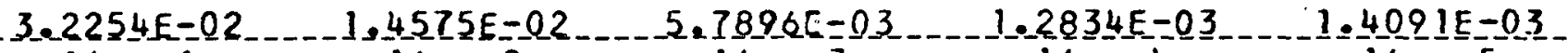

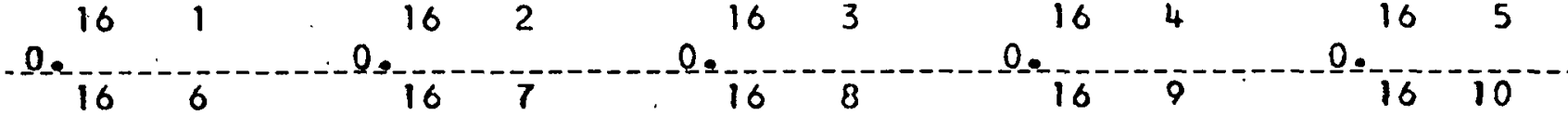

$0.16-16^{0} 16^{12}-16^{16}$

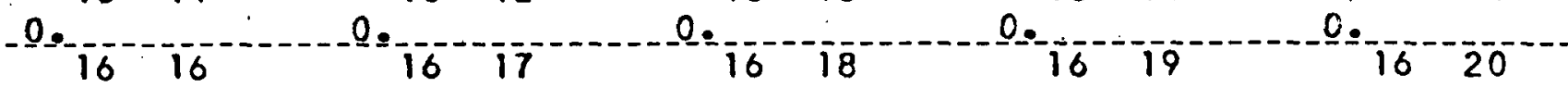
$.8 .6947 \mathrm{E} 00$

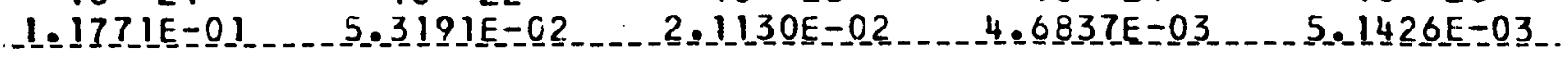


H FREE

N1 N2

SIGMA_SO_ (NI/N2)

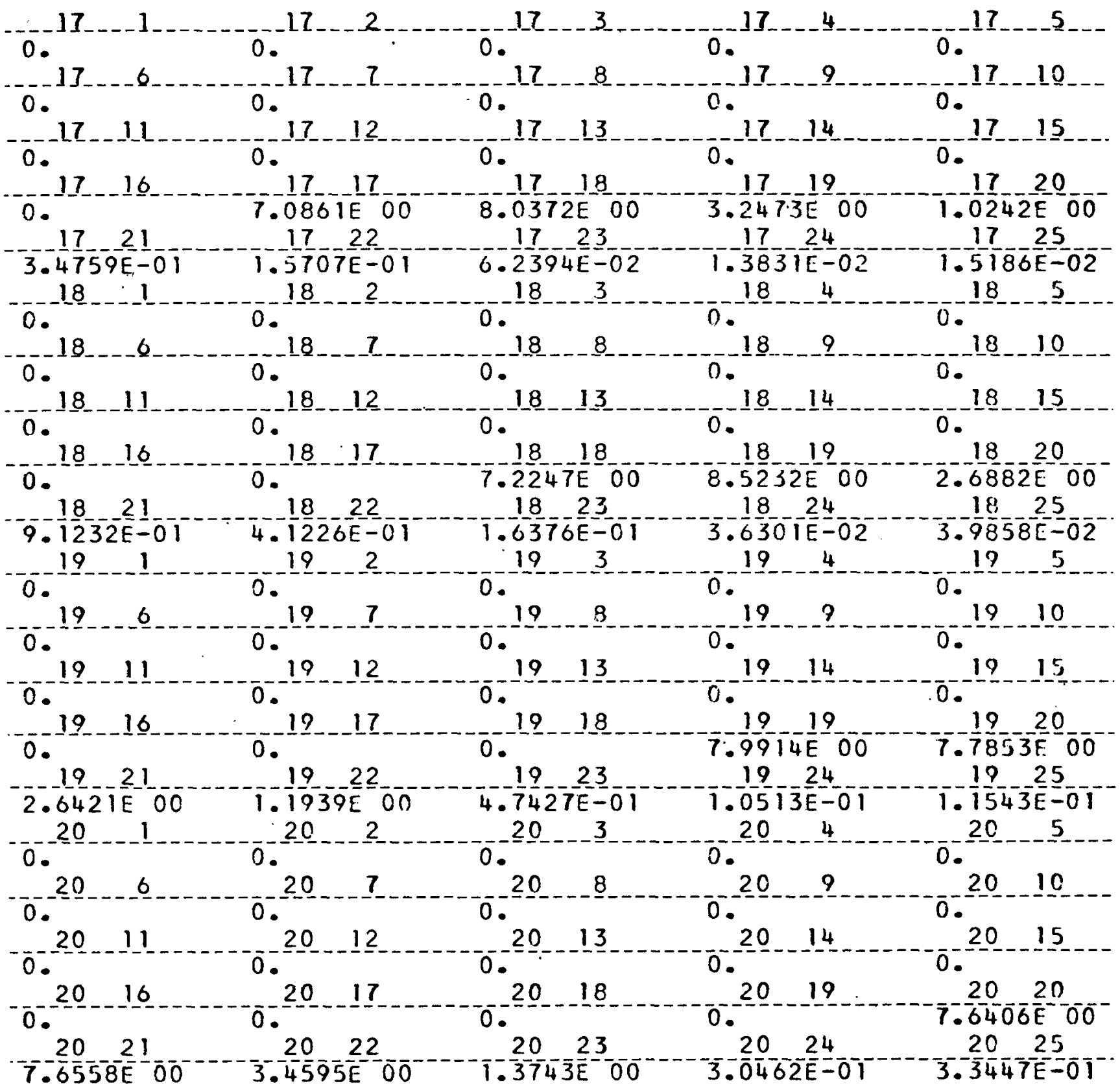




\section{H FREE}

TRANSFERS FROM GROUPS I THROUGH 4 TO GROUPS I THROUGH 8

$$
\text { Ni N2 }
$$

SIGMA_N,G_(N]IN2)

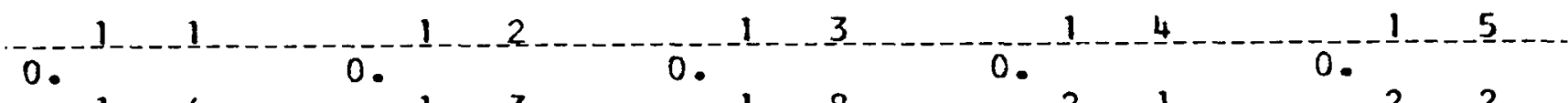
$0.1 .2183 E-0400.12 \ldots$

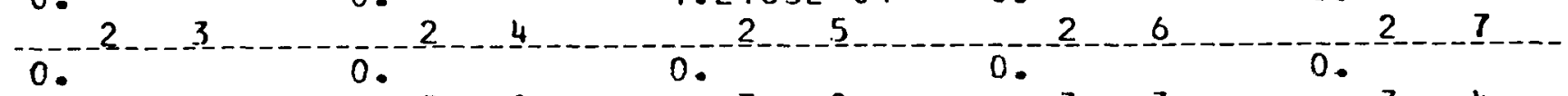

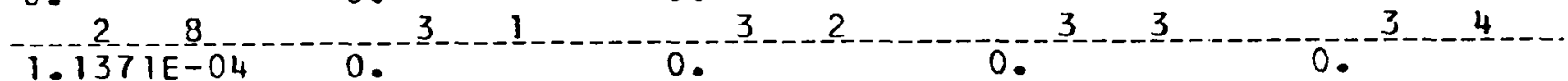

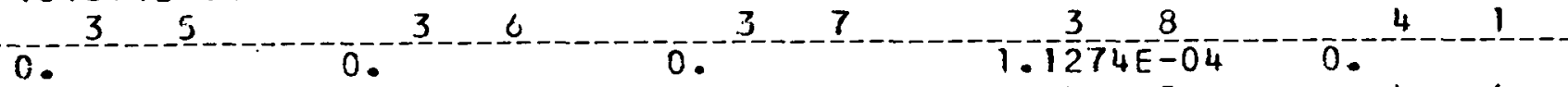

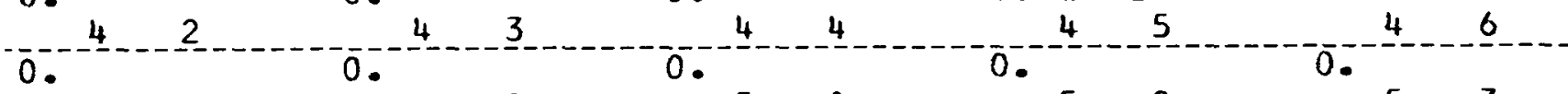

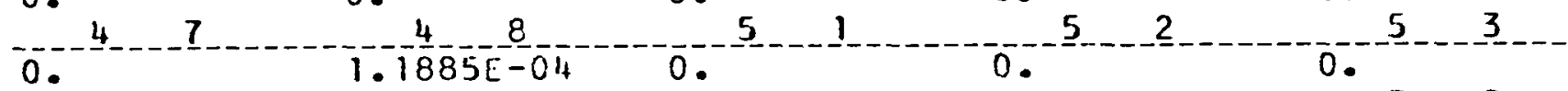

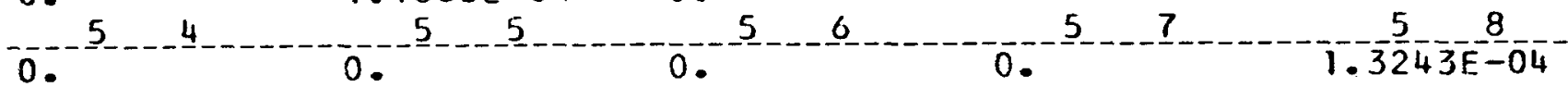

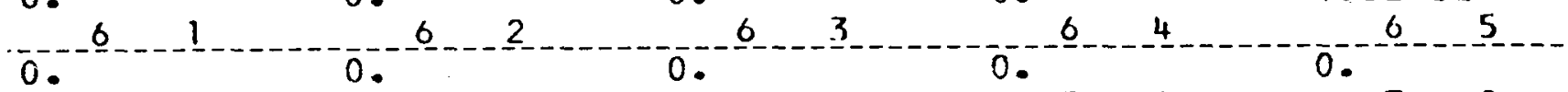

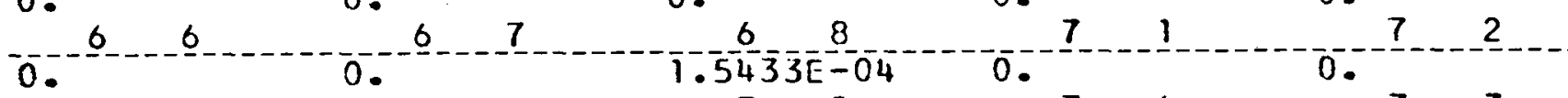

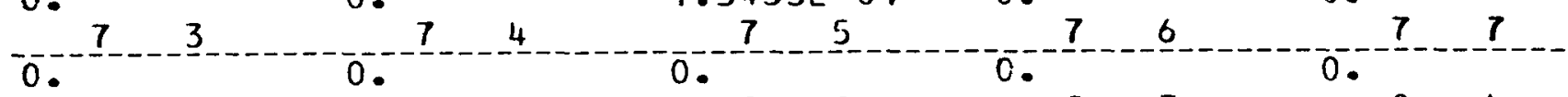

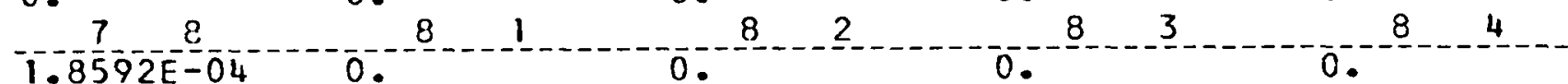

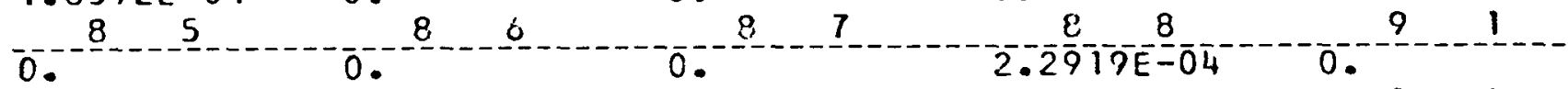

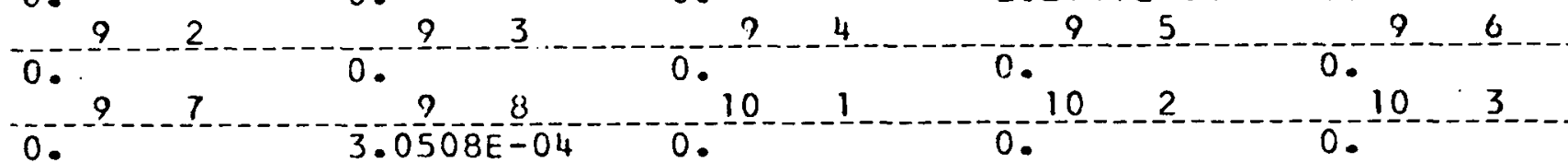

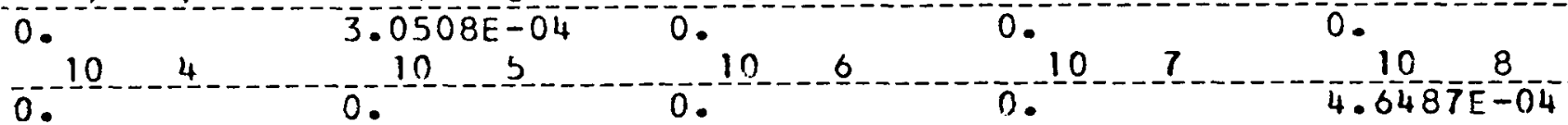

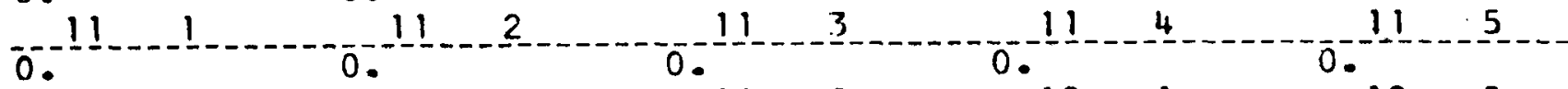

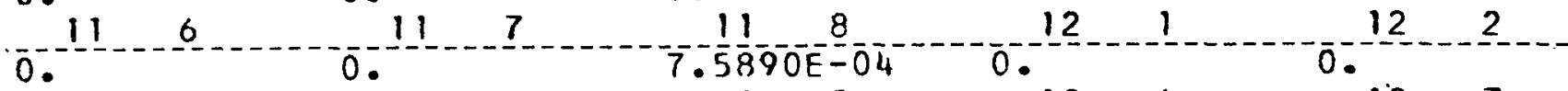

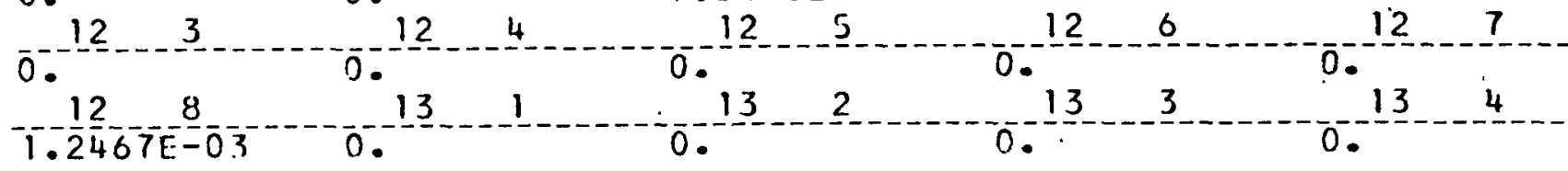


- H. FREE.

\section{SIGMA N.G (NITN2)}

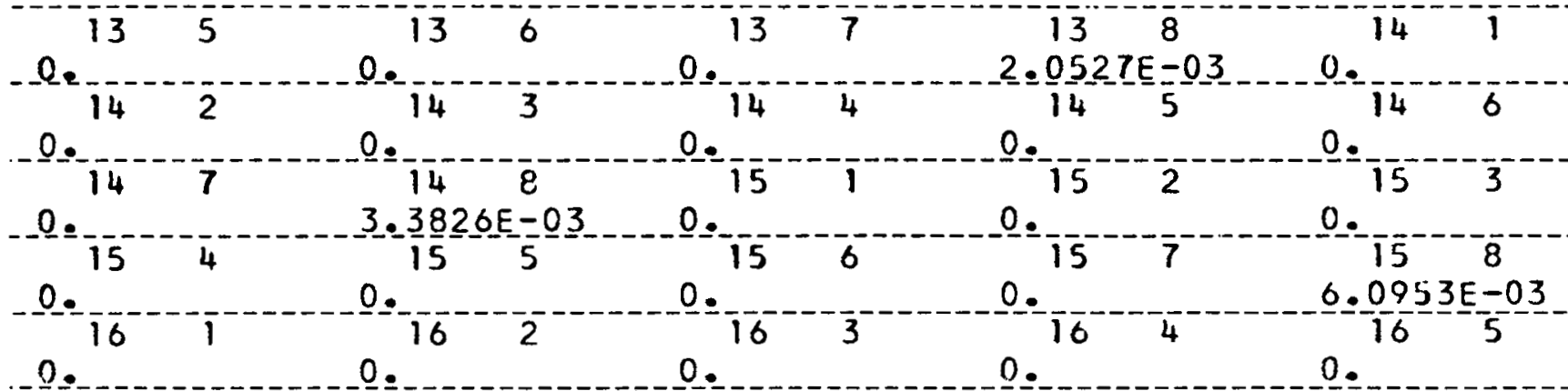

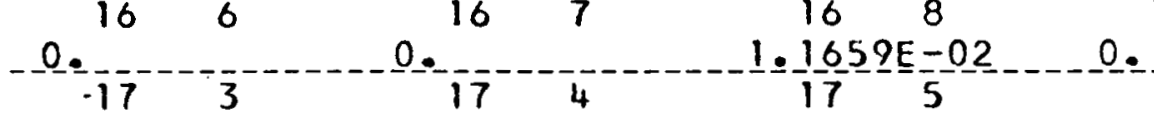

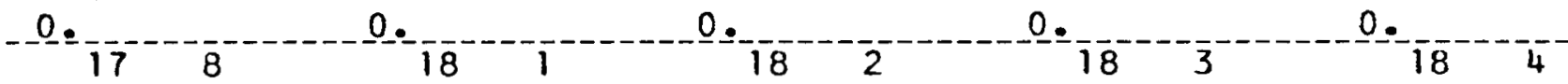

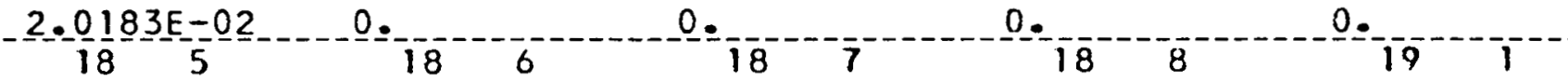

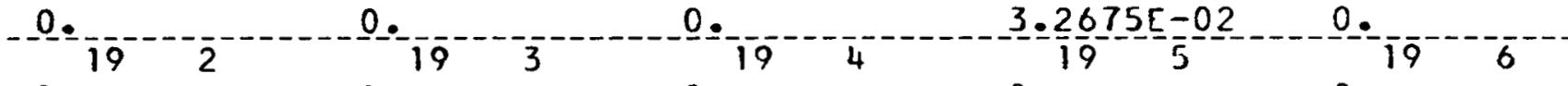

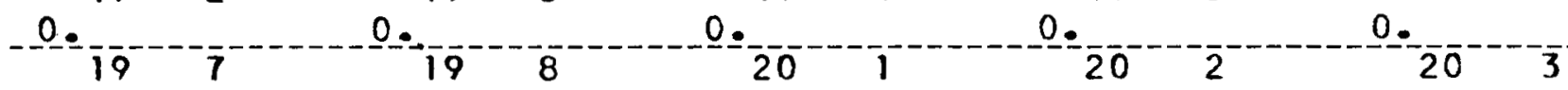

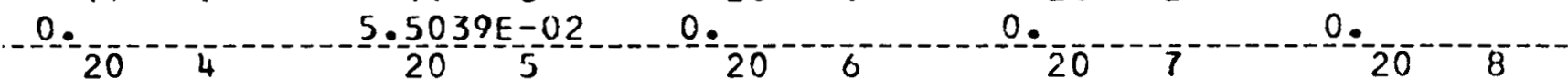

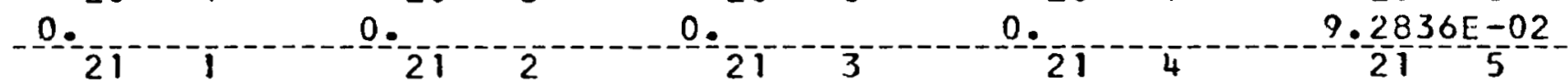

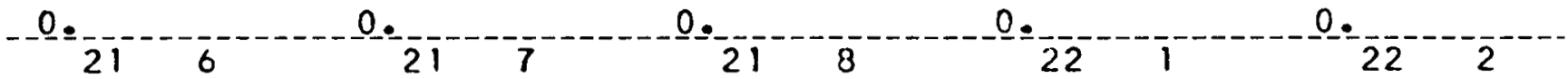

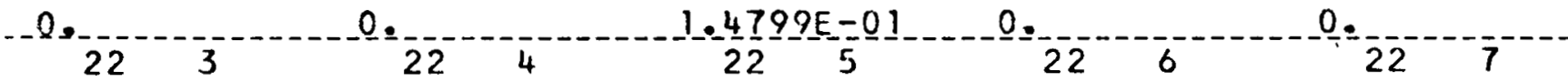

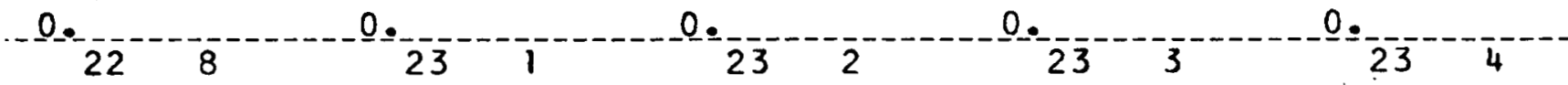

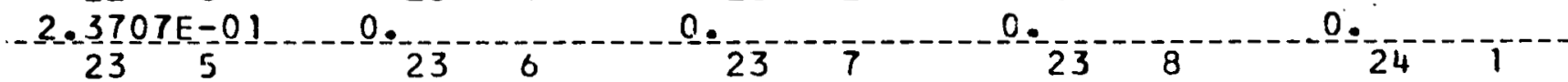

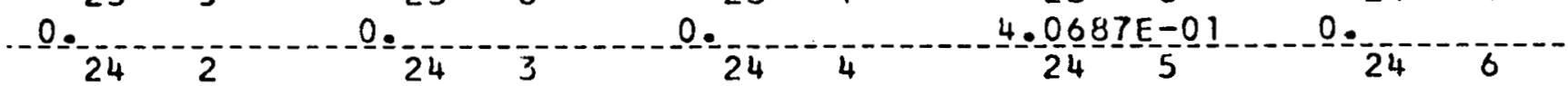

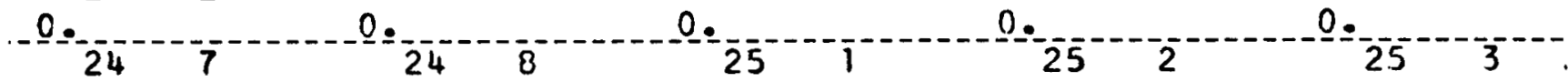

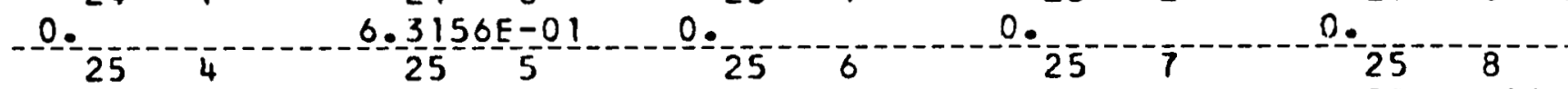

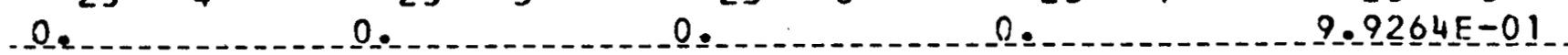


H FREE

SIGMA_N, G (NIIIN2)

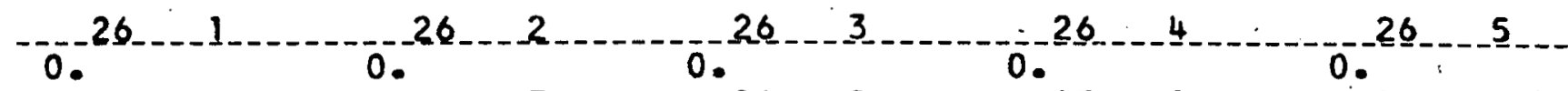

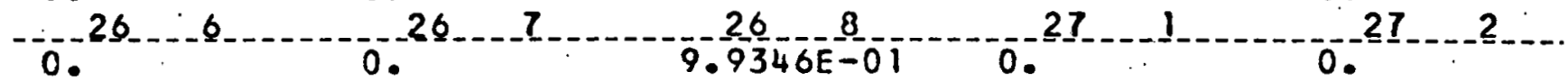

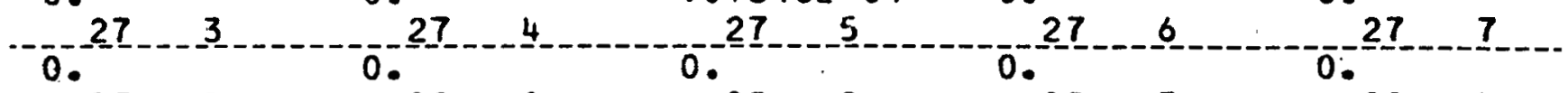
$-9.9446 \mathrm{E}-010.280 .28$

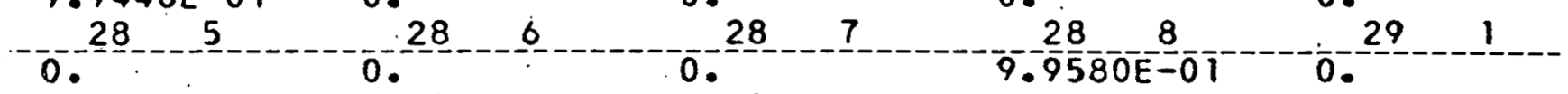
2920.292960

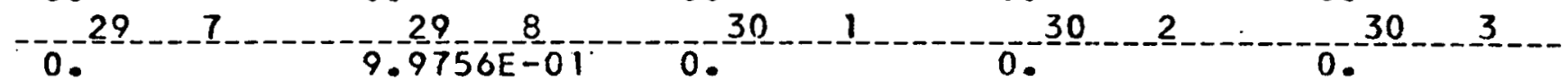

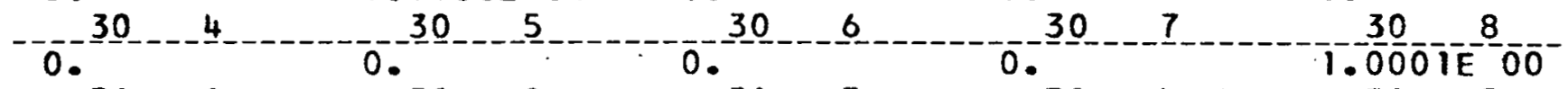

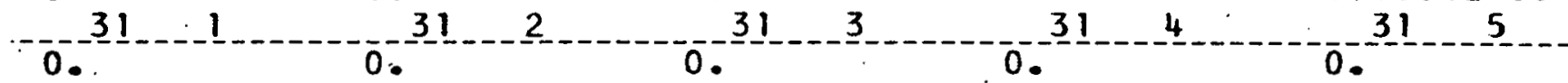

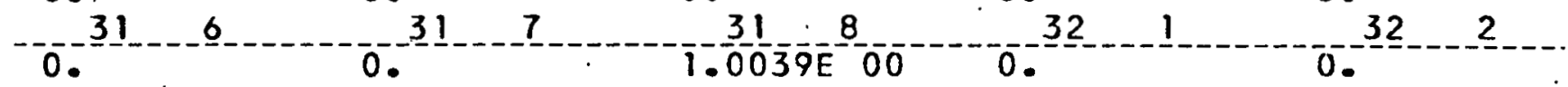

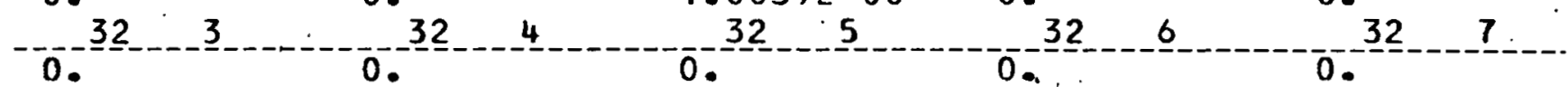

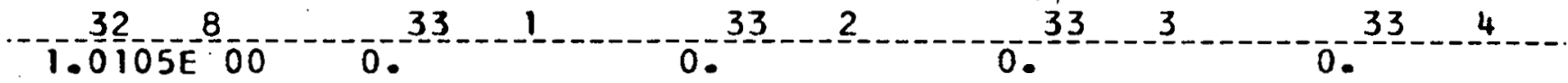

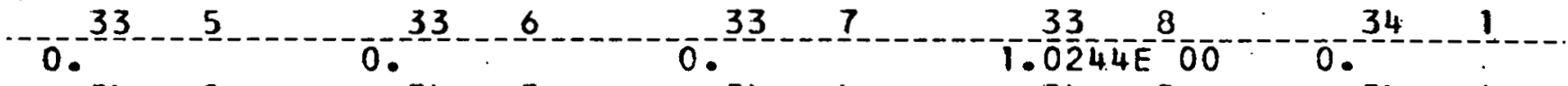

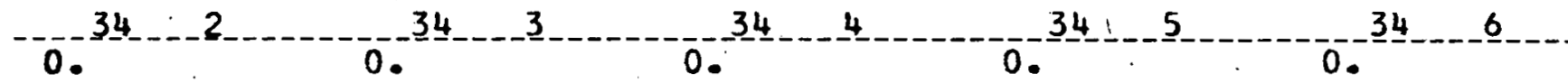
1.0609000 
DAIE 12/08/60.

DESIGNATION BORON 10
CODE NO.

5.1000
DENSITY FACTOR

$1.66249 \mathrm{E}$ O
SIGMA

LEVEL ENERGY SCATTER
XL_SIGMA

SIGMA SCATTER
TRANSPORT
SIGMA ABSORPTIO
$X I I S I G M A$

SCATTER 1

$1.000 E$ O $1.4418 \mathrm{E} 009.6946 \mathrm{E}-018.9348 \mathrm{E}-011.3295 \mathrm{E}-02-4.4194 \mathrm{E}-01$

2 6.065E 06 1.5061E_00 8.6203E-01_1.0003E_00 2. $7842 E=02=-4.0768 E=01$

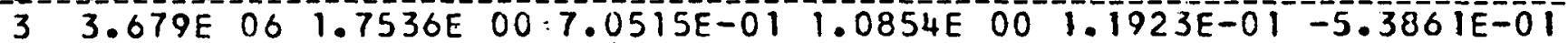

$42.231 \mathrm{E} 06-1.7641 \mathrm{E}-00 \quad 5.4703 \mathrm{E}-011.0968 \mathrm{E}-002.9118 \mathrm{E}-01-5.3779 \mathrm{E}-01$

5. $1.353 \mathrm{E} 062.0642 \mathrm{E} 004.4155 \mathrm{E}-011.2149 \mathrm{E} 002.1099 \mathrm{E}-01-6.8453 \mathrm{E}-01$

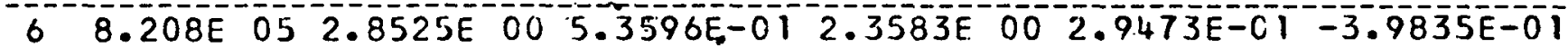

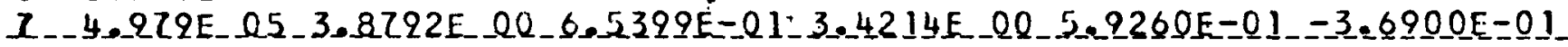

$8 \quad 3.020 E$ O5 4.0036E $007.0303 E-013.7549 \mathrm{~F} 00$ 8.4595E-01-2.0039E-01

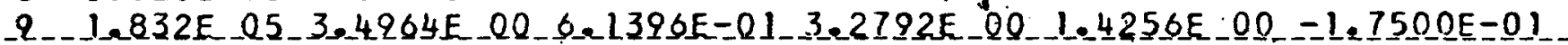

10 . 6.738E $042.2623 E .003 .9726 E-012.1218 E \quad 00 \quad 2.5507 E \quad 00-1.1323 E-01$

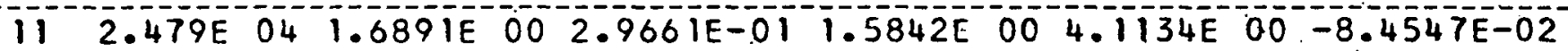

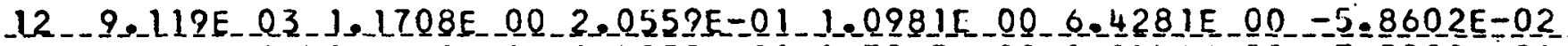

$133.355 \mathrm{E}$ 03 1.4781E. 00.2.5955E-0.1 1.3863E 00 1.0546E 01 $-7.3982 \mathrm{E}-02$

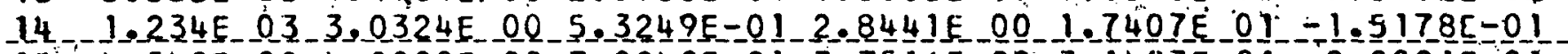

15 4.540E $024.0000 E$ O0 7.0240E-01 3.7516E $00.3 .1483 E 01-2.0021 E-01$

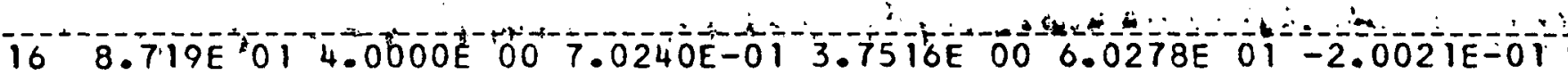

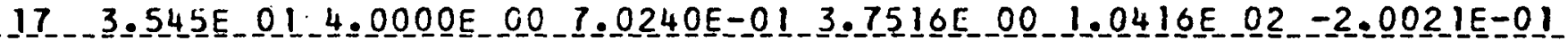

$181.304 \mathrm{E} 014.0000 \mathrm{E} 00-7.0240 \mathrm{E}-013.7516 \mathrm{E} 00-1.6855 \mathrm{E} 02-2.002 \mathrm{IE}=0 \mathrm{1}$

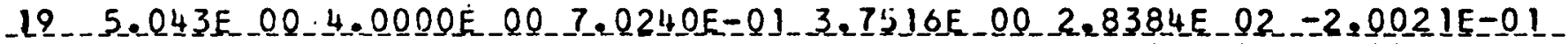

$20 \quad 1.445 E$ OO 4.00.00E $00 \quad 7.024 .0 E-013.7516 E .004 .7818 E 02-2.0021 E-01$

$216.826 E-014.0000 E 007.0240 E-013.7516 E 007.0222 E 02-2.0021 E-01$

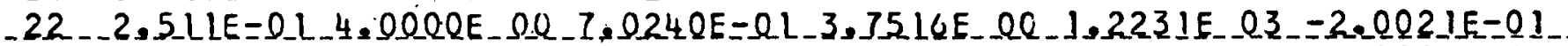

$239.237 E-024.0000 E$ 00 7.0240E-01 3.7516E 00 2.1035E $03-2.0021 E-01$

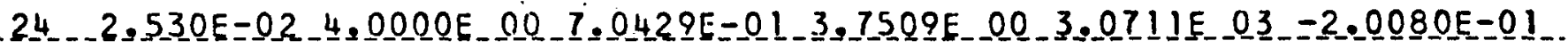

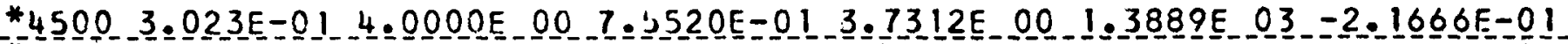

* $4000-2.718 E-0 T 4.0000 E-007.5520 E-013.7312 E-00-1.3889 E-03-2.1666 E-01$

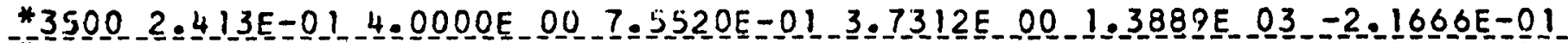

* 30002 2. $109 E-01.4 .0000 E$ O0 7.5520E-01 3.7312E 00 1.3889E 03 -2.1666E-01

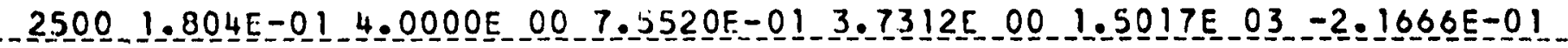

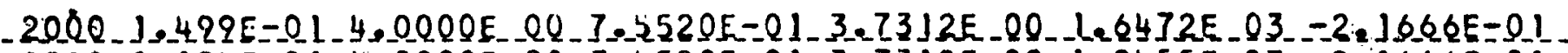
$15001.194 \mathrm{E}-014.0000 \mathrm{E}$ 00 $7.5520 \mathrm{E}-013.7312 \mathrm{E}$ 00 $1.8455 \mathrm{E} 03-2.166 .6 \mathrm{E}-01$

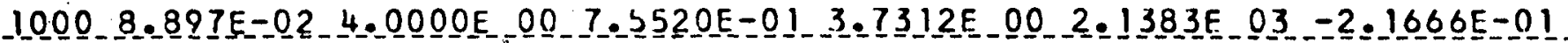
$5005.850 E-02$ 4.000OE 00 7.5520E-01 3.7312E 00 2.6371E 03-2. $1666 \mathrm{E}-01$.

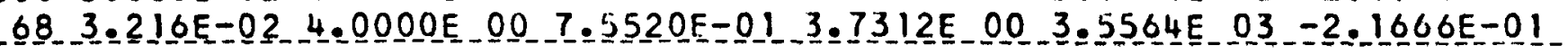

*Thermal cross Bections taken fram APEX-515:

NOTE: For a sumary of the sources of data see APEX-704. 
BORON 10

THERMAL TRANSFER MATRICES

TEMPERATURE
SIGMA

\begin{tabular}{|c|c|c|c|c|}
\hline-2$]_{-} .21$ & $21-22$ & 21 & 21,24 & 21 \\
\hline $\begin{array}{c}3.0771 E 00 \\
2221\end{array}$ & $\begin{array}{c}8.6846 E-01 \\
22\end{array}$ & $\begin{array}{c}5.1373 E-02 \\
2223\end{array}$ & $\begin{array}{c}2.2777 E-03 \\
22.24\end{array}$ & $\begin{array}{l}7.8066 \mathrm{E}-04 \\
22\end{array}$ \\
\hline $.0056 \mathrm{E}-01$ & $2.4909 \mathrm{E} 00$ & $5.5298 \mathrm{E}-01$ & $3.8879 E-02$ & $1.6718 \mathrm{E}-02$ \\
\hline $1.5431 \mathrm{E}-0 \mathrm{1}$ & $1.6058 \mathrm{E} 00$ & $1.8704 \mathrm{E} 00$ & $2.5603 E-01$ & $1.2141 \mathrm{E}-01$ \\
\hline $5.3221 E-02$ & $8.8121 E-01$ & $1.9370 \mathrm{E} O 0$ & $7.1126 E-01$ & $4.1730 \mathrm{E}-01$ \\
\hline$-5.8112 E-02$ & $1.2084 \mathrm{E} 00$ & $3.0240 \mathrm{E}-00$ & 1.3 & $1.4029 \mathrm{E} 00$ \\
\hline
\end{tabular}

TEMPERATURE
SIGMA

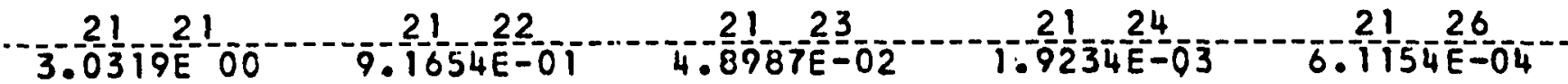

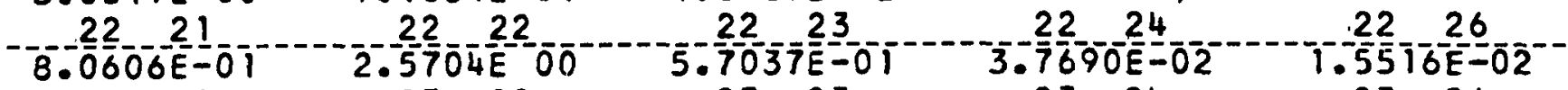

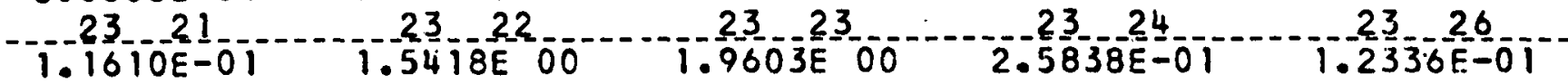

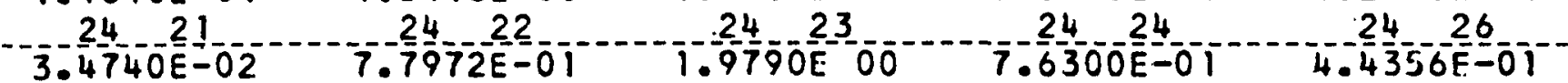

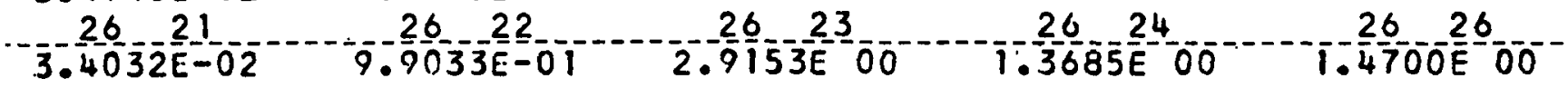


BORON 10

THERMAL TRANSFER MATRICES

TEMPERATURE
SSIGMA $\begin{gathered}\text { SIGMA } \\ \text { DEG }\end{gathered}$

\begin{tabular}{|c|c|c|c|c|}
\hline 21 & 21 & 21 & 24 & \\
\hline $.7974 \mathrm{E} 00$ & $1.1618 \mathrm{~B} 00$ & $3.9784 E-02$ & $8.6382 \mathrm{E}-04$ & $2 E-0$ \\
\hline$-22-21$ & 22 & 23 & 24 & 29 \\
\hline $\begin{array}{l}7144 \mathrm{E}=01 \\
23 \quad 21\end{array}$ & $2.8261 E 00$ & $6.5845 E-01$ & $2996 \mathrm{E}-02$ & $\begin{array}{l}1017 E-0 \\
23\end{array}$ \\
\hline $3.2362 \mathrm{E}-02$ & $1.3292 \mathrm{E} 00$ & $2.1791 \mathrm{E} 00$ & $3.2264 \mathrm{E}-0 \mathrm{~T}$ & $1.3670 \mathrm{E}-0$ \\
\hline 24 & 24 & $24-23$ & 24 & \\
\hline $\begin{array}{c}4.4031 E-03 \\
29\end{array}$ & $\begin{array}{c}4.2143 E-01 \\
29\end{array}$ & $\begin{array}{r}2.0452 \mathrm{E} \\
2923\end{array}$ & $8204 E-$ & $2 E-0$ \\
\hline $.6262 E-03$ & $8282 \mathrm{E}-01$ & - & $1.4888 \mathrm{E}$ & $7500 \mathrm{E}$ \\
\hline
\end{tabular}

TEMPERATURE

DEGe._F.-

2.0000E 03

5.6961E_OO

SIGMA

SCAIIER

SIGMA TRANSPORI

$5 \cdot 3133 E_{-} 00$

5.15896E_0 3

SIGMA

ABSORPIION
NU' SIGMÁ

EISSION

$\overline{\mathrm{N}} \mathrm{i} 2$

SI GMA S_. (N1 IN2)

2121

$2.6679 \mathrm{E} 00^{-1.2963 \mathrm{E}} 0 \mathrm{O}^{-3.5130 \mathrm{E}-02}$

- $-22-21$

2222

2223

2.9017E OO

$7 .-1425 \mathrm{E}-0 \mathrm{~T}$

...23 21.

$1.4830 \mathrm{E}-02$

- $-2.3250 \mathrm{E}-03 \mathrm{3}-2.9401 \mathrm{E}-0 \mathrm{~T}$

$1.1475 \mathrm{E} 00$

$2-\frac{23}{36}-23$

$-2 !-24$

$5 .-\frac{2}{3} \frac{1}{80}=\frac{24}{7 E}-0 \overline{4}$

ㅇ.

$6.0150 E-04 \quad 2.2384 E-01$

$2.3617 E$
24

$3.0939 \mathrm{E}-02$

$23396-02$

$3.4082 \mathrm{E}-01$

$24=24$

$1.0902 \mathrm{E} 00$

$-\frac{21}{560}-\frac{30}{\mathrm{E}-05}$

$22 \quad 30$

$9.1740 E-0 \overline{3}$ $2.0213 \mathrm{E} 00$

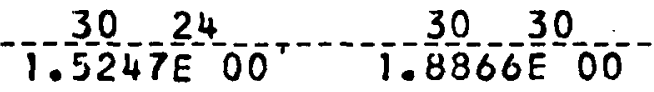
$2.0603 E 00$ 
BORON 10

- IHERMAL_TRANSFER MAIRICES

- LEMPERAIURE

SIGMA

S_LGMA

SIGMA

NU_ SIGMA

DEG. F. SCATTER

TRANSPORT

ABSORPTION

FISSION

$1.5000 \mathrm{E}$ O3 $5.3956 \mathrm{E} 00 \mathrm{5}$

\section{SIGMA S $(N) / N 2)$}

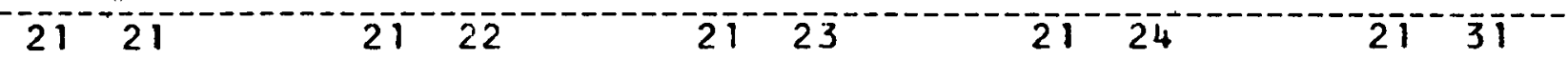

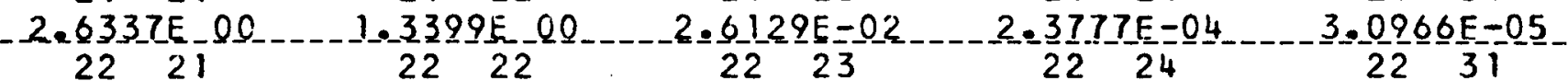

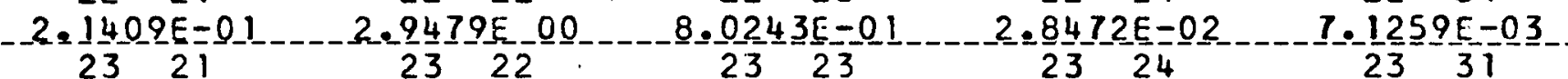

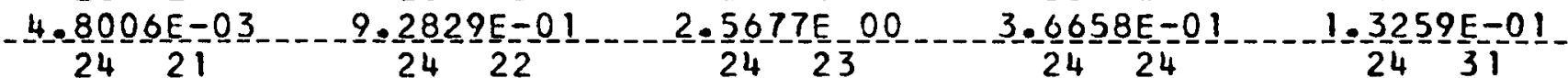

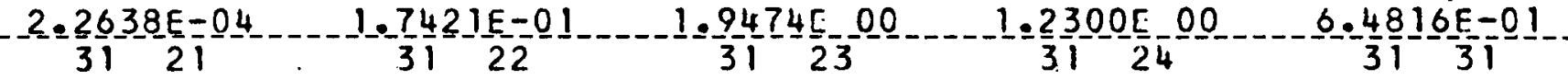

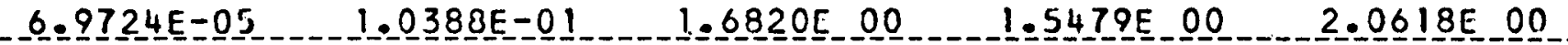

TEMPERATURE
DEG. FIGMA
F. SCATTER

SIGMA S $(N)$ NIN2)
$2 i-2 i$
$21^{-12}$
$\overline{2} \overline{1}=-\overline{3}$
$21^{24}$
$21^{-12}$
$2.0002 \frac{E}{2}=0$
$9 \cdot \frac{3}{2} \frac{867 E}{2}-0$
$3.4879 E=05$
$2.7434 E-006$
$2.917 \mathrm{SE}-\frac{O}{22}$
$9.5713 E-01$
2.5 186E-02
4. $8379 E-03$
$6.6250 E-01$
$2 . \frac{79}{24}-\frac{8}{2}-00$
$4.0914 E=01$
$2 \frac{3}{3} 2$
$3843 E=05$
2422
$.9616 \mathrm{E} 00$
2424
1. $-\frac{29}{24} 34 E-01$
$32=2 \mathrm{~T}$
$3 \frac{2}{2}$
$32-23$
$1=\frac{1704}{32} \mathrm{E}-\mathrm{QO}$
7. $\frac{8}{3} 505 E-01$
3. $1035 E-02$
$1.2136 \mathrm{E} 00$
$1.5372 E \quad 00$
2.2990 E 00

3.0905E 00

$-9.5 \frac{5289}{2}-02$

$-7-\frac{8}{2} \frac{54}{4}-\frac{E}{2}=04$

2. $1080 E-06$ 
BORON 10

THERMAL TRANSFER MATRICES

\begin{tabular}{|c|c|c|c|c|}
\hline $\begin{array}{l}\text { TEMPERATURE } \\
\text { DEG }\end{array}$ & $\begin{array}{l}\text { SIGMA } \\
\text { SCAIIER }\end{array}$ & $\begin{array}{l}\text { SIGMA } \\
\text { TRANSPORI }\end{array}$ & $\begin{array}{c}\text { SIGMA } \\
\text { ABSORPIION }\end{array}$ & $\begin{array}{l}\text { NU SIGMA } \\
\text { EISSION }\end{array}$ \\
\hline 02 & $4.25] 0 E-00$ & $4.4317 E_{-} 00$ & $=28433 E_{-} 03$ & O- \\
\hline & & $\begin{array}{l}\text { N2 } \\
\text { NII }\end{array}$ & & \\
\hline-21$]-21$ & $8.2159 E-01$ & $-\frac{21}{2.8229 E-03}$ & $1.6078 \mathrm{E}-06$ & 33 \\
\hline $4.8076 \mathrm{E}-02$ & $2.7384 E \quad 00$ & $1.1928 \mathrm{E} 00$ & $1.8501 \mathrm{E}-02$ & $-2.1885 E-03$ \\
\hline $2.3652 \mathrm{E}-05$ & $3.4530 \mathrm{E}-01$ & $3.0262 \mathrm{E} 00$ & $5.0116 \mathrm{E}-01$ & $\begin{array}{c}23 \\
1.2734 \mathrm{E}-01 \\
24\end{array}$ \\
\hline 0. & $1.5507 E-02$ & $1.4748 \mathrm{E} 00$ & $1.7067 \mathrm{E} 0$ & $8.0293 E-01$ \\
\hline & $3.2313 E-03$ & $6.6690 \mathrm{E}-01$ & $1.4317 \mathrm{E} O$ & $2.6491 \mathrm{O} 00$ \\
\hline
\end{tabular}

TEMPERATURE SIGMA SIGMA SIGMA

DEG _ F. - _ SCAITER _._._IRANSPORI _ _ ABSORPIION _ _ EISSION

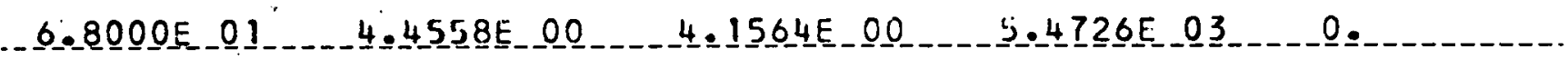

in

SIGMA S $($ N] $I N ?)$

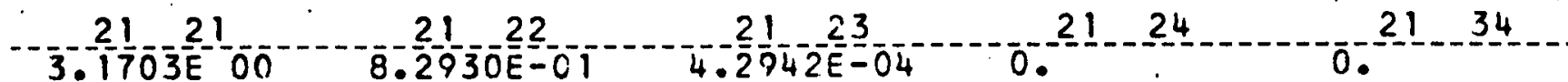

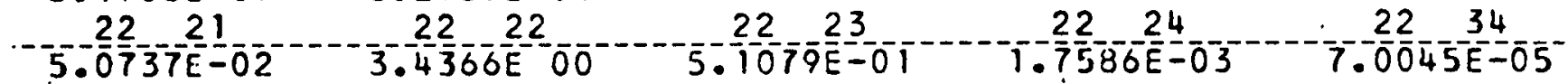

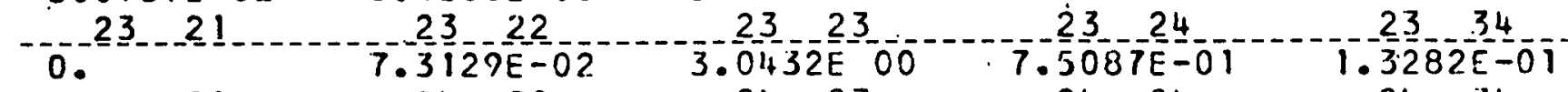

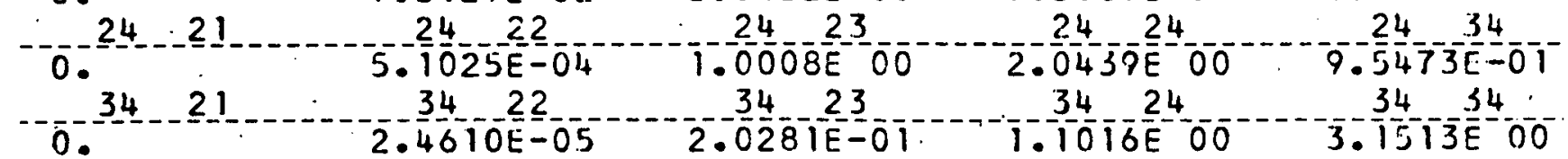




\section{SIGMA 50 (N) INAT)}

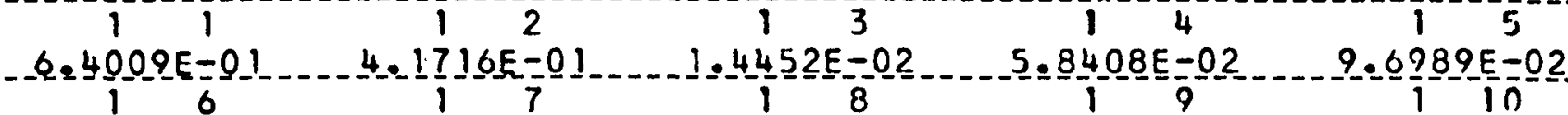
. $9.3810 E-02 \quad 0.2010 E-02 \quad 3.2744 E-02 \quad 1.9025 E-02$

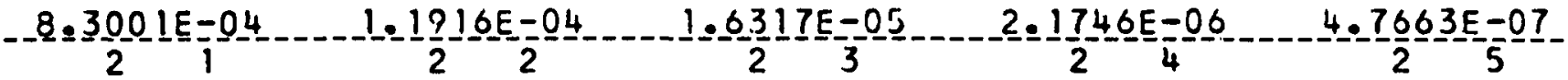
…

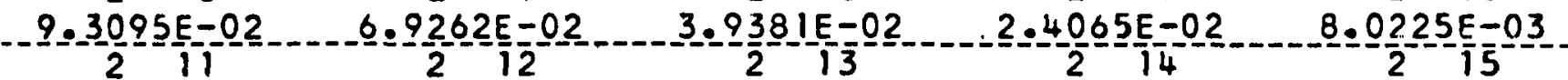

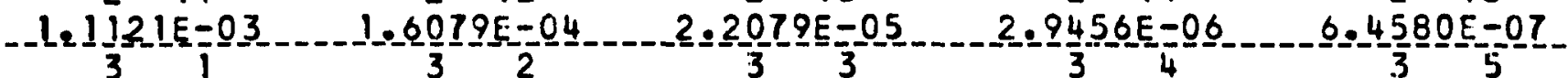
-0.

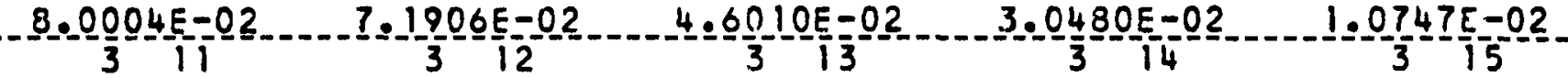

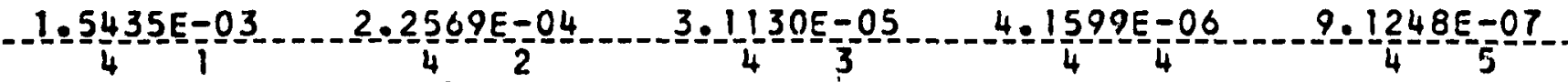
-0. ..5.5461E-02 $-6.3029 E-02 \quad-4.6775 E-02 \quad 3.4328 E-02 \quad 1.2993 E-02$

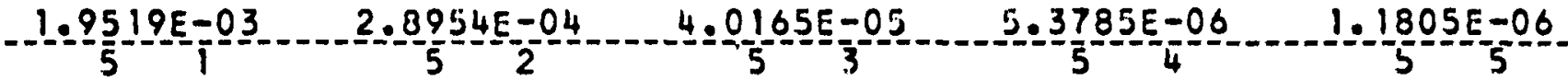

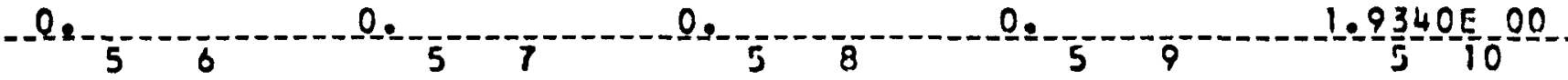

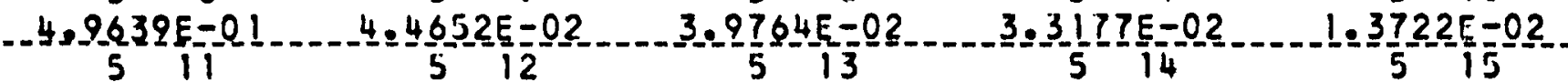

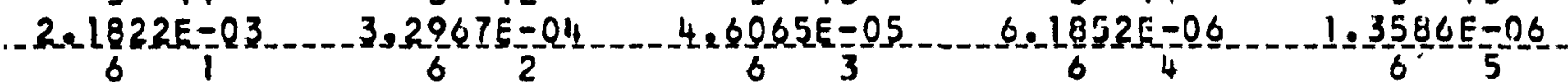
0. Q $02-6$ 0. $0:$ $1,7325 E 00 \quad 1.0215 E 00 \quad 2.40311 E=02 \quad 2.3517 E-02 \quad 1.08345-02$ $61^{1}$ 614 6 - 15

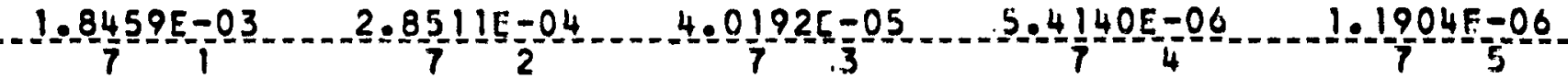

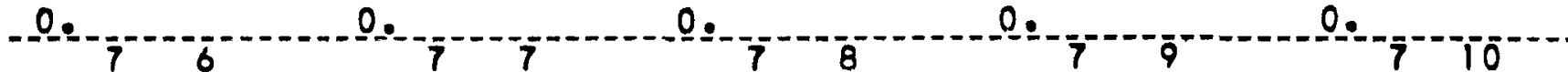
Q.

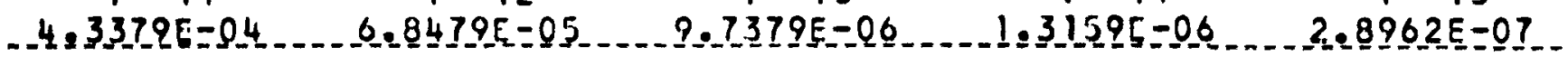


BORON 10

TRANSFERS FROM GROUPS I THROUGH 34 TO GROUPS I THROUGH 21

\section{Ni $\mathrm{N}^{2}$ \\ SIGMA N, G INIIN2)}

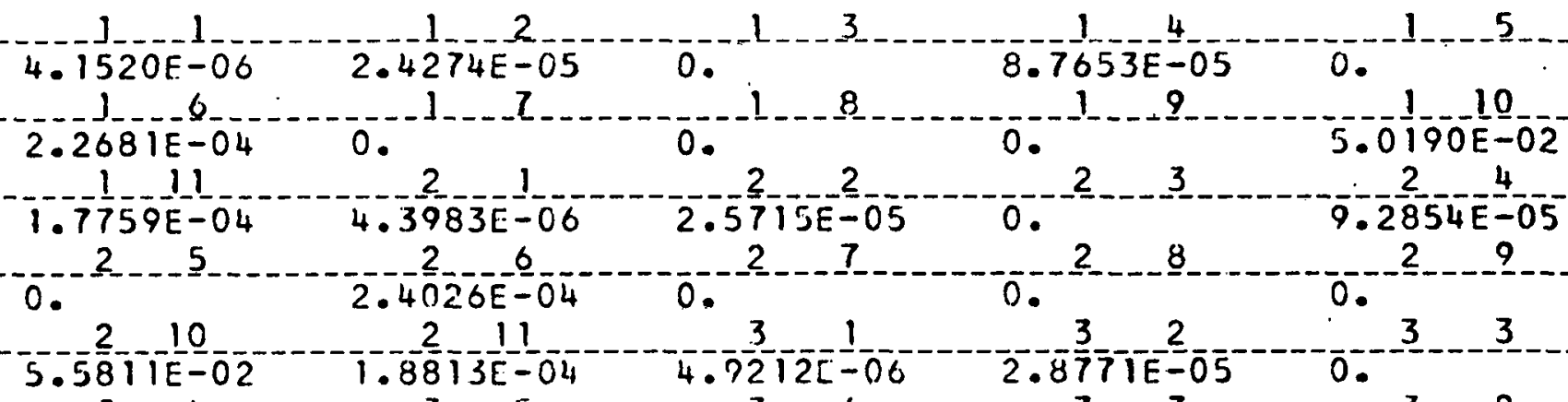

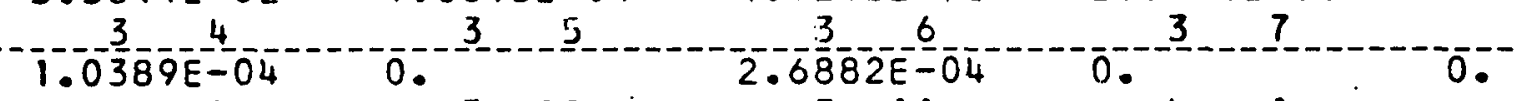

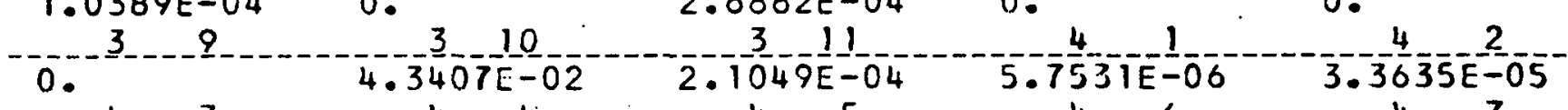

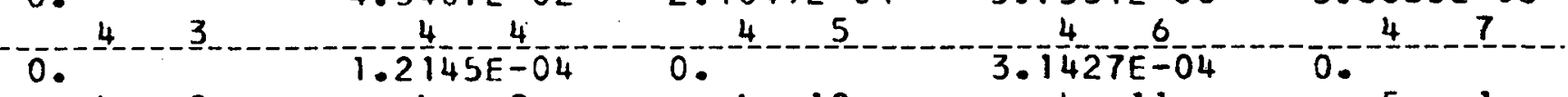

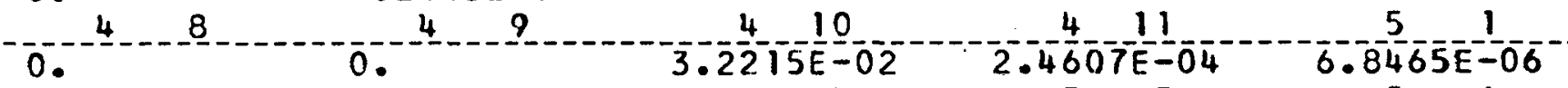

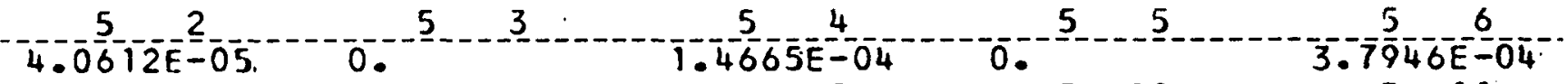

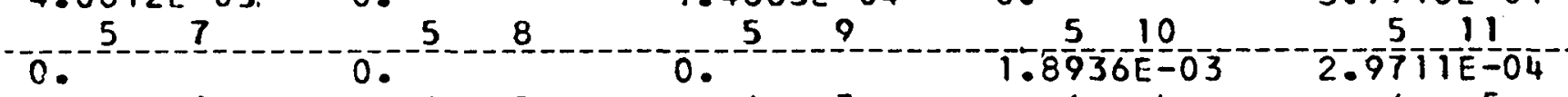

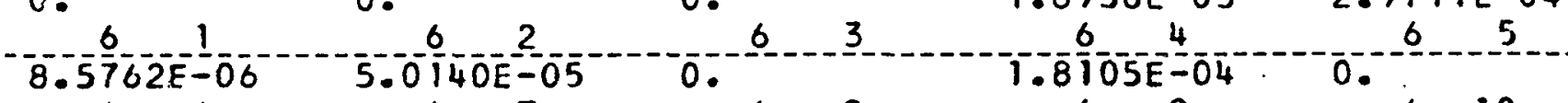

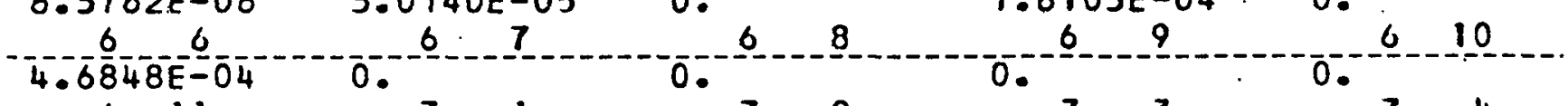

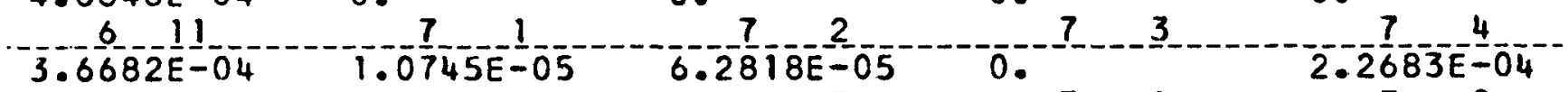

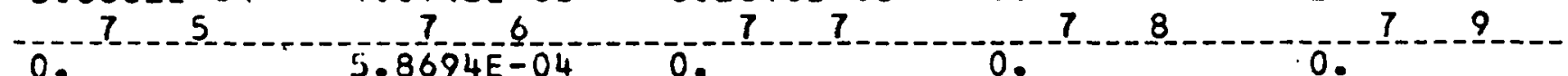

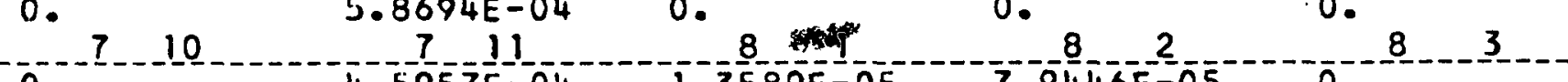

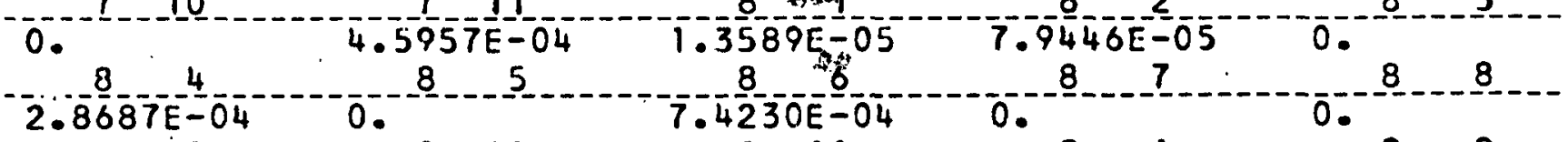

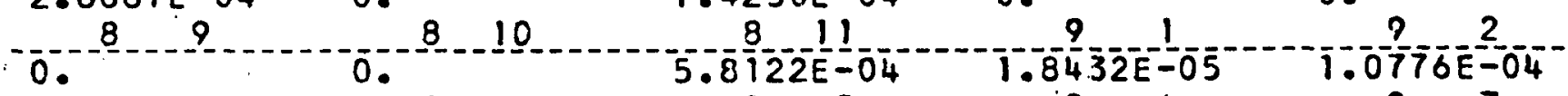
0.9
0.9
0. 
-BORON_ JO.

$\mathrm{N} 1$

SIGMA N,G (NIJN2)

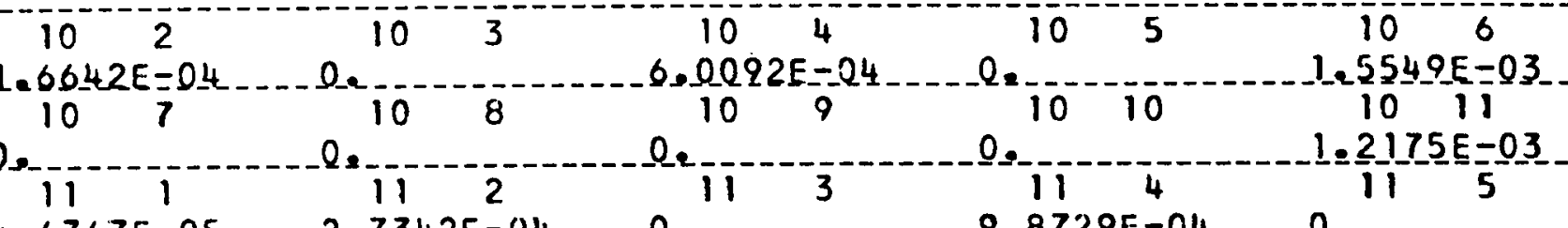

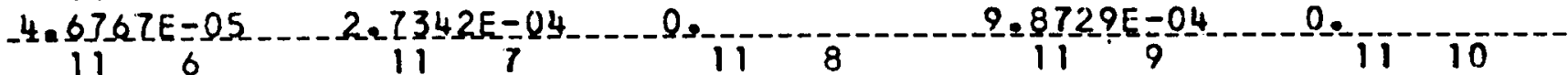

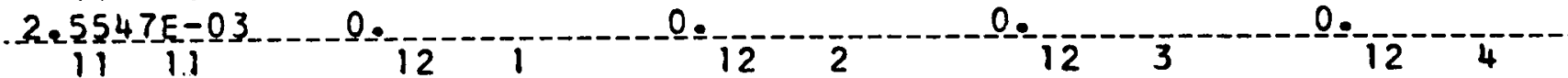

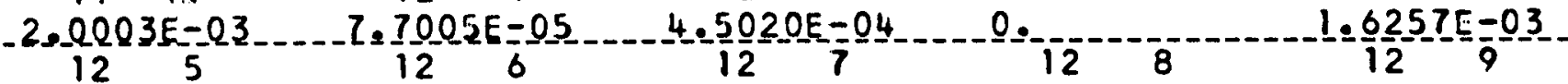

Q. 12 - 4.2065E-03 12 11 0.13

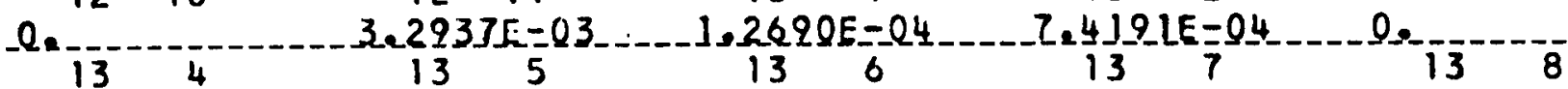

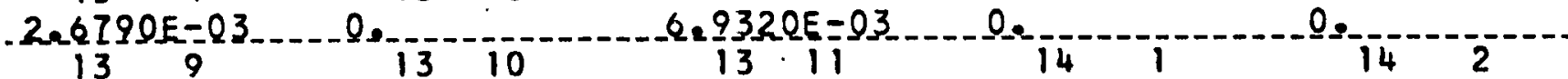

$Q_{2} 14$

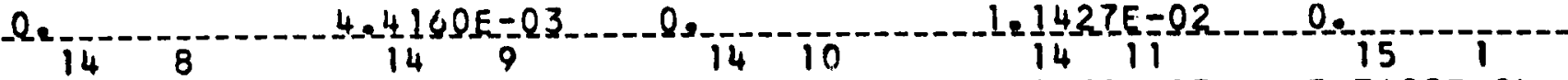

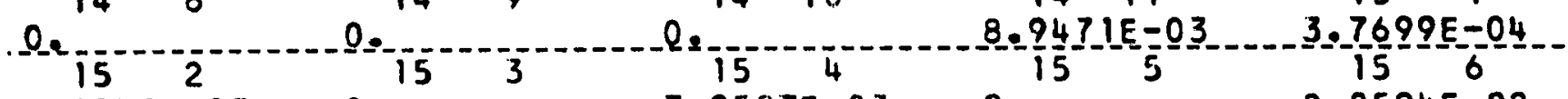

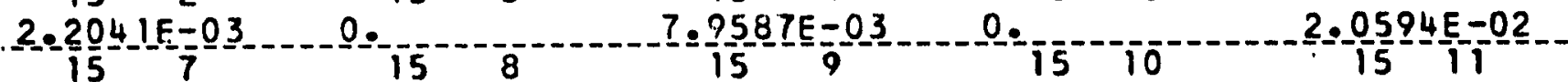

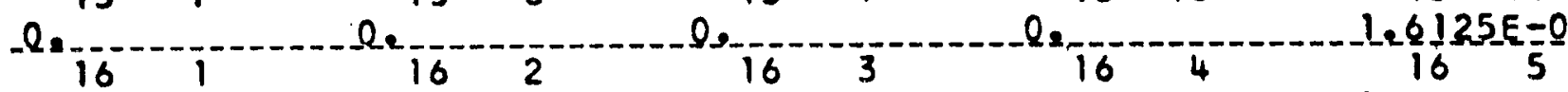

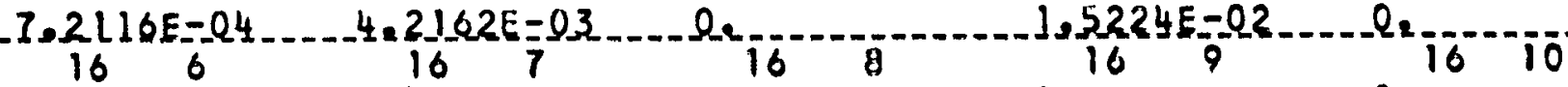

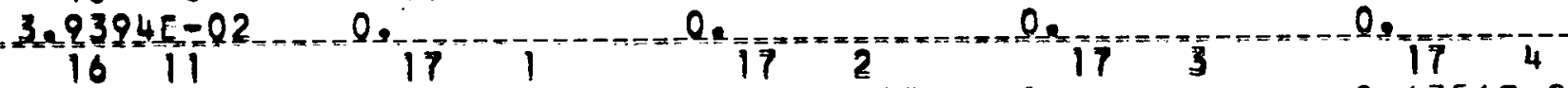

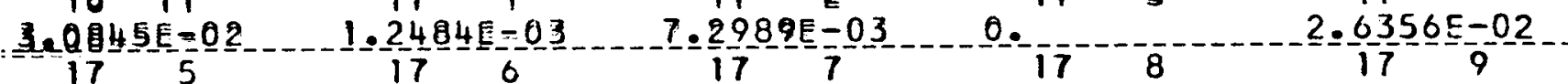

$0.0 .81975=02 \quad 0.1717$

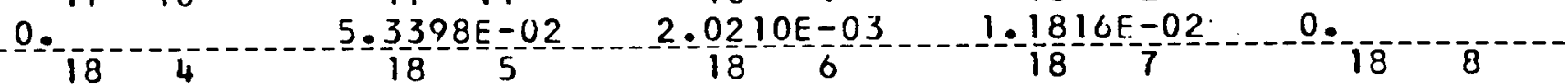

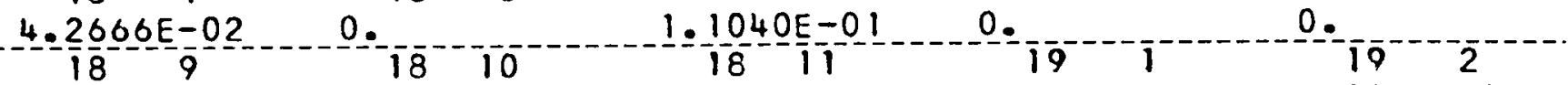

ㅇ.. 
BORON 10

$\mathrm{N} 1 \quad \mathrm{~N} 2$

SIGMA_N,G_(N_) N22)

\begin{tabular}{|c|c|c|c|c|}
\hline $0^{19}$ & $19-4$ & $0.12 \ldots 5$ & & 19 \\
\hline 0.19 & $\begin{array}{c}7.1869 E-02 \\
19\end{array}$ & $0.12 \quad 10$ & $\begin{array}{c}1.8596 \mathrm{E}-01 \\
19\end{array}$ & 0.20 \\
\hline 20 & 0 & 0. & $1.4561 E-01$ & $5.7423 E-0$ \\
\hline $20-2$ & & $20 \ldots--4$ & 5 & \\
\hline $\begin{array}{l}-3572 E-02 \\
-20\end{array}$ & 0. & $\begin{array}{c}1.2123 E-01 \\
20\end{array}$ & $0.20 \quad 10$ & $\begin{array}{c}3.1368 E-0 \\
20\end{array}$ \\
\hline 0.21 & 0 . & 0. & 0. & $\begin{array}{c}2.4561 \mathrm{E}-01 \\
21\end{array}$ \\
\hline $9.1541 E-03$ & $5.3519 E-02$ & 0. & $1.9325 \mathrm{E}-0 \mathrm{1}$ & 0.210 \\
\hline $5.0005 E-01$ & 0. & 0. & 0 & 0. \\
\hline$-21 \ldots 11$ & 22. & -2 & 22. & $22=-4$ \\
\hline $\begin{array}{c}3.9154 \mathrm{E}-01 \\
22\end{array}$ & $\begin{array}{c}1.4663 E-02 \\
2 ?\end{array}$ & $\begin{array}{c}8.5728 E-02 \\
22\end{array}$ & 0. & $3.0956 E-01$ \\
\hline & $8.0100 \mathrm{E}-01$ & 0. & 0. & 0. \\
\hline 10 & 11 & 23 & $-23-2-2$ & 3 \\
\hline 23 & $0.26180-01$ & 2360 & 0.4714 E -7 & \\
\hline $\begin{array}{c}5.3130 E-01 \\
23\end{array}$ & 0 & $\begin{array}{l}3748 \mathrm{E} \\
23\end{array}$ & 0. & 0. \\
\hline & 0 & $1.0764 \mathrm{E} 00$ & $3.9064 \mathrm{E}-02$ & $2.2839 E-01$ \\
\hline & $8.2469 E-0$ & & $2.1339 E^{0} 0$ & 0 \\
\hline 24 & -24 & 10 & $-\frac{2}{6}=-111$ & \\
\hline 25 & 0 . & & $.6709 E$ OE & $\begin{array}{r}6.1399 \mathrm{E}-02 \\
25 \\
-\end{array}$ \\
\hline $\begin{array}{c}3.5897 \mathrm{E}-01 \\
25\end{array}$ & & $1.2962 \mathrm{E} 00$ & 0 & $3540 E 00$ \\
\hline 0 & 0 & & 0 & $2.6262 \mathrm{E} 00$ \\
\hline $6.14 \frac{26}{50 E-0}$ & 26 & $=$ & $2 \underline{6}$ & 26 \\
\hline $\begin{array}{c}6.1450 E-02 \\
26\end{array}$ & $\begin{array}{c}3.5926 E-01 \\
26\end{array}$ & 0.26 & $\begin{array}{c}1.2973 E 00 \\
26\end{array} 9 \begin{array}{l}0 \\
0\end{array}$ & - \\
\hline $\begin{array}{c}3.3567 \mathrm{E} \\
26\end{array}$ & & & 0. & \\
\hline $2.6283 E 00$ & $6.1512 \mathrm{E}-02$ & $3.5963 \mathrm{E}-01$ & 0 & $-1-\frac{27}{2986} E^{4}-00$ \\
\hline 27 & $-\frac{2}{3}-6$ & 27 & 2 & 27 \\
\hline $27 \quad 10$ & $\begin{array}{r}3.3601 E 0 \\
27 \\
-\end{array}$ & & & 28 \\
\hline & $2.6310 \mathrm{E} O 0$ & 6.15 & 3.601 & -5 \\
\hline
\end{tabular}


-BORON _ 10

SIGMA $N, G(N I / N 2)$

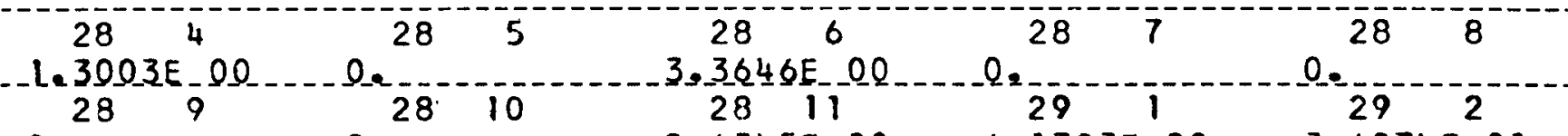

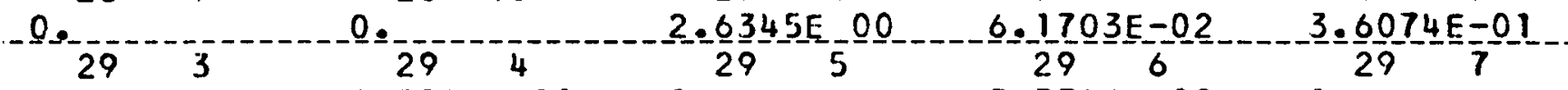

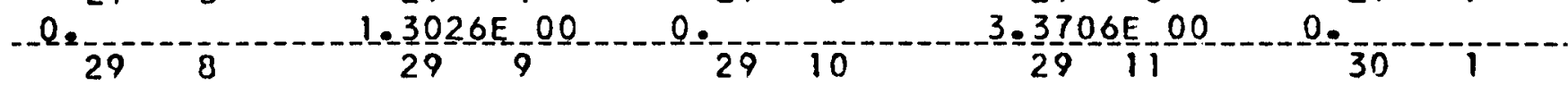

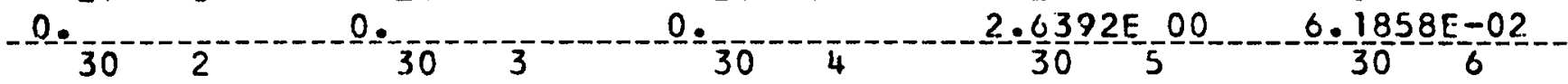

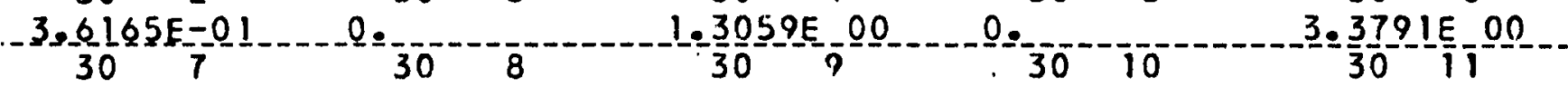
- $e_{3}$

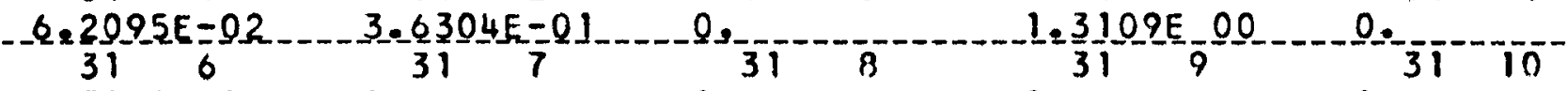

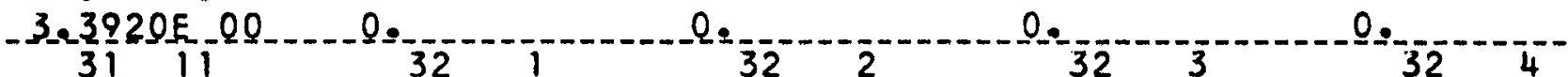

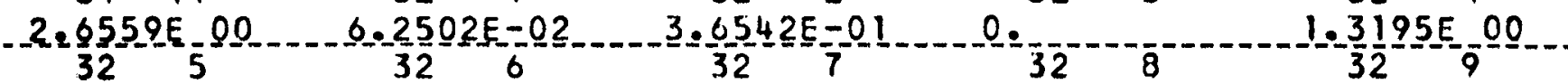

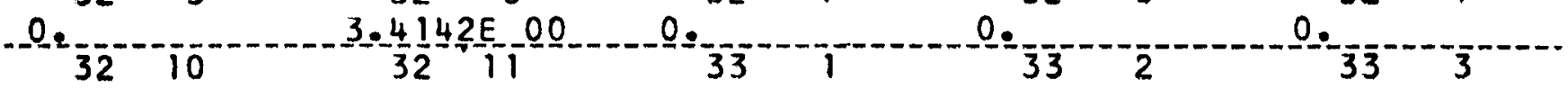

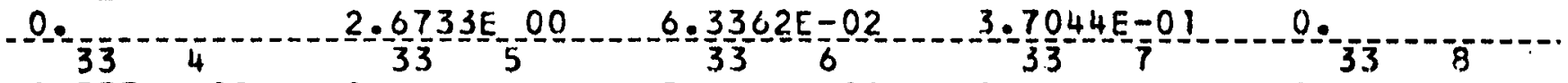

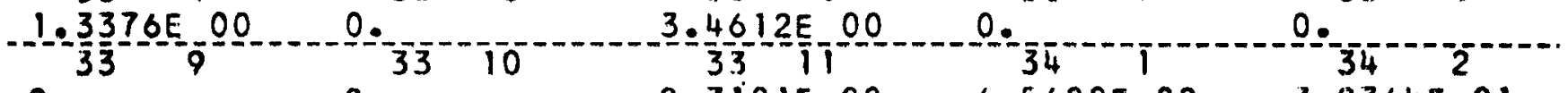

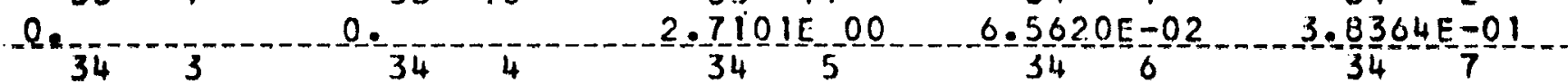

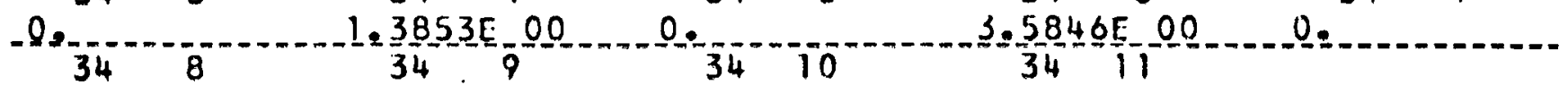
. 
DATE $12 / 08 / 60$

DESIGENAIIQN CODE NO. DEENSIIY EACTOR OXXYGEN 8.1600 $2.656040 \underline{0} E_{-} 01$

SIGMA
LEVEL

$1-1.000 E-07-9.2651 E=01 \quad 4=3426 E=017.0442 E=012.5054 E-01=1.9475 E-01$ $20.065 \mathrm{E} 061.2480 \mathrm{E} 002.3928 \mathrm{E}-011.0226 \mathrm{E} 00-1.6399 \mathrm{E}-01-1.9771 \mathrm{E}-01$ 3 3.679E 06 2. $1945 \mathrm{E} \quad 0022.3217 \mathrm{E}-01$ 1.7080E $002.6315 \mathrm{E}-02-4.2661 \mathrm{E}=01$ $42.231 \mathrm{EE} 06 \mathrm{1.3575E} 002.0891 \mathrm{E}-011.2630 \mathrm{E} 003.0000 \mathrm{E}-04-8.2930 \mathrm{E}-02$

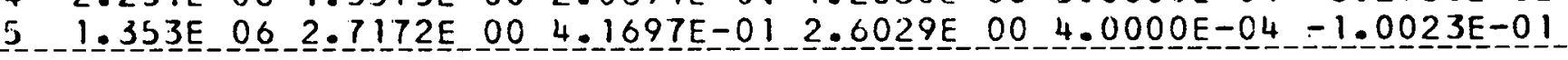

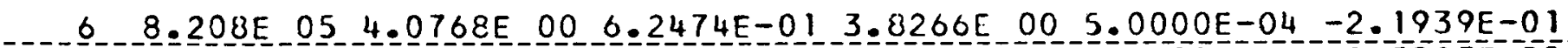
$7-4.979 \mathrm{C}-056.6931 \mathrm{E} 008.1527 \mathrm{E}=015.1179 \mathrm{E} 00 \mathrm{0} .0000 \mathrm{E}-04-1.3813 \mathrm{E}-0 \mathrm{O}$ 8 3.020E 05 3.7219E 00 5.9109E-01 3.8984E $007.0000 E-04-1.5473 \mathrm{E}-01$

$90.832 \mathrm{E} 053.4271 \mathrm{E} 004.1471 \mathrm{E}-013.2870 \mathrm{E}$ 00 $6.0307 \mathrm{E}-08-1.2286 \mathrm{E}=0 \mathrm{~T}$

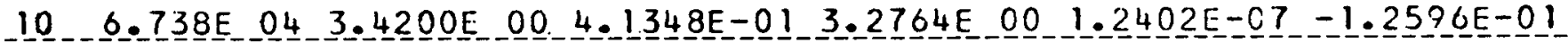

$11-2.479 E 043.4386 E-00 \quad 4.1573 E-013.2942 E$ EO $2.0425 E-C 7-1.2064 E-01$ $129.119 \mathrm{E} 033.6547 \mathrm{E} 004.4186 \mathrm{E}-013.5012 \mathrm{E}-00-3.3670 \mathrm{E}-07-1.3460 \mathrm{E}-0 \mathrm{~T}$ $13 \quad 3.355 \mathrm{E} \quad 03 \quad 3.7837 \mathrm{E}$ 00 $4.5745 \mathrm{E}-013.6248 \mathrm{E} \quad 00 \quad 5.5624 \mathrm{E}-07-1.3935 \mathrm{E}-01$

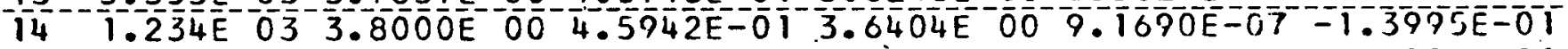

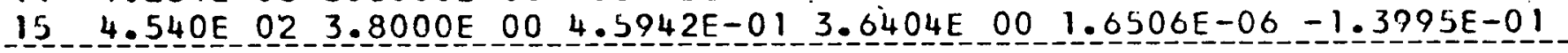

$-16-8.719 E-013.8000 E-004.5942 E-013.6404 E-00 \quad 3.1576 E-06-1.3995 E-01$

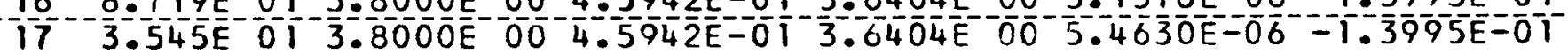
$18 \quad 1.304 E$ OI 3.8000 E $00 \quad 4.5942 E-01 \quad 3.6404 E$ E $00 \quad 8.8380 E-06-1.3995 E-01$

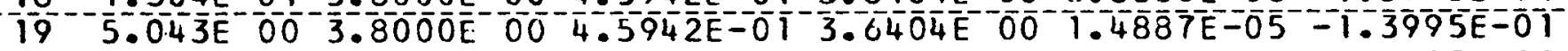
20 1.445E 00 3.8000E 00 4.5242E-01 3.6404E 00 2. $5072 E=05=1.3995 \mathrm{E}=01$

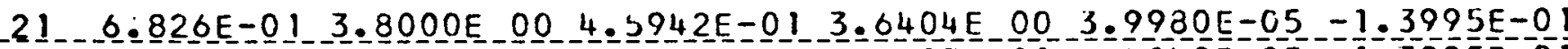
$222.511 E-01.3 .7999 \mathrm{E} 004.5941 \mathrm{E}-013.6403 \mathrm{E}-000.4148 \mathrm{E}-05=1.3995 \mathrm{E}-01$ $23-9.237 \mathrm{E}=02-3.8683 \mathrm{E}-004.6768 \mathrm{E}=01-3.7058 \mathrm{E}-001.1031 \mathrm{E}-04-1.4247 \mathrm{E}=01$ $242.530 \mathrm{E}-02$ 4.0546E $004.9020 \mathrm{E}-013.8843 \mathrm{E}-001.6085 \mathrm{E}-04=1.4933 \mathrm{E}-01$

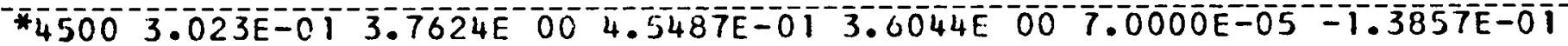
* $4000 \quad 2.718 \mathrm{E}-013.7624 \mathrm{E} \quad 00 \quad 4.5487 \mathrm{E}-013.6044 \mathrm{E}-00 \quad 7.0000 \mathrm{E}-05-1.3857 \mathrm{E}-01$

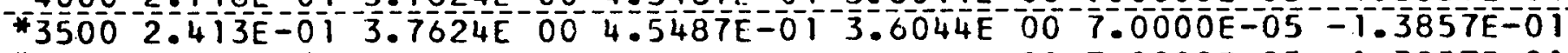
*3000 2. 109E-01 $3.7624 \mathrm{E}$ 00 $4.5487 \mathrm{E}-013.6044 \mathrm{E} \quad 00 \quad 7.0000 \mathrm{E}-05-1.3857 \mathrm{E}-01$ $25001.804 \mathrm{E}-01-3.7747 \mathrm{E}-004.5636 \mathrm{E}=01-3.6162 \mathrm{E}-00-0000 \mathrm{E}-05=1.3902 \mathrm{E}=01$

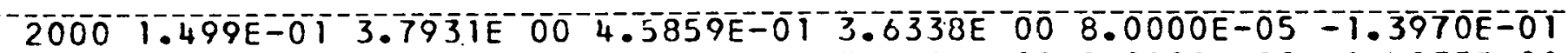
$15001.194 \mathrm{E}-013.8217 \mathrm{E} 00-4.6204 \mathrm{E}-013.6612 \mathrm{E}$ 00 $9.0000 \mathrm{E}-05=1.4075 \mathrm{E}-01$ $10008.897 \mathrm{E}-02-3.8699 \mathrm{E} 00-4.6787 \mathrm{E}-013.7074 \mathrm{E} 00-1.1000 \mathrm{E}-04-1.4253 \mathrm{E}=0 \mathrm{1}$ $500 \quad 5.850 \mathrm{E}-023.9634 \mathrm{E} 004.7918 \mathrm{E}-013.7969 \mathrm{E}$ 00 $1.3000 \mathrm{E}-04-1.4597 \mathrm{E}-01$ $683.216 \mathrm{E}-024.1624 \mathrm{E}-005.0323 \mathrm{E}-013.9876 \mathrm{E}-00-1.8000 \mathrm{E}-04-1.5330 \mathrm{E}-01$

*Thermal cross sections taken from APEX-515.

NOTE: For a summary of the sources of data see APEX-704. 
OXYGEN

IHERMAL_IRANSEER_MAIRICEIS_

-_IEMPERAIURE

DEG. F.

$4.5000 E \quad 03$
SISMA

SCATTER
SIGMA TRANSPORT
SI_MMA ABSORPIION
NU SIICMA

FISSION

\section{SIGMA S $\left(N \frac{N}{N} / \frac{N}{N} 2\right)$}
$2 i 2 i$
2122
$2 \overline{1} \overline{3}$
2124
$\overline{2} \overline{1} \overline{2}$

- 3.0816E_00 _-6.9895E=0

$22 \quad 21$

$22 \quad 22$

$1.896065=02$

$3.9428 E=04$

8. $7 ? \underline{7} \underline{7} 3 \mathrm{E}-0 \underline{0}$

- $T=16066$ E $=0$

2321

2. $7056 E-00$

$22 \quad 23$

$22 \quad 24$

$22 \quad 25$

$6.3295 \mathrm{E}-02$

2322

4. $-\frac{57}{23} \frac{6}{23}=\frac{61}{23}$

$2 \cdot \frac{1}{2} \frac{251 E}{2}=02$

7. $0368 \mathrm{E}-03$

2421

$24-22$

$2=\frac{2}{2} \frac{2}{4} 17 E \frac{0}{2}=$

2. $-\frac{6}{2} \frac{1}{4} 84 \mathrm{E}-01$

$1.0927 \mathrm{E}-01$

$I=2342 E-02$ 2521

6. $7069 E-01$

2. $5101 \mathrm{I}-0 \mathrm{O}$

$1.0902 E 00$

2425

$6.1935 E-03$

5. $0245 E-01$

$2.3727 E \quad 00$

$1.3442 \mathrm{E} \quad 00$

5. $-\frac{9}{2} \frac{3}{5} 1 E-01$.

1. $5093 \mathrm{E}$

IEMPERAIURE

DEG. F.

$4.0000 E$
SIGMA

SCATTER
SIGMA

TRANSPORT
SI GMA

ABSORPIION
NU SIGMA

FISSION

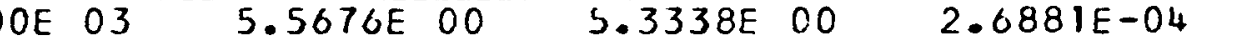

SIGMA $\left.-\frac{N}{S}(N) \frac{N 2}{N}-1\right)$

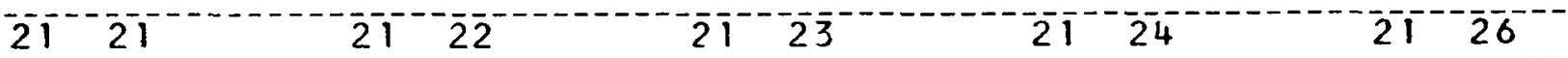

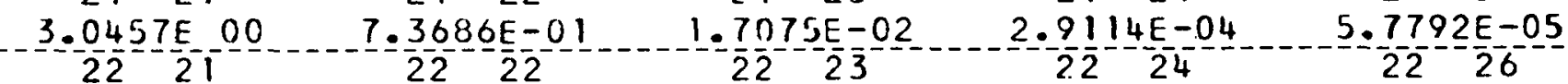

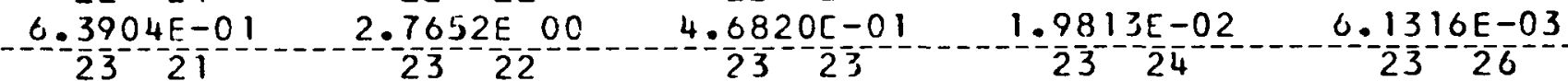

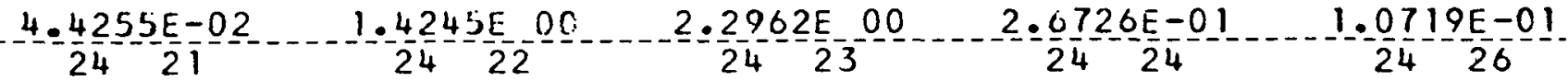

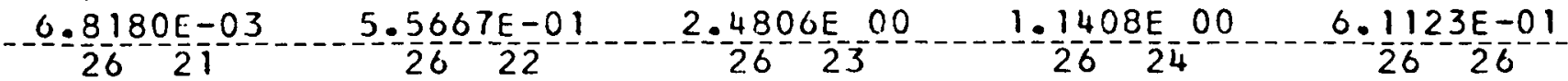

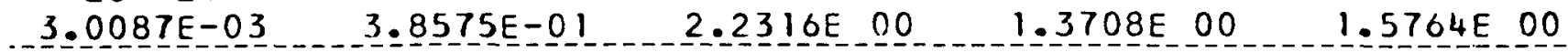


QXXYGEN

THERMAL TRANSFER MATRICES

TEMPERATURE
SIGMA $\begin{gathered}\text { SIGMA } \\ \text { SIGESIGMA }\end{gathered}$

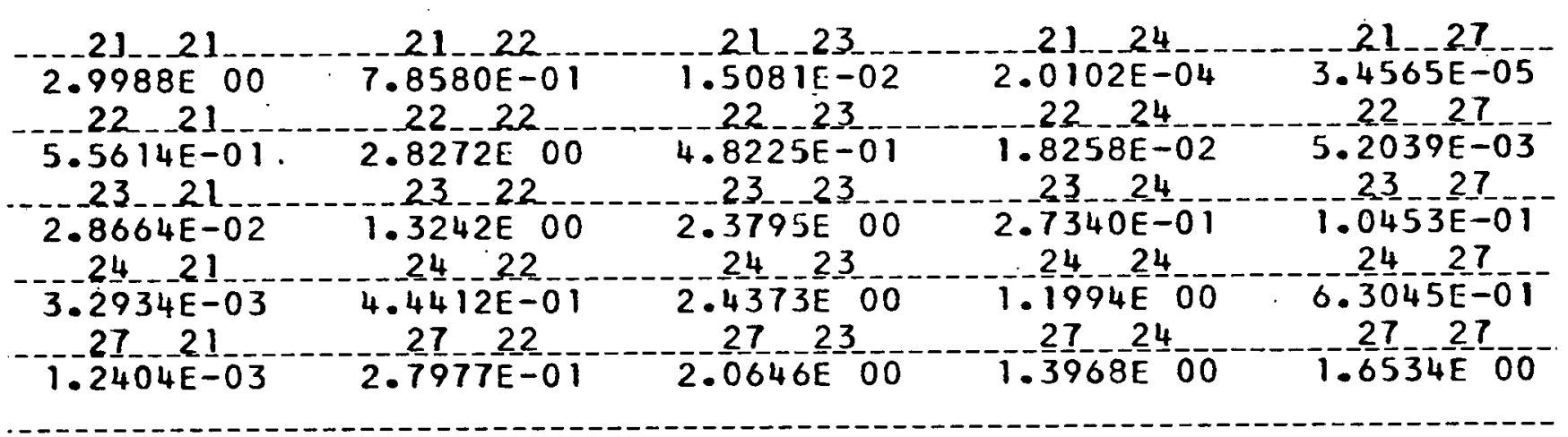

TEMPERATURE
SIIGMA

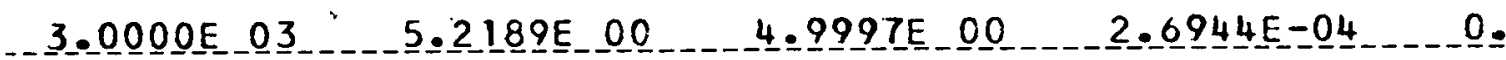

$\mathrm{N} \overline{\mathrm{T}} \mathbf{N}$

SI GMA S $($ NIIN2)

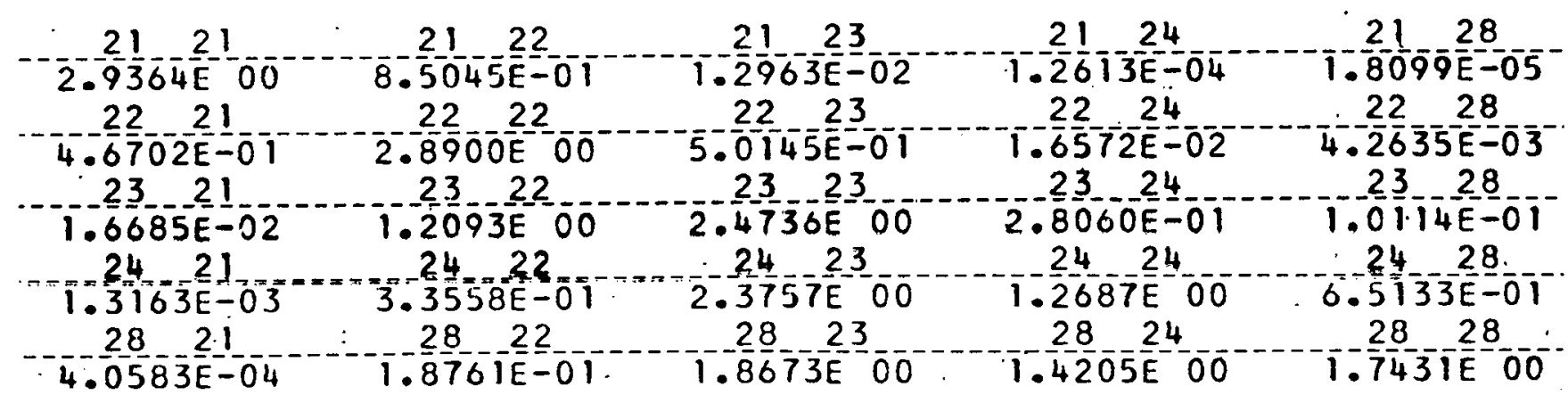


OXYGEN

IHERMAL_IRANSEER MAIRICES.

IEMPERAIURE

S I GMA

SIGMA

SIIGMA

NNU_SIGMA

DEG. F. SCATTER

TRANSPORT

ABSORPTIONS

FISSION

$2.5000 E^{0} 3$

$5.0368 \bar{E}-10$

$4.8252 E^{2} 00$

$2.699 \bar{E}-04$

o.

\section{SIGMA S $\left(\frac{N}{2}-\frac{N 2}{1 / N 2}\right)$}

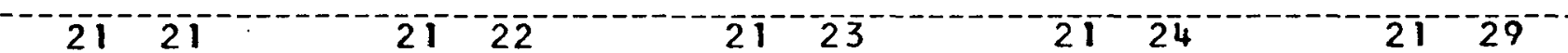

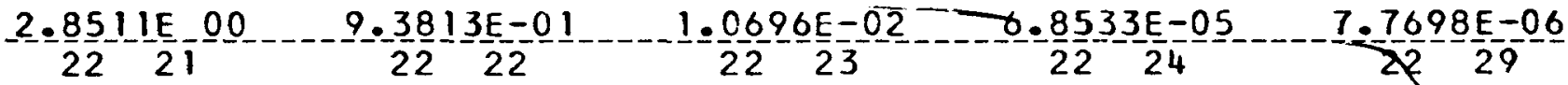

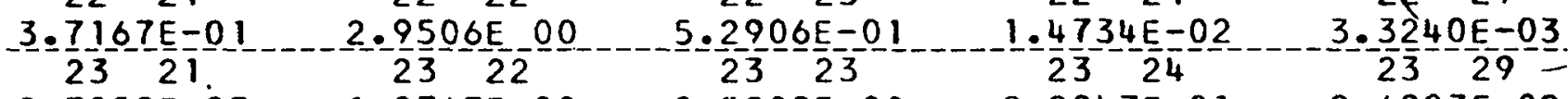

$8.3052 \mathrm{E}-03$

$3.9741 E-04 \quad 2.3446 E-01 \quad 2.2890 E-00 \quad 1.3523 E-200 \quad 6.7405 E-01$

$9.4308 \mathrm{E}-05 \quad 1.1233 \mathrm{E}-01 \quad 1.6355 \mathrm{E} 00 \quad 1.4393 \mathrm{E} 00 \quad 1.8496 \mathrm{E} 00$

IEMPERAIURE

DEG. F.
SCI IMA SCATTER

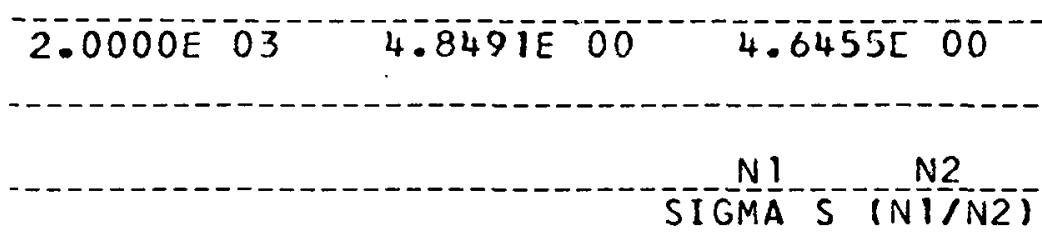

SIGMA

TRANSPORT
SIGMA

ABBSORPTION
NU SIGMA

FISSTON

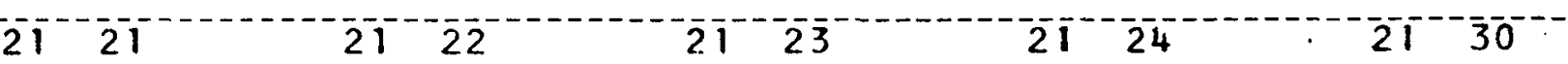

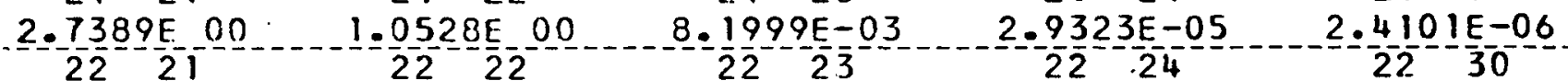

$2 .=\frac{7}{2} \frac{107 E-01}{3}-\frac{3.0020 E}{2} 1300 \quad 5.7125 E-01 \quad 1.2704 E-02 \quad 2.4048 E-03$

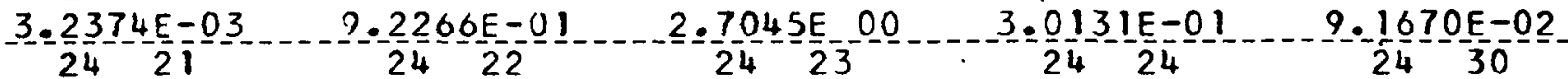

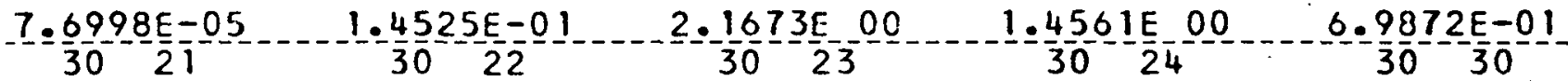
$1.2768 \mathrm{E}-05 \quad 5.6502 \mathrm{E}=02 \quad 1.3654 \mathrm{E} 00 \quad 1.4483 \mathrm{E}$. 00 1.9789E 00 
QXYGEN

THERMAL TRANSFER MATTRICES

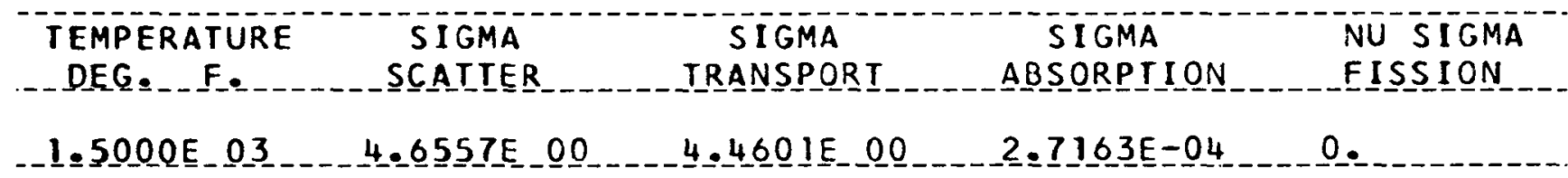

$\bar{N} \overline{1}-1-12$

SIGMA S $(N 1 / N 2)$

\begin{tabular}{|c|c|c|c|c|}
\hline 23 & 21 & 21 & 21 & \\
\hline $.6967 \mathrm{E} 00$ & $1.0982 \mathrm{E} \quad 00$ & $5.0217 E-03$ & $7.4879 \mathrm{E}-06$ & $3.8435 \mathrm{E}-07$ \\
\hline$-22-21$ & $-22 \underline{2}-22$ & 23 & $22-24$ & 31 \\
\hline $\begin{array}{l}6887 E-01 \\
23 \quad 21\end{array}$ & $\begin{array}{c}3.0276 E \text { OO } \\
2322\end{array}$ & $\begin{array}{c}6.4085 E-01 \\
2323\end{array}$ & $\begin{array}{l}.0408 E-02 \\
2324\end{array}$ & $\begin{array}{l}E-0 \\
31\end{array}$ \\
\hline $8.3077 \mathrm{E}-04$ & $7.4194 \mathrm{E}-01$ & $2.8472 \mathrm{E} 00$ & $1905 \mathrm{E}-$ & $8.5361 \mathrm{E}-0$ \\
\hline 24 & 22 & 23 & 24 & 24 \\
\hline $.9265 \mathrm{E}-06$ & $7.3511 E-02$ & $1.9951 \mathrm{E} 00$ & $1.5905 \mathrm{E} 00$ & $7.2545 E-01$ \\
\hline$--\frac{31}{6}-\frac{21}{1}-\overline{1}$ & $=3 \frac{1}{1}-22$ & $-\frac{31}{055}=\frac{23}{6}$ & $-\frac{3}{4} \frac{1}{3}-\frac{24}{6 F}-0$ & $-3 \frac{3}{14}-\frac{3}{3}=$ \\
\hline
\end{tabular}

$-1$

TEMPERATURE SIGMA

DEG. F.

SCAAITER

1.0000E 03 _ $4.4564 E_{1} 00$

\section{SIGMÁ}

IRANSPORI

1.0000E 03 _ $4.4564 E_{1} 00$

ABSORPIION

$1.0000 E\left[03 \quad 4.4564 E_{0} 00 \quad 4.2692 E 00 \quad 2.7341 E-04\right.$

NÜ SIGMÁ

FISSION

Ni

$\mathrm{N} 2$

SI GMA S $($ NIIN2)

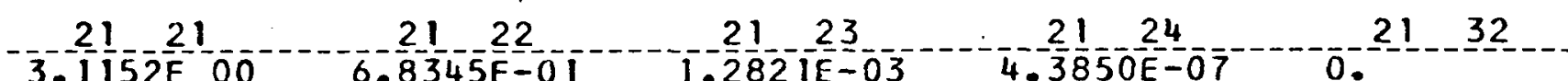

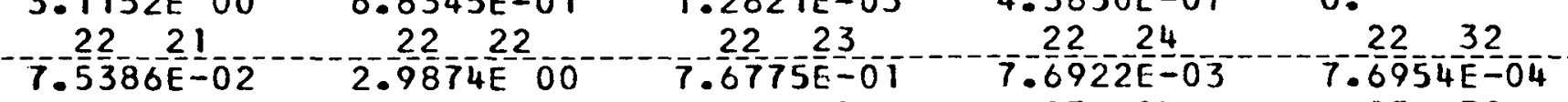

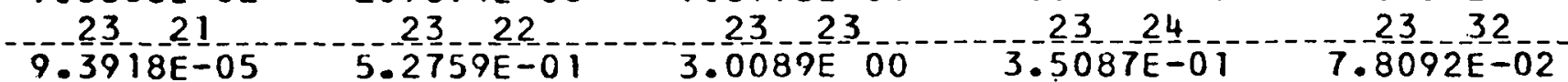
$\ldots 24 \quad 21 \ldots 24-22 \ldots 24$ $1.3626 \mathrm{E}-07 \quad 2.5228 \mathrm{E}-02 \quad 1.7474 \mathrm{E} 0 \mathrm{O}^{-1.7745 \mathrm{E} 00} 7.5432 \mathrm{E}-0 \mathrm{~T}$

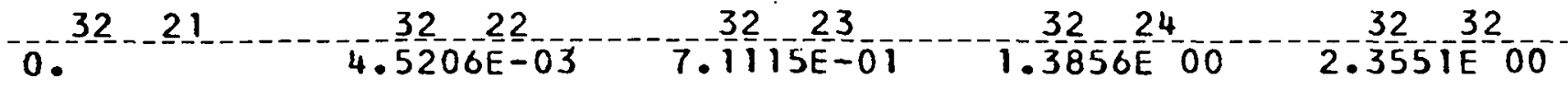


OXYGEN

IHERMAL IRANSFER MAIRICES

ILEPERATURE

DEG . $F$.

SI GMA

SIGMA

SIGMA

NNU SIIGMA

$5.0000 \mathrm{E} 02$

SCATIER

TRANSPORT

ABSORPTION

FISSION

\section{$5.0000 \mathrm{0} 2$}

$4.2515 \bar{E} 00$

4.073OE OO

$2.7717 \mathrm{E}-04$

0 .

\section{$S I G M A-\frac{N}{S}\left(N \frac{N 2}{N}\right)$}

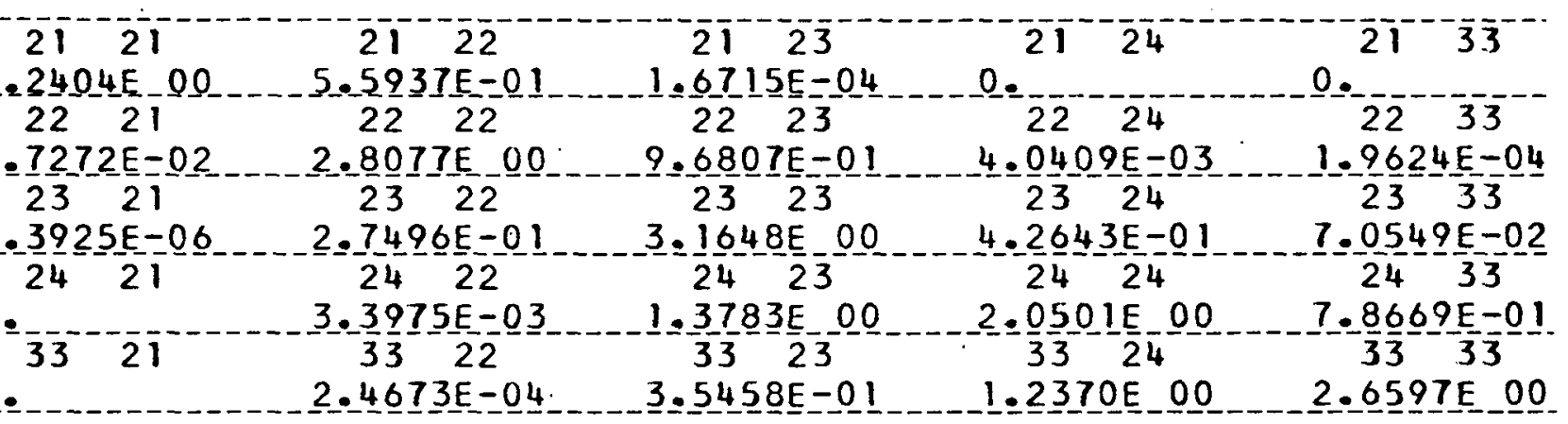

IEMPERAIURE

DEG. $F$.

$6.8000 \bar{O} 01$
SIGMA

SCATIER

$4.07 \overline{T E} \mathrm{CO}$
SIGMA TRANSPORT
SIGMA ABSORPTION
NU_ S_IGMA

\section{FISSION}

\section{SIGMA S $\left.(N) \frac{N}{N}-N 2\right)$}

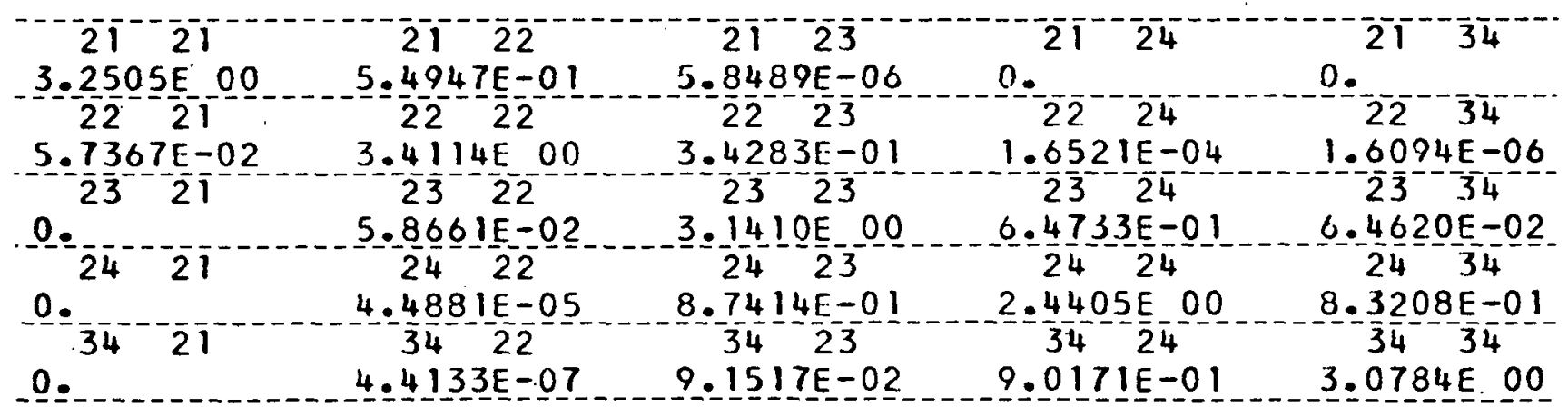


OXYGEN

TRANSFERS FF'M GROUPS 1 THEROUGH 2 TO GROUPS 1 THROUGH 15

$\mathrm{Ni} N 2$

SIGMA SO (N]IN2)

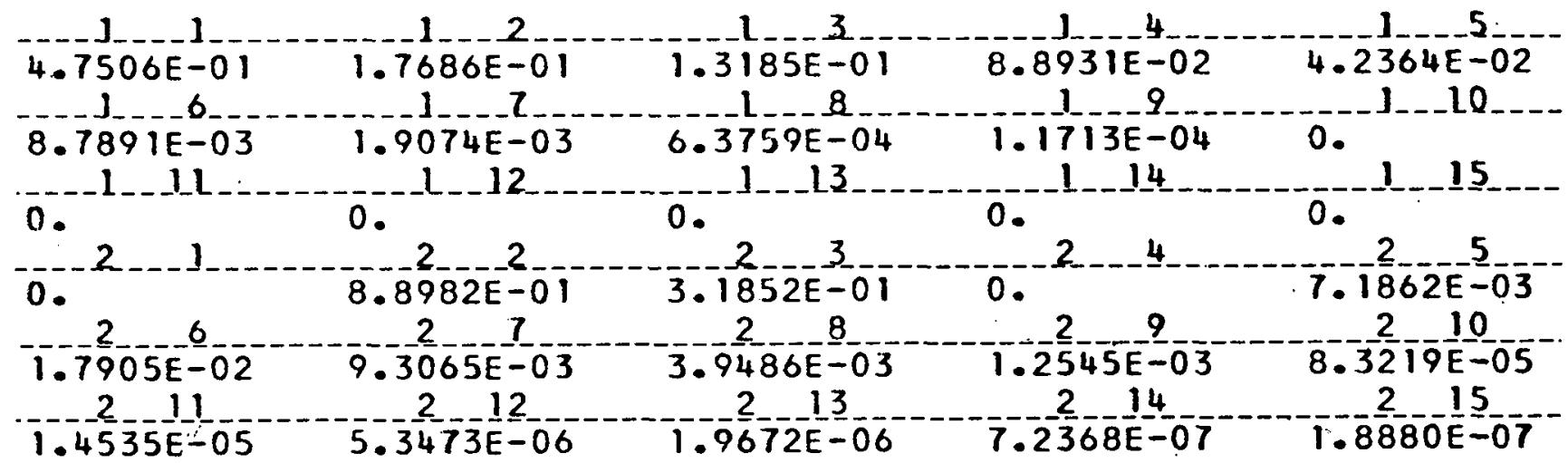


QXXYGEN

TRANGFERS FROM GROUPS I THROUGH 2 TO GROUPS I THROUGH 21

N. 1

SIGMA N,G (NIIN2)

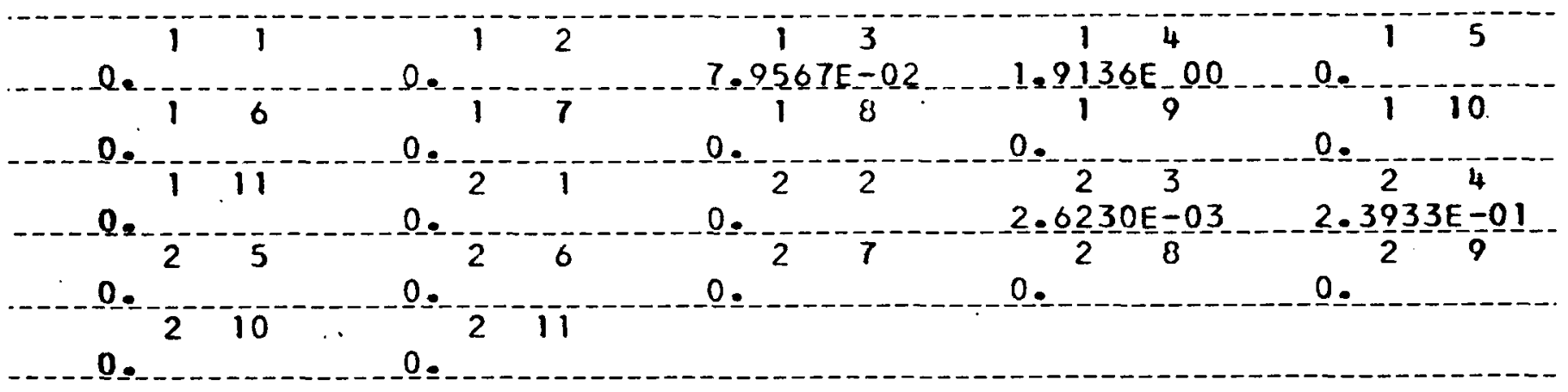


DATE $12 / 08 / 60$

DESLIGNATLON ALUMINUM
CODE_NO

13.2700
DENSIIYY EACTQR

$4=4782 \underline{3} E$

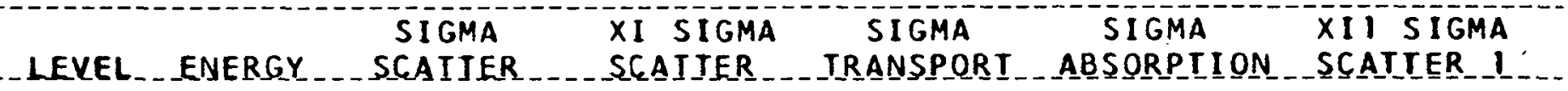

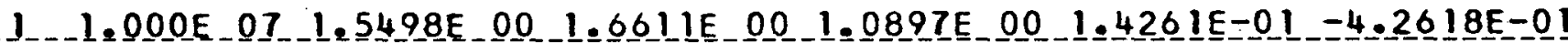

$20.065 \mathrm{E} 06 \quad 2.0550 \mathrm{E}$ 00 $1.5337 \mathrm{E} 00 \quad 1.3808 \mathrm{E} 005.6933 \mathrm{E}-02-6.2443 \mathrm{E}-0 \mathrm{~T}$

3 3.679E $06-2.4335 \mathrm{E}-000.10275 \mathrm{E}-00 \quad 1.7948 \mathrm{E} 00 \quad 1.0333 \mathrm{E}-02-5.9162 \mathrm{E}-01$

$42.231 \mathrm{E} 062.8664 \mathrm{E} 00-4.742 \mathrm{E}-011.7925 \mathrm{E} 003.9555 \mathrm{E}-03-9.9469 \mathrm{E}=0 \mathrm{~T}$

5 1.353E $06 \quad 3.1229 E_{0} 002.5212 \mathrm{E}-01$ 1.9497E $00 \quad 3.9392 \mathrm{E}-03-1.0866 \mathrm{E} \quad 00$

$6 \quad 8.208 E$ E $05 \quad 3.5846 E$ E $001.9387 E-01 \quad 2.4666 E$ E $009.1684 E-04-1.0355 E 00$ 7 4.979E $053.9120 \mathrm{E}-002.2151 \mathrm{E}=012.9594 \mathrm{E}-009.9564 \mathrm{E}=04-8.8236 \mathrm{E}-01$

8 3.020E 05 3.6077E $002.2151 E=012.9589 E$ E $001.5481 E-03-6.0096 E-01$

9. $1.832 \mathrm{E} 057.3047 \mathrm{E} 00-4.9973 \mathrm{E}-016.6755 \mathrm{E} 003.1905 \mathrm{E}-03-5.8284 \mathrm{E}-0 \mathrm{1}$

10 6.738E 04 4_.6342E 00 3.3818E-01 4.5172E 00 3.8965E-03 -1.0830E-01

$11 \quad 2.479 E \quad 04 \quad 5.9852 E \quad 00 \quad 4.3692 E-01 \quad 5.8361 E$ E $00 \quad 5.5038 E-03-1.3804 E-01$

$12-9.119 \mathrm{E} 031.4250 \mathrm{E}$ 00 $1.0402 \mathrm{E}-011.3895 \mathrm{E}-00-2.998 \mathrm{E}-03-3.2864 \mathrm{E}=02$

$\begin{array}{llllllllll}13 & 3.355 E & 03 & 1.4436 E & 00 & 1.0538 E-01 & 1.4077 E & 00 & 3.6453 E-03 & -3.3295 E-02\end{array}$

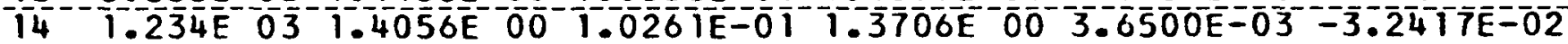

$15 \quad 4.540 E \quad 02 \quad 1.4014 E \quad 00 \quad 1.0230 E-01 \quad 1.3665 E$ O $00 \quad 3.6500 E-03-3.2320 E-02$

$\begin{array}{lllllllllll}16 & 8.719 E & 01 & 1.4024 E & 00 & 1.0237 E-01 & 1.3675 E & 00 & 3.9380 E-03 & -3.2343 E-02\end{array}$

$17-3.545 \mathrm{E}-0 \mathrm{1} 1.4035 \mathrm{E}-001.0246 \mathrm{E}-0 \mathrm{1} 1.3686 \mathrm{E}-00-0.400 \mathrm{E}-03-3.2370 \mathrm{E}=02$

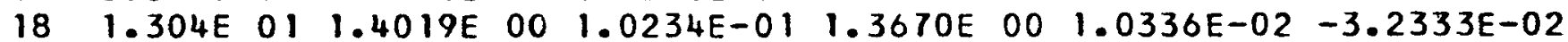

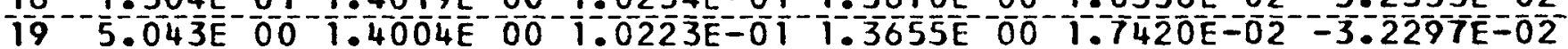

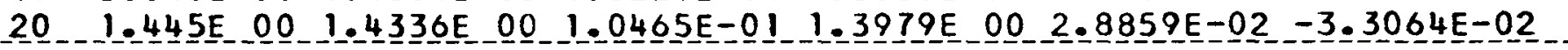

21 6.826E-01 1.4555E 00 1.0625E-01 1.4192E $00 \quad 4.6443 E-02-3.3568 E-02$

$22-511 \mathrm{E}-011.4822 \mathrm{E}-001.0820 \mathrm{E}-011.4453 \mathrm{E} 007.5450 \mathrm{E}-02=3.4185 \mathrm{E}-02$

$23 \quad 9.237 \mathrm{E}-02 \quad 1.4308 \mathrm{E}$ 00 $1.0445 \mathrm{E}-01 \quad 1.3952 \mathrm{E} \quad 00 \quad 1.2755 \mathrm{E}-01-3.3000 \mathrm{E}-02$

$24-530 \mathrm{E}-021.4062 \mathrm{E} 001.0266 \mathrm{E}-011.3712 \mathrm{E}-00-1.9767 \mathrm{E}-0 \mathrm{1}-3.2432 \mathrm{E}=02$

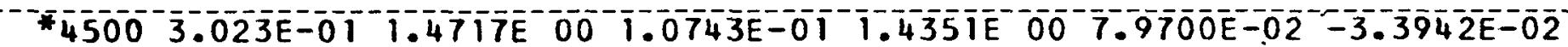

* $40002.718 E-01 \quad 1.4717 E$ 00 $1.0743 E-01 \quad 1.4351 E$ O0 $7.9700 E-02-3.3942 E-02$

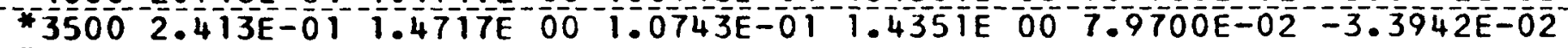

* $3000 \quad 2.109 \mathrm{E}-01 \quad 1.4717 \mathrm{E}$ 00 $1.0743 \mathrm{E}-01 \quad 1.4351 \mathrm{E}$ 00 $7.9700 \mathrm{E}-02$-3.3942E-02

$25001.804 \mathrm{E}-01-1.4735 \mathrm{E} 001.0757 \mathrm{E}-011.4368 \mathrm{E}-008.6100 \mathrm{E}-02-3.3984 \mathrm{E}-02$

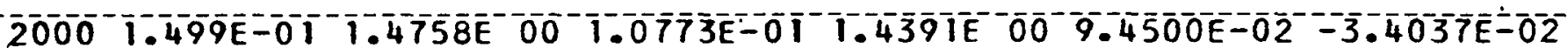

$15001.194 \mathrm{E}-011.4784 \mathrm{E} 00 \quad 1.0792 \mathrm{E}-01 \quad 1.4416 \mathrm{E}$ 00 $1.0590 \mathrm{E}-01-3.4097 \mathrm{E}-02$

$10008.897 \mathrm{E}-021.4815 \mathrm{E} 00$ 1.0815E-01 $1.4446 \mathrm{E} 001.2260 \mathrm{E}-01-3.4168 \mathrm{E}-02$

$500 \quad 5.850 E-02 \quad 1.4841 E$ E $00 \quad 1.0834 E-01 \quad 1.4471 E$ E $00 \quad 1.5130 E-01-3.4228 E-02$

$683.216 \mathrm{E}-02-1.4753 \mathrm{E}-001.0770 \mathrm{E}-01.4386 \mathrm{E}-002.0400 \mathrm{E}-01-3.4025 \mathrm{E}=02$

Thermal cross sections taken from APEX-515.

NOTE: For a summary of the sources of data see APEX-704. 
ALUMINUM

._THERMAL_IRANSFER_MAIRICES

-_LEMPERATURE

SIGMA

SIGMA

DEG. F. SCATIER

TRANSPORT

SIGMA

NU SIGMA - :

$4.5000 E^{-13}$

$1.6919 \mathrm{E} 00$

$1.6497 \mathrm{E} 00$

ABSORPTION

FISSION

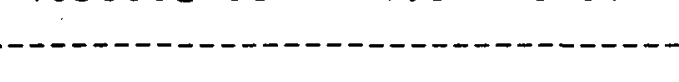

SIGMA S $\left(N \frac{N}{1} / N 2\right)$

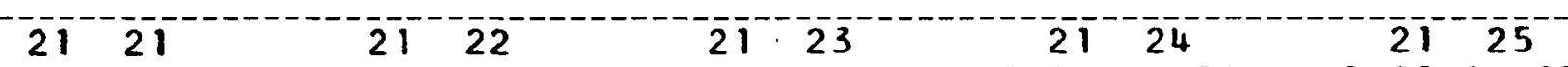

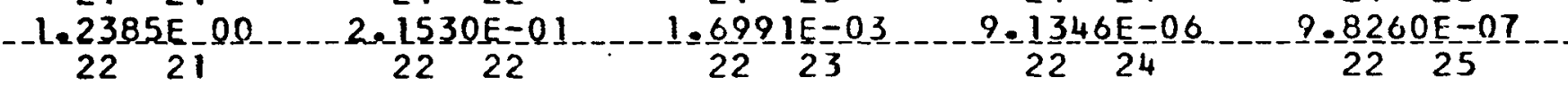

$-2.2234 \mathrm{E}=01 \quad 1.1337 \mathrm{E}-00 \quad 1.4718 \mathrm{E}-01$

$-5.2680 \mathrm{E}-03$

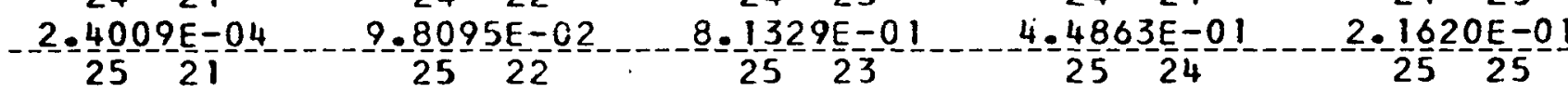

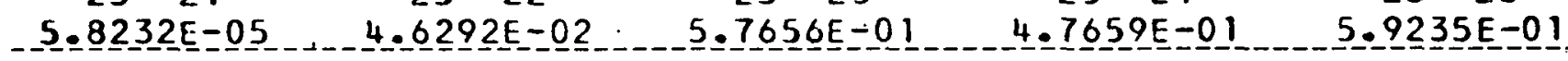

IEMPERAIIURE

DEG. F.

$4.0000 \mathrm{E} 03$
SIGMA

SCATTER
SIIGMA TRANSPORT
SIGMA

ABSORPTION

$\overline{3} . \overline{3} \overline{6} \overline{\mathrm{E}} \overline{\mathrm{C}}-\overline{0} \overline{1}$
NU SIGMA

FISSION

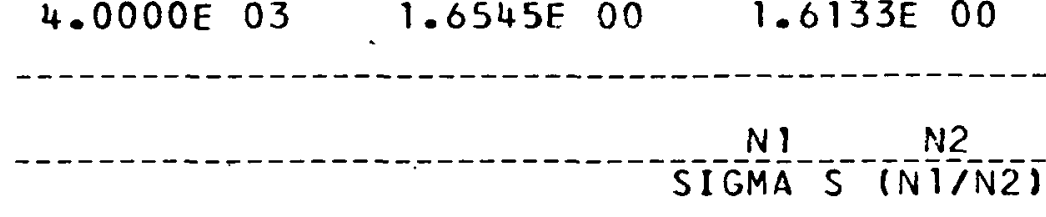

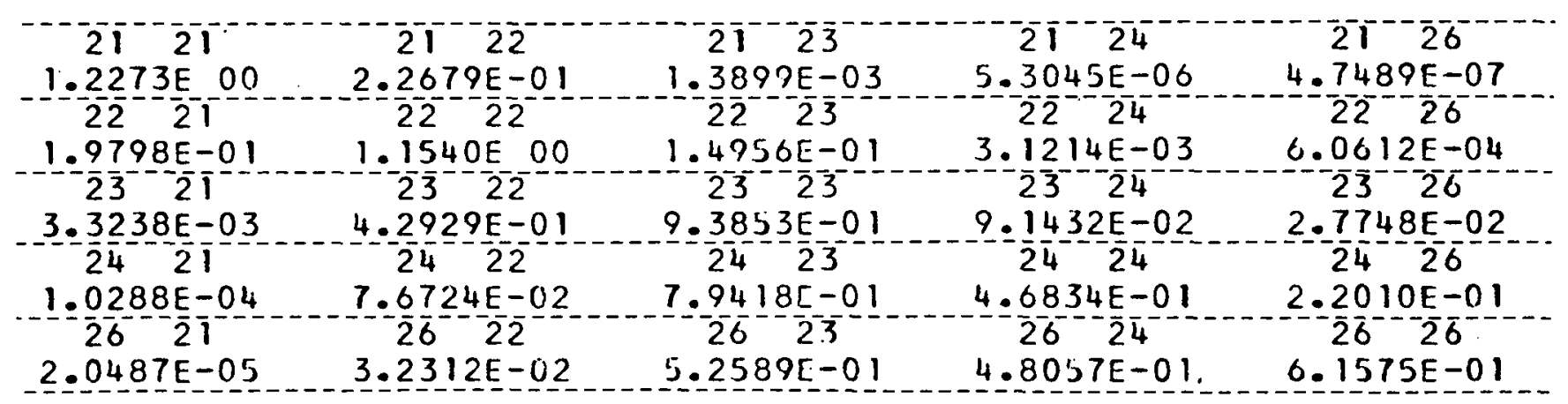


AL UMINUM

THERMAL TRANSFER MATRICES

TEMPERATURE

-_DEGe_f F.

$3.5000 E_{2} 03$ _. 1.6166E 00
SIGMA

SCAIIER
SIGMA

IRANSPORI

$1.5763 E_{-} 00$
SIGMA

$3.2424 E=01$
NU SIGMA

FISSION$$
\text { Ni }
$$

SIGMA_S (NIIN2)

\begin{tabular}{|c|c|c|c|c|}
\hline 2. & 21. & 21 & 24 & 21 \\
\hline $1.2125 \mathrm{E} \quad 00$ & $2.4185 \mathrm{E}-01$ & $1.0929 E-03$ & $2.7204 E-06$ & $1.9431 E-07$ \\
\hline$-22-21$ & $-22-22$ & $-\frac{22}{5}-23$ & $-\frac{22}{2}-2-24$ & $-\frac{22}{4-6}-\frac{27}{6 E-04}$ \\
\hline $23 \quad 21$ & $23-22$ & 2323 & $23 \quad 24$ & $\begin{array}{l}4.0100 t-04 \\
23\end{array}$ \\
\hline $1.8996 \mathrm{E}-03$ & $3.9606 \mathrm{E}-01$ & $9.6748 \mathrm{E}-01$ & $9.2355 \mathrm{E}-02$ & $2.6156 \mathrm{E}-02$ \\
\hline 24 & 24 & 23 & 24 & 27 \\
\hline $\begin{array}{l}.6260 E-0 \\
27 \quad 21\end{array}$ & $\begin{array}{l}5.7018 E-02 \\
27\end{array}$ & $7: 7024 E-01$ & $.9109 E-01$ & $.2402 E-01$ \\
\hline $5.6640 E-06$ & $2.0906 \mathrm{E}-02$ & $4.7010 E-01$ & $4.8329 E-01$ & $6.4226 \mathrm{E}-0 \mathrm{I}^{-}$ \\
\hline
\end{tabular}

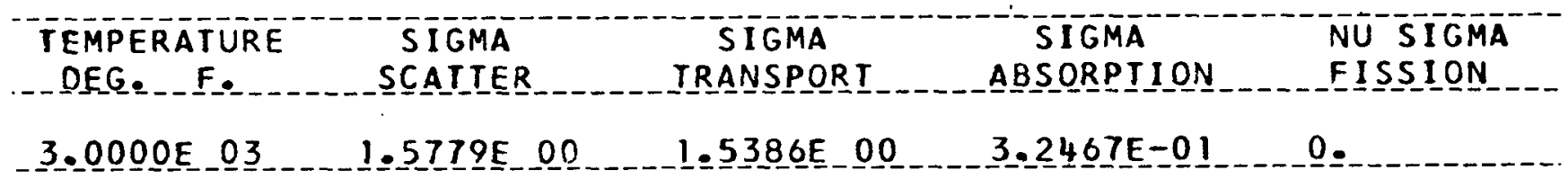

\section{SIGMA S (NIIN2)}

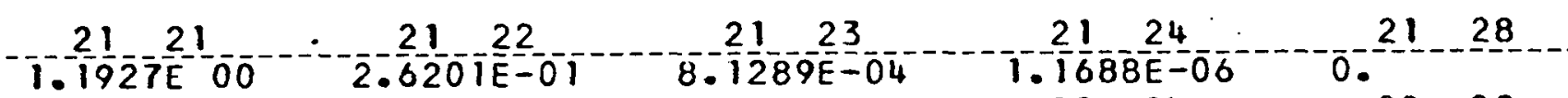

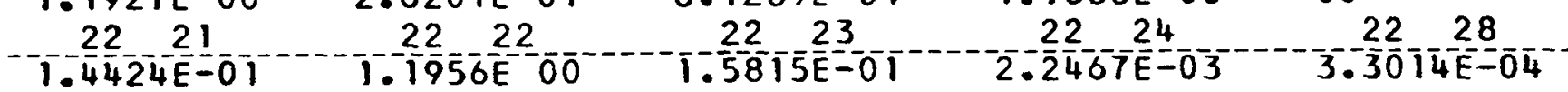

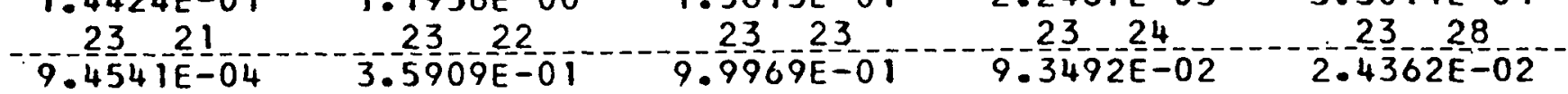

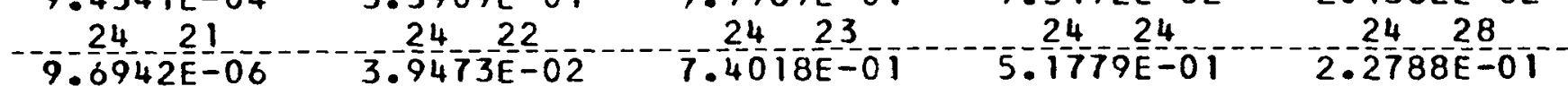

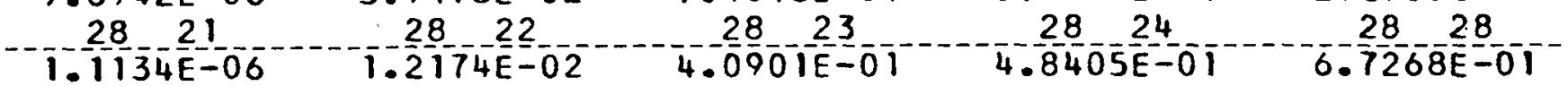


ALUMINUM

_. THERMAL_IRANSEER MAIRICES

IEMPERAIURE

SIGMA

SIGMA

DEG. $F$.

SCATTER

TRANSPORT

SIGMA

NU_ SIGMA.

$2.5000 \bar{O}$

$1.5389 \bar{E} 00$

$1.5005 \mathrm{E} 00$

$3.2525 \bar{E}-0 \overline{1}$

FISSION

$2.5000 E 03 \quad 1.5389 E 00$

\section{SIGMA $-\frac{N}{S}\left(\frac{N}{1}-N 2\right)$}

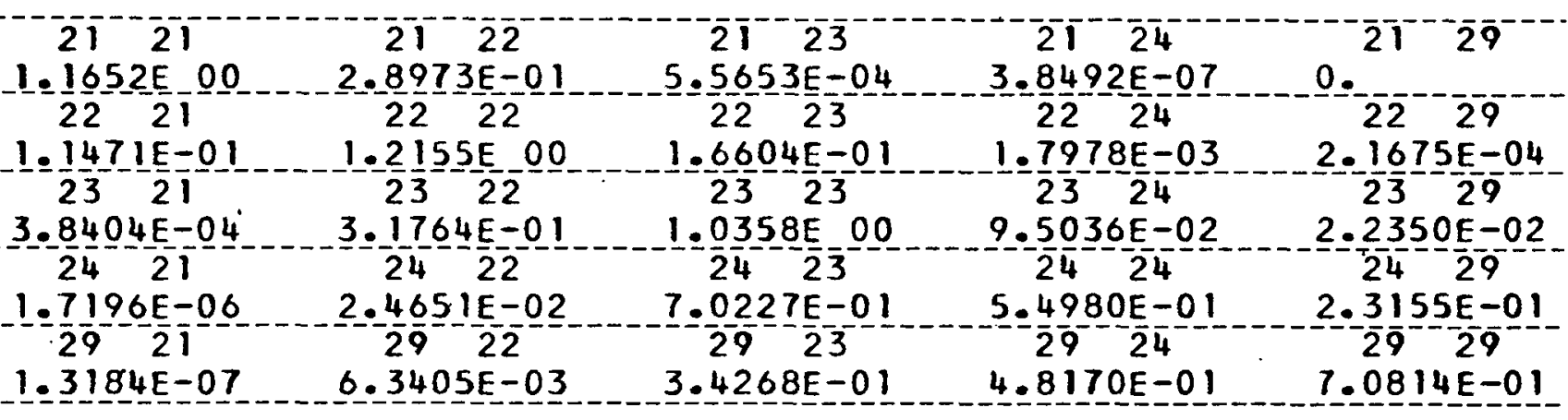

TEMPERAIURE

$2 . \overline{0 O O O} \overline{\mathrm{E}} \overline{\mathrm{O}} \overline{\mathrm{3}}$
SI IGMA

SCATTER
SIGMA

TRANSPORT
SIGMA

ABSORPTION
NU SIGMA

FISSION

$---\cdot$ $1 . \overline{9} 8 \overline{8} \bar{E}^{-} 0 \bar{O}$

3. $26 \overline{0} \overline{7} \bar{E}-\bar{O} \overline{1}$

0 .

\section{SIGMA $-\frac{N}{S}-\frac{N 2}{11 N 2)}$}
2121 $21-22$ $-\overline{2} \overline{1}^{--} \overline{2} \overline{3}$
1. $1285 \mathrm{E}-00$ $3.2667 E-01$ $3.3116 E-04$ 1.232 IE 00 $1.7869 \mathrm{E}-01$ $2 i^{-1}$
.3698E-02 $23-21$ I. $\frac{1}{2} \frac{3}{4}$ SOEE $=04$ 2.7068E-01 2422 $1.0766 E_{0} 00$ 1. $\frac{592}{30}-5 \mathrm{E}=07$ $1.3122 \mathrm{E}-02$
$30-22$ $2.4948 \mathrm{E}=03$
$6.5408 \mathrm{E}-01$ $30-23$ $2.7161 E-01$ 0. $22^{-12}$ o. $1.3464 E-03$ $1.2444 E-04$ $23-\frac{1}{30}$ $9.7404 E=02$ $24-24$ $5.8920 \mathrm{E}-01$ $30-\frac{2}{4}$ 2. $0107 E-02$ $2.3478 E-01$ $4.7429 \mathrm{E}-01$ o. 
ALUMINUM

THERMAL TRANSFFER MATRICES

TEMPERATURE
SIGMA

\section{$\overline{\mathrm{N}} \overline{\mathrm{N}} \mathbf{2}$ \\ SIGMA S (NIIN2)}

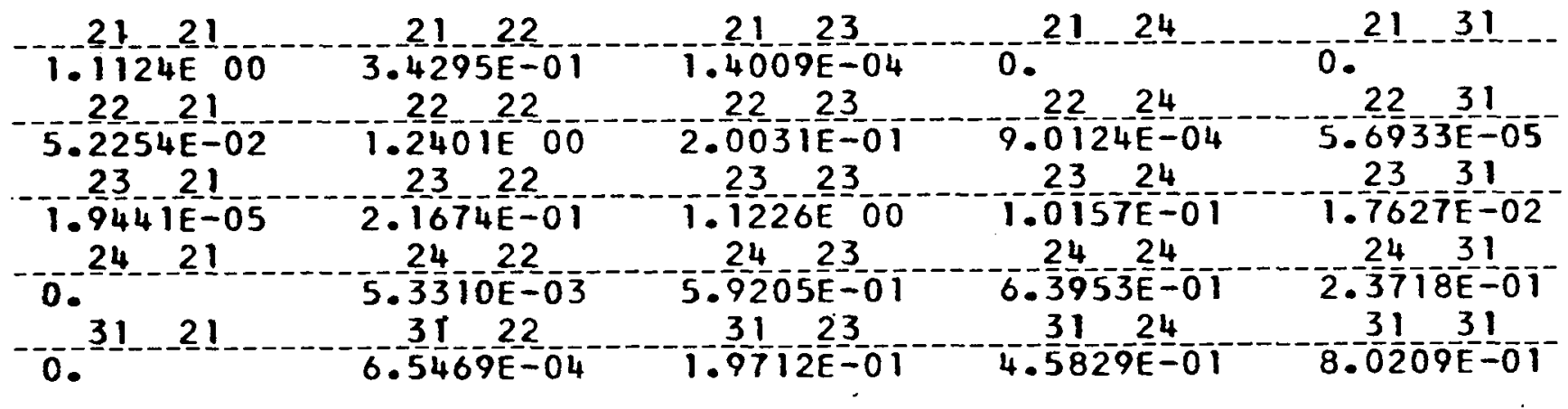

TEMPERATURE
SEEG SEMA
$N 1$
SI GMA S_(NIIN2)

\begin{tabular}{|c|c|c|c|c|}
\hline 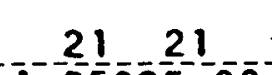 & -21 & -23 & 21 & 21 \\
\hline $\begin{array}{ll}509 \bar{E} & 0 \\
2 & 21\end{array}$ & $\begin{array}{r}2.0458 \mathrm{E}=0 \\
22 \quad 22\end{array}$ & $\begin{array}{l}9218-05 \\
22\end{array}$ & 0 & 2 \\
\hline $3457 \bar{E}=02$ & $1.22265 \bar{E} 0$ & $2.4082 \mathrm{E}-01$ & $4.8344 \mathrm{E}$ & $.6 \overline{6} 62 \overline{2}=0 \overline{5}$ \\
\hline $1.1380 \mathrm{E}-06$ & $1.5375 E-01$ & $1.1733 \mathrm{E} 00$ & $1.1024 \mathrm{E}-01$ & $1.4912 \mathrm{E}$ \\
\hline &.$\overline{2}$ & $5.1035 \mathrm{E}$ & $7.0725 \overline{\mathrm{E}}$ & 2.3812 \\
\hline $0-3$ & $7.8223 E-$ & $-\frac{32}{22}-1 \frac{23}{15 E-0}$ & $4-\frac{32}{26}=\frac{24}{00}$ & $8.6829=\frac{32}{E-1}$ \\
\hline
\end{tabular}


ALUMINUM

_IHERMAL IRANSFER_MAIRICES

IEUPERAIURE

SIGMA

SIGMA

DEG. $F$.

SCATIER

TRANSPORT

SIGMA

ABSORPIION

NU SIGMA..

$5.0000 E \quad 02$

$1.3764 \mathrm{E} 00$

$1.3421 E 00$

$3 . \overline{3} \overline{9} \overline{\mathrm{E}}-\overline{\mathrm{O}} \overline{1}$

FISSION

\section{SIGMA $\left.-\frac{N}{S}-(N) / N 2\right)$}

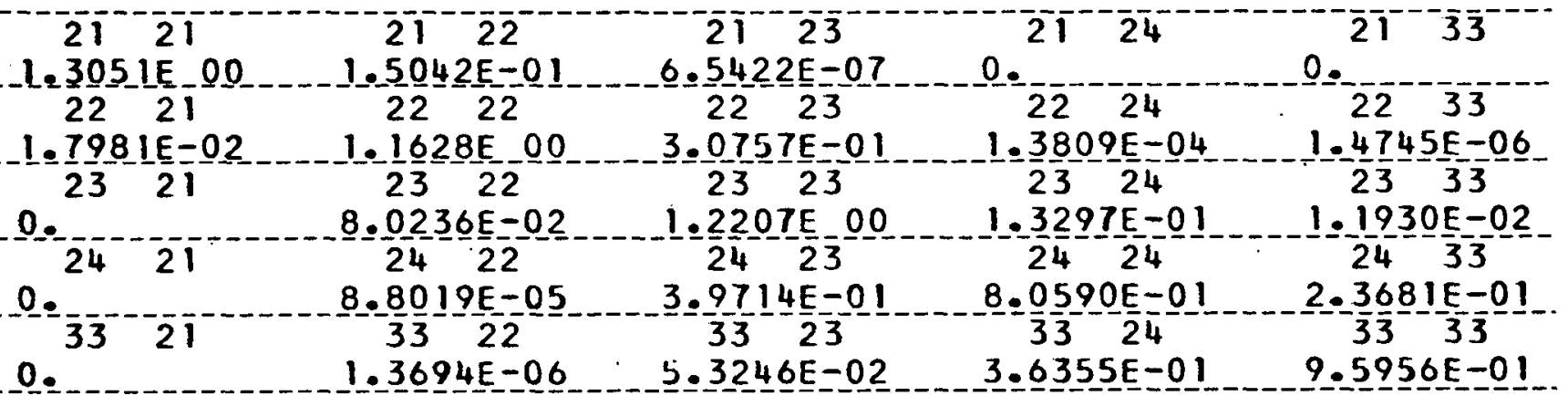

IEMPERATURE

DEG. $F$
SIGMA

SCATTER
SIGMA

TRANSPORT
SIGMA ABSORPTION
NU SIGMA

FISSION

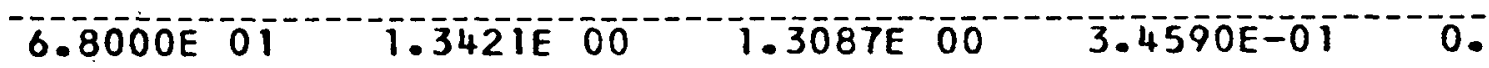

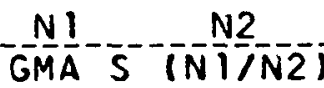

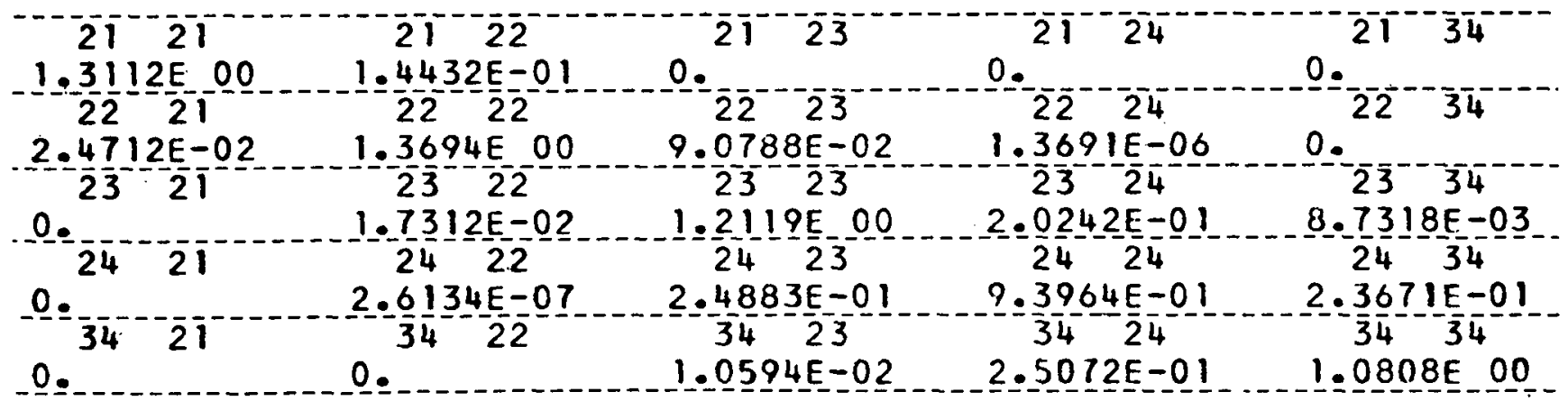


AL UMINUM

TRANSFFERS FROM GROUPS 1 THROUGH 5 TO GROUPS 1 THROUGH 16

$\mathrm{Ni} N 2$

SIGMA_SO (N1/N2)

\begin{tabular}{|c|c|c|c|c|}
\hline 1. & 2 & & 4 & \\
\hline $5.0487 E-01$ & $2.1854 \mathrm{E}-01$ & $1.0022 \mathrm{E}-01$ & $2.0274 E-01$ & $2.1645 \mathrm{E}-01$ \\
\hline $5391 E-01$ & $8.4819 E-02$ & $3.9948 \mathrm{E}-02$ & $2.1326 \mathrm{E}-02$ & $5.9693 \mathrm{E}-03$ \\
\hline $1 \ldots$ & 1 & 1 & 1 & 1 \\
\hline $6578 \mathrm{E}-04$ & $1.1956 \mathrm{E}-04$ & $1.6563 \mathrm{E}-05$ & $2.2032 \mathrm{E}-06$ & $3.2448 E-07$ \\
\hline 1 & & 2 & 2 & 2 \\
\hline $2.5091 E-08$ & 0. & $1.0480 E \quad 00$ & $2.2667 E-01$ & $1126 E-01$ \\
\hline $2 \frac{2}{420 E-01}$ & $1-2 \frac{6}{8}-6$ & $10897 \overline{7}-01$ & $-\frac{2}{047 E-0}-8$ & $2.9885 \bar{E}^{-9}-02$ \\
\hline $2 \quad 10$ & 2 & 2 & 213 & 2 \\
\hline $5808 \mathrm{E}-03$ & $1.2611 \mathrm{E}-03$ & $1.7502 E-04$ & $2.4290 \mathrm{E}-05$ & $3.2334 \mathrm{E}-06$ \\
\hline-15 & 16 & 1 & & \\
\hline $.7631 E-07$ & $3.6835 E-08$ & 0 . & 0. & 1.5197500 \\
\hline $4.0193 \mathrm{E}-01$ & $1.5426 \mathrm{E}-01$ & $1.5328 E-01$ & $1.0428 E-01$ & $5.5972 E-02$ \\
\hline $3 \ldots 9$ & 3.10 & 11 & & 13 \\
\hline $\begin{array}{c}3.2658 \mathrm{E}-02 \\
3 \quad 14\end{array}$ & $\begin{array}{c}9.7473 E-03 \\
3 \quad 15\end{array}$ & $\begin{array}{c}1.4617 E-03 \\
3 \\
16\end{array}$ & $\begin{array}{c}2.0439 E-04 \\
4\end{array}$ & $\begin{array}{c}2.8444 E-05 \\
4\end{array}$ \\
\hline $3.7904 \mathrm{E}-06$ & $5.5859 \mathrm{E}-07$ & $.3204 \mathrm{E}-08$ & 0 & 0. \\
\hline & $-5-\frac{4}{2}=-4$ & $-\frac{4}{3}-5$ & $-4 \frac{4}{26-1}-02$ & $5743 E-02$ \\
\hline & $\begin{array}{l}2.2877 E \\
-4\end{array}$ & $\begin{array}{r}3.7842 \mathrm{E}-01 \\
4 \quad 10\end{array}$ & $\begin{array}{l}-4626 E-02 \\
-4 \\
-\end{array}$ & $\begin{array}{l}43 t-02 \\
4 \quad 12\end{array}$ \\
\hline $\begin{array}{c}3.5489 \mathrm{E}-02 \\
4 \mathrm{~B}\end{array}$ & $2.2196 \mathrm{E}-02$ & $\begin{array}{c}6.9646 E-03 \\
4\end{array}$ & $\begin{array}{c}1.0719 E-03 \\
4\end{array}$ & 1.5757 \\
\hline $1140 E-05$ & $.8210 \mathrm{E}-06$ & $1593 \mathrm{E}-07$ & $3.2176 \bar{E}-0 \overline{8}$ & 0 \\
\hline $5 \quad 2$ & $5 \quad 2$ & 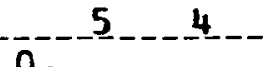 & $\frac{5}{67-1 F}=$ & \\
\hline & & -9 & $5 \quad 10$ & 5 \\
\hline $\begin{array}{c}1.4409 E-02 \\
-5 \quad 12\end{array}$ & $9.4395 \mathrm{E}-03$ & $6.3110 E-03$ & $2.0768 \mathrm{E}-03$ & $3.2762 E-04$ \\
\hline $.0696 \mathrm{E}-05$ & $6.5437 \mathrm{E}-06$ & $8.7435 E-07$ & $1.2897 \mathrm{~T}-07$ & $9.9793 \bar{E}-09$ \\
\hline
\end{tabular}


ALUMINUM.

SIGMA N,G $(N) / N 2)$

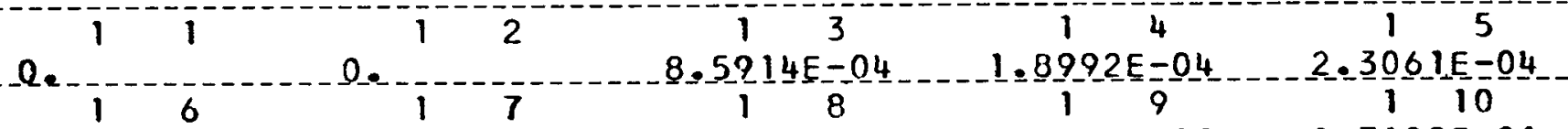

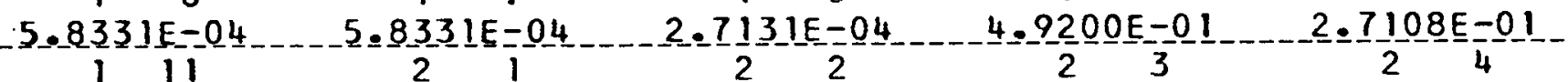

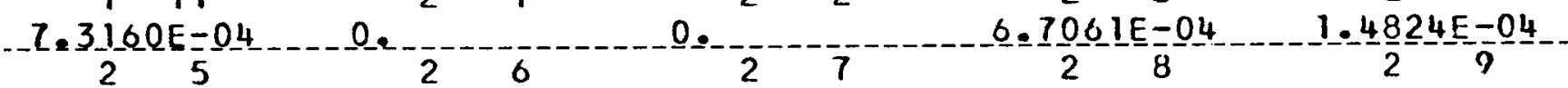
-1.80OU1E-04

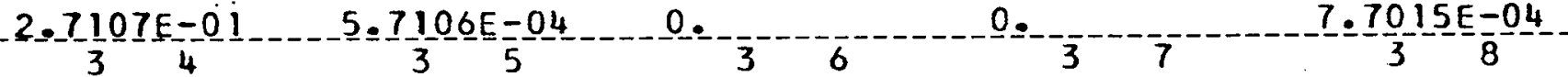

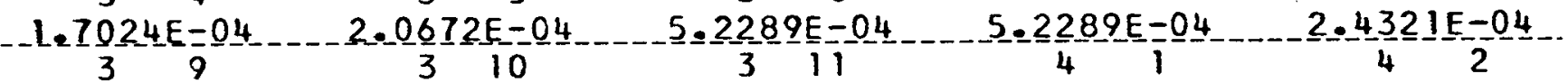
$6.0702 E-01$

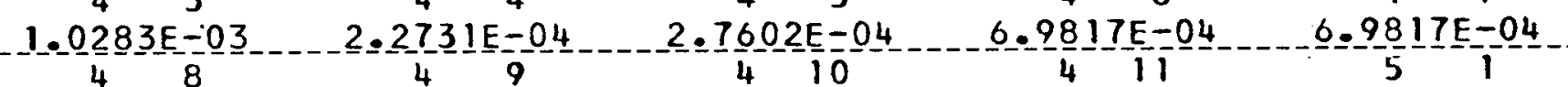

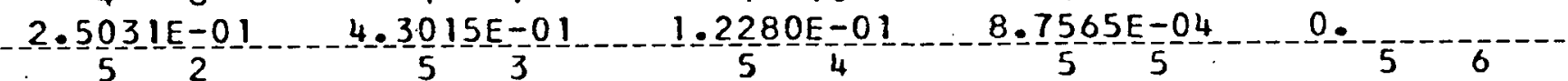
‥ -1 5.9470E-04 $64.7601 E-04 \quad 2.6011 E-01 \quad 1.1967 E-01$ $0.00 .101011 E-03 \quad 2.4339 E-04 \quad 2.9555 E-04$

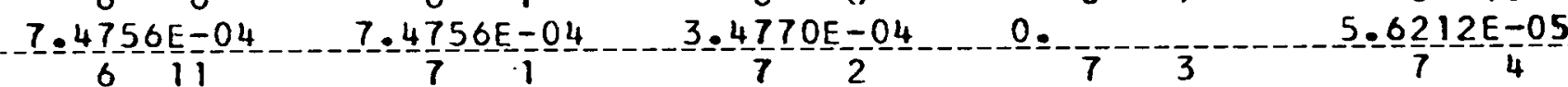

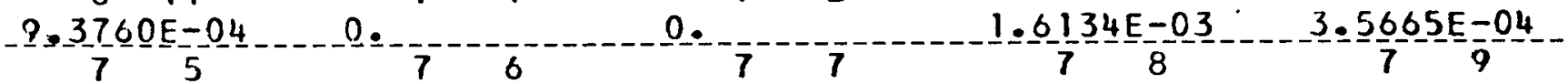
4. $3308 E-04 \quad 1.0954 E-03 \quad 1.0954 E-03 \quad 03.0950 E-04$

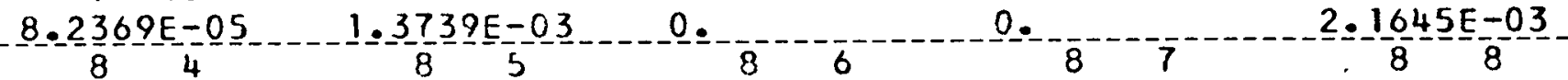
$4.7846 E-04 \quad 5.9098 E-04 \quad 1.4695 E-03 \quad 1.4695 E-03 \quad 6.8351 E-04$

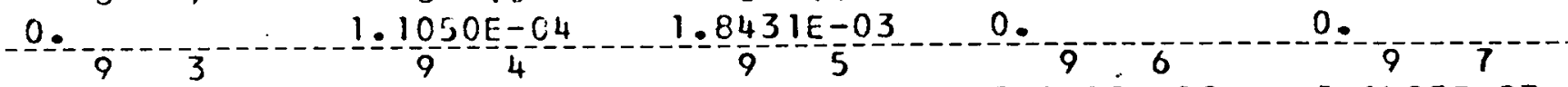
$4.6373 E-03 \quad 1.0251 E-03 \quad 1.2447 E-03 \quad 3.1485 E-03 \quad 3.1485 E-03$ $1.4644 E-03$ _ 0. 
ALUMINUM

TRANSFFERS FROM GROUPS 1 THROUGH 34 TO GROUPS 1 THROUGH II

N1 N2

SIGMA_N,G_INIIN2)

\begin{tabular}{|c|c|c|c|c|}
\hline 1 & $-10-3$ & $-10=-4$ & -10 & -10 \\
\hline 1 & $\begin{array}{c}8.3378 E-03 \\
10\end{array}$ & $\begin{array}{c}1.8431 \mathrm{E}-03 \\
10\end{array}$ & $\begin{array}{l}2.2380 \mathrm{E}-03 \\
10 \quad 10\end{array}$ & $\begin{array}{c}5.6609 \mathrm{E}-03 \\
10 \mathrm{11}\end{array}$ \\
\hline 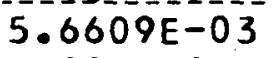 & $2.6330 E-03$ & 0. & $4.2567 E-04$ & $7.1000 E-03$ \\
\hline & & -11 & 11 & \\
\hline & 0. & $1.0885 E-02$ & $2.4063 E-03$ & $2.9219 \mathrm{E}-0$ \\
\hline 39 & $7.3907 \mathrm{E}-03$ & $-3 . \frac{11}{4}-\frac{1}{3} 75 E-03$ & 0 & $-5 . \frac{1}{5} \frac{1}{7}-\frac{10}{3 E}=0$ \\
\hline 11. & 12 & 12 & 12 & 124 \\
\hline 2 & 0. & 0 & $7.7157 \mathrm{E}-03$ & $1.7056 \mathrm{E}-\mathrm{C}$ \\
\hline & & 12 & 12 & 12 \\
\hline$-0 \overline{3}$ & $5.2386 \mathrm{E}-03$ & $5.2386 \mathrm{E}-03$ & $2.4365 E-03$ & 0 \\
\hline-1 & -12 & 13 & -13 & 13 \\
\hline$=-04$ & $6.5703 E-03$ & 0. & 0 & $1.2711 E-02$ \\
\hline-4 & 5 & 13 & 13 & 13 \\
\hline $8099 E-03$ & $3.4120 E-03$ & $8.6304 E-03$ & $8.6304 E-03$ & $4.0141 E-0$ \\
\hline-13 & $--\frac{13}{13}$ & $-\frac{1}{2} 1_{-}$ & -14 & -14 \\
\hline & $.4 \xi$ & $\begin{array}{l}.0824 E-02 \\
14\end{array}$ & • & $\bullet_{12}$ \\
\hline & $4.6312 E-0$ & $5.6237 \mathrm{E}-04$ & $1.4225 \mathrm{E}-03$ & $1.4225 \mathrm{E}-03$ \\
\hline 1 & 14 & 10 & 11 & $15 \quad 1$ \\
\hline $\mathrm{TE}-04$ & & $1.0696 \mathrm{E}=04$ & $1.7841 E-03$ & 0. \\
\hline & 15 & $-\frac{15}{34}=5=-4$ & 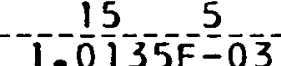 & $-\frac{15}{56}=-6$ \\
\hline 15 & 15 & 15 & $15 \quad 10$ & 1511 \\
\hline & 1.1924 & 0. & $1.9277 E=04$ & $3.2153 \mathrm{E}-03$ \\
\hline & -16 & $\frac{16}{2}$ & $-\frac{16}{5} 6-5=4$ & $-1 \frac{6}{2}-1-=5$ \\
\hline & & 8 & $16 \quad 9$ & $\begin{array}{c}1.938 r E-0 \\
16 \quad 10\end{array}$ \\
\hline $\begin{array}{l}9039 \mathrm{E}-0 \\
16 \quad 11\end{array}$ & $4.9039 E-03$ & $2.2809 E-03$ & 0 & $3.6874 \mathrm{E}=04$ \\
\hline 11 & 17 & 17 & & \\
\hline $\begin{array}{l}1505 E-03 \\
175\end{array}$ & 0. & 0 . & $1.2503 E-02$ & $2.7638 E-03$ \\
\hline$-\frac{5}{E-0}$ & -8.48 & $8.4889 E-03$ & $3.9 \frac{17}{48}-8$ & 0 \\
\hline 17 & 17 & 18 & 18 & \\
\hline $182+2$ & 1.064 & & 0 & $2.0241 E-02$ \\
\hline$-\frac{18}{4}$ & $=-\frac{18}{4}$ & $\overline{1-5 \pi}$ & 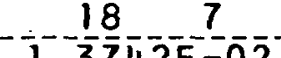 & $\frac{18}{30}=\frac{8}{2}$ \\
\hline & 18 & 1.. & $19 \quad 1$ & 19 \\
\hline & & & & \\
\hline
\end{tabular}


-ALUMLIVM.

N 1

\section{SIGMA N,G (NI/N2)}

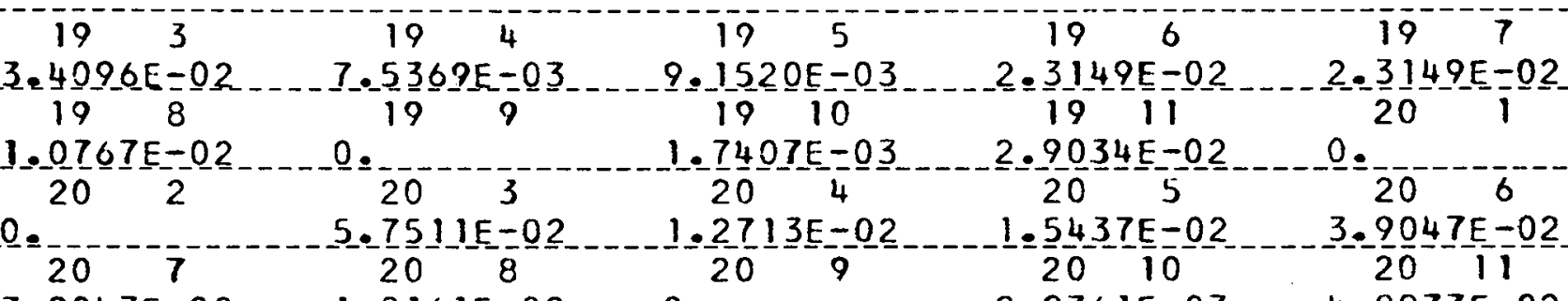

$-3.9047 E-02-1 \cdot \frac{8}{2} 161 E-022-0$.

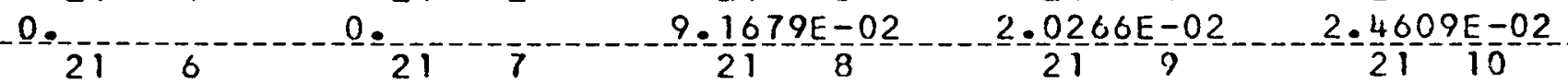

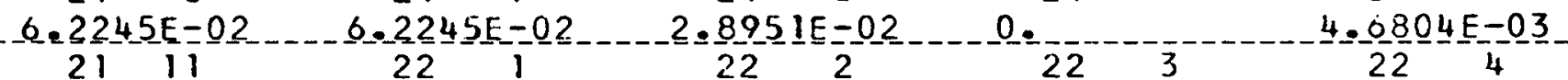

$3.9420 E=02$

$2210 \quad 22 \quad 112331233$

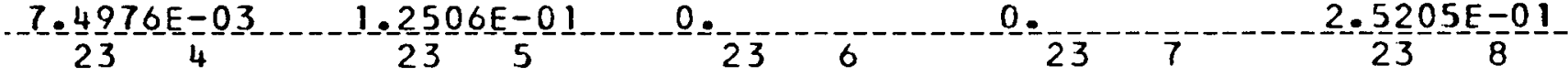

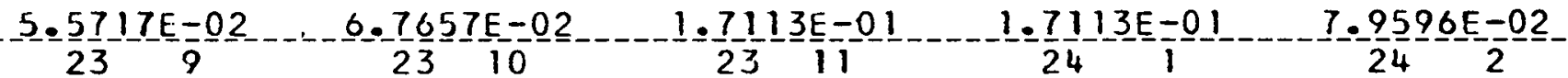

0.

$3.9 \frac{1}{2} 23 \mathrm{E}-01$

$-1.2355 E=01$

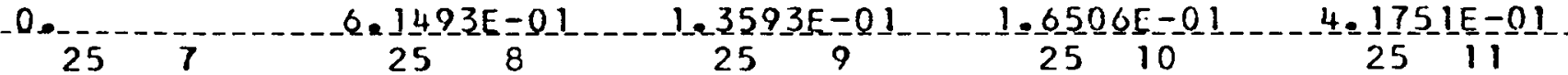

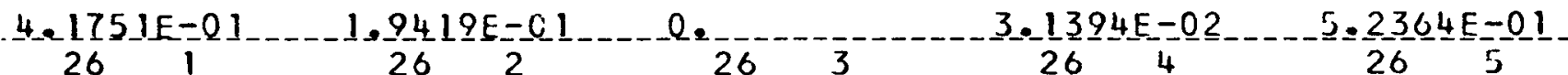

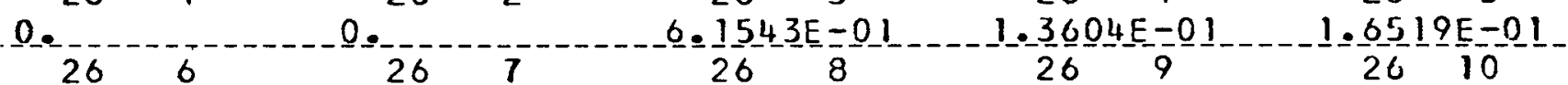

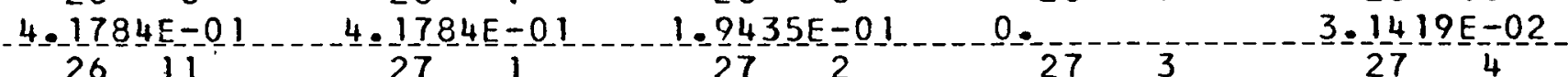

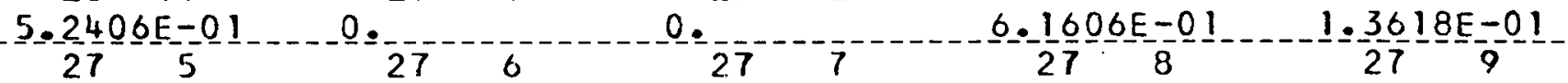

$1.6536 \mathrm{E}-01$

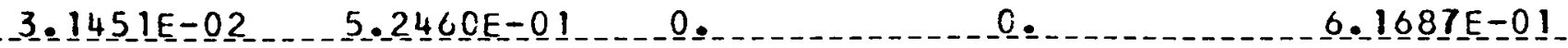




\section{ALUMINUM}

N1 N2

SIGMA N, G (NI/N2)

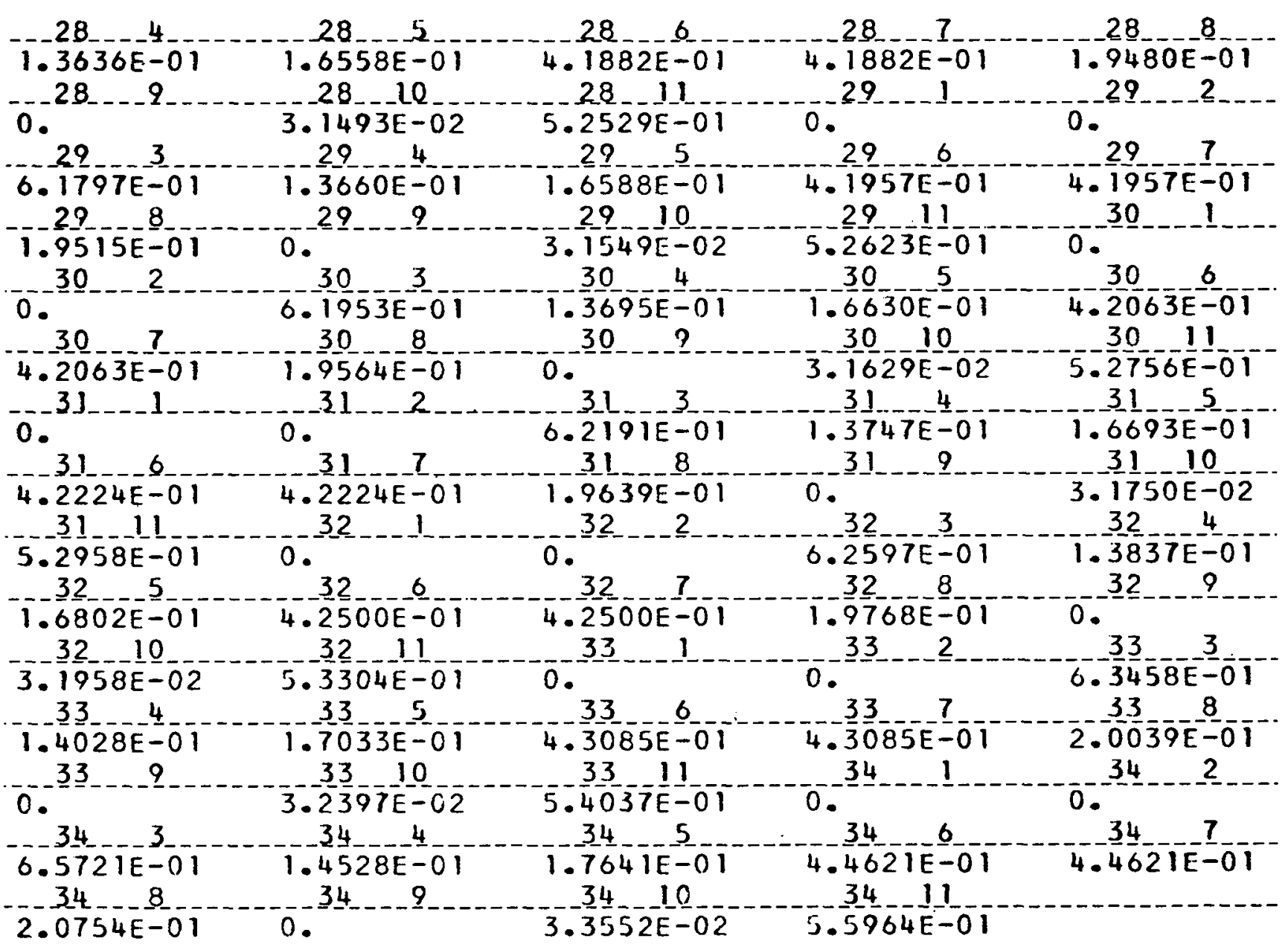


DAIE_ 12/08/60.

DESIGNATION CODE NO. DENSITY FACTOR

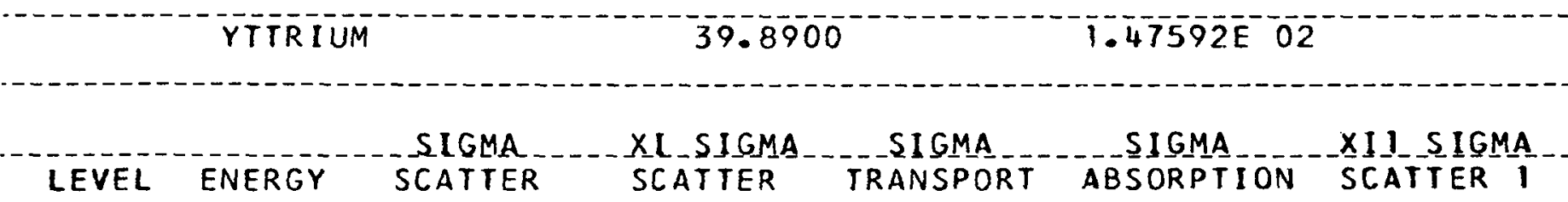

1 1.000E $073.9827 E$ OO $3.9295 \mathrm{E} 002.008 \mathrm{EE} 00 \quad 4.2567 \mathrm{E}-03-1.9475 \mathrm{E} 00$

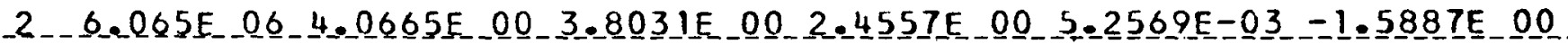

$3 \quad 3.679 E$ O6 4.2277 E $00 \quad 3.0305 E$ DO 2.8824E 00 6.0470E-03-1.3268E 00

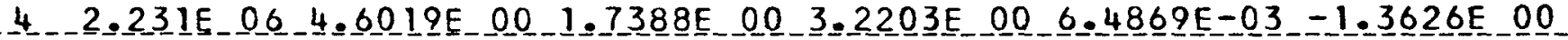

$51.353 \mathrm{E} 065.8895 \mathrm{E} 007.6949 \mathrm{E}-013.9401 \mathrm{E} 006.8167 \mathrm{E}-0.3-1.9227 \mathrm{E} 00$

6 8.208E O5 7.486 IE $002.2418 \mathrm{E}-015.0810 \mathrm{E}$ 00 $7.0740 \mathrm{E}-03-2.372 \mathrm{TE} 00$

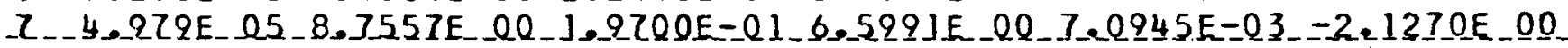

8 3.020E $05 \quad 8.4674 \mathrm{E}$ 00 1.9052E-017.2466E 00 6.9775E-03-1.2040E 00

$-2-1.832 \mathrm{E}-05-7.558 \mathrm{JE}-00 \_.2006 \mathrm{E}=01-6.8724 \mathrm{E}-00-6.8779 \mathrm{E}=03=6.7635 \mathrm{E}=01$

$\begin{array}{llllllllll}10 & 6.738 E & 04 & 7.4003 E & 00 & 1.6651 E-01 & 7.1017 E & 00 & 6.8278 E-03 & -2.9449 E-01\end{array}$

$112.479 \mathrm{E} 047.2938 \mathrm{E}$ 00 $1.6411 \mathrm{E}-017.1769 \mathrm{E}$ 00 $2.2046 \mathrm{E}-02-1.1527 \mathrm{E}-0 \mathrm{~T}$

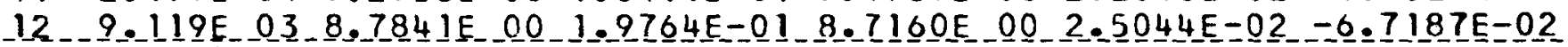

13 3.355E $037.6762 \mathrm{E} \quad 00$ 1.7272E-01 $7.6179 \mathrm{E}$ 00 8.2402E-02 $-5.7538 \mathrm{E}-02$

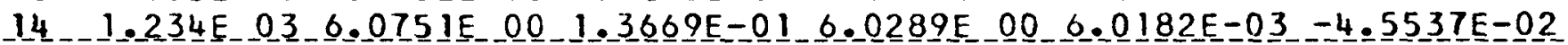

$154.540 \mathrm{E} 026.4193 \mathrm{E} 001.4443 \mathrm{E}-016.3705 \mathrm{E} 001.0888 \mathrm{E}-02-4.8116 \mathrm{E}-02$

$168.719 \mathrm{E} 016.7450 \mathrm{E}$ OO $1.5176 \mathrm{E}-016.6938 \mathrm{E} 002.0745 \mathrm{E}-02-5.0558 \mathrm{E}-02$

17 3.545E 01 7.0104E 00 1.5773E-01 6.9571E $00 \quad 3.6026 \mathrm{E}-02=5.2547 \mathrm{E}=02$

$18-1.304 \mathrm{E} 01-7.2785 \mathrm{E} 00-1.6377 \mathrm{E}-01-7.2232 \mathrm{E}-00-5348 \mathrm{E}-02-5.4557 \mathrm{E}-02$

19 5.043E $007.4547 \mathrm{E} 00-1.6773 \mathrm{E}-01$ 1.3980E $009.8183 \mathrm{E}=02-5.5878 \mathrm{E}=02$

$201.445 \mathrm{E}$ 00 $7.4005 \mathrm{E}$ 00 $1.6651 \mathrm{E}-017.3443 \mathrm{E} \quad 001.6580 \mathrm{E}-01-5.5472 \mathrm{E}-02$

$210.826 \mathrm{E}-0 \mathrm{~T} 7.4262 \mathrm{E} 00 \mathrm{0} 1.0709 \mathrm{E}-017.3697 \mathrm{E} 002.6252 \mathrm{E}-01-5.5664 \mathrm{E}-02$

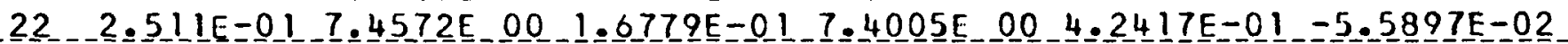

$239.237 E-027.3382 E$ DO $1.6511 E-017.2824 E$ E $007.2586 E-01-5.5004 E-02$

24 -2.530E-02 7.1187E 00 1.6017E-01 7.0646E 00 1.1207E 00_-5.3359E-02

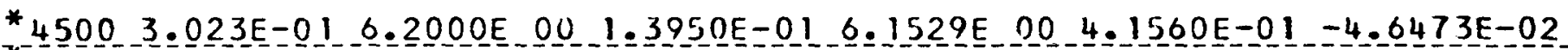

* $40002.718 \mathrm{E}-016.2000 \mathrm{E}-00-1.3950 \mathrm{E}-016.1529 \mathrm{E}-00-4.1560 \mathrm{E}-01-4.6473 \mathrm{E}-02$

* $35002.413 E-01 \quad 6.2000 E$ O0 $1.3950 E-01 \quad 6.1529 \mathrm{E}$ 00 $4.1560 \mathrm{E}-01-4.6473 \mathrm{E}-02$

* $30002.109 \mathrm{E}-016.2000 \mathrm{E}-00-1.3950 \mathrm{E}=016.1529 \mathrm{E} 00-4.1560 \mathrm{E}-01-4.6473 \mathrm{E}=02$ $25001.804 E-01$ 6.2000E 00 1.3950E-01 6.152?E $0044.4940 E-01-4.6473 E-02$

$20001.499 E-01-6.2000 E=001-3950 E=01-6.1529 E-00-4.9290 E=01-4=6473 E=02$ $15001.194 \mathrm{E}-016.2000 \mathrm{E} 001.3950 \mathrm{E}-016.1529 \mathrm{E} 00 \mathrm{5} .5230 \mathrm{E}-01-4.6473 \mathrm{E}-02$

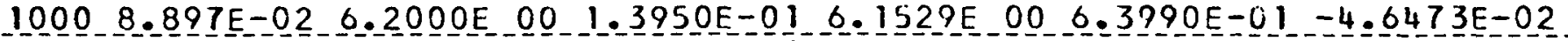
$5005.850 \mathrm{E}-026.2000 \mathrm{E}$ 00 $1.3950 \mathrm{E}-016.1529 \mathrm{C} 007.8920 \mathrm{E}-01-4.6473 \mathrm{E}-02$

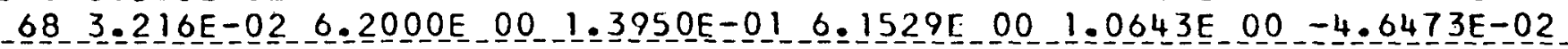

*Thermal cross sections taken from APEX-515.

NOTE: For a summary of the sources of data see APEX-704. 


\section{YITRIUM}

\section{THERMAL TRANSFER MATRICES}

TEMPERATURE
SIGMA

$\mathrm{N} 1{ }^{-12}$

SI GMA_S_ (NIIN2)

\section{TEMPERATURE}

DEG. F.

4..0000E 03
SIGMA SCAITER

7.9810E 00
$21-23$

2.2350E-05

$4.3944 \mathrm{E}-\mathrm{O} 1$

$5.6999 \mathrm{E} 00$

$24-23$

$2.9605 \mathrm{E} 00$

$8 .-\frac{25}{96} \div \frac{23}{1 \mathrm{E}-01}$
21

22

$5.9034 \mathrm{E}-04$

$-2 \frac{3}{3}-24$

$3.3818 E-01$

$3-\frac{24}{3}-24-10$

2524
$0.21-25$

$22 \quad 25$

$1.9294 E-05$

$23-25$

4. $1577 \mathrm{E}-02$

$24 \quad 25$

$1.0322 \mathrm{E} 0 \mathrm{O}^{-}$

2525 
YTTRIUM

- IHERMAL IRANSEER MAIRICES

...IEMPERAIURE

SI GMA

SIGMA

SCATTER TRANSPORT

SIGMA

ABSORPTION

NU S SIGMA

FISSION

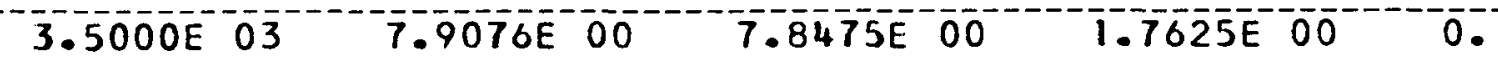

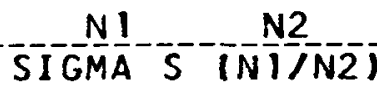

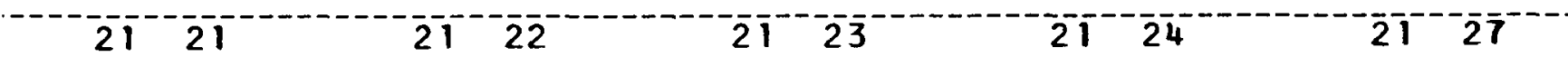

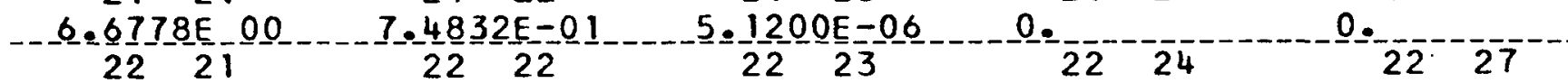
$-4=\frac{91}{23} \frac{28}{21}=01$

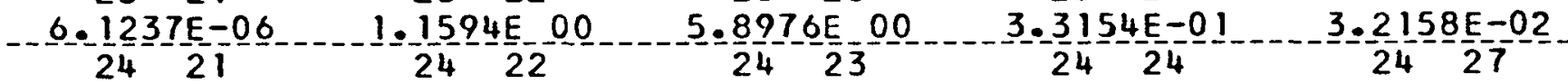

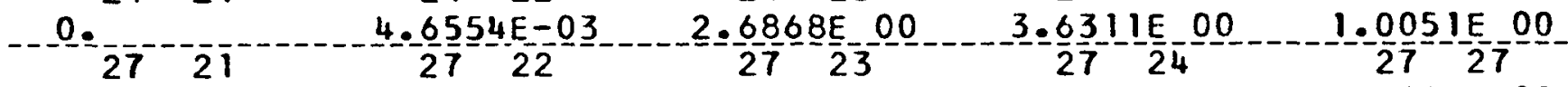
-..‥ $1.9173 E-04$ $6.2481 \mathrm{E}-01$ $2.4509 E$ EO 4.8316E 00

IEMPERAIURE

DEG. $F$.

$3.0000 \bar{E} 0 \overline{3}$
SCATTER

SIGMA

TRANSPORT
SIGMA

ABSORPTION
NU SIGMA

FISSION

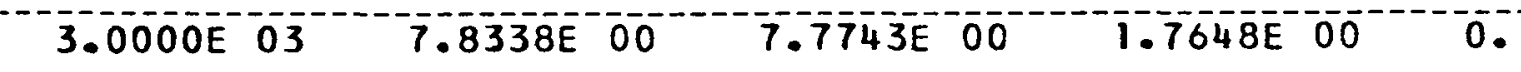

\section{SIGMA}

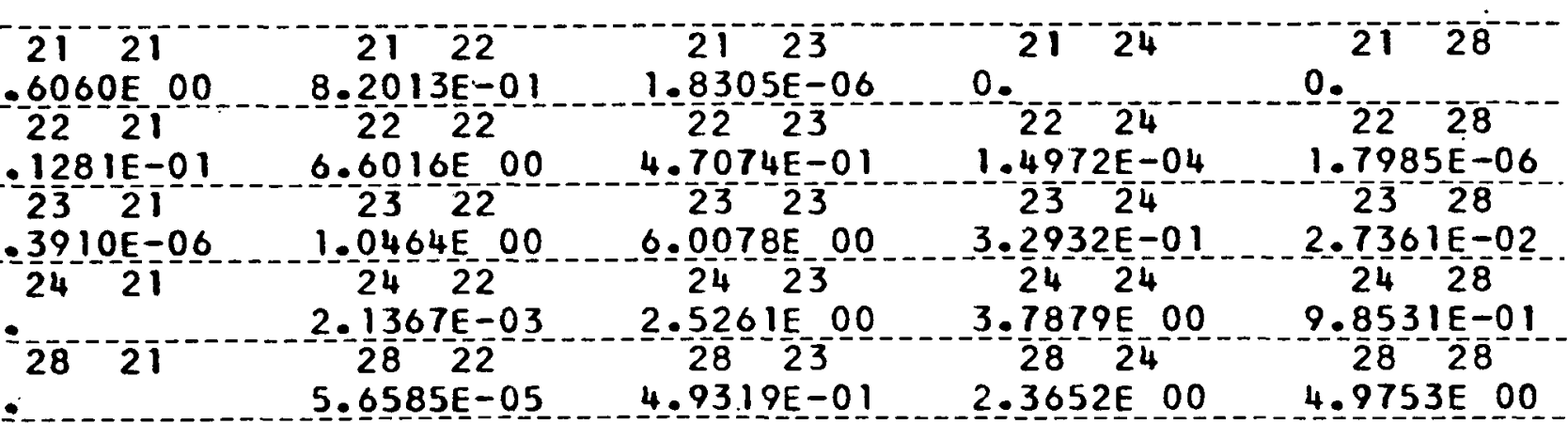


YIIRIUM

\section{THERMAL TRANSFER MATRICES}

\begin{tabular}{|c|c|c|c|c|}
\hline $\begin{array}{l}\text { TEMPERATURE } \\
\text { DEGQ_F_- }\end{array}$ & $\begin{array}{l}\text { SIGMA } \\
\text { SCAIIER }\end{array}$ & $\begin{array}{l}\text { SIGMA } \\
\text { TRANSPORI }\end{array}$ & $\begin{array}{l}\text { SIGMA } \\
\text { ABSORPIION }\end{array}$ & $\begin{array}{l}\text { NU SIGMA } \\
\text { EISSION }\end{array}$ \\
\hline $2.5000 E_{-} 03$ & 7.27597E_00 & $I=7007 E$ & 76729E_ & 0 \\
\hline & & NMA_S IN1II & & \\
\hline$\frac{21}{6.5048 E 00}$ & $\frac{21}{2134 E-01}$ & $-\frac{2 L}{4.7156 E-07}$ & $0.21 \ldots$ & ${ }_{0}-2 \mathrm{~L}_{-} 29$ \\
\hline 22 & $22-22$ & 22 & $22 \ldots 24$ & 29 \\
\hline $\begin{array}{l}3.2939 E-01 \\
23\end{array}$ & $\begin{array}{l}6.6560 E 00 \\
23.22\end{array}$ & $\begin{array}{c}4.9626 E-01 \\
2323\end{array}$ & $\begin{array}{c}7.3695 E-05 \\
23\end{array}$ & $\begin{array}{c}5.1305 E-07 \\
2329\end{array}$ \\
\hline $2.0065 \mathrm{E}-07$ & $9.2249 \mathrm{E}-01$ & $6.1271 \mathrm{E} 00$ & $3.2878 \mathrm{E}-01$ & $2.2541 \mathrm{E}-02$ \\
\hline & $\frac{24}{7.8058 E-04}$ & $\begin{array}{l}24 \\
2.3447 E \quad 00\end{array}$ & $3.9702 \mathrm{E} 00$ & $9.5960 \mathrm{E} 00$ \\
\hline 0 & $\frac{29}{1.1547 E-05}$ & $\frac{29}{3.6747 E-01}$ & $2.2555 \mathrm{E}-2$ & $5.1367 \mathrm{E} 00$ \\
\hline
\end{tabular}

SEMPERATURE
SIGMA

Ni 12

SIGMA S_ (NIIN2)

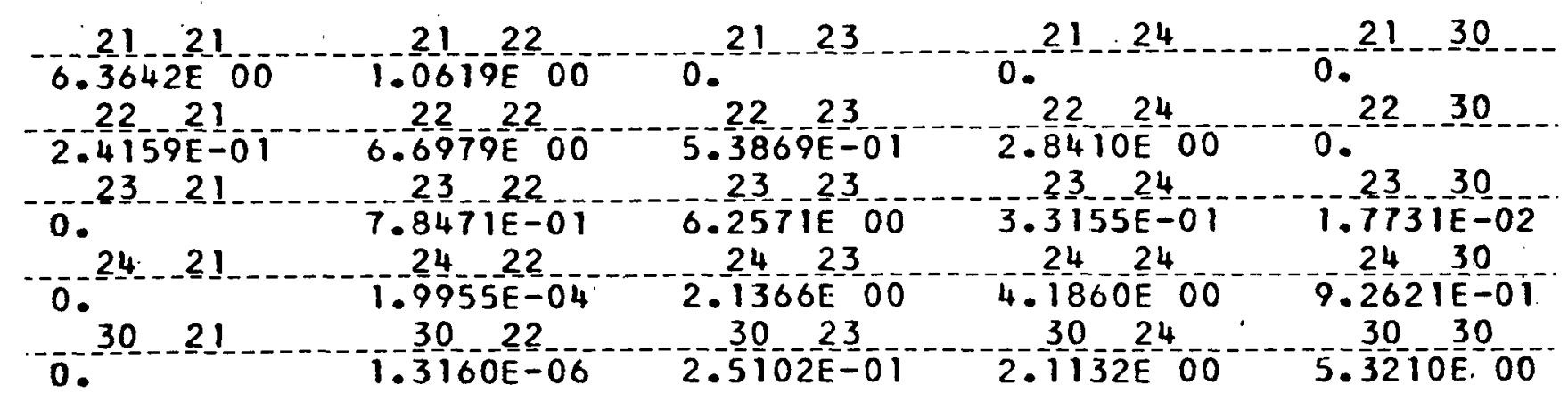


YTTR I UM

_LHERMAL_JRANSFER_MAJRICES_-

__LEMPERATURE SI GMA

SIGMA

SIGMA

NU_ SIGMA_-

DEG. F.

SCATTER

TRANSPORT

ABSORPTION

FISSION

$1.5000 \mathrm{E} \quad 03$

$7.6106 \mathrm{E} 00$

$7.5527 \mathrm{E} 00$

$1.7792 \mathrm{E} 00$

0 .

\section{SIGMA S (NI/N2)}

2121

6.2757E-00

$22 \quad 21$

$1.5210 E=01$

2321

2421

0.

‥
2122

$1.1505 E$

$22 \quad 22$

6.7091E 00

2322

$6.2855 \mathrm{E}-01$

2422

2. $7696 \mathrm{EE}=05$

0.
3122 3

$$
2 \overline{1}^{2}
$$

0.

2223

$-1343 \mathrm{E}=-0$

$6.3985 \mathrm{E}-00$

$1.8920 \mathrm{E} \quad 00$

3123

$1.4874 E-01$
$21^{24}$

$22-24$

$7.1632 E=06$

2324

$3.4 \frac{1}{24} 18 E-01$

$4=4481 E$

3124

$1.9252 \mathrm{E} 00$
0.2131

$0 .-13$

1.2987E-02

8. $8270 E-01$

3131 5. $5366 \mathrm{E}$ O0

IEMPERAIURE

DEG. $F$.
SCATTER

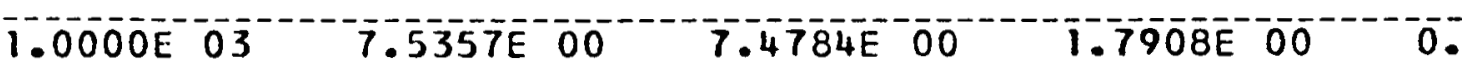

SIGMA

TRANSPORT
SIGMA

ABSORPTION
NU SIGMA

FISSION

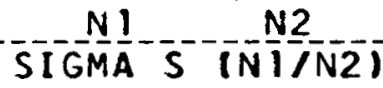

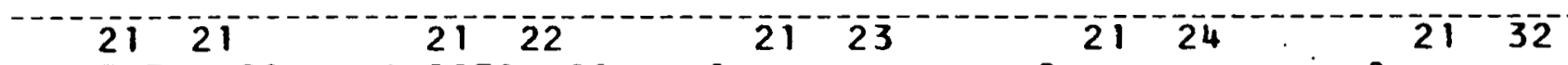

_6.7274E 00

222122

$2 \overline{2} \overline{3}$

$0 .-2 \overline{2}-24$

$22-32$

$6.9246 E-01$

$7.5889 \mathrm{E}-01$

$7.6549 \mathrm{E}-07$

$-\frac{6}{2} 3-2 \frac{1}{2}$

$2 \overline{3}-\overline{3} \overline{2}^{-\cdots}$

은

$4.4 \frac{4}{2} \frac{6 E}{2}-01$

$6.5481 \mathrm{E}-00$

$3.6716 \mathrm{E}=01$

8. $406606-03$

$-0 . \overline{3} \overline{2}$

$1.1610 E-06$

$1.5936 E \quad 00$

…

o.

$32-23$

$4=7769 E_{0} 00$

$8.2605 E-01$

$6.7677 E-02$

$1.6696 \mathrm{E} \quad 00$

5.7984 E 00 . 
YIJRIUM

THERMAL TRANSFER MATRICES

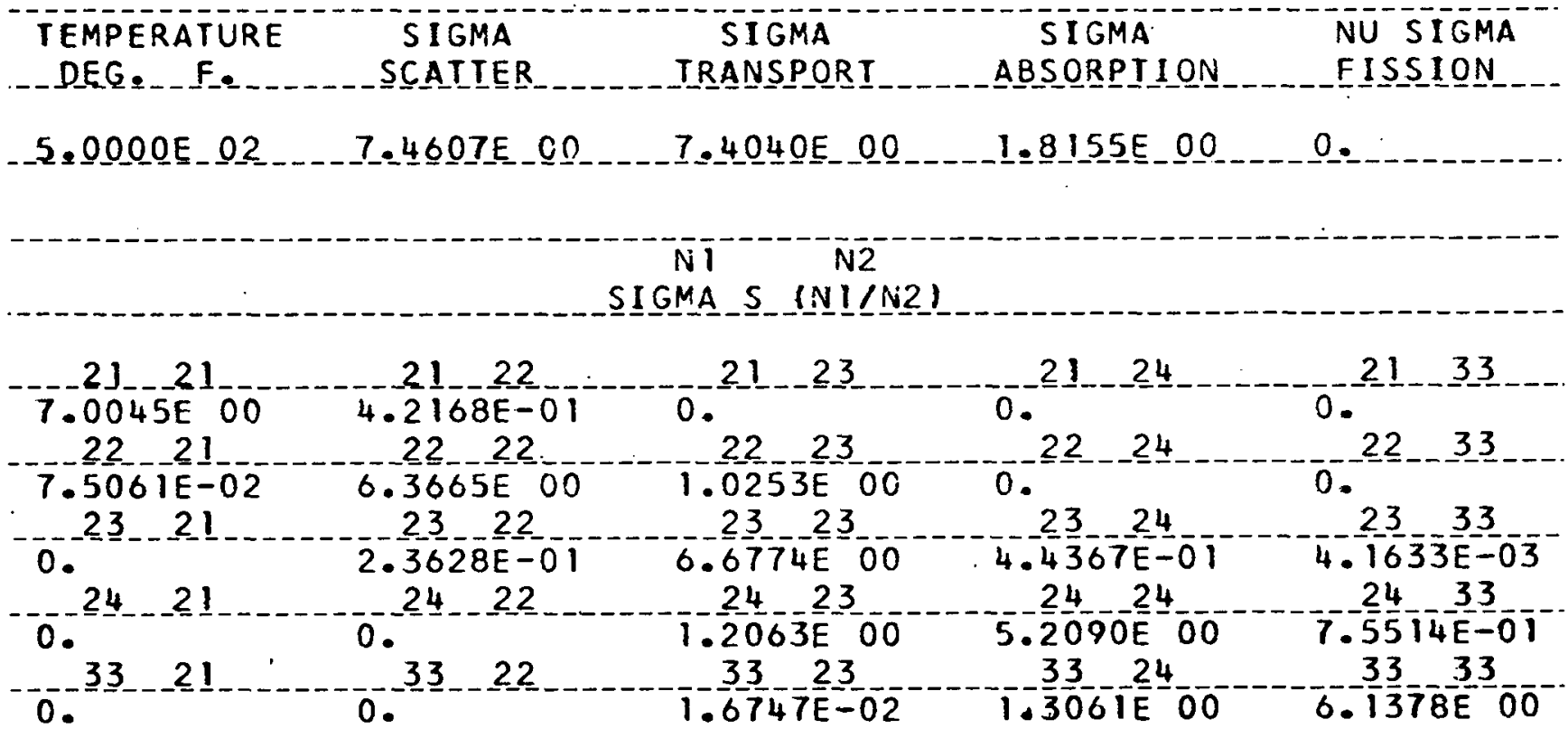

TEMPERATURE
SIGMA

$\mathrm{N} 1 \mathrm{~N} 2$

SI GMA S

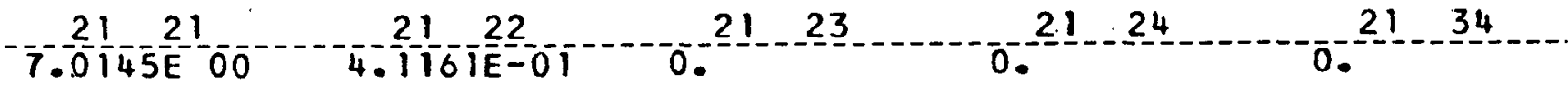

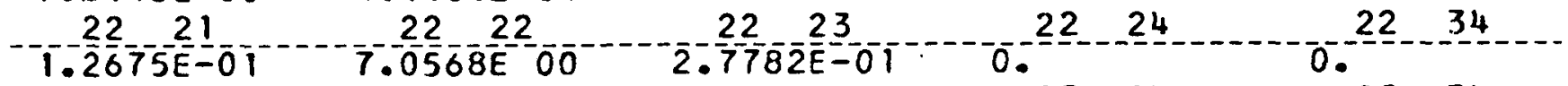

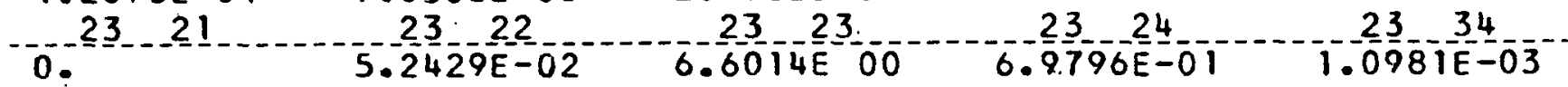

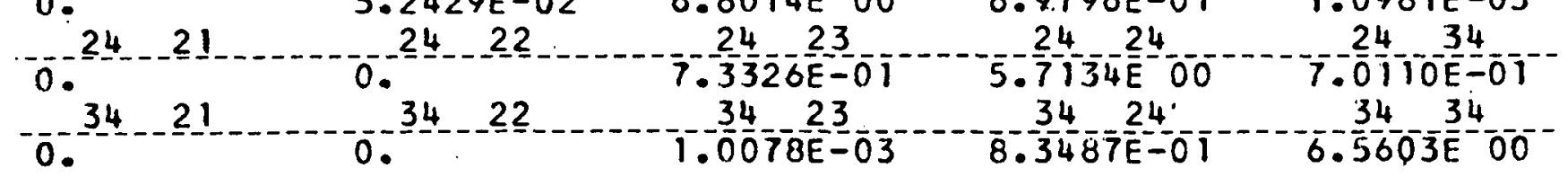


YIIRIUM

TRANSFERS FROM GROUPS I THROUGH 10 TO GROUPS 1 THROUGH 16

N1

N2

SIGMA SO (N1/NR2)

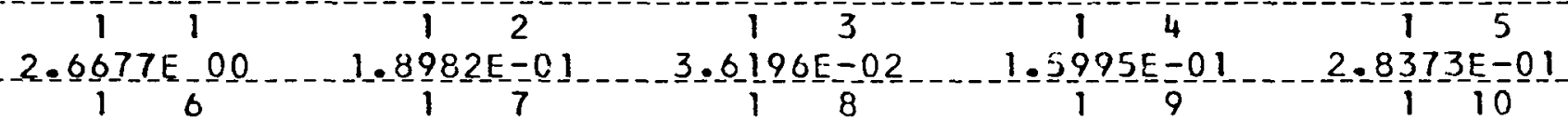

$-2.7866 \mathrm{E}-01 \quad 1.8787 \mathrm{E}=0 \mathrm{l} \quad 1.0019 \mathrm{E}-01 \quad 5.8189 \mathrm{E}-02$

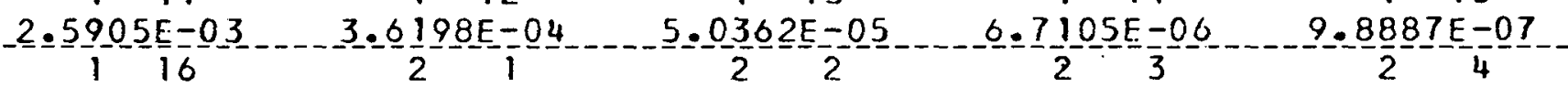

$7.6 \frac{4}{2} 84 \mathrm{E}-08$

$-2.4583 \mathrm{E}-01$

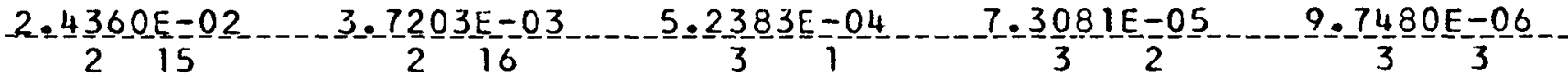

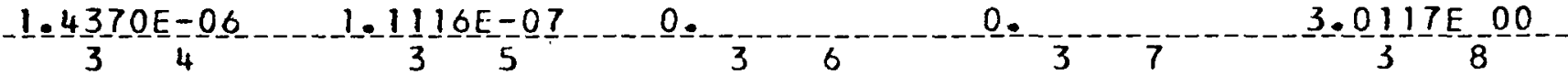

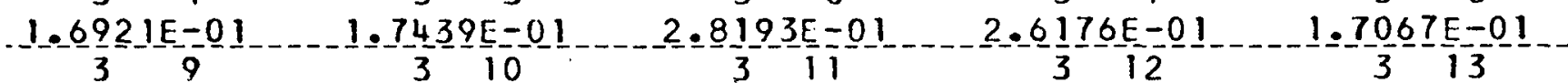

$1=1381 \mathrm{E}-01$

$1.5 \frac{72}{4} 9 \mathrm{E}-05$

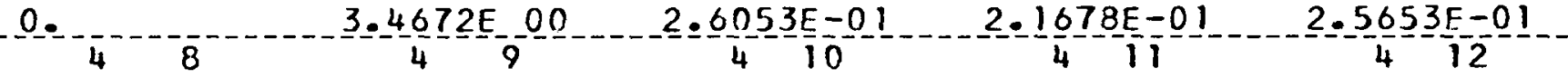

$1.9502 E-01 \quad 1.4468 E-01 \quad 0.1323 E-02 \quad 8.4208 E-03 \quad 1.2183 E-03$

$1.7164 \mathrm{E}-04 \ldots-2.2983 \mathrm{E}-05$

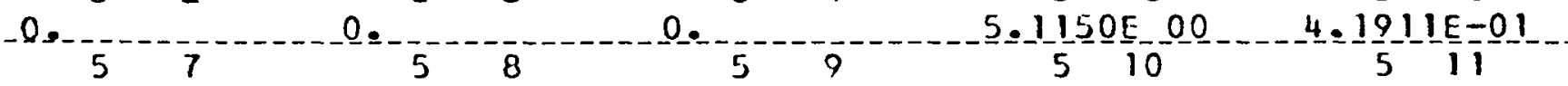

$1.1893 \mathrm{E}=01$ $\begin{array}{lllllllll}5 & 12 & 5 & 13 & 5 & 14 & 5 & 15 & 5 \\ 16\end{array}$ $-8.6 \frac{1}{6} 53 E=04 \ldots-1.2211 E=04-1=6388 E=05-2.4213 E-06-1.8746 E-07$

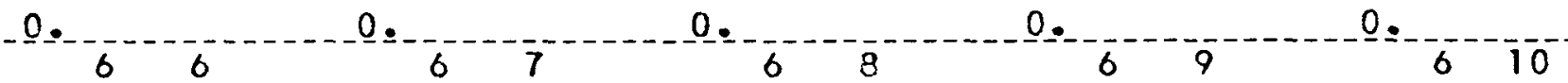
$7.1642 E-00 \quad 3.0997 E-01 \quad 4.9488 E-03 \quad 4.6550 E-03 \ldots 1.9540 E-03$ $3.4998 E-04 \quad 5.2348 E-05 \quad 7.4654 E-06 \quad 1.0044 E-06 \quad 1.4851 E-07$ $1.1502 E-08$ 


\section{YTTRIUM}

$\mathrm{N1} N 2$

SIGMA SO (N1IN2)

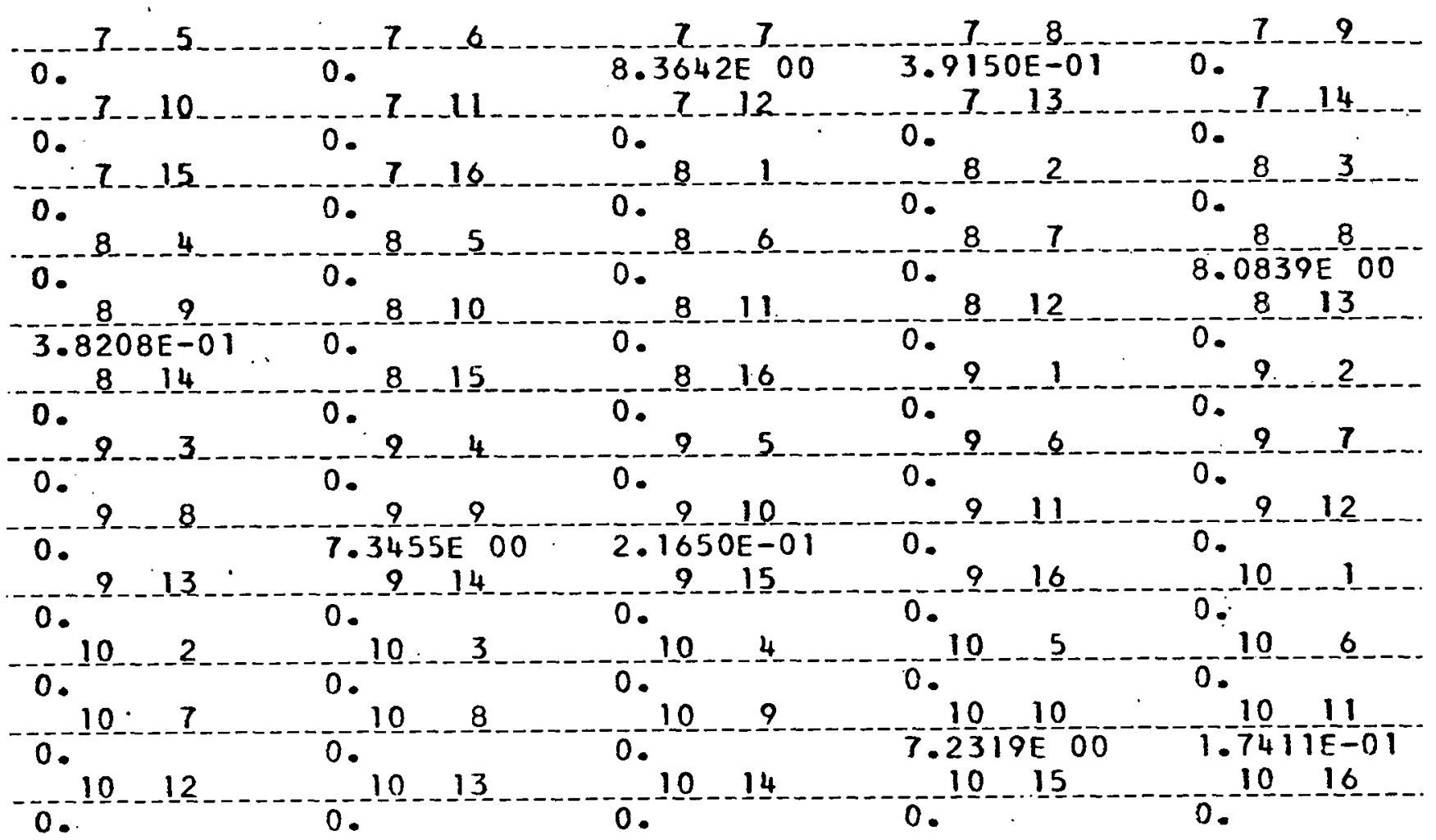


YIIRIUM

TRANSFERS FROM GROUPS I THROUGH 34 TO GROUPS 1 THROUGH II

N1

SIGMA N,G (NI/N2)

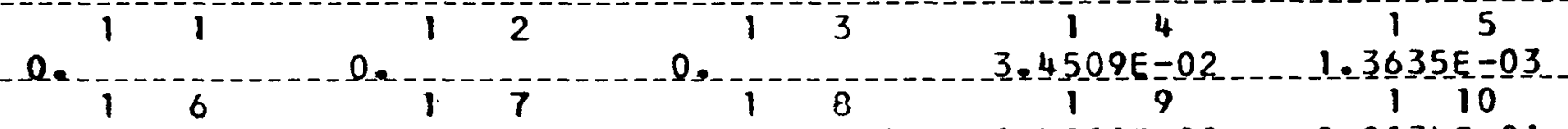

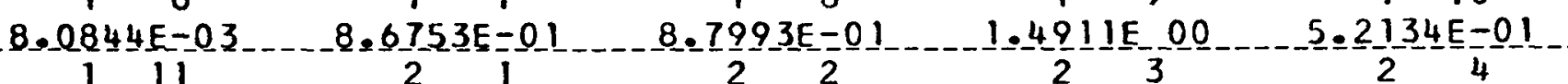

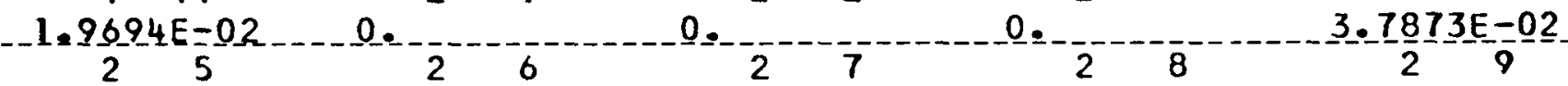

$1.4964 E=03$

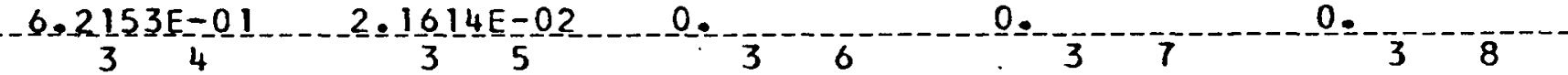

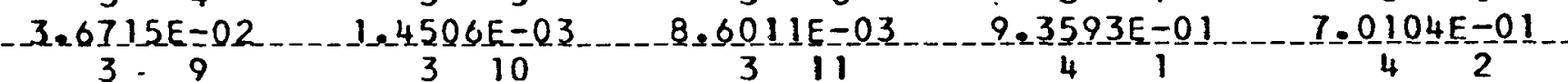

$\left.1.5512 E_{3} 00-5.23\right] 0 E-01-2.0953 E=02$

$0.4 .4836 E=02$

$$
48049
$$

$1.8027 E-03 \quad 3,3760 E-01$

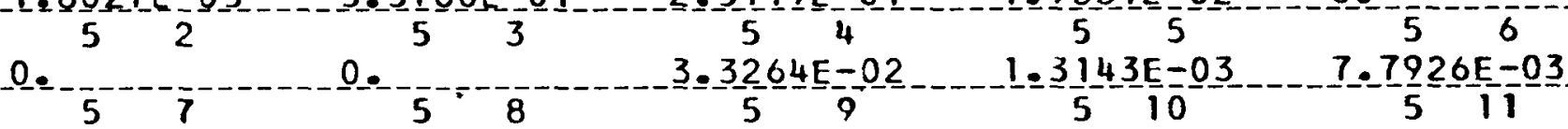

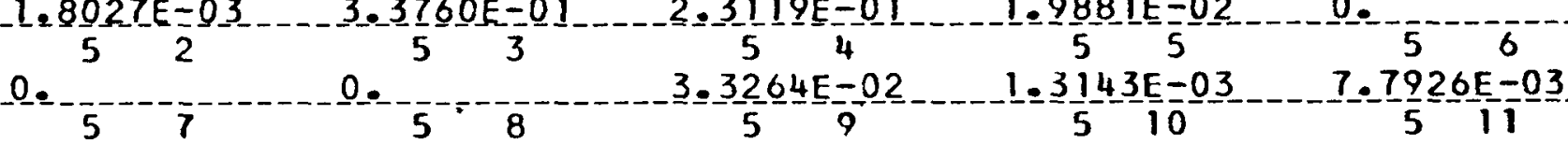

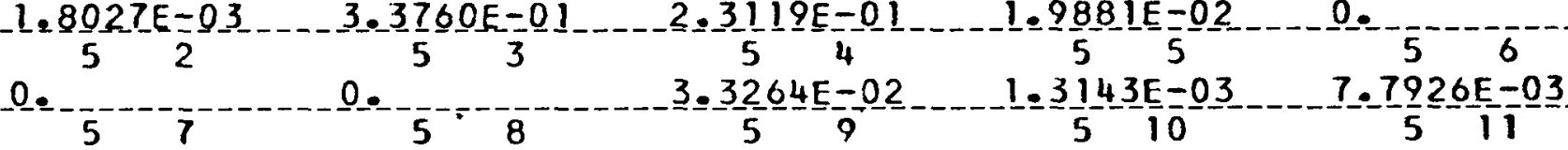

으.

4

0.

$1.2794 \mathrm{E}-02$

-0.

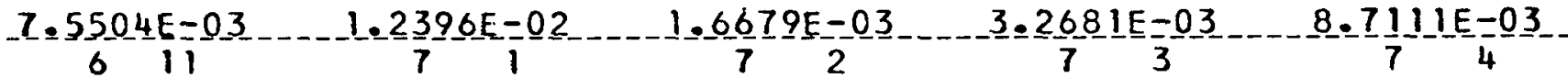

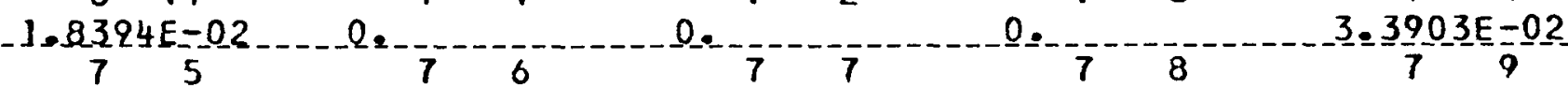

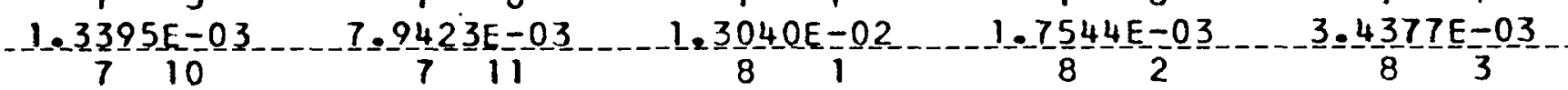

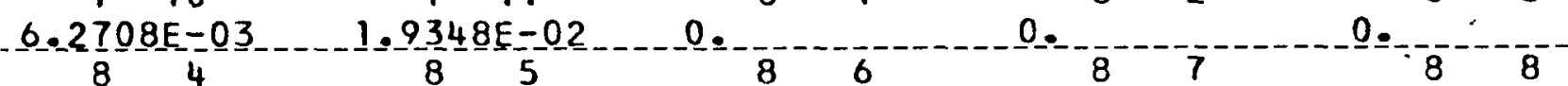

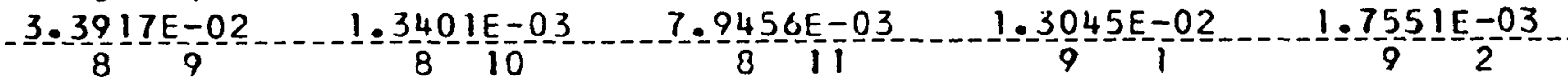

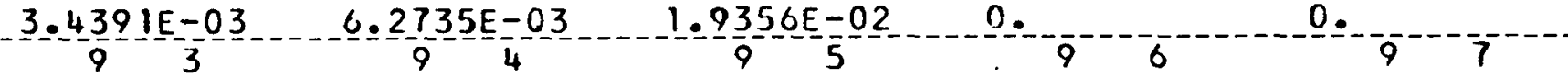

…

.3. $03466=03$ _ $5.9461 E=03 \ldots$ 
YTTRIUM

N1 N2

SIGMA_N,G_(N1IN2)

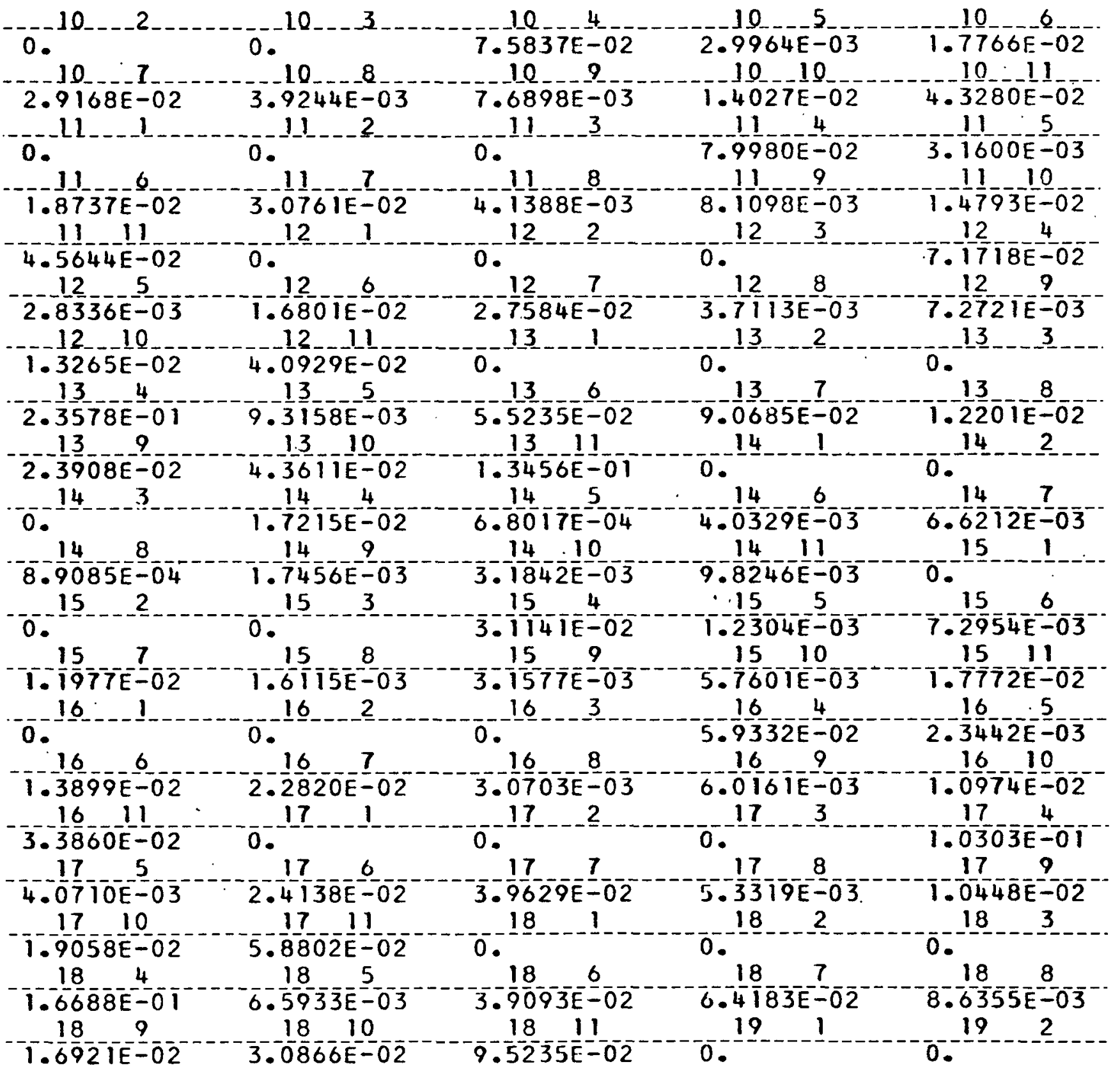


-YIJRLUM.

SIGMA N,G (NITN2)

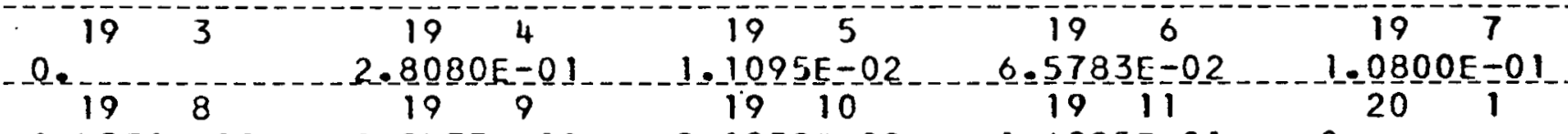

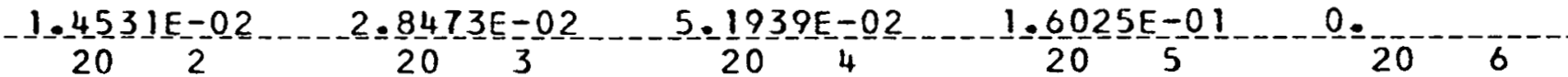

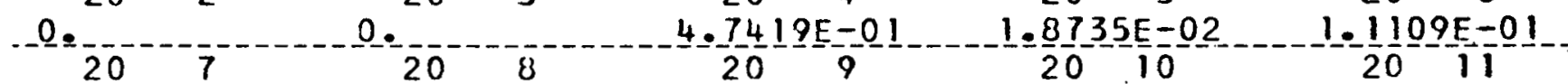

$-1.8238 E-01$

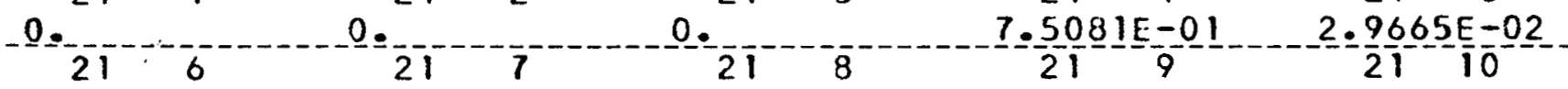

$1.7589 E=01 \quad 2.8877 E-01$ $2111 \quad 22 \quad 1 \quad 22 \quad 2 \quad 22 \quad 3 \quad 22$

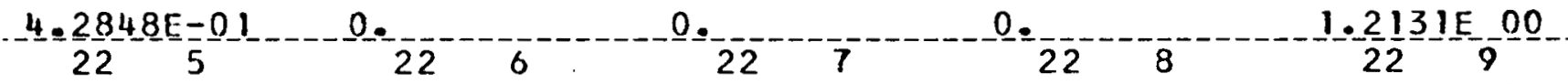

$-4.79 \frac{3}{22} 1 \mathrm{E}-022_{10}-\frac{2}{22} 19 \mathrm{E}-01$

$2.2 \frac{2}{2} 39 E-01$

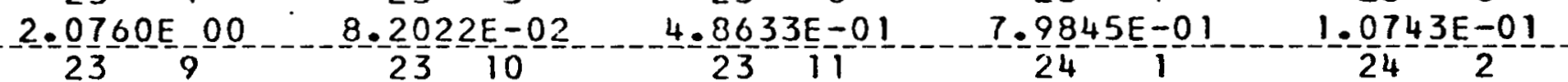

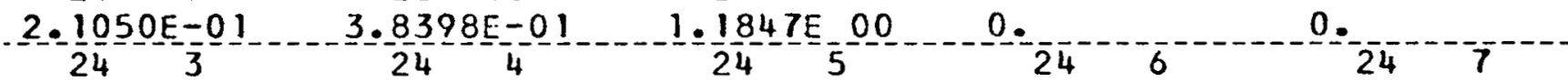
O. $24.3 .2012 E 00 \quad 1.2648 E-01 \quad 7.4993 E-01 \quad 1.2312 E-00$ $-1 . \frac{65}{25} 66 \mathrm{E}-01$

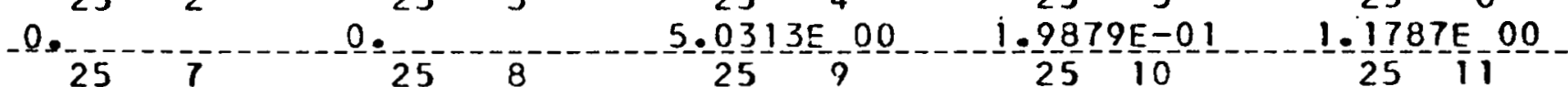
$1.9351 E-00$

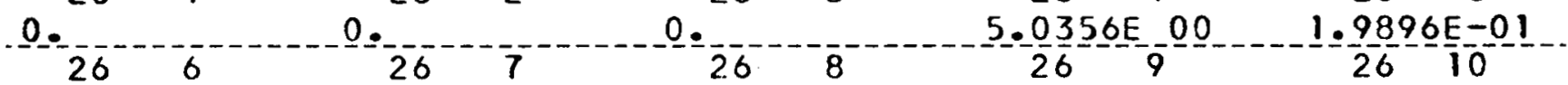

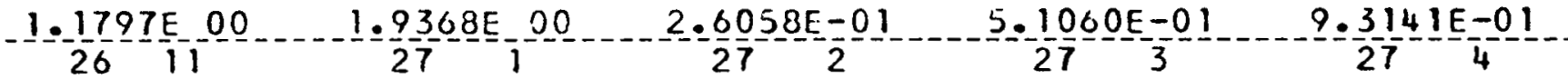

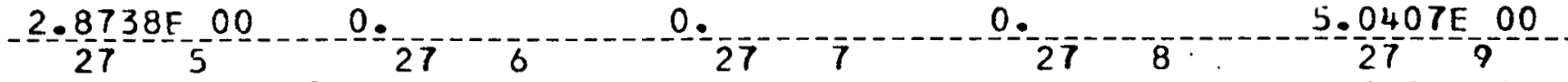
$\therefore 1.9916 \mathrm{E}-01 \cdot 1.1809 \mathrm{E}-00 \quad 1.9387 \mathrm{E}-00 \quad 2.6085 \mathrm{E}-01$ 9. 3236E-01 2.8767E 00 _ 0 . 
YTTRIUM

N1 N2

SIGMA_N,G INIIN2)

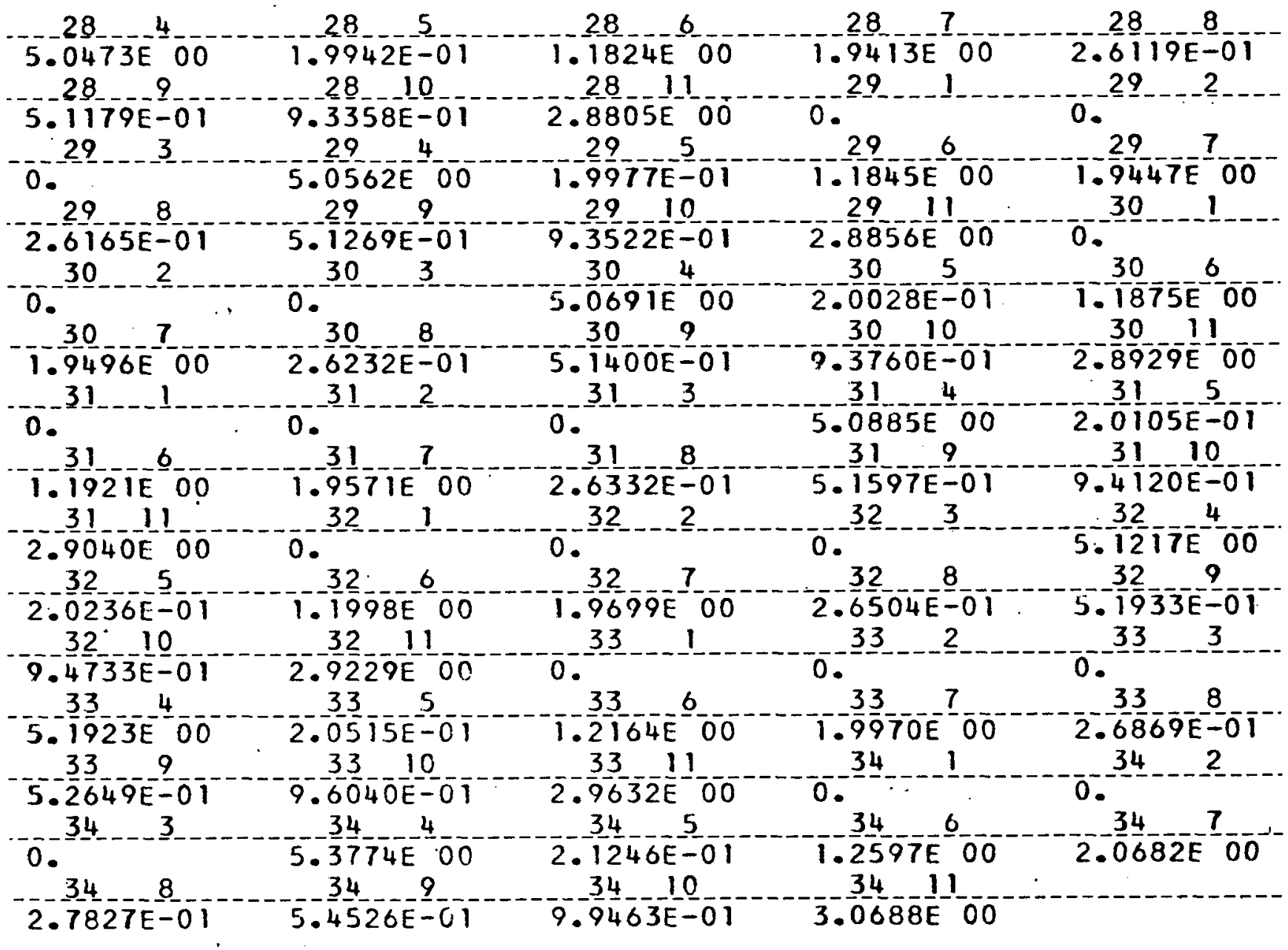


DAIE 12108180

DESIGNATION

ZIRCONIUM
CODE NO.

40.9100
DENSITY FACTOR

$\overline{1.5} \overline{14} \overline{10 E} 0 \overline{2}$

\section{$\begin{array}{ll} & \text { SIGMA } \\ \text { LEVEL ENERGY SCATTER SIGMA } & \text { SCATTER TRANSPORT ABSORPTION SCATIER I }\end{array}$}

1 1.0OOE OT 4.2378E OO 4.1804E $002.1372 \mathrm{E} 00$ 4.6133E-03-2.0607E 00

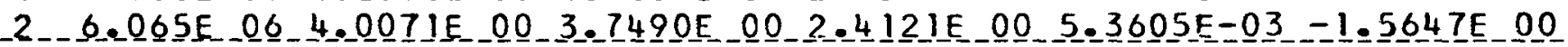

$3 \quad 3.679 \mathrm{E} 06 \quad 4.1420 \mathrm{E}$ 00 $2.9542 \mathrm{E}$ 00 2.8261E 00 6.5609E-03-1.2909E 00

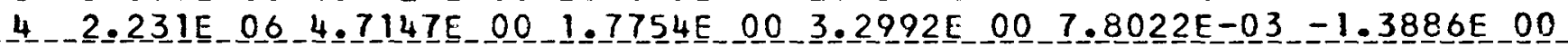

$51.353 \mathrm{E} 060.0945 \mathrm{E} 007.9343 \mathrm{E}-014.0768 \mathrm{E} 00-9.5087 \mathrm{E}-03-1.9794 \mathrm{E} 0 \mathrm{O}$

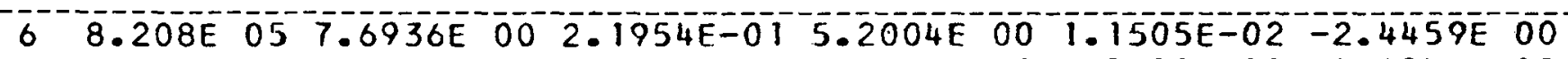

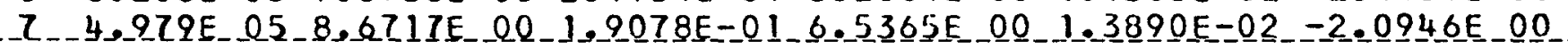

$8^{-} 3.020 E$ O5 $7.9743 \mathrm{E}$ 00 $1.7544 \mathrm{E}-016.8122 \mathrm{E}$ 00 $1.6830 \mathrm{E}-02-1.1401 \mathrm{E}$ OD

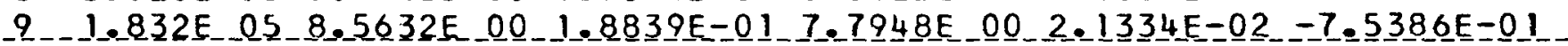

$10 \quad 6.738 E \quad 048.6817 \mathrm{E} \quad 00 \quad 1.9100 \mathrm{E}-018.3505 \mathrm{E} \quad 00 \quad 2.9573 \mathrm{E}-02-3.2491 \mathrm{E}-01$

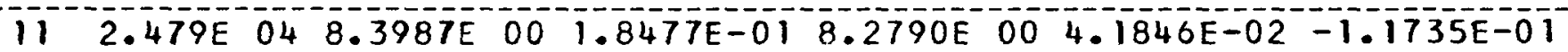

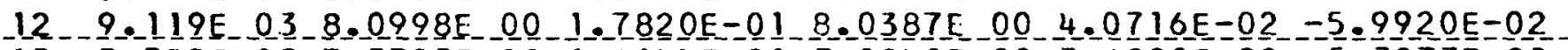

$133.355 \mathrm{E} \quad 037.3383 \mathrm{E}$ 00 $1.6144 \mathrm{E}-017.2840 \mathrm{E}$ 00 $3.6890 \mathrm{E}-02-5.3273 \mathrm{E}-02$

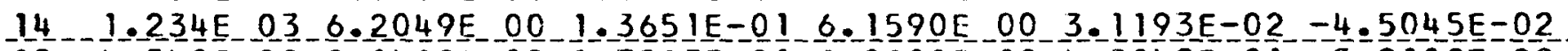

$154.540 \mathrm{E} 028.1422 \mathrm{E} 00-1.7913 \mathrm{E}-018.0820 \mathrm{E}-00-4.5049 \mathrm{E}-01-5.9109 \mathrm{E}-02$

$168.719 \bar{E} 01-1929 \bar{E} 00-1.3624 \bar{E}-016.147 \bar{E} 00-3.9649 \bar{E}-03-4.4958 \overline{0}=02$

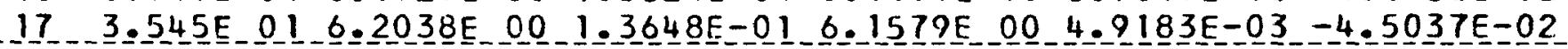

$180-304 \mathrm{E} 016.2135 \mathrm{E} 00-1.3670 \mathrm{E}-016.1676 \mathrm{E} 00-8.0150 \mathrm{E}-0 \mathrm{3}-4.5107 \mathrm{E}-02$

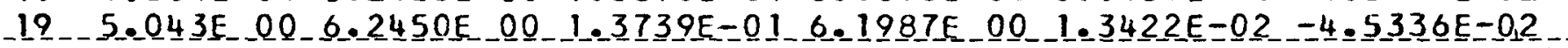

$20 \quad 1.445 E \quad 006.2745 E \quad 001.3804 E-016.2281 E$ OD $2.2755 E-02-4.5550 E-021$

$210.826 \mathrm{E}-016.1877 \mathrm{E} 001.3613 \mathrm{E}-016.1419 \mathrm{E} 003.6004 \mathrm{E}-02-4.4920 \mathrm{E}-02$

$22-2.511 E=01-6.1473 E-00-1.3524 E=0 L_{-} 6.1018 E-0005.7994 E-02-4.4626 E-02$

$239.237 \mathrm{E}-02 \quad 6.1054 \mathrm{E} \quad 00 \quad 1.3432 \mathrm{E}-01 \quad 6.0603 \mathrm{E} \quad 00 \quad 9.9540 \mathrm{E}-02-4.4323 \mathrm{E}-02$

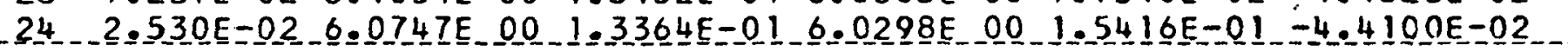

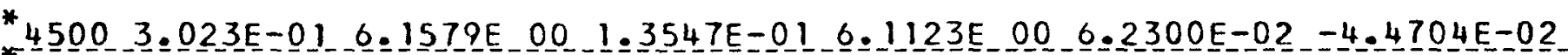

* $4000-2.718 \mathrm{E}-016.1579 \mathrm{E} 00-1.3547 \mathrm{E}-016.1123 \mathrm{E} 00-6.2300 \mathrm{E}-02-4.4704 \mathrm{E}-02$

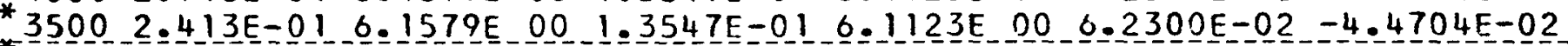

$30002.109 E=016.1579 E 001.3547 \mathrm{E}-016.1123 \mathrm{E} 006.2300 \mathrm{E}-02-4.4704 \mathrm{E}-02$

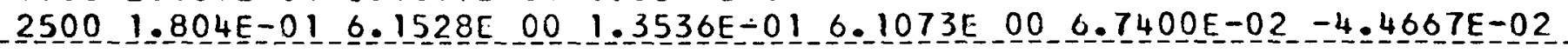

$2000-1.499 E-01-6.1460 E 00-1.3521 E-01-6.1005 E 00-7.3900 E=02-4.4617 E=02$

$15001.194 \mathrm{E}-01-6.1357 \mathrm{E} 00-1.3499 \mathrm{E}-01-6.0903 \mathrm{E} \quad 00-8.2800 \mathrm{E}-02-4.4542 \mathrm{E}-02$

$10008.897 \mathrm{E}-02$ 2. $1204 \mathrm{E} 00$ 1.3465E-01 6.0751E $00.9 .6000 \mathrm{E}=02-4.4431 \mathrm{E}=02$

$500-5.850 \mathrm{E}-026.09 \mathrm{TBE} 00 \mathrm{1}-3402 \mathrm{E}-016.0467 \mathrm{E} 00 \mathrm{1}-1840 \mathrm{E}-01-4.4224 \mathrm{E}-02$

68 3.216E 02 6.0253E 00 1.3256E-01 5.9807E 00 1.5960E-01-4.374 1E-02

*Thermal cross sections taken from APEX-515.

NOTE: For a summary of the sources of data see APEX-704. 
ZIRCONIUM

THERMAL TRANSFER MATRICES

TEMPERATURE
SIGMA

Ni 2

SIGMA S (N1/N2)

\begin{tabular}{|c|c|c|c|c|}
\hline $21-21$ & $-21-22$ & $-\frac{2}{5478 E-05}$ & 0 & 21 \\
\hline $\begin{array}{l}6463 E 00 \\
22 \quad 21\end{array}$ & $\begin{array}{c}5.4139 \mathrm{E}-0 \mathrm{1} \\
22 \\
\end{array}$ & $\begin{array}{r}1.3468-05 \\
22 \quad 23\end{array}$ & 22 & ${ }^{0 .} 22$ \\
\hline $5.1682 E-01$ & $5.3028 \mathrm{E} 00$ & $3.5829 E-01$ & $4.3910 E-04$ & $1.3513 \mathrm{E}-05$ \\
\hline$\frac{23}{3.5436 \mathrm{E}-05}$ & $1.1188 \mathrm{E}$ 00 & $4.7573 \mathrm{E} 00$ & $2.7898 E-0$ & $-\frac{23}{34} \div-\frac{25}{E-0}$ \\
\hline 24 & 24 & $24 \quad 23$ & 24 & 24 \\
\hline & $\begin{array}{c}1.1092 E-02 \\
7.859-22 \\
7.889-04\end{array}$ & $\begin{array}{l}2.5037 \mathrm{E} 00 \\
7-25333 \mathrm{~B}-01\end{array}$ & $\begin{array}{l}2.9005 \mathrm{E} 00 \\
2.1504 \mathrm{E}-100\end{array}$ & $\begin{array}{l}8.7712 E-0 \\
25 \\
3.8506 E-0\end{array}$ \\
\hline
\end{tabular}

TEMPERATURE
SIGMA

SIGMA S $($ N $N$ IIN2)

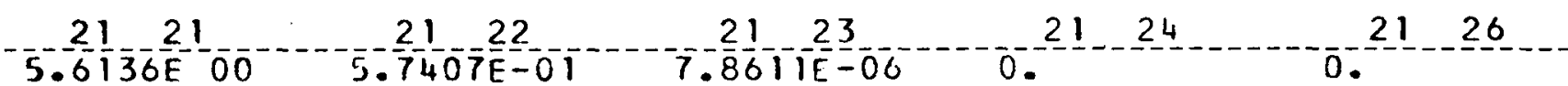

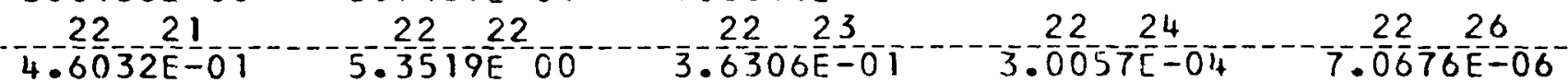

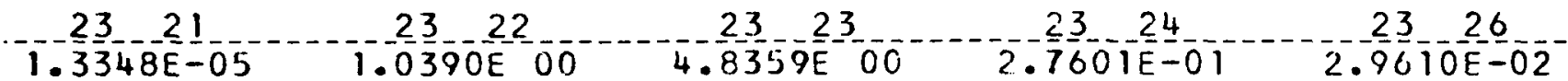

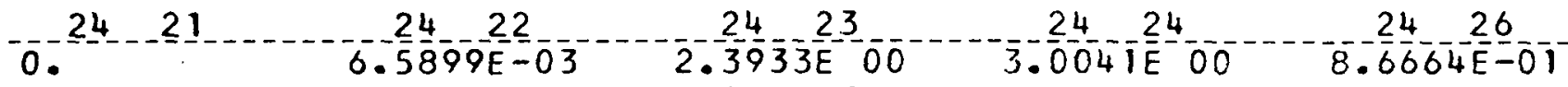

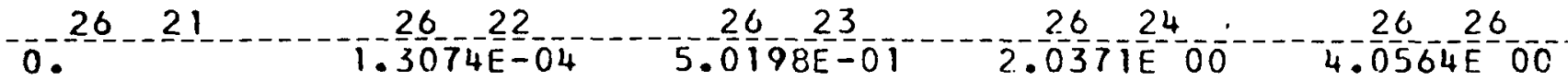


ZIRCONIUM

_. IHERMAL_IRANSFER _MAIRICES _-

-. TEMPERAIURE

DEG • $F$.
SIGMA

SCATTER
SIGMA TRANSPORT
SIGMA ABSORPTION
NU_SIGMA_..

FISSION
$3.5000 \mathrm{O} \quad \mathrm{3}$
$6.5956 \bar{E} 00$
$6.5468 \mathrm{E} 00$
$2.489 \overline{O E}-0 \overline{3}$
0 .

\title{
SIGMA $\frac{N 1}{S}\left(\frac{N 2}{N) / N 2)}\right.$
}

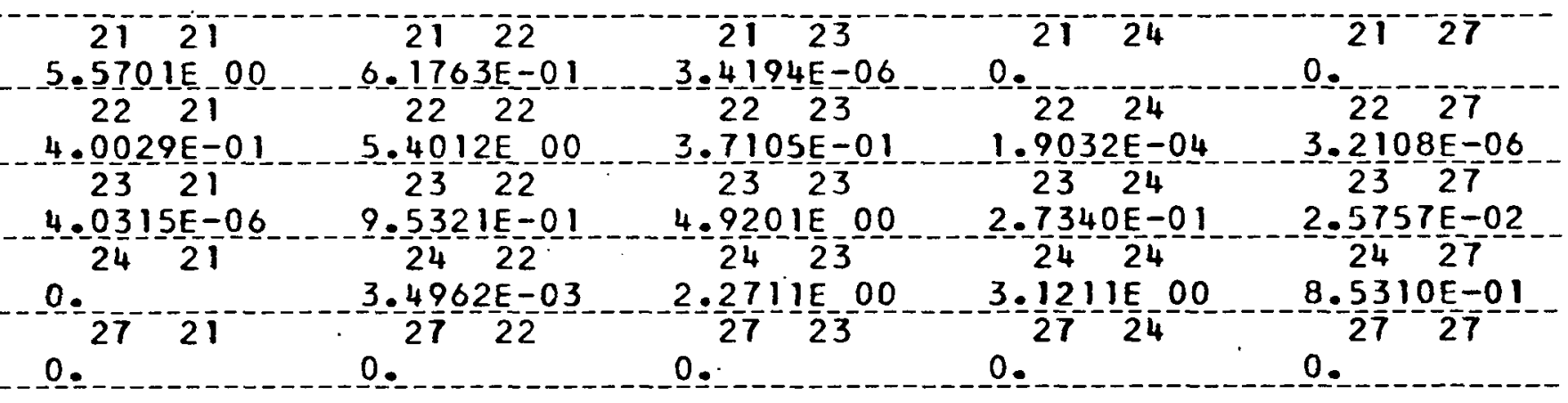

.. TEMPERA IURE

DEG. F.
SCATTER
TRANSPORT
SIGMA

ABSORPTION
NU SIGMA

FISSION
$6.5354 E^{-} 00$
$6.4 \overline{8} \mathrm{OE}$ OO
$3.0000 E^{\circ}$

$--1$

3.0000

\begin{abstract}
E 03
\end{abstract}
$-\cdots$

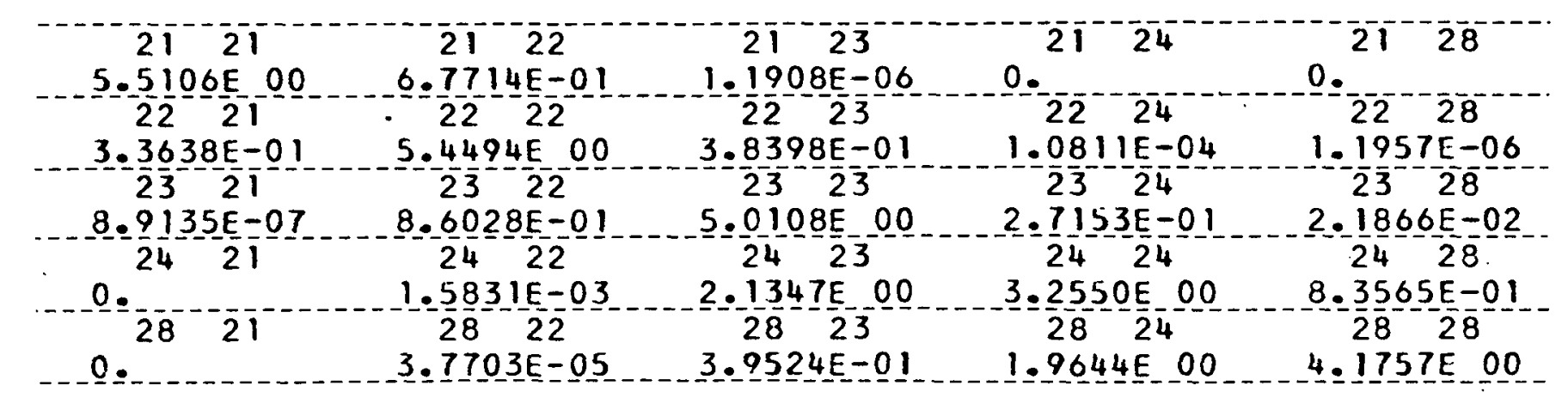


Z IRCONIUM

THERMAL TRANSFER MATRICES

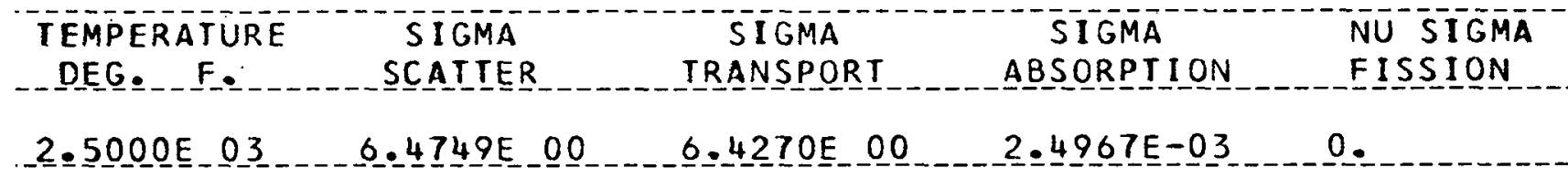

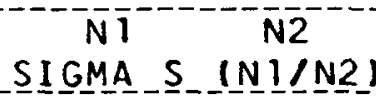

\begin{tabular}{|c|c|c|c|c|}
\hline$-2 J_{-}-21$ & $21-22$ & $-21-23$ & -21 & $0^{-21}$ \\
\hline 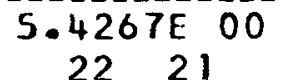 & $7.6106 \mathrm{E}-01$ & $2.9613 E-07$ & 0. & 0. \\
\hline $.6844 \mathrm{E}-01$ & $5.4936 \mathrm{E} 00$ & $4.0491 \mathrm{E}-01$ & $5.2367 E-05$ & $3.3130 E-07$ \\
\hline $2406 \mathrm{E}-0$ & 23 & 23 & $-23-24$ & -23 \\
\hline $\begin{array}{l}-244 \\
-24\end{array}$ & $\begin{array}{l}7.3845 E-01 \\
24-22\end{array}$ & $\begin{array}{l}0.10891 \\
24\end{array}$ & $\begin{array}{c}2.7105 E-01 \\
2424\end{array}$ & $\begin{array}{c}1.7964 \mathrm{E}-02 \\
2429\end{array}$ \\
\hline & $5.6816 \mathrm{E}-04$ & $1.9809 \mathrm{E} 00$ & $3.4105 \mathrm{E} 00$ & $8.1319 \mathrm{E}-01$ \\
\hline & $-\frac{29}{46}-\frac{22}{1 E-06}$ & $-\frac{29}{2.9355 E-01}$ & $1.8718 \mathrm{E} 00$ & $4.3096 \mathrm{E}-00$ \\
\hline
\end{tabular}

SEMPERATURE
SIGMA
DEG _ FIGMA

$2.0000 E_{03} 03.4142 E_{-00} 0.3667 E$ OO $2.5030 E=03$

N1 2

SI GMA S (N1 IN2)

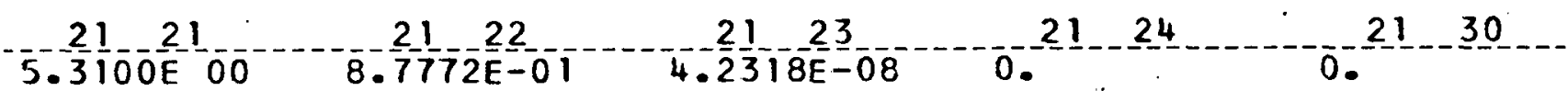

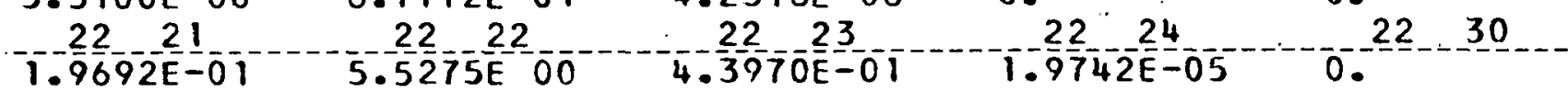

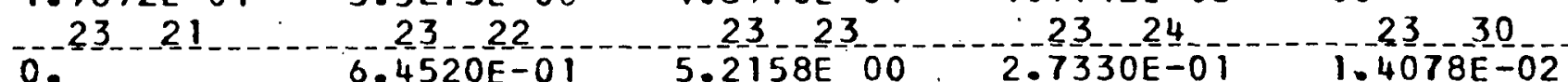

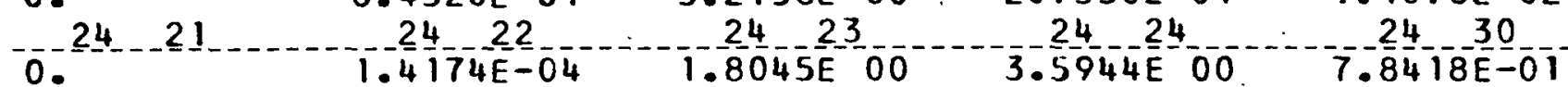

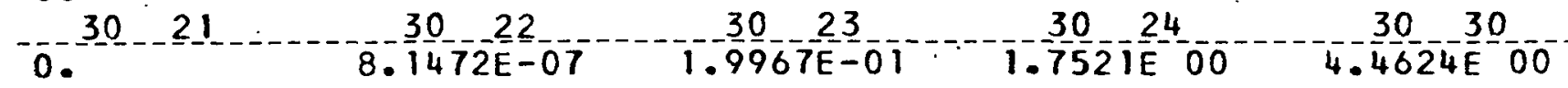




\section{ZIRCONIUM}

IHERMAL_JRANSEER_MAIRICES.

IEUPERATURE DEG . F.

SIGMA

SCATIER

$6.3533 \mathrm{E} 00$
SLGMA TRANSPORT
SIGMA ABSORPTION
NU_SIGMA.

FISSION

$1.5000 \mathrm{E} 03$

$6.3063 \mathrm{E} 00$

$2.5126 E-03$

0 .

SIGMA S $(N) / N 2)$

$21^{2} i^{2}-2 i^{2} i^{2}-2 i^{2}$

$5.2360 E_{-00}$

2221

$-1.2400 E=01$

2321

o.

2421

0. 31 - 21

ㅇ.

$9.517 J E=01$

2222

$5.5363 E_{0} 00$

2322

$5.1686 \mathrm{E}=01$

2422

1. $8975 E=05$

3122

0.
$0.22-23$

5. $0099 E=01$

2323

$5.3320 E$ OO

2423

$1.5974 \mathrm{E}$

3123

1.1즈미트이
Q. 22

4. $8130 E=06$ 2324

2. $8123 E-01$ 2424

3.8174 E 00

3124

$1.59466 \mathrm{E} 00$
0.2231

0. 2331 $1.0258 E-02$ 2431

$7.4657 \mathrm{E}-01$ 3131 4.64IIE 00

IEMPERAIURE

SIGMA

SCATTER
SIGMA TRANSPORT
SIGMA ABSORPTION
NU U SIGMA

FISSION
$1.0000 \mathrm{E} 03$
$6.2922 \mathrm{E} 00$
$6.2456 \mathrm{E} 0 \mathrm{O}$
$2.52 \overline{1 E}-0 \overline{3}$
0

\section{SIGMA $\left.S(N) \frac{N}{N}\right)$}

$21^{2} 11^{2} 21^{-13}$

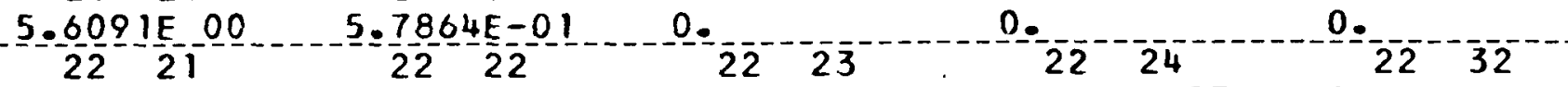

$5.6473 \mathrm{E}-02 \quad 5.4815 \mathrm{E} 00 \quad 6.2034 \mathrm{E}-0$
$2 \overline{3} \overline{\mathrm{i}}$
$2 \frac{3}{3} 2$
23

$4.856606-07$

2

ㅇ.

3. $6815 \mathrm{E}-01$

2422

$5 . \frac{45}{24} \mathrm{O} \frac{\mathrm{E}}{23} \mathrm{OO}$

3. $0267 \mathrm{E}=01$

$6.5875 \mathrm{E}-03$

$242 i$

$7.4935 E=07$

$1.3449 \frac{9 E}{3} 00$

$4.0968 E 00$

2432

O. …

5. $3003 E-02$

$1.3813 \mathrm{E} \quad 00$

ㅇ.- $\frac{97}{3} \frac{87}{2}-0-01$

ㅇ.

4. 8578 E 00 
ZIRCQNIUM

THERMAL TRANSFER MATRICES

TEMPERATURE
SCIGMA
DEG $5.0000 E$ IGMA 02

\begin{tabular}{|c|c|c|c|c|}
\hline $2 \jmath_{--21}$ & $21 \ldots 22$ & 23 & 2 & 21 \\
\hline $\begin{array}{c}5.8394 \mathrm{E} \\
22\end{array}$ & $\begin{array}{c}3.4834 E-01 \\
22\end{array}$ & $0.22-23$ & 0.22 & $0.22 \quad 33$ \\
\hline $\begin{array}{c}6.1560 E-02 \\
.23 \quad 21\end{array}$ & $\begin{array}{c}5.2541 \mathrm{E} \\
2320\end{array}$ & $\begin{array}{c}8.3935 E-01 \\
2323\end{array}$ & 0.23 & 0 \\
\hline 0. & $\begin{array}{c}1.9440 \mathrm{E}-01 \\
24 \quad 22\end{array}$ & $\begin{array}{c}5.5609 \mathrm{E} 00 \\
2423\end{array}$ & $\begin{array}{c}3.6591 \mathrm{E}-01 \\
24\end{array}$ & $\begin{array}{c}3.2136 E-03 \\
2433\end{array}$ \\
\hline 0. & & $\begin{array}{l}1.0176 \mathrm{E} 00 \\
33 \\
23\end{array}$ & $\begin{array}{l}4.4630 E 00 \\
33\end{array}$ & $\begin{array}{c}0.3727 E-01 \\
33\end{array}$ \\
\hline 0. & 0. & $1.2883 E-02$ & $1.07 .94 \mathrm{E} \quad 00$ & $5.1387 \mathrm{E}$ \\
\hline
\end{tabular}

TEMPERATURE
SIGMA
6.8000E 01 1 $6.1785 E$ E 00

\section{$N 1$ N2}

SI GMA S (N1/N2)

\begin{tabular}{|c|c|c|c|c|}
\hline$-\frac{1}{8}-\frac{2}{2}=-1$ & $-\frac{21}{405}=\frac{22}{F}-0$ & $021-23$ & 21 & $21-34$ \\
\hline $\begin{array}{l}8472 E 00 \\
22 \quad 21\end{array}$ & $\begin{array}{c}3.4055 E-0 \\
22 \quad 22\end{array}$ & 0. & 22 & $22 \quad 34$ \\
\hline $.0436 \mathrm{E}-1$ & $\begin{array}{l}5.8184 \mathrm{E} O \mathrm{OC} \\
2322\end{array}$ & $\begin{array}{c}.7823 \\
2323\end{array}$ & 2 & 0. \\
\hline & $4.3160 \mathrm{E}-02$ & $5.4969 E 00$ & $5.7650 \mathrm{E}-01$ & $8.1840 E-04$ \\
\hline & & $6.1838 E-01$ & $4.8892 \mathrm{E} 00$ & $5.99139 E-01$ \\
\hline & 0 & $7.4572 \mathrm{E}-$ & $6.8967 \mathrm{E}-01$ & $-5 . \frac{34}{4880 \mathrm{E}}-3 \frac{3}{\mathrm{C}}$ \\
\hline
\end{tabular}


ZIRCONIUM

TRAIISFERS FROM GROUPS I THROUGH 6 TO GROUPS I THROUGH 16

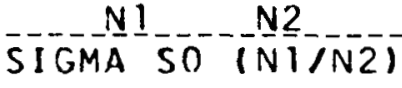

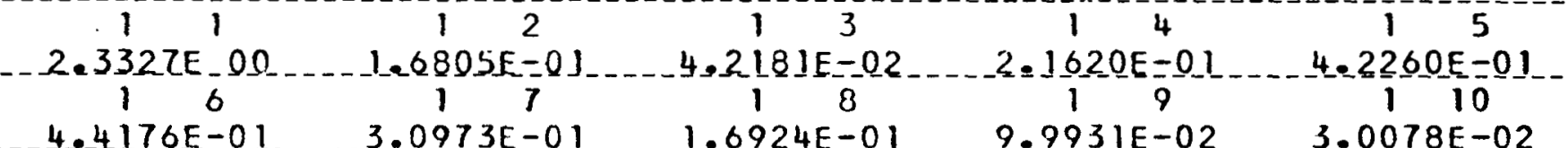
$4.4176 \mathrm{E}-01 \ldots-0.973 \mathrm{E}=01 \ldots$

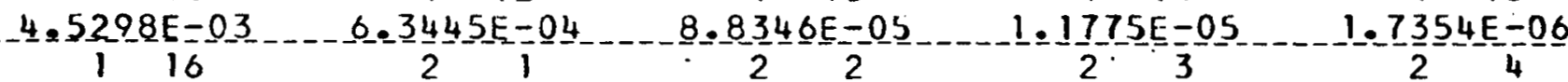
$1.3423 E=07 \quad 0.2-2169 E-00 \quad 1.2960 E-01-1.3109 E-01$

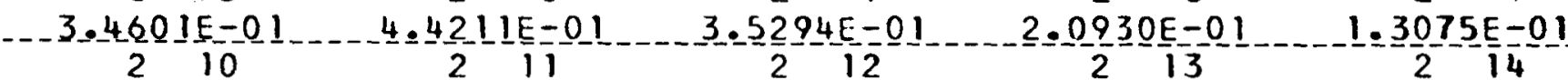

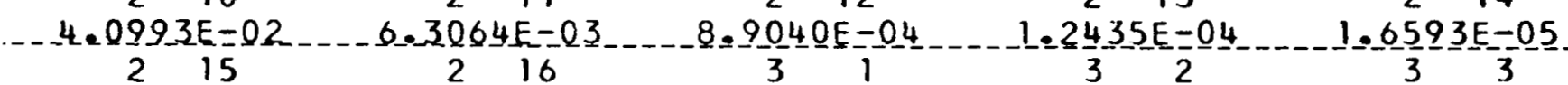
$2.4464 E=06 \quad 1.8925 E=07$

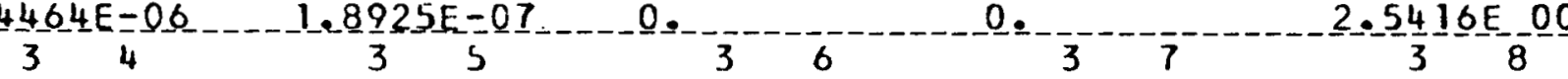
$1.5878 E=01 \ldots 2.1266 E=01 \quad 3.7492 E=01 \quad 3.6815 E-01-2.4866 E-01$ . $1.6992 \mathrm{E}-01 \ldots 5.6824 \mathrm{E}-02$ $2.4246 E-05 \quad 3.5771 E-06 \quad 2.7679 E-07 \quad 0$.

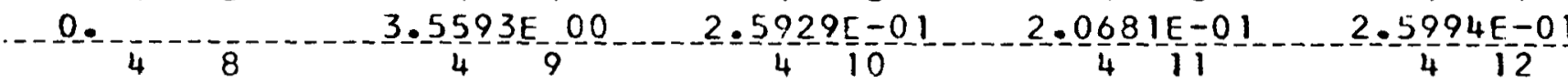

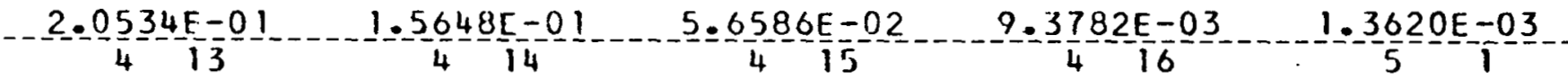

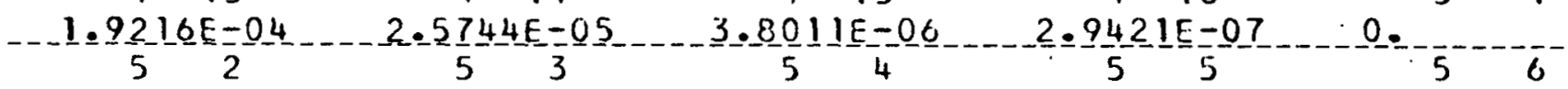

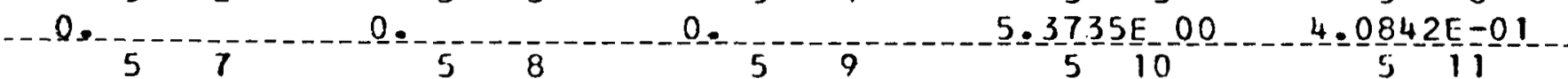

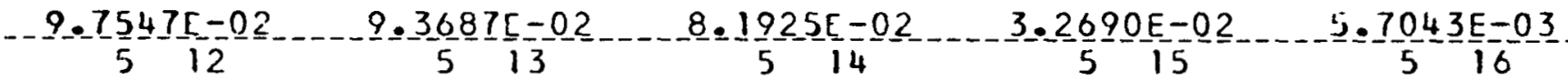

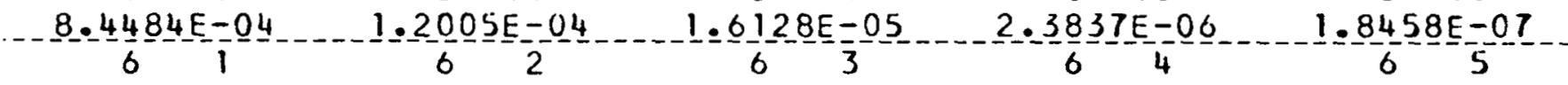

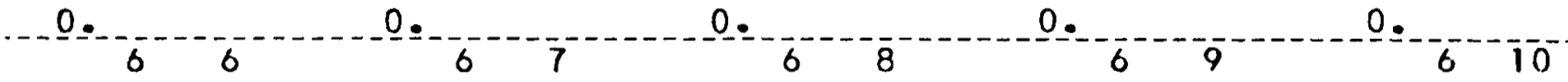
$7.3360 E-00 \quad 3.2934 E-01 \quad 1.1222 E-02 \quad 1.1155 E-02 \ldots$ $\ldots 8.9035 E-04 \quad 1.3423 E-04 \ldots \ldots 1.9197 E-05 \quad 2.5855 E-06 \quad 3.8248 E-07$ - 2.9626틈요 


\section{ZIRCONIUM}

RESONANCE
ENERGY
INTEGRAL


N1

SIGMA N,G (NITN2)

$1 i^{1}{ }^{2}$ Q.

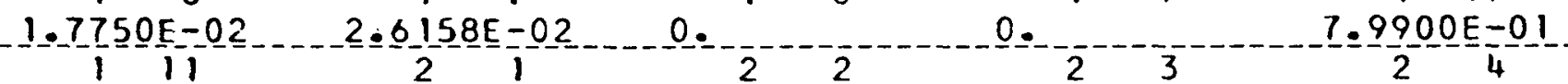
$1.3336 \frac{E}{2}-02 \quad 0.21 .0367 E-03 \quad 1.4137 E-03 \quad 1.0839 E-02$ $1.0085 \mathrm{E}-02$

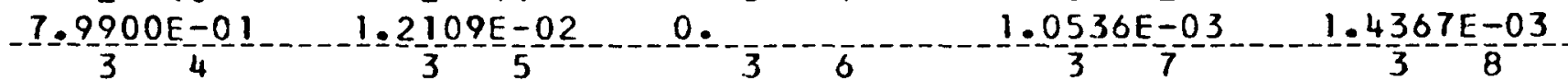

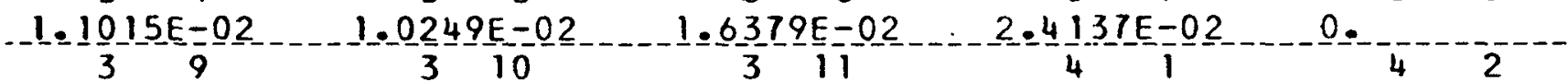

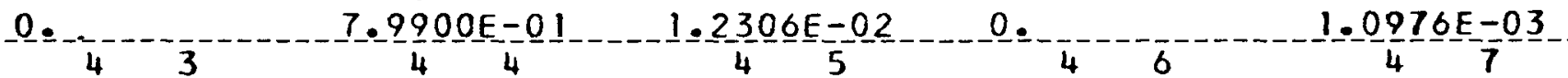

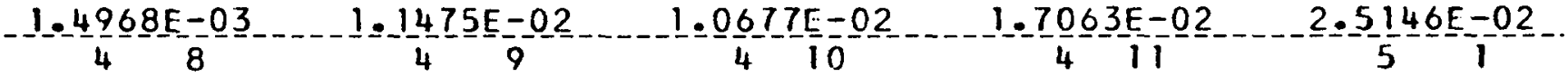

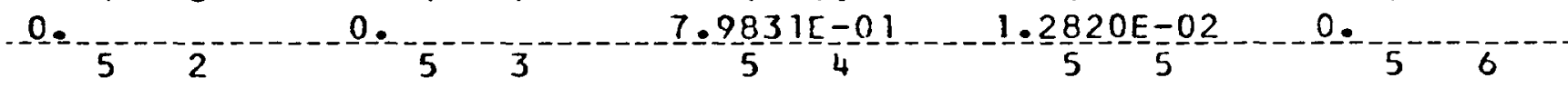

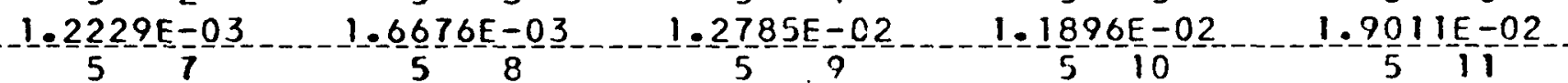

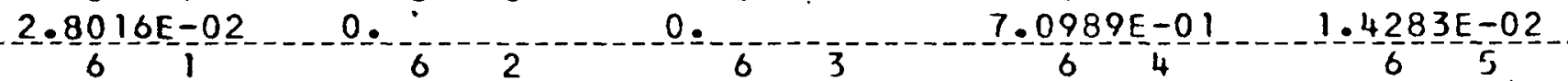
$0.01 .3954 E-03$ $2.1692 \mathrm{E}=02$ 61117

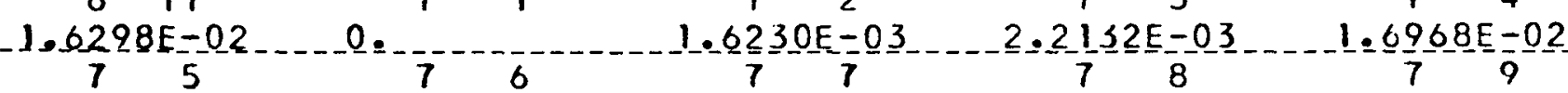
$1.5787 \mathrm{E}=02 \ldots-5230 \mathrm{E}=02 \ldots 3.718 \mathrm{IE}=02$ -0.

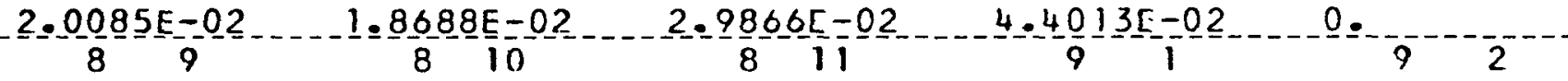

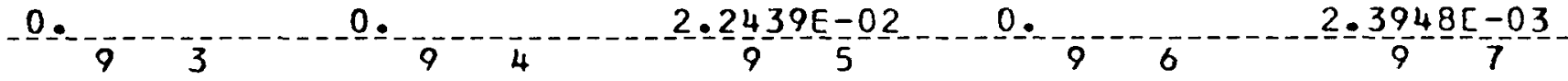
3. $2656 \mathrm{E}-03 \quad 2.5036 \mathrm{E}-02 \ldots 2.3295 \mathrm{E}-02 \quad 3.7228 \mathrm{E}-02 \quad 5.4862 E-02$ . 0. 0. 2. $79970 E=02$ 0. 
ZIRCONIUM

Ni $\mathrm{N} 2$

SIGMA N,G (N]IN2)

\begin{tabular}{|c|c|c|c|c|}
\hline $2813 \mathrm{E}-0$ & $\frac{10}{4.4745 E-03}$ & $3.4304 E-02$ & $\frac{10}{3.1918 E-02}$ & $5.1009 E-02$ \\
\hline SE-O & $\begin{array}{r}4.4845 t-03 \\
10\end{array}$ & $\begin{array}{l}3.4304 \\
-10\end{array}$ & - Mrioe-uz & 10 \\
\hline-0 & 0. & 0. & 0. & $3.8324 E-02$ \\
\hline & $4.6178 E-03$ & $6.2970 \mathrm{E}-03$ & $4.8277 \mathrm{E}-02$ & $4.4918 E-02$ \\
\hline 1$]$ & 11 & 1 & 11 & $10+2-2+2$ \\
\hline $\begin{array}{l}E-02 \\
11\end{array}$ & $1.0579 E-01$ & 0.12 & 0. & 0.12 \\
\hline $\begin{array}{l}-1 E-02 \\
5\end{array}$ & 0. & $4.4840 E-03$ & $6.1146 \mathrm{E}-03$ & $4.6878 \bar{E}-02$ \\
\hline 5 & $-12 \frac{6}{-12}$ & $-\frac{12}{3}=$ & 12 & 12 \\
\hline & 0.91211 & 13 & 13 & \\
\hline & $5.2372 E-02$ & 0 & $4.0597 E-03$ & $5.5359 E-03$ \\
\hline 24 & 13 & 으. & 13 & $1 \underline{3}$ \\
\hline $\begin{array}{l}2442 \\
13 \\
13-1\end{array}$ & $\begin{array}{r}3.94 \\
13\end{array}$ & $\begin{array}{c}6.3109 E-02 \\
13 \quad 11\end{array}$ & $\begin{array}{c}9.3003 E-0 \\
14\end{array}$ & $0 \cdot{ }_{14}$ \\
\hline & & $4.7415 \mathrm{E}-02$ & 0. & $3.4318 \bar{E}-03$ \\
\hline$\frac{14}{6797 E-03}$ & $=-\frac{1}{5}-378$ & 5 & $-\frac{1}{3} \frac{4}{3}-0=0$ & $-\frac{1}{8} \frac{4}{6}-9-7$ \\
\hline 14 & 14 & 14 & 14 & $15 \quad 1$ \\
\hline & 0.15 & & $4.0082 \mathrm{E}-02$ & 0. \\
\hline $9556 E-$ & $5.7577 E^{-15}-0$ & $=\frac{15}{180}=\frac{4}{5}-0$ & $-\frac{15}{8}-0-5=-0$ & $7.75 \frac{15}{8} \overline{8}=-0$ \\
\hline $1 \frac{15}{2}=-2$ & 15 & & $15 \quad 10$ & $15 \quad 11$ \\
\hline 13531 & & 0. & & $E=0 \overline{1}$ \\
\hline & $4.3615 E-04$ & $5.9474 \bar{E}-04$ & $4.5597 E-03$ & $4.2425 E-03$ \\
\hline $780 \mathrm{IE}-0$ & 16 & 16 & & 10 \\
\hline IE-O & $9.9917 E-03$ & & & 0. \\
\hline-109 & $0^{-17}$ & $5-\frac{17}{4}-02 \mathrm{E}-04$ & $7.3775 E-04$ & $5-\frac{17}{656}=\frac{4}{0}=0$ \\
\hline$-\frac{17}{6} \frac{7}{2}$ & $17=6$ & 17 & 17 & 179 \\
\hline 10 & $\begin{array}{c}8.4103 E-03 \\
1711\end{array}$ & $\begin{array}{c}1.2394 E-02 \\
18\end{array}$ & & \\
\hline & $\begin{array}{c}0.3189 \mathrm{E}-0 \\
185\end{array}$ & & $165 E=04$ & $.2023 E^{-103}$ \\
\hline$E-0$. & $i E=0$ & $-1 . \frac{18}{37}$ & $2.01 \overline{8}$ & 18 \\
\hline & 10 & 11 & 19 & 19 \\
\hline
\end{tabular}


- $I$ I RCONIUM.

\section{SIGMA $N, G(N)-N i N 2)$}

193019401906079

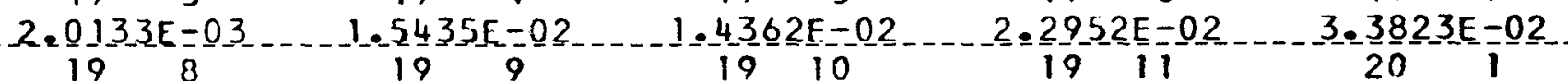

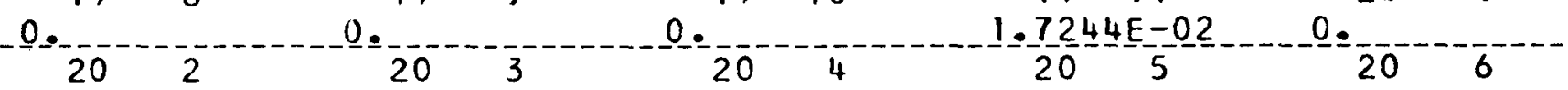

$2.5031 E=03-3=4133 E=03-2=6168 E=02-2.4348 E-02-3.8911 E-02$

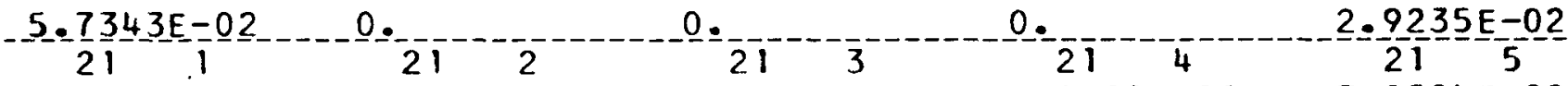

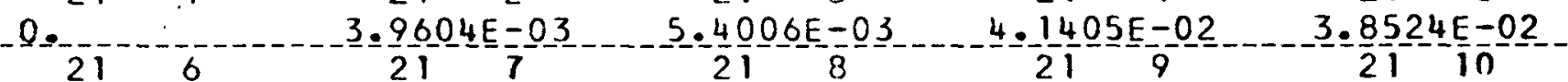

$6 . \frac{1}{21} 67 E=02 \quad 9.0730 E-02 \ldots 0.2211$

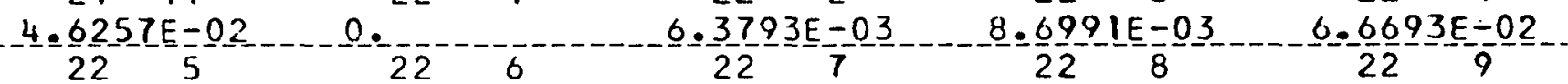

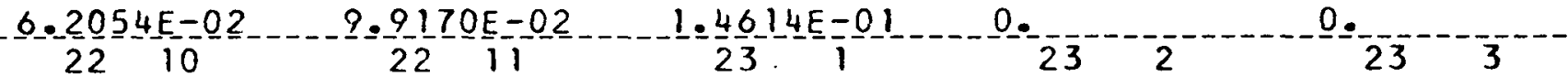

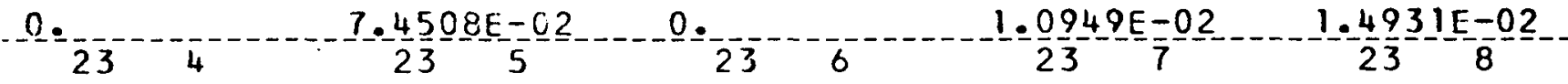

$1 . \frac{1}{2} 347 E-01 \quad 1.0651 E-01 \quad 1.7021 E-01 \quad 2.5084 E-01$

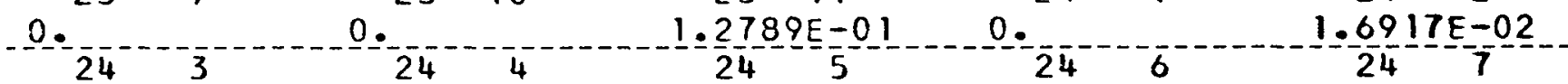

$2.3068 E-02 \quad 1.7686 E-01 \quad 1.6456 E-01 \quad 2.6298 E-01 \quad 3.8755 E-01$

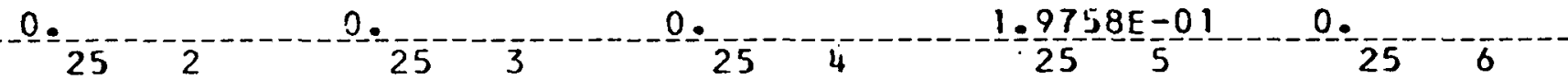

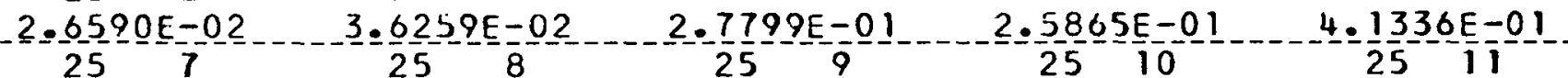

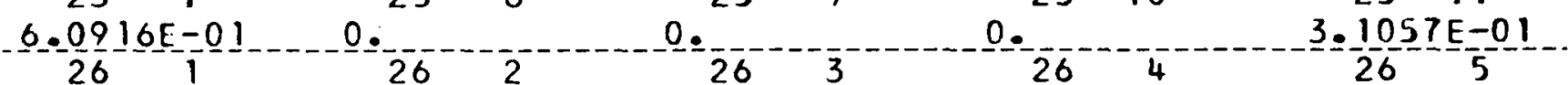

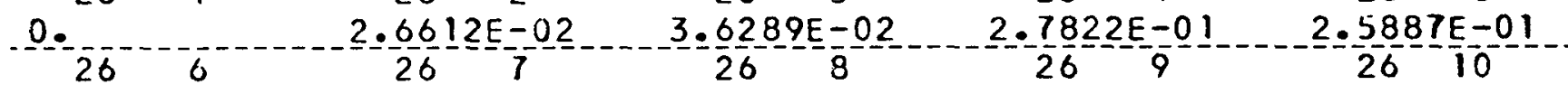

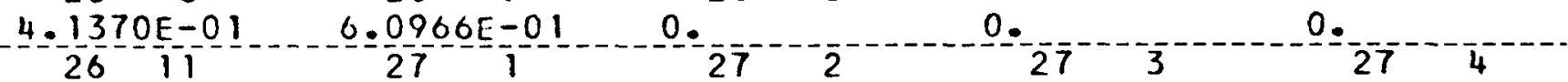

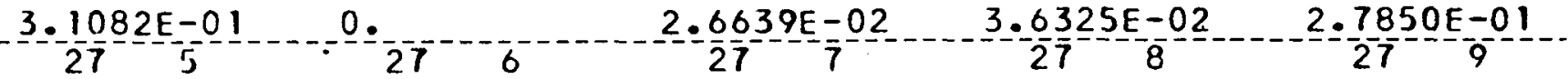

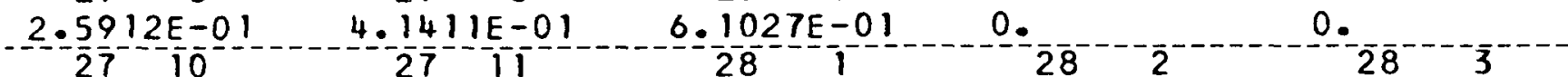

0.27 3.1113E-01 0.28 2.0674E-02 3.6373E-02 


\section{ZIRCONIUM}

N1 N2

SIGMA N,G (NIIN2)

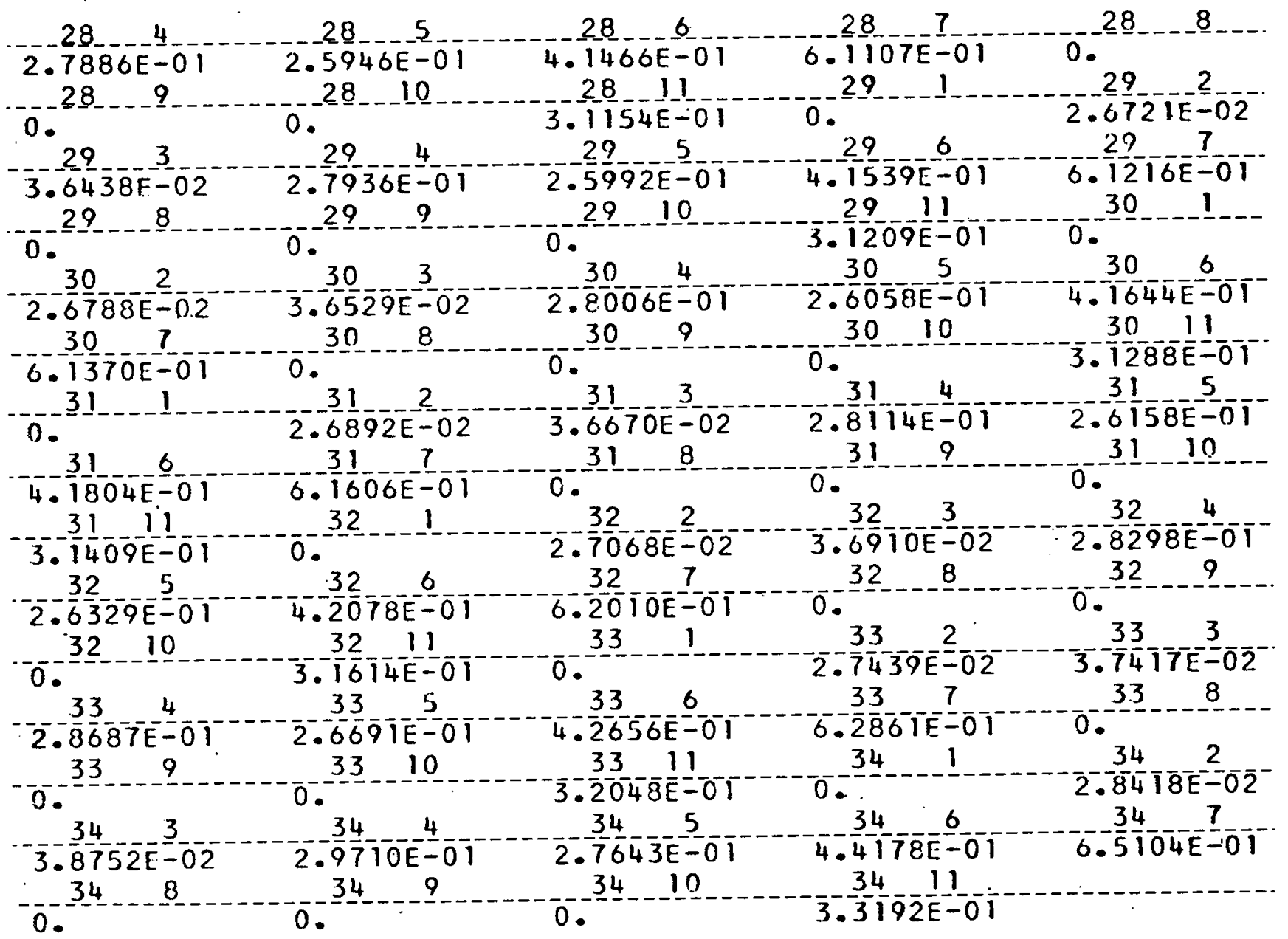


DESIGNAI_LQN MANGANESE
CODE NO.

25.5500
DENSIIY FACIOR

9.119 13E 01

\section{SI GMA}

XI SIGMA

SI SMA

SIGMA

XII SIGMA

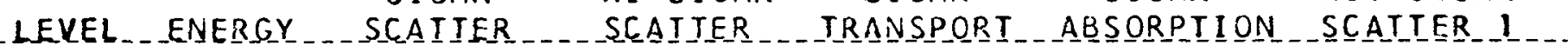

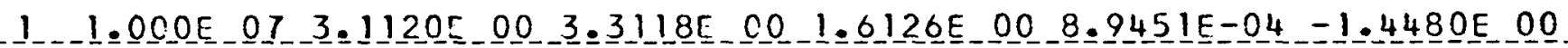

$2.6 .065 \mathrm{E} 00 \quad 3.5469 \mathrm{E} 003.0615 \mathrm{E}$ 00 $1.8194 \mathrm{E} 00 \quad 1.0805 \mathrm{E}-03-1.6682 \mathrm{E} 00$

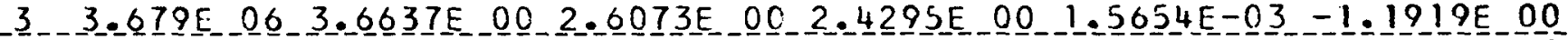

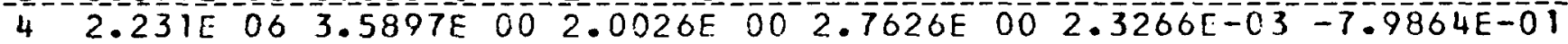

5 1.353E_06 3.3744E_00 1.3195E $0002.7562 \mathrm{E} 00$ 3.1797E-0.3 -5.9696E-0I

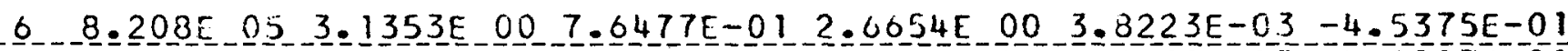

$7.979 E$
7

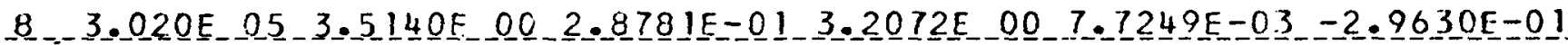

$7.832 \mathrm{E} 054.2162 \mathrm{E} 001.9879 \mathrm{E}-014.0274 \mathrm{E} 001.2538 \mathrm{E}-02-1.8233 \mathrm{E}-01$

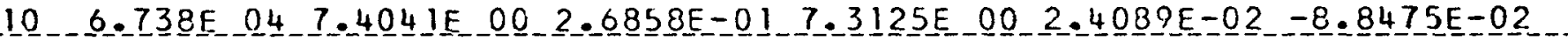

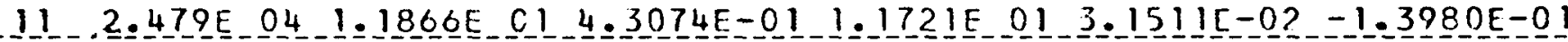

$129.119 \mathrm{E} 03-3.2075 \mathrm{E}$ O1 $1.1643 \mathrm{E}$ 00 3.1634E O1 3.7314E-02 -3.7790E-01

$13-3.355 \mathrm{~F} \quad 03 \quad 1.0839 \mathrm{E} \quad 02 \quad 3.9346 \mathrm{E} \quad 00 \quad 1.0707 \mathrm{E} \quad 02 \quad 1.5267 \mathrm{E}-01-1.2770 \mathrm{E} \quad 00$

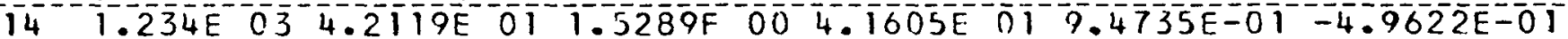

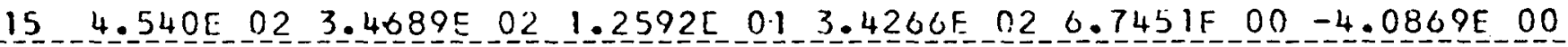

$10 \quad 8.719 E \quad 016.3845 E$ O $002.3176 E-01-6.3066 E \quad 003.6667 E-01-7.5219 E-02$

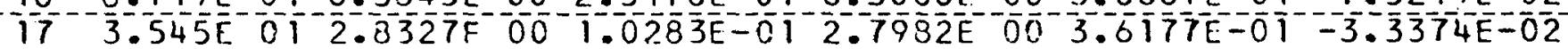

$18 \quad 1.304 E$ O $012.3977 E$ O $00 \quad 8.7036 E-02 \quad 2.3684 E$ O $00 \quad 5.8587 E-01-2.8248 E-02$

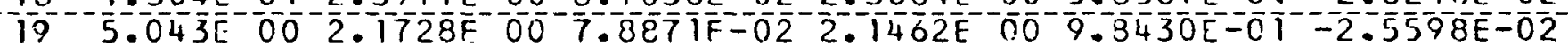

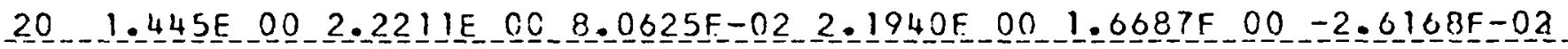

$21-6.826 E-012.2551 E-00-8.1861 E-02-2.2276 E 002.6453 E-00-2.6569 E-02$

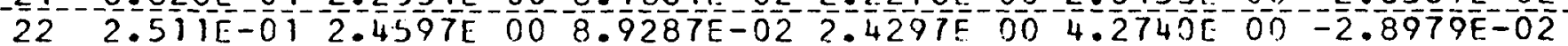

$23.9 .237 \mathrm{E}-022.7907 \mathrm{E} 00 \quad 1.0130 \mathrm{E}-012.7566 \mathrm{E} 00 \quad 7.3140 \mathrm{E} 00-3.2878 \mathrm{E}-02$

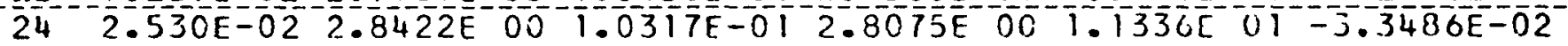

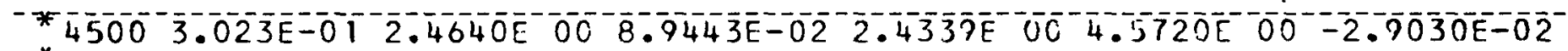

* $40002.718 E-012.4640 E$ OO $8.9443 E-02 \quad 2.4339[00 \quad 4.5720 E$ O $00-2.9030 E-02$

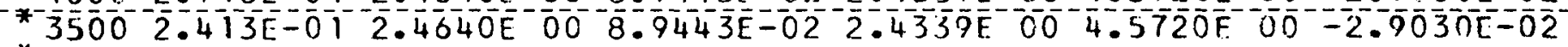

* $30002.109 E-012.4640 E$ O0 $8.9443 E-02 \quad 2.4339 E$ OC $4.5720 E$ ON $-2.9030 E-02$ $25 \mathrm{CO} 1.804 \mathrm{E}-0 \mathrm{1} 2.4960 \mathrm{E}-000$

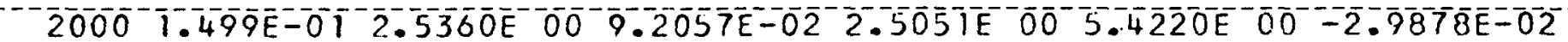

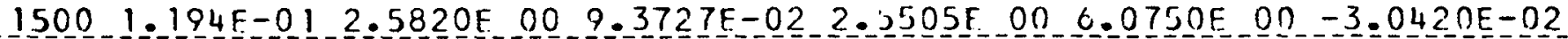

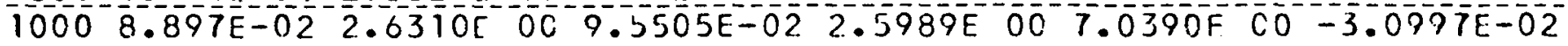
$5005.850 \mathrm{E}-022.6600 \mathrm{~F}$ 00 $9.0558 \mathrm{~F}-02-2.0275 \mathrm{E} \quad 00-8.6810 \mathrm{E} \quad 00-3.1339 \mathrm{~F}-02$

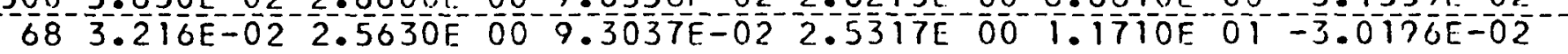

*Thermal cross sections taken from APEX-515.

NOTE: For a summary of the sources of data see APEX-704. 
MANGANESE

_IHERMAL IRANSEER_MAIRICES_-

IEUPERAIURE

SIGMA

SIGMA

SIGMA

NU SIGMA

DEG. $F$.

SCATTER

TRANSPORT

ABSORPTION

FISSION

$4.5000 E \quad 03$

$2.9113 \bar{E} 00$

$2 . \overline{8} \overline{58 E} 0 \overline{0}$

1.

0.

SIGMA S $-\frac{N}{1}-\frac{N 2}{1 / N 2)}$
2121
$2 \overline{1}^{-} \overline{2}$
$2 \overline{1} 2 \overline{3}$
$2 \overline{1}^{-1}$

$1.3710 E=04$

- 2. $0108 \mathrm{E} 00$

$2.4422 E-01$

$22 \quad 22$

$22 \quad 23$

으.

$22-24$

0

221
$6359 \mathrm{E}=0$

2321

2. $0358 E$ 음

2322

$1 . \frac{79}{23} \frac{8}{2}=-01$

$1.0 \frac{0}{2} 340 E \frac{0}{2}-0$

225

4. $7200 E-04$

2421

$6.5187 E=01$

$2=\frac{022}{2} \frac{3 E}{2}-00$

1. $-\frac{4}{24} \frac{999 E-01}{24}$

9. $-\frac{1}{2} \frac{297 \mathrm{E}-\mathrm{0}}{25} \mathrm{5}$

$-6=\frac{7}{25} \frac{52}{2}=\frac{E}{2}=0 ?$

$3.4162 \mathrm{E}-02$

$1.3880 E$ OO

$1.1528 E$ OO

$2.90 \frac{9}{24}-\frac{E}{25}-02$

으.

6. $1110 \mathrm{E}-03$

$5.7095 E-01$

$9.3076 \mathrm{E}-01$

4. $-\frac{3}{2} \frac{6}{5}-\frac{6 E}{25}-01$

$1.4035 E \quad 00$

TEMPERAIURE

DEG. F.

$4.0000 \overline{0}$
SIGMA

SCATTER
SIGMA

TRANSPORT
SIGMA

ABSORPTION
NU SIGMA

FISSION

2.872 IE 00

$2 . \overline{3} \overline{\mathrm{OOE}} \mathrm{OO}$

$1.774 \overline{1 E} 01$

0.

\section{SIGMA S (NIIN2)}
2121
$21^{22}$
$2 \overline{1} 2 \overline{3}$
$2 i^{24}$
$9.0524 \mathrm{E}=05$
0 .
$22^{--24}$
2126
1. $-997 \mathrm{IE}-0$
2222

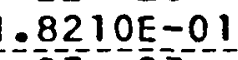
$8.3241 E-04$ $22^{-126}$
$2 \cdot \frac{3}{2} \frac{59 E}{2} \overline{1}$
$2.06 \frac{09 E}{2}-0$
6.-0594E-01
$2.0656 \frac{6}{24}-00$
$23-\frac{3}{4}$
$5.9 \frac{9}{2} \frac{4}{3}-\frac{05}{2} 6$
2. $-\frac{3}{24} \mathrm{~T}$ - $8 \mathrm{E}=0$$$
2422
$$
. $3358 E$ OOO
$1.4 \frac{9}{2} \frac{25}{2}=-01$
2. $-\frac{6}{24} \frac{5}{26}-\frac{02}{26}$
. $1=\frac{5458}{26}=07$
2. $3525 E-02$
$26 \quad 22$
$26-23$
$1=1984 \mathrm{E} 00$
$4 \cdot \frac{3}{26} \frac{4}{6}-\frac{3 E}{26}-01$
$3.4745 \mathrm{E}-03$
4.9895E-01
$9.2226 E-01$
$1.4474 \mathrm{E} 00$

- 
MANGANESE

\section{THERMAL TRANSFER MATRICES}

\begin{tabular}{|c|c|c|c|c|}
\hline $\begin{array}{l}\text { TEMPERATURE } \\
\text { DEG }\end{array}$ & $\begin{array}{c}\text { SIGMA } \\
\text { SCATIER }\end{array}$ & $\begin{array}{c}\text { SIGMA } \\
\text { TRANSPORI }\end{array}$ & $\begin{array}{c}\text { SIGMA } \\
\text { ABSSORPIION }\end{array}$ & $\begin{array}{l}\text { NU SIGMA } \\
\text { EISSION }\end{array}$ \\
\hline $5000 E_{-} 03$ & $2.8325 E_{-} 0 \mathrm{C}$ & IIIIE $O O$ & QEE_OI & 0 \\
\hline & & $N 2$ & & \\
\hline$-2 \mathrm{~J}-21_{-}$ & $-21-22$ & $2 L_{-} 23$ & _2] 24 & $-21 \ldots 27$ \\
\hline $\begin{array}{c}1.9790 E \text { OO } \\
22\end{array}$ & $\begin{array}{l}2.7606 E-01 \\
22\end{array}$ & $\begin{array}{c}5.4687 E-05 \\
22\end{array}$ & 0. & 0 \\
\hline $\begin{array}{l}2.0378 \mathrm{E}-01 \\
23\end{array}$ & $2.0864 \mathrm{E} 00$ & $1.8578 \mathrm{E}-01$ & $6.2083 E-04$ & $3.5972 \mathrm{E}-05$ \\
\hline $\begin{array}{l}1.0107 E-04 \\
24\end{array}$ & $5.5630 E-01$ & $2.1125 \mathrm{E} 00$ & $1.4865 \mathrm{E}-01$ & $2.3884 E-02$ \\
\hline & $\begin{array}{r}1.4961 \mathrm{E}-02 \\
27\end{array}$ & $\begin{array}{l}1.2761 \mathrm{E} \\
22 \\
23\end{array}$ & $\begin{array}{r}1.2506 \mathrm{E} 00 \\
27\end{array}$ & $\begin{array}{l}4.3556 \mathrm{E}-01 \\
27\end{array}$ \\
\hline & $1.7477 \mathrm{E}-03$ & $2500=-01$ & $\sin _{0}$ & $.4962 \mathrm{E} 00$ \\
\hline
\end{tabular}

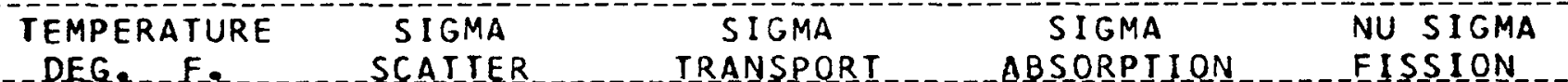

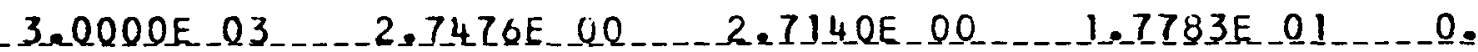

\section{$\mathrm{Ni} N 2$ \\ SIGMA S_IN IIN2)}

\begin{tabular}{|c|c|c|c|c|}
\hline $21-21$ & $=-21-22$ & $21-23$ & 21 & 21 \\
\hline $\begin{array}{c}1.9544 E \\
2221\end{array}$ & $\begin{array}{c}3.0070 E-01 \\
2222\end{array}$ & $\begin{array}{l}2.9112 \mathrm{E}-05 \\
2223\end{array}$ & 0. & 0. \\
\hline $\begin{array}{l}1.7100 E-01 \\
2321\end{array}$ & $2.1115 E 00$ & $\begin{array}{l}1.9172 \mathrm{E}-01 \\
23\end{array}$ & $4.3337 \mathrm{E}-04$ & $1.9152 \mathrm{E}-05^{2}$ \\
\hline $3.4938 \mathrm{E}-05$ & $5.0224 E-01$ & $2.1636 \mathrm{E} 00$ & $1.4835 \mathrm{E}-01$ & $2.1130 E-02$ \\
\hline & $8.5 \frac{4}{5} 152 E-03$ & $1.2075 \mathrm{E} 00$ & $-1 . \frac{24}{3} 11024$ & $-4 . \frac{24}{3} 32-\frac{28}{7 E}-0$ \\
\hline 2 & $7 . \frac{28}{4042 E-0}$ & $3 . \frac{28}{50}-2 \frac{23}{6 E-0}$ & $8.9060=\frac{28}{E}-0$ & $1 . \frac{28}{5509 E} 0$ \\
\hline
\end{tabular}


MANGANESE

_. IHERMAL IRANSEER_MAIRICES

\begin{tabular}{|c|c|c|c|c|}
\hline $\begin{array}{l}\text { IEUPERA TURE } \\
\text { DEG. F. }\end{array}$ & $\begin{array}{l}\text {-SIGMA } \\
\text { SCATIER }\end{array}$ & $\begin{array}{l}\text { SLGMA } \\
\text { TRANSPORT }\end{array}$ & $\begin{array}{l}\text { SLGMA } \\
\text { ABSORPTION }\end{array}$ & $\begin{array}{l}\text { NU SLGMA } \\
\text { FISSIION }\end{array}$ \\
\hline$O E E^{\circ}$ & $2.7523 \mathrm{E}$ & $2.7187 E$ OC & $1.7814 \mathrm{E} O 1$ & 0 . \\
\hline
\end{tabular}

$N 1$

SIGMA $S$ (NI/N2)

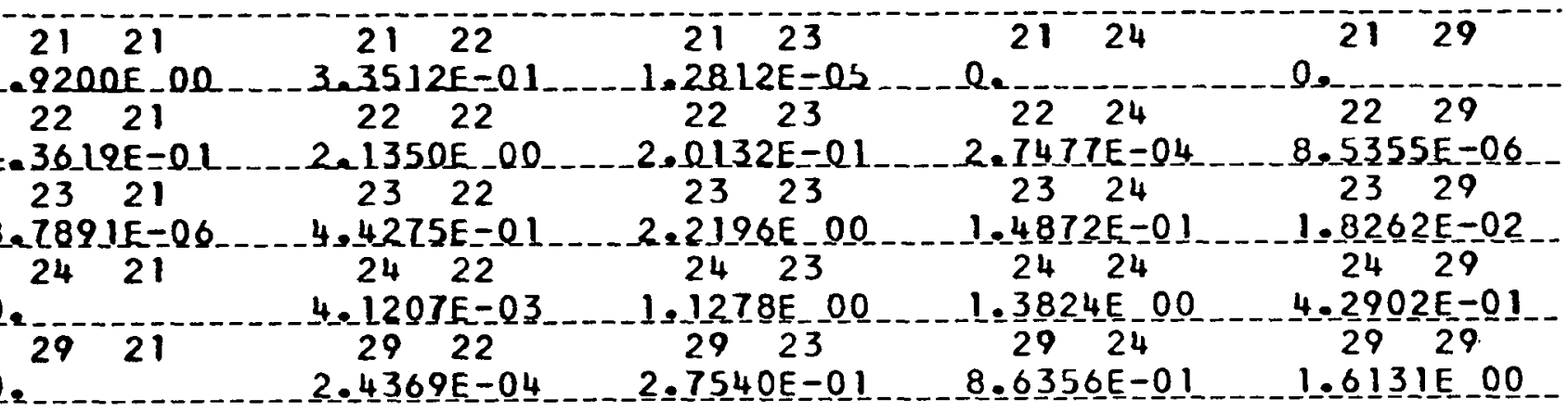

IEMPERAIURE

DEG. $F$.

$2.0000 \mathrm{E} 03$
SIGMA

SCATIER

-7TIBE OO
SIGMA TRANSPORT
SIGMA ABSORPTION
NU SIGMA

FISSION

SIGMA S (NIIN2)

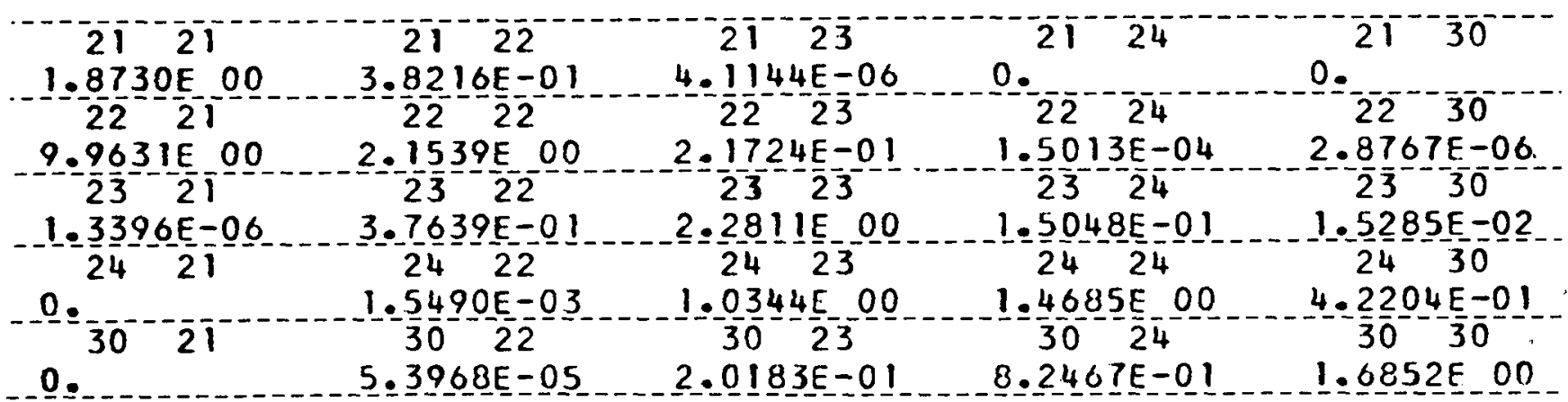


MANGANESE

THERMAL TRANSFER MATRICES

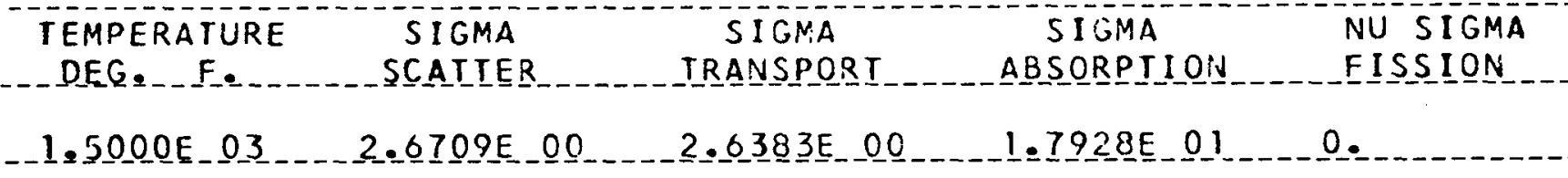

\section{$\mathrm{Ni} N 2$ \\ SIGMA S (NIIN2)}

\begin{tabular}{|c|c|c|c|c|}
\hline 21 & 21 & 21 & 2 & 31 \\
\hline $1.8471 \mathrm{E} 00$ & $4.0798 E-01$ & $6.8576 E-07$ & 0 & 0 \\
\hline $\begin{array}{l}-22 \\
.2486 E-02\end{array}$ & $--\frac{2}{2}-\frac{22}{16}$ & $-\frac{22}{45}-23$ & $--\frac{22}{3}-2-\frac{24}{4}-05$ & $-\frac{22}{5}-\frac{31}{45 E-0}$ \\
\hline 23 & $23 \quad 22$ & & $23 \quad 24$ & $23 \quad 31$ \\
\hline & $3.0100 \mathrm{E}-01$ & $2.3487 \mathrm{E} 00$ & $1.5522 \mathrm{E}-01$ & $1.2201 E-0$ \\
\hline $0-24$ & $--\frac{24}{3}-\frac{22}{70}=$ & $9-\frac{24}{2246 E-01}$ & $1 . \frac{24}{5} 755 \mathrm{E}-2$ & $4 .-\frac{24}{1} 17-\frac{31}{1}$ \\
\hline 31 & $31-22$ & 31 & 3 & 31 \\
\hline & $0.0528 \mathrm{~F}$ & 1.32 & 7.68 & $1.7708 E$ \\
\hline
\end{tabular}

TEMPERATURE
DEG SIGMA

Ni

SIGMA S (NIIN2)

\begin{tabular}{|c|c|c|c|c|}
\hline 21 & 21 & 21 & 21 & 21 \\
\hline $\begin{array}{l}2.0118 \mathrm{E} 00 \\
2221\end{array}$ & $\begin{array}{c}2.4332 \mathrm{E}-01 \\
2222\end{array}$ & & 22 & 22 \\
\hline $2.8275 \mathrm{E}=02$ & $2.1400 \mathrm{E} 00$ & $2.98750-01$ & $1.6262 \mathrm{E}-05$ & \\
\hline-23 & $2.1382 \mathrm{E}-01$ & $-\frac{23}{2.42110}-23$ & $1.6715 E-01$ & $9.0105 \mathrm{E}-03$ \\
\hline & $24 \quad 22$ & 24 & 24 & $-2 \leq 4$ \\
\hline 0. & $4.0274 E-05$ & $7.8330 E-0$ & $.1 .7143 \mathrm{E} 0$ & $3.9463 \mathrm{E}-01$ \\
\hline 0. & $1.7797 \mathrm{E}-07$ & $7.0154 \mathrm{E}-02$ & $0.8309 \mathrm{E}-01$ & $1.8765 \mathrm{E} 00$ \\
\hline
\end{tabular}


MANGANESE

IHERMAL IRANSFER MAIRICES.

- LEMPERAIURE

DEG. $F$.

$5.0000 \mathrm{E} \quad 02$
SI GMA

SCATTER
SIGMA

TRANSPORT
SIGMA

ABSORPTION
NU SIGMA

FISSION

$2.5885 \mathrm{E}^{-100} 2.5569 \mathrm{E} 00^{-1.8293 \mathrm{E}} 0 \mathrm{~T}^{-}$

\section{SIGMA $\left.-\frac{N}{2}-\frac{N 2}{2} 1 / N 2\right)$}

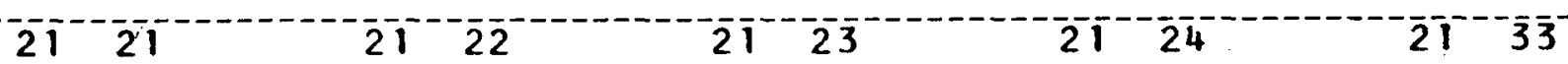

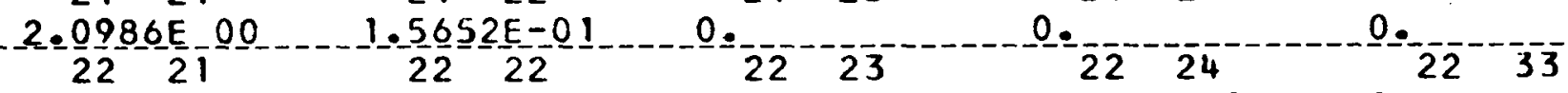

$-2=\frac{7}{2} 3 \frac{1}{6}-0 \mathrm{E}=02$

2. $0448 \mathrm{E} \quad 00$

2322

$3.9290 \mathrm{E}-01$

$1.0632 E-06$

$23 \overline{3} 3$

$-0$.

$1 .-1223 E-\frac{2}{24}=01$

$2=\frac{4861 E}{24}-\frac{10}{2}{ }^{3}$

$2.0105 E-01$

$5.686 \frac{3 E-03}{24}$

$\overline{3} \overline{3}^{--} \overline{2} \overline{1}$

$\left.5 \cdot 5 \frac{5}{3}-7 \mathrm{E}-\mathrm{E}-\mathrm{O}\right]$

$5=994 \div \frac{7 E}{2}-01$

$1.9053 \mathrm{E}-00$

$3.7083 \mathrm{E}-01$

$2.3181 E-02$

$5.4918 E-01$

$2.0162 E 00$
IEMPERAIURE

DEG. $F$.
S IGMA

SCATTER
SIGMA

TRANSPORT
SIGMA

ABSORPTION
NU SIGMA

FISSION

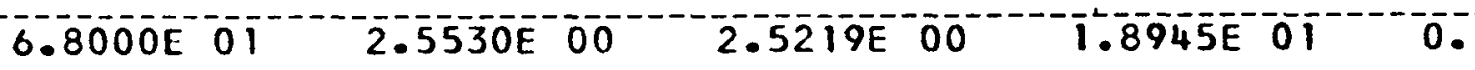

\section{SIGMA $-\frac{N}{2}-\left(N \frac{N}{2}-\frac{1}{N}\right)$}

$21^{2} 1^{2} 1^{2} 21^{2} 23^{-24}$

-2.1056E $2200 \quad 1.4955 E-01$

-4. $-\frac{24}{2} \frac{47 E}{2} 1$

-

$2.4597 \mathrm{E}-02$

$24^{-1} 1$

2422

$\overline{2} \overline{2}-\overline{2} \overline{3}$

$22 \overline{2}$

$222^{-} 34$

$1.0515 E-01$

$23-\frac{13}{3}$

ㅇ.

$2.4631 \mathrm{E} O 0$

$0=\frac{0}{34} \overline{1}$

$34-22$

2423

$3.0951 E-01$

$3.6775 E-01$

2424

ㅇ.-

$23^{-1} \overline{4}$

$2.5718 \mathrm{E}-03$ 2434

$2.1428 E \quad 00$

$34-24$

$3=5 \frac{048 E-01}{3} \frac{1}{3}$

2. $5939 \mathrm{E}-03$

$3.5734 E-01$

2. 1931 IE 00 


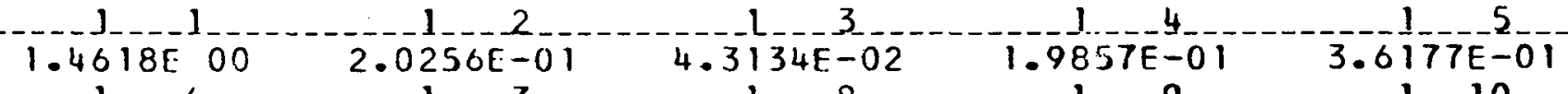
$-3-1-b_{-}$ $2.4633 \mathrm{E}-01$ $1.3225[-01$ $7.7158 \mathrm{E}-02$ $2.3024 \mathrm{E}-02$

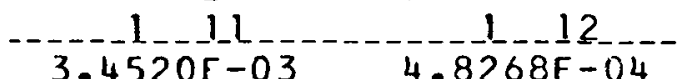
$6.7170 \mathrm{E}-05$ $1-14$ $1.3191 \mathrm{E}-06$ 16 $4.8268 E-04$ 6. 2 $8.9508 \mathrm{E}-06$

$1.0202 E-07$ $1.9380 E$ DO $-3.2432 \mathrm{E}-0$ 0. 2 $1.2647 \mathrm{E}-01$ $1.3263 E-01$ 3.2432

$3.3903 E-02$ $-2.0069 E-06$ $3.9424 E-01$ $3.0486 \mathrm{E}-01$ $1.77 \frac{2}{22 E-O}$ $1.0921 E-01$ - 3 $5.1890 \mathrm{E}-03$ $7.3122 E-04$ $1.0205 \mathrm{E}-04$ $1.3613 \mathrm{E}-05$ $1.9152 E-0$ $1.5525 E-07$ 0 . 3 0 . 103

$1.6733 E-01$ $-2.3353 \mathrm{E}-05$ $5.5280 E-02$ $3.9997 \mathrm{E}-01$ $3.7784 \mathrm{E}-01$ $8.7394[-03$ $3.4450 \mathrm{E}-06$ $2.6656 \frac{3}{6}-07$ $1.2467 E-0 \frac{3}{3}$ $4 \quad 4-9 \quad 4 \quad 10$ $2.7822 E-0 T-2.0824 E-0 T$ - 13 $2.4987 \mathrm{E}-04$ $3.3464 \mathrm{E}-05$ $7.4338 \mathrm{E}-02$ 0.4 3

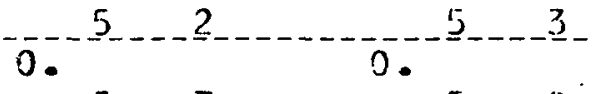
$4.9402 \frac{4}{2}-06$ $2.9 \frac{4}{94}-\overline{6}-\frac{6}{-0}-1$ $1.9717 \bar{E} 00$ 0. - 19 $4 \frac{3}{3}-1,-1$ $2507-5$ $\begin{array}{ccc}2.2837 E-01 & 2.1321[-01 & 1.8277 E-0 \\ 5 \quad 12 & 513 & 514\end{array}$ $1.8395 \mathrm{E}-0 \overline{3}-2.6112 \mathrm{E}-04$ 0 . (6)

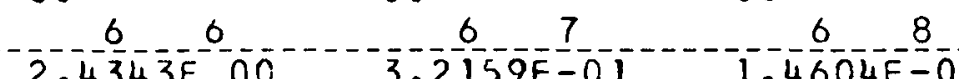

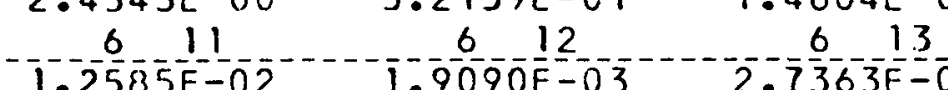

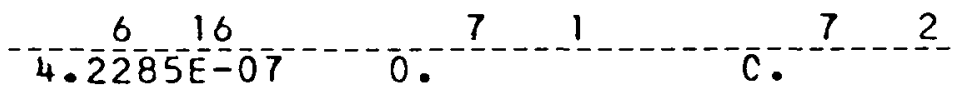
$3.5067 E-05$ $1.2237 \mathrm{E}-02$
$4 \quad 16$ $3.823 \overline{3} 7 \overline{0}-\overline{7}$ $2.4904 \mathrm{E}-0 \mathrm{~T}$ 
- MANGANESE.

$\operatorname{SigMA}-\operatorname{so}\left(\frac{N}{N} 1 / N 2\right)$

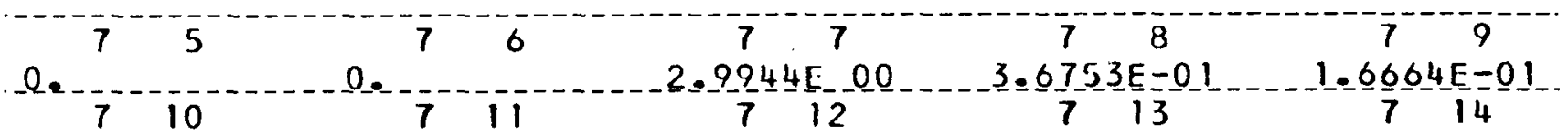

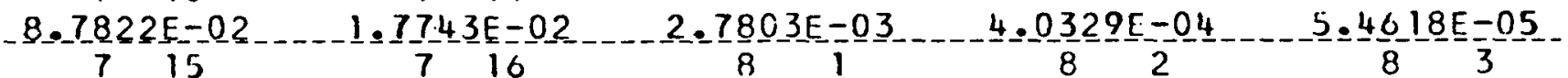

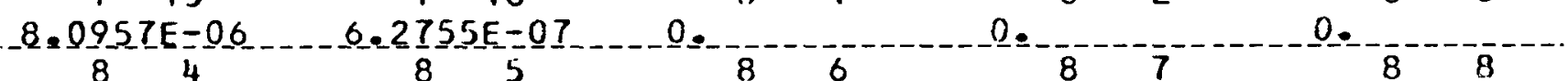

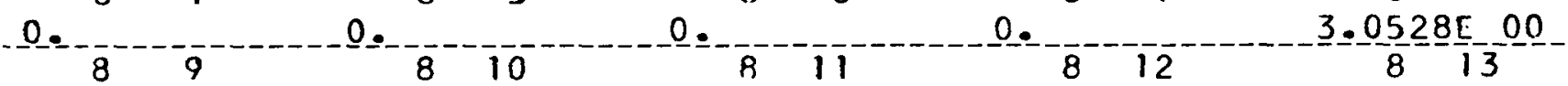
$-3.5003 E-01-14.7813 E=02 \quad 1.9656 E-02 \quad-3.2052 E-03-4.7177 E=04$ 6. $4259 E=05$

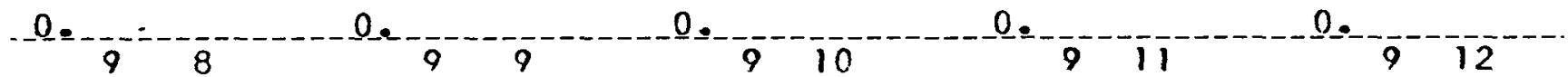

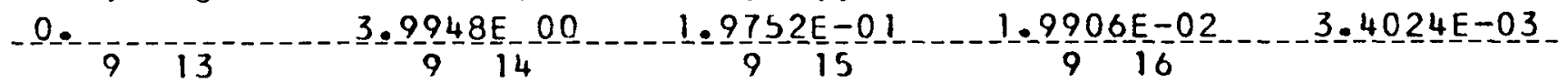
- 5. $0953 \mathrm{E}=04$ $0.9872 E=05$ 
MANGANESE

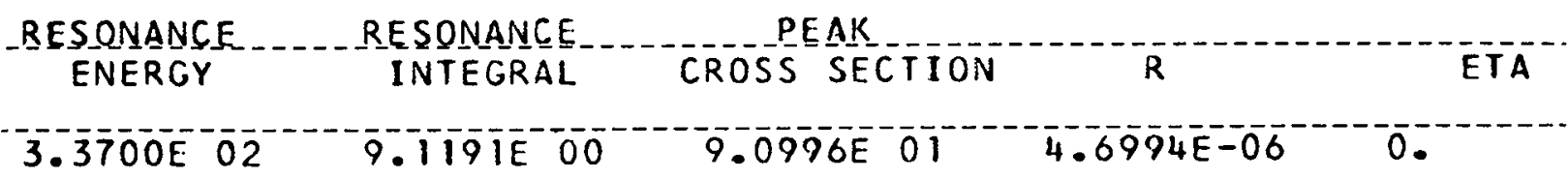


$\mathrm{N} 1$

SIGMA N,G (NIINZ)

-0. -2.6252E-0

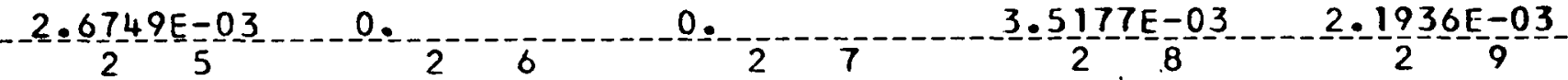
$-1.9367 \mathrm{E}-03$

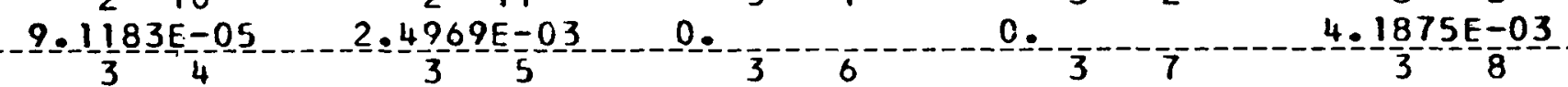
-2. $6113 \mathrm{E}-03$

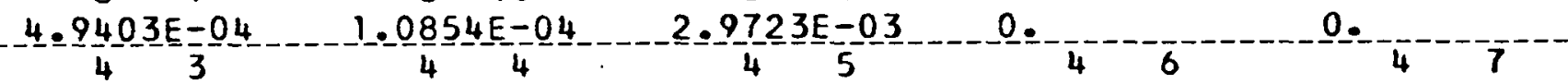

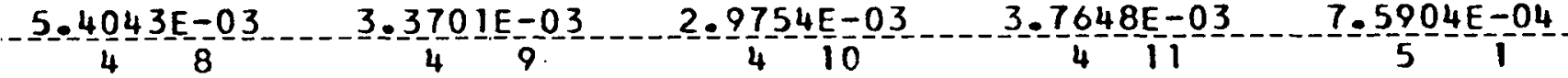
$1.1537 E-03 \quad-1925 E-01$

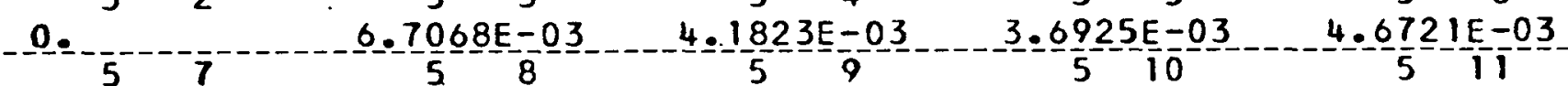
-9.4 $\frac{4}{6} 97 E-04 \quad 1.4318 E-03 \quad 06.4089 E-02 \quad 2.7835 E-01$ 0. $5.2714 \mathrm{E}-03 \quad 1.0628 \mathrm{E}=03$

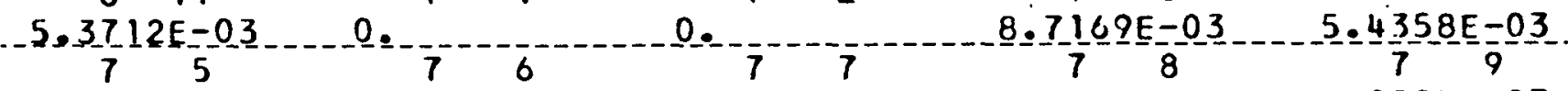

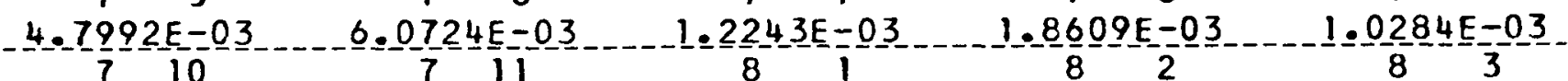
$7.4560 \mathrm{E}-02$ $8.9285 E-03 \quad 0.8829 E-03 \quad 0.9742 E-03 \quad 2.0109 E-03 \quad 03.0566 E-03$

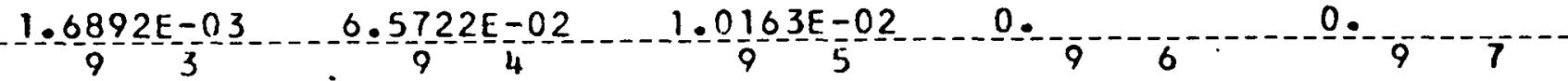
$2.2817 E-02 \quad 1.4229 E-02 \quad 1.2562 E-02 \quad 1.5895 E-02 \quad 3.2046 E-03$

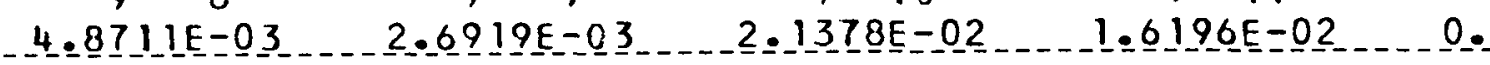


MANGANESE

N1 N2

SIGMA N, G (NIIN2)

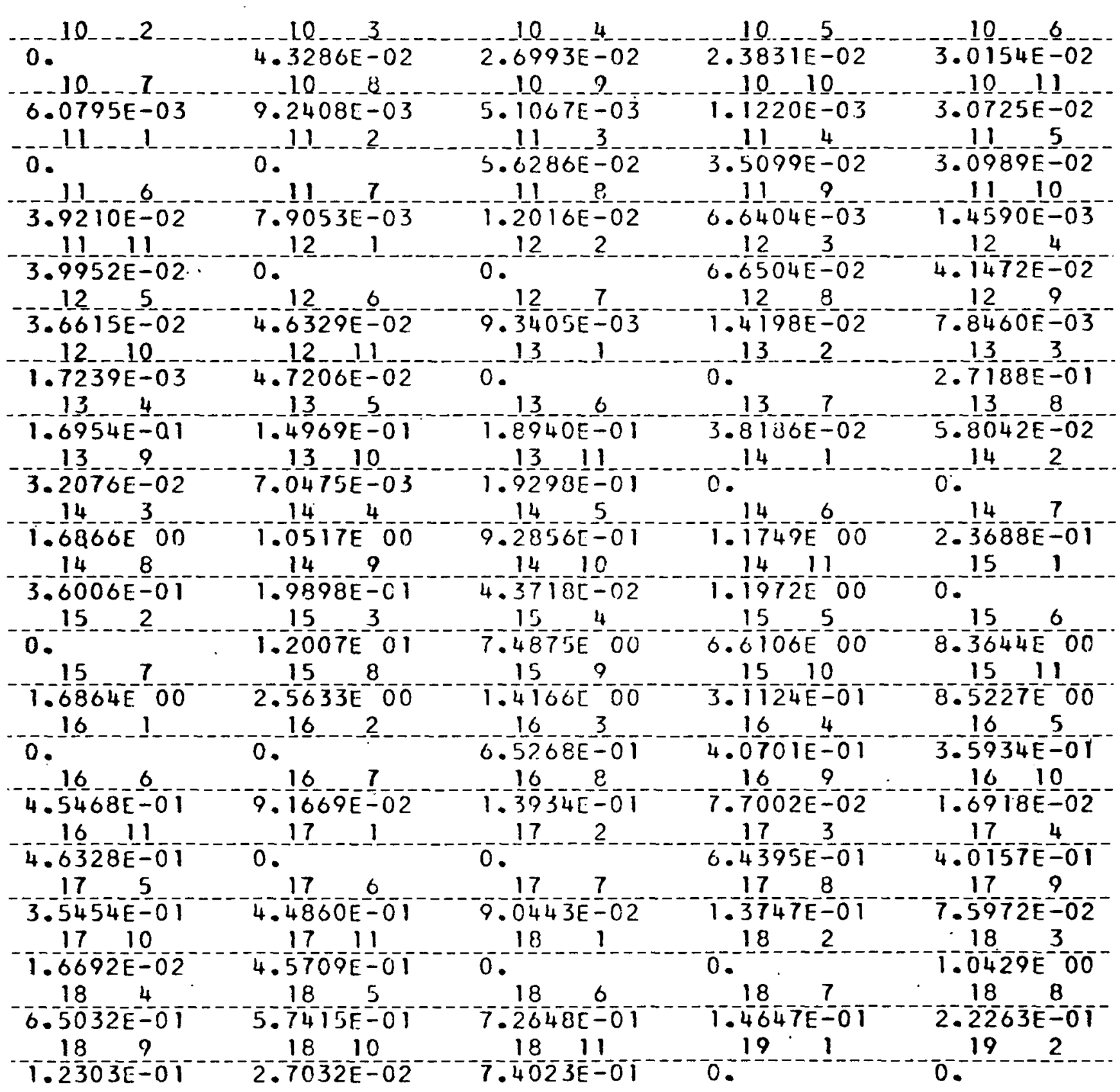


-MANGANESE.

N1 1

SIGMA N,G (NI/N2)

$1930194019-19067$

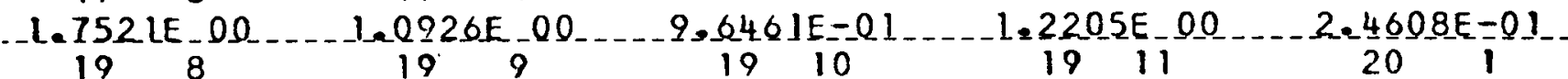

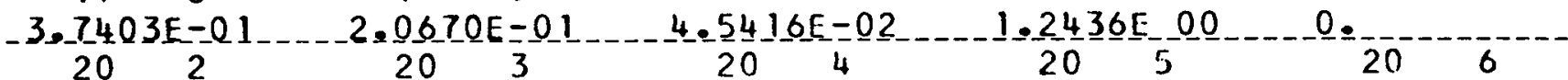

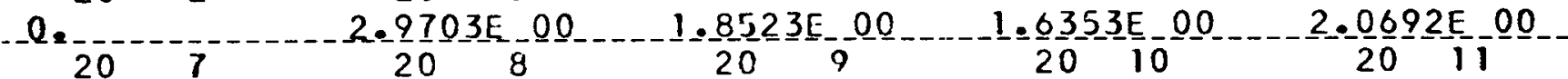
$4.1718 \mathrm{E}-01$

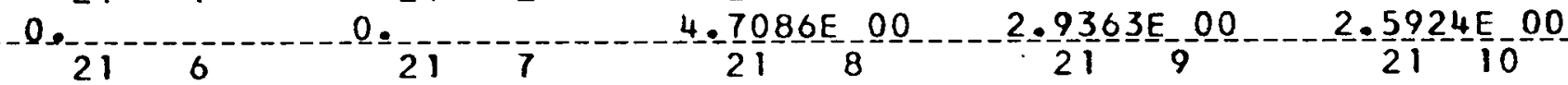

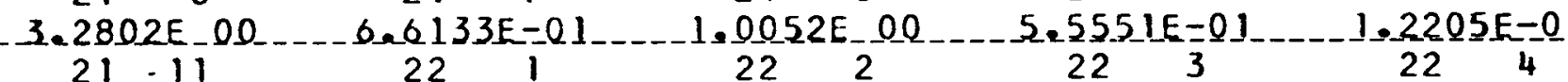

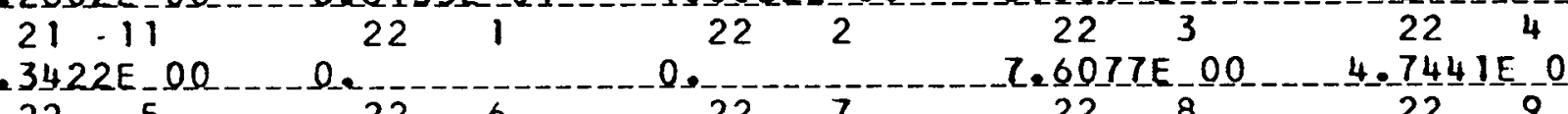

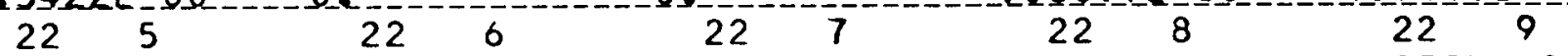

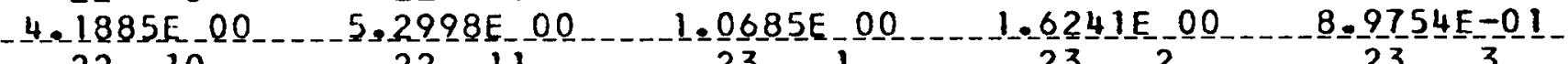
$221022 \quad 11232323$

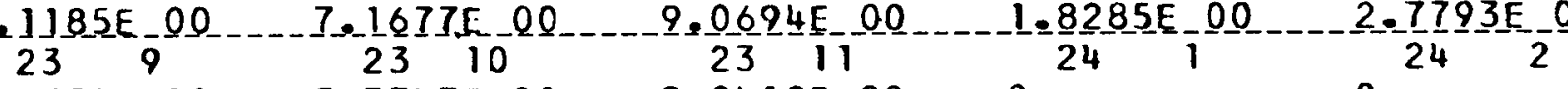
$-1.53596 E_{3} 00-3.3747 E-01$ $-2.0075 E 01$

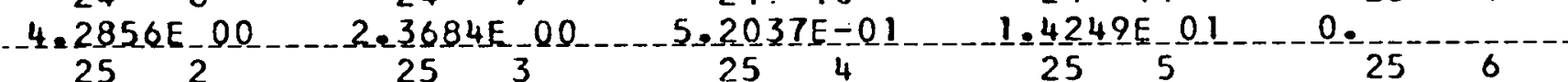
_0._._.

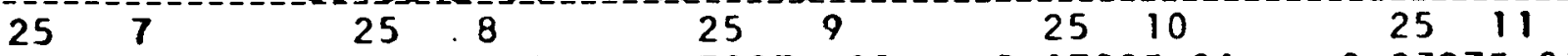
$-4.4317 E_{0} 00$

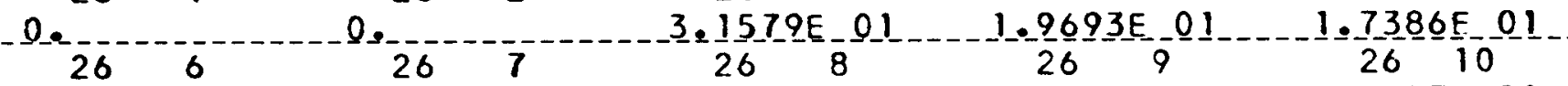

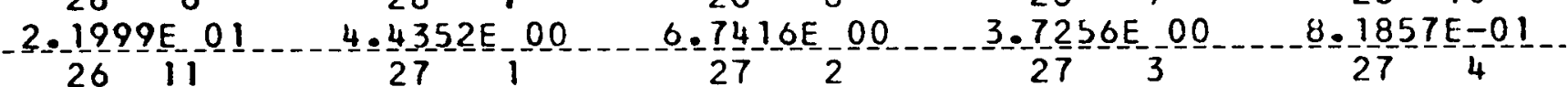

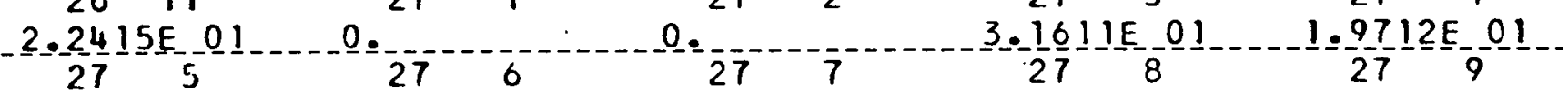
$\begin{array}{ll}27 & 5 \\ 7404 E^{2} 01 & 2.2021 E^{\circ}\end{array}$ 2710 2711 $4.4397 E-00$ $3.7294 E-00$ $8.1940 E-01 \quad 2.2438 E \quad 01$ _ 0. 3.1654E_01 
MANGANESE

$\mathrm{N1} N 2$

SIGMA N,G (N1/N2)

\begin{tabular}{|c|c|c|c|c|}
\hline $1.9739 E^{4} 01$ & $1.7427 E 01$ & $2.205 \mathrm{iE} 01$ & $4.4457 \mathrm{E} 00$ & $6.7575 E 00$ \\
\hline $\begin{array}{r}1.9854 \\
-28\end{array}$ & $\begin{array}{r}1.727 E \\
-28\end{array}$ & $\begin{array}{l}2.2051 \mathrm{~K} \\
28 \\
-2\end{array}$ & $\begin{array}{l}4.4457 \mathrm{E} 00 \\
29\end{array}$ & $\begin{array}{c}6.7575 E 00 \\
-29\end{array}$ \\
\hline $\begin{array}{r}3.7344 \mathrm{E} 00 \\
29\end{array}$ & $\begin{array}{c}8.2051 E-01 \\
29\end{array}$ & $\begin{array}{c}2.2468 E^{2} 01 \\
29\end{array}$ & 0. & 0.29 \\
\hline $3.1709 \mathrm{E} 01$ & $1.9774 E_{0} 01$ & $1.7458 \mathrm{E} 01$ & $2.2089 \mathrm{E} 01$ & $4.4535 \mathrm{E}, 00$ \\
\hline $\begin{array}{c}6.7693 \mathrm{E} 00 \\
30\end{array}$ & $\begin{array}{c}3.7409 E_{3} 00 \\
30\end{array}$ & $\begin{array}{c}8.2194 E-01 \\
30\end{array}$ & $\begin{array}{c}2.2507 E_{5} O 1 \\
30\end{array}$ & 306 \\
\hline & $3.1789 \mathrm{E} 01$ & $1.9823 \mathrm{E} 01$ & $1.7502 \mathrm{E} 01$ & $2.2145 \mathrm{E} O 1$ \\
\hline 30 & 30 & 30 & 30 & 30 \\
\hline $4.4647 E 00$ & $6.7864 \mathrm{E} 00$ & $3.7504 \mathrm{E} 00$ & $8.2401 E-01$ & $2.2564 \mathrm{E} 01$ \\
\hline-31 & -31 & $3-31-3$ & $1-\frac{3}{1}$ & -31 \\
\hline 31 & 31 & 31 & 31 & 31 \\
\hline $2.2231 E 01$ & $4.4820 \mathrm{E}, 00$ & $6.8126 \mathrm{E}, 00$ & $3.7649 \mathrm{E} 00$ & $8.2720 \mathrm{E}-01$ \\
\hline $2.265 \mathrm{IE} 01$ & 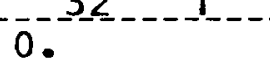 & 0 & $3.2120 \mathrm{OE}$ & $2.0030 E^{4} O$ \\
\hline 32 & & & 32 & 32 \\
\hline $\begin{array}{c}1.7684 \mathrm{E} \quad 01 \\
32 \quad 10\end{array}$ & $2.2376 \mathrm{E}$ 01 & $\begin{array}{l}4.5112 E, 00 \\
33\end{array}$ & $\begin{array}{c}6.8571 E 00 \\
33\end{array}$ & $3.7894 \mathrm{E} 00$ \\
\hline $3260 E-01$ & $2.2799 E 01$ & 0 & 0 & $3.2562 \mathrm{E} O \mathrm{1}$ \\
\hline & & & & \\
\hline $2.0305 E 01$ & $1.7927 \mathrm{E} 01$ & $2.2683 E 01$ & $4.5732 \mathrm{E} 00$ & $6.9513 E 00$ \\
\hline $3.8 \frac{3}{4} 15 \mathrm{E}^{-9} 0 \overline{0}$ & $8.4304-\frac{10}{2}=$ & $2-\frac{33}{3} 13 \frac{11}{01}$ & & 0.34 \\
\hline 34 & 34 & $3 \underline{4}$ & 34 & \\
\hline $3.3722 \mathrm{E} \quad 01$ & 2.1029501 & $1.8566 \mathrm{E} 01$ & $2.3492 \mathrm{E} 01$ & $4.7362 \mathrm{E} 00$ \\
\hline 0 & $3-\frac{34}{3784 \mathrm{~F}^{2}}-10$ & $8-34-12 F-01$ & $5-\frac{34}{39}=$ & \\
\hline
\end{tabular}


DAIE 12115160

DESIGNATION

COBALT
CODE NO.

$2 \overline{7} \cdot \overline{5} \overline{900}$
DENSITY FACTOR

$9.7850 \overline{3}$ O

LEVEL ENERGY SCATTER SCATTER TRANSPORT ABSORPTION SCATTER 1 -

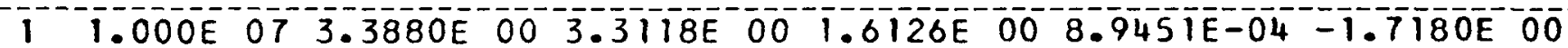
2 6.065E 06 3.6677E 00 3.0615E 00 1.8194E $00 \quad 1.0805 \mathrm{E}-03=1.7886 \mathrm{E}$ 00

3 3.679E $06 \quad 3.6172 \mathrm{E} \quad 002.6073 \mathrm{E} \quad 002.4295 \mathrm{E} \quad 001.5654 \mathrm{E}-03-1.1494 \mathrm{E} 00$ $4-2.231 \mathrm{E}-06-3.4062 \mathrm{E}-002.0026 \mathrm{E}-002.7626 \mathrm{E}-002.3266 \mathrm{E}-03-6.2274 \mathrm{E}-01$

$51.353 \mathrm{E} 063.3459 \mathrm{E} 001.3195 \mathrm{E} 002.7562 \mathrm{E} 003.1797 \mathrm{E}-03-5.7070 \mathrm{E}-01$

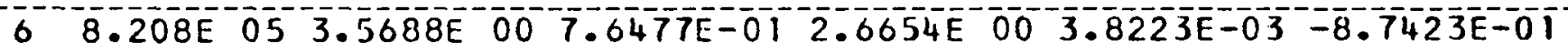

7 4.979E 05 3.9720E 00 4.5716E $=01$ 3. $1931 \mathrm{E}-004.585 \mathrm{EE}-03=7.5373 \mathrm{E}=01$

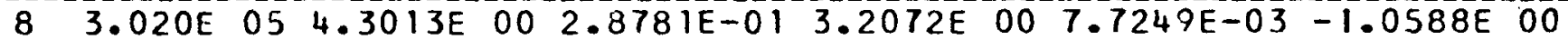

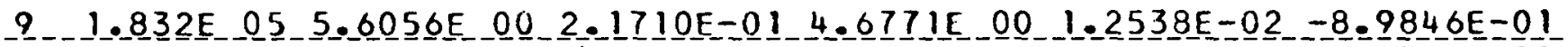

$106.738 \mathrm{E}$ O4 5.7003E OC $1.9265 \mathrm{E}-015.6318 \mathrm{E}$ 00 $2.4125 \mathrm{E}-02-6.6326 \mathrm{E}-02$

$112.479 \mathrm{E} 041.1486 \mathrm{E} 013.8823 \mathrm{E}-011.1355 \mathrm{E}$ 01 $3.0792 \mathrm{E}-02-1.267 \mathrm{IE}-0 \overline{1}$

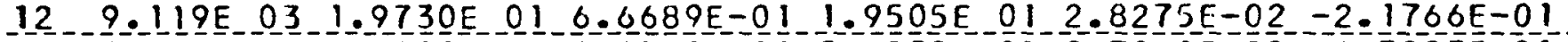

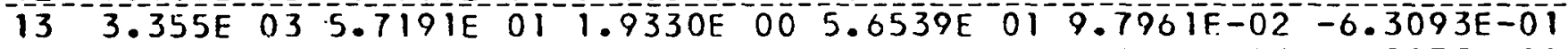

$14-1.234 E-03-3.5418 E-00-1.1971 E-0113.5015 E-006.8352 E-03-3.9073 E=02$

$154.540 \mathrm{E} 021.0191 \mathrm{E} 013.4446 \mathrm{E}-011.0075 \mathrm{E}-12.0355 \mathrm{E}=0 \mathrm{~T}-1.1243 \mathrm{E}=0 \mathrm{~T}$

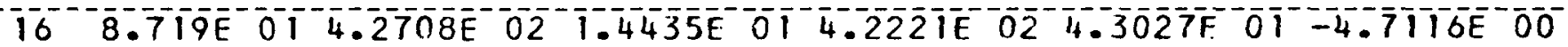

$173.545 \mathrm{E} 01 \quad 8.5954 \mathrm{E} 00 \quad 2.9053 \mathrm{E}-018.497 \mathrm{SE}$ 00 $1.0415 \mathrm{E} 00-9.4825 \mathrm{E}-02$

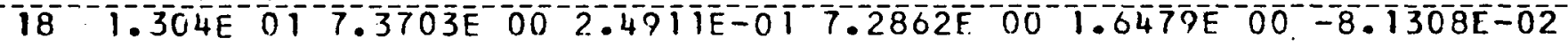

19 - 5.043E 00 0.8683E $002.321 \mathrm{EE}-016.7900 \mathrm{E} 002.7731 \mathrm{E} 00-7.5771 \mathrm{E}-02$

$201.445 E-006.7494 \mathrm{E} 002.2813 E-016.6724 \mathrm{E} \quad 004.6830 \mathrm{E} \quad 00=7.4459 \mathrm{E}-02$

$210.826 \mathrm{E}-016.2916 \mathrm{E} 0 \mathrm{O} 2.1265 \mathrm{E}-0 \mathrm{O} 6.2198 \mathrm{E}-007.4148 \mathrm{E}-00-0.940 \overline{\mathrm{E}}-02$

$22-2.511 E=01-6.2534 \mathrm{E}-002.1137 \mathrm{E}=01-6.1822 \mathrm{E}-00-1.1980 \mathrm{E} 01-6.898 \mathrm{EE}-02$

$239.237 \mathrm{E}-025.8223 \mathrm{E} 00 \mathrm{O} 1.9679 \mathrm{E}-015.7 \mathrm{~J} 59 \mathrm{E}$ DO $2.050 \mathrm{FF} 01-6.4231 \mathrm{C}-02$

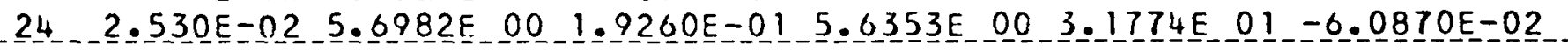

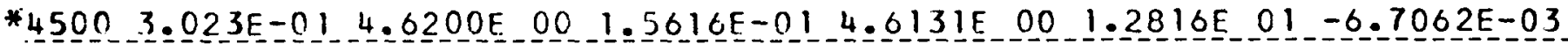

* $40002.718 \mathrm{E}-014.6200 \mathrm{E} 00-1.5616 \mathrm{E}-014.6131 \mathrm{E} 00-1.2816 \mathrm{E} 01-6.7062 \mathrm{E}=0 \mathrm{3}$

*3500 2.413E-01 4.6200E 00 1.5616E-01 4.6131E $00 \quad 1.2816 E \quad 01-6.7062 E-03$

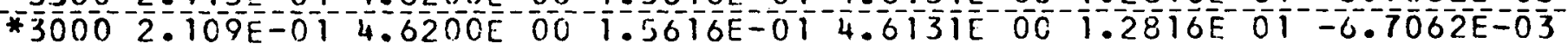
$25001.804 \mathrm{E}-014.3400 \mathrm{E}$ 00 $1.4669 \mathrm{~F}-01$. $3335 \mathrm{E}$ 00 1.3856E $01.6 .2998 \mathrm{E}-03$

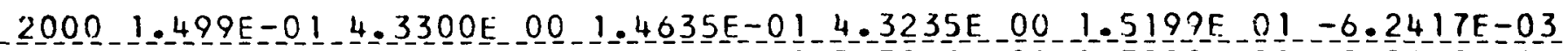
1500 1.194E-01 3.8000E 0C $1.2844 \mathrm{~F}-013.7943 \mathrm{E} 00$ 1.7028E 01 $-5.5160 \mathrm{E}-03$

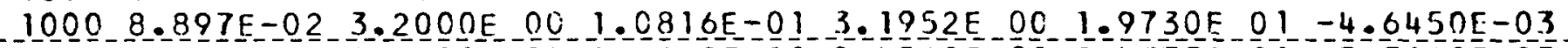
$5005.850 E-022.5600 \mathrm{E} 00-8.6548 \mathrm{E}-022.5562 \mathrm{E}-002.4333 \mathrm{E}-0 \mathrm{~T}=3.7160 \mathrm{E}=03$

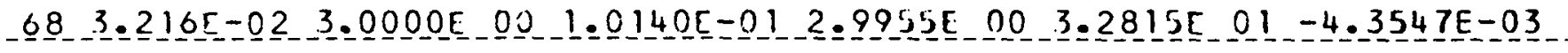

*Thermal cross sections taken from APEX-515.

NOTE: For a summary of the sources of data see APEX-704. 
COBALI

THERMAL TRANSFER MATRICES

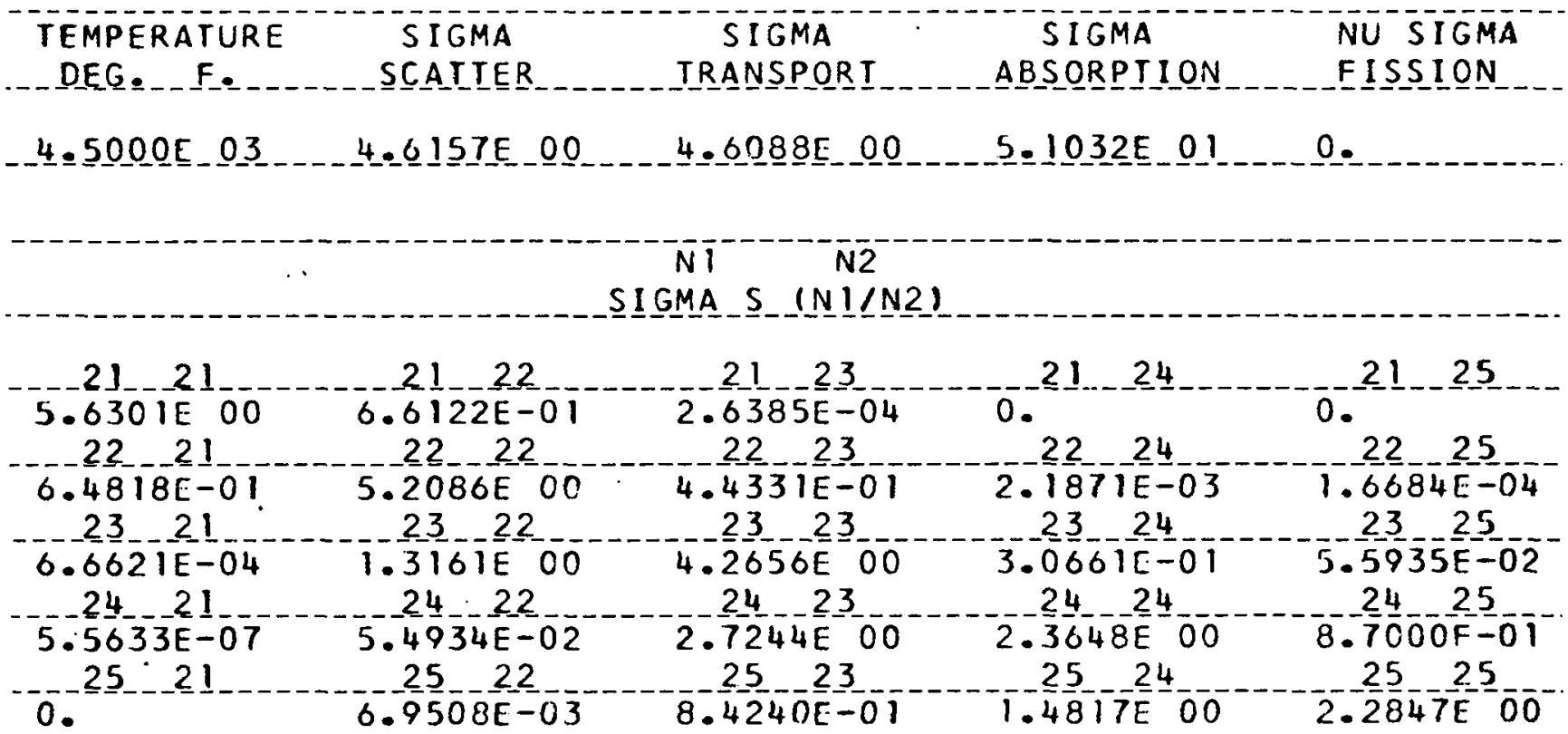

TEMPERATURE

DEG _. F....... SCAIIER

-4_-OOOOEE_03
SIGMA
TRANSPQR I

SIGMA

ABSORPIION
NU SIGMA

FI SSION

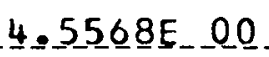
$4.550 Q E_{0} 00 \ldots 5.1073 E_{0} 01 \ldots$
- 4.OOOOOE

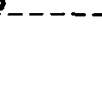

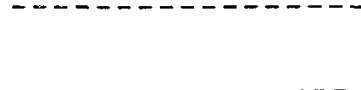

N1

$\mathrm{N} 2$

SIGMA S $(N 1 / N 2)$

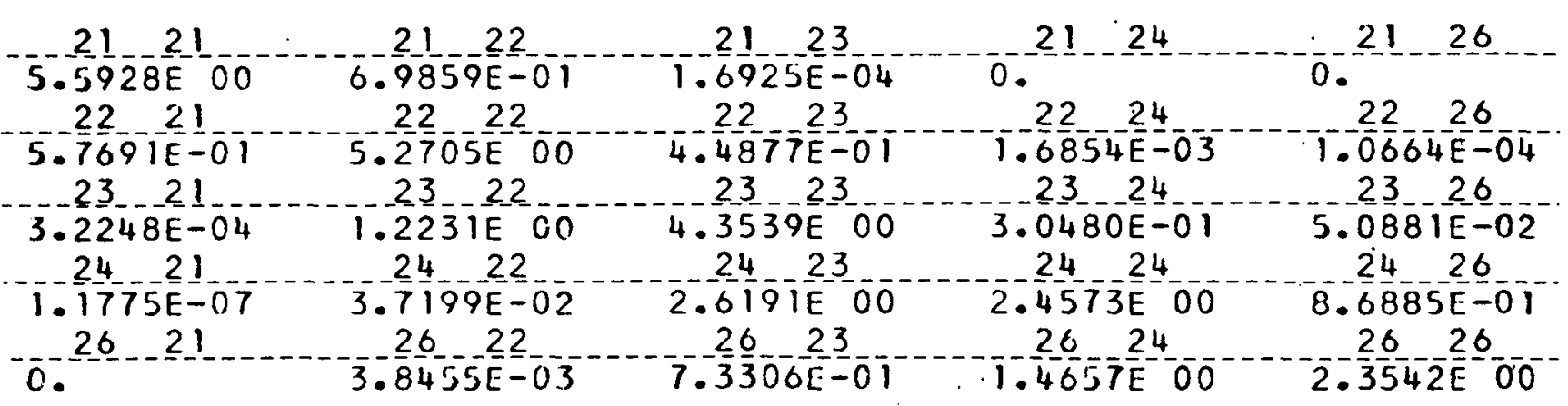




\section{COBALT}

\section{IHERMAL_IRANSEER_MAIRICES_}

LEMPERAIURE
DEG SIGMA

\section{$S I G M A-S(N) / N 2)$}

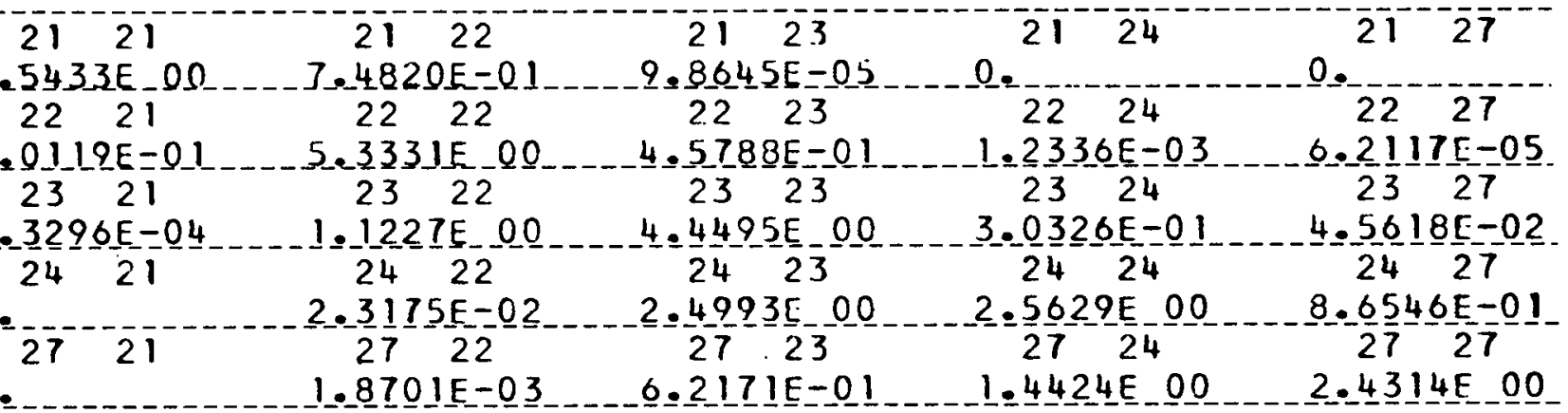

IEMPERAIURE

DEG. F.

$3.0000 E \quad 03$
SIGMA

SCATTER
SIGMA

TRANSPORT
SIGMA

ABSORPTION
NQU_SIGMA_.

FISSION

\section{$\left.S I G M A-\frac{1}{S}-(N)-N 2\right)$}

$21^{2} 1^{2} 21^{2} 21^{2} 21^{2}$

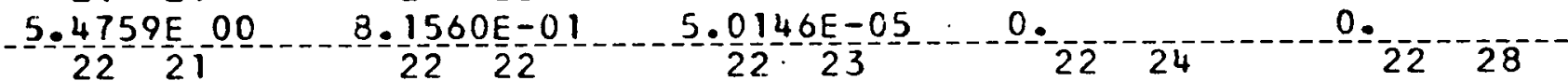
$-4 \cdot 2 \frac{20}{2} \frac{3 E}{2} 1-01$ $-4 \cdot \frac{3}{2} \frac{7}{4} 6 \mathrm{E}=05$ .

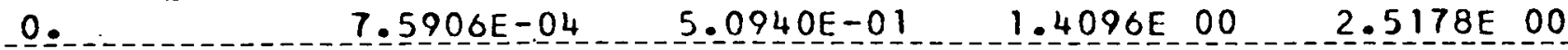


COBALI

THERMAL TRANSFER MATRICES

TEMPERATURE

DEG. _ F.

$2.5000 E_{-} 03$
SIGMA

SCAIIER

4. 3773E_00
SIGMA

IRANSPORI
SIGMA ABSORPIION $5=1284$ E
NU SIGMA

EISSION (1)$$
\mathrm{N1} N 2
$$

SI GMA S _ (N1 IN2)

\begin{tabular}{|c|c|c|c|c|}
\hline-8 & $211_{-} 22$ & 21 & $-1 \leq 1$ & $-1-2$ \\
\hline$\cdot 3$ & $9.0990 E-01$ & $2.0748 E-05$ & 0. & 0. \\
\hline $\begin{array}{l}21 \\
9 E-0\end{array}$ & $22--22$ & 32 & -22 & 21 \\
\hline $\begin{array}{l}3 \\
2\end{array}$ & $\begin{array}{c}5.4524 \mathrm{E} \\
23\end{array}$ & $\begin{array}{c}4.9649 E-01 \\
23\end{array}$ & $\begin{array}{c}5.1726 E-04 \\
2324\end{array}$ & $1.3443 E-05$ \\
\hline $1.0304 E-$ & $8.9330 E-01$ & $4.6668 \mathrm{E} 00$ & $3.0288 \mathrm{E}-01$ & $3.4482 \mathrm{E}-0$ \\
\hline 21 & $\ldots 2$ & 23 & & 24 \\
\hline 29 & $\begin{array}{r}6.0124 \mathrm{E} \\
-29 \quad 22\end{array}$ & $\begin{array}{r}2.20400 \\
29 \quad 23\end{array}$ & $\begin{array}{l}2.82845 \\
29\end{array}$ & $\begin{array}{r}0.40 \\
2\end{array}$ \\
\hline & $2.3609 E-04$ & $3.9767 E-C$ & $1.3634 E 00$ & $2.6160 E$ \\
\hline
\end{tabular}

TEMPERATURE

$\therefore$ DEE

2. $0000 E \quad 03$

\section{SIGMA}

SCAITER

$4.3163 E$ EO

$4.3707 E$ E 00

‥

$\overline{N 1}^{-1}{ }^{-12}$

\section{A}


COBALT

._. IHERMAL_IRANSFER_MAIRICES.

LEMPERAIURE

SIGMA

SIGMA

DEG. F.

SCATTER

TRANSPORT

SIGMA

NO SIGMA

$1.5000 \mathrm{E}$ O3

$4.2554 \mathrm{E}$

$4.2490 \mathrm{E} 00$

$5.1610 \mathrm{E}$ OI

FISSION

\begin{abstract}
-.-.-.....-.--
\end{abstract}

\title{
SIGMA S (NI/N2)
}

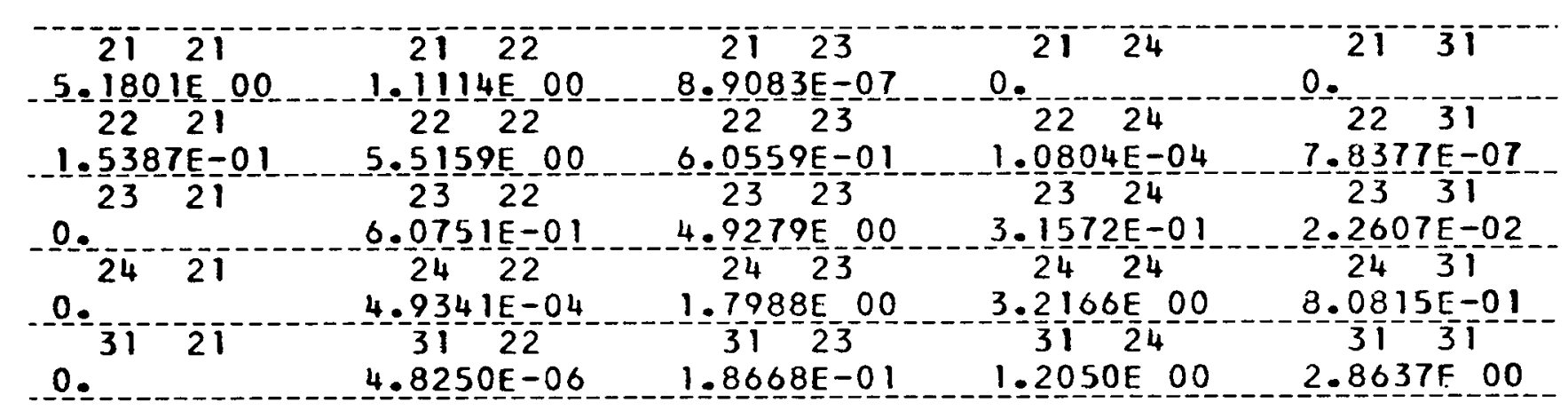

IEMPERA IURE

DEG. F.

$1.0000 \bar{E} 0 \overline{3}^{-1}$
SIGMA

SCATTER
SIGMA

TRANSPORT
SIGMA

ABSORPTION
0.
$4.1939 \bar{E} 00$
4. $1876 \mathrm{E} 00$
$5.1948 \bar{E} 01$

NU SIGMA

FISSION

\section{SIGMA $-\frac{N}{-}\left(N \frac{N 2}{N}\right)$}

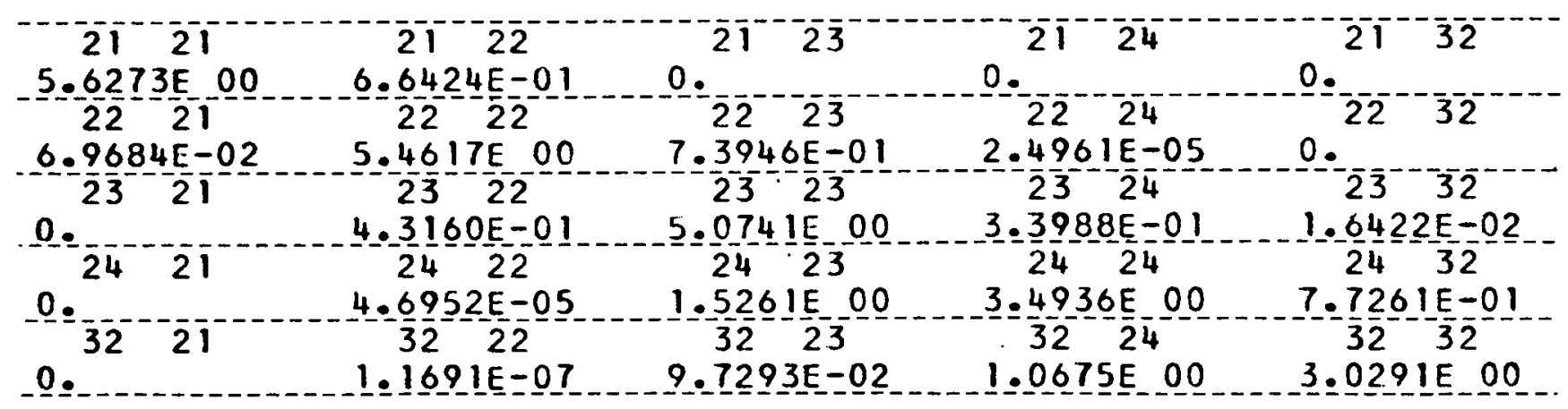


COBALI

\section{THERMAL TRANSFER MATRICES}

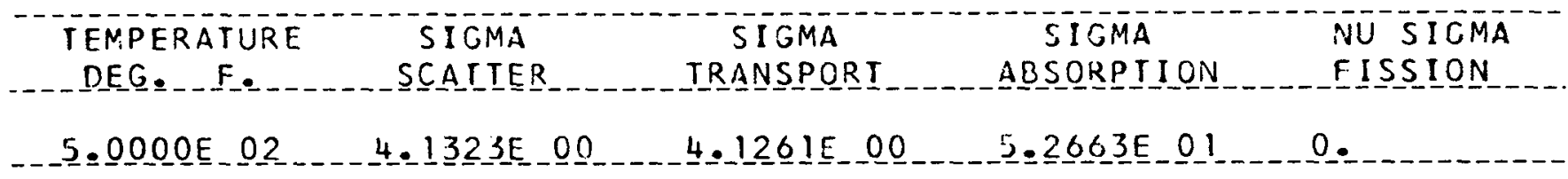

\section{$\mathrm{N} 1$ \\ SI GMA S (N1/N2)}

21

5.8689500

$22-21$

$6.8210 \mathrm{E}-02$

$-23-21$

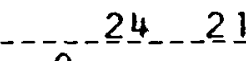

0 .

0.
4.2265E-01

22

$5.2215 \mathrm{E} 00$

$23-22$

$2.2670 \mathrm{E}-0 \mathrm{I}$ $24-22$

$5.2450 \mathrm{E}-07$

0.
33
21

0 .

$22-23$

$9.7593 E-01$ $23-23$

$5.2046 \mathrm{E} 00$ $24-23$

$1.1656 \mathrm{E} 00$

$33-23$

$3.103 \mathrm{IE}-02$
21.24

0 . $22-24$

$1.3257 \mathrm{E}-06$ $23-24$

$4.0892 \mathrm{E}-01$

$3.2 \frac{24}{3}-25 \mathrm{E}-2.0$

$33-24$

$8.5427 E=0$
21 33

0. 22 $23 \quad 33$ $1.0060 \mathrm{E}-02$ $24 \quad 33$ $7.2264 \mathrm{E}-0 \mathrm{1}$ $3.2470 \mathrm{E}-00^{-1}$

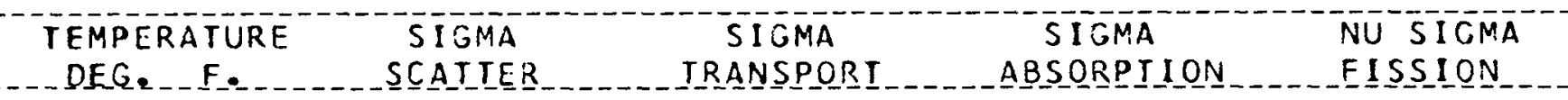

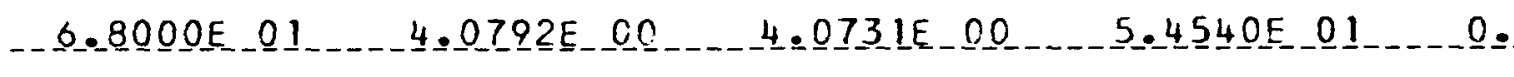

$\mathrm{Ni} N 2$

SIGMA S (N) IIN2)

\begin{tabular}{|c|c|c|c|c|}
\hline 2121 & 22 & 23 & 24 & $1-34$ \\
\hline $\begin{array}{l}8869 E 0 C \\
22 \quad 21\end{array}$ & $\begin{array}{c}4.0460 E-01 \\
2222\end{array}$ & 0.22 & 0.22 & $0_{22} \quad 34$ \\
\hline $\begin{array}{r}1.0782 \mathrm{E}-0 \\
23 \quad 21\end{array}$ & $\begin{array}{c}5.8900 E \\
2320\end{array}$ & $\begin{array}{l}2.6088 \mathrm{E}-01 \\
2323\end{array}$ & 0.23 & 0.23 \\
\hline$==-$ & $4.9766 \mathrm{E}-02$ & $5.15500 \quad 00$ & $6.3092 E-01$ & $4.2694 E-0$ \\
\hline $0 .-2$ & -24 & $-\frac{24}{.1379 E-01}$ & $-\frac{24}{3} 30-\frac{24}{0}$ & $-2 \frac{24}{8}-34$ \\
\hline-7 & -34 & $3 . \frac{34}{2} 210=-23$ & $\frac{34}{5}=24$ & $-\frac{34}{5} 25 \frac{34}{2}$ \\
\hline
\end{tabular}


COBAL I

TRANSFERS FROM GROUPS 1 THROUGH 7 TO GROUPS 1 THROUGH 16

\section{SIGMA $50\left(\frac{N}{2} 1 / N 2\right)$}

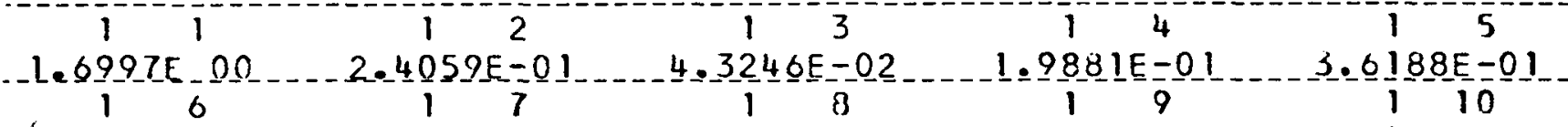

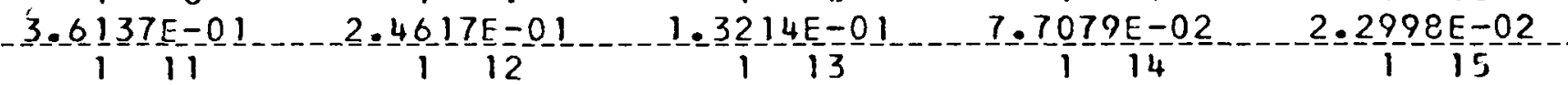

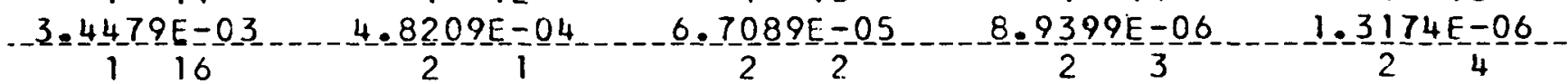

$1.0190 \mathrm{E}-0 \mathrm{Z}$

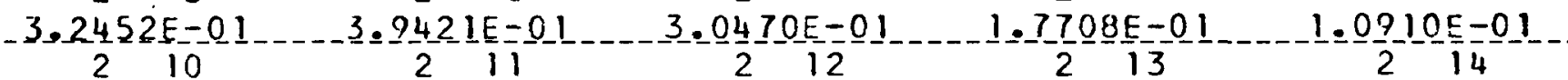

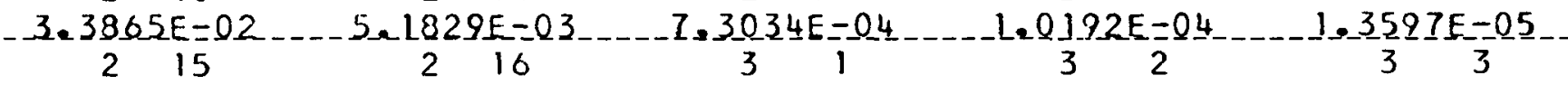

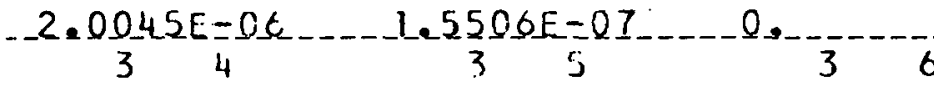

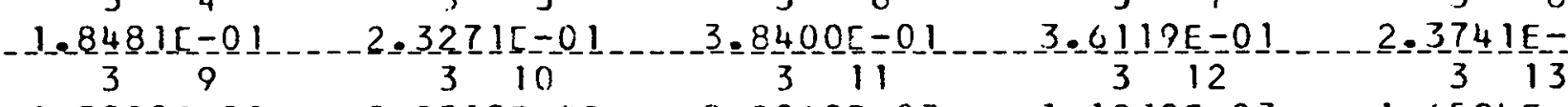

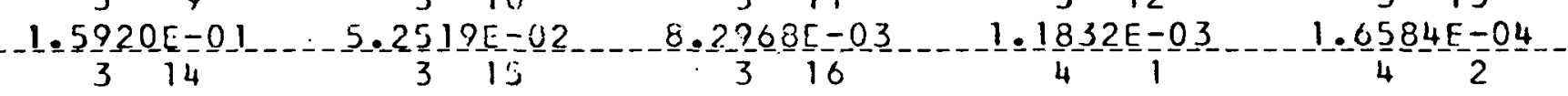

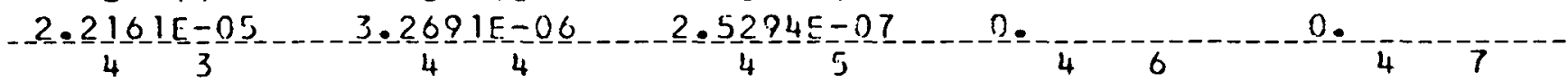

-.

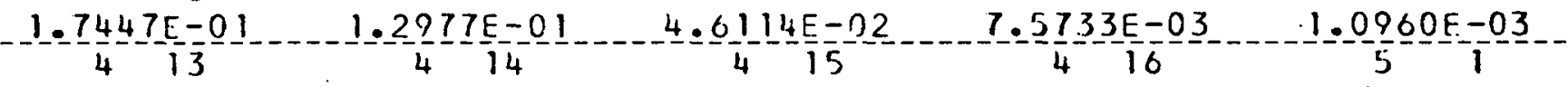

$-1.5444 E=04-2.0681 E-05$

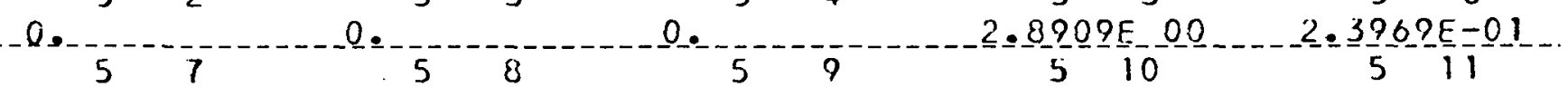

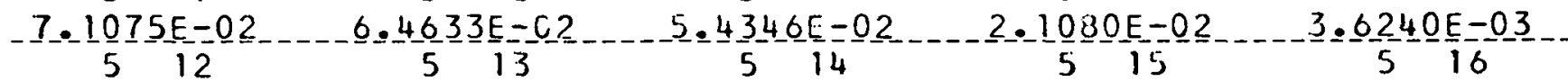

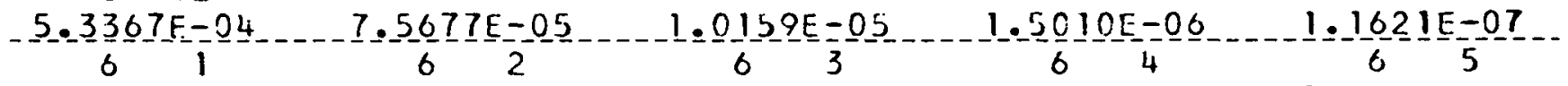

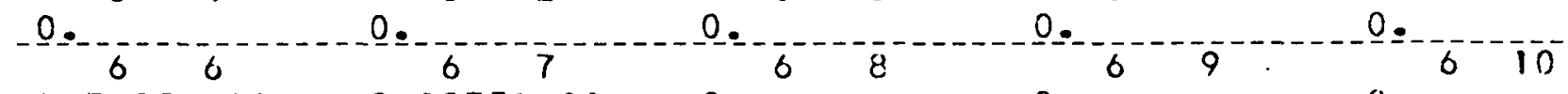

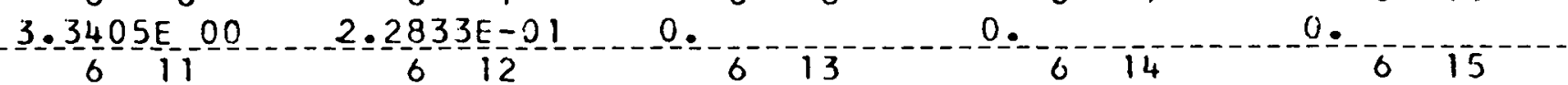

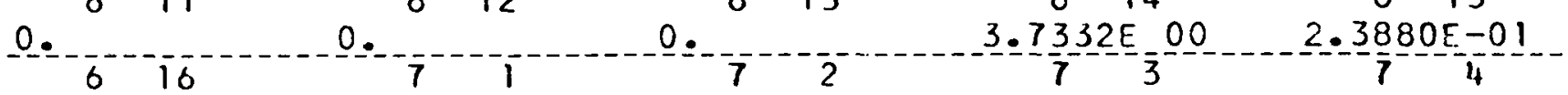

Cie 
240 .

\section{COBALT}

N1 N2

SIGMA SO_(N1/N2)

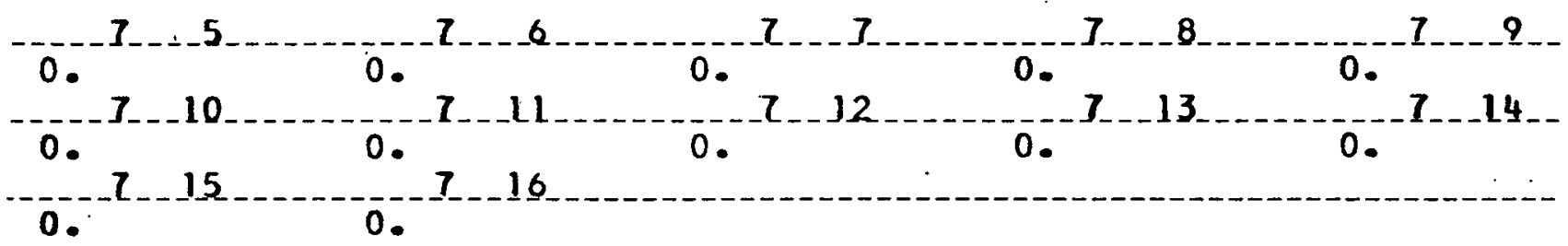


_. COBALI

RESONANCE
ENERGY


COBALT

TRANSFHRS FROM GROUPS I THROUGH 34 TO GROUPS 1 THROUGH 11

$N 1 \quad N 2$

SIGMA_N,G (N)INZ2)

\begin{tabular}{|c|c|c|c|c|}
\hline & & & & \\
\hline & & $3315 E-03$ & $3.9712 \mathrm{E}-03$ & $3.6675 \mathrm{E}-03$ \\
\hline $0323 E-03$ & $1.7987 \mathrm{E}-03$ & $1.8688 \mathrm{E}-04$ & $5.8400 E-04$ & $7.9425 E-04$ \\
\hline $0038 \mathrm{E}-03$ & 1 & 0. & $1.0319 \mathrm{E}-03$ & $3.0776 \bar{E}-03$ \\
\hline$=5$ & 6 & 2 & -2 & 2 \\
\hline $\begin{array}{c}8422 E-03 \\
210\end{array}$ & $.5750 E-03$ & $.3940 E-03$ & $1.4483 E-04$ & $4.5258 E-0$ \\
\hline $1551 E-0$ & $2.3279 E-03$ & & 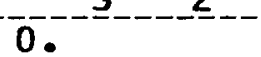 & $8.5015 \bar{E}-0$ \\
\hline & 5 & & 3 & 3 \\
\hline $5355 E-0$ & $2.3416 \mathrm{E}-03$ & $1.2976 \mathrm{E}-03$ & $1.1484 E-03$ & $1.1932 \bar{E}-02$ \\
\hline$=\frac{3}{7287 E-0 i}$ & 3 & $3=11$ & $-4^{4}$ & 2 \\
\hline $\begin{array}{l}E-01 \\
3\end{array}$ & $.0711 E-04$ & $\begin{array}{c}9179 E-03 \\
45\end{array}$ & 0. & 0. \\
\hline $4296 E-03$ & $4.2637 \mathrm{E}-03$ & $3.9377 \mathrm{E}-03$ & $2.1820 \mathrm{E}-03$ & $1.9312 \mathrm{E}-03$ \\
\hline-4 & $-\frac{4}{27}-\frac{9}{2}=-0$ & $274 \bar{E}-\mathrm{C}$ & $-\frac{4}{2}-1 \frac{1}{5}-0 \overline{3}$ & 5 \\
\hline & 5 & is & $5 \quad 5$ & U. \\
\hline & $.372 \mathrm{IE}-0 \overline{3}$ & $7.0748 \mathrm{~F}-\mathrm{c}$ & $6.5338 E=03$ & $3.6206 \mathrm{E}-0 \overline{3}$ \\
\hline $2045 E-03$ & $3 . \frac{5}{32} \overline{3 E E-04}$ & $\frac{5}{1.0404 \bar{E}-03}$ & $-1-\frac{5}{4}-50 \frac{10}{E}-0$ & $5 . \frac{5}{35}-\frac{11}{4 E^{2}-0}$ \\
\hline & & & $=4$ & $6 \quad 5$ \\
\hline & & & & $\begin{array}{l}E-C \\
10\end{array}$ \\
\hline $\begin{array}{c}1033 E-0 \\
611\end{array}$ & $3.6316 \mathrm{E}-0$ & $7 \overline{T E}=04$ & $1.1791 E-03$ & $1.6036 \mathrm{E}-03$ \\
\hline $0647 \mathrm{E}-03$ & 0 & 0 & $2.8437 \bar{B}-03$ & $8 . 4 8 \longdiv { 2 E } - 0$ \\
\hline$-8 \frac{7}{32}=\frac{5}{E}-03$ & -7 & 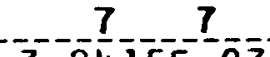 & $=8$ & $=\frac{7}{1-3}=9$ \\
\hline $\begin{array}{c}8327 E-03 \\
7 \quad 10\end{array}$ & $4.3404 E-03$ & $\begin{array}{c}3.8415 E-03 \\
8\end{array}$ & $\begin{array}{c}3.9912 E-04 \\
8\end{array}$ & $\begin{array}{c}1.2472 E-03 \\
8\end{array}$ \\
\hline $6962 \mathrm{E}-0$ & $6.4152 \mathrm{E}-03$ & 0. & 0. & $3.9052 \mathrm{E}-03$ \\
\hline$\frac{8}{16}-\frac{4}{47 E-0}$ & & & & \\
\hline $\begin{array}{c}1647 E-0 \\
8\end{array}$ & $1.0756 \bar{E}-02$ & $5.9605 \bar{E}-03$ & $5.2754 E-03$ & $5.4809 \bar{E}-04$ \\
\hline$-7 \frac{8}{12}=9-9$ & $-2.3 \frac{8}{294}-\frac{10}{E}-0$ & $8.8098 \mathrm{E}-0$ & 0 & 2 \\
\hline$\frac{3}{O E}-c$ & $=4$ & $5=$ & $\frac{9}{6}-\frac{6}{80}$ & $-\frac{9}{5}-\frac{7}{2}$ \\
\hline $\begin{array}{c}O E-0 \\
8\end{array}$ & 9 & 10 & 11 & $\begin{array}{c}5.7440 \mathrm{E}-0 \\
10\end{array}$ \\
\hline$\overline{0} \bar{A} \overline{70}=\overline{0}$ & $=-0$ & & & \\
\hline
\end{tabular}


-COBALI.

N 1

SIGMA NNG (NI/N2)

10

2

10

10

$10-5$

100

_.

4. $1393 E=02$

$3.8228 E=02$

2. 11 34E $=2$

-1.87495-02

$1.94795=0.3$

0.0 $0872 E-03$

$8.2787 E-03$

1011

$-11,1$

112

$1.7011 \mathrm{E}=02$

11

$3.1310 E=\frac{02}{5}$

$-2$.

1

7

$2.47318 E=03$

$5.2525 E=02$

4. $8509 E=02$

2. $-688 \geq 1[-02$

11 i1

$2.3791[-02$

7.? $7243 E-03$

1.0505E-02

-3. $-7 \frac{7}{12} \frac{0}{5}-02$

0.

ㅇ.

$12-3$

12

$-4.4448 E=02$

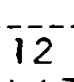

0

2

$1.6137 E-02$

$4.8 \frac{1}{12} 28 \mathrm{E}=02$

$12 \quad 10$

$2.4630 E-02$

$2.17929=02$

2. $2648 E=03$

7.0776E-03

$-9.6256 E=03$

0.

ㅇ..-

13

5 . $864 E-03$

134 $5387 \mathrm{E}-02$

$8.5265 E=03$

$7-\frac{5}{14} 65 \mathrm{E}-03$

$13=4 E-0.3$

$1.60601 E=02$

1310

$-2.4502[-03$

3. $322 \mathrm{E}-\mathrm{C} 3$

$1.2602 E-0.2$

$7.8405 E-04$

-3. $\frac{8}{14} 67 \mathrm{Z} E-0 \mathrm{0}$

1. $1622 E-02$

$.4691 E-04$

149

$1=07335=02$

$0 .-14-\frac{1}{6}$

0.

1

$1.7091 \mathrm{E}-\mathrm{C}$

$2.3244 E=03$

$5=94766 E=03$

147

0.

$1 .-1603 E-0$

$3.4605 E-01$

8. $7907 \mathrm{O}=03$

$5 \cdot 2 \frac{6}{2} 0 \mathrm{E}=03$

$1.5674 \mathrm{E}=01$

1. $6285 E-02$

5. $0890 E-02$

$3.19596=01$

0

$15+10501$

16 i

$-0 .-16$ -

ㅇ.

$2.4526 E \quad 01$

$6.92 ! 11 \varepsilon=02$

1. $7710 \mathrm{E}-01$ $7434 E_{01} \quad 3.3131 E \quad 01 \quad 3.4422 E \quad 00$ 1611 $-317-1]$

$3=\frac{4}{17}-22 E-200$

7.314 E 01 17 万 $17-17$ $-0$.

$9=00,11 \mathrm{E}-\mathrm{C}$

$8 \cdot 0196 \mathrm{~B}=01$ 169 $2=\frac{6}{16} 76[-0)$

_. $5328 E$ _ 1 175

$-1.6352500$ 1.3392500

0.

$1.0757 E$

$6.7553 E-01$

$3.5411 E-0$ 18 4

$2.85 \leq 7 E$ 은

1.4614500

- $4.1995 E=01 \ldots+5.71] 3 E-C 1 \ldots$

- $4.1995 E=01$

$5.9306 E-01$

$1.4629 E_{1} 01$

$83-320 \mathrm{E}-02$

1.7706 E 00

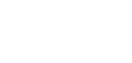


COEALT

$\mathrm{N} 1 \mathrm{N2}$

SIGMA N,G (NI/N2)

\begin{tabular}{|c|c|c|c|c|}
\hline $1.6128 \mathrm{E} 00$ & $4.8102 \mathrm{E} 00$ & $4.4423 \mathrm{E} 00$ & $2.4617 \mathrm{E} 00$ & $2.1787 \mathrm{E} 00$ \\
\hline$\ldots 19$ & 12 & $19-10$ & 19,11 & \\
\hline $\begin{array}{c}2.2636 E-01 \\
20\end{array}$ & $\begin{array}{c}7.0738 E-01 \\
20\end{array}$ & $\begin{array}{l}9.6203 E-01 \\
20\end{array}$ & $\begin{array}{l}3.6384 \mathrm{E} 00 \\
20\end{array}$ & 0. \\
\hline 0 & $2.7204 \mathrm{E} \quad 00$ & $8.1136 \mathrm{E} O 0$ & $7.4931 E 00$ & $4.1522 \mathrm{E} \quad 00$ \\
\hline $3.6750 \mathrm{E} 00$ & $3.8182 E-01$ & $1.1932 \mathrm{E} 00$ & $1.6227 E 00$ & $6.137 \mathrm{E}$ - 00 \\
\hline 21 & 21 & 21 & $21 \quad 4$ & $21 \quad 5$ \\
\hline & & $4.3367 \mathrm{E}$ & $1.2934 \mathrm{E} 01$ & $1.1945 \mathrm{E} 01$ \\
\hline $6.6191 \mathrm{E} 00$ & $-\frac{2}{5.8583 E}=0$ & $0.08866-\frac{2}{1}-01$ & $-\frac{2}{1}-9021{ }^{9}$ & $2-\frac{2}{5} 868 \frac{1}{10}=0$ \\
\hline$-2 \mathrm{~J}_{-} 11$ & 22 & 22 & 22 & $\begin{array}{l}2.3800 \mathrm{E} \\
22\end{array}$ \\
\hline $\begin{array}{l}9.7832 \mathrm{E} 00 \\
225\end{array}$ & 0. & 0. & $6.9472 E_{8} 00$ & $2.0720 E_{01}$ \\
\hline $1.9135 \mathrm{E} 01$ & $1.0604 \mathrm{E} 01$ & $9.3848 \mathrm{E} \quad 0 \mathrm{C}$ & $9.7504 \mathrm{E}-01$ & $3.04700^{-}$ \\
\hline $22-10$ & $-22=-11$ & _23 & 23 & $23=-3$ \\
\hline $\begin{array}{l}1439 E 00 \\
23\end{array}$ & $\begin{array}{r}1.5672 \mathrm{E} 01 \\
23\end{array}$ & & & $\begin{array}{c}1.1923 E 01 \\
238\end{array}$ \\
\hline $3.5559 \mathrm{E} 01$ & $3.2840 \mathrm{E} 01$ & $1.8198 \mathrm{E} \quad 01$ & 1.6106001 & $1.6734 \mathrm{E} 00$ \\
\hline $.2 \frac{3}{2}-29 E^{-9}$ & $=23-10$ & $23-11$ & -24 & 24 \\
\hline $\begin{array}{l}2292 \mathrm{E} \\
24 \\
-\end{array}$ & $24 \quad 4$ & $\begin{array}{c}2.6897 E \text { OI } \\
24 \quad 5\end{array}$ & 0.24 & • \\
\hline $1.8507 E_{8}$ & $5.5196 \mathrm{E} \mathrm{OI}$ & $5.0775 \mathrm{E}$ & $2.8247 \mathrm{E} 01$ & $2.5000 \mathrm{E} \mathrm{Oi}$ \\
\hline $2.5974 E^{-2} 00$ & 824 & $-24-10$ & $--\frac{24}{17}-1 \frac{1}{0}$ & 25 \\
\hline 25 & 25 & 25 & 25 & \\
\hline & $2.9080 E 01$ & $8.6754 \mathrm{E} 01$ & $8.0120 \mathrm{E} \quad 01$ & $4.4398 \mathrm{E} 01$ \\
\hline$-25-7$ & --25 & 25 & $25-10$ & $-25--11$ \\
\hline $\begin{array}{c}3.9295 E, 01 \\
26\end{array}$ & $4.0826 E 00$ & $1.2758 E_{3} 01$ & $1.7351 E 01$ & $6.5621 E_{5} 01$ \\
\hline & & $2.9112 E 01$ & $8.6824 E 01$ & $8.0185 \mathrm{E} 01$ \\
\hline-26 & 26 & -26 & 26 & $26=10$ \\
\hline $\begin{array}{l}4.4434 E 01 \\
26\end{array}$ & $\begin{array}{c}3.932 .6 E \mathrm{E} 01 \\
27\end{array}$ & $\begin{array}{c}4.0858 E \text { OC } \\
27\end{array}$ & $\begin{array}{c}1.2768 E \text { O } 01 \\
27\end{array}$ & $\begin{array}{c}1.7365 E \text { O } 01 \\
27\end{array}$ \\
\hline $6.5674 \mathrm{E} 01$ & & & $2.9141 \mathrm{E} O 1$ & $8.6912 \mathrm{E} 01$ \\
\hline $8.0266 E^{-27} 01$ & $-4.4477 E O T$ & $-3.9 \frac{27}{366 E} 01$ & $4.0900 \mathrm{E} 00$ & $1.2 \frac{27}{278} \mathrm{IE}^{9} \mathrm{O}$ \\
\hline $1 . \frac{27}{7382 E}-10$ & $27-11$ & 28 & 28 & -28 \\
\hline $1.7382 \mathrm{E} 0$ & $6.5741 E 01$ & 0. & & $2.9180 \mathrm{E}$ \\
\hline
\end{tabular}


COBALI

SIGMA N,G (NITN2)

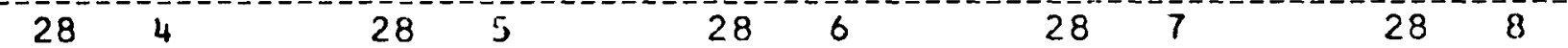

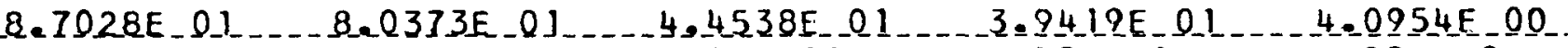

$\begin{array}{llllllll}28 & 9 & 28 & 10 & 28 & 11 & 29 & 1\end{array}$

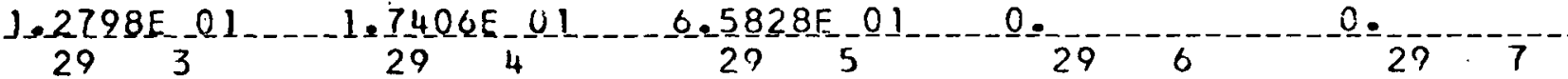

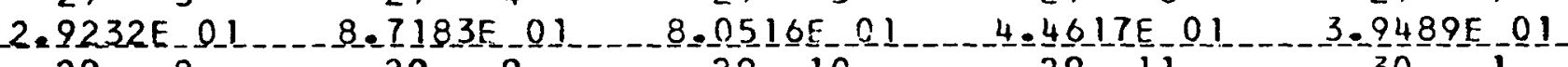

$29 \quad 8 \quad 29 \quad 99 \quad 10$

$4 \cdot \frac{1027 E}{30} 00$ $29 \quad 11$ 30

$6.5945 E$ E 01 $30-7-2=9305 E$

$3.9588 E_{-} 0.1$

$31 \quad 31 \quad 2 \quad 31$

Q. $310-3$

. 94 1 18E_ol

318

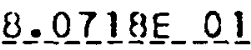

$4=\frac{4}{3} 729 \mathrm{E}-0.1$

$4.490 .1 E_{31} Q 1 \ldots 3.9740 E_{1} Q L_{1}-4.1288 E_{2}$

$6.6364 E_{2} 01$

325

320

$0 .-7$

$30 \quad 10$

$31.80 E_{-1} 01$

8.77372 O 01 _ $8.1028 E$ E 01

7373 - 310

$1.2902 E \quad 0$

$1.7547 \mathrm{E} \quad 01$

$32 \quad 10$

$.7662 E_{2} 0$

32. 11

$3340-335$

$8.9527 E-01$

$8.2681 \mathrm{E} 01$

339

$1.3166 E_{2}$ Q L L _..._Le?905E_QJ

343

$$
34
$$

4

$34.1088 E_{-1}$

$-9.27 .18 E^{4}$

$1.3635 E_{0} O$
33

0.

$33-6$

4 .5817E 01 33 i

6. $7718 E-01$

345

$8.5628 E_{0} 01$

$34 \quad 10$

$1.8544 \mathrm{E} 01$
32

\section{2}

$8.8312 E 01$ 32 음 $1.2 \frac{2}{3} 87 \mathrm{E}-\mathrm{O} 1$ $3.0018 E-01$ 0. 4.055 IE 01 $4 .-\frac{2}{34} 30 E-200$ (a) $0 .-34^{-1}$ $4=7450 E$ O I _ 4 . 1996E 01 $34 \quad 11$ $7.0132 \mathrm{E} \quad 01$ 
DATE $12 / 15 / 60$

QES_LGVAILLY LII I I U UM 6
CODE_NO.

DENSIIYY EACIOR

3.6000
$2 \cdot 9872 \underline{2}=0$
SIGMA XI SIGMA SIGMA SIGMA XII SIGMA SGAIJER _. _RRAOSP_QBI _ ABSORPIION _ SCAIIER_ 1..

LEKEL_ _ ENERSY _ _ SCAIJER

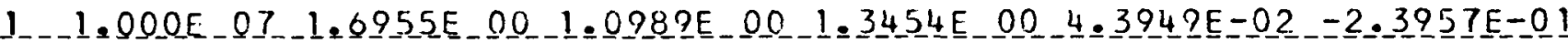

$26.065 \mathrm{E} 061.8615 \mathrm{E} 008.4400 \mathrm{~F}-011.4898 \mathrm{E} 006.8132 \mathrm{E}-02-2.5437 \mathrm{E}-01$

3 3.679E O6 1.8716E QC 5.4448E-01 1.5245E 00 1.2016E-01-2.3754E-01

4 2.231E तO 1.3196E DO 3.5150E-01 1.1516E 00 1.3982E-01-1.1491E-01

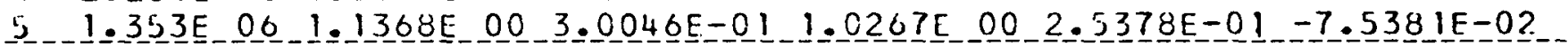

$6.8 .203 E-05-1.2757 E 0 C-3.3717 E-011.1521 E-00-3.2251 E-01-8.4592 E-02$

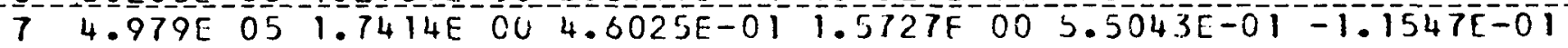

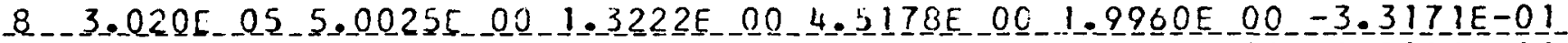

$91.832 \mathrm{E}$ o5 $2.4012 \mathrm{E}$ 00 $6.3464 \mathrm{E}-012.1635 \mathrm{E} 001.2158 \mathrm{E}$ 00 $-1.5921 \mathrm{E}-01$

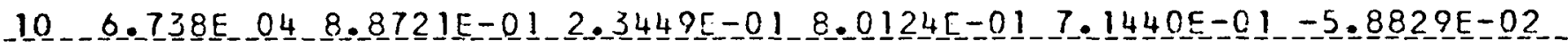

$11-2.479 E-04-1340 E-01-2.1498 E-01-7 \cdot 3458[-01-2.8158[-01-5.3934 E=02$

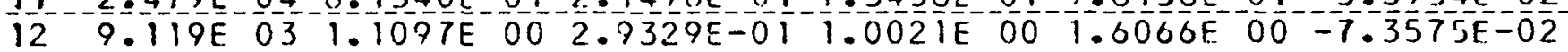

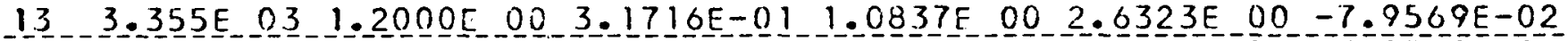

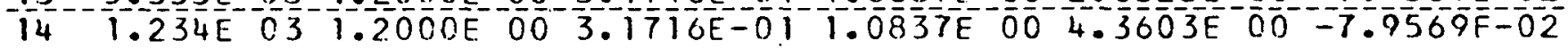

15 4.540E 02 1.2000E $003.1716[-01$ 1.083]E 00 7.8027E $00-7.9569 E-02$

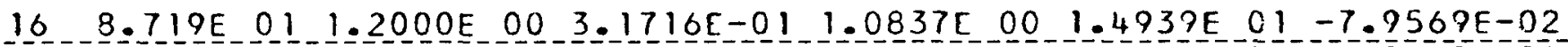

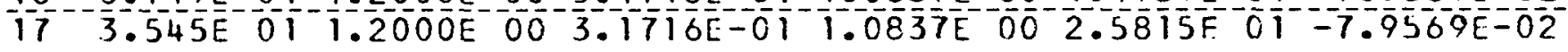

$18-1.304 E$ E 1 1.200OE $003.1716 \mathrm{E}-01$ 1.0837C $004.1773 \mathrm{~F} 01-7.9569 \mathrm{E}-02$

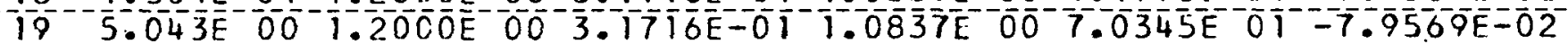

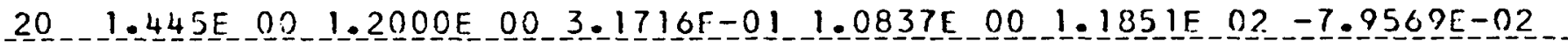

$21-6.826 E-011.2000 E-003.1716 E-011.0837 E-00-1=8891 E C 2-7.9569 E-02$

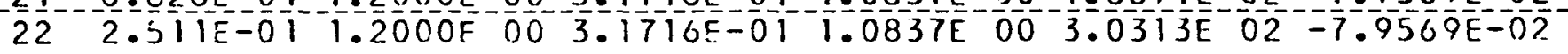

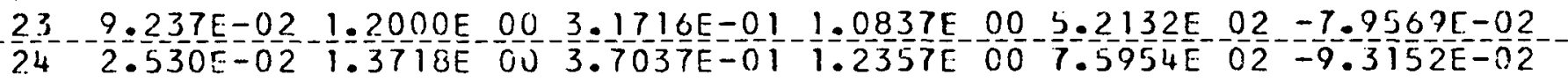

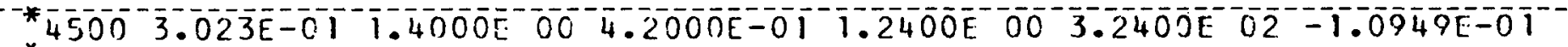

* $40002.718 E-011.4000 E$ 00 $4.2000 E-01$ 1.2400E $003.2400 E$ O2 - $1.0949 E-01$

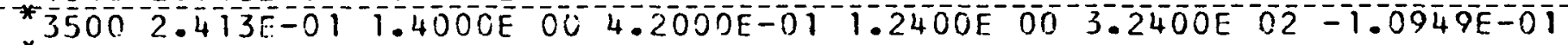

*3000 2.1C9E-01 1.4000E OU 4.2000E-01 $1.2400 E$ 0.0 3.2400E C2 $-1.0949 E-01$

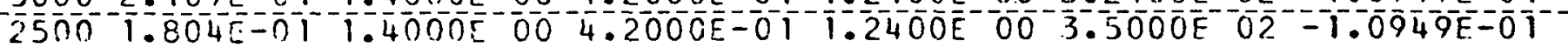

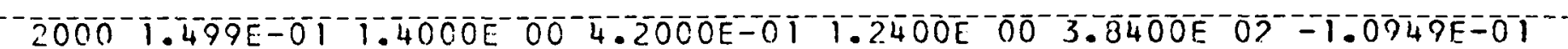

$15001.194 \mathrm{~F}-01$ 1.4000E $004.2000 \mathrm{E}-01$ 1.2400E $00.4 .3000 E-02-1.0949 E=01$

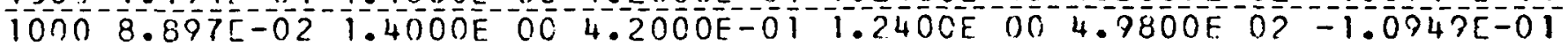

$5005.830 E-02 \quad 1.4000 E \quad 00 \quad 4.2000 E-01 \quad 1.2400 E-000.1400 E 02-1.0949 E-01$ $683.216 \mathrm{E}-02 \mathrm{1} .40 \mathrm{O} \mathrm{E}-0 \mathrm{C}$

*Thermal cross sections taken from APEX-515.

NOTE: For a summary of the sources of data see APEX-704. 
LITHIUM 6

IHERMAL IRANSEER MAIRI ICES

IEMPERAIURE

S I SMA

SIGMA

SISMA

NU SIGMA

DEG. $F$.

SCATTER

TRANSPORT

ABSORPTION

FISSION

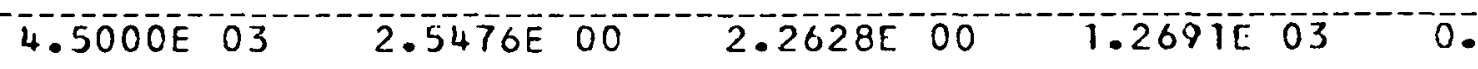

\section{$\mathrm{N} 1$ \\ SIGMA S (NNINZ)}

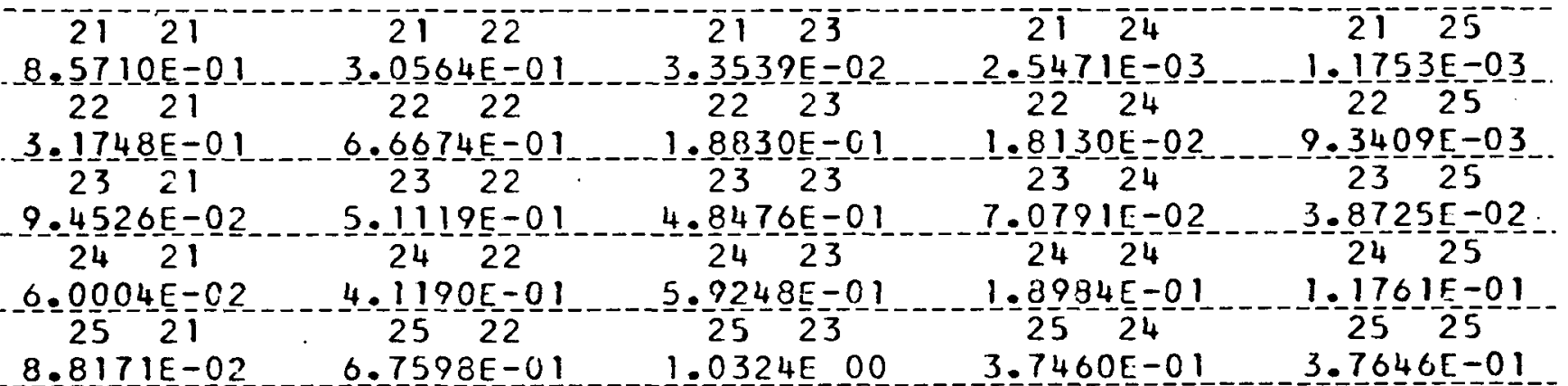

IEMPERAIURE

DEG. F.

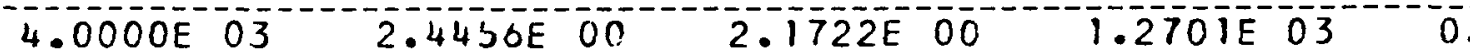

SI GMA

SCATTER
SIIGMA

TRANSPORT
SIGMA

A.BSORPTION
NU SICMA

FISSION

\section{SIGMA S (N) 1 N2)}

2121

- $3=\frac{39685-01}{22}=\frac{01}{21}$

$2 \cdot \frac{8}{2} 397 F-0 !$

- 7. $\frac{49}{24} 59 E=02$

$4.3636 E=02$

26 T

$5.9223 E-02$
$21^{22}$

$3=2337 \mathrm{E}-\mathrm{O} 1$

$6.9167 E-01$

$4.9778[-01$

2422

3. $8041 E-01$

$26-\frac{8}{2} 2$

$5.8602 E-01$
万人 25

$3 . \frac{3}{2} \frac{5}{2} 13 E-\frac{C 2}{2}$

$1.9598 \mathrm{E}-01$

5. $1201 E=01$ 24,23

6. $1559 \mathrm{E}-01$ $26-23$

1. CIIIE OC
2124

$2.3790 E-03$

$1.8210 E-02$

$7.48360=02$

$24-24$

$2.05383 E-01$

$26-\frac{1}{4}$

$3.87805-01$
0. 
LI THIUM 6

THERMAL TRANSFER MATRICES

TEMPERATURE
SIGMA

Ni

SI GMA_S__NI/N2)

\begin{tabular}{|c|c|c|c|c|}
\hline 21. & $21-.22$ & 23 & -21 & 21 \\
\hline $.1794 E-01$ & $3.4545 E-01$ & $3.3504 \mathrm{E}-02$ & $2.1887 E-03$ & $9.2130 \mathrm{E}-04$ \\
\hline 21 & 22 & 23 & -22 & 27 \\
\hline $2.4912 E-01$ & $7.1832 E-01$ & $2.0533 E-01$ & $1.8279 E-02$ & $8.9392 E-03$ \\
\hline 23 & 23 & 23 & 23 & 23 \\
\hline $5.6303 E-02$ & $4.7894 E-01$ & $5.4299 E-01$ & $7.9516 E-02$ & $4.2249 E-02$ \\
\hline 24 & -22 & 24 & $\ldots 24$ & 24 \\
\hline $2.9478 \mathrm{E}-02$ & $3.4239 \mathrm{E}-01$ & $6.3868 E-01$ & $2.2380 \mathrm{E}-01$ & $3 E-01$ \\
\hline $3.6287 \mathrm{E}-02$ & $\begin{array}{l}-27-22 \\
4.89935-01\end{array}$ & $9.927-23$ & $\frac{27}{4.0223 E-01}$ & $-4.1761 E-01$ \\
\hline
\end{tabular}

TEMPERATURE

. DEg. . . F.

3.QQ00E $03 \ldots 2.2273 E_{2}$
SIGMA

SCATIEB
SIGMA
IRANSPQRI

L.9783E 00
SIGMA

ABSQRPIILN
NU SIGMA

EISSION

$\mathrm{Ni}$ N2

SI GMA S $(N 1 / N 2)$ $1.2731 E$. 03

\begin{tabular}{|c|c|c|c|c|}
\hline 21 & 21 & 21.23 & 21 & \\
\hline $9018 E-01$ & $3.7357 \mathrm{E}-01$ & $3.3502 \mathrm{E}-02$ & $1.9706 E-03$ & $7.7583 \mathrm{E}-04$ \\
\hline $22-21$ & 22 & $22=23$ & $-22-24$ & -22 \\
\hline $\begin{array}{l}0981 \mathrm{lE}-\mathrm{C} \\
23.21\end{array}$ & $\begin{array}{c}7.4608 E-01 \\
2322\end{array}$ & 2323 & $\begin{array}{l}8344 E-02 \\
23 \quad 24\end{array}$ & $\begin{array}{l}6397 E-03 \\
23 \quad 28\end{array}$ \\
\hline $3.9202 E-02$ & $4.5298 E-01$ & $5.7853 E-0.1$ & $8.5033 E-02$ & $.4250 E-02$ \\
\hline 24 & 24 & 24 & 24 & \\
\hline $\begin{array}{c}1.7860 \mathrm{E}-02 \\
28 \quad 21\end{array}$ & $\begin{array}{l}2.9719 E-C 1 \\
2822\end{array}$ & $\begin{array}{l}E-01 \\
23\end{array}$ & $\begin{array}{c}-4600 E-0 \\
28 \quad 24\end{array}$ & $\begin{array}{l}5023 E-0 \\
28 \quad 28\end{array}$ \\
\hline $9546 \mathrm{E}-0$ & & $\therefore 2$ & $1794 \mathrm{E}-$ & -0 \\
\hline
\end{tabular}


LITHIUM 6

ILERMAL_JRANSFER_MAIRICES.

IEUPERATURE

SIGMA

SIGMA

SIGMA

NU SIGMA.

DEG. $F$.

SCATTER

TRANSPORT

ABSORPIION

FISSION

$2.5000 E 03$

2. $1098 \mathrm{E} 00$

$1.8739 E$ OO

$1.2753 \mathrm{E} 03$

0 .

$N 1$

SIGMA S (NIIN2)

2121

7. $53866=01$

2122

4. $1033 E=01$

$2 \overline{1} 2 \overline{3}$

$3.3470 \mathrm{E}=02$

2124

$2 \overline{1} 2 \overline{9}$

$22 \quad 21$

$22 \quad 22$

2223

$1.7168 \mathrm{E}=03$ 6. $1768 E-04$

- 1.67_LOE=01

$7.7352 \mathrm{E}=01$

$2.3271 \mathrm{E}=01$

2322

2323

$22 \quad 24$

$22 \quad 29$

2321

4. $1774 E-01$

$6=\frac{1}{24} \frac{969}{2}=01$

1. 84 15E $=02$

8. $-2505 E-03$

2421

$24 \quad 22$

9. 172 IE -01

$4.6436 E-02$

9. 2354 E $=02$

2. -44626 E -01

$6.7901 \mathrm{E}-01$

$2.7330 \mathrm{E}-01$

2429

2921

2.8767E-O 1

$9.0247 \mathrm{E}-01$

$4.3484 E=01$

1. $\frac{6}{29} \frac{56 E-01}{29}$

-8.7114E-0

2. $8767 \mathrm{E}-\mathrm{O}$

9.0247E 01

4. $7614 E-0 !$

TEMPERA IURE DEG. F.
SIGMA

SCATTER
SIGMA

TRANSPORT
SIGMA

ABSORPTION
NU SIGMA

FISSION

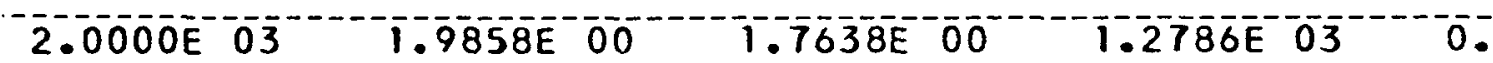

\section{SIGMA}

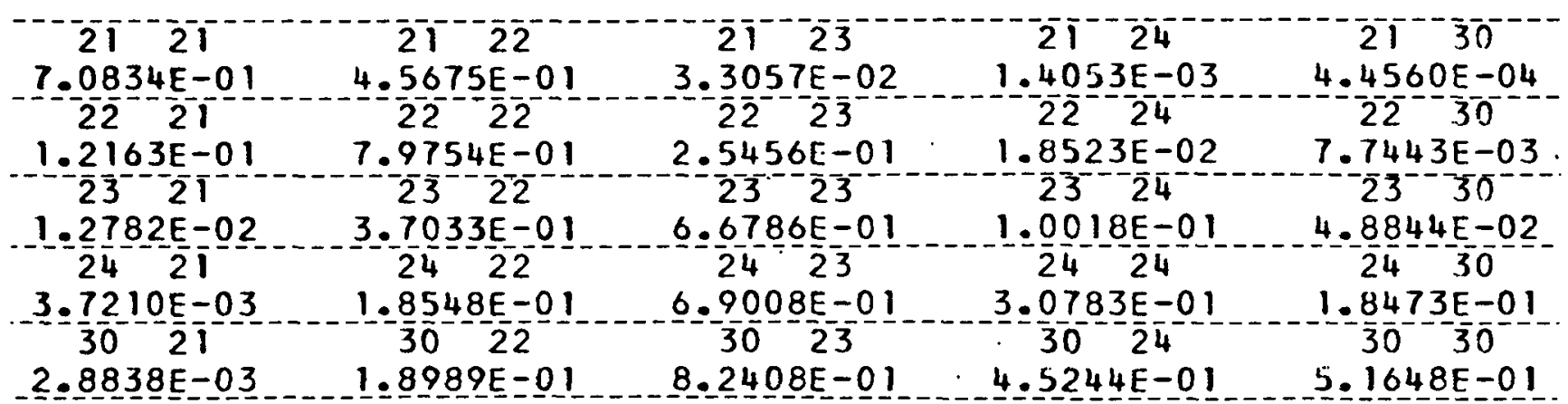


LI JHIUM 6

THERMAL TRANSFER MATRICES

TEMPERATURE
SIGMA

\section{$N 1$
SI
GMA S $(N 1 / N 2)$}

$21+21$

$6.9284 E-01$

22

$7.5339 E-02$

$-\ldots 23-21$

$4.9603 E-03$

- 24

$9.7474 \mathrm{E}-04$

$31-21$

$5.7284 E-04$
$421-22$

4.7698E-01

222

8. MIME-Ci $23-22$

$3.0713 \mathrm{E}-0$ $24-22$

$1.2257 \mathrm{E}-0$ $31-22$ $1.0390 \mathrm{E}-01$
$-21-23$

$2.9003 \mathrm{E}-02$

$2.8776 \mathrm{E}-01$

$23-23$

$7.2465 \mathrm{E}-01$ $24-23$

$6.8613 E-01$

-31 23
$-21-24$

$9.3168 E-04$

$1.8715 \mathrm{E}-02$

$23-24$

$1.1168 \mathrm{E}-01$

$24-24$

$3.5312 \mathrm{E}-01$

31
$2.4453 \mathrm{E}-04$

$22-31$

$7.0732 E-03$

5. $-2375 \frac{31}{5-02}$

$24 \quad 31$

$2.0903 \mathrm{E}-0 \mathrm{i}$

$5.6 \frac{3}{6}-\frac{3}{2}-1$

SIGMPERATURE
SIGATER

ii

SI GMA S (NIIN2)

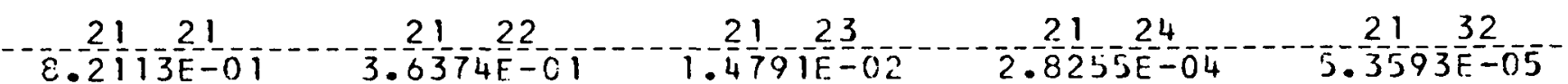

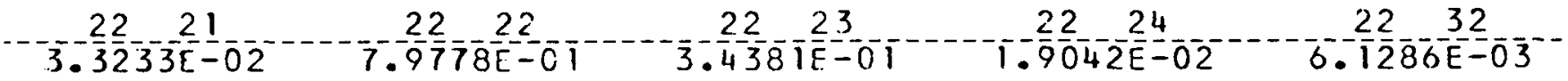

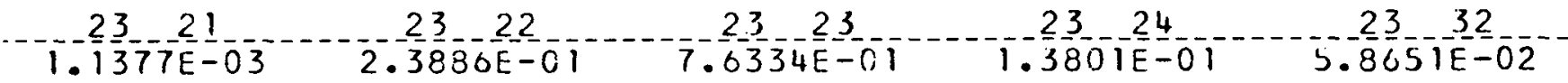
2421

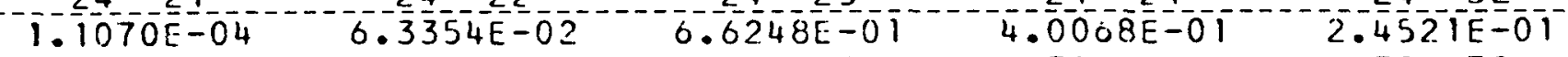
$32-21$

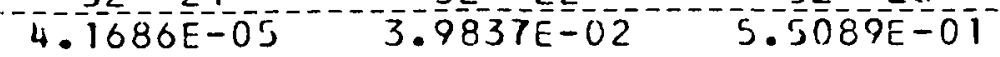
$4.7979 \frac{24}{3}-01$ $6 .-\frac{32}{4} 263 \frac{32}{3}-01-$ 


\section{LITHIUM 6}

_IHERMAL_IRANSFER_MAIRICES

-IEMPERATURE

DEG • $F$.
SIGMA

SCATTER
SIGMA TRANSPORT
SISMA ABSORPIION
NU SIGMA . .

\section{FISSION}

T.5619E OO

\section{$1 . \overline{3} \overline{7} 3 \mathrm{E} 0 \mathrm{O}$}

\section{$1.3096 \mathrm{E} 03$}

\section{0}

\section{SIGMA S $\frac{N 1}{N(N)}-\frac{N 2}{2}$}

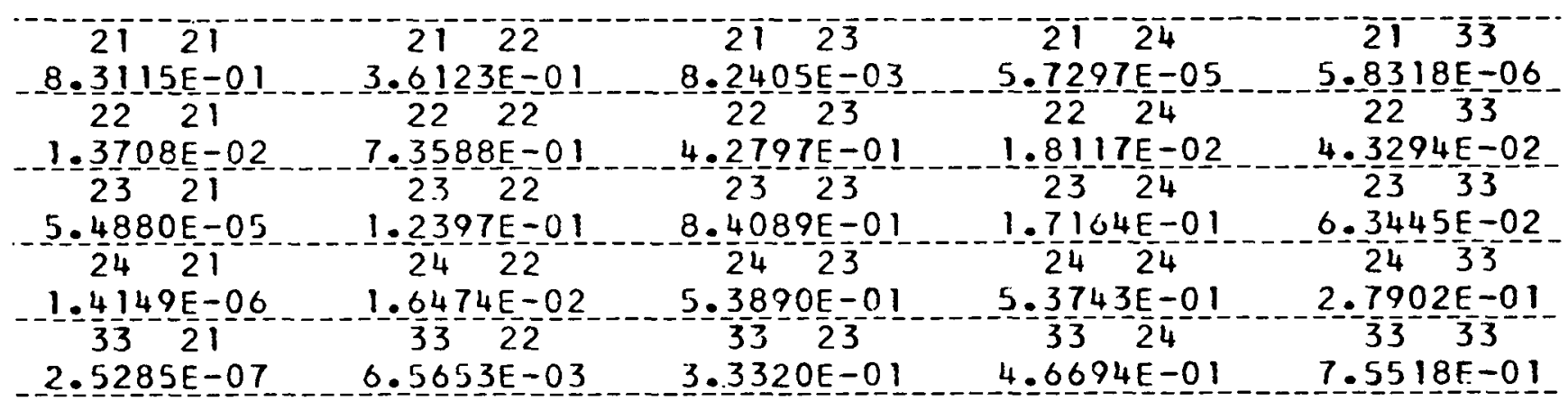

TEMPERAIURE
DEG. FIGMA
$6.8000 \mathrm{E} O \mathrm{O}$
$1.4225 \mathrm{E}^{-100}$
$1.263 \overline{5 E} 0 \bar{O}$
$1.356 \overline{3} \overline{\mathrm{E}} 0 \overline{3}$

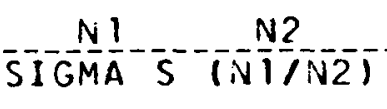

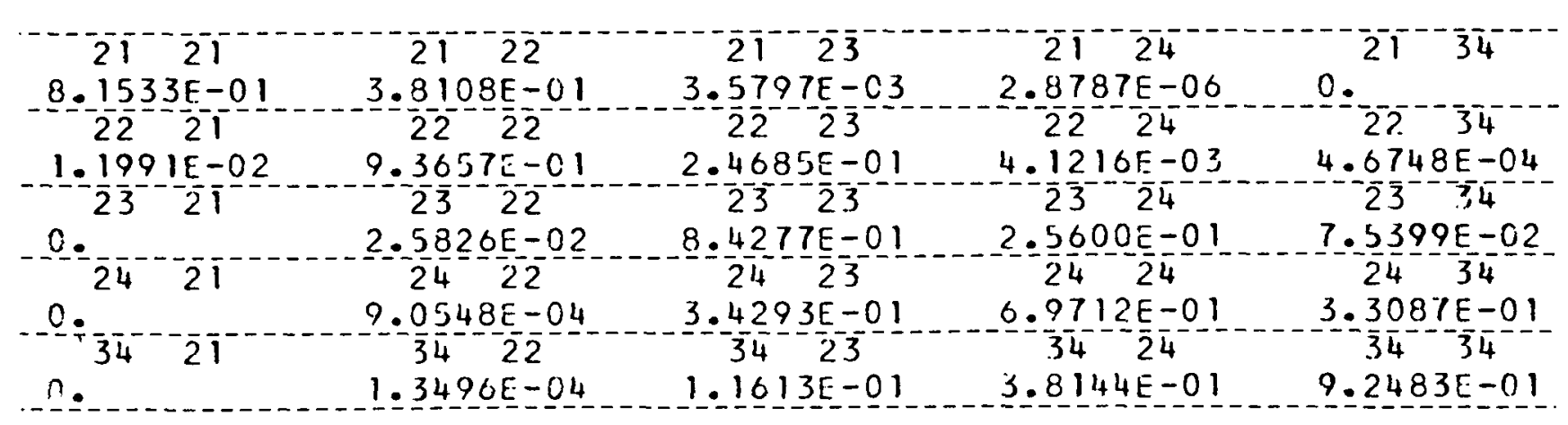


LITHIUM 6

TRANSFERS FROM GROUPS I THROUGH 4 TO GROUPB 1 THROUGH 13

$\mathrm{N} 1$ N2

SIGMA_ SO_ (NIIN2)

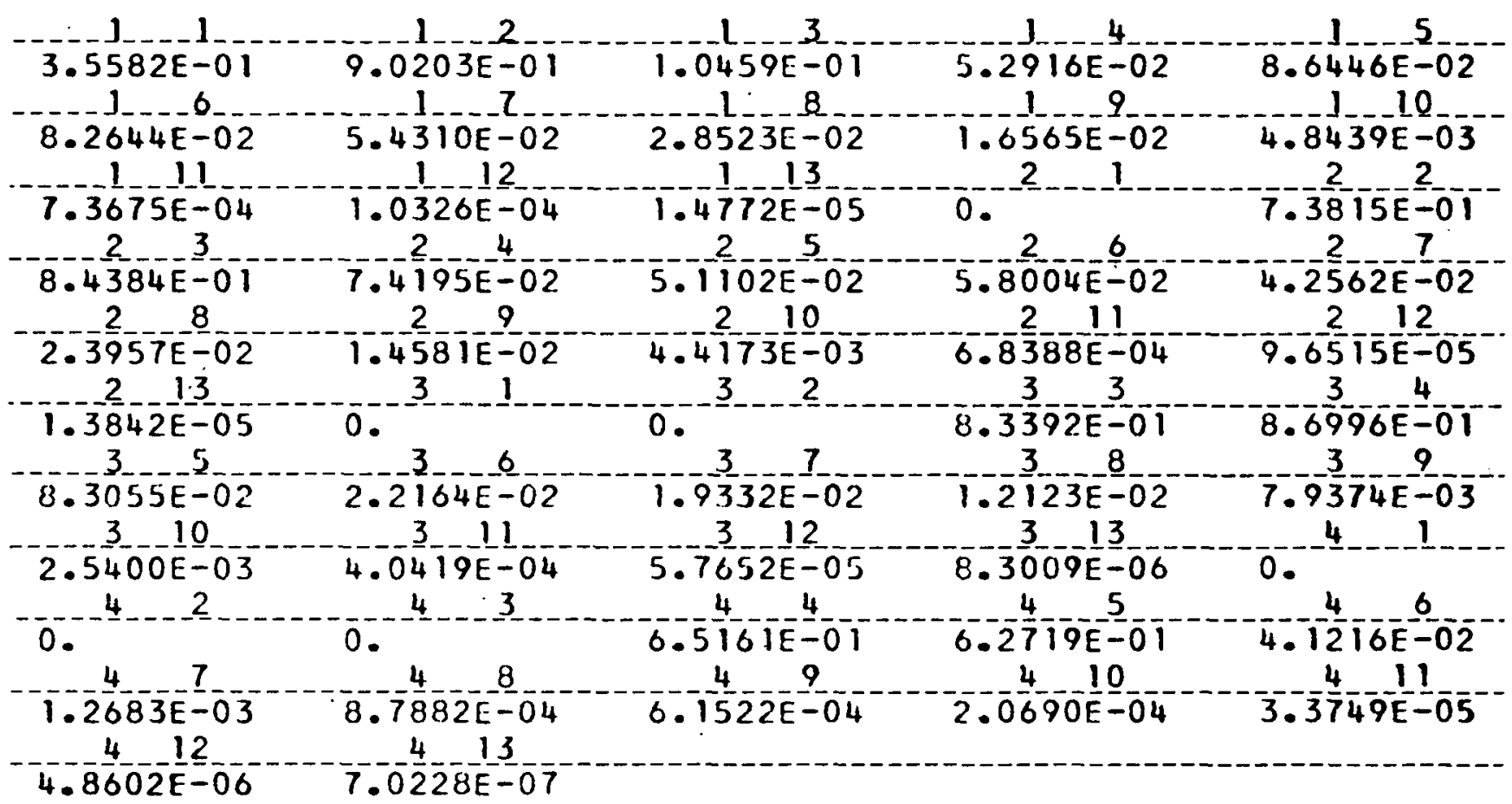


LIIHIUM 6

TRANSFERS FROM GROUPS 1 THROUGH 34 TO GROUPS 1 THROUGH 21

$\mathrm{N} 1$

SIGMA N,G (NITNZ)

Q

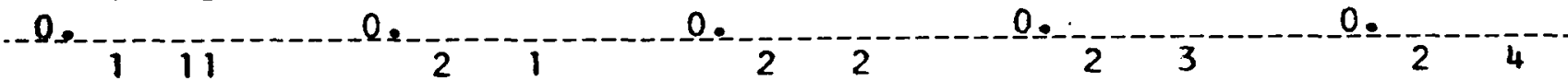

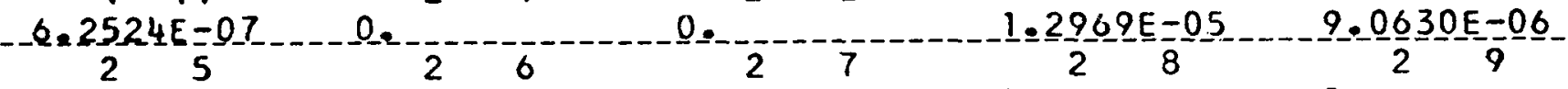

-0.

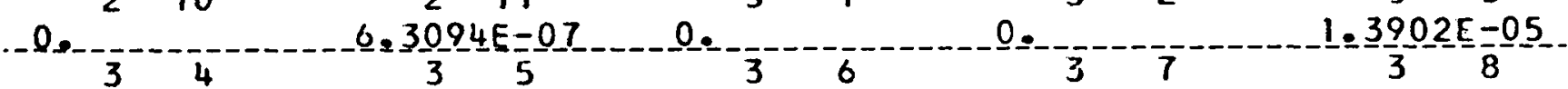

-2.7] $48 E=06$

390

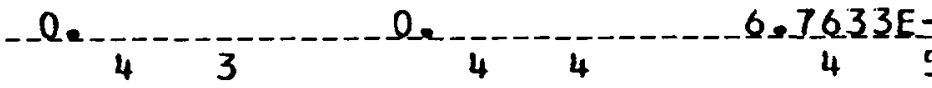

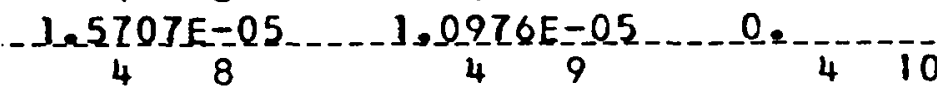

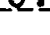

4

0

Q.

Q.

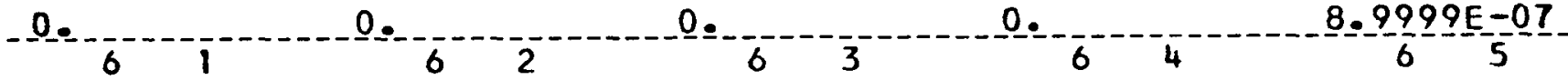

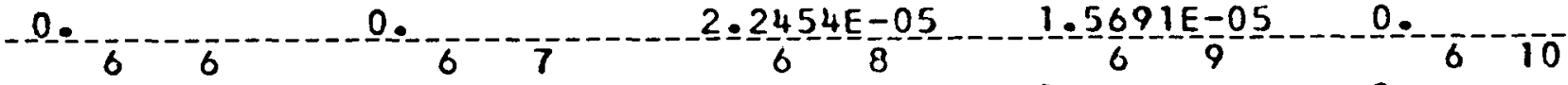

Q.

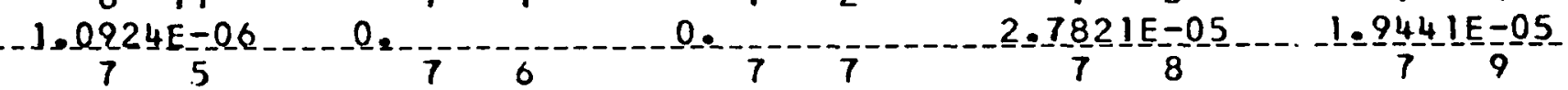

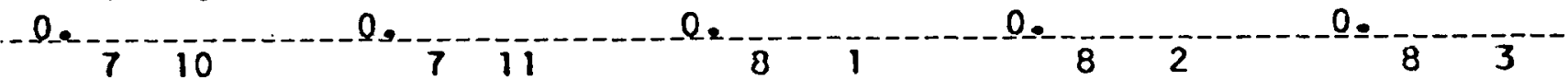

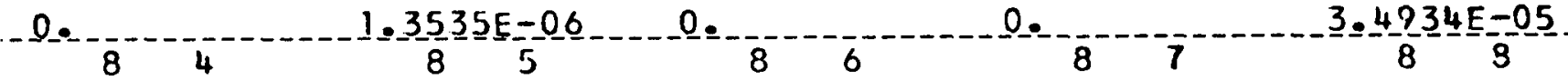

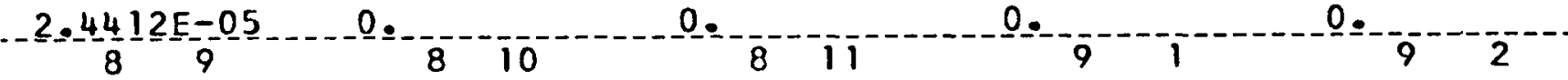

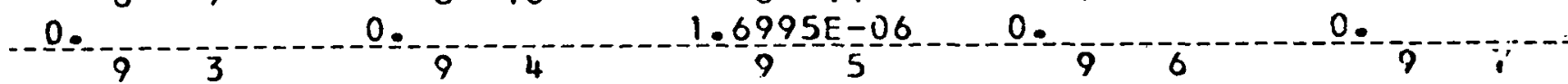

. $4.7134 \mathrm{E}-05$

...2.

0.

$2.2930 E=06$

0. 


\section{LITHIUM 6}

N1 N2

SIGMA_N,G_IN1/N2)

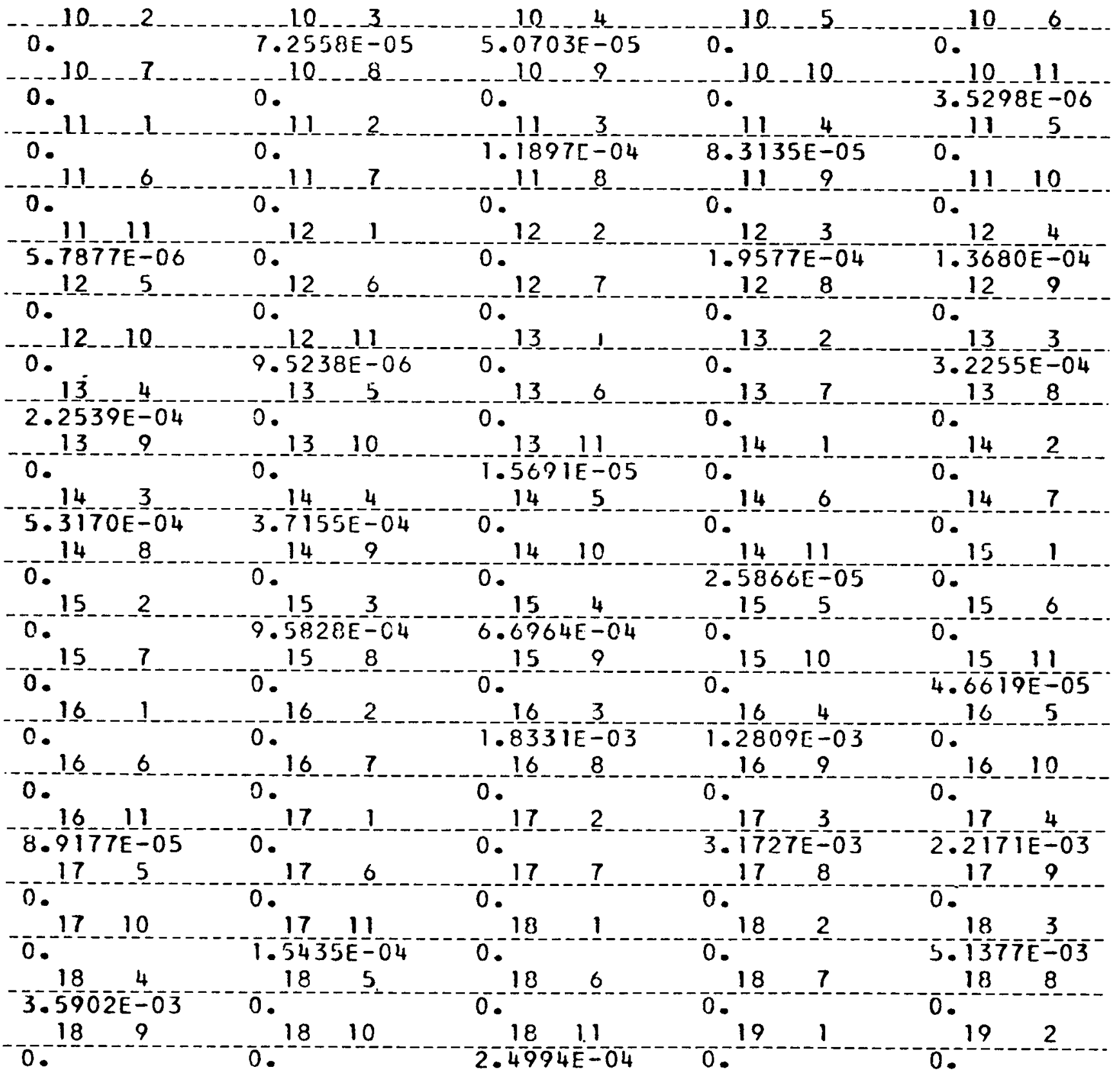


_-LIJHIUM_b.

\section{SIGMA N,G (NINN2)}

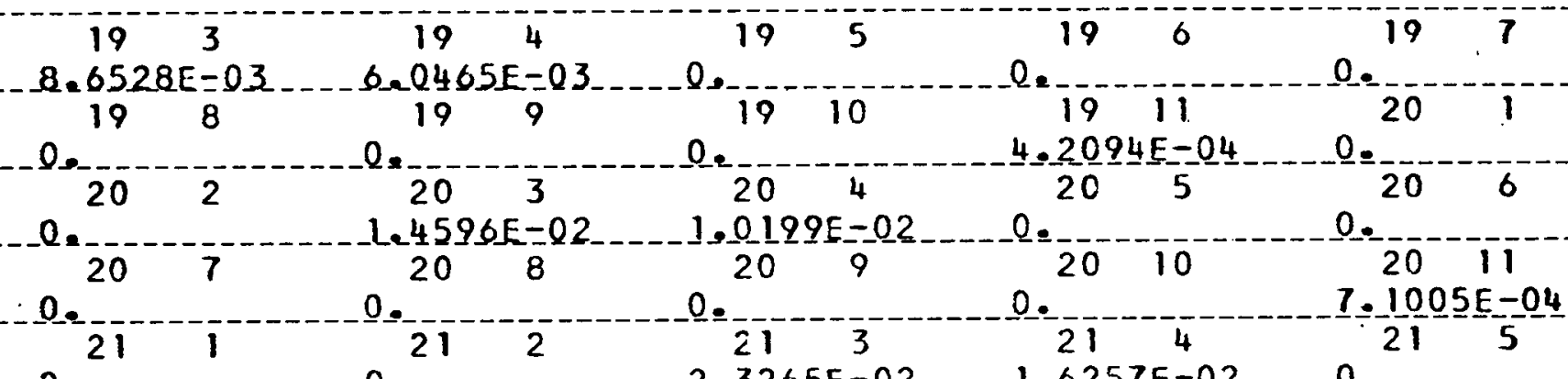

. 0 . 21

-. l.

$21-2.3265 E$

211

0.

0 .

$\frac{62}{21}=7 E=0$

0.

. $1318 E=03$

225

$22^{1}$

$22^{2}$

Q.

0 .

$3 . \frac{72}{2} 207 E=02$

224

-.0.

$22^{10}$

22

22

2. $-\frac{6}{22} 042 E-02$

...

22

$23^{0}$

$0 .-$

$23^{2}-\frac{0}{2} 3^{-}$

23

$1.8 \frac{1}{2} 30 E-03$

0.-

0

$6.3951 E-02$

$4=\frac{4}{2} 389 \mathrm{E}-02$

- 0.

24

0.3

$-0$.

ㅇ..

$24^{-\cdots}$

0 .

8

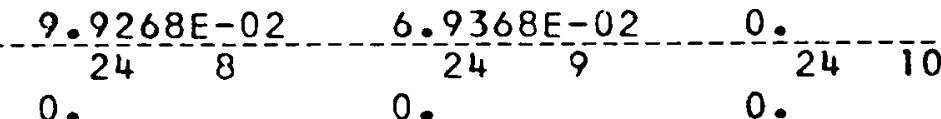

0.25

25

25

4

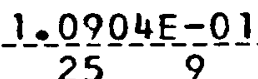

$\frac{0}{24}-\cdots$

$0 . \overline{2}^{-1}-1 \overline{1}$

$24^{-1-1-1}$

$25-\frac{0}{7}-\frac{5604 E}{8}$

$26-0.00 .25$

$0.000-26$

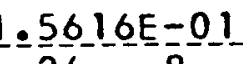

$4=8293 \mathrm{E}=03$

25

0.

2611

$7.5972 E-03 \quad 0.27$

-

$27^{-10}$

0.79

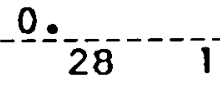

25

.

7.6053E-03

0.

$25-10$

-

11

0.

$26^{-1}$

$7 . \frac{59}{26} 11 \mathrm{E}-\frac{03}{5}$

$1.09 \frac{1}{26} 3 \mathrm{E}-\frac{0}{9}$

$26-10^{-\cdots}$

$0.7^{0}-7^{-} 7^{-1}$

$1.5633 E-01$

$0 . \frac{}{2} 8$

0 $2 \overline{8}^{-\cdots}$ 


\section{LITHIUM 6}

$N 1$
SIGMA N,

$1.0939 E-01$
0.28


DATE 12/15/60.

\section{DESIGNATION}

LับัHIUM
CODE NO.

$3 . \overline{7000}$

\section{DENSITY FACTOR}

\section{$1.15192 \mathrm{E} O \mathrm{I}$}

LEVEL ENERGY
SIGMA

SCATTER

XI SIGMA. SCATTER
SI GMA TRANSPORT
SIGMA ABSORPTION
XI 1 SIGMA SCATTER I

1 1.000E $071.8265 \mathrm{E}$ 00 $9.4559 \mathrm{E}-0 \mathrm{1} 1.4173 \mathrm{E} 00-3.8110 \mathrm{E}-03-2.9642 \mathrm{E}-01$

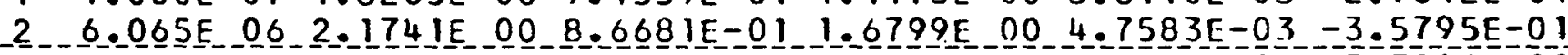

$3.679 \mathrm{E} 062.2447 \mathrm{E} 005.5596 \mathrm{E}-011.7780 \mathrm{E} 00 \mathrm{9}-0640 \mathrm{E}-03-3.3801 \mathrm{E}-01$

$4-2.23 \mathrm{IE} 06-1.7834 \mathrm{E}-005.0030 \mathrm{E}-011.5507 \mathrm{E} 001.4685 \mathrm{E}-02-1.6852 \mathrm{E}-01$

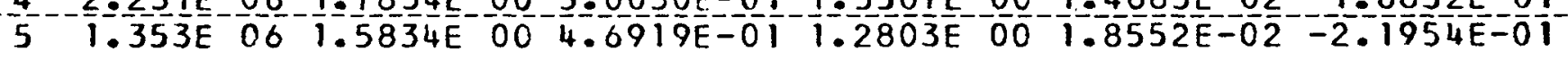

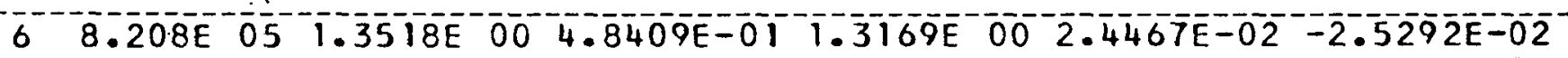

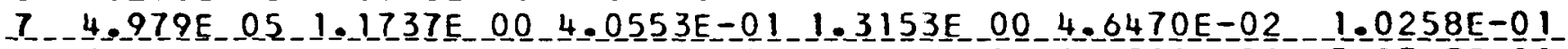

$83.020 \mathrm{E}$ O5 4.3260E $001.1434 \mathrm{E}$ 00 3.9068E $001.5319 \mathrm{E}-01-3.0363 \mathrm{E}-01$

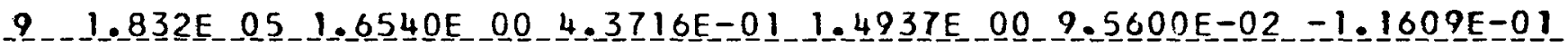

$106.738 \mathrm{E} 049.4660 \mathrm{E}-012.5019 \mathrm{E}-018.5487 \mathrm{E}-015.3533 \mathrm{E}-02-6.6435 \mathrm{E}-02$

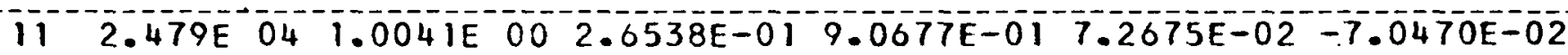

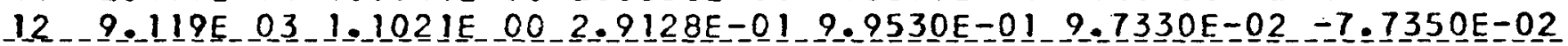

$133.355 \mathrm{E} 031.1200 \mathrm{E}$ 00 2.9602E-01 1.0115E.00 $9.7258 \mathrm{E}-02-7.8605 \mathrm{E}-02$

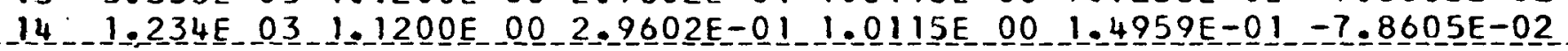

$15 \quad 4.540 \mathrm{E} 021.1200 \mathrm{E} 00-2.9602 \mathrm{E}-011.0115 \mathrm{E}-00-3.3426 \mathrm{E}-01-7.8605 \mathrm{E}-02$

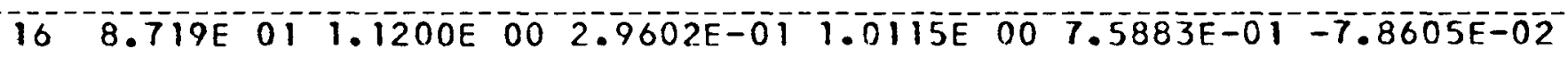

$173.545 E-011.1200 E-002.9602 E-011.0115 E 00 \quad 1.4764 E 00=7.8605 E=02$

$181.304 \mathrm{E} 011.1200 \mathrm{E} 002.9602 \mathrm{E}-0 \mathrm{1} 1.0115 \mathrm{E} 00-2.6447 \mathrm{E} 00-7.8605 \mathrm{E}=02$

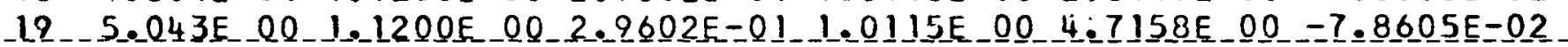

$20 \quad 1.445 \mathrm{E} \quad 001.1200 \mathrm{E} \quad 002.9602 \mathrm{E}-011.0115 \mathrm{E} 00 \quad 8.5617 \mathrm{E} 00-7.8605 \mathrm{E}-02$

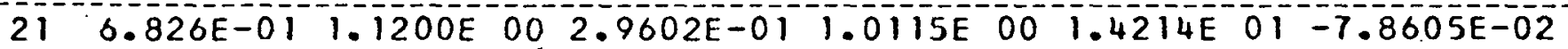

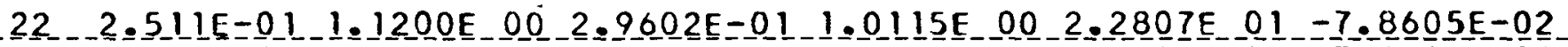

$23-9.237 \mathrm{E}-02 \quad 1.1200 \mathrm{E}$ 00 $2.9602 \mathrm{E}-011.0115 \mathrm{E} 003.9223 \mathrm{E}$ O $1-7.8605 \mathrm{E}-02$

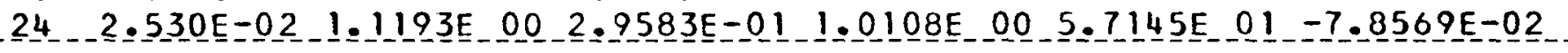

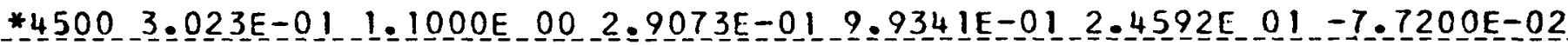

* $40002.718 \mathrm{E}-0 \mathrm{1} 1.1000 \mathrm{E} 002.9703 \mathrm{E}-019.9341 \mathrm{E}-012.4592 \mathrm{E} 01-7.7200 \mathrm{E}-0 \mathrm{2}$

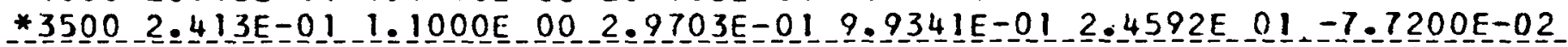

* $30002.109 \mathrm{E}-0 \mathrm{1} 1.1000 \mathrm{E} 002.9703 \mathrm{E}-019.9341 \mathrm{E}-012.4592 \mathrm{E} 01-7.7200 \mathrm{E}-02$

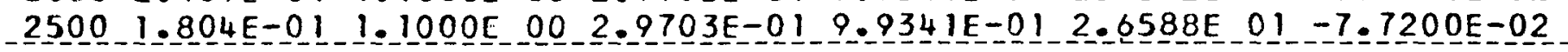

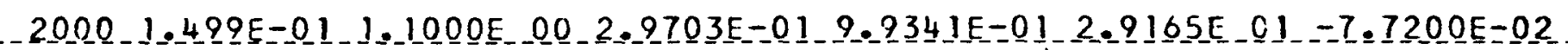

$15001.194 \mathrm{E}-0 \mathrm{I} 1.1000 \mathrm{E}$ 00 $2.9703 \mathrm{E}-019.9341 \mathrm{E}-0 \mathrm{i} 3.2676 \mathrm{E}$ 01 $-7.7200 \mathrm{E}-02$

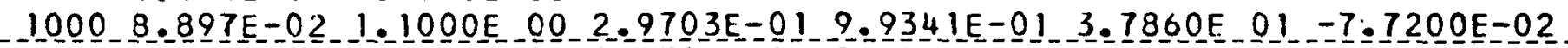

$5005.850 E-021.1000 E-002.9703 E-0179341 E-014.6692 E-01-7.7200 E-02$ 68 3.216E-02 1.1000E 00 2.9703E-01 ?.?341E-01 6.2968E 00 -7.7200E-02

*Thermal cross sections taken from APEX-515.

NOTE: For_a summary of the sources of data see APEX-704. 
LIJHIUM

THERMAL TRANSFER MATRICES

TEMPERATURE
SIGMA

\begin{tabular}{|c|c|c|c|c|}
\hline 2. & 22 & 23 & $2 \underline{4}$ & $\leq 1$ \\
\hline $8.1800 E-0$ & $2.7373 E-01$ & $\begin{array}{c}2.5797 E-02 \\
2 ?\end{array}$ & $1.7260 E-03$ & $+411 E-04$ \\
\hline .8 & $6.4310 E-01$ & $1.7000 E-01$ & $1.5178 E-02$ & $7.5040 E-03$ \\
\hline 2 & 22 & $-\underline{=}$ & 2 & \\
\hline-2 & 22 & 2 & 24 & 2 \\
\hline $4 E-02$ & $35 E-01$ & $5.0097 \mathrm{E}-01$ & $1.6598 \mathrm{E}-01$ & $1.0152 E-01$ \\
\hline$\overline{02}$ & $5 \bar{E}-0$ & $3451 \mathrm{E}-0$ & $15 \bar{E}=\overline{0}$ & $3.6098 E-0$ \\
\hline
\end{tabular}

TEMPERATURE
SI IGMA

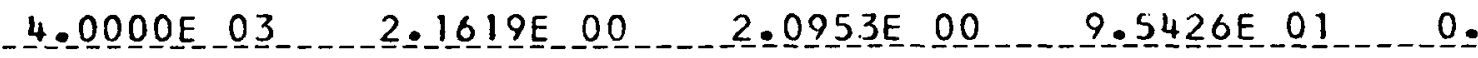

N

SIGMA S (N1/N2)

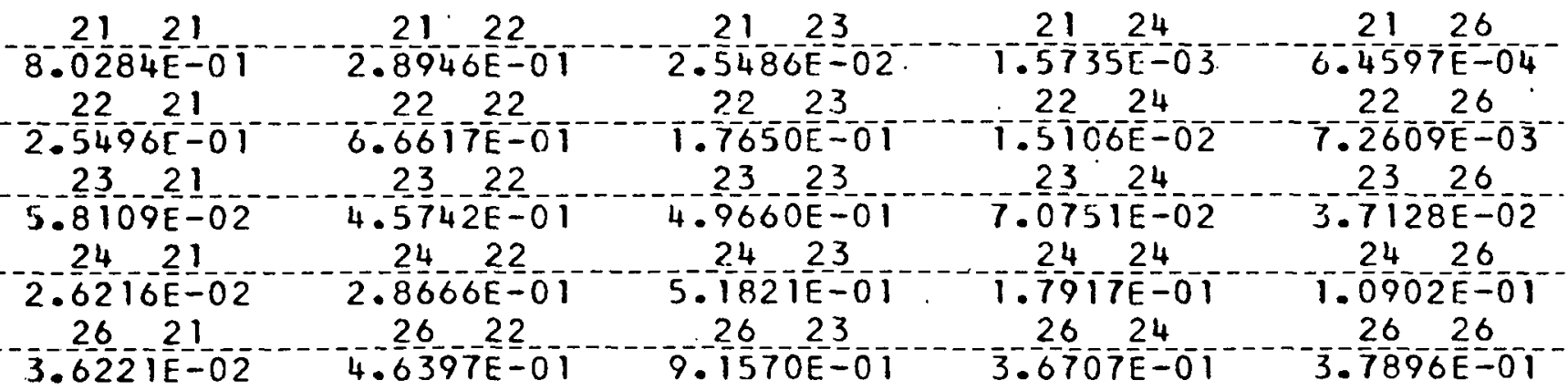


LI THIUM

IHERMAL_IRANSFER_MAIRICES

_LEMPERAIURE

SIGMA

SIGMA

SCATTER

TRANSPORT

SILMA

ABSORPTION

NU SIGMA

DEG. F.

$2.0709 \mathrm{E}^{-} \mathrm{OO}$

$2.0071 E$ CO

$9.552 \overline{4}$ O 1

0 .

\section{$S I G M \frac{N}{A}-\bar{S}\left(N \frac{N}{2}-N 2\right)$}

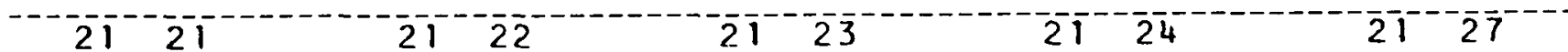

$7 .-\frac{8}{2} 378 \mathrm{E}=01$

$2=\frac{2}{2} \frac{28}{2}-\frac{E}{2}=01$

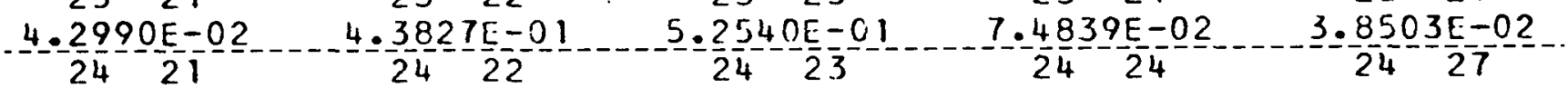

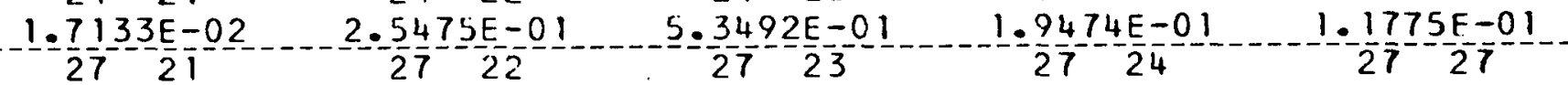

$2.1324 E-02 \quad 3.8149 E-01 \quad 8.8820 E-01 \quad 3.7998 E-01 \quad 3.9992 E-01$

IEMPERAIURE DEG. F.
SIGMA

SIGMA SCATTER TRANSPORT
SI GMA ABSORPTION
INU SIGMA

FISSION

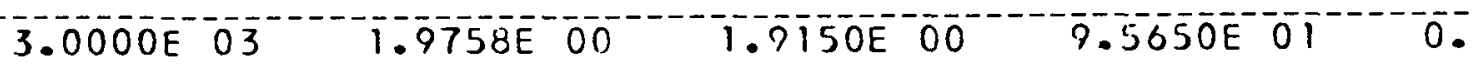

\section{$N 1$ \\ $\mathrm{N} 2$ \\ SIGMA S (NITNZ)}

$21-11$

$7.59260-01$

1. $-\frac{8}{2} \frac{7}{3} 65 \mathrm{E}-01$

2.9396E-02

$242 i$

$9.9819 E=02$

$282 i$

1.0930E-02
$2 \overline{1}^{-1} \overline{3}$

$2.4742 E-02$

$22-\frac{34}{2} 2$

7. $1631 \mathrm{E}-01$

$2 \frac{1}{2} 2$

4. $1271 E-01$

2422

2. $1787 E-01$

$2.9729 E-01$
$1.9457 E-01$

$23-23$

$5.58325-01$

2423

5. $5000 E-01$

$.4893 E-01$
$2 \overline{1}$

$1.2 \frac{2}{2} \frac{13 E}{2}-03$

1. $4878 \mathrm{E}-02$

$\frac{1}{3} \frac{8}{2} \frac{1}{4}$

$7.9629 \mathrm{E}=02$

2424

- 134 OE -01

$28-24$

$3.9387 E-01$
2128 4. $3516 \mathrm{E}-04$ 22 28 $6.5989 E-03$ $2 \overline{3}^{2} \overline{8}$ $.5512 E-02$ 2428 $1 \cdot 2802 E-01$ $4.2482 E-01$ 
LIIHIUM

THE

TEMPERATURE
DEEG. SIGMA

$\bar{n} \overline{1} \bar{n} \overline{2}$

SIGMA S $($ N 1 IN2)

$-21-21-21-22-21-23-21-29$

$\begin{array}{lllll}7.2695 \mathrm{E}-01 & 3.6747 \mathrm{E}-\mathrm{C} 1 & 2.4231 \mathrm{E}-02 & 1.0156 \mathrm{E}-03 & 3.2527 \mathrm{E}-04\end{array}$

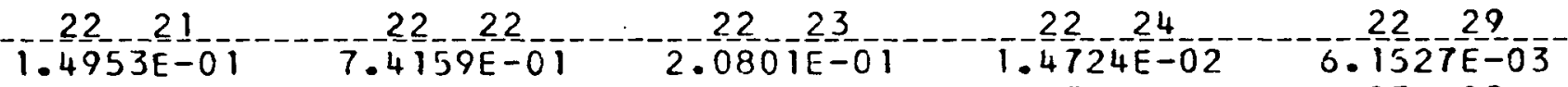

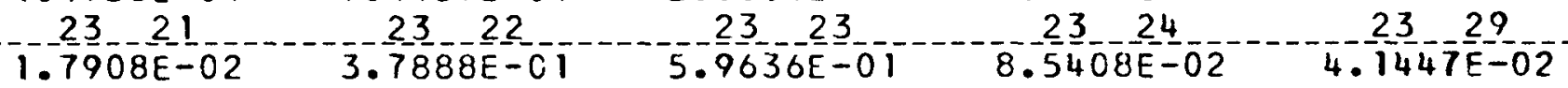

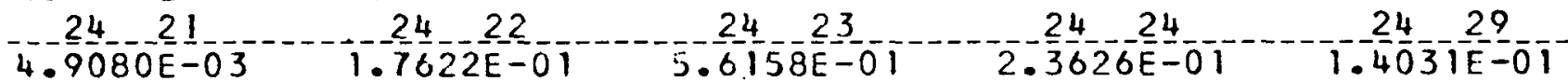

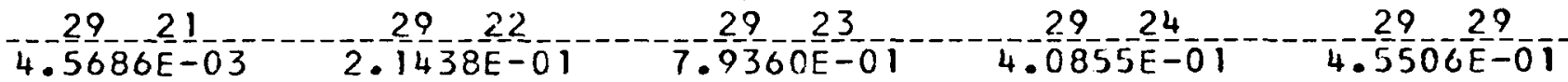

TEMPERATURE
SICMA

N1 12

SIGMA S (N1/N2)

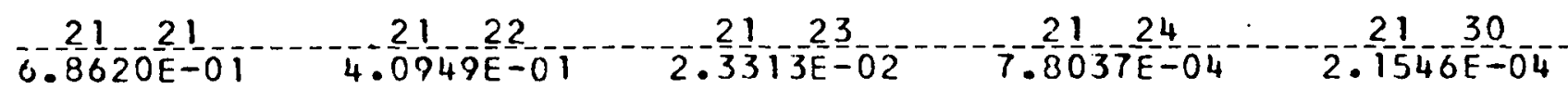

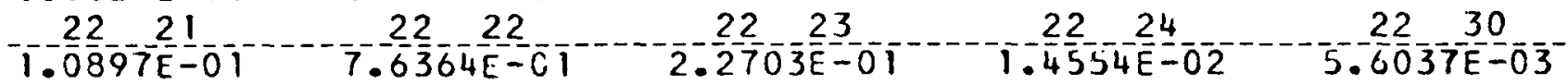

$9.1053 E-03-3.3441 E-010.4077 E-0109.2705 E-02-4.3002 E-02$

- 2421

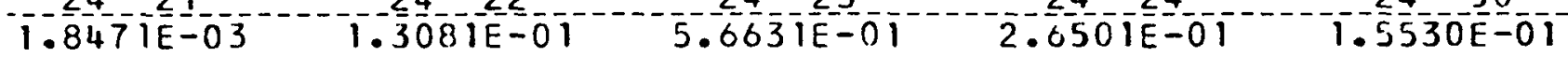

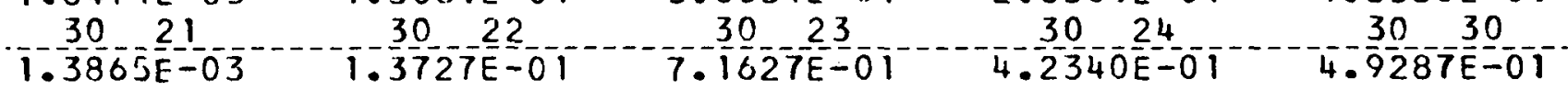


LI THI UM

IHERMAL_IRANSFER_MAIRICES.

IEMPERAIURE

SIGMA

SIGMA

DEG • $F$.

SCATTER

TRANSPORT

NU SIIGMA

SIGMA

FISSION

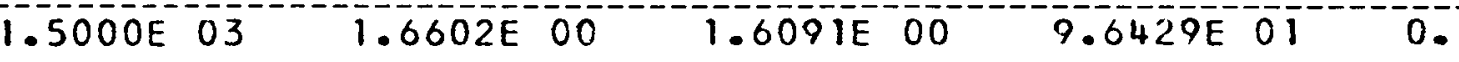

N

N2

SIGMA S (NIIN2)

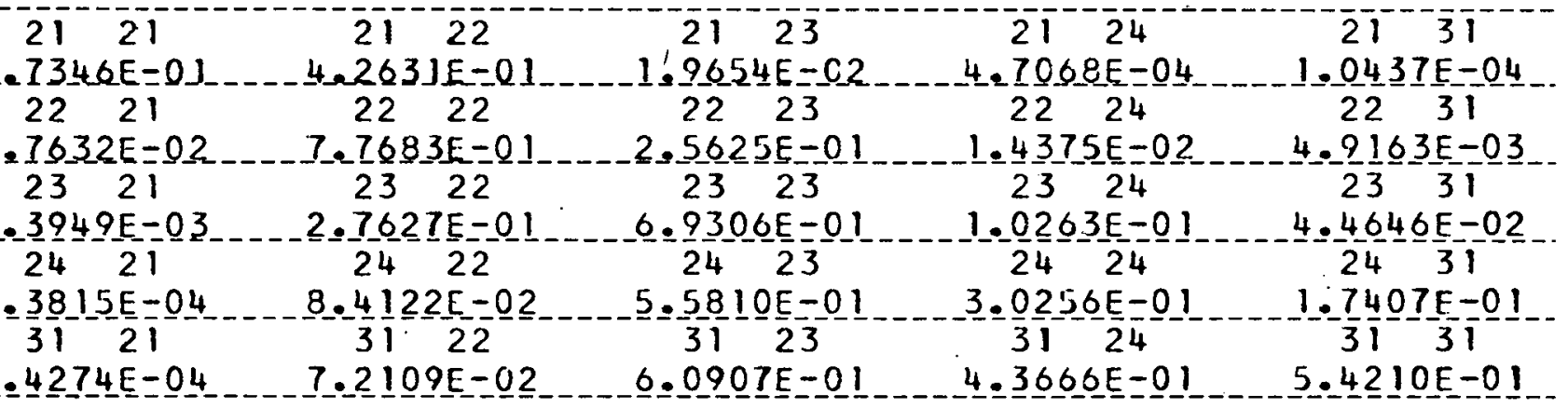

IEMPERAIURE

DEG. F.

SI GMA

SIGMA

SIGMA

NU SIGMA

SCATTER

TRANSPORT

ABSORPTION

FISSION
$1.000 \bar{O} 0 \overline{3}$
$1.5422 \mathrm{E} 00$
$1.4947 E 00$
$9.706 \overline{1 E} 0 \overline{1}$
0 .

SIGMA S (N)

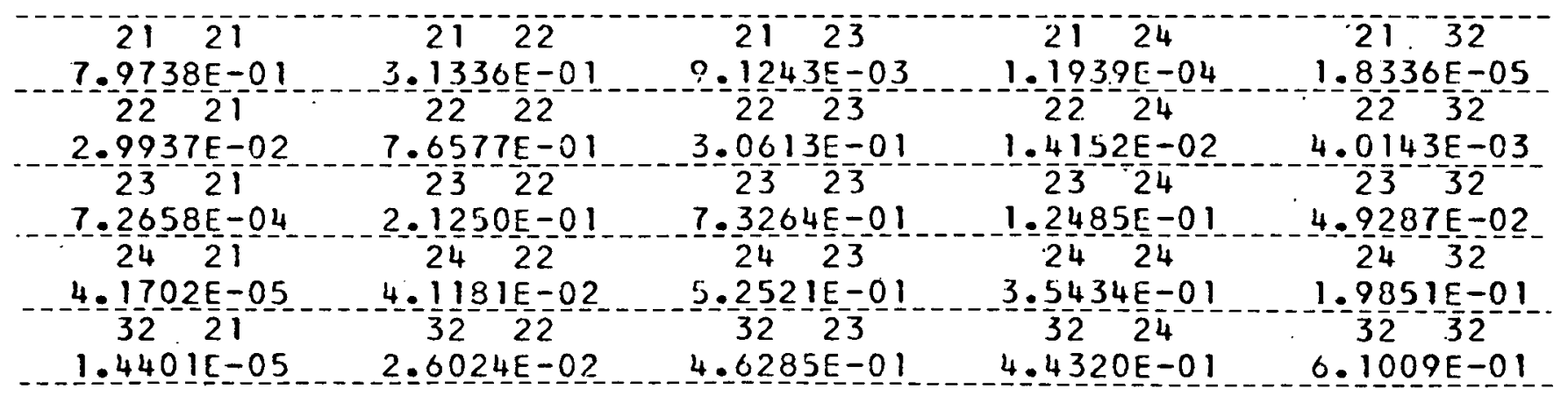


LIJHLUM

THERMAL TRANSFER MATRICES

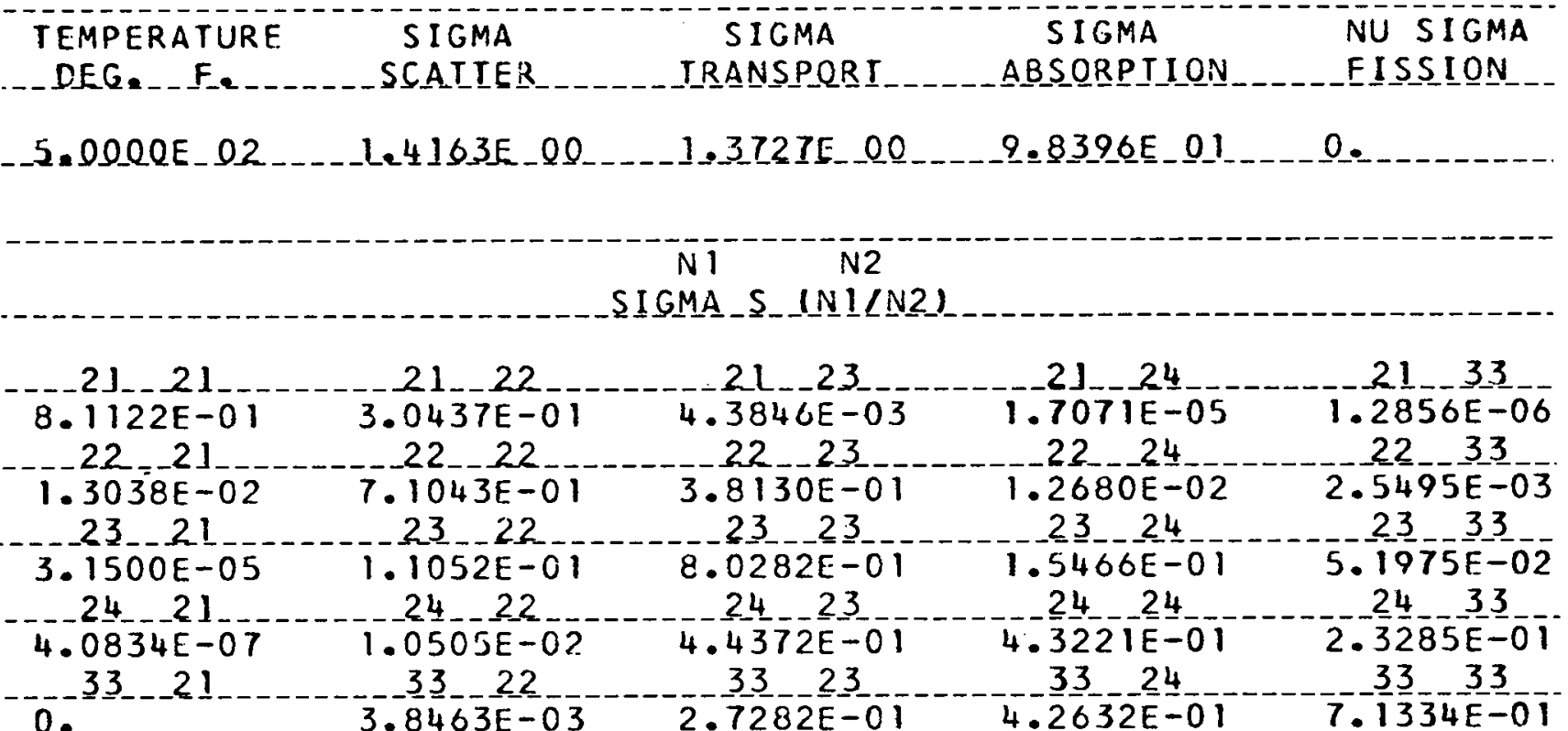

TEMPERATURE

DEG 2 - F .

6. 8000 EE 01

\section{SIGMA}

SCAIIER

$1.3014 E_{2} 00$
SIGMA IRANSPORI $1.2613 E$

\section{SIGMA ABSORPIION}

$1.0190 E$ O2 20.
NU SIGMA FISSION

\section{$\mathrm{N} 1 \mathrm{~N} 2$}

SIGMA S (NIIN2)

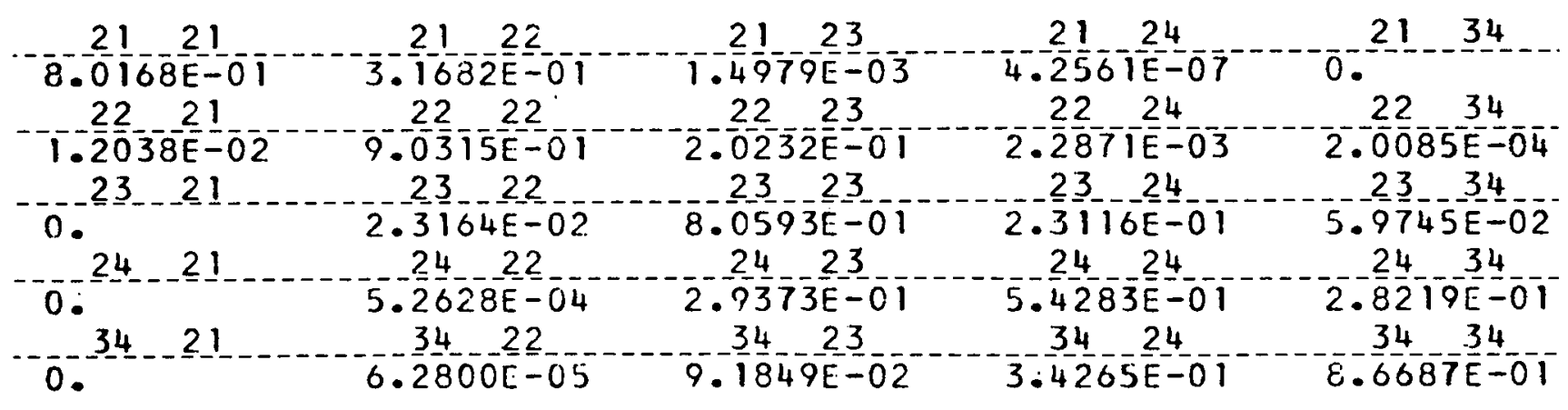


LI I I I I U

TRANSFERS FROM GROUPS I THROUGH 7 TO GROUPS I THROUGH 15

SIGMA

4. $\begin{gathered}1 \\ 1\end{gathered}$

$-6.217] E-02-4.0622 E-02 \quad 2.1273[-02 \quad 1.2330 E-02-3.6002 E-03$

$-5.47-17 E=04 \quad 7.6670 E-05 \quad 1.0967 E-05$

-0.

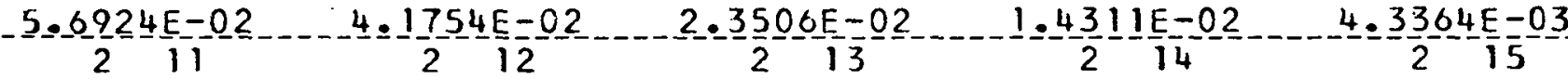

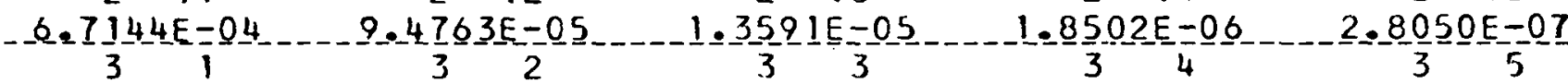

-0.

- $4.7501 E-02 \ldots 4.2018 E-02 \quad 2.6589 E-02 \ldots 1.7526 E-02$

311313

$8.9927 E-04-1.28 \frac{3}{4} 9 E-04-1.8494 E-05-2.5218 E-06-3.8256 E-07$

$0.40 .0 .0316 E-01$

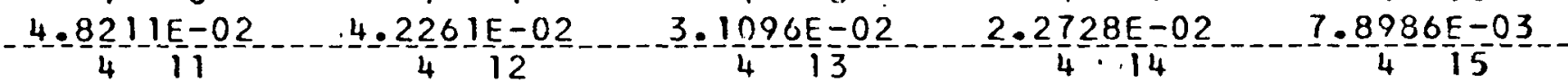

$1.3102 E-03 \quad 1.8991 E-04 \quad 2.7508 E-05 \quad 3.7593 E-06 \quad 5.7076 E-07$

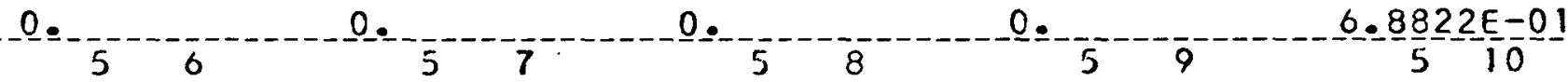

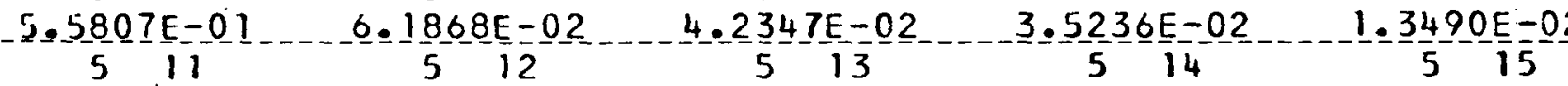

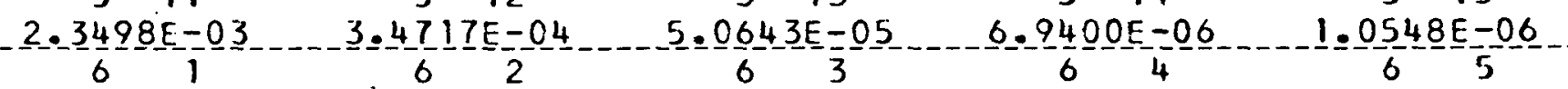

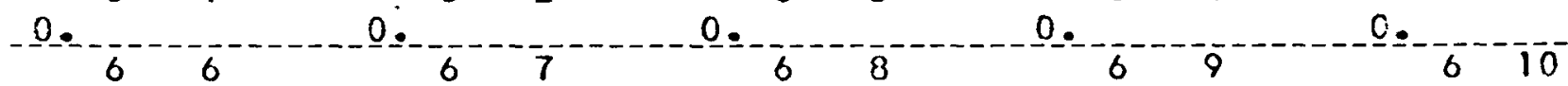
$6.0737 \mathrm{E}-01 \ldots 0.4763 \mathrm{E}=01 \quad 3.7927 \mathrm{E}-02 \quad-2.4862 \mathrm{E}-02 \ldots 1.0594 \mathrm{E}-02$

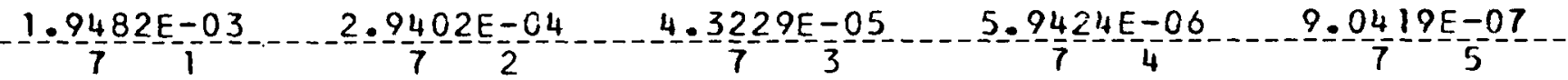

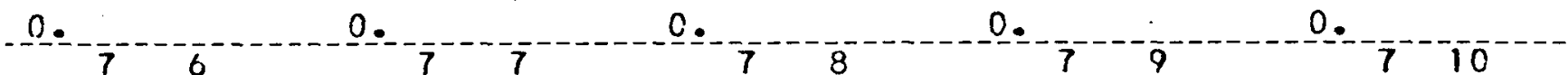

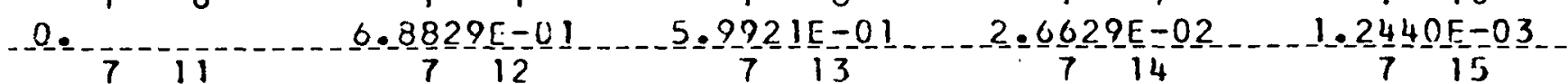

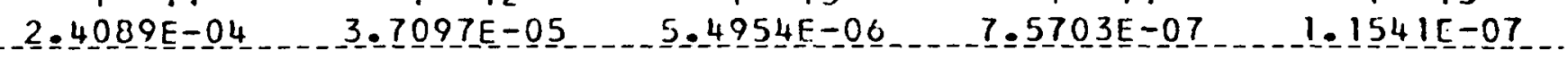


LITHIUM

TRANSFERS FROM GROUPS 1 THROUGH 34 TO GROUPS 1 THROUGH 11

\section{$\mathrm{N1} N 2$}

SIGMA N, G $(N] / N 2)$

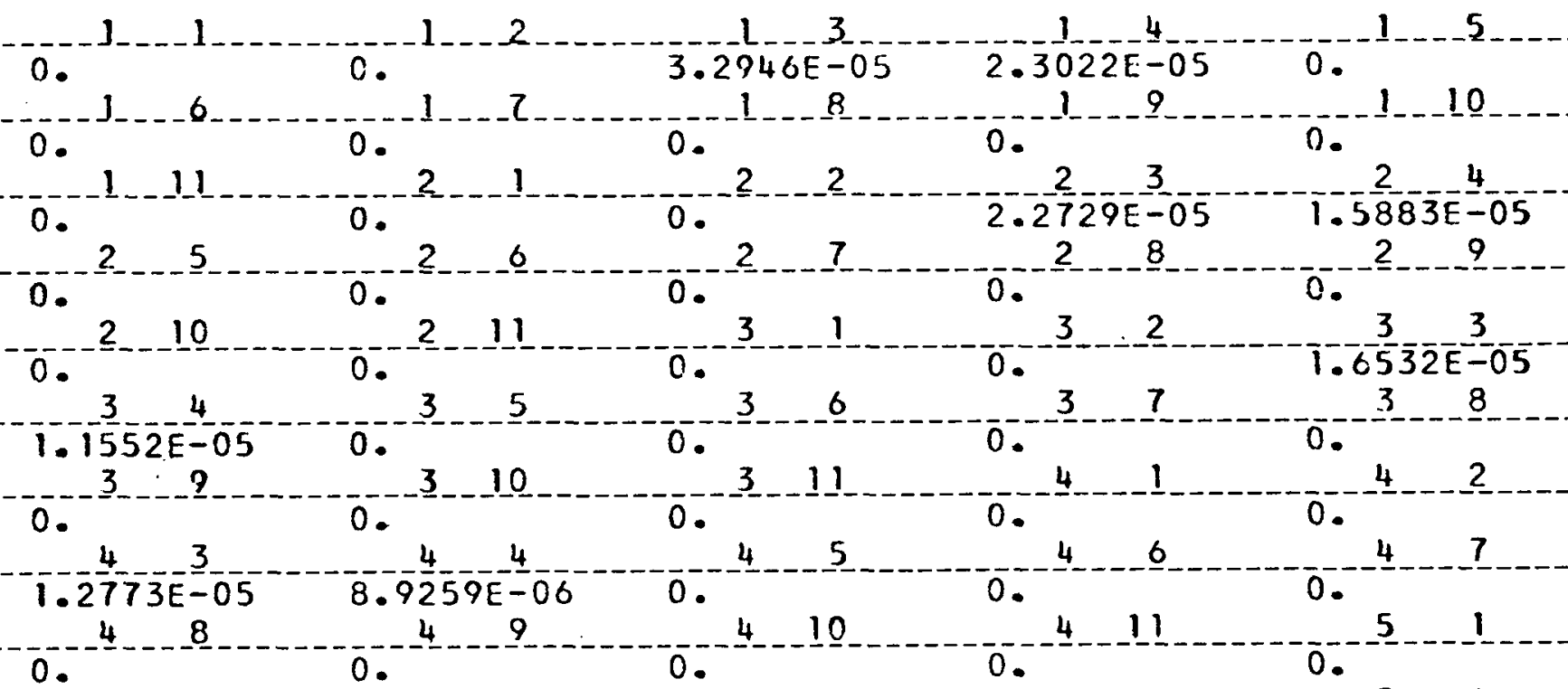

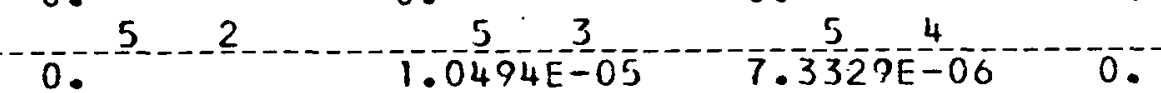

0.

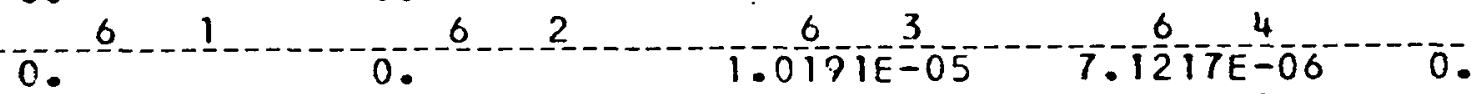

o.

0. 5.9

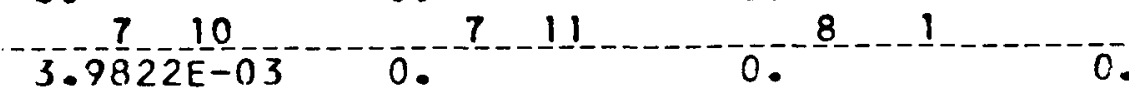

0.8

$3.9822 E-03$
8

$2.5899 \mathrm{E}-05^{-10} 0$.

0. 8

$-\frac{9}{3} .4861 \mathrm{E}-05^{-}-\frac{9}{2}-\frac{4}{360 \mathrm{E}-05}-0.9$

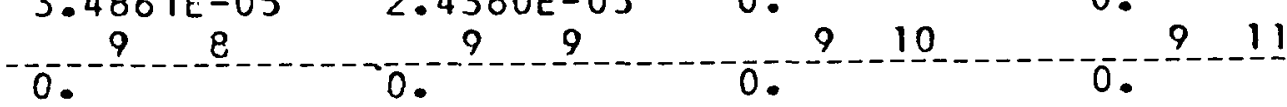

$-8$

$3 .-706 \overline{3}=\frac{3}{-05}$ 
-_LIJHLUM_..

N 1

SIGMA N,G (NIINZ)

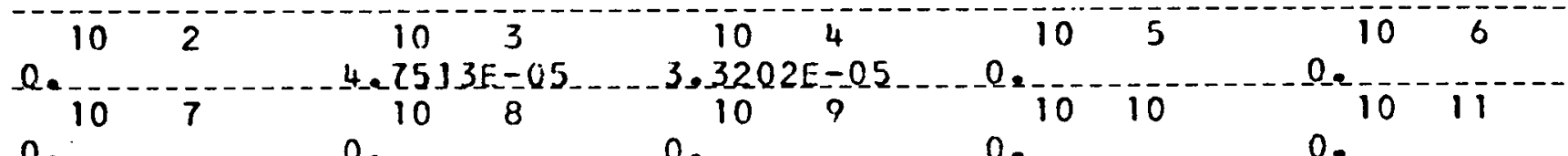

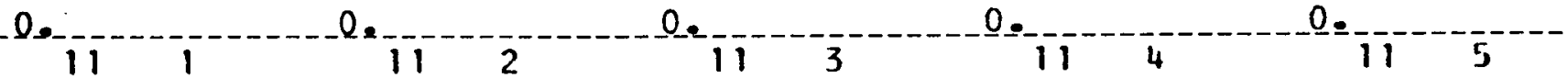

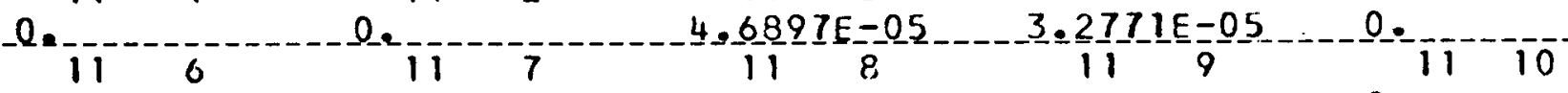

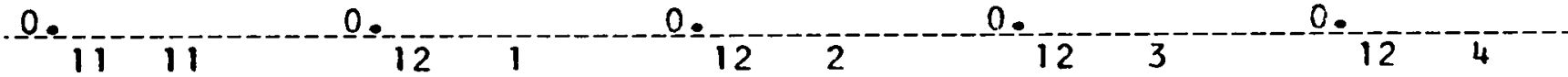

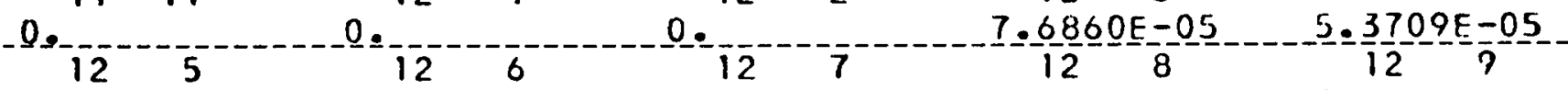

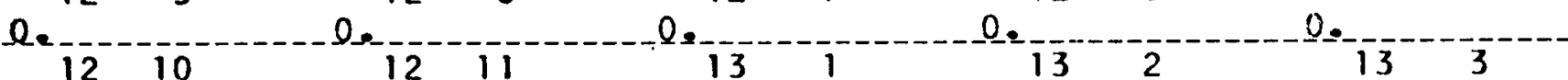

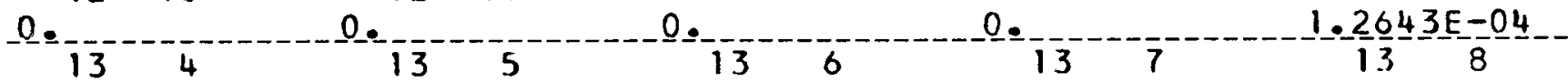

8. 835 J $13=05$

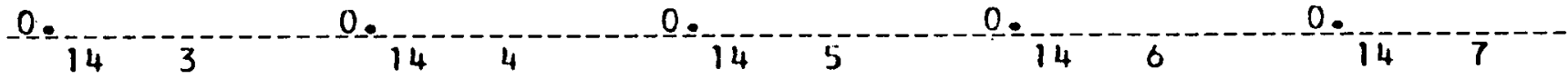

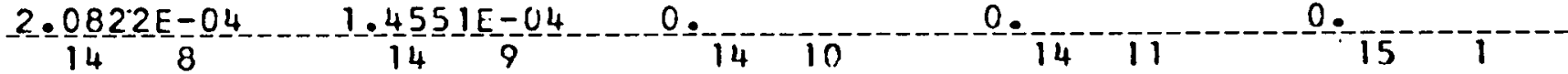

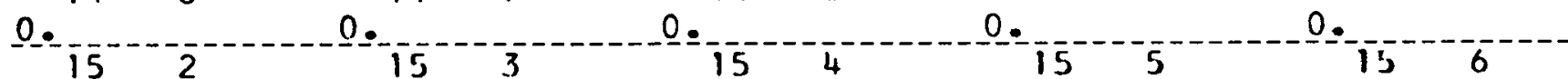

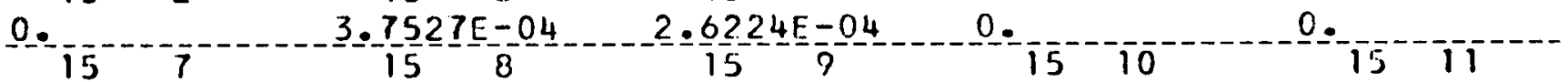

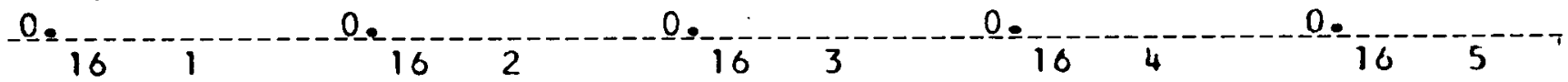

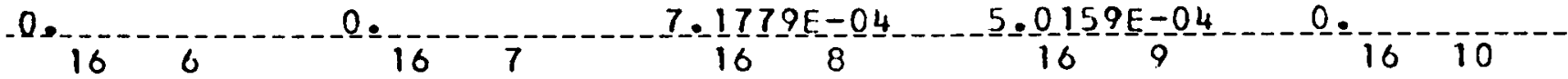

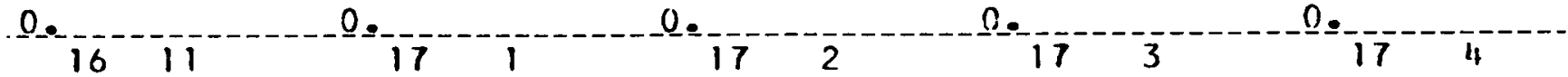

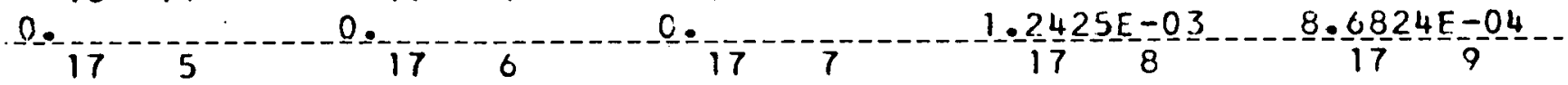

$0.1710^{0}-17^{17}$

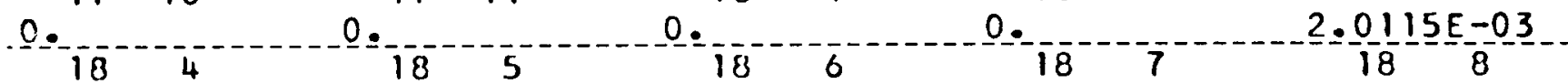

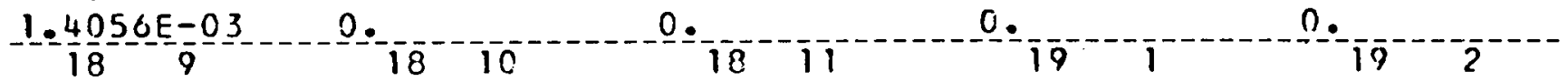

Q 
LITHIUM

Ni $\mathrm{N} 2$

SIGMA N,G INI/N2]

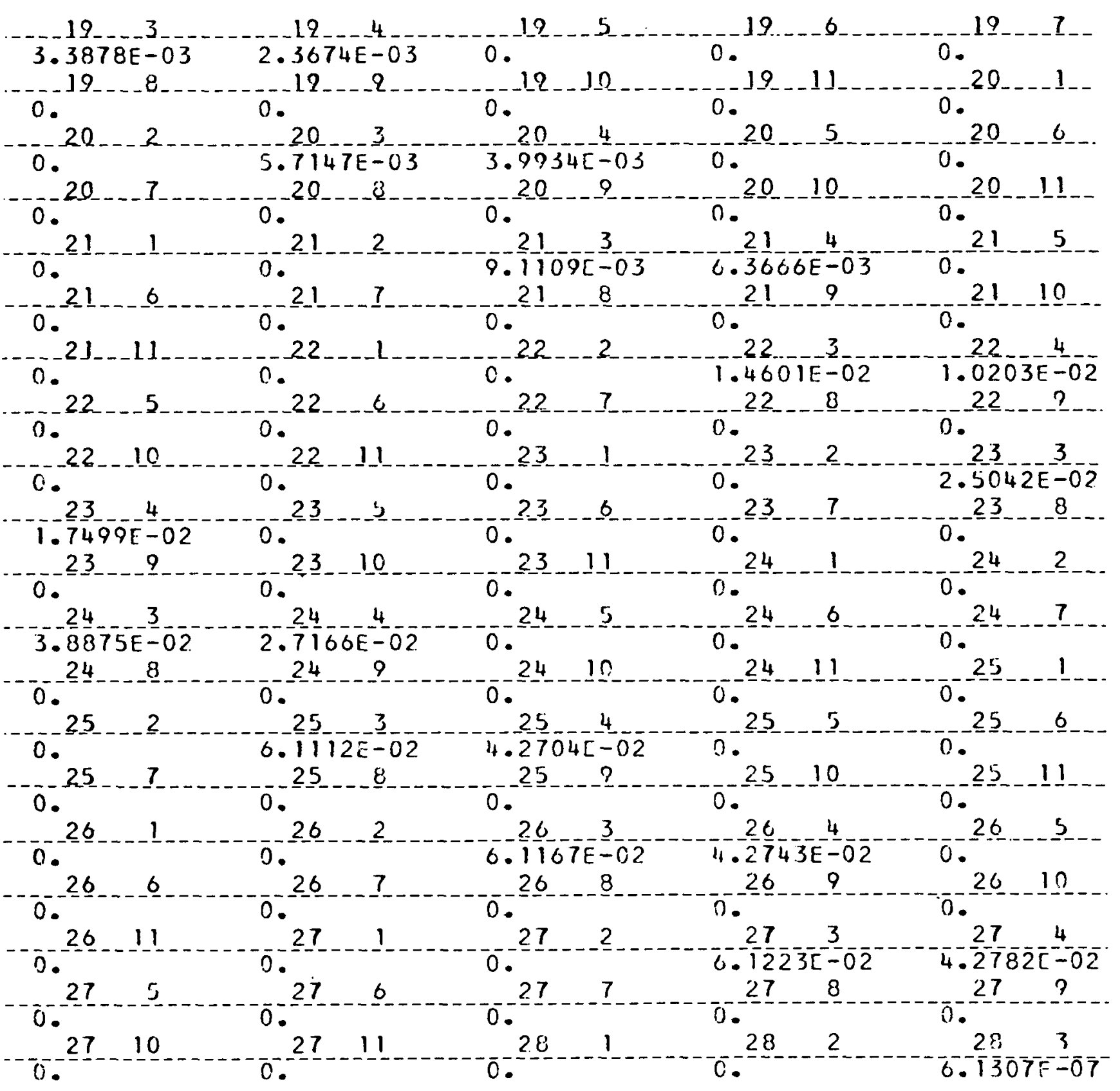




\section{-LIJHLUM.}

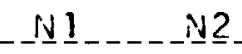

SIGMA N,S (INI/N2)

28428,2806728

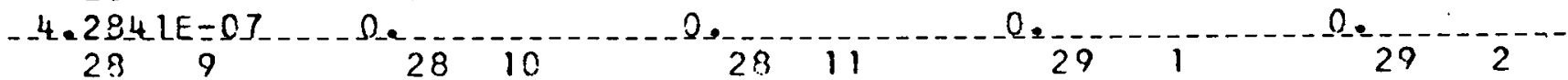

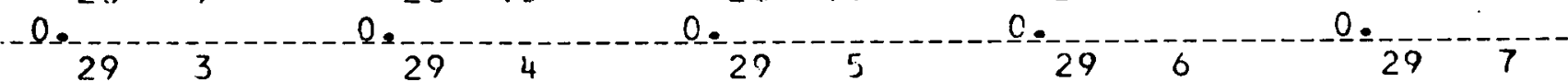

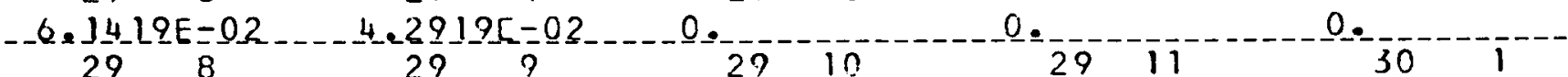

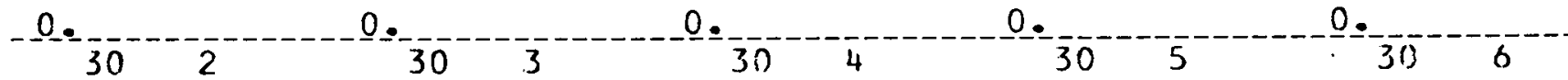

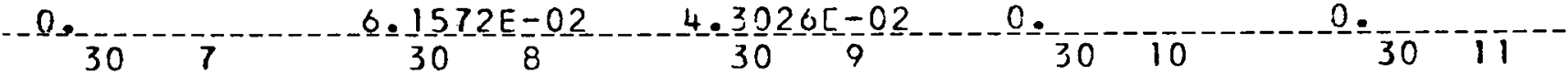
- 31 . $31^{2}$

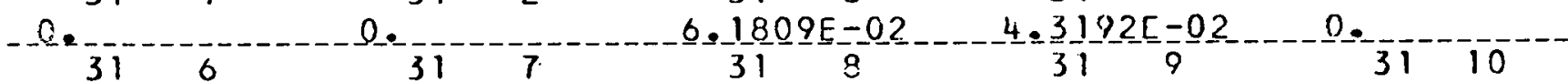

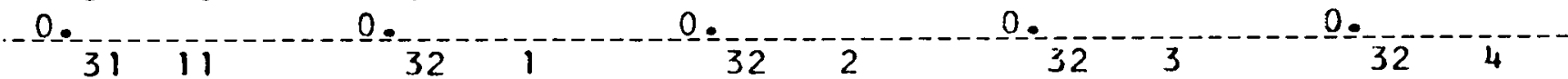

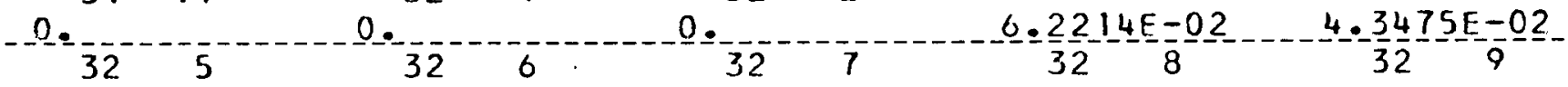
- . 32

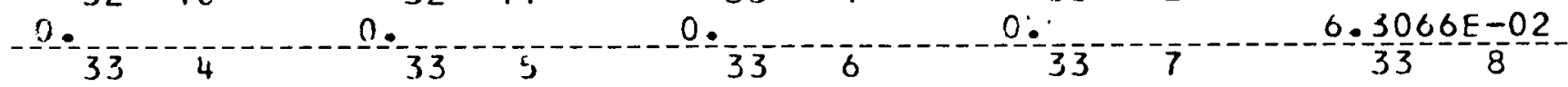

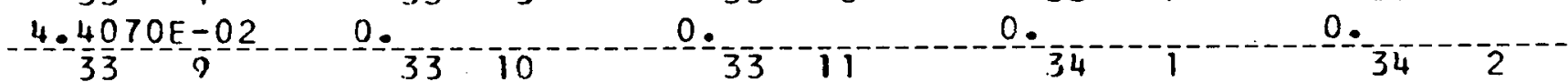

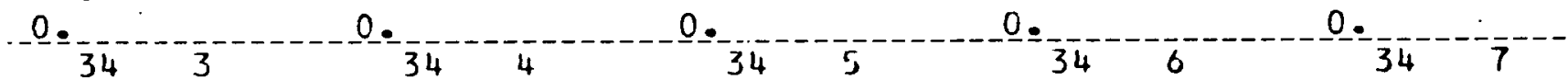

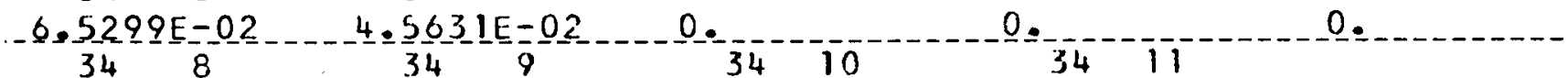
0. ㅇ. 
DESIGNAILON BORQN
CODE_NO.

5.1100
DENSIIIY_FAC_IQR $1.79594 E_{-} 01$

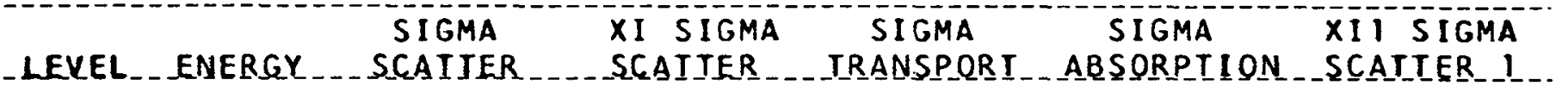

1 1.000E $07-1.3581 E-00 \quad 1.1805 E-001.1361 E-00 \quad 5.8735 E-02-1.8208 E-01$

$26.065 \mathrm{E} \quad 06 \quad 1.4598 \mathrm{E} 00 \quad 8.2095 \mathrm{E}-011.1586 \mathrm{E} \quad 006.0964 \mathrm{E}-02-2.4697 \mathrm{E}-01$

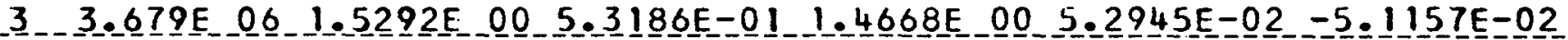

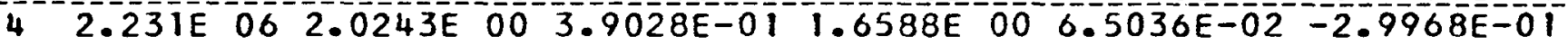

5 - 1.353E_06_2.4675E_00_2.7935E-01 1.2621E_00 4.3127E-02_-9.8833E-01.

$6.8 .208 E$ E $051.9717 E-002.6419 E-011.3628 E 005.3098 E=02-4.9930 E-01$

7 4.979E $053.6623 \mathrm{E} 006.3940 \mathrm{E}-013.4151 \mathrm{E} 00-1.2850 \mathrm{E}-0 \mathrm{~T}-2.027 \mathrm{IE}-0 \mathrm{~T}$

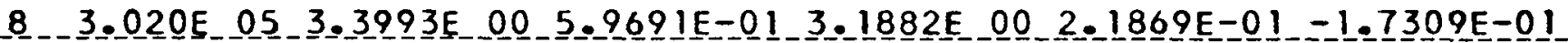

$9 \quad 1.832 \mathrm{E} \quad 05 \quad 3.7554 \mathrm{E} \quad 00 \quad 6.5944 \mathrm{E}-013.5221 \mathrm{E} \quad 002.9448 \mathrm{E}-01-1.9122 \mathrm{E}-01$

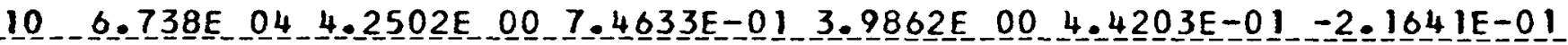

$11-2.479 \mathrm{E}-044.5250 \mathrm{E}-007.9459 \mathrm{E}-014.2440 \mathrm{E} 007.1209 \mathrm{E}-01-2.3041 \mathrm{E}-01$

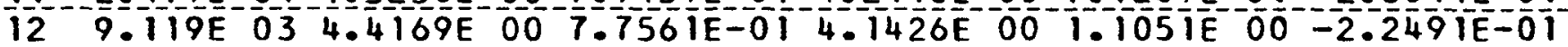

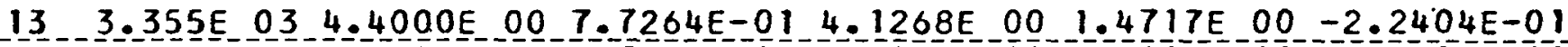

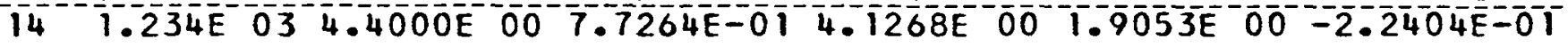

15 4.540E 02 4.4000E 00 7.7264E-01 4.1268E 00 3.0247E $00-2.2404 \mathrm{E}-01$

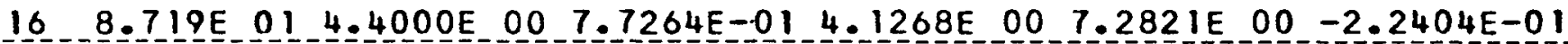
$17-545 \mathrm{E}-1-4000 \mathrm{E} 0 \mathrm{O}-7264 \mathrm{E}-0 \mathrm{1}-1268 \mathrm{E}$ $18 \quad 1.304 E$ OI $4.4000 E$ O0 $7.7264 E-014.1268 E \quad 002.8759 E \quad 01-2.2404 E-01$

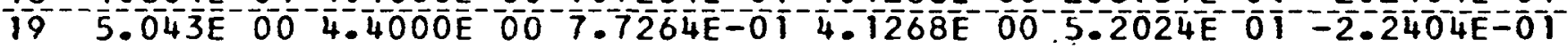

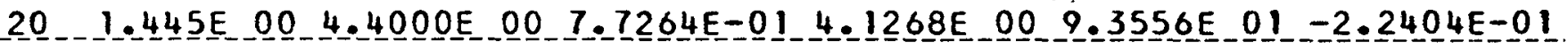

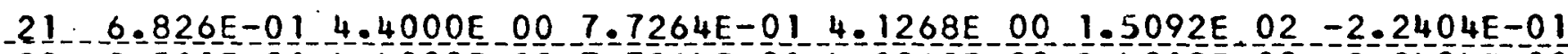
22 2.511E-0 $4.4000 E$ 00 $7.7264 \mathrm{E}-014.1268 \mathrm{E} 002.4219 \mathrm{E} 02-2.2404 \mathrm{E}-01$

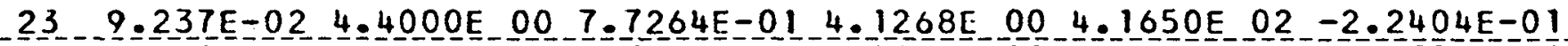
$242.530 E-024.3857 \mathrm{E} 00-7.7013 \mathrm{E}-014.1133 \mathrm{E} 00-6.0685 \mathrm{E}-02-2.2331 \mathrm{E}-01$

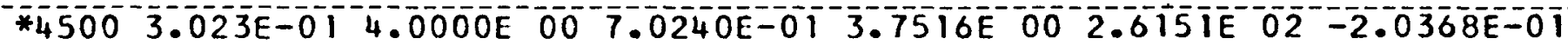
*4000 2.718E-01 4.0000E $00 \quad 7.0240 E-013.7516 E$ 00 2.6151E $02-2.0368 E-01$

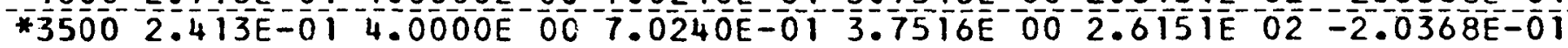
*3000 2.109E-01 4.0000E 00 7.0240E-01 3.7516E 00 2.6151E 02 -2.0368E-01

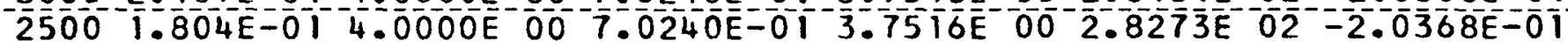

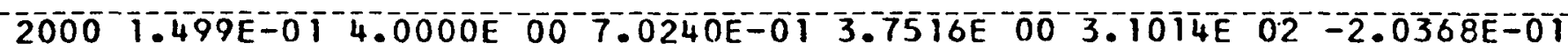

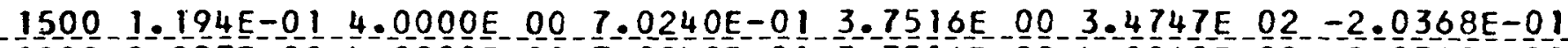
$10008.897 \mathrm{E}-024.0000 \mathrm{E}$ 00 $7.0240 \mathrm{E}-0,3.7516 \mathrm{E}$ 0O $4.0260 \mathrm{E} 02-2.0368 \mathrm{E}-01$ 500 5. $850 \mathrm{E}-02$ 4.0000E 00 7.0240E-01 3.7516E $004-9652 \mathrm{E} 02-2.0368 \mathrm{E}-01$ 68 3.2 $16 \mathrm{E}-02-4.0000 \mathrm{E}$ 00 $7.0240 \mathrm{E}-013.7516 \mathrm{E}-00-6.6959 \mathrm{E}-02-2.0368 \mathrm{E}-01$

Thermal cross sections taken from APEX-515.

NOTE: For a summary of the sources of data see APEX-704. 
BORQN

THERMAL TRANSFER MATRICES

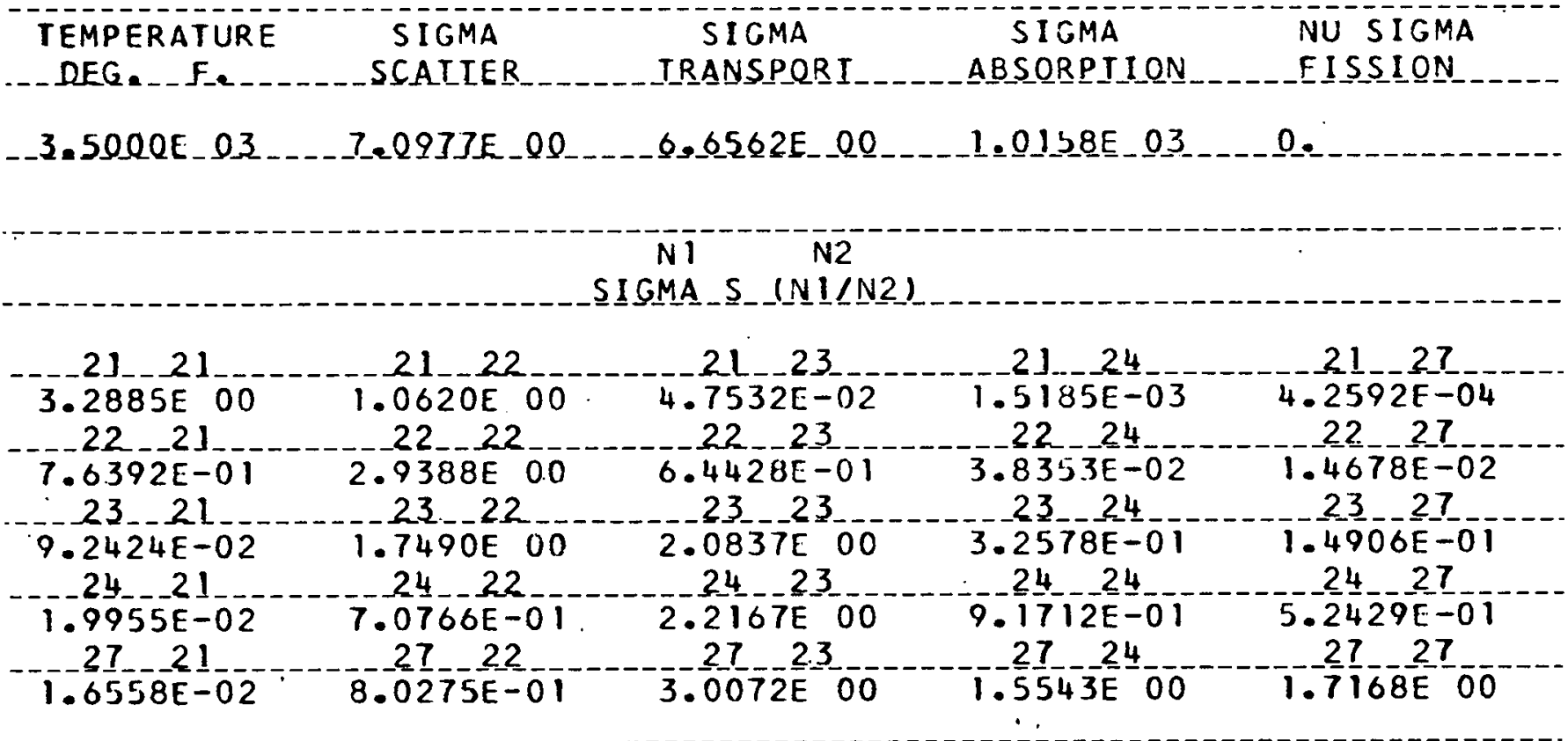

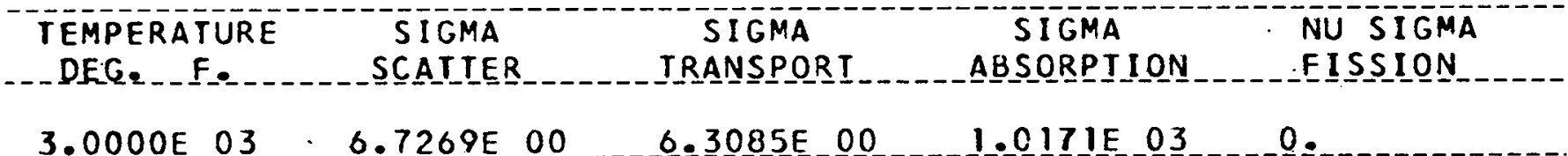

Ni

SI GMA S (N I IN2)

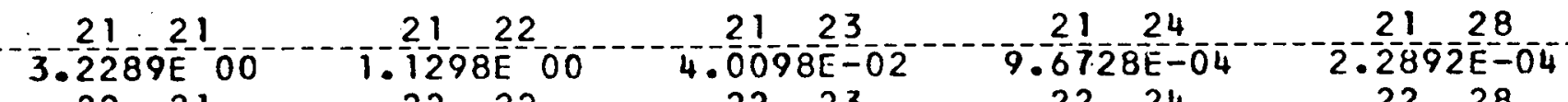

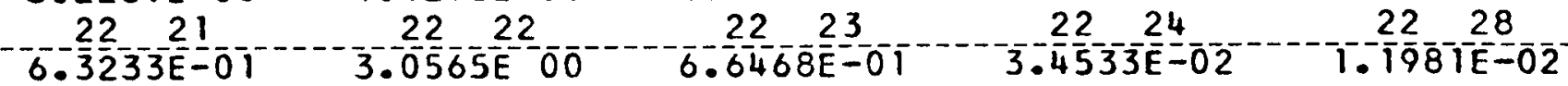

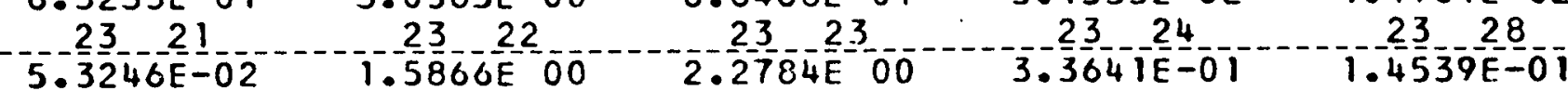

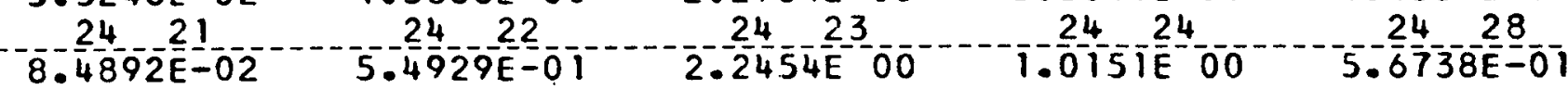

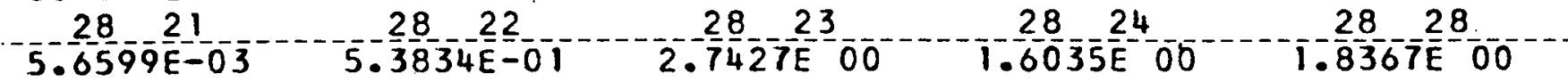


BORON

_LHERMAL _ IRANSFER_MAIRLCES - .

.LEMPERATURE

SIGMA

SIGMA

SIGMA.

NU SIIGMA

DEG. F. SCATTER

TRANSPORT

ABSORPTION

FISSION

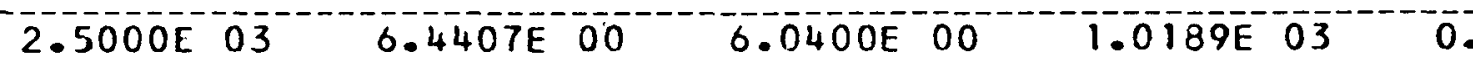

SIGMA S $(\mathrm{N} 1 / \mathrm{N} 2)$

$2121 \quad 2122$

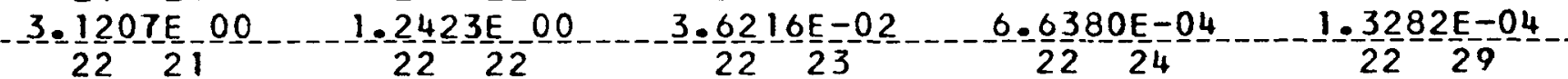

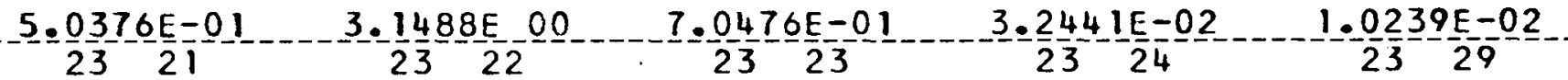

$-2.9394 \mathrm{E}-02-1.4223 \mathrm{E}-00 \mathrm{O}-2.4536 \mathrm{E}-00 \quad 3.5095 \mathrm{E}-01-1.4373 \mathrm{E}-01$

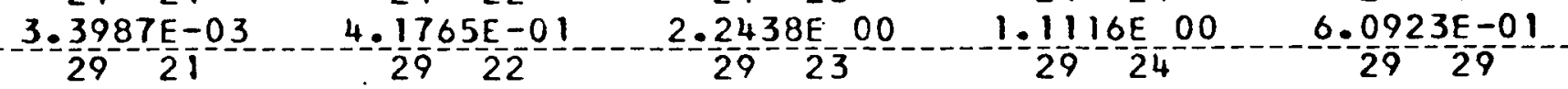

$1.8268 \mathrm{E}-03 \quad 3.5548 \mathrm{E}-01 \quad 2.4804 \mathrm{E} 00 \quad 1.6443 \mathrm{E} 00 \quad 1.9587 \mathrm{E}$

IEMPERATURE

DEG. F.

$2.000 \bar{O} \overline{0} \overline{3}$
S IGMA

SCATTER
SIGMA

TRANSPORT
SIGMA ABSORPTION
NU SIGMA

FISSION

\section{$5 . \overline{6} \overline{0} \overline{6} \mathrm{E} 0 \overline{0}$}

$1.0215 E^{-} 0 \overline{3}$
0

\section{SIGMA S $\left.(N) \frac{N}{N} \frac{1}{N}-\bar{N} 2\right)$}

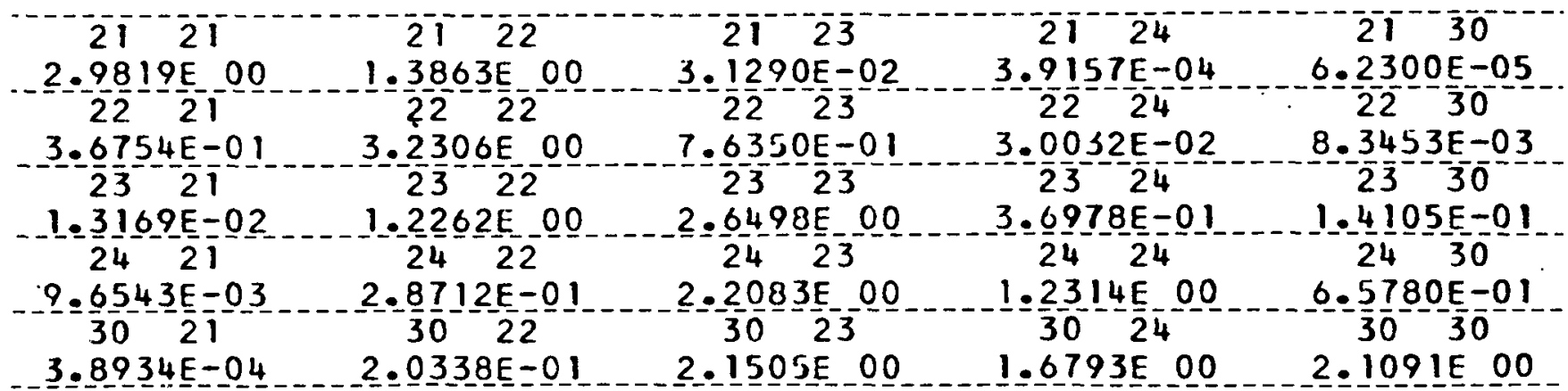


BORON

THERMAL TRANSFER MATRICES

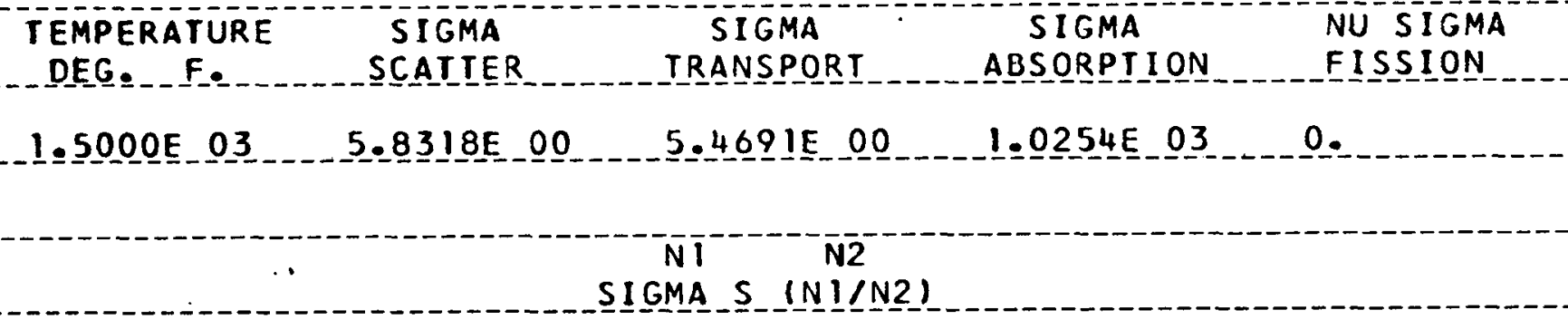

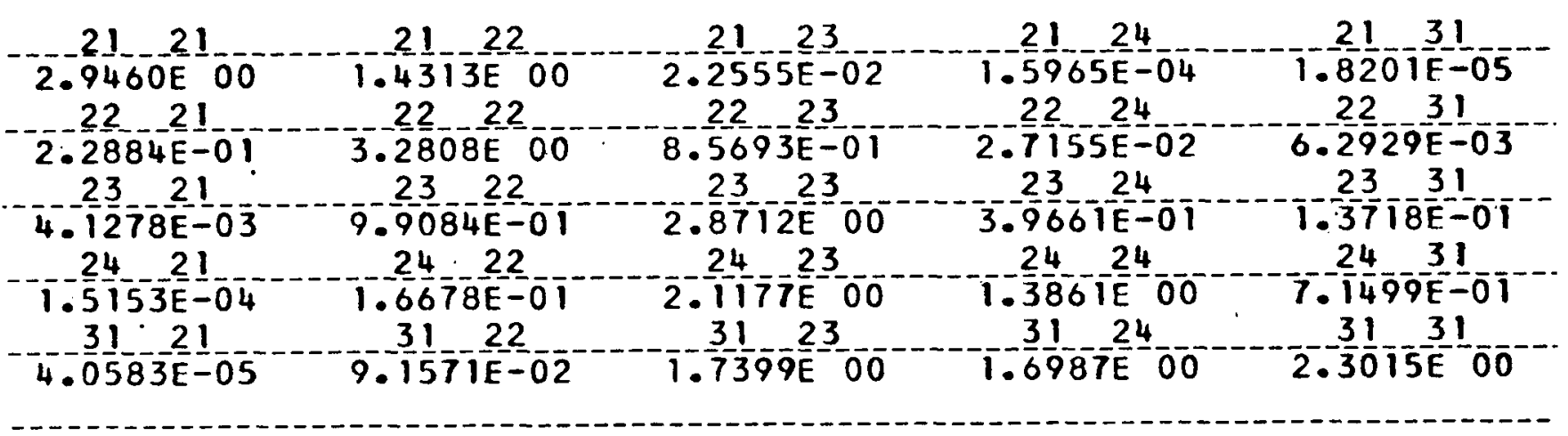

TEMPERATURE
SIGMA

$\mathrm{Ni}-\bar{N}^{2}$

SIGMA S (NIIN2)

\begin{tabular}{|c|c|c|c|c|}
\hline $21-21$ & 21 & $21-23$ & $21-24$ & 32 \\
\hline $3.447 \mathrm{E} O \mathrm{O}$ & $9.4518 \mathrm{E}-0$ & $\begin{array}{l}7.6456 \mathrm{E}-03 \\
22\end{array}$ & $\begin{array}{c}2.0432 \mathrm{E}-05 \\
2224\end{array}$ & $\begin{array}{l}3574 E-06 \\
22\end{array}$ \\
\hline$-0194 E-0$ & $3.2489 \bar{E} 00$ & $1.0217 \mathrm{E} 00$ & $2.3 \frac{3}{3} 74 \mathrm{E}-\overline{0}$ & $4.0807 \mathrm{E}-0 \overline{3}$ \\
\hline $\begin{array}{r}23 \\
0.4188 E-04\end{array}$ & $-23-22$ & $23-23$ & $-4-23-24$ & $1.3234 E-01$ \\
\hline 24 & 24 & 24 & 24 & $24 \quad 32$ \\
\hline $7.3728 \mathrm{E}-06$ & $7.0430 \mathrm{E}-02$ & $1.9408 \mathrm{E} 00$ & $1.6023 \mathrm{E} 00$ & $7.8644 E-01$ \\
\hline$-1.0301 \frac{32}{10-06}$ & $-3.6096 E-1$ & $1.2414 E^{3}-\frac{23}{0}$ & $1-\frac{32}{678}=\frac{24}{E}$ & $2-\frac{32}{5607 \mathrm{E}}-\frac{32}{00}$ \\
\hline
\end{tabular}


BORON

IHERMAL_IRANSFER MAIRICES

_LEMPERATURE DEG. F.

$5.0000 \bar{E} 02$
SIGMA

SCATTER
SIGMA TRANSPORT
SIGMA ABSORPTION
NU__ SIGMA

FISSION

\section{-}

$5.167 \overline{7}$ OO

4.8463500

$1.046 \overline{3 E} 0 \overline{3}$

0 .

\section{SIGMA $S(N) \frac{N 2}{N}$}

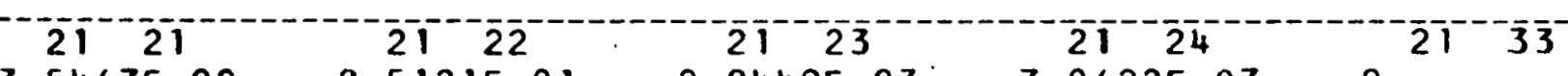

$3.5467 E$ OOO

2221

8. $5121 \mathrm{I}-01$

503089 E $=02$

2222

$2=\frac{04}{22}-\frac{9 E}{2}=-03$

$7=\frac{0}{2} \frac{6}{2}-2 \frac{2}{2}=07$

$22-3 \overline{3}$

2321

3. $0555 \underline{5}-0 \underline{0}$

$1.2734 \mathrm{E}$ OOO

$1.6 \frac{6}{2}-12 E=\frac{02}{2}$

1. $-\frac{6}{2} \frac{9}{3}-\frac{9 E}{3}-0.3$

_. $7558 \mathrm{E}-05$

2421

- $0 .-\frac{1}{3} \overline{3} \overline{1}$

2422

$3.3639 \mathrm{E}-00$

S. $-39 \frac{395 E}{24}=01$

$1=\frac{28}{24}-\frac{3}{3}-0-1$

0.

$1.3 \frac{3}{3} \frac{7 O E}{3}-02$

$1 . \frac{59}{3} 3 \frac{3}{2}-\frac{0}{3}-0$

$1.9 \frac{9}{3} 3 \frac{2}{2}-\frac{0}{24}-0$

$8 \cdot-\frac{74}{3} \frac{4}{3}-\frac{8 E}{3}-01$

2. $4851 E-03$

6. 7172 E -01

$1.5525 E$
TEMPERAIURE

DEG. F.
SI GMA

SCATTER
SIGMA TRANSPORT
SIGMA ABSORPTION
NU SIGMA

FISSION

\section{SIGMA S $\left(\frac{N}{2} 1 / N 2\right)$}

$2 i^{2} i^{-12}$

3.5455E 00

22

. $.7 \frac{7}{2} \frac{2}{3}-\frac{E}{2}-02$

.

$7 \cdot 8139 E-02$

$34^{--} \overline{2} \overline{1}$

$3.9863 E=04$

34

- 0.
$2 \cdot \frac{5}{2} \frac{1}{2}-1 E-\frac{2}{2} \overline{3}$

$5.222 \frac{9}{2}=01$

$3.3829 \mathrm{E}-00$

1. $-0761 E-\frac{00}{34}$

$1.9939[-01$
$1.0836 \mathrm{E} 03 \mathrm{O}$ 
BORON

TRANSFERS FROM GROUPS 1 THROUGH 6 TO GROUPS 1 THROUGH 15

$\mathrm{NI} N 2$

SIGMA_SO_LNIIN2)

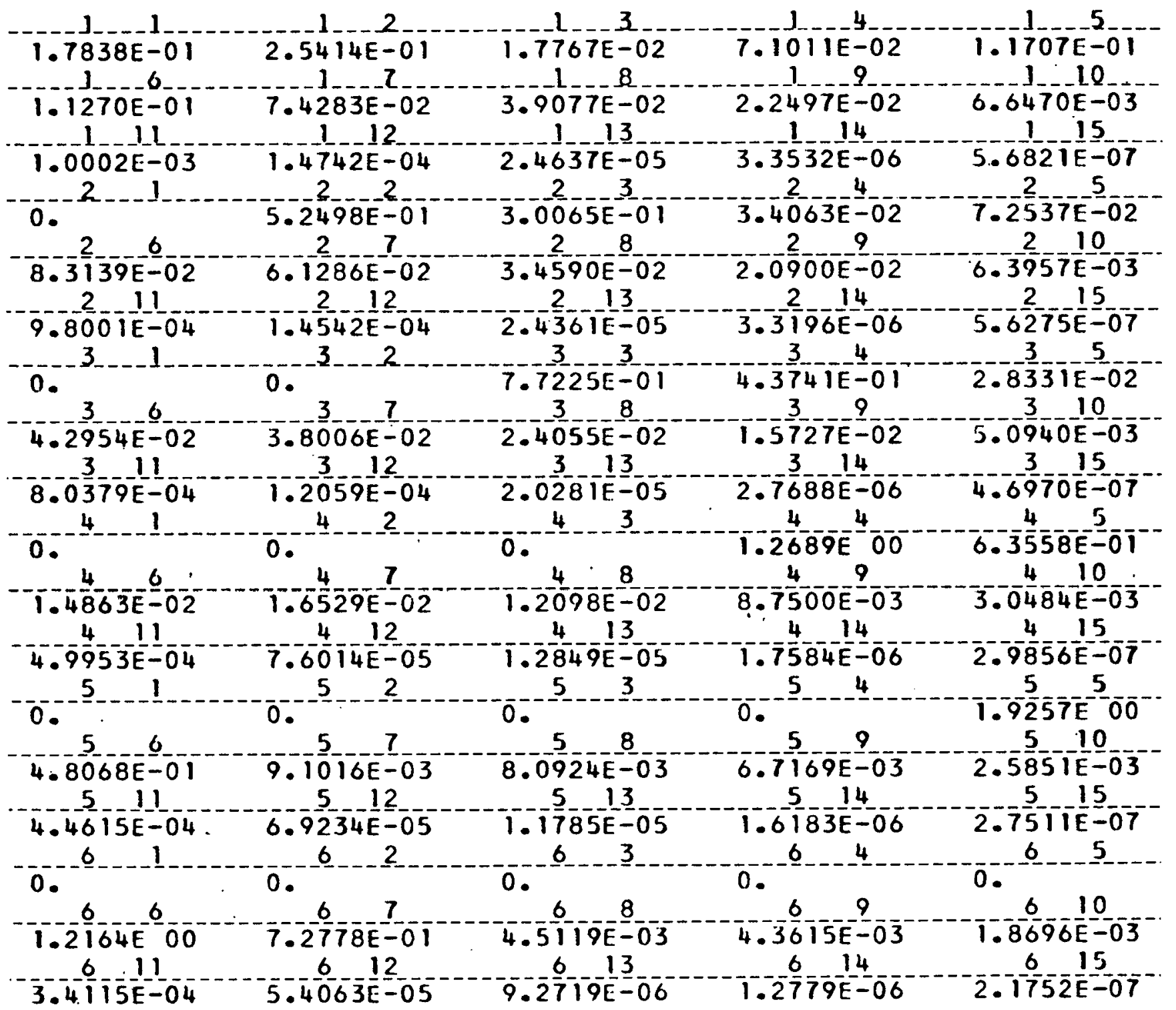


DATE $=01 / 11 / 61$

DESIGNATION

THOR IUM 232
CODE NO.

90.2320
DENSITY FACTOR

3. $8516 \overline{4} \overline{0}$

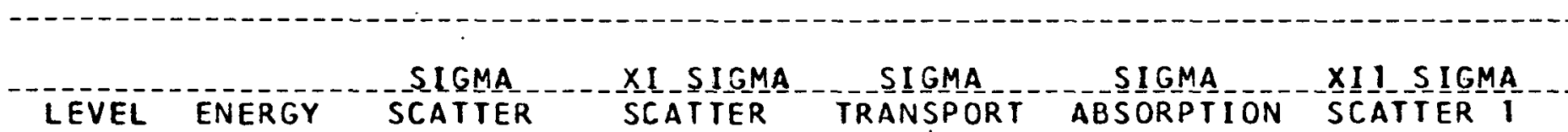

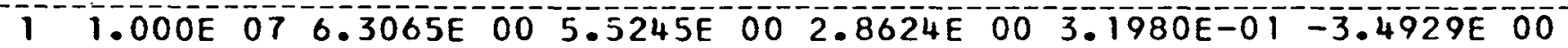

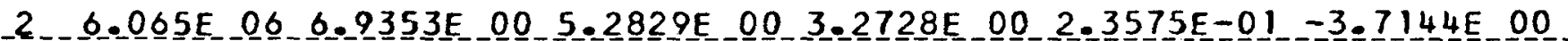

3 3.679E $06 \quad 7.2214 \mathrm{E} 004.8137 \mathrm{E}$ 00 $3.8085 \mathrm{E} 00 \quad 1.6935 \mathrm{E}-0 \mathrm{1}-3.4612 \mathrm{E}$ 00

$4.2 .231 \mathrm{E}-06-6.6523 \mathrm{E}-004.1088 \mathrm{E}-004.1133 \mathrm{E}-001.6930 \mathrm{E}-01-2.5749 \mathrm{E}-00$

5 1.353E $066.4994 \mathrm{E} 002.7469 \mathrm{E} 004.2790 \mathrm{E} 00-4513 \mathrm{E}-01-2.2518 \mathrm{E}$

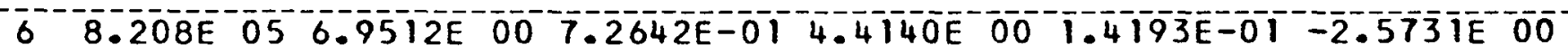

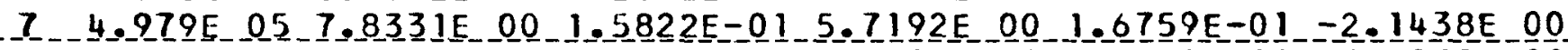

$8 \quad 3.020 \mathrm{E}$ O5 $9.244 \mathrm{IE} 008.9143 \mathrm{E}-028.9191 \mathrm{E}$ 00 $1.9260 \mathrm{E}-01-3.2959 \mathrm{E}-01$

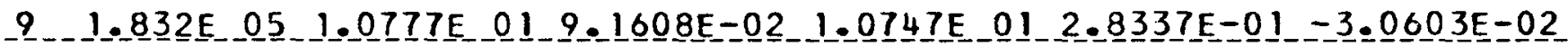

$10.6 .738 \mathrm{E} 041.2288 \mathrm{E} 011.0445 \mathrm{E}-0 \mathrm{1} 1.2254 \mathrm{E} 014.4001 \mathrm{E}-01-3.4893 \mathrm{E}-02$

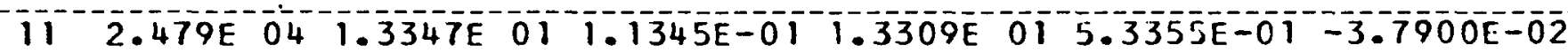

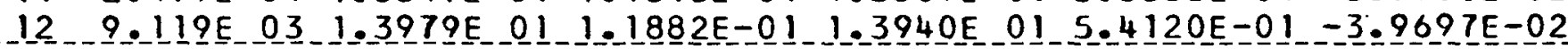

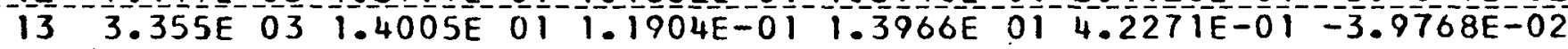

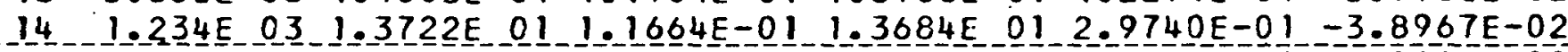

$154.540 \mathrm{E} 021.732 \mathrm{IE} 011.4723 \mathrm{E}-011.7272 \mathrm{E} 012.2628 \mathrm{E} 00-4.9186 \mathrm{E}-02$

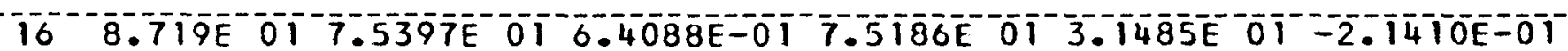

17 . $345 \mathrm{E} 01$ 1.4600E $011.2410 \mathrm{E}-01.1 .4559 \mathrm{E} 01.4 .6923 \mathrm{E} 01-4.1461 \mathrm{E}=02$

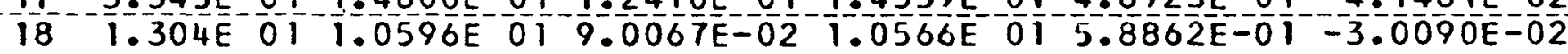

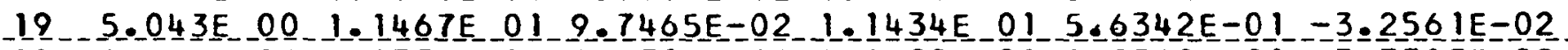

$201.445 \mathrm{E} 001.173 \mathrm{IE} 019.9716 \mathrm{E}-021.1698 \mathrm{E} 019.5569 \mathrm{E}-01-3.3313 \mathrm{E}-02$

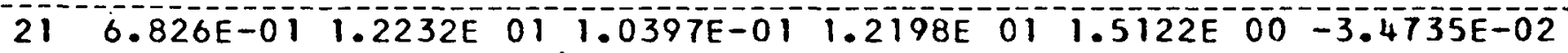

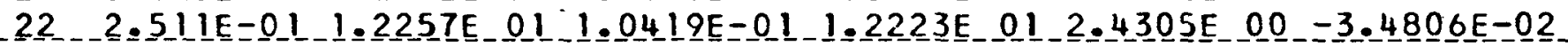

$239.237 \mathrm{E}-021.2112 \mathrm{E} 011.0295 \mathrm{E}-011.2078 \mathrm{E} 014.1751 \mathrm{E} 00-3.4392 \mathrm{E}-02$

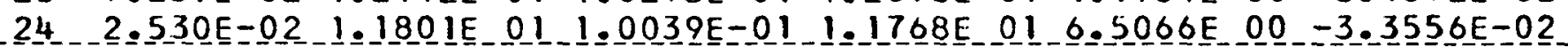

* 4500 3.023E-01 1.2439E 01 1.0786E-01 1.2403E 01 2.4225E 00 -3.6584E-02

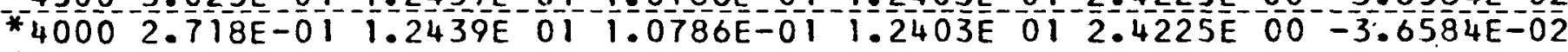

* $35002.413 \mathrm{E}-01$ 1.2439E 01 1.0786E-01 1.2403E 01 2.4225E $00-3.6584 \mathrm{E}-02$

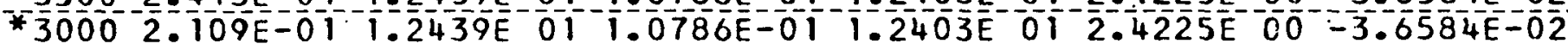

$25001.804 \mathrm{E}-01$ 1.2444E 01 1.0790E-01 1.2408E 01 2.6207E $00-3.6599 \mathrm{E}-02$

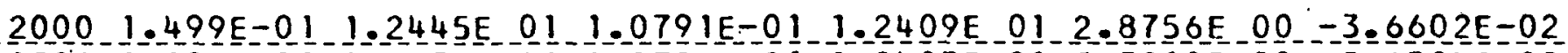

$15001.194 \mathrm{E}-011.2439 \mathrm{E} 011.0786 \mathrm{E}-011.2403 \mathrm{E}$ 01.3.3210E $00-3.6584 \mathrm{E}-02$

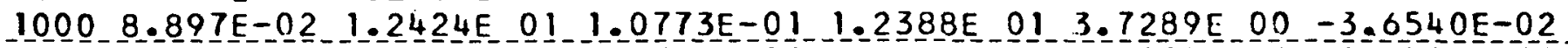

$5005.850 \mathrm{E}-02 \mathrm{2} 1.2405 \mathrm{E}$ O $1.0756 \mathrm{E}-0 \mathrm{1} 1.2369 \mathrm{E} 0 \mathrm{1}-4.5919 \mathrm{E} 00.3 .6484 \mathrm{E}-02$

$683.216 \mathrm{E}-02$ 1.2401E 01 1.0753E-01 1.2365E 01 . $0.1823 \mathrm{E}$ 00 - $3.6472 \mathrm{E}-02$

*Thermal cross sections taken from APEX-515.

NOTE: For a summary of the sources of data see APEX-704. 


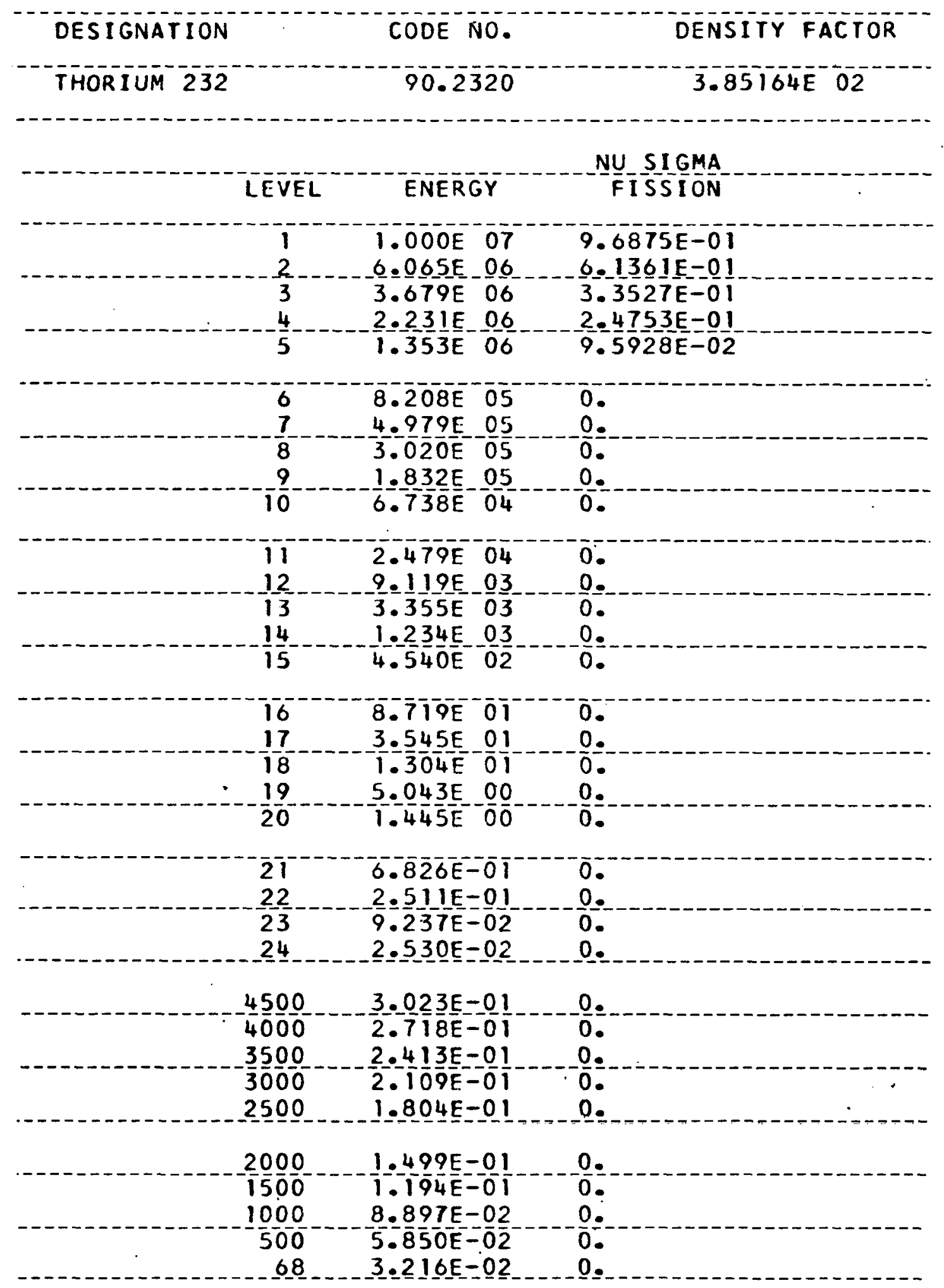


THERMAL TRANSFER MATRICES

TEMPERATURE
SEG IGMA
SE

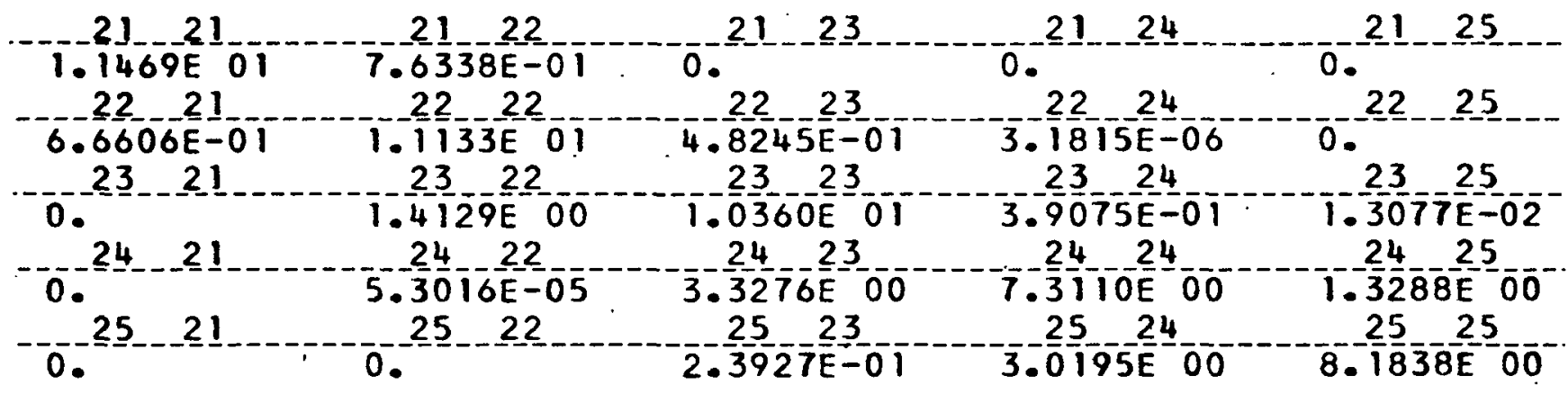

\section{TEMPERATURE}

... DEG. . F.

- 4.0000E_03

\section{SIGMA}

SSAIIER

$1399 \mathrm{E} 01$
SIGMA IRANSPORI

1.13666E_O_

SIGMA
ABSORPIION

$1.0161 E$ 으는
NU SIGMA

FI SSION
$\mathrm{Ni} N 2$

SIGMA S_IN I IN2)

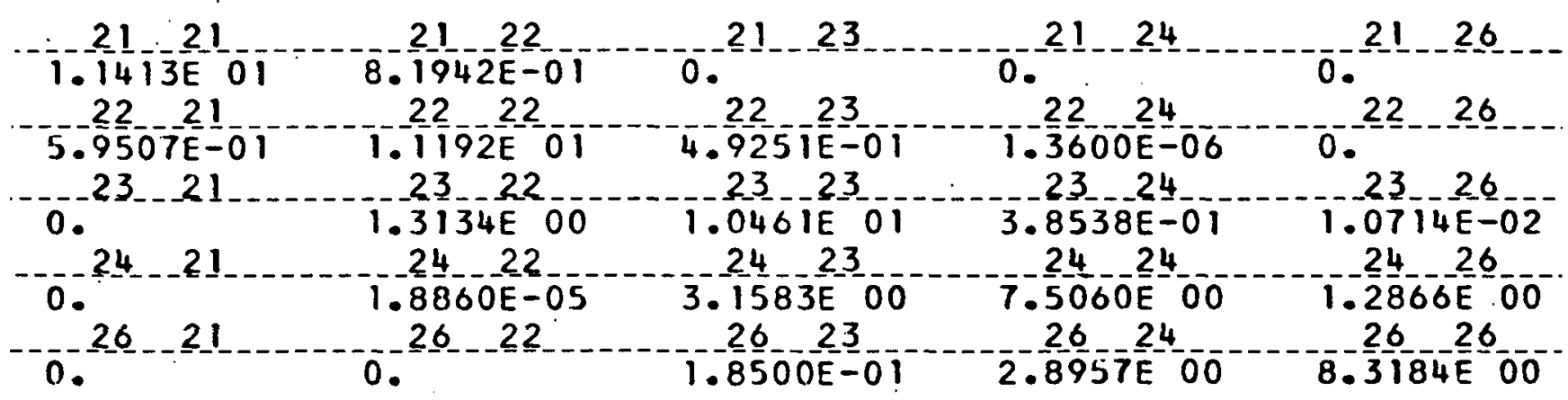


-_IHERMAL IRANSFER MAIRICES.

- IEMPERATURE DEG. F.
SIGMA

SCATTER
SIGMA IRANSPORT
SIGMA ABSORPTION
NU_SIGMA . .

FISSION

$3.5000 \mathrm{E} 03$

$1.1356 \mathrm{E} 01$

I. $1323 \mathrm{O}$ OT

1.0 ITTE OI

o.

\section{SIGMA $S\left(N \frac{N}{N}\right)$}
2121
$212 \overline{2}$
$2 \overline{1} 2 \overline{3}$
$2 i^{-14}$
$21-27$

. $1338 E_{-1} 01$

8. $2455 \mathrm{E}=01$

Q. 22

Oe 22

2221

$-5.1942 E=01$

2321

2421

. -0.27

...ㅇ..

2222

5.0805E $=01$

$4.7864 \mathrm{E}=07$

2322

$1.2067 \mathrm{E} \quad 00$

1.0568501

5. $3040 \mathrm{OE}-06$

2.9747 E -00

0 .
$8078 \mathrm{E}-01$

2424

$7=7204 E-00$
2724

$1.3615 E=01$

2.7555E_00
$22-27$

0. 2327 $8=4851 E-03$ 2427 $1.2390 E 00$ 2727 8.46니트므.
-LEMPERAIURE

DEG . F.
SIGMA

SCATIER
SIGMA

TRANSPORT
SIGHA

ABSORPTION
NNU SIGMA

FISSION
SIGMA

1.1280E OI $1.0185 \bar{O}^{-10}$

\section{o.}

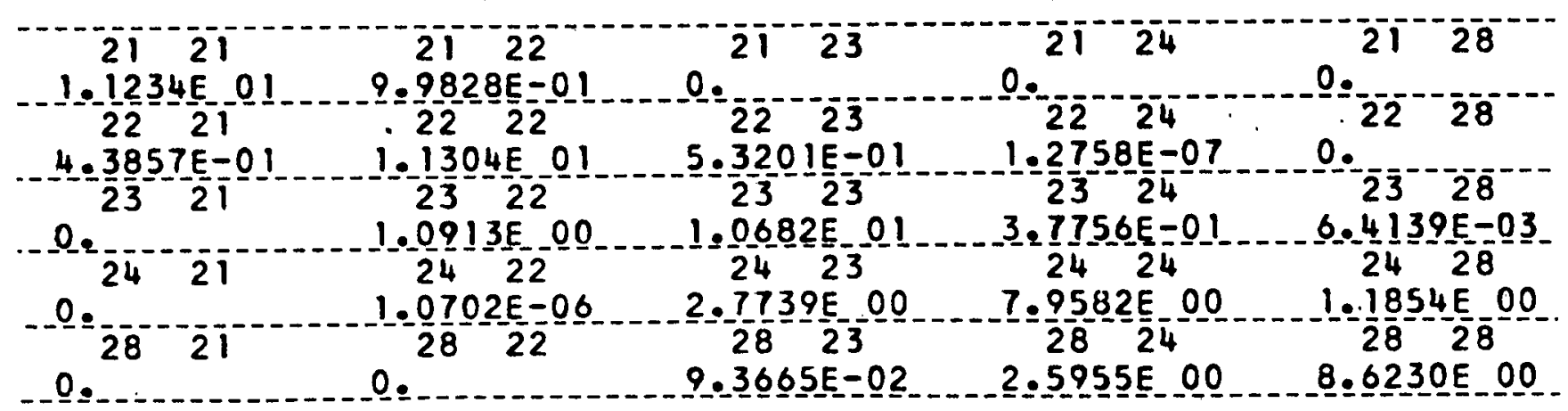


IHORLUM_ 232

THERMAL TRANSFER MATRICES

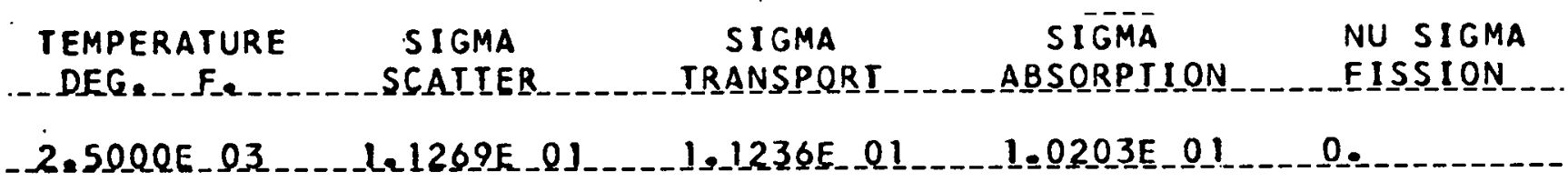

\section{Ni $1^{-2}$ \\ S1GMA S (N1IN2)}

\begin{tabular}{|c|c|c|c|c|}
\hline$-21-21$ & $-21-22$ & $2 L-23$ & $1 . \ldots 24$ & -29 \\
\hline $\begin{array}{l}1.1085 \mathrm{E} 01 \\
22-21\end{array}$ & $\begin{array}{l}1.1467 \mathrm{E} 00 \\
22\end{array}$ & 0.22 & 0.2 & 0.2 \\
\hline $\begin{array}{l}3.5218 E-01 \\
23221\end{array}$ & $\begin{array}{l}1.1351 E \\
23\end{array}$ & $\begin{array}{c}5.6966 \mathrm{E}-01 \\
23 \quad 23\end{array}$ & & 0. \\
\hline & $\begin{array}{l}9.6489 \mathrm{E}-01 \\
2422\end{array}$ & $\begin{array}{c}1.0805 E \text { OI } \\
24\end{array}$ & $\begin{array}{l}3.7680 \mathrm{E}-01 \\
2424\end{array}$ & $\begin{array}{r}4.5458 \mathrm{E}-\mathrm{C} \\
24 \quad 2 ?\end{array}$ \\
\hline & $\begin{array}{l}1.3192 E-07 \\
29 \\
22\end{array}$ & $\begin{array}{l}2.5519 \mathrm{E} 00 \\
29\end{array}$ & $\begin{array}{l}8.2241 \mathrm{E} 00 \\
29\end{array}$ & $\begin{array}{r}1.1248 \mathrm{E} \\
229 \\
-29\end{array}$ \\
\hline 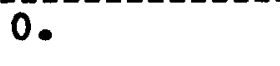 & 0. & $5.8459 E-02$ & $2.4118 \mathrm{E} 00$ & 8.7 \\
\hline
\end{tabular}

TEMPERATURE

DEGe -.F-2

-.2.0000E_03
SIGMA SSAIIER

$1,1225 E$
SIGMA IRANSPORI

$1.1193 E_{-01}$
SIGMÁ

ABSORPIION
NU' SIGMA'

EI SSIION

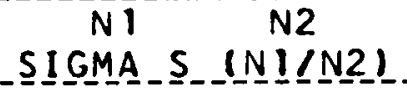

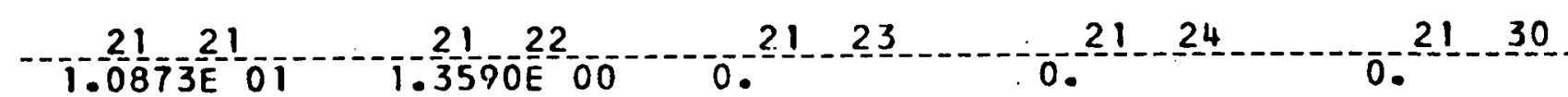

22
$2.6055 \mathrm{E}-01$
$1.1378 \mathrm{E} 01$

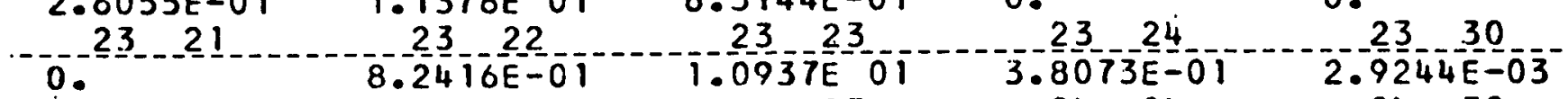

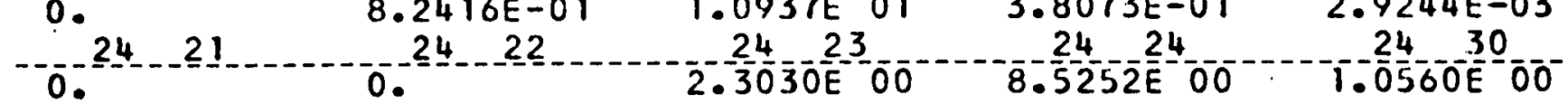

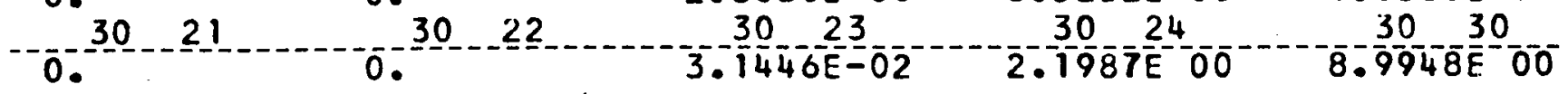


THORIUM 232

IHERMAL_IRANSEER _MATRICEES_-

- IEMPERAIURE

SIGMA.

SIGMA

SIGMA

NU SIGMA.

DEG • $F$.

SCATTER

TRANSPORT

ABSORPTION

FISSION

1.5000E 03

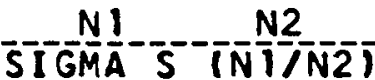

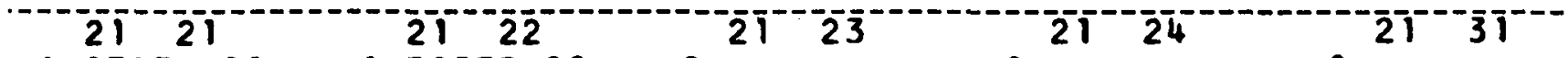
L 0707E 01
2221
I. $22253 \mathrm{E} 22$
Q. 22
Q. 22
o.
$\overline{2} 2$

$\ldots 1.66 \frac{6}{2} 30 \mathrm{O}=01$

$7.4030 \frac{3}{23}=0$

ㅇ. $=-\frac{1}{2}--\frac{-1}{24}$

$-0$.

$1 \cdot \frac{1}{2} \frac{079 E}{2} \overline{3}$

$3 .-\frac{9}{2} 432 E-0.1$

$-\overline{3}--\frac{5}{3} \bar{i}^{--}$

- 0.

$\overline{3} \bar{i}-\overline{2}$

Q.- $31--\frac{2}{22}$

$2.0181 E-\frac{00}{3}$

$8.8712 E \frac{00}{2}$

$1.6019 \mathrm{E}-03$

- 0.

ㅇ..

$1.3210 E-02$

$1.9478 E 00$

9. $-\frac{7}{3} \frac{19 E-0 !}{3} \div$

9.2202E 00

- TEMPERA A UURE

DEG. F.

SIGMA

SIGMA

TRANSPORT

1. 1TOSE Ōi
SIGMA

ABSSORPTION

$1.0 \overline{3} \overline{35}$ E OT
NU SIGMA

FISSTON

'1.00000

\section{STGMA}

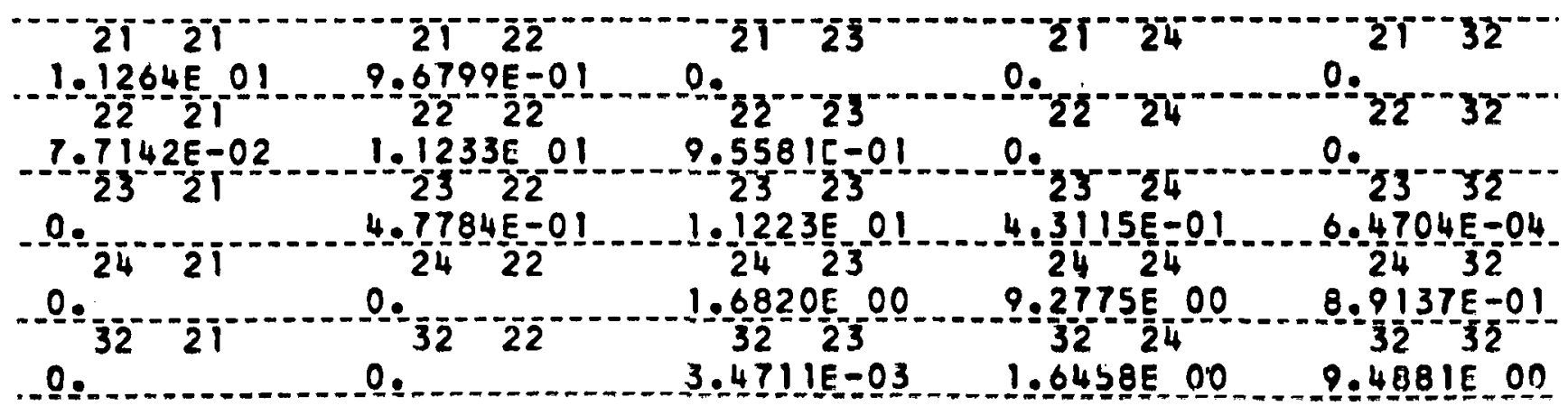


IHORIUM 232

THERMAL TRANSFER MATRICES

TEMPERATURE
SEGIGMA
SIGMA S $(N 1)$ N2)

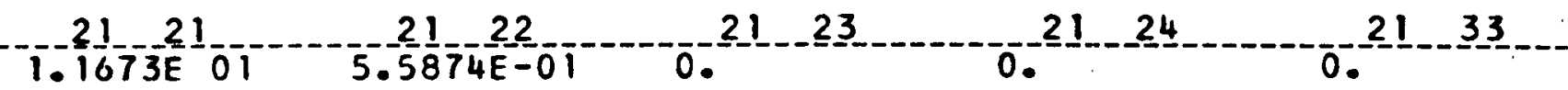

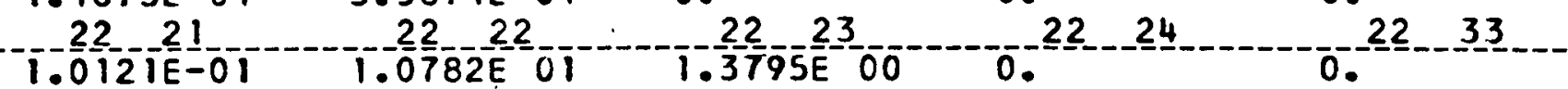

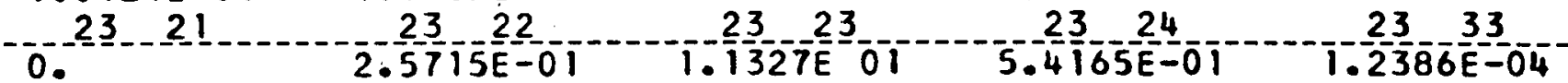
-0. - 0.33

TEMPERATURE
SIGMA
DEG

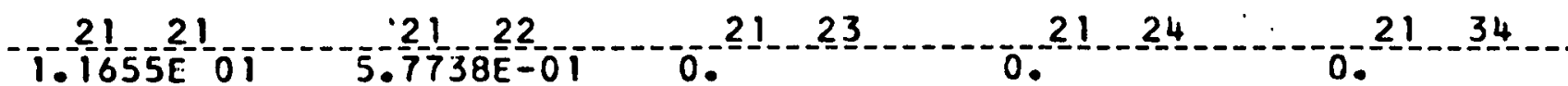

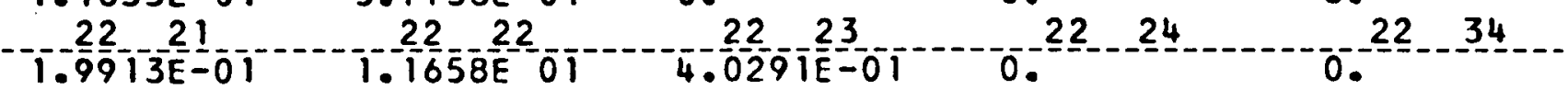

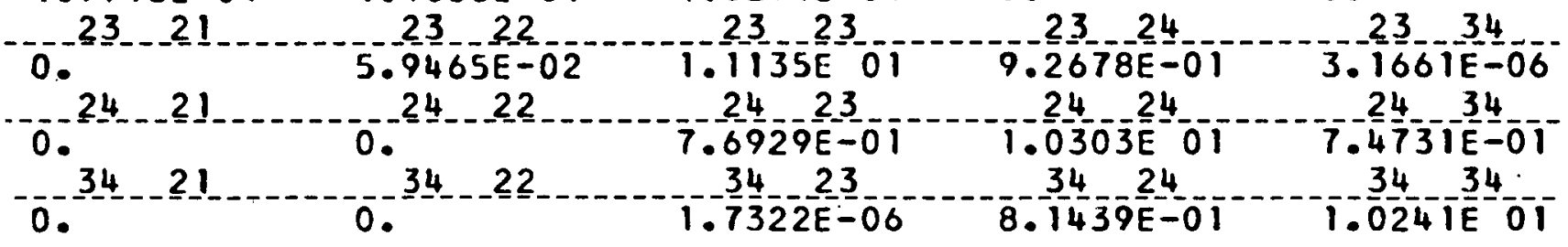


THORIUM 232

IRANSFERS FROM GROUPS 1 THROUGH $\rightarrow$ TO GROUPS 1 THROUGH 16

N1 1

SIGMA SO (NI/N2)

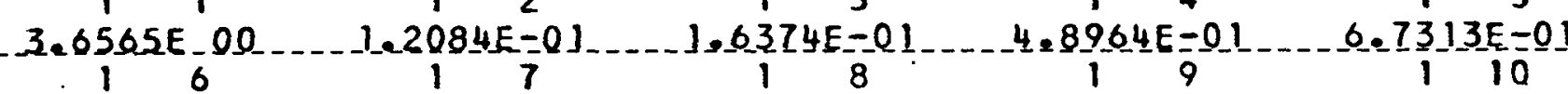

$5.6180 E=01 \ldots 3.4205 E=01 \ldots 1.7129 E=01$

111 I 12 13

114

15

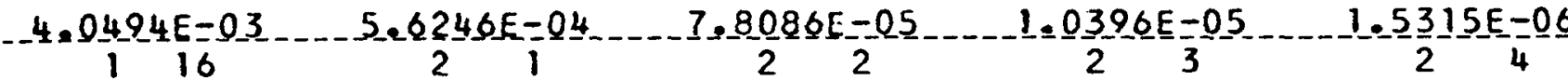

$1.1844 E-07$

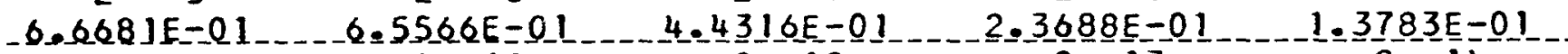
210
211
212
213
214

-4. $105 Z E=02$

6. $1504 E=03$

8.5-220E $=04$

$1.19 .62 E=04$

1.5240E -05

215

2. 34요트 $=06$

34.

1. $8168 \mathrm{E}=07$

35

$5.3229 E=Q$

6. $2533 E=02$

39

$2.1548 E=0$

314

$-2.7328 \mathrm{E}=05$

4. $0292 E=06$

$0.2-1$

$3.9463 E$

$4-\frac{4}{4} 3 E$

$3.1233 E-01$

$4-13$

4. $45400=05$

$-3.33-2.9 E=02$

0 .

5

0.

58

$4.3480 E=01$

513

512

$-03$

5
$2.8750 E-03$

..2.8750E-03 $6.4 .0561 E=04$

0.

32

33

$0 .-13$

$4.4287 E-00$

$2.3014 \mathrm{E}=0.1$

5. $8230 E=$

$1.0388 E=02$

$1.4665 E=03$

$3.4509 \mathrm{E}-0$

$=01$

$20480 E-0$

316

$3.1170 E=07$

$4=4477 E=01$

410

$1.0441 E=0$

415

$6.5711 E=06$

$0 .-$

4

6. $9052 E-01$

0.

$\frac{0480 E-0}{2}$

6

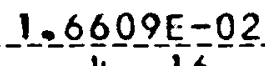

6.7738E-01

$2.3749 E-03$

$5.0847 E-07$

55

0 .

56

0.

$-2=6043 E=01$

\section{0}

5 . 11

$3.3088 \mathrm{E}=0.1$

$1.1233 \mathrm{E}=01$

515

$1.9801 E-02$

- 0 .

606

$0=-\frac{1}{6}$

$5.4 \frac{339 E}{6}=05$

8.023IE-06

$5 \quad 16$

6.2856 E 00

6 11

$2.85599=01$

ㅇ.--

8

0.

$6.2100 E-07$

$9.8905 E-03 \quad 1.4619 E-0$

6 16

$4619 E-03$

$1.6718 \mathrm{E}-01$

$1.4420 E-01$

$\underline{0}$

60

$3.1903 E=07$

$2.0759 E-04$

$2.7881 \mathrm{E}-05$

$5.6976 \frac{6}{6}-02$

$4 \cdot 1204 E-06$

$-\frac{4}{7}-1$

o. 
THORIUM 232

N1 N2

SIGMA_ SO_(N1/N2)

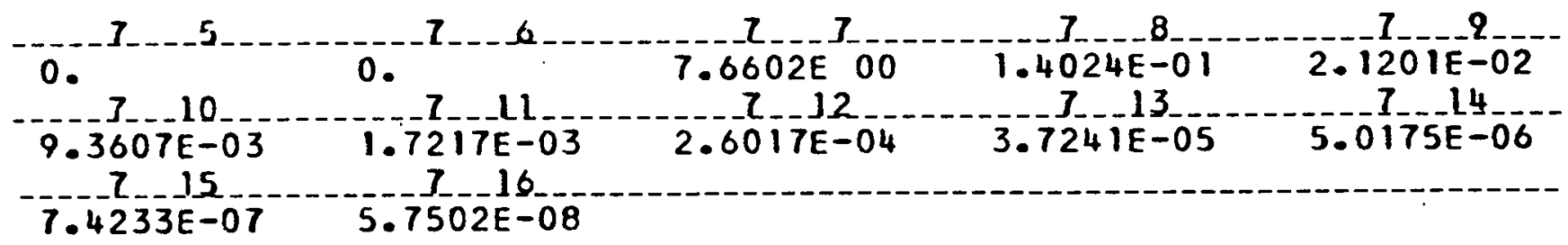


_- IHORI UM 233 _.

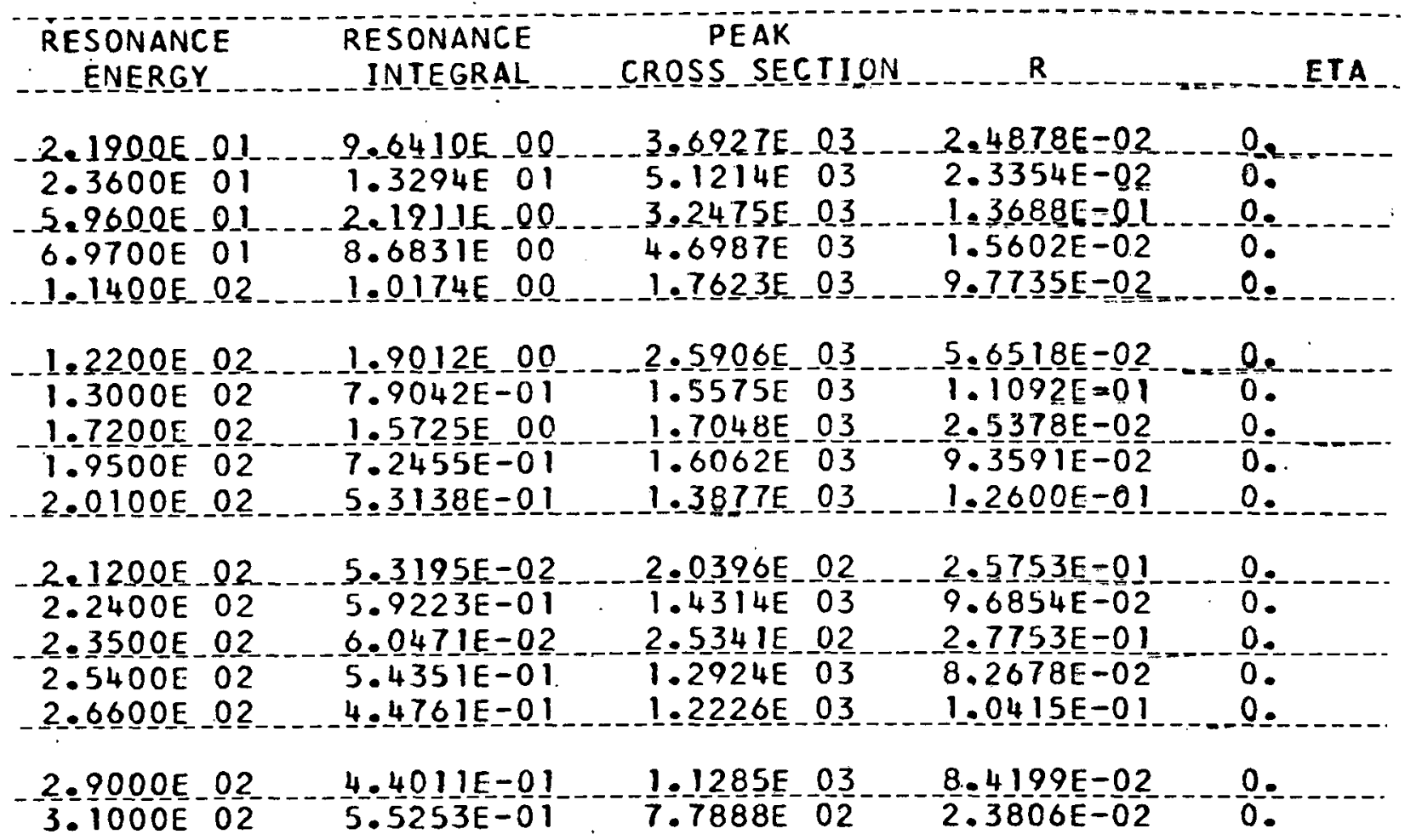


DATE $01 / 11 / 61$

DESIGNAIION GADOLINIUM
CODE NO.

64.1570
DEENSIIYY FACILOR

2.60428 E -02

\section{SIGMA XI SIGMA SIGMA SIGMA XI SIGMA}

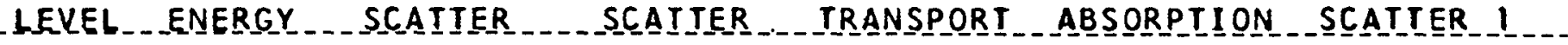

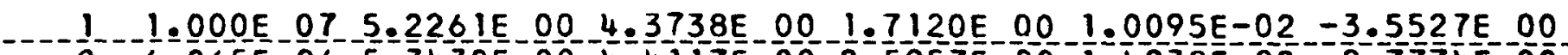

$20.065 \mathrm{E} 065.3430 \mathrm{E} 004.47 \mathrm{TE} 002.5957 \mathrm{E} 00-1.4938 \mathrm{E}-02-2.7774 \mathrm{E}$

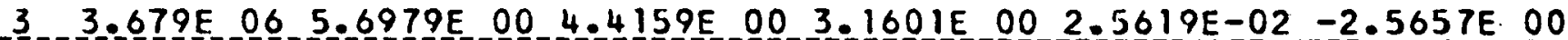

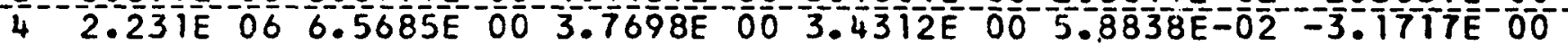

5 1.353E 06 ? 1973 E 00 2.6320E 00 4.0365E 00 1.2198E-01 -3.1956E 00

$6 \quad 8.208 E$ E $7.2459 \mathrm{E} \quad 00 \quad 1.2804 \mathrm{E} 00$ 5.2907E $00 \quad 1.8110 \mathrm{E}-01-1.9766 \mathrm{E}-00$

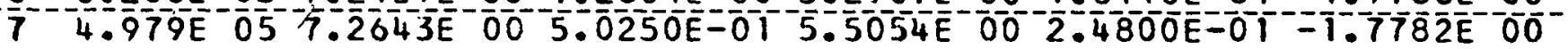

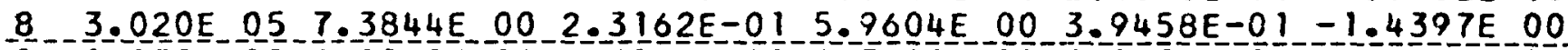

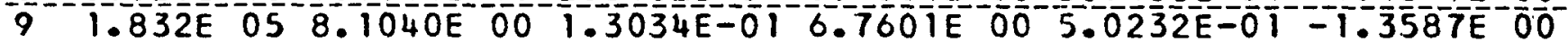

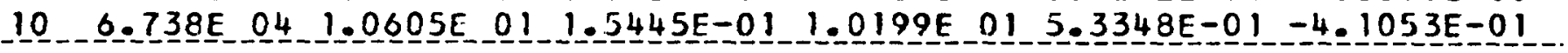

11 2.479E 04 1.1499E $01 \quad 1.3754 \mathrm{E}-01 \quad 1.1447 \mathrm{E} \quad 011.1341 \mathrm{E} \quad 00-5.3478 \mathrm{E}-02$

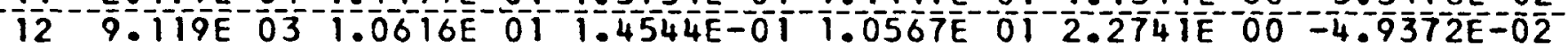

$13 \quad 3.355 E \quad 03 \quad 1.2720 E \quad 01 \quad 1.7426 E-01 \quad 1.2661 E \quad 014.1162 E \quad 00-5.9153 E-02$

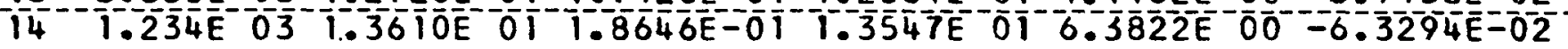

15 4.54OE 02 1.8120E 01 2.4824E 01 1.8036E 01 1.3758E 01 -8.4268E-02

$16 \quad 8.719 E \quad 01 \quad 1.7173 E .012 .3527 E-01 \quad 1.7094 E \quad 012.4235 E \quad 01-7.9866 E-02$

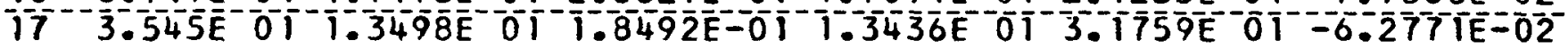

$18 \quad 1.304 \mathrm{E} \quad 01.1 .6010 \mathrm{E} \quad 01 \quad 2.1934 \mathrm{E}-011.5937 \mathrm{E} \quad 013.9795 \mathrm{E} \quad 01-7.4457 \mathrm{E}-02$

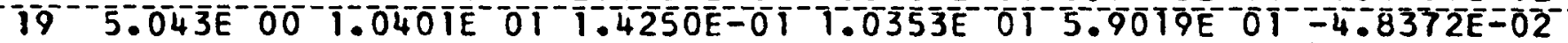

20 1.445E 00 1.3605E 01 1.8638E-01_1.3542E 01 1.2018E $02-6.3270 \mathrm{E}-02$

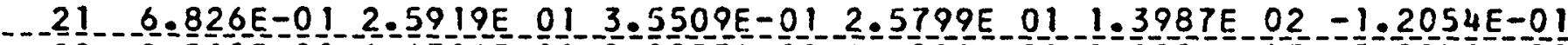

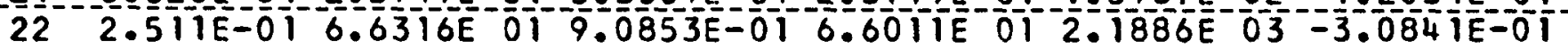

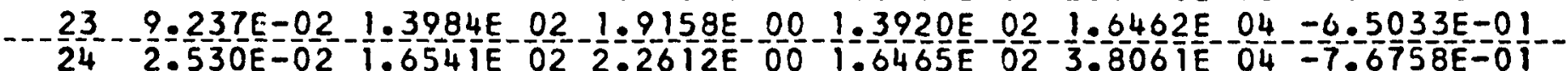

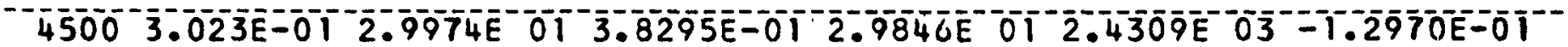
$40002.718 E-013.3228 E$ O $14.2452 E-013.3086 E \quad 012.8761 E \quad 03-1.4378 E-01$

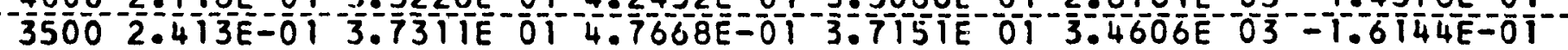

3000 2.109E-01 4.2544E $015.4355 E-014.2362 E$ O1 $4.2501 E-03-1.8410 E-01$

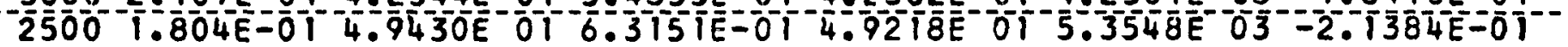

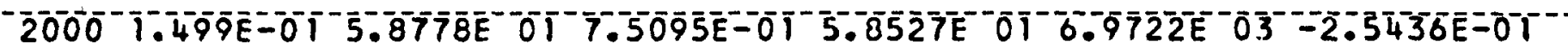

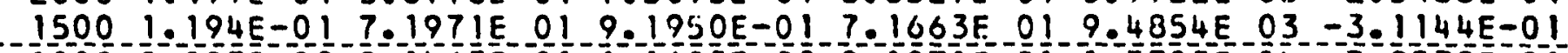

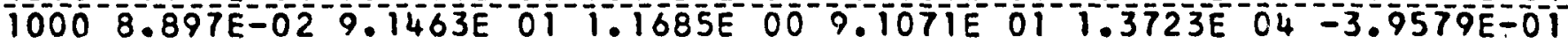

500 5.850E -02 1.2160E 02 1.5536E 00 1.2108E 02 2.1769E $04-5.2672 \mathrm{E}-01$

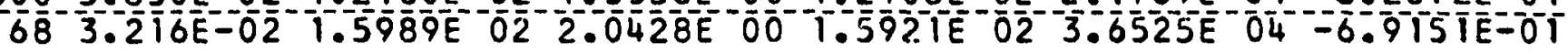

NOTE: For a summary of the sources of data see APEX-704. 
GADOLINIUM

IHERMAL_IRANSEER_MAIRICES

IEMPERATURE DEG F . SCATTER 4.
SIGMA

SCATTER

SIUMA

TRANSPORT
SIGMA

ABSORPTION
NU_ SILMA_-

FISSION

$4.5000 E$ O3

$1.0948 \mathrm{E} \quad 02$

$1.6875 E \quad 02$

$0.0519 \mathrm{E} 04$

0.

$N 1-N 2$

SIGMA S (NIIN2)

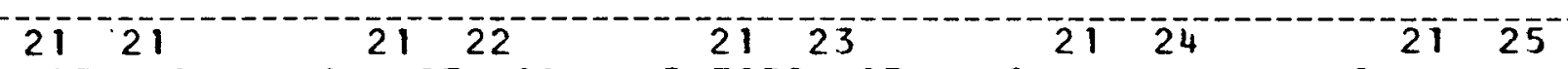

$2.4076 E 0$

2221

1. $8423 \mathrm{E}-\underline{0} \underline{0}$

$3.70396-07$

O.

2224

$2.9348 \mathrm{E}-04$

2324

$22 \quad 25$

$4.3147 E-00$

5.9 14 IE 01

2321

2322

$3.0555 E-00$

5. $\frac{24}{24} 18 \mathrm{E}-00$

1. $6187 \mathrm{E}-06$

3. $4744 E=06$

$1=96960 \mathrm{E}-00$

$1=\frac{1}{2} \frac{5}{4}-\frac{8}{2}{ }^{3}-2$

2424

1. $5631 E-02$

$25-\frac{1}{21}$

o.

2522

$5 . \frac{5}{25}-30 E-01$

$25-\frac{2}{4}$

4. 9700E 01

$23 \quad 25$

$3.2356 \mathrm{E}-01$

$1.8261 E-C 4 \quad 7.7789 E \quad 00$

U.

2425

2.- $\frac{1}{2} \frac{1}{5} 03 E-\frac{0}{25}-$

1. 120OE 02 .

IEMPERAI URE

DEG. F.

4.000OE $0 \overline{3}$
SCATTER

$1.6855 \mathrm{E} 02$
SIGMA

TRANSPORT
SIGMA

ABSORPTION
NU SIGMA

FISSION
0 .

\section{SIGMA $\frac{N 1}{S}\left(N \frac{N 2}{N}\right.$}

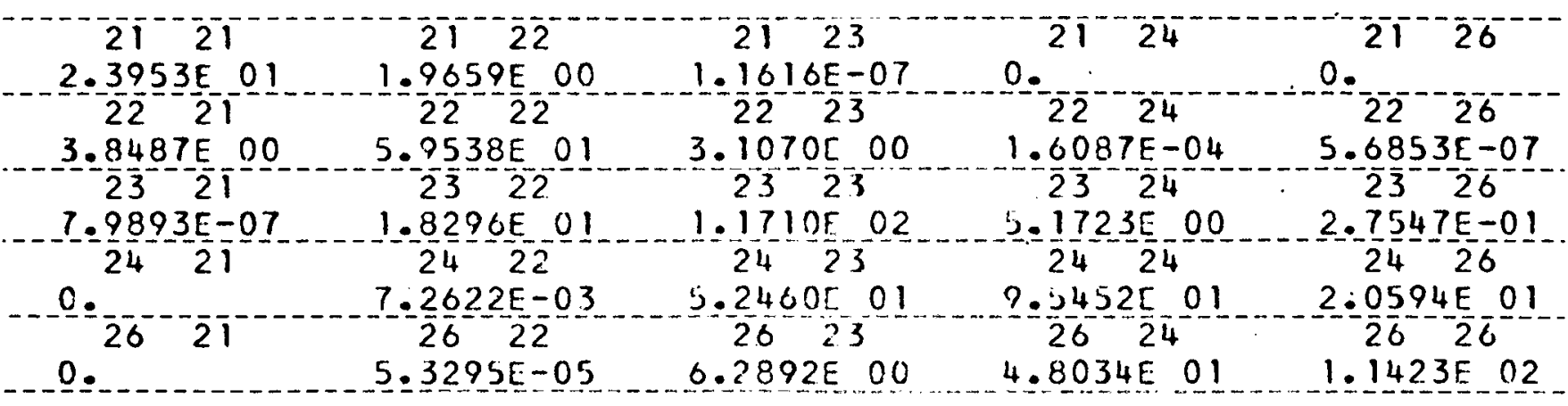


GADOLINIUM

\section{THERMAL TRANSFER MATRICES}

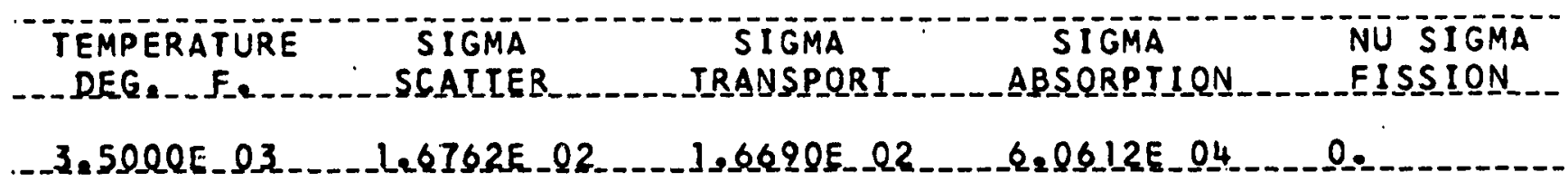

$\mathrm{N} i-1-12$

SIGMA_S_(N1LN2)

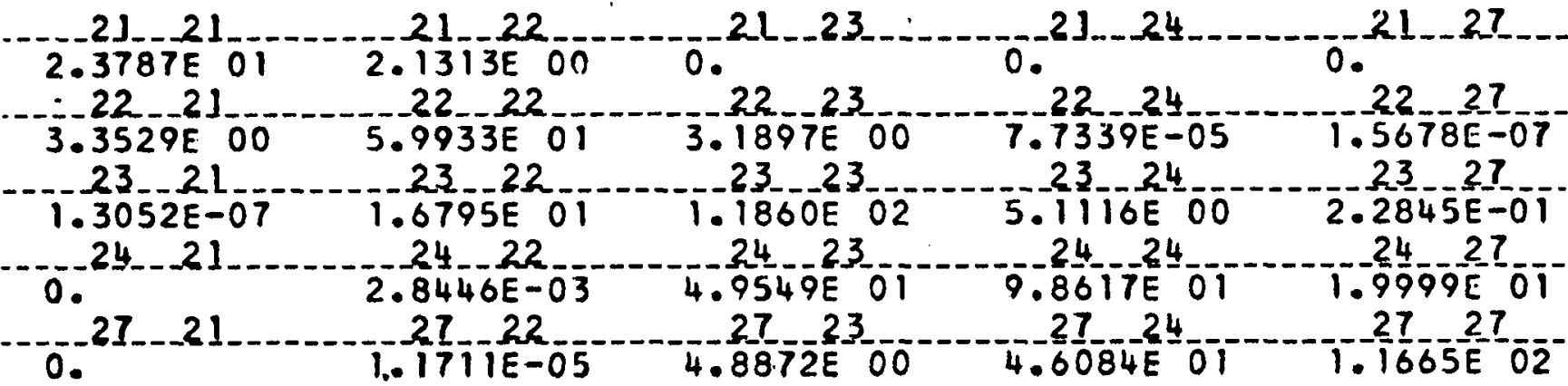

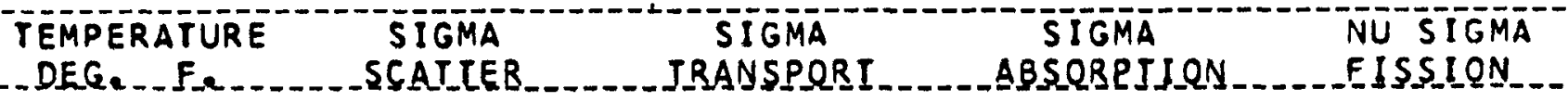

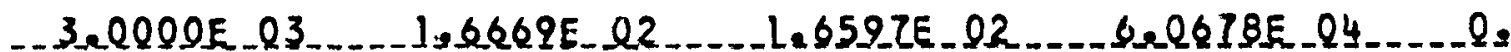

SIGMA S. SNILN2L

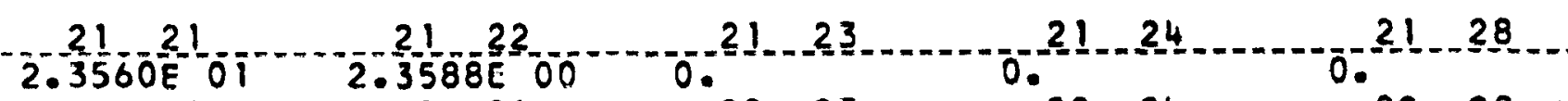

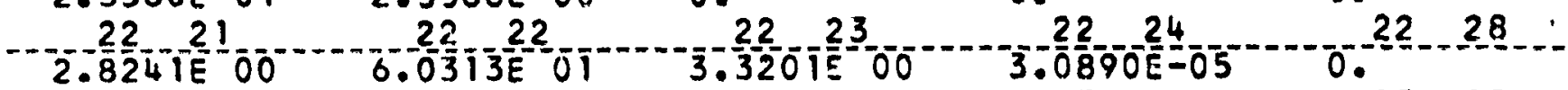

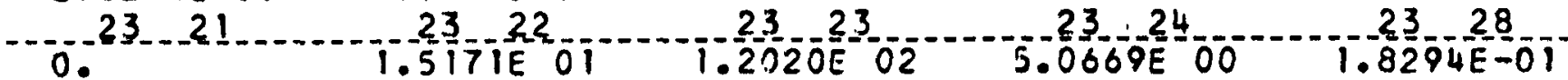

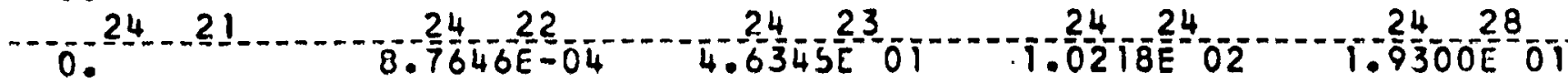

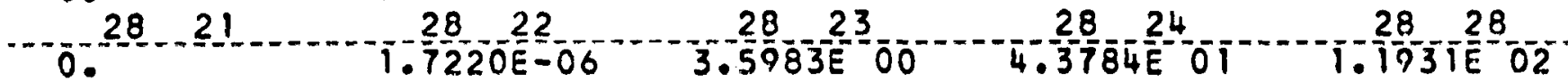


GADOL INIUM

_IHERMAL_IRANSEER_MAIRICES

IEMPERATURE

SIGMA

SIGMA

DEG. F. SCATTER

TRANSPORT

SIGMA

NU SIIGMA.

ABSORPTION

FISSION

$2.500 \overline{O E} 0 \overline{3}$

$1.6576 \mathrm{E} 02$

$1.6504 \mathrm{E} 02$

N!

N2

SIGMA S (NITN)

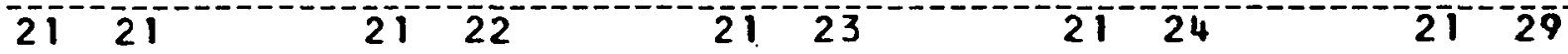

$2.3236 E-01$

$222 i$

$2.6824 \mathrm{E} 00$

$22 \quad 22$

$\underline{0}$ $22-23$ $6.0651 E$ OI

$2.2605 E_{-} 00$

2321

2322

-

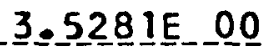

2323

1.2 $2193 \mathrm{E}-02$

$=\frac{2}{4}-\frac{1}{3}$

$6.0768 \mathrm{E} 04$

$\overline{0}$

O. $252--\frac{1}{24}$

ㅇ..

$-\frac{3}{2} 337-\frac{3}{2}=06$

…

5. $0514 \mathrm{E}-00$

2424

$-0=\frac{0}{29} \overline{1}$

$1.89942 E-04$

4. $\frac{2775 E}{29}-\frac{01}{23}$

ㅇ..

1. $3928 E-07$

$2.4521 \mathrm{E} 00$

1. $\frac{395}{24}$ I $\frac{E}{29}=01$

1.0622E 02

I. $\frac{8}{2} \frac{7}{9}-\frac{8 E}{29} 01$

4. $1048 E 01$

1.2226E 02 .

IEMPERAIURE

DEG. $F$.

SIGMA

SIGMA

SCATTER

TRANSPORT

SIGMA

NU SIGMA

$2.0000 \bar{O}$

$1.6 \overline{82} \overline{\mathrm{E}} \mathrm{02}$

$1.6 \overline{11 E} 02$

$6.08 \overline{8} \overline{\mathrm{E}} \mathrm{0}-\overline{4}$

0

\section{$S\left(G M A-N \frac{N}{S}(N) \frac{N 2}{N}-\right.$}

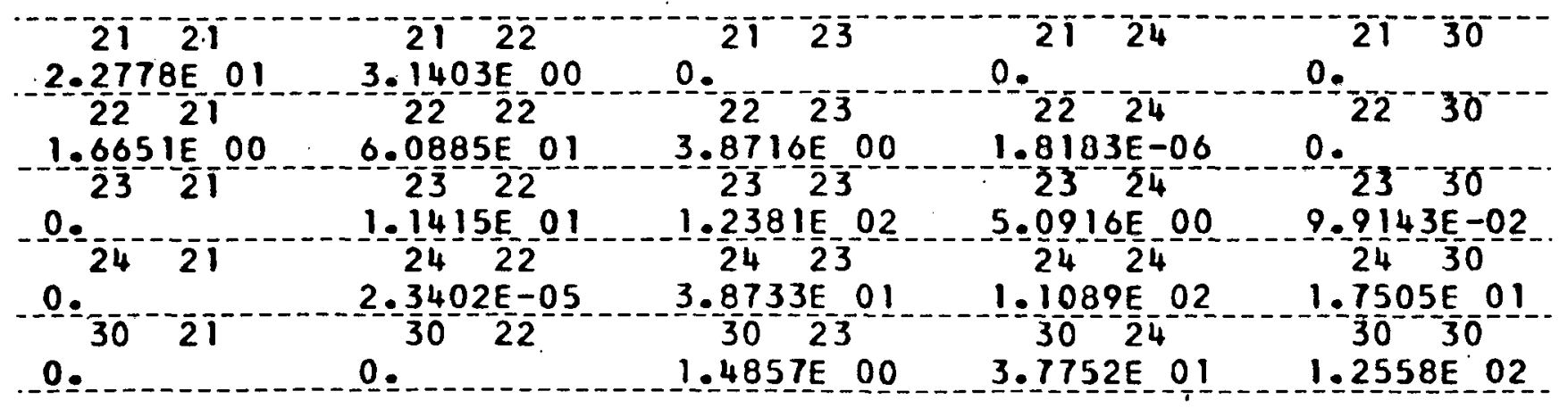


GADOLINIUM

THERMAL TRANSFER MATTICES

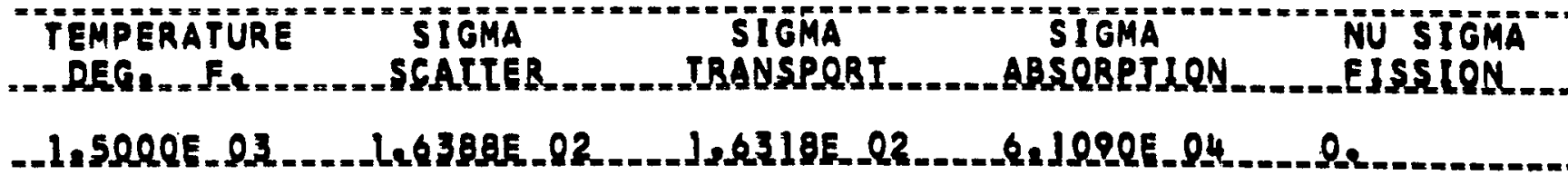

Ni

SIGMA_S_.(NILNR)

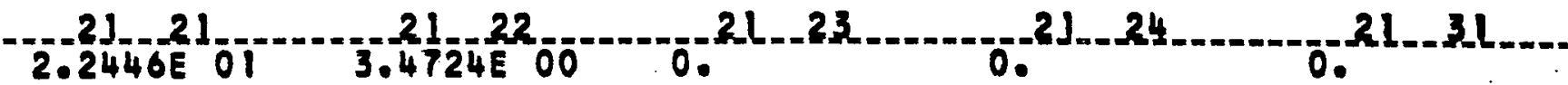

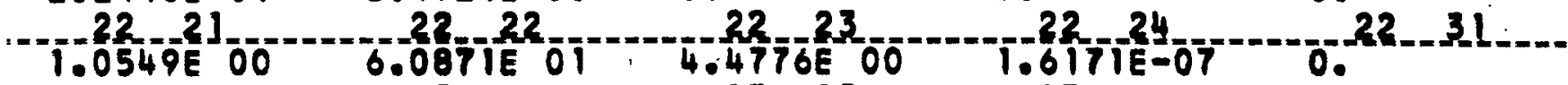
-.23 2L.

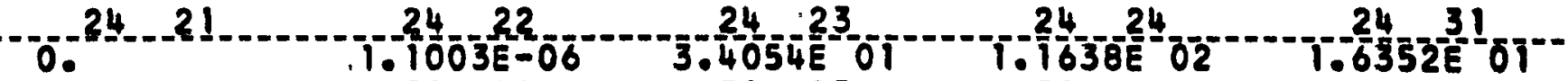

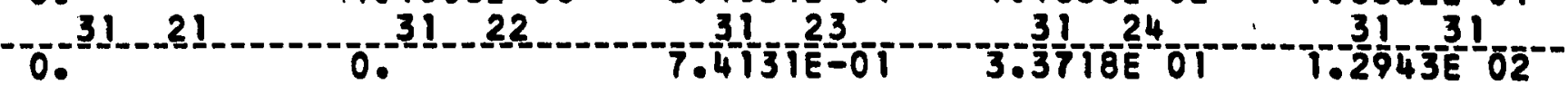

TEMPERATURE
SEG STMA
$\bar{N} \overline{\mathrm{N}}$ SIGMA S_(NIIN2)

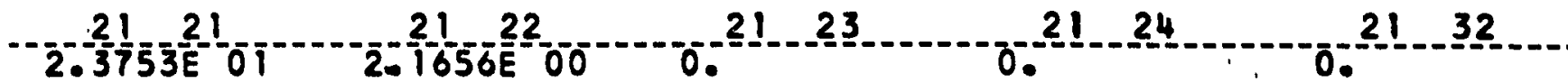

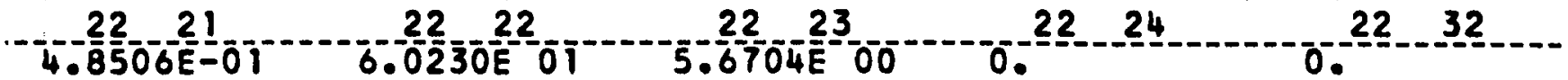

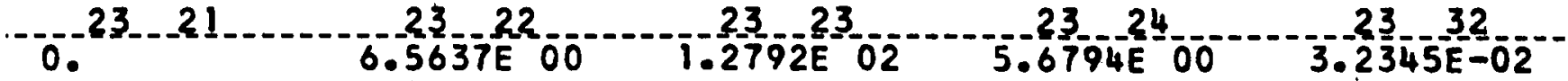
- 0.24

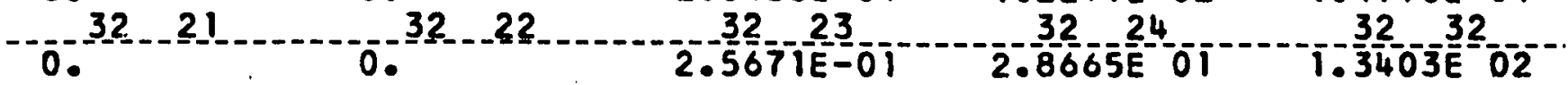


GADOL INIUN

TRANSFERS FROM GROUPS I THROUGH 10 TO GROUPS 1 THROUGH 16

$\mathrm{N} 1$ N2

SIGMA_SO_(NIIN2)

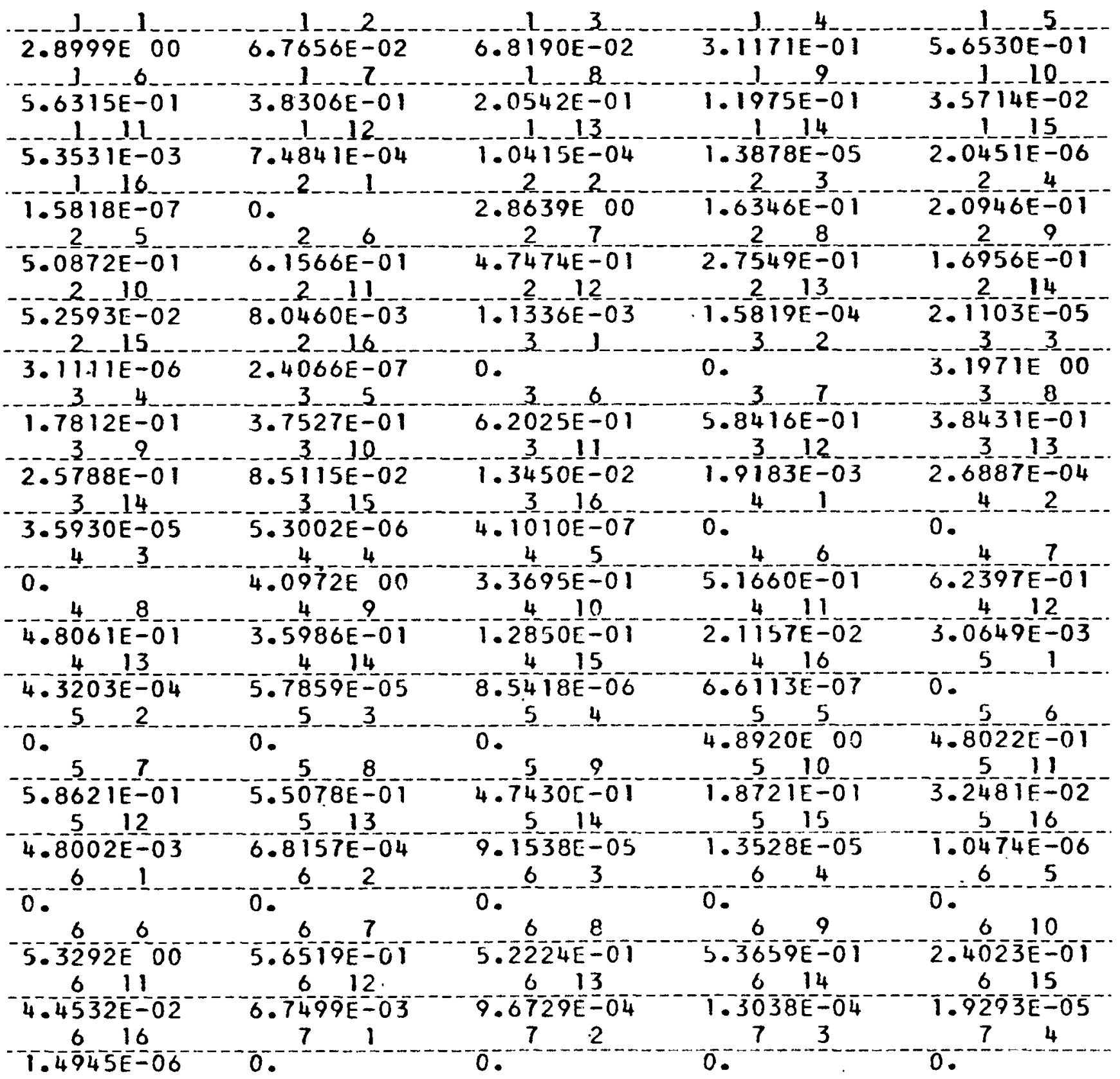


.-GADOLINIUM .

SIGMA $\operatorname{SO}^{-1}(\mathrm{~N} 1 / \mathrm{N} 2)$

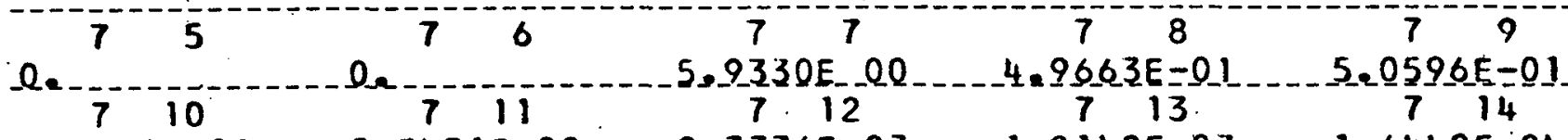

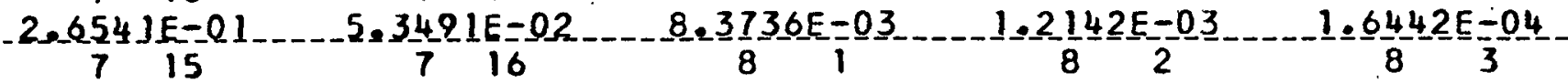

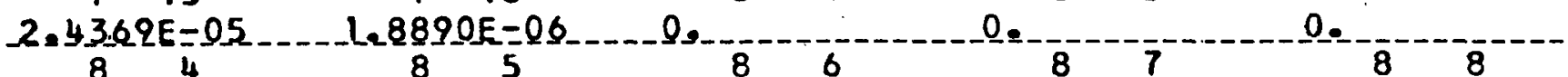
Q.

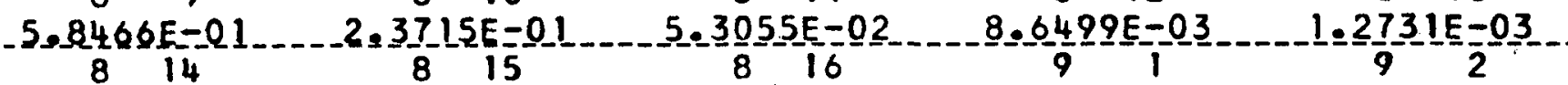

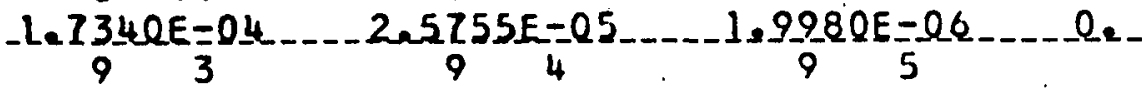

Q.

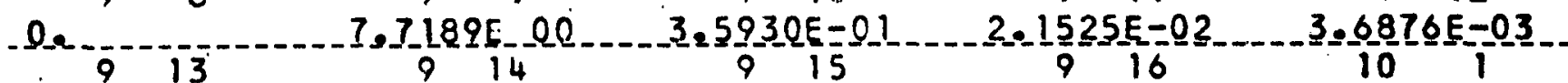

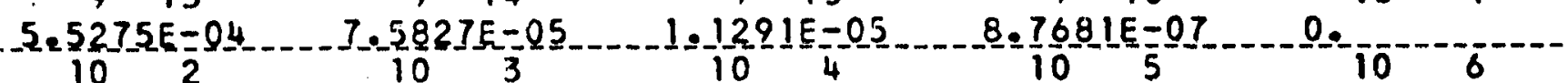
${ }^{0} 10^{10} 7^{0} 10^{0}$

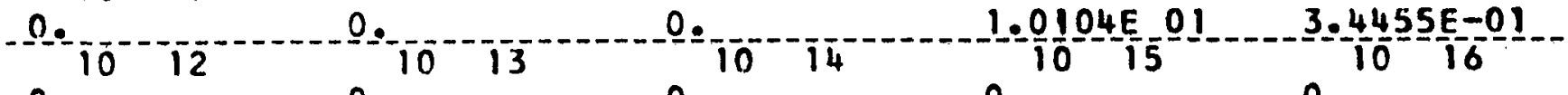
ㅇ.. o. ㅇ.. 
GADOL INIUM

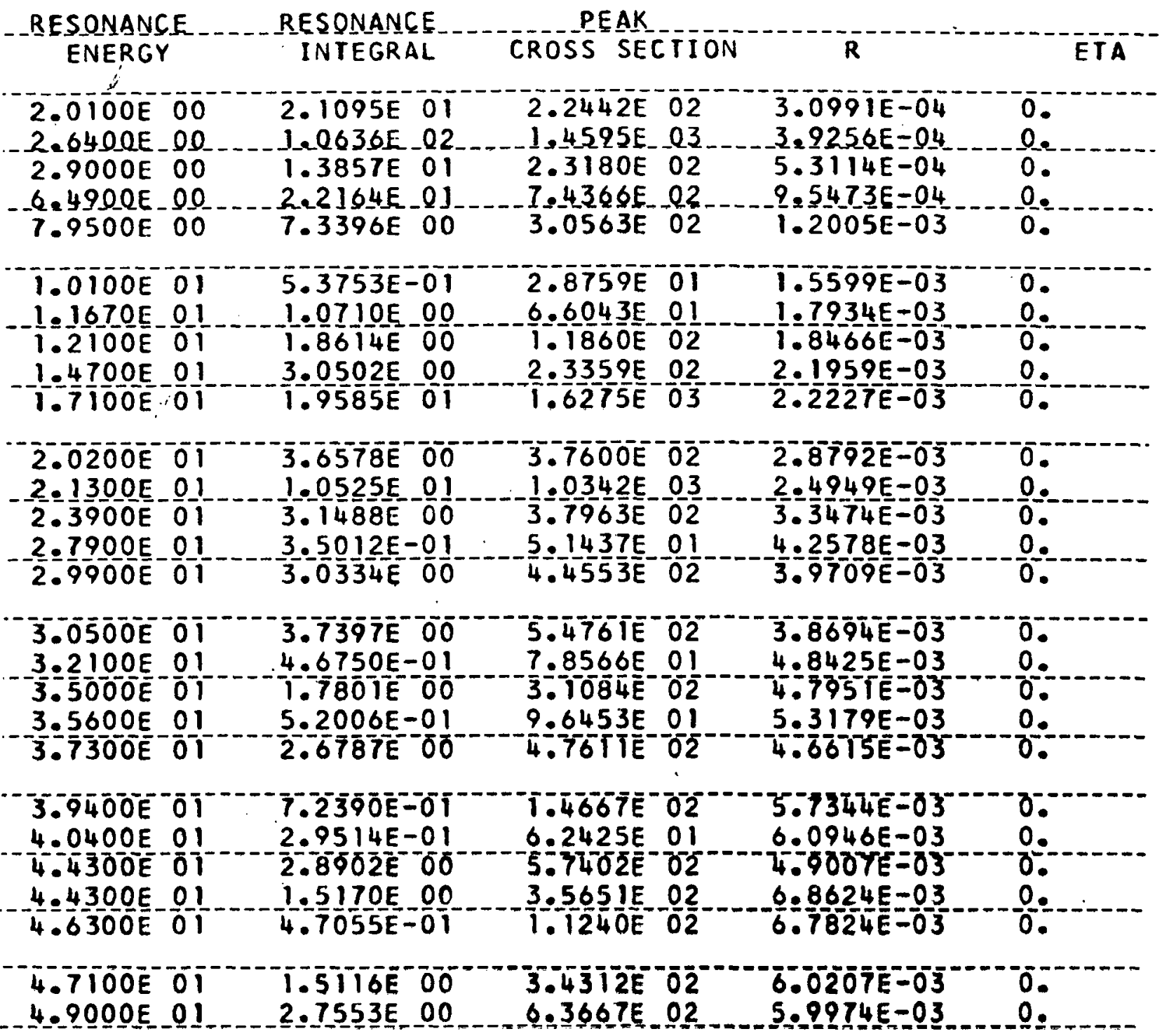


GADOLI NIUM :

TRANSFERS FROM GROUPS I THROUGH 34 TO GROUPS I THROUGH 11

SIGMA N,G $(N I T N 2)$

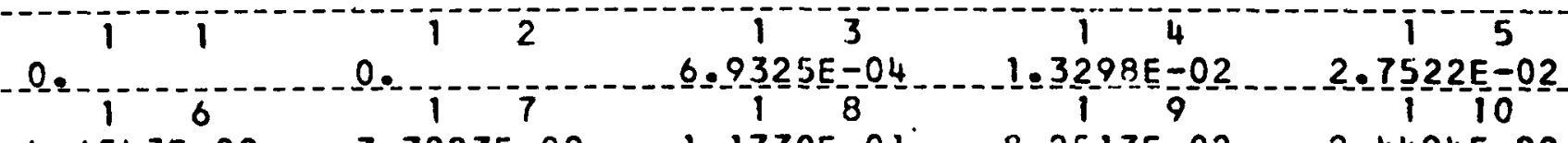

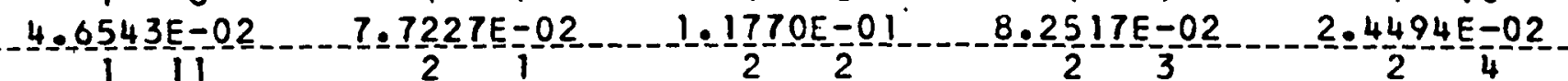
- $4.8360 E-04$

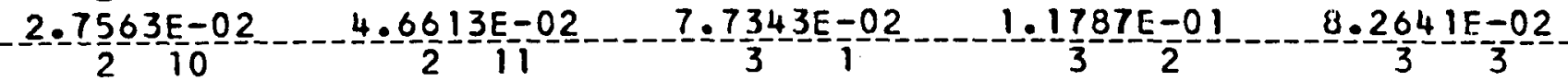

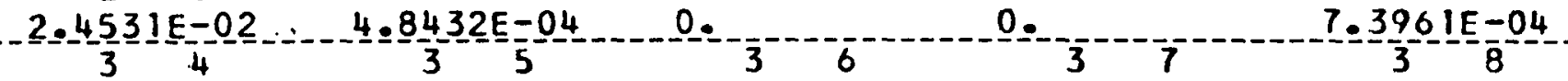

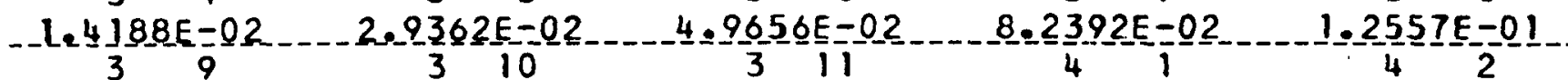
$8.8036 E-02 \quad 2.6 \frac{1}{4} 33 E=02$ $8.3419 E-04$ 4 4 $3.311725=02$ $1.4162 E=01 \ldots 9.9294 E-02$ 5 5.3 -0. 54 $-14-\cdots$ ㅇ.4 ‥ $1.0988 E-01 \ldots 1.6746 E-01 \ldots 1.1741 E-01$ -0.

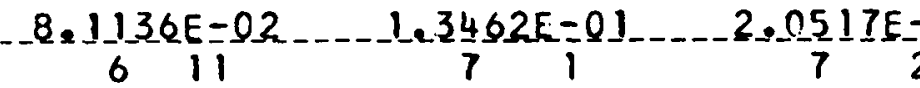
..8.-4302E=04 75 7 $0 .-7$ 5 5 $6006 E-02$ $9.2928 E-02$ $0.0265 E=02$ 710 $1.01925=0$ 5. 3635 S $5=02$ 3. $71896-02$ $1.05892=03$ $1.6910 E=01$ $5.8192 \mathrm{E}=04$ 0 . 5 
GADOLINIUM

Ni 2

SIGMA N, G INIIN I I

10
0.10
0.10


-GADOLINIUM .

SIGMA $N, G(N)-N(N 2)$

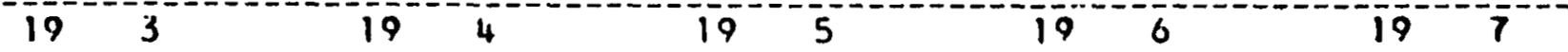

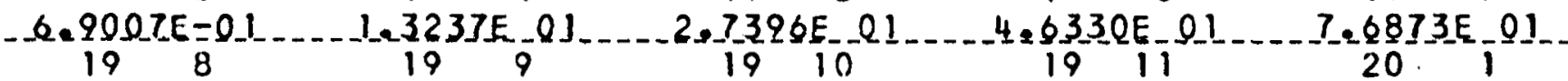

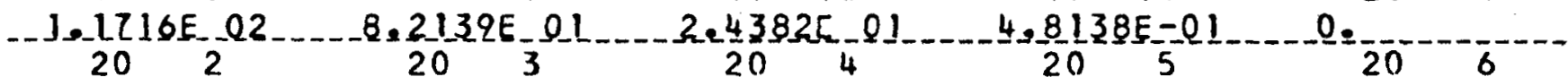

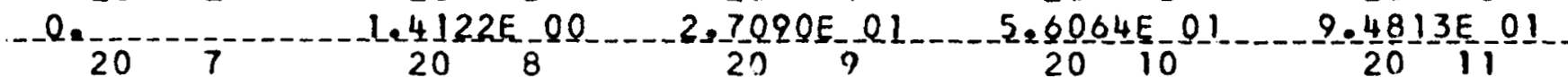

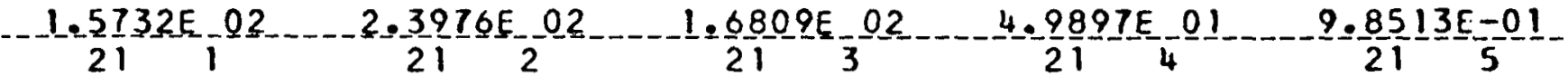

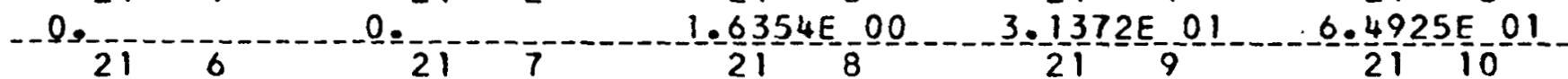

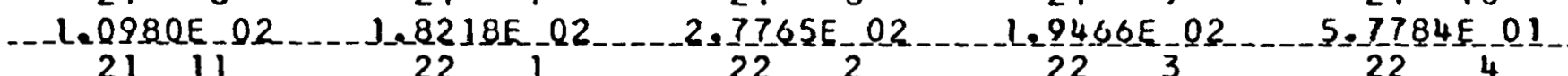

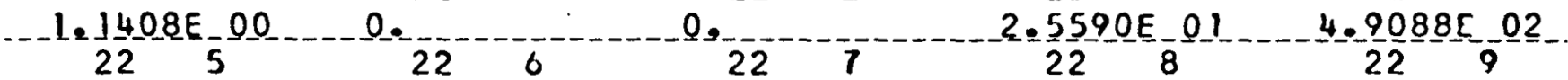

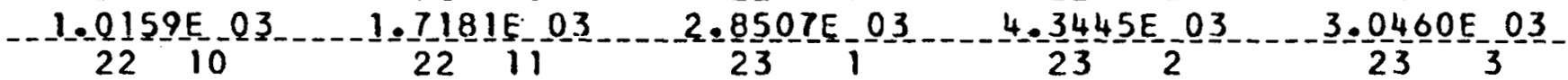

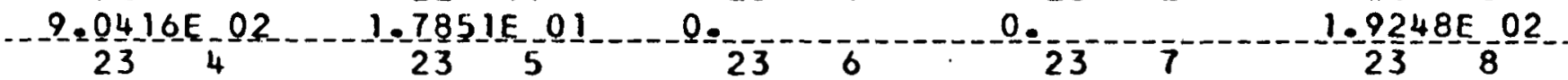

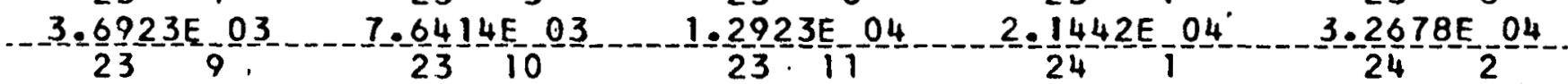

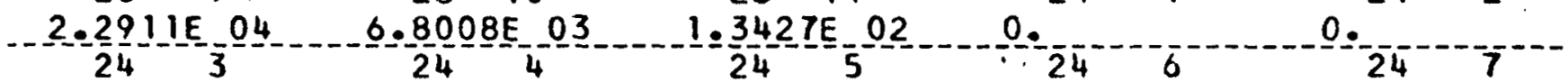

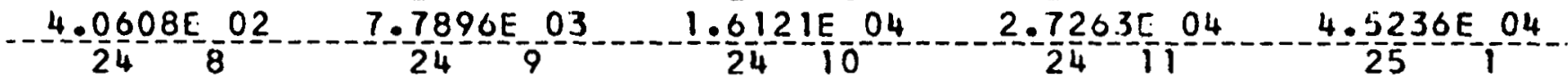

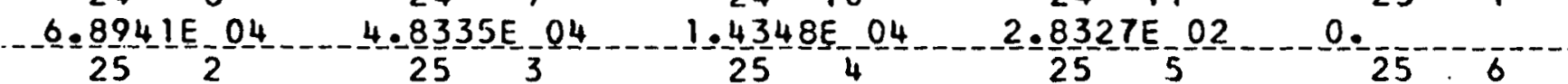

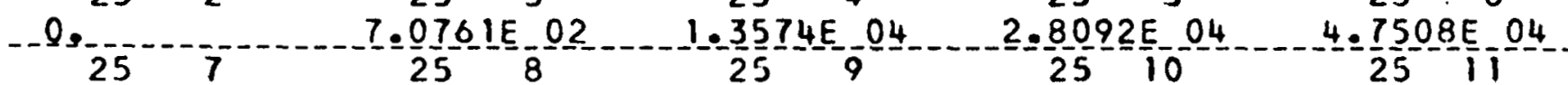

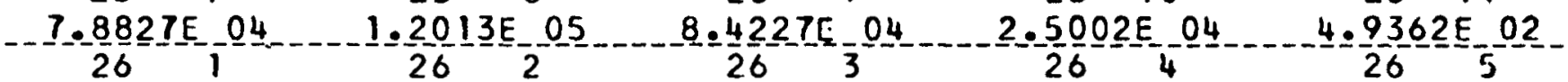

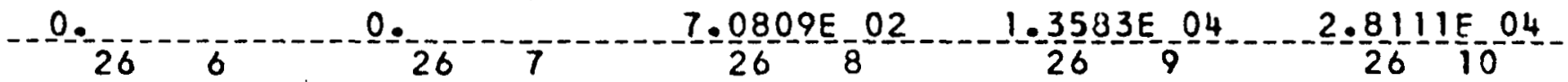

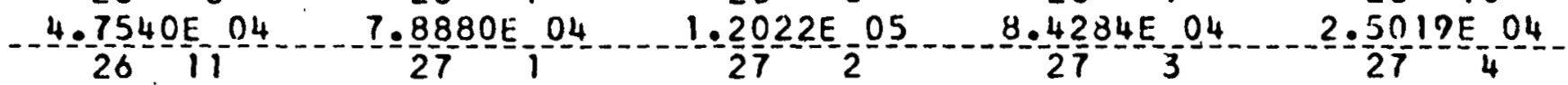

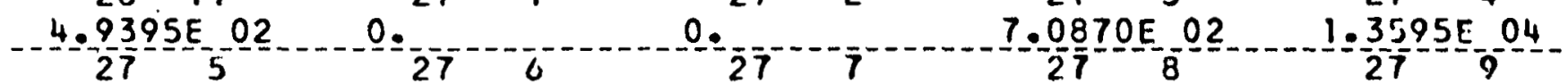

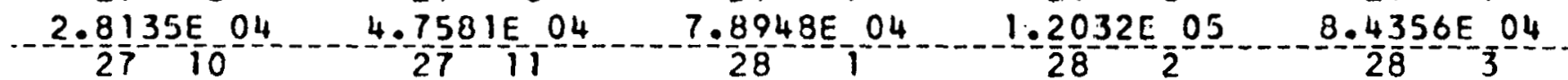

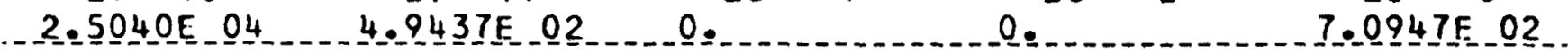


GADOL INIUM

\section{SIGMA N.G INIIN2)}

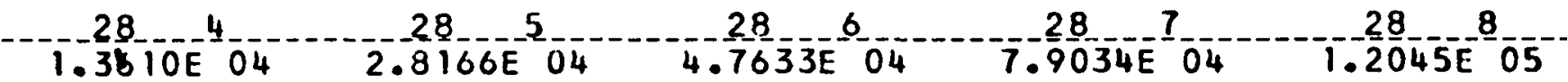

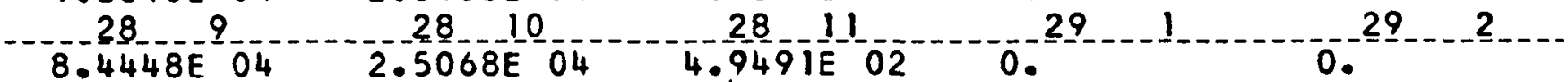

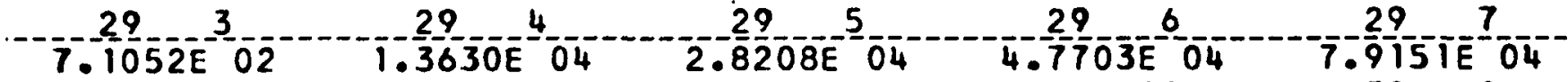

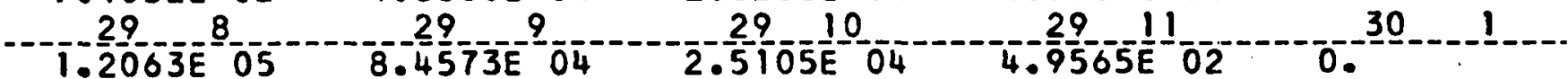

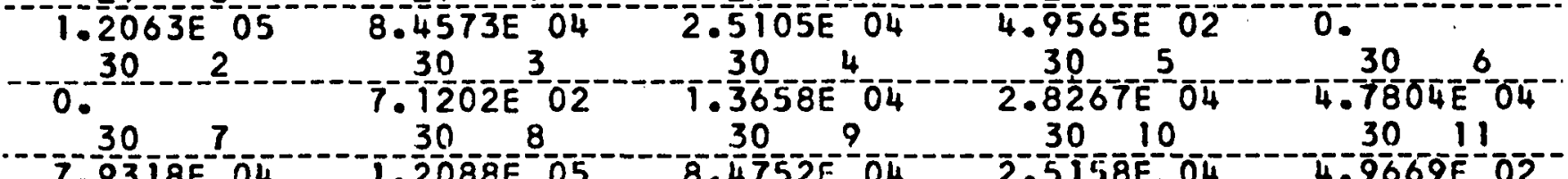

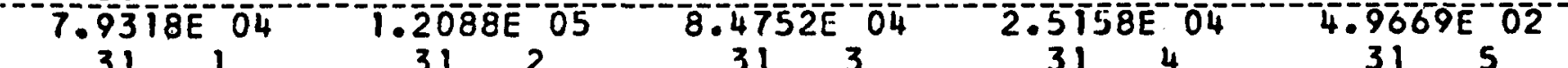
-31.

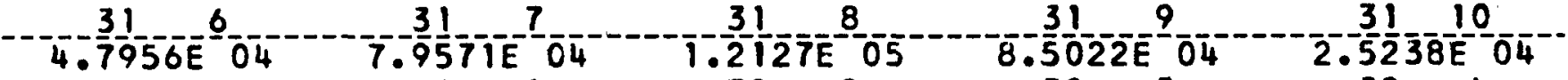

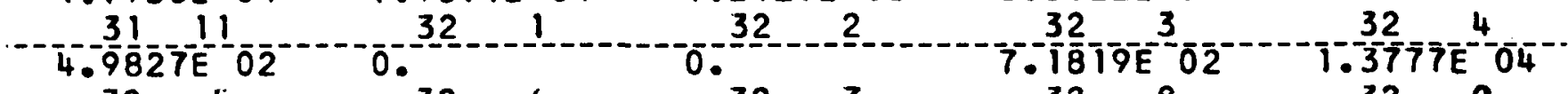

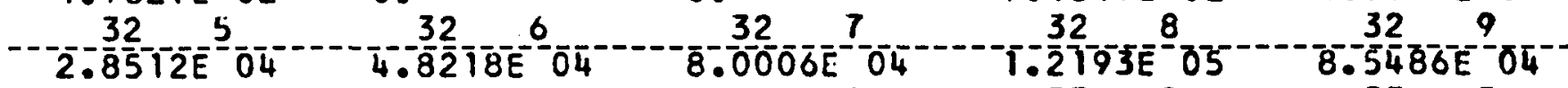

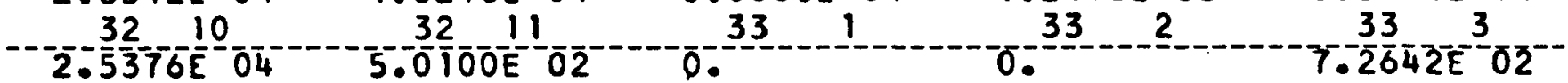

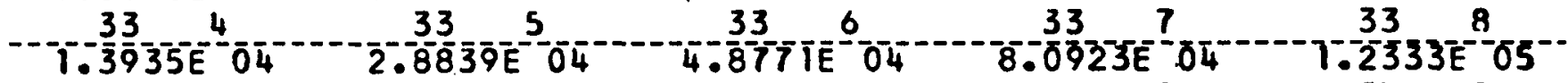

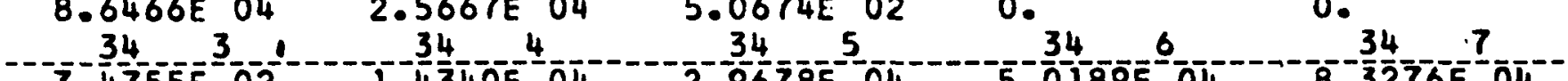

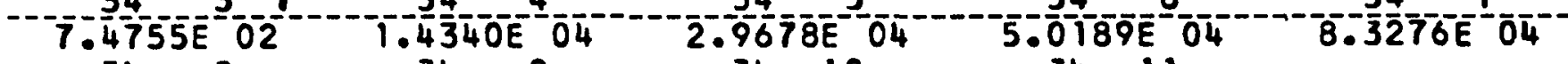

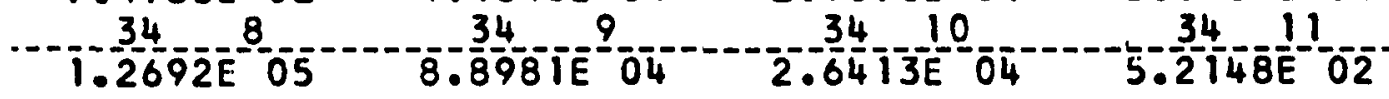


DAIE

DESIGNATION

EUROPIUM
$\overline{C O D E} \bar{N} \bar{O}^{-}$

$6 \overline{3} \cdot \overline{1} \overline{2} 20$

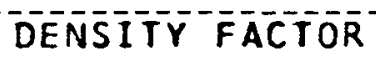

$\overline{2} \cdot \overline{5} \overline{2} \overline{2} \overline{\overline{5}} \overline{\mathrm{E}} \overline{\mathrm{O}} \overline{2}$

\section{LEVEL ENERGY SCATTER SCATIER TRANSPORT ABSORPTION SCATTER}

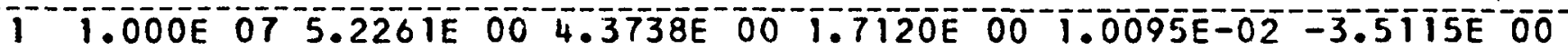

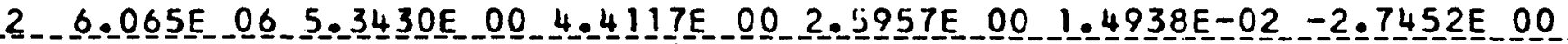

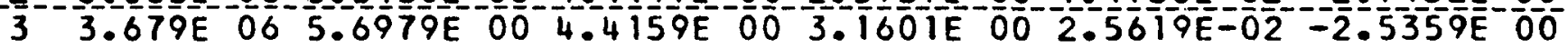

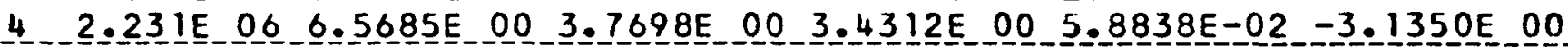

5 1.353E $067.1973 \mathrm{E} 00 \mathrm{2} .6320 \mathrm{E} 00-4.0365 \mathrm{E} 0 \mathrm{1}$

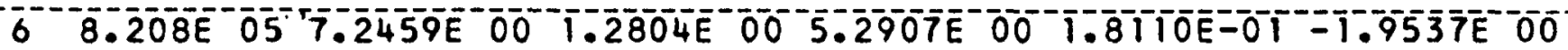

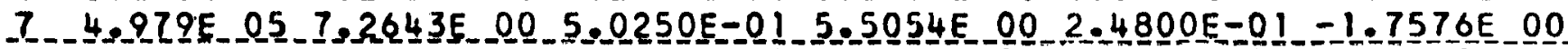

8 3.020E $057.3844 \mathrm{E} 00$ 2.3162E-0 $5.9604 \mathrm{E} 00 \quad 3.9458 \mathrm{E}-0 \mathrm{1}-1.4230 \mathrm{E} 00$

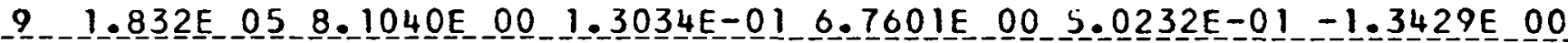

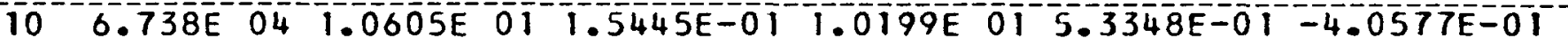

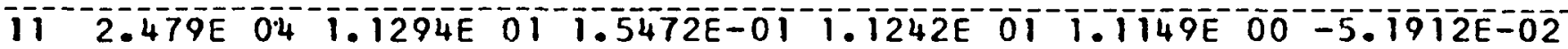

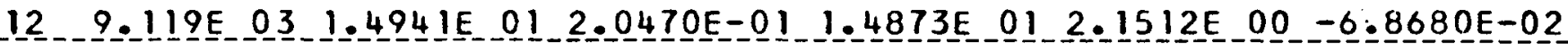

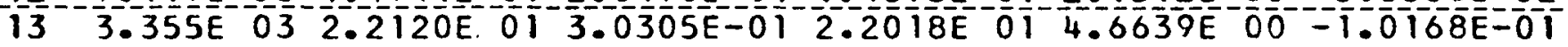

$14.1 .234 \mathrm{E} 03$ 2.9710E 01 4.0703E-01 2.9574E 01 9.5585E $00-1.3657 \mathrm{E}-01$

$154.540 \mathrm{E} 024.2574 \mathrm{E}$ OI $5.8327 \mathrm{E}-0 \mathrm{~T} 4.2378 \mathrm{E} 0 \mathrm{1} 2.3725 \mathrm{E} 0 \mathrm{~T}-1.9569 \mathrm{E}=0 \mathrm{~T}$

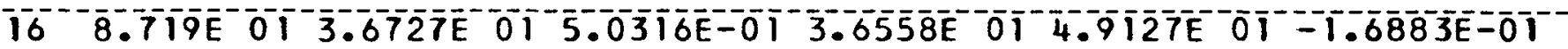

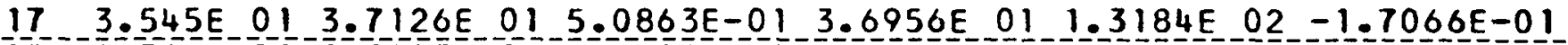

$18-1.304 \mathrm{E} 01.7593 \mathrm{E} 012.410 \mathrm{E}-0 \mathrm{1}-75 \mathrm{~T}$

19 5.043E $0001.4280 \mathrm{E} 01$ 1.9564E-01_1.4214E 01_4.7670E $02=6.5640 \mathrm{E}-02$

$20-1.445 \mathrm{E}$ 00 $9.1859 \mathrm{E} 001.2585 \mathrm{E}-0 \mathrm{~T} 9.1436 \mathrm{E} 00-4.5825 \mathrm{E}-02-4.2225 \mathrm{E}-02$

$210.826 \mathrm{E}-0 \overline{3} .1194 \mathrm{E}$ O

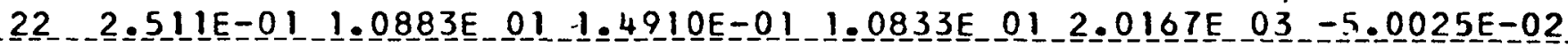

$23-9.237 \mathrm{E}-028.5506 \mathrm{E}$ 00 $1.1714 \mathrm{E}-018.5113 \mathrm{E}$ 00 $1.4610 \mathrm{E} 03-3.9304 \mathrm{E}-02$

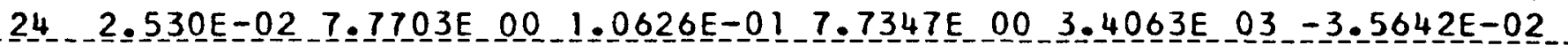

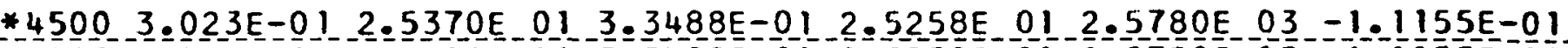

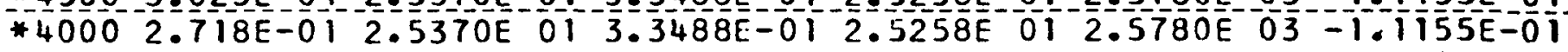

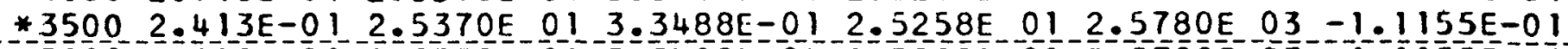

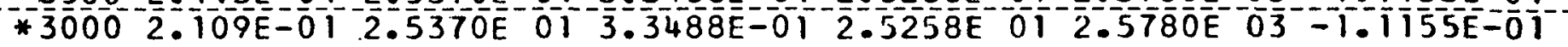

$25001.804 \mathrm{E}-012.3170 \mathrm{E} 013.0584 \mathrm{E}-012.3068 \mathrm{E} 012.4920 \mathrm{E} 03-1.0187 \mathrm{E}=01$

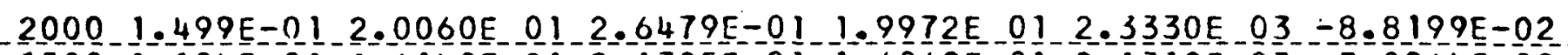

$15001.194 \mathrm{E}-011.6140 \mathrm{E} 012.1305 \mathrm{E}-011.6069 \mathrm{E} 01.2 .1310 \mathrm{E} 03-7.0964 \mathrm{E}-02$

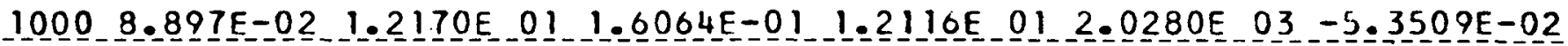

$5005.850 \mathrm{E}-029.8800 \mathrm{E}$ 00 $1.3042 \mathrm{E}-0 \mathrm{1} 9.8365 \mathrm{E} 002.3970 \mathrm{E} 03-4.3440 \mathrm{E}-02$

68 3.216E-02 1.0560E $011.3939 \mathrm{E}-01$ 1.0514E 01 3.8070E $03-4.6430 \mathrm{E}-02$

* Thermal cross sections taken from APEX-515.

NOTE: For a summary of the sources of data see APEX-704. 
EUROP I UM

\section{THERMAL TRANSFER MATRICES}

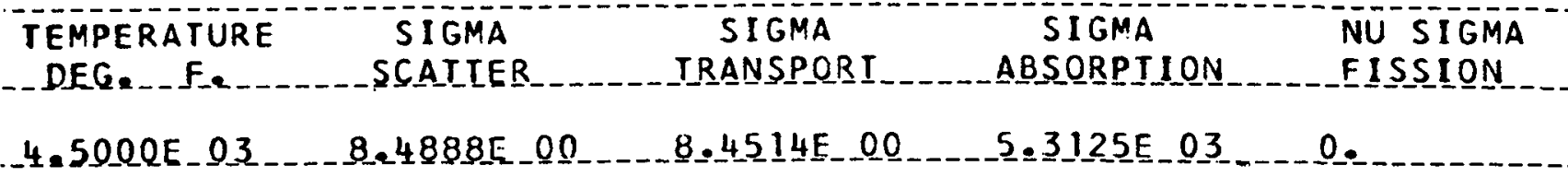

$\mathrm{Ni} N 2$

SI GMA S_ (NIIN2)

\begin{tabular}{|c|c|c|c|c|}
\hline$-2 \mathrm{~J}_{-}-21$ & $2.2424 \mathrm{E} 00$ & $\begin{array}{c}2 L-23 \\
6.4418 E-07\end{array}$ & $21 \ldots 24$ & 21 \\
\hline $\begin{array}{r}2.8951 \\
22\end{array}$ & $\begin{array}{r}2.24245 \\
-22\end{array}$ & 0.4223 & 22 & 0. \\
\hline $7.1855 E-01$ & $9.6896 \mathrm{E} 00$ & $5.0809 E-01$ & $5.8407 \mathrm{E}-05$ & $3.6492 \mathrm{E}-0$ \\
\hline $2 \mathrm{~L}$ & $23-22$ & 23 & 23 & 23 \\
\hline $3.1264 E-07$ & $1.2228 \mathrm{E} 00$ & $7.0524 \mathrm{E} \quad 00$ & $3.2434 E-01$ & $2.0916 \mathrm{E}$ \\
\hline & $9.0277 \mathrm{E}-04$ & $2.6236 \mathrm{E} O 0$ & $4.3129 E 00$ & $1.00010^{2}$ \\
\hline 0. & $1.2756 \mathrm{E}-05$ & $4.1218 E-01$ & $2.5067 \mathrm{E}=0$ & $-\frac{25}{5699-25}$ \\
\hline
\end{tabular}

TEMPERATURE
SIGMA

$4.0000 E_{-} 03$

$\mathrm{N} 1 \mathrm{~N} 2$

SIGMA_S_ (N IIN2)

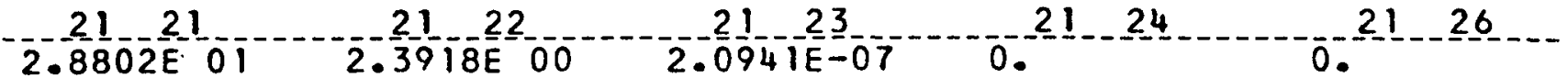

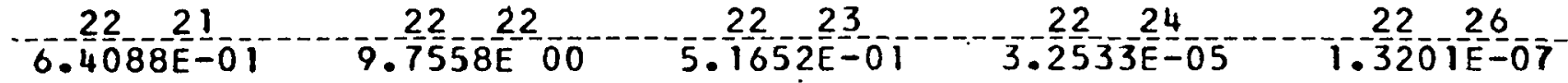

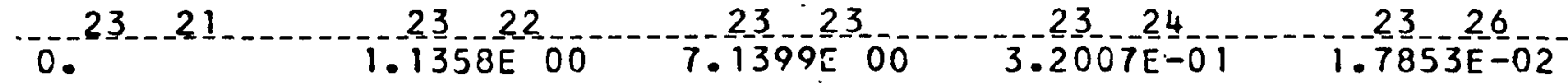

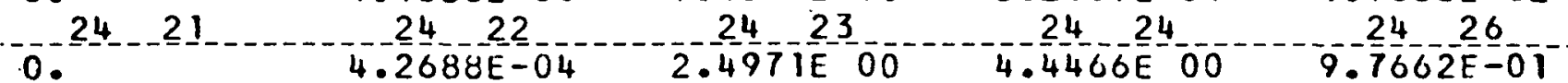
26
0. 
EUROP I UM

_LHERMAL_IRANSFER_MAIRICES

- LEMPERAIURE

SIGMA

SIGMA

SIGMA

NU_ SIGMA_-

OEG. $F$.

SCATTER

TRANSPORT

ABSORPTION

FISSION

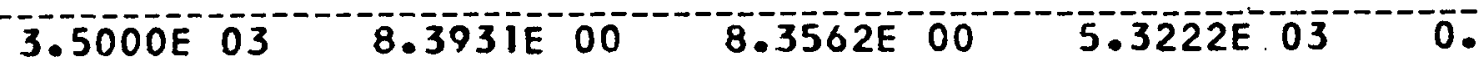

N 1

N2

SIGMA S (NIIN2)

$2 i 2 i$

$2.8602 E_{01}$ 2221

$5.5825 E-01$ 2321

$24-2 i$

$2 \frac{0}{27}$

0.
2122

2.5217E_00

$22 \quad 22$

$9.8219 \mathrm{E} \quad 00$

2322

1,04266

2422

$1.7090 \mathrm{E}=04$

2722

$8.7674 E-07$
$2 \overline{1}^{-2} \overline{3}$

$0 .-22$

5. 3009E $=01$

2323

$7.2332 E$ EO

2423

$2.3591 \mathrm{E}-\mathrm{O}$

$2.6075 E=01$
- 2

$22-24$

$1.5959 E-05$

2324

3.1633E-01

2424

$4=\frac{5956}{27} 24$

2. 3275E_00
$27-27$

0. $22-27$ $23-27$ $1.4855 \mathrm{E}-02$ 2427 $9-4 \frac{4907 E}{2}=01$ 5.8048E 00
- IEMPERAIURE

DEG. $F$.
SIGMA

SCATIER
SIGMA TRANSPORT
SIGMA ABSORPTION
NU SIGMA

FISSION

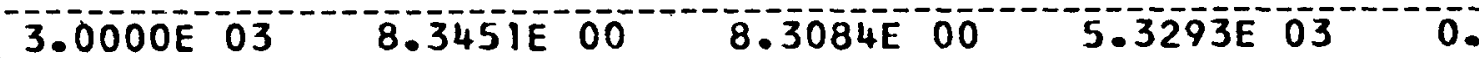

N!

N1-

SIGMA S (NITN2)

$21^{2} 1^{2} 1^{2} 21^{2} 21^{2} 21^{2}$

$2.8327 E$

22

22

$2 \overline{2}-\overline{2} \overline{3}$

0.

$0 .-22$

$-4 \cdot \frac{70}{2} 3 \frac{13 E}{2}-0$

$9.8855 \mathrm{E} \quad 00$

$5.5153 E-01$

$6.5408 E-06$

0

- 0.

$23-22$

$7.3330 \mathrm{E}$ OO

3.1359 E $=01$

2424

$1 .-\frac{194}{24}-\frac{E}{2}-02$

$-0$.

$24 \frac{1}{22}$

2423 .

$4.7 \frac{7}{2} 84 \frac{E}{2} \bar{O}^{0}$

$9 \cdot \frac{1667 E}{2} 67=01$

. 0.

$-28-22$

$28-23$

$2.2132 \mathrm{E} 00$

5.939IE 00 
EUROPIUM

THERMAL TRANSFER MATRICES

TEMPERATURE
SIGMA $\begin{gathered}\text { SIGMA } \\ \text { SIGMA }\end{gathered}$
SIGMA_S (N1 N1

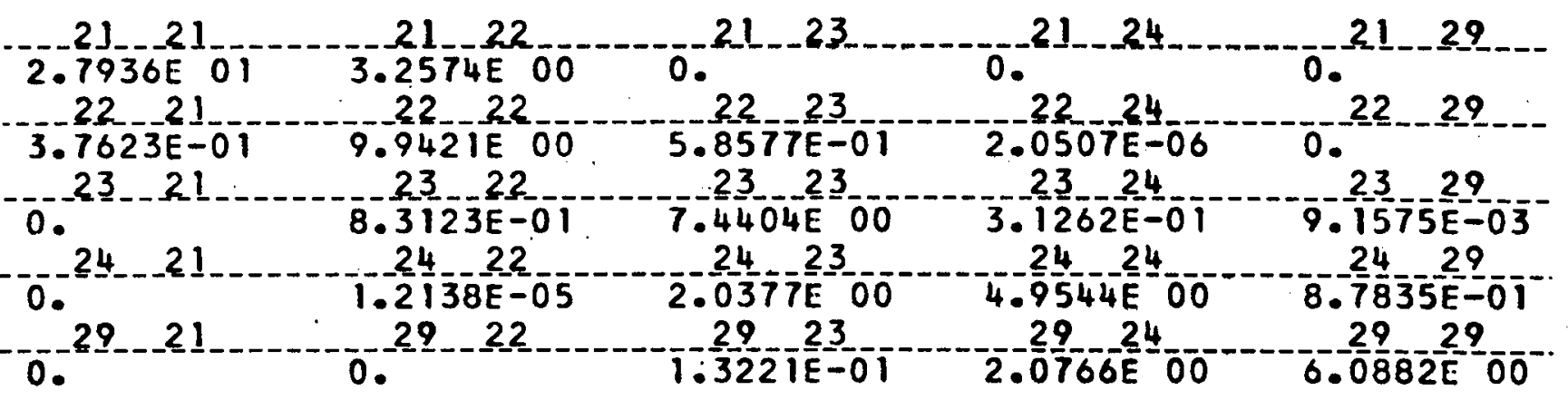

SEMPERATURE
SIGMA $\begin{gathered}\text { SIGMA } \\ \text { DEGE }\end{gathered}$

SIGMA S (NIIN2)

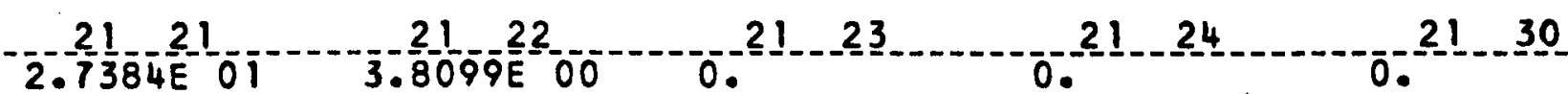

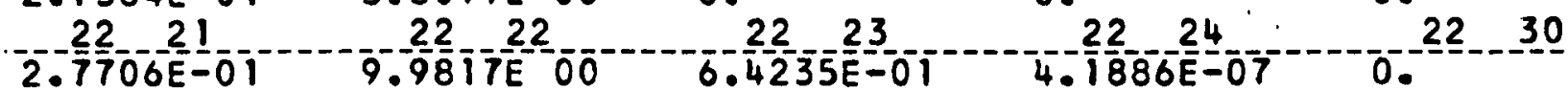

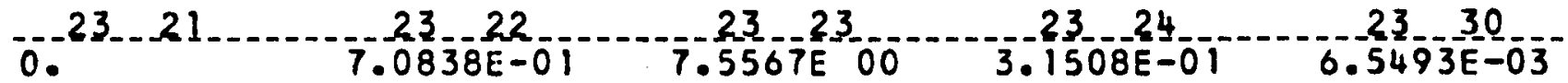

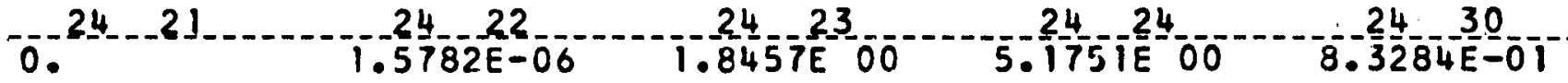

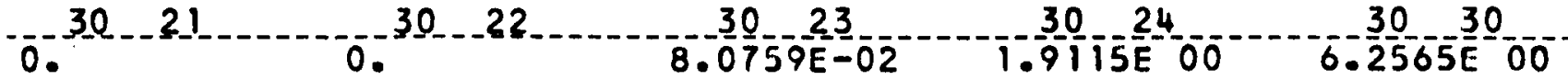


EUROPIUM

_LHERMAL_JRANSEER_MATRICES

LEMPERATURE

SIGMA

SIGMA

SISMA

DEG. F. SCAITER

TRANSPORT.

ABSORPTION

NU_SLGMA -

FISSION

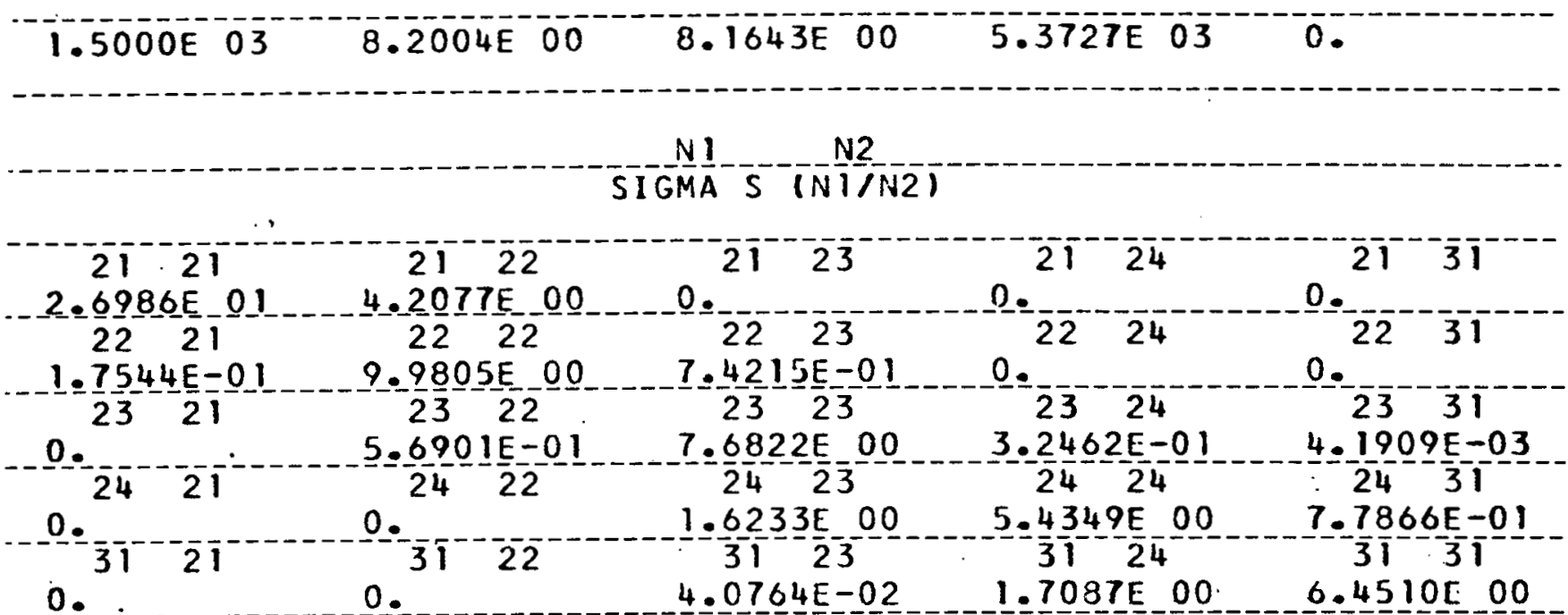

TEMPERATURE

DEG. F.

$1.0000 \bar{E} 0 \overline{3}$
SI GMA

SCATTER
SI GMA TRANSPORT
SIGMA ABSORPTION
NU SIGMA

FISSION
$8.1520 \mathrm{E}, 00$

8. 1 TOTE 00

$5.4079 \bar{E} 03$

0.

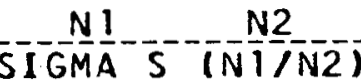

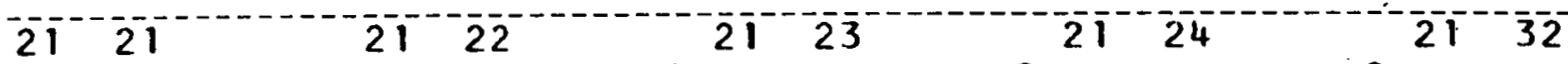

$-2.8574 \frac{0}{2} 21$

$8.06 \frac{6}{2} 19 E-02$

0.

0.

$4.0711 \mathrm{E}-01$

$2 \overline{4}$

2422

24

$3.5118 \mathrm{E}-01$

O.

-

1.3570 C 00

2424

$\overline{2} \overline{3}^{--} \cdot \overline{3} \overline{2}^{-\cdots}$

- $\underline{0}$.

ㅇ.

32 . 23

$\frac{7}{32}-\frac{85}{2} \frac{0}{4}$

$2 \frac{1}{3}-15 E-03$

$1.4381 E-02$

$1.4537 \mathrm{E} \quad 00$

$-\frac{1}{3} \frac{4}{2}-6 \frac{6}{3} \overline{2}=0$

6.6839500 
EUROPI I UM

\section{THERMAL TRANSFER MATRICES}

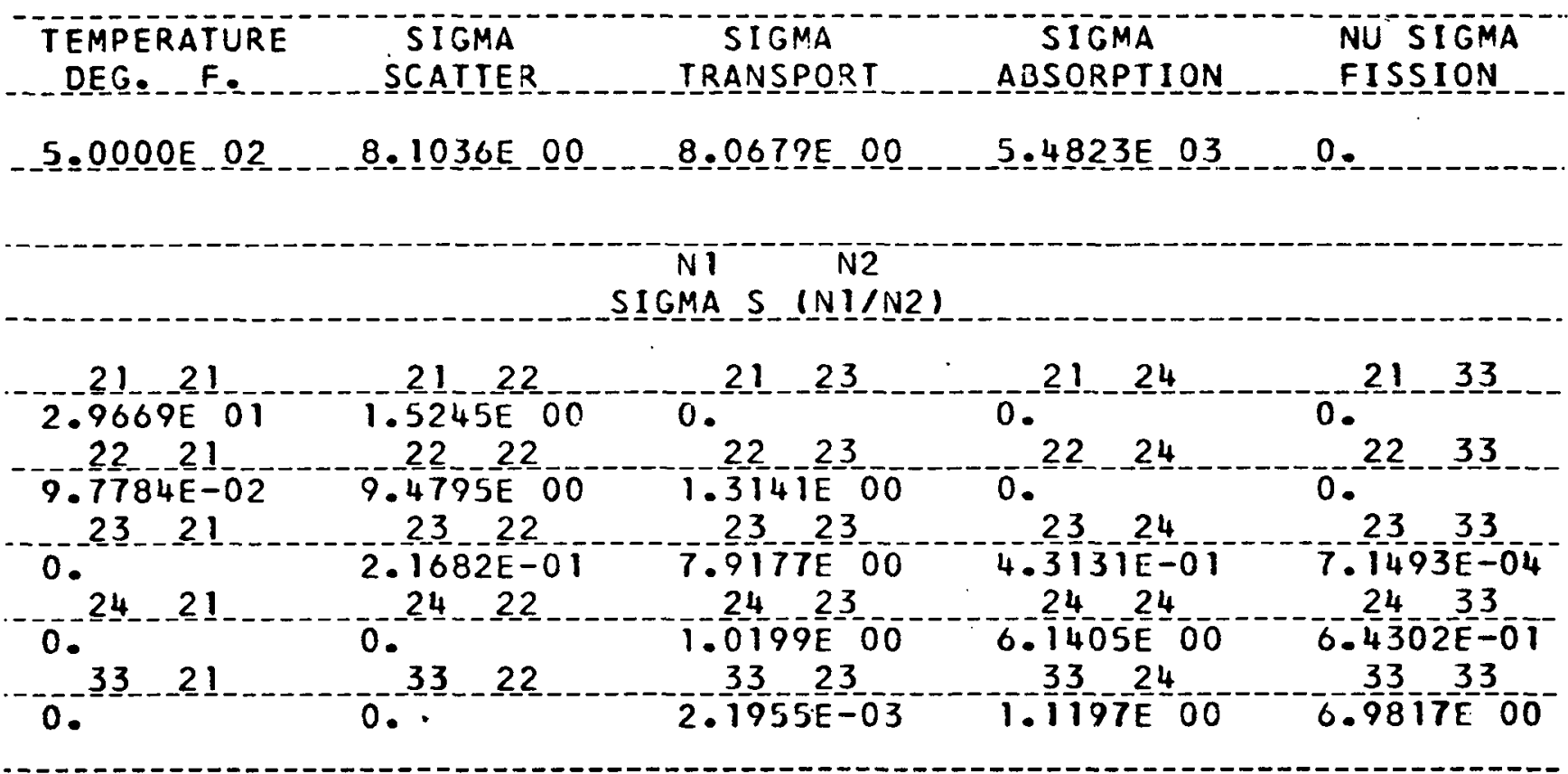

TEMPERATURE

DEG. _. F.
SIGMA

SCAIIER
SIGMA IRANSPORI
SIGMA

ABSORPIION
NU SIGMA

EISSIION
-6.8000E_01
$8.0618 E$
8. $0263 E_{-} 00$
5.67]7E 03
ㅇ.

\section{$\mathrm{N} 1 \mathrm{~N} 2$}

SIGMA_S (N] IN2)

\begin{tabular}{|c|c|c|c|c|}
\hline 21 & 22 & 21 & 21 & 21 \\
\hline $\begin{array}{r}2.9650 \mathrm{E} \\
22 \quad 21\end{array}$ & $\begin{array}{l}1.5435500 \\
22 \quad 22\end{array}$ & & & \\
\hline $\begin{array}{c}1.7987 \mathrm{E}-01 \\
23 \quad 21\end{array}$ & $\begin{array}{l}1.0335 \mathrm{E} 01 \\
2322\end{array}$ & $\begin{array}{c}3.7173 \mathrm{E}-01 \\
2323\end{array}$ & & \\
\hline 0. & $4.9043 E-02$ & $7.8043 \mathrm{E} 00$ & $7.0732 \mathrm{E}-01$ & $6.8563 \mathrm{E}-05$ \\
\hline-2 & 024 & $\begin{array}{c}24 \\
6.1900 \mathrm{E}-01 \\
3423\end{array}$ & $\begin{array}{c}24 \\
6.5740 \mathrm{E} \\
34 \quad 24\end{array}$ & $\begin{array}{c}2 \frac{24}{3}-34 \\
3434 \mathrm{E}-01\end{array}$ \\
\hline & & 4.57 & $7.1467 \mathrm{E}-1$ & $7.3471 E 00$ \\
\hline
\end{tabular}


EUROPI UM

TRANSFERS FROM GROUPS I THROUGH 10 TO GROUPS 1 THROUGH 16

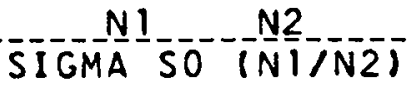

11013

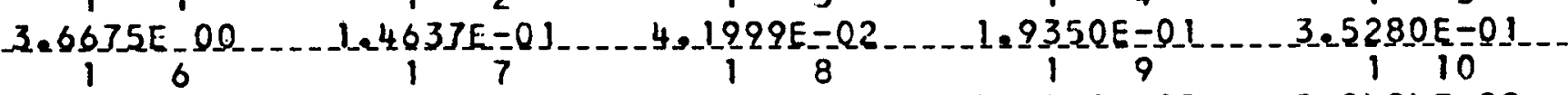

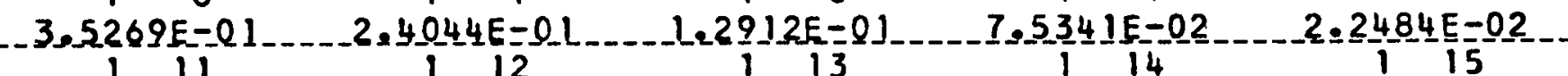

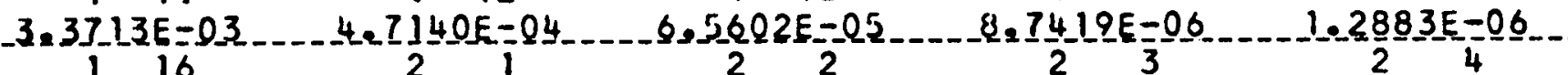

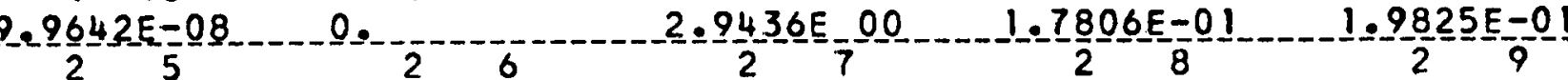

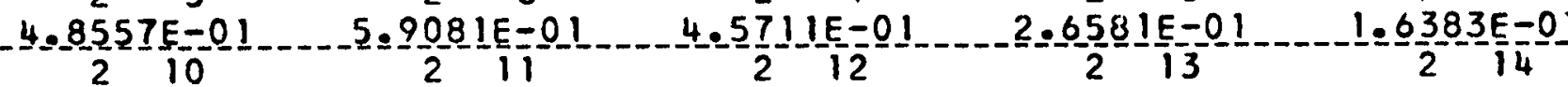

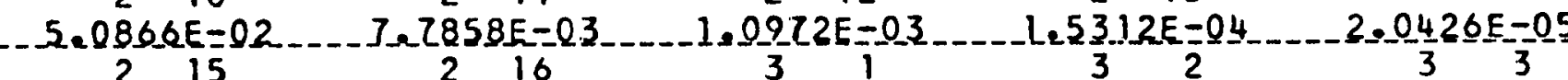

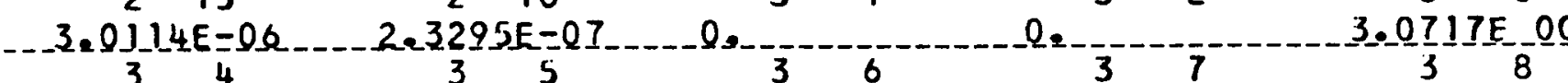

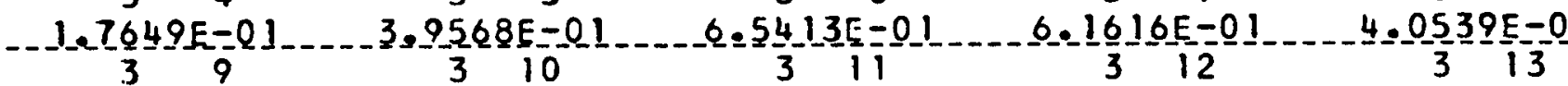

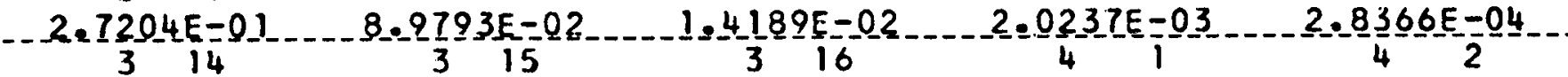

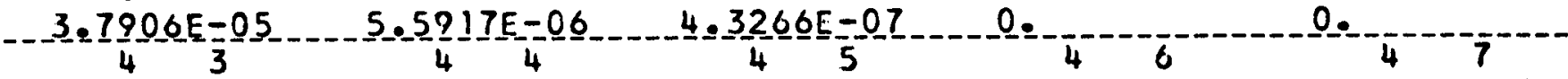

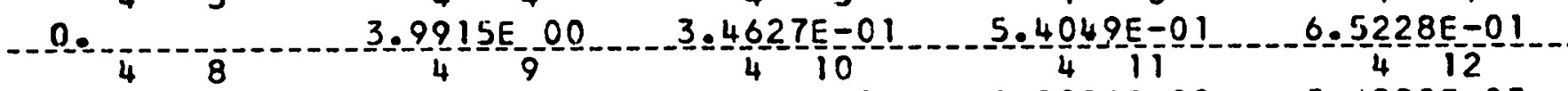
- $5.0214 E-01 \quad \ldots 3.7583 E-01 \quad 1.34 \frac{6}{4} 16 E-01 \quad 2.2086 E-02 \quad 3.1992 E-03$ - $4.5096=0.04$

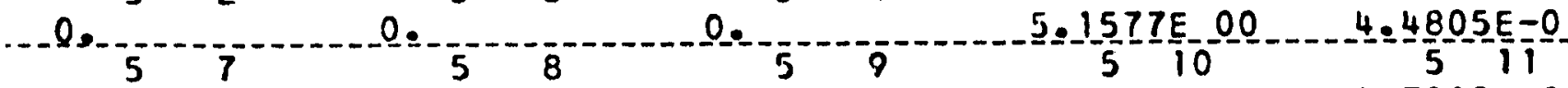

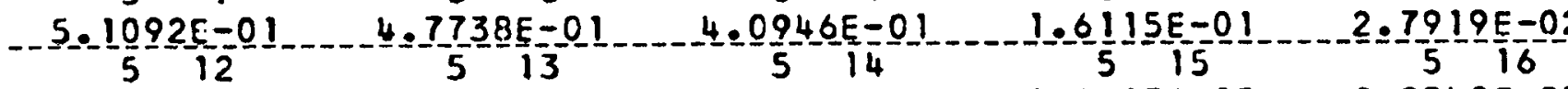

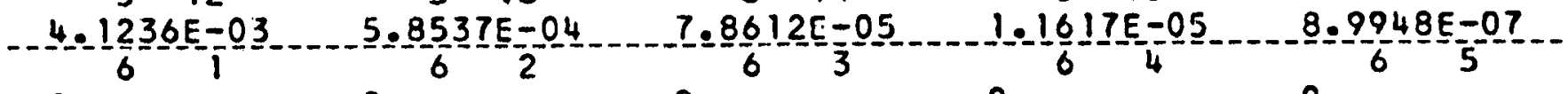

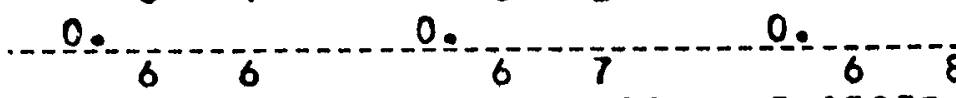

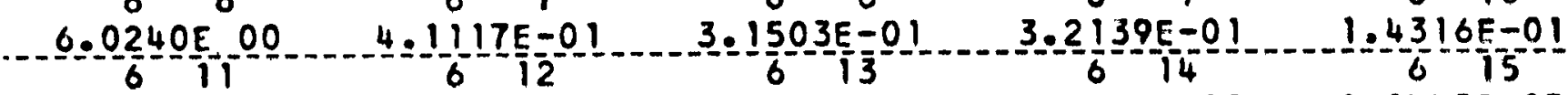
- 2.6469E-02 $64.0080 E-03 \quad 5.7415 E-04 \quad 7.7378 E-05 \quad 1.1449 E-05$ 8.869 ] $0.00-100$ 
EUROPIUM

$\mathrm{N} 1 \quad \mathrm{~N} 2$

S1 GMA SO (NIIN2)

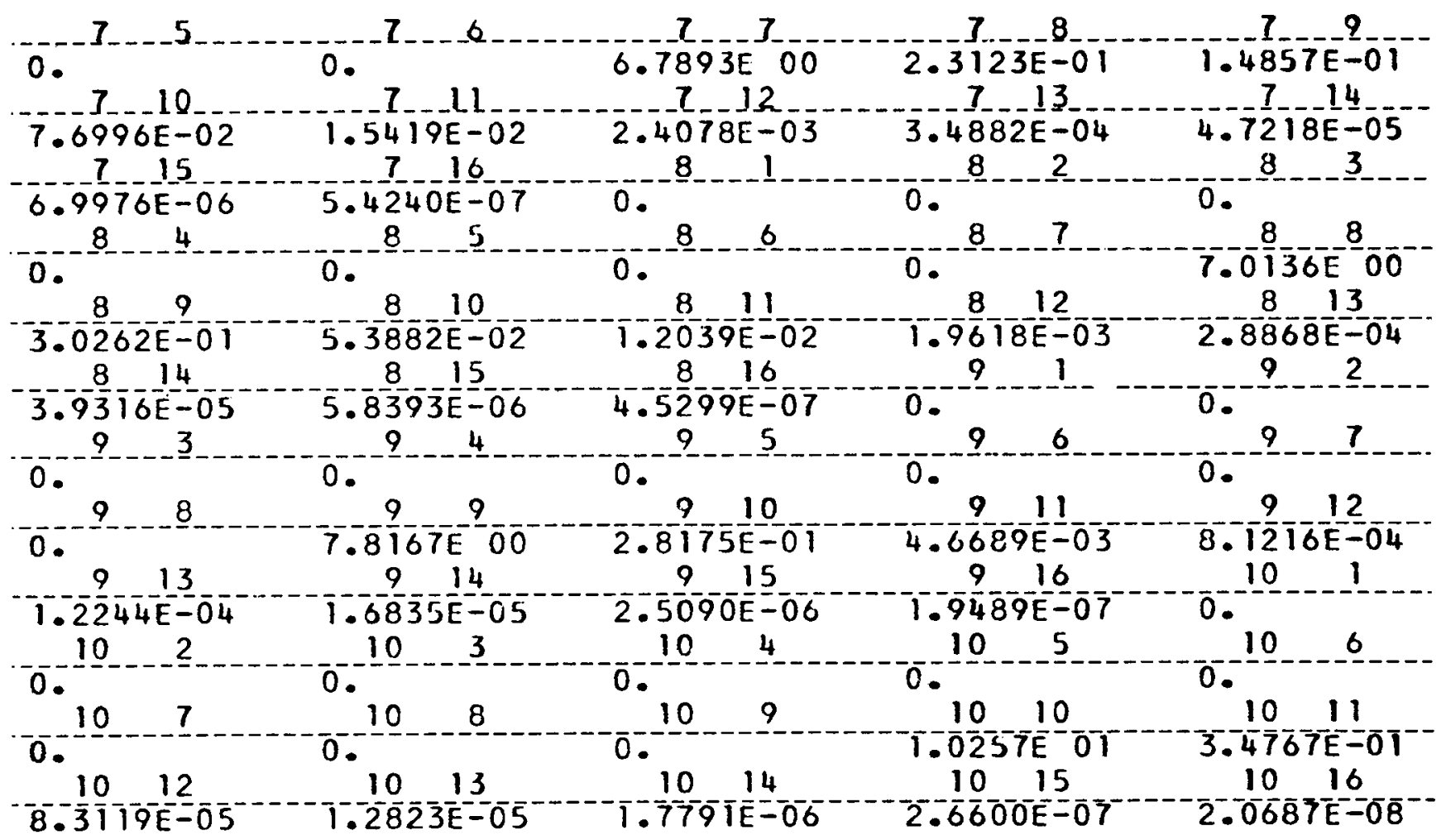


_EUROPIUM .

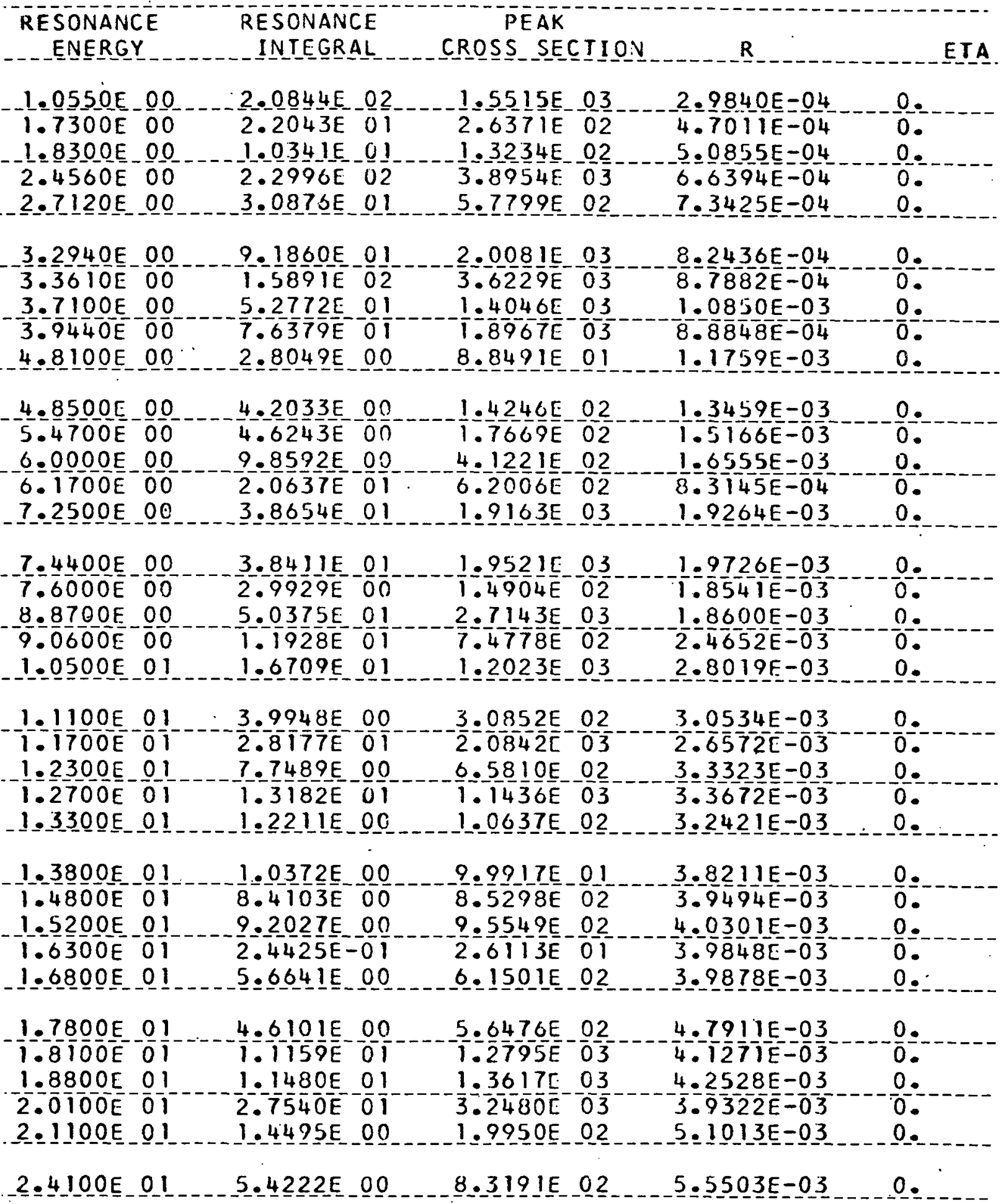


EUROPIUM

TRANSFERS FROM GROUPS 1 THROUGH 34 TO GROUPS 1 THROUGH

$$
\text { Ni N } 2
$$

SI GMA N, G IN] N 2 ?

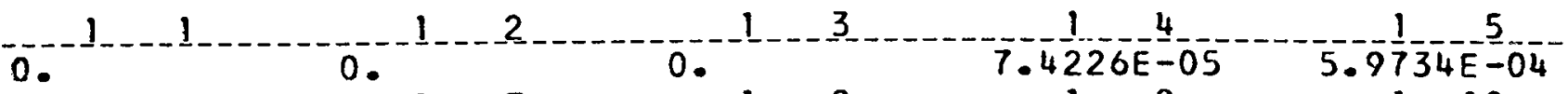

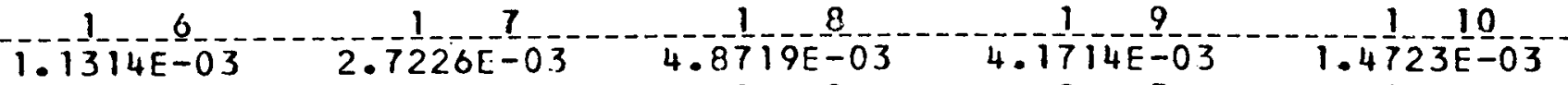

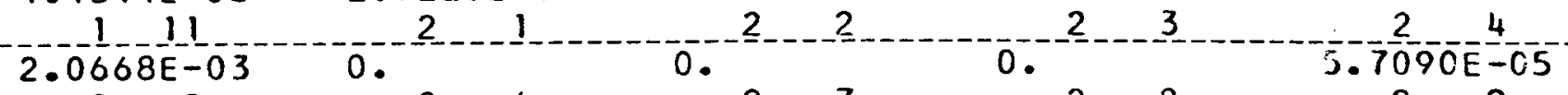

$4.5944 E-04 \quad 8.7021 E-04 \quad 2.0941 E-03-3.7472 E-033-\frac{2}{3.2084 E-03}$

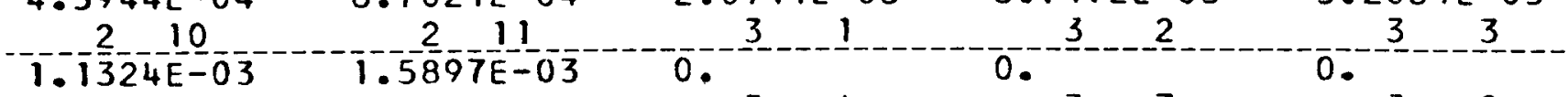

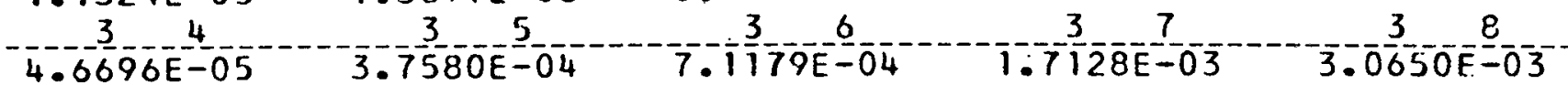

-

$2.6243 E-03 \quad 9.2624 E-04 \quad 1.3003 E-03$

${ }_{0 .}$

$4 \quad 4$

$1.8039 \bar{\varepsilon}-02$

0 .

-

$1.4712 E-0101.2597 E-01$ 4.4401E-02

0.

0

$\frac{5}{4.424} 1 E^{4}-03$

$3.4167 \mathrm{E}-02$

$0.24 \frac{4}{16 E-0}-\frac{1}{1}$

$8.22195-02$

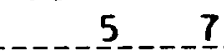

0.

5.8

5.9.

$\frac{5}{3.5604 E-02}$

0. 5

0.

1

$2.4863 \mathrm{E}-0 \mathrm{~T}$

$5 \quad 10$

$6.7 \frac{5}{3} \overline{36}-\frac{6}{0}-\overline{2}$ 0 . 6

$8.7754 \mathrm{E}-02$

$1.2 \frac{5}{3} 19 \mathrm{E}-011$

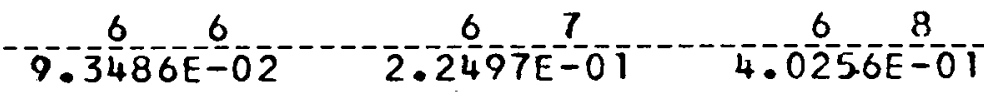

- $60-11$

$1.7078 \mathrm{E}-01$

$-7$

2.

$6.1 \frac{6}{3} \overline{3} 1-\frac{4}{2}-\overline{0} \overline{3}$

$-\frac{6}{4.7} \overline{3} \overline{7} \overline{7}-\overline{0}-\overline{0} \overline{2}$

$\begin{array}{cc}6.3609 \mathrm{E}-02 & 1.2048 \mathrm{E}-0 \\ 7 & 10\end{array}$

0 .

0.

$3.4 \frac{6}{4} 68 \mathrm{8E}=0 \overline{1}$

$6 \quad 10$

$1.5678 \mathrm{E}-012009 \mathrm{E}-01$

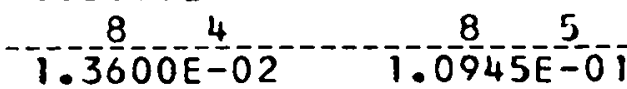

89

$7.043 \mathrm{IE}-\mathrm{O}$

- 92

$8-10$

0.

$-\frac{9}{1.672} \overline{8} \bar{E}-02$

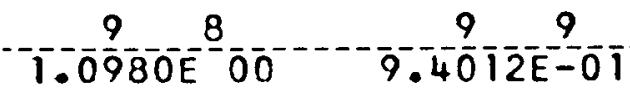

$-2.8 \frac{7}{9}-2 \mathrm{E}-\overline{0} \mathrm{I}$

0.

$1.2165 \mathrm{E}-0 \mathrm{~T}$

$7.90 \frac{7}{1-1 E}-0 \overline{3}$

$5.1880 \frac{8}{4}-01-\frac{7}{442} 1-\frac{9}{E}-0$

0 .

8

0.

8 ( 3

$2.0 \frac{8}{730 E-01}$

4.98

$3.7870 \mathrm{E}-11$

$1.3 \frac{9}{4} 62 \bar{E}-\overline{0}$

910

$3.3181 E-01$

0.

0 .

$\frac{8}{8.9266}=\frac{8}{-0}-01$ 
- EUROPIUM

SI GMA $\left.-N, G-\frac{N}{N}(N)-N \frac{1}{N}\right)$

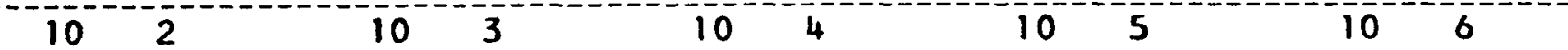

-.$=10$

8. $8456 \mathrm{E}-01 \ldots$

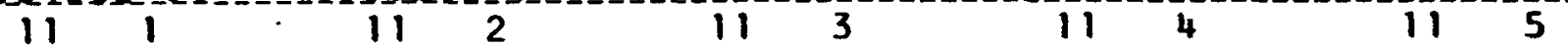

Q.

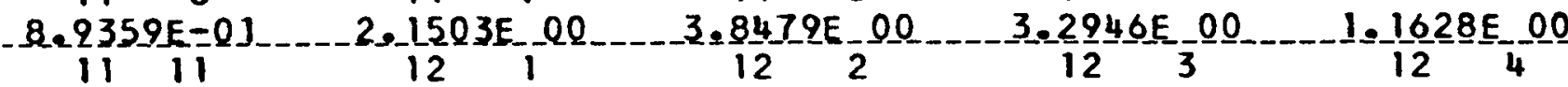

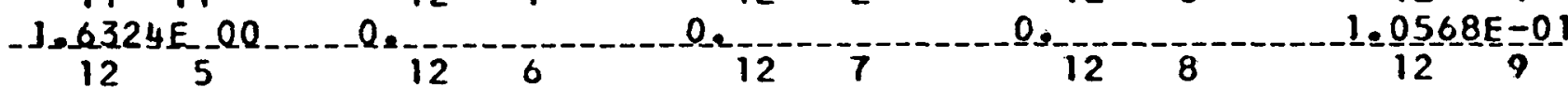

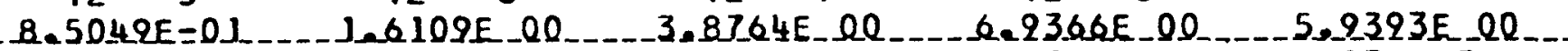

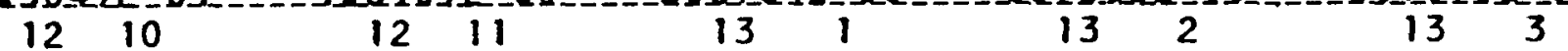

$2.0962 E_{1} D Q_{13}-2.2428 E_{5} 00 \ldots-0$.

13

13.

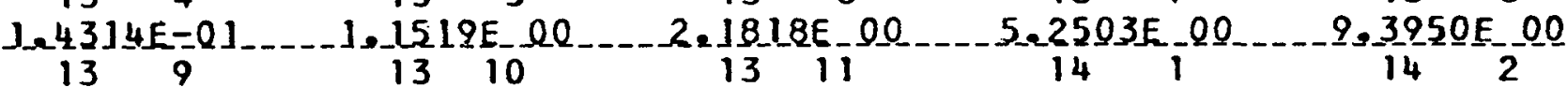

8. $0442 E_{-} 0 D_{2}$

$3.9857 E_{-} \mathrm{O}$

1430.924

1488149

_l. $\frac{92}{15} 49 E_{2} 01 \ldots 1.6481 E_{3}$

$2.3601 \mathrm{E} 00$

Q2 14

$0.7 \overline{7}^{-1}$

147016

1. $0757 E$ 임

$14 \quad 10$

1411

0.

-

0.

15

$5.8169 E_{-} 00$

$8=1660 E_{-10}$

$150-\frac{1}{5}$

$I=\frac{27}{15}-1 \mathrm{E}=0 \mathrm{O}$

5. 8572 E 00

15

$2.6696 E_{-} 01$

$1.4436 E_{-} 01$

$1.109 \underline{15}-1$

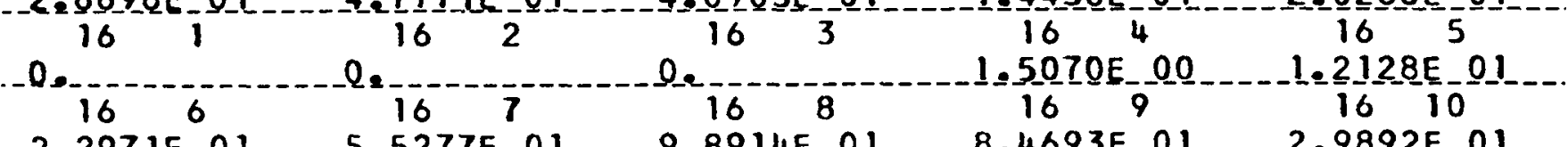

$2.2971 E_{1} 01-5.5277 E_{1} 01-2.8914 E_{2} 01$

$4-\frac{1}{17} 63 E_{5} 01$

3.25 $27 \mathrm{E} 01$

8.02 $18 \mathrm{E}-01$

-6. $\frac{75}{18} 5 \mathrm{E}-00 \ldots$

3. $7964 \mathrm{E}$. 02

ㅇ..- 
EUROP I UM

N1 N2

SIGMA_N,G_(NI/N2)

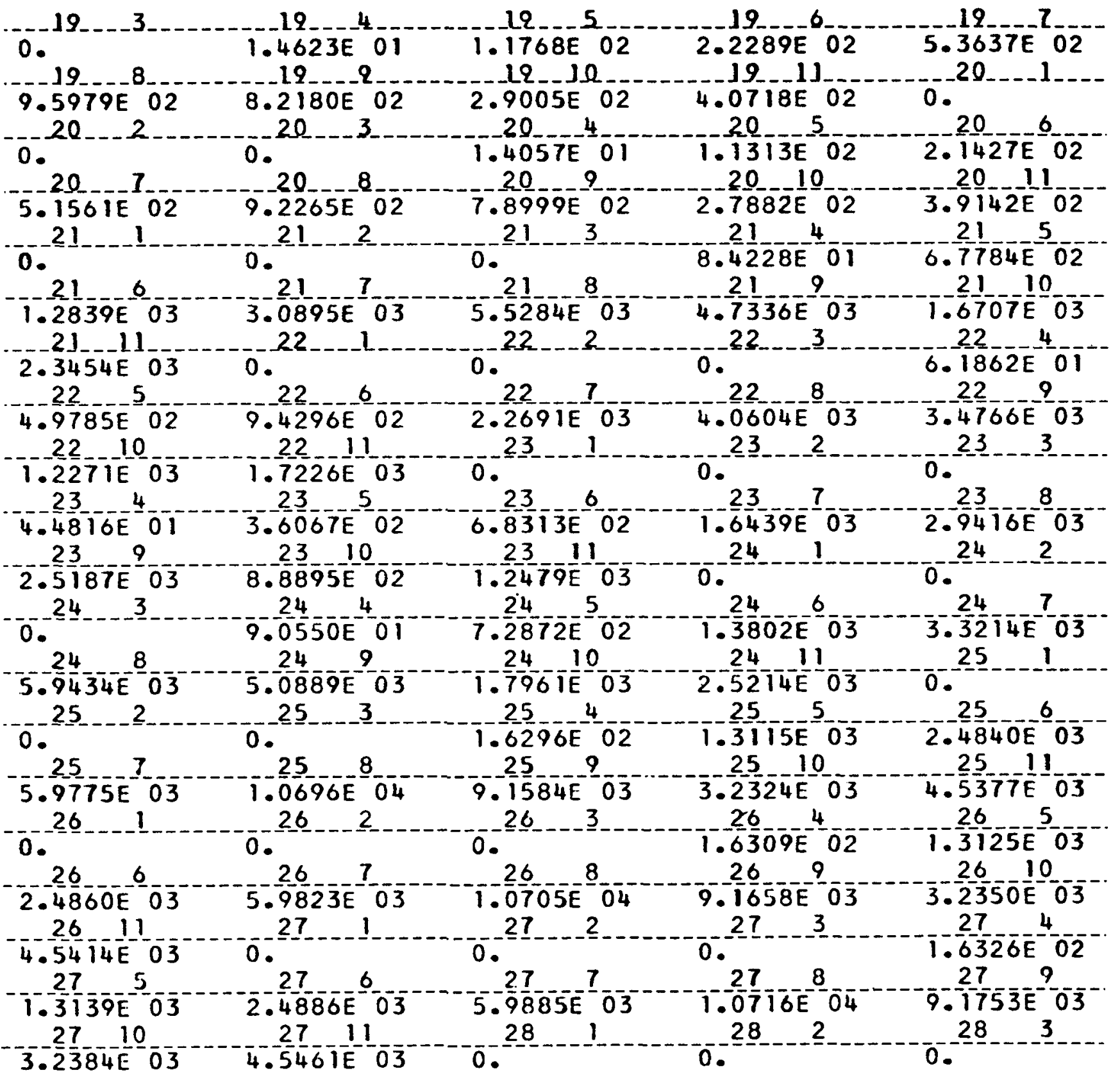


-EURQPIUM.

SIGMA N,G $(N I / N 2)$

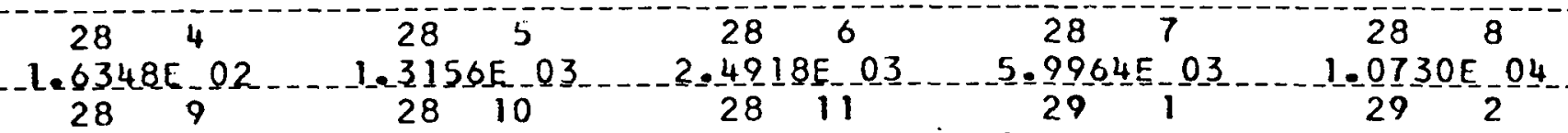

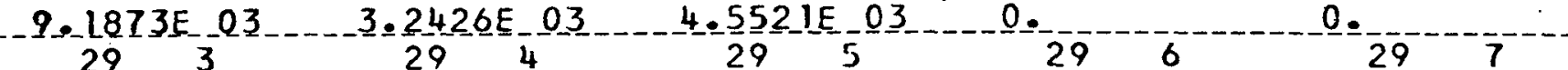

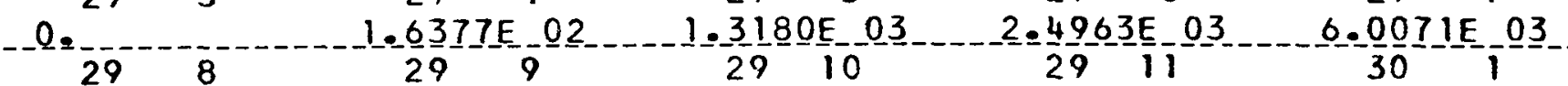

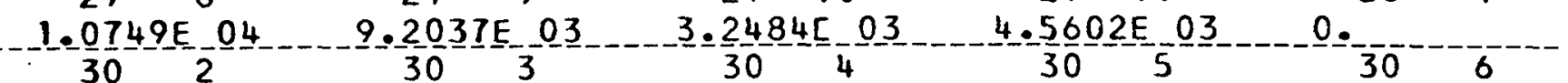

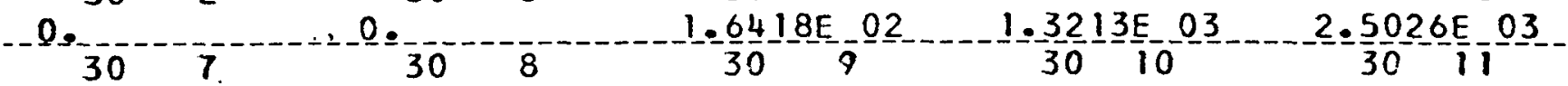

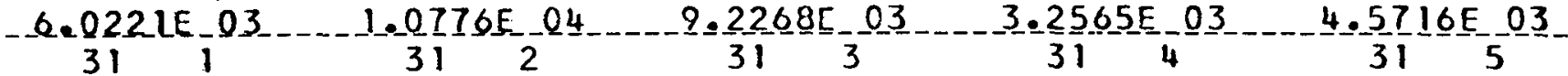

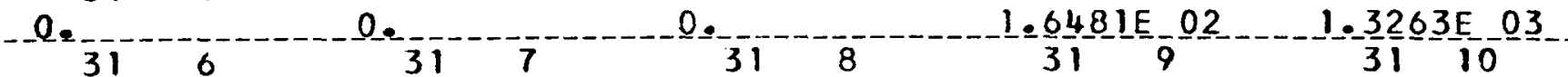

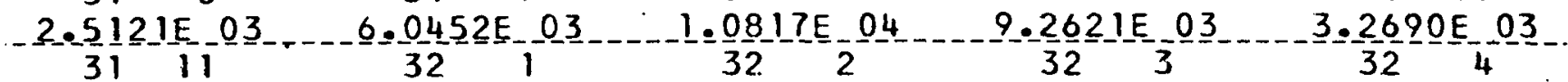

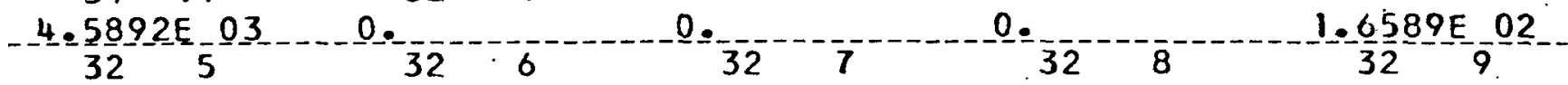

$-1=\frac{33}{32} 50 \mathrm{E}-03-2.52 \frac{86}{32} \mathrm{E}-03-6.0848 \mathrm{E}-03 \quad 1.0888 \mathrm{E}-04$

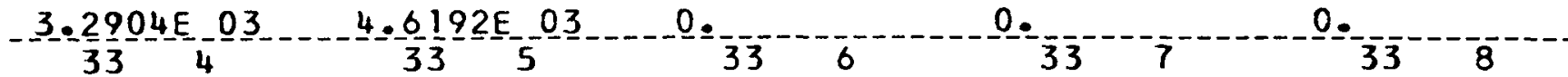

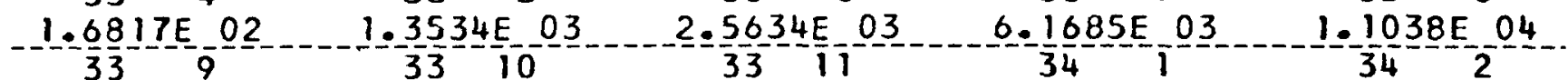

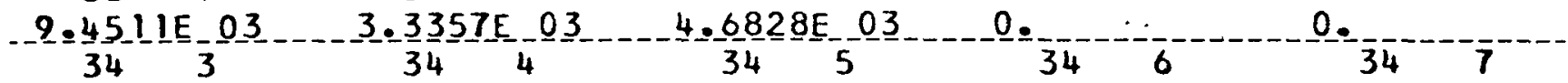

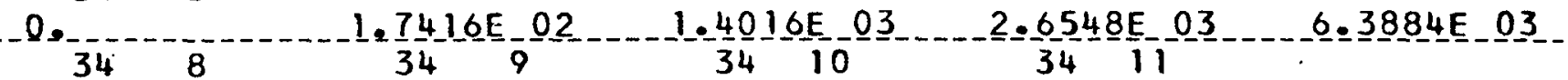
_.1.1432E $04 \ldots$ 
DESIGNAI_LON SAMARIUM 149
CODE_NO 62.1490
DENSIIY_EACIOR

2.47254 E 02

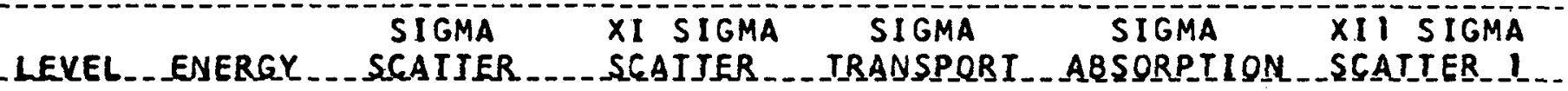

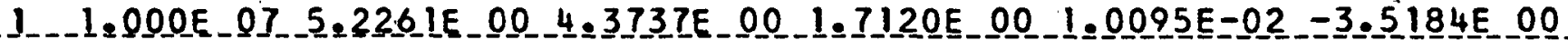

$26.065 \mathrm{E} 06$ 5.3430E $00 \quad 4.4117 \mathrm{E} 002.5957 \mathrm{E}$ 00 $1.4938 \mathrm{E}-02-2.7506 \mathrm{E}$ 00

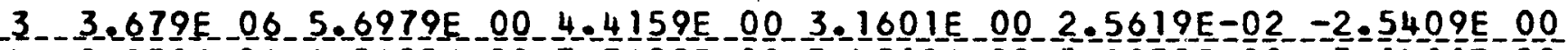

$42.231 E$ O6 $6.5685 \mathrm{E} \quad 00$ 3.7698E $003.4312 \mathrm{E}$ 00 5.8838E-02 -3.1411E 00

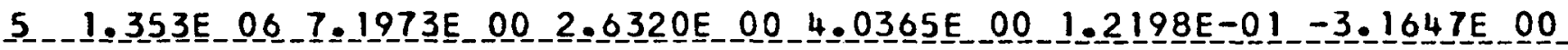

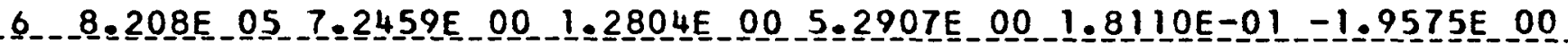

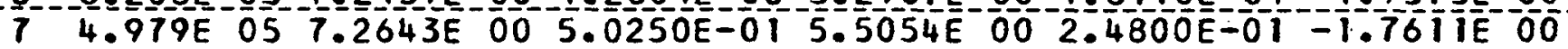

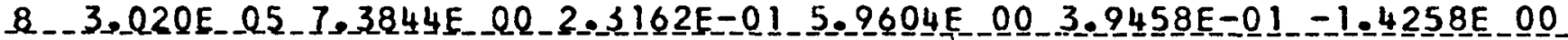

$9 \quad 1.832 \mathrm{E}$ 05 8.1040E $001.3034 \mathrm{E}-016.760 \mathrm{IE} 00 \mathrm{C}$.0232E-01-1.3455E 00

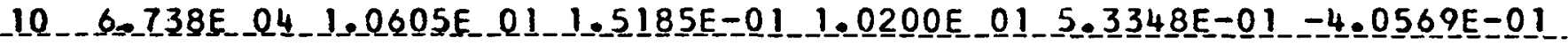

$11-2.479 E-041.1294 E-01-1.5133 E=011.1243 E-01-1.1149 E-00-5.0862 E=02$

12 9.119E 03 1.5473E $012.0734 \mathrm{E}-011.5403 \mathrm{E}$ 01 $2.0947 \mathrm{E}$ 00 $-6.9685 \mathrm{E}-02$

$13 \quad 3.355 E \quad 032.9880 E$ E $014.0039 E-012.9745 E$ O $013.6492 E$ E $00-1.3456 E-01$

$14-1.234 \mathrm{E} 034.6288 \mathrm{E}$ 01 $6.2026 \mathrm{E}-014.6079 \mathrm{E}$ 01 $6.2916 \mathrm{E}$ 00 $-2.0855 \mathrm{E}-0 \mathrm{~T}$

15 4.540E 02 8.6532E 01 1.1595E 00 8.6143E 01 1.2163E 01 -3.8988E-01

$16-8.719 \mathrm{E}-012.6294 \mathrm{E}-02 \quad 3.5233 \mathrm{E} 002.6175 \mathrm{E}-02 \quad 1.2892 \mathrm{E} 02-1.1854 \mathrm{E} 00$ $17-3.55 \mathrm{E} 016.0723 \mathrm{E} 018.1369 \mathrm{E}-016.0450 \mathrm{E} 01 \mathrm{1}-7136 \mathrm{E} 02-2.7353 \mathrm{E}=01$

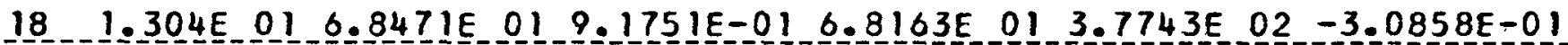
$19-043 \mathrm{E} 00-1.932 \mathrm{IE} 012.6007 \mathrm{E}-011.9234 \mathrm{E} 0 \mathrm{~T} 2.1144 \mathrm{E} 02-8.7006 \mathrm{E}=02$

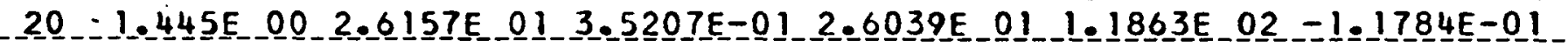

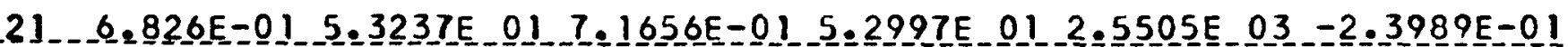
$220.511 E-011.2206 \mathrm{E}$ 02 $1.6429 \mathrm{E} 001.215 \mathrm{IE} 025.7013 \mathrm{E} 03-5.4967 \mathrm{E}-01$

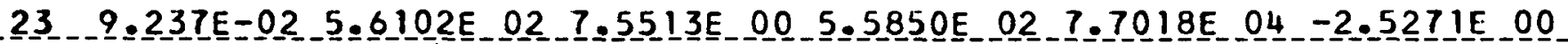
$242.530 \mathrm{E}-02$ 1.1530E $021.5520 \mathrm{E} 001.1478 \mathrm{E} 02-1063 \mathrm{E} 04-5.1963 \mathrm{E}-01$

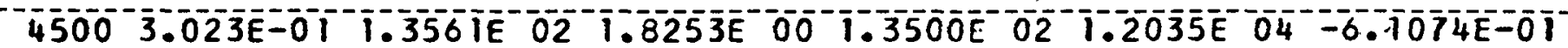

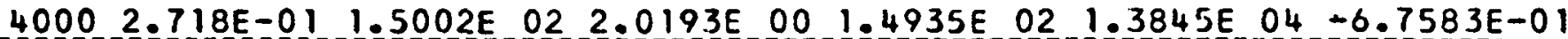
$35002.413 \mathrm{E}=0 \mathrm{~T}-1.6824 \mathrm{E}-02-2645 \mathrm{E}-001.6748 \mathrm{E}-02-1.6176 \mathrm{E}-04-7.5792 \mathrm{E}=01$ $30002.109 E-011.9580 E-02 \quad 2.6350 E-001=9492 E-02-1.9424 E-04-8.8207 E-01$

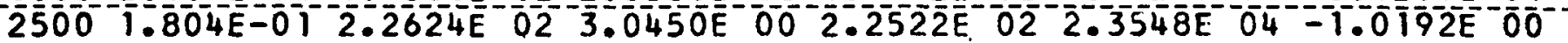

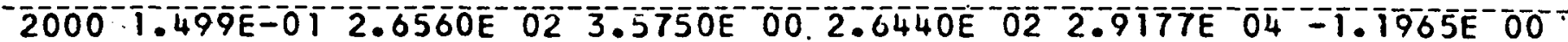

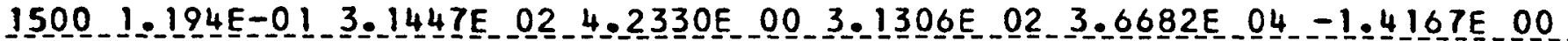
$10008.897 \mathrm{E}-02-3.6623 \mathrm{E} 02-4.9300 \mathrm{E} 00.3659 \mathrm{E} 024.6994 \mathrm{E} 04-1.6500 \mathrm{E} 00$ $500 \quad 5.850 E-02 \quad 3.8192 E$ E $02.5 .1410 E$ O0 3.8020E $02 \quad 5.7322 E \quad 04-1.7211 E 00$ $683.216 \mathrm{E}-022.5853 \mathrm{E}$ 02 $3.4800 \mathrm{E} 002.5736 \mathrm{E} 025.5879 \mathrm{E} 04=1.1644 \mathrm{E} 0 \mathrm{O}^{-}$

NOTE: For a summary of the sources of data for energles E > $21.5 \mathrm{ev}$, see APEX-704; for energles $\mathrm{E} \leq 21.5 \mathrm{ev}$, see XDC $61-1-113$. 
SAMARIUM 149

. IHERMAL_IRANSFER_MATRICES .

-_LEMPERAIURE

SIGMA

SIGMA.

DEG. F. SCATTER

TRANSPORT

SIGMA

ABSORPTION

NU SIGMA

FISSION

$4.5000 E 03$
$6.2206 E$

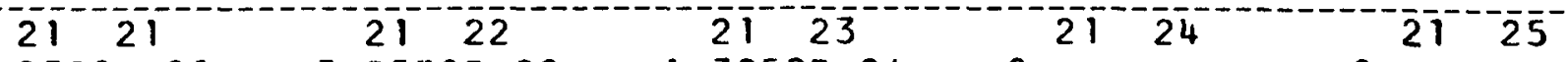

$4.9382 E\left[01 \ldots 3.8550 E \_00\right.$ 2221

$22 \quad 22$

$22 \quad 23$

ㅇ..

$22-24=-22$

$8 \cdot 1355 E_{-} 00$

2321

1.08 056 E_ 02

$5.74 \underline{1} \underline{3} \underline{E}-\underline{0} \underline{0}$

2322

2323

I. $3982 \mathrm{E}=04$

$4.9992 E-06$

- 2. $6163 \mathrm{E}-05$

8. $1019 \mathrm{E}-01$

$4.6181 \mathrm{E}-02$

2324

$23 \quad 25$

242

$24 \quad 22$

2423

2. $1443 \mathrm{E}-01$

$1.4216 \mathrm{E}-00$

$-0 . \frac{0}{25}-\frac{1}{2}$

1. -526$]$ IE -02

$3 . \frac{72}{25} 5 \frac{5}{23}-\mathrm{O}$

$6.36396-01$

I. $-\frac{4}{2} \frac{2}{5}-\frac{3 E}{25}-01$ ...

1. $1525 E-04$

3. $1303 E$ OOO

$1.8448 E \quad 01$

4.0627E_01.

_IEMPERAIURE

DEG. F.

$4.0000 E$ OB
SIGMA

SCATTER
SIGMA

TRANSPORT
SIGMA

ABSORPTION
NUU SIGMA

FISSION

$4.0000 E$ O3

$6.1814 \mathrm{E}$ OT

$6.1536 E$ O

$4.1125 \overline{8}$

0

SIGMA S $(N 1 / N 2)$
2121
2122
$2 \overline{1} 2 \overline{3}$

4. $9126 E$ E 01

$22-21$

$4=1108 E$ OO

222

7.2556E 00

$2 \frac{1}{2} 1$

$1.0931 \mathrm{E}-02$

$4.6 \frac{6085 E}{22}=07$

$21^{--74}$

O.

$22^{-24}$

$. \frac{84}{2} \frac{7 E}{2}-00$

$4 \cdot 1627 E-04$

4.67615 은

$-2 \frac{5255 E}{24}-0$

2423

$-\frac{1}{2} \frac{1}{4} 62 \mathrm{E}-01$

$3.7367 E-\frac{0}{26}$

$6 \cdot \frac{5625 E}{26}-\frac{01}{24}$ 1. $7840 E$ O 01

$21 \cdot 2 \overline{6}$ 0. $25-\overline{25}$ $1.84225-06$ $2158 E 00$

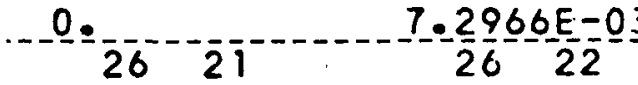
2. $5422 \mathrm{E} \quad 00$ $3.5348 E=05$ $24-\frac{1}{26}$ $.4579 E 00$ - $\underline{0}$. 
SAMARI UM 149

THERMAL TRANSFER MATRICES

TEMPERATURE
STAMA

\begin{tabular}{|c|c|c|c|c|}
\hline $2 J_{-}-21$ & $21-22$ & $2 L-23$ & $21 \ldots 24$ & 21 \\
\hline $\begin{array}{l}8784 E 01 \\
2221\end{array}$ & $\begin{array}{c}4.4529 E 00 \\
22\end{array}$ & $\begin{array}{c}1.1757 E-07 \\
22\end{array}$ & & 0.2 \\
\hline $\begin{array}{l}3196 \mathrm{E} 00 \\
23 \quad 21\end{array}$ & $1.1006 \mathrm{E} 02$ & $5.9938 \mathrm{E} \quad 00$ & 2.0679 & $5.3961 \mathrm{E}-0$ \\
\hline$-1214 \mathrm{E}-06$ & $-6.9076 \mathrm{E} 01$ & $4.7379 \mathrm{E} \quad 02$ & $2.0917 E^{24} 01$ & $1.0135 \mathrm{E} \mathrm{O}$ \\
\hline (c) & -24 & 24 & 24 & 24 \\
\hline & $\begin{array}{l}2.9614 E-03 \\
-27 \\
8.2632 E-06\end{array}$ & $\begin{array}{l}3.5308 \mathrm{E} \quad 01 \\
1.27 \\
1.9862 \mathrm{E}\end{array}$ & $\begin{array}{c}6.7840 E \text { O1 } \\
1.57124 E \quad 01\end{array}$ & $\begin{array}{r}1.4175 E 0 \\
-2-\frac{27}{3}-27 \\
4.23040\end{array}$ \\
\hline
\end{tabular}

SEMPERATURE
SIGMA

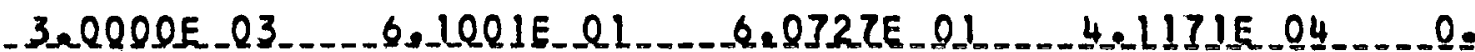

$\bar{N} \overline{\mathrm{T}}-\mathrm{N} 2$

SIGMA S (N] IN2)

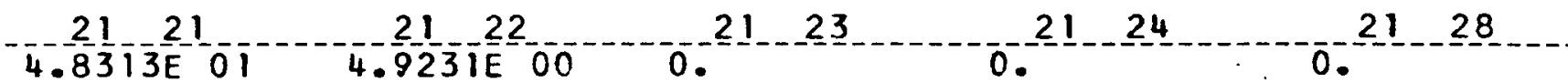

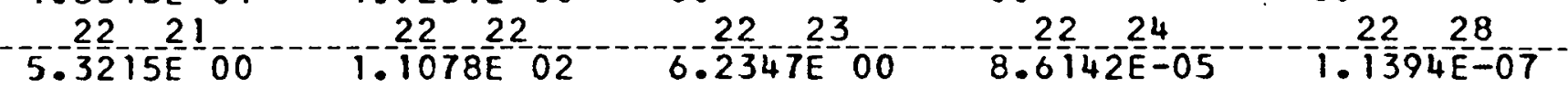

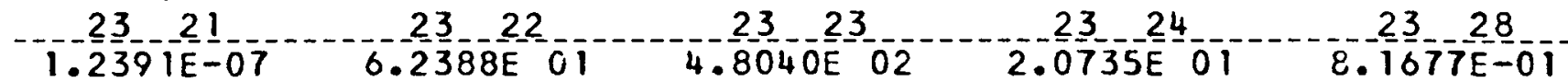

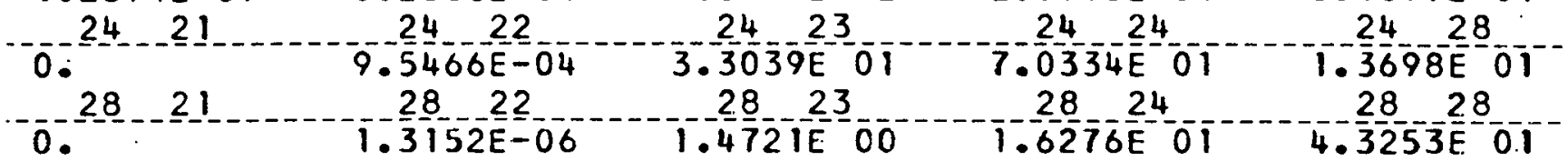


SAMARIUM 149

..LHERMAL_IRANSFER MAIRICES.

._LEMPERATURE

SIGMA

SIGMA

SIGMA

NU_SLGMA_-

DEG. F. SCATTER

TRANSPORT

ABSORPTION

FISSION

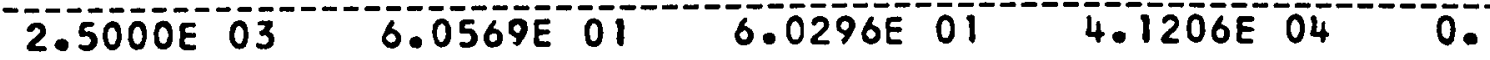

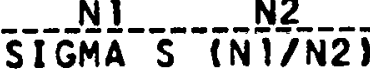

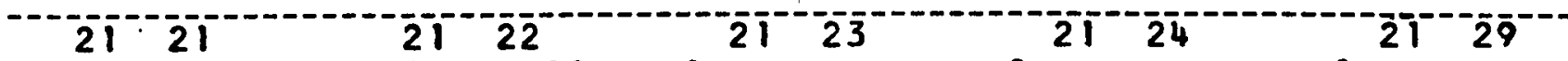

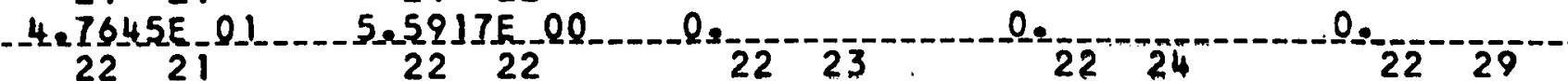
$4=2581 E 00$

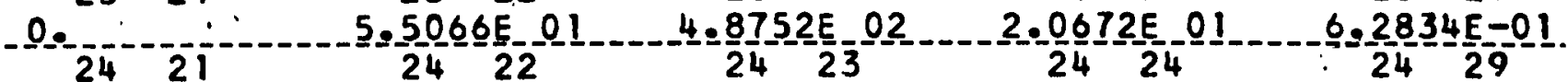
을

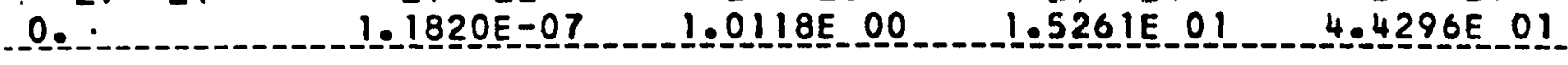

TEMPERATURE
DEG. FIGMA 2.0000E $0 \overline{3}^{-0.0 T O T E \mathrm{O}^{-}}$

STGMÁ S

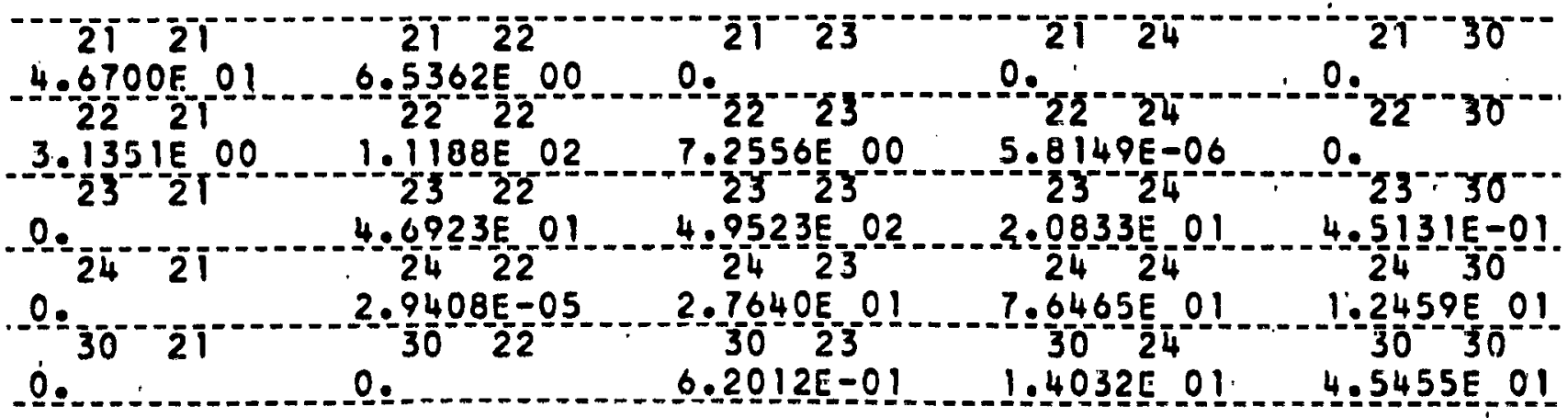


TEMPERATURE

DEG._F.

-1.5000E_ 03

$$
\text { (1) }
$$$$
-
$$

\begin{tabular}{|c|c|c|c|c|}
\hline $4.0023 E 01$ & $-\frac{21}{7.2131 \mathrm{E} 00}$ & $-21-23$ & --2 & 0.21 \\
\hline-22 & 22 & $22-23$ & -22 & 22 \\
\hline $\begin{array}{c}1.9847 E \quad 00 \\
23\end{array}$ & $\begin{array}{l}1.1187 E 02 \\
23\end{array}$ & $\begin{array}{l}8.3776 \mathrm{E} \text { OO } \\
23\end{array}$ & $\begin{array}{l}5.8459 E-07 \\
23\end{array}$ & 0.23 \\
\hline & $\begin{array}{c}3.7686 \mathrm{E} 01 \\
24 \quad 22\end{array}$ & $\begin{array}{l}5.0355 \mathrm{E}{ }^{02} \\
24\end{array}$ & $\begin{array}{l}2.14610^{2} 1 \\
24 \quad 24\end{array}$ & $\begin{array}{r}2.9056 \mathrm{E} \\
24 \quad 31\end{array}$ \\
\hline & $\begin{array}{l}1.5658 \mathrm{E}-06 \\
31\end{array}$ & $\begin{array}{r}2.4315 \mathrm{E} 01 \\
31 \quad 23\end{array}$ & $\begin{array}{r}0.0341 E \\
31 \\
-24\end{array}$ & $\begin{array}{r}1655 \mathrm{E} \\
31 \\
\end{array}$ \\
\hline & & $3.1450 \mathrm{E}-01$ & $1.2518 \mathrm{E}$ & $4.6760 \mathrm{E} 01$ \\
\hline
\end{tabular}

N 12

SI GMA S (N1/N2) 
SAMARIUM 149

_IHERMAL_IRANSFER_MAIRICES

IEMPERAIURE

SIGMA

SLGMA

DEG. $F$.

SCATTER

TRANSPORT

SIGMA

NU SIGMA_-

ABSORPTION

FISSION

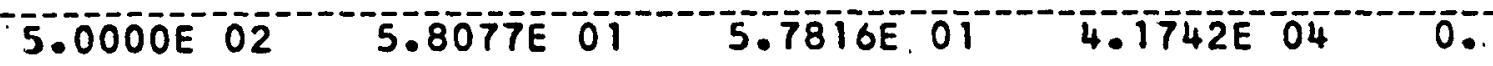

\section{$\operatorname{sigmA} S\left(\frac{N}{S}-\frac{N 2}{1 / N 2}\right.$}
2121
$2 \overline{1} \overline{2}$
21.23
$3^{-12} i^{-24}$

5.0624E_01_-_2.6128E_00

$22 \quad 21$

$22 \quad 22$

2223

$0 .-\frac{1}{22}$

0.

$1.1014 E_{-} 00$

1.06266 E 02

2322

$1.4 \frac{4}{23}-292$

$23-\overline{2} 4$

$22-33$

\section{1}

$1.4351 \mathrm{E}-01$

$5 \cdot \frac{1}{2} \frac{2}{4} 1 \mathrm{E}-02$

$2.84799 E_{2}-0$

$2 \overline{3} 3 \overline{3}$

…ㄴ..- 24

$-\frac{1}{3}--\overline{2}$

$1 . \frac{52}{33} 82 \mathrm{E}=01$

$9=\frac{0888}{3} \frac{8}{2}=01$

$24-\frac{1}{3}=2$

$-0.53--\frac{1}{2} \overline{1}$

으.

1.?287E-02

8 .0985E_00

\section{$=$ IEMPERATURE \\ QEQ. $F$. \\ SIGMA \\ FRANSPOR \\ SEATTEA \\ NY SLCMA $=$}

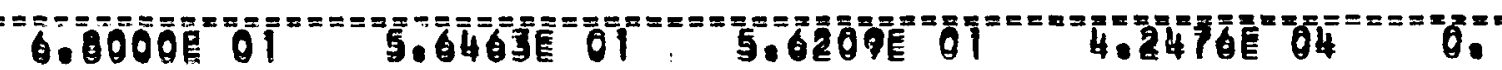

"6.

$==\mathbf{=}=$

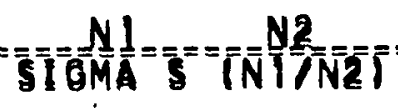

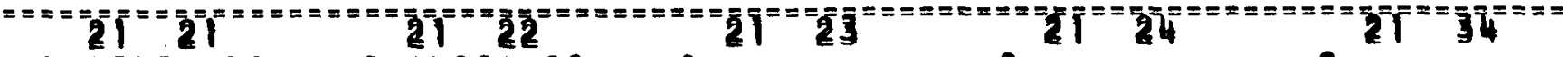

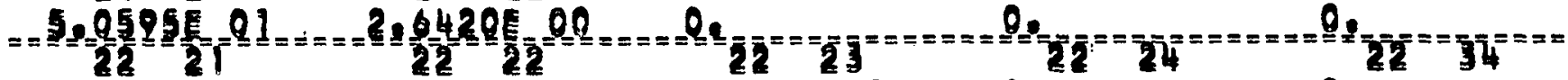

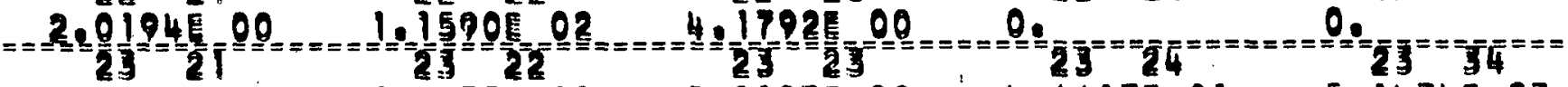

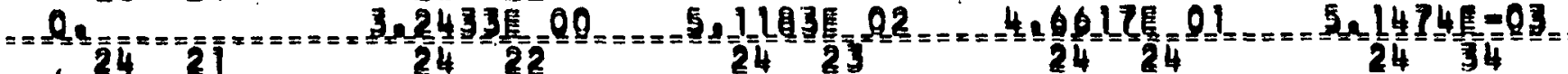

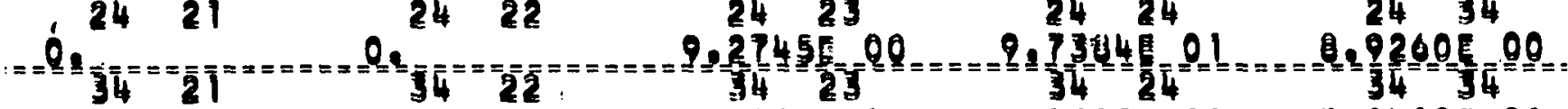

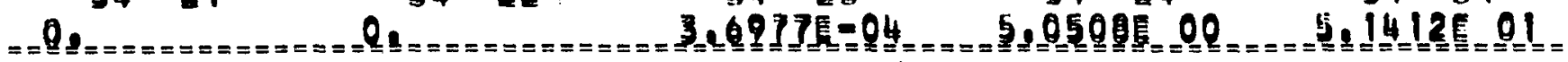


SAMARIUM 149

N1 N2

SIGMA_SO_L L I/N2L

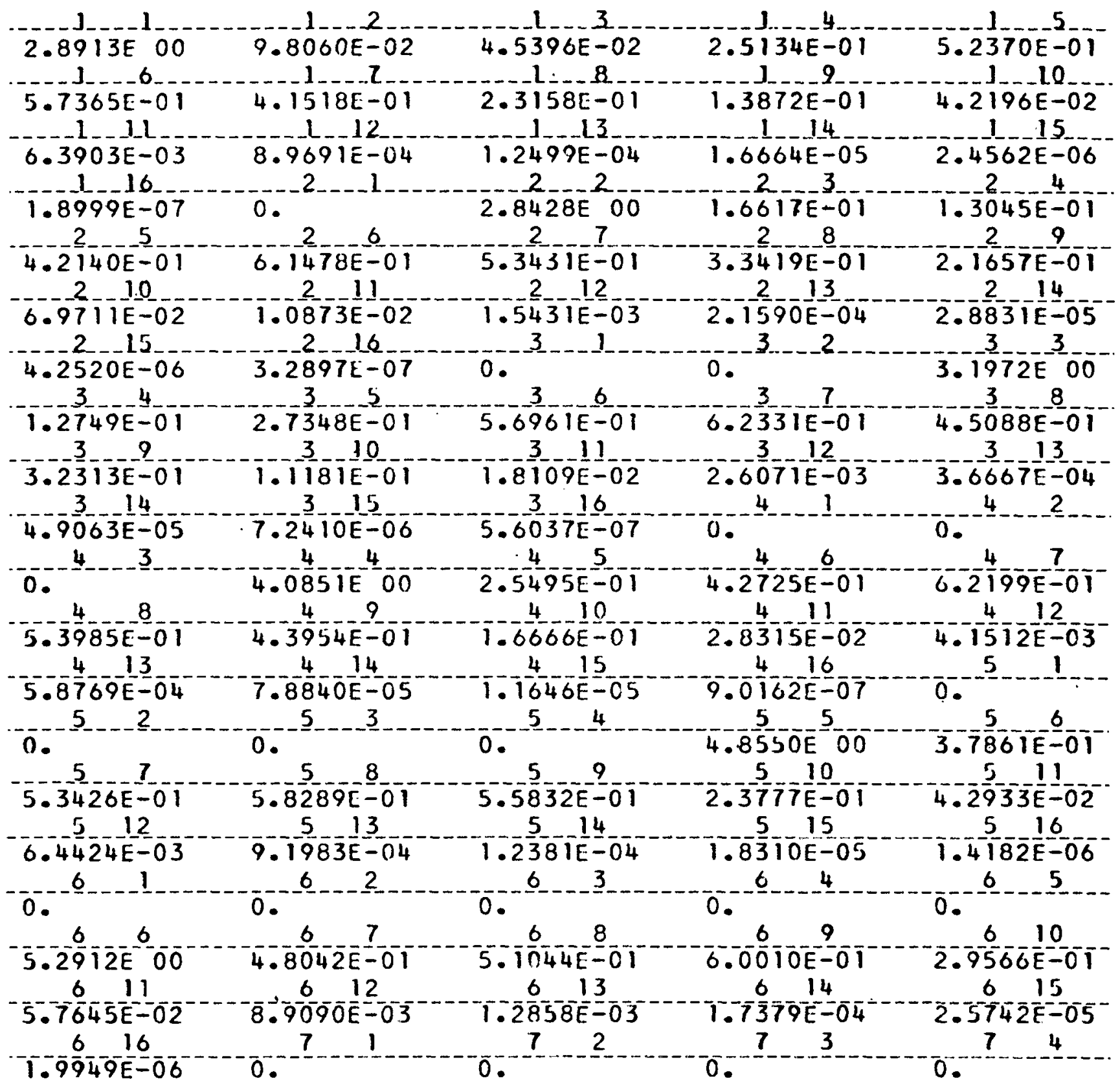


SIGMA $-\mathrm{SO}^{-2}(\mathrm{~N} I / \mathrm{N} 2)$

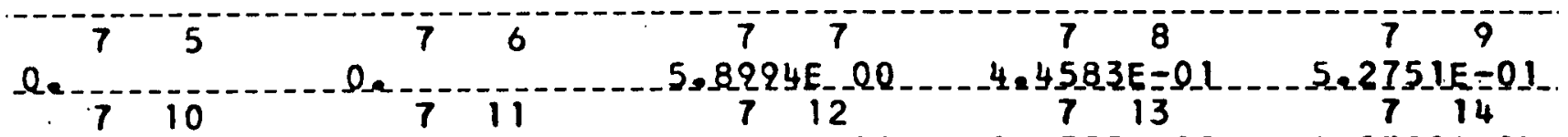

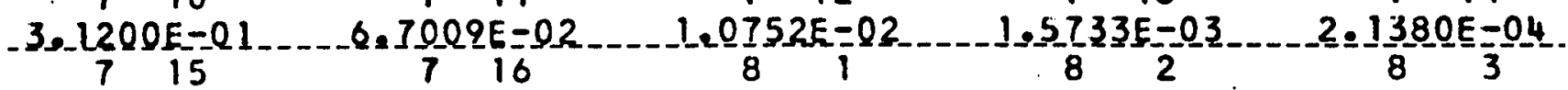

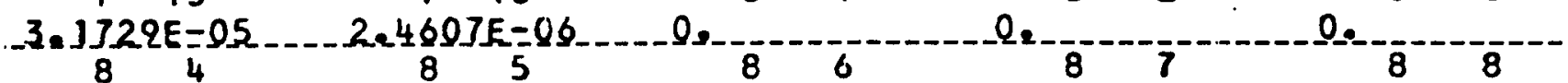

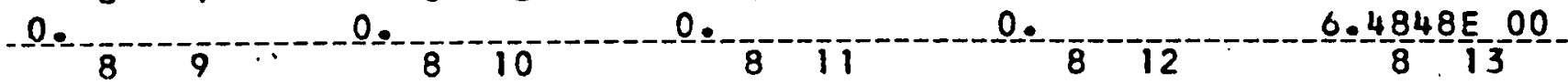

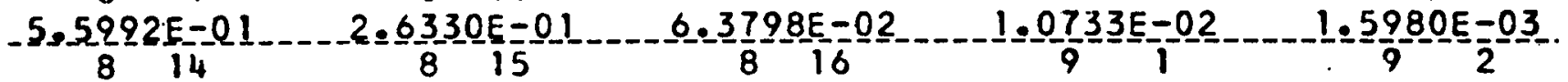

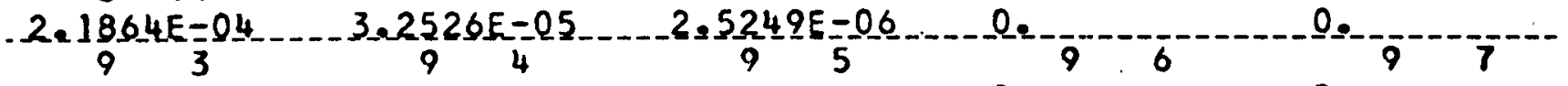
Q. -0. .66.6932E-04 $9.2345 E-05$ 
SAMARIUM 149

RESONANCE RESONANGE PEAK ENERGY

INTEGRAL CROSS SECTION

$\mathbf{R}$ ETA

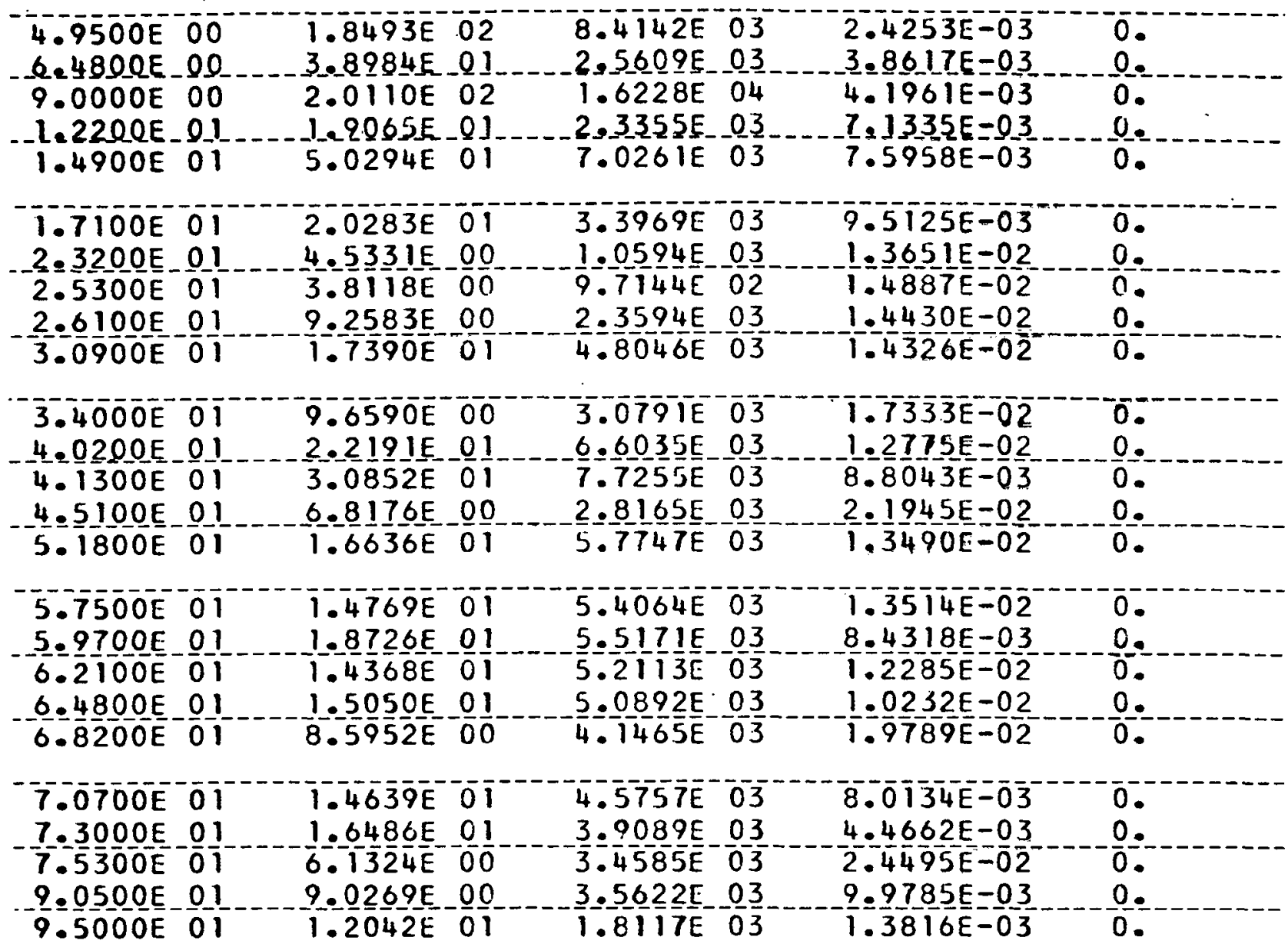

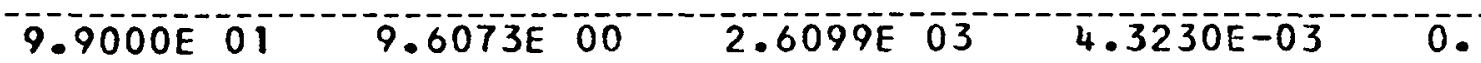


SAMAR I UM 149

TRANSFERS FROM GROUPS I THROUGH 34 TO GROUPS I THROUGH 11

\section{SIGMA N,G $(N) / N 2)$}

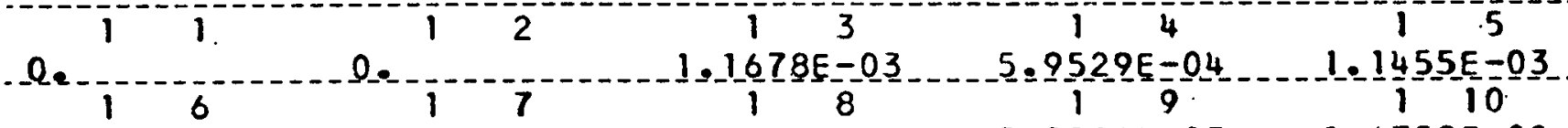

1.4Q16E-03

111 $0=-2-1$

$0 .-2$ $5=9816 \underline{6}=03$ $1.67599-\frac{02}{4}$ $1.5966 E_{2}=0.1$ - 1. $3332 E=03$ 210

$1.9505 \mathrm{E}=02$ $1.85825=01$ $0 .-7$ $1.3591 E=03$ $0.9279 E-04$

34

$-9.2252 \mathrm{E}=04$

$-2.9730 E=03$

1. $2100 \mathrm{E}=03$

ㅇ.

$3-1$

$0 .-\frac{1}{3}$

$2.33692=03$

$2.7243 \mathrm{E}=02$

3. $9370 \mathrm{E}=03 \ldots 2.0070 E=03$

48

- .

49

$2.0166 E-02$

53

$0.25-4 \frac{8}{5} 98 \mathrm{E}=03$

$2.6621 E=01$

45

$3.8621 E=03$

410

$5.65503 E=02$

$3.8180 E=03$

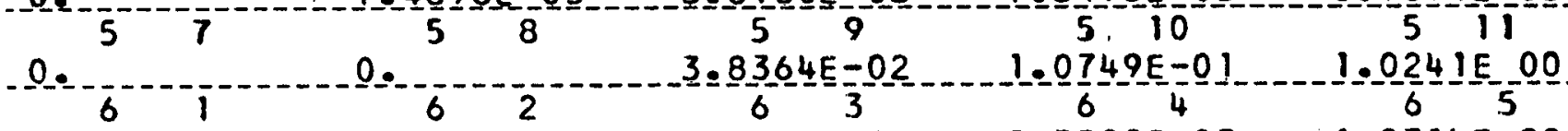

$0.0 .0515 E-02 \quad 5.3599 E-03 \quad 1.0314 E-02$

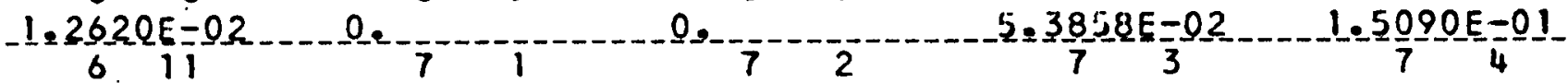

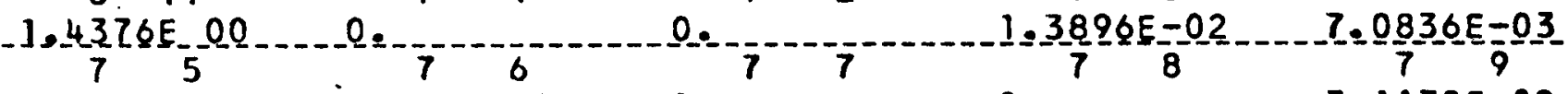

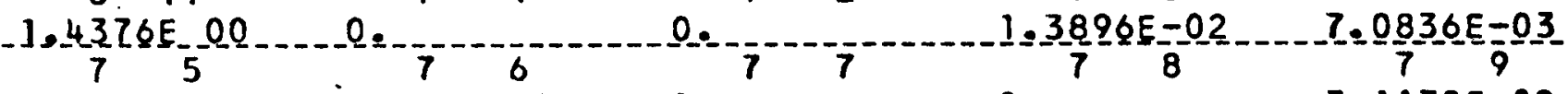

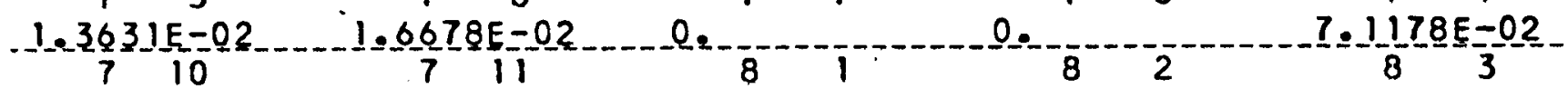

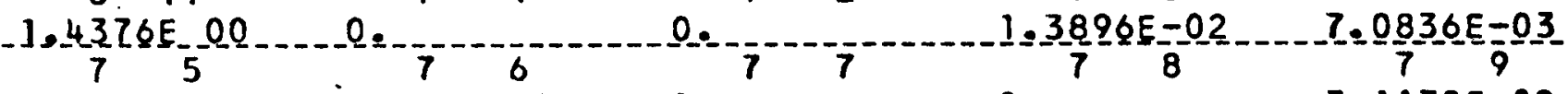

Q.

$02-4$

4. $7253 E=03$

4 II

$5=382-9 E-01$

$7=3473 E=03$

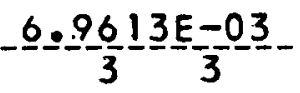

$1=9470 E-03$

0.0.

0.

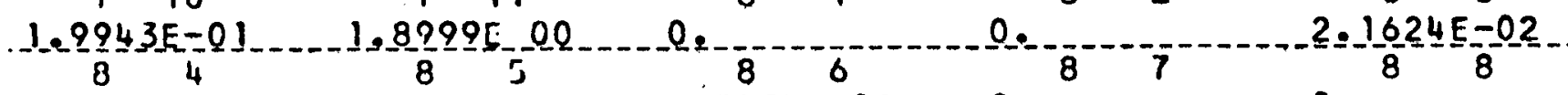

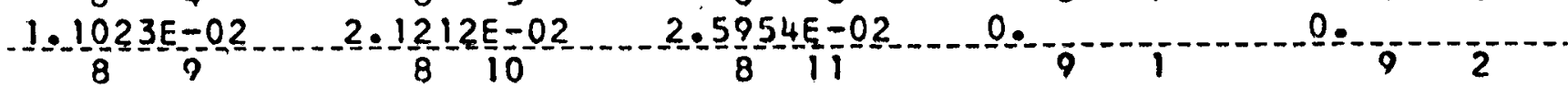

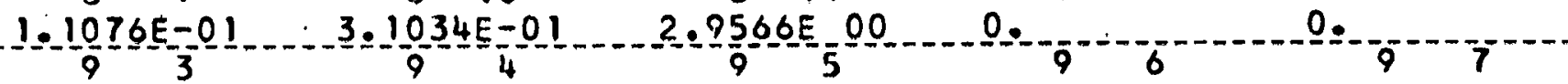

$2.7093 E-02 \ldots 1.3811 E-02 \quad 2.6577 E-02 \quad 3.2517 E-02 \quad 02$

…

$1.3877 E-01 \ldots 3.8882 E=01$

3.7042500

0. 
SAMARIUM 149

N1 N2

SIGMA_N,G_IN]/N2)

10
0.10
0.10


_SAMARIUM_L49:-

\section{SIGMA N,G (NIIN2)}

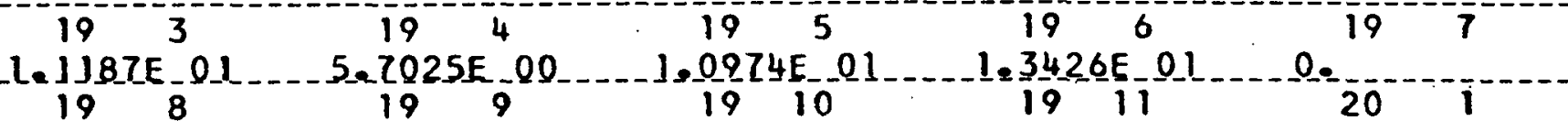

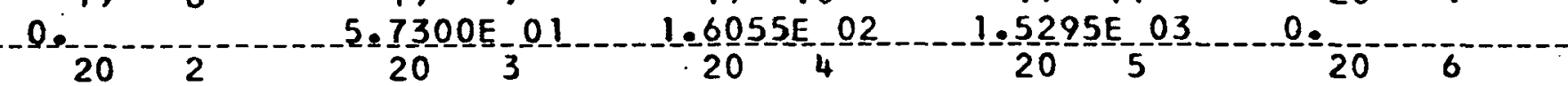

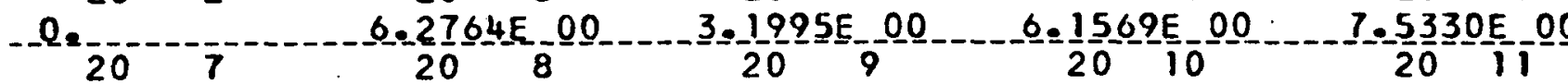

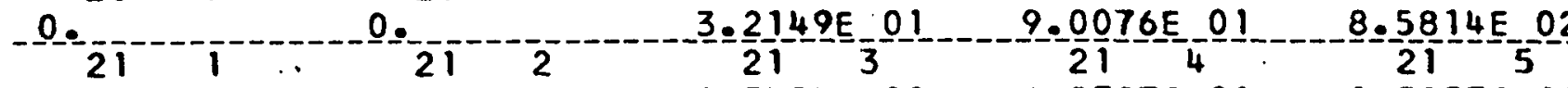

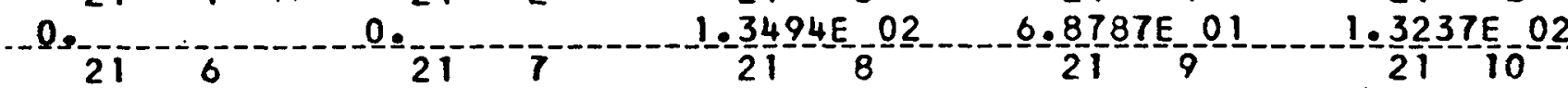

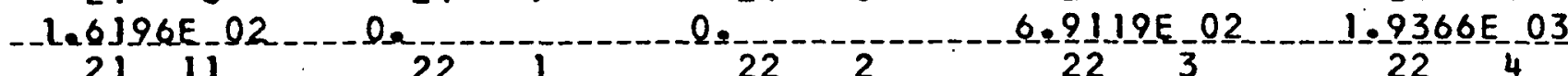

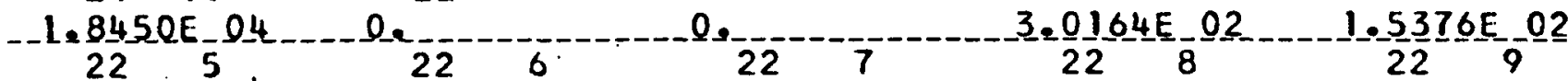

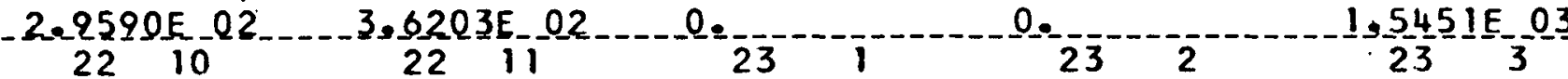

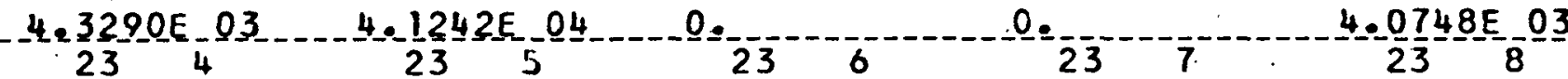
$2.0772 E_{2} 03$

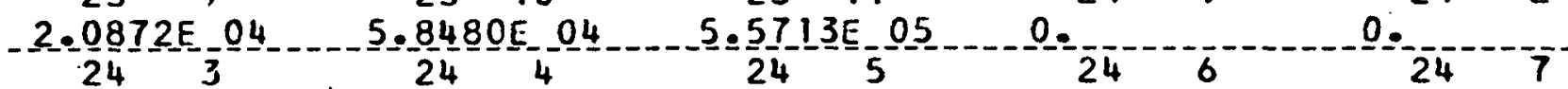
$-2.2043 \mathrm{E}-03$

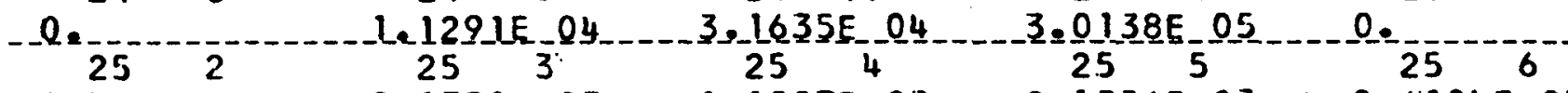

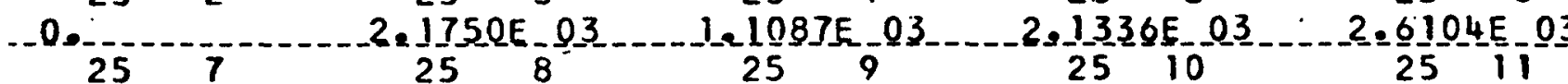

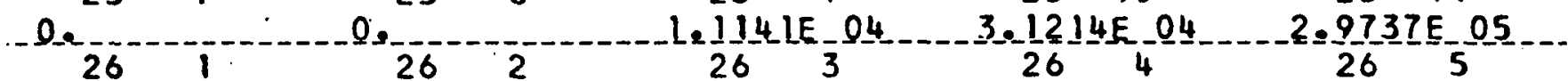

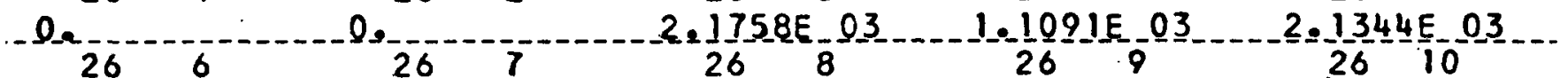

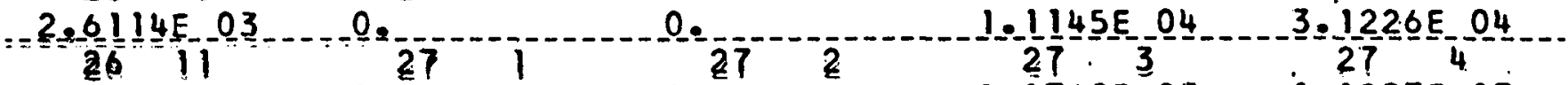

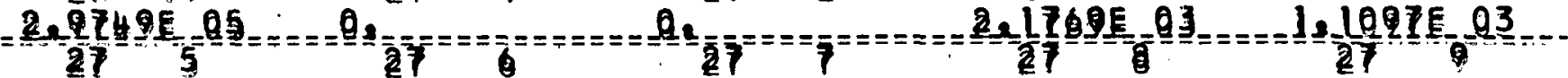

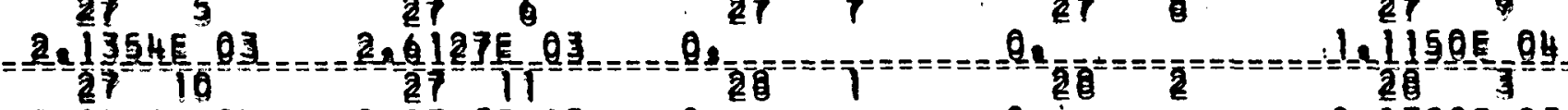

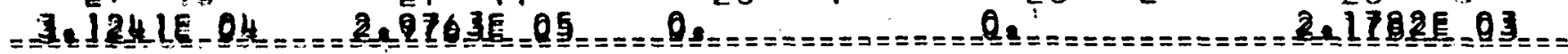


SAMARIUM 149

$N 1$
SIGMA N, (N1/N2)

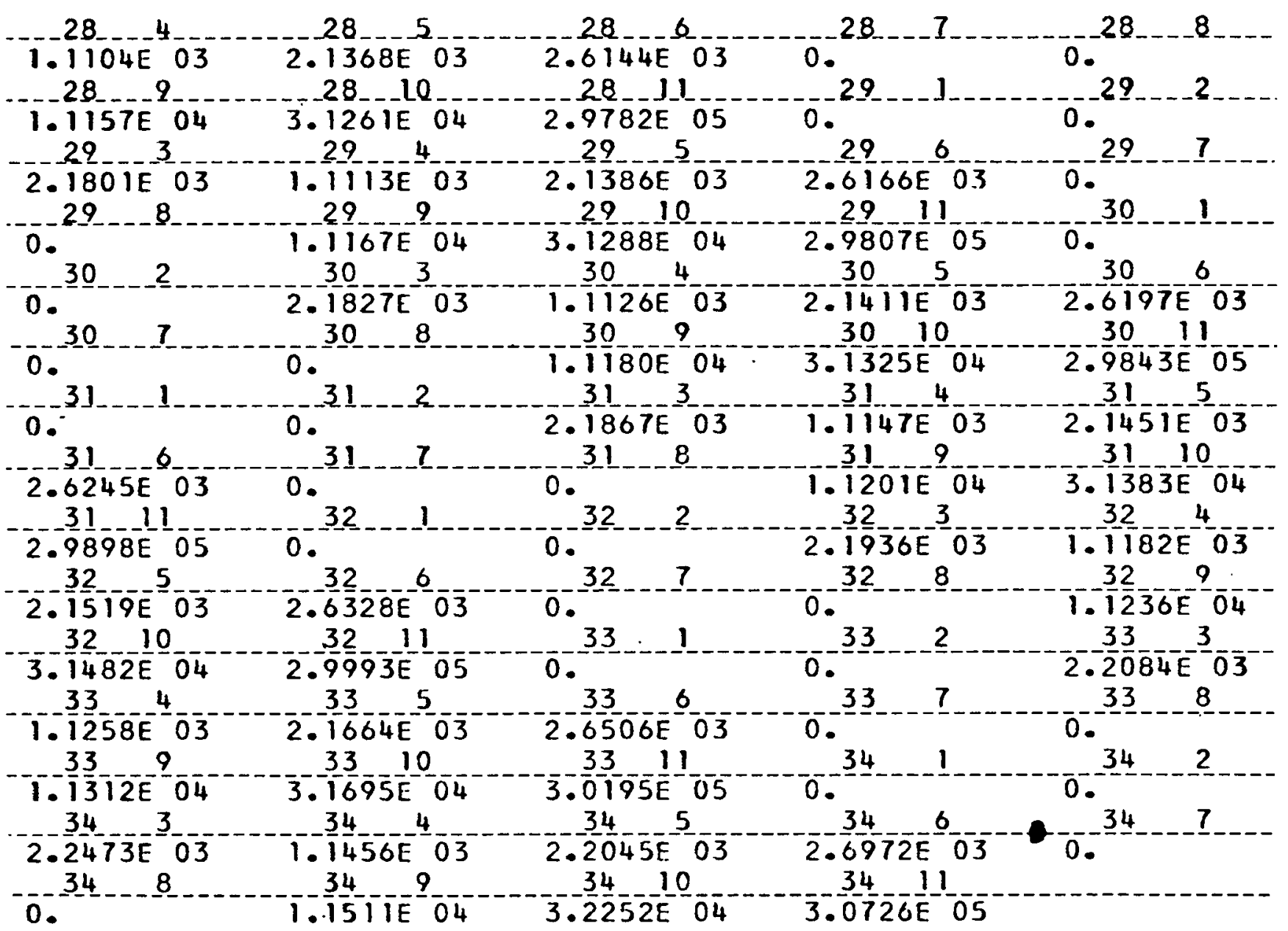


DAIE_ 2/21 1/6]

DESIGNATION

RHODIUM
CODE NO.

45.1030
DENSITY FACTOR

I. $70858 \mathrm{E} 02$
SIGMA LEVEL ENERGY SCATTER
XI_SIGMA SCATTER
SI GMA TRANSPORT
SIGMA. ABSORPTION
XII SIGMA SCATTER 1

$1.000 E$ OT $4.3373 \mathrm{E} 00 \quad 4.0956 \mathrm{E}$ 00 $1.9402 \mathrm{E} 00$ 1.6464E-02 $-2.3728 \mathrm{E} 00$

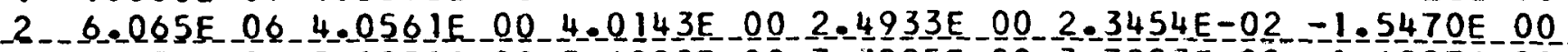

$\begin{array}{llllllllllll}3 & 3.679 E & 06 & 5.105 & & \end{array}$

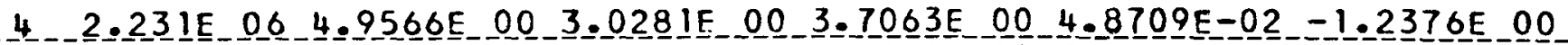

5 1.353E $065.996 \mathrm{IE} 002.3843 \mathrm{E} 004.3780 \mathrm{E} 007.0175 \mathrm{E}-02=1.6017 \mathrm{E} 0 \mathrm{O}$

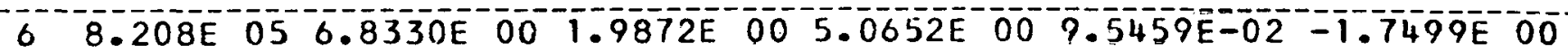

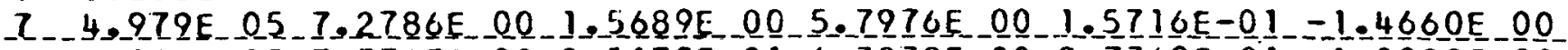

$8 \quad 3.020 E$ O5 $7.3363 E$ O0 2.5179E-01 $6.3232 \mathrm{E} \quad 002.7369 \mathrm{E}-01-1.0028 \mathrm{E} 00$

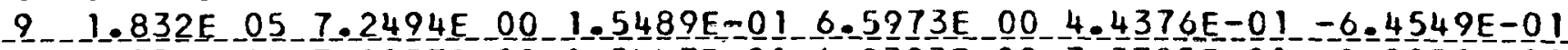
$100.738 \mathrm{E}$ 04 7.1157E 00 1.5447E-01 6.8303E 00 7.3785E-01-2.8251E-0

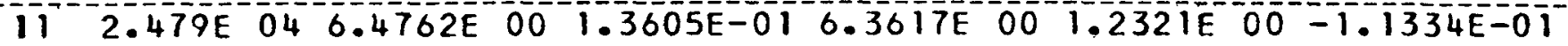

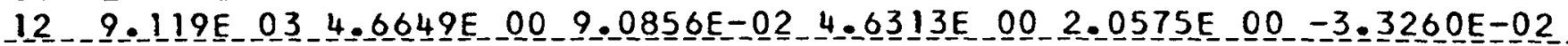
$133.355 \mathrm{E}$ 03 3.2500E $00-6.3299 \mathrm{E}-02 \quad 3.2287 \mathrm{E}$ 00 3.2222E $00-2.1084 \mathrm{E}-02$ 14 1.234E $03+1.843 \mathrm{IE} 00$ 3.5898E-02 1.8311E $004.7280 \mathrm{E} 00-1.1878 \mathrm{E}=02$. $154.540 \mathrm{E} 023.4556 \mathrm{E} 006.7303 \mathrm{E}-023.4330 \mathrm{E}$ 00 $3.4919 \mathrm{E} 00=2.2371 \mathrm{E}=02$

16 8. TिवE O

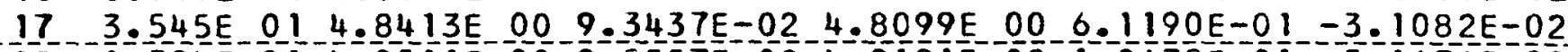
$181.304 \mathrm{E} 014.9511 \mathrm{E} 00-9.5557 \mathrm{E}-02-4.9191 \mathrm{E}-00-1.0678 \mathrm{E}-0 \mathrm{1}-3.1676 \mathrm{E}-02$

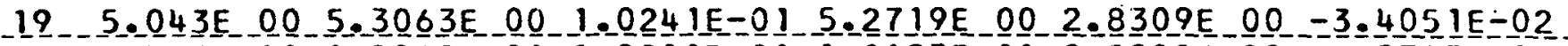

$201.445 E$ O0 $1.0260 E$ OI $1.9801 E-011.0193 E$ O1 $8.8922 E 02-6.5727 E-02$

$210.826 \mathrm{E}-0 \mathrm{1}-1597 \mathrm{E}$ 00 6.0982E-02 3.1392E 00 1.5530E $02-2.0292 \mathrm{E}-02$

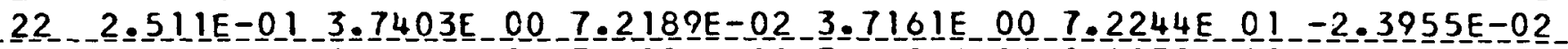

$239.237 \mathrm{E}-02 \quad 3.8858 \mathrm{E}$ 00 $7.4996 \mathrm{E}-023.8606 \mathrm{E}$ 00 $9.0238 \mathrm{E} 01-2.4945 \mathrm{E}-02$

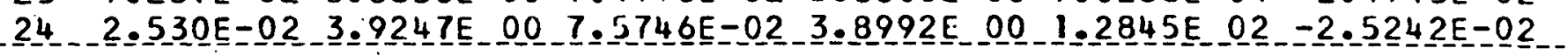

$45003.023 E=01 \quad 4 \cdot 1375 E-007.9853 E-02 \quad 4 \cdot 11075002.2261 E 02-2.6529 E-02$ $40002.718 \mathrm{E}-0 \mathrm{1}-3.9609 \mathrm{E}-0 \mathrm{O}-6445 \mathrm{E}-02-3.9352 \mathrm{E}-00-1.8232 \mathrm{E}-02-2.5440 \mathrm{E}-0 \overline{2}$

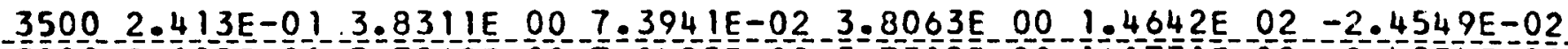

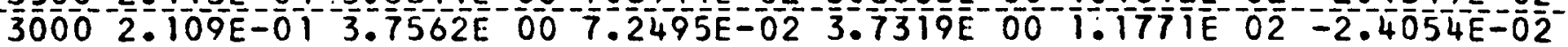
2500 I.804E-01 3.7355E 00 7.2095E-02 3.7113E 00 9.8258E 01 -2.3955E-02

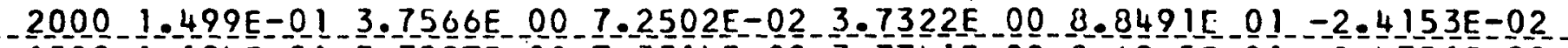
1500 1. $194 \mathrm{E}-013.7987 \mathrm{E} 00-7.3314 \mathrm{E}-02 \mathrm{3} .774 \mathrm{IE} 00-8.6965 \mathrm{E} 01-2.4351 \mathrm{E}-02$

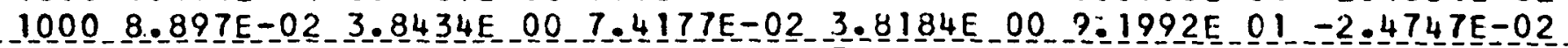
$500-5.850 \mathrm{E}-023.8833 \mathrm{E}$ 00 $7.4948 \mathrm{E}-02-3.8582 \mathrm{E} 00 \mathrm{0}-0530 \mathrm{E}-02-2.4846 \mathrm{E}-02$

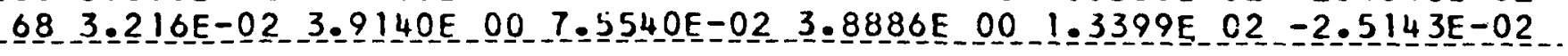


THERMAL TRANSFER MATRICES

SEMPERATURE
SIGMA $\begin{gathered}\text { SIGMA } \\ \text { DIGMA }\end{gathered}$

\section{$\mathrm{N} 1{ }^{-1} \mathrm{~N}$}

SI GMA S (N1/N2)

\begin{tabular}{|c|c|c|c|c|}
\hline $2.8964 \mathrm{E} 00$ & & $=\frac{21-23}{3.0240 E-06}$ & 24 & 25 \\
\hline $\begin{array}{l}2.8964 \mathrm{E} 0 \\
-\quad 22-21\end{array}$ & $\begin{array}{r}2.6335 \mathrm{E}-01 \\
-22 \\
-22\end{array}$ & $\begin{array}{l}3.0240 E-06 \\
-22 \\
-2\end{array}$ & 22 & 0.2 \\
\hline $2.9686 E-01$ & $3.2535 \mathrm{E} 00$ & $2.0663 E-01$ & $1.5722 E-04$ & $3.5327 E-06$ \\
\hline$\frac{23}{1484 \mathrm{E}-06}$ & $--\frac{23}{2}-22$ & $-23-23$ & $-23-24$ & 25 \\
\hline $\begin{array}{l}1484 \mathrm{E}-06 \\
-24 \\
-2\end{array}$ & $\begin{array}{c}0.122 \\
2422\end{array}$ & $24 \quad 23$ & $24 \quad 24$ & $\begin{array}{r}18 \\
24\end{array}$ \\
\hline-25 & $\begin{array}{l}4.0907 E-03 \\
25 \\
2\end{array}$ & $\begin{array}{l}1.5463 \mathrm{E} 00 \\
25\end{array}$ & $\begin{array}{l}1.9448 \mathrm{E} 00 \\
25\end{array}$ & 25 \\
\hline & $2.1015 \mathrm{E}-04$ & $3.8993 E-01$ &.$\overline{35}$ & $32 \mathrm{E}$ \\
\hline
\end{tabular}

\section{TEMPERATURE}

DEGe_F.

4.0000E_03

$4.2554 E 00$
SIGMA

SCAIIER

\section{SIGMA}

IRANSPORI
SIGMA
ABSORPIION

1.93프은
NU SIGMA

EISSION 4.2277E 00

$\underline{0}=$

Ni N2

SIGMA S (N1/N2)

\begin{tabular}{|c|c|c|c|c|}
\hline$-21-21$ & & $21-23$ & -21 & -2 \\
\hline 2.88010 & $\begin{array}{c}2.7960 \mathrm{E}-01 \\
22 \quad 22\end{array}$ & $.4106 E$ & 0. & \\
\hline $\begin{array}{l}2.6448 E-01 \\
23 \quad 21\end{array}$ & $\begin{array}{r}3.2815 \mathrm{E} O 0 \\
23\end{array}$ & $\begin{array}{c}2.0951 \mathrm{E}-01 \\
23\end{array}$ & $\begin{array}{l}1.0331 \mathrm{E}=04 \\
23\end{array}$ & $\begin{array}{l}1.72058 \\
23\end{array}$ \\
\hline $2.8073 E-06$ & $6.2358 E-01$ & $3.1207 \mathrm{~F}$ & $1.6820 E-01$ & $1.5693 \mathrm{E}-02$ \\
\hline & $2.3225 E-03$ & $1.4763 E 00$ & $2.0122 \mathrm{E}-00$ & $5 . \frac{24}{5}-94-\frac{26}{9}-0$ \\
\hline & $8.6 \frac{26}{6723 E-05}$ & $-\frac{26}{3} \frac{6}{6}-\frac{2}{92}-\frac{3}{-0}-0$ & $1.3187 \mathrm{E}=0$ & $-\frac{26}{2.6098 \mathrm{E}} 0$ \\
\hline
\end{tabular}


RHODIUM

IHERMAL_IRANSFER_MAIRICES

_LEMPERAIURE DEG. F.
SIGMA SCATIER
SIGMA TRANSPORT
SIGMA ABSORPTION
NU SIGMA

FISSION

\section{$3.5000 E 03$ \\ $4.2210 E 00$ \\ $4.1936 \mathrm{E} 00$ \\ I. $9371 E 02$ \\ 0 .}

N 1

N2

SIGMA S (NIIN2)

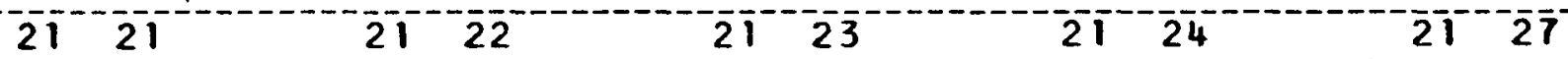

$2.8584 E 00$ 3. $01265-01$ $5.5132 E-07$

ㅇ. $22 \quad 21$ 2222 2223

$22 \quad 24$ $22-27$

-2. 3006E $=01$

3.30960 . 00

$-\frac{14}{23}=\frac{9 E-0}{23}$

6. 2209E-01

$$
2321
$$

2322

$3.1715 \mathrm{E} \quad 00$

$==\frac{\theta}{24}=-\frac{1}{3} 1$

$5.7213 \mathrm{E}=01$

2422

2423

2324

7. $1513 E-07$

1. 1652 를 $=03$

27 年

1,3923

$1.6649 \mathrm{E}-01$

2327

$==0$

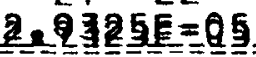

年

2424

$1.3523 \frac{3 E}{24}=02$

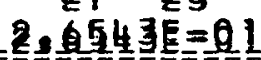

$28 \frac{8925}{2}$

5. $3579 E=01=-$

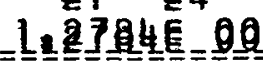

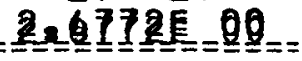

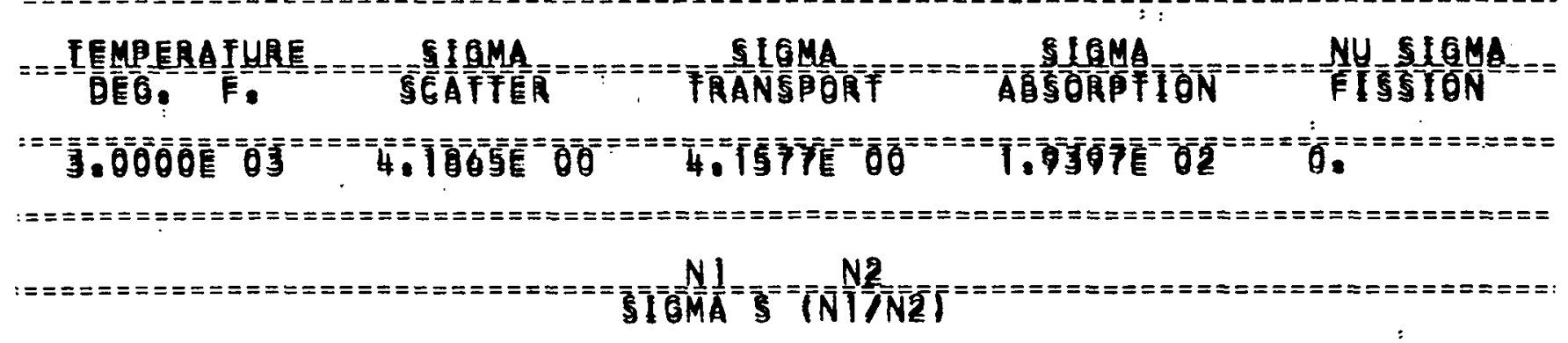

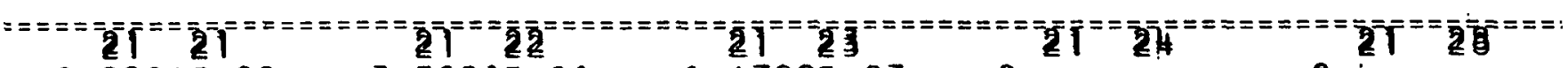
2.000

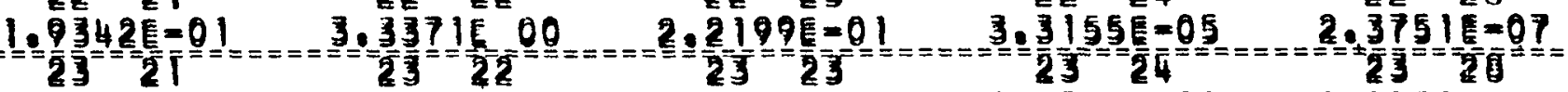

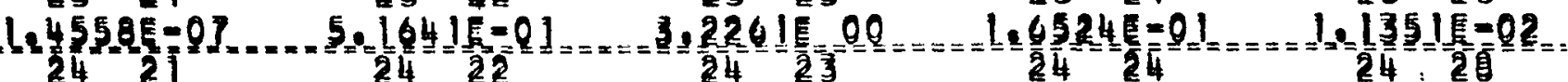
$==0$.

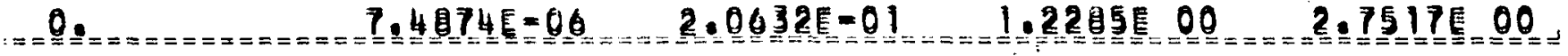


RHOD_LUM

\section{THERMAL TRANSFER MATRICES}

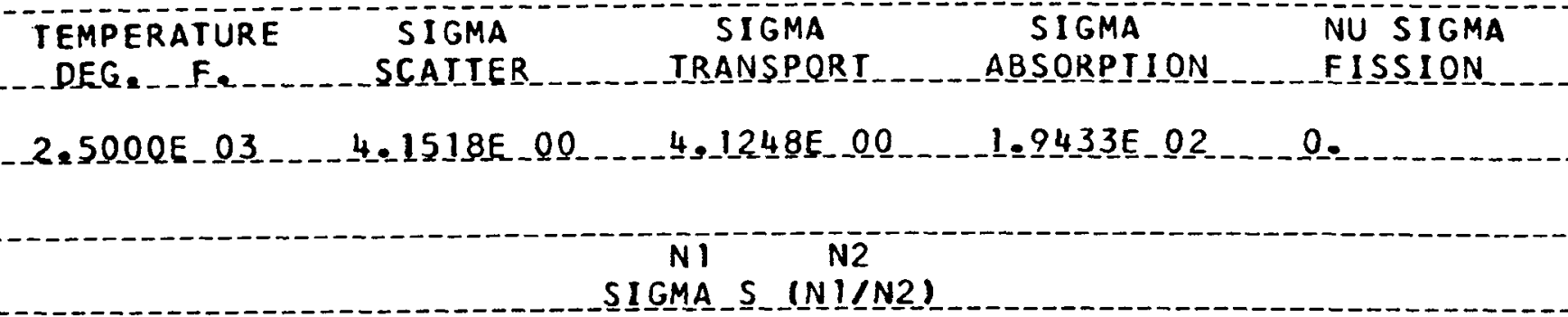

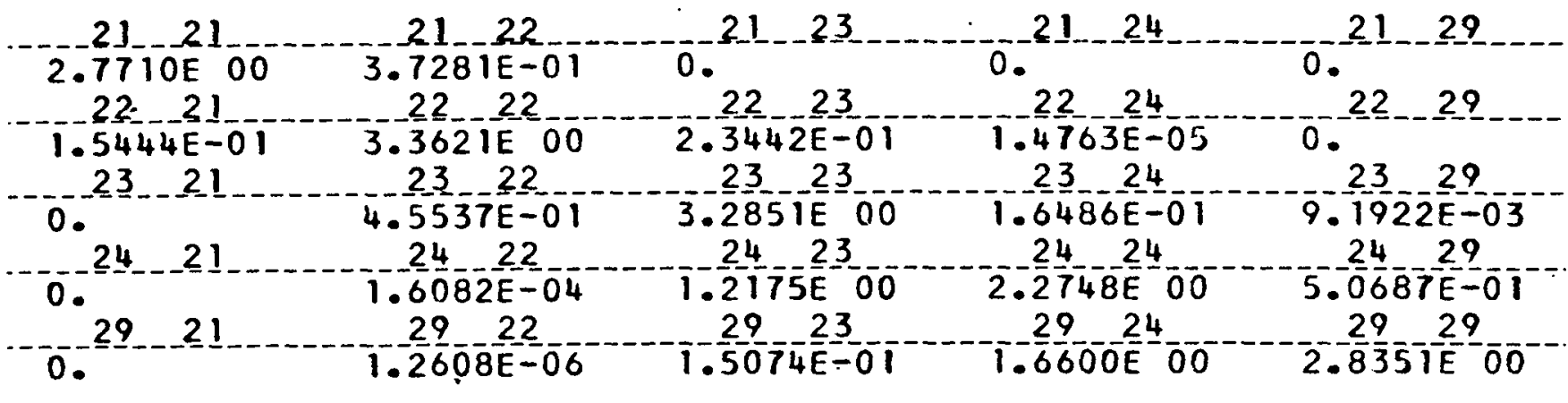

TEMPERATURE

DEG. $F$.

- 2.0000E 03

4.1170E OOO
SI'GM̄A IRANSPORI

4.0902E 00
SIGMA
ABSORPIION

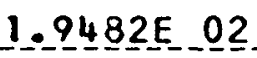

NU SIGMA

FISSION

\section{$N 1$
SIGMA S $(N 21 / N 2)$}

$-\frac{2}{2} \frac{21}{16} \frac{21}{3 \mathrm{E}} 00^{-1}-\frac{21}{312} \frac{22}{2}-01$

0

$22 \quad 21$

$22 \quad 22$

$22 \quad 23$

2224

$22 \quad 30$

0.

$3.8751 \mathrm{E}-0$

$3.3493 \mathrm{E} \cdot 00$

$-024-21 \quad 2 \frac{24}{3} 5305 E-05$

$30-21$

24
$3.5305 E-05$
30
$1.0994 E-07$

$1.1075 E^{2} 00$

$30-23$

$1.0031 E-01$

$4.9474 \mathrm{E}=06-10$.

$23-24$

$23 \quad 30$

$1.6617 \mathrm{E}-01-7.0687 \mathrm{E}-03$

$2 . \frac{24}{3926 \mathrm{E}} 00$

$4.8660-\frac{30}{8}=0 \overline{1}$

$30-24$

$1.0867 \mathrm{E} 0 \mathrm{O}^{-}$

$30 \quad 30$

$2.9300 \mathrm{E} 00^{-1}$ 
RHODIUM

THERMAL TRANSFER MATRTICES

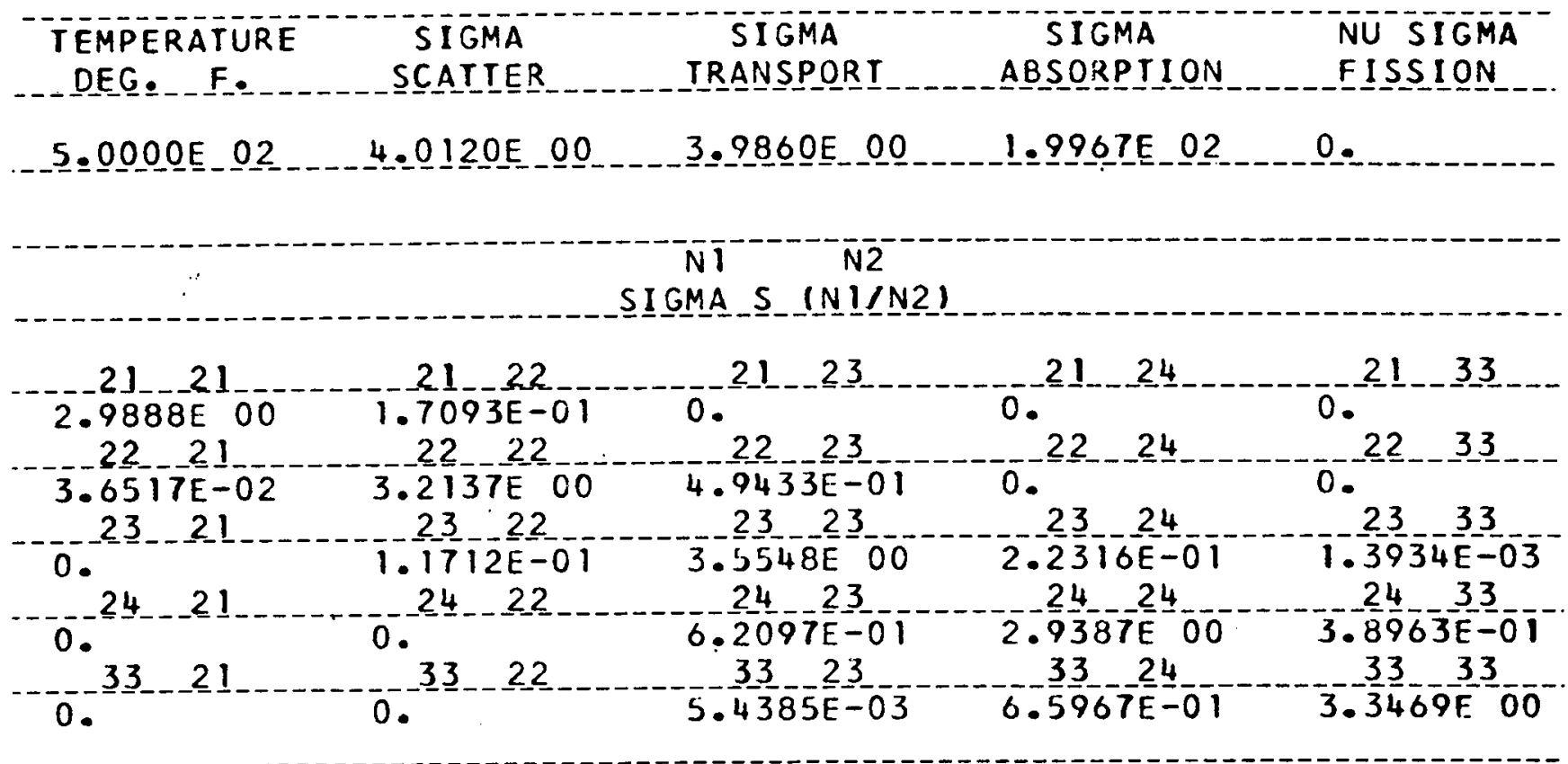

TEMPERATURE

DEG. .. Fe.

_6.8000E 01 _ 3.9816E_00___3.9558E_00 _-2.0678E_02

SIGMA

SCATIER
SIGMA IRANSPQRI
SIGMA

ABSORPIION
NU SIGMA

EI SSION

\section{$N T^{N}$ \\ S1 GMA S (N1/N2)}

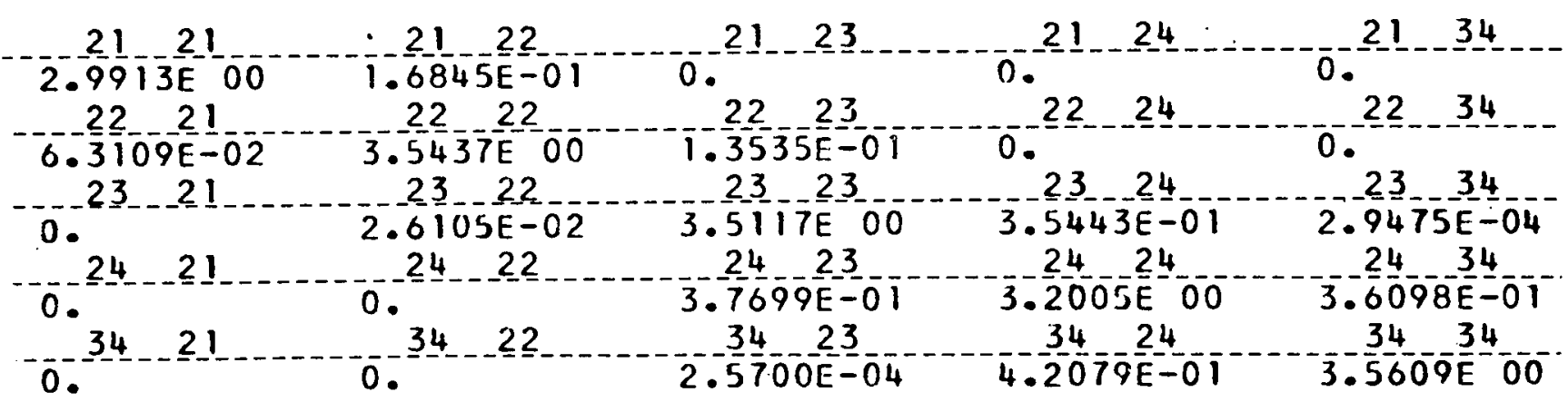


1
1
$2.6605 E[D Q$

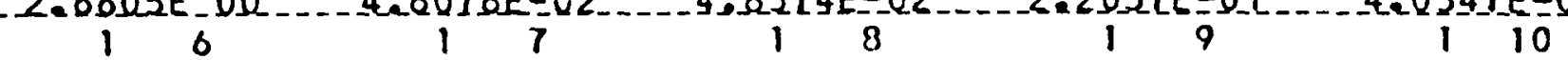

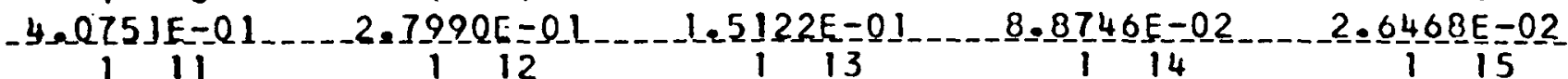

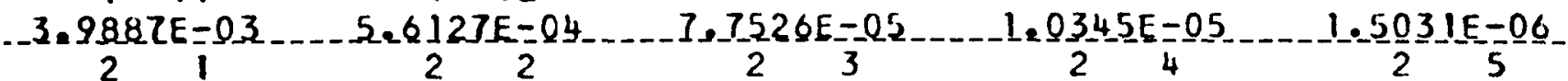

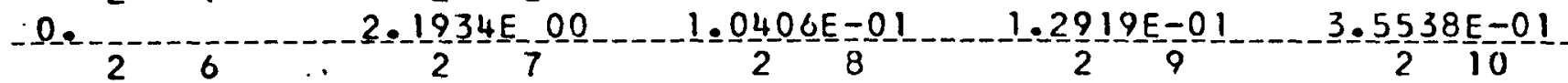
$4.6284 \mathrm{E}-01 \ldots-3.8039 \mathrm{E}=01$

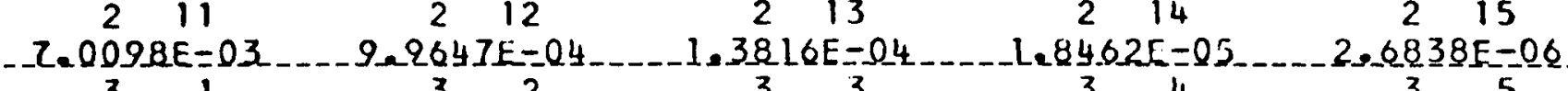

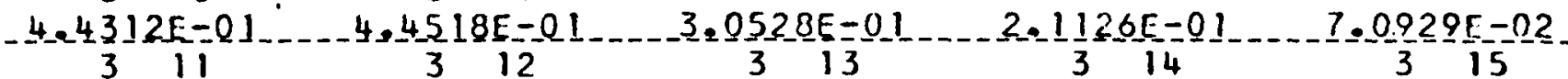
$-1 \cdot 1368 E=02 \ldots 1.637] E=03$

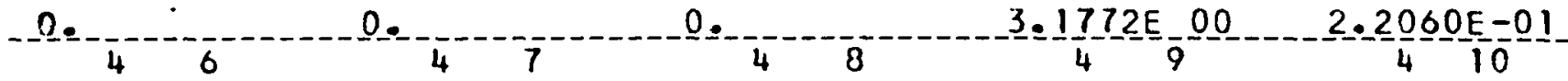
-3.4 $181 E-01 \ldots 4.4754 E-01 \ldots 3.6316 E-01 \quad 2.8273 E-01 \ldots 1.0329 E-01$ $1.7301 E-02$ …

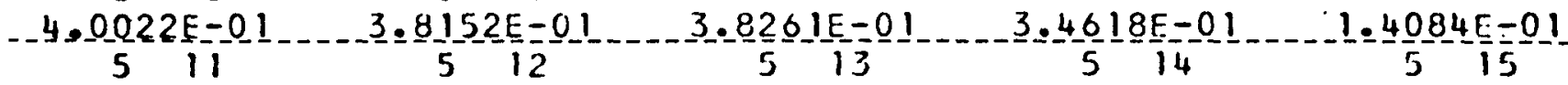

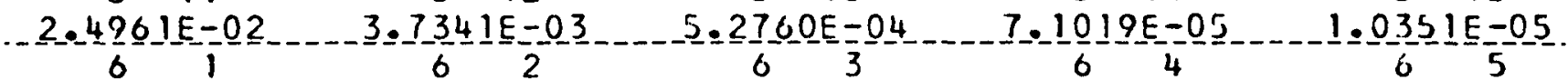

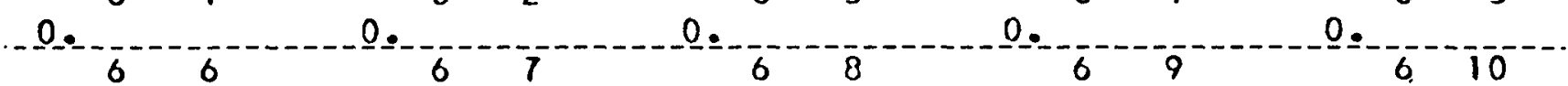
. $5.2785 E-00 \quad 4.5858 E-01 \quad 4.0050[-01 \quad-4.4178 E-01 \quad-2.0700 E-01$.

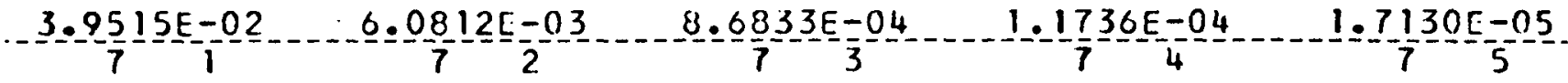

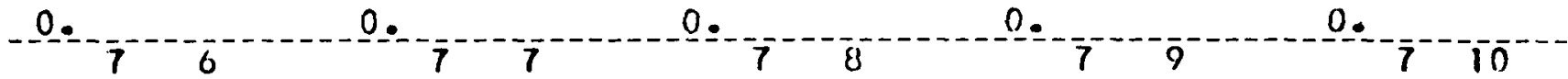
_. 
RHODIUM

Ni N2

SIGMA SO INI/N2)

$3.7559 \mathrm{E}-02$
$0.4933 \mathrm{E}-03$


_BHODLUM.

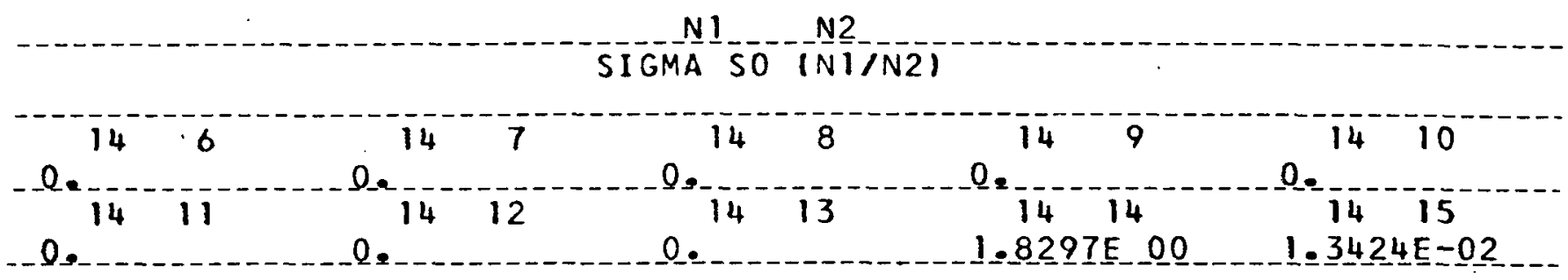


RHODIUM

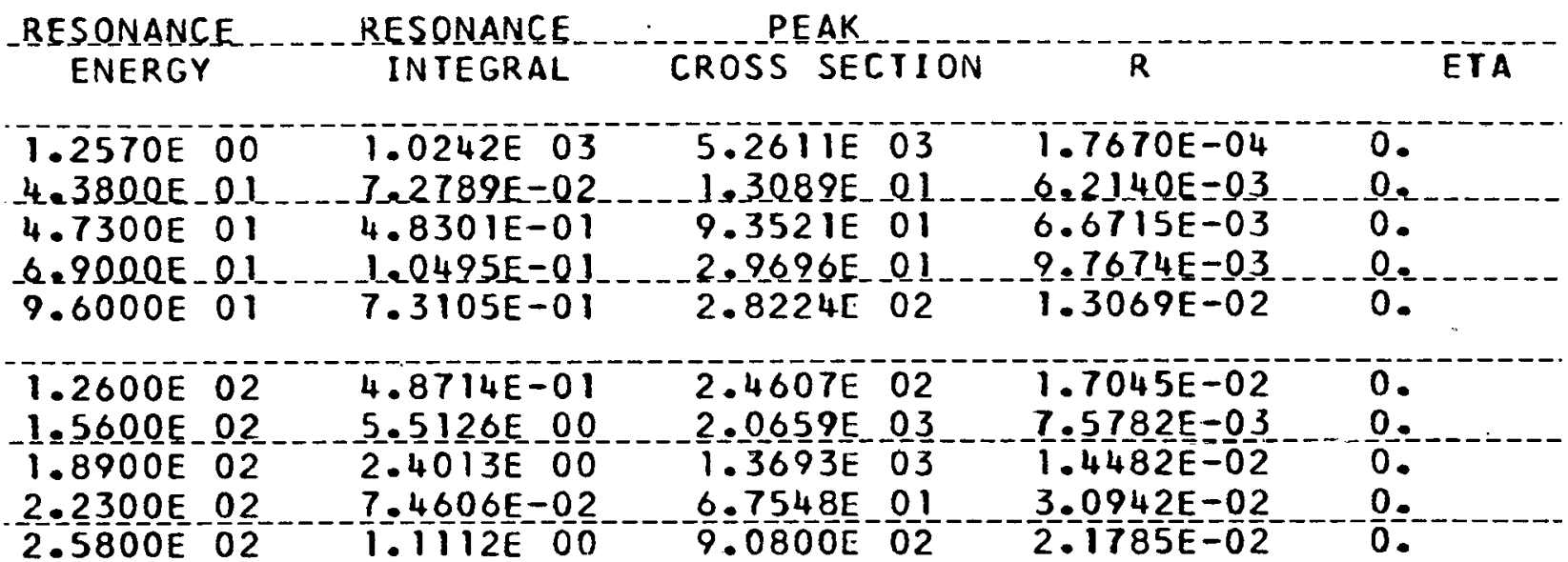


BHODIUM

TRANSFERS FROM GROUPS 1 THROUGH 34 TO GROUPS 1 THROUGH 11

SIGMA

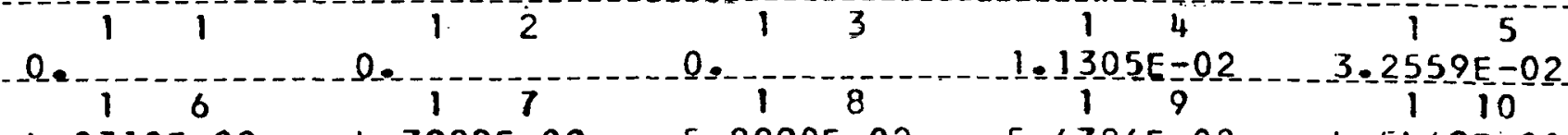

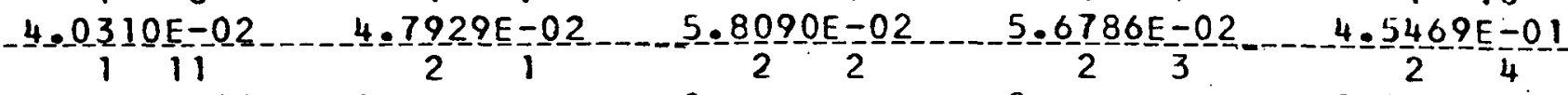

$2.76 \frac{64}{2}=\frac{02}{5}$

$3.5 \frac{8}{2} 90 \mathrm{E}-02$

$-4.5591 \mathrm{E}-01$

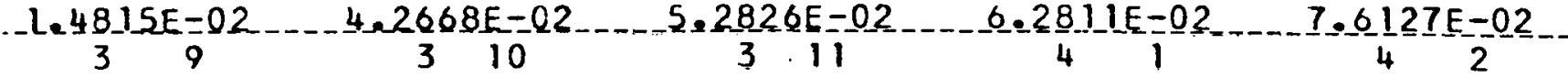

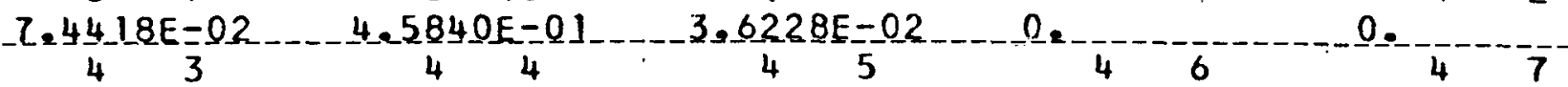

$0.4 .8508 E=02 \quad-5.3305 E-02 \quad 0.5994 E-02-7.8469 E-02$

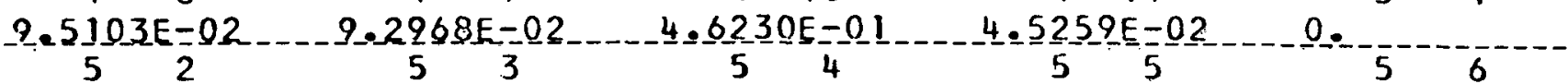

Q.

$1.0274 \mathrm{E}=01$

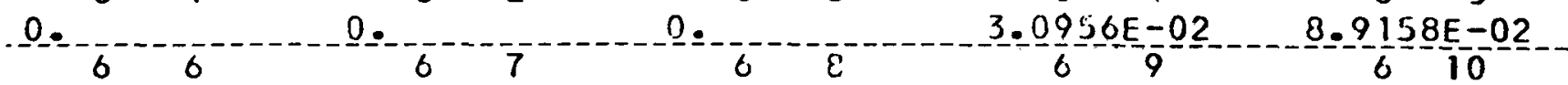

1. $1038 E=01$

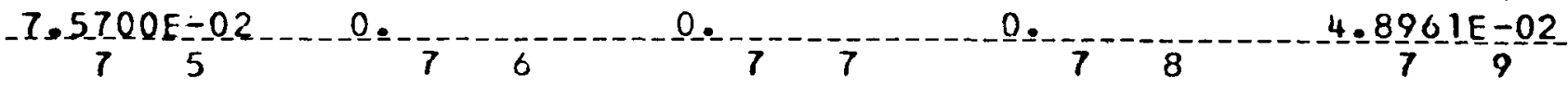

$1.4 \frac{1}{7} \frac{02 E-01}{10}-1.7459 \mathrm{E}=01 \ldots 2.07 \mathrm{~S} 9 \mathrm{E}=01$

$4.47265-01$

$-8.3148 E-02 \ldots 2.3948 E-01 \quad 2.9649 E-01 \quad 3.5253 E-01-104.2727 E-01$

4. $1767 E-01 \ldots$

$0.0 .3239 E-01 \quad 3.8132 E-01 \quad 4.7209 E-01 \quad 5.6133 E-01$

-6. $8032 E=01 \ldots$ 
RHODIUM

N1 N2

SIGMA_N,G_(N]IN2)

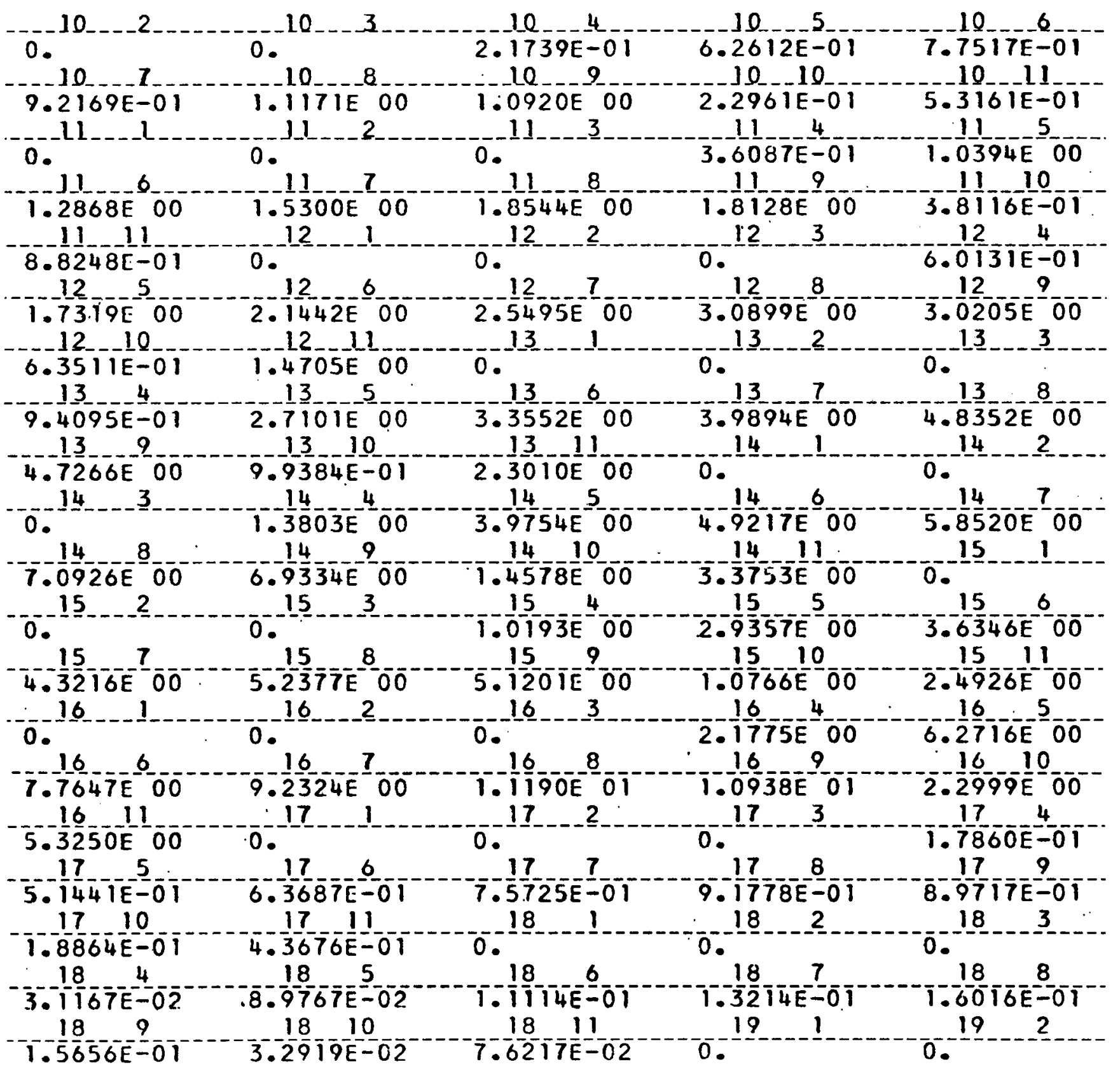


-3HORLUM.

N1

SIGMA N, G (NITNZ)

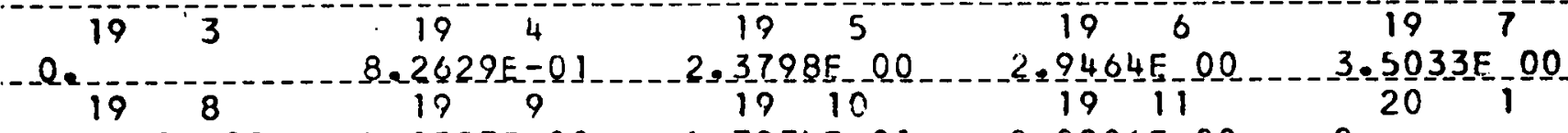

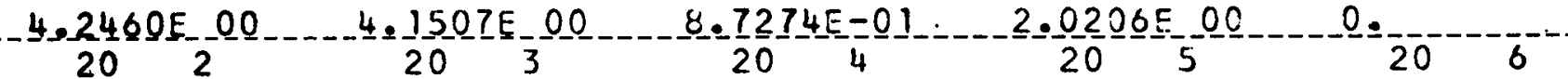

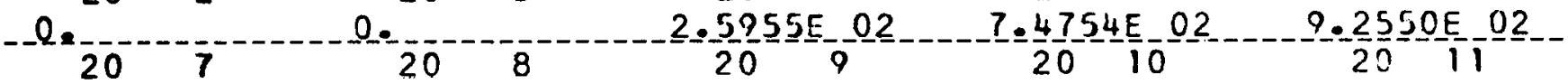

$1.1004 E_{1} 03 \quad 1.3337 E_{2} 03 \quad 1.3038 E_{2} 03 \quad 2.7414 E-02-6.3470 E-02$

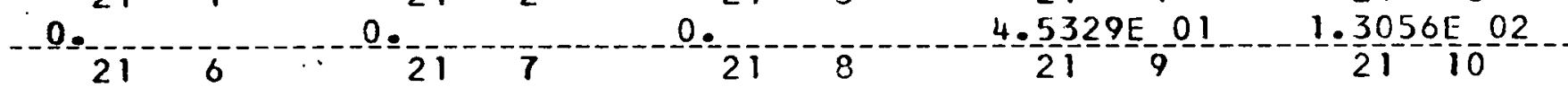
L. $6164 E_{-02} 02$ _.9219E_02___ $23293 E_{0} 02$ $211122 \quad 22 \quad 22 \quad 32$

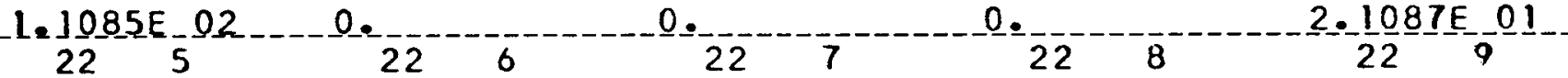

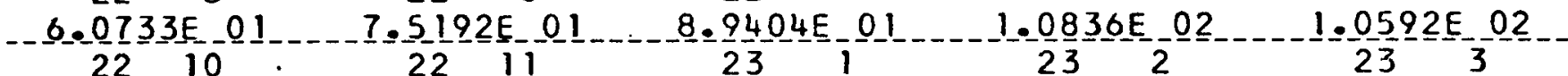
$2.2272 E_{4} 01$ $2.6339 E_{0} 01-7.5860 E-01$

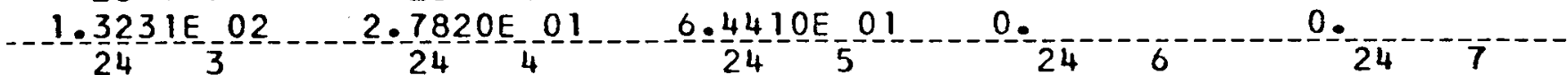
-0.

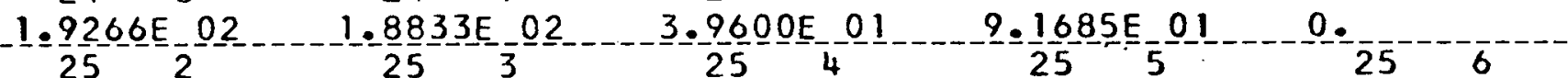

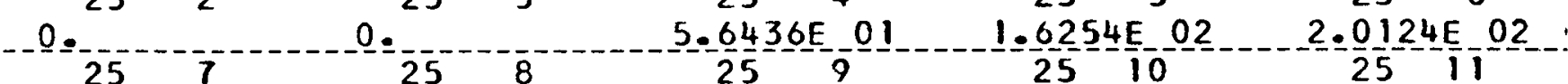
-2. $-\frac{3}{2} 928 E_{1} 02$

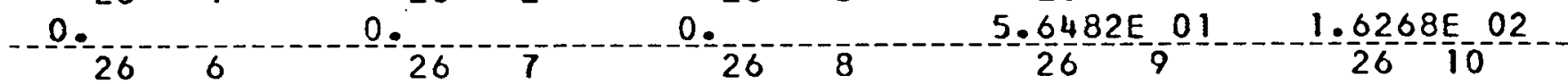
$-2.0141 E-02 \quad-2.3947 E-02 \quad 2.9024\left[\begin{array}{l}02 \\ 2\end{array}\right.$

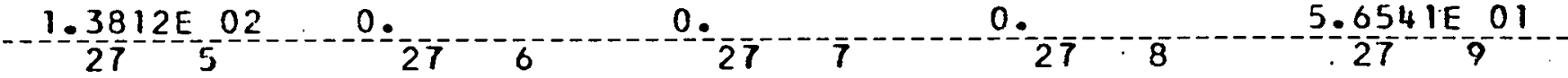
$1.6285 \mathrm{E} 02 \quad 2.0161 \mathrm{E} 02 \quad 2.3972 \mathrm{E}-02 \quad 2.9054 \mathrm{E}-02 \quad 2.8402 \mathrm{E} 02$ -5.9?19E 01 ㅇ..…… 0 
RHODIUM

N1 N2

S1GMA_No_G_ (N]/N2)

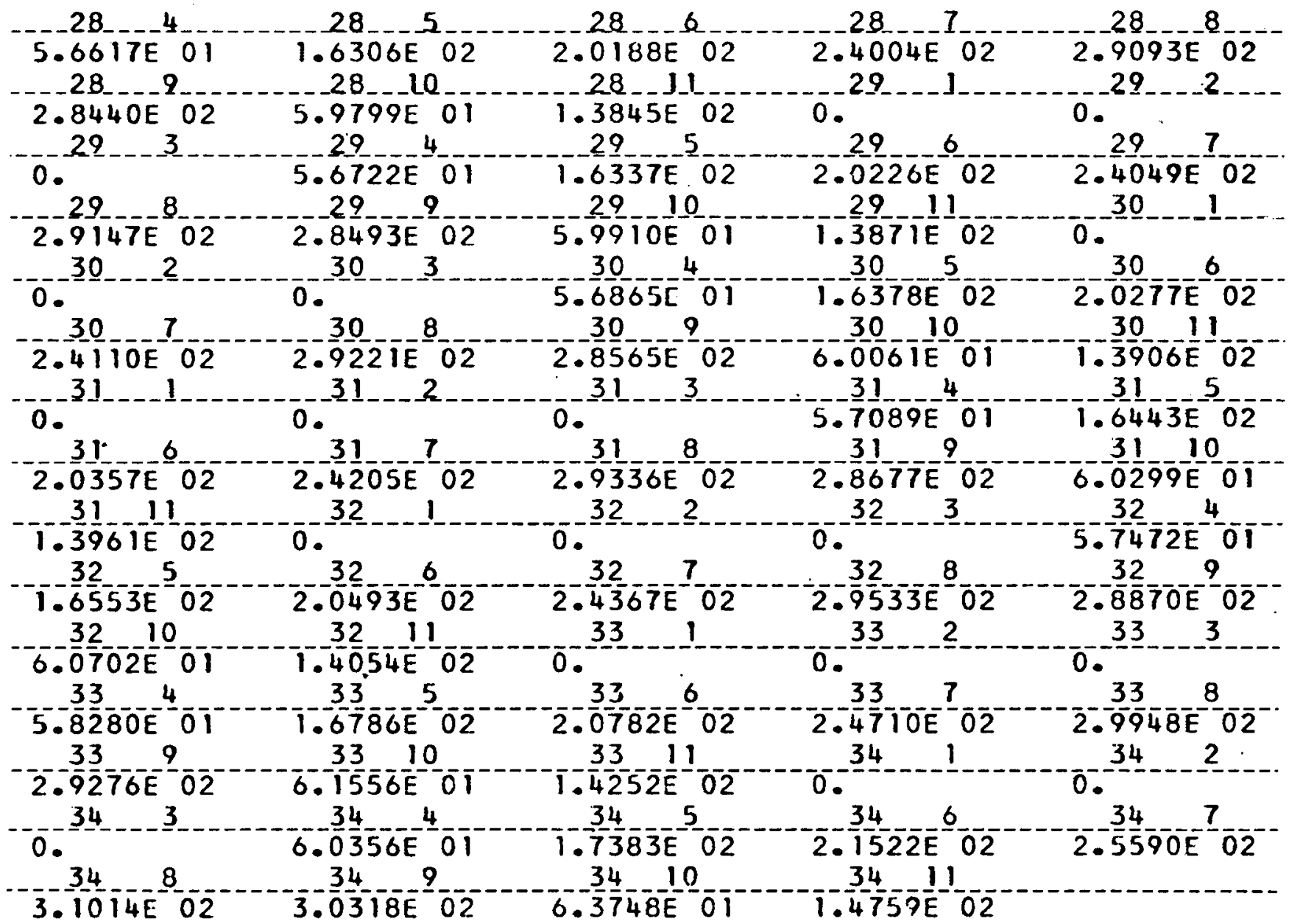


DAIE_2/21/6I.

DESIGNATION

PLATTINUM
CODE NO.

78.1950
DENSITY FACTOR

$3 . \overline{3} 9 \overline{0} \bar{O} \overline{\mathrm{O}} \overline{\mathrm{O}} \overline{2}$

\section{SIGMA

LEVEL ENERGY SCATTER SCATTER TRANSPORT ABSORPTION SCATTER I

1.000E 075.1831E 00 1. 1506E 00 5. 1052E 00-8. 1167E-04-1.7713E-02

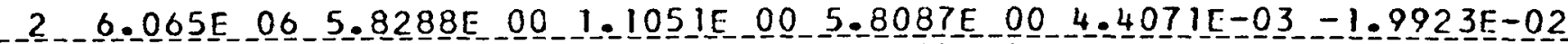

$30.679 \mathrm{E} 060.3522 \mathrm{E} 009.8486 \mathrm{E}-016.3303 \mathrm{E} 001.3415 \mathrm{E}-02-2.1708 \mathrm{E}-02$

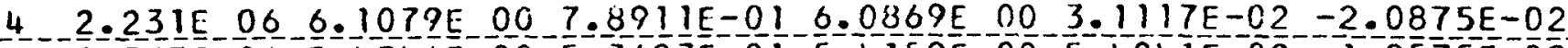

5 1.353E $065.4346 \mathrm{E} 00-\frac{1}{5} .3623 \mathrm{E}-015.4159 \mathrm{E} 00-\frac{1}{5.404} \mathrm{IE}-02-1.8575 \mathrm{E}=02$

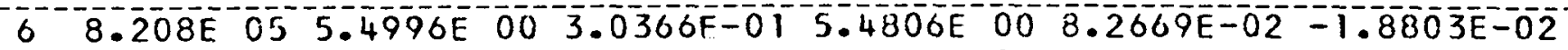

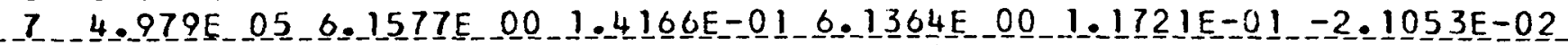

8 3.020E O5 7.2260E 00 7.4485E-02 7.2011E 00 1.6290E-01-2.470 IE-02

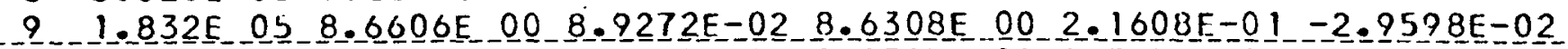

$106.738 \mathrm{E} 041.0791 \mathrm{E} 011.1123 \mathrm{E}-011.0754 \mathrm{E} 012.7856 \mathrm{E}-01-3.6873 \mathrm{E}-02$

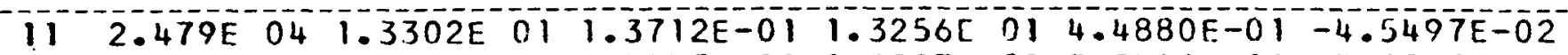

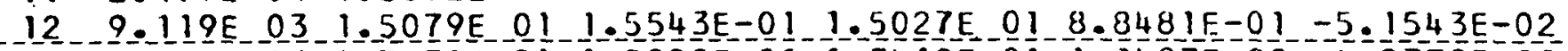

$133.355 \mathrm{E} 031.7470 \mathrm{E} 011.8008 \mathrm{E}-011.7410 \mathrm{E}$ 01 $1.8487 \mathrm{E} 00-5.9770 \mathrm{E}-02$

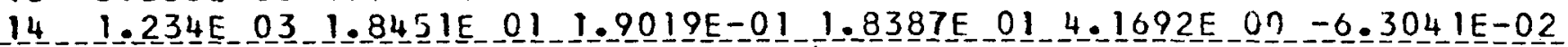

15 4.540E $021.9548 \mathrm{E} 012.0149 \mathrm{E}-011.9480 \mathrm{E} 015.5579 \mathrm{E} 00-6.6808 \mathrm{E}-02$

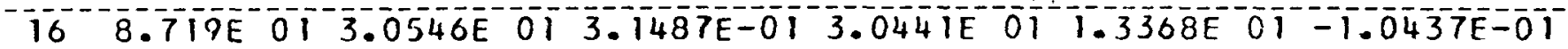

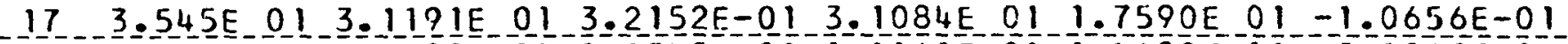

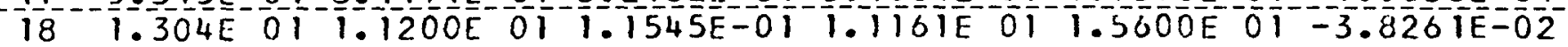

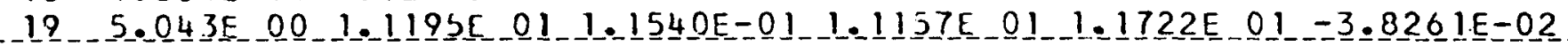

$2.01 .445 E$ E $1.1432 E$ O $1.1784 E-011.1393 E 011.0509 E 00-3.9054 E-02$

$216.826 \mathrm{E}-0 \mathrm{~J} 1.1578 \mathrm{E} \mathrm{C} 1.1934 \mathrm{E}-0 \mathrm{~T} 1.1138 \mathrm{E} 0 \mathrm{1} 1.6898 \mathrm{E} 00-3.9549 \mathrm{E}-02$

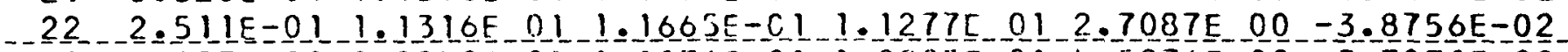

$230.237 \mathrm{E}-021.0842 \mathrm{E} 011.1176 \mathrm{E}-011.0805 \mathrm{E} 014.5876 \mathrm{E} 00-3.7071 \mathrm{E}-02$

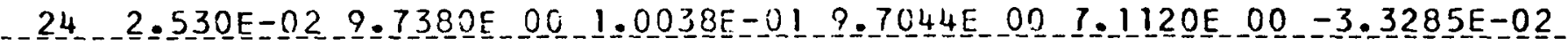

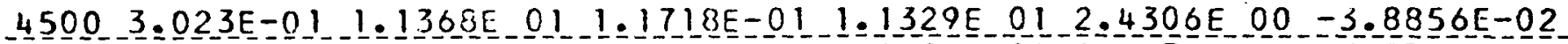
$40002.718 \mathrm{E}-011.1344 \mathrm{E} 011.1693 \mathrm{E}-011.1305 \mathrm{E}-012.5637 \mathrm{E} 00-3.8756 \mathrm{E}-02$

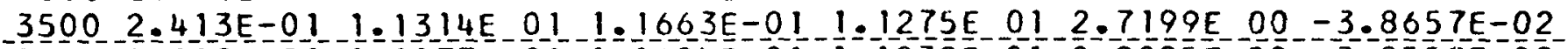
$30002.109 \mathrm{E}-011.1277 \mathrm{E} 011.1624 \mathrm{E}-011.1238 \mathrm{E} 012.9095 \mathrm{E}-00-3.8558 \mathrm{E}=02$ $25001.804 \mathrm{E}-01$ 1.1229E 01 1.1575E-01 1.1191E 01 3.1456E $00-3.8360 \mathrm{E}-02$

$20001.499 E-01,1.1169 E-01-1.1512 E-011.1130 E-01-3.4505 E-00-3.8162 E-02$ $15001.194 \mathrm{E}-011.1089 \mathrm{E}$ o1 $1.1431 \mathrm{E}-011.1051 \mathrm{E} 013.8658 \mathrm{E}$ 00 $-3.7864 \mathrm{E}-02$

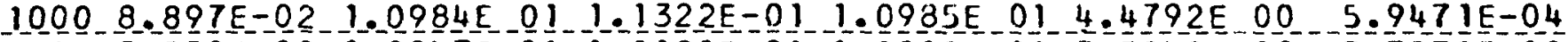

$5005.850 \mathrm{E}-021.0847 \mathrm{E}$ 01 $1.1181 \mathrm{E}-011.0810 \mathrm{E}$ 01 $5.5242 \mathrm{E} 00-3.707 \mathrm{EE}-02$ 68 3.216E-02 1.0785E 01 1.1117EE-01 1.0748E 01 7.4498E 00 -3.6873E-02

NOTE: Basic cross section data were obtained from BNL 325 , Second Edition and from Supplement 1 to BNL-325, Second Edition. 
PLATINUM

THERMAL TRANSFER MATRICES

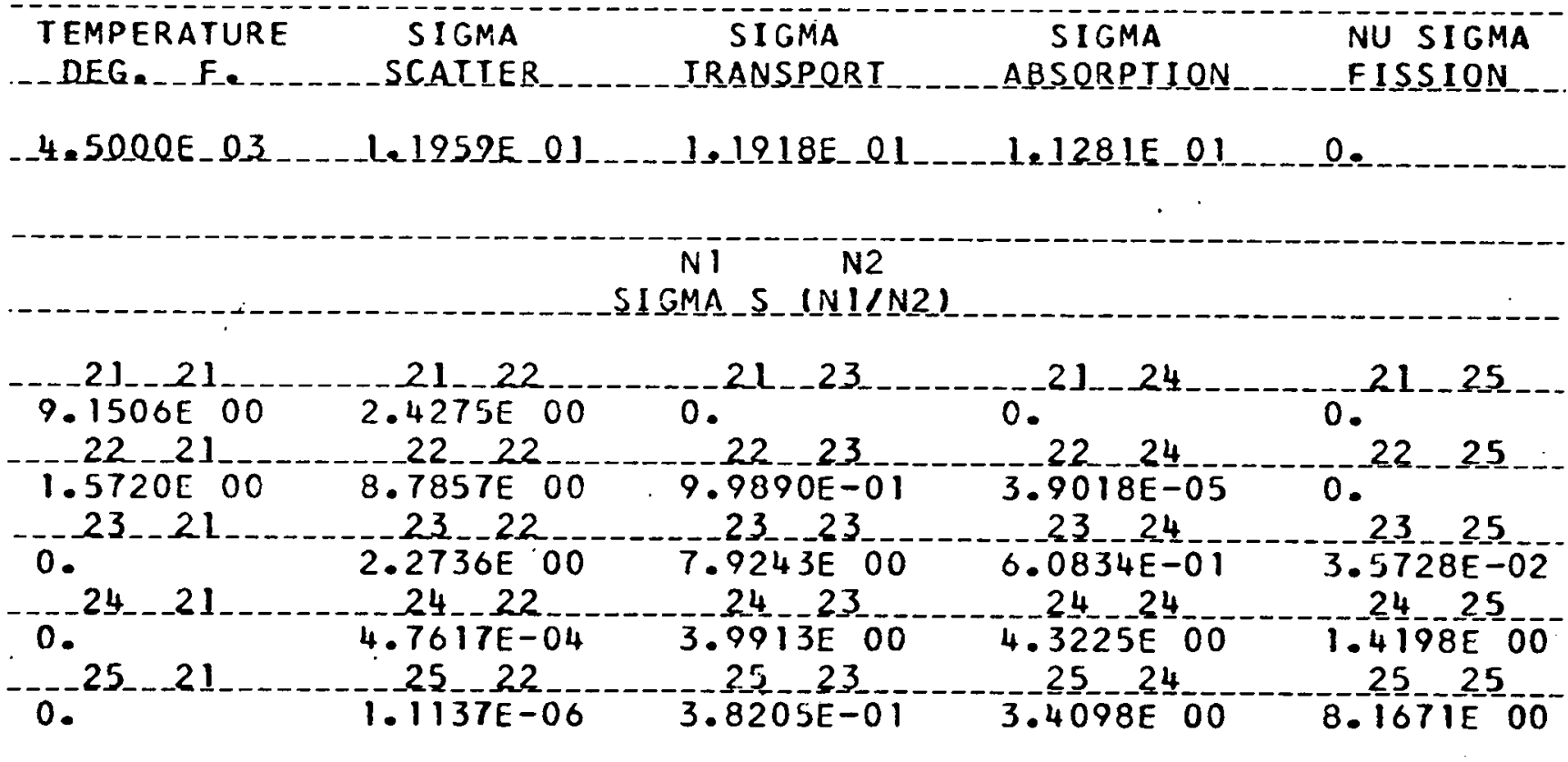

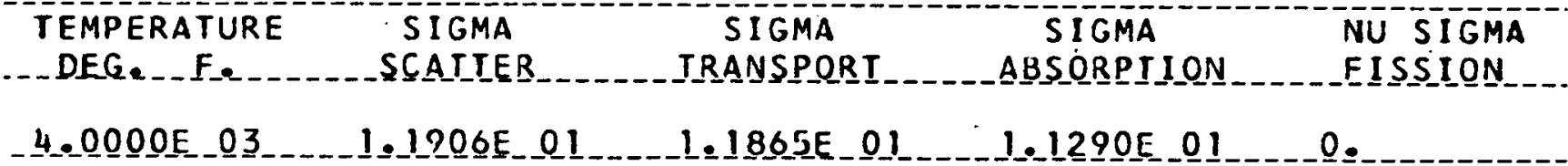

\section{Ni \\ SIGMA S (NIIN2)}

\begin{tabular}{|c|c|c|c|c|}
\hline 21 & $21-22$ & 21 & 21 & $21-26$ \\
\hline $\begin{array}{l}.9175 \mathrm{E} 0 \\
22 \quad 21\end{array}$ & $\begin{array}{c}2.6606 \mathrm{E} \\
2200\end{array}$ & 0. & 0 & \\
\hline $\begin{array}{r}1.4612 \mathrm{E} \\
23\end{array}$ & $\begin{array}{c}8.7931 E 00 \\
2322\end{array}$ & $\begin{array}{c}1.0618 \mathrm{E} 00 \\
2323\end{array}$ & $\begin{array}{l}1.9368 \mathrm{E}-0 \\
2324\end{array}$ & \\
\hline & $2.1911 \mathrm{E} 00$ & $7.9964 \mathrm{E} \quad 00$ & $6.2677 \mathrm{E}-0$ & $3.1672 F$ \\
\hline $0 .-24$ & $2.1661 \mathrm{E}-\mathrm{C} 4$ & $4.0464 \mathrm{E} 00$ & 4.21310 & $-1.47883 \mathrm{E}-0$ \\
\hline${ }_{0}^{26}$ & $2.5700 \mathrm{E}-07$ & $3.0 \frac{26}{377 E-0}$ & $3.2945 \mathrm{E}$ & $--\frac{26}{3079}-\frac{26}{E}$ \\
\hline
\end{tabular}


PLATINUM

__LHERMAL_ JRANSFER_UAIRICES.

_LEUPERATURE.

SIGMA

SIGMA.

SIGMA

NU SIGMA..

DEG . $F$.

SCATTER

TRANSPORT

ABSORPTION

FISSION

\begin{tabular}{|c|}
\hline $3.5000 E \quad 03 \quad 1.1854 E \quad 01 \quad 1.1813 E 01$ \\
\hline
\end{tabular}

$\left.S I G M \frac{N}{S}-\bar{S}-(\bar{N})-N 2\right)$

21

_. $8.6258 E 00$

2221

$2.9523 E-00$

$-\underline{0}$

$22-\frac{1}{23}$

$2 i^{--\frac{2}{4}}$

$\ldots 1=\frac{3}{23} 15 E-00$

$22 \quad 22$

$1.1446 \frac{6 E}{23}-00$

$22-24$

$21^{2} \overline{7}$

2.333

$8=\frac{1}{2} 350 E=06$

$22-27$

- 0.

$24-\frac{1}{2}$

$2.0993 \frac{\mathrm{E}}{24}-00$

$8.06 \frac{36}{2} \frac{\mathrm{E}}{2} \overline{\mathrm{B}} \mathrm{0}-\mathrm{O}$

$6 \cdot 5 \frac{5}{2} \frac{69 E}{24}-0 !$

$2 \overline{3}^{-1} \overline{7}$

-. 0.

$2 \overline{7}-\overline{2} \overline{1}$

7.8359E-0 5

$27=-22$

$3.9450 E$ OE

4. $3055 \mathrm{E} \quad 00$

$27-\frac{1}{3}$ $\frac{74}{24}-\frac{3}{2} 7-02$

……

0.

2.3119E-01

3.1619E 00

1. $-\frac{4}{2} \frac{866 E}{27}=0$

$8.4606 E 00$

IEMPERAIURE

DEG. $F$.

SI GMA

SCATTER
SIGMA

TRANSPORT
SIGMA

ABSORPTION
NU SIGMA

FI'SSION

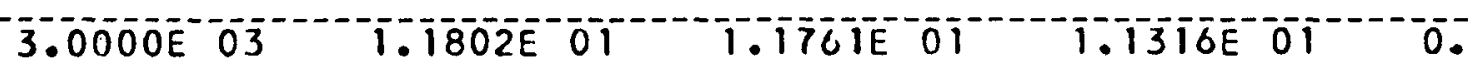

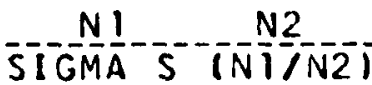

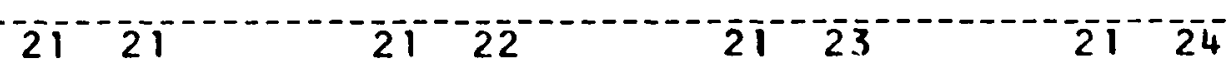

$8.2565 \mathrm{E}-00$

$\overline{2} \overline{2}^{--} \overline{2} \overline{3}$

$0 .-12-15 \overline{2}$ $21^{2} 8$

$1=\frac{1}{2} \frac{7}{3} 79 \mathrm{E}-\frac{00}{2}$ $8.8820 E \quad 00$

$1.2564 \mathrm{E} \quad 00$

$2.6900 \mathrm{E}-06$ $22-28^{-\cdots}$

...

2421

$1.9943 \mathrm{E}-00$

$23-23$

$2 \frac{1}{3} 24$ $2 \overline{3}^{-2} \overline{8}^{-\cdots}$

$-0 .-18 \overline{2} 1$

2. $1574 E-05$

$8.1386 E_{2}=0$

$0.8610 E=01$

$2.2987 \mathrm{E}=02$

…

ㅇ.

$3.8359 \mathrm{E}-00$

$4=\frac{40}{28} 19 E-\frac{0}{2} 4$

1. $50.02 \mathrm{E} 00$

$1.6571 E-01$

$3.0078 E \quad 00$

$8.6285 E 00$ 
PLATLINUM

THERMAL TRANSFER MATRICES

TEMPERATURE

SIGMA

SIGMA

SIGMA

NU SIGMA

.-DEG 2 _. F.

SCATIER

TRANSPORI

ABSORPIION

FISSION

2. 500006

1.175IE 01

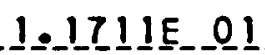

$1=1336$ 트요 1

ㅇ..

Ni

SIGMA S (N1/N2)

-..21__ 21

$7.7864 \mathrm{E} 00$

$3.7916 \mathrm{E} \quad 00$

$22-21$

$9.9431 E-01$

$22-22$

$21-23$

$8.9087 \mathrm{E} 00$

0.

$1 . \frac{22}{4}-\frac{23}{3}=$

-21..2 24

-21 - 29

0 .

$1.8698 \mathrm{E} 00$

$8.2188 \mathrm{E} 00$

22

$6.2000 \mathrm{E}-07$

22 29

0. $24-21$

$3.9500 E-06$

$7-\frac{2}{3} \frac{3}{5}-\frac{24}{10 E}-0$

0.

2329

$1.8342 \mathrm{E}-02$

$0.29-21$

$0.29-22$

$3.7166 \mathrm{E} 00$

$4 .-\frac{4}{9}-25 \mathrm{E}$

$1 . \frac{24}{52} 19 \frac{29}{0}-$

$1.0893 \mathrm{E}-01$

$2.8270 \mathrm{E} 00$

8.8153500

TEMPERATURE SIGMA
SIGMA

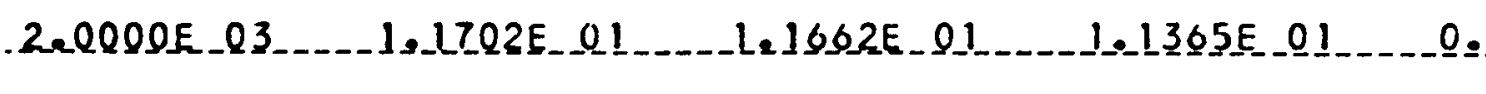

Ni $\mathrm{N} 2$

SI GMA S (N1/N2)

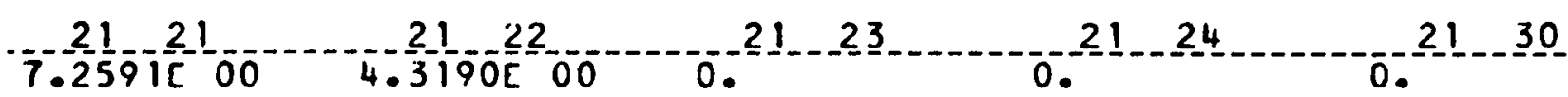

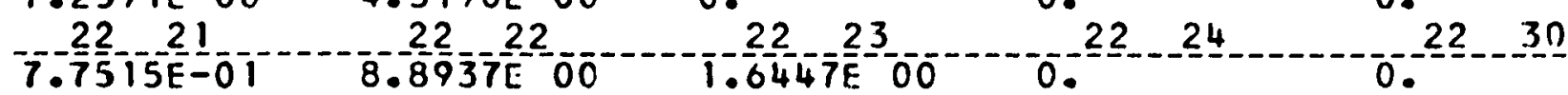

0.

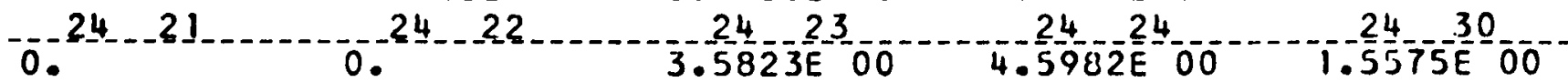

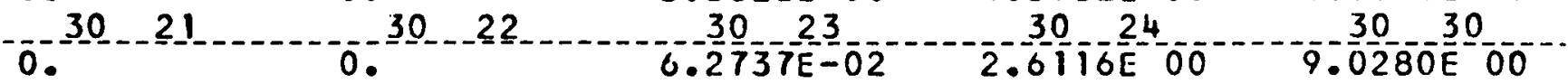


PLATINUM

THERMAL IRANSEEER MAIRILEES.

- TEMPERAIURE

SI GMA

SIGMA

DEG. F.

SCATTER

TRANSPORT

NU SIGMA

FISSION

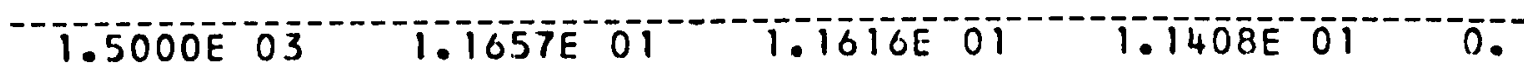

in 1

N2

SIGMA S INTINZT

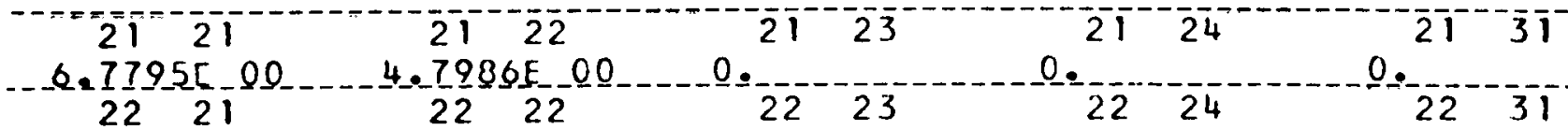

_5.2031E 01

0. 2321 2323 $23-2 \frac{1}{4}$ $23-31$ 24,21 $1.5079 \mathrm{E}-01$ $8.4003 E-00$ 2423 $9.2 \frac{25}{24}-2 \mathrm{E}=0$ 8.76 58 E -03

…… 2422 $3.4234 \mathrm{E} O 0$ 3123 4. $69696 \mathrm{E}$ - 00 3124 2431 ……

$31-22$ 2.9016E-02 2. 3495E 00

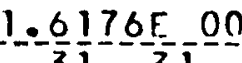
o. 9.278 IE 00

IEMPERAIURE DEG. $F$. SCATTER
SIGMA TRANSPORT
SIGMA ABSORPIION
NU SIGMA

FISSION 1. $1618 \mathrm{E} O \mathrm{~T}$
$1.0000 E \quad 03$
$1.1578 \mathrm{E} O \mathrm{~T}$
$1.1483 \mathrm{E}$ OI
0 .

\section{SIGMA S (NIIN2)}

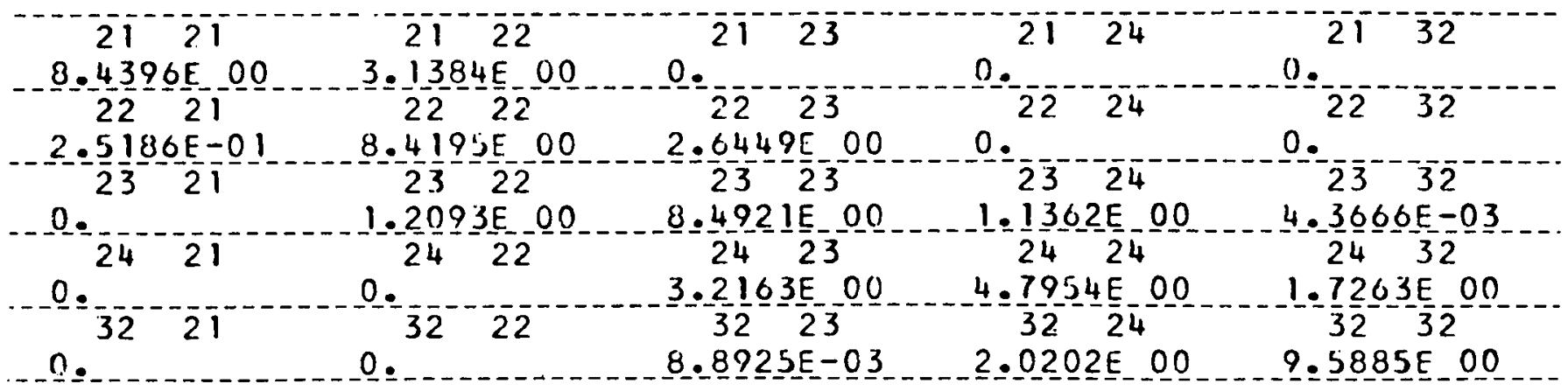


PLATINUM

THERMAL TRANSFER MATRICES

TEMPERATURE _. DEG. _. F.

SIGMA

SI GMA

SIGMA

NU SIGMA

SCAILER IRANSPQRI ABSQRPIILN

EISSI ION

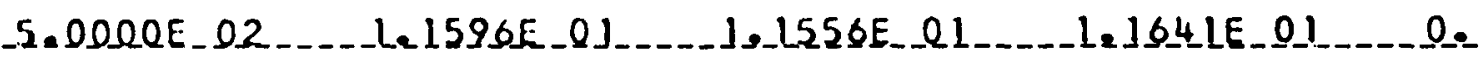

Ni N2

SIGMA S_ (NILN2)

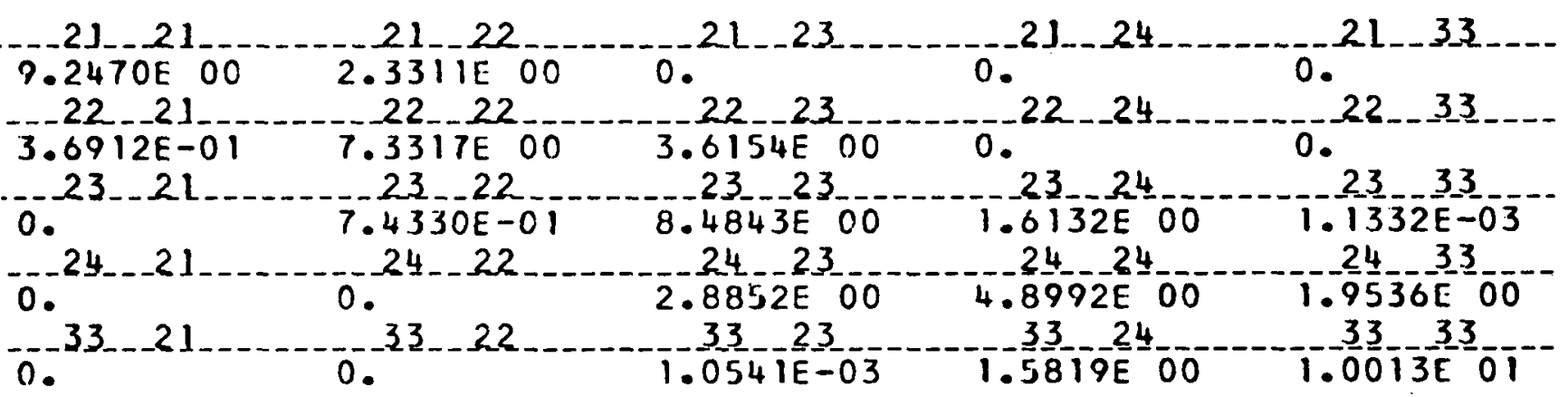

TEMPERATURE

- DEG . F Fe.

$6.8000[0]$

$1.1627 E-21$
SIGMA IRANSPQRI

$15860^{2}$
SIGMA A.BSQRPJIQN 1.2056E 01
NU SIGMA

EISSLLN
N $1^{2}$

SI GMA S__(N1/N2)

$9.1207 \mathrm{E} 00-2.4574 \mathrm{E} 00^{2}$

$0.21-24$

- $22-21$

$22 \quad 22$

1. $-\frac{22}{31}-\frac{2}{2}-\frac{3}{4}-00$

$9.0137 \mathrm{O} 0 \overline{0}$

$0-22-2$ 24

$23-23$

0 .

23

$0.23-21.23$ 1. 22

$7.7860 \mathrm{E} 00$

$2.8665 \mathrm{E} 00$

o.

$0.24--21$

0 .

$-34-21$

$2.2406 \mathrm{E} 00$

5.013 IE 00

$1.2894 \mathrm{E}-05$

$0 .-34-22$

$1.0310 \mathrm{E}$ OO

0. $21-34$

0.

22

-23 34

$4.9895 E-05$

2.4843500 1.0595 E 
PLALLNUM

TRANSFERS FROM GROUPS I THROUGH 7 TO GROUPS 1 THROUGH 15

SIGMA $20-(N 2)-N 2)$

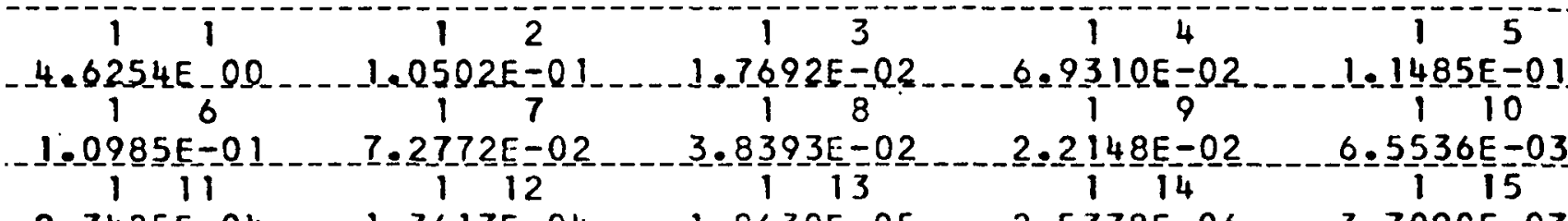

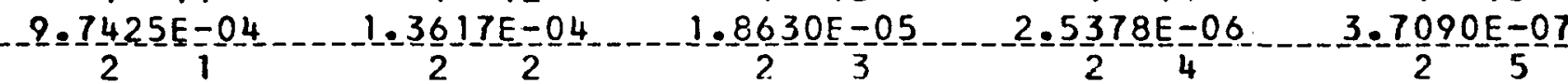
-0. $1.2567 E-01$ - L. $60648 E=03$ 3 0. $1.2102 E-01$

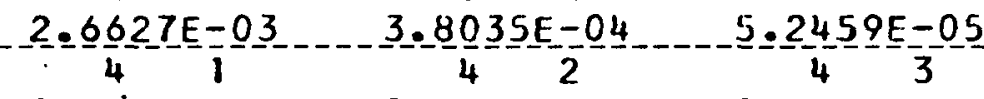
0. $3.2250 E=05-4.3 \frac{3990 E}{3}=06$ $6 .-\frac{4}{3} 27 \frac{2}{5}-07$ $3=\frac{79}{3} 45 \mathrm{E}=\frac{0}{9}$ 7.-2 $2831 \mathrm{E}-02$ $-02$ $5.1083 E-02$ $1.6898 \mathrm{E}-02$ $\frac{6}{3}-\frac{8 E}{15}$

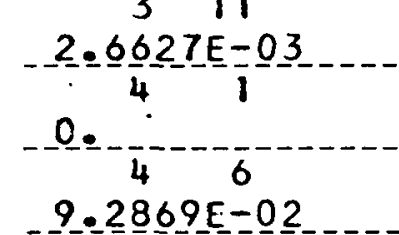
9.2869E-02

4
4
$-3.8579 E-03$
5 $7 \cdot 1670 \mathrm{E}=06$ 1. $-0486 \mathrm{~F}-\frac{06}{5}$ $5 .=\frac{2005 E}{4}-00$ 5.-20 $\frac{20}{4} 3 \mathrm{E}-0 \mathrm{O}$ -

$-\frac{0}{5}-\frac{1}{6}$

$4.2103 E=01$ 511 4. $7008 E-03$ 61 -0. 6 S. $1043 E-00$ 611 $4.5758 E-03$ -0.-$\therefore 0 .-71$ $-2.5980 E=03$ 0. $8.7318 E-02$

$8.4854 E=02$

$$
512
$$
$6.9557 E-04$

$$
62
$$

$0 .-7$

$2.5597 E-01$ $6 \quad 12$

$7=76392=05$ O. $5630 E-02$ 1. $-06300-05$ 2. $3491 E-02$<smiles>[C+]1CCCC1</smiles>
5 o. 59 6.8735 E -02 514 $1.55660-06$ 7.96692E $=02$ 513 -9. $\frac{7197 E}{6}=05$ 1. $3343 \mathrm{E}-05$ 0. $5.3862 E-02$ 0. $4.7476 E-00$ $2.7166 \mathrm{E}-02$ 5 15 $1.955925-06$ 6 $0=-10$ $0.9406 E-04$ $5.5337 \mathrm{E}-02$ $2: 4768 \mathrm{E}-02$ $1.3482 E-05 \quad 1.9788 E-06$ 0. 0.000 $5.9366 E_{12} 00$ $5.7720 E-05$ $2: 5440 \mathrm{E}=02$ 714 $1.3074 E-02$ 4. $0507 E-04$ 7.9808E-06 715 1. 1730E-06 
PLATINUM

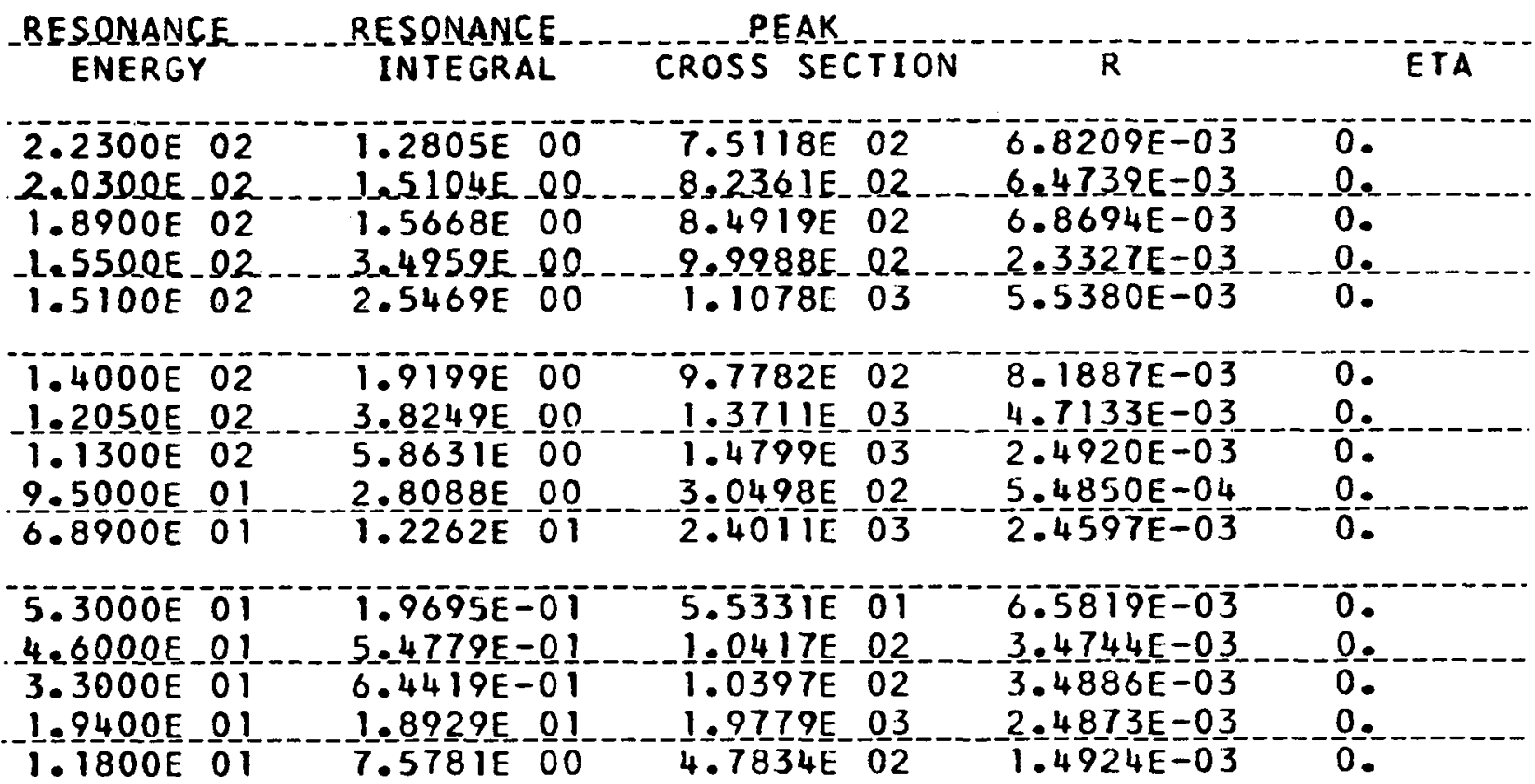


DAIE 3III6I

DESIGNATION

XENONON-T̄ $\overline{3} \overline{5}$
CODE NO.

$54 . \overline{1350}$
DENSITY FACTOR

$2 . \overline{39} \overline{5} \overline{\mathrm{E}} 0 \overline{2}$

\section{LEVEL ENERGY SCATTER SCATTER TRANSPORT ABSORPTION SCATTERT}

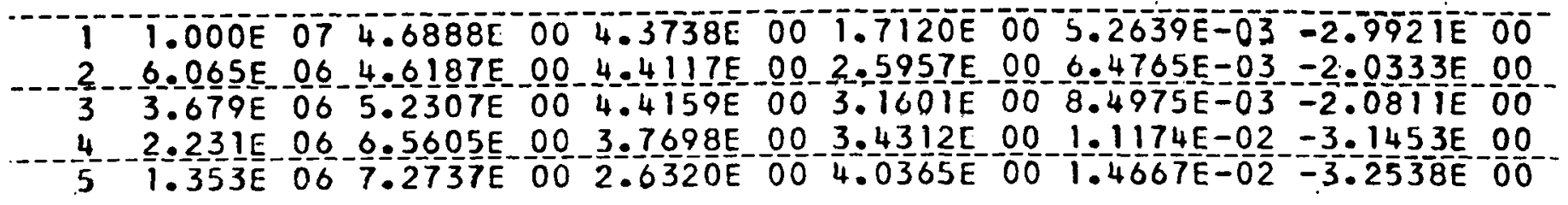

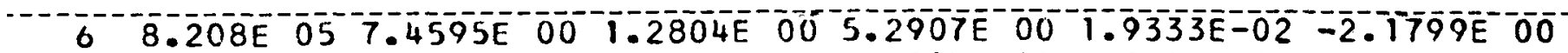

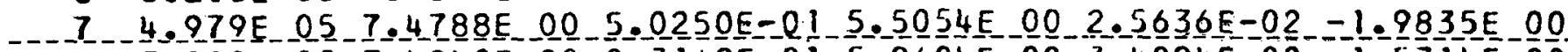

$83.020 \mathrm{E} 057.4840 \mathrm{E} 002.3162 \mathrm{E}-0 \mathrm{~T} 5.9604 \mathrm{E} 003.4094 \mathrm{E}-02-1.5314 \mathrm{E} 00$

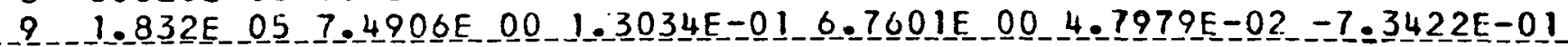

$100.738 E-047.7174 E-001.2975 E-017.6868 E$ O0 $7.8083 E-02-3.0769 E-02$

$112.479 \mathrm{E} 048.1764 \mathrm{E} 00$ 1.2510E-01 $9.1347 \mathrm{E} 00$ 1. $3642 \mathrm{E}-0 \mathrm{~T}=4.1913 \mathrm{E}-02$

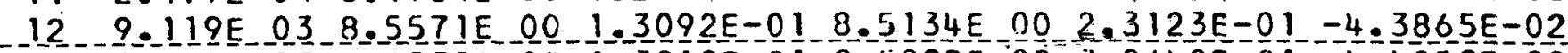

$13-3.355 \mathrm{E} 038.6339 \mathrm{E} 00 \mathrm{O} 1.3210 \mathrm{E}-018.5899 \mathrm{E} 00 \mathrm{O} 3.9642 \mathrm{E}-01-4.4259 \mathrm{E}-02$

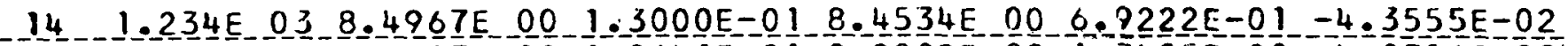

$154.540 \mathrm{E} 02,8.2623 \mathrm{E} 00 \mathrm{O} 1.264 \mathrm{IE}-018.2202 \mathrm{E} 00 \mathrm{1} 1.3405 \mathrm{E} 00-4.2356 \mathrm{E}=02$

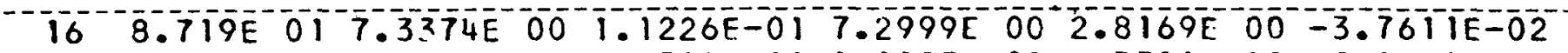

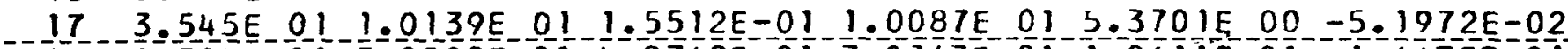

18 1.304E $013.2529 \mathrm{E} 014.9769 \mathrm{E}-013.2363 \mathrm{E}$ 01 $1.0611 \mathrm{E} 01-1.6675 \mathrm{E}-0 \mathrm{1}$

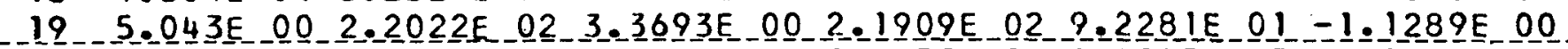
20 1.445E $001.4732 \mathrm{E} 032.254 \mathrm{IE} 011.4657 \mathrm{E} 031.2893 \mathrm{E} 03-7.552$ IE 00

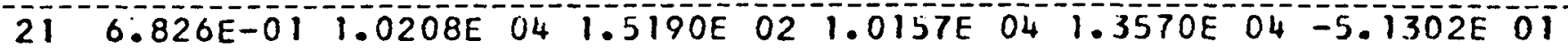
$22-2.511 E=01-1.1343 E-051.6879 E-03-1.1286 E-05-2.5607 E-05-5.7006 E 02$ $239.237 E-026.3107 E$ O5 $9.3903 E$ 03 6.2791E O5 2.2669E $06-3.1715 E 03$ -24 - 2. $530 \mathrm{E}=02$-4.5405E 05

$4500 \quad 3.023 E=01 \quad 1.1773 E-05-1.7519 E-03 \quad 1=1714 E-053.6242 E-05-5.9169 E-02$ $40002.718 \mathrm{E}-011.3564 \mathrm{E} 012.0184 \mathrm{E} 031.3497 \mathrm{E}$ 05 $4.2351 \mathrm{E} 05-6.8148 \mathrm{E} 02$

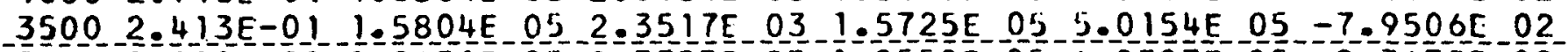
$30002.109 \mathrm{E}-01.1 .865 \mathrm{TE}-052.7753 \mathrm{E} 03-1.8558 \mathrm{E}-05-6.0327 \mathrm{E}-05-9.3678 \mathrm{E}-02$

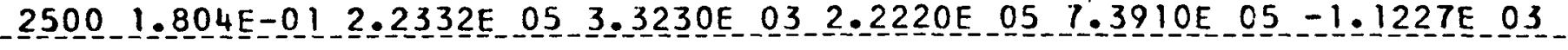

-2000 1.499E-01 2.7162E 05 4.04 18E 03 2.7027E 05 9.2531E 05 - 1. 3650E 03 $15001.194 \mathrm{E}-013.3542 \mathrm{E}$ OS $4.9911 \mathrm{E} 033.3374 \mathrm{E}$ 05 $1.1875 \mathrm{E}$ 06 $-1.6856 \mathrm{E} 03$

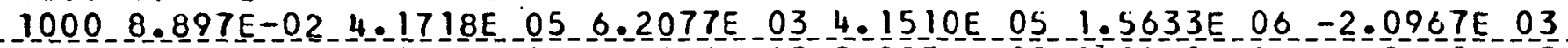
$5005.850 \mathrm{E}-025.0288 \mathrm{E} 057.4828 \mathrm{E} 035.0036 \mathrm{E}-052.0840 \mathrm{E} 06-2.5269 \mathrm{E}-03$

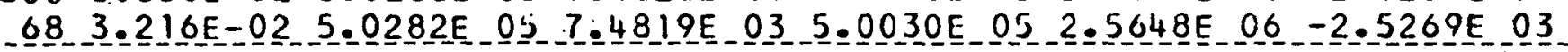

NOTE: For a summary of the sources of data see APEX-704. 
XENON-135

THERMAL TRANSFER MATTICES

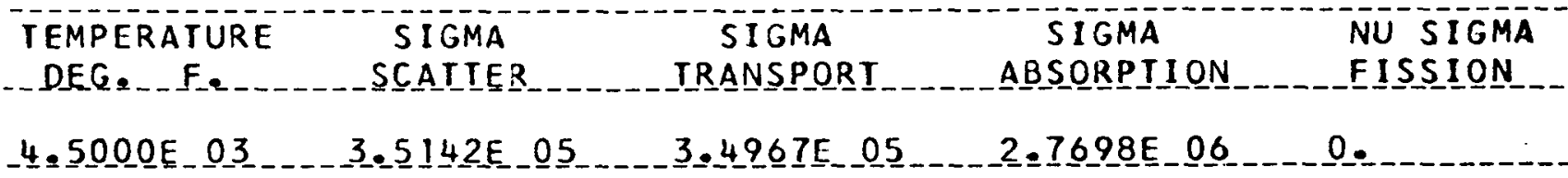

SIGMA $N$ S $(N 1] N 2)$

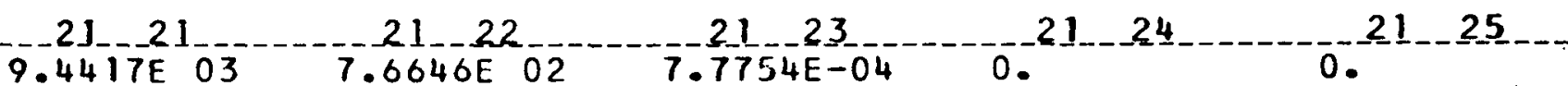

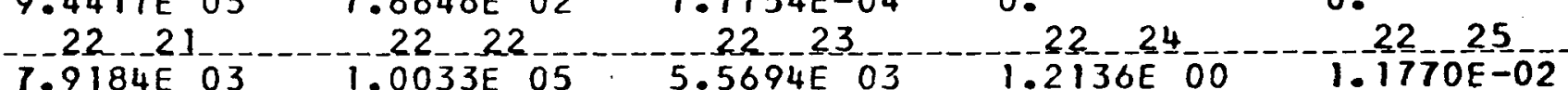
$.8919 \mathrm{E} 03$

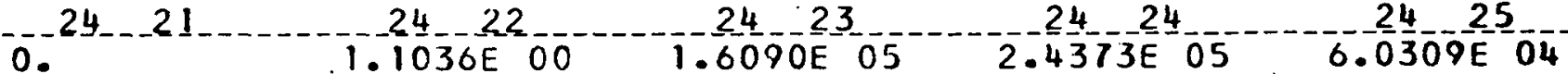

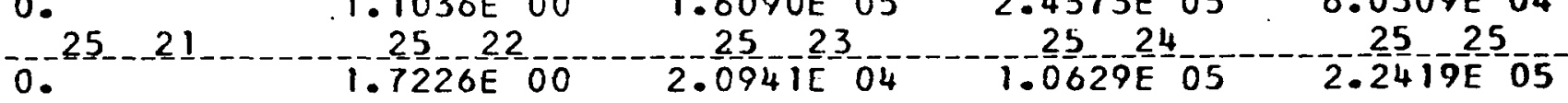
0 .

SEMPERATURE
SIGMA

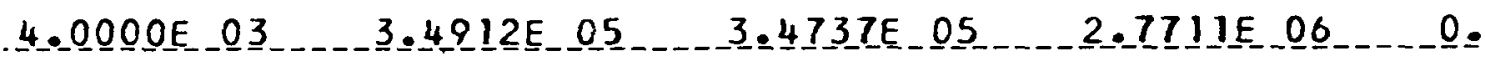

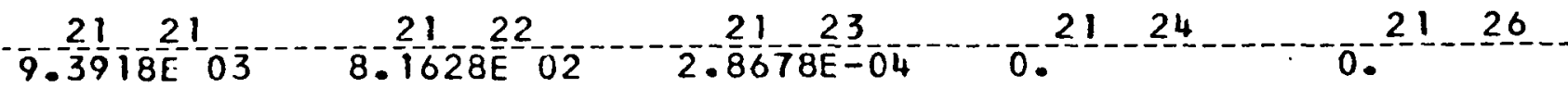

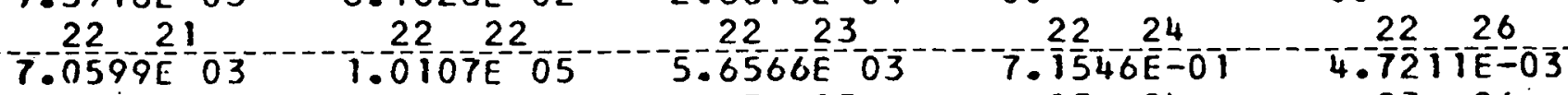

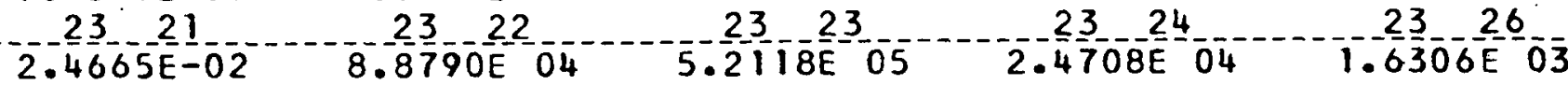

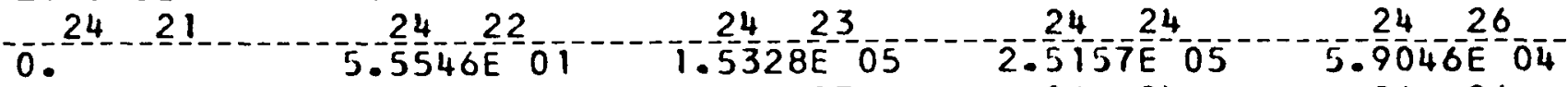
$-26-21 \quad 1.7167 \mathrm{E} 04-1.0304 \mathrm{E} 05-2.2892 \mathrm{E}$ 
XENON-135

THERMAL_IRANSFER MAIRICEES.

- IEMPERAIURE

SIGMA

SIGMA

SIGMA

NU SIGMA

DEG. $F$

SCATTER

TRANSPORT

ABSORPTION

FISSION

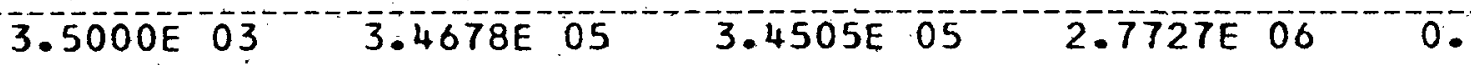

\section{SIGMA $-\frac{N}{2}\left(\frac{N 2}{1 / N}\right)$}

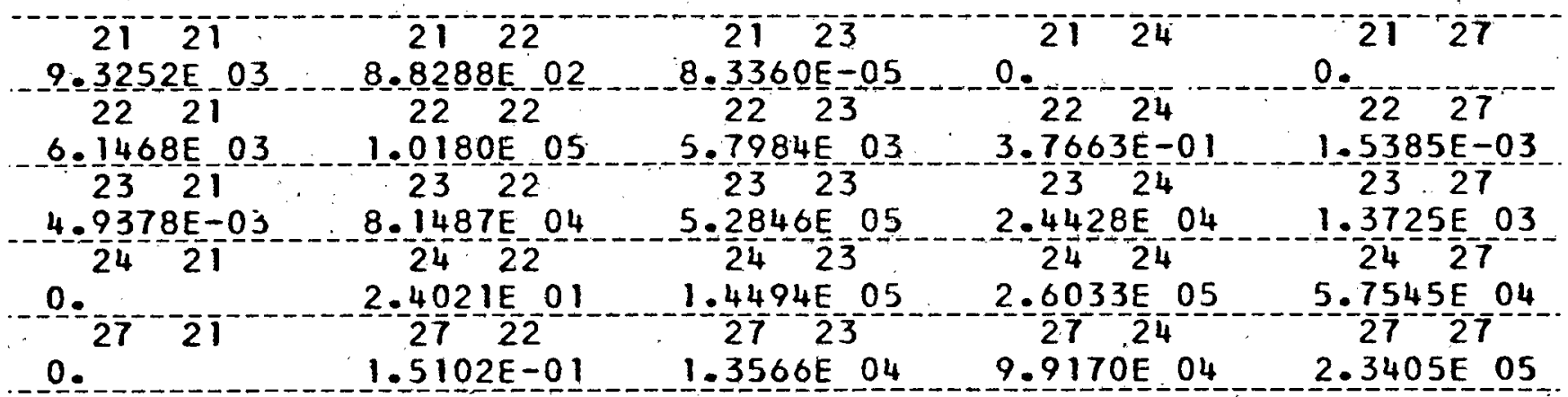

TEMPERAIURE

DEG. $F$.

$3.0000 E^{0}$
SIGMA

SCATIER
SIGMA TRANSPORT
SIGMA

ABSORPTION

$2.7749 \bar{E} 06$
NU SIGMA

FISSTION

\section{SIGMA S $-\frac{N}{2}-\frac{N 2}{1-N}$}

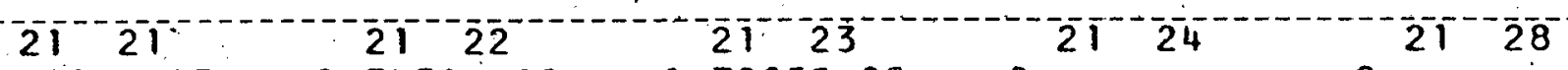

$-9.2338 \mathrm{E}-03$

$5 \cdot \frac{1735 E}{2} 33-1.0251 \mathrm{E} \quad 05$

0.024 OE 03

$\frac{024}{23}-\frac{0 E}{23}$

7. $-3582 E-04$

$5.3627 \mathrm{E}-05$

$8 . \frac{3940 E}{2} \frac{00}{22}$

1. $3574 \mathrm{E}-05$

1.0198E 04
0.

$1.6903 E-01$

$-\frac{6}{2} 303-\frac{1}{2} 4$

$2.4 \frac{22}{2} 1 E-04$

$2.7024 E-05$

$9.4541 \mathrm{E} 04$
- 0. 2. $7729 \mathrm{E}-02$
0. 
XENON=135

THERMAL TRANSFER MATRICES

TEMPERATURE
SIGMA
SIGMA S (NIIN2)

\begin{tabular}{|c|c|c|c|c|}
\hline$-\frac{21}{1040 E} 0$ & $-21-22$ & $-2 \frac{21}{2}-\frac{23}{148 \mathrm{~F}-0}$ & 21 & 0 \\
\hline $\begin{array}{l}9.1040 \mathrm{E} \\
-22\end{array}$ & $22 \quad 22$ & $\begin{array}{l}22 \\
2\end{array}$ & 22 & \\
\hline $\begin{array}{l}1371 E 03 \\
23\end{array}$ & $1.0315 \mathrm{E} \quad 05$ & $6.3856 \mathrm{E} \quad 03$ & $5.9867 \mathrm{E}-02$ & $5.8058 \mathrm{E}-05$ \\
\hline$-\frac{23}{4539 E-05}$ & $-\frac{23}{6.4927 E}-\frac{22}{04}$ & $5.4468 \mathrm{E}-05$ & $2.8158 E$ O5 & $\frac{23}{3598-29}-04$ \\
\hline $24 \quad 21$ & 24 & $-24-23$ & 24 & 29 \\
\hline 29 & $\begin{array}{c}2.1425 \mathrm{E} 00 \\
29 \quad 22\end{array}$ & $\begin{array}{c}1.2546 E 05 \\
29 \quad 23\end{array}$ & $\begin{array}{r}2.8158 \mathrm{E} \\
29 \quad 24\end{array}$ & $\begin{array}{l}3 E^{0} \\
29 \\
\end{array}$ \\
\hline & & & & $.4590 E^{05}$ \\
\hline
\end{tabular}

SEMPERATURE
SIGMA $\begin{gathered}\text { SIGMA } \\ \text { 2.0000E_03 }\end{gathered}$

nit $\mathrm{N} 2$

SIGMA S (NIIN2)

\begin{tabular}{|c|c|c|c|c|}
\hline $2 !$ & 21 & 21 & 21 & 2130 \\
\hline $.9213 \mathrm{E} 03$ & $1.2868 \mathrm{E} 03$ & $1.1155 \mathrm{E}-0$ & 0 & , \\
\hline $0434 \mathrm{E} 03$ & $1.0361 \mathrm{E} 0$ & $6.9844 \mathrm{E} 03$ & $1.4533 \mathrm{E}-02$ & $4.4317 \mathrm{E}-0 \overline{6}$ \\
\hline $1176 \mathrm{E}-06$ & $5.5303 E 04$ & $-5.5379 \mathrm{E} 05$ & $2.4334 \mathrm{E} 04$ & $6.4029 \overline{0} 02$ \\
\hline 21 & & $24-23$ & $-24-24$ & $24-30$ \\
\hline & 30 & 30 & 3024 & $30 \quad 30$ \\
\hline & $1.4258 \mathrm{E}$ & $1=03$ & $8.2086 E \quad 04$ & $2.5289 E \quad 05$ \\
\hline
\end{tabular}


XENON-135

THERMAL IRANSFER MATRICES.

-_IEMPERAIURE SIGMA

SI GMA SI GMA ABSORPIION NU SIGMA DEG. $F$. SCATIER TRANSPORT

$2 . \overline{7} \overline{7} \overline{9} \overline{\mathrm{O}} \overline{0}$ FISSION

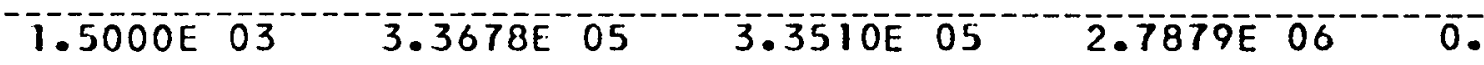

\section{SIGMA}

2121

$8.7933 E_{-} 03$

22 i

$1.9243 E-03$

23 2

$-0$.

0.

‥

(3)
$21 \overline{1}^{-12}$

$1.4149 \mathrm{E} 03$

2222

1.- $\frac{03}{2} \frac{6}{3}-\frac{2}{2}-05$

$4=-4390 E-04$

$2.2 \frac{1}{3} \frac{57 E}{1}-02$

$1.5893 E-06$
$2 i^{--} 2 \overline{3}$

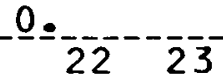

8. $0405 \mathrm{E}-00$

$5.6 \frac{3}{2} 64 E^{2}-05$

$1.0021 E-05$

$2.3556 \mathrm{E} \quad 03$ $-\frac{1}{2} i^{-} \overline{2} 4$

0.

$1.8139 \mathrm{E}-03$

50532 O4

$24-24$

$3 \cdot 1037 \mathrm{E}-05$

$7.3541 E .04$ $\overline{2} i^{--} \overline{3} i^{-\cdots}$ 은 ‥ $2 \overline{3}^{-1}$ $4.2427 \mathrm{E}-02$ 4. $7854 E \quad 04$ $31-31$ 2. $6088 E$ E 05
TEMPERATURE

DEG. $F$.

SCATTER
SIGMA

SCATTER
SIGMA

TRANSPORT
SI GMA

ABSORPTION
NU SIGMA

FISSION

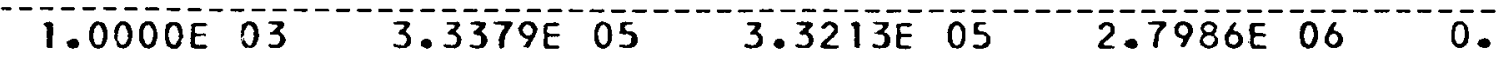

SIGMA S (N) $\left(\frac{N}{2}-\frac{1}{2} 2\right)$

$2121-2122-21^{2}-21^{2}$

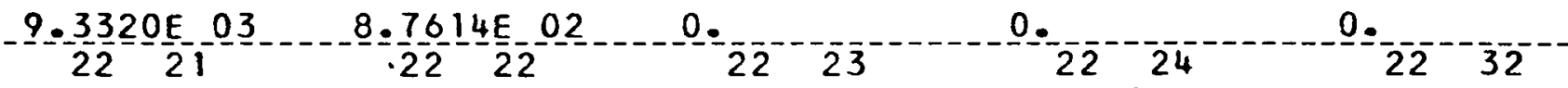
$-8.8212 E-02 \quad 1.0257 E-05 \quad 1.0113 E-04 \quad 5.8429 E-05 \quad 0$. $-0=2421$. $-0$. 을... $8.8581 E$. 02 2. 7028 E 05 
XENON=135

THERMAL TRANSFER MATRICES

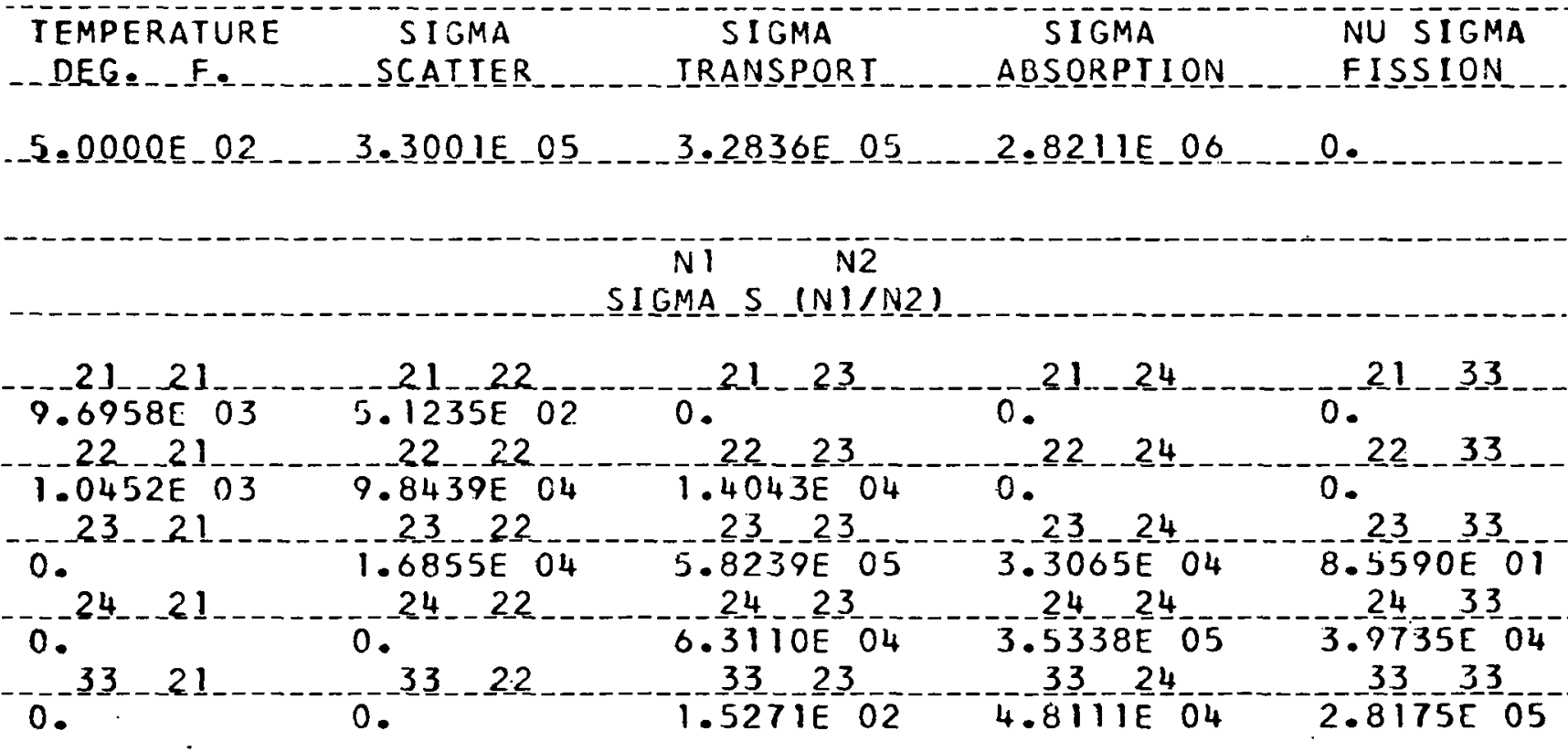

TEMPERATURE

DEG. . F.
SIGMA

SCATIER
SIGMA IRANSPORI
SIGMA ABSORPIION
NU SIGMA

EI SSION
$-6.8000 E_{-} 01$ $3.2431 E_{-} 05$ $3.2269 E_{0} 05$ $2.8800 E$ 0.

$\mathrm{Ni} N 2$ SI GMA S (NN1IN2)

$-9.6935 \mathrm{E} 03-5.1463 \mathrm{E} 02-0.21023$

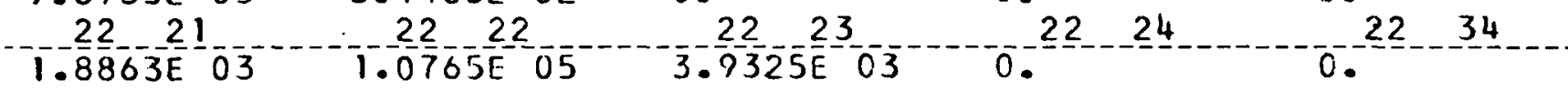

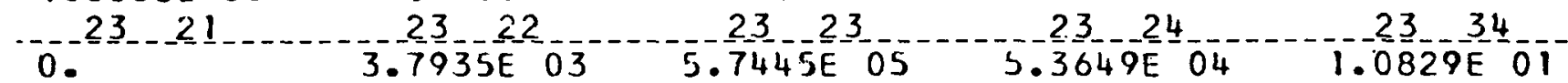
$0.24-21-204-22$ 0 $3.8284 \mathrm{E}$ O4 $3.8019 \mathrm{E}-05$ $4.1589 \mathrm{E} 0 \mathrm{O}^{-3.0322 \mathrm{E}} 04 \mathrm{2} .9399 \mathrm{E}$ $3.6793 \mathrm{E} 04$ 
DAIE $03 / 28 / 61$

DESIGNATION

TANTALUM
CODE NO.

$7 \overline{3} . \overline{18} 10$
DENSITY FACTOR

\section{$3.00435 E^{02}$}

\section{- \\ XI_SIGMA \\ SCATTER \\ SCATTER \\ SIGMA \\ SIGMA \\ TRANSPORT \\ ABSORPTION \\ XI I SIGMA}

LEVE

1 1.000E $074.9066 \mathrm{E} 00 \mathrm{5} .5242 \mathrm{E} 002.0944 \mathrm{E} 00 \mathrm{1} .8313 \mathrm{E}-02-2.1636 \mathrm{E} 0 \mathrm{O}^{-}$

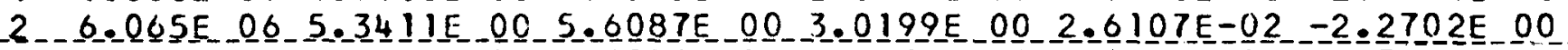

$3 \quad 3.679 \mathrm{E} \quad 06 \quad 6.3682 \mathrm{E} \quad 00 \quad 5.3891 \mathrm{E} \quad 00 \quad 3.5376 \mathrm{E} \quad 00 \quad 3.9556 \mathrm{E}-02-2.7684 \mathrm{E} 00$

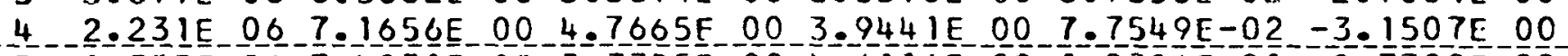

5 1.353E $067.403 \mathrm{IE} 003.7325 \mathrm{E} 00-4.6116 \mathrm{E} 00-1.2326 \mathrm{E}-0 \mathrm{~T}-2.7302 \mathrm{E} 0 \mathrm{O}$

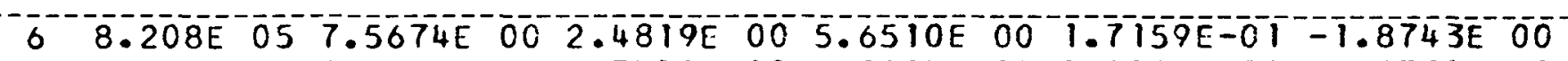

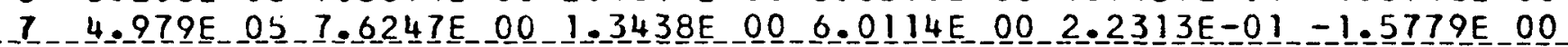

8 3.020E $057.7310 \mathrm{E}$ 00 5.9900E-01 6.3571E $002.8074 \mathrm{E}-01-1.3437 \mathrm{E} 00$

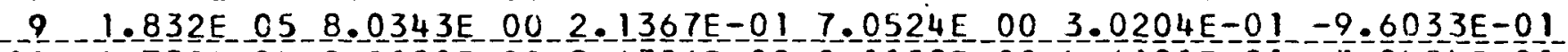

$10.6 .738 \mathrm{E} 048.5190 \mathrm{E} 009.6306 \mathrm{E}-028.1159 \mathrm{E} 004.6191 \mathrm{E}-01-3.9424 \mathrm{E}-01$

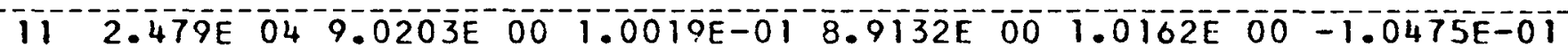
12 - $119 \mathrm{E} 03$ 1.0208E 01 1.1338E-01 1.0167E $012.1453 \mathrm{E} 00-4.0490 \mathrm{E}=02$

$13-3.355 \mathrm{E} 031.2820 \mathrm{E} 011.4239 \mathrm{E}-011.2772 \mathrm{E}-1 \mathrm{1}-1.0486 \mathrm{E} 00-4.6847 \mathrm{E}=02$

14 1.234E $0.31 .5701 E$ E 1 1.7439E-01 1.5643E 01 7.8679E $00=5.7117 \mathrm{E}=02$

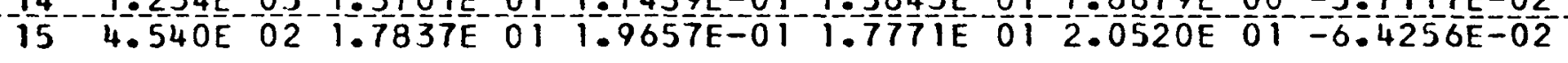

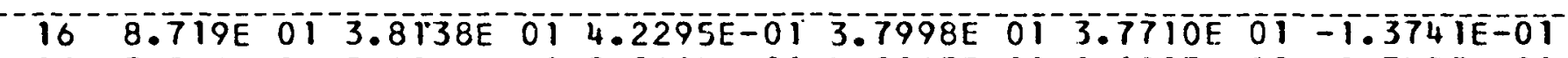

17 3.545E $015.0244 E$ E $015.5614 \mathrm{E}-01$ 4.9963E 01 1.1293E $02-2.7453 \mathrm{E}-01$

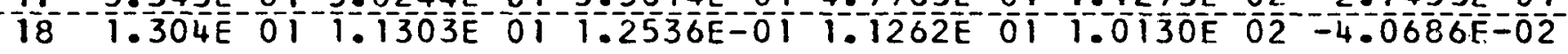

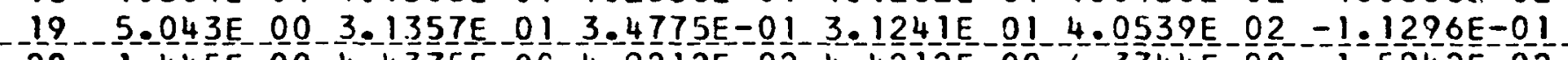

$20 \quad 1.445 \mathrm{E}$ OO $4.4375 \mathrm{E} \quad 00 \quad 4.9212 \mathrm{E}-02 \quad 4.4212 \mathrm{E} 006.3744 \mathrm{E} 0 \mathrm{D}-1.5942 \mathrm{E}-02$

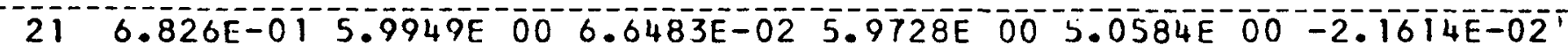

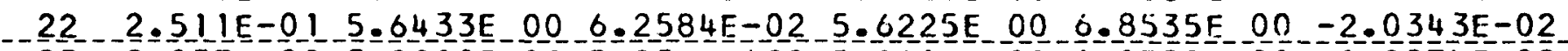

$23-9.237 \mathrm{E}-025.2810 \mathrm{E} 00-5.8566 \mathrm{E}-025.2616 \mathrm{E} 00-1.1592 \mathrm{E} 01-1.8974 \mathrm{E}-02$

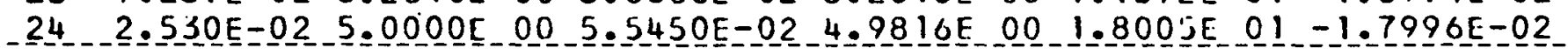

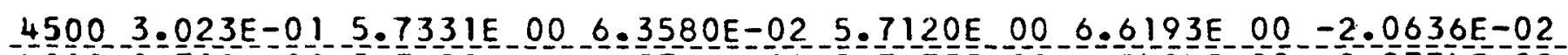

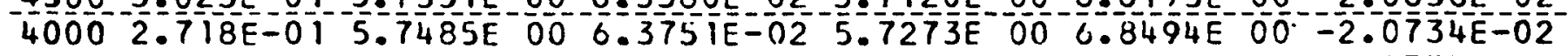

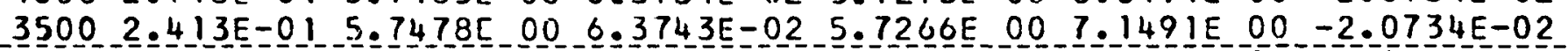

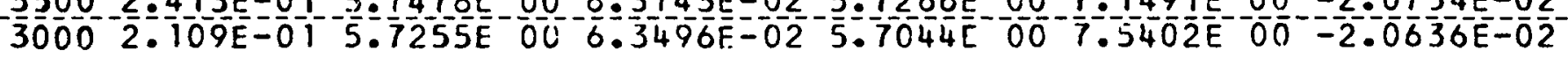

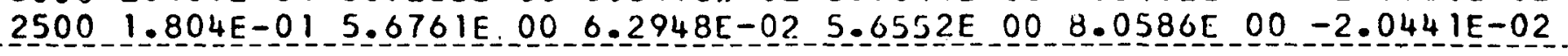

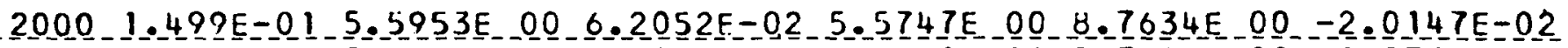

$15001.194 \mathrm{E}-015.4843 \mathrm{E}$ 00 $6.082 \mathrm{IE}-025.4641 \mathrm{E}-00-9.7600 \mathrm{E}-00-1.9756 \mathrm{E}-02$

$10008.897 \mathrm{E}-025.3558 \mathrm{E}-00 \mathrm{5} .9396 \mathrm{E}=025.3361 \mathrm{E}-001.1265 \mathrm{E} 01-1.9267 \mathrm{E}-02$

$500-5.850 \mathrm{E}-025.2284 \mathrm{E} 00 \mathrm{5} .7983 \mathrm{E}-02502091 \mathrm{E} 001.3845 \mathrm{E} 0 \mathrm{1}-1.8876 \mathrm{E}-02$

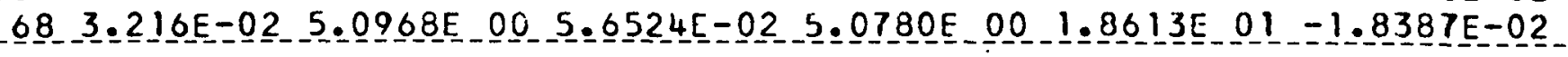

NOTE: For a summary of sources of data see XDC 61-4-35. 


\section{THERMAL TRANSFER MATRICES}

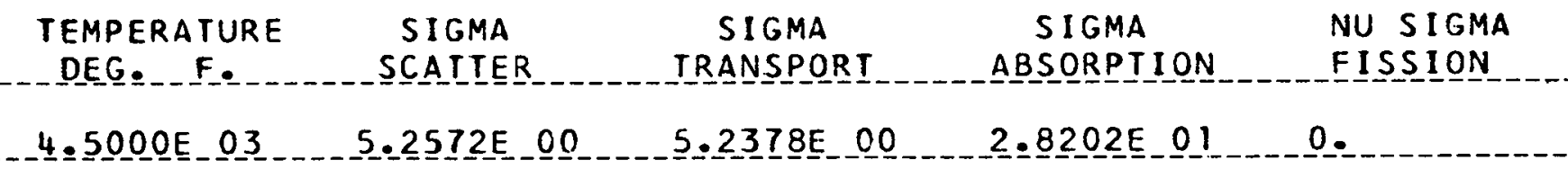

\section{$\bar{N} \overline{1}-\bar{N}$ \\ SI GMA S (N) I N2)}

\begin{tabular}{|c|c|c|c|c|}
\hline-21 & $21-22$ & 21 & $-21 \ldots 24$ & $-21-25$ \\
\hline $\begin{array}{l}.5895 \mathrm{E} 00 \\
22 \quad 21\end{array}$ & $\begin{array}{c}4.0540 E-01 \\
2222\end{array}$ & 0.22 & 0 & 0.2 \\
\hline $\begin{array}{c}3.4360 \mathrm{E}-01 \\
23\end{array}$ & $\begin{array}{c}5.0690 \mathrm{E} \\
23 \quad 220\end{array}$ & $\begin{array}{c}2.4510 \mathrm{E}-01 \\
2323\end{array}$ & $\begin{array}{l}9.7896 \mathrm{E}-06 \\
2324\end{array}$ & \\
\hline & $6.9420 \mathrm{E}-01$ & $4.4261 \mathrm{EO} 00$ & $1.8748 \mathrm{E}-01$ & $0.3984 \bar{E}$ \\
\hline - & $1.7295 E-04$ & $1 . \frac{24}{5697 \mathrm{E}}=\frac{23}{00}$ & $-2 .-\frac{2}{9089}-2 \frac{24}{00}$ & $6.1 \frac{2}{148 E-0}$ \\
\hline & $1.1124 \mathrm{E}-06$ & $1.8457 \mathrm{E}-01$ & $1 . \frac{25}{4} 895 \mathrm{E}-\frac{24}{00}$ & $-\frac{25}{58}-25$ \\
\hline
\end{tabular}

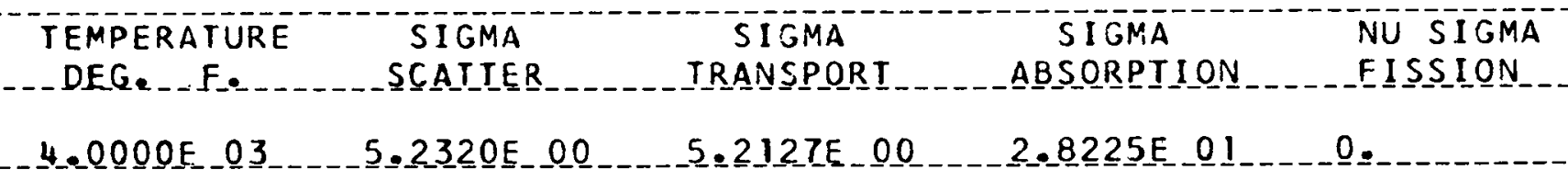

Ni 2

SI GMA S $($ N I I N2)

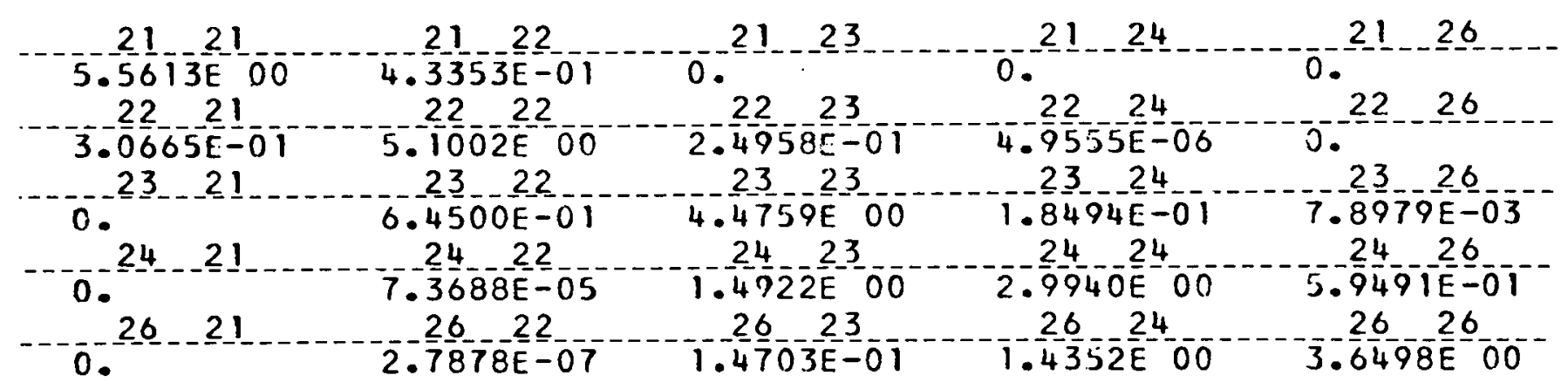


TANTALUM

_. IHERMAL_TRANSFER_MAIRICES.

. IEMPERATURE

SIGMA

SLGMA

SIGMA

NU SIGMA

DEG. F. SCATTER

TRANSPORT

ABSORPTION

FISSION

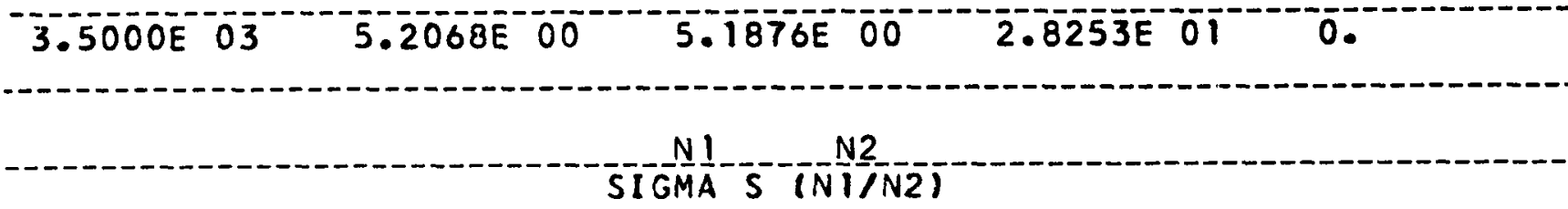

$21^{2} i^{2} 21^{2}-2 i^{2}$

$5.5238 E_{00}$

4..2114E-01

2221

$22 \quad 22$

$22-23$

$22-24$

$22-27$

$-2.6732 E=0$

5.1311600

$2.5663 \mathrm{E}=01$

2. $1567 E=06$

2321

2322

2323

2324

2327

0.2401

$5.92260=01$

2422

$4.5288 E-00$

$1.8273 E=01$

$0.44490 E=03$

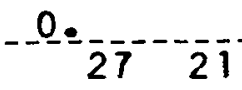

$2.592727 E-05$

2423

2424

‥

o.

$-\frac{4}{27} 79 \frac{7}{23}-00$

$27-\frac{1}{2} 4$

$5.75866-01$

1.1222 E -01

1.37266 E 00

3. 7220 E 00

TEMPERAIURE

DEG. $F$.
SIGMA

SCATTER
SI IGMA

TRANSPORT
SIGMA ABSORPIION
NU SIGMA

FISSION

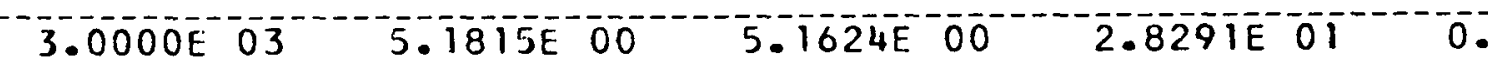

SIGMA S $(N) / N 2)$

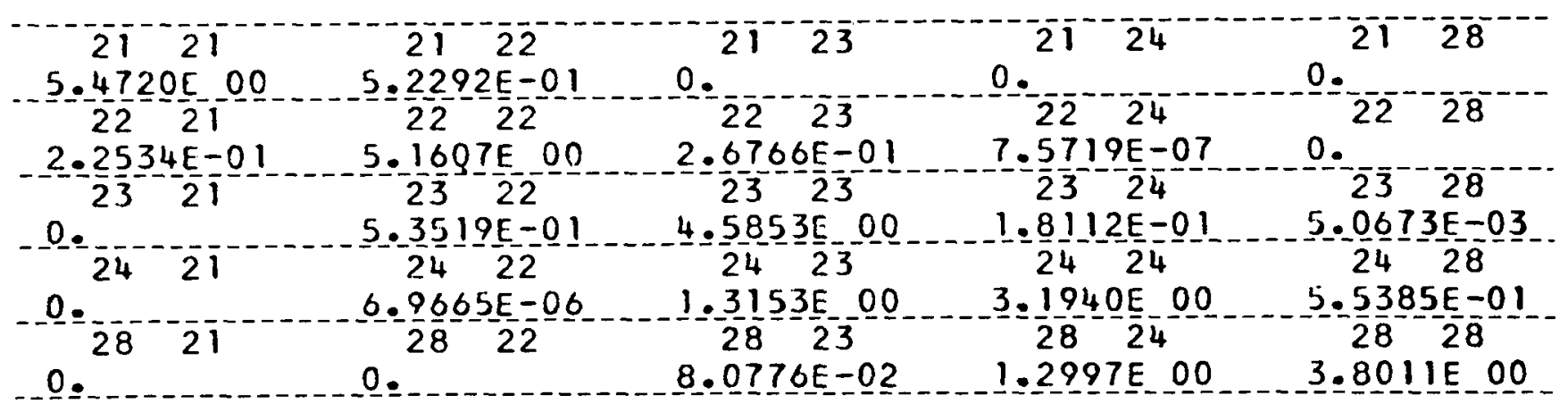


IANTALUM

\section{THERMAL TRANSFER MATRICES}

TEMPERATURE
SIGMA

\section{N1 12 \\ SIGMA S (NIIN2)}

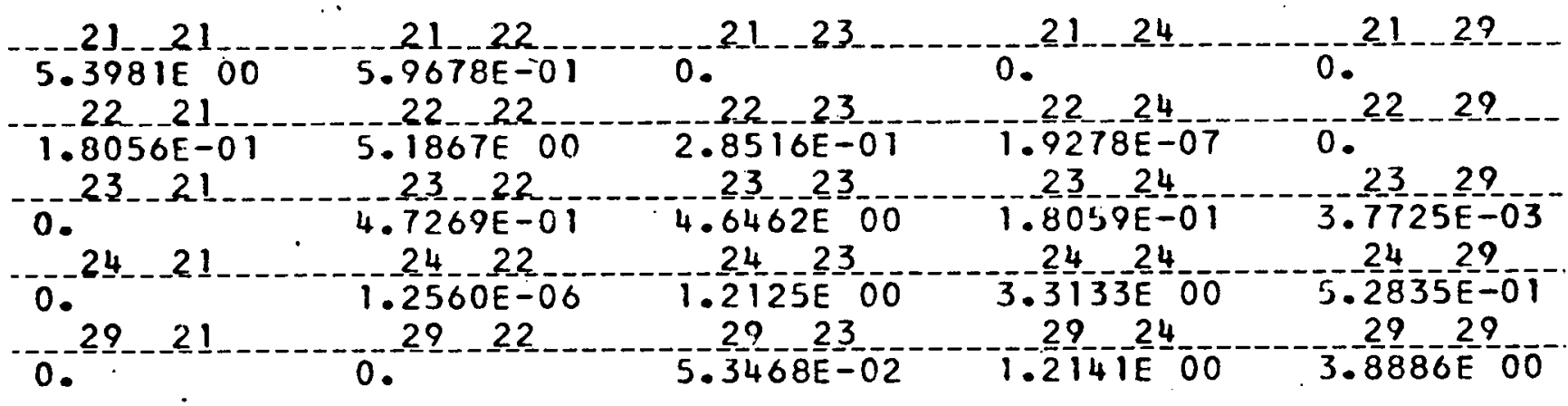

TEMPERATURE
SIGMA

nit

SIGMA S (NIIN2)

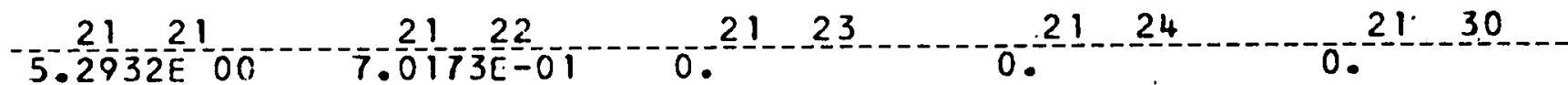

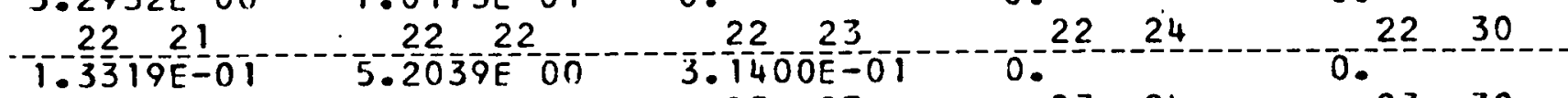
O.

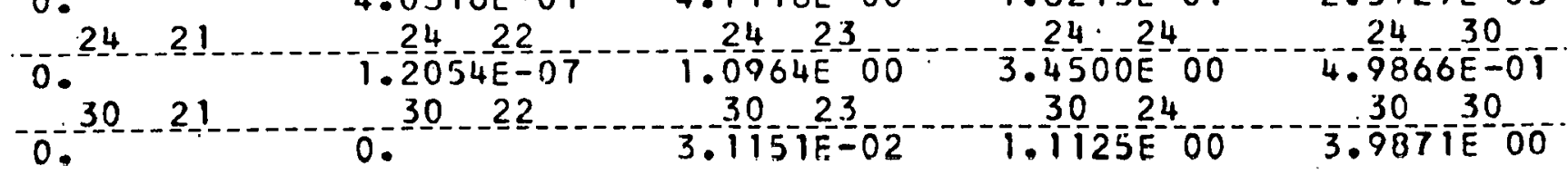


TANTALUM

IHERMAL_IRANSEER MAIRICES.

IEMPERA IURE

DEG. F.

1.5000E 03
SIGMA

SCATTER
SIGMA TRANSPORT
SIGMA ABSORPTION
NU SIIGMA

FISSION
$5.1054 \mathrm{E} 00$
$5.0866 E^{2} 00$
$2.8521 \mathrm{E}$ OT
0 .

\section{SIGMA $-\frac{N}{S}\left(\frac{N 2}{1 / N 2}\right)$}

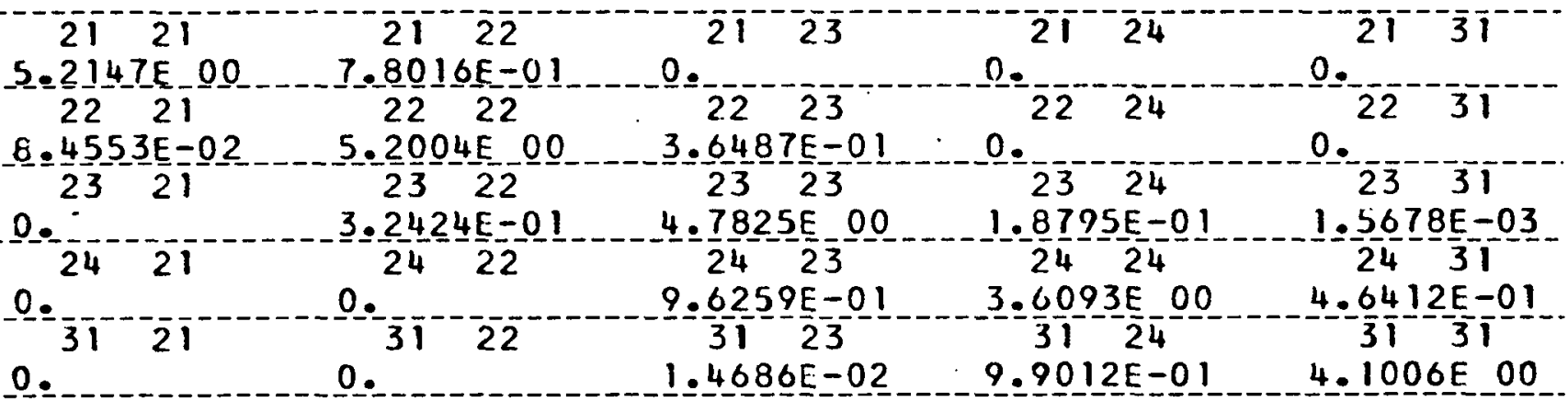

TEMPERATURE

DEG. F.

$1.0000 \mathrm{E} 03$
SI GMA

SCATTER
SIGMA

TRANSPORT
SIGMA ABSORPTION
NU SIGMA

FISSION

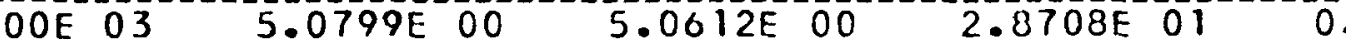

\section{SIGMA S (NIIN2)}

$21^{2} 1$

5

$4.8980 E-01$

…-

$\frac{0}{2} 2^{-}-\frac{1}{24}$

$\frac{0}{22}-\overline{3} \overline{2}$

$3 \cdot \frac{9}{2} \frac{007 E}{3}-02$

$5.1442 E-200$

$4.0522 E-01$

$0 \cdot \frac{0}{2} \overline{3}^{--} \overline{2} \overline{4}$

0 . $-\overline{3}-13 \overline{2}^{-}$

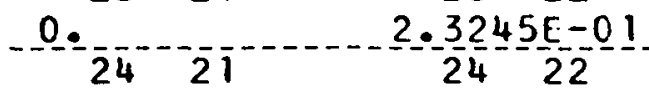

$-0 \cdot 32-\overline{2}$

O. $3 \overline{2}-\overline{2} \overline{2}$

$4.8555 \mathrm{E} 00$

$24-\frac{1}{3}$

$2.04 \frac{04}{24}-\frac{6}{24}-01$

$7 \cdot-\frac{4}{24} \frac{65}{32}-04$

20

$\underline{0}$
$3.7992 \mathrm{E}-00$

$32-\frac{2}{4}$

8.3925E-01
4. $2434 E-01$

4. 2360 E 00 
IANTALUM

THERMAL TRANSFER MATRICES

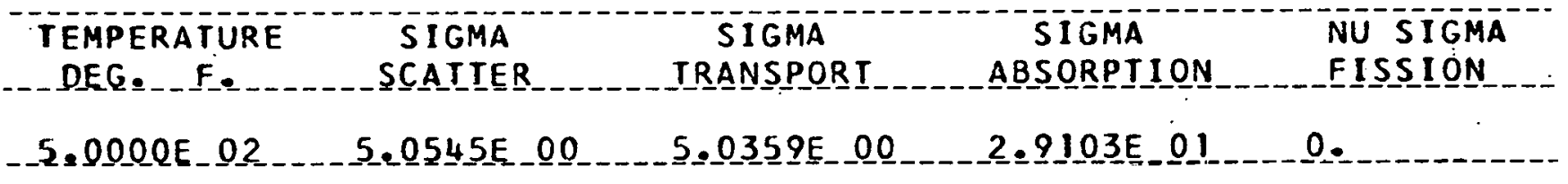

SIGMA S (NIIN2)

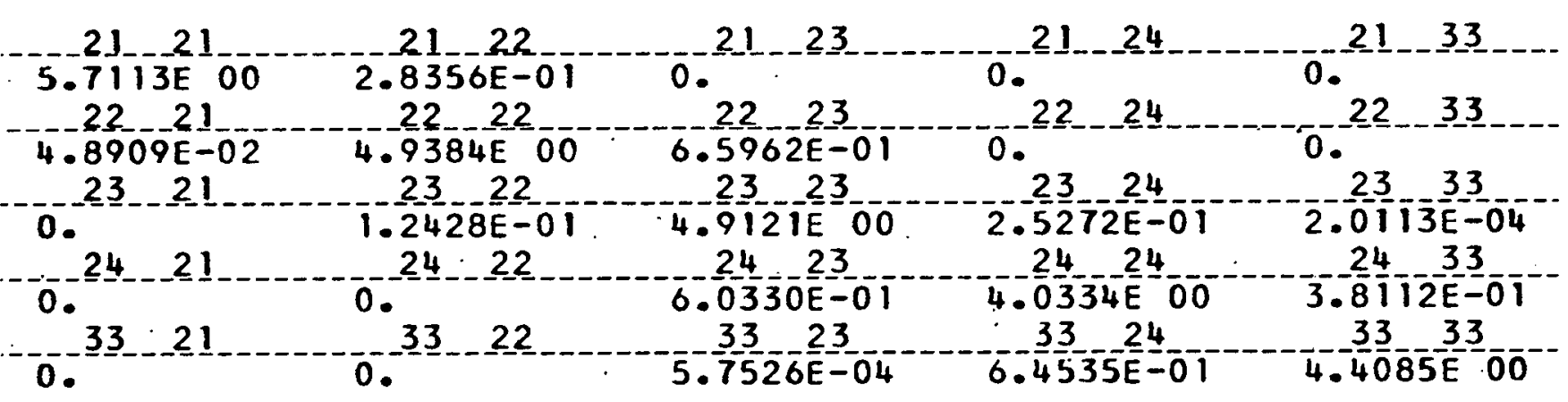

SEMPERATURE
SEGGA

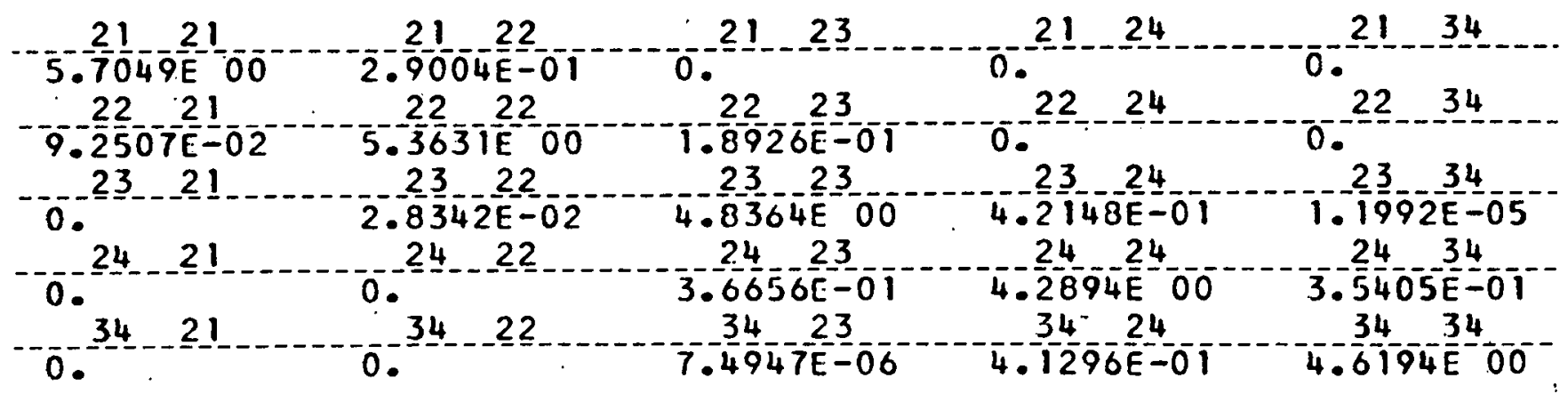


IANTALUM

TRANSFFRS FROM GROUPS 1 THROUGH 12 TO GROUPS 1 THROUGH 14

SIGMA

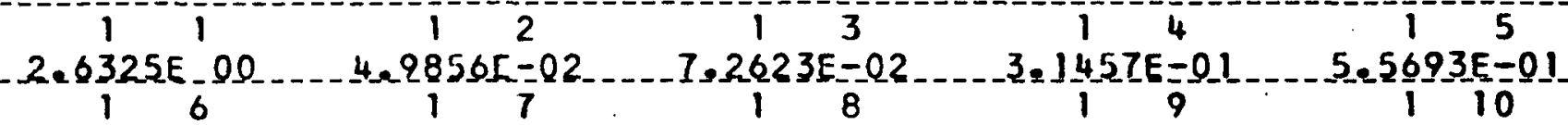

$5.5037 \mathrm{E}-01 \ldots 3.7286 \mathrm{E}=0 \mathrm{~L}$

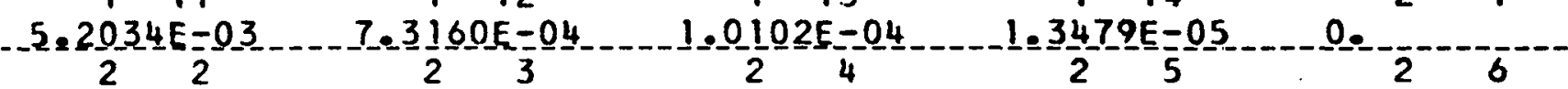

$2.6961 E-00-1.5625[-01 \quad 1.9624 E-01 \quad 5.1740 E-01$

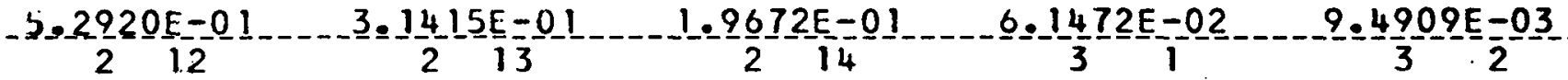

- L. 347 J Z

$-3.62 \mathrm{LEE}-00 \quad 1.2178 \mathrm{E}-01 \quad 3.8525 \mathrm{E}=01 \quad 0.8135 \mathrm{E}-01$

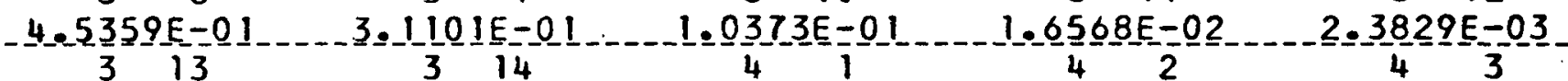

$3.3180 E-04-4.4 \frac{4}{4} 13 E-05-0.00-4$

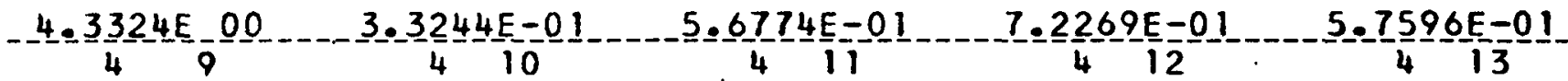

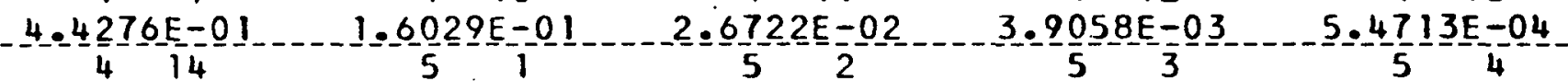

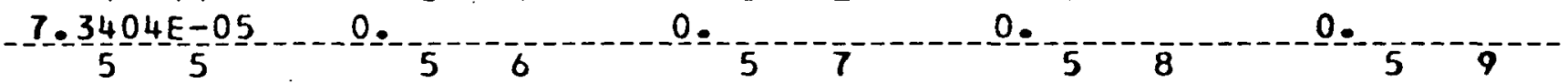

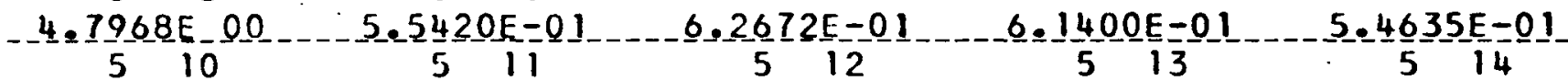

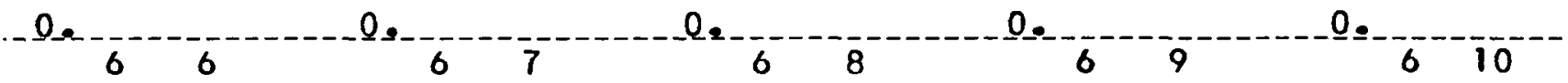
$5.6653 E-00 \quad 5.2603 E-01 \quad 5.1617 E-01 \quad 5.5132 E-01-12-5247 E-01$

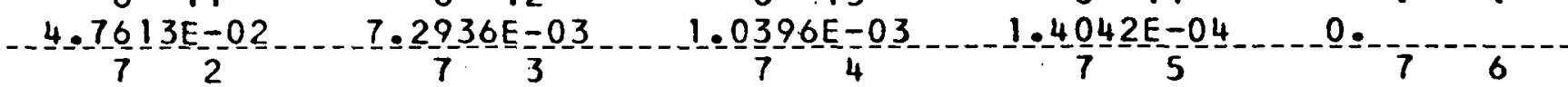

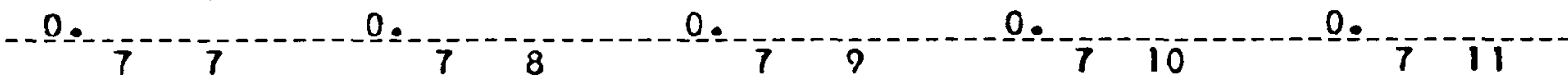

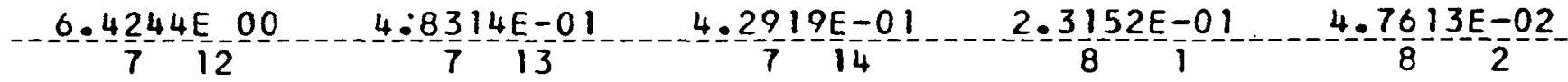
- $7.5404 E=03 \ldots 1.0882 E-03 \ldots$ 0. 
TANTALUM

$$
\text { N1 N2 }
$$

SIGMA SO (NI/N2)

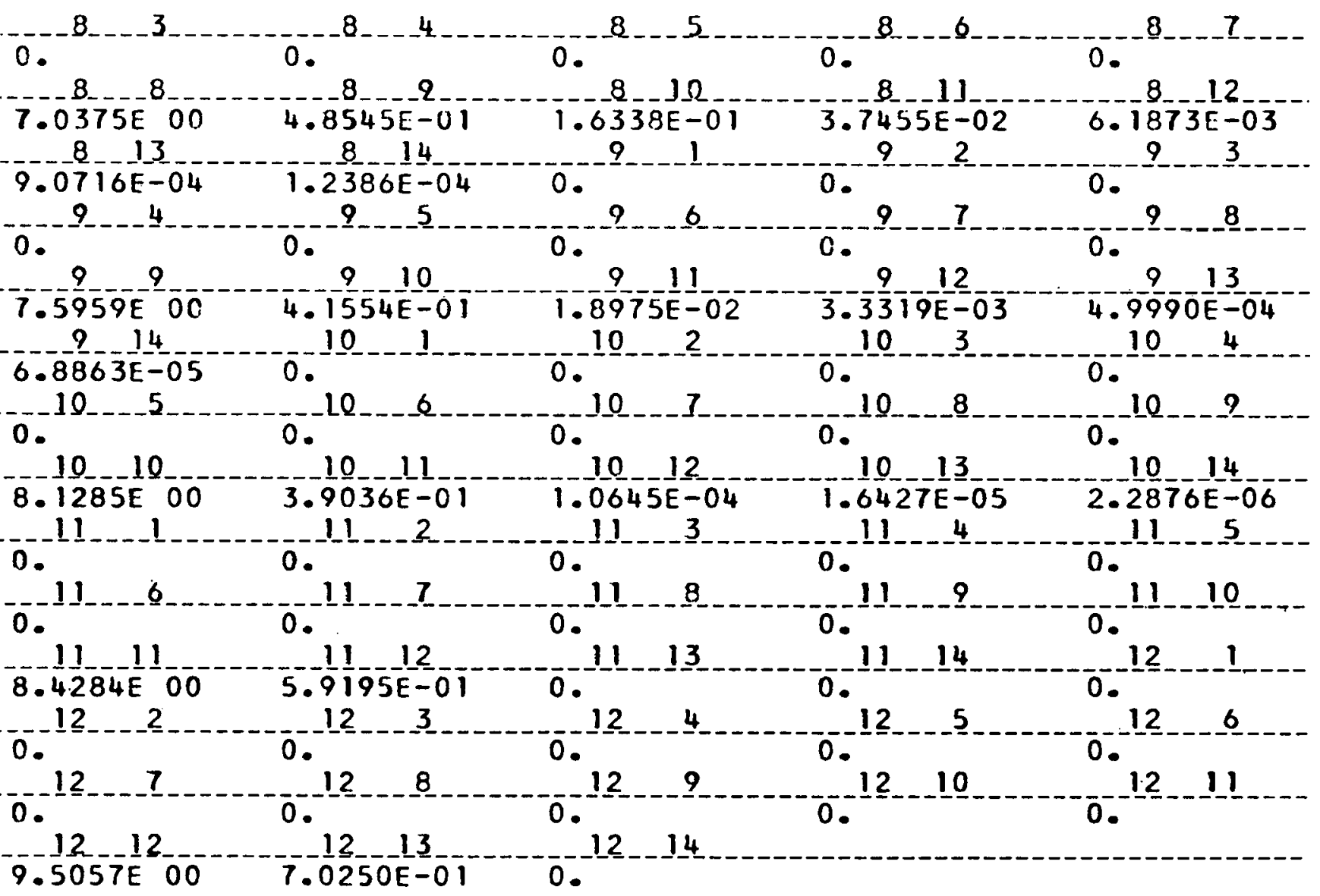


IANTALUM _

RESONANCE
ENERGY
INTEGRAL

$2.28 Q 0 E-0 . \mathrm{L}$

$2.4100 E$ OI $2.0620 E$ OI

$2.9900 E-01$

$3.5200 E 01 \quad 1.8891 \mathrm{E}$ O1

$3.5200[$ Q 1

LeQ2530E_O

$9.3054 \mathrm{E}=03$

-

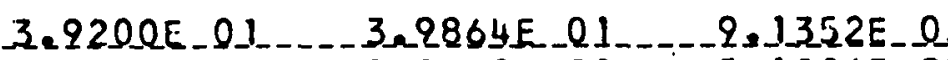

$4.9100 E$ O 1 9.1669E-01 5.1086E 0

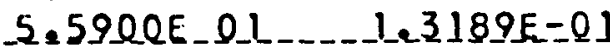

$5.7500 E$ OI $1.8667 E-01$ 7.?285E_01

6.3000E_01____.2.9319E_.0Q

$1.1523 \mathrm{E} 02$

$1.74999 E_{0}$

L.677Z7E $=02$

4.6939E 03

$1.7470 E$

$5.6520 E$ O3

$1.0249 E-02$

$1.5918 \mathrm{E}=02$

$1.2122 E-02$

0 .

$9.46560=03$

0 .

$\underline{0}$.

Z26900E-01__- 3.4182E_Q0

$2,4323 E-03$

7.7700 E 01

$1.7230 E 00$

$1.3214 \mathrm{E} 03$

7.29000E 01

$7.0267 E=01$

$5=7744$ E -02

6.38.6QE $=03$

$3.0152 E-02$

$3.0815 E=02$

3.1591E-02

2. $69253 E-02$

o.

$8.3000 E 01$

$3.5960 E$ OO

$2.5993 \mathrm{E} 03$

$3.1387 E=02$

3.6083E-02

0.

8. 5200 E_ $01 \ldots$

9.978.1E_02

$4.0750 E=02$

3.0008E-02

$4.0951 E-02$

0 .

$8.5800 E-01$

$8.9700 \mathrm{E}-\frac{1}{1}$

-9. 15OQE 01

$8.3835 \mathrm{E}-02$

$7.7222 \mathrm{E}$

$4.7139 E=02$

$8.2545 \mathrm{E} 02$

$9.7100 E$ OI $5.6955 E-01$

9.94QQE 01

$8.2151 E-01$

$5.4034 E$ E2

$8.0607 \mathrm{E} 02$

$5.0638 \mathrm{E}=02$

0.

$1.0360 \mathrm{E} 02$

$7.4710 E$

2.28 10E 03

$4.6890 E=02$

$4.72055-02$

$0.0449 \varepsilon=03$

0.

0.

0.

$1.0560 \mathrm{E} 02$

$1.1520 E-02$

$1.1840 \mathrm{E} 02$

$1.8944 E=01$

$2.0823 E-02$

$5.5578 E-02$

3.0159E 03

$3.3533 \mathrm{E}-02$

2.8260 E 03

$2.9404 E=02$

.352SE 00

4.0149502

1.26600E 02 3 . 13966E 음

2. $5821 \mathrm{IE} 03$

$6.0874 E=02$

0.

$1.3600 E \quad 02$

$1.3850 \mathrm{E} 02$

$1.4430 E \quad 02$

$1.4850 \mathrm{E} 02$

$2.5467 \mathrm{E}=0$ ?

ㅇ. ㅇ.

LLE950E 0

$1.6000 E \quad 02 \quad 2 \quad 2.8102 E=02$

$1.6650 \mathrm{E} 02$ 5.8242E=01

$1.7520 \mathrm{E}-02$

$1.7600 \mathrm{E} 02$ $2.4006 \mathrm{E}-00$

$1.7870 \mathrm{E} \quad 02$

$2.07035-03$

$4.1235[-02$

$1.3000 \mathrm{E}-03$

$4.2874 \mathrm{E}=02$

$2.4832 E-02$

$7.5604 \mathrm{E}-02$

$7.048 \overline{3}=02$

7,3773 E 02

$0.83625=02$

$-\frac{0}{0}=$

$-\frac{0}{0}$.

4. $1620 \mathrm{E}-01$

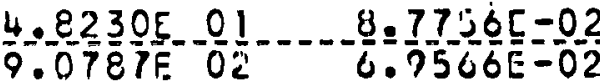

O.: 1.7970E 03

$1.52465-02$

1.8570503

$2.03617 \mathrm{E}-02$

$6.3670 E=02$

$1.20725 \quad 02$

$9.5901 E-02$

o.

0. 
TANTALUM

- RESONANCE

RESONANGE

PEAK

ENERGY

INTEGRAL

CROSS SECTION

$\mathrm{R}$

ETA

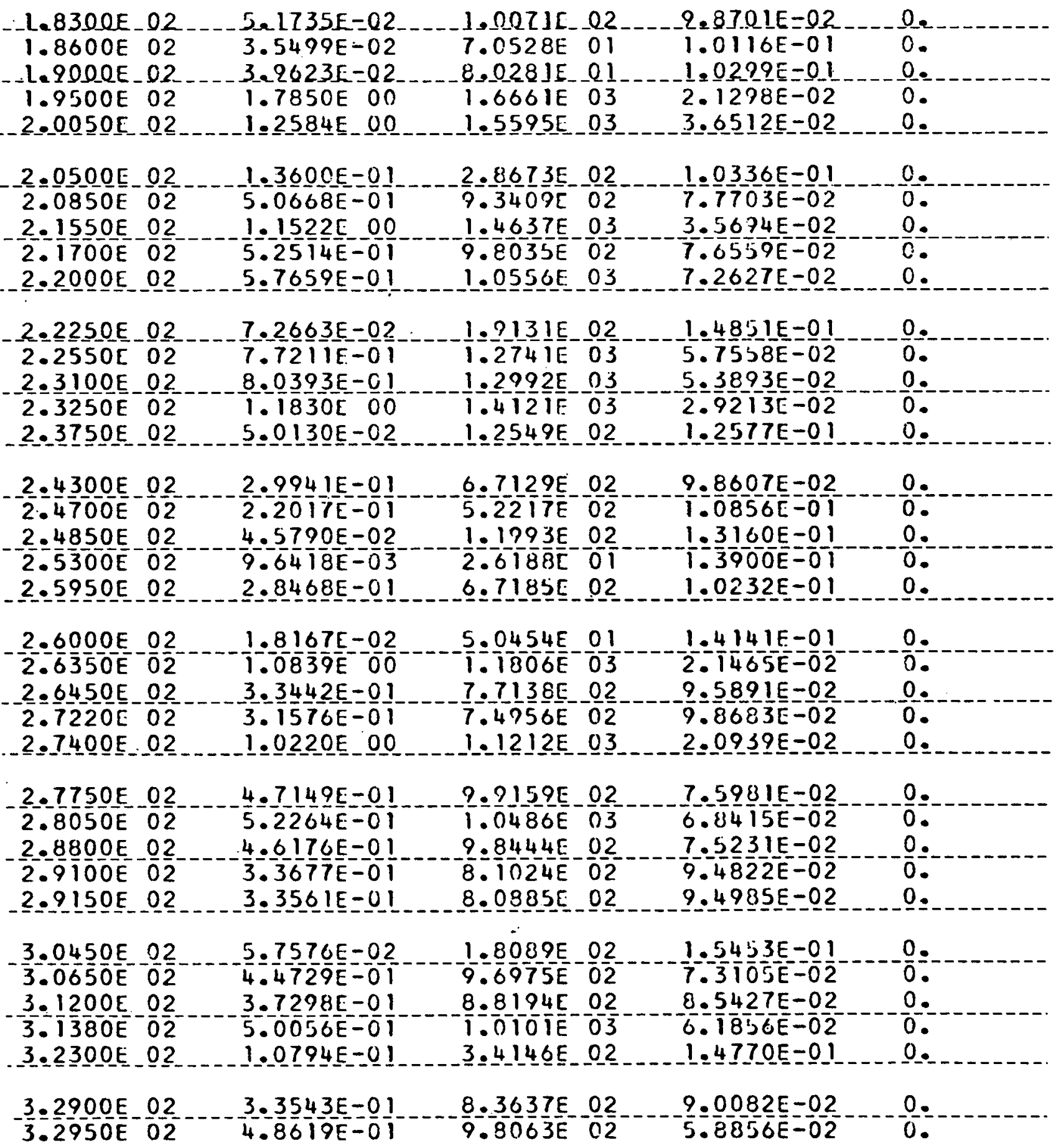




\subsection{Macroscopic Cross Sections}

MACROSCOPIC CROSS SECIIONS.

CONSIIIUENI TABLES

MACROSCOPIC COOE NOE
CONSIITUENI
NOE 1000 OEESIGNAIION
40.9100


DAIE $02 / 15161$

HYDRIDED ZIRCONIUM

DESTIGNATION DODE NO'.

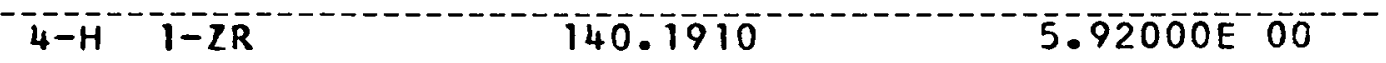

LEVEL ENERGY SCATTER SCATTER TRANSPORT ABSORPTION SCATTER 1-

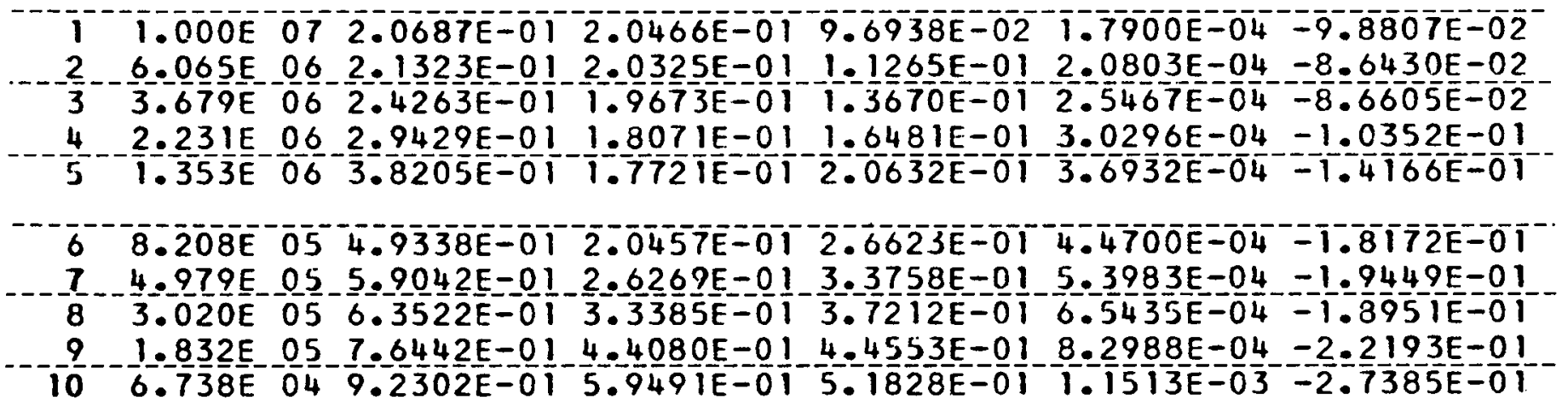

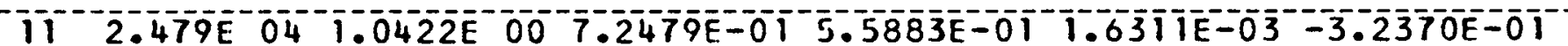
$129.119 E$ E 03 1.1023E $007.9616 \mathrm{E}-01$ 5.7339E-01 $1.5965 \mathrm{E}-03-3.5333 \mathrm{E}-01$

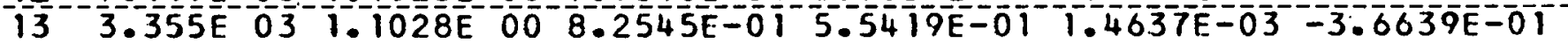
$141.234 E-03 \quad 1.0656 E$ E $008.3106 E-01.5 .1290 E-01,1.2682 E-03-3.6899 E-01$ $15-4.540 \mathrm{E} 021.1404 \mathrm{E} 00-3265 \mathrm{E}-015.8720 \mathrm{E}-0101.7521 \mathrm{E}-02 \overline{2}-3.695 \mathrm{TE}=0 \mathrm{~T}$

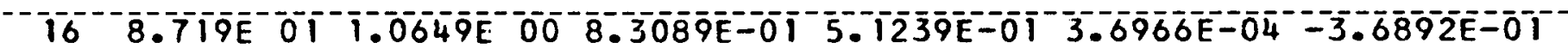
$17 \quad 3.545 \mathrm{E} 01,1.0652 \mathrm{E}-00 \quad 8.3074 \mathrm{E}-015.1276 \mathrm{E}-015.6452 \mathrm{E}-04-3.6885 \mathrm{E}-01$ $18-1.304 \mathrm{E} 01-1.0660 \mathrm{E}-008.3115 \mathrm{E}-01-5.1326 \mathrm{E}-01-9.1552 \mathrm{E}=04-3.6903 \mathrm{E}=0 \mathrm{~T}$

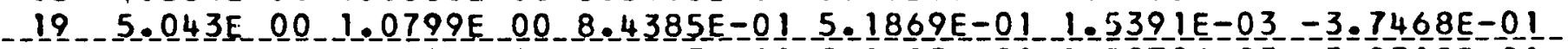
$201.445 \mathrm{E} 001.1001 \mathrm{E} 008.6287 \mathrm{E}-015.2623 \mathrm{E}-012.5979 \mathrm{E}-03-3.8312 \mathrm{E}-01$

$210.826 \mathrm{E}-019.0935 \mathrm{E} 007.9568 \mathrm{E}-015.6795 \mathrm{E}-014.1318 \mathrm{E}-03-3.5096 \mathrm{E}-0 \mathrm{1}$

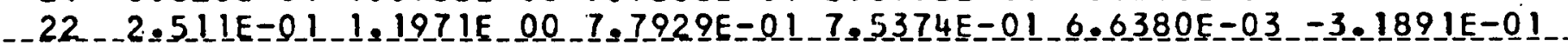
$23-9.237 E-021.4812 \mathrm{E}-007.5543 \mathrm{E}-011.0519 \mathrm{E} 001.1408 \mathrm{E}-02-2.6269 \mathrm{E}-01$

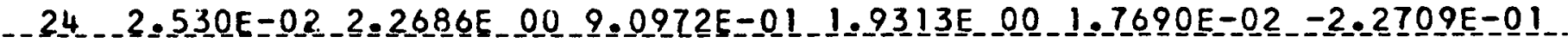

$45003.023 E=01$ 1. 3380E $00.8=7485 E=01-7.7630 E=01-7.1271 E-03-3.7501 E-01$

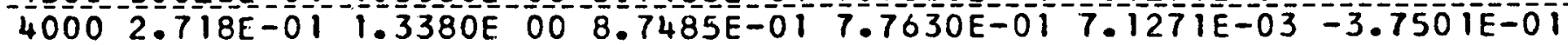

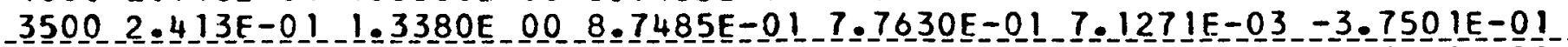
$30002.109 \mathrm{E}-0 \mathrm{~T} 1.3380 \mathrm{E}-008.7485 \mathrm{E}=0 \mathrm{~T} 7.7630 \mathrm{E}-01-7.1271 \mathrm{E}-03-3.750 \mathrm{TE}-01$

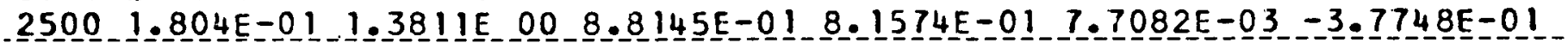

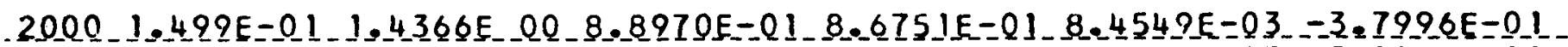
$15001.194 \mathrm{E}-011.5118 \mathrm{E} 008.9837 \mathrm{E}-019.3896 \mathrm{E}-019.4719 \mathrm{E}-0.3-3.8243 \mathrm{E}-01$

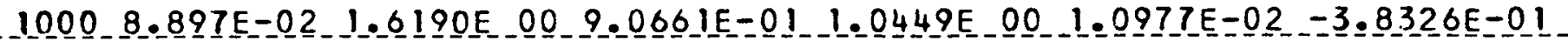

$5005.850 \mathrm{E}-02$ 1.79.46E $009.1154 \mathrm{E}-011.2243 \mathrm{E} 001.3536 \mathrm{E}-02-3.8077 \mathrm{E}-01$

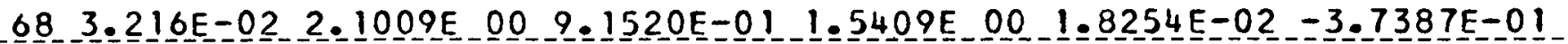


$-4=\mathrm{H}-1=2 \mathrm{R}$

THERMAL TRANSFER MATRICES

TEMPERATURE

DEG. F.

-4. 50.00E- 0.3

o.
SIGMA ABSORPIION
NU SIGMA FISSION. TRANSPQR I 0. 0. ㅇ.

\section{N1 N2}

SIGMA S INIIN2)

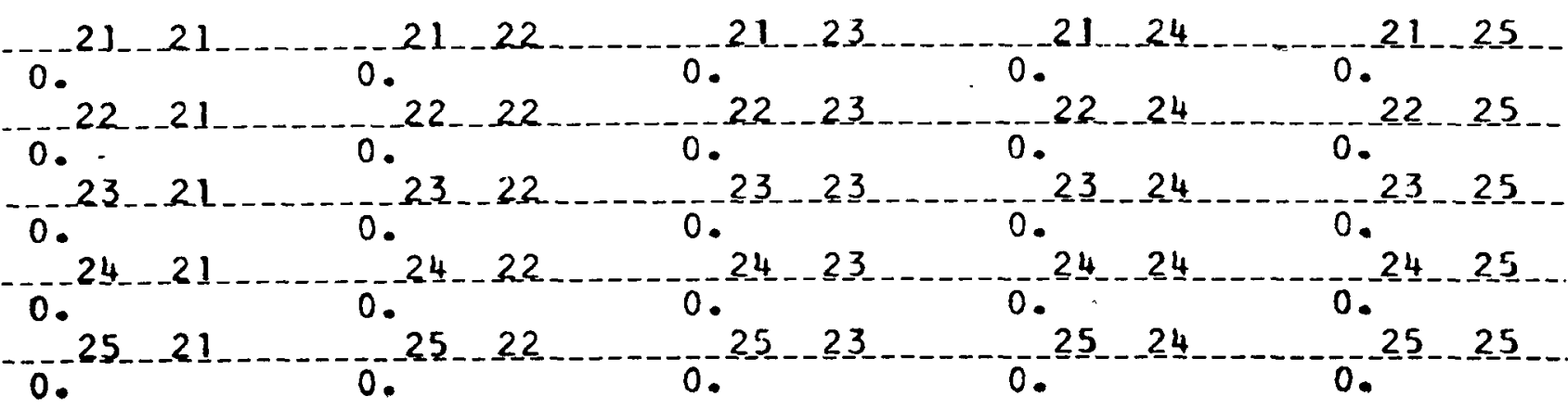

SEMPERATURE
SIGMA

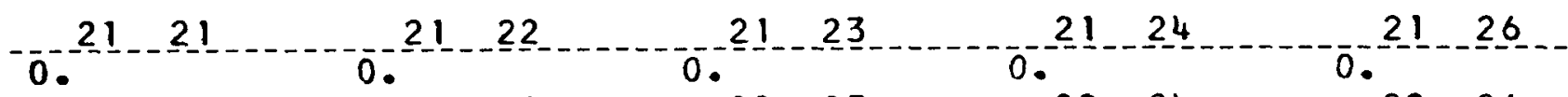
0 0. 0.-2ㄴ.-ㄴ. 26

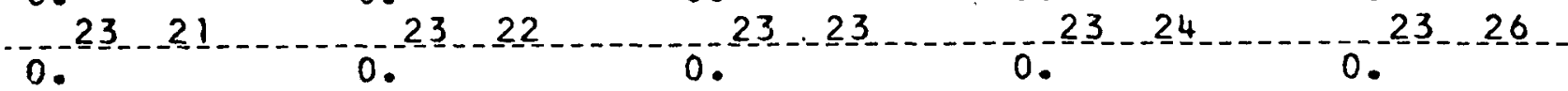
02421

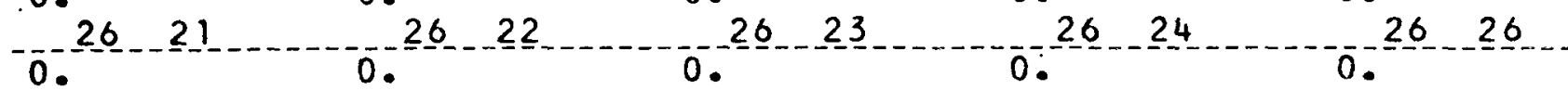


$4-H \quad 1-Z R$

_.IHERMAL_IRANSEER_MAIRICES.

IEMPERAIURE

SIGMA

SIGMA.

DEG. F.

SCATIER

TRANSPORT

SJGMA

ABSORPIION

NQ S_IGMA . .

$3.5000 \mathrm{E}^{2} \mathrm{O}$.

0.

0 .

0.

\section{$\operatorname{SIGMA} S\left(\frac{N}{-1}-\frac{N 2}{1 / N 2}\right)$}

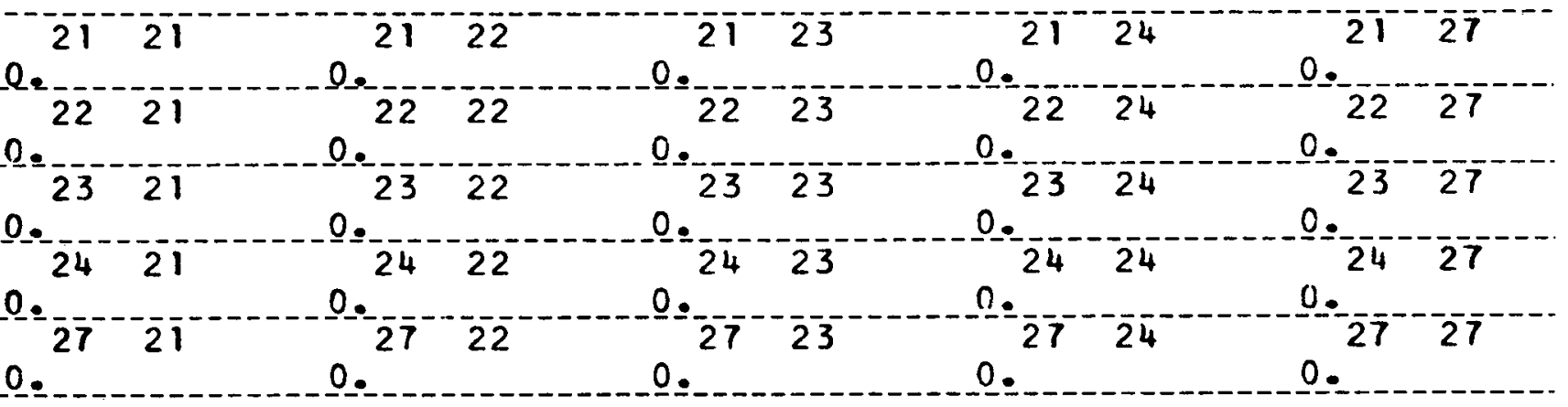

IEMPERAIURE

DEG. $F$.
SIGMA

SCATTER
SIGMA

TRANSPORT
SISMA

ABSORPTION
NU SIIGMA

FISSION

$3.0000 \mathrm{E} 03^{-1-10}$

0.

0.

0.:

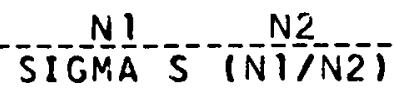

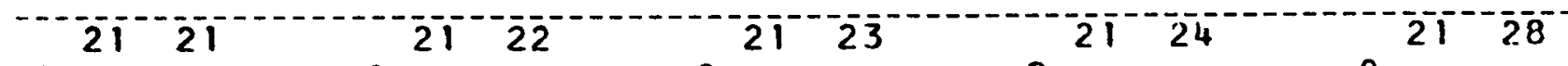

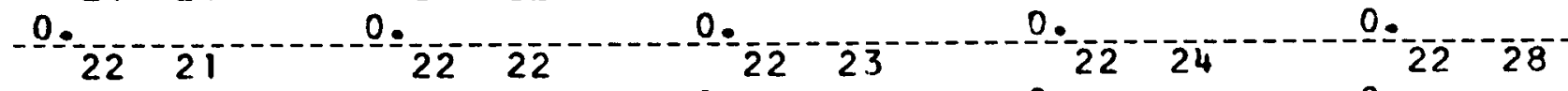

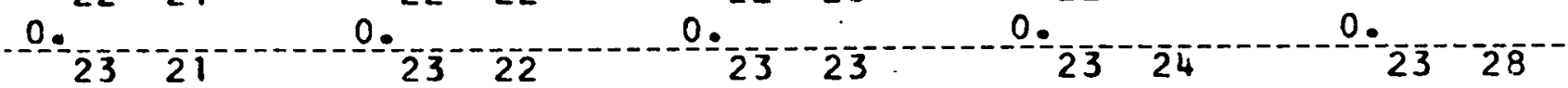

0.242100 .242424

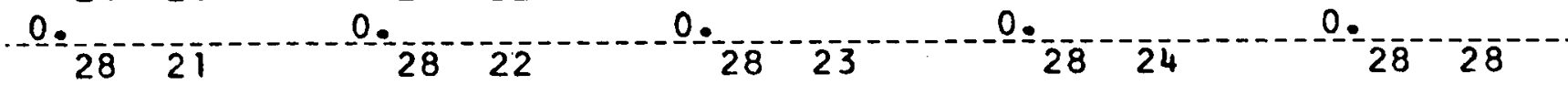

… 
$-4=-H+1=2 R$.

THERNAL TRANSFER MATRICES

TEMPERATURE
SIGMA $\begin{gathered}\text { SIGMA } \\ \text { SIGMA }\end{gathered}$ NU SIGMA

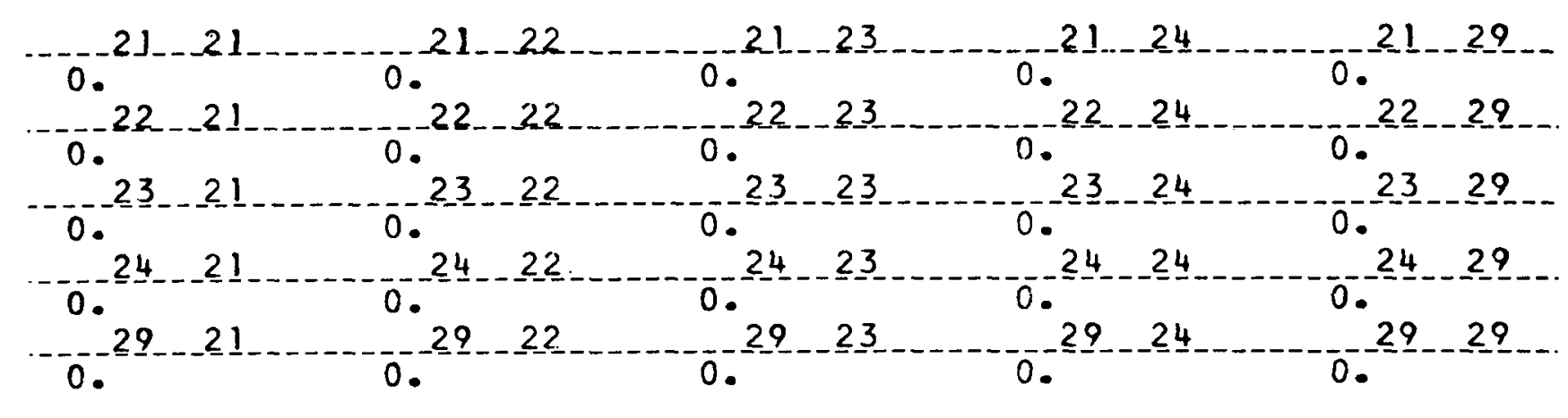

TEMPERATURE
SIGMA
$2.00000_{0} 03$. 0 .

SIGMA S $(N 1 / N 2)$

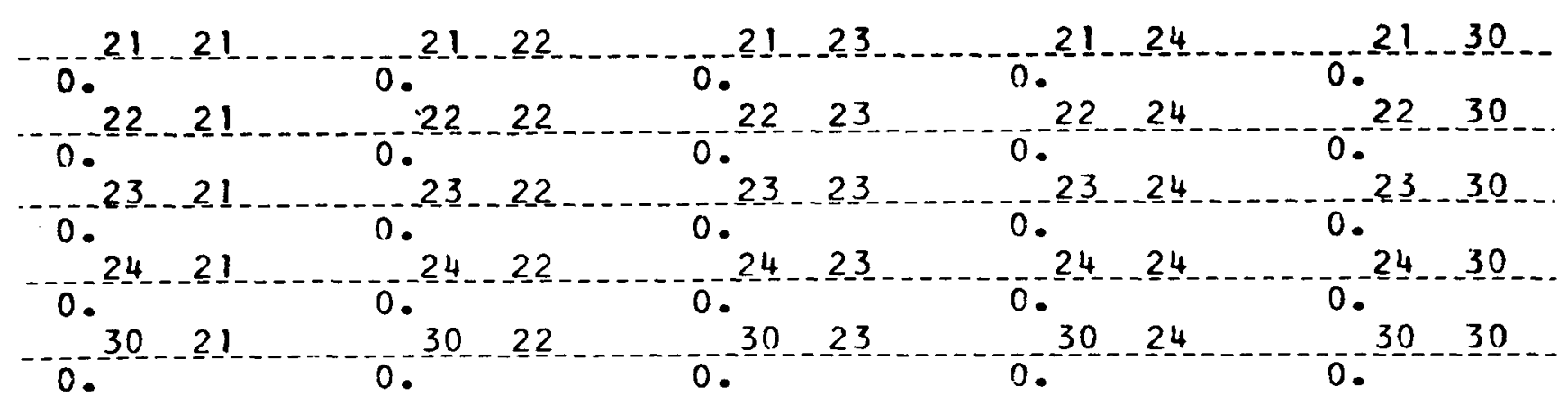


4-H I-ZR

-_LHERMAL_IRANSFER MAIRICES.

- IEMPERAIURE

SIGMA

SIGMA

SIISMA TRANSPORT

ABSORPIION

NQ SIIGMA

DEG. F. SCATTER

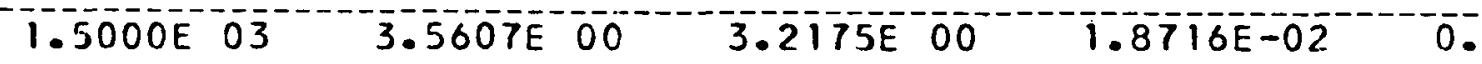

FISSION

SIGMA S $\left.(N) \frac{N 2}{N}-\frac{1}{N} 2\right)$
$2 i-1$
21
$2 \overline{1}^{--} \overline{2} \overline{3}$
$2 i^{24}$
2人一行

$-5=9558 E=01$

3. $8373 E=0$

$1=1345 \mathrm{E}=01$

1. $93375 \mathrm{E}=02$

$1.2700 E-02$

-6. $6784 E=02$

$23^{21}$

8.22 248E-0 1

$2.76 \frac{7}{23}-\frac{E}{23}=01$

5.9788 E $=02$

3. $3448 E-02$

1.94 $15 \mathrm{E}=02$

$3.4082 E-01$

2421

2422

$1=\frac{1}{24} \frac{3}{2} \mathrm{OE} \frac{\mathrm{E}}{2} \mathrm{O}$

1. $-\frac{09}{24} 33 E-01$

$3728 E-02$

$-2.5679 E=02$

4.-6604E-01

$3.96692=01$

$1.3346 E$ OOO

$31-\frac{1}{4}$ $24-\frac{1}{31}$

$312 i$

3122

$312 \overline{3}$

$5.2964 \mathrm{E}-01$

$3.1503 \frac{3 E}{3}=01$

$3.4904 \mathrm{E}=02$

5.3059E-01

4.035IE-01

2. $0621 E 00$

TEMPERA IURE

DEG. F.

SI GMA

SIGMA

SCATTER

TRANSPORT

SIGMA

NU SIGNA

$1.0000 \overline{0} 0 \overline{3}$

$3.2770 E-00$

$2.9903 E^{-} 00$

ABSORPTION

FISSION

\section{SIGMA S (NITNE)}

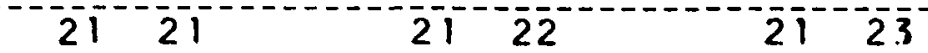

-5.8307E-0!

$3.7375 E=01$

$1.2 \frac{259}{2} 2-\frac{6}{2}-0$

$1.8859 \bar{E}-02$

0.

22

$-3 \cdot \frac{30}{2} \frac{62 E}{2}=0 ?$

8.0490E-01

$23 \frac{1}{22}$

2. $1254 E=01$

2421

$24 \quad 22$

$2.8203[-01$

32 - 21

3222

I. $4323 E=03$

$2 \cdot \frac{905}{23}-\frac{2}{23}-01$

$1.2170 \mathrm{E}-00$

2423

$3.2 \frac{2}{3} 2 \frac{2}{2}-0$.

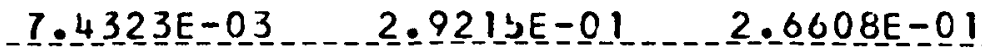

2124

$2-1 \frac{1}{2} \geq \frac{2}{2}-\frac{2}{24}-02$

$0 . \frac{5}{2} \frac{4}{3}-\frac{9 E}{2}=02$

$1.0878 E=0$

$1.4918 E-00$

4.8422E-01
2132

$1.446 \frac{5 E}{22}-02$.

3. $\frac{6}{2} 317 E-02$

2. 3 I $67 E-02$ 2432

3. $\frac{1}{3} \frac{2}{2}-\frac{8 E}{3}=$

$2.2271 \mathrm{E}$ 
$4=H-1=2 R$

THERMAL TRANSFER MATRICES

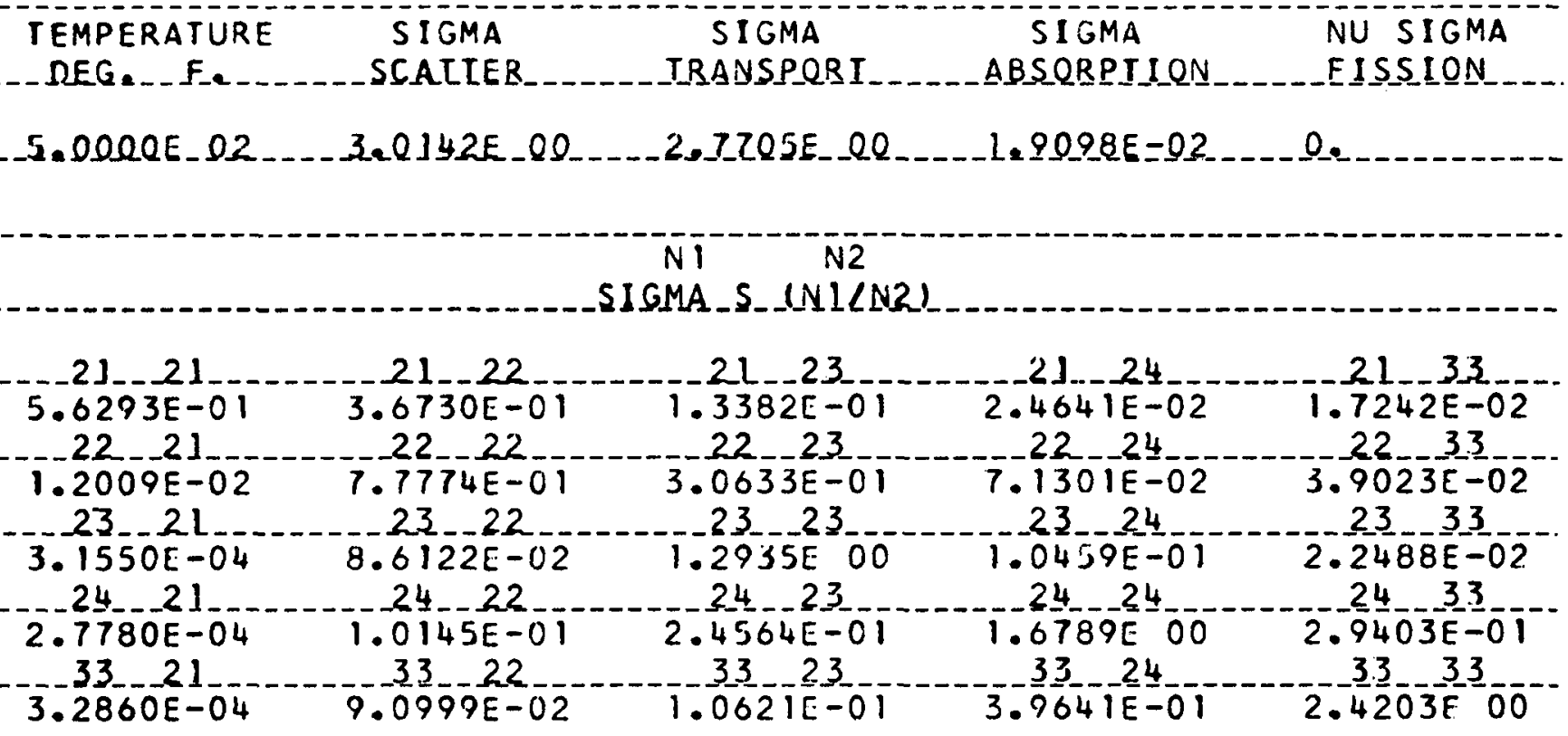

SEMPERATURE
SIGMA

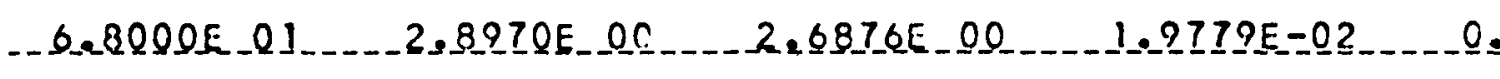

Nin

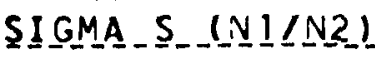

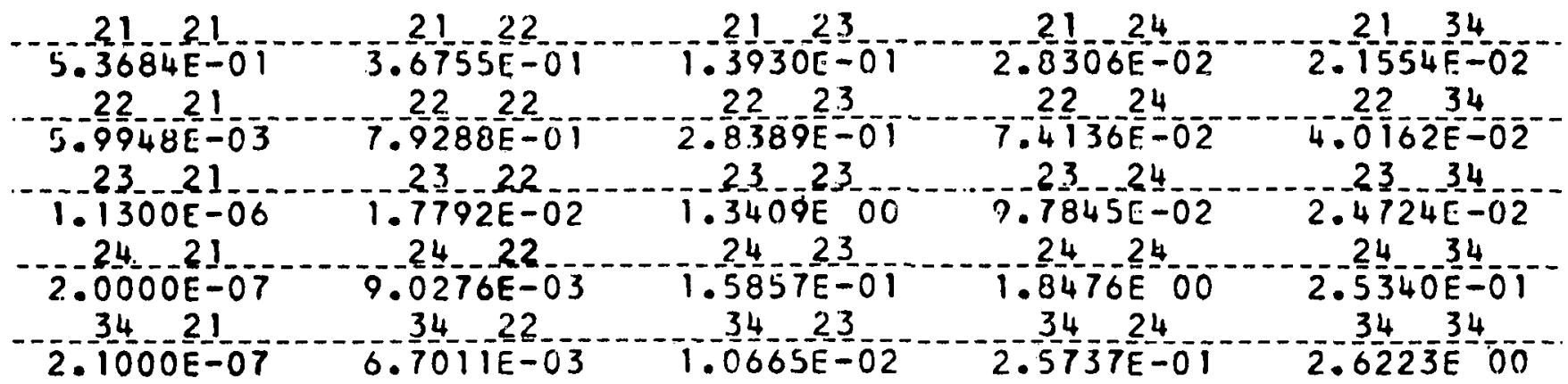


$4-H-1=2 R$

TRANSFERS FROM GROUPS I THROUGH 20 TO GROUPS 1 THROUGH 25

$\operatorname{SIGMA}$ SO (N) $1 / \mathrm{N} 2)$

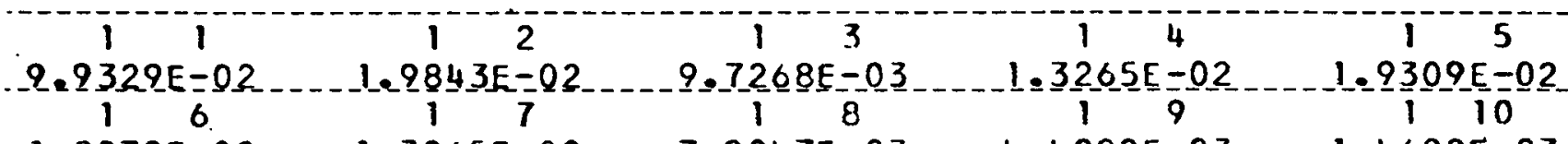
$-1.8878 E-02 \ldots-1=3065 E=02 \quad 7.2047 E-03 \quad-4.4022 E-03$

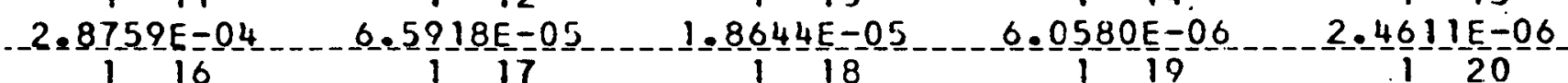

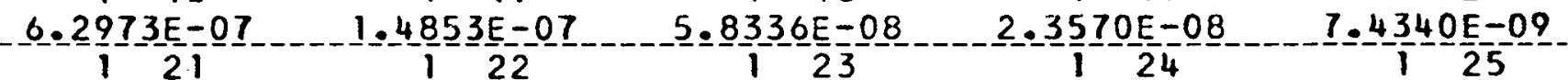

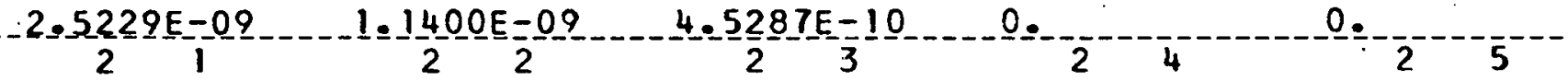

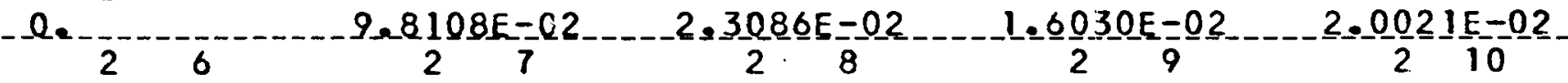

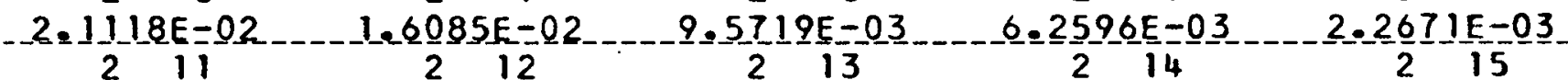
$-4.9496 E-04-1.2684 E=04-3.8810 E=05 \quad 1.3151 E-05$

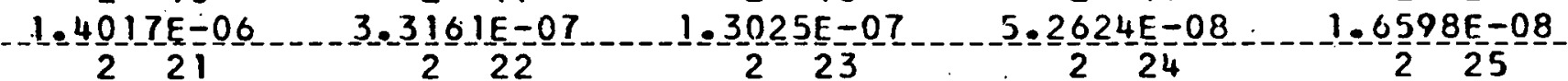
$5.6 \frac{32}{3} 8 \mathrm{E}=09$ -0.

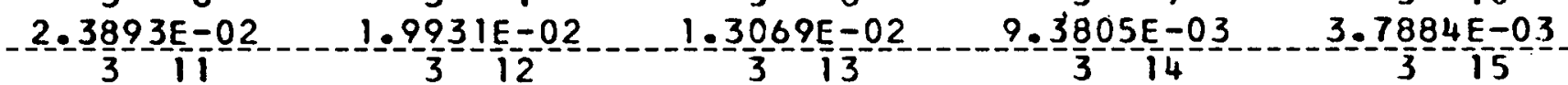

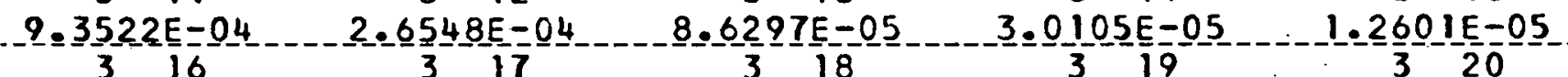

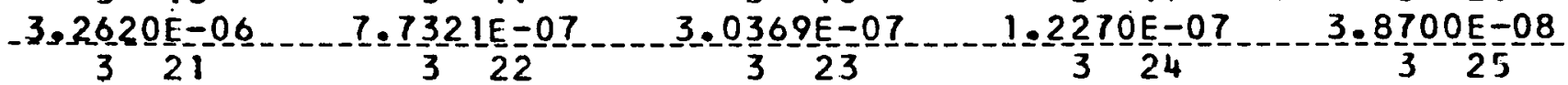

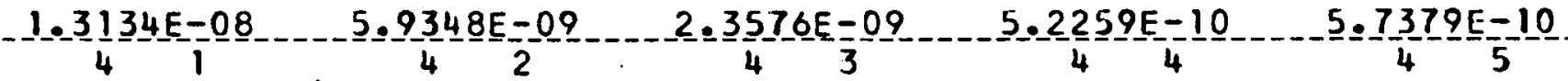

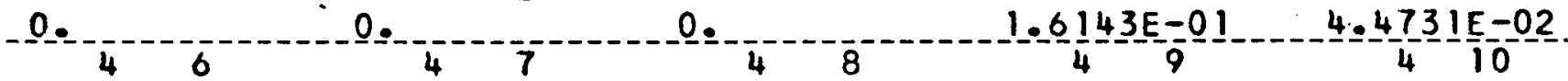
$-2.9045 E-02 \quad 2.2814 E-02 \quad 1.5680 E-02 \quad 1.2346 E-02 \quad 5.7516 E-03$

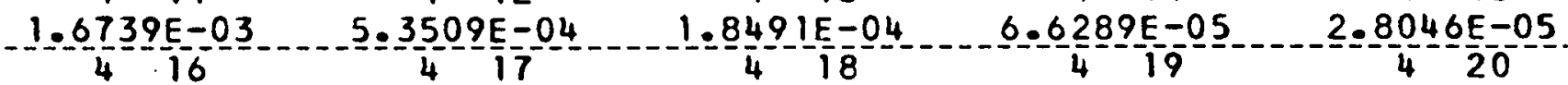

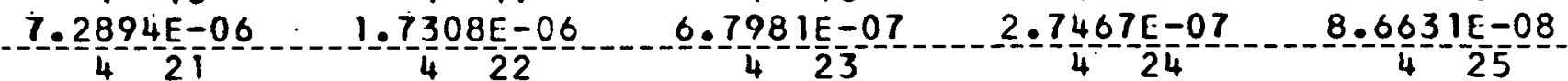
. 2. $94000 E=08 \ldots$ 
$4-H \quad 1-2 R$

N1 N2

SIGMA_SO (NIIN2)

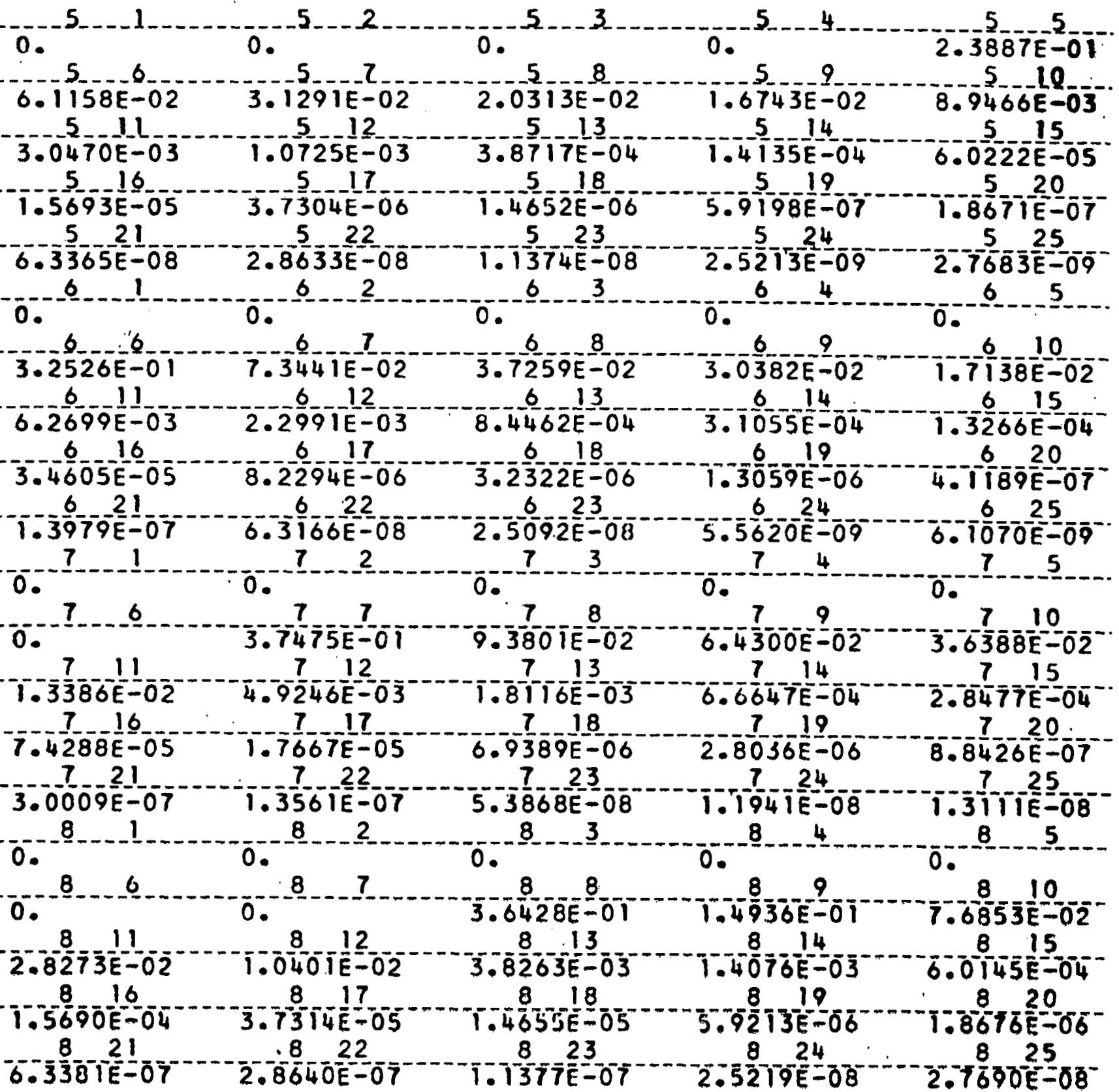


$--4=H-\ldots 1=2 R$

SIGMA $-\frac{N 1}{20}(N 1 / N 2)$

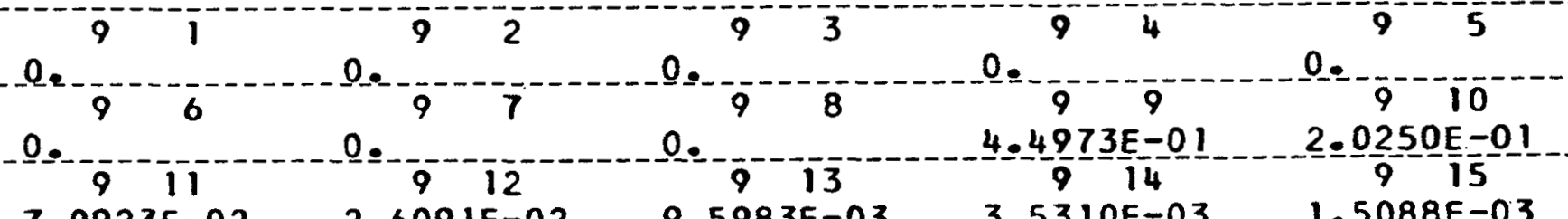

_7. $0923 \mathrm{E}=02 \ldots 2.6091 \mathrm{E}-02$

3. $9 \frac{359}{9} \frac{E}{2}-04$

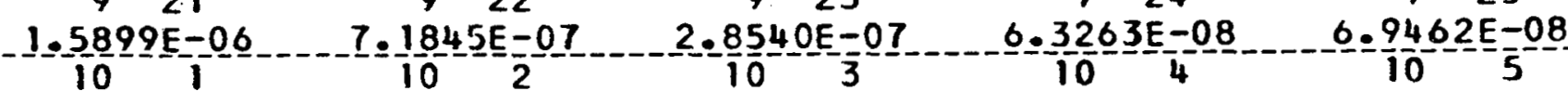

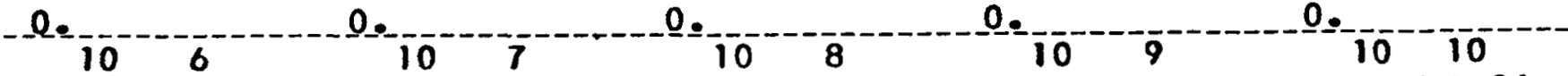

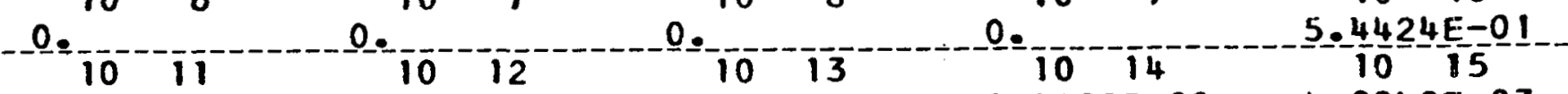

$2.4214 E-01$

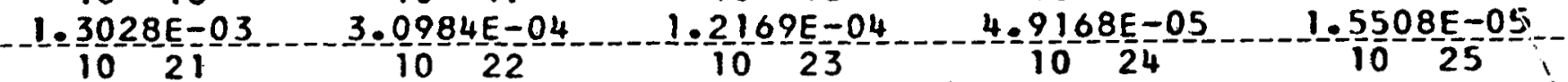

5. $26292 \mathrm{E}-06$

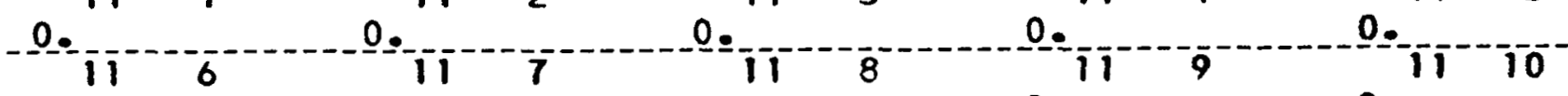

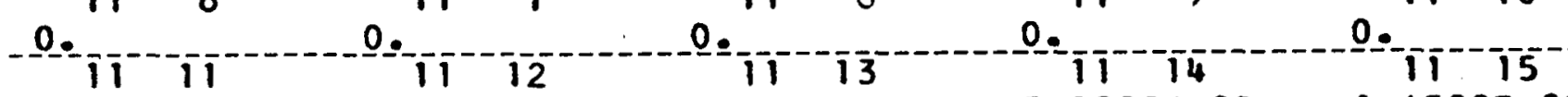

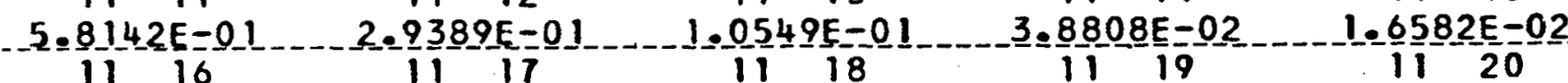

$4.3258 \mathrm{E}-03 \quad 1.0287 \mathrm{E}-03$ _ $4.0405 \mathrm{E}-04$
1121
1122
1123
1124
1125

$1.7474 E-05-7.8962 E=06-3=1367 E=06-6.9529 E-07$

$0.12-12-12-12$

$0.12110^{0} 12^{12}$

…

$1.2932 E-02 \quad 3.0755 E-03$

$12-2 i$

$12=22$

$12-\frac{2}{3}$

$7.63426=07$

0 -

125

$0=-12-10$

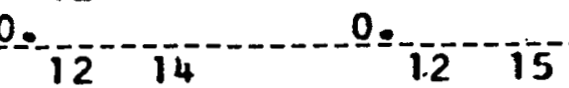

12

$1602 E-01$

$\begin{array}{ccc}1221 & 1222 & 12 \\ 5.2241 E-05 & 2.3607 E-05 & 9.3775 E=0\end{array}$

$4.8805 E-04$

$1.5393 E-04$

12

125

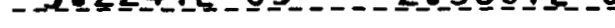

$2.0787 E=06$

2.2823E $=06$ 
$4-H \quad 1-2 R$

N1 N2

SIGMA_SO (N1/N2)

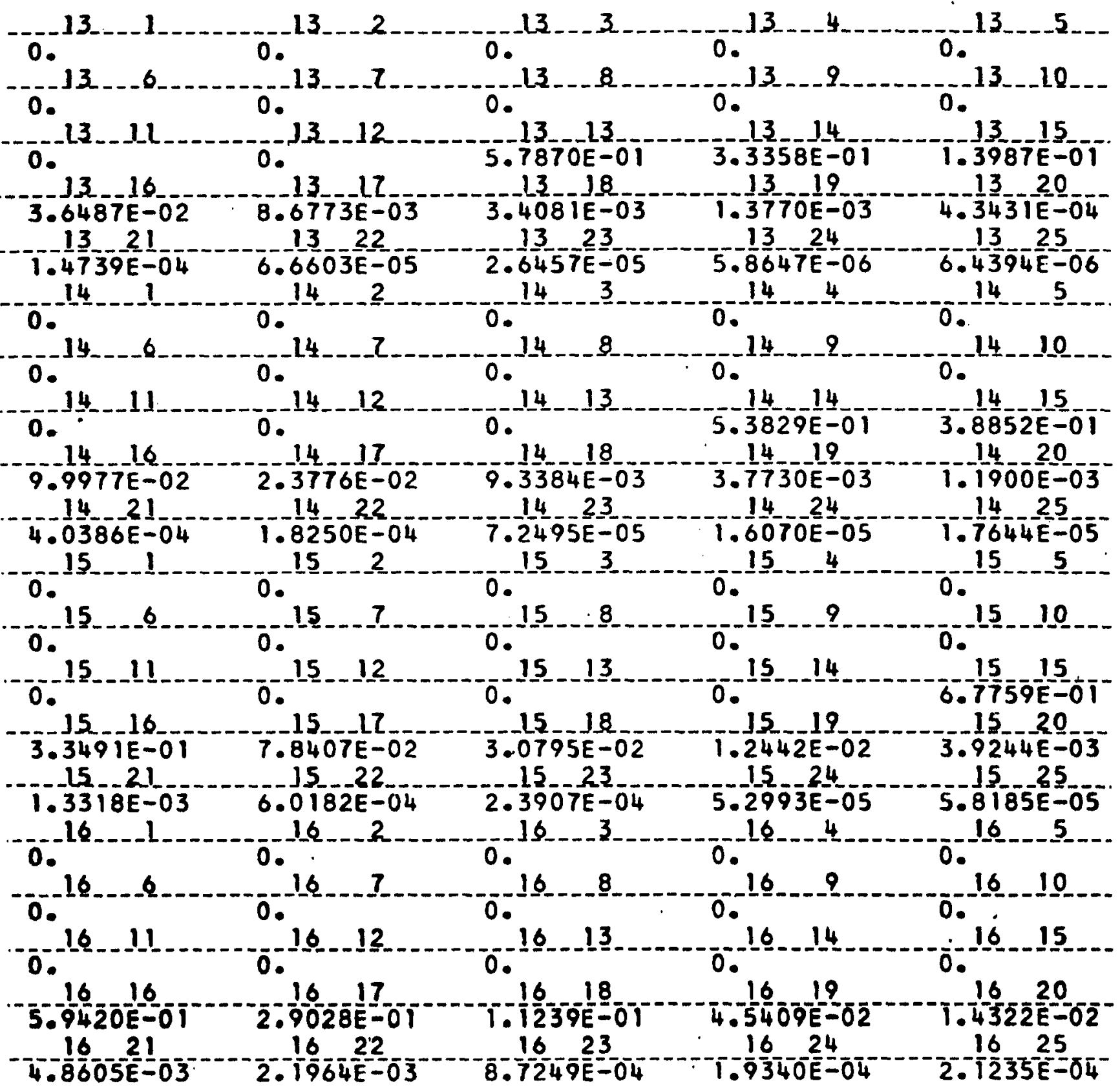


$--4=H-1=2 R$.

SIGMA SO (NI/N2)

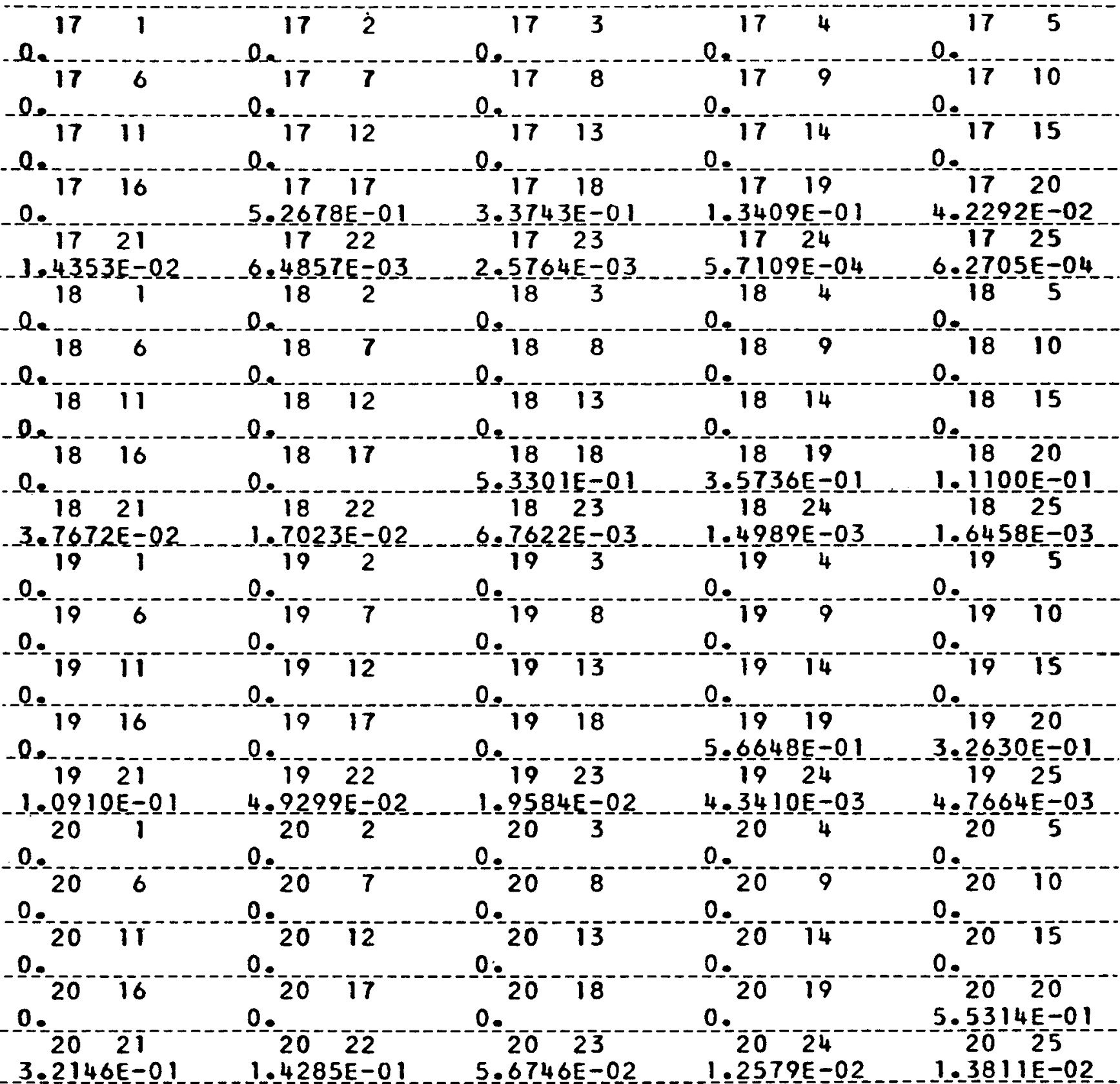




$$
-4=H-1=2 R .
$$

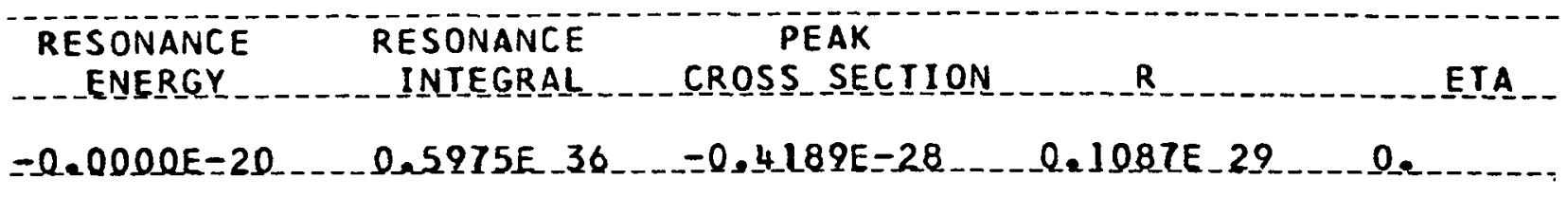


$4-H \quad 1-2 R$

TRANSFERS FROM GROUPS I THROUGH 34 TO GROUPS 1 THROUGH 11

N1 N2

SIGMA N, G INIIN2)

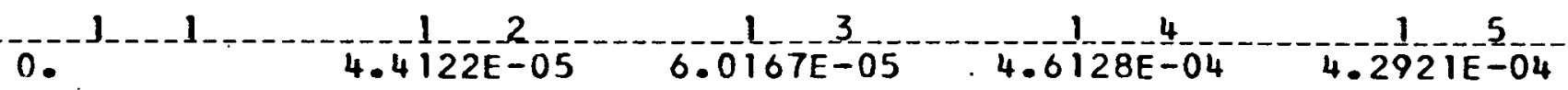

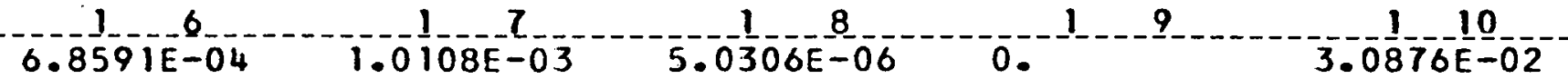

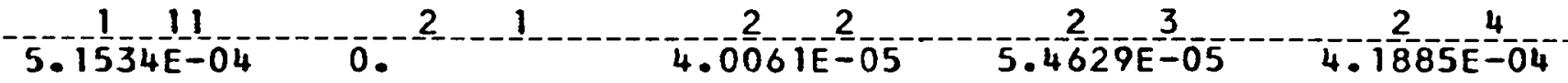

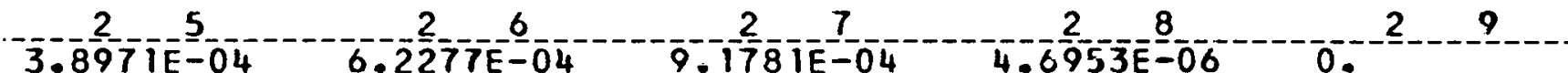

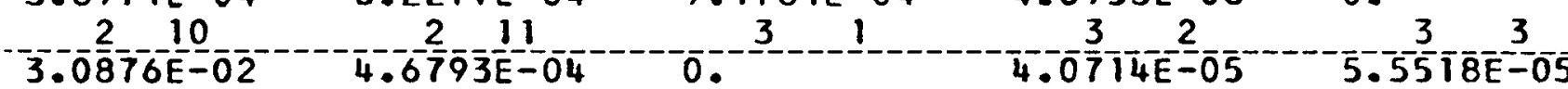

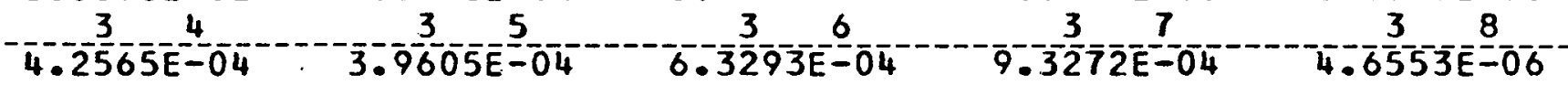

0.

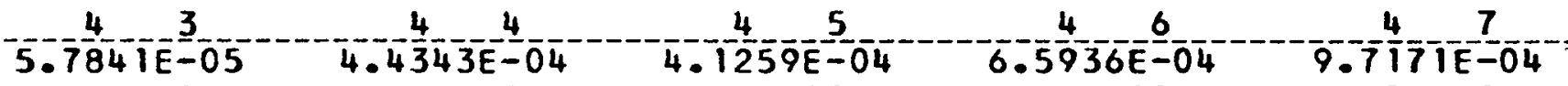

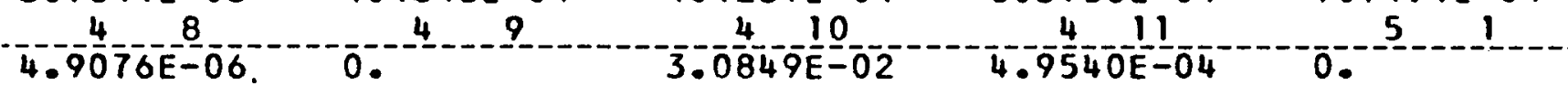

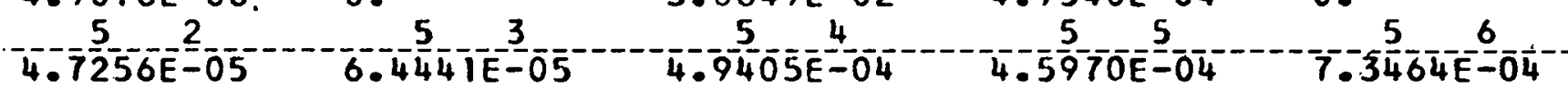

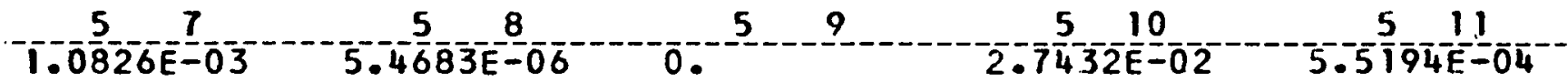

0.

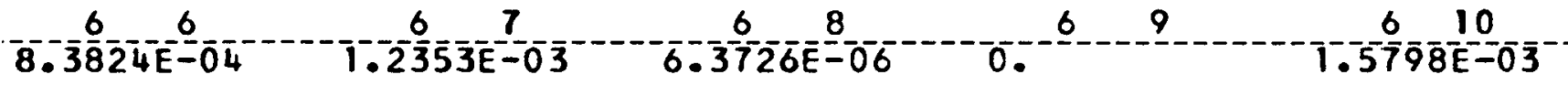

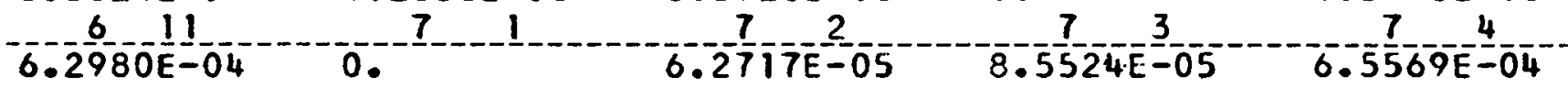

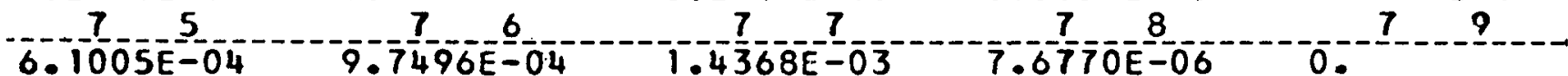

0.

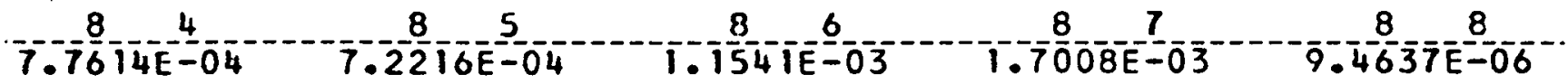

0.

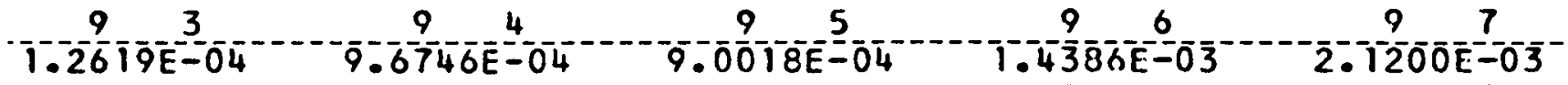

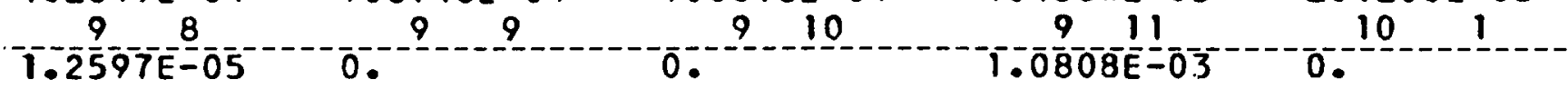


$-\underline{4}=\underline{H}--1=\underline{Z}-\underline{R}$

SIGMA N,G $(N I J N 2)$

$10^{2} 10^{3} 10^{-3} 0^{-10}$

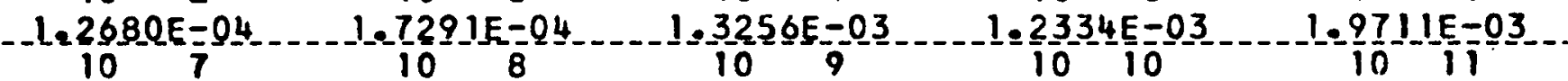

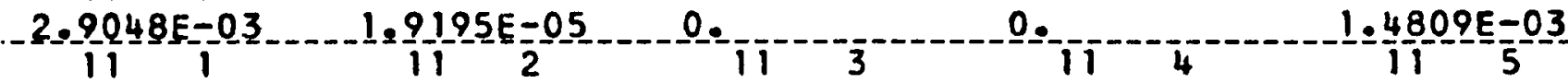

$-02711.7844 E-04 \quad 2.4333 E-04 \quad 1.8656 E-03 \quad 1.7358 E-03$

$-2.7740 E-03 \quad 4.0880 E-03 \quad 3.1337 E-05 \quad 0.12$

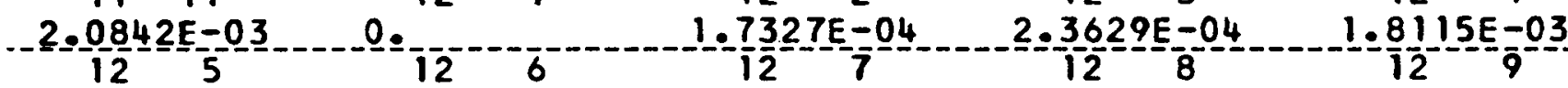

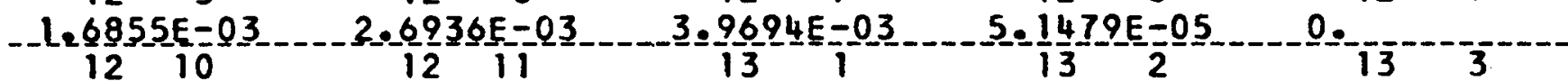

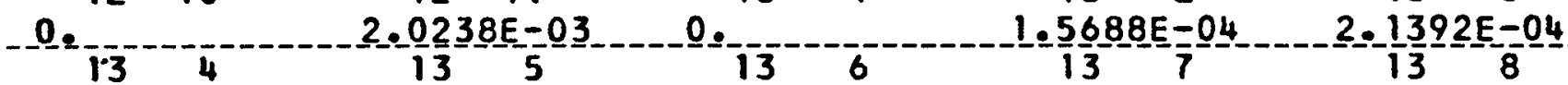

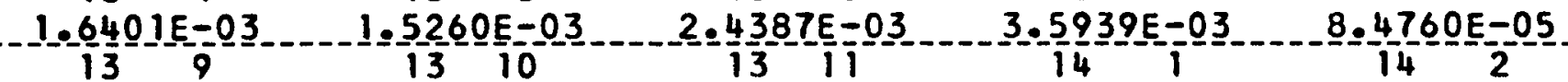

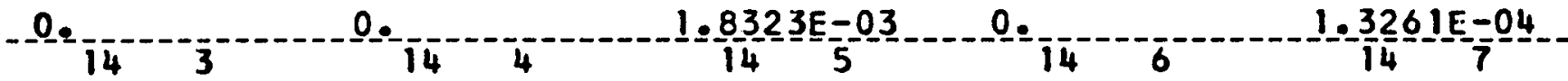

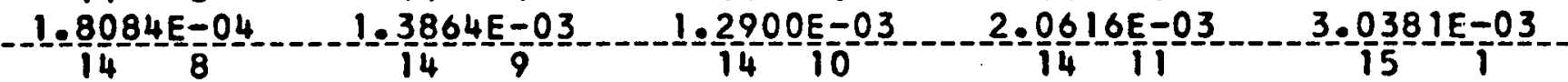

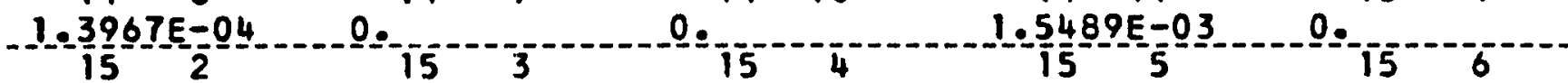

‥9150E-03

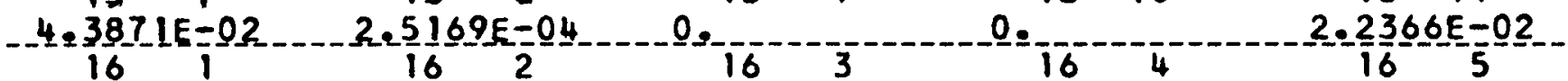

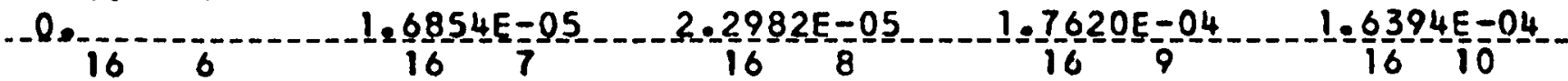

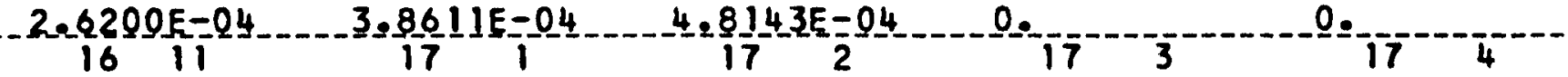

-1.9685E-04

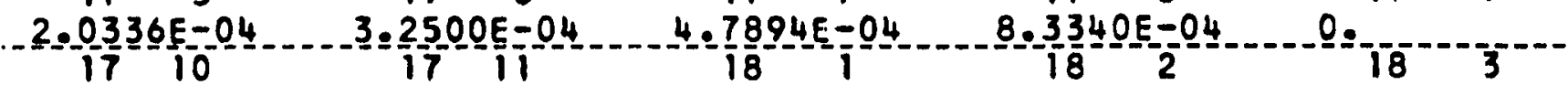

.0.

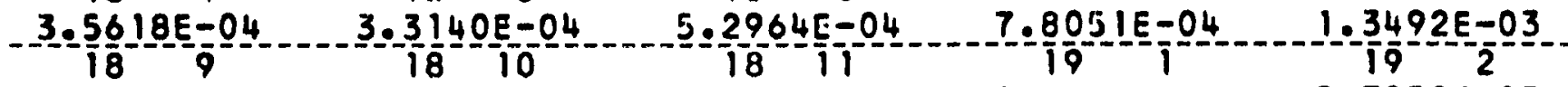

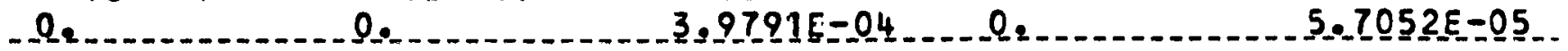




\section{N1 N2 \\ SIGMA_N,G_ (NIIN2)}

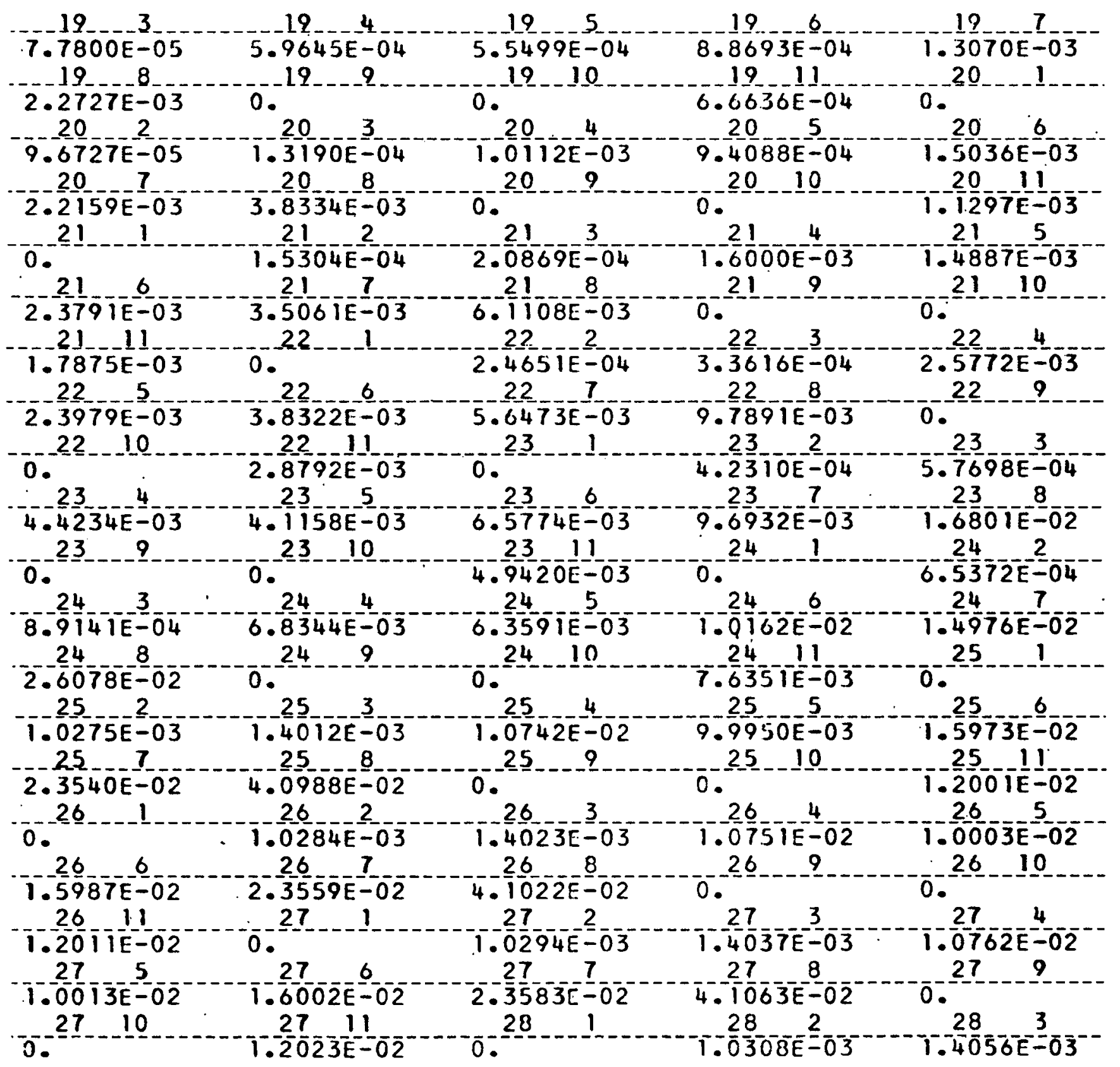


- $4-H$

\section{SIGMA N,G (NIIN2)}

$284285288^{2}-287^{8}$

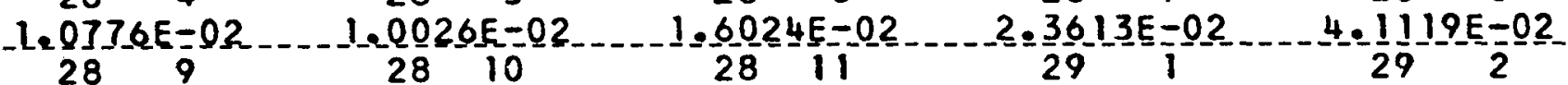

0.

Q.

1.2039E-02

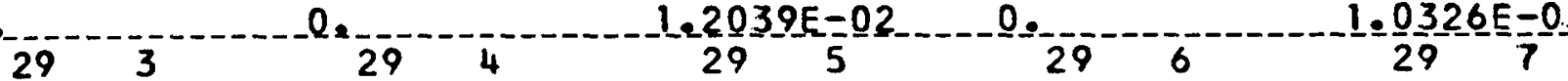

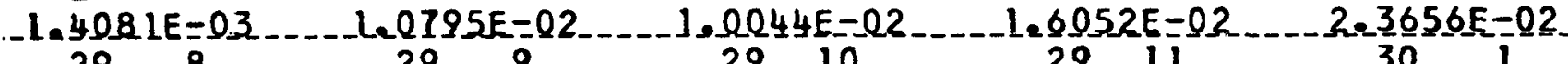
$\begin{array}{llllllll}29 & 8 & 29 & 9 & 29 & 10 & 29 & 11\end{array}$

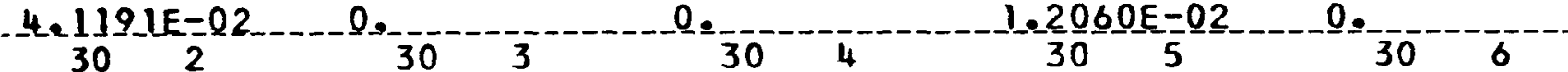

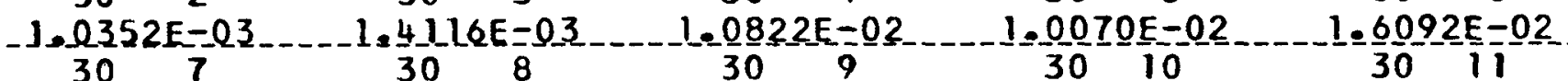

2. 3715E=0.2 - 4. 2296E $=02$

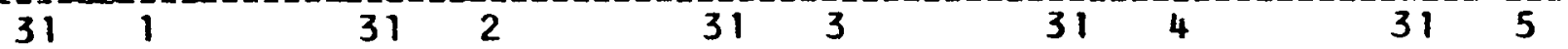

Q. $\begin{array}{llllllll}31 & 6 & 31 & 7 & 31 & 8 & 31 & 9\end{array} 310$

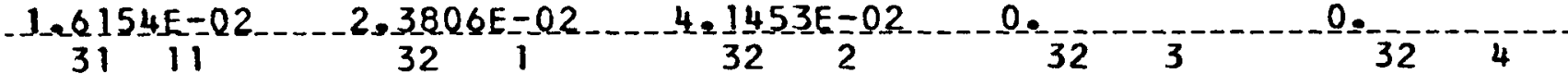

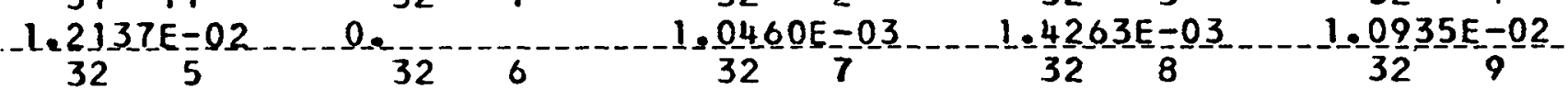

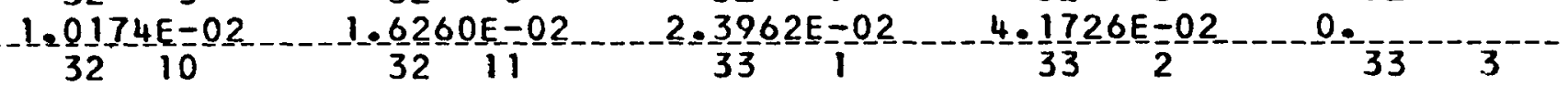

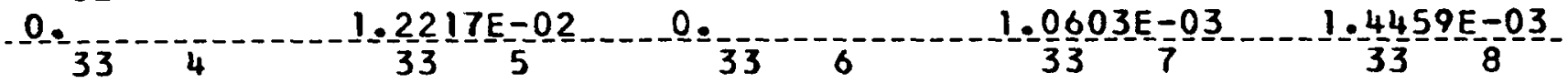

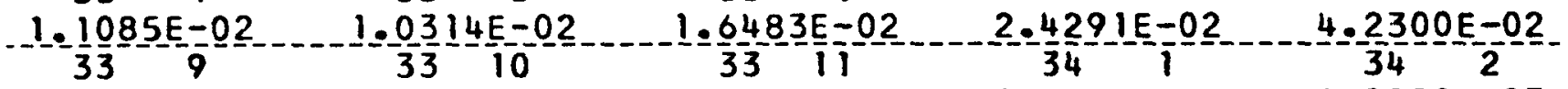

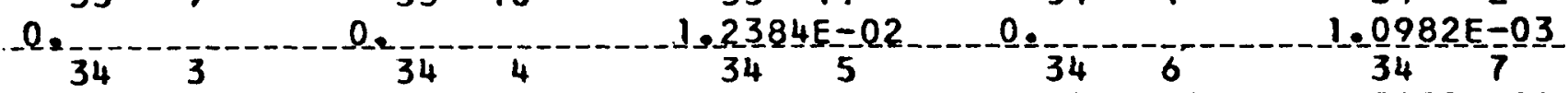

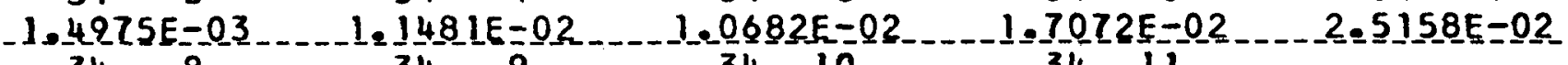
$\begin{array}{lllllll}34 & 8 & 34 & 9 & 34 & 10 & 34\end{array}$

4_.3807E-02 
AIRCRAFT NUCLEAR PROPULSION DEPARTMENT GENERAL (9g:) ELETRIC 